\title{
$65^{\text {th }}$ International Congress and Annual Meeting of the Society for Medicinal Plant and Natural Product Research (GA 2017)
}

Date/Venue:

3.-7. September 2017, Basel, Switzerland
Chair:

Prof. Dr. Matthias Hamburger

Prof. Dr. Veronika Butterweck, Basel

\section{Editorial}

\section{Abstracts of the $65^{\text {th }}$ Annual Meeting of the Society for Medicinal Plant and Natural Product Research (GA)}

From September 3 rd to 6th 2017 over 600 participants from more than 30 countries gathered in Basel, Switzerland, for the $65^{\text {th }}$ Annual Meeting of the Society for Medicinal Plant and Natural Product Research.

The main scientific topics of the conference were

- Bioactive Natural Products

- Dermatology and Dermatocosmetics

- Functional Foods

- Biosynthesis and Biotechnology

- Sustainable Use of Natural Products

- Natural Product Formulation and Nanotechnolology

- Analytical Studies, Natural Product Chemistry \& Computational approaches

- Quality control methods for herbal extracts, starting materials and natural products

- Phytopharmacology/Extract Pharmacology

- Animal health care and Veterinary phytomedicine

The scientific programme of the Main Conference included 5 plenary lectures by invited speakers, 4 award lectures, 96 contributed short lectures and 545 posters. The short lectures were held in three parallel sessions, and the two poster sessions on Monday and Tuesday provided a forum for lively scientific discussions.

On Sunday, $3^{\text {rd }}$ September, three pre-congress events took place. The Young Researchers Workshop offered a platform for young scientists to present their latest findings, and the Regulatory Affairs Workshop served as a forum for industry to update on the latest trends in regulation of phytomedicines and related areas. A Pre-congress Symposium on Veterinary Phytotherapy was organized by the Swiss Medical Society for Phytotherapy (SMGP), in cooperation with the GA Networking Group on Animal Healthcare and Veterinary Medicine. On Tuesday, $5^{\text {th }}$ September, the session 'Herbals for health worldwide - WHO strategy and Herbal Regulation for Meeting People's Need' provided an excellent forum for all participants to discuss the needs for international educational, regulatory and research activities.

We would like to take the opportunity to express our sincere thanks and appreciation to all participants of the conference, and to the sponsors and exhibitors. They all contributed significantly to make this meeting a success. We also would like to thank the members of the organizing committee for all the efforts during the preparation phase of the congress, and to our staff members and students who volunteered to assist us in various organizational tasks during the conference.

Last, but not least, we would like to thank Thieme Publishers for their generous support, and for publishing the conference abstracts in Planta Medica International Open.

Prof. Dr. Matthias Hamburger \& Prof. Dr. Veronika Butterweck

On behalf of the Organizing Committee

\section{Veterinary Medicine Satellite Symposium} Basel, Sunday, September 3, 2017

Abstracts of the Veterinary Medicine Satellite Symposium - in order of appearance

\section{Su-Veterinary Medicine Satellite Symposium-KNL-01 Recent challenges in veterinary pharmacotherapy - could medicinal plants be an option?}

Authors Naegeli $\mathrm{H}^{1}$, Mevissen $\mathrm{M}^{2}$, Walkenhorst $\mathrm{M}^{3}$, Ayrle $\mathrm{H}^{3}$, Kupper $\rfloor^{1}$ Institute 1 Institute of Veterinary Pharmacology and Toxicology, University of Zurich, Zurich, Switzerland; 2 Division of Veterinary Pharmacology and Toxicology, University of Berne, Berne, Switzerland; 3 Department of Livestock Sciences, Research Institute of Organic Agriculture (FiBL), Frick, Switzerland DOI 10.1055/s-0037-1608012

One major challenge in veterinary pharmacotherapy is the currently still excessive use of antimicrobials. European countries banned the misuse of antimicrobials as growth promoters, but it is recognized that their remaining widespread application for the prevention and treatment of diseases in farm as well as companion animals is a main driver of antibiotic resistance. A primary concern is that the antimicrobial use in veterinary medicine contributes to reduce efficacies in humans. Cases of transmission of acquired resistances from animals to humans have been described, although the importance and extent of this resistance spread has yet to be quantified. Another concern is the increasing antibiotic resistance detected in zoonotic bacteria, but the potential for transmissions extends to commensals and opportunistic bacteria. These issues are further aggravated by the fact that many antimicrobials used in veterinary medicine are critically important for the management of life-threatening infections in humans. Even in the presence of a bacterial infection, the use of antimicrobials is not always justified. Stewardship programs advocate that the treatment of infections should not necessarily involve antimicrobials in diseases that are self-limiting or can be controlled with symptomatic therapy. In these cases, the application of medicinal plants with anti-inflammatory, analgesic, antiseptic, antimicrobial, antidiarrheal or immune-stimulatory activity may provide a valuable alternative widely accepted by animal owners. To assist veterinary practitioners in the fit-to-purpose use of medicinal plants, we launched a decision support system describing their organoleptic/pharmacologic properties and the applied preparations/ doses in domestic animal species. The system also provides botanical characteristics of medicinal plants, their composition and safety profile, falsifications, legal aspects for administrations to food-producing animals and, if applicable, indications on their doping relevance. This decision support system can be accessed under "www.phytoarznei.ch" taking advantage of various userfriendly search functions. The underlying database is being expanded and updated regularly. 


\section{Su-Veterinary Medicine Satellite Symposium-KNL-02 Recent challenges in veterinary pharmacotherapy - could medicinal plants be an option?}

\author{
Authors Fink-Gremmels ] \\ Institute 1 Utrecht University, IRAS, Utrecht, Netherlands \\ DOI 10.1055/s-0037-1608013
}

The use of medicinal plants has a long tradition all over the world, both in human and animal health care. Recently, the challenge to overcome the global emergence of antimicrobial resistance, has initiated numerous research activities to understand the benefits (and limitations) of herbal remedies in the treatment of infectious diseases, which can be discussed along the following lines:

1. In contrast to common antibiotics, which are generally single small molecules, plants produce complex mixtures of related substances with antimicrobial activity. These mixtures of active ingredients, impair bacterial metabolism and replication at multiple sites and hence do not easily induce the expression of specific resistance genes. This has been very well documented for many essential oils, and their different chemical classes of active ingredients.

2. In a clinical situation, bacterial infections are commonly associated with the formation of bacterial biofilms. Plants express various substances that interfere with biofilm formation, inhibiting the initial phase of quorum sensing. By this activity, herbal remedies can significantly improve and maintain the efficacy of common antibiotics in the treatment of acute and chronic infections.

3. Most herbal extracts possess not only antibacterial activity, but also antioxidant and anti-inflammatory properties, which contribute to an uneventful recovery from infections.

In the light of these mechanisms-based evidence for their beneficial effects, traditional plant remedies should be re-considered in the treatment of infectious diseases as they contribute to resistance avoidance and improve recovery and wellbeing of animals.

\section{Su-Veterinary Medicine Satellite Symposium-KNL-03 Medicinal Plants for Chronic Disease in Small Animals}

Authors Fougere $\mathrm{B}^{1}$

Institute 1 All Natural Vet Care, Sydney, Australia

DOI 10.1055/s-0037-1608014

A major contributing factor in the development of chronic diseases and age related diseases is oxidative stress associated with risk factors such as stress, environmental pollutants, diet, radiation and infection that interact with cells through the generation of reactive oxygen species. In animals an association between oxidative stress and chronic disease has been demonstrated in many conditions including: inflammatory bowel disease in dogs; degenerative myelopathy in dogs; cancer in dogs; inflammatory skin disease in dogs; immunemediated hemolytic anemia in dogs; periodontitis in dogs; cataracts in poodles; canine chronic hepatitis; canine dementia; chronic kidney disease cats and dogs; myxomatous mitral valve disease in dogs; FIV in cats; pancreatitis in small animals and hyperadrenocorticism.

Medicinal plants are composed of phytochemical constituents with varied phytochemistry and physiological actions with many such plants having significant antioxidant, anti-inflammatory, anti-neoplastic, anti-fibrotic and circulation improving properties. The antioxidant properties and other actions of herbs can be exploited in the treatment and support of common conditions. For example Crataegus sp. in chronic heart conditions; Withania somnifera in degenerative myelopathy; Salvia miltiorrhiza, Curcumin, Cordyceps sinensis, Astragalus, Rehmannia glutinosa, Bupleurum falcatum and Angelica sinensis in chronic renal disease; Coptis chinensis, Glycyrrhiza uralensis, Silybum marianum, Bupleurum chinensis, Salvia miltiorrhiza and Scutellaria baicalensis in chronic liver disease and curcumin in hyperadrenocorticism. The potential of medicinal plants, alone or in combination with conventional treatment represent pro- mising tools for the development of new treatment concepts for chronic disease of small animals.

\section{Su-Veterinary Medicine Satellite Symposium-SL-01 How to gather information from veterinary practise? The "Idialoog System" for assessing the use of Guides with information on natural products}

Authors Groot $\mathrm{M}^{1}$, Kissels $\mathrm{W}^{2}$, Feller $\mathrm{R}^{3}$

Institute 1 RIKILT Wageningen University \& Research, Wageningen, Netherlands; 2 Kissels Consulting, Bilthoven, Netherlands; 3 NewCurrent, Hilversum, Netherlands

DOI 10.1055/s-0037-1608015

Objective was to assess the use by veterinarians of the Guides for farmers to keep their animals healthy with natural products. These booklets are published by the Dutch government to help in reducing the use of antibiotics in livestock by veterinarians. A questionnaire according to the iDialo og methodology, was distributed among all approved veterinarians that take part in a quality assurance program for a specific species. This Idialoog methodology is a proven research technique that allows for a high response and delivers relevant information on the primary question for the target group. Additional information on use of natural products and the role of the veterinarian was collected. All responses (answers and opinions) are anonymised and grouped. The participants receive this as feedback via email (figure 1). Within iDiaLoog, all participants are equal and can participate at any time that suits them best. Because of its near-real-time feedback properties it is attractive for participants to join and thus ensuring a high response rate. iDiaLoog is a simple, effective and efficient manner to gather and share relevant and actual information about specific topics. This provides practitioners with insight of their peer group.

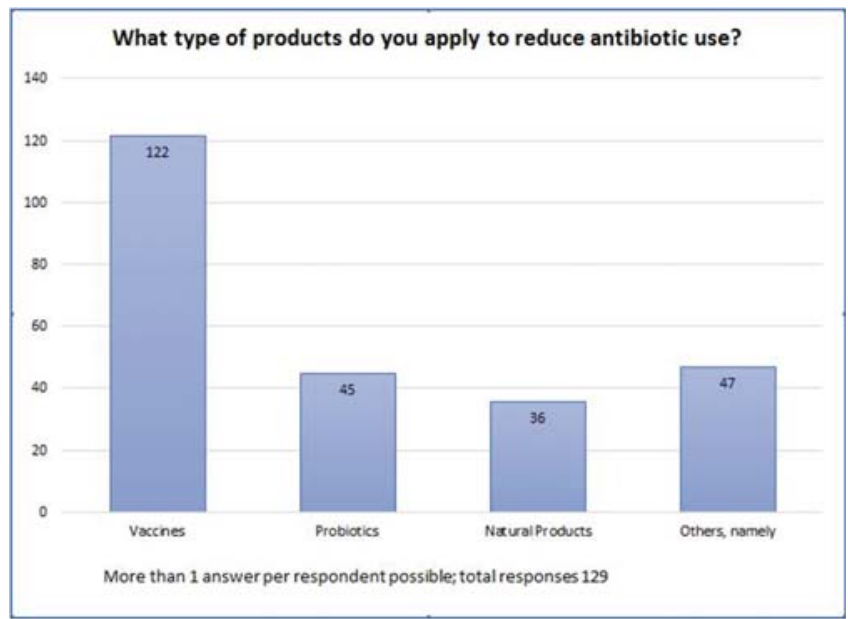

Fig. 1: example of results of the questionnaire

\section{Su-Veterinary Medicine Satellite Symposium-SL-02 Plant extracts and isolated compounds that are as active as commercial acaricides in controlling ticks in vitro and in vivo}

Authors Eloff $]^{1}$, Nyahangare $\mathrm{E}^{2}$, Mvumi $\mathrm{B}^{2}$, Adenubi $\mathrm{O}^{1}$, Naidoo $\mathrm{V}^{1}$, McGaw L ${ }^{1}$

Institute 1 Phytomedicine Programme Faculty of Vetrinary Science University of Pretoria, Pretoria, South Africa; 2 University of Zimbabwe, Harare,

Zimbabwe

DOI 10.1055/s-0037-1608016

World-wide ticks cause such major problems in tropical areas that if ticks are not controlled by chemical acaricides commercially viable animal production 
Tab. 1: Some common practices of farmers

\begin{tabular}{|c|c|c|c|}
\hline Name of plant & Disease & Part used & Method of application \\
\hline $\begin{array}{l}\text { Coriandrum sati- } \\
\text { vum }\end{array}$ & Stomatitis & Leaves & $\begin{array}{l}\text { Feed a handful of fresh leaves with the } \\
\text { fodder for small and large niminants }\end{array}$ \\
\hline $\begin{array}{l}\text { Trigonella foenum } \\
\text { graecum }\end{array}$ & Diarrhea & Seeds & $\begin{array}{l}\text { Soak } 25 \text { gms seeds in water. Mix with } \\
300 \mathrm{ml} \text { buttermik and drench. this quan- } \\
\text { tity is good for large ruminants. Use half } \\
\text { the quantity for small ruminants }\end{array}$ \\
\hline Curcuma longa & $\begin{array}{l}\text { Wounds, ulcers, } \\
\text { lesions }\end{array}$ & Root powder & $\begin{array}{l}\text { Mix } 1 \text { tsp powder with fresh butter, ghee } \\
\text { or coconut oil and apply on wound or le- } \\
\text { sion }\end{array}$ \\
\hline Ocimum sanctum & $\begin{array}{l}\text { Wounds and in- } \\
\text { flammations }\end{array}$ & Leaf juice & $\begin{array}{l}\text { Cmsh sufficient leaves in vour hand and } \\
\text { applv on wound and on the inflamed area }\end{array}$ \\
\hline Annona squamosa & External parasites & Leaves and seeds & $\begin{array}{l}\text { Crush \& grind sufficient leaves and seeds } \\
\text { of fruit. Mix with coconut oil and apply on } \\
\text { body. For small ruminants dissolve } 100 \\
\text { gms plant material in } 10 \text { litres water and } \\
\text { spray on the animal }\end{array}$ \\
\hline Azadarichta indica & Skin disorders & Leaves & $\begin{array}{l}\text { Crush \& grind sufficient leaves. Mix with } \\
\text { coconut oil and apply on body. For small } \\
\text { ruminants dissolve } 100 \text { gms plant mate- } \\
\text { rial in } 10 \text { litres water and spray on the } \\
\text { animal. }\end{array}$ \\
\hline Moringa oleifera & $\begin{array}{l}\text { Dietary supple- } \\
\text { ment }\end{array}$ & $\begin{array}{l}\text { Leaves and flow- } \\
\text { ers }\end{array}$ & $\begin{array}{l}\text { Offer } 250 \text { gms fresh leaves and flowers to } \\
\text { small ruminants and } 500 \text { gms to large } \\
\text { ruminants along with feed every day }\end{array}$ \\
\hline Psididium guajava & Astringent & Bark and leaves & $\begin{array}{l}\text { Boil } 100 \mathrm{gms} \text { of clean leaves in } 10 \text { liters } \\
\text { water, cool the water, strain out the } \\
\text { leaves and use the water to wash wounds }\end{array}$ \\
\hline Emblica officinalis & $\begin{array}{l}\text { Fresh, bleeding } \\
\text { wounds }\end{array}$ & Leaves & $\begin{array}{l}\text { Apply crushed leaves and leaf juice on } \\
\text { fresh bleeding wounds, bandage the area }\end{array}$ \\
\hline $\begin{array}{l}\text { Tridax procum- } \\
\text { bens }\end{array}$ & Fresh wounds & Whole plant & $\begin{array}{l}\text { Crush the entire plant and apply on } \\
\text { wounds and bandage the area }\end{array}$ \\
\hline Vitex negundo & External parasites & Leaves & $\begin{array}{l}\text { Crush \& grind sufficient leaves.Mix with } \\
\text { coconut oil and apply on body. For small } \\
\text { niminants dissolve } 100 \text { gms plant material } \\
\text { in } 10 \text { litres water and spray on the animal }\end{array}$ \\
\hline
\end{tabular}

\section{Su-Veterinary Medicine Satellite Symposium-SL-04 Ethnoveterinary herb use in the Netherlands - between ethnobotany and zoopharmacognosy}

Authors van Asseldonk $T^{1}$, Lans $C^{1}$, Kleijer $G^{1}$

Institute 1 Institute for Ethnobotany and Zoopharmacognosy (IEZ), Beek, Netherlands

DOI 10.1055/s-0037-1608019

The Institute for Ethnobotany and Zoopharmacognosy (IEZ) in the Netherlands, founded in 1996, combines academic research with teaching and consultancies on the subjects of ethnobotany (including herbs for animals) and zoopharmacognosy.

With help of more than 80 students we made a survey of ethnoveterinary practices in the Netherlands at the start of the 21st century, before these newly trained animal-naturopaths came into practice. Remedies used most often we found to be preparations from Linum usitatissimum, Urtica spp. and Allium sativum.

Traditional use of herbs for animals in the Netherlands can be found in textbooks from circa 1500 - 1900. Also human traditional herbalism and observations of wild plant consumption of free ranging ( $\mathrm{farm}$ ) animals were important roots for the herbal ethnoveterinary traditions we documented. We found several similarities, both in our methodologies and in the herbs, with the ethnoveterinary traditions in British Columbia (Canada), described by Lans. Lans compaired the latter also with first nation folk medicine, unvailing 64 herbs with comparitive uses.

For an experimental validation of herbal remedies some pilot studies were performed. Examples are: feeding herbs to sows to improve piglet health; for better growth of pigs; to improve meat taste; or feeding herbs to boars to diminute aggression.
Recommended Readings

[1] Van Asseldonk \& Beijer. Herbal folk remedies for animal health in the Netherlands. Proceedings ICEB 2005.

[2] Van Asseldonk. Case study Netherlands. Ch 16. in Ethnoveterinary Botanical Medicine, 2010 (CRC, ed Katerere/Luseba).

[3] Lans. Ethnoveterinary medicines British Columbia, 2012.

[4] Lans. J of Ethnopharmacology 192 (2016) 53 - 66.

[5] Van Asseldonk, Kleijer, Vernooij. Kruiden in de Kraamstal 2005. http:// www.ethnobotany.nl/wp-content/uploads/2017/04/E V-Klei-As-Verslag-KrKrProvincie-Ov2005.pdf

[6] Kleijer el al. I lerbal products for pig health. WOCMAP conference 2008

[7] Van Asseldonk http://edepot.wur.nl/266411

\section{Su-Veterinary Medicine Satellite Symposium-KNL-05 What could we learn from primates for the maintenance of domestic and zoo animal health?}

Authors Huffman $\mathrm{M}^{1}$

Institute 1 Primate Research Institute, Kyoto University, Inuyama, Japan DOI 10.1055/s-0037-1608020

The field of ethnoveterinary medicine looks to traditionally living humans for alternative or complimentary solutions to maintaining the health of our domesticated animals. The origins of herbal medicine have their roots deep within human history, with evidence dating back to the ancient human Neanderthal culture. Medicine may have been used not only to treat ourselves, but also the animals we domesticated. The dog is said to have been the first domesticated vertebrate, established across Eurasia before the end of the Late Pleistocene era $(2,588,000$ to 11,700 years ago), predating agriculture. Pastorlist have looked to the self-medicative behavior of wild ungulates and indeed their own livestock for clues to treating disease. It seems that perhaps humans have long been interested in veterinary medicine, but more likely then not, the interest in animal health goes back even further. Even in traditional societies today, healers are known to look to the behavior of wild animals for answers to health problems. For example, chimpanzees and humans co-existing in subSaharan Africa are known to use Vernonia amygdalina for the control of parasite infections. In recent years, phytochemical studies have demonstrated a wide array of biologically active properties in this species. The compounds common to the entire genus infact are known for their antihelmintic (Oesophagostomum, Toxicara, Ancylostoma, Schistosoma, Entamoeba), antimalarial ( $P$. falciparum), and anticancer properties, to name but a few (Table1). In light of the growing resistance of parasites and pathogens to synthetic drugs, the study of animal self-medication may offer a new, biologically relevant and culturally unbiased line of investigation to provide new compounds for the treatment of domestic and zoo animals.

Acknowledgements: Warm thanks to many colleagues who collaborated in this work over the years. In memory of a dear friend and collaborator, Mohamedi Seifu Kalunde, traditional Tongwe healer and park ranger par excellence.

Recommended Readings:

Engel C. Wild Health - How animals keep themselves well and what we can learn from them. Boston: Houghton Mifflin Co, 2002.

Huffman MA. Seifu M. Observations on the illness and consumption of a possibly medicinal plant Vernonia amygdalina by a wild chimpanzee in the Mahale Mountains, Tanzania. Primates 1989; 30: 51 - 63.

Ohigashi H, Huffman MA, Izutsu D, Koshimizu K, Kawanaka M, Sugiyama $H$, Kirby GC, Warhurst DC, Allen D, Wright CW, Phillipson JD, Timmon-David P, Delmas F, Elias R, Balansard G. Toward the chemical ecology of medicinal plant use in chimpanzees: The case of Vernonia amygdalina, a plant used by wild chimpanzees possibly for parasite-related diseases. J Chem Ecol 1994; 20 : $541-553$.

Huffman MA. Self-medicative behavior in the African Great Apes: An evolutionary perspective into the origins of human traditional medicine. Bioscience 2001; 51: $651-661$. 
Mukherjee JR, Chelladurai V, Ronald J, Rawat GS, Mani P, Huffman MA (2011). Do animals eat what we do? Observations on medicinal plants bused by humans and animals of Mudanthurai Range, Tamil Nadu. pp. 179-195. In: Medicinal Plants and Sustainable Development (ed. CP Kala), Nova Science Publications, New York.

\section{Su-Veterinary Medicine Satellite Symposium-SL-05 Are horses able to choose the right remedy - a practice report}

Authors Vollstedt $S^{1}$

Institute 1 Praxis für Traditionelle-Chinesische Pferdemedizin, Bokholt-

Hanredder, Germany

DOI 10.1055/s-0037-1608021

Most horses like dried herbs, however, it is of interest to investigate if they discriminate among herbs according to their health conditions. The observations are based on the treatment of horses of all breeds and ages over the last 4.5 years. Some horses were seen on a monthly basis, others only when there was a problem. In general, the herbs were adapted to the presented problem. When there was not a clinical problem present the selection was based on the time of year or on the management conditions. Some herbs are eaten without any apparent symptoms: milk thistle (Silybum marianum), stinging nettle (Urtica dioica), horsetail (Equisetum arvense), vervain (Verbena officinalis), shepherd's purse (Capsella bursa-pastoris) and ribwort (Plantago lanceolatum) support the general metabolism and organ functions. Therefore, it does not seem curious that they were taken up as supplements for general health. Horses with conditions which render them warm like Equine Metabolic Syndrome or the early phase of Equine Cushing's Disease prefer hemp-nettle (Galeopsis segetum). However, when horses have a chronic disease such as Cushing's in the later phase they accept cinnamon (Cinnamomi cortex). Horses with skin conditions such as eczema will willingly take pansy (Viola tricoloris) and marigold (Calendula officinalis). Although horses without any noticeable problems also eat it, it seems that the preference increases slightly when when these plants are needed. Ribwort (Plantago lanceolatum) and common lungwort (Pulmonaria officinalis) are taken with or without apparent lung problems. Common mallow (Malva sylvestris) is preferred by horses with dry cough, while common horehound (Marrubium vulgare) is only eaten by horses with mucilage in the airways. As long as horses have enough roughage and do not get too much processed food, they are able to distinguish herbs if they are benevolent to their health.

Tab. 1

\begin{tabular}{ll|l|l|l} 
English name & Latin name & Function & Acceptance & $\begin{array}{l}\text { Clinical } \\
\text { symptoms }\end{array}$ \\
\hline Milkthistle & Silybummarianum & metabolism & +++++ & ++ \\
\hline Stinging nettle & Urtica dioica & metabolism & +++++ & + \\
\hline Horsetail & Equisetum arvense & metabolism & ++++ & ++ \\
\hline Hemp-nettle & Galeopsissegetum & metabolism & ++ & ++++ \\
\hline Cinnamon & Cinnamomi cortex & metabolism & + & ++++ \\
\hline Vervain & Verbena officinalis & blood & +++++ & + \\
\hline Shepherd's purse & Capsella bursa-pastoris & blood & +++++ & + \\
\hline Pansy & Viola tricoloris & skin & +++ & +++ \\
\hline Marigold & Calendula officinalis & skin & +++ & +++ \\
\hline Ribwort & Plantago lanceolatum & lung & +++++ & + \\
\hline Common lungwort & Pulmonaria officinalis & lung & ++++ & ++++ \\
\hline Common mallow & Malvasylvestris & lung & +++ & +++ \\
\hline Common horehound & Marrubium vulgare & lung & + & ++++ \\
\hline
\end{tabular}

Su-Veterinary Medicine Satellite Symposium-SL-06 The benefits of employing saponin-rich plants as feed additives in cattle

Authors Mendel $\mathrm{M}^{1}$, Chłopecka $\mathrm{M}^{1}$

Institute 1 Warsaw University of Life Sciences Faculty of Veterinary Medicine Division of Pharmacology and Toxicology, Warsaw, Poland

DOI 10.1055/s-0037-1608022
Once called anti-nutritional agents, today saponins belong to the favorable phytogenic feed additives (PFA) due to numerous pharmacological activities they exhibit. Saponins are a substantial group of low molecular weight plant secondary metabolites containing either a tetracyclic steroidal or a pentacyclic triterpenoid aglycone with one or more sugar chains. They are widely distributed in higher plants, and to some extent in lower marine animals and bacteria. Two major commercial sources of saponins are Yucca schidigera and Quillaja saponaria. The constantly growing attention paid to saponins as PFA results from their immunostimulatory, anti-inflammatory, hepatoprotective, antibacterial, antiviral and antifungal activity [1]. In recent years, there have been satisfactory in vitro and in vivo data revealing the ability of saponins to inhibit methane production and demonstrating their anti-protozoal activity. Saponins are shown to modify rumen fermentation through direct influence on the composition and activity of rumen protozoa, bacteria, fungi and archaea [2]. Besides, some saponins alter nutrient digestibility by reducing $\mathrm{NH}_{3}$ $\mathrm{N}$ concentration in rumen liquor and increasing the concentration of volatile fatty acids. In addition to the extensive use as methane production modifiers, saponins are considered as potent antiparasitic agents [3].

Although saponins undergo profound degradation in the rumen it is believed that sufficient amount, predominantly in the form of aglycones, gets absorbed and induces systemic effects. Extracts of saponin-rich plants are currently used as dietary additives for livestock and companion animals.

[1] Sprag SG, Light ME, van Staden J. J Ethnopharmacol 2004; 94: 219 - 243

[2] Patra AK, Saxena J. Nutr Res Rev 2009; 22: 204 - 219

[3] Mehlhorn H, Al-Quraishy S, Al-Rasheid KAS, Jatzlau A, Abdel-Ghaffar F. Parasitol Res 2011; 108: 1041 - 1046

\section{Regulatory Affairs Workshop}

Basel, Sunday, September 3, 2017

Abstracts of the Regulatory Affairs Workshop

- in order of appearance

\section{Su-Regulatory Affairs Workshop I-KL-01 Regulation of herbal and complementary medicines in Switzerland}

Authors Ziak $\mathrm{M}^{1}$

Institute 1 Swissmedic, Swiss Agency for Therapeutic Products, Berne, Switzerland

DOI 10.1055/s-0037-1608023

Complementary medicines are popular and widely used in Switzerland. According to the latest Swissmedic Annual Report for 2016, around 21\% of the 8,300 authorised medicinal products are complementary and herbal medicines. These figures highlight the need to take account of the Swiss public's demand for complementary and herbal medicines in addition to medicinal products from conventional medicine.

The principles for the approval/authorisation of complementary and herbal medicines are clearly defined in therapeutic products legislation.

Swissmedic assesses herbal medicines for human use that contain either known active pharmaceutical ingredients or new active substances in accordance with the Instructions for submitting applications for authorisation of herbal medicinal products for human use ("Phyto instructions"). According to these instructions, herbal medicinal products may only contain active pharmaceutical ingredients consisting of substances or preparations of plant origin. Herbal medicines with known active pharmaceutical ingredients can be authorised in a simplified procedure, i.e. the preclinical and clinical documentation can include references to published literature data. This simplified authorisation procedure can be used only if therapeutic or pharmaceutical equivalence with a therapeutic product already authorised by the Agency is demonstrated. No simplification is possible for the quality documentation. For complementary medicines with an indication, the proof of safety and efficacy of a preparation can be provided in the form of published data 
(bibliographical evidence) without the need to conduct controlled clinical trials. The review of complementary medicines without an indication focuses on quality and safety. Proof of efficacy is not required. Complementary medicines without an indication are used for individual treatment and may be dispensed only by authorised persons.

The revised Therapeutic Products Act, which will enter into force at the start of 2019, is designed to promote the availability of a wide range of complementary and herbal medicines by further simplifications in the authorisation procedure.

\section{Su-Regulatory Affairs Workshop I-KL-02 Industry's perspective Particular challenges for a pharmaceutical company}

Authors Halbsguth $C^{1}$, Zahner $C^{1}$

Institute 1 Zeller AG, Romanshorn, Switzerland

DOI 10.1055/s-0037-1608024

Different challenges for industry may occur before and throughout the registration process with a known herbal active substance in Switzerland. Using an EU-CTD-dossier it will be lined out what changes an applicant has to implement before a submission of a registration application in Switzerland (e. g. the "helvetisation" of module 1).

If the applicant is also marketing authorization holder in other European and non-European countries special challenges exist with regard to the CMC part (module 3). Country-specific quality requirements of different health authorities have to be considered and feasible and reasonable specifications have to be set up.

Furthermore as an example, in the non-clinical part (module 4) for known herbal active substances Swissmedic may request genotoxicity tests as well as interaction studies.

For the clinical part (module 5), especially the conduct of clinical trials The Human Research Act (HRA) and its corresponding ordinances are in force in Switzerland since 2014. The Ordinance of Clinical Trials in Human Research (ClinO) as well as the Human Research Ordinance (HRO), Ordinance on Human Research with the Exception of Clinical Trials and their peculiarities will be outlined. The author shares some insight in first experiences of human research projects belonging to both ordinances and compares the Swiss situation to the European/international requirements for clinical trials.

In Switzerland - in contrast to many other countries - there exists pharmaceutical reimbursement and pricing policies for herbal medicines. The authors will outline these policies more in detail and share own experiences with the audience.

\section{Su-Regulatory Affairs Workshop I-KL-03 Contributions to regulation of herbal medicinal products from scientific institutions}

Authors Wolfram $\mathrm{E}^{1}$

Institute 1 ZHAW, ICBT, Research Group of Phytopharmacy and Natrual

Products, Wädenswil, Switzerland

DOI 10.1055/s-0037-1608025

In contrast to mono-substance pharmaceuticals, the compliant assurance of quality, safety and efficacy of herbal medicinal products requires a much more complex and process oriented approach. The scope for standardization, relevant data acquisition and documentation starts on the field and in the natural habitats with Good Agricultural and Collection Practice (GACP) and not only later at the doors of a GMP certified factory. In addition to common physicochemical identity testing, botanical aspects are assessed. In contrast to analytical quality control methods searching for peaks of known active pharmaceutical ingredient (API) molecules with a characteristic spectrum or molecular mass, the valid analysis of the multi-compound natural product mixture (herbal extract) as the API requires a multivariate and wider approach for standardization. The scientific institutions are capable to offer regulatory authorities and industry from a non-profit and independent position important competences and academic methodologies in assessment of e. g. API content, activity, toxicology and contaminants.

Moreover, the academic sector provides education and training of professionals in the area of quality management, quality control methods and regulatory affairs. Special study and advanced study programs are in need for the herbal sector, to meet the demand of industry and authorities for competent staff within this special field.

Selected case studies from own experience of collaboration with Swissmedic, EDQM and herbal medicinal industry of challenges in analytical method development for monographs and common technical documents (CTD) provide for hands-on insights about the current and potential value of the academic sector for regulation. Among other examples, the focus will be on HPTLC identification by flavonoid fingerprints and HPLC assay of anthraquinones and their aglycones in Senna leaves and pods.

\section{Lecture Session - ESA Gold Award Lecture Monday, 4th September, 2017 \\ Montreal - 09:45 - 10:30 \\ Mo-Egon Stahl Gold Award Lecture From Egon Stahl to Metabolomics}

Authors Verpoorte $\mathrm{R}^{1}$

Institute 1 Natural Products Laboratory, Institute of Biology Leiden, Leiden University, Leiden, Netherlands

DOI 10.1055/s-0037-1608026

After a century of microscopy, the advent of chromatography changed the field of Pharmacognosy completely: from botany to chemistry. Particularly Professor Dr. Egon Stahl played a major role in the 1960ties by developing and standardizing thin layer chromatography. Due to his pioneering work TLC became a standard method in all laboratories working with natural products. About 40 years later with the advent of metabolomics it became clear how important his clear vision on the need for standardization is. Now we may ask the question: what will be the next breakthrough for natural products research and Pharmacognosy? At least the 2015 Nobel prize for Medicine and Physiology should be seen as an important gesture from the medical world. It is the recognition of natural products as an important resource for developing novel medicines. Moreover the changing economic reality results in shifting scientific interests. A more sustainable production of food and other products is urgently needed. This raises the interest in learning more about natural resistance in crop plants to be able to reduce the use of agrochemicals. That means insight in biosynthesis of secondary metabolites is needed. But also increasing appreciation of other medical systems is important to note. Medical systems based on a more holistic approach, require a systemic approach to understand their traditional medications. The paradigm of "single compound - single target" will not help much to understand the meaning of making mixtures of plants. Instead synergy and prodrugs needs to be taken into account. The omics and particularly metabolomics will play an important role in the natural products research. Chromatography and spectroscopic methods will be major tools, as well as molecular biology and microscopy. All research will be connected with biological activities, e. $\mathrm{g}$. in plant resistance or as health affecting compounds. So a lot of work waiting for us! 
Lecture Session - Plenary Lecture 1

Monday, 4th September, 2017

Montreal - 11:00-11:45

\section{Mo-Plenary Lecture Session 1-PL-01 Opportunities and challenges of plant biotechnology for the production of active substances}

\author{
Authors Warzecha $\mathrm{H}^{\top}$ \\ Institute 1 Plant Biotechnology and Metabolic Engineering, Technische \\ Universität Darmstadt, Darmstadt, Germany \\ DOI 10.1055/s-0037-1608027
}

Plants are used as remedies against various diseases since the early days of human history, and their large repertoire of chemically complex natural products still serves as blueprint for numerous modern medicines. With the advent of genetic engineering and biotechnology, this classical view is rapidly changing. Novel technologies are not only enabling the elucidation of the biosynthetic capacity of plants but also the deliberate reconstruction of metabolic pathways. Moreover, even biopharmaceuticals like Taliglucerase alpha or specific antibodies are now being produced in plants, exemplifying the great potential of plants as production and delivery system for modern therapeutics. This lecture will summarize the state of the art in plant made pharmaceuticals and biologics and also discuss future potential of metabolic engineering of medicinal plants.

\section{Lecture Session - Plenary Lecture 2 \\ Monday, 4th September, 2017 \\ Montreal - 11:45-12:30}

\section{Mo-Plenary Lecture Session 2-PL-01 Rooibos and honeybush phenolics as drivers of product development - impact on a sustainable South African herbal tea industry}

Authors Joubert $\mathrm{E}^{1}$

Institute 1 Plant Bioactive Group, Agricultural Research Council (InfruitecNietvoorbij), Post-Harvest \& Wine Technology Division, Stellenbosch, South Africa; Department of Food Science, Stellenbosch University, Matieland, South Africa

DOI 10.1055/s-0037-1608028

The indigenous South African medicinal fynbos plants, Aspalathus linearis (rooibos) and multiple Cyclopia species (honeybush), largely found traction as herbal teas on the domestic and global markets during the last century $[1,2]$. Investigations into their phenolic composition have brought the focus back to their medicinal properties, driven from the perspective of present day lifestyle diseases [3]. Opportunities arose for nutraceutical product development as aspalathin and mangiferin, the main phenolic compounds of these herbal teas demonstrated potential in ameliorating features of the metabolic syndrome and delaying its development towards serious metabolic diseases. Given the global prevalence of non-communicable lifestyle diseases, exploitation for nutraceutical production will provide the South African herbal tea industry with new vehicles to expand their economic viability. The commercialisation of rooibos and honeybush represents two different scenarios. The rooibos industry is well established and production depends almost exclusively on cultivation of one particular species, while the honeybush industry is still under development and largely dependent on wild harvesting of a number of Cyclopia species. Increasing demand for honeybush as herbal tea has already escalated unsustainable harvesting practices, placing stress on endangered Cyclopia species. The threat of climate change has renewed interest in wild rooibos types with higher drought resistance and non-utilised Cyclopia species. Genotypes superior in target phenolic compounds may lessen the demand for biomass by the nutraceutical market, but could impact negatively on their consumer acceptance as herbal teas. Several of the UN's Sustainable Development Goals [4] deserve consideration by industry and are relevant to contextualise the potential of these herbs as sources of polyphenols for nutraceutical product development.

[1] Joubert E et al. S Afr J Bot 2011; 77: $887-907$

[2] Joubert E, De Beer D. S Afr J Bot 2011; 77: 869 - 886

[3] Chellan et al. Planta Med 2014; 80: $622-629$

[4] https://sustainabledevelopment.un.org/? menu $=1300$

\section{Lecture Session - Bioactive Natural Products I}

Monday, 4th September, 2017

Montreal - 14:00 - 16:00

\section{Mo-Bioactive Natural Products I-SL-01Flavonoids from Friesodielsia desmoides and their NO production inhibitory}

Authors Laphookhieo $S^{1}$, Meesakul $\mathrm{P}^{1}$, Pudhom $\mathrm{K}^{2}$, Pyne $\mathrm{S}^{3}$

Institute 1 Natural Products Research Laboratory, School of Science, Mae Fah Luang University, Chiang Rai, Thailand; 2 Department of Chemistry, Faculty of Science, Chulalongkorn University, Bangkok, Thailand; 3 School of Chemistry, University of Wollongong, Wollongong, Wollongong, Australia DOI 10.1055/s-0037-1608029

Friesodielsia is a small genus belonging to the Annonaceae family which is distributed in Africa and Asia. Many types of secondary metabolites are produced from this genus including flavonoids, chalcones, alkaloids, benzyl benzoate derivatives and sesquiterpenes [1,2]. Some of these compounds show interesting biological properties, such as antiplasmodial and cytotoxicity activities. F. desmoides (Craib) Steenis is a small tree or shrub which is grown as an ornamental plant in Thailand. To the best of our knowledge, this is the first report of phytochemical investigations of this plant. The crude extracts of the leaves and twigs of $F$. desmoides were separated by column chromatography using various stationary phases to yield three new flavan-flavanone hybrids, friesodielsones A-C (1-3), along with 18 known compounds [3]. The structures of compounds 1-3 were elucidated by intensive analysis of spectroscopic data and their absolute configurations were determined by CD spectroscopy and biosynthesis considerations. Two compounds significantly exhibited NO production with the $\mathrm{IC}_{50}$ values of $10.21 \pm 0.074$ and $7.56 \pm 0.087 \mu \mathrm{M}$, two others were moderate inhibitors $\left(\mathrm{IC}_{50}=28.14-37.21 \mu \mathrm{M}\right)$.

[1] Prawat U, Phupornprasert D, Butsuri A, Salae AW, Boonsri S, Tuntiwachwuttikul P. Phytochemitry Lett 2012; 5, 809-813.

[2] Fleischer TC, Waigh RD, Waterman PG. Phytochemistry 1997; 44, 315 318.

[3] Meesakul P, Pudhom K, Pyne SG, Laphookhieo S. RSC Adv 2017; 7 , $17545-17550$

\section{Mo-Bioactive Natural Products I-SL-02Four new cytotoxic polyprenylated acylphloroglucinols from Hypericum barbatum Jacq.}

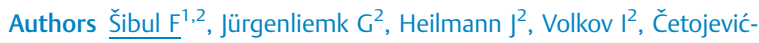
Simin $D^{3}$, Orčić $D^{1}$, Mimica-Dukić $N^{1}$

Institute 1 Department for Chemistry, Biochemistry and Environmental protection, Faculty of Sciences, University of Novi Sad, Novi Sad, Serbia; 2 Chair for Pharmaceutical Biology, Faculty of Chemistry and Pharmacy, University of Regensburg, Regensburg, Germany; 3 Oncology Institute Sremska Kamenica, Faculty of Medicine, University of Novi Sad, Novi Sad, Serbia

DOI 10.1055/s-0037-1608030

Polycyclic polyprenylated acylphloroglucinols (PPAPs) are one of the most interesting compounds from Hypericum species. Their biological and pharma- 
cological activity is varying largely due to the possibilities of prenylation, cyclization and oxydation. Among other activities, antidepressant, analgesic, antioxidative, anti-inflammatory, antimicrobial and cytotoxic are predominantly related with PPAPs[1]. This increases the interest for discovery and isolation of new acylphloroglucinols and testing their biological abilities. $H$. barbatum Jacq. is a plant distributed widely throughout Europe, from Austria to Greece, through Balkans and Mediterranean regions[2]. Being poorly investigated regarding its PPAP spectrum, it represents an interesting source for phytochemical research. Using ' ${ }^{1} \mathrm{H}$-NMR guided fractionation of its aerial parts' petrol ether extract, four novel tricyclic polyprenylated, endoperoxide bridgecontaining acylphloroglucinols, hyperibarbins A-D (1-4) were isolated. Their structures were elucidated using 2D NMR spectroscopic data and HRESIMS. Cytotoxic activity was evaluated against HepG2 liver tumor cells, HeLa cervix epitheloid carcinoma, PC-3 prostate tumor cells, A549 lung tumor cell lines and MRC- 5 healthy human fetal lung cells, with the four compounds exhibiting moderate cytotoxicity against all examined cell lines. $I C_{50}$ values were 9.22 $17.0 \mu \mathrm{M}$ for HepG2, 12.8-21.8 $\mu \mathrm{M}$ to inhibit growth of HeLa cells, 14.4$27.8 \mu \mathrm{M}$ for PC-3 tumor cells and $20.2-27.1 \mu \mathrm{M}$ for A549 lung tumor cells. Unfortunately, they were also toxic for the human fibroblast lung cell line MRC-5, with $\mathrm{IC}_{50} 13.4-20.4 \mu \mathrm{M}$. Similarity in action is noted among the analogous hyperibarbins A (1) and C (3), as well as B (2) and D (4).
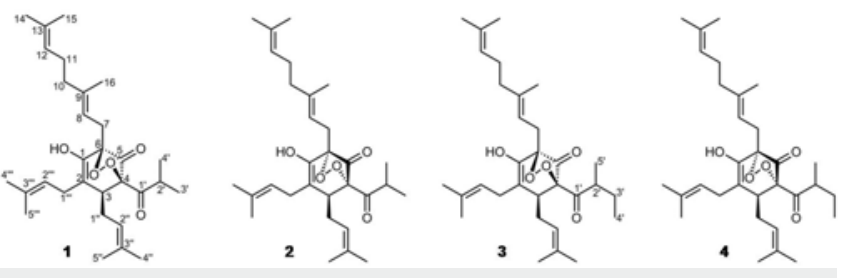

Fig. 1

[1] Zhao J, Liu W, Wang JC. Chem Biodiv 2015; 12: 309 -349;

[2] Robson NKB. Phytotaxa 2012; 72: 1 - 111.

\section{Mo-Bioactive Natural Products I-SL-03hERG channel blockers in medicinal plants: Hysteria or serious problem?}

Authors Grienke $U^{1}$, Mair $\mathrm{CE}^{1}$, Kratz JM $\mathrm{M}^{1}$, Rollinger JM $\mathrm{M}^{1}$

Institute 1 Department of Pharmacognosy, University of Vienna, Austria, Vienna, Austria

DOI 10.1055/s-0037-1608031

A blockage of the human Ether-à-go-go Related Gene (hERG) channel is associated with QT interval prolongation in the electrocardiogram and an increased risk of fatal arrhythmias. In early drug discovery and development, candidate compounds are tested against this important antitarget in a standard procedure. Surprisingly, the hERG channel blocking profile of natural compounds present in frequently consumed botanicals, such as dietary supplements, spices, and herbal medicinal products, is not routinely assessed. In the recently accomplished EU funded project 'hERG related risk assessment of botanicals' (PIRSES-GA-2011-295174), a consortium of nine partners from three different continents committed themselves to the evaluation of putatively hERG channel blocking properties of commonly consumed herbal remedies. The project aimed at the critical assessment of hERG channel related toxicity to improve consumer and patient safety. Intriguingly, of more than 1,200 extracts probed in this endeavour, only $\sim 2.5 \%$ reduced the hERG channel peak tail current by $\geq 30 \%$ at $100 \mu \mathrm{g} / \mathrm{mL}$ in a voltage-clamp assay using Xenopus oocytes.

Here, examples and strategies for the identification of hERG blockers from medicinal plants will be presented $[1-3]$. This includes also a critical view on the relevance of these findings and a concluding discussion of the outcome of the hERGscreen project.
This work was supported by a Marie Curie IRSES Fellowship within the Seventh European Community Framework Programme (hERGscreen, 295174).

[1] Kratz JM, Schuster D, Edtbauer M, Saxena P, Mair CE, Kirchebner J, Matuszczak B, Baburin I, Hering S, Rollinger JM. J Chem Inf Model 2014; 54: $2887-2901$

[2] Grienke U, Mair CE, Saxena P, Baburin I, Scheel O, Ganzera M, Schuster D, Hering S, Rollinger JM. J Agric Food Chem 2015; 63: 5634 - 5639

[3] Mair CE, de Miranda Silva C, Grienke U, Kratz JM, Carreño F, Zimmermann ES, de Araújo BV, Dalla Costa T, Rollinger JM. Planta Med 2016; 82: 1030 1038

\section{Mo-Bioactive Natural Products I-SL-04Search for new antiplasmodial leads in the oleo-gum-resin of Boswellia serrata Roxb.}

Authors Greve $\mathrm{H}^{1}$, Kaiser $\mathrm{M}^{2,3}$, Brun $\mathrm{R}^{2,3}$, Schmidt $\mathrm{T}^{1}$

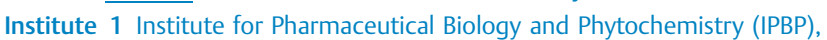
WWU Münster, Münster, Germany; 2 Swiss Tropical and Public Health Institute (Swiss TPH), Basle, Switzerland; 3 University of Basle, Basle, Switzerland DOI 10.1055/s-0037-1608032

Malaria is a vector-borne disease caused by pathogens of the genus Plasmodium that are transmitted by the bite of infected female Anopheles mosquitoes. Worldwide this infectious disease caused almost 430,000 deaths in 2015 [1]. Despite some improvements in prevention and therapy, especially increasing drug resistances remain a big problem [2]. As part of our continued search for natural products with antiprotozoal activity, Indian frankincense, the oleogum-resin of Boswellia serrata (Burseraceae) showed activity against Plasmodium falciparum (NF54 strain) in vitro. Successive extraction with solvents of increasing polarity showed best results for the dichloromethane extract $\left(\mathrm{IC}_{50}=2.58 \mu \mathrm{g} / \mathrm{ml}\right)$. Bioactivity-guided fractionation led to the isolation of 22 compounds that were characterized by spectroscopic measurements. 17 compounds could be identified by comparison with literature data, three of which were obtained as natural products for the first time. The five remaining constituents were identified as rel-(1S,3R,7E,11S,12R)-1,12-epoxy-4-methylenecembr-7-ene-3,11-diol, 3-epi-neoilexonol, isoflindissol lactone, rel-

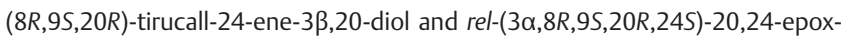
ytirucalla-3,25-diol. To the best of our knowledge, these compounds are not described in literature so far. All substances were tested for antiplasmodial activity in vitro using established protocols [3]. Isoflindissone lactone showed the best activity with an $\mathrm{IC}_{50}$ value of $2.1 \pm 0.3 \mu \mathrm{M}$.

This project is an activity within the Research Network Natural Products against Neglected Diseases (ResNet NPND; http://www.resnetnpnd.org/). HLG thanks Cusanuswerk for a doctoral scholarship.

[1] WHO. Fact sheet: Malaria. Online: http://www.who.int/mediacentre/factsheets/fs094/en/; cited: March 20, 2017

[2] Schmidt T] et al. Curr Med Chem 2012; 19: $2128-2175$

[3] Nour AMM et al. Planta Med 2009; 75: $1363-1368$

\section{Mo-Bioactive Natural Products I-SL-05The role of $\beta$-glucuronidase in disposition and anti-inflammatory effects of urolithins- gut microbiota-derived metabolites of ellagitannins.}

Authors Piwowarski JP ${ }^{1}$, Stanisławska I ${ }^{1}$, Granica $S^{1}$, Kiss AK ${ }^{1}$, Moeslinger $\mathrm{T}^{2}$ Institute 1 Department of Pharmacognosy and Molecular Basis of Phytotherapy, Medical University of Warsaw, Faculty of Pharmacy, Warsaw, Poland; 2 Institute of Physiology, Center for Physiology and Pharmacology, Medical University of Vienna, Vienna, Austria

DOI 10.1055/s-0037-1608033

The gut microbiota metabolism of orally applied natural products has been in recent years indicated as an important factor significantly influencing their therapeutic effects. One of the most extensively studied groups of compounds are ellagitannins, which are metabolized to urolithins- compounds 
having good bioavailability and being detected in plasma and urine at micromolar concentrations following oral intake of ellagitannin-containing products. In vivo studies conducted for these natural products clearly indicate beneficial health effects towards inflammation-related diseases, which are associated with the formation of urolithins. However, since the majority of in vitro experiments was conducted for urolithin aglycones, they occur incongruent with the results of recent pharmacokinetic studies clearly indicating that glucuronide conjugates are the dominant metabolites present in plasma, tissue and urine [1].
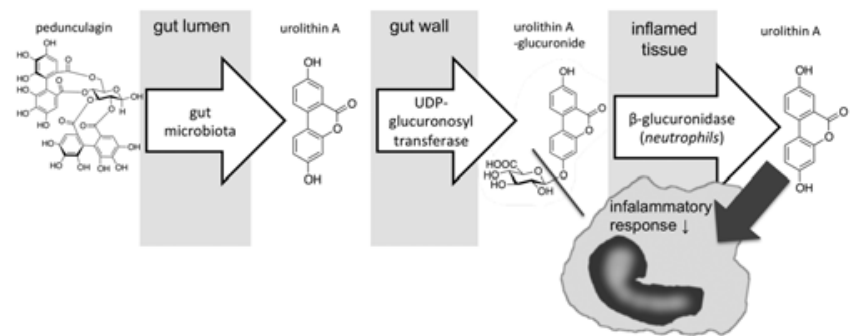

Fig. 1

The aim of this study was to isolate and structurally characterize urolithin conjugates from the urine of volunteers ingesting ellagitannin-containing natural products and to evaluate the potential role of human $\beta$-glucuronidase in urolithin disposition. Glucuronides of urolithin $A$, iso-urolithin $A$ and urolithin $B$ were isolated and shown to be cleaved by the $\beta$-glucuronidase released by neutrophils from azurophilic granules upon f-MLP stimulation. The comparative studies of urolithins and their glucuronides on bone marrow derived macrophages and BV2 microglial cells have shown, that urolithins were significantly more active than respective conjugates towards inhibition of LPSinduced nitric oxide production and iNOS mRNA and protein synthesis.

These results justify the hypothesis that the selective activation of urolithin glucuronides by $\beta$-glucuronidase, which is present at high abundance at inflammation site, could locally increase the concentration of biologically active urolithin aglycones.

The project was financially supported by a Polish Ministry of Science and Higher Education research grant, luventus Plus [IP2015 062274] and Foundation for Polish Science scholarship [START 84.2016].

[1] Tomas-Barberan F.A, Mol Nutr Food Res 2017, doi: 10.1002/ mnfr.201500901

\section{Mo-Bioactive Natural Products I-SL-06South African endophytes- potential antimicrobial agents}

Authors Aboobaker $Z^{1}$, van Vuuren $S^{1}$, Viljoen $A^{2}$, Crous $\mathrm{P}^{3}$

Institute 1 Department of Pharmacy and Pharmacology, Faculty of Health Sciences, University of the Witwatersrand, 7 York Road, 2193, Parktown, South Africa; 2 Department of Pharmaceutical Sciences, Faculty of Sciences,

Tshwane University of Technology, Private Bag X680, 0001, Pretoria, South Africa; 3 Westerdijk Fungal Biodiversity Institute, P.O. Box 85167, 3508 AD, Utrecht, Netherlands

DOI 10.1055/s-0037-1608034

Endophytes have been found in every plant species examined to date and have been recognized as potential sources of novel antimicrobials. The antimicrobial potential of endophytes isolated from South African medicinal plants have, however, been poorly explored. The aim of this study was therefore to investigate the antimicrobial activity of endophytes isolated from the roots of three popular South African medicinal plant species (Pelargonium sidoides, Hypoxis hemerocallidea and Gunnera perpensa) and explore the chemical diversity of the isolated endophytes. Fungal cultures were isolated from the roots, and extracts were prepared and tested independently and in combination with the host plant to determine if any synergistic interactions are apparent. Antibacterial studies were undertaken using the minimum in- hibitory concentration (MIC) assay against Gram-positive (Staphylococcus aureus ATCC 25923 and Enterococcus faecalis ATCC 29212) and Gram-negative (Escherichia coli ATCC 8739 and Pseudomonas aeruginosa ATCC 27853) bacteria. A total of 19 endophytic fungi were isolated from the P. sidoides root. Four fungal extracts exhibited antibacterial activity either alone or in combination with the host plant. Noteworthy results were exhibited by a fungal isolate from Pelargonium sidoides root with a MIC value of $30 \mu \mathrm{g} / \mathrm{mL}$ against S. aureus and $60 \mu \mathrm{g} / \mathrm{mL}$ against E. coli. Six endophytic fungi could be isolated from the roots of $\mathrm{H}$. hemerocallidea, and five from the roots of $\mathrm{G}$. perpensa. Hypoxis hemerocallidea and G. perpensa both had one endophytic fungal strain that exhibited antibacterial activity against $\mathrm{S}$. aureus with MIC values of $250 \mu \mathrm{g} / \mathrm{mL}$ and $500 \mu \mathrm{g} / \mathrm{mL}$ respectively. Results indicate that endophytic fungi from these South African medicinal plants yield a complex chemistry and could be a promising source of antimicrobial metabolites. Furthermore, selected species exhibit a synergistic antimicrobial role with the host plant demonstrating a unique anti-infective role for medicinal plants.

\section{Mo-Bioactive Natural Products I-SL-07Bilirubin Decreases Macrophage Cholesterol Efflux and ABCA1 Protein Expression}

Authors Wang $D^{1,2}$, Tosevska $A^{3,4,5}$, Heiß $E^{1}$, Ladurner $A^{1}$, Mölzer $C^{4,6}$, Wallner $\mathrm{M}^{4,7}$, Bulmer $\mathrm{A}^{8}$, Wagner $\mathrm{KH}^{3,4}$, Dirsch $\mathrm{V}^{1}$, Atanasov $\mathrm{A}^{1,2}$

Institute 1 Department of Pharmacognosy, University of Vienna, Vienna,

Austria; 2 Institute of Genetics and Animal Breeding of the Polish Academy of Sciences, Jastrz? biec, Poland; 3 Research Platform Active Ageing, University of Vienna, Vienna, Austria; 4 Department of Nutritional Sciences, University of Vienna, Vienna, Austria; 5 Department of Molecular, Cell and Developmental Biology, UCLA, Los Angeles, United States; 6 School of Medicine, Medical

Sciences and Nutrition, Institute of Medical Sciences, University of Aberdeen, Aberdeen, United Kingdom; 7 Institute of Dietetics and Nutrition, University of Applied Sciences, FH JOANNEUM, Graz, Austria; 8 School of Medical Science and Menzies Health Institute Queensland, Gold Coast, Australia

DOI 10.1055/s-0037-1608035

Mild, yet chronically elevated circulating unconjugated bilirubin (UCB) is associated with reduced total and LDL-cholesterol concentration, which correlates with reduced cardiovascular disease risk [1, 2]. We aimed to investigate whether UCB influences macrophage cholesterol efflux, as one potential mechanism for the altered circulating lipoprotein concentrations observed in hyperbilirubinemic individuals.

Cholesterol efflux from THP-1 macrophages was assessed using plasma obtained from normo- and hyperbilirubinemic (Gilbert's Syndrome) humans ( $n=60 / 60)$ or (heterozygote/homozygote Gunn) rats $(n=20 / 20)$ as an acceptor. Hyperbilirubinemic plasma from Gilbert's Syndrome individuals and Gunn rats induced a significantly reduced cholesterol efflux compared to normobilirubinemic plasma. UCB ( $3-17.1 \mu \mathrm{mol} / \mathrm{L})$ exogenously added to plasma- or apolipoprotein A1 (apo A1)-supplemented media also decreased macrophage cholesterol efflux in a concentration- and time-dependent manner. We also show a reduced protein expression of the ATP-binding cassette transporter $A 1$ ( $A B C A 1$ ), a transmembrane cholesterol transporter involved in apo A1-mediated cholesterol efflux, in THP-1 macrophages treated with UCB and in peripheral blood mononuclear cells (PBMCs) obtained from hyperbilirubinemic individuals. Furthermore, we demonstrate that bilirubin accelerates the degradation rate of the ABCA1 protein in THP-1 macrophages.

Cholesterol efflux from THP-1 macrophages is decreased in the presence of plasma obtained from humans and rats with mild hyperbilirubinemia. A direct effect of UCB on cholesterol efflux was demonstrated, which is associated with a decreased $A B C A 1$ protein expression. These data improve our knowledge concerning bilirubin's impact on cholesterol transport and represent an important advancement in our understanding of its role in cardiovascular disease.

[1] Mayer M. Clinical Chemistry. 2000; 46: 1723 - 1727. 
[2] Bulmer AC, Verkade H] and Wagner KH. Progress in Lipid Research. 2013; 52: $193-205$.

\section{Mo-Bioactive Natural Products I-SL-08In silico identification and in vitro evaluation of new flavonoid inhibitors of pteridine reductase I from Leishmania major (LmPTR1)}

Authors Herrmann Fabian $\mathrm{C}^{1}$, Sivakumar $\mathrm{N}^{1}$, Schmidt Thomas $]^{1}$ Institute 1 Institute of Pharmaceutical Biology and Phytochemistry (IPBP), University of Münster, PharmaCampus Correnstraße 48, D-48149 Münster, Germany, Münster, Germany

DOI 10.1055/s-0037-1608036

Continuing our computational studies on a variety of target proteins from parasites causing neglected tropical diseases [1,2], the present study aimed at the in silico identification and subsequent in vitro evaluation of natural products (NPs) as potential lead structures for the development of novel inhibitors of pteridine reductase I from Leishmania major (LmPTR1), a peculiar enzyme of the folate metabolism in Leishmania species.

The in silico study was carried out employing a pharmacophore based virtual screening followed by molecular docking into the substrate site of LmPTR1 using about 1300 compounds from a commercial NP database and led to the identification of 11 flavonoids as potential inhibitors. In vitro evaluation of the in silico hits on recombinant LmPTR1 revealed significant inhibitory activity (> $20 \%$ inhibition at $50 \mu \mathrm{M}$ ) for $8(73 \%)$ of the 11 tested flavonoids. Three of these compounds displayed $I_{50}$ below $50 \mu \mathrm{M}$ against $L$ mPTR1, with sophoraflavanone $G$ being the most active $\left(I_{50}=19.2 \mu \mathrm{M}\right)$. The significant in vitro activity of flavonoids is in agreement with very recent data by Borsari et al. [3]. The compounds identified in the present study represent further promising lead structures for inhibitor development. Additionally, the obtained data allowed the elucidation of basic structure activity relationships of the tested flavonoids against LmPTR1.
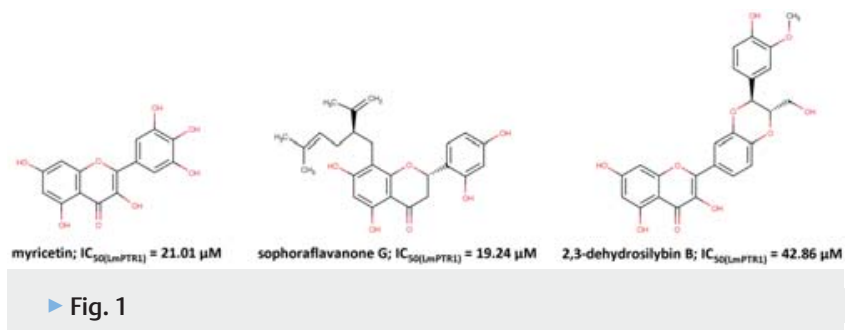

Test compounds were kindly donated by Phytolab GmbH, Vestenbergsgreuth, Germany. Bacteria containing the expression plasmid for LmPTR1 were kindly provided by M.P. Costi (Modena, Italy).

Support of CCG (Montreal, Canada) is gratefully acknowledged.

This work is part of the activities of ResNetNPND: http://www.uni-muenster. de/ResNetNPND/.

[1] Herrmann F. C, Schmidt T. J. Planta Med. 2013; 791132

[2] Herrmann F. C. et al. Molecules 2015; 20, $16154-69$

[3] Borsari C. et al, J. Med. Chem. 2016; 597598 - 7616

\section{Lecture Session - Analytical Studies \& Natural Products Chemistry I \\ Monday, 4th September, 2017 \\ Osaka/Samarkand - 14:00 - 16:00}

\section{Mo-Analytical Studies \& Natural Products Chemistry I- SL-01 Implementing metabolomic tools in the search for new cytotoxic agents from the endophyte Chaetomium subaffine}

Authors Tabaza $\mathrm{Y}^{1,2}$, Edrada-Ebel RA ${ }^{1}$, Young $\mathrm{L}^{1}$

Institute 1 Strathclyde Institute of Pharmacy and Biomedical Science, 161 Cathedral Street, G4 ORE, Glasgow, United Kingdom; 2 Department of Pharmaceutical Sciences, Faculty of Pharmacy, The University of Jordan, P.O. Box: 11942, Amman, Jordan

DOI 10.1055/s-0037-1608037

In the search for new anticancer agents of natural origin, plant-associated endophytes could be a good source for bioactive secondary metabolites. The endophytic fungus Chaetomium subaffine was obtained from the plant Anthemis palestina and cultured in liquid and rice media for different time periods to optimize its growth and production of compounds employing both NMR and mass spectrometry-based metabolomics. Culturing the fungus on rice gave better yield and more chemically diverse extract. Therefore, this type of medium was chosen for scaling-up purposes. The scaled-up extract was subjected to partitioning between lipophilic and hydrophilic phases. The lipophilic phase which is mainly composed of fatty acids was discarded while the hydrophilic phase was fractioned using a flash-chromatography system and yielded 12 fractions. These fractions were tested in-vitro against both ZR75 and A549 cell lines and analyzed using both proton nuclear magnetic resonance (NMR) and liquid chromatography-high resolution Fourier transform mass spectrometry (LC-HRFTMS). The HRFTMS data was processed with MZmine then subjected to Partial Least Square-Discriminant Analysis (PLSDA). The PLS-DA results pinpointed the biologically active secondary metabolites. Metabolomic-guided isolation work targeted the bioactive secondary metabolites. Six compounds were isolated and elucidated by employing 1D and 2D NMR. Stereochemistry was determined by Mosher's method. These compounds included four new orsillides as well as the already known compounds acremonisol A and cochliodinol.

\section{Mo-Analytical Studies \& Natural Products Chemistry I- SL-02 Discovery of Novel Bioactive Compounds from Rhamnaceae Plants: The Initial Steps}

Authors Kang Kyo B ${ }^{1}$, Park $]^{1}$, Hahn $\mathrm{H}^{1}$, Han Byung $\mathrm{W}^{1}$, Lee $\mathrm{W}^{1}$, Sung Sang $\mathrm{H}^{1}$

Institute 1 College of Pharmacy and Research Institute of Pharmaceutical Sciences, Seoul National University, Seoul, Korea, Republic of (South) DOI $10.1055 / \mathrm{s}-0037-1608038$

The Rhamnaceae family, commonly called the buckthorn family, contains approximately 55 genera and 950 species of flowering plants. Phylogenetic analysis revealed that Rhamnaceae plants can be categorized to three clades, rhamnoid, ampeloziziphoid, and ziziphoid [1]. Enormous phytochemicals including triterpenoids, flavonoids, alkaloids, and quinones have been reported from Rhamnaceae plants. Among these, ceanothane-type triterpenoids have been mainly reported from the ziziphoid species, while quinone derivatives have been isolated from the rhamnoids. Recently, our group has endeavored to establish a chemical library from Rhamnaceae plants and to screen their bioactivities. Herein, the early results of our project will be briefly described. Unprecedented yellow phenyl polyene triglycoside pigments, named berchemin A-C, were isolated from the unripened fruits of Berchemia berchemiifolia. Their chemical structures were established by spectroscopic and chemical analysis. The evaluation of their bioactivities is still ongoing. 
Eighteen new ceanothane- and lupane-type triterpenoids and three novel catechin-bound ceanothane-type triterpenoid derivatives, along with twelve previously reported ones, were isolated from the roots of Z. jujuba [2, 3]. A LCMS dereplication was performed based on them, and six new triterpenoids were additionally isolated from Hovenia dulcis. These triterpenoids were subjected to various bioactivity screening assays. 2-O-phenolic ester derivatives of alphitolic acid inhibited the proteasome activity and effectively induced the killing of human myeloma RPMI8226 cells resistant to carfilzomib, a FDAapproved proteasome inhibitor drug. The results from the molecular docking simulation suggest that the deubiquitinase USP7 is a potential target for these compounds, and further validation efforts are ongoing.

[1] Richardson JE et al, American Journal of Botany 2000; 87: 1309-1324.

[2] Kang KB et al, Journal of Natural Products 2016; 79: $2364-2375$.

[3] Kang KB et al, Journal of Natural Products 2017; 80: 1048- 1054.

\section{Mo-Analytical Studies \& Natural Products Chemistry I- SL-03 Targeted Dereplication of Microbial Natural Products by High-Resolution MS and Predicted LC-Retention Time}

Authors Chervin J $]^{1}$, Stierhof $\mathrm{M}^{1}$, Tong Ming $\mathrm{H}^{1}$, Peace $\mathrm{D}^{1}$, Østnes Hanssen $\mathrm{K}^{3}$, Urgast Dagmar $\mathrm{S}^{1}$, Andersen Jeanette $\mathrm{H}^{3}$, $\mathrm{Yu} \mathrm{Y}^{4}$, Ebel $\mathrm{R}^{1}$, Kyeremeh $\mathrm{K}^{5}$, Paget $\mathrm{V}^{6}$, Cimpan $\mathrm{G}^{6}$, Salbert $\mathrm{T}^{7}$, Deng $\mathrm{H}^{1}$, Jaspars $\mathrm{M}^{1}$, Tabudravu $\mathrm{JN}^{1,2}$ Institute 1 The Marine Biodiscovery Centre, Department of Chemistry, University of Aberdeen, Aberdeen, United Kingdom; 2 School of Biological and Chemical Sciences, Faculty of Science, Technology and Environment, The University of the South Pacific, Suva, Fiji; 3 Marbio, UiT The Arctic University of Norway, Tromsø, Norway; 4 Key Laboratory of Combinatory Biosynthesis and Drug Discovery (Ministry of Education), School of Pharmaceutical Sciences, Wuhan University, Wuhan, China; 5 Marine and Plant Research Laboratory of Ghana, Department of Chemistry, University of Ghana, Accra, Ghana; 6 Advanced Chemistry Development (ACD/Labs) UK Ltd, Bracknell, United Kingdom; 7 Advanced Chemistry Development (ACD/Labs) Germany GmbH, Frankfurt am Main, Germany

DOI 10.1055/s-0037-1608039

A new strategy for the identification of known compounds in Streptomyces extracts that can be applied in the discovery of natural products is presented. The strategy incorporates screening a database of 5.555 natural products including 5.098 structures from Streptomyces sp, using a high throughput LCMS data processing algorithm that utilizes HRMS data and predicted LC retention times (tR) as filters for rapid identification of known compounds in the natural product extract. The database named StrepDB contains for each compound, the structure, molecular formula, molecular mass, and predicted $\mathrm{LC}$ retention time. All identified compounds are annotated and color coded for easier visualization. It is an indirect approach to quickly assess masses (which are not annotated) that may potentially lead to the discovery of new or novel structures. In addition, a spectral database named MbcDB was generated using $\mathrm{ACD} /$ Spectrus DB Platform. MbcDB contains 665 natural products, each with structure, experimental HRESIMS, MS/MS, UV, and NMR spectra. StrepDB was used to screen a mutant Streptomyces albus extract that led to the identification and isolation of two new compounds: legonmaleimides $A$ and $B$, the structures of which were elucidated with the aid of MbcDB and spectroscopic techniques. The structures were confirmed by Computer Assisted Structure Elucidation (CASE) methods using ACD/Structure Elucidator Suite. The developed methodology suggests a pipeline approach to the dereplication of extracts and discovery of novel natural products.

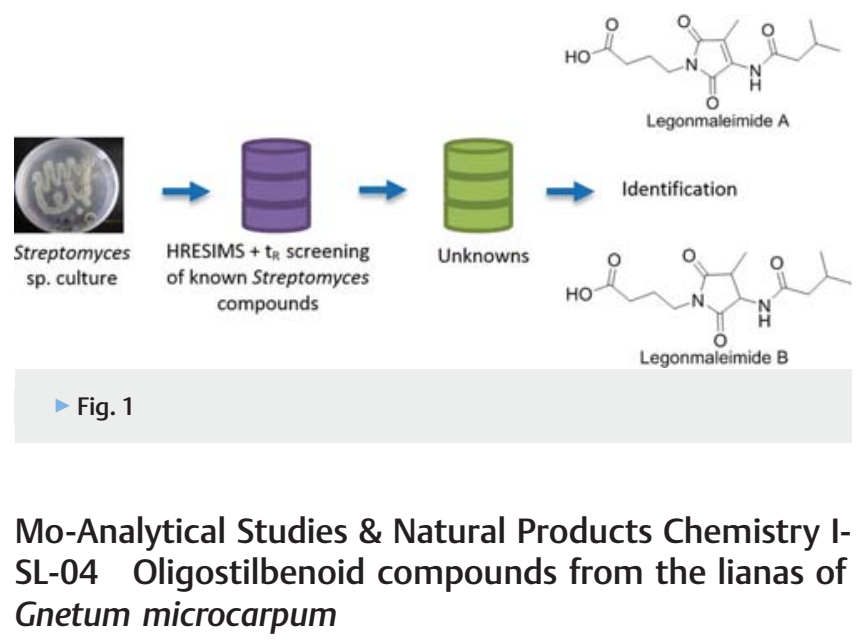

Authors Ahmat $N^{1}$, Nik Azmin Nik $\mathrm{F}^{1}$

Institute 1 Universiti Teknologi MARA, Shah Alam, Malaysia

DOI 10.1055/s-0037-1608040

The family Gnetaceae with a sole genus, Gnetum is well known as a rich source of plant-derived stilbenoids which possess unique structures and multi-faceted biological activities[1]. One of the species, Gnetum microcarpum Blume is a large-fruited species of climber which is reported from western Indochina (Thailand, Myanmar) and Malaya Peninsula to Sumatra[2] and has been found in open margins and canopy of secondary forest, on hill slopes and in heath forest. Gnetum microcarpum Blume is not recorded in folk medicines and previously a new stilbenoid compound has been reported from the lianas of the plant [3]. The isolation and purification was carried out on the lianas of this plant by a combination of repetitive radial and preparative thin layer (pTLC) chromatographic techniques to afford a new oligostilbenoid trimer, compound A (1) which consist of two unit of resveratrol and one unit of oxyresveratrol together with ten known stilbenoids $(2-11)$. Malaysianol D (3) [4], a trimer resveratrol which was previously reported as a new compound from Dryobalanops beccarii by our group was also isolated from Gnetum Microcarpum for the first time in Gnetum plants. The structures of compounds were determined on the basis analysis of spectral evidence and comparison with the published data. The plausible biopathway of the new compound was also proposed. Some of the isolated compounds were tested for DPPH radical scavenging and PGE2 inhibitory activities and their structure-activity relationship study was discussed.
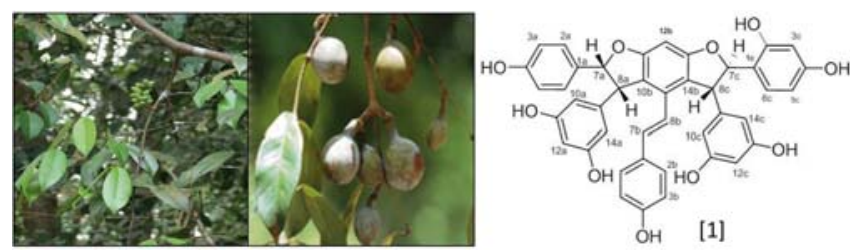

Fig. 1

UniversitiTeknologi MARA is acknowledged for financial and technical support. [1] Sotheeswaran S, Pasupathy, V. Phytochemistry1993, 32:1083-1092.

[2] Azmin NFN, Ahmat N, Syah YM, Zawawi NKNA, Yusof MIM. Natural Product Communications: 2014, 9:1743-1744.

[3] Wibowo A, Ahmat N, Hamzah AS, Latif FA, Norrizah JS, Khong HY, Takayama H. Phytochemistry Letters: 2014,9:117 - 122. 


\section{Mo-Analytical Studies \& Natural Products Chemistry I- SL-05 Utilization of high resolution UPLC-Orbitrap-MS and quantitative UPLC-QqQ-MS/MS technique in proanthocyanidin analysis}

Authors Leppä $\mathrm{M}^{1}$, Karonen $\mathrm{M}^{1}$, Tähtinen $\mathrm{P}^{1}$, Engström $\mathrm{M}^{1}$, Salminen $\mathrm{P}^{1}$ Institute 1 University of Turku, TURKU, Finland DOI 10.1055/s-0037-1608041

Tannins are a group of large secondary metabolites produced by plants. Proanthocyanidins (PAs, i.e. condensed tannins) have the basic flavan-3-ol structure as the core monomeric unit [1,2]. Depending on the flavan-3-ol type, the oligomers and polymers formed are called procyanidins or prodelphinidins, or their mixtures (Fig. 1). The main difference in procyanidins and prodelphinidins is in the hydroxylation degree of the B-ring (Fig. 1). Oligomeric and further polymeric structures can be formed via interflavan bonds either by $\mathrm{C}-\mathrm{C}$ bonds (B-type PAs) or by both $\mathrm{C}-\mathrm{C}$ bonds and additional ether bonds (A-type PAs). These structures may contain only procyanidin units, but also variable numbers of prodelphinidin units. As a result of this structural diversity, proanthocyanidins usually occur as a complex mixture of complex structures in a plant cell.<smiles>Oc1cc(O)c2c(c1)OC(c1cc(O)c(O)c(O)c1)[C@H](O)C2c1c(O)cc(O)c2c1OC(c1ccc(O)c(O)c1)[C@H](O)C2</smiles>

Fig. 1: B-type proanthocyanidin dimer consisting of procyanidin (PC) and prodelphinidin (PD) units.

The fundamental trouble with PA analysis is the fact that all PA molecules cannot be effectively separated from each other by reversed phase liquid chromatography. Instead, PA polymers tend to produce a chromatographic hump that covers tens and hundreds of individual PA structures. In this study we have utilized a new approach in PA analysis by combining UPLC high resolution mass spectrometry and tandem mass spectrometry with a novel PA separation tool in an innovative way. With the new separation tool, we have the opportunity to separate distinguished PA fractions from the complete PA mixture and thus investigate the composition of the PA mixture more accurately than before.

[1] Waterman, P. G. and Mole, S, Oxford, Blackwell Scientific Publications; 1994: 1 - 35

[2] Haslam, E, Cambridge, UK: Cambridge University Press; 2005: 10 - 83.

\section{Mo-Analytical Studies \& Natural Products Chemistry I- SL-06 Metabolomics-based chemotaxonomic classification of the subfamily Barnadesioideae (Asteraceae)}

Authors Ccana-Ccapatinta GV ${ }^{1}$, Padilla-Gonzalez $\mathrm{GF}^{1}$, Monge $\mathrm{M}^{2}$, Semir $]^{2}$, Ferreira $\mathrm{PL}^{3}$, Groppo $\mathrm{M}^{3}$, Da Costa $\mathrm{FB}^{1}$

Institute 1 AsterBioChem Research Team, Laboratory of Pharmacognosy, School of Pharmaceutical Sciences of Ribeirão Preto, University of São Paulo, Ribeirão Preto, Brazil; 2 Department of Plant Biology, Institute of Biology, University of Campinas, Campinas, Brazil; 3 Department of Biology, Faculdade de Filosofia Ciências e Letras de Ribeirão Preto, University of São Paulo, Ribeirão Preto, Brazil

DOI $10.1055 / \mathrm{s}-0037-1608042$
The subfamily Barnadesioideae (Asteraceae) encompasses more than 90 species distributed in nine genera entirely restricted to South America. This group constitutes the earliest diverging lineage of the family Asteraceae[1]. Some reports describe Barnadesioideae as a group with "simple" phytochemistry, and associated this possible character to its basal position[2]. This study aimed to employ untargeted metabolomics as a chemotaxonomic tool to reveal the intergeneric relationships of the subfamily Barnadesioideae and explore its phytochemical composition. Representative samples of six genera of the subfamily Barnadesioideae were processed for obtaining their metabolic fingerprints by LC-ESI-HRMS. Two different extraction solvents and chromatographic methods were tested to assess their impact in the final chemotaxonomic classification of Barnadesioideae. The negative mode datasets were treated for baseline correction, chromatogram deconvolution, peaks alignment, and data normalization before exploratory (HCA and PCA) and supervised (OPLSDA) analyses. The cluster groups observed in the HCA and PCA resembled the intergeneric relationships proposed by Gruenstaeudl et al, 20091 based on morphological and molecular markers, demonstrating that untargeted metabolomics can be used as an auxiliary tool to study the intergeneric relationships of the subfamily Barnadesioideae. Based on the OPLS-DA, phenolic compounds (Fig. 1) were recognized as possible chemotaxonomic markers. Contrary to previous reports, the metabolome annotation of the subfamily Barnadesioideae revealed a complex phytochemical scenario. Furthermore, the extraction solvent and chromatographic elution program displayed a significant impact in the metabolomics-based chemotaxonomic classification of the subfamily Barnadesioideae.
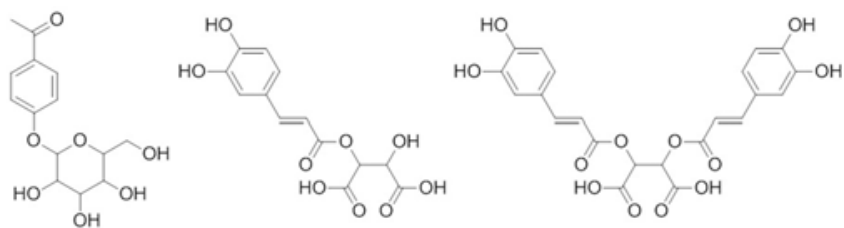

Fig. 1: Phenolic compounds in the subfamily Barnadesioideae

FAPESP (Grants\# 2014/ 16850 - 6 and 2014/26866 - 7) for funding.

[1] Gruenstaeudl M, Urtubey E, Jansen RK et al. Mol Phylogenet Evol 2009; 51:572- 587 .

[2] Calabria LM, Emerenciano VP, Ferreira MJP et al. Nat Prod Commun 2007; 2:277- 285

\section{Mo-Analytical Studies \& Natural Products Chemistry I- SL-07 Sesquiterpenes from Petasites hybridus rhizomes and determination absolute configuration by circular dichroism}

Authors Nejad Ebrahimi $\mathrm{S}^{1}$, Asadi $\mathrm{H}^{1}$, Esmaeili $\mathrm{H}^{3}$, Karami $\mathrm{A}^{3}$, Hadian $\mathrm{J}^{2}$, Hamburger $\mathrm{M}^{5}$, Chaanin $\mathrm{A}^{4}$

Institute 1 Department of Phytochemistry, Medicinal Plants and Drugs Research Institute, Shahid Beheshti University, Tehran, Iran; 2 Department of Agriculture, Medicinal Plants and Drugs Research Institute, Shahid Beheshti University, Tehran, Iran; 3 Department of Horticulture, Faculty of Agriculture, Shiraz University, Shiraz, Iran; 4 VitaPlant AG, Romanshornerstar. 39, CH8592, Uttwil, Switzerland; 5 Pharmaceutical Biology, Department of Pharmaceutical Sciences, University of Basel, Uttwil, Switzerland DOI 10.1055/s-0037-1608043

Petasites hybridus, the butterbur is species belongs to the Asteraceae family is widely vegetated in Europe, Northwest Asia and North America [1]. It has been reported that this plant are treatment of headache, spasm of gastrointestinal, disturbances of the respiratory tract like bronchial asthma and many other illnesses [2]. Phytochemical investigation of n-hexane extract of the rhizome of $P$. hybriduse collected from north part of Iran has been carried out using normal phase column chromatography and RP-HPLC. Three known 
(1-3) together with two new (4-5) sesquiterpenoids have been isolated, purified and identified. The complete structures elucidated by application extensive 1D, 2D NMR and HRMS spectroscopy. Also the absolute configuration of 2-5 were achieved by comparison of experimental ECD spectra with simulated ECD data for possible stereoisomers, by using time dependent density function theory (TDDFT). The results shows that plants growing wild in Iran are new chemo type and these class of compounds are not reported from P. hybridus growing in Europe. Also $P$. hybridus known for presence pyrrolyzidine alkaloids. The amount of these compounds quantified by application of UPLC MRM ESIMS and showed high amount of senecionin- $\mathrm{N}$-oxid and senecionin.
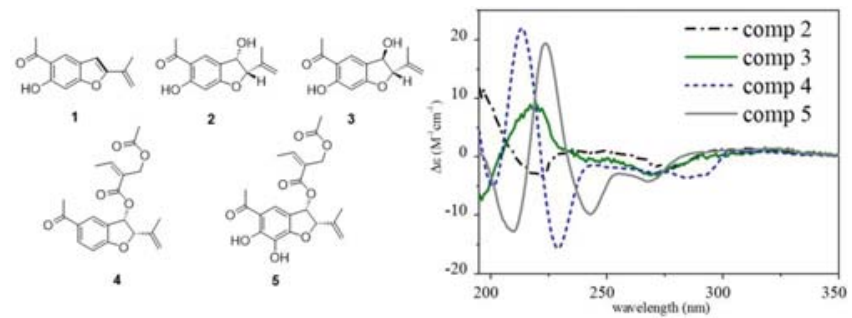

Fig. 1

[1] Khaleghi F, Din LB, Charati FR, Yaacob WA, Khalilzadeh MA, Skelton B, Makha M. Phytochemistry Letters 2011; 4: 254 - 258

[2] Lipton R, Göbel H, Einhäupl K, Wilks K, Mauskop A. Neurology 2004; 63 : $2240-2244$

\section{Mo-Analytical Studies \& Natural Products Chemistry I- SL-08 Metabolomic evidence for allopatric segregation in Espeletiinae (Asteraceae): a new perspective of an Andean adaptive radiation in sky islands}

Authors Padilla-Gonzalez $\mathrm{G}^{1}$, Diazgranados $\mathrm{M}^{2}$, da Costa $\mathrm{F}^{1}$

Institute 1 AsterBioChem Research Team, Laboratory of Pharmacognosy, School of Pharmaceutical Sciences of Ribeirão Preto, University of São Paulo, Ribeirão Preto, SP, Brazil, Ribeirao Preto, Brazil; 2 Natural Capital and Plant Health Department, Millennium Seed Bank, Royal Botanic Gardens Kew, Ardingly, West Sussex, RH17 6TN, UK, West Sussex, United Kingdom DOI 10.1055/s-0037-1608044

The biogeography of subtribe Espeletiinae, Asteraceae (Frailejones) and the effect of altitudinal barriers as evolutionary constrains in the Andes have been the focus of great debate. Several studies suggest that the Andean topography and past climatic history are important events for the development of current plant diversity in South America. Some clear evidence for geographic isolation of Andean-endemic groups has been recently obtained by genetic markers in an attempt to explain the explosive radiation of plant lineages in the páramos: a high altitude ecosystem that biogeographically functions as "sky islands" of open grassland vegetation isolated by deep valleys. The páramos topography along with the Pleistocene climatic fluctuations influenced the migration routes of Espeletiinae through the Andes and determined the evolution of the group, as demonstrated by their molecular phylogeny. However, molecular markers are too coarse to reveal subtle biogeographical trends in Espeletiinae and metabolomic evidence for allopatric segregation in plants has never been reported. In this work, we present for the first time a metabolomics approach based on UHPLC-HRMS and multivariate analyses to reveal subtle biogeographical trends in the diversification of the Frailejones. We used an untargeted metabolomics approach to study the metabolic profile of 210 samples of Espeletiinae belonging to five different genera. We demonstrate that different lineages of Espeletiinae can be distinguished by means of different metabolic profiles correlating to páramo massif of origin on a regional scale and to páramo complex on a local scale. In summary, our results suggest that Espeletiinae lineages modified their metabolic capacities as they evolved independently after the Pleistocene glaciations and that secondary metabolites may have played an important role in the diversification and adaptive success of the Frailejones to the Andean páramos, offering a new perspective of an Andean adaptive radiation in sky islands.

\section{Lecture Session - Phytopharmacology \& \\ Extract Pharmacology I \\ Monday, 4th September, 2017 \\ Singapore $-14: 00-16: 00$}

\section{Mo-Phytopharmacology \& Extract Pharmacology I-SL- 01 Switch to a motile lifestyle: Possible mechanism for antiadhesive effects of Orthosiphon stamineus Benth. aqueous extract against uropathogenic E. coli}

Authors Srashar $S^{1}$, Hensel $A^{1}$, Brandt $S^{1}$, Stork $C^{2}$, Dobrindt $U^{2}$ Institute 1 Institute of Pharmaceutical Biology and Phytochemistry, Corrensstraße 48, D-48149, Muenster, Germany; 2 University of Münster, Institute of Hygiene, Robert-Koch-Str. 41, D-48149, Münster, Germany DOI 10.1055/s-0037-1608045

Adhesion is an essential phase for uropathogenic E. coli (UPEC) to efficiently deploy on host cells. Hence, if bacteria-host cells attachment is inhibited in advance, infection is not productive. Therefore, targeting UPEC adhesion becomes an alternative approach to prevent UTIs. Interfering with adhesive surface structures such as pili, could potentially decrease the bacterial adhesion. This study investigated the mechanism of the antiadhesive potential of a quantified aqueous extract (OWE) from the leaves of O. stamineus against UPEC, considering the effects of OWE on the adhesion-related genes.

OWE was quantified by UHPLC-UV on the content of rosmarinic acid, cichoric acid, and caffeic acid. Under in vitro conditions phenolic compounds depleted OWE (OWEøPC) exerts a dose-dependent antiadhesive activity against different UPEC strains. Furthermore, 3 and 5-day treatment of mice with OWE $(750 \mathrm{mg} / \mathrm{kg}$ ) after transurethral infection with UPEC CFT073 reduced the bacterial load in bladder and kidney, similar to norfloxacin [1].

Transcriptome analysis by next generation sequencing and cross-validation of these data by RT-PCR indicated that OWEøPC down-regulated the genes responsible for chaperone-mediated protein folding/unfolding and pilus assembly process (leading to decrease of porin activity) while flagellar assembly responsive genes were up-regulated as claimed by mRNA-Seq analysis (Fig. 1). So, on one hand OWE increases the motility of UPEC which makes it tougher for bacteria to attach to the host cells and on the other hand OWE disturbs the expression of relevant adhesive proteins. Therefore, it is concluded that OWE transforms the sessile lifestyle of bacteria to a motile one and therefore disables the bacterial surface attachment.

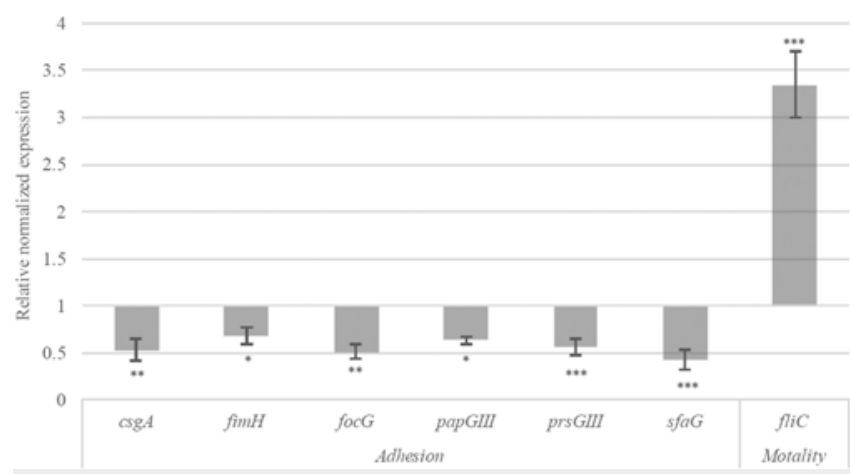

Fig. 1: Relative gene expression of selected virulence factors (VF) from E. coli strain UTI89 determined by Illumina RNA-Seq. considering the effects of $O W E^{\oplus P C}[2 \mathrm{mg} / \mathrm{mL}]$ on VFs regulations after $2 \mathrm{~h}$ incubation in urine. All the expressions are related to untreated control adjusted at one. 
[1] S. Sarshar, S. Brandt, M.R. Asadi Karam, M. Habibi, S. Bouzari, M. Lechtenberg, U. Dobrindt, X. Qin, F.M. Goycoolea, A. Hensel. Phytomedicine 2017; 28 : $1-9$

\section{Mo-Phytopharmacology \& Extract Pharmacology I-SL- 02 Effects of WS ${ }^{\circledR} 1541$ in a transgenic mouse model of benign prostatic hyperplasia (BPH)}

\author{
Authors Pigat $N^{1}$, Koch $E^{2}$, Goffin $\mathrm{V}^{1}$ \\ Institute 1 PRL/GH Pathophysiology Laboratory, Inserm U1151, Institut \\ Necker Enfants Malades, Paris, France; 2 Preclinical Research, Dr. Willmar \\ Schwabe Pharmaceuticals, Karlsruhe, Germany \\ DOI 10.1055/s-0037-1608046
}

WS ${ }^{\circledR} 1541$ (trade product: Prostagutt ${ }^{\circledR}$ forte) is a phytopharmaceutical drug combination containing a lipophilic extract from fruits of Sabal serrulata (WS ${ }^{\circledR}$ 1473 ) and an aqueous ethanolic extract from roots of Urtica dioica (WS ${ }^{\circledR}$ 1031). It is approved in Germany and several other countries for the symptomatic treatment of early stages of BPH in men. The aim of the present study was to investigate whether WS ${ }^{\circledR} 1541$ exerts growth-inhibitory and anti-inflammatory actions in the prostate of Probasin-Prolactin (Pb-PRL) transgenic mice. The overexpression of prolactin in the prostate of these animals is accompanied by several features of the human disease, e. g. tissue hypertrophy, inflammation and lower urinary tract symptoms, indicating that it is a suitable animal model of BPH (Bernichtein et al, Prostate 75:706, 2015).

Male heterozygous Pb-PRL mice at an age of 6 months were randomly distributed (11-12 animals/group) to three doses of WS ${ }^{\circledR} 1541(300,600$, $900 \mathrm{mg} / \mathrm{kg} /$ day), finasteride $(5 \mathrm{mg} / \mathrm{kg} /$ day) or vehicle (olive oil $5 \mathrm{ml} / \mathrm{kg} /$ day) and treated orally for 28 consecutive days. Administration of WS ${ }^{\circledR} 1541$ was well tolerated and caused a dose-dependently reduction of prostate weight that was statistically significant at the two higher doses. While WS ${ }^{\circledR} 1541$ exerted an effect on all three lobes, the 5 -alpha reductase inhibitor finasteride - which is widely used for the treatment of BPH - reduced the weight only of the ventral prostate. The growth-inhibitory activity of WS ${ }^{\circledR} 1541$ was accompanied by an anti-inflammatory action as evidenced by the reduced infiltration of cells expressing the leukocyte common antigen CD 45. In contrast, finasteride significantly increased the inflammatory status in all lobes. Since application of WS ${ }^{\circledR} 1541$ did not interfere with transgene expression, the effects observed are entirely attributable to the intrinsic pharmacological action of the drug combination supporting the established therapeutic use of WS ${ }^{\circledR}$ 1541 in the treatment of $\mathrm{BPH}$.

\section{Mo-Phytopharmacology \& Extract Pharmacology I-SL- 03 Effects of Cimicifuga Extract Ze 450 on mitochondrial resilience}

Authors Rabenau $\mathrm{M}^{1}$, Unger $\mathrm{M}^{2}$, Drewe $\mathrm{J}^{2}$, Culmsee $\mathrm{C}^{1}$

Institute 1 Institute for Pharmacology and Clinical Pharmacy, BiochemicalPharmacological Center Marburg, University of Marburg, Marburg, Germany; 2 Preclinical Research, Max Zeller Soehne AG, Romanshorn, Switzerland DOI 10.1055/s-0037-1608047

Cimicifuga racemosa extract is currently used in the therapy of menopausal symptoms. The mechanisms of Cimicifuga extract underlying its multiple therapeutic effects are not well defined. Recent studies showed that Cimicifuga extract Ze 450 activates AMP-activated protein kinase suggesting beneficial metabolic effects, e.g. for the treatment of type II diabetes [1]. In the pathogenesis of metabolic, immunological and age-related diseases, mitochondrial dysfunction is an emerging focus of research, since these organelles are key regulators of energy metabolism and cellular homeostasis. Increasing evidence suggests that mitochondrial resilience can provide protection against age-related pathologies [2].

In this study, we investigated the effects of Ze $450(1-200 \mu \mathrm{g} / \mathrm{ml})$ on mitochondrial integrity and function, and cell viability in models of oxidative stress in neuronal cell lines and in liver cells. The effects of Ze 450 in control condi- tions and after oxidative stress were analyzed using FACS for detecting lipid peroxidation (BODIPY), mitochondrial ROS formation (MitoSOX) and cell death (AnnexinV/PI staining). Furthermore, we determined ATP levels and mitochondrial respiration (oxygen consumption rates; Seahorse).

In the models of oxidative stress, Ze $450(50-200 \mu \mathrm{g} / \mathrm{ml})$ preserved mitochondrial morphology, ATP levels, and prevented mitochondrial ROS formation and cell death in neuronal cells. Further, cell death was remarkably inhibited by Ze 450 in a dose-dependent manner in liver cells, whereas under standard culture conditions Ze 450 itself did not affect liver or neuronal cell viability.

In conclusion, our data show that Cimicifuga extract Ze 450 did not affect cell viability under control conditions and supported mitochondrial resilience and preserved cell viability in neuronal and liver cells exposed to oxidative stress. Therefore, Ze 450 offers promising therapeutic potential in ageing processes and related diseases involving mitochondrial damage and increased ROS formation.

[1] Moser C. et al, Phytomedicine. 2014; 21: 1382 - 1389

[2] Held N.M. et al, Bioessays. 2015; 37: $867-876$

\section{Mo-Phytopharmacology \& Extract Pharmacology I-SL- 04 Eurycoma longifolia Jack and Its Quassinoids Stimulate Lipolysis in 3T3-L 1 Adipocytes}

Authors Lahrita $L^{1}$, Hirosawa $R^{1}$, Kato $E^{1}$, Kawabata J

Institute 1 Hokkaido University, Sapporo, Japan

DOI 10.1055/s-0037-1608048

In recent years, the prevalence of obesity has increased globally, due to changes in lifestyle and eating habits. Medicinal plants from local biodiversity in Southeast Asian region represent one of the most promising resources to address this prevailing metabolic disease. This study aims to investigate antiobesity property of Eurycoma longifolia Jack and elucidate the active compounds, as well as to demonstrate their cellular mechanism of actions.

Fifty percent methanolic extract of E. longifolia was tested for its activity to reduce lipid accumulation in 3T3-L 1 adipocytes. While, measurement of glycerol released from the cells to culture medium was conducted to investigate lipolysis stimulation. The results showed that the E. longifolia extract reduced lipid accumulation and increased glycerol release in the adipocytes. Isolation of active compounds was then conducted based on the stimulation of glycerol release. Two active compounds were successfully isolated and identified as Eurycomanone and 13ß,21-epoxyeurycomanone, unique quassinoids contained in E. longifolia.

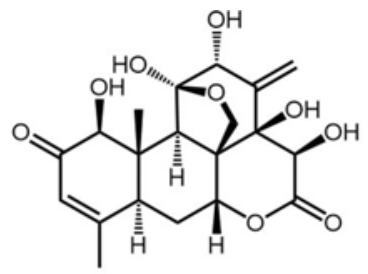

Eurycomanone (1)

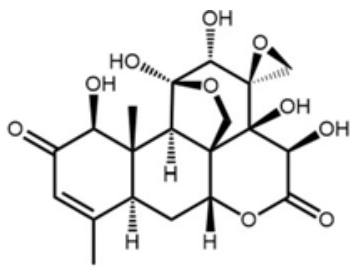

13ß,21-epoxyeurycomanone (2)
Fig. 1

To investigate the mechanism of actions, the extract of $\mathrm{E}$. longifolia and the isolated compounds were subjected to inhibitory challenges on the stimulation of glycerol release. Inhibitory challenges demonstrated that the extract stimulated lipolysis through CAMP-dependent protein kinase (PKA) and extracellular signal-regulated kinase (ERK) pathways, while the quassinoids worked through PKA pathway. Western blotting analysis of these potential kinases also confirmed the activation of PKA and ERK by the extract, and PKA by the isolated quassinoids.

These findings provide scientific basis of lipid reduction activity of plant-based traditional medicine, E. longifolia. Since this plant species has not been exten- 
sively studied for its anti-obesity property, this work promotes further investigation for its use in the treatment of obesity.

Lahrita L, Kato E, Kawabata J. J Ethnopharmacol 2015; 168, 229 - 236.

\section{Mo-Phytopharmacology \& Extract Pharmacology I-SL- 05 Anti-hypertensive effect of Moringa oleifera}

Authors Hernandez $\mathrm{C}^{1}$, Acuram Lovely $\mathrm{K}^{1}$

Institute 1 Institute of Chemistry, University of the Philippines Diliman,

Quezon City, Philippines

DOI 10.1055/s-0037-1608049

Hypertension is the fifth leading cause of morbidity according to the Department of Health's statistics. Untreated hypertension may lead to stroke, blindness, heart attack, kidney and heart failure. ${ }^{1}$ However great the success in the therapy of hypertension has been gained today by recent drugs, the definite healing of patients has not yet been attained. ${ }^{2}$ Plants continue to be an excellent source of lead compounds. Previous efforts in our laboratory lead to the isolation of bioactive compounds. ${ }^{3}$ Our results established the antihypertensive activity of $M$. oleifera using the angiotensin-converting enzyme or ACE inhibitory assay and revealed the concentration of the activity in the ethyl acetate portions. Two flavonoid glycosides were reported to have significant \%inhibition, comparable with the positive control captopril. The results obtained in vivo indicated that mice treated with crude methanol and ethyl acetate extracts of $M$. oleifera significantly decrease the systolic blood pressure of $\mathrm{N}^{\omega}$-nitro- ${ }^{-}$-arginine methyl ester or L-NAME induced-hypertensive mice. In either case, the effect was established after four weeks of treatment and there was no significant difference in BP values between high $(0.3 \mathrm{~g} / \mathrm{kg} /$ day) and low $(0.01 \mathrm{~g} / \mathrm{kg} /$ day $)$ doses. The study shows that $M$. oleifera extracts are effective in the inhibiton of two major pathways in hypertension study which are correlated to ACE inhibition and to nitric oxide synthesis. The results of this study support that $M$. oleifera extracts can be developed as a potential antihypertensive health product.

[1] Chobanian AV, et al. JAMA 2003.

[2] Petkov, V. Am J Chin Med 1979; 7:197- 236.

[3] Chichioco-Hernandez C, Villasenor I, Schroeder F, Paulus H, Clardy J. J Herbs Spices Med Plants 2016; 22: 94 - 102.

\section{Mo-Phytopharmacology \& Extract Pharmacology I-SL-} 06 Complex data in herbal medcinal product research: Multi-step clustering of preclinical data for a herbal combination, STW 5

Authors Abdel-Aziz $\mathrm{H}^{1}$, Lorkowski $\mathrm{G}^{2}$, Kelber $\mathrm{O}^{3}$

Institute 1 Medical and Clinical Affairs Phytomedicines, Innovation and

Development, Phytomedicines Supply and Development Center, Bayer

Consumer Health Division, Steigerwald Arzneimittelwerk GmbH, Darmstadt, Germany, Darmstadt, Germany, Darmstadt, Germany; 2 GL Pharma

Consulting Research \& Development, Gauting, Germany; 3 Innovation and

Development, Phytomedicines Supply and Development Center, Bayer

Consumer Health Division, Steigerwald Arzneimittelwerk GmbH, Darmstadt, Germany; 4 Pharmaceutical Institute, Leipzig University, Leipzig, Leipzig,

Germany; 5 Center for Endoscopy, Starnberg, Germany

DOI 10.1055/s-0037-1608050

A multitude of causes and likewise also targets for therapeutic interventions have been identified in functional gastrointestinal diseases (FGDs) [1]. Therefore, a multi-target approach is a promising therapeutic strategy, as is exemplified by the herbal combination medicinal product STW 5 (Iberogast), which has been proven to be effective in a large number of randomized controlled clinical studies, while likewise exhibiting a highly favourable safety profile [2]. The fact, that STW 5 consists of nine plant extracts, makes the combination an ideal candidate for the use of the newly described method of step-wise cluster analysis, a standardized procedure to transfer heterogeneous pharmacological data, from different models, into effect size categories.
This method is based on a step-wise cluster formation starting from the level of single tests up to the level of different pathomechanisms involved in the development of a certain disease, in this case functional dyspepsia (FD) and irritable bowel syndrome (IBS).

The result is based on an overview on the pharmacological data on STW 5 and its single components, analysed using step-wise cluster formation. The different modes of action address multiple etiologic factors involved in functional dyspepsia and irritable bowel syndrome, like hyper- and hypomotility, acidity, inflammation and hypersensitivity, and also inflammatory gastrointestinal diseases. The results on STW 5 and the contribution of the single constituents to the overall multi-target action of this herbal combination preparation are evaluated and visualized by 2D histograms.

Thus, by making the allocation of specific actions to the different components of STW 5 manageable, the results also give support to the clinical use of the combination in patients with different symptom clusters.

[1] Allescher et al. 2006, Phytomedicine 13 SV: 2 -11;

[2] Storr et al. 2016, Pharmakon 4: 356 - 364

\section{Mo-Phytopharmacology \& Extract Pharmacology I-SL- 07 Modulation of Neurological Pathways through Salvia officinalis depends on manufacturing process and plant parts used.}

Authors Tober $\mathrm{C}^{1}$, Schoop $\mathrm{R}^{2}$

Institute 1 Rent-a-lab, Reutlingen, Germany; 2 A. Vogel Bioforce AG, Roggwil (TG), Switzerland

DOI 10.1055/s-0037-1608051

Hot flushes and excessive sweating are cardinal post-menopausal symptoms which occur with hormonal fluctuations during climacterium. Frequency and severity of complaints however correlate with presence of neurotransmitters rather than estrogen levels in blood and serum. Neurogenic processes are not only involved in thermoregulation by the hypothalamus and sweating, but also in mood and cognitive impairment.

Salvia officinalis has traditionally been used for the treatment of extensive sweating and hot flushes, it reduced intensity and frequency of hot flushes significantly in clinical trials. The mode of action of Salvia is still unclear and we investigated Menosan ${ }^{\circledR}$, a hydroalcoholic extract from freshly harvested Salvia off. leaves and manufacturing variants thereof on receptors and transporters involved in thermoregulation.

Competition binding of Menosan ${ }^{\circledR}$ with specific radioligands was measured at recombinant human receptors and findings confirmed on native receptors in hypothalamic tissue originating from 37- and 51-year old women. High binding affinities (low $\mathrm{IC}_{50}$-values = dry mass concentration to reduce the specific binding of the radioligand by $50 \%$ ) were observed for adrenergic $\alpha_{2 A}$ receptors $\left(\mathrm{IC}_{50}=13 \mu \mathrm{g} / \mathrm{ml}\right) ; \mu$-opioid receptors $\left(\mathrm{IC}_{50}=18 \mu \mathrm{g} / \mathrm{ml}\right)$; muscarinic $\mathrm{M} 3$ receptors $\left(\mathrm{IC}_{50}=28 \mu \mathrm{g} / \mathrm{ml}\right)$ and serotonin $5-\mathrm{HT}_{1 \mathrm{~A}}$ receptors $\left(\mathrm{IC}_{50}=22 \mu \mathrm{g} / \mathrm{ml}\right)$. In addition, Serotonin re-uptake was inhibited with an $\mathrm{IC}_{50}$-value of $32 \mu \mathrm{g} / \mathrm{ml}$. Usage of freshly harvested Salvia leaves resulted in a much higher binding affinity compared to material from dried plants or stipes, e.g. at the adrenergic $\alpha_{2 \mathrm{~A}}$ receptor an about 4 -fold higher binding affinity was measured.

In conclusion, we suggest modulation of receptors (adrenergic, serotonergic, muscarinic and $\mu$-opioid) and transporters (Serotonin reuptake) involved in thermo- and mood-regulation as mode of action for the effectiveness of Salvia off. In addition, we provide evidence that the production process (i. e. usage of fresh Salvia off. leaves) is crucial for maximal activity. 
Poster Session

Monday, 4th September, 2017

San Francisco - 16:00 - 18:00

\section{Mo-Poster Session 1-PO-02 Cryptotanshinone from Salvia miltiorrhiza Bunge lowers the Cytokeratin 1/10 expression in primary human Keratinocytes in vitro}

Authors Esch $S^{1}$, Hensel $A^{1}$, Brandt $S^{1}$, König $S^{2}$

Institute 1 University of Münster, Institute of Pharmaceutical Biology and Phytochemistry, Münster, Germany; 2 University of Münster, Interdisciplinary Centre for Clinical Research, Core Unit Proteomics, Münster, Germany DOI 10.1055/s-0037-1608052

Salvia miltiorrhiza Bunge (danshen) is commonly used in TCM to treat vascular and coronary heart diseases and is also applied in psoriasis therapy. Cryptotanshinone, one out of four characteristic tanshinones found in danshen, shows an activity versus hypertrophic scars [1]. This inspired subsequent research on potential effects of $S$. miltiorrhiza extract and cryptotanshinone on the differentiation process of primary human keratinocytes (NHEK).

A quantified EtOH $50 \%$ extract from S. miltiorrhiza roots $(50 \mu \mathrm{g} / \mathrm{mL})$ reduced cytokeratin 10 (KRT10) expression in NHEK over seven days nearly to the limit of detection (Western blot analysis), whereas involucrin (IVL) expression remained unchanged. This dramatic changes in keratinocyte differentiation by the extract could be assigned to the presence of cryptotanshinone, which suppresses the KRT1/10 expression at $1 \mu \mathrm{M}$ nearly completely. This was also proven by gene expression analysis by qPCR: The gene expression for CK1 / 10 after $60 \mathrm{~h}$ under the influence of cryptotanshinone $(1 \mu \mathrm{M})$ was reduced to $>90 \%$.

At higher concentrations crytotanshinone influenced cell vitality negatively (MTT vitality assay $\mathrm{IC}_{50}=14.4 \mu \mathrm{M}$, BrdU proliferation assay $\mathrm{IC}_{50}=8.2 \mu \mathrm{M}$ ).

To determine possible target structures in the differentiation signaling pathway, Drug Affinity Responsive Target Stability (DARTS) was used. By comparing the protein stability of $\mathrm{HaCaT}$ total cell lysate versus thermolysin with and without cryptotanshinone preincubation and consecutive protein sequencing, heat shock protein 90 was identified as a potential major molecular target for cryptotanshinone. An inhibition of this chaperon is known to result in reduced KRT1/10 expression [2]. From these data the use of cryptotanshinone and S. miltiorrhiza extract for skin diseases seems to be rationalized.

[1] Qi Ye et al. Evidence-Based Complementary and Alternative Medicine

[2] Sadanori Miyoshi et al. FEBS openbio

\section{Mo-Poster Session 1-PO-03 How natural products act on molecular level: understanding the effect of cryptotanshinone on keratinocyte differentiation using $\mathrm{qPCR}$, DARTS and proteomics}

\footnotetext{
Authors Esch $S^{1}$, Hensel $A^{1}$, Brandt $S^{1}$, König $S^{2}$

Institute 1 University of Münster, Institute of Pharmaceutical Biology and Phytochemistry, Münster, Germany; 2 University of Münster, Interdisciplinary Centre for Clinical Research, Core Unit Proteomics, Münster, Germany DOI 10.1055/s-0037-1608053

Cryptotanshinone (CTS), one of the main compounds from the roots of Salvia miltiorrhiza Bunge was investigated on its potential influence on the differentiation process of human keratinocytes (NHEK) in order to rationalize the traditional use of this plant for psoriasis treatment.

In vitro studies indicated strong influence of CTS $(1 \mu \mathrm{M})$ on keratinocyte differentiation: CTS suppresses the differentiation-specific keratins KRT1/10 nearly completely on protein level. Gene expression analysis by qPCR proved $>90 \%$ reduction of $\mathrm{CK} 1 / 10$ after $60 \mathrm{~h}$.

At higher concentrations CTS influenced cell vitality negatively (vitality assay $\mathrm{IC}_{50}=14.4 \mu \mathrm{M}$, proliferation assay $\left.\mathrm{IC}_{50}=8.2 \mu \mathrm{M}\right)$.
}

For pinpointing the signaling pathways induced by CTS $(1 \mu \mathrm{M})$ qPCR screening plates (BioRad) and CDNA from NHEK, treated with CTS $(1 \mu \mathrm{M})$ for $60 \mathrm{~h}$, were used. As expected distinct pathways towards keratin expression were reduced, indicating that this kind of qPCR screening plates can be a powerful tool for identifying signaling pathways.

To determine possible target structures in the differentiation-specific signaling pathways, Drug Affinity Responsive Target Stability (DARTS) in combination with proteomic approach was used. Stability of proteins, obtained from a total cell lysate from $\mathrm{HaCaT}$ keratinocytes with and without CTS treatment, versus the proteases thermolysin and pronase, was determined by using colloidal Coomassie staining. This method allows a distinguished and sensitive protein staining with low background and no interference with the subsequent protein sequencing. The full proteome analysis leads to the identification of Heat shock protein 90 as a possible major molecular target for CTS. An inhibition of this chaperon is known to result in reduced KRT1/10 expression [1]. From these data the use of CTS for skin diseases like lamellar ichthyosis seems to be rationalized.

[1] Sadanori Miyoshi et al. FEBS openbio

\section{Mo-Poster Session 1-PO-04 $\mathrm{Y}$-Propoxy-sulfo-lichenin, a semisynthetic polysaccharide of Lichenan shows strong activity in keratinocyte differentiation}

Authors Esch $S^{1}$, Hensel $A^{1}$, Brandt $S^{1}$

Institute 1 University of Münster, Institute of Pharmaceutical Biology and Phytochemistry, Münster, Germany

DOI 10.1055/s-0037-1608054

$\mathrm{Y}$-Propoxy-sulfo-lichenin ( $\mathrm{Y}$-PSL) is a semisynthetic polysaccharide obtained by etherification of lichenan, a $\beta-1,3 / 1,4$-glucan from Cetraria islandica $L$. and propansulton. Substitution of the glucan with 3-propoxy sulfuric acid was shown by NMR studies predominantly at position 0-2.

$\mathrm{Y}$-PSL did not show any signs of toxicity against human kerationcytes up to $100 \mu \mathrm{g} / \mathrm{mL}$ (MTT vitality assay, $\mathrm{IC}_{50}=143 \mu \mathrm{g} / \mathrm{mL}$ ), but significantly increased keratinocyte differentiation: the typical differentiation-specific proteins cytokeratin $1 / 10$, involucrin and loricrin were upregulated by $\mathrm{Y}$-PSL. This induction of differentiation was much stronger compared to the unsubstituted lichenan [1].

Also on gene level stimulation of cell differentiation was observed. An increased gene expression of Cytokeratin $1 / 10$, involucrin, fillagrin, loricrin and transglutaminase 1 could be proven by time-dependent qPCR up to 60 hours. By use of qPCR screening plates for keratinocyte differentiation signalling (Bio-Rad) $y$-PSL was characterized to act via calcium depended signalling pathway towards a changed differentiation process. Cell cycle analysis was not influenced by $\mathrm{Y}$-PSL. Scratch assay over $72 \mathrm{~h}$ did not indicate a significant influence of $Y$-PSL $(100 \mu \mathrm{g} / \mathrm{mL})$ on cell migration behaviour.

Summarizing $Y$-PSL is a strong and specific inductor of keratinocyte differentiation, rationalizing the potential use of this polysaccharide for improved wound healing and specific directed differentiation. $y$-PSL is significantly more potent in this indication as unsubstituted lichenan, which is traditionally used for its moderate activity for treatment of irritated skin and epithelia.

[1] D. Zacharski (2014) Lichenan from Cetraria islandica (L.) ACH. and xyloglucan from Tropaeolum majus $\mathrm{L}$. as inductors of cellular differentiation 


\section{Mo-Poster Session 1-PO-05 Molecular rationalization of antioxidant properties of bioactive compounds from Aerides rosea Lodd. ex. Lindl. \& Paxton (Orchidaceae)}

Authors Cakova $\mathrm{V}^{1,2}$, Ossman $\mathrm{T}^{3}$, Rajnochova Svobodova $\mathrm{A}^{4}$, Vostalova $\mathrm{J}^{4}$ Delecolle $J^{5}$, Groh $C^{5}$, Bonté $F^{1}$, Heintz $D^{5}$, Ulrichova $J^{4}$, Trouillas $\mathrm{P}^{3,6}$, Lobstein $\mathrm{A}^{2}$

Institute 1 LVMH Recherche, Saint Jean de Braye, France; 2 Université de Strasbourg, CNRS, LIT UMR 7200, Illkirch Graffenstaden, France; 3 UMR 850, INSERM, Univ. Limoges, Faculty of Pharmacy, Limoges, France; 4 Department of Medical Chemistry and Biochemistry, Faculty of Medicine and Dentistry, Palacký University, Olomouc, Czech Republic; 5 Institut de Biologie

Moléculaire des Plantes, Plateforme métabolomique, CNRS-UPR 2357, Strasbourg, France; 6 RCPTM, Faculty of Science, Palacký University, Olomouc, Czech Republic

DOI 10.1055/s-0037-1608055

Dihydrophenanthrene derivatives (imbricatin and methoxycoelonin), bibenzyls (gigantol and batatasin III), and dihydroconiferyl dihydro-p-coumarate (DDPC) were isolated for the first time from the stems of a tropical orchid Aerides rosea (Figure 1). Imbricatin, methoxycoelonin and gigantol have been shown to display in vitro antioxidant and anti-inflammatory activities [1]. However, up to now, their redox reactivity was insufficiently characterized.

The in vitro antioxidant activity of the isolated compounds is described as their capacity i) to scavenge the $\mathrm{DPPH}$ and $\mathrm{ABTS}^{+\cdot}$ free radicals and ii) to inhibit $t$ butyl hydroperoxide-induced lipid peroxidation (LPx). Quantum chemistry calculations (based on Density Functional Theory) were achieved to predict thermodynamic descriptors, mainly O-H bond dissociation enthalpies (BDEs). Molecular dynamics simulations were used to rationalize the differences in activity, in particular to predict the capacity of the compounds to insert lipid bilayers [2].

Imbricatin and methoxycoelonin exhibited relatively low BDE values, providing great free radical scavenging capacity. With relatively deep insertion inside lipid bilayer membranes, they allow efficient inhibition of the propagation stage of LPx. DDPC was confirmed to be an efficient free radical scavenger. However, it was less efficient as LPx inhibitor than the two dihydrophenanthrenes. This is possibly due to the negative charge and the pKa values which may partially prevent insertion into lipid bilayer. Despite exhibiting a relatively low O-H BDE value (so a relatively low $\mathrm{IC}_{50}$ in DPPH scavenging), gigantol was a less efficient LPX inhibitor than batatasin III. This was attributed to a deeper insertion inside lipid bilayer of the latter compared to the former derivative. These new data allowed to establish clear structure-activity relationships in terms of free radical scavenging of studied compounds, also shedding light on their mechanisms of action.

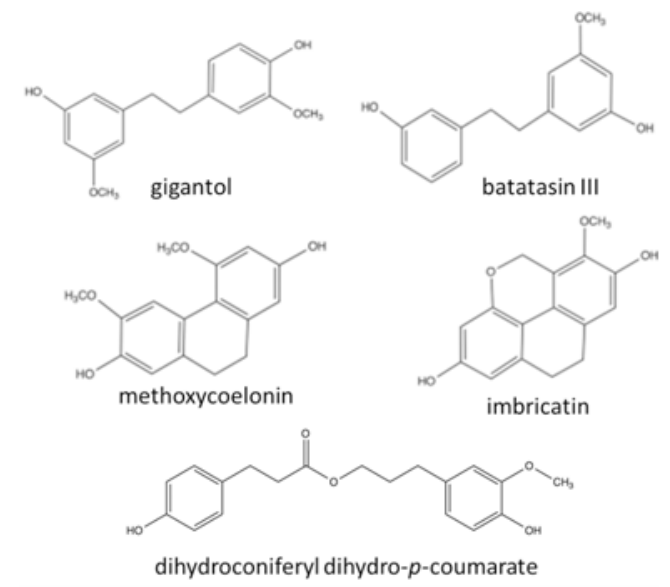

Fig. 1
[1] Simmler C. et al. Plos One 2010, 5:e13713.

[2] Anouar el H. et al. J Comput Aided Mol Des 2013, 27:951 - 64

\section{Mo-Poster Session 1-PO-06 Bioactive Compounds and Antioxidant in Hibiscus sabdariffa Leaf}

\section{Authors Rithichai $\mathrm{P}^{1}$, Jirakiattikul $Y^{1}$, Sriboonthai $C^{1}$, Itharat $A^{2}$} Institute 1 Division of Agricultural Technology, Faculty of Science and Technology, Thammasat University, Rangsit Campus, Pathumthani, Thailand; 2 Department of Applied Thai Traditional Medicine, Faculty of Medicine, Thammasat University, Rangsit Campus, Pathumthani, Thailand DOI 10.1055/s-0037-1608056

The Hibiscus sabdariffa leaf is usually discarded, but some countries consume it as vegetable. Many studies have demonstrated that an extract of $H$. sabdariffa leaves shows several bioactivities including antioxidant, antihyperlipidemic, anti-inflammation, anti-atherosclerotic and anti-proliferation [1, 2]. The purpose of this study was to determine bioactive compounds and antioxidant in the leaf of $\mathrm{H}$. sabdariffa. The mature leaves of fourteen accessions were collected at 70 days after sowing. According to leaf morphology, two clusters were classified as nine accessions containing medium leaf lobing and five accessions having deep leaf lobing. Both clusters exhibited the similar contents of bioactive compounds and antioxidant capacities. Total phenolic and carotenoid contents were $55.77 \pm 4.02 \mathrm{mg} \mathrm{GAE} / \mathrm{g}$ dry extract and 6.66 $\pm 1.15 \mu \mathrm{g} / \mathrm{g}$ dry weight in the medium leaf lobing cluster, $54.27 \pm 4.31 \mathrm{mg}$ GAE/g dry extract and $7.01 \pm 1.93 \mu \mathrm{g} / \mathrm{g}$ dry weight in the deep leaf lobing group, respectively. The antioxidant activity was measured by DPPH radical scavenging assay, giving $58.08 \pm 2.41$ and $59.42 \pm 2.67 \%$ scavenging activities in the medium and deep leaf lobing clusters, respectively.

[1] Chen JH, Wang C], Wang CP, Sheu JY, Lin CL. Food Chemistry. 2013; 141: $397-406$.

[2] Zhen J, Villani TS, Guo Y. Qi Y, Chin K, Pan MH, Ho CT. Food Chemistry. 2016; 190: $673-680$.

\section{Mo-Poster Session 1-PO-07 New Cembranoids from the Formosan Soft Coral Lobophytum sp. and Their Anti-Inflammatory Activity}

Authors Su JH $\mathrm{H}^{1,2}$, You WJ ${ }^{1,2}$, Liu $\mathrm{Cl}^{3}$, Chao $\mathrm{YH}^{4}$

Institute 1 National Museum of Marine Biology \& Aquarium, Pingtung,

Taiwan; 2 Graduate Institute of Marine Biology, National Dong Hwa University, Pingtung, Taiwan; 3 Department of Nursing, Meiho University, Pingtung,

Taiwan; 4 Institute of Biomedical Science, National Chung Hsing University, Taichung, Taiwan

DOI 10.1055/s-0037-1608057

Three new cembranoids, lobophylides A-C (1-3), along with three known compounds (4-6) were isolated from the Formosan soft coral Lobophytum $\mathrm{sp}$. The structures of these compounds were elucidated by means of IR, MS and NMR techniques and comparison of the NMR data with those of known analogues. Among these natural products, compound 2 is rarely found in cembrane diterpenes possessing an isopropyl moiety with an epoxide group. Cytotoxicity study of 1-6 against the growth of K562, Molt-4 and HL-60 cancer cell lines showed that compounds 1-6 were not cytotoxic (IC $50>20 \mu \mathrm{g} / \mathrm{mL}$ ) toward the above three cancer cell lines. The in vitro anti-inflammatory effects of compounds 1-5 were also tested. The results showed that compounds 2 and 3 significantly suppressed the accumulation of pro-inflammatory proteins, iNOS and COX-2. 

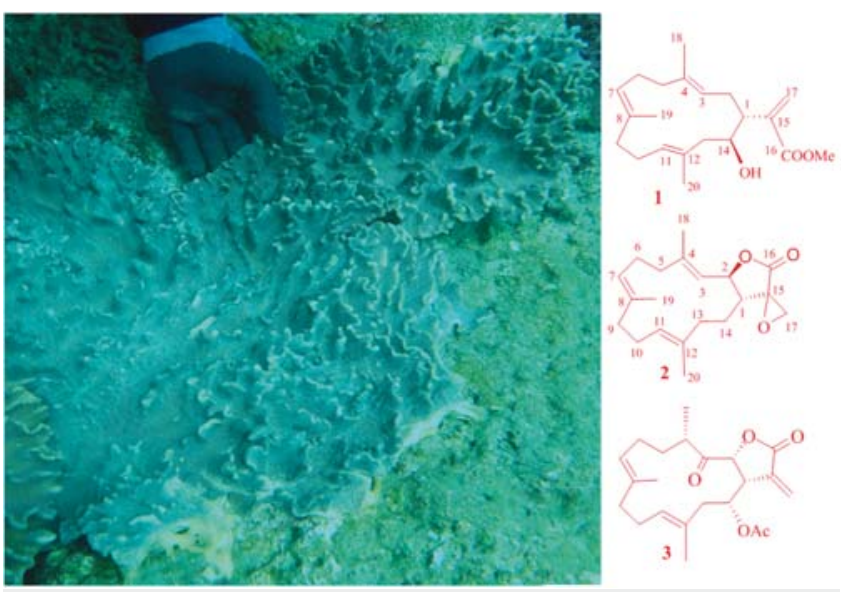

Fig. 1
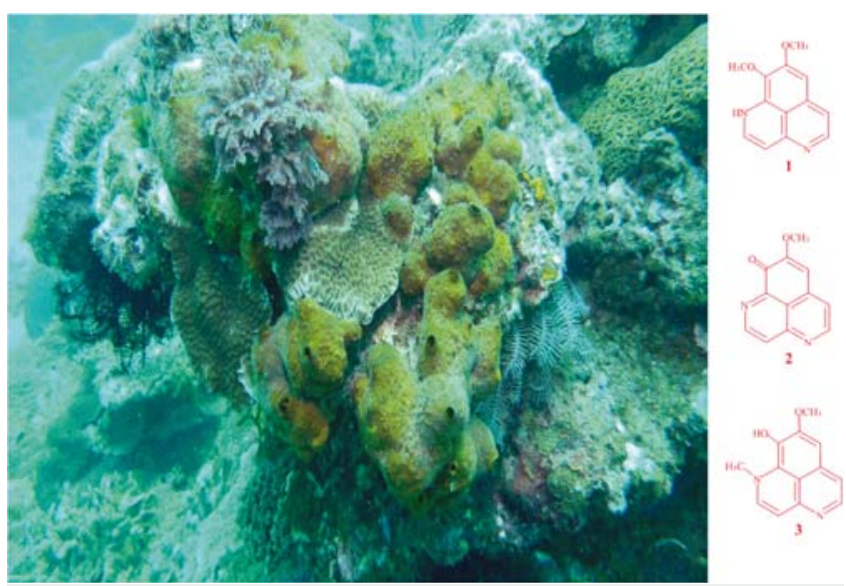

Fig. 1

\section{Mo-Poster Session 1-PO-08 Isoaaptamine Induces t-47D Cells Apoptosis and Autophagy via Oxidative Stress}

Authors Su $\mathrm{JH}^{1,2}$, Shih $\mathrm{SP}^{1,2}$, Chen $\mathrm{YC}^{1,2}$, Sung PJ ${ }^{1,2}$, Lu $\mathrm{MC}^{1,2}$, Chiu $\mathrm{CW}^{3}$, Wu YC ${ }^{4}$

Institute 1 Graduate Institute of Marine Biology, National Dong Hwa University, Pingtung, Taiwan; 2 National Museum of Marine Biology and Aquarium, Pingtung, Taiwan; 3 Department of Marine Biotechnology and Resources, National Sun Yat-sen University, Kaohsiung, Taiwan; 4 Graduate Institute of Natural Products, Kaohsiung Medical University, Kaohsiung, Taiwan DOI 10.1055/s-0037-1608058

Bioactivity-guided fractionation of the marine sponge Aaptos sp. extract led to the isolation of aaptamine (1), demethyloxyaaptamine (2) and isoaaptamine (3). The cytotoxic activity of the isolated compounds (1-3) was evaluated revealing that compound 3 was the most potent cytotoxic against breast cancer T47D cells. In a dose dependent manner, 3 inhibited the growth of T47D cells as indicated by the short- (MTT) and long-term (colony formation) assays. The cytotoxic effect of $\mathbf{3}$ was mediated through apoptosis which was suggested by DNA ladder formation, caspase-7 activation, XIAP inhibition and PARP cleavage. Furthermore, TEM and flow cytometric analysis using acridine orange dye indicated that that 3 treatment could induce t-47D cells autophagy. Immunoblot assays demonstrated that 3 treatment significantly activated autophagy marker proteins such as the increase in Type II LC-3. In addition, 3 treatment enhanced the activation of DNA damage ( $\mathrm{yH} 2 \mathrm{AX})$ and ER stress-related proteins (IRE1aand BiP). Moreover, the use of 3 resulted in a significant increase in the generation of reactive oxygen species (ROS) as well as in the disruption of mitochondrial membrane potential (MMP). The pretreatment of t-47D cells with an ROS scavenger, NAC, attenuated apoptosisand MMP disruption-induced by 3 up to $90 \%$.Taken together, these findings suggest that the anticancer effect of 3 is associated with the induction of apoptosis and autophagy through oxidative stress. Accordingly, our data indicated that 3 exhibited as an outstanding lead for the development of marine derived anti-breast cancer agent.

\section{Mo-Poster Session 1-PO-09 Cytotoxic proteins from the seeds of Gypsophila elegans M. Bieb}

Authors Weng $A^{1}$, Keresztes $F^{1}$, Weise $C^{2}$, Kokorin $A^{1}$, Melzig $M^{1}$ Institute 1 Institut für Pharmazie, Königin-Luise-Str. 2+4, Freie Universität Berlin, Germany, Berlin, Germany; 2 Institut für Chemie und Biochemie, Thielallee 63, Freie Universität Berlin, Germany, Berlin, Germany DOI 10.1055/s-0037-1608059

A considerable number of plants from the carnation family (Caryophyllaceae) are known to contain $\mathrm{N}$-glycosidases with cytotoxic properties. This study was intended to investigate the cytotoxic activity of a seed extract from Gypsophila elegans M. Bieb. (Caryophyllaceae). For this purpose, Gypsophila elegans M. Bieb was cultured and seed material was harvested in autumn 2015. Seeds were grinded and defatted by Soxhlet extraction using petrol ether. The defatted seed material was extracted by phosphate buffered saline (PBS). The seed extract showed enzymatic activity against nucleic acid resulting in the fragmentation of hering sperm DNA. In combination with an isolated and characterized triterpenoid saponin from Saponaria officinalis L. the seed extract exhibited a strong cytotoxic activity against glioblastoma cells. This was demonstrated by MTT-based endpoint as well as by impedance- based live cell imaging assays. In order to identify the active principle, the seed extract was analyzed by SDS-PAGE and MALDI-TOF-MS.

\section{Mo-Poster Session 1-PO-10 Subtilisin-like protease Mauritanicain and Phorbol-12-myristat-13-acetate stimulate production of interleukine- 8 in $\mathrm{HaCaT}$ keratinocytes and juvenile primary human fibroblasts}

Authors Günther $\mathrm{F}^{1}$, Melzig $\mathrm{MF}^{1}$

Institute 1 Freie Universitaet Berlin, Institute of Pharmacy - Pharmaceutical Biology, Koenigin-Luise-Str. 2+4, D-14195 Berlin, Germany, Berlin, Germany DOI 10.1055/s-0037-1608060

Originally discovered in bacteria, subtilisin-like proteases are also abundant described in plants such as Oryza sativa L, Arabidopsis thaliana (L.) Heynh. and Solanum lycopersicum L. Proteases have the potential to induce inflammation through activation of protease-activated receptors (PAR) [1]. It is known, that plant latices induce strong skin inflammation. Therefore, we investigate the plant latex from plants of Euphorbia mauritanica L. (Euphorbiaceae Juss.) which contains amongst to other secondary plant ingredients diterpenes [2] and proteins [3]. We focused on the inflammatory potential of the combination with diterpenes and proteases. Mauritanicain, a Subtilisin-like protease from E. mauritanica, was isolated with anion exchange chromatography, followed by size exclusion chromatography. Fibroblasts were isolated from juvenile human foreskin. Various concentrations were tested on $\mathrm{HaCaT}$ keratino- 
cytes and the isolated fibroblasts. $\mathrm{PAR}_{2}$ selective agonist peptide 2-FluorylLIGRO-amide, diterpene Phorbol-12-myristat-13-acetate (PMA) and Subtilisin were used as single treatments. PAR positive studies were made with PCR. IL-8 was detected with ELISA. The results show, that the combination of Mauritanicain and the diterpene PMA increased the release of IL- 8 both, in HaCaT cell line (1.5-fold after $48 \mathrm{~h}$ compared with Mauritanicain) and in primary fibroblasts (4-fold after $24 \mathrm{~h}$ - compared with PMA), compared to single treatments with Mauritanicain, Subtilisin, or PMA.

[1] Guenther F, Melzig MF. J Pharm Pharmacol 2015; 67: 1623 - 1633

[2] Vasas A, Hohmann J. Chem Rev 2014; 114: 8579- 8612

[3] Sytwala S, Guenther F, Melzig MF. Plant Physiology and Biochemistry 2015; 95: $35-40$

\section{Mo-Poster Session 1-PO-11 New Dioxopiperazine and peptide derivatives, isolated from the leaves of Breynia Nivosa.}

\author{
Authors Umeokoli Blessing $\mathrm{O}^{1,2}$, Onyegbule Felix $\mathrm{A}^{1}$, Wang $\mathrm{H}^{2}$, Liu $\mathrm{Z}^{2}$, \\ Proksch $\mathrm{P}^{2}$ \\ Institute 1 Department of Pharmaceutical and Medicinal Chemistry, Nnamdi \\ Azikiwe University, Awka, Nigeria, Awka, Nigeria; 2 Institut für \\ Pharmazeutische Biologie und Biotechnologie, Heinrich-Heine-Universität \\ Düsseldorf, Germany, Dusseldorf, Germany \\ DOI 10.1055/s-0037-1608061
}

In its first report, Chemical investigation of the ethylacetate fraction of the leaves of Breynia nivosa from the eastern part of Nigeria, led to the isolation of two new Dioxopiperazine derivatives,Breynivosin A (1), Breynivosin B (2) and two new peptides Breynivosamide A (4), Breynivosamide B (5) along with other seven known compounds, Asperphinamate (3), Cristatin A (6), breynioside A (7), Robostacide A (8), (E)-p- Coumaric acid (9), Blumenol A (10), and Ligodinolide (11),. Chemical characterization, with detailed interpretation of 1D and 2D NMR spectroscopic and mass spectrometry data analysis were used to establish the structures of these compounds. All compounds are isolated from this plant for the first time.

\section{Mo-Poster Session 1-PO-12 Ginger (Zingiber officinale) Extract Promotes Telomere Shortening and Induces Cellular Senescence in A549 Lung Cancer Cells}

\footnotetext{
Authors Kaewtunjai $N^{1}$, Pompimon $\mathrm{W}^{2}$, Tuntiwechapikul $\mathrm{W}^{1}$

Institute 1 Department of Biochemistry, Faculty of Medicine, Chiang Mai University, Chiang Mai, Thailand; 2 Department of Chemistry, Faculty of Science and Center of Innovation in Chemistry, Lampang Rajabhat University, Lampang, Thailand

DOI 10.1055/s-0037-1608062

Cellular senescence caused by telomere shortening is considered a tumor suppressor mechanism. ${ }^{1}$ However, more than $85 \%$ of cancers maintain their telomeres by reactivating telomerase to escape the replicative senescence. ${ }^{2}$ Previously, we reported that the crude ethyl acetate fraction of Zingiber officinale extract (ZOE) suppressed the expression of the hTERT and c-Myc proteins, leading to the reduced telomerase activity in A549 lung cancer cells. ${ }^{3}$ In this report, we demonstrate that long-term treatment with subcytotoxic doses of ZOE, which allowed A549 cells to proliferate normally, induced telomere shortening corresponding with ZOE-induced telomerase suppression. The telomere shortening consequently induced cellular senescence in these cells, which was shown by the increase in the senescence associated $\beta$-galactosidase positive cells and the reduction in clonogenicity. In order to find the telomerase suppressors in ZOE, we employed assay-guided fractionation and GC/MS analysis to identify the chemical components. We found that the major compounds in all active subfractions contained paradols and shogaols of different chain lengths. We have concluded that the telomerase suppressors in ZOE are likely to be paradols and shogaols. Altogether, these results suggest ZOE could potentially be used as a dietary phytochemical for cancer prevention.
}

[1] Ohtani N, Mann DJ, Hara E. Cancer Science 2009; 100: 792 - 797.

[2] Kim NW, Piatyszek MA, Prowse KR, et al. Science 1994; 266: 2011 - 2015.

[3] Tuntiwechapikul W, Taka T, Songsomboon C, Kaewtunjai N, Imsumran A, Makonkawkeyoon L, Pompimon W, Lee TR. J Med Food 2010; 13: 1347 1354.

\section{Mo-Poster Session 1-PO-13 Major compounds of the anti-dengue active leaf methanol extracts from Faramea hyacinthina and Faramea truncata (Rubiaceae)}

Authors Barboza Rodolfo $\mathrm{S}^{1}$, Valente Ligia $\mathrm{MM}^{1}$, Wolff $\mathrm{T}^{1}$, Miranda Iranaia $\mathrm{A}^{2}$, Neris Rômulo $\mathrm{SL}^{2}$, Gomes $\mathrm{M}^{3}$

Institute 1 Universidade Federal do Rio de Janeiro, Instituto de Química, Av. Athos da Silveira Ramos 149, C.T, Bl. A, 21941 - 909, Rio de Janeiro, Brazil; 2 Universidade Federal do Rio de Janeiro, Instituto de Microbiologia Paulo Góes, Av. Carlos Chagas Filho 373, C. C. S, Bl. I, 21941 - 902, Rio de Janeiro, Brazil; 3 Instituto de Pesquisas Jardim Botânico do Rio de Janeiro, R. Jardim Botânico 1008, 22470 - 180, Rio de Janeiro, Brazil DOI 10.1055/s-0037-1608063

The $\mathrm{MeOH}$ extracts from the species Faramea hyacinthina and F. truncata (Rubiaceae) have shown in vitro non-cytotoxity and anti-dengue virus serotype 2 (DENV2) activity in human hepatocarcinoma cell lineage (HepG2) [1]. The comparison of the HPLC-DAD profiles of these extracts with that of $F$. bahiensis showed the common presence of the bioactive flavanone isosakuranetin-7-O$\beta$-D-apiofuranosyl- $(1 \rightarrow 6)-\beta$-D-glucopyranoside $(1)[1,2]$. The partition of the extracts between hexane: $\mathrm{MeOH} / \mathrm{H}_{2} \mathrm{O}$ led to $\mathrm{MeOH} / \mathrm{H}_{2} \mathrm{O}$ active fractions which when submitted to a previously developed RP-SPE method [3] allowed yielding in addition to compound (1), non-cytotoxic sub-fractions with different bioactivity profile. The $\mathrm{C}-18 \mathrm{CC}$ of these sub-fractions led to the isolation of the diasteroisomeric epimer pair $2 S+2 R$ of the new 5,3',5'-trihydroxy-flavanone7-O- $\beta$-D-apiofuranosyl $(1 \rightarrow 6)-\beta$-D-glycopiranoside $(2 \mathrm{a} / 2 \mathrm{~b})$ from $F$. hyacinthina; the known narigenin-7-O- $\beta$-D-apiofuranosyl $(1 \rightarrow 6)-\beta-D-g l y c o p i r a n o s i d e ~(3)$ from both species; the known rutin (4) and quercetin-4'- $\beta$-D-O-glucopiranosyl-3-O-rutinoside (5) from $F$. hyacinthina, and the known kaempferol-3-Orutinoside (6), erythroxyloside $A$ (7) and asperuloside (8) from $F$. truncata. Structural determinations were performed by NMR, HRMS, UV and OR. The presence of the bioactive flavanone (1) seems to play an important role in the anti-dengue activity of the species.

IPPN/UFRJ and FAPERJ.

[1] Barboza RS, Valente LMM, Nascimento AC, Miranda IA, Gomes M. Planta Med 2015; 81: 32.

[2] Nascimento AC, Valente LMM, Gomes M, Barboza RS, Wolff T, Neris RLS, Figueiredo CM, Assunção-Miranda I. Phytochem Lett 2017; 19: 220 - 225.

[3] Barboza RS, Valente LMM, Wolff T, Miranda IA, Neris RLS, Gomes M. Planta Med 2016; 82: 340.

\section{Mo-Poster Session 1-PO-14 Influence of Soil Flooding with Organic Matters Amendment on Reducing the Root Rot Disease and Content of Ginsenosides in Ginseng Crops}

Authors $\underline{\mathrm{Kim} \mathrm{SI}}{ }^{1}$, Kim $\mathrm{HH}^{1}$, Seong BJ ${ }^{1}$, Jee $\mathrm{MG}^{1}$, Lee $\mathrm{KS}^{1}, \mathrm{Kim}^{\mathrm{H}}{ }^{2}$, Kweon $\mathrm{KB}^{3}$

Institute 1 Ginseng \& Medicinal Plant Research Institute, CNARES, Geumsangun, Korea, Republic of (South); 2 Department of Applied Biology, Chungnam National University, Daejeon, Korea, Republic of (South); 3 Department of Herbal Crop Research, NIHHS, RDA, Eumseong, Korea, Republic of (South) DOI 10.1055/s-0037-1608064

Ginseng is mostly grown in Republic of Korea and China, and has been traditionally used as a medicinal plant for treating diseases including hypertension, diabetes mellitus, liver and kidney dysfunction and mental disorders. It is cultivated for three to six years. One of the most serious problems in ginseng cultivation is that, when ginseng is cultivated in a soil damaged by successive 
cropping, the root rot caused by Cylindrocarpon destructans disables replanting for the following decade. In this study, as a solution to soil sickness by continuous cropping, flooding treatment using organic matters (rice straw, cocopeat, bark) was applied to a paddy field from which ginseng was harvested for two years, and only rice straw was used for the non-treatment field. Regarding the prevalence of root rot according to the organic matter used for flooding treatment, in the rice straw group, the prevalence was reduced to $27.9 \%$ among 3-year ginseng and to $13.7 \%$ among 4 -year ginseng, while, in the cocopeat group, it was reduced from $22.6 \%$ to $12.7 \%$ among 3 -year ginseng. Also, in the bark group, it was reduced from $12.1 \%$ to $7.9 \%$ among 3 -year ginseng. In the non-treatment group, the prevalence was high, $98.7 \%$ among 3-year ginseng and 100\% in 4-year ginseng. The yield increased, in the rice straw group, from $626 \mathrm{~kg} / 10$ a for 3-year ginseng to $825 \mathrm{~kg} / 10$ a for 4 -year ginseng, and, in the bark group, $531 \mathrm{~kg} / 10 \mathrm{a}$ for 3 -year ginseng to $791 \mathrm{~kg} / 10 \mathrm{a}$ for 4-year ginseng, while, in the cocopeat group, it increased from $321 \mathrm{~kg} / 10 \mathrm{a}$ for 3-year ginseng to $770 \mathrm{~kg} / 10$ a for 4 -year ginseng. Among ginsenoside components, Rg1, Rf, Re, and Rd decreased in 4-year ginseng compared to 3-year ginseng in all organic matter groups, while Rc+Rb2 increased in the 4year ginseng compared to 3-year ginseng. The Rb1 content was similar between 3-year ginseng and 4-year ginseng.

\section{Mo-Poster Session 1-PO-15 CYTOTOXIC ALKALOIDS FROM ROOTS OF GONIOTHALAMUS LANCEOLATUS (BÂN) MAT-SALLEH}

Authors Ismail Nor $\mathrm{H}^{1}$, Rasol Nurulfazlina $\mathrm{E}^{1}$, Ahmad Fasihuddin $\mathrm{B}^{2}$, Mai $\mathrm{CW}^{3}$ Institute 1 Atta-ur-Rahman Institute for Natural Products Discovery,Universiti Teknologi MARA, Selangor, Malaysia; 2 Department of Chemistry, Faculty of Resourse Science and Technology, Universiti Malaysia Sarawak, Sarawak, Malaysia; 3 School of Pharmacy, International Medical University, Kuala Lumpur, Malaysia DOI 10.1055/s-0037-1608065

Goniothalamus lanceolatus (Bân) Mat-Salleh is an ethnomedicinal plant indigenous to Sarawak, Malaysia used as traditional remedy for cancer by the local people but has not been previously studied, chemically or biologically. Interestingly, comprehensive UHPLC-MS profiling of the root extract detects presence of alkaloidal components in addition to the well-known styryl lactones and acetogenins, usually present in Goniothalamus species. Evaluation of fractions obtained from the dichloromethane root extract showed cytotoxic activities against three panels of cancer cell lines, which are the human breast cell lines (MCF-7, MDA-MB-231 and MDA-MB-468), human colon cell lines (HT29, HCT116 and SW48) and lung cancer cell lines (A549, NCl-H23, and NCl$\mathrm{H} 1299$ ). Guided by the cytotoxic activities, herein we report isolation of lactam alkaloid 1 and quinone alkaloid 2, together with a known styryl lactone, 9-deoxygoniopypyrone 3 , from the roots $G$. lanceolatus. Compound 1 showed strong cytotoxic effect on human lung and human colon cancer cell lines with $\mathrm{IC}_{50}$ values ranging from 5.32 to $9.91 \mu \mathrm{M}$.

\section{Mo-Poster Session 1-PO-16 Urolithins, gut microbiota metabolites of ellagitannins, in prostate cancer chemoprevention.}

Authors Stanisławska I ${ }^{1}$, Piwowarski I , Granica $S^{1}$, Kiss A ${ }^{1}$

Institute 1 Department of Pharmacognosy and Molecular Basis of

Phytotherapy, Medical University of Warsaw, Warsaw, Poland

DOI 10.1055/s-0037-1608066

Urolithins are well bioavailable products of ellagitannin degradation by gut microbiota. These compounds have numerous biological activities, including anti-cancer [1].

In this study, we focused on the effects of urolithins on androgen receptor activity, which is crucial in prostate cancer development and progression.

We incubated androgen-dependent LNCaP cells with urolithins A, B and C (5$50 \mu \mathrm{M}, 72 \mathrm{~h}$ ) and their mixtures with $10 \mu \mathrm{M}$ bicalutamide, a clinically used non- steroidal antiandrogen. Cell proliferation was determined by measurement of DNA-Hoechst 33258 complexes fluorescence in cells lysates. Apoptosis was detected by flow cytometry after annexin V-FITC and PI staining. PSA secretion was measured with PSA total ELISA kit. Androgen receptor localization was examined by flow cytometry. Urolithin $A$ and $B$ showed similar anti-proliferative activity with $\mathrm{IC}_{50}=32.6 \pm 2.7 \mu \mathrm{M}$ and $\mathrm{IC}_{50}=35.7 \pm 0.9 \mu \mathrm{M}$, respectively, while urolithin $C$ had slightly weaker effect $\left(I_{50}=45.5 \pm 3.7 \mu \mathrm{M}\right)$. Bicalutamide had additive anti-proliferative effect with urolithin $\mathrm{A}(\mathrm{Cl}=0.86$ $\pm 0.08)$ and $\mathrm{B}(\mathrm{Cl}=1.06 \pm 0.08)$ and antagonistic with urolithin $\mathrm{C}(\mathrm{Cl}=2.27$ \pm 0.95 ). Urolithins induced apoptosis of LNCaP cells. Urolithin A was the most and urolithin $C$ the least active metabolite $(40 \mu \mathrm{M}, 25.7 \pm 0.9 \%$ and 16,13 $\pm 1.0 \%$ of apoptotic cells, $p<0.05$ respectively). Bicalutamide attenuated apoptosis induced by urolithin A and B. Urolithin A and urolithin C decreased dihydrotestosterone-stimulated PSA secretion to $56.9 \pm 3.3 \%$ and $81.0 \pm 5.6 \%$, respectively. Urolithin B counteracted bicalutamide-induced decrease in PSA secretion $(p<0.05)$. Moreover, urolithin A promoted cytoplasmic localization of androgen receptor irrelevant of the presence of bicalutamide. Our results show that urolithins may contribute to chemopreventive activity of ellagitannin rich preparations. However, observed interactions with clinically used drug warrant further study to determine safety of ellagitannin supplementation during hormonal therapy of prostate cancer.

[1] Espin JC, Larrosa M, Garcia-Conesa MT, Tomas-Barberan F. Evid Based Complement Alternat Med 2013; 2013: Article Number 270418

\section{Mo-Poster Session 1-PO-17 Anti-metastatic and anti-allergic spirostanol saponins from Solanum macaonense and S. torvum}

Authors Lee $\mathrm{CL}^{1,2}$, Yang $\mathrm{JC}^{2}$, Peng $\mathrm{CY}^{2}$, Wu $\mathrm{YC}^{2,3,4,5}$

Institute 1 Department of Cosmeceutics, China Medical University, Taichung, Taiwan; 2 Chinese Medicine Research and Development Center, China Medical University Hospital, Taichung, Taiwan; 3 Graduate Institute of Natural Products, Kaohsiung Medical University, Kaohsiung, Taiwan; 4 Research Center for Natural Products \& Drug Development, Kaohsiung Medical University, Kaohsiung, Taiwan; 5 Department of Medical Research, Chung-Ho Memorial Hospital, Kaohsiung Medical University, Kaohsiung, Taiwan DOI 10.1055/s-0037-1608067

Twenty-five steroidal saponins isolated from Solanum macaonens (1-15) [1] and S. torvum (16-25) [2] were assayed for cytotoxicity, anti-metastatic, and anti-allergic activities. Spirostanols 1, 2, 5, and furostanol 10 have moderate cytotoxicities toward human lung (A549), breast (MCF-7), ovarian (SKOV3), colon (HCT116), prostate (DU145), and oral (Ca922) cancer cell lines ( $\mathrm{IC}_{50}$ $29.6-48.2 \mu \mathrm{M})$. Spirostanols $1-5$ and 7 showed inhibition against A549 cancer cell migrations at the concentration of $50 \mu \mathrm{M}$, notably 1 and 2 that could remarkably suppressed wound migration by $71.7 \%$ and $73.0 \%$ in comparison with control cells, respectively. However, 1-5 and 7 did not have effect at the lower concentration of $25 \mu \mathrm{M}$. In addition, 18 and 19 could lead to $26.0 \%$ and $60.3 \%$ healing at $20 \mu \mathrm{g} / \mathrm{mL}$, respectively. Compounds $1-6$ and $18-19$ which showed antineutrophilic inflammatory effects $[1,2]$, were not able to inhibit immediately allergic degranulation in rat basophilic leukemia RBL-2H3 cells, but 2, 4-6, and 18 could be significantly against interleukin-4 (IL-4) release at the concentration of $3.1 \mu \mathrm{M}$ that is a late-phase response of allergic inflammation reaction. In our studies, spirostanol saponins 2 and 18 have both antimetastatic and anti-inflammatory effects in accordance with anti-inflammatory phytochemicals are potential candidates against tumor metastasis. Spirostanols 2 and 18 could be as the promising lead compounds against inflammation-associated tumors.

[1] Lee CL, Hwang TL, Yang JC, Cheng HT, He W], Yen CT, Kuo CL, Chen C], Chang WY, Wu YC. J Nat Prod 2014; 77: 1770 - 1783.

[2] Lee CL, Hwang TL, He W], Tsai YH, Yen CT, Yen HF, Chen C], Chang WY, Wu YC. Phytochemistry 2013; 95: 315 - 321. 


\section{Mo-Poster Session 1-PO-18 New bufadienolides from the whole plants of Helleborus foetidus and their apoptosis-inducing activity}

\author{
Authors Yokosuka $\mathrm{A}^{1}$, Iguchi $\mathrm{T}^{1}$, Mimaki $\mathrm{Y}^{1}$ \\ Institute 1 Tokyo University of Pharmacy and Life Sciences, Hachioji, Tokyo, \\ Japan \\ DOI 10.1055/s-0037-1608068
}

The genus Helleborus with about 20 species belongs to the family Ranunculaceae. Helleborus foetidus is an evergreen perennial plant native to Europe. Although anemonin, a steroidal glycoside, and a few phenolic compounds including a quercetin glycoside were isolated from $\mathrm{H}$. foetidus, there has been no systematic chemical work concerning the secondary metabolites of the plant. Therefore, we conducted a phytochemical examination of $H$. foetidus and evaluated the cytotoxicity and apoptosis-inducing activity of the isolated compounds.

The whole plants of Helleborus foetidus were extracted with $\mathrm{MeOH}$. The concentrated $\mathrm{MeOH}$ extract was passed through a Diaion HP-20 column, successively eluted with $30 \% \mathrm{MeOH}, 50 \% \mathrm{MeOH}, \mathrm{MeOH}$, EtOH, and EtOAc. The $50 \%$ $\mathrm{MeOH}$ and $\mathrm{MeOH}$ eluate fractions showed cytotoxicity against $\mathrm{HL}-60$ leukemia cells with $\mathrm{IC}_{50}$ values of 0.15 and $0.23 \mu \mathrm{g} / \mathrm{mL}$, respectively. Then, $50 \% \mathrm{MeOH}$ eluate fraction was subjected to column chromatography on silica gel and octadecylsilanized silica gel, giving compounds 1-5.

The structures of the new bufadienolides ( 1 and 2 ) were determined by spectroscopic analysis. Compounds 3-5 were identified as $11 \alpha$-hydroxyscilliglaucoside (3), desacetylscillicyanosid (4), and hellebortin A (5), respectively, based on their physical and spectroscopic data.

Compounds 1-5 and the aglycones (4a and 5a) were evaluated for their cytotoxicity against HL-60 cells. All compounds exhibited cytotoxicity against $\mathrm{HL}-60$ cells with $\mathrm{IC}_{50}$ values ranging from 0.0076 to $3.6 \mu \mathrm{M}$.

Compound 1 is a new bufadienolide with cytotoxicity against HL-60 cells with an $\mathrm{IC}_{50}$ value of $0.035 \mu \mathrm{M}$. HL-60 cells treated with 1 displayed the typical characteristics of apoptosis, such as the nuclear chromatin condensation, accumulation of sub-G1 cells, and activation of caspase-3. Furthermore, 1 was shown to induce the intracellular events associated with the mitochondrial-dependent apoptotic pathway, as was evident from the loss of the mitochondrial membrane potential and release of cytochrome $c$ to the cytosol.
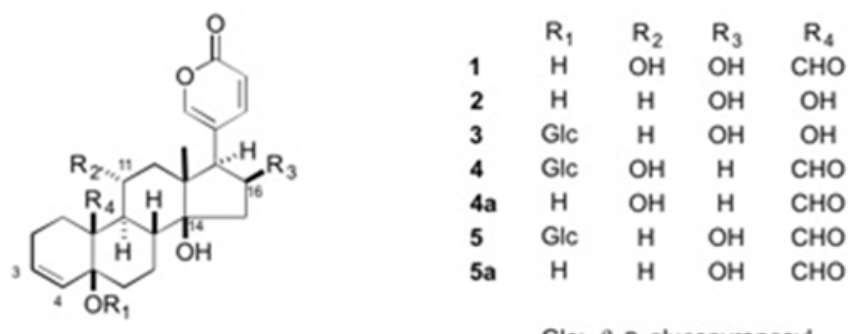

Fig. 1

\section{Mo-Poster Session 1-PO-19 Comparative gene expression study in WM164 melanoma cells treated with dimethylacrylshikonin.}

Authors Kretschmer $\mathrm{N}^{1}$, Deutsch $\mathrm{A}^{2}$, Rinner $\mathrm{B}^{3}$, Scheideler $\mathrm{M}^{4,5,6}$, Bauer $\mathrm{R}^{1}$ Institute 1 University of Graz, Institute of Pharmaceutical Sciences, Department of Pharmacognosy, Graz, Austria; 2 Division of Hematology, Medical University of Graz, Graz, Austria; 3 Core Facility Alternative Biomodels and Preclinical Imaging, Medical University of Graz, Graz, Austria; 4 Institute for Diabetes and Cancer, Helmholtz Zentrum München, German Research Center for Environmental Health, Munich, Germany; 5 Joint Heidelberg-IDC Translational Diabetes Program, Heidelberg University Hospital, Heidelberg,
Germany; 6 German Center for Diabetes Research (DZD), Neuherberg, Germany

DOI 10.1055/s-0037-1608069

Currently, one in every three cancers diagnosed is a skin cancer. Melanoma accounts for only $4 \%$ of all skin cancers but is responsible for ca. $79 \%$ of skin cancer deaths. Moreover, the incidence of malignant melanoma is rising faster than any other solid tumor type. Even though the survival rate is $100 \%$ at a very early stage, it drops dramatically at an advanced stage and leaves melanoma then as one of the most aggressive and incurable types of solid cancer. This reflects the need for the discovery and development of new respective anti-cancer drugs. In a previous study [1], we discovered that dimethylacrylshikonin, isolated from the roots of Onosma paniculata Bur.\&Franch. (Boraginaceae), exhibited the lowest $I C_{50}$ of several isolated shikonin derivatives and was most active towards melanoma cell lines. Therefore, it was chosen for more in-depth investigations. We did a comprehensive gene expression study using the metastatic melanoma cell line WM164. Cells were treated with $8 \mu \mathrm{M}$ dimethylacrylshikonin for $24 \mathrm{~h}$ and the gene expression signature was compared to vehicle-treated control cells (vehicle: 0.5\% DMSO). 1192 distinct mRNAs could be identified as expressed in all three biological replicates out of which 89 were 2-fold differentially expressed $(n=3)$. Testing for significantly differentially expressed genes following the Benjamini-Hochberg correction for multiple testing resulted in 31 distinct mRNAs. The most interesting candidates (among other things sequestosome 1) are known to be involved in apoptosis, cell proliferation, survival and/or drug resistance and were validated and confirmed by semi-quantitative real-time PCR. Additional experiments are in progress to study the changes in different signaling pathways in more detail to reveal the exact mode of action.

[1] Kretschmer et al, J Nat Prod, 2012, 75:865 - 9.

\section{Mo-Poster Session 1-PO-20 Stellettin B Suppresses Human Hepatocellular Cacinoma Cell Migration and Invasion through MAPK and FAK/PI3K/AKT/mTOR Signaling Pathways}

Authors Wu YJ ${ }^{1}$, Neoh $\mathrm{CA}^{2}$, Chang $\mathrm{HT}^{3}$, Su JH${ }^{4}$, Liu $\mathrm{Cl}^{5}$

Institute 1 Department of Biological Technology, Meiho Universtiy, Pingtung, Taiwan; 2 Department of Research, Pingtung Christian Hospital, Pingtung,

Taiwan; 3 Graduate Institute of Applied Healthy and Biotechnology, Meiho

University, Pingtung, Taiwan; 4 National Museum of Marine Biology and

Aquarium, Pingtung, Taiwan; 5 Department of Nursing, Meiho University, Pingtung, Taiwan

DOI 10.1055/s-0037-1608070

Stellettin B, isolated by wild-type sponge Stelletta sp. from the ocean, exhibited potent antiprolifertive activities on various tumor cells. In this study, we use demonstrated the stellettin $B$ inhibited the migration and invasion of the hepatocellular carcinoma cell HA22T/VGH. We found that the stellettin B inhibited the invasion and migration of hepatocellular carcinoma cells in a dose-dependent manner. The results of zymography assay showed that stellettin B suppressed the activities of matrix metalloproteinase MMP-9 and MMP-2. Moreover, protein levels of MMP-9, MMP-2, and urokinase-type plasminogen activator (UPA) were reduced by stellettinB in a dose-dependent manner. Stellettin B also exerted an inhibitory effect on phosphorylation of c-Jun N-terminal kinase (JNK), extracellular signal-regulated kinases (ERK), phosphatidylinositol 3-kinase (PI3K) and Akt. Taken together, these results demonstrated that stellettin B could inhibit hepatocellular carcinoma cell migration and invasion and alter HA22T/VGH cell metastasis by reduction of uPA, MMP-2 and MMP-9 expression through the suppression of MAPKs and FAK/ $\mathrm{PI} 3 \mathrm{~K} / \mathrm{AKT} / \mathrm{mTOR}$ signaling pathway. These findings suggest that stellettin $\mathrm{B}$ merits further evaluation as a chemotherapeutic agent for human hepatocellular carcinoma. 


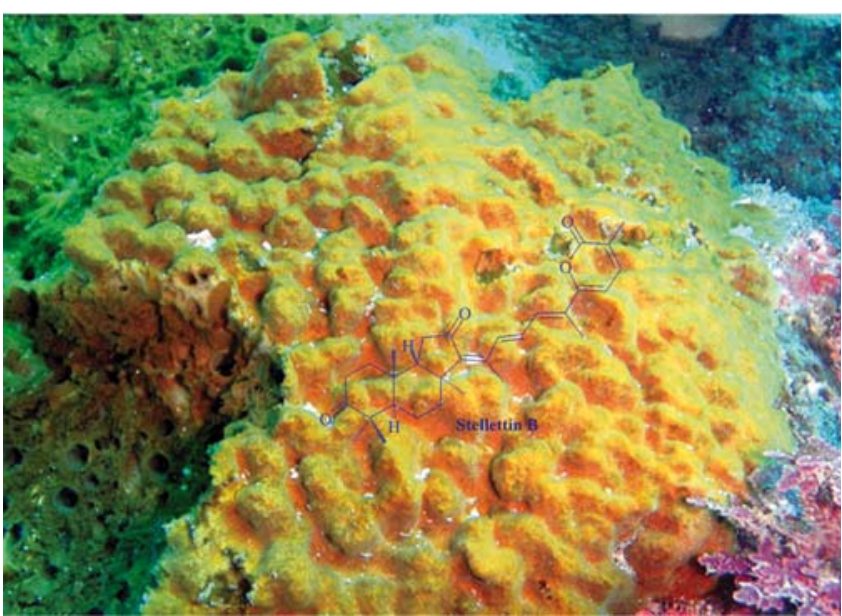

Fig. 1

\section{Mo-Poster Session 1-PO-21 Cholestane glycosides from Ornithogalum saundersiae bulbs and their apoptosis-inducing activity via a mitochondria-independent pathway}

\section{Authors Iguchi $\mathrm{T}^{1}$, Yokosuka $\mathrm{A}^{1}$, Kuroda $\mathrm{M}^{1}$, Mimaki $\mathrm{Y}^{1}$}

Institute 1 Tokyo University of Pharmacy and Life Sciences, Hachiouji, Japan DOI 10.1055/s-0037-1608071

Ornithogalum saundersiae (Liliaceae) is native to South Africa and cultivated as an excellent garden plant in the world. We have previously reported a total of 29 cholestane glycosides isolated from the $\mathrm{MeOH}$ extract of $O$. saundersiae bulbs and their cytotoxic activity against malignant tumor cells. Among the cholestane glycosides, OSW-1 showed exceptionally potent cytotoxic activity against various tumor cells. In this presentation, we wish to report further cytotoxic cholestane glycosides isolated from the $\mathrm{MeOH}$ extract of $O$. saundersiae bulbs.

The concentrated $\mathrm{MeOH}$ extract of $O$. saundersiae bulbs was passed through a Diaion HP-20 column eluted with $20 \% \mathrm{MeOH}$, EtOH and EtOAc, successively. The $\mathrm{EtOH}$ eluate fraction was subjected to column chromatography on silica gel and ODS silica gel, as well as preparative HPLC to give 40 cholestane glycosides including 20 new naturally occurring compounds. The structures of the new compounds were determined by $1 \mathrm{D}$ and 2D NMR spectroscopic analysis and the results of hydrolysis.

Cholestane glycosides 1-40 exhibited cytotoxic activity against HL-60 human promyelocytic leukemia cells with $\mathrm{IC}_{50}$ values ranging from $0.0019 \mathrm{nM}$ to $7.72 \mu \mathrm{M}$. HL-60 cells treated with (22S)-33,22-dihydroxycholest-5,24-dien$16 \beta$-yl $\alpha$-L-rhamnopyranoside (17), which had an $\mathrm{IC}_{50}$ value of $0.16 \mu \mathrm{M}$, displayed the typical characteristics of apoptosis, such as the nuclear chromatin condensation, DNA laddering, accumulation of sub-G1 cells, and activation of caspase-3. The loss of the mitochondria membrane potential and release of cytochrome $c$ to the cytosol were not observed in the $\mathrm{HL}-60$ cells treated with 17, suggesting that 17 may induce apoptosis in HL-60 cells via a mitochondria-independent pathway. Similar experiments were conducted for several other compounds.

\section{Mo-Poster Session 1-PO-22 Interaction of essential oil isomers carvacrol and thymol with the antibiotics doxycycline and tilmicosin against pathogenic respiratory tract bacteria.}

Authors Kissels $W^{1,2}$, Wu $X^{3}$, Santos $R^{4}$

Institute 1 Ecostyle B.V, Oosterwolde, Netherlands; 2 Kissels Consulting B.V, Bilthoven, Netherlands; 3 Hubert Department of Global Health, Emory
University, Atlanta, United States; 4 Institute for Risk Assessment Sciences, Utrecht University, Utrecht, Netherlands

DOI 10.1055/s-0037-1608072

Essential oils are candidates for adjuvant or replacement for antibiotics due to their antimicrobial properties ${ }^{1}$. This study evaluates 4 essential oil components as antibacterial agents or adjuvants for antibiotics against major respiratory pathogens. Respiratory disease is a problem in calves leading to reduced animal welfare and economic losses. Often antibiotic therapy is necessary. Bacterial resistance against antibiotics is an increasing problem. The current policy focusses on resistance prevention and reduction of antibiotic usage. Combined treatment of antibiotics with essential oils might offer a solution. Objective was to evaluate 4 essential oil components carvacrol, thymol, transanethole, and 1,8 cineole as antibacterial agents or adjuvants for doxycycline and tilmicosin against M. haemolytica and P. multocida. Bacteria were cultured according to standard protocols and determined for minimum inhibitory concentration (MIC) and minimum bactericidal concentration. A checkerboard assay was applied to detect interactions between components, between antibiotics, and between components and antibiotics. The fractional inhibitory concentrations (FIC) were calculated.

Tab. 2: Fractional inhibitory concentration (FIC) values of the components against Pasteurella multocida and Mannheimia haemolytica

\begin{tabular}{|l|l|l|l|l|}
\hline Combination & Bacteria strains & FIC & FIC index & Remark \\
\hline Dox + Til $^{2}$ & P. multocida & $0.25 / 1.0$ & 1.25 & Indifferent \\
\hline Dox + Cvc $^{3}$ & M. haemolytica & $0.5 / 0.5$ & 1.0 & Indifferent \\
\hline & P. multocida & $0.125 / 0.125$ & 0.25 & Synergy \\
\hline Dox + Thy & M. haemolytica & $0.0625 / 0.0625$ & 0.125 & Synergy \\
\hline & P. multocida & $0.5 / 0.0625$ & 0.5625 & Additive \\
\hline Til + Cvc & M. haemolytica & $0.5 / 0.0625$ & 0.5625 & Additive \\
\hline & P. multocida & $0.5 / 0.0625$ & 0.5625 & Additive \\
\hline Til + Thy & M. haemolytica & $0.0625 / 0.5$ & 0.5625 & Additive \\
\hline & P. multocida & $0.0625 / 0.5$ & 0.5625 & Additive \\
\hline Cvc + Thy & M. haemolytica & $0.5 / 0.25$ & 0.75 & Additive \\
\hline & P. multocida & $0.25 / 0.125$ & 0.375 & Synergy \\
\hline 'Dox: doxycycline. & M. haemolytica & $0.25 / 0.125$ & 0.375 & Synergy \\
\hline 2Til: tilmicosin. & & & & \\
\hline 3Cvc: carvacrol. & & & & \\
\hline Thy: thymol. & & & & \\
\hline
\end{tabular}

Only carvacrol and thymol were selected for checkerboard assay because no antibacterial effect of trans-anethole or 1,8-cineole was observed. MIC values for $P$. multocida and $M$. haemolytica, were 2.5 and $1.25 \mathrm{mM}$ for Carvacrol and 1.25 and $0.625 \mathrm{mM}$ for thymol respectively. Carvacrol and thymol presented additive effect when each was combined with tilmicosin. Additive effect for thymol was observed when combined with doxycycline. Synergism was observed when carvacrol was combined with doxycycline or with thymol. The study shows additive and synergistic effect of carvacrol and thymol when combined with each other, or with doxycycline or tilmicosin against $P$. multocida and $M$. haemolytica. These findings indicate both components as potential adjuvants during antibiotic therapy. Economic justification for selection of essential oil components can be subject for further studies.

[1] Kissels, Wu, Santos.Journal of Dairy Science, 2017

\section{Mo-Poster Session 1-PO-23 Dietary isothiocyanates reduce anoikis resistance of non-small cell human lung cancer cells}

Authors $\mathrm{Wu} \mathrm{CC}^{1}$, Tsai JY $\mathrm{Y}^{1}$, Tsai $\mathrm{SH}^{1}$

Institute 1 Kaohsiung Medical University, Kaohsiung City, Taiwan DOI 10.1055/s-0037-1608073

Normal cells rapidly undergo apoptosis when lose adhesion or relocate to an inadequate environment, in a process termed anoikis. In cancer, anoikis-resistant ability is a key feature that promotes survival of metastatic cancer cells. Isothiocyanates (ITCs) are natural compounds present in cruciferous vegetables like broccoli, cabbage, and cauliflower. Previous studies showed that ITCS exhibit a protective effect against various types of cancer such as lung cancer, esophagitis, breast cancer, hepatocellular carcinoma, and colon cancer. In the present study, three common ITCs, sulforaphane (1), benzyl isothiocyanate 
(2), and phenethyl isothiocyanate (3) were investigated for the their ability to reduce anoikis resistance in non-small cell lung cancer (NSCLC) A549 and CL 1 - 5 cells. A549 and CL $1-5$ cells were cultured in ultra-low attachment dishes to prevent cell attachment and treated with ITCs for 72 hours. Among them, 1 was the most potent in inhibiting cell viability in suspending A549 and CL $1-5$ cells. Of note, 1 had a less effect on the monolayer growth of adherent cells, indicating a selective inhibition of anchorage-independent cell growth. In addition, SFN induced anoikis of suspending A549 and CL 1-5 cells, which was identified by nuclear condensation, annexin $\mathrm{V}$ binding, activation of caspases, and cleavage of PARP. Investigating the action of mechanism of SFN revealed that SFN inhibited focal adhesion kinase (FAK) and Akt activation, upregulated the cyclin-dependent kinase inhibitor p21 as well as the proapoptotic proteins Bax and Bad. Taken together, our results indicate that dietary ITCS, especially 1 , are able to restore the sensitivity of NSCLC to anoikis, and suggest a potential benefit of ITCs for prevention or treatment of metastatic lung cancer.

\section{Mo-Poster Session 1-PO-24 Dereplication of Bioactive Markers from the Leaves of Anthocleista nobilis: Searching for new Bioactive Metabolites}

Authors Uzor $\mathrm{PF}^{1}$, Nnadi $\mathrm{CO}^{1,2}$

Institute 1 Department of Pharmaceutical and Medicinal Chemistry, Faculty of Pharmaceutical Sciences, University of Nigeria Nsukka, 410001 Nsukka Enugu State Nigeria, Nsukka, Nigeria; 2 Institute of Pharmaceutical Biology and Phytochemistry, University of Muenster, PharmaCampus, Corrensstr. 48, D-48149, Muenster Germany, Muenster, Germany

DOI 10.1055/s-0037-1608074

Anthocleista nobilis is a small to medium-sized tree up to $18-30 \mathrm{~m}$ tall which occurs in several African countries including Nigeria. Various parts of the plant are used for the treatment of several ailments including venereal diseases, constipation, hernia, haemorrhoids, leprosy, malaria and menstrual problems [1]. The plant belongs to the genus Anthocleista which are known to be rich source of bioactive secondary metabolites such as alkaloids, xanthones, phthalides, secoiridoids and terpenes, many of which have been isolated and reported [2]. Nevertheless, there are still yet-to-be isolated metabolites which could be implicated by dereplication approaches. Dereplication strategy was applied to discriminate between previously known and potential novel compounds. Ultra-high performance liquid chromatography/electro-spray ionization-quadrupole time-of-flight mass spectrometry (UHPLC/ESI-qTOF MS) technique was adopted for dereplication of secondary metabolites of the leaves of A. nobilis. Based on ultra-violet (UV) profiles, chromatographic retention times (tR) and positive mode-ESI mass spectra data, five previously reported compounds $(1-5)$ (Figure 1 ) were successfully dereplicated from a pool of sixtyeight queried molecular ion masses and common adducts. The compounds were known to be a secoiridoid (1), a dibenzo- $\alpha$-pyrone (2), a a triterpene (3), a xanthone (4), and a phytosterol (5). Interestingly, the remaining sixty-three masses could be potential novel bioactive compounds from the genus Anthocleista and could provide template for guided isolation and identification.

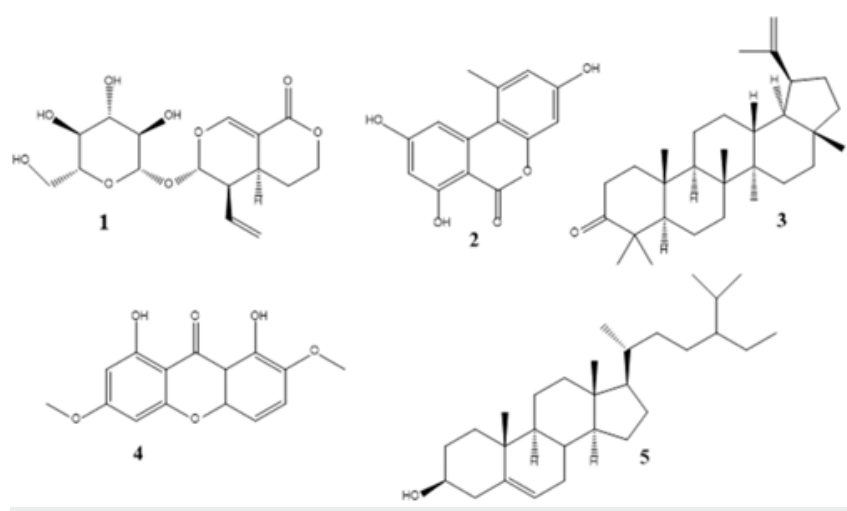

Fig. 1: Compounds dereplicated from UHPLC/ESI-qTOF MS of $A$. nobilis leaf

Acknowledgement: The authors are grateful for the opportunity to use the LC/MS facility of the Institute of Pharmaceutical Biology and Phytochemistry, University of Münster, Germany.

[1] Burkill HM. The useful plants of West Tropical Africa. Vol. 3. Families J-L. Kew; Royal Botanic Gardens; 2000: 32 - 50

[2] Anyanwu GO, Rehman N, Onyeneke CE, Rauf K. J Ethnopharmacol, 2015; 175: $648-667$.

\section{Mo-Poster Session 1-PO-25 New PTP1B inhibiting ellagitannins from the rhizome of Thonningia sanguinea}

Authors Pompermaier $L^{1}$, Schwaiger $S^{1}$, Lautenschläger $T^{2}$, Mandombe José $\mathrm{L}^{3}$, Waltenberger $\mathrm{B}^{1}$, Stuppner $\mathrm{H}^{1}$

Institute 1 Institute of Pharmacy/Department of Pharmacognosy, University of Innsbruck, Center for Molecular Biosciences Innsbruck, Innrain $80-82$, Innsbruck, Austria; 2 Department of Biology, Faculty of Science, Institute of Botany, Technische Universität Dresden, Dresden, Germany; 3 Faculty of Agronomy, Universidade Kimpa Vita, Uíge, Angola

DOI 10.1055/s-0037-1608075

The protein tyrosine phosphatase 1B (PTP1B) is considered as negative regulator of insulin receptor signalling and thus a promising target for the development of new antidiabetic drugs [1]. The crude methanol extract of the rhizome of Thonningia sanguinea showed strong inhibition (13.3\% residual PTB1B activity) at $30 \mu \mathrm{g} / \mathrm{ml}$ in an in vitro enzyme assay with human recombinant PTP1B compared to the DMSO vehicle control. Bioactivity guided isolation using different chromatographic techniques including RP-MPLC, SEC (Sephadex LH-20) and semipreparative HPLC yielded 10 different compounds (Fig. 1). All isolated compounds consist of a central chalcone-glucoside-unit differing in the connection position of the sugar unit (position 2' or 4' of the chalcone-unit) and the further substitution of the glucose moiety with gallic acid and/or hexahydroxydiphenic acid (HHDP). LC-MS- and 1D- and 2D-NMR experiments were used for structure-elucidation while the presence of DGlucose in the molecules was confirmed by hydrolysis and GC-MS-analysis of the corresponding thiazolidine derivative. Analysis of the PTP1B inhibition of the isolated compounds at $30 \mu \mathrm{M}$ (positive control: sodium orthovanadate $10 \mu \mathrm{M}$, residual PTB1B activity $21.7 \%$ ) showed the highest activity for the HHDP-substituted compounds 4,5 and 8 along with Thonningianin A and B (Fig. 1). The strongest effect was observed for Thonningianin $A$ with a residual PTB1B activity of $1.9 \%$. This study is the first description of compounds $2,3,4$, 5,7 and 8 and revealed a new biological activity of the previously isolated compounds of Thonningia sanguinea, Thonningianin A and B [2]. 


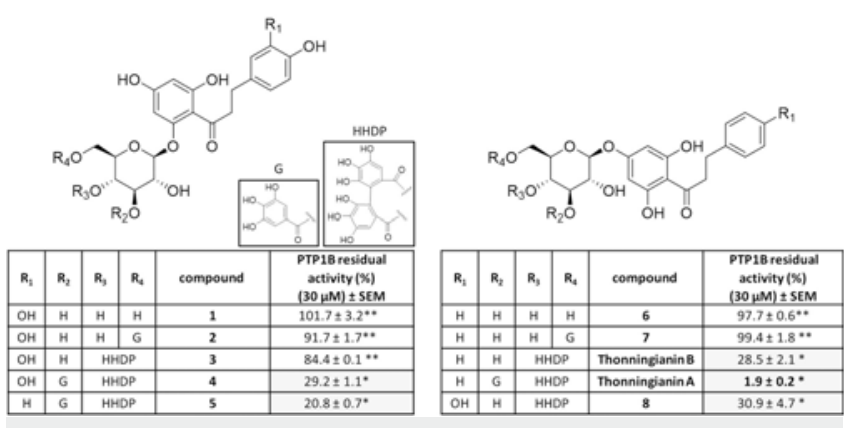

Fig. 1: Postive control: SOV $(10 \mu \mathrm{M})=21.7 \%$ residual activity vs. DMSO control $(\mathrm{n}>3),{ }^{*} \mathrm{n}=3,{ }^{* *} \mathrm{n}=2$

The autors thank DAAD (Deutscher Akademischer Austauschdienst) and the program "Strategische Partnerschaften" of the TU Dresden for the financial support.

[1] Combs AP. J Med Chem 2010; 53: 2333 - 44

[2] Ohtani II, Gotoh N, Tanaka J, Higa T, Gyamfi A, Aniya Y, J. Nat. Prod. 2000; 63: $676-679$

\section{Mo-Poster Session 1-PO-26 Anti-inflammatory activity of Alpinia nantoensis extracts}

Authors Wang $\mathrm{HC}^{1}$, Lin JW' $\mathrm{W}^{1}$, Wang $\mathrm{SY}^{2}$

Institute 1 Graduate Institute of Natural Products/College of Pharmacy Kaohsiung Medical University, Kaohsiung City, Taiwan; 2 Department of Forestry, National Chung Hsing University, Taichung, Taiwan DOI 10.1055/s-0037-1608076

Innate immune cells defense harmful stimuli such as bacteria endotoxin through inducing a complex inflammatory response; however, unrestrained inflammation leads to a range of inflammatory diseases, including asthma, atherosclerosis, rheumatoid arthritis, inflammatory bowel disease, and cancers. Controlling inflammatory processes is considered as one of approach to limit the progression of these diseases. In this study, we investigated the aerial and underground parts extract of Alpinia nantoensis F. Y. Lu \& Y. W. Kuo (zigiberaceae) for anti-inflammatory activity in the lipopolysaccharide (LPS)stimulated macrophage. Our results showed the aerial part of ethanolic extract and the underground part of hexanoic extract have more potent activity on inhibition of LPS-induced nitric oxide (NO) production, with a half-maximal inhibitory $\left(\mathrm{IC}_{50}\right)$ values of $1.83 \pm 0.2 \mu \mathrm{g} / \mathrm{ml}$ and $9.6 \pm 0.4 \mu \mathrm{g} / \mathrm{ml}$, respectively. Compounds cardamonin and pinostrobin (PSB) from active fractions showed have potent activity on inhibition of NO formation than 3,5-dimethoxystilbene (35DS), with an $\mathrm{IC}_{50}$ concentration of $3.28 \pm 1.2 \mu \mathrm{M}, 83.40 \pm 0.8 \mu \mathrm{M}$, and $>100 \mu \mathrm{M}$, respectively. The levels of NO formation by extracts or pure compounds treatment was paralleled with the levels of inducible nitric oxide synthase (iNOS) protein expression. Other pro-inflammatory factors, cyclooxygenase-2 (COX-2), IL-6, and TNF-alpha were also investigated. Compounds cardamonin, PSB, and 35DS displayed the different extent of inhibition on LPSinduced COX-2, IL-6, and TNF-alpha expression, as well as PGE2 formation. Our study suggests that the constituents of $A$. nantoensis have the potential to development anti-inflammatory drugs.

[1] Kuo YW, Lu FY, Duh MH, Deng SL. Taiwan J For Sci 2008; 23: 93 - 7, 2008

\section{Mo-Poster Session 1-PO-27 Diterpenoids from Astrodaucus orientalis (L.) Drude - Antiprotozoal activity}

Authors Nejad Ebrahimi $\mathrm{S}^{1}$, Hashempour $\mathrm{H}^{2}$, Herfati $\mathrm{F}^{2}$, Valizadeh $\mathrm{H}^{2}$, Mahmoudi-Kordi $\mathrm{F}^{3}$, Kaiser $\mathrm{M}^{5}$, Hamburger $\mathrm{M}^{4}$

Institute 1 Department of Phytochemistry, Medicinal Plants and Drug Research Institute, Shahid Beheshti University, G. C, Tehran, Iran;

2 Department of Chemistry, Faculty of Basic Sciences, Azarbaijan Shahid

Madani University, Tabriz, Iran; 3 Department of Biology, Faculty of Basic
Sciences, Azarbaijan Shahid Madani University, Tabriz, Iran; 4 Division of Pharmaceutical Biology, Department of Pharmaceutical Sciences, University of Basel, Klingelbergstrasse 50, 4056, Basel, Switzerland; 5 Swiss Tropical and Public Health Institute, Socinstrasse 57, 4002, Basel, Switzerland DOI 10.1055/s-0037-1608077

Astrodaucus orientalis (L.) Drude belongs to Apiaceae family. It is called 'Havij-ekohi' meaning 'mountain carrot' in Iran. The plant has been used as a food additive, vegetable and a salad in Iran and Turkey. The chemical compositions of essential oils from different parts of $A$. orientalis have been reported previously but there are no reports on nonvolatile phytochemical. Fractionation of an $n$-hexane extract of Astrodaucus orientalis (L.) Drude petals resulted on isolation, purification and identification of four new diterpenoids (1-4) and two known compounds namely 2-epilaserin (5) and $\alpha$-Angeloyloxylatifolone (6). Structure elucidation was achieved by 1D and 2D NMR experiments and HRMS spectroscopy. Relative configurations 1-4 were established on the basis of ${ }^{3} / \mathrm{H}-\mathrm{H}$ coupling constants and NOE difference spectra. The absolute configuration of 1-4 were achieved by comparison of experimental ECD spectra with simulated ECD data for possible stereoisomers, by using time dependent density function theory (TDDFT). Compounds tested in vitro for antiprotozoal activity against Plasmodium falciparum, Trypanosoma brucei rhodesiense, and Leishmania donovani with $\mathrm{IC}_{50}$ values ranging from 15.9 to $37.4 \mu \mathrm{M}, 10.4$ to $61.4 \mu \mathrm{M}$, and 5.8 to $78.5 \mu \mathrm{M}$ respectively. The cytotoxicity of isolated compounds tested against L6- rat myoblast cell line and showed no toxicity.

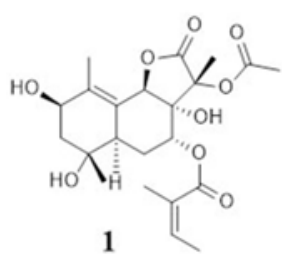

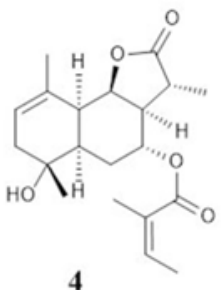
4

Fig. 1<smiles>C/C=C(\C)C(=O)O[C@H]1C[C@H]2C(=C(C)[C@H](O)C[C@H]2O)C2OC(=O)[C@H](C)[C@H]21</smiles><smiles>C/C=C(/C)C(=O)OC(C)C(OC(=O)/C(C)=C\C)c1cc(OC)c2c(c1)OCO2</smiles>

5

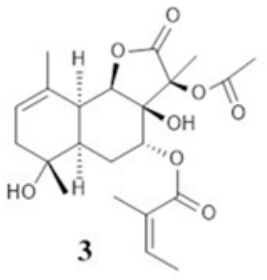<smiles>C/C=C(/C)C(=O)O[C@@H](C)C(=O)c1cc(OC)c2c(c1)OCO2</smiles>

6

\section{Mo-Poster Session 1-PO-28 Inhibitors of lactate dehydrogenase (hLDH5) from Polygala flavescens subsp. flavescens}

Authors Braca $A^{1}$, De Leo $M^{1}$, Peruzzi $L^{2}$, Granchi $C^{1}$, Tuccinardi $T^{1}$, Minutolo $\mathrm{F}^{1}$, De Tommasi $\mathrm{N}^{3}$

Institute 1 Dipartimento di Farmacia, Università di Pisa, via Bonanno 6 and 33, 56126, Pisa, Italy; 2 Dipartimento di Biologia, Università di Pisa, via Derna 1, 56126, Pisa, Italy; 3 Dipartimento di Farmacia, Università degli Studi di Salerno, via Giovanni Paolo II 132, 84084, Fisciano (SA), Italy DOI 10.1055/s-0037-1608078

The human isoform 5 of lactate dehydrogenase ( $h \mathrm{LDH} 5)$ is an enzyme upregulated in tumor tissues since cancer cells depend mainly on anaerobic respiration and their glycolytic rate is up to 200 times higher than that of the normal tissue. hLDH5 inhibition should cause cancer cell death by starvation, without interfering with healthy cells that normally use oxidative phosphorylation for ATP generation. Inhibition of LDH is so considered as a promising target in cancer treatment, since it is possible to cause a starving of cancerous cells by reducing glycolysis or by inhibiting the conversion of glu- 
cose to lactate. In the course of our research program on the $h \mathrm{LDH} 5$ inhibitory activity of natural products [1], a chemical study of $P$. flavescens subsp. flavescens was carried out. Polygala L. genus (Polygalaceae) is well known to contain phenolic oligosaccharides, xanthones, lignans, and triterpenic saponins and it's largely used in the traditional medicine [2]. Ten new compounds were isolated from the $n-\mathrm{BuOH}$ residue of the aerial parts through Sephadex and RP-HPLC separations, including four flavonol glycosides, two oligosaccharides, one $\alpha$-ionone, and three triterpenoidic saponins, together with two known oligosaccharides and two flavonol glycosides. All structures were elucidated on the basis of their spectroscopic and spectrometric data. The isolates were assayed for their inhibitory activity against $h \mathrm{LDH} 5$ and 3,6'-di-O-sinapoylsucrose showed an $\mathrm{IC}_{50}$ value of $90.4 \mu \mathrm{M}$. Modeling studies were carried out to suggest the putative interaction mode of this compound in the enzyme active site. This analysis highlighted that 3,6'-di-O-sinapoylsucrose shows a high number of $\mathrm{H}$-bonds and interacts with enzyme regions rarely explored by the known hLDH5 inhibitors.

[1] Bader A, Tuccinardi T, Granchi C, Martinelli A, Macchia M, Minutolo F, De Tommasi N, Braca A. Phytochemistry 2015; 116: $262-268$.

[2] Klein LC Jr, Faloni de Andrade S, Cechinel Filho V. Chem. Biodivers. 2012; 9 : $181-209$.

\section{Mo-Poster Session 1-PO-29 Antiplasmodial leads in oleo-gum-resins from Burseraceae: bioactivity-guided fractionation of myrrh}

Authors Greve $\mathrm{H}^{1}$, Kaiser $\mathrm{M}^{2,3}$, Brun $\mathrm{R}^{2,3}$, Schmidt $\left.\mathrm{T}\right]^{1}$ Institute 1 Institute for Pharmaceutical Biology and Phytochemistry (IPBP), WWU Münster, Münster, Germany; 2 Swiss Tropical and Public Health Institute (Swiss TPH), Basle, Switzerland; 3 University of Basle, Basle, Switzerland DOI 10.1055/s-0037-1608079

Malaria is a life-threatening infectious disease caused by Plasmodium spec. that are transmitted to human blood by the bite of Anopheles mosquitoes. In the course of our continued search for antiprotozoal natural products, an alcoholic extract of Commiphora myrrha showed antiplasmodial activity in vitro [1]. Thus myrrh, the oleo-gum-resin exsuded by species of the genus Commiphora, was subjected to a systematic investigation. Antiplasmodial tests were conducted according to established protocols [2]. A dichloromethane extract showed an $\mathrm{IC}_{50}$ value of $1.03 \mu \mathrm{g} / \mathrm{mL}$ in vitro against Plasmodium falciparum (NF54 strain). In a bioactivity-guided fractionation, several chromatographic separation steps resulted in the isolation of 12 sesquiterpenoids, 3 nortriterpenoids of the mansumbinane-type and one triterpene, 20-hydroxydammar-24-en-3,16dione. Isolated compounds were characterized and identified by spectroscopic means and comparison to literature data. Among the sesquiterpenoids 3 compounds are, to the best of our knowledge, reported for the first time, namely, rel-(5S,6R,7aS)-6-((E)-2-methoxyvinyl)-3,6-dimethyl-5-(prop-1-en-2yl)-5,6,7,7a-tetrahydrobenzofuran-2(4H)-one, rel-(5S,6R,7aR)-6-((E)-2-methoxyvinyl)-3,6-dimethyl-5-(prop-1-en-2-yl)-5,6,7,7a-tetrahydrobenzofuran-2(4H)one and rel-(4aR,5S,7S,7aS)-7-methoxy-3,5,8-trimethyl-5,6,7,7a-tetrahydroazuleno[6,5-b]furan-4(4aH)-one. The dammarane triterpene showed the best antiplasmodial activity so far with an $\mathrm{IC}_{50}$ value of $6.8 \mu \mathrm{M}$. Further compounds are still under isolation and will also be tested.

This project is an activity within the Research Network Natural Products against Neglected Diseases (ResNet NPND; http://www.resnetnpnd.org/). HLG thanks Cusanuswerk for a doctoral scholarship.

[1] Llurba Montesino N et al. Molecules 2015; 20: $14118-14138$

[2] Nour AMM et al. Planta Med 2009; 75: $1363-1368$

\section{Mo-Poster Session 1-PO-30 Biological activities of extracts and constituents of Salvia tingitana Etl. (Lamiaceae)}

Authors Bisio $\mathrm{A}^{1}$, Castagnola $\mathrm{P}^{2}$, Panfoli I ${ }^{1}$, Schito Anna $\mathrm{M}^{3}$, Pedrelli $\mathrm{F}^{1}$, Ruffoni $\mathrm{B}^{5}$, De Tommasi $\mathrm{N}^{4}$

Institute 1 Università degli Studi di Genova - Dipartimento di Farmacia Viale Cembrano 4, 16148, GENOVA, Italy; 2 IRCCS AOU, San Martino - IST, Largo Rosanna Benzi, 10, 16132, Genova, Italy; 3 Università degli Studi di Genova - Dipartimento di Scienze Chirurgiche e Diagnostiche Integrate, Sezione di Microbiologia - Largo Rosanna Benzi 8, 16145, Genova, Italy; 4 Università di Salerno - Dipartimento di Farmacia, Via Giovanni Paolo II 132, 84084, Salerno, Italy; 5 CREA OF - Centro di ricerca Orticoltura e

Florovivaismo, San Remo (IM), Italy

DOI 10.1055/s-0037-1608080

The dichloromethane extract obtained from the aerial parts of Salvia tingitana [1] was investigated for its anticancer and antimicrobial properties following a bioassay-oriented fractionation approach. Twelve known compounds were isolated i.e. two polymethoxylated flavonoids, three labdane and one isopimarane diterpenes and six sesterterpenes, along with six new sesterterpenes, identified by IR, 1D and 2D NMR and HR-MS analysis. The extract and the semi-purified fractions inhibited both the ATP synthetic and hydrolytic activity of retinal rod outer segment homogenates (OS). The modulatory activity of the OS ectopic FoF1-ATP synthase of two of the isolated compounds, manool and salvileucolide methylester, was comparable with that of polyphenols like resveratrol [2]. The extract and the isolated compounds were then investigated for anticancer properties. In particular, manool and one novel sesterperpene, both assayed at $75 \mu \mathrm{M}$, inhibited the $48 \mathrm{~h}$ survival of the BT474 human breast cancer cells at the $13.7 \%$ and $40.0 \%$ of controls, respectively. The antimicrobial activity was analyzed on several Gram positive multiresistant bacterial strains of clinical origin (Staphylococcus aureus, S. epidermidis, S. capitis, S. lugdunensis, S. saprophyticus, S. haemolyticus, Enterococcus faecium, E. faecalis, E. durans, E. gallinarum, E. casseliflavus, E.gallolyticus). The extract and the compounds showed moderate antimicrobial activity against Gram positive bacteria, with the exception of manool and sclareol, which exhibited MIC values ranging from 4 to $64 \mu \mathrm{g} / \mathrm{mL}$. Moreover, methanolic fractions of the root extract were particularly active, displaying MIC values ranging from 2 to $32 \mu \mathrm{g} / \mathrm{mL}$. Results indicate that S. tingitana can be considered a plant active against important Gram positive human pathogens.

[1] Foley, MJY, Hedge, IC, Möller, M. Willdenowia 2008; 38:41 - 59

[2] Calzia, D, Oneto, M, Caicci, F, Bianchini, P, Ravera, S, Bartolucci, M, Diaspro, A, Degan, P, Manni, L, Traverso, CE, Panfoli, I. Brit J Pharmacol 2015; $172: 3890-903$

\section{Mo-Poster Session 1-PO-31 Cytotoxicity of aloe-emodin towards several drug sensitive cells and their resistant counterparts}

Authors Ozenver $\mathrm{N}^{1}$, Saeed $\mathrm{M}^{2}$, Demirezer Lutfiye $\mathrm{O}^{1}$, Efferth $\mathrm{T}^{2}$ Institute 1 Department of Pharmacognosy, Faculty of Pharmacy, Hacettepe University, Sihhiye, 06100, Ankara, Turkey; 2 Department of Pharmaceutical Biology, Institute of Pharmacy and Biochemistry, Johannes Gutenberg University, Staudinger Weg 5, 55128, Mainz, Germany

DOI 10.1055/s-0037-1608081

Cancer is one of the leading causes of death in the world. Despite there are notable improvements in cancer in the past few decades, many cancer patients still cannot be cured due to the development of multi drug resistance (MDR) [1]. Anthraquinones were shown to inhibit tumor growth on various cell lines in previous studies [2,3]. Main anthraquinone aglycons are significant due to their structural similarities to anthracyclines well established anticancer drugs. Therefore aloe-emodin was searched on various cell lines including drug-sensitive CCRF-CEM and multidrug resistant P-glycoprotein-over-expressing CEM/ADR5000 leukemia cells, MDA-MB-231-pcDNA3 breast cancer cells 
and their transfectant subline MDA-MB-231-BCRP clone 23, HCT116 $\left(\mathrm{p} 53^{+/+}\right)$ colon cancer cells and their knockout clone HCT116 ( $553^{-1-}$ ), U87.MG glioblastoma multiform cells and their transfectant subline U87.MGAEGFR as well as HEK293 human embryonic kidney cells transfected with or without a cDNA for $A B C B 5$. The $I C_{50}$ values ranged from $21.20 \mu \mathrm{M}$ to $37.32 \mu \mathrm{M}$ for drug-sensitive and wild-type cell lines. For drug-resistant and transfected sublines, $I C_{50}$ values were ranged from $14.68 \mu \mathrm{M}$ to $66.31 \mu \mathrm{M}$. Collateral sensitivity (hypersensitivity) to aloe-emodin was observed in HCT116 ( $553^{--}$) knockout cells (0.69 fold). Aloe-emodin having a notable cytotoxicity on few cancer cells needs to be conducted further researches on to clarify the background mechanisms behind its cytotoxicity.

This study was supported by The Scientific and Technological Research Council of Turkey (TÜBITAK) 2214-A scholarship.

[1] Efferth T. Resistance to Targeted ABC Transporters in Cancer. New York: Springer; 2014

[2] Chen YC, Shen SC, Lee WR, Hsu FL, Lin HY, Ko CH, Tseng SW. Biochem Pharmacol 2002; 64: 1713 - 1724

[3] Cichewicz RH, Zhang Y], Seeram NP, Nair MG. Life Sci 2004; 74: 1791 1799

\section{Mo-Poster Session 1-PO-32 Structure determination and preliminary cytotoxicity researches of the compounds isolated from Rumex acetosella $\mathrm{L}$. on leukemia cells}

Authors Ozenver $\mathrm{N}^{1}$, Saeed $\mathrm{M}^{2}$, Kauhl $\mathrm{U}^{3}$, Guvenalp $\mathrm{Z}^{4}$, Demirezer Lutfiye $\mathrm{O}^{1}$, Opatz $\mathrm{T}^{3}$, Efferth $\mathrm{T}^{2}$

Institute 1 Department of Pharmacognosy, Faculty of Pharmacy, Hacettepe University, Sihhiye, 06100, Ankara, Turkey; 2 Department of Pharmaceutical Biology, Institute of Pharmacy and Biochemistry, Johannes Gutenberg

University, Staudinger Weg 5, 55128, Mainz, Germany; 3 Institute of Organic Chemistry, Johannes Gutenberg University, Duesbergweg 10 - 14, D-55128, Mainz, Germany; 4 Department of Pharmacognosy, Faculty of Pharmacy, Ataturk University, 25240, Erzurum, Turkey

DOI 10.1055/s-0037-1608082

Rumex acetosella L. (Polygonaceae) has a usage in folk medicine for the cancer treatment taking a part in the composition of Essiac tea in Canada [1]. Traditional uses of its extracts were also confirmed in previous study [2]. The constituents of the plant may account for its cytotoxicity. In the present study, we searched the cytotoxicities of the isolated compounds RAN-2, RAN-6, RAB4, RAT-1, RAA-2, RAA-3, RAA-4 from the roots of $R$. acetosella. The compounds were gained by using various chromatography methods as Sephadex column chromatography (SPH), normal phase silica gel column chromatography (SK), reverse phase column chromatography including vacuum liquid chromatography (VLC) or medium pressure liquid chromatography (MPLC) and determined by NMR, IR, mass spectras and optical rotations measurements. They were elucidated as (E)-piceid, musizin-/nepodin-8-O- $\beta$-glucopyranosid, rumejaposide $\mathrm{G} / \mathrm{H}$ diastereomeric mixture, catechin/flavan-3-ol, emodin, emodin-8-O- $\beta$-D-glucosid and the mixture of chrysophanol-8-O- $\beta$-D-glucopyranoside and physcion-8-O- $\beta$-D-glucopyranoside, respectively. Initially we investigated their cytotoxicity with preliminary studies. Therefore we conducted Resazurin reduction assay on CCRF-CEM and CEM/ADR5000 leukemia cells at $10 \mu \mathrm{g} / \mathrm{ml}$. None showed notable cytotoxicity to deserve going further researches on.

This study was supported by The Scientific and Technological Research Council of Turkey (TÜBITAK) 2214-A scholarship.

[1] Leonard SS, Keil D, Mehlman T, Proper S, Shi, XL, Harris GK. J Ethnopharmacol 2006; 103: $288-296$

[2] Wegiera M, Smolarz HD, Bogucka-Kocka A. Acta Pol Pharm 2012; 69: $487-499$

\section{Mo-Poster Session 1-PO-33 Aloe-emodin's cytotoxicity against CCRF-CEM cells: NF-KB as a major player induces apoptosis}

Authors Ozenver $\mathrm{N}^{1}$, Saeed $\mathrm{M}^{2}$, Demirezer Lutfiye $\mathrm{O}^{1}$, Efferth $\mathrm{T}^{2}$ Institute 1 Department of Pharmacognosy, Faculty of Pharmacy, Hacettepe University, Sihhiye, 06100, Ankara, Turkey; 2 Department of Pharmaceutical Biology, Institute of Pharmacy and Biochemistry, Johannes Gutenberg

University, Staudinger Weg 5, 55128, Mainz, Germany

DOI 10.1055/s-0037-1608083

NF-KB family as being significant in inflammation and immunity also has a notable role in cancer initiation, progression and inhibition of programmed cell death [1-3], which indicates it's a crucial target for cancer. A main anthraquinone aglycone of Rumex and other genera (e.g. Aloe, Rheum and Rhamnus) aloe-emodin has cytotoxic effect against CCRF-CEM leukemia cells with the $\mathrm{IC}_{50}$ value of $11.95 \mu \mathrm{M}$. In the present study, we focused on the background mechanisms behind its cytotoxicity on the basis of pharmacogenomic approach. Therefore we performed microarray-based mRNA profiling which was validated by $\mathrm{qPCR}$ analysis. According to the Ingenuity Pathway Analysis of microarray data, we observed that NF-kB and the target genes of whom were downregulated causing apoptosis by aloe-emodin. We additionally conducted gene promoter binding motif analysis. NF-KB binding motifs were screened $75 \mathrm{~kb}$ upstream of the transcription start site of the genes of interest. To test aloe-emodin real act on NF-KB, we carried out NF-kB reporter assay and observed NF-kB downregulation by aloe-emodin in dose dependent manner which was upward of $50 \%$ with the treatment of $75 \mu \mathrm{M}$ or $100 \mu \mathrm{M}$ aloe-emodin. Inhibition of NF-KB was further proven by docking analysis. We specificied NF-KB as one of the major player determining the cytotocity of aloe-emodin against CCRF-CEM cells in addition to other cellular and molecular factors determined in our previous studies.

This study was supported by The Scientific and Technological Research Council of Turkey (TÜBITAK) 2214-A scholarship.

[1] Hoesel B, Schmid JA. Mol Cancer 2013; 12: 86

[2] Karin M, Cao Y, Greten FR, Li ZW. Nat Rev Cancer 2002; 2: 301 - 310

[3] Liu ZG, Hsu H, Goeddel DV, Karin M. Cell 1996; 87: 565 - 576

\section{Mo-Poster Session 1-PO-34 The First Plant Seco-Steroid and a New lignan from the Australian Arid Zone}

Authors Tan Yuen $\mathrm{P}^{1}$, Savchenko $\mathrm{A}^{1}$, Broit $\mathrm{N}^{2}$, Boyle $\mathrm{G}^{2}$, Parsons $\mathrm{P}^{2}$, Williams $\mathrm{C}^{1}$

Institute 1 University of Queensland, Brisbane, Australia; 2 QIMR Berghofer Medical Research Institute, Brisbane, Australia

DOI 10.1055/s-0037-1608084

Terrestrial plants from Australia's arid zone and deserts have received little attention in terms of bioactive profiling and secondary metabolite discovery. The Australian desert shrub Frankenia foliosa and Eremophila macdonnellii from the Simpson Desert were chosen as a novel species for investigation. F. foliosa has revealed the first B-ring 5,6-seco-steroid (1) isolated from plant and displayed inhibitory activity against head and neck SCC cancer cell lines that contain the $\operatorname{cdc} 25 \mathrm{~A}$ phosphatase protein with $\mathrm{IC} 50=\sim 8.0 \mu \mathrm{M}$. E. macdonnellii has isolated one new, and six known, furofuran lignans. The new lignan (2) displayed moderate anti-cancer activity against the breast cancer cell line (MCF-7) and hypopharyngeal cancer cell line (FaDu) with IC 50 $=10.0 \mu \mathrm{M}$ and IC $50=20 \mu \mathrm{M}$, respectively.

[1] Tan Y.P, Savchenko A.I, Broit N, Boyle, G.M, Parsons, P.G, and Williams C. Eur. J. Org. Chem. 2017; 11:1498-1501. 


\section{Mo-Poster Session 1-PO-35 Alkaloid composition and antimicrobial activity of Chelidonium majus L. from natural habitats and in vitro cultures}

Authors Zielinska $\mathrm{S}^{1}$, Wojciak-Kosior $\mathrm{M}^{2}$, Junka $\mathrm{A}^{3}$, Sowa $\mathrm{I}^{2}$, Wlodarczyk M4, Chmara $S^{5}$, Matkowski $A^{1}$

Institute 1 Department of Pharmaceutical Biology, Wroclaw Medical University, Poland, Wroclaw, Poland; 2 Department of Analytical Chemistry, Medical University of Lublin, Poland; Lublin, Poland; 3 Department of Pharmaceutical Microbiology, Wroclaw Medical University, Poland, Wroclaw, Poland; 4 Department of Pharmacognosy, Wroclaw Medical University, Poland, Wroclaw, Poland; 5 Student Scientific Club no 84, Department of Pharmaceutical Biology, Wroclaw Medical University, Poland, Wroclaw, Poland DOI 10.1055/s-0037-1608085

Chelidonium majus L (Greater celandine) - is an ancient and popular European folk medicinal herb. It grows spontaneously in rich synanthropic habitats and is also cultivated for needs of herbal industry. Major pharmacologically relevant components of $\mathrm{Ch}$. majus are several isoquinoline alkaloids: chelidonine, chelerythrine, sanguinarine, berberine, and coptisine. The compounds are stored and secreted from laticifers - highly specialized elongated cells. However, we also observed a characteristic orange-yellow color of latex in dedifferentiated tissue culture. RP-DAD-HPLC analysis of Ch. majus extracts obtained from roots, immature fruits, and leaves showed differences in the quality and quantity of all analyzed alkaloids. Aerial parts (leaves and immature fruits contained lesser amounts of alkaloids (averaging at $15 \mathrm{mg} / \mathrm{g} \mathrm{dw}$ ) and the major of them were chelidonine in leaves and coptisine along with chelidonine in fruits. In roots, sanguinarine was the predominating compound followed by chelidonine and chelerythrine and the alkaloid content (sum of 7 compounds) reached $40 \mathrm{mg} / \mathrm{g}$.

To assess antimicrobial activity, we used standard microdilution method according to EUCAST guidelines. In case of Gram-positive pathogens, i.e. Staphylococcus aureus and Enterococcus faecalis, $\mathrm{MIC}$ of $\mathrm{MeOH}$ extracts was $62.5 \mathrm{mg} / \mathrm{L}$ and also a strong reduction of bacterial biomass in comparison to untreated bacteria was observed when $31.25 \mathrm{mg} / \mathrm{L}$ of extract was used $-49 \%$ and $68 \%$ for S.aureus and E.faecalis, respectively. All extracts also inhibited Candida growth by 70 to $80 \%$, depending on the fungal strain. For example, leaf $\mathrm{MeOH}$ extract treated C. glabrata cells were reduced by $73 \%$ at the concentration of only $10 \mathrm{mg} / \mathrm{L}$. At $60 \mathrm{mg} / \mathrm{L}$, the leaf extract reduced $80 \%$ of biomass of $C$. parapsilosis and C. krusei. Ch. majus extracts might therefore find an application in eradication of Gram-positive cocci, but are especially promising against Candida pathogenic strains other than C. albicans, including those with significant resistance against standard antimycotic drugs.

\section{Mo-Poster Session 1-PO-36 Anti-inflammatory Diterpenoids from the Formosan Soft Coral Lobophytum varium}

Authors Teng $\mathrm{WT}^{1}$, Huang $\mathrm{CY}^{1}$, Sheu $\mathrm{JH}^{1,2}$

Institute 1 Department of Marine Biotechnology and Resources, National Sun Yat-sen University, Kaohsiung, Taiwan; 2 Frontier Center for Ocean Science and Technology, National Sun Yat-sen University, Kaohsiung, Taiwan DOI 10.1055/s-0037-1608086

In order to discover bioactive secondary metabolites, we have investigated the chemical constituents from the organic extracts of the soft coral Lobophytum varium, which was collected from Jihui Fish Port, Taitung, Taiwan. This study led to the isolation of twelve natural compounds, including five new lobane diterpenes [1], lobovarols A-E (1-4), and an eudesmane derivative [2] lobovarol E (5), along with seven known compounds 6-12. The structures and relative configurations of all metabolites had been established by detailed spectroscopic analyses including 1D and 2D NMR (COSY, HSQC, HMBC and NOESY) data in association with MS experiments. The anti-inflammatory activities of compounds 1-12 on neutrophil pro-inflammatory responses were evaluated by measuring their ability in suppressing $\mathrm{fMLP} / \mathrm{CB}$-induced superoxide anion
$\left(\mathrm{O}_{2}^{-}\right)$generation and elastase release in human neutrophils. From the results, 17,18-epoxyloba-8,10,13(15)-trien-16-ol (11) [3] showed significant inhibition $(45.3 \pm 4.1 \%)$ toward elastase release generation at $10 \mu \mathrm{M}$.

[1] Raju BL, Subbaraju GV, Rao CB, Trimurtulu G. J Nat Prod 1993, 56: 961 966.

[2] Matthée GF, Körrig GM, Wright AD. J Nat Prod 1998, 61: 237 - 240.

[3] Dunlop RW, Wells R. Aust J Chem 1979, 32: 1345-1351.

\section{Mo-Poster Session 1-PO-37 AGEs Formation Inhibitory Compounds of Euphorbia pekinensis and Their Effects on Vessel Dilation in Larval Zebrafish In Vivo}

Authors Lee $\mathrm{k} \mathrm{S} \mathrm{S}^{1}$, Kim Jin $\mathrm{S}^{1}$

Institute $1 \mathrm{KM}$ Convergence Research Division, Korea Institute of Oriental Medicine, Daejeon, Korea, Republic of (South)

DOI 10.1055/s-0037-1608087

This study attempted to identify the active compounds in Euphorbia pekinensis extract for diabetic complications. E. pekinensis extract was applied to a Diaion HP-20 column and was divided into four fractions (I-IV), on the basis of the peaks of UV absorption. Of the four fractions, fraction II, which most significantly inhibited AGEs formation, was purified by preparative RP-HPLC, leading to the isolation of one new ellagic acid derivative, 3,3'-di-O-methylellagic acid 4-O-(6"-O-galloyl)- $\beta$-D-galactopyranoside (1), along with three known compounds, geraniin (2), 3,3'-di-O-methylellagic acid 4-O- $\beta$-D-xylopyranoside (3), ellagic acid 3,3'-dimethyl ether (4). The structure of the new compound was established by extensive spectroscopic studies and chemical evidence. The inhibitory effects of isolated compounds (1-4) on AGEs formation were examined. All compounds markedly inhibited AGE formation with $\mathrm{IC}_{50}$ values of $0.41-12.33 \mu \mathrm{M}$, compared with that of a positive control, aminoguanidine $\left(I C_{50}=1122.34 \mu \mathrm{M}\right)$. In addition, the effects of these isolates on the dilation of hyaloid-retinal vessels induced by high glucose (HG) in larval zebrafish were also investigated. Of the tested compounds, compound 2 significantly reduced the dilation of HG-induced hyaloid-retinal vessels. This compound reduced the diameters of HG-induced hyaloid-retinal vessels by about $82.3 \%$ and $84.6 \%$ at 10 and $20 \mu \mathrm{M}$, respectively, versus the HG-treated control group, whereas the positive control, VEGFR inhibitor, exhibited $77 \%$ inhibition at a concentration of $1 \mu \mathrm{M}$.

\section{Mo-Poster Session 1-PO-38 Selective in vitro and in silico butyrylcholinesterase inhibitory activity of diterpenes and polyphenols from traditional Asian medicinal plants}

Authors Ślusarczyk $\mathrm{S}^{1,2}$, Sezer Senol $\mathrm{F}^{5}$, Matkowski $\mathrm{A}^{2}$, Perez Garrido $\mathrm{A}^{3}$, Girón Rodríguezd $\mathrm{F}^{4}$, Cerón-Carrasco José $\mathrm{P}^{3}$, den Haan $\mathrm{H}^{3}$, Peńa García $\mathrm{J}^{3}$, Pérez Sánchez $\mathrm{H}^{3}$, Erdogan Orhan $\mathrm{I}^{5}$, Kowalczyk $\mathrm{M}^{1}$, Stochmal $\mathrm{A}^{1}$ Institute 1 Department of Biochemistry and Crop Quality,IUNG-Institute of Soil Science and Plant Cultivation, Pulawy, Poland; 2 Department of Pharmaceutical Biology and Botany, Wroclaw Medical University, Wroclaw, Poland; 3 Bioinformatics and High Performance Computing Research Group, Universidad Catolica San Antonio de Murcia (UCAM), Murcia, Spain;

4 Department of Food and Nutrition Technology, Universidad Catolica San Antonio de Murcia (UCAM), Murcia, Spain; 5 Department of Pharmacognosy, Faculty of Pharmacy, Gazi University, Ankara, Turkey DOI $10.1055 / \mathrm{s}-0037-1608088$

Cholinesterase inhibition is one of the treatment strategies against Alzheimer's disease $(A D)$ where metal accumulation is also strongly associated with $A D$ pathogenesis. In this study, we assessed inhibition of acetyl-(AChE) and butyrylcholinesterase (BChE) and metal-chelating capacity of 17 diterpenes from roots of Perovskia and Salvia species and 8 isoflavonoids isolated from Belamcandae chinesis rhizoma. Terpenoids included 13 known (arucadiol, mil- 
tirone, tanshinone Ila, 1-oxomiltirone, cryptotanshinone, 1,2-didehydromiltirone, 1,2-didehydrotanshinone Ila, 1b-hydroxycryptotanshinone, 15,16-dihydrotanshinone, tanshinone I, isotanshinone II, 1(S)-hydroxytanshinone Ila, isogradifoliol) and three new natural diterpenes (1-acetyloxy-cryptotanshinone, 1-acetyloxy-tanshinone Ila and 3,10,10-trimethyl-2,3,9,10-tetrahydroanthra [1,2-b]furan-4,5,7(8 H)-trione (Figure). All these compounds were isolated from Perovskia atriplicifolia Benth. and Salvia glutinosa L. The compounds were tested at $10 \mathrm{mg} / \mathrm{mL}$ using ELISA microtiter assays against AChE and BChE. QSAR and molecular docking studies have been also performed on the active compounds. Most of the compounds showed significant inhibition of BChE in vitro with $\mathrm{IC}_{50}$ between 1.12 and ca. $6 \mathrm{mg} / \mathrm{mL}$ (e. g, $\mathrm{IC}_{50}=1.12 \mathrm{mg} / \mathrm{mL}$ for 1,2 didehydromiltirone, $1.15 \mathrm{mg} / \mathrm{mL}$ for cryptotanshinone, $1.20 \mathrm{mg} / \mathrm{mL}$ for arucadiol) markedly stronger than galanthamine $\left(\mathrm{IC}_{50}=12.56 \mathrm{mg} / \mathrm{mL}\right)$, whereas only 15,16-dihydrotanshinone moderately inhibited AChE (65.17 $\pm 1.39 \%)$. 1,2-Didehydrotanshinone Ila (48.94 $\pm 0.26 \%$ ) and 1 (S)-hydroxytanshinone lla (47.18 $\pm 5.10 \%$ ) had the highest metal-chelation capacity. The present study affords an evidence for the fact that selective BChE inhibitors should be further investigated as promising candidate molecules for $A D$ therapy.

The study was supported by the Polish National Science Center postdoctoral grant" to S. Ślusarczyk (No. FUGA - DEC/2014/12/S/NZ9/00715)

\section{Mo-Poster Session 1-PO-39 Cytotoxic effects of novel semisynthetic shikonin derivatives on melanoma cells}

Authors Durchschein $C^{1}$, Kretschmer $N^{1}$, Rinner $B^{2}$, Deutsch $A^{3}$, Stallinger $A^{2}$, Huefner $A^{4}$, Bauer $R^{1}$

Institute 1 Institute of Pharmaceutical Sciences, University of Graz, Graz,

Austria; 2 Core Facility Alternative Biomodels and Preclinical Imaging, Medical University of Graz, Graz, Austria; 3 Division of Hematology, Medical University of Graz, Graz, Austria; 4 Institute of Pharmaceutical Sciences, Department of Pharmaceutical Chemistry, University of Graz, Graz, Austria

DOI 10.1055/s-0037-1608089

One of the most challenging problems in medicine is treatment of cancer, which is worldwide the second common cause of death. Malignant melanoma is an incurable type of cancer because of its mostly unnoticeable metastatic spreading and aggressive growth rate. Enhancements in the understanding of melanoma biology, the identification and development of novel therapeutic strategies are still urgent research objectives. In the development of new anticancer drugs, natural products are of big relevance. About $87 \%$ of all newly approved anti-cancer drugs are natural products or derived from them [1]. Zicao, the roots of various members of the Boraginaceae family, have been used in TCM to treat macular eruption, measles and burns. Naphthoquinones, the main constituents of the roots, possess antitumor, wound healing, antiphlogistic and antibacterial activities [2]. In this study, the structure of shikonin was altered in order to obtain even more potent and specific derivatives. Previous investigations showed that the naphthoquinone moiety is more crucial for the entire activity than the side chain. There is evidence that the side chain can modify the total activity and can influence pharmacokinetics [3]. The cytotoxic potential of nine semisynthetic shikonin derivatives with modified side chain have been investigated by a XTT based viability assay in several melanoma cell lines (SbCl2, WM164, WM9 and MugMel2) at different concentrations. The identity and purity of the novel semisynthetic compounds has been checked by HPLC and NMR measurements. The most active compounds will be examined in more detail in future experiments.

[1] Newman et al, Journal Natural Products 2016, 79:629-661.

[2] Kretschmer et al, Journal Natural Products 2012, 75:856 - 869.

[3] Damianakos et al, Molecules 2012, 17:14310 - 14322.

\section{Mo-Poster Session 1-PO-40 Anti-Angiogenic diketopiperazines from the Endophytic Fungus Diaporthe arecae Associated with Mangrove Kandelia obovate}

Authors Cheng $\mathrm{YB}^{1,2,3}$, Lu YY ${ }^{1}$, Chang FR', Wang SW${ }^{4}$, Chen $\mathrm{CY}^{5}$, Wu YC $\mathrm{C}^{1,2}$ Institute 1 Graduate Institute of Natural Products, College of Pharmacy, Kaohsiung Medical University, Kaohsiung, Taiwan; 2 Research Center for Natural Products \& Drug Development, Kaohsiung Medical University, Kaohsiung, Taiwan; 3 Center for Infectious Disease and Cancer Research, Kaohsiung Medical University, Kaohsiung, Taiwan; 4 Department of Medicine, Mackay Medical College, New Taipei City, Taiwan; 5 Department of Physical Therapy, Tzu-Hui Institute of Technology, Pingtung, Taiwan

DOI 10.1055/s-0037-1608090

Mangrove is littoral shrub distributes worldwide in subtropics and tropics. The mangrove ecosystem is a high humidity, high salinity, and hypoxia tolerant ecosystem which supports the existence of many halotolerant and halophilic microorganisms. Mangrove-derived endophytes are regarded as abundant sources of bioactive natural products and have aroused great interests of natural product chemists [1]. For instance, chamigrane sesquiterpenes isolated from basidiomycetous fungus showed cytotoxic effect against gastric cancer [2], while polyketides purified from Nectria sp. inhibited $\alpha$-Glucosidase [3]. In our screening of Taiwanese mangrove-derived endophytes, twenty species of mangrove-derived endophytes were successfully purified and cultured. The EtOAc extracts of all isolated endophytes were evaluated for anticancer activity. As a result, the EtOAc extract of Diaporthe arecae isolated from mangrove Kandelia obovate was found to have potent cytotoxic activity against A549, HepG2, and MDA-MB-231 human cancer cell lines. Accordingly, a series of bioassay-guided fractionation for this endophyte was carried out. After repeated chromatography, twenty three diketopiperazines (1-23), were isolated from the culture broth of $D$. arecae. The structures of all isolateds were determined by their mass and NMR (COSY, HMBC, and HSQC) spectroscopic data. In the anti-angiogenic assay, cordysinin A (4) displayed mild inhibited effect by suppressing tube formation with $\mathrm{IC}_{50}$ values of $15.2 \mu \mathrm{g} / \mathrm{mL}$.
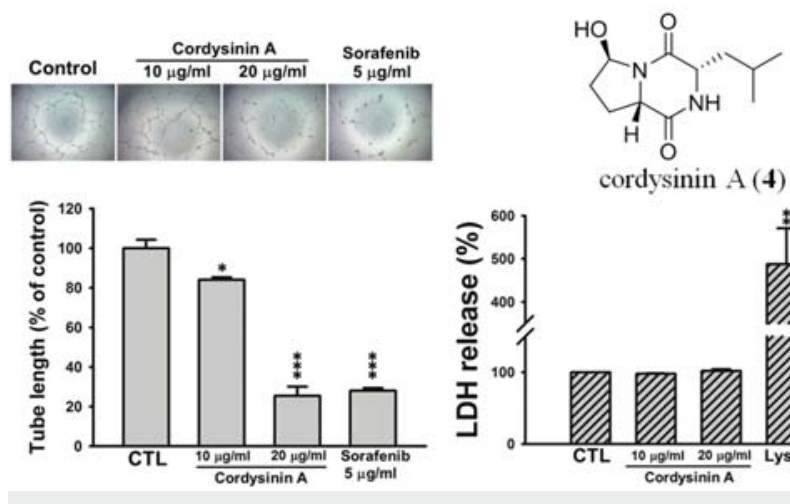

cordysinin $A(4)$

Fig. 1

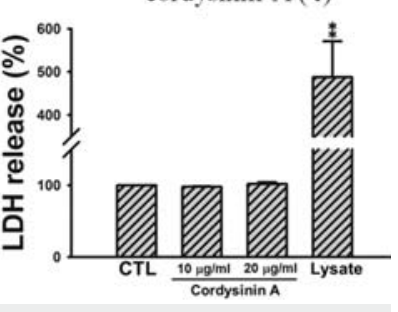

[1] Xu J. RSC Adv 2015; 5: 841

[2] Choodej S, Teerawatananond T, Mitsunaga T, Pudhom K. Mar Drugs 2016; 14: 132

[3] Cui H, Liu Y, Nie Y, Liu Z, Chen S, Zhang Z, Lu Y, He L, Huang X, She Z. Mar Drugs 2016; 14: 86

\section{Mo-Poster Session 1-PO-41 New Alkaloids from Taiwanese Zoanthid Palythoa tuberculosa}

Authors Cheng $\mathrm{YB}^{1,2,3}$, Su Cl$]^{1}$, Chen $\mathrm{SR}^{1}$, Chen $\mathrm{CY}^{4}$

Institute 1 Graduate Institute of Natural Products, College of Pharmacy, Kaohsiung Medical University, Kaohsiung, Taiwan; 2 Research Center for 
Natural Products \& Drug Development, Kaohsiung Medical University, Kaohsiung, Taiwan; 3 Center for Infectious Disease and Cancer Research, Kaohsiung Medical University, Kaohsiung, Taiwan; 4 Department of Physical Therapy, Tzu-Hui Institute of Technology, Pingtung, Taiwan DOI 10.1055/s-0037-1608091

Zoanthid is a radial symmetry cnidarian with two rows of tentacles and is usually found on the rocky coast of subtropical and tropical areas. The various colors of zoanthids were due to the symbiotic relationships with single-celled zooxanthellae, such as dinoflagellates and green algae. Due to its fascinating colors, this sessile benthic organism often becomes ornamentals in aquariums. In addition, zoanthids are also regarded as rich sources of novel secondary metabolites with diverse bioactivities. For example, ecdysones isolated from Palythoa mutuki suppress dengue virus production [1], while alkaloids purified from Zoanthus kuroshio inhibit superoxide anion generation and elastase release [2]. In our bioactive screening of Taiwanese zoanthids, the high polarity fraction of Palythoa tuberculosa was found to have potent cytotoxic and moderate anti-inflammatory activities. Therefore, a series of bioassayguided fractionation for these animal materials was carried out. The animal material of $P$. tuberculosa $(4.0 \mathrm{~kg})$ were extracted by ethanol and separated by consecutive column chromatography. As a result, three new pyrazines (1-3) and five known alkaloids (4-8) were isolated. The structures of all isolates were determined by analyzing their IR, Mass, NMR, and UV spectroscopic data. Cytotoxicity against a human breast cancer cell line (MDA-MB231) and anti-inflammatory activities of the isolated compounds were evaluated.

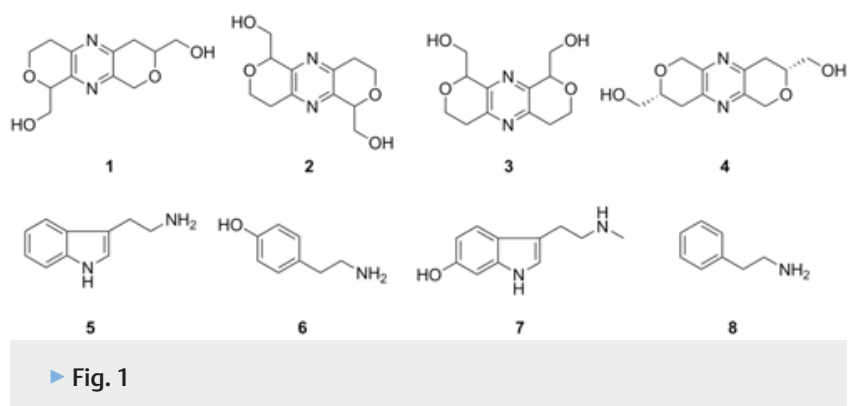

[1] Lee JC, Chang FR, Chen SR, Wu YH, Hu HC, Wu YC, Backlund A, Cheng YB. Marine Drugs 2016; 14: 151.

[2] Hsu YM, Chang FR, Lo IW, Lai KH, El-Shazly M, Wu TY, Du YC, Hwang TL, Cheng YB, YC Wu. J Nat Prod 2016; 79: 2674-2680

\section{Mo-Poster Session 1-PO-42 Identification of mistletoe (Viscum album L.) miRNAs and their putative roles in medicinal applications}

Authors Xie $\mathrm{W}^{1}$, Melzig $\mathrm{M}^{1}$

Institute 1 Institute of Pharmacy, Freie Universität Berlin, Berlin, Germany DOI 10.1055/s-0037-1608092

MicroRNAs (miRNAs) are a class of approximately 22 nucleotides singlestranded non-coding RNA molecules that play crucial roles in gene expression. Zhang et al. suggested that the rice miRNAs might enter mammlian bloodstream and have a functional role in human metabolism [1], which provided thrilling clues that miRNAs might be one of the hidden bioactive compounds in medicinal plants [2].

Viscum album L. (Loranthaceae, European mistletoe) has been widely used for the treatment of cancer and cardiovascular diseases, but its functional compounds have not been well characterized. We assumed that miRNAs might be involved in the pharmacological activities of V. album. In total, 699 conserved miRNAs and 44 novel miRNAs have been identified from $V$. album. Based on the combination of Targetscan, miRanda, PITA, and RNAhybrid methods, the intersection of 30697 potential human genes have been predicted. These putative human targets were mapped to KEGG database. Interestingly, highly enriched KEGG pathways were associated with some human diseases, especially cancer (e.g. VEGF signaling pathway, transcriptional misregulation in cancer, pathways in cancer and MAPK signaling pathway) and cardiovascular diseases (e.g. VEGF signaling pathway, type II diabetes mellitus, calcium signaling pathway and MAPK signaling pathway), which might explain the clinical use as well as folk medicine use of mistletoe. However, further experimental validation is necessary to confirm these human targets of mistletoe miRNAs. In summary, our study provides indirect evidence for the medicinal use of $\mathrm{V}$. album, more importantly, increases the knowledge regarding the miRNAs mediated cross-kingdom regulation between plants and mammalian.

[1] Zhang et al. Cell Res 2012; 22: $273-274$

[2] Xie W, Weng A, Melzig M. Planta Med 2016; 82:1153-1162

\section{Mo-Poster Session 1-PO-43 The anti-inflammatory action of the Amaryllidaceae alkaloid narciclasine is based on the inhibition of leukocyte-endothelial cell interaction}

Authors Stark $A^{1}$, Zuchtriegel $C^{2}$, Reichel $C^{2}$, Bischoff $I^{1}$, Fürst $R^{1}$ Institute 1 Institute of Pharmaceutical Biology, Biocenter, Goethe University, Max-von-Laue Str. 9, 60438, Frankfurt am Main, Germany; 2 Department of Otorhinolaryngology, Head and Neck Surgery and Walter Brendel Centre of Experimental Medicine, University of Munich, Marchioninistr. 15, 81366,

Munich, Germany

DOI 10.1055/s-0037-1608093

Despite the great number of approved anti-inflammatory drugs, the treatment of chronic inflammatory diseases, such as multiple sclerosis or rheumatoid arthritis, is still a therapeutical challenge. Thus, there is an ongoing need for new anti-inflammatory lead compounds. In this context, narciclasine, an Amaryllidaceae alkaloid, was found to exert anti-inflammatory properties. ${ }^{1}$ The goal of our project is the preclinical characterization of narciclasine as an anti-inflammatory agent, in particular, the identification of its mode of action. In vivo, narciclasine inhibited the rolling, firm adhesion and transmigration of leukocytes into the TNF $\alpha$-activated cremaster muscle of C57BL/ 6 mice (intravital microscopy). In vitro, narciclasine significantly reduced the TNFotriggered adhesion of T cells (Jurkat), monocytic cells (THP-1) and primary mononuclear cells to primary endothelial cells. As adhesion was reduced, we investigated the influence of narciclasine on the expression of endothelial cell adhesion molecules and chemokines. Narciclasine significantly downregulated endothelial ICAM-1 (IC50: 54 nM), VCAM-1 (IC 50: 30 nM), E-selectin (IC 50: $51 \mathrm{nM}$ ) and $\mathrm{CX} 3 \mathrm{CL} 1$ protein expression (flow cytometry, western blot analysis). Interestingly, narciclasine did neither influence the activation of the mitogen-activated protein kinases p38 and JNK, nor the NF-KB activation pathway (IKB $\alpha$ expression, p65 translocation). However, narciclasine strongly inhibited the NF-KB-dependent gene expression (reporter gene assay), indicating a transcriptional or translational mechanism. Taken together, the anti-inflammatory property of narciclasine could be based on the inhibition of leukocyteendothelial cell interaction. This action is due to a downregulation of the expression of endothelial cell adhesion molecules and chemokines. The precise molecular mechanism of action of narciclasine will be the subject of our further investigations.

[1] Fuchs S, Hsieh LT, Saarberg W, Erdelmeier CA, Wichelhaus TA, Schaefer L, Koch E, Fürst R. J Cell Mol Med 2015; 19: 1021 - 1032 


\section{Mo-Poster Session 1-PO-44 Anti-hyperglycemic/ antidiabetic activity of Serjanic acid obtained from Cecropia telenitida.}

Authors Balcazar-Morales $N^{1}$, Guillen $A^{1}$, Munoz Diana $L^{1}$, Ramirez-Pineda Jose $R^{1}$, Montoya-Perez Guillermo $L^{2}$, Acin-Martinez $S^{1}$

Institute 1 School of Medicine, Universidad de Antioquia, Calle 62 \# 52 - 59, Medellin, Colombia; 2 School of Natural Science, Universidad ICESI. Calle 18

No. $122-135$, Cali, Colombia

DOI 10.1055/s-0037-1608094

Cecropia telenitida is restricted to the central and northern sections of the Andes. The principal ethno-pharmacological use comprises anti-inflammatory properties, and the treatment of diabetes type 2 [1]. Serjanic acid is one of the most abundant pentacyclic triterpenes present in the roots of $C$. telenitida [2]. In this study, we evaluated the anti-hyperglycemic potential of Serjanic acid by using an insulin resistance mouse model. An ethyl acetate extract of roots of C. telenitida was partitioned with hexane and ethanol. The ethyl acetate was concentrated, diluted in methanol and purified by Sephadex LH-20. The fractions containing triterpenes were pooled and used for analytical and biological assays. We evaluated the effect of Serjanic acid on hyperglycemia, glucose intolerance and insulin blood levels in C57BL/6 J mice fed with a high-fat diet (HFD). After 10 Serjanic acid oral doses, animals were sacrificed. Blood samples were taken to evaluate glucose, insulin, lipid and adipokine plasma levels. We demonstrated that Serjanic acid was effective in ameliorating the hyperglycemia and glucose intolerance in mice fed with HFD. Results showed no change in triglycerides and LDL cholesterol levels. However, serjanic acid reduced total cholesterol, increased HDL and reduced significantly plasma leptin levels in mice fed with a HFD. In addition, Serjanic acid increased insulin levels and this could contribute to reduce the hyperglycemia and improve glucose tolerance.

[1] Aragão DM, Guarize L, Lanini J, da Costa JC, Garcia RM, Scio E. J Ethnopharmacol. 2010; 128:629- 33.

[2] Guillermo L. Montoya Peláeza, Jelver A. Sierra, Fernando Alzate, Ulrike Holzgrabee, José R. Ramirez-Pineda. Rev. Bras. Farmacogn. 2013, 23;754 - 761

\section{Mo-Poster Session 1-PO-45 Phenolic Compounds and Antioxidant Activity of Scorzonera pygmaea Sibth. \& Sm. Subaerial Parts}

Authors Sarı $\mathrm{A}^{1}$, Şahin $\mathrm{H}^{1}$, Özsoy $\mathrm{N}^{2}$

Institute 1 Istanbul University, Faculty of Pharmacy, Department of

Pharmacognosy,? stanbul, Turkey; 2 Istanbul University, Faculty of Pharmacy, Department of Biochemistry,? stanbul, Turkey

DOI 10.1055/s-0037-1608095

The genus Scorzonera is a member of the family Asteraceae, subfamily Liguliflorae, tribe Lactuceae and is represented by around 160 species in the world. There are 52 species in Turkey and 31 of them are endemic. Scorzonera species are used in traditional medicine as analgesic, antirheumatic, anthelmintic, diuretic, wound healer, in the treatment of infertilitiy, gout, pulmonary edema, diarrhea, gastric ulcer, malign gastric cancer and hypertension $(1,2)$. Previously dihydroisocoumarins, benzyl phthalides, flavonoids, lignans, neolignans, bibenzyl derivatives, phenolic acid derivates, kavalactones, sesquiterpenes and triterpenes were reported from Scorzonera species $(2,3)$. In this study, S. pygmaea, the endemic species of Turkey was investigated. Three dihydroisocoumarins (thunberginol C, scorzocreticoside I and II), two phenolic acid derivatives (chlorogenic acid, 3,5-di-O-caffeoylquinic acid) were isolated and identified from subaerial parts using chromatographic and spectral methods. These compounds are reported for the first time from S. pygmaea. Thunberginol $C$ is new for the genus Scorzonera. Total phenolic content and antioxidant capacity of the petroleum ether, choloroform, ethyl acetate and nbutanol fractions of the ethanol extract of subaerial parts of S. pygmaea were investigated via 5 different methods (ferric reducing/antioxidant power assay, inhibition of lipid peroxidation, scavenging activity of DPPH, superoxide and
ABTS radicals). Ethyl acetate fraction showed the highest phenolic content and antioxidant capacity. This is the first report on the antioxidant activity of $S$. pygmaea.

[1] Coşkunçelebi K, Makbul S, Gültepe M, Okur S, Güzel ME. Turk J Bot 2015; 39: $76-87$

[2] Sarı A, Zidorn C, Ellmerer EP, Özgökçe F, Ongania K-H, Stuppner H. Helv Chim Acta 2007; 90: $311-317$

[3] Granica S, Lohwasser U, Johrer K, Zidorn C. Food Chem 2015; 173: 321 331

\section{Mo-Poster Session 1-PO-46 Polyketides and Anti-inflammatory Activities of the Endophytic Fungus Aspergillus ochraceopetaliformis Isolated from Anthurium brownii}

Authors Chang $\mathrm{FR}^{1,2}$, Yang DY ${ }^{1}$, Cheng $\mathrm{YB}^{1,2,3}$, Wu YC $\mathrm{C}^{1,3}$

Institute 1 Graduate Institute of Natural Products, College of Pharmacy, Kaohsiung Medical University, Kaohsiung, Taiwan; 2 Center for Infectious Disease and Cancer Research, Kaohsiung Medical University, Kaohsiung, Taiwan; 3 Research Center for Natural Products \& Drug Development, Kaohsiung Medical University, Kaohsiung, Taiwan

DOI 10.1055/s-0037-1608096

The genus Aspergillus contains a large number of diverse species, and can produce a variety of secondary metabolites [1]. Natural products isolated from Aspergillus are usually nitrogen containing ingredients which demonstrated cytotoxic, antimicrobial, antioxidant and insecticidal activities [2]. Therefore, fungus belonging to Aspergillus genus is regarded as an interesting research topic for new drug development. In the current research, six new polyketides, named asperochralactone A-D (1-4), isoaspyronol (5), 8-demethylaspyronol (6), together with eleven known polyketides 7-17, were isolated from the ethyl acetate layer of the endophyte Aspergillus ochraceopetaliformis Bat. \& Maia (Trichocomaceae), which was isolated and cultured from the leaves of Anthurium brownii Mast. (Araceae). The structures of these compounds were determined by spectroscopic methods, and the relative configurations of the new compounds were elucidated especially by the information of their proton coupling constants and NOESY spectra. Furthermore, the absolute configurations were determined by circular dichroism spectrum (CD) and electronic circular dichroism (ECD) analyses. All isolates were evaluated for anti-inflammatory and cytotoxic activities.

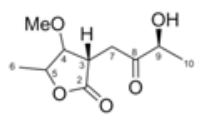

$1^{*} 4 S, 5 S$ $2^{*} 4 R, 5 R$<smiles></smiles>

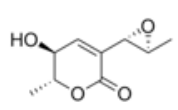

Fig. 1

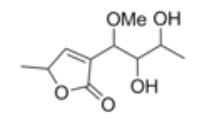

$$
3^{*}
$$

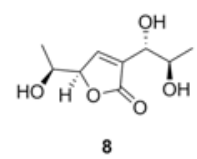

8
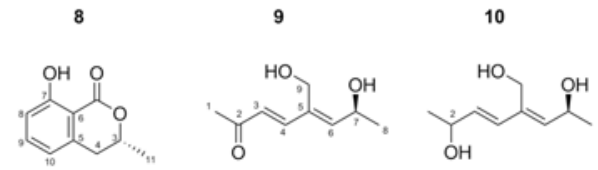

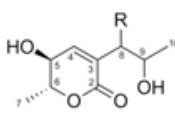

$5^{*} \mathrm{R}=\mathrm{OCH}_{3} ; 8 R$, $9 \mathrm{~S}$ $6^{*} \mathrm{R}=\mathrm{OH} ; 8 \mathrm{~S}, 9 \mathrm{R}$ $12 \mathrm{R}=\mathrm{OCH}_{3} ; 8 \mathrm{~S}, 9 \mathrm{R}$ $13 \mathrm{R}=\mathrm{H} ; 9 \mathrm{~S}$
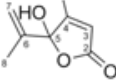

10
Fig. 1

[1] Frisvad JC, Larsen TO. Appl Microbiol Biotechnol 2015; 99: 7859 - 7877

[2] Zhao C, Liu H, Zhu W. Acta Microbiologica Sinica 2016; 56: 331 - 362 


\section{Mo-Poster Session 1-PO-47 Screening for Anti-allergic Activity of Natural Products}

Authors Korinek $\mathrm{M}^{1,2}$, El-Shazly $\mathrm{M}^{1,3}$, Tsai $\mathrm{YC}^{1}$, Wang $\mathrm{LC}^{1}, \mathrm{Yu} \mathrm{ML} \mathrm{M}^{1}$, Wu YC $\mathrm{C}^{1,4}$, Chen $\mathrm{BH}^{2,5}$, Chang $\mathrm{FR}^{1,6}$

Institute 1 Graduate Institute of Natural Products, College of Pharmacy, Kaohsiung Medical University, Kaohsiung, Taiwan; 2 Department of

Biotechnology, College of Life Science, Kaohsiung Medical University,

Kaohsiung, Taiwan; 3 Department of Pharmacognosy and Natural Products

Chemistry, Faculty of Pharmacy, Ain-Shams University, Cairo, Egypt;

4 Research Center for Natural Products \& Drug Development, Kaohsiung

Medical University, Kaohsiung, Taiwan; 5 Department of Medical Research,

Kaohsiung Medical University Hospital, Kaohsiung, Taiwan; 6 Research Center for Environmental Medicine, Kaohsiung Medical University, Kaohsiung, Taiwan DOI 10.1055/s-0037-1608097

Increasing prevalence of allergic diseases with inadequate variety of treatment drives forward search for new alternative drugs. The untapped potential of natural products as an inexhaustible mine of biologically active compounds encouraged us to investigate the potential of separating natural products with promising anti-allergic activity. We utilized degranulation assay [1], which is based on $\beta$-hexosaminidase release from RBL-2H3 cells activated either by calcium ionophore A23187 or antigen (IgE plus DNP-BSA). Over 200 samples were screened for anti-allergic activity revealing a potential of several plants. For instance, ornamental plant Dietes bicolor, seeds of Aquilaria malaccensis, aerial parts of Pandanus amaryllifolius and Liriope platyphylla, leaves of Carica papaya, Artabotrys vinhensis, or nonpolar extracts of Typhonium blumei and $T$. roxburghii [2] exerted promising anti-allergic activity. The fractionation of these extracts, aiming to purify and identify the active compound, yielded various results, with each tested material developing its own unique story. For example, pursuing bioactivity-guided fractionation, anti-allergic phorbol ester was isolated from the seeds of Aquilaria malaccensis [3]. In contrast, the component of active fraction of Liriope platyphylla was never identified, while the ethanol layer of Carica papaya leaves was much more active than any of the isolated pure compounds. Interestingly, some of the chlorophyll-rich fractions showed potent activity, however, the toxicity of the samples raised upon light exposure, thus limiting their utilization. The active compound or mixture may serve as a new candidate for further anti-allergic evaluation and mechanism of action study.

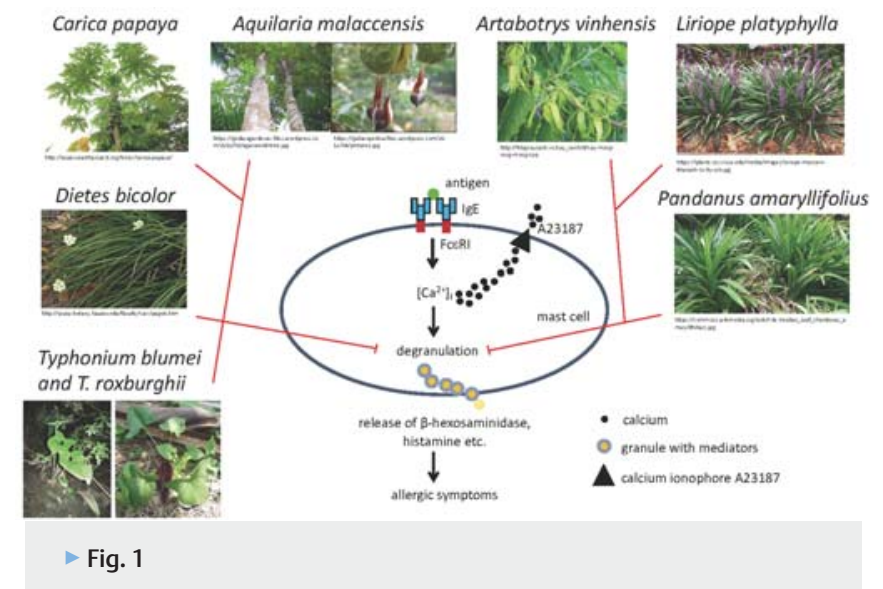

[1] Chen BH, Wu PY, Chen KM, Fu TF, Wang HM, Chen CY. J Nat Prod 2009; 72: $950-953$.

[2] Korinek M, Wagh VD, Lo IW, Hsu YM, Hsu HY, Hwang TL, et al. Int ] Mol Sci 2016; 17: 398.

[3] Korinek M, Chen KM, Jiang YH, El-Shazly M, Stocker ], Chou CK, et al. Phytomedicine 2016; 23: 1706 - 1715.

\section{Mo-Poster Session 1-PO-48 Compounds from Artemisia argyi as potential leads for the development of new immunosuppressive drugs}

Authors Klemd $\mathrm{AM}^{1}$, Reinhardt $\mathrm{JK}^{2}$, Hamburger $\mathrm{M}^{2}$, Gründemann $\mathrm{C}^{1}$ Institute 1 Institute for Environmental Health Sciences and Hospital Infection Control, Faculty of Medicine, University of Freiburg, Freiburg, Germany; 2 Pharmacenter, Department of Pharmaceutical Sciences, University Basel, Basel, Switzerland

DOI 10.1055/s-0037-1608098

The need for novel drugs for the treatment of autoimmune diseases is rising since available pharmaceuticals are often characterized by severe side effects and a limited efficacy [1]. Therefore, we investigated the immunosuppressive potential of a library of 435 extracts prepared from plants used in Traditional Chinese Medicine.Immunosuppressive activity of extracts was assessed in a proliferation-based assay utilizing physiologically relevant anti-CD 3 and antiCD 28 stimulated primary human T lymphocytes [2]. Among others, an ethyl acetate extract of Artemisia argyi (Asteraceae) was found to be highly active. The observed inhibitory effect on $T$ cell proliferation was not based on toxic effects of the extract, as shown by apoptose-/necrosis induction analysis of $T$ lymphocytes. The A. argyi extract was submitted to HPLC-based activity profiling [3]. A T cell proliferation assay identified two active regions in the chromatogram, and a highly active peak with an $\mathrm{IC}_{50}$ of $0.25 \mu \mathrm{g} / \mathrm{ml}$ was identified in the first region. Fractionation of this peak finally determined $(1 R, 2 S, 3 R, 4 S, 5 S, 6 S, 7 S, 10 R)$-canin (1) and two seco-tanapartholides as constituents responsible for most of the activity. $I C_{50}$ values of $0.71 \mu \mathrm{g} / \mathrm{ml}$ for 1 , and $0.24 \mu \mathrm{g} / \mathrm{mland} 0.28 \mu \mathrm{g} / \mathrm{ml}$, respectively, for the $(4 R, 5 R, 6 S, 7 S)$ - and $(4 S, 5 S, 6 S, 7 S)$-seco-tanapartholides (2 and 3), respectively, qualify these compounds as potential immunosuppressive leads. Further investigations focusing on the mode of action are underway.

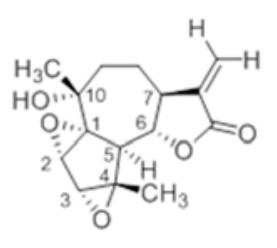

1

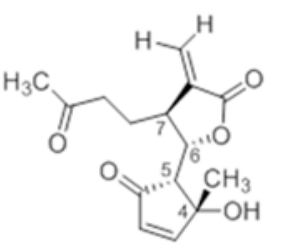

2

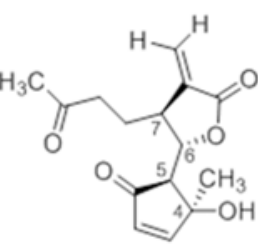

3
Fig. 1

[1] Steinman L. Immune Science 2004; 305: 212

[2] Quah BJC, Parish CR. J Visualized Exp 2010: 2259

[3] Potterat O, Hamburger M. Planta Med 2014; 80: 1171 - 1181

\section{Mo-Poster Session 1-PO-49 Antileishmanial Activity and Cytotoxicity in U937 cells of Essential Oils From Aromatic Plants Commonly Used in Traditional Medicine}

Authors Robledo $\mathrm{SM}^{1}$, Upegui $\mathrm{YA}^{1,2}$, Echeverri $\mathrm{F}^{2}$, Vélez $\mathrm{ID}^{1}$ Institute 1 Programa de Estudio y Control de Enfermedades TropicalesPECET, Facultad de Medicina, Universidad de Antioquia, Medellín, Colombia; 2 Medellín, Colombia. Quimica Orgánica de Productos Naturales -QOPN, Instituto de Química, Universidad de Antioquia, Medellín, Colombia DOI 10.1055/s-0037-1608099

The essential oils from aromatic plants Rosmarinus officinalis, Mentha piperita, Schinus molle, Cinnamomum zeylanicum, Citrus sinensis, Pinus pinea, Lavandina abrial, Hyssopus officinalis, Lippia alba, Cimbopogon nardus, Eletaria cardamomum and Aloysia polystachya are used in traditional medicine because their antibacterial and anti-inflammatory properties. However, there are no available reports of activity against Leishmania parasites. In the present study we evaluated the antileishmanial activity and cytotoxicity of essential oils ex- 
tracted from these plants. The essential oils were solubilized in DMSO not exceeding $0.5 \%$. The cytotoxicity was evaluated on U-937 macrophages using the 3-(4,5-Dimethylthiazol-2-yl)-2,5-Diphenyltetrazolium Bromide (MTT) colorimetric method while the antileishmanial activity was evaluated on $L$. panamensis amastigotes by flow cytometry. The results are expressed as median Lethal Concentration $\left(\mathrm{LC}_{50}\right)$ for cytotoxicity and median Effective Concentration $\left(E_{50}\right)$ for effectiveness, both values calculated by Probit [1]. The most effective essential oil were $M$. piperita and $S$. molle with an $\mathrm{EC}_{50}<3$ and $15.2 \mathrm{mg} / \mathrm{mL}$, respectively while $E$. cardamomun showed moderate antilaeishmanial activity ( $\mathrm{EC}_{50} 43.9 \mathrm{mg} / \mathrm{mL}$ ). None of the essential oils was cytotoxic to the U-937 cells. This is the first report of antileishmanial activity to M. piperita, S. molle and E. cardamomun. Since the essential oils of these plants with high or moderate antileishmanial activity are traditionally used for management of skin lesions, the development of a formulation that combines the antileishmanial and anti-inflammatory properties could be a possible alternative for the management of uncomplicated cutaneous leishmaniasis.

[1] Duque-Benítez SM, Ríos-Vásquez LA, Ocampo-Cardona R, Cedeño DL, Jones MA, Vélez ID, Robledo SM. Molecules 2016; 21:381.

\section{Mo-Poster Session 1-PO-50 Zanthoxylum zanthoxyloides - an African medicinal tree with antimalarial effects}

Authors Wangensteen $\mathrm{H}^{1}$, Hoang $A n \mathrm{~T}^{1}$, Goodman Christopher $\mathrm{D}^{2}$, Diallo $\mathrm{D}^{3}$, Malterud Karl $\mathrm{E}^{1}$

Institute 1 School of Pharmacy, section Pharmacognosy, University of Oslo, Oslo, Norway; 2 School of Biosciences, University of Melbourne, Melbourne, Australia; 3 Department of Traditional Medicine, Bamako, Mali

DOI 10.1055/s-0037-1608100

Malaria is a serious tropical disease and an important cause of death among African children. Zanthoxylum zanthoxyloides is a West African tree that is used against malaria and other diseases by traditional healers. Usually, the bark is used, but it is not well known which constituents are responsible for the antimalaria effect. The anti-malaria effects of substances isolated from the root bark and stem bark extracts of this tree were tested. Twenty substances were isolated and identified from the extracts. Seven of these have not been reported previously from this plant: The alkaloids buesgenine, 6-hydroxydihydrochelerythrine, bis-dihydrochelerythrinyl ether, dictamnine and (-)-p-synephrine, the coumarin 6,7,8-trimethoxycoumarin, and neochlorogenic acid. The dichloromethane extracts of the root bark and stem bark and the methanol extract of the stem bark killed the protozoa Plasmodium falciparum, the organism that gives malaria, with $\mathrm{IC}_{50}$ values between 1 and $10 \mu \mathrm{g} / \mathrm{ml}$. This effect was observed both towards chloroquine sensitive and chloroquine resistant protozoa. Among the isolated substances, the alkaloids skimmianine, $\gamma$ fagarine and bis-dihydrochelerythrinyl ether showed good antiplasmodial effect.

\section{Mo-Poster Session 1-PO-51 Inflammatory response of spilanthol on Human oral cells}

Authors Freitas Blanco Veronica $\mathrm{S}^{1,2}$, Rodrigues Rodney Alexandre $\mathrm{F}^{2}$, Carvalho Joao $\mathrm{E}^{3}$, Alonso Isabella $\mathrm{G}^{4}$, Pastre Julio $\mathrm{C}^{4}$, Murata Ramiro $\mathrm{M}^{5}$ Institute 1 Pharmacology, Anesthesiology, and Therapeutics, Department of Physiological Sciences, Piracicaba Dental School, University of Campinas, Piracicaba, Brazil; 2 Agricultural, Biological, and Chemical Research Center (CPQBA), Department of Chemistry of Natural Products, University of Campinas, Campinas, Brazil; 3 Faculty of Pharmaceutical Sciences, University of Campinas, Campinas, Brazil; 4 Chemistry Institute, University of Campinas, Campinas, Brazil; 5 Department of Foundational Sciences, School of Dental Medicine, East Carolina University, Greenville, United States

DOI 10.1055/s-0037-1608101

Acmella oleracea (L.) R.K. Jansen, a herb native from Brazil, it is traditionally used in the culinary and to treat toothaches. Spilanthol is believed to be responsible for the anesthetic and anti-inflammatory effects presented by this species and therefore possess high therapeutic value [1]. Our study aimed to evaluate the cytotoxicity and the effect of spilanthol in human gingival fibroblast (HGF-1) focusing on the inflammatory pathway. Human gingival fibroblasts, HGF-1 (ATCC ${ }^{\circledR}$ CRL-2014) were seeded in 96 well plates and incubated with different concentrations of spilanthol $(1-1000 \mu \mathrm{M})$ for 24,48 , and $72 \mathrm{~h}$. Toxicity was evaluated using fluorometric quantification of cellular viability (Promega CellTiter-Blue). Cytokine production was analyzed by Human Inflammatory Cytokine Multi-Analyte ELISArray (Qiagen, MEH-004A) and gene expression profile was performed by $\mathrm{RT}^{2}$ Profiler PCR Array using The Human Inflammatory Response \& Autoimmunity (Qiagen, PAHS-077Z). A decrease in cell viability was observed in cells exposed at the highest concentration tested $(1000 \mu \mathrm{M})$ and in a time-dependent manner (Figure 1). Five out of 84 genes related to inflammatory and immune response were down-regulated when the cells were treated with $20 \mu \mathrm{M}$ of spilanthol (fold change $\geq 2$ and p-value $<0.05$ ). The expression of some genes (e.g, SELE, CCL17, IL-9) were significantly down-regulated by spilanthol. However, Spilanthol (20 and $200 \mu \mathrm{M})$ did not affect the cytokine and chemokines levels in HGF-1 supernatant when compared to control. In conclusion, Spilanthol is a promising naturally occurring agent displaying low toxicity and anti-inflammatory activity. The putative pathway by which spilanthol affect inflammation process may involve the downregulation of cytokines and chemokine receptors.

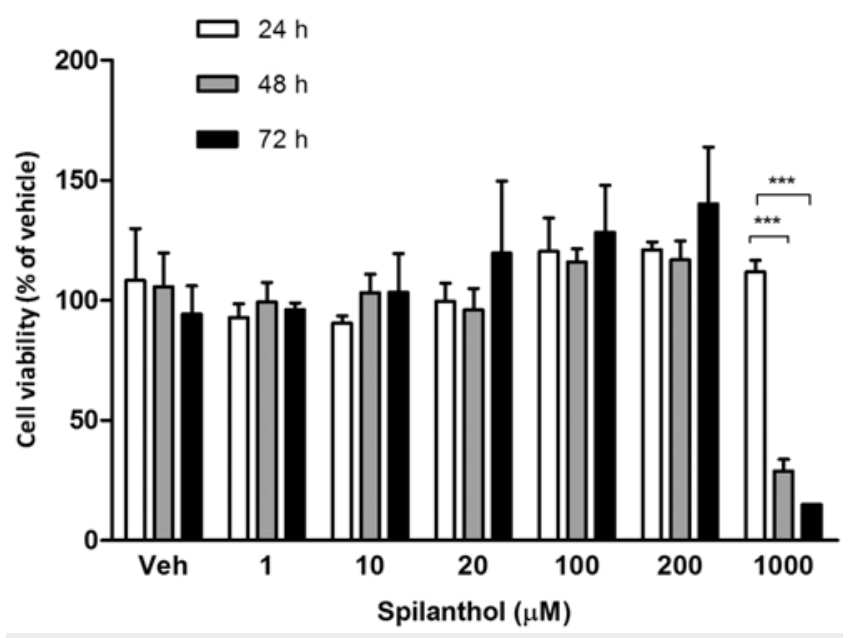

Fig. 1: The cytotoxic effects of spilanthol on human gingival fibroblast (HGF-1) cells.

Capes (grant \# 006211/2015 - 01) and Fapesp (grant \#2014/06461 - 2). [1] Barbosa AF, de Carvalho MG, Smith RE, Sabaa-Srur AUO. Braz. J. Pharmacog. 2016; 26:128-33.

\section{Mo-Poster Session 1-PO-52 Potentiating Activity of Shikonin with Membrane-permeabilizing agents or ATPase inhibitors against Methicillin-resistant Staphylococcus aureus.}

Authors Lee YS ${ }^{1}$, Lee Jae $W^{1}$, Choi Doo J $J^{1}$, Kim GS ${ }^{1}$, Seo Kyung $H^{1}$, Kim Hyung $D^{1}$, Lee Seung $E^{1}$, Kwon $D Y^{2}$, Lee $D Y^{1}$

Institute 1 Department of Herbal Crop Research, National Institute of Horticultural and Herbal Science, Rural Development Administration (RDA), Eumsung, Chungbuk, Korea, Republic of (South); 2 Department of Oriental Pharmacy, College of Pharmacy and Wonkwang-Oriental Medicines Research Institute, Wonkwang University, Iksan, Jeonbuk, Korea, Republic of (South) DOI 10.1055/s-0037-1608102

Shikonin (SKN), alkannin, and their derivatives are highly liposoluble naphthoquinone pigments isolated from the root of the plants belonging to the Boraginaceae family, including Lithospermum erythrorhizon Sieb. et Zucc, Al- 
kanna tinctoria, Arnebia euchroma (Royle) Johnst, and Arnebia guttata Bunge. L. erythrorhizon is a well-known herbal crop in Republic of Korea used in traditional oriental medicine to treat burns, ulcers, hemorrhoids, infected crusts, bedsores, external wounds, and oozing dermatitis. In addition, L.erythrorhizon has been reported to exhibit wound healing, anti-inflammatory, antithrombotic, and antitumor effects, and antimicrobial activity. The aim of this study was to examine Shikonin antibacterial activity against methicillin-resistant Staphylococcus aureus (MRSA). The Shikonin was analyzed in combination with membrane-permeabilizing agents TRIS and Triton X-100, ATPase inhibitors Sodium azide and N,N'-dicyclohexylcarbodiimide, and Staphylococcus aureus-derived peptidoglycan; the effects on MRSA viability were evaluated by the broth microdilution method, time-kill test, and transmission electron microscopy. Addition of membrane-permeabilizing agents or ATPase inhibitors together with a low dose of Shikonin potentiated Shikonin anti-MRSA activity, as evidenced by the reduction of MRSA cell density by $75 \%$ compared to that observed when Shikonin was used alone; in contrast, addition of peptidoglycan blocked the antibacterial activity of Shikonin. The results indicate that the anti-MRSA effect of Shikonin is associated with its affinity to peptidoglycan, the permeability of the cytoplasmic membrane, and the activity of ATP-binding cassette $(A B C)$ transporters. This study revealed the potential of Shikonin as an effective natural antibiotic and of its possible use to substantially reduce the use of existing antibiotic may also be important for understanding the mechanism underlying the anti-bacterial activity of natural compounds.

\section{Mo-Poster Session 1-PO-53 The active constituents of Lamium album as inhibitors of cytokines secretion in human neutrophils}

\author{
Authors Czerwińska $\mathrm{M}^{1}$, Świerczewska $A^{1}$, Granica $\mathrm{S}^{1}$ \\ Institute 1 Department of Pharmacognosy and Molecular Basis of \\ Phytotherapy, Medical University of Warsaw, Warsaw, Poland \\ DOI 10.1055/s-0037-1608103
}

Preparations from Lamium album aerial parts were traditionally used in the treatment of chronic bronchitis, skin and vaginal inflammations. The plant material has been reported to be the valuable source of phenylpropanoids, such as lamiusides, which have not been identified in this species to date [1]. Phenylpropanoids are considered to possess anti-inflammatory activity as well as antithrombotic, wound healing and cardioprotective properties [2]. The aim of the study was a determination of inhibitory potential of constituents from $L$. album against the secretion of inflammatory mediators, such as cytokines (IL-8, TNF- $\alpha$ ) and reactive oxygen species (ROS) by human neutrophils, which are the cells of immune system involved in the inflammation. The compounds were isolated from L. album as it was previously described [1]. Isolated compounds were identified based on UV-Vis, MS and NMR spectra. The inhibition of ROS production was analyzed after f-MLP (formyl-met-leuphenylalanine) stimulation, using luminol-dependent chemiluminescence. The effect of extracts on cytokines, such as IL- 8 and TNF- $\alpha$, secretion after LPSinduced stimulation was established using ELISA tests. Among identified compounds from the groups of phenylpropanoids (lamiusides, phlinoside D, verbascoside), iridoids (caryoptoside, lamiridoside) and flavonoids (tiliroside), in particular phenylpropanoids with methyl groups in a caffeoyl moiety exerted efficacy in the inhibition of IL-8 secretion by neutrophils. The cells treated with glucomartynoside, phlinoside D and lamiuside B produced $29.5 \pm 7.1 \%, 52.4$ $\pm 3.9 \%$ and $64.7 \pm 5.2 \%$ of IL- 8 , respectively, compared to not treated control, whereas they did not inhibit the secretion of TNF- $\alpha$. Simultaneously, the inhibition of ROS production by identified compounds was determined. In conclusion, the cytokine-release inhibitory activity of the methylated phenylpropanoids is proposed as a potential anti-inflammatory mode of their action.

[1] Czerwińska et al, Planta Med. 2017, DOI: 10.1055/s-0043- 107031

[2] Di Paola et al, Biochem Pharmacol. 2009, 77: $845-857$

\section{Mo-Poster Session 1-PO-54 A Dibromotyrosine Derivative from Pseudoceratina sp. Suppresses TGF- $\beta$ Responsiveness by Inhibiting TGF- $\beta$ type I Receptor Kinase Activity}

Authors Chen $\mathrm{CL}^{1}$, Kao $\mathrm{YC}^{1}$, Sung $\mathrm{PJ}^{2,3}$, Wen $\mathrm{ZH}^{4}$

Institute 1 National Sun Yat-sen University, Department of Biological Science, Kaohsiung, Taiwan; 2 National Museum of Marine Biology and Aquarium, Pingtung, Taiwan; 3 National Dong Hwa University, Graduate Institute of Marine Biology, Hualien, Taiwan; 4 National Sun Yat-sen University, Department of Marine Biotechnology and Resources, Kaohsiung, Taiwan DOI 10.1055/s-0037-1608104

For clinical application, there is a great need for small molecule inhibitors (SMIs) that could control pathogenic effects of transforming growth factor (TGF- $\beta$ ) and/or modulate effects of TGF- $\beta$ in normal responses. Selective SMIs of the TGF- $\beta$ signaling pathway developed for therapeutics will also be powerful tools in experimentally dissecting this complex pathway, especially its cross-talk with other signaling pathways. In this study, we characterized ( $1{ }^{\prime}$ R,5'S,6'S)-2-(3',5'-dibromo-1', 6'-dihydroxy-4'-oxocyclohex-2'-enyl) acetonitrile (DT), a member of a new class of small molecule inhibitors related to bromotyrosine derivate from Pseudoceratina sp, which inhibits the TGF- $\beta$ type I receptor serine/threonine kinase known as activin receptor-like kinase (ALK) 5 . The inhibitory effects of DT on TGF- $\beta$-induced Smad signaling and epithelialto-mesenchymal transition (EMT) were investigated in epithelial cells using in vitro kinase assay, luciferase reporter assays, immunoblotting, confocal microscopy, and wound healing assays. The novel ALK5 inhibitor, DT, inhibited the TGF- $\beta$-stimulated transcriptional activations of 3TP-Lux. In addition, DT decreased phosphorylated Smad2 levels and the nuclear translocation of Smad2 increased by TGF- $\beta$. In addition, DT inhibited TGF- $\beta$-induced EMT and wound healing of A549 cells. Our results suggest that DT is a potential therapeutic approach for fibrotic disease and cancer treatment.

\section{Mo-Poster Session 1-PO-55 Hepatoprotective Principles and Other Chemical Constituents from the Mycelium of Phellinus linteus}

Authors Shiow-Chyn $\mathrm{H}^{1}$, Ping-Chung $\mathrm{K}^{2}$, Hsin-Yi $\mathrm{H}^{2}$, Tai-Long $\mathrm{P}^{3}$, TianShung $\mathrm{W}^{2}$

Institute 1 Department of Pharmacy, Chia-Nan University of Pharmacy and Science, Tainan 717, Taiwan, Tainan, Taiwan; 2 School of Pharmacy, National Cheng Kung University Hospital, College of Medicine, National Cheng Kung University, Tainan 701, Taiwan, Tainan, Taiwan; 3 School of Traditional Chinese Medicine, Chang Gung University; Research Center for Industry of Human Ecology, Chang Gung University of Science and Technology; Liver Research Center, Chang Gung Memorial Hospital, Taoyuan 333, Taiwan, Taoyuan, Taiwan

DOI 10.1055/s-0037-1608105

The ethanol extract of cultured mycelium of Phellinus linteus exhibited protective effect against hepatic fibrosis in experimental animals. In the present study, ten new ionone derivatives, phellinulins D-M (4-13), were characterized from the mycelium of $P$. linteus and their structures were established on the basis of spectroscopic and spectrometric analysis. Part of the purified compounds were subjected to the examination of their effects on activated rat hepatic stellate cells and several isolates exhibited significant inhibition of hepatic fibrosis. The results indicated that the mycelium of P. linteus had potential to be developed as hepatoprotective drugs or healthy food in the future. 


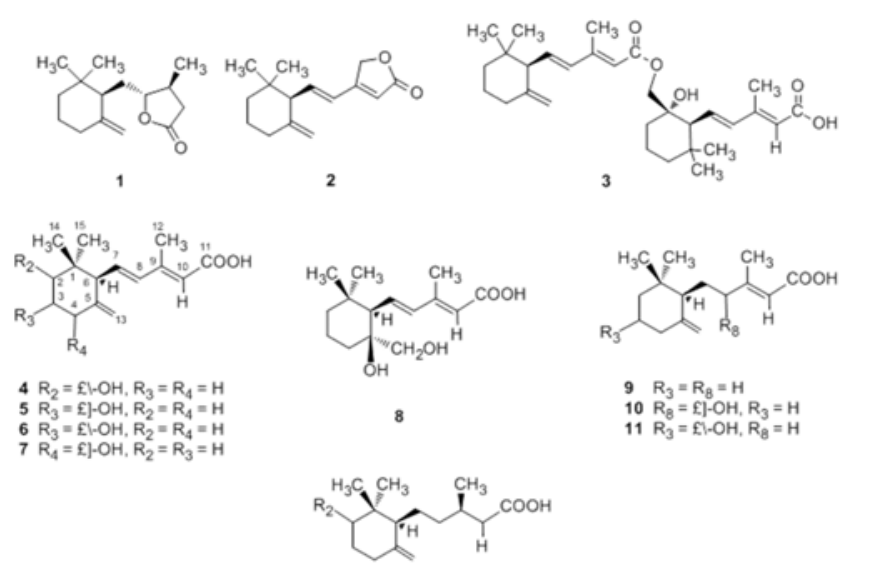

12. $\mathrm{R}_{2}=\mathrm{H}$

Fig. 1: Structures of compounds $1-13$.

Mo-Poster Session 1-PO-56 Hungarian mushrooms as untapped source of natural products: from screening studies to biologically active metabolites

Authors Ványolós $A^{1}$, Kovács $B^{1}$, Béni $Z^{3}$, Dékány $M^{3}$, Krámos $B^{3}$, LiktorBusa $\mathrm{E}^{1}$, Zomborszki Zoltán $\mathrm{P}^{1}$, Zupkó $\mathrm{I}^{2}$, Hohmann J'

Institute 1 Department of Pharmacognosy, University of Szeged, Szeged, Hungary; 2 Department of Pharmacodynamics and Biopharmacy, University of Szeged, Szeged, Hungary; 3 Spectroscopic Research, Gedeon Richter Plc, Budapest, Hungary

DOI 10.1055/s-0037-1608106

Mushrooms have long been recognized for their therapeutic value, particularly in the traditional medicines of several countries in the Far East. Hungary thanks to its varied relief is one of the European countries with higher biodiversity in wild mushrooms: more than 3000 species are indigenous to Hungary, but to date, however, there has been little rigorous scientific study of these mushrooms in terms of their potential pharmacological benefits. In our research program started in 2012, we aimed to identify fungal species with remarkable antiproliferative, antioxidant and antibacterial activities. More than 40 species were screened for their pharmacological properties; several species demonstrated significant biological activity with moderate to high potential. Species exhibiting outstanding biological activity e.g. Gymnopus fusipes and Tapinella atrotomentosa were selected for further detailed mycochemical and pharmacological analyses. Bioactivity-guided isolation led to the identification of two novel cyclopeptides from G. fusipes. Gymnopeptides A and B are the largest cyclic peptides of mushroom origin: they are constituted of 18 monomers, compared to the well-known cyclic peptides of the poisonous Amanita and Omphalotus species, which have 7-12 monomers in their molecules. The antiproliferative property of the isolated compounds was investigated on different human cancer cell lines. Gymnopeptides A and B exhibited outstanding cell growth inhibitory property with IC 50 values in nanomolar range. Detailed investigation of $T$. atrotomentosa, a species with antioxidant and antimicrobial potentials in preliminary studies revealed terphenyl quinone type compounds with notable antioxidant activity in ORAC assay. Our results highlight the importance of further in-depth studies to explore the fairly neglected field of macrofungi.

\section{Mo-Poster Session 1-PO-57 Inhibition of angiogenic key features: the Amaryllidaceae alkaloid narciclasine diminishes proliferation, migration, tube formation and sprouting of human endothelial cells}

Authors Bräutigam $J^{1}$, Nguyen Dieu $L^{2}$, Heiss $E^{2}$, Bischoff $\mathrm{I}^{1}$, Fürst $\mathrm{R}^{1}$ Institute 1 Institute of Pharmaceutical Biology, Biocenter, Goethe University, Frankfurt/Main, Germany; 2 Department of Pharmacognosy, University of Vienna, Vienna, Austria

DOI 10.1055/s-0037-1608107

Narciclasine, an isocarbostyril alkaloid from Narcissus and Haemanthus species, exhibits potent in vitro and in vivo cytotoxicity against cancer cells. Surprisingly, potential effects of narciclasine on endothelial cells in the context of tumor angiogenesis have been neglected so far. Thus, we aimed to elucidate the action of narciclasine on in vitro key features of angiogenesis, i.e. on the proliferation, migration, tube formation and angiogenic sprouting of human primary endothelial cells (ECs). Treatment of ECs with narciclasine up to a concentration of $300 \mathrm{nM}(48 \mathrm{~h})$ did not affect the metabolic activity, while cell proliferation was concentration-dependently reduced (IC $\left.\mathrm{C}_{50}: 61 \mathrm{nM}\right)$. Moreover, narciclasine (300 $\mathrm{nM}$ ) reduced the undirected migration of ECs (scratch assay) by $54 \%$ and the chemotactic migration (Boyden chamber) by $77 \%$. In a 2D migration assay, narciclasine lowered the accumulated (50\%) and euclidean distance (44\%), diminished the velocity $(50 \%)$ and attenuated the $y$ forward migration index by $30 \%$. Most importantly, the alkaloid reduced both the formation of endothelial tube-like structures on Matrigel and the growth factor-induced sprouting of EC spheroids (100 and $300 \mathrm{nM}$ ). Regarding the underlying mode of action, narciclasine did neither inhibit the activation of Erk and Akt (Western blot analysis), nor interfere with endothelial nitric oxide production (arginine/citrulline conversion). Interestingly, ECs partially recovered their capacity to migrate when the Rho-associated protein kinase (ROCK) was inhibited, indicating that narciclasine might act by ROCK activation. Correspondingly, microscopical analysis of the cytoskeleton revealed that narciclasine increased the formation of F-actin stress fibers, which is mediated via the Rho-ROCK signaling pathway. Taken together, the isocarbostyril alkaloid narciclasine effectively decreases key features of angiogenesis in ECs. Thus, narciclasine might represent a promising anti-angiogenic lead compound. Moreover, we suggest that the compound's mode of action partially depends on the activation of the ROCK signaling pathway.

\section{Mo-Poster Session 1-PO-58 Bioactive coumarin derivatives from Murraya exotica}

Authors Liu $\mathrm{B}^{1}$, Zhang $\mathrm{C}^{1}$, Zeng $\mathrm{K}^{1}$, Tu $\mathrm{P}^{1}$, jiang $\mathrm{Y}^{1}$

Institute 1 State Key Laboratory of Natural and Biomimetic Drugs, Peking University, Beijing, China

DOI 10.1055/s-0037-1608108

Murraya exotica is a dwarf tree native to the tropical and subtropical areas of Asia with white petals. As one of the source plants of Murrayae Folium et Cacumen listed in Chinese Pharmacopoeia (2015 Edition), this herb has been extensively used for the treatment of toothache, eczema, and rheumatism. Previous chemical and pharmacological studies showed that the main active components in M. exotica are coumarins, possessing anti-inflammatory, analgesic, and antitumor activities. As an ongoing search for more new active natural products from Murraya genus, the $95 \%$ aqueous ethanol extract of the roots of $M$. exotica was investigated to afford 50 coumarin derivatives, including two novel heterodimers of isopentenyl-substituted indole and coumarin derivatives linked through a new fused heptacyclic ring system $(1-2)$, three novel terpenophenylpropanoids formed by two isopentenyl substituted 4-hydroxycinamyl alcohol through Diels-Alder [4+2] cycloaddition and cyclic hemiacetal formation ( $3-5)$, and ten new coumarin derivatives ( 6 - 15), along with 35 known analogues $(16-50)$. Their absolute configurations were determined by Mosher's method, exciton chiral method, ECD spectrum calculation, singlecrystal X-ray diffraction, as well as ECD data of the in situ formed transition 
metal complex. The biosynthetic path way of compounds $1-5$ were proposed based on the structural characters. Compounds 1, 4, 30, 35, and 37 showed potent inhibition against LPS-induced NO production in BV-2 microglial cells with IC 50 values of $9.2 \pm 0.2,8.6 \pm 0.3,11.8 \pm 0.9,15.5 \pm 0.9$, and $16.9 \pm 1.0$ $\mathrm{mM}$, respectively. Structure-activity relationship analysis indicated that the polarity and the stereo-configuration are the main factors related to the activity. Compound 31 showed remarkable inhibitory effect to ion channel protein of transient receptor potential A1 (TRPA1). In conclusion, these results revealed the possible pharmacodynamic material for the anti-inflammation and analgesic effects of $M$. exotica, and supplied a valuable reference for the further researches of this drug.

\section{Mo-Poster Session 1-PO-59 Anti-melanogenesis components from Leonurus japonicus}

Authors Lai $\mathrm{KY}^{1}$, Lee $\mathrm{CL}^{1}$, Chiang $\mathrm{HM}^{1}$

Institute 1 Department of Cosmeceutics, China Medical University, Taichung, Taiwan

\section{DOI 10.1055/s-0037-1608109}

Leonurus japonicus (Lamiaceae), a traditional Chinese medicine (TCM), could inhibit the melanin synthesis on B16F10 melanoma cells. No anti-melanogenesis phytochemical study of this TCM has been reported to date. Therefore, the species was selected as a lead candidate of anti-melanogenesis drugs developments to investigate its active components and mechanism of action. The methanol extracts (LJM) of dried $L$. japonicus showed anti-melanogenesis effects, and then the LJM were partitioned into EtOAc (LJME) and aqueous extracts (LJMW). The LJME fraction has significant inhibitory effects against B16F10 cells at the concentrations of 50 and $100 \mu \mathrm{g} / \mathrm{mL}$, but cytotoxicity at concentration of $200 \mu \mathrm{g} / \mathrm{mL}$. Additionally, the LJMW fraction showed antimelanogenesis effects in a dose-dependent manner. The LJME fraction was further isolated into $95 \% \mathrm{MeOH}$ (LJMEM)- and hexane- (LJMEH) soluble fractions. Chromatographic fractionation of the LJMEM led to isolation 24 compounds, nine diterpenes $1-9$, four monoterpenes $10-13$, four flavones $14-$ 17 , four benzenoids $18-21$, and two lignans $22-23$. The isolates $1-7$ were elucidated as new compounds via various spectroscopic data. Anti-melanogenesis effects of $1-23$ (IC50<50 $\mathrm{g} / \mathrm{mL}$ ) were evaluated to clarify the potential activity of $L$. japonicus. Overall, our studies identified $L$. japonicus as a promising plant source of new agents for whitening drugs and cosmetic research.

\section{Mo-Poster Session 1-PO-60 Effects of glycyrrhizin pre-treatment on transient middle cerebral artery occlusion induced brain injury in mice}

Authors Chiyeon $L^{1}$, Sehyun $L^{2}$, Suin $C^{3}$

Institute 1 College of Medicine, Dongguk University, Koyang, Korea, Republic of (South); 2 School of Public Health, Far East University, Chungcheongbukdo, Korea, Republic of (South); 3 School of Korean Medicine, Pusan National University, Yangsan, Korea, Republic of (South)

DOI 10.1055/s-0037-1608110

Glycyrrhizin is the main sweet component in licorice and has a number of pharmacological activities, which include neuro-protective, anti-fungal, and anti-cariogenic activities. Ischemia induced brain damage is the leading cause of adult disability and the fifth leading cause of death, and thus, the development of anti-apoptotic neuro-protective therapeutic agents is viewed as an attractive developmental strategy. This study was undertaken to investigate the effects of glycyrrhizin on ischemia-induced brain damage. In infarct volumes and the levels of several apoptosis-related proteins, caspase-3, -8, 9, $\mathrm{BCl}-\mathrm{xL}, \mathrm{BCl}-2$, and their activities in the brains of middle cerebral artery occlusion (MCAO) treated mice were measured using western blotting methods. Single pre-treatment with glycyrrhizin $(10-100 \mathrm{mg} / \mathrm{kg})$ at 2 hours before MCAO significantly reduced infarct volumes at $24 \mathrm{~h}$ after MCAO. In addition, glycyrrhizin effectively inhibited the activations of caspase- 3 and -9 and the down-regulation of Bcl-xL protein caused by MCAO. The neuro-protective effect of glycyrrhizin was found to be due to its regulation of apoptosis-related proteins signals.

[1] Kim SW, Jin Y, Shin JH, Kim ID, Lee HK, Park S and Lee JK. Neurobiology of Disease 2012;46:147 - 156 .

[2] Faraji ], Lehmann H, Metz GA and Sutherland RJ. Neuroscience Letters 2009;462:248- 252.

\section{Mo-Poster Session 1-PO-61 Isolation and biological evaluation of prenylated flavonoids from Maclura pomifera}

Authors Ross $S^{1,4}$, Orazbekov $\mathrm{Y}^{1,2,3}$, Srivedavyasasri $\mathrm{R}^{1}$, Mombekov $\mathrm{S}^{2}$, Datkhayev $\mathrm{U}^{2}$, Makhatov $\mathrm{B}^{3}$

Institute 1 National Center for Natural Products Research, University of Mississippi, MS, University, United States; 2 Kazakh National Medical

University, Almaty, Kazakhstan; 3 South Kazakhstan Pharmaceutical Academy, Al-Farabi square, Shymkent, Kazakhstan; 4 Department of BioMolecular Science, University of Mississippi, MS, University, United States DOI 10.1055/s-0037-1608111

Maclura pomifera (Moraceae), is native to Southwestern United States, and known as Osage orange. Various Maclura species are worldwide used in folkloric medicine [1]. M. pomifera and its components possess several biological activities including cytotoxic, antitumor, antibacterial, estrogenic, antifungal, antiviral and antimalarial activities [2 -4]. Several prenylated flavonoids with potent pharmacological effects were previously reported from $M$. pomifera. Herein, we report the isolation, characterization, and biological evaluation of four new (I-IV) and nine known compounds: warangalone, auriculasin, osajin, pomiferin, artocarpesin, tonkinensisol, corchoionoside $\mathrm{B}$, stigmasterol and $\beta$ sitosterol from the fruits of $M$. pomifera growing in Kazakhstan. The total extract showed significant activity towards cannabinoid receptors (CB1103.4, CB2 $-68.8 \%$ displacement) and possibly allosteric towards $\delta$ and $\mu$ opioid receptors (-49.7, and $-53.8 \%$ displacement, respectively). Compound I exhibited possible allosteric activity in $\mathrm{k}$ and $\mu$ opioiod receptors $(-88.4$, $27.2 \%$ displacement, respectively), and showed moderate activity (60.5\%displacement) towards $\mathrm{CB} 1$ receptor.

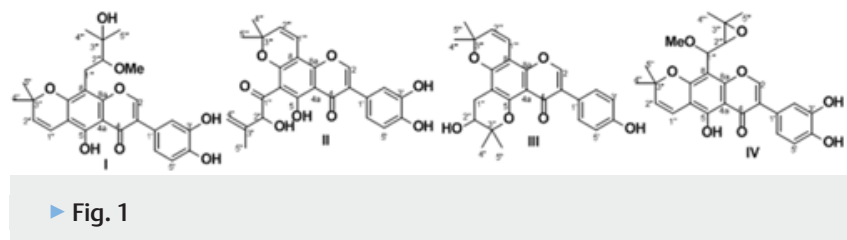

The project was supported by Kazakhstan government and National Center for Natural Product Research, USA. We would like to acknowledge Award Number P20GM104932 from the National Institute of General Medical Sciences, USA for bioassay results.

[1] Bourdy G, de Michel LRC, Roca-Coulhard A. J. Ethnopharmacol. 2004; 91: $189-208$.

[2] Bunyapraphatsara N, Dechsree S, Yoosook C, Herunsalee A, Panpisutchai Y. Phytomedicine 2000; 6: 421 - 424.

[3] Peterson CF, Brockemeyer EW. Am. J. Pharm. Sci. Suppl. Pub. Hlth. 1953; 125: $303-310$

[4] Jones JM, Soderberg F. Cell Immunol. 1979; 42: 319- 326. 


\section{Mo-Poster Session 1-PO-62 Isolation of potent tripanocidal compounds - primin and miconidin - through a bioguided study of Miconia wildenowii}

Authors Rosa $\mathrm{W}^{1}$, Santana Caldas I ${ }^{1}$, Aparecida Chagas-Paula D ${ }^{1}$, Luiz Baldim J ${ }^{1}$, Pedro Costa Elias J ${ }^{1}$, Gomes Soares $\mathrm{M}^{1}$

Institute 1 Federal University of Alfenas, UNIFAL-MG, Alfenas, Brazil

DOI 10.1055/s-0037-1608112

Trypanosoma cruzi affects about 8 to 9 million people all over the world, leading to approximately 14,000 deaths annually, therefore considered one of the most troublesome disease among those known like neglected. The demand for more effective medicines against $T$. cruzi parasites has rised, leading to search for new compounds mainly natural products plant derivatives. From a screening of crude extracts of different species of plants, Miconia wildenowii, an endemic Brazilian rainforest specie, presented high biological activity against epimastigote forms, strain $\mathrm{Y}$, of $T$. cruzi. Then, its crude extract was partitioned into hexane, ethyl acetate and mixture ethanol: water. The three fractions were submitted to the same anti-epimastigote assay showing that the ethyl acetate fraction was the most active. This fraction was subjected to silica gel column chromatography and eluted with a gradient of solvents like hexane, ethyl acetate and methanol by gradually increasing the polarity, yielding 12 subfractions. These also were evaluated against $T$. cruzi epimastigotes forms in which three of them presented higher antichagasic activity. HPLC-UV-DAD profile of these active subfractions exposed the same two compounds. Isolation was performed in the same equipament and identification could be made by NMR and mass data of the compounds, in comparison with data on literature, characterized as primin and miconidin. The anti-epimastigote assay with both primin $(E C 50=2,59 \mu \mathrm{M})$ and miconidin $(E C 50=5,70 \mu \mathrm{M})$ showed that they are more efficient than the reference drug benznidazole $(\mathrm{EC} 50=9,46 \mu \mathrm{M})$. Both isolated compounds presented cytotoxic effects only in higher concentration, evidencing the anti-chagasic potential of them.

FAPEMIG, FINEP, CAPES and CNPq.

Paucar R. et al. Curr Med Chem. 2016;23:3154-3170.

\section{Mo-Poster Session 1-PO-63 Effect of melittin from Apis mellifera venom on bronchogenic carcinoma cell proliferation and tumor-associated macrophage differentiation}

\footnotetext{
Authors Chanchao $C^{1}$, Tipgomut $C^{1}$, Takeo $E^{2}$, Wongprommoon $A^{3}$, Ittiudomrak $\mathrm{T}^{4}$, Puthong $\mathrm{S}^{5}$

Institute 1 Department of Biology, Faculty of Science, Chulalongkorn University, 254 Phayathai Road, Bangkok 10330, Thailand, Bangkok, Thailand; 2 Department of Biotechnology, Graduate School of Engineering, Osaka University, 2 - 1 Yamadaoka, Suita, Osaka, 565 - 0871, Japan, Osaka, Japan; 3 Homerton College, University of Cambridge, Hills Road, Cambridge CB2 $8 \mathrm{PH}$, United Kingdom, Cambridge, United Kingdom; 4 Program in Biotechnology, Faculty of Science, Chulalongkorn University, 254 Phayathai Road, Bangkok 10330, Thailand, Bangkok, Thailand; 5 Institute Biotechnology and Genetic Engineering, Chulalongkorn University, 254, Phayathai Road, Bangkok 10330, Thailand, Bangkok, Thailand

DOI 10.1055/s-0037-1608113

Bronchogenic carcinoma or lung cancer is the most leading cause to death nowadays. Although paclitaxel extracted from Taxus brevifolia (Pacific yew) has commonly used as drug to treat lung cancer patients, drug resistance has become a serious problem. Thus, an alternative anti-cancer drug is challenging. Here, melittin, a water-soluble toxic peptide, from Apis mellifera venom, is the interest. Due to the half maximum concentration from MTT assay, melittin was more cytotoxic to human bronchogenic carcinoma cells (Chago K-1) than human lung fibroblasts (CRL-1947) as normal control cells. By annexin $\mathrm{V}$ and propidium iodide staining and flow cytometry analysis, melittin could induce Chago K-1 to undergo apoptosis. In addition, it caused the cell
}

cycle arrest of only Chago K-1 at S phase. To investigate tumor microenvironment which directly affects the survival of cancer cells, the activation of circulating monocytes (THP-1) to differentiate into tumor-associated macrophages (TAMs) was investigated. By sandwich-ELISA used to measure interleukin-10, a molecule secreting out of TAMs, the result showed that melittin could inhibit the differentiation of THP-1 into TAMs. Since cathepsin S (cat S) is significantly involved with the severity of cancer in term of proliferation, migration and angiogenesis, here, the expression of cat $S$ was observed by quantitative RT PCR. It was found that the expression of cat $\mathrm{S}$ was decreased in melittin treated Chago K-1. Also, by flat plate colony formation assay to observe cell proliferation and migration, melittin could reduce the number of colonies forming in Chago K-1 cells, comparing to untreated cells. However, by ELISA assay for secretion of vascular endothelial growth factor (VEGF), melittin could not affect angiogenesis of Chago $\mathrm{K}-1$. In conclusion, melittin could induce apoptosis and arrest the cell cycle of Chago K-1. Furthermore, it could inhibit the differentiation of THP-1 into TAMs and decrease the expression of cat $\mathrm{S}$.

\section{Mo-Poster Session 1-PO-64 STEROIDAL ALKALOIDS AS POTENT AGENTS AGAINST Trypanosoma brucei rhodesiense}

Authors Nnadi $\mathrm{CO}^{1,2}$, Nwodo $\mathrm{NJ}^{2}$, Kaiser $\mathrm{M}^{3,4}$, Brun $\mathrm{R}^{3,4}$, Schmidt Thomas J Institute 1 Institute of Pharmaceutical Biology and Phytochemistry (IPBP), University of Münster, PharmaCampus, Corrensstraße 48, D-48149, Münster, Germany; 2 Department of Pharmaceutical and Medicinal Chemistry, Faculty of Pharmaceutical Sciences, University of Nigeria Nsukka, 40001 Nsukka, Enugu State, Nigeria; 3 Swiss Tropical and Public Health Institute (Swiss TPHI), Basel, Switzerland; 4 University of Basel, Socinstr. 57, Basel CH-4051, Basel, Switzerland

DOI 10.1055/s-0037-1608114

Against the backdrop of our previous preliminary report on steroid alkaloids with promising antitrypanosomal activity from the leaves of West African Holarrhena africana [1], we have extended our search for such alkaloids to the stem bark. Bioactivity-guided isolation by repeated column chromatographic separation and purification of various alkaloidal fractions yielded six $(1-6)$ steroidal alkaloids from the leaves [1], and eleven (7-17) steroidal alkaloids as well as one (18) nitrogen-free steroid from the stem bark. The structures were resolved by comparison of HRMS, 1D and 2D NMR spectra with reported data [2, 3]. All compounds were tested in vitro against Trypanosoma brucei rhodesiense and for cytotoxicity to mammalian cells ( $\mathrm{L} 6$ cell line) and some structure-activity relationships became evident. The presence of a basic amino group at position 3 was found to be necessary for high antitrypanosomal activity of the steroid alkaloids. Pairwise comparison of the $I_{50}$ values $(\mu \mathrm{M})$ of $1(0.40 \pm 0.28)$ and $3(0.08 \pm 0.01), 12(0.42 \pm 0.09)$ and 13 $(0.17 \pm 0.11), 15(1.66 \pm 0.39)$ and $14(0.12 \pm 0.08)$ showed that monomethylation at the 3 -amino group represents an optimum for antitrypanosomal activity. Comparison of the $I_{50}$ values of $4(1.20 \pm 0.75)$ and $5(0.67 \pm 0.03)$ furthermore indicates that the $\mathrm{C}-3 \beta$ amino substitution enhances activity. Overall, antitrypanosomal potency increases in the order $\mathrm{O}<\mathrm{NH}<\mathrm{nh} 2<\mathrm{N}$ $(\mathrm{CH}<\mathrm{n}(\mathrm{ch} 3) 2<\mathrm{NHCH}<\mathrm{nhch} 3$ with respect to substitution at $\mathrm{C}-3$. The antitrypanosomal activities of these steroid alkaloids have not been reported before. Further studies on structure-activity relationships in terms of QSAR are currently in progress and will represent the basis for eventual lead optimization.<1 nhch $<$ n $(\mathrm{ch}</$ nh

[1] Nnadi CO, Nwodo NJ, Brun R, Kaiser M, Schmidt TJ. Planta Medica. 2016; 82(S 01):S1-S381.

[2] Zirihi GN, Grellier P, Guede-Guina F, Bodo B, Mambu L. Bioorg Med Chem Lett. 2005; 15:2637 - 2640.

[3] Leboeuf M, Cavé A, Goutarel R. Ann Pharm Fr. 1969; 27:217 - 28. 


\section{Mo-Poster Session 1-PO-65 Anti-trypanosomal activity of flavones and chlorogenic acids from Moquiniastrum floribundum (Asteraceae)}

Authors Indy Tamayose $\mathrm{C}^{1}$, Mesquita $\mathrm{JT}^{2}$, Lima $\mathrm{ML}^{2}$, Galuppo $\mathrm{MK}^{2}$, Tempone $\mathrm{AG}^{2}$, Roque $\mathrm{N}^{3}$, Ferreira MJP1

Institute 1 University of São Paulo, São Paulo, Brazil; 2 Adolfo Lutz Institute, São Paulo, Brazil; 3 Bahia Federal University, Salvador, Brazil

DOI 10.1055/s-0037-1608115

Asteraceae is one the largest families of Eudicots comprising several species used for economic and medicinal purposes, and characterized by the production of various bioactive metabolites ${ }^{1}$. Among the 1700 genera of the family, Moquiniastrum (Cabrera) G. Sancho is restricted to South America and comprises 21 species, 12 of them endemic to Brazil ${ }^{1}$. The aim of this work is to report the phytochemical study of polar phases from Moquiniastrum floribundum and the phytotoxic activities of their isolated compounds. Aerial parts (421.7 g) of M. floribundum were extracted with hexane and subsequently with methanol. Methanol extract was partitioned successively with hexane, dichloromethane, ethyl acetate and butanol. The partition phases were subjected to silica gel and preparative HPLC, respectively, furnishing the compounds $1-\mathbf{8}$. The structures were elucidated by ${ }^{1} \mathrm{H}$ and ${ }^{13} \mathrm{C}$ NMR, gHMBC and comparison with literature data. Thus, the compounds were identified as three flavones: genkwanin (1), hispidulin (2) and cirsimaritin (3), and five chlorogenic acid derivatives: 4,5-(4), 3,5-(5) and 3,4-(6) dicaffeoylquinic acids, and 3,5-dicaffeoylquinic acid methyl ester (7) and 3,4,5-tricaffeoylquinic acid methyl ester (8). The anti-trypanosomal activity of compounds was tested against trypomastigotes of Trypanosoma cruzi. Compound 1 and 8 showed significant activity exhibiting $50 \%$ inhibitory concentration $\left(\mathrm{IC}_{50}\right)$ values of $72.6 \mu \mathrm{M}$ and $40.6 \mu \mathrm{M}$, respectively. Additionally, the compounds were evaluated for cytotoxicity against NCTC cells and they did not display cytotoxicity $\left(\mathrm{CC}_{50}\right.$ superior to $200 \mu \mathrm{M})$.

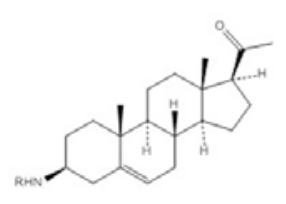

1: $\mathrm{ReH}, 2: \mathrm{R}=\mathrm{COCH}_{3}, 3: \mathrm{R}=\mathrm{CH}_{2}$
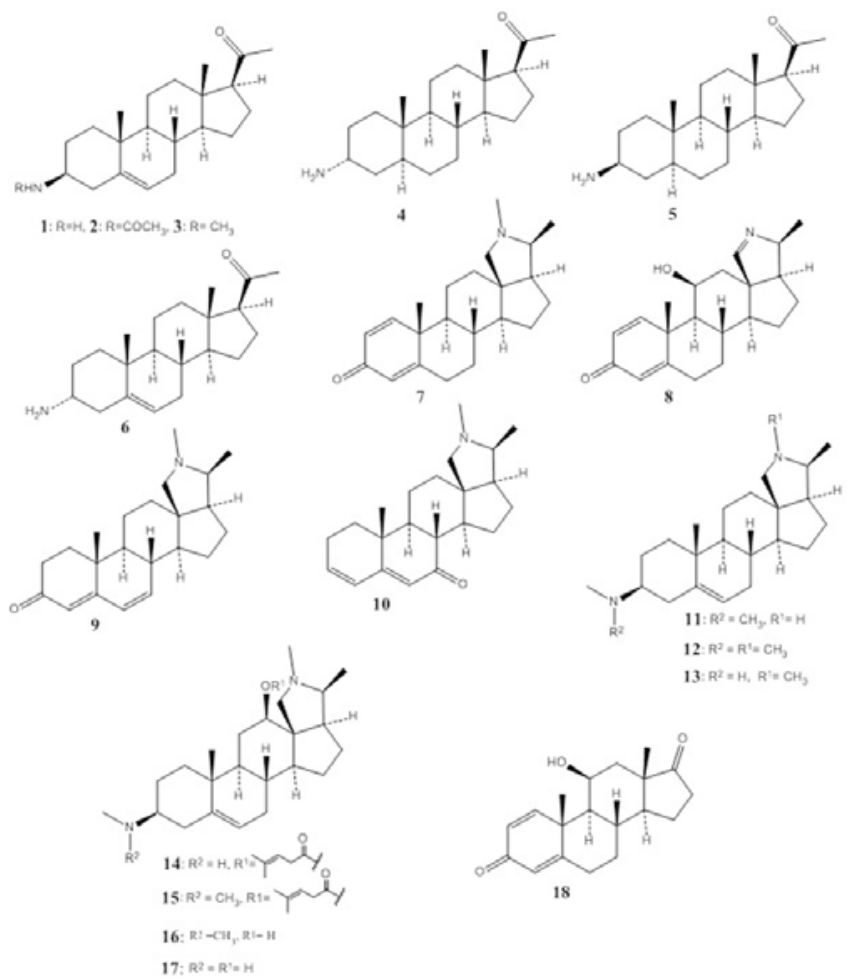

Fig. 1: Steroidal alkaloids and steroidal non-alkaloid isolated from the leaves $(1-6)$ and stem bark (7 - 18) of Holarrhena africana

[1] Sancho G, Funk VA, Roque N. Phytotaxa 2013; 147: 26 - 34

\section{Mo-Poster Session 1-PO-66 HIV-integrase inhibitory activity of natural flavonoids}

Authors Hohmann ${ }^{1,2}$, Hunyadi $A^{1,2}$, Musat MG $^{3,4}$, Matei $A^{3}$, Ciubotaru $M^{3,5}$ Institute 1 Institute of Pharmacognosy, University of Szeged, Szeged,

Hungary; 2 Interdisciplinary Centre of Natural Products, University of Szeged, Szeged, Hungary; 3 Department of Immunology, Colentina Clinical Hospital Research Center, Bucharest, Romania; 4 Department of Biochemistry, Faculty of Pharmacy, "Carol Davila" UMF, Bucharest, Romania; 5 Department of Life and Environmental Physics, National Institute for Physics and Nuclear Engineering Horia Hulubei, Bucharest-Magurele, Romania

DOI 10.1055/s-0037-1608116

HIV integrase inhibitors (INi) are among the most effective agents used in the treatment of HIV infection. They target HIV IN, the enzyme that inserts the viral genome into the chromosomes of the host cells. IN binds to sequences, flanking the viral DNA called LTR (Long Terminal Repeats) and cleaves specific phosphodiester bonds at $3^{\prime}$ ends (the $3^{\prime}$ processing reaction). In earlier studies, $\beta$-ketoenol structure and coplanar aromatic rings have been recognised as pharmacophores of IN inhibitors, and some flavonoids, lignans and phenylpropanoids, fitting this requirement were found as promising INis [1]. The present work reports the screening of 33 natural flavonoids (4 flavanones, 13 flavones, 15 flavonols and catechin), including many previously uninvestigated compounds, in fluorescence assays testing HIV-IN LTR vDNA binding and 3 ' single strand processing catalytic inhibition. Selected hydroxy-, methoxy- and prenylsubstituted compounds and glycosides were investigated in order to gain deeper insight in the structural requirement of INis. For binding and 3 '-processing assays, purified recombinant HIV-1 integrase and a 27 bp synthetic double stranded DNA with the viral $5^{\prime}$ LTR sequence were used. The compounds were tested at $100 \mu \mathrm{M}$ concentration in comparative tests using positive controls unlabeled 5' LTR viral DNA for IN DNA binding, and L708 906 for catalytic $3^{\prime}$ processing inhibition assay. It was found that flavonol-type compounds with $3^{\prime}, 4^{\prime}$-dihydroxy substitution, such as quercetin, physetin, and rhamnetin have the highest viral DNA binding inhibitory effect. Glycosylation at position 7 had no influence, but at position 3 reduced this activity. In case of flavonol 7-O-monoglycosides, moderate catalytic inhibition was also observed. The most interesting compounds were gossypitrin and herbacitrin with double inhibitory activity.

The present work was supported by PN-II-PT-PCCA-2013-4-0930, European cooperation ERA-NET HIVERA contract 11/2016 and NKFIH NN 118176.

[1] Maurin C, Bailly F, Cotelle P. Curr Med Chem 2003; 10: 1795 - 1810

\section{Mo-Poster Session 1-PO-67 Zebrafish bioassay-guided isolation of the anticonvulsant compounds from Cyperus articulatus}

Authors Brillatz $\mathrm{T}^{1}$, Jacmin $\mathrm{M}^{2}$, Challal $\mathrm{S}^{1}$, Marcourt $\mathrm{L}^{1}$, Slacanin $\mathrm{I}^{3}$, Crawford $A D^{2}$, Petit $C^{1}$, Merle $T^{1}$, Ngo Bum $E^{4}$, Petersen $F^{5}$, Schmitt $E^{5}$, Krastel $\mathrm{P}^{5}$, Queiroz $\mathrm{EF}^{1}$, Wolfender $\mathrm{LL}^{1}$

Institute 1 School of Pharmaceutical Sciences, University of Geneva, University of Lausanne, rue Michel Servet 1, CH-1211, Geneva, Switzerland; 2 Luxembourg Centre for Systems Biomedicine (LCSB), University of Luxembourg 6, avenue du Swing, 4367, Luxembourg, Luxembourg; 3 Ilis Institut \& laboratory, chemin de la passerelle 17, CH-2503, Bienne, Switzerland; 4 Department of Biological Sciences, Faculty of Sciences, University of Ngaoundéré, P.O. Box 454, Ngaoundéré, Cameroon; 5 Novartis Pharma Ltd, Research, CH-4002, Basel, Switzerland

DOI 10.1055/s-0037-1608117

Epilepsy is a common CNS disorder usually treated by commercial antiepileptic drugs (AEDs) in western countries. A large number of AEDs are available and preferentially used according to the type of seizures. However, $30 \%$ of patients suffering from epilepsy remain pharmaco-resistant to the current treatments. Medicinal plants are also widely used in low and middle-income countries to treat epilepsy and the decoction of Cyperus articulatus roots is a 
known and traditional remedy to heal epilepsy in Cameroon. Previous studies have demonstrated its in-vivo activity in a mice model [1]. In order to identify the active principles, a zebrafish epilepsy model well suited for bioguidedassay fractionation was chosen [2]. Extraction of the roots by solvents of increasing polarity indicated a high anticonvulsant activity in the hexanic extract (HE). Microfractionation of the HE by normal phase flash chromatography combined with a zebrafish bioguided-assay enable to localize LC-peaks responsible for the bioactivity. The isolation of these compounds at larger scale was targeted based on their physico-chemical profiles and resulted in the isolation of four bioactive terpenoids reducing drastically zebrafish induced seizures up to $81 \%$. Their chemical structures were characterized by classical spectroscopic methods including HRMS and NMR. Additionally, the parallel artificial membrane permeability (PAMPA) absorption of the HE has been performed and confirmed the passive permeation of major active compounds in the gastrointestinal tract (GIT) as well as in the blood brain barrier (BBB). This study provides further proof supporting the traditional use of this plant in the treatment of epilepsy.

[1] Ngo Bum E, Rakotonirina A, Rakotonirina SV, Herrling P. J Ethnopharmacol 2003; $87: 27-34$

[2] Challal S, Queiroz EF, Debrus B, Kloeti W, Guillarme D, Gupta MP, Wolfender JL. Planta Med 2015; 81: 1636 - 1643

\section{Mo-Poster Session 1-PO-68 Anticonvulsant Principle Isolation of the Marine Diatom Skeletonema marinoi}

Authors Brillatz $\mathrm{T}^{1}$, Lauritano $\mathrm{C}^{2}$, Jacmin $\mathrm{M}^{3}$, Khamma $\mathrm{S}^{1}$, Marcourt $\mathrm{L}^{1}$, Righi $D^{1}$, Romano $\mathrm{G}^{2}$, Esposito $\mathrm{F}^{2}$, lanora $\mathrm{A}^{2}$, Crawford $A D^{3}$, Queiroz $\mathrm{EF}^{1}$, Wolfender $\mathrm{LL}^{1}$

Institute 1 School of Pharmacy, University of Geneva, University of Lausanne, rue Michel Servet 1, CH-1211, Geneva 4, Switzerland; 2 Stazione Zoologica Anton Dohrn, Villa Comunale, 80121, Napoli, Italy; 3 Luxembourg Centre for Systems Biomedicine, University of Luxembourg, 6, avenue du Swing, 4367 Belvaux, Luxembourg, Luxembourg

DOI 10.1055/s-0037-1608118

Neuroscience is in needs of new drugs to overcome risen CNS disorders because of the increasing human life expectancy [1]. Marine organisms constitute an unexplored source compared to terrestrial plants in the search of novel type of anticonvulsant natural products [2]. In the context of a screening of marine diatoms in a zebrafish epilepsy model with seizures induced by the $\mathrm{GABA}_{\mathrm{A}}$ antagonist pentylenetetrazole, the methanolic extract of Skeletonema marinoi revealed a significant activity. A bioassay-guided fractionation was undertaken by reverse phase flash chromatography after enrichment with vacuum liquid chromatography in order to isolate the bioactive compounds [3]. This rapid approach leads to the identification of the nucleoside inosine as the main active antiepileptic constituent. Inosine is known for its anticonvulsant activities but was never isolated from diatoms. Structures of isolated compounds were elucidated by spectroscopic methods including evaporative light scattering detector (ELSD), nuclear magnetic resonance (NMR) and high resolution mass spectrometry (HRMS). Additionally, the extract of Skeletonema marinoi was submitted to a metabolite profiling by UHPLC-HRMS. It reveals an interesting chemical diversity and different algal metabolites were annotated together with additional other putative compounds.

[1] Grosso C, Valentao P, Ferreres F, Andrade PB. Mar. Drugs 2014; 12: 2539 2589

[2] Montaser R, Luesch H. Future Med Chem 2011; 3: 1475 - 1489

[3] Challal S, Queiroz EF, Debrus B, Kloeti W, Guillarme D, Gupta MP, Wolfender JL. Planta Med 2015; 81: $1636-1643$

\section{Mo-Poster Session 1-PO-69 Sesquiterpenes from Neurolaena lobata with antiproliferative activity}

Authors Vasas $A^{1}$, Lajter $I^{1}$, Forgó $P^{1}$, Kúsz $N^{1}$, Bózsity $N^{2}$, Zupkó $I^{2}$, Nagy $N^{2}$, Krupitza $\mathrm{G}^{3}$, Frish $\mathrm{R}^{4}$, Hohmann $\mathrm{J}^{1,5}$

Institute 1 Institute of Pharmacognosy, University of Szeged, Szeged, Hungary; 2 Institute of Pharmacodynamics and Biopharmacy, University of Szeged, Szeged, Hungary; 3 Clinical Institute of Pathology, Medical University of Vienna, Vienna, Austria; 4 Institute of Ethnobiology, Playa Diana, San José/ Petén, Guatemala; 5 Interdisciplinary Centre of Natural Products, University of Szeged, Szeged, Hungary

DOI 10.1055/s-0037-1608119

Neurolaena lobata L. (Asteraceae) is a perennial herb native to tropical part of America. It has been used in traditional medicine for the treatment of different types of cancer, ulcers, inflammatory skin disorders and diabetes. The main constituents of the plant are sesquiterpene lactones, which can be responsible for its different pharmacological effects. Our previous studies revealed the presence of sesquiterpenes of the germacranolide, eudesmanolide and furanoheliangolide types, and some of them proved to be active against cancer and inflammation in vitro and in vivo. In continuation of our work, further sesquiterpenes were isolated from $N$. lobata and their antiproliferative activity was investigated on human cancer cell lines ( $\mathrm{SiHa}$, HeLa and $\mathrm{C} 33 \mathrm{~A}$, at 10 and $30 \mu \mathrm{M}$ concentrations) using the MTT assay. The aerial parts of the plant were extracted with methanol. After evaporation, the extract was subjected to solvent-solvent partition with $n$-hexane and $\mathrm{CH}_{2} \mathrm{Cl}_{2}$. The $\mathrm{CH}_{2} \mathrm{Cl}_{2}$ fraction was chromatographed by a combination of different methods, including CC, VLC and PLC. The structure elucidation of the compounds was carried out by means of MS, 1D and 2D NMR spectroscopy. The results allowed the identification of five sesquiterpenes, four of them esterified with isovaleric acid at C-8 or C-9. The new compounds are sesquiterpene lactones, the dimer lobatolide $\mathrm{A}$, the calyculatolide derivative lobatolide $\mathrm{B}$, and the germacranolide-type lobatolides $C$ and D. Furthermore, one known guaiane-type sesquiterpene $[(+)$-aphanamol I] were also isolated from the plant. With the exception of the known compound, all component possessed remarkable antiproliferative activities (inhibition \% \pm SEM between $60.92 \pm 1.73$ and $96.45 \pm 0.27$ ) on at least two cell lines, at $30 \mu \mathrm{M}$ concentration. Lobatolide A proved to be the most active.

This work was supported by GINOP-2.3.2-15-2016-00012, ÚNKP-16-3 and a János Bolyai Research Scholarship of the Hungarian Academy of Sciences.

\section{Mo-Poster Session 1-PO-70 Phytochemical and pharmacological investigation of Sideritis montana $\mathrm{L}$.}

Authors Tóth $\mathrm{B}^{1}$, Kúsz $\mathrm{N}^{1}$, Bózsity $\mathrm{N}^{2}$, Zupkó $\mathrm{P}^{2,4}$, Csizmadia $\mathrm{V}^{2}$, Pinke $\mathrm{G}^{3}$, Hohmann $\mathrm{J}^{1,4}$, Vasas $A^{1,4}$

Institute 1 Institute of Pharmacognosy, University of Szeged, Szeged, Hungary; 2 Institute of Pharmacodynamics and Biopharmacy, Szeged, Hungary; 3 Department of Botany, University of West Hungary, Mosonmagyaróvár, Hungary; 4 Interdisciplinary Centre of Natural Products, University of Szeged, Szeged, Hungary

DOI 10.1055/s-0037-1608120

The genus Sideritis (Lamiaceae) includes more than 150 species, which are distributed widely in the Mediterranean area. Sideritis species are traditionally used for the treatment of several disorders, e.g. ulcers, spasm and pain. Although Sideritis species are considered to be abundant source of diterpenoids, until now only siderol was described from S. montana L. Previously, iridoid glycosides (ajugol, ajugoside and melittoside), diosmetin, and verbascoside have also been isolated from the plant. The present study focused on the phytochemical and pharmacological investigation of $S$. montana. From the methanol extract of the plant, four abietane-type diterpenes (1-4) were isolated. The compounds are substituted with hydroxyl, methyl and isopropyl groups, two of them, sideritins A and B $(1,2)$, are new natural products. 
Further diterpenes [9 $\alpha, 13 \alpha$-epi-dioxiabiet-8(14)-en-18-ol (3) and pomiferin E (4)], a lignan (paulownin, 5), a flavonoid (6-methoxysakuranetin, 6), a megastigmane (3-oxo- $\alpha$-ionol, 7) and a phenol-glucoside (dictamnoside A, 8) were also identified. All of the compounds were isolated for the first time from the plant. Several diterpenoids have been reported to exhibit remarkable antiproliferative activity, but there were no previous data of the cytotoxic effects of metabolites from S. montana. All of the isolated compounds were tested in vitro on human cervical cancer cell lines (HeLa, SiHa and C33A) using the MTT assay. Among the isolated compounds, pomiferin E (4) and 6-methoxysakuranetin (6) showed considerable activity [inhibition (\%) \pm SEM: $46.93 \pm 2.35$ on HeLa (4), and $51.52 \pm 2.45$ on C $33 \mathrm{~A} \mathrm{(6)]} \mathrm{at} 30 \mu \mathrm{M}$ concentration.

This work was supported by the GINOP-2.3.2 - 15 - 2016 - 00012, and a János Bolyai Research Scholarship of the Hungarian Academy of Sciences.

\section{Mo-Poster Session 1-PO-71 Identification of a triterpenoid as a novel PPARy activator derived from Formosan plants}

Authors Weng JR ${ }^{1}$

Institute 1 Department of Marine Technology and Resources, National SunYat-sen University, Kaohsiung, Taiwan

DOI 10.1055/s-0037-1608121

Peroxisome proliferator-activated receptory (PPARY), one of the transcription factors that regulate lipid metabolism and energy use in tumor cells, is a viable target for cancer therapy. In our search for potential PPARy activator, extracts from five Formosan plants were tested. Among them, Momordica charantia showed the highest ability to activate PPARy, which led us to identify its potential constituents. Among the seven compounds isolated from M. Charantia, a triterpenoid (Compound 1), was identified as a PPARy activator with an $\mathrm{IC}_{50}$ of $10 \mu \mathrm{M}$ in MCF-7 breast cancer cells. Flow cytometric analysis indicated that compound 1 induced G1 cell cycle arrest which might be attributable to the modulation of phosphorylation and expression of numerous key signaling effectors, including cyclin D 1, CDK6, and p53. Taken together, these findings suggest that compound 1 may have therapeutic applications in cancer treatment through PPARy activation.

\section{Mo-Poster Session 1-PO-72 A triterpenoid from wild bitter gourd inhibits prostate cancer cells}

\begin{abstract}
Authors Weng JR ${ }^{1}$
Institute 1 Department of Marine Technology and Resources, National SunYat-sen University, Kaohsiung, Taiwan

DOI 10.1055/s-0037-1608122

Wild bitter gourd, a vegetable, is also used as folk medicine because of its anti-diabetic and chemopreventive effects. The antitumor activity of a triter-

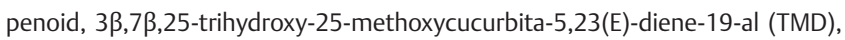
isolated from wild bitter gourd in prostate cancer cells was investigated. TMD suppressed the proliferation of PC-3 prostate cancer cells with $\mathrm{IC}_{50}$ value at $48 \mathrm{~h}$ of $24 \mu \mathrm{M}$, respectively. TMD induced cell apoptosis accompanied with pleiotrophic biological modulations including down-regulation of Akt, p38 mitogen-activated protein kinase and ERK, and increased reactive oxygen species generation. Together, these findings provided the translational value of TMD as an antitumor agent for patients with prostate cancer.
\end{abstract}

\section{Mo-Poster Session 1-PO-73 Secondary metabolites with anti-platelet aggregation activity from the insect Tenebrio molitor}

Authors Park IW' ${ }^{1}$, Kim DY ${ }^{1}$, Bae JS ${ }^{2}$, Na MK ${ }^{1}$

Institute 1 College of Pharmacy, Chungnam National University, Daejeon, Korea, Republic of (South); 2 College of Pharmacy, CMRI, Research Institute of
Pharmaceutical Science, Kyungpook National University, Daegu, Korea,

Republic of (South)

DOI 10.1055/s-0037-1608123

Recently, researches for novel food and medicinal materials have focused on insect resources. Tenebrio molitor is an edible insect and recognized to have various biological properties, such as antimicrobial, anticancer, antidementia, and antihypertensive effects. The aim of this study was to identify previously unreported bioactive anti-platelet compounds from T. molitor larvae. Chemical investigation on the larvae resulted in the isolation of ten secondary metabolites. The structures of these entities were identified as cyclo(L-Val-L-Pro) (1), cyclo(L-Ala-L-Pro) (2), cyclo(L-Pro-L-Tyr) (3), cyclo(L-Phe-L-Tyr) (4), cyclo(ValTyr) (5), cyclo(Ala-Ile) (6), hydroxytyrosol (7), N-acetyldopamine (8), N-acetyltyramine (9), and oleracein E (10) based on spectroscopic data analysis. All the isolates were evaluated for anti-platelet aggregation properties by monitoring clotting time. Cyclo(L-Pro-L-Tyr) (3), hydroxytyrosol (7), and N-acetyltyramine (9) were shown to have platelet aggregation inhibitory activities. Collectively, these results show that compounds 3,7 , and 9 could serve as candidates and provide scaffolds for the development of new anti-platelet drugs.

\section{Mo-Poster Session 1-PO-74 The first investigation on chemical constituents and biological activity of Rubia philippinensis}

Authors Khong $\mathrm{Q}^{1}$, Park $\mathrm{HS}^{1}$, Myung $\mathrm{CS}^{1}$, Na MK ${ }^{1}$

Institute 1 College of Pharmacy, Chungnam National University, Daejeon,

Korea, Republic of (South)

DOI 10.1055/s-0037-1608124

Despite the genus Rubia being researched to identify valuable commercial and medicinal virtues, Rubia philippinensis has rarely been investigated. In the present study, seventeen arborinane-type triterpenoids, including nine new compounds (1-9), were isolated from the underutilized plant species. Structures of new compounds were determined as 3-O-Acetylrubiarbonol L (1), 3-O-Acetylrubianol C (2), 28-Deoxyrubiarbonone E (3), 19-Dedihydrorubiarbonol B (4), 2-Deoxyrubianol F (5), 3-O-Acetylrubiarbonol B (6), 3-O-Acetylrubiarbonol A (7), 19-O-Acetylrubiarbonol B (8), and 19-O-Acetyl-28-deoxyrubiarbonone (9). The new chemical entities were assessed for their inhibitory potential on platelet derived growth factor-induced proliferation and the migration of vascular smooth muscle cells. Treatment with $5 \mu \mathrm{M}$ of compounds 5 (62.6 $\pm 10.7 \%)$ and $9(41.1 \pm 4.7 \%)$ impeded proliferation without exerting cytotoxicity. Compound 7, meanwhile, exhibited antimigration activity in a dose-dependent manner $(38.5 \pm 3.0 \%$ at $10 \mu \mathrm{M}$ and $57.6 \pm 3.2 \%$ at $30 \mu \mathrm{M}$, respectively). These results suggest that the arborinane-type triterpenoids may be a pertinent starting point for the development of cardiovascular drugs capable of preventing neointimal hyperplasia of vascular smooth muscle cells.

\section{Mo-Poster Session 1-PO-75 A novel mycosporin like amino acid in an undescribed alga of the Prasiolaceae family}

Authors Rainer $\mathrm{B}^{1,2}$, Schwaiger $\mathrm{S}^{1}$, Dapra $\mathrm{L}^{2}$, Remias $\mathrm{D}^{3}$, Stuppner $\mathrm{H}^{1}$, Trockenbacher $\mathrm{A}^{2}$, Griesbeck $\mathrm{C}^{2}$

Institute 11 Institute of Pharmacy/Department of Pharmacognosy, University of Innsbruck, Center for Chemistry and Biomedicine, Innrain 80 82, Innsbruck, Austria; 2 MCI-Management Center Innsbruck,

Maximilianstraße 2, Innsbruck, Austria; 3 FH OÖ Fakultät für Technik und Angewandte Naturwissenschaften, Stelzhamerstraße 23, Wels, Austria DOI 10.1055/s-0037-1608125

In the course of a national program ((co)Operation SKD) for the identification of secondary metabolites in algae, specimens of the culture collection of algae at the University of Innsbruck (ASIB 505) were investigated. A promising candidate (V224; [1]) producing a mycosporin like amino acid (MAA) - tentatively assigned by the observed absorption maximum at $320 \mathrm{~nm}$ in LC-DAD- 
MS extract screening - was investigated in detail. Beside morphological classification genetically data were generated and compared with the NCBI database. Due to the lack of large sequence analogies with database entries, we assume that the investigated alga has not been sequenced before. For a further phylogenetic classification the marker genes rbcL and $18 \mathrm{~S}$ rDNA were analyzed. Phylogenetic trees based on $r b c \mathrm{~L}$ sequences revealed a close relation to the genus Diplosphaera, while the ones based on the $18 \mathrm{~S}$ sequences pointed to a closer relationship with the genus Stichococcus. Based on these results the investigated microalga was identified as member of Prasiolaceae and is situated between the genera Stichococcus and Diplosphaera. Isolation and structure elucidation of the initially detected MAA by 1D- and 2D-NMR and (HR)-LC-MS experiments identified the novel compound as $\mathrm{N}^{2}$-(4,5-dihydroxy-5-(hydroxymethyl)-2-methoxy-3-oxocyclohex-1-en-1-yl)- $\mathrm{N}^{2}$-methylasparagine (figure 1), which is structurally related to the recently published Prasiolin isolated from Prasiola calophylla [2] and supports thereby the proposed phylogenetic classification.

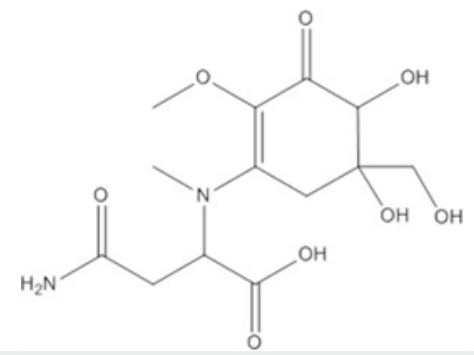

Fig. 1: $\mathrm{N}^{2}$-(4,5-dihydroxy-5-(hydroxymethyl)-2-methoxy-3-oxocyclohex-1-en-1-yl)- $\mathrm{N}^{2}$-methylasparagine

The authors thank FFG Project number 845423 for the financial support. [1] Gärtner G. ASIB - the Culture Collection of Algae at the Botanical Institute of the University at Innsbruck (Austria) Catalogue of Strains, Ber. nat.-med. Verein Innsbruck, Band 83, 1996: 45-69

[2] Hartmann A, Holzinger A, Ganzera M and Karsten U. Planta 2016, 243

\section{Mo-Poster Session 1-PO-76 N-acetyldopamine dimers from the edible grasshopper Oxya chinensis sinousa}

Authors Lee $\mathrm{HS}^{1}$, Min $\mathrm{BS}^{2}$, Na MK${ }^{1}$

Institute 1 College of Pharmacy, Chungnam National University, Daejeon, Korea, Republic of (South); 2 College of Pharmacy, Catholic University of Daegu, Daegu, Korea, Republic of (South)

DOI 10.1055/s-0037-1608126

Recently, research regarding novel food and medicinal materials has focused on insect resources, demonstrating their potential value. Oxya chinensis sinuosa Mistshenko, an edible grasshopper, belongs to the phylum Arthropoda. Traditionally in Korea, it is known as the "famine relief insect" and has been used to treat cough, whooping cough, asthma, bronchitis, paralysis, and seizures. The rice field grasshopper has been registered as a food in the Korean Food Standards Codex, Korea Food \& Drug Administration (KFDA). In this study, five $\mathrm{N}$-acetyl dopamine dimers, componds (1) and (2) were identified as new chemicals with chiral center at $\mathrm{H}-2$ and $\mathrm{H}-3$ of the benzo-1,4-dioxane structure, (2R,3S)-2-(3',4'-dihydroxyphenyl)-3-acetylamino-6-(N-acetyl-2"'aminoethyl)-1,4-benzodioxane (3), (2R,3S)-2-(3',4'-dihydroxy-phenyl)-3-acetylamino-7-( $N$-acetyl-2"'-aminoethyl)-1,4-benzodioxane (4), (2R,3S)-2-(3',4'-dihydroxyphenyl)-3-acetylamino-7-( $\mathrm{N}$-acetyl-2"-aminethylene)-1,4-benzodioxane (5) were isolated from Oxya chinensis sinuosa. The structures of compounds 1-5 including absolute configurations were determined by $1 \mathrm{D}$ and 2D NMR, HRESIMS, circular dichroism (CD) spectroscopic data analysis.

\section{Mo-Poster Session 1-PO-77 Antithrombotic and antiplatelet activities of indole alkaloids isolated from the edible insect Protaetia brevitarsis seulensis (Kolbe)}

Authors Lee $\mathrm{JI}^{1}$, Bae $\mathrm{JS}^{2}$, Min $\mathrm{BS}^{3}$, Na MK'

Institute 1 College of Pharmacy, Chungnam National University, Daejeon, Korea, Republic of (South); 2 College of Pharmacy, CMRI, Research Institute of Pharmaceutical Sciences, BK21 Plus KNU Multi-Omics based Creative Drug Research Team Kyungpook National University, Daegu, Korea, Republic of (South); 3 College of pharmacy, Catholic University of Daegu, Daegu, Korea, Republic of (South)

DOI 10.1055/s-0037-1608127

Edible insects have been reported to produce metabolites showing various pharmacological activities, recently emerging as rich sources of health functional food and medicines. In particular, Protaetia brevitarsis seulensis (Kolbe) has been temporarily registered as a food material by the Ministry of Food and Drug Safety of Korea (MFDS). The current study aimed to discover small antithrombotic molecules from this edible insect. Five indole alkaloids, 5-hydroxyindolin-2-one (1), (1R,3S)-1-methyl-1,2,3,4-tetrahydro-beta-carboline-3carboxylic acid (2), (1S,3S)-1-methyl-1,2,3,4-tetrahydro-beta-carboline-3-carboxylic acid (3), (3S)-1,2,3,4-tetrahydro-beta-carboline-3-carboxylic acid (4), and L-tryptophan (5) were isolated from the insect. Among them, compounds 1 and 2 prolonged the activated partial thromboplastin time and prothrombin time and inhibited the activity and production of thrombin and activated factor $X$ on HUVEC surface. Moreover, these compounds inhibited platelet aggregation. Antithrombotic effects of compounds 1 and 2 were further confirmed in pre-clinical models of pulmonary embolism and arterial thrombosis. Collectively, these results demonstrated that compounds 1 and 2 could be effective antithrombotic agents and serve as new scaffolds for the development of antithrombotic drug.

\section{Mo-Poster Session 1-PO-78 Chemical constituents and biological activities of parasitic plant Cuscuta japonica Choisy on Dimocarpus longans Lour.}

Authors Huang $\mathrm{HC}^{1}$, Cheng $\mathrm{JC}^{2}$, Hwang $\mathrm{SY}^{3}$, Kuo $\mathrm{YH}^{1}$ Institute 1 Department of Chinese Pharmaceutical Sciences and Chinese Medicine Resources, China Medical University, Taichung, Taiwan; 2 Department of Medical Laboratory Science and Biotechnology, China Medical University, Taichung, Taiwan; 3 Endemic Species Research Institute, Council of Agriculture, Nantou, Taiwan

DOI 10.1055/s-0037-1608128

Cuscuta japonica is a unique parasitic vine that belongs to the family Convolvulaceae, which is obligatory dependent upon a host plant for nourishment in order to survive. We studied on the parasite plants $C$. japonica growing on different plants including, Dimocarpus longans Lour, Litchi chinensis Sonner, Ficus microcarpa L. f. and Duranta repens L. exhibited different constituents which have been detected by HPLC analyses. Determination of the major metabolites present in the parasites showed a clear relationship with compounds. Three new phenolic glycosides (1-3) and 21 known compounds were isolated and characterized from the methanol extract of the parasite plant $C$. japonica on $D$. longan Lour. The structures were established by spectroscopic analyses and comparisons with data from related references. Two phenolic glycosides (1-3), two caffeoylquinic acid derivatives (4-5), and two fatty acids (23-24) exhibit significant activities against influenza virus A (H1N1). 


\section{Mo-Poster Session 1-PO-79 Anti-inflammatory activity of procyanidins isolated from Tiliae flos.}

Authors Czerwińska $\mathrm{M}^{1}$, Pawłowska $\mathrm{K}^{1}$, Dudek $\mathrm{M}^{2}$, Prus|' $\mathrm{A}^{1}$, Granica S Institute 1 Department of Pharmacognosy and Molecular Basis of Phytotherapy, Faculty of Pharmacy, Medical University of Warsaw, Warsaw, Poland; 2 Centre of Molecular and Macromolecular Studies PAS, Lodz, Poland DOI 10.1055/s-0037-1608129

Tiliae flos is a pharmacopeial plant material used in the form of infusions in the threatmant of feverish colds and upper respiratory tracts irritations. Previous studies have shown that lime flowers contain significant amounts of flavonoids, mucilage and essential olis [1]. Recent research revealed that lime flowers also contain procyanidins from which only few compounds were identified [2]. One kilogram of dried plant material was extracted with acetone: methanol:water (3:1:1) to be next separated by liquid-liquid extraction. Ten flavan-3-ol derivatives were isolated using a combination of column chromatography on Diaion HP-20, Sephadex LH20 or Toyopaerl HW40F as well as preparative HPLC on C-18 column. Compounds were identified using 1D and 2D NMR experiments. One monomer, four dimers, one trimer, two tetramers and two pentamers were isolated. Compounds were identified as epicatechin, procyanidin $A 2$, procyanidins $B 1, B 2$ and $B 5$, epicatechin- $(4 \beta->6)$-epicatechin$(4 \beta->8)$-epicatechin, epicatechin- $(4 \beta->8)$-epicatechin- $(4 \beta->8)$-epicatechin$(4 \beta->8$-epicatechin, epicatechin- $(4 \beta->6)$-epicatechin-( $4 \beta->8)$-epicatechin-

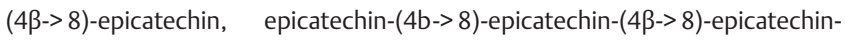
$(4 \beta->8)$-epicatechin- $(4 \beta->8)$-epicatechin and epicatechin-( $4 \beta->6)$-epicate-

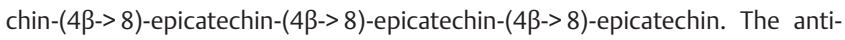
inflammatory potential, of isolated was evaluated using human neutrophils model. Compounds were tested in the concentration range of $1-20 \mu \mathrm{M}$. The influence on the production of reactive oxygen species, secretion of IL-8, TNFalpha, MIP-1alpha and MIP-1beta was checked. Compounds (excluding pentameric procyanidnins) were not toxic for neutrophils up to $20 \mu \mathrm{M}$. All compounds were able to inhibit the production of reactive oxygen species at concentrations of 1,5 and $20 \mu \mathrm{M}$. Tertrameric and pentameric procyanidins exhibited the strongest activity. Tested procyanidins were able to decrease the release of IL- 8 from stimulated neutrophils but did not influence the secretion of other tested inflammatory mediators. The contribution of procyanidins to the anti-inflammatory potential of Tiliae flos was established.

[1] Wichtl, M. Herbal Drugs and Phytopharmaceuticals. A hand book for practice on a sceintific basis. London, CRC Press, 2004

[2] Karioti, A, Chirabini, L, Fawaz Chehna, M, Vincieri, F.F, Bilia, A.R. J Pharmaceut Biomed Anal 2014, 100: 205 - 214

\section{Mo-Poster Session 1-PO-80 Synergistic effect of $\alpha$-mangostin on antibacterial activity of tetracycline and erythromycin against acne involved bacteria}

\section{Authors Panichayupakaranant $\mathrm{P}^{1,3}$, Ahmad Md I ${ }^{1}$, Keach JE ${ }^{2}$}

Institute 1 Phytomedicine and Pharmaceutical Biotechnology Excellence

Center, Faculty of Pharmaceutical Sciences, Prince of Songkla University, HatYai, Thailand; 2 Research and Horticulture Department, Gardens by the Bay, Singapore, Singapore; 3 Department of Pharmacognosy and Pharmaceutical Botany, Faculty of Pharmaceutical Sciences, Prince of Songkla University, HatYai, Thailand

\section{DOI 10.1055/s-0037-1608130}

During the last decade, bacterial resistance to antibiotics has become a public health crisis throughout the world. $\alpha$-Mangostin is a major bioactive xanthone found in the pericarps of Garcinia mangostana L. It has been used as an antibacterial agent, especially in anti-acne preparations made from the extract of $G$. mangostana. The aim of the present study was to conduct a synergistic evaluation of $\alpha$-mangostin with both tetracycline and erythromycin against bacteria involved in acne production, namely: Propionibacterium acnes and Staphylococcus aureus. In this study, $\alpha$-mangostin was purified from the dichloromethane extract of G. mangostana pericarp using silica gel column chromatography. A broth microdilution method was used to determine the minimum inhibitory concentration (MIC) of $\alpha$-mangostin and the antibiotics. Synergistic effects on antibacterial activity were determined at their own MIC using a checkerboard method and a time-kill assay at $37^{\circ} \mathrm{C}$ for $24 \mathrm{~h}$. $\alpha$-Mangostin showed antibacterial activity against $P$. acnes and $S$. aureus with MIC values of 0.78 and $3.13 \mu \mathrm{g} / \mathrm{mL}$, respectively. Tetracycline and erythromycin exhibited antibacterial activity against $P$. acnes and S. aureus with MIC values of 1.56 and 0.12 , and 0.39 and $0.78 \mu \mathrm{g} / \mathrm{mL}$, respectively. The results of the checkerboard assay showed that $\alpha$-mangostin produced synergistic effects with both antibiotics against $P$. acnes and $S$. aureus, with a fractional inhibitory concentration index $(\mathrm{FICl})$ ranging from 0.076-0.31. Moreover, time-kill curve data indicated that $\alpha$-mangostin increased the antibacterial activity of tetracycline and erythromycin against the tested bacteria. These findings suggest that $\alpha$-mangostin may be used to enhance the antibacterial activity of tetracycline and erythromycin against bacteria involved in acne production.

\section{Mo-Poster Session 1-PO-81 Antitumor activity of tricin, a flavone isolated from leaves of Casearia arborea (Salicaceae)}

Authors Sartorelli $\mathrm{P}^{1}$, Santos $\mathrm{A}^{1}$, Figueiredo $\mathrm{C}^{2}$, Lago $\mathrm{J}^{1}$, Soares $\mathrm{M}^{3}$ Institute 1 Institute of Environmental, Chemical and Pharmaceutical Sciences, Federal University of São Paulo, São Paulo, Brazil, São Paulo, Brazil; 2 Departament of Microbiology, Immunology and Parasitology, Federal University of São Paulo, São Paulo, Brazil, Diadema, Brazil; 3 Institute of Chemistry - Alfenas Federal University - MG, Brazil, Alfenas, Brazil DOI 10.1055/s-0037-1608131

Metastasis is one of the most important processes for the cancer progression, consisting to a set of events related to the tumor cell migration [1]. Considering that many chemotherapeutic agents used for treatment of cancer have side effects, active principles from plants played an important role in cancer chemotherapy [2]. Casearia Jaquin represents one of the largest genus in the Salicaceae family and accumulates bioactive clerodane type diterpenes [3]. $C$. arborea, was reported presence of casearborins A-E, from roots exhibiting cytotoxic activity against LOX and SF539 cell lines [4]. Aiming to isolate active compounds, $\mathrm{MeOH}$ extract from leaves of $C$. arborea was evaluated to cytotoxic activity against B16F10 and then fractioned to afford a flavone tricin. In order to evaluate the anti-proliferative activity of tricin, it was submitted to the clonogenic assays. Thus after 72 hour of incubation with tumor cells, was observed significant inhibition of colony formation, which may be related to a decreased proliferation profile of tumor cells. Also, was assessed the migration profile of murine melanoma cells after incubation of tricin and was observed that tricin significantly inhibited more than $60 \%$ the migration of melanoma cells. Thus for $C$. arborea the antitumor activity was due to the presence of a flavonoid that presents a more restricted occurrence in Casearia species. Additionally substances related to flavonoids have already been described with anti-migratory activity, and the description of tricin with this activity in the present work contributes to the development of prototypes that act as inhibitors of tumor cells.

[1] Talmadge J.E. et al. Cancer Res. 2010, 70, 5649.

[2] Newman D.J. et al. J. Nat. Prod. 2016, 79, 629.

[3] Xia L. et al. Phytochem. Rev. 2015, 14, 99.

[4] Beutler J.A. et al, J. Nat. Prod. 2000, 63, 657.

\section{Mo-Poster Session 1-PO-82 New Isoflavones from the Fuits of Psoralea corylifolia and their Anti-inflammatory Activity}

Authors Chen J] ${ }^{1}$, Chen $\mathrm{CH}^{2}$, Hwang $\mathrm{TL}^{3}$, Chang $\mathrm{TH}^{4}$

Institute 1 Faculty of Pharmacy, School of Pharmaceutical Sciences, National Yang-Ming University, Taipei 112, Taiwan; 2 Department of Pharmacy, Tajen University, Pingtung 907, Taiwan; 3 Graduate Institute of Natural Products, College of Medicine, Chang Gung University, Taoyuan 333, Taiwan; 
4 Department of Medical Education and Research, Kaohsiung Veterans General Hospital, Kaohsiung 813, Taiwan

DOI 10.1055/s-0037-1608132

Psoralea corylifolia L. (Leguminosae) is an annual herb distributed in India, Malay peninsula, Indonesia, China, and Taiwan [1]. Dry fruit of P. corylifolia Linn is one of the most popular Traditional Chinese Medicine (TCM) and officially listed in Chinese Pharmacopoeia [2]. This crude drug has been used for the treatment of spermatorrhea, nephritis, asthma, pollakiuria, and various inflammatory diseases. Various flavonoids, isoflavones, meroterpenes, coumestans, and their derivatives were isolated from this plant in previous studies. Many of these compounds exhibit anti-inflammatory, phytoestrogen, anti-nitric oxide synthase (iNOS), anti-platelet aggregation, antibacterial, and antiHelicobacter pylori activities. Three new isoflavone derivatives, 7-O-methylcorylifol A (1), 7-0-isoprenylcorylifol A (2), and 7-O-isoprenylneobavaisoflavone (3), have been isolated from the fruits of $P$. corylifolia, together with 9 known compounds (4-12). The structures of these new compounds were determined through spectroscopic and MS analyses. Among the isolated compounds, 7-Omethylcorylifol A (1) and psoralen (5) exhibited potent inhibition ( $\mathrm{IC}_{50}$ values $\leq 10.89 \mu \mathrm{M}$ ) of superoxide anion generation by human neutrophils in response to $\mathrm{N}$-formyl-L-methionyl-L-leucyl-L-phenylalanine/cytochalasin B (fMLP/CB). 7O-Isoprenylcorylifol A (2), 7-O-isoprenylneobavaisoflavone (3), and 12,13-dihydro-12,13-epoxybakuchiol (7) inhibited $\mathrm{fMLP} / \mathrm{CB}$-induced elastase release with $\mathrm{IC}_{50}$ values $\leq 14.30 \mu \mathrm{M}$. In addition, compounds 2 , bakuchiol (6), 7, and psoralidin (10) showed potent inhibition with $\mathrm{IC}_{50}$ values $\leq 36.65 \mu \mathrm{M}$, against lipopolysaccharide (LPS)-induced nitric oxide (NO) generation.

[1] Huang TC, Ohashi H. 1993. Leguminosae. In: Flora of Taiwan, 2nd edition. Editorial Committee of the Flora of Taiwan: Taipei, Taiwan; 1993; 3: 160 - 396. [2] Pharmacopoeia of the People's Republic of China. Chemical Industry Publications: Beijing; 2005; 1: 129-130.

\section{Mo-Poster Session 1-PO-83 Cardioprotective effect of (-)- $\alpha$-Bisabolol in animal model of myocardial infarction}

Authors Ojha $\mathrm{S}^{1}$, Azimullah $\mathrm{S}^{1}$, Al Taee $\mathrm{H}^{1}$, Meeran MFN ${ }^{1}$

Institute 1 College of Medicine and Health Sciences, United Arab Emirates University, AL AIN, United Arab Emirates

DOI 10.1055/s-0037-1608133

Myocardial infarction characterized by an imbalance of coronary blood supply and demands in myocardial cell death involves oxidative stress and subsequent inflammation. Despite of tremendous development in interventional cardiology and pharmacological agents, novel agents are needed for the prevention and treatment of myocardial infarction. In such efforts, (-)- $\alpha$-Bisabolol, a sesquiterpene alcohol has received enormous interest being present in the essential oil of a variety of dietary plants, including chamomile. Recently, it showed gastroprotective, antimicrobial, anticancer and anti-inflammatory properties. Thus, the present study was aimed to investigate the cardioprotective activity of Bisabolol in a clinically relevant animal model of myocardial infarction induced by isoproterenol. Being a synthetic catecholamine, isoproterenol at supra-physiological doses induces myocardial injury similar to that of human myocardial infarction. In rats, two subcutaneous injections of isoproterenol $(85 \mathrm{mg} / \mathrm{kg})$ at an interval of 24 hour induced myocardial injury and Bisabolol $(20 \mathrm{mg} / \mathrm{kg})$ was administered orally for 15 days. The injections of isoproterenol induced depletion of endogenous non enzymatic and enzymatic antioxidants (glutathione, superoxide dismutase, catalase, glutathione cycle enzymes), and serum marker enzymes (creatine phosphokinase-MB, lactate dehydrogenase) from myocardium along with raised lipid peroxidation (malondialdehyde) and enhanced levels of pro-inflammatory cytokines (IL-1 1 , IL-6, TNF- $\alpha$ ). Isoproterenol also produced hemodynamic (arterial pressures and heart rate) impairment and caused histopathological alterations in myocardium. Treatment with (-)- $\alpha$-Bisabolol prevented the depletion of endogenous antioxidants, augmented enzymatic antioxidants and restored myocardial en- zymes, therefore reduced myocyte injury marker enzymes and inhibited lipid peroxidation. Bisabolol treatment also corrected the altered hemodynamics, reduced induction of pro-inflammatory cytokines and salvaged myocardium evidenced by reduced myonecrosis, edema and infiltration of inflammatory cells. The present study demonstrates that Bisabolol exerts cardioprotective effect by mitigating oxidative stress, augmenting endogenous antioxidants and maintaining structural integrity. The results indicate that dietary available Bisabolol may be useful to prevent the onset and progression of myocardial injury.

\section{Mo-Poster Session 1-PO-84 Caspase-dependent apoptosis induced by 5,6-dihydroxy-2,4- dihydrophenanthrene in human lung adenocarcinoma A549 cells}

Authors Hansakul $\mathrm{P}^{1}$, Aree $\mathrm{K}^{2}$, Duangprompo $\mathrm{W}^{1}$, Itharat $\mathrm{A}^{3}$

Institute 1 Biochemistry and Molecular Biology, Department of Preclinical

Science, Faculty of Medicine, Thammasat University, Pathumthani, Thailand; 2 Microbiology and Immunology, Department of Preclinical Science Faculty of Medicine, Thammasat University, Pathumthani, Thailand; 3 Department of Applied Thai Traditional Medicine, Faculty of Medicine, Thammasat University, Pathumthani, Thailand

DOI 10.1055/s-0037-1608134

5,6-dihydroxy-2,4-dimethoxy-9,10-dihydrophenanthrene (HMP) is an active compound isolated from the rhizome extract of Dioscorea membranacea Pierre, a Thai medicinal plant that has been traditionally used in cancer treatment [1]. This research aimed to study the cytotoxic effect of HMP and to further analyze its apoptosis-inducing capacity in A549 cells. The cytotoxicity studies using the sulforhodamine B assay revealed that HMP exerted the potent cytotoxic activity against A549 cells with $50 \%$ growth inhibition $\left(\mathrm{IC}_{50}\right)$, total growth inhibition (TGI), and $50 \%$ lethal concentration ( $\mathrm{LC}_{50}$ ) values of $9.22 \pm 1.26,56.86 \pm 3.99$, and $93.85 \pm 2.50 \mu \mathrm{M}$, respectively. Moreover, cell cycle analysis using flow cytometry showed that HMP at $50 \mu \mathrm{M}$ (nearly the TGI value) and $25 \mu \mathrm{M}$ (the half TGI value) significantly increased the sub- $\mathrm{G}_{1}$ peak (apoptotic cells) ranging from $26.51-32.90 \%$ after 48 and $72 \mathrm{~h}$, along with the decreased percentage of $\mathrm{G}_{2} / \mathrm{M}$-phase cells. Using FITC-labeled Annexin $\mathrm{V}$ and PI staining followed by flow cytometry, the time-dependent effect of HMP at 25 and $50 \mu \mathrm{M}$ on the percentage of early and late apoptotic cells was observed in A549 cells, with approximately $36 \%$ and $41 \%$ of these early and late apoptotic cells combined at $72 \mathrm{~h}$, respectively. Treatment with $25 \mu \mathrm{M} \mathrm{HMP}$ for 24,48 , and $72 \mathrm{~h}$ resulted in changes of caspase- 3 activity that reached its highest level at $48 \mathrm{~h}$. Such activity was completely abolished with pretreatment of $50 \mu \mathrm{M}$ Z-VAD-fmk (an irreversible pan-caspase inhibitor) for $6 \mathrm{~h}$ prior to HMP incubation. Additionally, Western blot analysis detected the active form of caspase-3 and its well-established substrate, PARP. Remarkably, HMP-treated cells exhibited chromatin condensation and nuclear fragmentation, the terminal events of apoptosis, using fluorescence microscopy and gel electrophoresis, respectively. Altogether, these data indicate that HMP induced caspase-dependent apoptosis and support its anticancer potential for lung cancer treatment.

[1] Itharat A, Thongdeeying P, Ruangnoo S. J. Ethnopharmacol 2014.

\section{Mo-Poster Session 1-PO-85 Suppression of airway inflammation by luteolin via upregulation of regulatory $\mathrm{T}$ cells}

Authors Shin Han J ${ }^{1}$, Han Chang $\mathrm{K}^{1}$

Institute 1 2017, Daejeon, Korea, Republic of (South)

DOI 10.1055/s-0037-1608135

In recent study, we used a mouse model of airway inflammation to examine the suppressive activity of luteolin-induced CD 4+CD 25+ regulatory T cells in vivo. We studies provide compelling evidence that induction of Treg cell populations by luteolin treatment can facilitate the suppression of allergic in- 
flammation and AHR through increased foxp3 and/or TGF-b1 production. Balb/c mice were sensitized with OVA and, upon OVA aerosol challenge, developed airway eosinophilia, mucus hypersecretion, elevation of OVA-specific $\operatorname{lgE}$, Th 2 cytokines and chemokine levels, up-regulation of eotaxin 2 or CCR3 expression, and airway hyperresponsiveness. Intraperitoneal administration of luteolin, identified from the root of Salvia plebeia $\mathrm{R}$. $\mathrm{Br}$, and cyclosporine $\mathrm{A}$ (CsA), significantly inhibited OVA-induced increases in immune cell counts, eosinophil counts, and IL-4, IL-5, IL-13, and eotaxin levels recovered in bronchoalveolar lavage fluid (BALF) in OVA-sensitized mice. Luteolin and CSA also substantially reduced the OVA-specific IgE, eotaxin 2 levels, and CCR3 expression of Bronchoalveolar lavage fluid (BALF). In contrast, luteolin also substantially $(p<0.05, p<0.01)$ increased the IL-10, IFN-g level, and IL-10 or TGF-b1 mRNA expression of BALF. Histological studies showed that luteolin and CsA dramatically inhibited eosinophilia, and infiltration lymphocytes in lung tissues. An in vitro also, CD 4+CD25- T cells of luteolin treatment contained elevated numbers of luteolin-induced CD 4+CD 25+ Treg (ciTreg) cells, higher TGF-b1 and Foxp3 mRNA expression, and produced strong interleukin-2, IL10, TGF-b response. Transfer of ciTreg cells to OVA-sensitized mice reduced airway hyperreactivity (AHR), recruitment of eosinophils, eotaxin, IgE, and T helper type 2 (Th2) cytokine expression, and increased the IFN-g production in the BALF after allergen challenge, and significantly improved pathologic lesion scores of the lungs. Furthermore, adoptive transfer of ciTreg cells prevented disease in a CD 25-depleted mouse asthma model. These studies show that immune suppression via the induction of foxp3 and CD 4+CD 25+ Treg cells may represent a new strategy in the development of therapies for managing asthma and other inflammatory diseases.

\section{Mo-Poster Session 1-PO-87 Anti-inflammatory and anti-tubercular properties screening of natural products from Plectranthus species}

Authors Marçalo J $]^{1}$, Garcia $C^{1}$, Custódio $L^{2}$, Nicolai $\mathrm{M}^{1}$, Reis $\mathrm{C}^{1,3}$, Rodrigues Maria $\mathrm{J}^{2}$, Romagnoli $\mathrm{A}^{4}$, Petruccioli $\mathrm{E}^{4}$, Goletti $\mathrm{D}^{4}$, Monteiro Rodrigues $\mathrm{L}^{1}$, Faustino $C^{5}$, Fimia Gian $M^{4,6}$, Rijo $P^{1,5}$

Institute 1 Universidade Lusófona's Research Center for Biosciences and Health Technologies (CBIOS), Lisboa, Portugal; 2 Centre for Marine Sciences (CCMAR), University of Algarve, Campus of Gambelas, Faro, Portugal;

3 Biophysics and Biomedical Engineering Institute (IBEB), Faculty of Sciences, University of Lisbon (ULisboa), Lisbon, Portugal; 4 National Institute for the Infectious Diseases “Lazzaro Spallanzani”, Rome, Italy; 5 Research Institute for Medicines (iMed.ULisboa), Faculty of Pharmacy, University of Lisbon (ULisboa), Lisbon, Portugal; 6 Department of Biological and Environmental Sciences and Technologies (Di.S.Te.B.A.), University of Salento, Lecce, Italy

DOI 10.1055/s-0037-1608136

Medicinal plants of the Plectranthus genus are widely used in traditional medicine for their anti-inflammatory and anti-infective properties [1]. Previous reports [2,3] directed this study for the production and release of Nitric Oxide, and for the CFU analysis of Mycobacterium tuberculosis H37Rv (Mtb) growth inhibition, of Plectranthus spp. non-cytotoxic compounds.

The natural compounds isolated from Plectranthus spp. using bioassay-guided fractionation of extracts, were: $6 \beta, 7 \alpha$-dihydroxyroyleanone and 6,7-dehydroroyleanone ( $P$. madagascariensis); $\beta$-sitosterol, stigmasterol, oleanolic and urso-

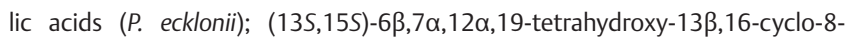
abietene-11,14-dione ( $P$. porcatus); $\left(11 R^{*}, 13 E\right)$-11-acetoxyhalima-5,13-dien15-oic acid, Plectrornatin C, 1,6-di-O-acetylforskolin and 1,6-di-O-acetyl-9deoxyforskolin ( $P$. ornatus); $\alpha$-amyrin and $\beta$-amyrin (P. neochilus); chlorogenic acid (P. saccatus) and rosmarinic acid (all aqueous extracts).

The compounds were unable to reveal in vitro anti-inflammatory activity neither in LPS-stimulated RAW 264.7 cells nor in the S-nitroso- $N$-acetylpenicillamine assay. Nonetheless, regarding the $M$ tb growth inhibition, one semisynthetic halimane derivative $\left(11 R^{*}, 13 E\right)$-halima-5,13-diene-11,15-diol) previously prepared [4], revealed promising results $\left(2.1 \times 10^{5} \mathrm{CFU} / \mathrm{mL}\right)$, with an effect similar to the anti-tubercular drugs ethambutol $\left(2.0 \times 10^{5} \mathrm{CFU} / \mathrm{mL}\right)$ and isoniazid $\left(1.2 \times 10^{5} \mathrm{CFU} / \mathrm{mL}\right)$.

To the best of our knowledge, this report is the first scientific validation on Plectranthus spp. isolated compounds concerning their anti-inflammatory activity for NO production and release, and concerning their anti-mycobacterial activity for Mtb growth inhibition. Further studies regarding other inflammatory mediators are under study.

[1] Lukhoba CW, Simmonds MS, Paton AJ. J. Ethnopharmacol 2006; 103: 1 24;

[2] Rijo, P, Simões MF, Francisco AP, Rojas R, Gilman RH, Vaisberg AJ, Rodríguez B, Moiteiro C. Chem. Biodivers 2010; 7: 922 - 932;

[3] Rijo, P, Gaspar-Marques C, Simões MF, Jimeno ML, Rodríguez B. Biochemical Systematics and Ecology 2007; 35: 215 - 221.

\section{Mo-Poster Session 1-PO-88 Discovery of novel cosmeuceutical agents from endophytic microorganisms of Spanish biodiversity}

Authors Georgousaki K1, Tsafantakis $N^{1}$, Cheilari $\mathrm{A}^{2}$, Gumeni $\mathrm{S}^{3}$, González $\mathrm{I}^{4}$, González $\mathrm{V}^{4}$, Tormo José $\mathrm{R}^{4}$, Genilloud $\mathrm{O}^{4}$, Foteinos $\mathrm{S}^{2}$, Trougakos $\mathrm{I}^{3}$, Fokialakis $\mathrm{N}^{1}$

Institute 1 Department of Pharmacognosy and Natural Products Chemistry, Faculty of Pharmacy, National and Kapodistrian University of Athens, Athens, Greece; 2 Lavipharm SA, Athens, Greece; 3 Department of Cell Biology and Biophysics, Faculty of Biology, National and Kapodistrian University of Athens, Athens, Greece; 4 Fundacion MEDINA, Granada, Spain

DOI $10.1055 / \mathrm{s}-0037-1608137$

In order to explore the potential of endophytic microorganisms to produce secondary metabolites with applications in the cosmeceutical industry, more than 120 potential candidate endophytic fungi and actinomycetes originated from Spanish biodiverse arid areas were selected to be studied. In order to broadly express the production of different secondary metabolites, four nutritional conditions have been used for the cultivation of the selected strains in liquid media. In total 1120 extracts have been generated and cell-based bioassays have being incorporated for the evaluation of their skin-protecting and skin-whitening activity. The whitening activity was determined using the tyrosinase assay, while the skin-protecting activity was determined by evaluating the elastase inhibitory activity. Among the initial 1120 extracts, approximately 30 were selected as promising bioactive samples and have been further evaluated in cell-based assays for their skin whitening (bleaching) activity in mouse melanocytes (B16F10 cell line), as well as for elastase inhibition in normal human fibroblasts (BJ cells). In parallel cytotoxicity evaluation (MTT method at the MCF-7 and HepG2 cell lines) was performed to the selected bioactive extracts in order to exclude those that exert cytotoxic effects. UHPLC-HRMS in bioactive extracts was used for profiling and metabolomics analysis in order to correlate the different secondary metabolites produced with each production condition and with the activity that each strain demonstrated. In addition a dereplication campaign for identification of known metabolites using commercial databases was performed. The noncytotoxic extracts that demonstrated significant activity in both cell-free and cell-based assays have been selected for cultivation in larger scale and bioguided fractionation and isolation of bioactive compounds using both classical chromatographic methods (HPLC, MPLC), as well as innovative techniques, like SFC-MS and FCPC has been performed. The full set of spectroscopic data (MS and NMR) were recorded for all isolated compounds in order to unambiguously elucidate their structure. 


\section{Mo-Poster Session 1-PO-89 Seco-cassine diterpenoids and cytotoxic activities of the constituents from the roots of Acacia farnesiana}

Authors Chen $\mathrm{YC}^{1}$, Liu $\mathrm{HH}^{2}$, Lee $\mathrm{HZ}^{3}$

Institute 1 School of Pharmacy, College of Pharmacy, Kaohsiung Medical University, Kaohsiung, Taiwan; 2 Department of Chinese Pharmaceutical Science and Chinese Medicine Resources, College of Pharmacy, China Medical University, Taichung, Taiwan; 3 School of Pharmacy, College of Pharmacy, China Medical University, Taichung, Taiwan

DOI 10.1055/s-0037-1608138

Acacia farnesiana (L.) Willd (Leguminosae) is an erect pantropic shrub with forking prickles and widely distributed in the southern part of Taiwan [1]. The preliminary study showed the methanolic extract of its root owning selected cytotoxicity against the $\mathrm{HSC}-3$ cancer cell line. The $\mathrm{MeOH}$ extract was partitioned into $n$-hexane, EtOAc, $n$ - $\mathrm{BuOH}$ and $\mathrm{H}_{2} \mathrm{O}$-soluble layers. Investigation of the active $n$-hexane- and EtOAc-soluble fractions of $A$. farnesiana led to the isolation of six new diterpenoids, farnesiranes $\mathrm{Cl}, \mathrm{CII}$, DI, DII, El, and Ell, together with 18 known compounds. Among these compounds, farnesirane $\mathrm{Cl}$ in $30 \mu \mathrm{M}$ showed the cytotoxic activity against HSC-3 cell line. The structures of these compounds were determined through spectral data.

[1] Huang TC, Ohashi H. Leguminosae in Flora of Taiwan, 2nd edition, Vol. 2. Taipei: Editorial Committee of the Flora of Taiwan; 1993: 162-396

\section{Mo-Poster Session 1-PO-90 Anticancer effect of cucurbitacin B in human cholangiocarcinoma cells}

Authors Senggunprai $L^{1}$, Klungsaeng $S^{1}$, Kukongviriyapan $V^{1}$, Prawan $A^{1}$ Institute 1 Department of Pharmacology, Faculty of Medicine, and Cholangiocarcinoma Research Institute, Khon Kaen University, Khon Kaen, Thailand, Khon Kaen, Thailand

DOI 10.1055/s-0037-1608139

Cholangiocarcinoma (CCA) is one of devastating cancers with very poor prognosis and is associated with high mortality. At present, the development of new strategies for prevention and treatment of CCA is highly desired. Several phytochemical compounds are known to possess anticancer activities. Cucurbitacin B is a compound derived from plants of Cucurbitaceae family. In this study, the cytotoxic effect of cucurbitacin B against CCA cells (KKU-100) and the underlying mechanisms mediating its effect were investigated. Sulphorhodamine B assay showed that cucurbitacin B had a potent cytotoxicity on CCA cells with $I_{50}$ value in the low micromolar range. To confirm that cell death affected by cucurbitacin B could involve apoptotic induction, CCA cell apoptosis was assessed by flow cytometry using FITC-annexin V/propidium iodide staining. The results showed that cucurbitacin B induced apoptosis in KKU-100 cells in a dose-dependent manner. To further investigate the mechanism underlying apoptosis-inducing effect of cucurbitacin B, the expression of proteins responsible for regulating apoptosis pathway, anti-apoptotic $\mathrm{BCl}-2$ and $\mathrm{BCl}-\mathrm{XL}$, were then analyzed using Western blot analysis. The results demonstrated that the decrease in the level of $\mathrm{BCl}-2$ and $\mathrm{BCl}-\mathrm{XL}$ proteins was implicated in cucurbitacin B-induced apoptosis. Because the activation of caspases plays an important role in apoptosis, the effect of cucurbitacin B on caspase- 9 activity was evaluated. The results showed that treatment with cucurbitacin B resulted in the activation of the initiator caspase- 9 . These data revealed anticancer activity of cucurbitacin B against CCA so the agent might be an effective phytochemical for prevention or treatment of CCA.

\section{Mo-Poster Session 1-PO-91 Antiproliferative effect of all-trans-retinoic acid in cholangiocarcinoma}

Authors Prawan $\mathrm{A}^{1}$, Butsri $\mathrm{S}^{1}$, Kukongviriyapan $\mathrm{V}^{1}$, Senggunprai $\mathrm{L}^{1}$, Kongpetch $\mathrm{S}^{1}$,

Institute 1 Department of Pharmacology, Faculty of Medicine, Cholangiocarcinoma Research Institute, Khon Kaen University, Khon Kaen,

Thailand

DOI 10.1055/s-0037-1608140

Cholangiocarcinoma (CCA) is an aggressive and potentially fatal bile duct cancer. This cancer responds poorly to current radiotherapy and chemotherapy, consequently, searching for novel therapeutic options of CCA is an urgent need. All-trans-retinoic acid (atRA), vitamin A derivative, is well-known to regulate cell behavior and to play key roles in determination of cell fate. Clinically, atRA is considered as a potential anti-tumor agent against human hematopoietic cancers. Up to now, little is known about the effect of atRA on treating human CCA. We aimed to investigate the potential of atRA in suppressing the proliferation of human CCA cells. In vitro experiments including sulforhodamine B (SRB) and colony-forming assays were performed in KKU100 CCA cells. The result showed that, in a dose-dependent manner, atRA significantly reduced cell viability of cholangiocarcinoma cells. The long-term reproductive survival of CCA cells were also suppressed by atRA treatment. Our data demonstrate the anti-proliferative effect of atRA on CCA cells and suggest that atRA may be a potential therapeutic agent for the treatment of CCA. Therefore further studies investigating to understand the molecular mechanisms underlying its effects and in vivo anticancer benefit of atRA for the treatment of CCA are warranted.

\section{Mo-Poster Session 1-PO-92 Antifungal Activity against Pythium insidiosum of Compounds from Scaevola taccada}

Authors Suthiwong J $]^{1}$, Yenjai $C^{1}$, Thongsri $Y^{2}$

Institute 1 Natural Products Research Unit, Department of Chemistry, Faculty

of Science, Center of Excellence for Innovation in Chemistry, Khon Kaen

University, Khon Kaen, Thailand; 2 Cellular and Molecular Immunology

Research Unit (CMIRU), Department of Medical Technology, Faculty of Allied

Health Science, Naresuan University, Phitsanulok, Thailand

DOI 10.1055/s-0037-1608141

A new coumarin, scataccanol (1) and ten known compounds, including five coumarins; ent-ammirin (2), nodachenetin (3), marmesin (4), xanthyletin (5) and umbelliferone (6), a benzaldehyde derivative; 4-formylsyringol(7), two iridoids; 6-hydroxy-7-methyl-1-oxo-4-carbomethoxy octahydro-cyclopenta[c] pyran (8) and loganetin (9), a lignan; matairesinol (10) and a cinnamoyl ester; 2-(4-hydroxyphenyl) 3-(3,4-dihydroxyphenyl)-2-propenoate (11) were isolated from the fruits of Scaevola taccada (Gaertn.) Roxb [1]. All compounds were evaluated for antifungal activity against Pythium insidiosum [2]. Xanthyletin (5) and 4-formylsyringol (7) showed strong antifungal activity with minimum inhibitory concentration values of 5 and $10 \mu \mathrm{g} / \mathrm{mL}$, respectively [3]. Structural determination of all compounds was accomplished by 1D and 2D-NMR, IR and MS. 


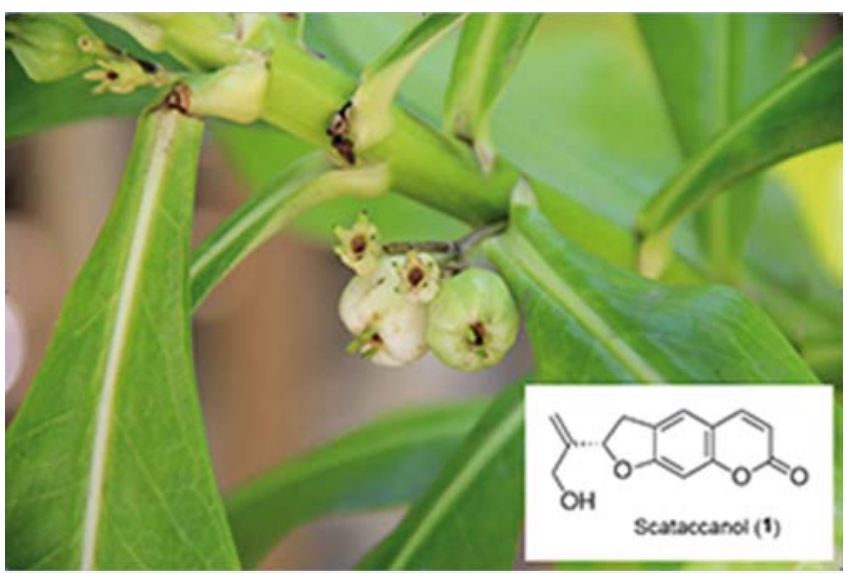

Fig. 1

[1] Ruangrungsi N, Mangkhla KT. Thai herbs, 1st edition. Thailand: Than Printing Company Limited; 2004: 259.

[2] Fonseca AOS, Pereira DIB, Jacob RG, Maia Filho FS, Oliveira DH, Maroneze BP, Valente JSS, Osório LG, Botton SA, Meireles MCA. Mycopathologia 2015; 179: $253-258$

[3] Suthiwong J, Thongsri Y, Yenjai C. Nat Prod Res 2017; 31: 453 - 459.

\section{Mo-Poster Session 1-PO-93 Benzoyltyramine Alkaloids Atalantums A-G with Their Cytotoxicity against Cholangiocarcinoma Cell Line from the Peels of Atalantia monophylla}

Authors Sribuhom $\mathrm{T}^{1}$, Boueroy $\mathrm{P}^{2}$, Hahnvajanawong $\mathrm{C}^{2}$, Phatchana $\mathrm{R}^{3}$, Yenjai $C^{1}$

Institute 1 Natural Products Research Unit, Center of Excellence for Innovation in Chemistry, Department of Chemistry, Faculty of Science, Khon Kaen University, Khon Kaen 40002, Thailand, Khon Kaen, Thailand;

2 Department of Microbiology, Liver Fluke and Cholangiocarcinoma Research Center, Faculty of Medicine, Khon Kaen University, Khon Kaen 40002, Thailand, Khon Kaen, Thailand; 3 Department of Chemistry, Faculty of Engineering,

Rajamangala University of Technology Isan, Khon Kaen Campus, Khon Kaen 40000, Thailand, Khon Kaen, Thailand

DOI 10.1055/s-0037-1608142

The extraction and isolation of the peels of Atalantia monophylla using chromatographic methods afforded seven new benzoyltyramines, atalantums A-G $(1-7)$, along with five known compounds [1]. The structures of these compounds were elucidated based on the analysis of spectroscopic data. Compound 5 displayed the strongest cytotoxicity against KKU-M156 cells, with an $\mathrm{IC}_{50}$ value of $1.97 \pm 0.73 \mu \mathrm{M}$, an approximately 4 .7-fold higher activity than that of the ellipticine standard [2]. Compound 1 was active against KKU-M214 cells, with an $\mathrm{IC}_{50}$ value of $3.06 \pm 0.51 \mu \mathrm{M}$. For KKU-M213 cell line, compounds 2,4 , and 11 displayed stronger cytotoxicity than the ellipticine standard, with $\mathrm{IC}_{50}$ values of $2.36 \pm 0.20,5.63 \pm 0.22$, and $2.71 \pm 0.23 \mu \mathrm{M}$, respectively. Compounds 1,5 , and 7 displayed cytotoxicity against KKU-M214 cells, with $\mathrm{IC}_{50}$ values of $3.06 \pm 0.51,8.44 \pm 0.47$, and $7.37 \pm 1.29 \mu \mathrm{M}$, respectively [3].
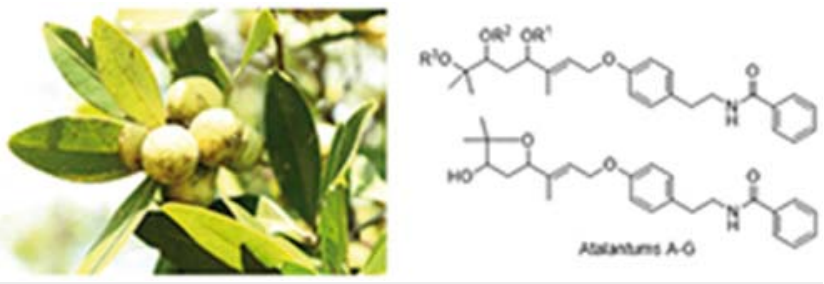

Fig. 1
[1] Panda H. Handbook on Medicinal Herbs with Uses: Asia Pacific Business Press Inc; 2004: 166 - 167.

[2] Loilome W, Yongvanit P, Wongkham C, Tepsiri N, Sripa B, Sithithaworn P, Hanai S, Miwa M. Mol. Carcinog 2006; 45: 279 - 287.

[3] Sribuhom T, Boueroy P, Hahnvajanawong C, Phatchana R, Yenjai C. J. Nat. Prod 2017; 80: $403-408$.

\section{Mo-Poster Session 1-PO-94 In vitro angiotensin-converting enzyme inhibition by phenolic compounds isolated from the root bark of Morus nigra}

Authors Zoofishan $Z^{1}$, Kúsz $N^{1}$, Zomborszki Zoltán $\mathrm{P}^{1}$, Csorba $\mathrm{A}^{1}$,

Hunyadi $A^{1,2}$

Institute 1 Institute of Pharmacognosy, University of Szeged, Szeged,

Hungary; 2 Interdisciplinary Centre for Natural Products, University of Szeged, Szeged, Hungary

DOI $10.1055 / \mathrm{s}-0037-1608143$

Hypertension is a major health issue across the world, with a high prevalence and progressive chronical effects. The root bark of Morus nigra has been traditionally utilized to treat hypertension ${ }^{[1]}$, yet not many studies explained the antihypertensive effect of this plant. This study was conducted to test the potential antihypertensive activity of isolated phenolic compounds of Morus nigra root bark as inhibitors of the angiotensin converting enzyme (ACE) in vitro.

The methanolic extract of Morus nigra was processed by various chromatographic techniques leading to the isolation of seven compounds. Their chemical structures were elucidated by 1- and 2D-NMR spectroscopy and HRMS, identifying them as morusin, kuwanon $\mathrm{U}$, kuwanon $\mathrm{E}$, albanol $\mathrm{A}$ and $\mathrm{B}$, and moracin $\mathrm{P}$ and $\mathrm{R}$ (1-7, respectively). All c ompounds were tested for their ACE inhibitory activity. Compound 4 exerted a complete ACE inhibition at $65 \mu \mathrm{M}$, whereas 3, 1 and 2 showed $90 \%, 60 \%$ and $50 \%$ inhibition at 85,86 , and $83 \mu \mathrm{M}$, respectively. $I C_{50}$ values for 1,3 , and 4 were determined as 34,43 and $45 \mu \mathrm{M}$, respectively, revealing 3 as an over two-times more potent ACE inhibitor as compared to 2 .<smiles>COc1cc(O)c(C2CC(=O)c3c(O)cc(O)cc3O2)cc1CC=C(C)CCC=C(C)C</smiles><smiles>CC(C)=CCC/C(C)=C/Cc1cc(C2CC(=O)c3c(O)cc(O)cc3O2)c(O)cc1O</smiles>

Fig. 1

Our results showed that presence of a 4'-OH group on the B ring is important for a significant ACE inhibition by the geranylflavanone kuwanons ( 2 and 3 ). This finding is in good agreement with previous reports on related SAR of other types of flavonoids ${ }^{[2]}$

This work was supported by the NKFIH, Hungary (K119770), the EU-funded Hungarian grant EFOP-3.6.1-16-2016-00008, and GINOP-2.3.2-152016 - 00012. A.H. acknowledges the János Bolyai fellowship of the Hungarian Academy of Sciences and the Kálmán Szász Prize.

[1] Ziaei SA et al. Journal of Kerman University of Medical Sciences, 2009; 16: $134-142$.

[2] Guerrero L et al. PloS One, 2012; 7: e49493. 


\section{Mo-Poster Session 1-PO-95 Isolation of endophytic fungi from South African plants and their medicinal significance}

Authors Abdalla $\mathrm{M}^{1}$, Aro $\mathrm{A}^{1}$, Famuyide $\mathrm{I}^{1}$, McGaw $\mathrm{L}^{1}$

Institute 1 Phytomedicine Programme Department of Paraclinical Sciences Faculty of Veterinary Science University of Pretoria Private Bag X04

Onderstepoort 0110 South Africa, Pretoria, South Africa

DOI 10.1055/s-0037-1608144

The growing resistance of pathogenic microorganisms to antibiotics has resulted in an increasing need worldwide for new candidate drugs to combat antibiotic resistance. It is widely acknowledged that microorganisms are a prolific source of highly potent antibacterial, antifungal and anticancer compounds, owing to their capacity for producing interesting natural products [1]. Endophytes residing in medicinal plants have excellent bioprospecting potential, particularly when combined with the remarkable pharmacological properties of many South African medicinal plants. These are highly under-investigated sources of potentially useful endophytic microbes. In this research project several endophytic fungi were obtained from the leaves of South African medicinal plants, including Cotyledon orbiculata L, Gomphocarpus fruticosus (L.) W.T.Aiton, Podranea ricasoliana (Tanfani) Sprague, Ficus elastica Roxb. ex Hornem, Catha edulis (Vahl) Endl. and Psychotria zombamontana (Kuntze) E.M.A. Petit. In preliminary microbial inhibition investigation, the isolated endophytes displayed promising antifungal activity against Cryptococcus neoformans and Candida albicans. Two endophytic fungi, named zom-gr and zom-cot obtained from Psychotria zombamontana, showed similar minimum inhibitory concentration (MIC) values of 0.625 to $1.25 \mathrm{mg} / \mathrm{ml}$ against Cryptococcus neoformans and Candida albicans. Catha edulis also afforded endophytic fungal strains with activity against Cryptococcus neoformans and Candida albicans with MIC values in the same range. In the antibacterial assays, endophytic fungi from Catha edulis had antibacterial activity against Staphylococcus aureus and Salmonella Typhimurium with MIC $=1.25 \mathrm{mg} / \mathrm{ml}$. Moreover they exhibited moderate activity against Enterococcus faecalis and Escherichia coli. Further experiments are in progress to identify the isolated fungal strains and to study their anticancer activity against different cancer cell lines. It is worth considering further investigation of their bioactive secondary metabolites [2], which will be the future focus of this study.

[1] Abdalla MA, Helmke E, Laatsch H. Nat Prod Comm 2010; 5: 1917 - 1920

[2] Abdalla MA, Matasyoh JC. Nat Prod Bioprospect 2014; 4: 257 - 270

\section{Mo-Poster Session 1-PO-96 Bioactive chemical constituents isolated from the root of Neolitsea acuminatissima}

Authors $\mathrm{Ko} \mathrm{HH}^{1,2}$, Chang $\mathrm{CC}^{1}$, Lin $\mathrm{CH}^{2,3}$, Kuo $\mathrm{YH}^{4,5}$, Chen $\mathrm{IS}^{3}$, Chang $\mathrm{HS}^{2,3}$ Institute 1 Department of Fragrance and Cosmetic Science, Kaohsiung Medical University, Kaohsiung, Taiwan; 2 Research Center for Natural Products and Drug Development, Kaohsiung Medical University, Kaohsiung, Taiwan; 3 School of Pharmacy, Kaohsiung Medical University, Kaohsiung, Taiwan; 4 Department of Chinese Pharmaceutical Science and Chinese Medicine Resources, China Medical University, Taichung, Taiwan;

5 Department of Biotechnology, Asia University, Taichung, Taiwan DOI 10.1055/s-0037-1608145

Neolitsea acuminatisima (Hayata) Kanehira \& Sasaki is genus of Neolistea (Lauraceae), an endemic species in Taiwan. In fact, the chemical constituents of the root of this plant have seldom been investigated previously. It is worth to verify the phytochemistry and secondary metabolites of the root of $N$. acuminatissima. One new carboline alkaloid, demethoxydaibucarboline A (1) and three new eudesmanolide type sesquiterpenes, methylneolitacumone $A(2)$, neolitacumone $E(3)$, and dineolitacumone $C$ (4), along with twelve known compounds neolitacumones A-C (5-7), b-sitosterol (8), quercetin (9), dihydroquercetin (10), epicatechin (11), oplopanone (12), zeorin (13), linderaggrine A (14), clovane-2 $\beta, 9 \alpha$-diol (15), and stigmast-5-ene-3b-yl formate (16) were isolated from the dichloromethane and ethyl acetate-soluble layers of the root of N. acuminatissima. All compounds were obtained by column chromatography and their structures were spectroscopically determined by $1 \mathrm{D}, 2 \mathrm{D}$ NMR and MS. In this present study, the bioactivity of the isolates was also reported.

\section{Mo-Poster Session 1-PO-97 Novel MDR-modulating Diterpenes from Euphorbia taurinensis}

Authors Kúsz $N^{1}$, Sátori $G^{1}$, Kincses $A^{2}$, Spengler $G^{2}$, Barina $Z^{3}$,

Hohmann $]^{1,4}$, Rédei $D^{1}$

Institute 1 Department of Pharmacognosy, University of Szeged, Szeged,

Hungary; 2 Department of Medical Microbiology and Immunobiology,

University of Szeged, Szeged, Hungary; 3 Hungarian Natural History Museum,

Budapest, Hungary; 4 Interdisciplinary Centre of Natural Products, University

of Szeged, Szeged, Hungary

DOI 10.1055/s-0037-1608146

Multidrug resintance (MDR) is a major obstacle in the treatment of cancer. MDR cells are less sensitive against structurally diverse chemotherapeutic agents, caused by the overexpression of P-glycoprotein (MDR1 or ABCB1 protein) in the cell membrane. These ATP dependent efflux proteins prevent the drug molecules from reaching the effective intracellular concentration by pumping them out from the cytoplasm. Despite the great efforts, the inhibition of multidrug resistance still remained an unresolved problem. Diterpenes isolated from Euphorbiaceae species represent a promising group of natural products due to their potent MDR-modulating properties.

The methanol extract of the whole plant of Euphorbia taurinensis All. was subjected to liquid-liquid partition with chloroform. Multistep chromatographic purification (CC, VLC, PLC, HPLC) yielded six pure compounds. The structure determination was carried out by means of HRMS and 1D and 2D NMR methods. The MDR modulating effects of the isolated compounds were evaluated on L5178 mouse T-lymphoma cell line using rhodamine 123 accumulation assay. The fluorescence activity ratios (FAR) between the MDR1transfected cell populations over-expressing P-glycoprotein and its parental counterparts were measured at two concentrations $(2 \mu \mathrm{M}$ and $20 \mu \mathrm{M})$ by flow cytometry.

As a result of the first phytochemical investigation of $E$. taurinensis, two novel and four known diterpenes were obtained $[1,2]$. One of the new compounds possesses the rare segetane skeleton. Two diterpenes belong to the jatrophane and three compounds to the ingenane group. The occurrence of such various types of diterpenes in one species is uncommon in the genus. Four of the isolated compounds exerted substantial MDR-reversing activity on the Tlymphoma cells with FAR values between 34 and 59 at $20 \mu \mathrm{M}$ concentration. The research was supported by the European Regional Development Fund under the project GINOP-2.3.2-15-2016-00012.

[1] Jakupovic J, Morgenstern T, Marco JA, Berendsohn W. Phytochemistry 1998; 47: $1611-1619$

[2] Jakupovic J, Jeske F, Morgenstern T, Tsichritzis F, Marco JA, Brendsohn W. Phytochemistry 1998; 47: $1583-1600$..

\section{Mo-Poster Session 1-PO-98 Preparation and chemo-sensitizing activity of nitrogen-containing ecdysteroid derivatives: 6-oximes, oxime ethers, and a lactam}

Authors Vágvölgyi $\mathrm{M}^{1}$, Martins $\mathrm{A}^{2}$, Kulmány Á ${ }^{3}$, Zupkó $\mathrm{I}^{3}$, Tóth $\mathrm{C}^{1,4}$, Hunyadi $A^{1,5}$

Institute 1 Institute of Pharmacognosy, University of Szeged, Szeged, Hungary; 2 Department of Medical Microbiology and Immunobiology, Faculty of Medicine, University of Szeged, Szeged, Hungary; 3 Department of Pharmacodynamics and Biopharmacy, University of Szeged, Szeged, Hungary; 4 Department of Inorganic and Analytical Chemistry, NMR Group, Budapest University of Technology and Economics, Budapest, Hungary; 
5 Interdisciplinary Centre for Natural Products, University of Szeged, Szeged, Hungary

DOI 10.1055/s-0037-1608147

Ecdysteroids are a group of natural hydroxysteroids, widespread in plants and invertebrates, exerting a broad spectrum of biological functions. These nontoxic compounds are commonly valued for their beneficial, non-hormonal bioactivities also in mammals including humans. Recent studies of our group revealed that less polar ecdysteroids can strongly sensitize various cancer cell lines, including multi-drug resistant (MDR) and drug susceptible ones, to chemotherapeutics. ${ }^{[1-3]}$ Oxime functionalities can increase the antitumor activity of certain steroids; ${ }^{[4]}$ however, no related studies on ecdysteroid oximes have been performed.

Here we report the synthesis and bioactivity testing of a set of nitrogencontaining ecdysteroid derivatives. By optimizing previously published methodology $^{[5]}, 14$ compounds including 12 new derivatives (oximes, oxime ethers and a lactam) were prepared from the semi-synthetic modification of 20hydroxyecdysone 2,3;20,22-diacetonide.

All compounds were tested for their antiproliferative activity against various cancer cells followed by determination of the chemo-sensitizing potential towards doxorubicin on a P-glycoprotein (Pgp) transfected MDR cancer cell line and on its sensitive counterpart. Moderate to strong anti-proliferative activities were observed. Each compound could considerably sensitize the studied cancer cell lines, with a noticeable selectivity towards the MDR one. The most promising results were obtained for a new ecdysteroid lactam, which showed remarkably strong chemo-sensitizing potential without interfering with the efflux function of Pgp.

This work was supported by the NKFIH, Hungary (K119770), the EU-funded Hungarian grant EFOP-3.6.1-16-2016-00008, and GINOP-2.3.2-152016 - 00012. A.H. acknowledges the János Bolyai fellowship of the Hungarian Academy of Sciences and the Kálmán Szász Prize.

[1] Martins A et al. J Med Chem 2012; 55: 5034 - 5043.

[2] Martins A et al. Biomed Res Int, 2015; 895360.

[3] Cui JG et al. Steroids 2009; 74: 62 - 72 and 2009; 74: 989- 995.

[4] Galyautdinov IV et al. Russ J Org Chem 2006; 42: 1333 - 1339.

[5] Shafikov RV Russ J Org Chem 2009; 45: 1456 - 1463.

\section{Mo-Poster Session 1-PO-99 Modulation of chemotherapeutic drug sensitivity by frankincense.}

\section{Authors Alhabib I ${ }^{1}$, Bhambra $A^{1}$, Haris $\mathrm{P}^{1}$, Al Salmani $\mathrm{K}^{2}$, Al-Harrasi $\mathrm{A}^{3}$,} Patel $\mathrm{R}^{4}$, Evans $\mathrm{M}^{1}$

Institute 1 School of Allied Health Sciences, Faculty of Health \& Life Sciences, De Montfort University, Leicester, United Kingdom; 2 Department of Cancer Studies, University of Leicester, Leicester, United Kingdom; 3 Biological Sciences \& Chemistry - Chemistry Section, College of Arts \& Sciences, University of Nizwa, Nizwa, Oman; 4 Department of Molecular \& Cell Biology, University of Leicester, Leicester, United Kingdom

DOI 10.1055/s-0037-1608148

The development of chemoresistance is a serious problem applicable to many types of cancer; ovarian cancer is a particular tumour type that can show intrinsic and acquired resistance to first-line chemotherapy. Second-line chemotherapy is used to treat recurrent ovarian cancer, e. g. liposomal doxorubicin (Jayson et al, 2014). A number of dose dependent side effects are reported from prolonged and repeated exposure to liposomal doxorubicin (Iwamoto, 2013). Reduction of the required dose for doxorubicin treatment using another agent that could also potentiate doxorubicin anti-cancer activity could be therapeutically valuable. Frankincense (from Boswellia sp.) has been used in traditional medicine for centuries primarily to treat diseases with an inflammatory aetiology. Bioactive components unique to Boswellia sp. (pentacyclic triterpenes in particular boswellic acids) responsible for many of the therapeutic actions of frankincense have been investigated more recently in the context of anticancer activity. Some reports show that 3-O-acetyl-11-keto- $\beta$-bos- wellic acid (AKBA) can modulate chemotherapeutic drug toxicity, although this has not been well explored. The identification of individual components in frankincense that can modify the anti-cancer actions of existing chemotherapeutic drugs or otherwise yield unexpected anticancer effects would be useful. This work examines the potential utility and mechanisms of action of AKBA for modifying cancer chemotherapeutic drug activity, using ovarian cancer cells as a model. Preliminary data (Fig. 1) show that a minimally cytotoxic dose of AKBA in combination with doxorubicin significantly $(p<0.01)$ enhances the EC50 of doxorubicin on A2780 and A280cis (cisplatin resistant) ovarian cancer cells. Work is funded by The Saudi Arabian Cultural Bureau: PhD scholarship awarded to IAH.

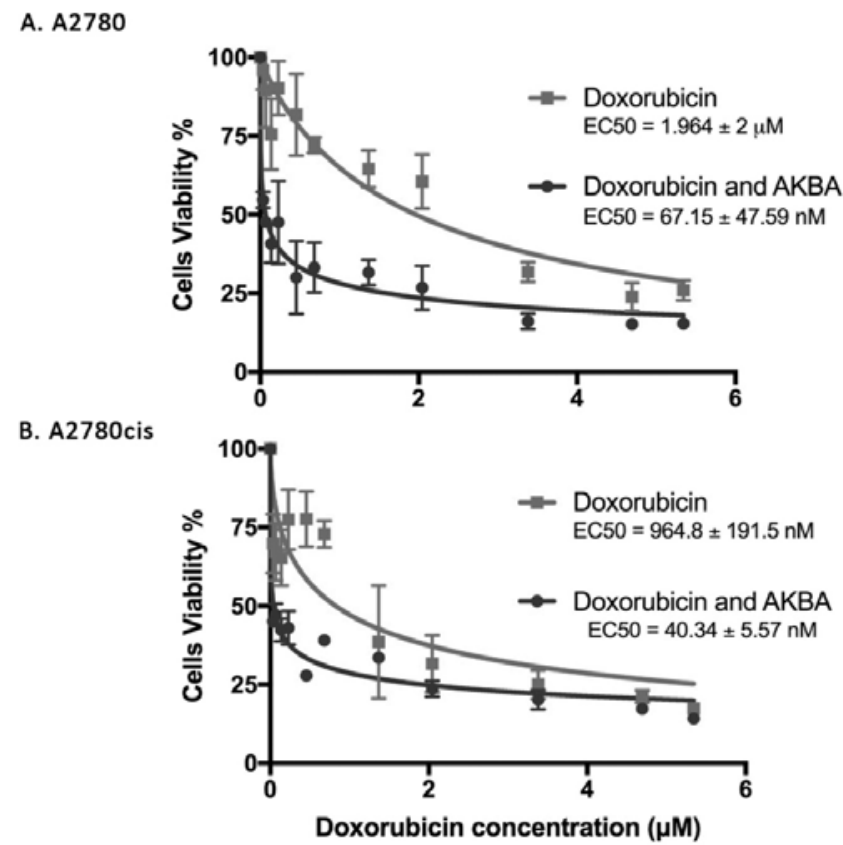

Fig. 1: Cytotoxicity of doxorubicin on ovarian cancer A2780 cells treated with and without AKBA. Cells were treated with doxorubicin alone (squares) or simultaneously with $26 \mu \mathrm{M}$ AKBA and doxorubicin (circles) for 24 hours at $37^{\circ} \mathrm{C}, 5 \% \mathrm{v} / \mathrm{v} \mathrm{CO}_{2}$. Viability assessed using the MTT assay. The mean \pm SEM for $n=3$ independent experiments shown.

Iwamoto, T, Biology Pharmacy Bulletin, 2013, 36, 715 - 718. doi: 10.1248/ bpb.b12- 01102.

Jayson, G. C, Kohn, E. C, Kitchener, H. C. and Ledermann, J. A, The Lancet. Elsevier Ltd, 2014, 384(9951), 1376 - 1388. doi: 10.1016/S0140 - 6736(13) $62146-7$.

\section{Mo-Poster Session 1-PO-100 Anti-hyperglycemic constituents from the leaves of Cinnamomum macrostemon}

Authors Yang $\mathrm{SS}^{1}$, Huang $\mathrm{YH}^{2}$, Lai $\mathrm{HC}^{2}$, Hsu $\mathrm{LC}^{3}$, Guh $\mathrm{JH}^{3}$, Cheng MJ ${ }^{4}$,

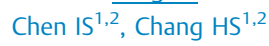

Institute 1 Graduate Institute of Natural Products, College of Pharmacy, Kaohsiung Medical University, Kaohsiung 807, Taiwan, Kaohsiung, Taiwan; 2 School of Pharmacy, College of Pharmacy, Kaohsiung Medical University, Kaohsiung 807, Taiwan, Kaohsiung, Taiwan; 3 School of Pharmacy, National Taiwan University, Taipei 106, Taiwan, Taipei, Taiwan; 4 Bioresource Collection and Research Center, Food Industry Research and Development Institute, Hsinchu 300, Taiwan, Hsinchu, Taiwan

DOI 10.1055/s-0037-1608149

Glucose transport and metabolism are tightly associated with diseases including diabetes. Sodium-coupled glucose co-transporters (SGLTs), SGLT1 and 
SGLT2, are important for intestinal absorption and renal reabsorption of glucose, respectively. The previous study reported that dual SGLT1/SGLT2 inhibitor as an adjunct therapy to insulin in type 1 diabetes could improve glycemic control and overcome the side effect, such as hypoglycemia and weight loss. Approximately 60 species of Lauraceae plants have been screened for the SGLT inhibitory activity and Cinnamomum macrostemon Hay. (Lauraceae) was shown as the most active species. The methanolic extract of the leaves of $C$. macrostemon was partitioned into the water-soluble and ethyl acetate layers. Water-soluble layer showed the potent activity toward SGLT inhibitory. Till now, the chemical constituents of water-soluble layer and bioactivity from $C$. macrostemon have never been investigated. Bioassay-guided fractionation of the active water-soluble layer from the leaves of $C$. macrostemon led to the isolation of one new megastigmane, cinnamacroside (1), along with 14 known compounds, including one benzenoid, one coumarin, 11 flavonoid glycosides, and one lignan. Twenty-one known compounds were also isolated from the ethyl acetate layer of the leaves of $C$. macrostemon.

Among the isolates, kaempferol 3-O- $\beta$-D-glucopyranosyl-( $1 \rightarrow 4)-\alpha$-L-rhamnopyranosyl-7-O- $\alpha$-L-rhamnopyranoside and kaempferol 3-O-(2"'- $\beta$-D-glucopyranosyl)- $\alpha$-L-rhamnopyranoside showed potent inhibitory activity toward SGLT, which $\mathrm{IC}_{50}$ values $475 \pm 34.2$ and $123 \pm 11.6 \mathrm{nM}$ for SGLT1, and $68.8 \pm 14.2$ and $12.5 \pm 2.95 \mathrm{nM}$ for SGLT2, respectively.

\section{Mo-Poster Session 1-PO-101 Anti-Escherichia coli $\beta$-glucuronidase activity constituents from the root of Neolitsea konishii}

\author{
Authors Huang $\mathrm{HK}^{1}$, Cheng $\mathrm{TL}^{2}$, Lin $\mathrm{CH}^{1}$, Wu $\mathrm{HC}^{3}$, Chen $\mathrm{IS}^{1,3}$, Gan $\mathrm{KH}^{1}$, \\ Chang $\mathrm{HS}^{1,3}$
}

Institute 1 School of Pharmacy, College of Pharmacy, Kaohsiung Medical University, Kaohsiung 807, Taiwan, Kaohsiung, Taiwan; 2 Department of Biomedical Science and Environmental Biology, Kaohsiung Medical University, Kaohsiung, Taiwan; 3 Graduate Institute of Natural Products, College of Pharmacy, Kaohsiung Medical University, Kaohsiung, Taiwan

DOI 10.1055/s-0037-1608150

Diarrhea is one of the most common side effects of chemotherapy. The Escherichia coli $\beta$-glucuronidase $(\mathrm{e} \beta \mathrm{g})$ in intestinal caused chemotherapy-induced diarrhea (CID) and decreased patient's chemotherapeutic efficacy. About sixty-three species of Formosan indigenous Lauraceous plants have been screened under the drug-screening platform of ebg inhibitors against CID. The methanolic extract of the root of Neolitsea konishii (Hay.) Kanehira \& Sasaki can specifically inhibit the intestinal $\mathrm{e} \beta \mathrm{g}$-activity but not affect the human bg $(\mathrm{h} \beta \mathrm{g})$. Therefore, the aims of this study are the isolation of chemical constituents and evaluation of their anti-e $\beta g$ activities.

$N$. konishii is a small evergreen tree and grows in low to medium altitudes forest in Ryukyu and Taiwan. The methanolic extract of the root of N. konishii was partitioned into ethyl acetate-soluble and water-soluble layers. Bioassayguided fractionation of the active ethyl acetate-soluble layer of the root of $N$. konishii led to the isolation of two new alkanoids, konishienone $A(1)$ and konishienone B (2), one new lignan, 9,9'-O-di-(E)-feruloyl-(-)-5,5'-dimethoxysecoisolariciresinol (3), and one new sesquiterpenoid, neolikonisin (4), along with 28 known compounds, including 17 sesquiterpenoids, one alkaloid, two amides, one steroid, five lignans, one flavonoid, and one fatty acid. The structures of these new compounds (1-4) were elucidated by 1D, 2D NMR, UV, IR, ESIMS, and HRESIMS analysis. Among the isolates, litseaculane, aesculitannin $C$, and daibucarboline $A$ can specifically block intestinal e $\beta$ g-activity but not affect $\mathrm{h} \beta \mathrm{g}$-activity. These three compounds exhibit the potential in developing anti-e $\beta g$ drug in the future.

\section{Mo-Poster Session 1-PO-102 Alnus viridis: a potential source of constituents with anti-ageing effects}

Authors Revoltella $S^{1}$, Waltenberger $B^{1}$, Baraldo $G^{2}$, Moesslacher $]^{3}$, Pagitz $\mathrm{K}^{4}$, Kohl $\mathrm{R}^{3}$, Jansen-Dürr $\mathrm{P}^{2}$, Stuppner $\mathrm{H}^{1}$

Institute 1 Institute of Pharmacy/Pharmacognosy and Center for Molecular Biosciences Innsbruck (CMBI), University of Innsbruck, Innrain 80 - 82,

Innsbruck, Austria; 2 Institute for Biomedical Aging Research and CMBI, University of Innsbruck, Rennweg 10, Innsbruck, Austria; 3 Cura Marketing GmbH, Dr.-Franz-Werner-Straße 19, Innsbruck, Austria; 4 Institute of Botany, University of Innsbruck, Sternwartestraße 15, Innsbruck, Austria

DOI 10.1055/s-0037-1608151

Alpine plants represent a valuable source for the identification of novel bioactive natural products with anti-ageing effects, especially substances that protect plants against UV radiation [1], which is also known to contribute to the ageing of human skin [2]. NADPH oxidase 4 (NOX4) has recently been implicated as driving force in cellular senescence [3].

Within this study, 150 plant species, mainly from the Alpine region, were selected, collected, and extracted. All extracts were investigated for their ability to inhibit NOX4 in HEK 293 cells.

Alnus viridis (Betulaceae) was identified as one of the most interesting plants. The methanolic extract of the leave material showed a significant inhibitory activity on NOX4, with $80 \%$ luminescence quenching and $112 \%$ cell vitality at a concentration of $25 \mu \mathrm{g} / \mathrm{mL}$. Therefore, it was selected for more detailed phytochemical analyses, aiming at the isolation and identification of the active principle(s).

Bioactivity-guided fractionation of the active Alnus viridis extract led to the isolation of three flavonoids (quercetin-3-O-ß-glucopyranoside, quercetin 3-O$\alpha$-arabinopyranoside, and quercetin 3-O- $\beta$-rhamnopyranoside). Preliminary pharmacological results suggest that they are responsible for the NOX4 inhibitory activity of the leave extract.

Moreover, also the bark material of Alnus viridis was studied. So far, five diarylheptanoids (5-hydroxy-1,7-bis(4-hydroxyphenyl)heptan-3-yl ß-apiofuranosyl-(1 $\rightarrow 6)$-ß-glucopyranoside, platyphylloside, aceroside VIII, platyphyllonol, and platyphyllenone) were isolated from the methanolic bark extract. All isolated compounds will be tested on their NOX4 inhibitory activity.

In conclusion, our study demonstrates that Alnus viridis might represent a valuable source of constituents with NOX4 inhibitory activity and potential anti-ageing effects.

Supported by the Austrian Forschungsförderungsgesellschaft (FFG) (BRIDGE 1 project 848474).

[1] Turunen M, Latola K. Environ Pollut 2005; 137: 390 - 403

[2] Yaar M, Gilchrest BA. Br J Dermatol 2007; 157: 874-887

[3] Weyemi U et al. Oncogene 2012; 31: 1117 - 1129

\section{Mo-Poster Session 1-PO-103 Chemical constituents and cytotoxic activities from the root of Cryptocarya concinna}

Authors Chang $\mathrm{HS}^{1,2}$, Wang $\mathrm{CS}^{2}$, Lin $\mathrm{CH}^{1}$, Chen $\mathrm{IS}^{1,2}$, Chen $\mathrm{YF}^{2}$ Institute 1 School of Pharmacy, College of Pharmacy, Kaohsiung Medical University, Kaohsiung, Taiwan; 2 Graduate Institute of Natural Products, College of Pharmacy, Kaohsiung Medical University, Kaohsiung, Taiwan DOI 10.1055/s-0037-1608152

Cryptocarya concinna Hance (Lauraceae) is a medium-sized evergreen tree and distributed in altitude $500-1,500 \mathrm{~m}$ broad-leaved forests in Taiwan and southern China. In our cytotoxic screening, the methanolic extract from the root of $C$. concinna exhibited cytotoxic activities against human cervical cancer SiHa cells, human ovarian cancer SKOV-3 cells, and human osteosarcoma U2OS cells. Here we investigated the chemical constituents from the root of this plant and studied their cytotoxic activities. Furthermore, the results of cytotoxic activity were determined by Alamar Blue assay. 
The methanolic extract of the root of $C$. concinna was partitioned into the ethyl acetate-soluble and water-soluble layers. The ethyl acetate-soluble layer showed potent cytotoxic activities against various cancer cell lines, including $\mathrm{SiHa}, \mathrm{SKOV}-3$, and U2OS. The bioassay-guided fractionation of the active ethyl acetate-soluble layer led to the isolation of 22 compounds, including three new chalcones, concichalcones A-C (1-3), together with 19 known compounds, including 16 flavonoids, two chalcones, and a steroid. The structures of these new compounds were elucidated by $1 \mathrm{D}$ and 2D NMR, UV, IR, ESIMS, and HRESIMS analyses. Most importantly, three pairs of flavonoid stereoisomers, cryptoflavanones $A \& B(8,9)$, cryptoflavanones $C \& D(14,15)$, and cryptoflavanones $E$ \& $F(20,21)$ were separated for the first time. Among these isolates, cryptocaryone (4), infectocaryone (5), and a mixture of cryptocaryanones A \& B $(11,12)$ exhibited potent cytotoxic activities against U2OS cancer cell line, with $\mathrm{IC}_{50}$ values of $2.81 \pm 0.07,3.90 \pm 0.50$, and 3.90 $\pm 0.50 \mu \mathrm{M}$, respectively.

\section{Mo-Poster Session 1-PO-104 Structure virtual screening of two cytotoxic compounds isolated from Papaver lacerum}

Authors Bayazeid $\mathrm{O}^{1}$, Bedir $\mathrm{E}^{2}$, Yalçın $\mathrm{F}^{1}$

Institute 1 Hacettepe University, Faculty of Pharmacy, Dept. of Pharmacognosy, ANKARA, Turkey; 2 Izmir Institute of Technology, Faculty of Engineering, Dept. of Bioengineering, Izmir, Turkey

DOI 10.1055/s-0037-1608153

Papaver species have been used to treat several diseases in Anatolia [1]. Chromatographic investigations on the $P$. lacerum methanolic extract $(\mathrm{MeOH})$ resulted in isolation of a phenolic compound (I) and a new mevalonic acid derivative (II). Structures of the compounds were elucidated as tyrosol-1-O$\beta$-D-xylopyranosyl-( $1 \rightarrow 6)-O-\beta$-D-glucopyranoside and 5-O-(6-O- $a$-L-rhamnopyranosyl- $\beta$-D-glucopyranosyl mevalonic acid by spectroscopic analysis. A ligand based virtual screening technique (Rocs \& EON) was used to screen I and II in drug like libraries, which provided similar structures [2]. For compound I, the most similar active ligand was EPIRUBICIN which is used to treat breast cancers [3]. ROCS TanimotoCombo was 0.665. EON ET combo was 0.911. For compound II, the most similar active ligand was VISMODEGIB, which is used to treat basal-cell carcinoma [3]. ROCS TanimotoCombo was 0.794. EON ET combo was 0.85 . MTT cytotoxicity assay was carried out to assess cytotoxicity of I and II on HeLa cell line, which revealed moderate activity. In Figure A "I" $(100 \mu \mathrm{M})$ is cytotoxic to HeLa cells by more than $40 \%(p<0.0001)$. As shown in Figure B "II" $(100 \mu \mathrm{M})$ is cytotoxic to HeLa cells by $65 \%(p<0.0001)$, whereas $P$. lacerum $\mathrm{MeOH}$ extract exhibited more cytotoxicity with $17 \%$ cell viability at $100 \mu \mathrm{g} / \mathrm{mL}(p<0.0001)$. Cisplatin $30 \mu \mathrm{M}$ was used as a positive control.
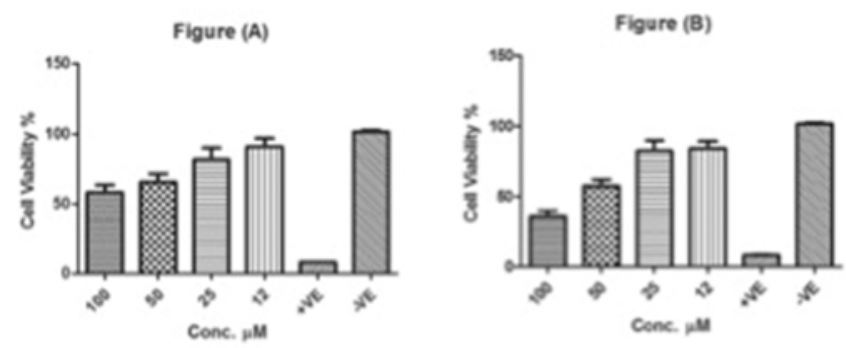

Fig. 1

[1] Mukemre M, Behcet L, Cakilcioglu U. J Ethnopharmacol 2015; 166:361 74.

[2] Hawkins PC, Skillman AG, Nicholls A. J Med Chem. 2007; 50:74-82.

[3] Law V, Knox C, Djoumbou Y, Jewison T, Guo AC, Liu Y. Nucleic Acids Research. 2014; 42 (Database issue): D 1091-D 7.
Mo-Poster Session 1-PO-105 Bioactive potential and role of secondary metabolites within the microorganism community of the sea grass Posidonia oceanica

Authors Alfattani $\mathrm{A}^{1}$, Blanchet $\mathrm{E}^{2}$, Oliveira Da Silva $\mathrm{I}^{2}$, Leoni $\mathrm{S}^{3}$, Allard $\mathrm{PM}^{1}$, Queiroz $E^{1}$, Roy $\mathrm{M}^{4}$, Chave $\mathrm{J}^{4}$, Lami $\mathrm{R}^{2}$, Perron $\mathrm{K}^{3}$, Stien $\mathrm{D}^{2}$, Wolfender $\mathrm{JL}^{1}$ Institute 1 School of Pharmaceutical Sciences, EPGL, University of Geneva, University of Lausanne, Geneva, Switzerland, Geneva, Switzerland; 2 Sorbonne Universités, UPMC Univ Paris 06, CNRS, Laboratoire de Biodiversité et Biotechnologies Microbiennes (LBBM), Observatoire Océanologique, Banyulssur-Mer, France, Banyuls-sur-Mer, France; 3 Microbiology Unit, Department of Botany and Plant Biology, University of Geneva, CH-1211 Geneva, Switzerland, Geneva, Switzerland; 4 Université Toulouse 3 Paul Sabatier, CNRS, ENFA, UMR5174 EDB (Laboratoire Évolution \& Diversité Biologique), 118 route de Narbonne, 31062 Toulouse, France, Toulouse, France

DOI 10.1055/s-0037-1608154

Posidonia oceanica a dominant herb of marine grassland from the Mediterranean has been selected for this project as a unique model system for studying its microbiota. This species is known for its longevity and is potentially the host for a large endophyte community (1). The aim of the project was to comprehensively profile the endophyte community of this peculiar marine plant, use it as a model for deciphering microbiome interactions and investigate if this can inspire the discovery of new antibacterial agents.

The diversity of endophytic microbial communities from leaves and rhizomes was assessed by high throughput sequencing approaches and 23 strains of fungi and bacteria were isolated and identified. These strains were cultivated and extracted by ethyl acetate for metabolite profiling and antimicrobial activity screening.

All samples were dereplicated by UHPLC-HRMS/MS and molecular networking for a preliminary survey of their secondary metabolite composition. Surprisingly some of the screened species presented unexpected high amounts of fatty acids. Among all fungal strains Lulwoana sp. were found very specific to the marine environment. Their metabolite profiles reveal the presence of original corymbiferan lactones and their targeted isolation reveal the presence of a new methylated derivative. Extensive profiling in different cultivation media also demonstrated the diversity of such compounds in these endophytes.

In parallel the extracts were screened for their antimicrobial activity against Staphylococcus aureus, Propionibacterium acnes and Pseudomonas aeruginosa. Penicillium and Fusarium spp. strains were found to display interesting antimicrobial activities and a correlation between their chemical profiles and activities will be presented.

All the results obtained provide a first survey of chemical and bioactivity profiles in view of studying interactions between the members of this unique community.

This work has benefited from a SECIL grant from ANR (15-CE21 - 0016) and SNF (310030E-164289/1).

Vohník M, et al. Mycorrhiza. 2015;25:663- 72.

\section{Mo-Poster Session 1-PO-106 HPLC-based activity profiling for GABAA receptor modulators from Homalomena occulta rhizomes using zebrafish larval locomotor activity assay}

Authors Akaberi $\mathrm{M}^{1,2}$, Moradi-Afrapoli $\mathrm{F}^{2}$, Danton $\mathrm{O}^{2}$, Hamburger $\mathrm{M}^{2}$ Institute 1 Department of Pharmacognosy, Faculty of Pharmacy, Mashhad University of Medical Sciences, Mashhad, Iran; 2 Division of Pharmaceutical Biology, Department of Pharmaceutical Sciences, University of Basel, Basel, Switzerland

DOI $10.1055 / \mathrm{s}-0037-1608155$ 
Gamma-aminobutyric acid type $\mathrm{A}\left(\mathrm{GABA}_{\mathrm{A}}\right)$ receptors are the major inhibitory neurotransmitter receptors in the central nervous system, and a key target for numerous clinically important drugs used to treat anxiety, insomnia and epilepsy. A dichloromethane extract from rhizomes of Homalomena occulta (Lour.) Schott (Araceae) potentiated GABA-induced chloride currents in a two-microelectrode voltage clamp assay with Xenopus laevis oocytes expressing human $G A B A_{A}$ receptors. The same extract was tested in a zebrafish larval locomotor activity assay in which larval locomotion is provoked with the $\mathrm{GABA}_{\mathrm{A}}$ receptor antagonist pentylenetetrazol (PTZ) [1]. A significant lowering of PTZ-provoked locomotion was observed in larvae pretreated with the extract for $3 \mathrm{~h}$. GABAergic activity in the extract was tracked by HPLC-based activity profiling. Reduced larval locomotion was observed with the microfraction collected between 24-27 min (A). Compounds in this time window were purified by a combination of normal and reverse phase chromatography, and their structures were established by 1D and 2D NMR and HRMS. Compounds were identified as 3-substituted phenols bearing alkyl/alkenyl chains or benzoalkyl/benzo-alkenyl moieties as represented in 1 and 2 , respectively (B).

A

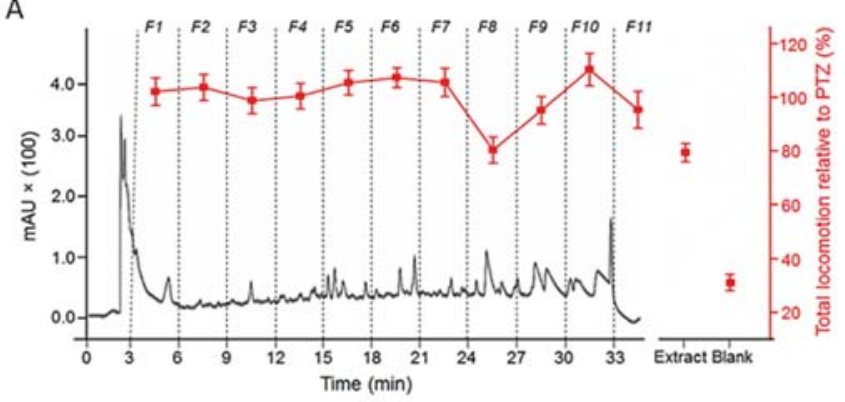

B<smiles>[R]CCCc1cccc(O)c1</smiles>

1

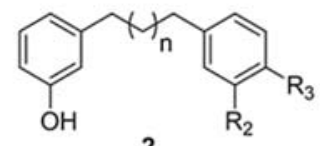

2

Fig. 1

[1] Moradi-Afrapoli F, Ebrahimi SN, Smiesko M, Hamburger M. J Nat Prod 2017; In press, doi: 10.1021 /acs.jnatprod.7b00081.

\section{Mo-Poster Session 1-PO-107 The putative PAINs nostotrebin 6 and derivatives from Nostoc sp. inhibit the trypanosomal cysteine protease rhodesain}

Authors Kossack $R^{1}$, Breinlinger $S^{1}$, Nguyen $T^{2}$, Schirmeister $T^{3}$, Enke $H^{4}$, Niedermeyer $\mathrm{T}^{1}$

Institute 1 Institute of Pharmacy, RG Biogenic Drugs, Martin-LutherUniversität Halle-Wittenberg, Halle (Saale), Germany; 2 Interfaculty Institute of Microbiology and Infection Medicine, Eberhard Karls Universität Tübingen, Tübingen, Germany; 3 Institute of Pharmacy und Biochemistry, Johannes Gutenberg-Universität Mainz, Mainz, Germany; 4 Cyano Biotech GmbH, Berlin, Germany

DOI 10.1055/s-0037-1608156

The trypanosomal cysteine protease rhodesain plays a major role during parasitic infection by Trypanosoma brucei, known as human African trypanosomiasis. The enzyme is regarded as a promising target for new drugs [1]. Thus, a collection of about 670 cyanobacteria extracts was screened for inhibitory activity against rhodesain using an established assay. The screening revealed 21 media extracts with an inhibition higher than $80 \%$ at $0.2 \mathrm{mg} / \mathrm{mL}$. The most promising extract from a Nostoc sp. strain was fractionated using flash and high-performance liquid chromatography, resulting in the isolation of 14 inhibitory compounds. Structure elucidation by nuclear magnetic resonance spectroscopy and high-resolution mass spectrometry revealed one of the compounds to be Nostotrebin 6 [2] while the others are new intriguing struc- turally related compounds composed of two basic building blocks that form monomeric, dimeric, or trimeric structures. Nostotrebin 6 acts as an inhibitor of acetylcholinesterase (IC50 $6 \mu \mathrm{M}$ ) and butyrylcholinesterase (IC50 $7 \mu \mathrm{M})$ [2] and exhibited further bioactivity; e.g. cytotoxicity and pro-apoptotic activity [3]. Due to its broad bioactivity spectrum and as, to our knowledge, no structure-activity relationship has been established so far, we assume Nostotrebin 6 , a polyphenolic compound, to be a pan-assay interference (PAIN) compound.

[1] Ettari R, Tamborini L, Angelo IC, Micale N, Pinto A, Micheli C de, Conti P. The Journal of Medicinal Chemistry 2013; 56: 5637 - 5658

[2] Zelik P, Lukesova A, Cejka J, Budesinsky M, Havlicek V, Cegan A, Kopecky J. J Enzyme Inhib Med Chem 2010; 25: 414-420

[3] Vacek J, Hrbáč J, Kopecký J, Vostálová J. Molecules 2011; 16: 4254-4263

\section{Mo-Poster Session 1-PO-108 Neolignans from leaves of Nectandra leucantha (Lauraceae) display in vitro antitrypanosomal activity via plasma membrane and mitochondrial damages}

Authors Lago J ${ }^{1}$, Grecco $\mathrm{S}^{1}$, Costa-Silva $\mathrm{T}^{1}$, Sousa $\mathrm{F}^{2}$, Tcacenco $\mathrm{C}^{3}$,

Andrade $C^{5}$, Sartorelli $P^{2}$, Tempone $A^{4}$

Institute 1 Federal University of ABC, Santo Andre, Brazil; 2 Federal

University of Sao Paulo, Diadema, Brazil; 3 Maua Institute of Technology, Sao Caetano do Sul, Brazil; 4 Adolfo Lutz Institute, Sao Paulo, Brazil; 5 Federal University of Goias, Goiania, Brazil

DOI $10.1055 / \mathrm{s}-0037-1608157$

Natural products isolated from plants are considered an important source of prototypes for the development of new drugs against tropical neglected protozoan diseases [1]. As a continuation of our previous studies with Brazilian plants with antitrypanosomal activity [2], the n-hexane extract from leaves of Nectandra leucantha (Lauraceae) displayed in vitro activity against T. cruzi. This extract was analyzed by HPLC/HRESIMS and subjected to a bioactivity-guided fractionation to afford four related neolignans: dehydrodieugenol B (1), 1-(8propenyl)-3-[3'-methoxy-1'-(8-propenyl)-phenoxy]-4,5-dimethoxybenzene (2), 1-[(7S)-hydroxy-8-propenyl]-3-[3'-methoxy-1'-(8'-propenyl)-phenoxy]-4hydroxy-5-methoxybenzene (3), and 1-[(7S)-hydroxy-8-propenyl]-3-[3'-methoxy-1'-(8'-propenyl)-phenoxy]-4,5-dimethoxybenzene (4). Chemical structures were determined by analysis of NMR, IR, UV and HRESIMS data. Isolated compounds were tested against intracellular amastigotes and extracellular trypomastigotes of $T$. cruzi and for mammalian cytotoxicity. Neolignan 4 showed the higher selectivity index $(\mathrm{SI})$ against trypomastigotes $(>5)$ and amastigotes (>13) of $T$. cruzi. The investigation of the mechanism of action demonstrated that neolignan 4 caused substantial alteration of the plasma membrane permeability, together with mitochondrial dysfunctions in trypomastigote forms. In silico studies of pharmacokinetics and toxicity (ADMET) properties predicted that all compounds were non-mutagenic, non-carcinogenic, non-genotoxic, weak hERG blockers, with acceptable volume of distribution $(1.66-3.32 \mathrm{~L} / \mathrm{kg})$, and low rodent oral toxicity $\left(\mathrm{LD}_{50} 810-2,200 \mathrm{mg} /\right.$ $\mathrm{kg})$. Considering some clinical events of cerebral Chagas disease, compounds 1 - 4 also demonstrated favorable properties, such as blood-brain barrier penetration. Unfavorable properties were also predicted as high promiscuity for P450 isoforms, high plasma protein binding affinity (> 91\%), and moderate-tolow oral bioavailability. Finally, none of the compounds was predicted as interference compounds (PAINS). Considering the promising chemical and biological properties of the isolated neolignans, these compounds could be used as starting points to develop new lead compounds for Chagas disease. [1] Newman DJ, Cragg GM. J Nat Prod 2016; 79: 629-661.

[2] Grecco SS, Costa-Silva TA, Jerz G et al. Phytomedicine 2017; 15: 62 - 67. 


\section{Mo-Poster Session 1-PO-109 Terpene ester derivatives of the roots of Ferula hezarlalehzarica}

Authors Alilou $\mathrm{M}^{1,3}$, Schwaiger $\mathrm{S}^{1}$, Khoddami $\mathrm{M}^{2}$, Troppmair $\mathrm{J}^{3}$, Stuppner $\mathrm{H}^{1}$ Institute 1 Institute of Pharmacy/Department of Pharmacognosy, University of Innsbruck, Center for Molecular Biosciences Innsbruck, Innrain 80 - 82, Innsbruck, Austria; 2 Herbal And Traditional Medicines Research center, kerman University of Medical Sciences, Kerman, Iran; 3 Daniel Swarovski Research Laboratory Department of Visceral, Transplant and Thoracic Surgery, Innsbruck Medical University, Innsbruck, Austria

DOI 10.1055/s-0037-1608158

The genus Ferula (Apiaceae) comprises about 180 species spreading throughout the world and some species are used in folk medicine of different countries for treatment of stomachache, hysteria and others. In Iranian flora, genus Ferula is represented by 30 species including 15 endemic representatives [1]. In Iranian traditional medicine Ferula species are highly appreciated e.g. the well-known representative $F$. assa-foetida of which the gum has been used as a remedy for abdominal pain, constipation, diarrhea, spasms and others. Ferula hezarlalehzarica Ajani, identified in 2008 [2], is an endemic species growing in Kerman province, Iran. This plant is used by the local people for the treatment of gastrointestinal disorders.

Phytochemical investigation of the dichloromethane extract of the roots of $F$. hezarlalehzarica resulted in the isolation of thirteen compounds. One daucane sesquiterpene and one fenchol derivatives were identified as new compounds, 6-hydroxy-1,3,3-trimethylbicyclo[2.2.1] heptan-2-yl 4-hydroxybenzoate (1) and 1,8-dihydroxy-1-isopropyl-3a,6-dimethyl-1,2,3,3a,4,5,8,8a-octahydroazulen-5-yl 4-hydroxybenzoate (2). The two remaining compounds were identified as known borneol derivatives.

An initial pharmacological investigation of the isolated compounds in a colorimetric assay (MTT) in MDA-MB-231 cells revealed two active compounds, the p-hydroxy benzoic acid as well as the 3-methoxy-4-hydroxy benzoic acid analog of ferutinol, which showed identical $I C_{50}$ values of $42 \mu \mathrm{M}$ after $24 \mathrm{~h}$ incubation.

[1] Sattar Z, Iranshahi M. Iran J Basic Med Sci. 2017; 20: 1 - 8.

[2] Ajani A, Ajani M. Edinb. J. Bot. 2008; 65: 425 - 431.

\section{Mo-Poster Session 1-PO-110 Pancreatic Lipase Inhibitors from Roselle - Natural Obesity Treatment}

Authors Buchholz $\mathrm{T}^{1}$, Görick $\mathrm{C}^{1}$, Wolber $\mathrm{G}^{2}$, Melzig $\mathrm{MF}^{1}$ Institute 1 Department of Pharmaceutical Biology, Freie Universitaet Berlin, 14195 Berlin, Germany; 2 Department of Medicinal and Pharmaceutical Chemistry, Freie Universitaet Berlin, 14195 Berlin, Germany

DOI 10.1055/s-0037-1608159

Overweight and obesity are leading risk factors for a number of chronic diseases such as diabetes mellitus type II [1,2]. Due to rapid lifestyle changes, their prevalence has increased in recent years [3].

In order to find new, plant based pancreatic lipase ( $\mathrm{PL}$, pancreatic triacylglycerol lipase (EC: 3.1.1.3) inhibitors for the treatment of obesity, in vitro tests and in silico docking experiments were carried out. Initially, methanolic and aqueous extracts of 23 medicinal plants with weight-reducing, serum glucosereducing, or related potential were investigated for anti-lipase activity in vitro. Our findings have shown that both the aqueous and methanolic extract of Hibiscus sabdariffa $\mathrm{L}$. (Malvaceae) showed a very strong inhibition $\left(\mathrm{IC}_{50}: 35.8\right.$ $\pm 0.8 \mu \mathrm{g} / \mathrm{mL}$ ) of the enzyme. Subsequently, the methanolic extract of the plant was analyzed by HPLC and ESI-Q-TOF-MS for isolation and identification of inhibitory active compounds. Hibiscus acid and hibiscus acid glucoside were identified by comparison with literature data. In silico docking experiments of hibiscus acid to the crystal structure of porcine pancreatic lipase (PDB code: $1 \mathrm{ETH})$ showed strong interactions with the active site of the enzyme.
[1] Kopelman PG. Nature 2000; 404:635 - 643

[2] Trigueros L, Pena S, Ugidos AV, Sayas-Barbera E, Perez-Alvarez JA, Sendra EJ. Food Sci. Nutr. 2013; 53: $929-942$

[3] World Health Organization. 2015; Factsheet 311

\section{Mo-Poster Session 1-PO-111 Anti-diabetic Activity of Oscarellin Isolated from Oscarella stillans in Zebrafish, a Model of Type 2 Diabetes}

Authors Jeon $\mathrm{Y}^{1}$, Nam Youn $\mathrm{H}^{2}$, Kang Tong $\mathrm{H}^{2}$, Schmitz Fj ${ }^{3}$, Kwak Jong $\mathrm{H}^{1}$ Institute 1 School of Pharmacy, Sungkyunkwan University, Suwon, Korea, Republic of (South); 2 Department of Oriental Medicine Biotechnology,

College of Life Sciences, Kyung Hee University, Gyeonggi-do, Korea, Republic of (South); 3 Department of Chemistry and Biochemistry, University of Oklahoma, Norman, OK, United States

DOI $10.1055 / \mathrm{s}-0037-1608160$

A new anthranilic acid derivative (1) was isolated from a Philippine sponge, Oscarella stillans (family Plakinidae. phylum Porifera, class Demospongiae, order Homosclerophorida) [1]. The structure of compound 1, named oscarellin, was determined as 2-amino-3-(3'-aminopropoxy)benzoic acid from spectral data, and confirmed by synthesis. We examined the anti-diabetic activity of compound 1 in zebrafish model for type 2 diabetes. The zebrafish model for type 2 diabetes was induced by exposure to excess insulin. Following exposure of excess insulin, the pancreatic islet size and fluorescence intensity were measured [2]. When compared to the insulin-treated group, the pancreatic islet size in oscarellin (1)-treated group was significantly increased at concentrations of 1 and $5 \mu \mathrm{M}$. Glucose uptake was evaluated in zebrafish treated with compound 1 by detecting the uptake of 2-NBDG fluorescence within the pancreatic islets. Oscarellin (1)-treated group revealed significantly increased glucose uptake at concentration of $5 \mu \mathrm{M}$. Compound 1 led to the recovery of pancreatic islet size and glucose uptake. In conclusion, oscarellin (1) possessed anti-diabetic activity for type 2 .

This work was supported by a grant from Marine Biotechnology Program (PJT200620, Genome Analysis of Marine Organisms and Development of Functional Applications) funded by Ministry of Oceans and Fisheries.

[1] Kwon I-S, Kwak JH, Pyo S, Lee H-W, Kim A, Schmitz F]. Oscarellin, J Nat Prod 2017; 80: 149-155

[2] Yang X, Mei S, Gu H, Guo H, Zha L, Cai J, Li X, Liu Z, Cao W. J Endocrinol 2014; 221: $469-480$

\section{Mo-Poster Session 1-PO-112 Ameliorative Effect of Methyl 2-(4'-Methoxy-4'-oxobutanamide) Benzoate on Insulin-induced Pancreatic Islet Damage in Zebrafish}

Authors Jeon $\mathrm{Y}^{1}$, Nam Youn $\mathrm{H}^{2}$, Kang Tong $\mathrm{H}^{2}$, Kwak Jong $\mathrm{H}^{1}$ Institute 1 School of Pharmacy, Sungkyunkwan University, Suwon, Korea, Republic of (South); 2 Department of Oriental Medicine Biotechnology, College of Life Sciences, Kyung Hee University, Gyeonggi-do, Korea, Republic of (South)

DOI 10.1055/s-0037-1608161

Methyl 2-(4'-methoxy-4'-oxobutanamide) benzoate (1) was isolated from $\mathrm{He}$ lianthus tuberosus and identified from its spectroscopic data. Compound 1 was previously reported from a dark brown alga, Jolyna laminarioides [1]. In previous biological investigations, compound 1 has been reported to exhibit inhibitory effect on chymotrypsin, and antibacterial and anti-inflammatory activities [2]. The zebrafish model for type 2 diabetes was induced by exposure to excess insulin. Following exposure of excess insulin, the pancreatic islet size and fluorescence intensity were measured [3]. Glucose uptake was evaluated in zebrafish by detecting the uptake of 2-NBDG fluorescence within the pancreatic islets. We evaluated the recovery effect of compound 1 on insulininduced pancreatic islet damage in zebrafish. Pancreatic islet size of the compound-treated group was significantly increased. Compound 1 treatment led 
to a great increase in pancreatic islet size at concentrations of 0.05 and $0.1 \mu \mathrm{M}$. Glucose uptake of the compound-treated group also was significantly higher compared to the normal group at concentrations of 0.05 and $0.1 \mu \mathrm{M}$. In conclusion, compound 1 revealed potent anti-diabetic activity for type 2 .

[1] Choudhary MI, Majeed A, Shabbir M, Ghani U, Shameel M. Phytochemistry 1997; 46: $1215-1218$.

[2] Jung Y], Kim BO, Kwak JH, Pyo S. J Agric Food Chem 2016; 64: 9317 - 9325 [3] Yang X, Mei S, Gu H, Guo H, Zha L, Cai J, Li X, Liu Z, Cao W. J Endocrinol 2014; 221: $469-480$

\section{Mo-Poster Session 1-PO-113 A New Anthranilic Acid Derivative from Oscarella stillans as Regulator of Inflammatory Cytokines in Macrophages}

Authors Kwon $\mathrm{I}^{1}$, Kwak Jong Hwan $\mathrm{K}^{1}$, Pyo $\mathrm{S}^{1}$, Kim AR², Schmitz FJ ${ }^{2}$ Institute 1 School of Pharmacy, Sungkyunkwan University, Suwon, Korea, Republic of (South); 2 Department of Chemistry and Biochemistry, University of Oklahoma, Norman, OK, United States

DOI 10.1055/s-0037-1608162

A new anthranilic acid derivative (1) was isolated from a Philippine sponge, Oscarella stillans (family Plakinidae. phylum Porifera, class Demospongiae, order Homosclerophorida) [1]. The structure of compound 1, designated oscarellin, was determined as 2-amino-3-(3'-aminopropoxy)benzoic acid from spectral data, and confirmed by synthesis. We examined the immunomodulating effect of compound 1 and its mechanism in lipopolysaccharide (LPS)-stimulated RAW 264.7 macrophages. LPSs, a bacterial endotoxin, induce the production of inflammatory mediators such as iNOS, COX-2, TNF- $\alpha$, IL-1, and IL-6 in macrophages [2]. Our data indicated that the expression of tumor necrosis factor- $\alpha$ (TNF- $\alpha$ ) and interleukin (IL)- 6 were significantly reduced by the pretreatment of compound $1(0.1-10 \mu \mathrm{M})$ for $2 \mathrm{~h}$. In addition, oscarellin (1) suppressed activation of c-Jun NH2-termimal kinase (JNK) and extracellular signal-regulated kinase $1 / 2$ (ERK1/2), but not p38 mitogen-activated protein kinase (MAPK) in LPS-stimulated RAW 264.7 cells. Compound 1 abrogated LPS-induced nuclear factor-KB (NF-KB) and activator protein-1 (AP-1) activities, whereas the induction of activating transcription factor-3 (ATF-3) was increased. Taken together, our results suggest that oscarellin (1) attenuates pro-inflammatory cytokines through the inhibition of JNK, ERK, AP-1, NF-KB and the activation of ATF- 3 signaling pathway. In conclusion, the present data suggest that oscarellin (1) is a potential therapeutic agent to treat inflammatory-related diseases.

This work was supported by a grant from Marine Biotechnology Program (PJT200620, Genome Analysis of Marine Organisms and Development of Functional Applications) funded by Ministry of Oceans and Fisheries.

[1] Bergquist PR, Kelly M. New Zeal J Mar Fresh 2004; 38: 51 - 66

[2] Hinz B, Brune K. J Pharmacol Exp Ther 2002; 300: 367 - 375

\section{Mo-Poster Session 1-PO-114 Looking for specific natural inhibitors targeting aberrant proliferation signaling in melanoma}

Authors Garo $\mathrm{E}^{1}$, Dobrzyński M², Rossberg $\mathrm{F}^{1}$, Fertig O $\mathrm{O}^{1}$, Pertz $\mathrm{O}^{2}$, Hamburger $\mathrm{M}^{1}$

Institute 1 Department of Pharmaceutical Sciences, University of Basel, Basel, Switzerland; 2 Institute of Cell Biology, University of Bern, Bern, Switzerland DOI 10.1055/s-0037-1608163

The incidence of melanoma, the most fatal dermatological cancer, is dramatically rising. In more than $50 \%$ of malignant melanomas the BRAF V600E mutation is present, leading to aberrant MAPK signaling and uncontrolled proliferation. Vemurafenib, a specific inhibitor of the V600E mutated form of B-Raf, has been approved in 2011 for the treatment of metastatic melanomas. Despite spectacular initial results, patients treated with vemurafenib relapse within 2-18 months due to drug resistance. Studies on the mechanism of drug resistance suggest that the development of combination therapies is needed to address this problem. Novel inhibitors targeting aberrant proliferation signaling (MAPK and PI3K pathways) in melanoma are therefore urgently needed.

To identify novel compounds for targeted therapy we combined our natural product lead discovery platform with an innovative high-content screening (HCS) assay that quantifies MAPK/PI3K signaling in A2058 melanoma cells. The HCS assay was first validated with known specific inhibitors, prior to its use for an initial screening of 88 plant extracts. An EtOAc extract from leaves of Casearia arborea was found to inhibit both the MAPK and PI3K pathways in A2058 cells. HPLC-based activity profiling combined with an efficient dereplication strategy identified Cucurbitacins Q (1) and B (2) to be responsible for the activity in the extract. These results were obtained in analytical scale using less than $5 \mathrm{mg}$ of extract. A scale-up isolation will allow for the full structural assignment of the compounds, and for further characterization of mechanisms of action. The current results provide a proof of concept for our approach that is now used for the screening and profiling of a library of $>800$ EtOAc extracts.

\section{Mo-Poster Session 1-PO-115 Pro-convulsant alkenyl amides from Piper nigrum fruits identified with a zebrafish larvae locomotor activity model}

Authors Syafni $\mathrm{N}^{1}$, Moradi-Afrapoli $\mathrm{F}^{1}$, Danton $\mathrm{O}^{1}$, Hamburger $\mathrm{M}^{1}$

Institute 1 Department of Pharmaceutical Sciences, University of Basel, Basel, Switzerland

DOI 10.1055/s-0037-1608164

A petroleum ether extract of Piper nigrum (Piperaceae) fruits was previously found to potentiate GABA induced chloride currents in a two-microelectrode voltage clamp assay using Xenopus laevis oocytes transiently expressing human $G A B A_{A}$ receptors, and piperine was identified as a positive $G A B A_{A}$ receptor modulator interacting at a benzodiazepine-independent allosteric bindingsite [1]. When testing the same extract in a zebrafish-larvae locomotor activity assay [2], it significantly increased larval locomotion provoked by the $G A B A_{A}$ receptor antagonist pentylenetetrazol (PTZ). Pro-convulsant activity in the extract was tracked by HPLC-based activity profiling. Increased larval locomotion was observed in two distinct micro-fractions, while the fraction containing piperine (1) decreased PTZ provoked locomotion. The constituents responsible for the pro-convulsant effect of the extract were purified, and their structures established by 1D and 2D NMR and MS. Geometry of double bonds was resolved by 2D JRES spectra. The pro-convulsant amides were identified as $2 E, 4 E$-alkenyl amides with a common structure feature as shown in 2 . In the zebrafish larval locomotor assay, the alkenyl amides increased PTZ provoked larval convulsions in a concentration-dependent manner (120 to $130 \%,<6$ $\mathrm{mM}$ ) when compared to the PTZ-only group.

[1]. Zaugg J, Barburin I, Strommer B, Kim H-J, Hering S, Hambueger M. J. Nat. Prod 2010; 73: 185 - 191.

[2].Moradi-Afrapoli F, Ebrahimi SN, Smiesko M, Hamburger M. J Nat Prod 2017, advance online publication 9 May 2017; doi:10.1021/acs.jnatprod.7b00081.

\section{Mo-Poster Session 1-PO-116 Anti-psoriatic Effects of Wannachawee Recipe on TNF- $\alpha$ - and IFN- $\gamma$-Induced Inflammatory Cytokine production in $\mathrm{HaCaT}$ Human Keratinocytes}

Authors Na Takuathung $M^{1,4}$, Wongnoppavich $A^{2}$, Panthong $A^{1}$, Khonsung $\mathrm{P}^{1}$, Chiranthanut $\mathrm{N}^{1}$, Soonthornchareonnon $\mathrm{N}^{3}$, Sireeratawong $\mathrm{S}^{1}$ Institute 1 Department of Pharmacology, Faculty of Medicine, Chiang Mai University, Chiang Mai, Thailand; 2 Department of Biochemistry, Faculty of Medicine, Chiang Mai University, Chiang Mai, Thailand; 3 Department of Pharmacognosy, Faculty of Pharmacy, Mahidol University, Bangkok, Thailand; 4 Department of Pharmacology (Doctor of Philosophy Program in 
Pharmacology), Faculty of Medicine and Graduate School, Chiang Mai

University, Chiang Mai, Thailand

DOI 10.1055/s-0037-1608165

Psoriasis is a common immune-mediated chronic inflammatory skin disease characterised by thick, erythema and scales. The psoriasis pathogenesis involves T cells activated via the IL-23/Th 17 axis. Conventional treatments of psoriasis have adverse events that influence patients' adherence. Wannachawee Recipe (WCR) is known to be an effective folk remedy for psoriasis patients, however, preclinical evidence defining how WCR works is still lacking. This study investigated specific mechanisms underlying the therapeutic potential of WCR on anti-proliferation and anti-inflammatory effects in HaCaT human keratinocytes.

An anti-proliferative activity of WCR was measured by using SRB and WST-8 assays. The distribution of cell cycle phases was determined by cell cycle analysis. The TNF- $\alpha$ and IFN- $\gamma$ induced mRNA expression of anti-inflammatory cytokines was detected by qRT-PCR, whereas the protein production of IL-17, IL-22 and IL-23 was confirmed by ELISA.

WCR exhibited a significant anti-proliferative effect $\left(I_{50} 600 \mu \mathrm{g} / \mathrm{mL}\right)$. Three non-toxic concentrations of WCR $(25,50$ and $75 \mu \mathrm{g} / \mathrm{mL})$ and acitretin had no effect on the induction of cell cycle arrest, while methotrexate significantly induced S-phase arrest. The expression of IL-1b, IL-6, IL-8, IL-17, IL-22, IL-23 and TNF- $\alpha$ was dramatically decreased by acitretin and WCR treatment in TNF$\alpha$ and IFN- $\gamma$-induced $\mathrm{HaCaT}$ cells. At the protein level, the treatment with $100 \mu \mathrm{g} / \mathrm{mL}$ of $\mathrm{WCR}$ and $6.5 \mu \mathrm{g} / \mathrm{mL}$ of acitretin significantly reduced the TNF$\alpha$ and IFN- $\gamma$-stimulated cytokine production compared with only TNF- $\alpha$ and IFN- $\gamma$ treatment group $(p<0.05)$.

WCR at low concentration $(<100 \mu \mathrm{g} / \mathrm{mL})$ was proved to possess potent antiinflammatory effects without toxicity or cell cycle arrest on the keratinocyte cells. This makes WCR a promising candidate for further investigation and development for psoriasis therapy. More sophisticated studies need to be verified to evaluate the precise mechanisms of action in relevant animal models to be certain that WCR is safe and effective for use in psoriasis patients.

\section{Mo-Poster Session 1-PO-117 Effect of fermented ginseng berry extract, enriched in Ginsenoside Rg2 on suppressing melanin and whitening activity in HDF and B16BL 6}

Authors $\mathrm{Yi}^{1} \mathrm{E}^{1}$

Institute 1 Gyeonggi-do Agricultural Research \& Extension Services, Yeoncheon, Korea, Republic of (South)

DOI 10.1055/s-0037-1608166

This study was carried out to determine the time of fruit harvest which does not affect the growth of 4 years and 5 years ginseng roots for the commercialization of fruit as a by - product of ginseng. Saponin content, total phenolic compound, acid polysaccharide content and antioxidant effect were determined in each sample during the fruit ripening process. The saponin content in berry of 4 years was relatively high at DAF 14 and 5 years specimens were at DAF 7. The most abundant ginsenoside Re in berry was converted into ginsenoside $\mathrm{Rg} 2$ by an enzyme bgp1 via mass production of recombinant enzyme bgp1. After bioconversion, the metabolites were checked for their cytotoxicity evaluation in human dermal fibroblast and murine melanoma B16BL 6 skin cell lines. In vitro cell viability assay has shown that it was non-toxic to human dermal fibroblast (HDF) and murine melanoma B16BL6 (B16) cell lines. However, fermented green ginseng berry (100 ug/ $\mathrm{ml}$ ) was found to be toxic to B16. The present results clearly suggested that the bio converted green ginseng berry was found to be more effective in suppressing melanin content and enhancing whitening activity in HDF and BL6BL 1.

[1] Md. Amdadul Huq a, Yeon-ju Kimb, Fayeza Md. Siraj a, Deok-Chun Yang a Biotechnology and Applied Biochemistry 2016;63:532 - 538.
[2] Yan Jin, Yeon-Ju Kim*, Ji-Na Jeon, Chao Wang, Jin-Woo Min, Hae-Yong Noh, Deok-Chun Yang* Plant Foods for Human Nutrition.2015;70:141 - 145.

\section{Mo-Poster Session 1-PO-118 Turkish medicinal and aromatic plants for the treatment of skin diseases both human and animals}

Authors Saltan Fatma $Z^{1}$

Institute 1 Anadolu University, Faculty of Pharmacy, Department of Pharmacognosy, Eskisehir, Turkey

DOI 10.1055/s-0037-1608167

Medicinal and aromatic plants used in traditional medicine to cure skin disorders in different provinces of Turkey have been updated by scanning 60 literatures based on their medicinal importance. This study determined the use of 61 families and 142 plant taxa in folk medicine. Leaves (as powdered, poultice or decoction) were the most widely used for wound, haemorrhoids, callus, abscess, hair loss, eczema, wart, psoriasis and cellulite in human and foot health as antimycotic in animals. Secondly, flowers (as infusion, essential oil or mixture with olive oil or combination with other plants) are taken for acne, wounds, rash or anti-inflammatory in human and antiparasitic on skin's animals. Roots (boiled, dried or powdered, mixture with barks) are applied for fast wound closure and treatment of burn in human while anti-inflammatory in animals. In addition to these, seeds are generally used (as essential oil, gel, extract, with a root or fruit) for hair loss, antibacterial and antifungal in human [1]. Essential oils are mostly antiviral (HSV-2 etc) in human or antiseptic for animals in medical uses. Moreover, hesperidin is important against skin stains but gallic acid and glabridin have antithyrosinase effect and arbutin is antiseptic to people while linalool, 1,8-cineole, carvacrol, limonene are allergic or irritant in human skin, [2-3]. As a conclusion, conscious use of herbs for dermatological diseases and their clinical or veterinary studies on human or animal should be enhanced through the public education.

[1]Tuzlacı E, Şifa Niyetine. Türkiye'nin Bitkisel Halk İlaçları, İstanbul: Alfa Yayınları; 2006: 15 - 400 .

[2] Başer KHC, Franz C, Handbook of Essential Oils. Science, Technology and Applications, USA: CRC Press; 2010: 881 - 894.

[3] Burlando B, Verotta L, Cornara L, Bottini-Massa E, Botanical Compounds and Their Dermatologic and Cosmetic Uses, USA: CRC Press; 2010: 9 - 27.

\section{Mo-Poster Session 1-PO-119 Phytochemistry and cosmetic potential of plants traditionally used for skincare and beauty in South Africa}

Authors Thibane Vuyisile $\mathrm{S}^{1,2}$, Abdelgadir $\mathrm{HA}^{2}$, Finnie $\mathrm{JF}^{1}$, Ndhlala Ashwell $\mathrm{R}^{2}$, Van Staden J ${ }^{1}$

Institute 1 Research Center for Plant Growth and Development, School of Life Sciences, University of KwaZulu-Natal Pietermaritzburg, Private Bag X01, 3209, Scottsvile, South Africa; 2 Agricultural Research Council, Vegetable and Ornamental Plant, Private Bag X293, 0001, Pretoria, South Africa DOI 10.1055/s-0037-1608168

The prolonged exposure of the skin to sun radiation can have undesired consequences, resulting in skin damage and darkening [1]. Plant extracts that can offer protection to the skin are important in skin rehabilitation. Therefore, the aim of the study was to test plant extracts for their phytochemical and cosmetic potential. Extracts of 16 plants from the Eastern Cape Province, South Africa traditionally used for skincare and beauty were evaluated for their total phenolic content, flavonoid content, anti-tyrosinase activity and sun protection factor (SPF). Methanolic extracts from Ruta graveolens L, Aloe ferox Mill, Ilex mitis (L.) Radlk, Cassipourea flanaganii (Schinz) Alston and Rorippa nasturtium (L.) Hayek had high total phenolic content of $29.98(0.40), 37.88$ (0.19), 44.15 (0.14), 49.45 (0.21) and 53.86 (0.18) mg GAE/g, respectively. The flavonoid content was the highest for Plantago lanceolata $\mathrm{L}$ at 5.06 (0.14) $\mathrm{mg} \mathrm{CE} / \mathrm{g}$. The percentage inhibition of the tyrosinase enzyme ranged between $29-74 \%$ for all tested plant extracts. Importantly, cosmetic products 
are categorised according to their SPF values, with minimal protection being (SPF < 12), moderate protection (SPF $12-30$ ) and high protection (SPF > 30). Plant extracts from P. lanceolata, C. flanaganii, Acokanthera oblongifolia (Hochst), I. mitis, Arctotis arctotoides (L.F) O. Hoffm and A. ferox exhibited moderate protection against ultraviolet (UV) radiation with SPF values 22.19 (2.177), 20.80 (0.696), 20.293 (0.417), 19.67 (2.144), 17.61 (0.329) and 13.56 (0.967), respectively. A number of the tested plant extracts showed good potential for use in cosmetic formulation and their combined use can enhance their activity.

[1]. Lorencini M, Brohem C.A, Dieamant G.C, Zanchin N.I.T, Maibach H.I. Ageing Res Rev 2014; 15:100-115.

\section{Mo-Poster Session 1-PO-120 Antimicrobial efficacy of selected natural preservatives in a cosmetic emulsion}

Authors Kočevar Glavač $N^{1}$, Kreft $S^{1}$, Lunder $M^{1}$

Institute 1 Faculty of Pharmacy, University of Ljubljana, Ljubljana, Slovenia DOI 10.1055/s-0037-1608169

Microbiological quality of cosmetic products is a significant issue for the cosmetic industry in terms of the excessive contamination [1] and contact allergies caused by cosmetic preservatives [2]. The term 'natural preservative' has evoked great interest in recent years in consumers, marketing and research. It is not an officially defined term, but rather a reflection of a broader concept of the so-called natural cosmetics presented by different certification standards. As data on the antimicrobial efficacy of natural preservatives are scarce, the aim of the present study was to investigate 13 preservatives (11 accepted as natural and 2 conventional preservatives as controls) at 2 concentrations in an o/w cosmetic emulsion.

The total aerobic bacterial count and total combined yeast count (analysed according to the European Pharmacopoeia 8.0) were below the acceptable limit for all formulations. Challenge tests were performed according to the ISO 11930 standard. The criterion A was fulfilled by $p$-anisic, benzoic, dehydroacetic, salicylic and sorbic acid, dehydroacetic acid/benzyl alcohol, glyceryl caprylate and ethanol. Grapefruit seed extract was efficient only at a higher concentration, while levulinic acid and Lactobacillus ferment failed to protect the formulation. Phenoxyethanol and methylparaben/propylparaben were inefficient at minimum concentrations.

The results offer important comparative data on the level of antimicrobial protection within the group of natural preservatives and in reference to some typical, widely used conventional preservatives. Moreover, further research must be encouraged with regard to cosmetic over-preservation.

[1] Lundov MD, Zachariae C. Int J Cosmet Sci 2008; 30:471 - 474.

[2] Schwensen JF, White IR, Thyssen JP, Menne T, Johansen JD. 2015; 73:133 141.

\section{Mo-Poster Session 1-PO-121 Studies In Vitro and In Vivo of antileishmanial activity and Differential Cytotoxicity of Cannabis spp.}

Authors Robledo $\mathrm{SM}^{1}$, Restrepo $\mathrm{A}^{1}$, Yepes $\mathrm{LM}^{1}$, Fernandez $\mathrm{M}^{1}$, Vélez ID Institute 1 Programa de Estudio y Control de Enfermedades TropicalesPECET, Facultad de Medicina, Universidad de Antioquia. Medellín, Colombia, Medellín, Colombia

DOI 10.1055/s-0037-1608170

Current research looks at the scientific findings on the potential of Cannabis to treat skin lesions, discovering the role played by the endocannabinoid system in maintaining healthy skin and wound healing, and validating the potential of Cannabis in the topical treatment of cutaneous lesions. Due to its anti-inflammatory and healing properties, the study aimed to confirm the in vitro and in vivo antileishmanial effect of Cannabis spp. Three ethanol extracts from Cannabis Nicole Kush strain ( $75 \%$ C. indica and $25 \%$ C. sativa) were prepared by percolation. The cytotoxicity was evaluated on different cell lines and primary culture cells using the 3-(4,5-Dimethylthiazol-2-yl)-2,5-Diphenyltetrazolium Bromide (MTT) colorimetric method while the antileishmanial activity was evaluated on $L$. braziliensis amastigotes by flow cytometry. The results were expressed as median Lethal Concentration $\left(\mathrm{LC}_{50}\right)$ for cytotoxicity and the median Effective Concentration ( $E C_{50}$ ) for effectiveness [1]. The content of cannabinoids was determined by chromatography. A cream and a lotion formulation were prepared and tested in hamsters experimentally infected with L. braziliensis [2]. The most active extract in vitro $\left(\mathrm{EC}_{50} 24 \mathrm{mg} / \mathrm{mL}\right)$ was the extract with the higher percentage of THC (18\%). The remained two extracts had moderate activity ( $E_{50} 28.2$ and $32.8 \mathrm{mg} / \mathrm{mL}$, respectively). No differential cytotoxicity was observed. The cream formulation of the most active extract showed cure $80 \%$ of hasmters after treatment. This is the first report of antileishmanial activity in Cannabis spp. Since this plant has been associated with anti-inflammatory effects, is possible to think that Cannabis extract could be a good alternative for the management of uncomplicated cutaneous leishmaniasis.

[1] Duque-Benítez SM, Ríos-Vásquez LA, Ocampo-Cardona R, Cedeño DL, Jones MA, Vélez ID, Robledo SM. Molecules 2016; 21: 381.

[2] Robledo SM, Carrillo LM, Daza A, Restrepo AM, Muñoz DL, Tobón J, et al. J Vis Exp. 2012; 62, 3533.

\section{Mo-Poster Session 1-PO-122 Determination and comparison of sun protection factors (SPF) of main anthraquinones and their glycosides}

Authors Demirezer Lutfiye $\mathrm{O}^{1}$, Uzun $\mathrm{M}^{1}$, Guvenalp $\mathrm{Z}^{2}$

Institute 1 Department of Pharmacognosy, Faculty of Pharmacy, Hacettepe University, 06100, Ankara, Turkey; 2 Department of Pharmacognosy, Faculty of Pharmacy, Ataturk University, Erzurum, Turkey

DOI 10.1055/s-0037-1608171

Sun protection factor (SPF) is a value, shows effectiveness of sunscreen and a higher SPF means more protection from UV radiation [1]. UV-A and UV-B are mainly responsible for skin hazards such as sunburn, cutaneous degeneration, photosensitivity and skin cancer in intense exposure [2]. The aim of the present study was to determine SPF values of main anthraquinones emodin, aloeemodin, rhein, physcion, chrysophanol and their glycoside forms.

The SPF's of main anthranoids were determined according to the in vitro spectrophotometric method described by Mansur et al [3]. All anthranoids were prepared at $100 \mu \mathrm{g} / \mathrm{mL}$ and $200 \mu \mathrm{g} / \mathrm{mL}$ concentrations with ethanol, scanned at wavelength $290-320 \mathrm{~nm}$ and three replicates were made. SPF values calculated by Mansur equation. All of the anthranoids showed high UV protection capability between SPF $4.408-30.6$, indicating that they have potentially protective against UV rays. Emodin showed highest UV capability with SPF $20.23(100 \mu \mathrm{g} / \mathrm{mL})$ and SPF $30.59(200 \mu \mathrm{g} / \mathrm{mL})$. SPF's of the glycosides were found lower than aglycones. Therefore, the sun protection effect decreased as the polarity increased. These results have shown that anthranoids can be used as sunscreen agents in formulations for various skin types. This study was supported by The Scientific and Technological Research Council of Turkey (TUBITAK) (Project No: SBAG-214S147)

[1] Kaur CD, Saraf S. Pharmacognosy Res 2010, 2: $22-25$

[2] D’orazio J, Jarrett S, Amaro-Ortiz A, Scott T. Int J Mol Sci 2013, 14:12222 48

[3] Mansur JS, Breder MN, Mansur MC, Azulay RD. An Bras Dermatol 1986, 61: $121-124$ 
Mo-Poster Session 1-PO-123 The potential of the essential oil from the Brazilian spice Xylopia sericea A. St.-Hil. (Annonaceae) in the treatment of skin inflammation

\begin{abstract}
Authors Mendes $\mathrm{R}^{1}$, Pinto $\mathrm{N}^{1}$, Silva $\mathrm{J}^{1}$, Chedier $\mathrm{L}^{2}$, Scio $\mathrm{E}^{1}$
Institute 1 Laboratory of Bioactive Natural Products, Department of

Biochemistry, Federal University of Juiz de Fora, Juiz de Fora, Brazil;

2 Department of Botany, Federal University of Juiz de Fora, Juiz de Fora, Brazil DOI 10.1055/s-0037-1608172

The fruits of Xylopia sericea, known as "embiriba", are popularly used for medicinal purposes, and as a condiment in food preparation [1]. The aim of this study was to investigate the anti-inflammatory potential of the essential oil extracted from its fruits. The oil (OXS) was obtained by hydrodistillation using a Clevenger type apparatus. The inflammation was induced in Swiss mice by topical application ( $20 \mu \mathrm{L} / \mathrm{ear}$ ) of croton oil $2.5 \%$ on the right ear of each mouse [2]. On the left ear was applied the vehicle (acetone). After 15 min, the animals $(n=8)$ received the following topical treatments: acetone, dexamethasone $(0.1 \mathrm{mg})$ or OXS $(0.5 \mathrm{mg})$. After $6 \mathrm{~h}$, theywere euthanized and identical punch biopsies were obtained from both ears. Data were subjected to analysis of variance (ANOVA) followed by Newman-Keuls test. The chemical composition of OXS was already described in details. The sesquiterpenes spathulenol (16.42\%), guaiol (13.93\%), and germacrene D (8.11\%) were the major constituents [3]. The results were expressed as difference in weight between the ears: $6.2 \pm 0.7 \mathrm{mg}$ for vehicle; $0.7 \pm 0.6 \mathrm{mg}$ (\% 88.1) for dexamethasone, and $2.8 \pm 1.6 \mathrm{mg}$ (\% 54.2) for OXS, demonstrating the significant anti-inflammatory activity $(p<0.001)$ of the oil. The antioxidant activity already reported [3] may be contributing to this action. The results evidenced the potential of the essential oil of $X$. sericea in the treatment of skin inflammation.
\end{abstract}

This work was supported by the grants from FAPEMIG, CAPES and CNPq.

[1] Di Stasi LC, Hiruma-Lima CA. Plantas medicinais na Amazônia e Mata Atlântica. UNESP; 2002

[2] Romay C, Ledón N, González R. Inflamm Res 1998; 47: 334 - 338

[3] Mendes RF, Pinto NCC, Silva JM, Silva JB, Hermisdorf RSC, Fabri R, Chedier LM, Scio E. J Pharm Pharmacol 2017; 69:341 - 348

\section{Mo-Poster Session 1-PO-124 Antipsoriatic potential of Pereskia aculeata Miller leaves}

Authors Pinto $\mathrm{N}^{1}$, Mendes $\mathrm{R}^{1}$, Silva $\mathrm{J}^{1}$, Duque Ana $\mathrm{P}^{1}$, Castañon Maria $\mathrm{C}^{2}$, Scio $\mathrm{E}^{1}$

Institute 1 Laboratory of Bioactive Natural Products, Department of Biochemistry, Federal University of Juiz de Fora, Juiz de Fora, Brazil;

2 Department of Morphology, Institute of Biological Sciences, Federal University of Juiz de Fora, Juiz de Fora, Brazil

DOI 10.1055/s-0037-1608173

Preliminary studies had shown the marked anti-inflammatory potential of Pereskia aculeata leaves [1]. Psoriasis is a chronic inflammatory disease which affects $1-3 \%$ of the world Caucasian population [2], which encouraged the investigation of $P$. aculeata antipsoriatic potential by the mouse tail test [3]. Topical pharmaceutical formulations $(20 \mathrm{mg}$ ) containing $6 \%$ or $12 \%$ of hexane fraction from $P$. aculeata leaves (HF), vehicle or Psorex ${ }^{\mathrm{TM}}$ were topically applied on the tail of Swiss mice $(n=5)$ and histometric analysis were performed as follows: (1) the total length of the scale region; (2) the length of the granular layer of the scale region; (3) the orthokeratosis degree, which was calculated by the ratio of (2)/(1). ANOVA followed by the Newman-Keuls test was used for statistical analysis (COBEA - Protocol $n^{\circ}$ 028/2014). The orthokeratosis degree presents high correlation with the antipsoriatic activity on human skin. The naive group and the topical pharmaceutical formulations containing vehicle, HF $6 \%$, HF $12 \%$ or Psorex ${ }^{\mathrm{TM}}$ showed, respectively, 49.62 $\pm 3.1,52.25$ $\pm 4.5,72.16 \pm 3,3^{* * *}, 78.14 \pm 3.1^{* * *}$, and $86.83 \pm 2.5^{* * *}$ of orthokeratosis degree $\left({ }^{* *} p<0.001\right.$ vs naive). Photomicrographs of the naïve and treated tissues are shown in Figure 1. Our findings strongly suggested that $P$. aculeata is endowed with antipsoriatic potential.

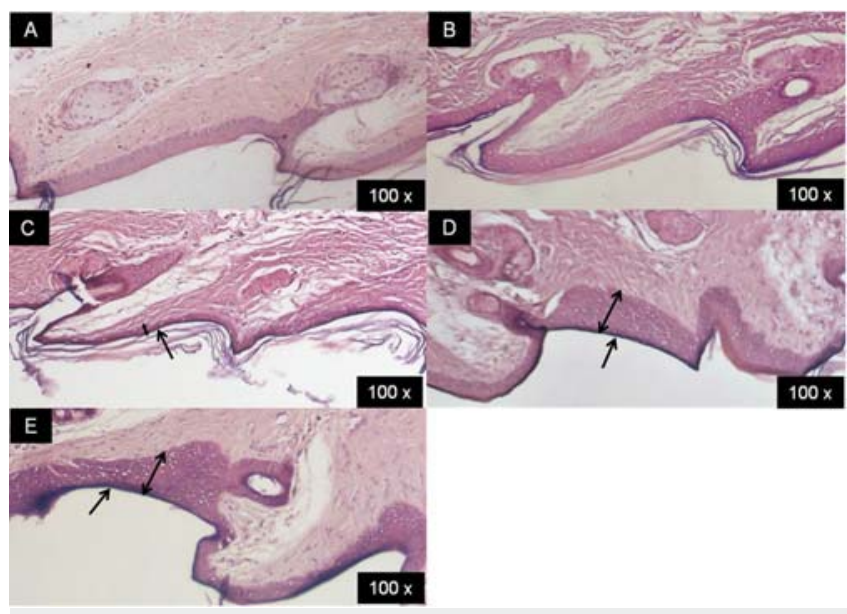

Fig. 1: (A) naive, (B) Vehicle, (C) Psorex ${ }^{\mathrm{TM}}$, (D) HF 6\%, (E) HF 12\%. The arrows indicate the granular layer induced by sample application. The double arrows evidenced the epidermal thickness. In most cases, the increase of the epidermal thickness is correlated to the antipsoriatic potential of a tested drug.

This work was supported by the grants from FAPEMIG, CAPES and CNPq. [1] Pinto NCC, Machado DC, Da Silva JM, Conegundes JL, Gualberto AC, Gameiro J, Chedier LM, Castañon MC, Scio E. J Ethnopharmacol 2015; 173 : 330 337

[2] Uva L, Miguel D, Pinheiro A, Antunes J, Cruz D, Ferreira J, Filipe P. Int J Endrocrinol 2012; 2012: 1 - 16.

[3] Bosman B, Matthiesen T, Hess V, Friderichs E. Skin Pharmacol 1992; 5: $41-48$.

\section{Mo-Poster Session 1-PO-125 Skin antiaging effect of Lavandula officinalis extract determining by Matrix Metalloproteinase- 1 inhibition assay}

Authors Gonulalan Ekrem $\mathrm{M}^{1}$, Uzun $\mathrm{M}^{1}$, Demirezer $\mathrm{LO}^{1}$

Institute 1 Department of Pharmacognosy, Faculty of Pharmacy, Hacettepe University, 06100, Sihhiye, Ankara, Turkey

DOI 10.1055/s-0037-1608174

Nowadays, there is a great interest in using herbal extracts as active ingredients of functional cosmetic products. Although the cosmetic products containing lavender have been shown to improve the appearance of the face skin, this claim has not yet been proven (1).

Matrix metalloproteinase-1 (MMP-1) break down collagen and elastin, leading to degenerative changes of the extracellular matrix, accelerating the formation of wrinkles and saggings and causing the skin to age (2). The discovery of MMP-1 enzyme inhibitors in preventing skin elasticity and wrinkle formation is important.

The present study was undertaken to evaluate the possible inhibitory effects of Lavandula ethanolic extracts on matrix metalloproteinase-1. MMP-1 inhibitory effects were measured in $25 \mu \mathrm{g} / \mathrm{mL}, 50 \mu \mathrm{g} / \mathrm{mL}, 100 \mu \mathrm{g} / \mathrm{mL}, 250 \mu \mathrm{g} / \mathrm{mL}$ and $500 \mu \mathrm{g} / \mathrm{mL}$ concentrations at $412 \mathrm{~nm}$. All samples showed the best efficacy at $500 \mu \mathrm{g} / \mathrm{mL}$ concentration. Inhibition of MMP-1 by the Lavandula officinalis methanolic extract, linalool and linalool-free extracts at $500 \mathrm{mg} / \mathrm{mL}$ was found to be $80.12 \%, 90.20 \%$ and 79.59 , respectively, while positive control NNGH inhibited MMP-1 $90.8 \%$ in 1,3 $\mu \mathrm{M}$ concentration. In this study, linalool was found the most effective among them but all extracts showed significant activity.

[1] Baumann LS. Dermatol Therapy 2007; 20: 330 - 342 
[2] Fisher G], Kang S, Varani J, Bata-Csorgo Z, Wan Y, Datta S. Arch Dermatol 2002; 138: $1462-1470$.

\section{Mo-Poster Session 1-PO-126 Does the parthenolide free Feverfew extract exhibit the similar effect as parthenolide containing extract?}

Authors Gonulalan Ekrem $\mathrm{M}^{1}$, Uzun $\mathrm{M}^{1}$, Demirezer LO ${ }^{1}$

Institute 1 Department of Pharmacognosy, Faculty of Pharmacy, Hacettepe University, 06100, Sihhiye, Ankara, Turkey

DOI 10.1055/s-0037-1608175

Matrix metalloproteinase-8 (MMP-8) from the collagenases group, break down collagen and elastin, leading to degenerative changes of the extracellular matrix, accelerating the formation of wrinkles and saggings and causing the skin to age (1). Feverfew (Tanacetum parthenium (L.) Schulz Bip. (Asteracae)) extracts have skin soothing properties and are not suitable for topical applications because of the allergic effect of the parthenolide (a sesquiterpene lactone) $(2,3)$.

The main purpose of this study was to determine the effect of parthenolide on the skin aging retardant effect. MMP-8 inhibitory effect was tested on parthenolide containing and removed extracts from Tanacetum parthenium. At the same time, we have also determined the MMP- 8 inhibitory effect of parthenolide.

MMP-8 inhibitory effect was measured in $25 \mu \mathrm{g} / \mathrm{mL}, 50 \mu \mathrm{g} / \mathrm{mL}, 100 \mu \mathrm{g} / \mathrm{mL}$, $250 \mu \mathrm{g} / \mathrm{mL}$ and $500 \mu \mathrm{g} / \mathrm{mL}$ concentrations at $412 \mathrm{~nm}$. All samples showed the best efficacy at $500 \mu \mathrm{g} / \mathrm{mL}$ concentration. Inhibition values were for parthenolide containing and depleted Tanacetum parthenium extracts and parthenolide, $72.28 \%, 74.55 \%$ and $81.80 \%$ respectively. Positive control NNGH inhibited MMP-8 $93.88 \%$ in $1.3 \mu \mathrm{M}$ concentration. As a result, although parthenolide alone has a high MMP-8 inhibition rate, parthenolite-free extracts inhibit MMP-8 as much as parthenolide-containing extracts. This suggests that the effect is due to the synergism of the other substances carried by the plant.

[1] Fisher G], Kang S, Varani J, Bata-Csorgo Z, Wan Y, Datta S Arch Dermatol 2002; 138: $1462-1470$.

[2] Sur R, Martin K, Liebel F, Lyte P, Shapiro S, Southall M. Inflammopharmacology 2009; 17:42 - 49 .

[3] Hausen BM, Osmundsen PE. Acta Derm Venereol 1983; 63: 308 - 314

\section{Mo-Poster Session 1-PO-127 Determination of Sun Protection Factor (SPF) and antioxidant capacity of Tanacetum parthenium}

Authors Gonulalan Ekrem $\mathrm{M}^{1}$, Uzun $\mathrm{M}^{1}$, Ozenver $\mathrm{N}^{1}$, Demirezer $\mathrm{LO}^{1}$ Institute 1 Department of Pharmacognosy, Faculty of Pharmacy, Hacettepe University, 06100, Sihhiye, Ankara, Turkey

DOI 10.1055/s-0037-1608176

The aim of the present study was to determine SPF values and antioxidant capacity of Tanacetum parthenium (Asteraceae), parthenolide and parthenolide free T. parthenium.

Products, with high SPF values protect the skin from harmful UV rays (1). SPF were determined according to Mansur et al (2). 100 and $200 \mu \mathrm{g} / \mathrm{mL}$ concentrations of samples were scanned at wavelength $290-320 \mathrm{~nm}$ and three replicates were made and results were obtained between $0.88-2.34$ $(200 \mu \mathrm{g} / \mathrm{mL})$.

Products with antioxidant capacity delay skin aging. In this study, antioxidant capacities of methanolic extracts prepared at 25, 50, 100, 250 and $500 \mu \mathrm{g} / \mathrm{mL}$ concentrations of parthenolide -containing or -free Tanacetum parthenium and parthenolide were tested with 4 different methods. The most effective concentration was found at $500 \mu \mathrm{g} / \mathrm{mL}$. Values are given in the table below.
Tab. 1

\begin{tabular}{|l|l|l|l|}
\hline & $\begin{array}{l}\text { Tanacetum parthe- } \\
\text { nium }\end{array}$ & Parthenolide & Parthenolide free T. parthenium \\
\hline DPPH & $87.8 \%$ & $8.12 \%$ & $80.33 \%$ \\
\hline ABTS & $85.5 \%$ & $21.99 \%$ & $80.95 \%$ \\
\hline NO & $37.26 \%$ & $36.64 \%$ & $39.75 \%$ \\
\hline Phosphomolibdate & $88.91 \%$ & $32.37 \%$ & $35.92 \%$ \\
\hline
\end{tabular}

As a result, it was determined that, removal of parthenolite from Tanacetum parthenium did not change antioxidant capacity and the unprocessed extract was found to be most effective. SPF results of all tested materials have not been found significant.

This study was supported by The Scientific and Technological Research Council of Turkey (TUBITAK) (Project No: SBAG-214S147)

[1] Kaur CD, Saraf S. Pharmacognosy Res 2010; 2: 22 - 25.

[2] Mansur JS, Breder MN, Mansur MC, Azulay RD. An Bras Dermatol 1986; 61: $121-124$.

\section{Mo-Poster Session 1-PO-128 Anti-Acne Inducing Bacteria and Free Radical Scavenging Activities of Turmeric Rhizome Extracts Prepared Using Different Solvents}

Authors Nurficahyanti $\mathrm{R}^{1}$, Charoenwiwattanakij $\mathrm{P}^{2}$, Wattanalai $\mathrm{R}^{2}$, Pothitirat $\mathrm{W}^{2}$

Institute 1 Faculty of Pharmacy, Ahmad Dahlan University, Yogyakata, Indonesia; 2 Faculty of Pharmacy, Siam University, Bangkok, Thailand DOI 10.1055/s-0037-1608177

Turmeric (Curcuma longa Linn.) of the family Zingiberaceae is one of the most popular medicinal plant used in food, drugs and cosmetics for a long time. It contains high amount of curcuminoid, especially curcumin which is a major constituent and responsible for numerous biological activities [1]. The purpose of this study is to compare anti-acne inducing bacteria and antioxidant activities of turmeric rhizome extracts which were prepared using different solvents i.e, hexane, dichloromethane, ethanol and water. All extracts were tested for antibacterial activities against bacteria causing acne, including Propionibacterium acnes, Staphylococcus epidermidis and Staphylococcus aureus, using broth microdilution method [2]. They were also evaluated for antioxidant activity using DPPH scavenging assay [3]. Additionally, the content of curcumin content of each extract was analyzed by HPLC [4]. The dichloromethane and ethanol extract exhibited the strongest antibacterial effect with MIC values for all bacterial species at $78 \mu \mathrm{g} / \mathrm{ml}$ and MBC values at $156.25 \mu \mathrm{g} /$ $\mathrm{ml}$. Based on DPPH scavenging method, it indicated that the dichloromethane extract of turmeric rhizome promoted the strongest DPPH scavenging effect $\left(I C_{50}=7.16 \mu \mathrm{g} / \mathrm{ml}\right)$, while the water extract showed poor antioxidant activity $\left(\mathrm{IC}_{50}=375.29 \mu \mathrm{g} / \mathrm{ml}\right)$. By HPLC, the dichloromethane extract yielded the highest content of curcumin $(18.87 \% \mathrm{w} / \mathrm{w})$ followed by the ethanol extract $(9.18 \%$ $\mathrm{w} / \mathrm{w})$, the hexane extract $(0.54 \% \mathrm{w} / \mathrm{w})$, and the water extract $(0.01 \% \mathrm{w} / \mathrm{w})$, respectively. These findings suggest that the dichloromethane extract of turmeric rhizome should be a potential source of natural anti-acne inducing bacteria and antioxidant agents for further development of pharmaceutical preparations.

[1] Hamid N, et al. J HerbMed Pharmacol 2014; 3: 5-8.

[2] Chomnawang MT, et al. J Ethnopharmacol 2005; 101: 330 - 333.

[3] Sithisarn P, et al. Evid Based Complement Alternat Med 2015: doi: $10.1155 / 2015 / 908242$

[4] Wichitnithad W, et al. Phytochem Anal, 2009; 20: 314-319. 


\section{Mo-Poster Session 1-PO-129 Topical anti-inflammatory activity of the hydroethanolic extract from Kalanchoe brasiliensis Cambess. (Crassulaceae) leaves}

Authors Mayorga $\mathrm{O}^{1}$, Florencio $\rfloor^{1}$, Santos $\mathrm{B}^{1}$, do Amaral Corrêa Joś $\mathrm{O}^{2}$, Sousa $\mathrm{O}^{2}$, Alves Maria $\mathrm{S}^{2}$

Institute 1 Graduate Program in Pharmaceutical Sciences, Federal University of Juiz de Fora, Rua José Lourenço Kelmer s/n, Campus Universitário, Bairro São Pedro, CEP 36036 - 900, Juiz de Fora, Brazil; 2 Department of Pharmaceutical Sciences, Faculty of Pharmacy, Federal University of Juiz de Fora, Rua José Lourenço Kelmer s/n, Campus Universitário, Bairro São Pedro, CEP 36036 900, Juiz de Fora, Brazil

DOI 10.1055/s-0037-1608178

Kalanchoe brasiliensis Cambess. (Crassulaceae), known as "saião", is a plant used in folk medicine for the treatment of inflammation, injuries, abscesses, and enlarged ganglia. ${ }^{1,2}$ The present study evaluated the topical anti-inflammatory effect of the hydroethanolic extracts EHJ50 (50\%) and EHJ70 (70\%) obtained from $K$. brasiliensis leaves. Using doses of $0.10,0.50$ and $1.0 \mathrm{mg} /$ ear of the extracts, the anti-inflammatory activity was assessed by Croton oilinduced acute ear edema method ${ }^{3}$, determination of inflammatory enzymes (MPO and NAG) and proinflammatory mediators (NO, IL-6 and TNF- $\alpha$ ) and histopathological analysis. After $24 \mathrm{~h}$ of treatment with EHJ50 and EHJ70, the inhibition of ear edema ranged from 62.03 to $80.34 \%$. These extracts also reduced MPO (55.27 to $81.74 \%$ ) and NAG (69.04 to $80.82 \%$ ), while the NO levels decreased from 46.74 to $67.43 \%$. The dose of $1.0 \mathrm{mg} /$ ear was able to inhibit IL-6 $(E H J 50=20.59 \%$ and EHJ70 $=22.78 \%)$ and TNF- $\alpha(E H J 50=16.71 \%$ and $\mathrm{EH} 70=23.71 \%$ ). These results suggest that $K$. brasiliensis has topical antiinflammatory effect and can be a promising source for the development of phytotherapics for skin disorders.

This investigation was supported by UFJF, FAPEMIG, CAPES, OEA and GCUB.

[1] Costa SS, Jossang A, Bodo B, Souza ML, Moraes VL. J Nat Prod 1994; 57 : $1503-1510$.

[2] Mourão RH, Santos FO, Franzotti EM, Moreno MP, Antoniolli AR. Phytother Res 1999; 13: $352-354$.

[3] Schiantarelli P, Cadel S, Acerbi D, Pavesi L. J Drug Res1982; 32:230-235.

\section{Mo-Poster Session 1-PO-130 Therapeutic effect s of Ginkgo beloba leaf preparations on psoriasis}

Authors Lin $Y^{1}$, Li S $S^{1}$, Tamaru $N^{1}$, Iwasaki $S^{1}$, Wang $R^{2}$, Kobayashi $Y^{3}$ Institute 1 Medical Corporation Soujikai, Osaka, Japan; 2 Zhejiang CONBA Pharmaceutical \& Drug Research Development Corporation, Hangzhou, China; 3 Faculty of Medicine, Shimane University, Izumo, Japan DOI 10.1055/s-0037-1608179

Therapeutic effects of Ginkgo beloba leaf preparations on psoriasis were studied. Eight patients with psoriasis were attended to the study. Psoriasis area and severity index (PASI) scores of the patients were 12 or more, and the surface of the eczema area was more than $10 \%$. These patients have been regularly or irregularly used steroids. All the patients stopped to use steroids before the start of Chinese medicine treatments. Patients were divided into groups A and B. Group A was 3 case and they have been treated for more than six months with general traditional Chinese medicines other than ginkgo leaves. Group B was 8 cases and they have been treated with the general traditional Chinese medicine plus oral Ginkgo leaf preparations $80 \mathrm{mg} \times 3 /$ day for more than six months. After 12 weeks and 52 weeks, we evaluated the therapeutic effects by the improvement of PASI points of both groups. After 12 weeks, there was 1 case whose PASI improved to $25 \%$ in group A and 6 cases $(75 \%)$ in group B. After 52 weeks, average of improvement of group A was $50 \%$ and that of group B was $82.5 \%$. Ginkgo leaf preparation showed possibly curative effects on the treatment of psoriasis. And we have not found obvious side effects on blood biochemical analyses such as liver and kidney function and on others.

\section{Mo-Poster Session 1-PO-131 The whitening components separated from a traditional Chinese medicine - Radix Trichosanthis (Tian Hua Fen)}

\author{
Authors Weng $\mathrm{IT}^{1}$ \\ Institute 1 China Medical University, Taichung, Taiwan \\ DOI 10.1055/s-0037-1608180
}

Radix Trichosanthis, so called Tian Hua Fen is the roots of Trichosanthes and has been used on swelling and hyperactivity therapy as a chinese medicine for thousands years. "Tang Materia Medica" mentioned that Radix Trichosanthis have the effect on whitening of skin[1]. However, research on the whitening effect and the mechanism of compounds in Radix Trichosanthis has not been investigated deeply. It is worth mentioning that the crude extracts of Radix Trichosanthis shows dose-dependent inhibition effect of melanin. So we try to investigate the whitening components in Radix Trichosanthis.

Radix Trichosanthis will be extracted by methanol, then the extracts will be purified and separated by column chromatography and high performance liquid chromatography. The structure of compounds we get will be identified by the Nuclear Magnetic Resonance and followed by assay for their capacity to affect melanogenesis. Thirteen compounds be separated, including benzoic acid, steroids and triterpenoids. Bryonolic, one of the triterpenoids get from Radix Trichosanthis shows the effective of melanin inhibition. The following mechanism of whitening effect and melanogenesis of components in Radix Trichosanthis still need more research to confirm.

[1] Tang Materia Medica, Su Jing et al. written in 659 AD, its a traditional Chinese medicine literature

\section{Mo-Poster Session 1-PO-132 Search for novel cosmeuceutical agents from the Actinobacteria Micromonospora sp.}

Authors Peyrat LA ${ }^{1}$, Georgousaki K${ }^{1}$, Tsafantakis $N^{1}$, Gumeni $S^{2}$, OvesCostales $\mathrm{D}^{3}$, González $\mathrm{I}^{3}$, Almeida $\mathrm{C}^{3}$, Genilloud $\mathrm{O}^{3}$, Trougakos $\mathrm{IP}^{2}$, Fokialakis $\mathrm{N}^{1}$

Institute 1 Department of Pharmacognosy and Natural Products Chemistry, Faculty of Pharmacy, National and Kapodistrian University of Athens, Athens, Greece; 2 Department of Cell Biology and Biophysics, Faculty of Biology, National and Kapodistrian University of Athens, Athens, Greece; 3 Fundación MEDINA, Granada, Spain

DOI 10.1055/s-0037-1608181

In the frame of MICROSMETICS project, 614 extracts from 110 actinobacteria strains were evaluated for their anti-ageing activity. Anti-oxidant, skin-protecting and skin-whitening bioactivities were analyzed and 12 actinobacteria strains were selected. Different cell-based analyses have been performed to evaluate their anti-ageing activity. The extracts were screened for tyrosinase inhibition effect (skin whitening) in mouse melanocytes (B16F10 cell line), as well as for their capacity to activate proteostasis and autophagy system in normal human skin fibroblasts, ensuring anti-ageing mechanisms [1]. One of the most active strains, Micromonospora sp. CA-129255 has been selected for large-scale cultivation, fractionation, dereplication and identification of bioactive compounds.

Biomass was first extracted with Hexane, followed by EtOAc and EtOH/ $\mathrm{H}_{2} \mathrm{O}$. Preliminary biological evaluation of the $\mathrm{EtOH} / \mathrm{H}_{2} \mathrm{O}$ extract showed statistically significant increasing of cathepsin activity $(56 \%$ at $1 \mu \mathrm{g} / \mathrm{ml})$ of human fibroblast exposed to the extract while the EtOAc extract showed inhibitory effects against tyrosinase activity on melanocyte cells $(66 \%$ at $300 \mu \mathrm{g} / \mathrm{ml})$. The dereplication of the extract was performed using UHPLC-HRMS and the isolation of secondary metabolites was realized using classical chromatographic methods. In order to elucidate the structure of all isolated compounds, the full set of spectroscopic (1 \& 2D NMR) and spectrometric data (HRMS/MS) were recorded. A series of indole derivatives, including the chlorinated compound $\mathrm{N}$-acetyl-6-chloro-L-tryptophan as well as a series of thymidine and guanosine derivatives were isolated from the $\mathrm{EtOH} / \mathrm{H}_{2} \mathrm{O}$ extract. All the isolates are 
currently under investigation for their anti-ageing properties in order to standardize the extract on specific biomarkers that can be used for cosmeceutical industry.

This work has been financially supported by EU under the frame of MICROSMETICS project (FP7-PEOPLE-IAPP 2013, Grant agreement: 612276).

[1] Georgousaki K et al. Planta Medica 2015; 81 - PM_234

\section{Mo-Poster Session 1-PO-133 Metabolite profile and antiproliferative effects in HaCaT cells of a Salix reticulata extract}

Authors Corradi $\mathrm{E}^{1}$, Schmidt $\mathrm{N}^{2}$, Räber $\mathrm{N}^{1}$, De Mieri $\mathrm{M}^{1}$, Hamburger $\mathrm{M}^{1}$, Potterat $\mathrm{O}^{1}$, Butterweck $\mathrm{V}^{2}$

Institute 1 Department of Pharmaceutical Sciences, University of Basel, Basel, Switzerland; 2 Institute for Pharma Technology, School of Life Sciences, University of Applied Sciences Northwestern Switzerland, Muttenz, Switzerland DOI 10.1055/s-0037-1608182

Phenolic constituents of Salix reticulata (Salicaceae) and antiproliferative activity of an extract and individual compounds was investigated in immortalized human non-tumorigenic keratinocytes $(\mathrm{HaCaT})$. A MeOH extract from aerial parts afforded several flavonoids, including luteolin and apigenin glycosides (2-5 and 9) and catechin (1), two procyanidin fractions, and the phenolic glucosides picein (6), triandrin (7), and salicortin (8). In an ATP assay, the $\mathrm{MeOH}$ extract reduced cell viability by approx. $60 \%$ at a concentration of $100 \mu \mathrm{g} / \mathrm{mL}$. Cell proliferation was assessed with a BrdU incorporation ELISA assay. The extract inhibited proliferation of HaCaT cells in a concentration dependent manner, with an approx. 50\% inhibition at $100 \mu \mathrm{g} / \mathrm{mL}$. In time lapse assays, the extract showed distinct inhibitory effects on cell migration at concentrations of $12.5,25$ and $50 \mu \mathrm{g} / \mathrm{mL}$. The activity of selected constituents was also determined. Luteolin-7-O- $\beta$-glucuronide (3) significantly inhibited cell proliferation at concentrations of 10 and $50 \mu \mathrm{M}$. In contrast, luteolin7-O- $\beta$-glucopyranoside (2) and a procyanidin fraction (P1) had only weak effects, and picein (6) and salicortin (8) did not affect cell proliferation. Luteolin-7-O- $\beta$-glucuronide $(10 \mu \mathrm{M})$ and, to a lesser extent, the procyanidin fraction $(10 \mu \mathrm{g} / \mathrm{mL})$ also inhibited cell migration.

\section{Mo-Poster Session 1-PO-134 Diversity of diterpenoids and polyphenols in Salvia glutinosa L. analyzed using targeted metabolomics based on LC-qTOF-MS and ${ }^{1} \mathrm{H}-\mathrm{NMR}$}

Authors Ślusarczyk $S^{1,2}$, Jas|'pińska $]^{2}$, Pecio $\iota^{1}$, Stochmal A $A^{1}$, Gille $E^{3}$, Matkowski $\mathrm{A}^{2}$

Institute 1 Dept. Biochemistry, IUNG-Institute of Plant Cultivation and Soil Science, Pulawy, Poland; 2 Dept. Pharmaceutical Biology, Botanical Garden of Medicinal Plants, Wroclaw Medical University, Poland, Wroclaw, Poland;

3 National Institute of Research and Development for Biological Sciences/

"Stejarul" Biological Research Center, Piatra-Neamt, Romania

DOI 10.1055/s-0037-1608183

Salvia glutinosa L. (Sticky sage) is a vigorous perennial associated with lower montane deciduous forests in Europe and its roots can be an alternative source of tanshinones. To date, it has largely been ignored as a medicinal plant. Also, despite a couple of reports about its phytochemistry based on single samples, the natural diversity of potentially active constituents in this plant is little documented. Here, we used two analytic approaches, ${ }^{1} \mathrm{HNMR}$ and UHPLC-qTOF-MS to get insight into the major and subtle differences in diterpenoid and phenylpropanoid profiles. Plant samples from three populations were tested - two East Carpathian - (1) from Romanian Bucovina, (2) from Ukrainian Skolivs'ki Beskydy, and (3) from the ex situ collection in the Botanical Garden of WMU in Wroclaw, Poland - started from material obtained in a westernmost Carpathian habitat in Beskid-Slaski. Additionally, we performed microplot experiment to test the influence of salt stress on the plant growth and phytochemical profile. Analytic data were processed using chemometric methods.

Samples of different origins were well separated by OPLS discriminant analysis both using NMR and LC-MS profiling. However, in NMR, the signals responsible for the differences were not assignable to any of specific metabolites. In LCMS, the main differences were quantitative, such as in the content of salviaflaside or melitric acid B, and to lesser extent to tanshinone-like nor-abietanoids. The salt stress induced by 50 and $100 \mathrm{mmol} \mathrm{NaCl}$ caused growth retardation and lower biomass (between 10 and 15\%) without any significant impact on specialized metabolites composition. The addition of silica alleviated negative impact of excess salt in the soil.

Salvia glutinosa root is a source of pharmacologically relevant tanshinones and phynylpropanoid. The samples from various Carpathian in situ and ex situ populations are not significantly different from the viewpoint of phytochemical value even if they can be easily discriminated using multivariate statistics. Financial support: Polish National Science Center FUGA - DEC/2014/12/S/ NZ9/00715.

\section{Mo-Poster Session 1-PO-137 Changes on polystyrene surface and biofilm formation as indication of biodegradation by soil microorganisms}

Authors Mohd Fadhil Mohd $\mathrm{F}^{1}$, Sharifah Aminah Syed $\mathrm{M}^{1}$, Siti Norazura $\mathrm{f}^{2}$ Institute 1 Faculty of Applied Sciences, Universiti Teknologi MARA, 40450 Shah Alam, Selangor, Malaysia; 2 Faculty of Applied Sciences, Universiti Teknologi MARA Negeri Sembilan, 72000 Kuala Pilah, Negeri Sembilan, Malaysia

DOI 10.1055/s-0037-1608184

It was reported that an amount of 5.475 million tons of solid waste which was about $0.81 \mathrm{~kg}$ per capita per day was estimated to generate in Malaysia [1] while in main cities in Malaysia, the amount increased to $1.7 \mathrm{~kg}$ per capita per day [2] in 2001. Solid waste increased due to the used of plastics especially polystyrene in every field of life. However, polystyrene can be degraded through biodegradation. The aim of the study is to observe the changing in polystyrene's surface and also biofilm formed by microorganisms from different soil samples using Electron Scanning Microscope (ESEM). Scanning electron microscopy is a tool to observe cavities and grooves formed on the plastic film, which directly reflected the extent of microbial colonization and degradation [3]. In this study, water filtrate from soil samples obtained from one landfill area, two waste disposal sites and one from soil burial method were collected and incubated with sterilized polystyrene at $30^{\circ} \mathrm{C}$ for 8 weeks before observation using ESEM was done. Formation of biofilm and changes in polystyrene's surface; crack, pit/cavity, erosion/rough indicated that the degradation have taken place due to colonization and adhered by bacteria on polystyrene which act as sole carbon for their survival due to carbon starvation. [1] Ngoc, U and H. Schnitzer. "Sustainable solutions for solid waste management in Southeast Asian countries." Waste Management 2009, 29:1982 1995.

[2] Kathirvale S. Comprehensive Characteristics of the Municipal Solid Waste Generated In Kuala Lumpur. Engineering Faculty, University Kebangsaan Malaysia and Technical Services Division, Malaysian Institute for Nuclear Technology Research. 2003.

[3] Sekhar V. C, Nampoothiri K. M, Mohan A. J, Nair N. R. Journal of Hazardous Materials. 2016, $347-354$.

\section{Mo-Poster Session 1-PO-138 Evaluation of antioxidant potential and cytotoxic effects of alcoholic extracts of Diospyros kaki leaves}

Authors Castro $\mathrm{V}^{1,3}$, Gomes $\mathrm{A}^{2}$, Botelho $\mathrm{C}^{2}$, Dias $\mathrm{A}^{3}$ Institute 1 DYNAMIKLIZARD LDA, Avenida Alves Figueiredo, 1100. 4780 163 Santo Tirso, Santo Tirso, Portugal; 2 CBMA - Centro de Biologia Molecular e Ambiental, Department of Biology, University of Minho, Braga, Portugal, 
Braga, Portugal; 3 Centre for the Research and Technology of AgroEnvironment and Biological Sciences (CITAB-UM), AgroBioPlant Group, Department of Biology, University of Minho, Portugal, Braga, Portugal DOI 10.1055/s-0037-1608185

Diospyros kaki (Dk) leaves have been used in China, Korea and Japan as a delicate, pleasant beverage. Additionally, it is used as a folk remedy for hypertension, ischemic stroke, angina and internal hemorrhage. However, in EU and particularly in Portugal Dk leaves are considered an agriculture waste. The aim of this study was to characterize methanolic Dk leaf extract concerning its in vitro antioxidant proprieties and evaluate eventual cytotoxic effects, using HepG2 cells (hepatocytes). HPLC-DAD profiles of Dk leaves showed that extracts are mainly composed by phenolic compounds (flavonoids and phenolic acids derivatives), known antioxidants. To assess antioxidant potential, we used several in vitro tests including DPPH scavenging, iron chelation, inhibition of nitric oxide and superoxide scavenging. Results showed that Dk have a high capacity to scavenge superoxide radicals $\left(E_{50} 8.94 \mu \mathrm{g} / \mathrm{ml}\right)$, even better than quercetin (EC $5018.73 \mu \mathrm{g} / \mathrm{ml}$ ), and DPPH radicals $\left(E_{50} 15.27 \mu \mathrm{g} / \mathrm{ml}\right)$. For iron chelating activity and inhibition of nitric oxide production Dk extract had lower potential with and $\mathrm{EC}_{50}$ of 1376 and $1027 \mu \mathrm{g} / \mathrm{ml}$, respectively. So, we can conclude that Dk leaf extract is a good radical scavenger and might protect cells from oxidative stress. Since Dk leaves are used in beverages and liver is always a key target organ for metabolization and detoxification, we tested possible cytotoxicity on HepG2 cells. Results did not show significant cytotoxic effects of Dk extracts on HepG2 cells (using MTS evaluation), up to $1 \mathrm{mg} / \mathrm{ml}$, for 3 and $24 \mathrm{~h}$ incubation times. Hence, we can infer that Dk leaf extracts have good antioxidant potential and no significant toxicity (at least on hepatocytes), however more tests are required with other cellular models.

This work was supported by project HEALTHKAKI, N 010672, Programa Operacional Regional do Norte, no âmbito do Portugal 2020.

\section{Mo-Poster Session 1-PO-139 Eudesmane sesquiterpenes from Verbesina lanata with inhibitory activity against major agricultural pathogen}

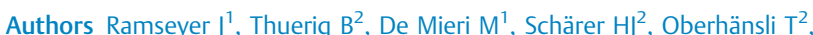
Gupta $\mathrm{MP}^{3}$, Tamm L ${ }^{2}$, Potterat $\mathrm{O}^{1}$, Hamburger $\mathrm{M}^{1}$

Institute 1 Department of Pharmaceutical Sciences, University of Basel, 4056 Basel, Switzerland; 2 Institute of Organic Agriculture FiBL, Department of Crop Sciences, 5070 Frick, Switzerland; 3 Center for Pharmacognostic Research on Panamanian Flora, Faculty of Pharmacy, University of Panama, Panama, Panama

DOI 10.1055/s-0037-1608186

In organic agriculture, there is a growing demand to replace copper by environmentally safer substitutes. In this context, an in-house library of more than 3000 extracts of plant and fungal origin was screened against the major plant pathogens Venturia inaequalis, Phytophthora infestans, and Plasmopara viticola. As one of the hits, the ethyl acetate extract from inflorescences of Verbesina lanata Rob. \& Greenm. (Asteraceae) showed significant inhibitory activity in vitro against $P$. viticola with a $\mathrm{MIC}_{100}$ value of $35 \mu \mathrm{g} / \mathrm{mL}$. In vivo activity was assessed on grapevine seedlings. A dry formulation with $50 \mathrm{mg} / \mathrm{g}$ of extract was developed to enhance the solubility. A $5 \mathrm{mg} / \mathrm{mL}$ solution of the formulation lowered leaf surface infestation to $18 \%$ compared to $79 \%$ infestation with the blank formulation.

With the aid of HPLC-based activity profiling, the activity could be correlated with a series of lipophilic compounds. Preparative isolation by a combination of chromatographic techniques, including silica gel column chromatography and preparative HPLC, afforded eight new and eight known eudesmane sesquiterpenes. Nine compounds were obtained in sufficient quantities to be tested in vitro, and were found to inhibit the growth of $P$. viticola with MICs 100 ranging from 4 to $50 \mu \mathrm{g} / \mathrm{mL}$. The two major compounds 1 and 2 showed MICs $_{100}$ of $31 \mu \mathrm{g} / \mathrm{mL}$ and $5 \mu \mathrm{g} / \mathrm{mL}$, respectively.
Our results demonstrate that plant derived compounds could serve as potential alternatives to copper in organic farming.

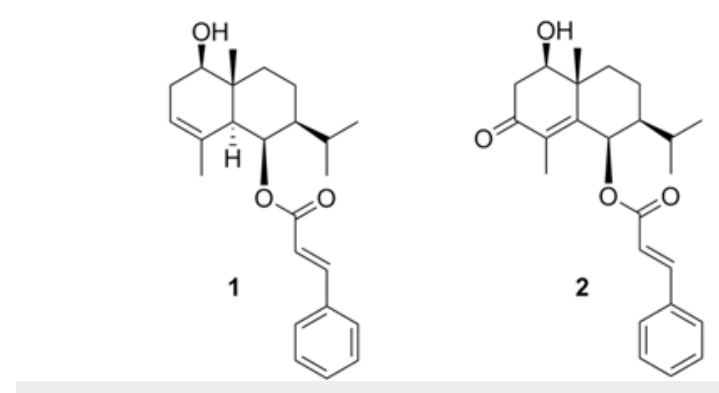

Fig. 1

\section{Mo-Poster Session 1-PO-140 Isolation and identification of flavonoids, saponins and two new flavolignans from Cecropia obtusifolia leaves collected in Panama}

Authors Rivera Mondragón $A^{1}$, Bijttebier $S^{1}$, Foubert $K^{1}$, CaballeroGeorge $C^{2}$, Pieters $L^{1}$

Institute 1 Natural Products \& Food Research and Analysis (NatuRA), Department of Pharmaceutical Sciences, University of Antwerp, Universiteitplein 1, 2610, Wilrijk, Belgium; 2 Group of Pharmaceutical Research, Institute of Scientific Research and High Technology Services (INDICASAT-AIP), Building 219, City of Knowledge, Panama, Panama DOI 10.1055/s-0037-1608187

Several plant species of the genus Cecropia (Urticaraceae) are traditionally used in Latin America for the treatment of a variety of diseases [1]. A wellknown example is dried leaves of $C$. obtusifolia, which are consumed as an antidiabetic and anti-inflammatory agent [2], and are commercially available as food supplements. The range of therapeutic properties attributed to these plants has been correlated to their content of phenolic acids, flavonoids, proanthocyanidins, triterpenoids and steroids [3]. However, until now there is no clear evidence on the nature of the active compounds, and there is lack of quality control of the herbal supplements. The aim of this study is to characterize the phytochemical constituents from leaves of $C$. obtusifolia collected in Panama. The plant material was exhaustively extracted with 70\% ethanol by maceration. A semi-preparative HPLC-MS system was used to isolate 11 compounds and their structures were elucidated based on HRESIMS, 1D and 2D NMR spectroscopic analysis (Figure 1). Seven flavone glycosides were obtained $(1-7)$, two saponins $(8-9)$ and two new flavolignans (1011). Isoorientin and isovitexin O-glycosides $(2-7)$, and the flavolignans (1011) were reported for the first time from the genus Cecropia. Development and validation of analytical methods for quality control and quantification of major constituents is in progress.

[1] Costa GM, Schenkel EP, Reginatto FH. Nat Prod Comun 2011; 6, 913 - 920.

[2] Pérez-Guerrero C, Herrera MD, Ortiz R, Alvarez De Sotomayor M, Fernández MA. J Ethnopharmacol 2001; 76, 279-284.

[3] Rivera-Mondragón A, Ortiz OO, Bijttebier S, Vlietinck A, Apers S, Pieters L, Caballero-George C. Pharm Biol 2017; 55, 1500 - 1512. 


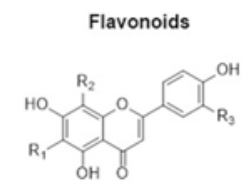

$1 R_{1}=H, R_{2}=\mathrm{Glu}, \mathrm{R}_{3}=\mathrm{OH}$ $2 R_{1}=$ Glu-2:-O-Xyl. $R_{2}=H, R_{3}=O H$ $3 R_{1}=$ Glu- $4^{*} \cdot 0-x_{y 1}, R_{2}=H, R_{3}=O H$ $4 \mathrm{R}_{1}=$ Glu-2:-O-Rha, $\mathrm{R}_{2}=\mathrm{H}, \mathrm{R}_{3}=\mathrm{OH}$ $5 R_{1}=$ Glu-2:-O-Xyl, $R_{2}=H, R_{3}=H$ $6 R_{1}=$ Glu-2:-O-Glu, $R_{2}=H \cdot R_{3}=H$ $7 R_{1}=$ Glu-2:O-Rha, $R_{2}=H, R_{3}=H$
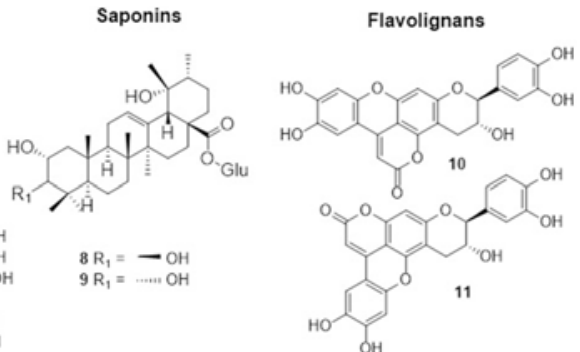

- Fig. 1: Compounds isolated from Cecropia obtusifolia Orientin (1). isoorientin-2"-O-xyloside (2), isoorientin-4"-O-xyloside (3), isoorientin2"-O-rhamnoside (4), isovitexin-2"-O-xyloside (5), isovitexin-2"-O-glucoside (6), isovitexin-2"-O- rhamnoside (7). tormentic acid 28-O-glucoside (8), euscaphic acid 28-O-glucoside (9), mururin A (10) and vaccinin $A(11)$ stereoisomers.

\section{Mo-Poster Session 1-PO-141 Optimization of extraction conditions for total flavonoids, chlorogenic acid and flavolignans contents from Cecropia sp. leaves using design-of-experiments methodology}

Authors Rivera-Mondragón $A^{1}$, Broeckx $G^{2}$, Bijttebier $S^{1}$, Fourbert $K^{1}$, Caballero-George $C^{3}$, Vander Heyden $Y^{4}$, Pieters $L^{1}$

Institute 1 Natural Products \& Food Research and Analysis (NatuRA),

Department of Pharmaceutical Sciences, University of Antwerp,

Universiteitplein 1, 2610, Wilrijk, Belgium; 2 Laboratory of Pharmaceutical

Technology and Biopharmacy, Department of Pharmaceutical Sciences,

University of Antwerp, Universiteitplein 1, 2610, Wilrijk, Belgium; 3 Group of Pharmaceutical Research, Institute of Scientific Research and High Technology Services (INDICASAT-AIP), Building 219, City of Knowledge, Panama, Panama; 4 Department of Analytical Chemistry and Pharmaceutical Technology, Center for Pharmaceutical Research (CePhaR), Vrije Universiteit Brussel-VUB,

Laarbeeklaan 103, B-1090, Brussels, Belgium

DOI 10.1055/s-0037-1608188

Plants of the genus Cecropia sp. are available on the market as food supplements with very little information on methods for warranting their quality [1]. Selection of suitable extraction methods plays a critical role in the quantification of phytochemicals for quality control purposes. The aim of this study was to optimize the extraction process for total flavonoids (TF), chlorogenic acid (CA) and flavolignans (FL) from six Cecropia sp. leaves using design of experiments methodology. Extractions were performed using ultrasound assisted extraction; methanol/water mixtures (v/v) and acetone were selected as extraction solvents. Chemical composition of Cecropia sp. was analyzed by HPLCDAD. From screening results using a Fractional Factorial Design (FFD) [2], time of extraction was set at 30 minutes, plant:solvent ratio at 1:50 (w/v), particle size at $\leq 125 \mu \mathrm{m}$, and number of extractions with methanol/water $(\mathrm{v} / \mathrm{v})$ and acetone at 3 and 1 , respectively. A central composite design (CCD) [2] was used to optimize methanol concentration ( $\mathrm{v} / \mathrm{v}$, in water) and ultrasonic bath temperature. The results showed that the use of methanol $70 \%(\mathrm{v} / \mathrm{v})$ combined with a temperature of $64^{\circ} \mathrm{C}$ gave maximal contents of TF, CA and $\mathrm{FL}$ (Figure 1). Under these conditions, the predicted and experimental yields were similar. These results proof that optimization of extraction conditions is important for obtaining reliable quantifications, which is further useful for the validation of an analytical methodology for assessing the quality of Cecropia sp. derived herbal products.
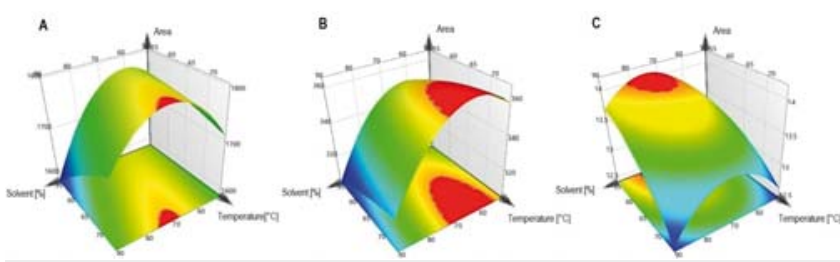

- Fig. 1: Response surface plots demonstrating the influence of methanol concentration (\%) and ultrasonic bath temperature $\left({ }^{\circ} \mathrm{C}\right)$ on the peak areas of flavonoids (A), chlorogenic acid (B) and flavolignans (C).

[1] Rivera-Mondragón A, Ortiz OO, Bijttebier S, Vlietinck A, Apers S, Pieters L, Caballero-George C. Pharm Biol 2017; 55, 1500 - 1512.

[2] Klein-Júnior LC, Viaene J, Salton J, Koetz M, Gasper AL, Henriques AT, Vander Heyden Y. J Chromatogr A 2016; 1463:60 - 70.

\section{Mo-Poster Session 1-PO-142 Phytochemical and Biological Investigation of Jatropha pelargoniifolia Root Indigenous to Saudia Arabia}

Authors Aati $\mathrm{H}^{1}$, Oliver $\mathrm{K}^{2}$, ElGamal $\mathrm{A}^{1,3}$

Institute 1 Department of Pharmacognosy, Faculty of Pharmacy, King Saud University, Riyadh11488, Saudi Arabia; 2 Department of Biochemical and Chemical Engineering, TU Dortmund, Emil-Figge.str.66 dortmound, Germany; 3 Department of Pharmacognosy, Faculty of Pharmacy, Mansoura University, El-Mansoura35516, Egypt

DOI 10.1055/s-0037-1608189

Jatropha pelargoniifolia (Euphorbiaceae), widely known as "Obab" is a medicinal plant widely used throughout Africa, Yamen \& Saudi Arabia. Genus Jatropha growing worldwide and comprising about 175 species $^{(1)}$. Reviewing the current literature for the phytochemical and biological importance of the plants belonging to genus Jatropha revealed many biological interests such as wound inflammation \& infection, skin ulcer, jaundice \& hemorrhoids but nothing was reported regarding the titled plants ${ }^{(2)}$. Thus, it was of interest to explore the active constituents and the biological activities for J. pelargoniifolia. Previous phytochemical investigation on different plants belonging to genus Jatropha revealed the presence of several classes of secondary metabolites including, terpenoids, flavonoids, alkaloids etc ${ }^{(3)}$. Repeated chromatographic separation and purification of petroleum ether, dichloromethane \& ethyl acetate extracts of $J$. pelargoniifolia root, using different chromatographic techniques resulted in the isolation of several compounds. The structures of the isolated compounds have been elucidated by using different spectroscopic techniques including 1\&2 D NMR and identified as; $\beta$.sitosterol, $\beta$-sitosterol glucoside, curcusons C\&D, naringenin, apigenin, pyrimidine-2,4-(1 H,3 H)dione, 2,3-dihydro-1-methyl-1 H-pyrrolo[2,3-b] pyridine-6-Ol, cleomiscosins $A \& B$ and propacin. Furthermore, hepatoprotective, analgesic, anti-inflammatory, antidiabetic \& anti-oxidant activities were conducted on methanolic extract of different plant parts and showed significant hepatoprotective, analgesic \& anti-oxidant activities. Perhaps, the obtained biological results were due to the presence of flavonoids, alkaloids, diterpenes \& non-conventional coumarinolignans in high yield. 

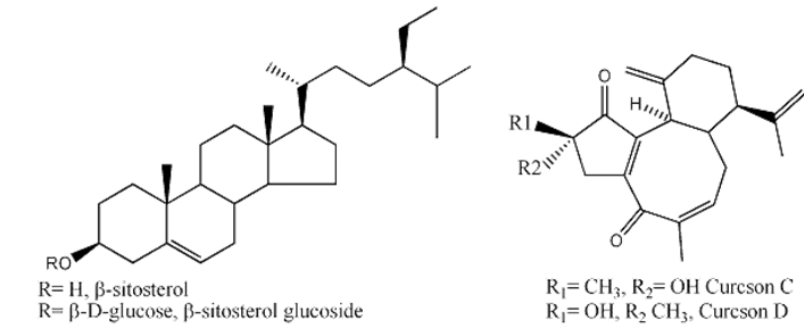

$\mathrm{R}=\beta$-D-glucose $\beta$-sitosterol glucoside<smiles>O=C1CC(c2ccc(O)cc2)Oc2cc(O)cc(O)c21</smiles><smiles></smiles><smiles>C1CCCCC1</smiles>

$$
\begin{aligned}
& \mathrm{R}_{1}=\mathrm{CH}_{3}, \mathrm{R}_{2}=\mathrm{OH} \text { Curcson C } \\
& \mathrm{R}_{1}=\mathrm{OH}, \mathrm{R}_{2} \mathrm{CH}_{3}, \text { Curcson D }
\end{aligned}
$$<smiles>[Tl]</smiles><smiles>O=c1cc[nH]c(=O)[nH]1</smiles>

Pyrimidine-2,4-(1H,3H)-dione<smiles>CN1CCc2ccc(O)nc21</smiles>

2,3-dihydro-1-methyl-1 $H$-pyrrolo[2,3-b]pyridin-6-ol<smiles>COc1cc([C@@H]2Oc3c(OC)cc4ccc(=O)oc4c3O[C@@H]2CO)ccc1O</smiles><smiles>[R]C1Oc2c(c(OC)cc3ccc(=O)oc23)O[C@@H]1c1ccc(O)c(OC)c1</smiles>

$\mathrm{R}=\mathrm{CH}_{3}$, Propacin $\mathrm{R}=\mathrm{CH}_{2} \mathrm{OH}$, Cleomiscosin $\mathrm{A}$

Fig. 1

[1] Trivedi S, Gaudani H, Gupta M, Gupta N, Patil P, Gupta G, Krishna V \& Reddy MP. The Int J Agric Sci 2012; 1:11- 20.

[2] Schmelzer GH \& GuribFakim A. Jatropha Pelargoniifolia. Record from PROTA4U. PROTA Plant Resources of Tropical Africa/Ressources végétales de l'Afrique tropicale, Wageningen, Netherlands 2007.

[3] Zhang XP, Zhang ML, Su XH, Huo CH, Gu YC\& Shi QW. Chemistry \& Biodiversity 2009;2166 - 2183.

\section{Mo-Poster Session 1-PO-143 Investigations on SOD in extremophile and non-extremophile plants}

Authors Woith $\mathrm{E}^{1}$, Stintzing $\mathrm{F}^{2}$, Melzig MF ${ }^{1}$

Institute 1 Freie Universität Berlin, Berlin, Germany; 2 WALA Heilmittel

GmbH, Bad Boll/Eckwaelden, Germany

DOI 10.1055/s-0037-1608190

Reactive oxygen species (ROS) can cause multiple damage to diverse biological structures, but they can also have beneficial effects, e. g. in signaling pathways [1]. Antioxidants scavenge cells from ROS and adjust the physiological steady state between oxidative and antioxidative processes. As one highly important agent of the enzymatic antioxidant system [2], we investigated the activity of superoxide dismutase (SOD) in 27 extremophile and non-extremophile plant species. The highest SOD activities were found in the extremophile plants Crassula multiflora Schönland \& Baker f, Crassula mesembryanthemoides D. Dietr, Aeonium haworthii Salm-Dyck ex Webb \& Berthel., and Aeonium viscatum Bolle. However, other extremophile species like Mesembryanthemum crystallinum L, Trichodiadema densum Schwantes, and Aloe juvenna Brandham \& S. Carter showed lower SOD activities than the investigated non-extremophile plants. Thus, we did not find any correlation between SOD activity and extremophilicity.

[1] Holmström KM, Finkel T. Nat Rev Mol Cell Biol 2014; 15: 411 - 421

[2] Alscher RG, Erturk N, Heath LS. J Exp Bot 2002; 53: 1331 - 1341

Mo-Poster Session 1-PO-144 In vitro and in vivo study of the absorption and gastrointestinal and hepatic biotransformation of the cyclopeptide alkaloid hymenocardine

Authors Tuenter $E^{1}$, Bijttebier $S^{1}$, Foubert $K^{1}$, Breynaert $A^{1}$, Apers $S^{1}$,

Hermans $N^{1}$, Pieters $L^{1}$

Institute 1 University of Antwerp, Department of Pharmaceutical Sciences, Natural Products \& Food Research and Analysis (NatuRA), Antwerp, Belgium DOI 10.1055/s-0037-1608191

Hymenocardine is a cyclopeptide alkaloid present in the root bark of Hymenocardia acida Tul. (Phyllanthaceae). In traditional African medicine, the leaves and roots of this plant are used to treat malaria, and moderate in vitro antiplasmodial activity has been reported for hymenocardine.[1] However, in view of its peptide-like nature, potential biotransformation after oral ingestion has to be taken into account when considering in vivo experiments. In this study, the gastric and small intestinal stability of hymenocardine was assessed using an in vitro gastrointestinal model (GIM).[2] In addition, potential biotransformation in the liver was investigated in vitro by incubation of hymenocardine with a human $\mathrm{S} 9$ fraction. Moreover, hymenocardine was administered to rats per os, and blood and urine samples were collected until $48 \mathrm{~h}$ and $24 \mathrm{~h}$ after administration, respectively. All samples resulting from these three experiments were analyzed by liquid chromatography-high resolution mass spectrometry (LC-HRMS). Analysis of the fractions obtained from the GIM indicated that hymenocardine is stable in the stomach and small intestine, in spite of its peptide-like nature. The in vitro $\$ 9$ biotransformation experiment resulted in the identification of several hymenocardine metabolites, the major ones being formed by the reduction and/or the loss of an $\mathrm{N}$-methyl group. The in vivo study showed that hymenocardine is absorbed unchanged from the gastrointestinal tract, since it could be identified in both rat plasma and urine, together with hymenocardinol, its reduction product (Figure).

[1] Tuenter E, Exarchou V, Baldé A, Cos P, Maes L, Apers S, Pieters L. J Nat Prod 2016; 79: $1746-1751$

[2] Breynaert A, Bosscher D, Kahnt A, Claeys M, Cos P, Pieters L, Hermans N. Planta Med 2015; 81: $1075-1083$

[3] Tuenter E, Bijttebier S, Foubert K, Breynaert A, Apers S, Hermans N, Pieters L. Planta Med; published online (DOI: 10.1055/s-0043-102494)

\section{Mo-Poster Session 1-PO-145 ANTIPROTOZOAL SESQUITERPENE LACTONES AND OTHER CONSTITUENTS FROM Schkuhria pinnata,} Tarchonanthus camphoratus AND Vernonia lasiopus

Authors Kimani $\mathrm{N}^{1}$, Matasyoh $\mathrm{J}^{2}$, Kaiser $\mathrm{M}^{3,4}$, Brun $\mathrm{R}^{3,4}$, Schmidt $\mathrm{T}^{1}$ Institute 1 Institute of Pharmaceutical Biology and Phytochemistry (IPBP), University of Münster, PharmaCampus, Corrensstraße 48, Münster D-48149, münster, Germany; 2 Department of Chemistry, Egerton University, P.O. Box 536, Egerton 20115, Egerton, Kenya; 3 Swiss Tropical and Public Health Institute (Swiss TPH), Socinstr. 57, Basel CH-4051, Basel, Switzerland; 4 University of Basel, Petersplatz 1, Basel CH-4003, Basel, Switzerland DOI 10.1055/s-0037-1608192

In our continued search for new antiprotozoal agents in plants of the family Asteraceae [1], we have investigated Schkuhria pinnata, Tarchonanthus camphoratus and Vernonia lasiopus. Bioassay-guided chromatographic fractionation and isolation from the dichloromethane extracts of the three plants led in addition to pectolinarigenin (5) and benzenepropanol (18) to the identification of three new $(13,14,20)$ and 18 known sesquiterpene lactones (STLs) 
from S. pinnata. Six elemanolide type STLs $(25-30)$ from V. lasiopus and two further STLs from $T$. camphoratus $(1-2)$ were also isolated (Figure 1). The compounds were identified by HR-MS and 1D and 2D NMR in comparison with literature data [2,3]. All these compounds showed interesting in vitro antitrypanosomal activity. Compounds (19) was the most active from S. pinnata with an $\mathrm{IC}_{50}$ value of $0.10 \mu \mathrm{M}$ against Trypanosoma brucei rhodesiense (Tbr) trypomastigotes and selectivity index (SI) of 21.5. From T. camphoratus, 3oxo-1,2-dehydrocostic acid (2) displayed interesting activity with $\mathrm{IC}_{50}$ values of $2.8 \mu \mathrm{M}$ and $0.18 \mu \mathrm{M}$ against $\mathrm{Tbr}$ and axenic grown L.donovani amastigotes and SI values of 6.2 and 95.4 respectively. Vernolepin (26), from V. lasiopus, displayed an $\mathrm{IC}_{50}$ value of $0.19 \mu \mathrm{M}$ against $\mathrm{Tbr}$ and an $\mathrm{SI}$ value of 14.5 . In vivo tests and determination of vernolepin (26) mechanism of action are currently in progress. To the best of our knowledge, elemanolides have not previously been reported to possess anti-trypanosomal activity. These bioactivity data complement previous data obtained in our group and give more insights into the structure- anti-trypanosomal activity relationships of STLs [1].
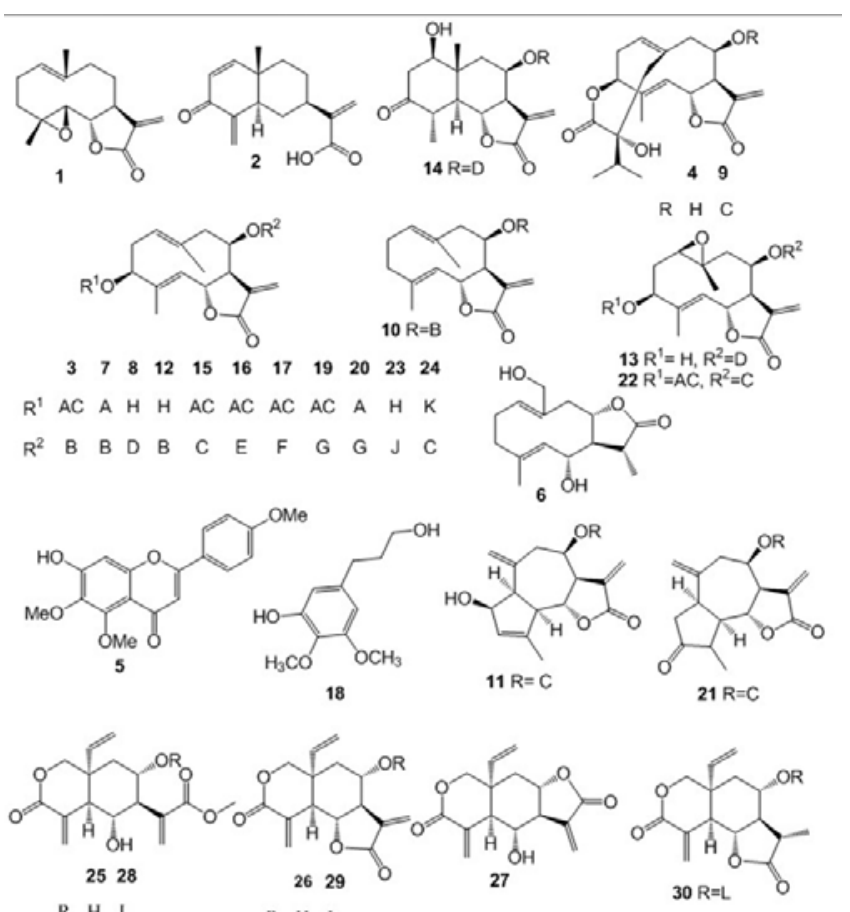

R H L

R H L<smiles>CCC(C)C(C)C(=O)C(C)CO</smiles>

Fig. 1: Compounds isolated from T. camphoratus $(1-2)$, S. pinnnata $(3-24)$ and $V$. lasiopus $(25-30)$

[1] Schmidt, T. J. et al. Antimicrob. Agents Chemother. 2014, 58, 325 - 332.

[2] Ganzer, U. et al. Phytochemistry 1990, 29, 535 - 539.

[3] Jakupovic, J. et al. Planta Med. 1985, 51, $378-380$.

\section{Mo-Poster Session 1-PO-146 The amazing in vivo multistep rearrangement of bilobalide to furopyranons and cyclopentadifurans}

Authors Schötz $\mathrm{K}^{1}$, Bacher $\mathrm{S}^{1}$, Kulic $\mathrm{Z}^{1}$, Koch $\mathrm{E}^{1}$

Institute 1 Preclinical Research, Dr. Willmar Schwabe Pharmaceuticals, 76227

Karlsruhe, Germany

DOI 10.1055/s-0037-1608193

Bilobalide 1, the principal terpenlactone of the Ginkgo special extract EGb $761^{\circledR}$, is a trilactone substituted with a very rare tertiary butyl alcohol group. The compound has several modes of action, which may act together in neuroprotection [1]. The absolute oral bioavailability is about $60-80 \%$ in rats and humans [2,3]. In rats, $31 \%$ of bilobalide is excreted unchanged in urine [3]. Therefore, we were interested in the yet unknown $69 \%$ metabolites of bilobalide.

In order to evaluate the metabolism of bilobalide, rats were treated orally either with pure $1(10 \mathrm{mg} / \mathrm{kg})$ or with EGb $761^{\circledR}(300 \mathrm{mg} / \mathrm{kg})$. Urine was collected during $0-4 \mathrm{~h}$ and $4-8 \mathrm{~h}$ after administration and the samples were analyzed after work-up by solid phase extraction with an ion trap LC-MS ${ }^{2}$. In agreement with former results no phase II metabolites were observed beside some very minor oxidation products. In contrast, several hydrolysis products characterized in the mass spectrum by added 18 Da to the parent mass of 1 were detected.

Since urine samples of treated animals contain only low concentrations of metabolites, the hydrolysis of bilobalide was mimicked in vitro by incubation of 1 under weak basic condition. Indeed, this reaction produced a large variety of compounds that were in agreement with the in vivo products. Preparative isolation and subsequent detailed 2D-NMR studies established an amazing multistep rearrangement of 1 . The product formation is probably initiated by a retro-aldol reaction in combination with hydrolysis and condensation steps (partially presented in Fig. 1) which results for example in furopyranons 2 and cyclopentadifurans 3.
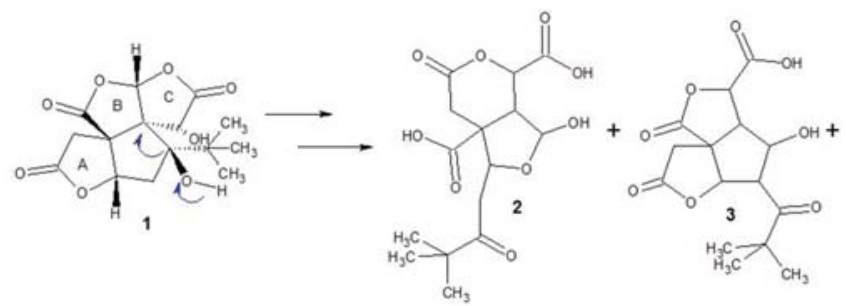

Fig. 1

[1] Defeudis FV. Pharmacol Res 2002; 46: 565 - 568.

[2] Dew TP, Wang G, Williamson G. Biofactors 2014; 40: 268 - 274.

[3] Biber A. Pharmacopsychiatry 2003; 36 Suppl 1: S32-S37.

\section{Mo-Poster Session 1-PO-147 Detection of metabolites in rat heart tissue after oral treatment with the Crataegus special extract WS ${ }^{\circledR} 1442$}

Authors Vogel $\mathrm{S}^{1}$, Schötz $\mathrm{K}^{1}$, Schneider $\mathrm{H}^{1}$, Koch $\mathrm{E}^{1}$

Institute 1 Dr. Willmar Schwabe GmbH \& Co. KG, Karlsruhe, Germany

DOI 10.1055/s-0037-1608194

$W^{\circledR} 1442$ is an aqueous ethanolic (45\% w/w; DER 4-6.6:1) extract from leaves with flowers of officinal Crataegus species registered as a traditional herbal medicinal product to support cardiovascular function. It is standardized for the content of oligomeric B-type procyanidins (OPCs) in the range of 17 to $20 \%$. These ingredients are supposed to be important for the reported cardioand vasoprotective effects. However, it is known that higher oligomers do not reach the blood circulation as such, but are metabolized to smaller entities by intestinal bacteria. Until now it is unknown, whether procyanidin monomers or intermediate metabolites (e.g. $\mathrm{y}$-valerolactones) or even further metabo- 
lized compounds (e.g. phenolic acids) reach the systemic circulation and target tissues where they are possibly responsible for the observed pharmacological activity.

Therefore, in a pilot experiment rats were treated twice daily with $300 \mathrm{mg} / \mathrm{kg}$ WS ${ }^{\circledR} 1442$ for a period of three days. Heart, aorta and liver tissue as well as plasma samples were collected at different time points after the last treatment on day 3. Tissue samples were homogenized in a phosphate buffer and centrifuged. Homogenate supernatants and plasma samples were applied onto a reversed phase column and adsorbed metabolites were subsequently eluted with acidic methanol. After removal of the organic solvent, the residue was taken up and submitted to HPLC-MS/MS and HPLC-MS2 analysis.

In contrast to a previous report (1), only phase II metabolites of $\mathrm{y}$-valerolactone but not the aglycones were found in all three tissues. Further studies are ongoing to more precisely define the pharmacokinetics of these and other metabolites and to investigate their pharmacological properties with respect to the clinical use of WS ${ }^{\circledR} 1442$.

[1] Serra A, Macia A, Romero MP, Angles N, Morello JR, Motilva M]. Food Funct 2011; 2:562- 568

\section{Mo-Poster Session 1-PO-148 Bioactive steroids against ovarian cancer cell line isolated from Malaysian green propolis}

\section{Authors Md Yusoff $Y^{1}$, Edrada-Ebel $R^{1}$}

Institute 1 Strathclyde Institute of Pharmacy and Biomedical Sciences,

University of Strathclyde, Glasgow, United Kingdom

DOI 10.1055/s-0037-1608195

Propolis has shown evidence of its effectiveness in various biological studies such as anticancer, antioxidant, anti-inflammatory, antibiotic and antifungal agents [1].

In this study, we apply metabolomics tools utilising nuclear magnetic resonance (NMR) and liquid chromatography-high resolution mass spectrometry (LC-HRMS) data as a promising approach in targeting the isolation of the bioactive metabolites. The green propolis sample was collected from Malaysia (B-B Town Sdn. Bhd. Malacca, Malaysia) which was colonized by Trigona sp. bees. The bees tend to visit different kind of the host plants, one is Tectona grandis, which is natively found in Southeast Asia including Thailand, Vietnam and later was introduced in Malaysia.

The propolis sample $(800 \mathrm{mg})$ was subjected to extraction using 1:1 water to methanol ratio, followed by using acetone and methanol to obtain the crude extract. All extracts were screened for anticancer activity against the ovarian cancer cell line (A2780). The obtained HRLCMS data was processed with MZmine 2.10 and dereplicated with in-house Excel macro coupled to the Dictionary of Natural Products 2016 (DNP). A supervised multivariate analysis was done by orthogonal partial least squares discriminant analysis (OPLS-DA) in SIMCA-P 14.0 to predict and pinpoint the plausible bioactive components. Based on the mass spectral data, there were three bioactive compounds that were successfully isolated from the predicted bioactive metabolites. All compounds were steroid and triterpenoid derivatives, which were confirmed with 1D and 2D NMR experiments.

[1] Watanabe M. A. E, Amarante, M. K, Conti B. J, Sforcin J. M. Journal of Pharmacy and Pharmacology; 2011: 63, 1378-1386

\section{Mo-Poster Session 1-PO-149 Phytochemical investigations of Berardia subacaulis}

Authors Schwaiger $\mathrm{S}^{1}$, Stuppner $\mathrm{H}^{1}$, Gafner $\mathrm{F}^{2}$

Institute 1 Institute of Pharmacy/Department of Pharmacognosy, University of Innsbruck, Center for Molecular Biosciences Innsbruck, Innrain 80 - 82, Innsbruck, Austria; 2 Mibelle Biochemistry, Bolimattstrasse 1, Buchs, Switzerland

DOI 10.1055/s-0037-1608196
Berardia subacaulis Vill. (syn.: Berardia lanuginosa (Lam.) Fiori) represents a monospecific genus within the Tribus Cynareae of the sunflower family and is endemic in the South-western Alps. Up to now no phytochemical investigations of this rare species, which grows exclusively on high-altitudes (>1700 m asl) were performed. In order to protect the rare natural populations, our investigations used exclusively material of cultivated sources. Investigation of a lipophilic extract $\left(\mathrm{CH}_{2} \mathrm{Cl}_{2}\right)$ of the leaves by HPLC-MS showed two major compounds at $205 \mathrm{~nm}$ which after isolation were identified by 1D- and 2DNMR and LC-MS as artemisiifolin [1] and its ester in position 6 with 3-hydroxy2-methyl-propanoic acid, representing a new natural compound. Investigations of different plant parts by HPTLC and HPLC-DAD showed that the identified sesquiterpene lactones can be found in leaves (green and mature) and mature flower heads without fruits but are absent in roots/rhizomes and fruits. Phytochemical analysis of a methanolic extract (after $\mathrm{CH}_{2} \mathrm{Cl}_{2}$ extraction) of the leaves revealed the presence of chlorogenic acid and 1,5-dicaffeoylquinic acid as well as rutin and isoquercitin as major compounds.

[1] Jimeno ML, Apreda-Rojas MC, Cano FH, Rodríguez B. Magn. Reson. Chem. 2004; 42: $474-483$

\section{Mo-Poster Session 1-PO-150 A supercritical $\mathrm{CO}_{2}$ based workflow for the fast extraction, isolation, and quantification of polar styrax constituents}

Authors Scheuba J ${ }^{1}$, Wronski VK ${ }^{1}$, Rollinger $\mathrm{JM}^{1}$, Grienke $\mathrm{U}^{1}$

Institute 1 Department of Pharmacognosy, University of Vienna, Vienna,

Austria

DOI 10.1055/s-0037-1608197

Recent instrumental advances of $\mathrm{CO}_{2}$ based supercritical fluid (SFx) technology have heralded a new era in natural product labs [1]. Despite the technology's poor reputation of being only suitable for non-polar compounds, the number of studies corroborating its suitability for a large variety of compound classes is increasing. However, so far, the polarity limit has not been reached. Hence, the aim of this study was to develop the first SFx based protocol for the extraction, analysis, and isolation of six polar compounds including ovanillin (1), styracin (2), vanillin (3), trans-cinnamic acid (4), vanillic acid (5), and shikimic acid (6)[2].

After initial method optimisation on an analytical SFC device $\left(\operatorname{UPC}^{2}\right)$, the compounds were successfully baseline separated within 4 min. A supercritical fluid extraction (SFE) protocol was established for eight authentic styrax products (R1-R8) known to contain compounds 2-6. The compounds were quantified and the method was validated according to current $\mathrm{ICH}$ guidelines. Scaling up to preparative SFC allowed for a fast separation and isolation of the selected constituents 2 and 4 from R6 within 7 min.

In sum, this is the first report of a complete SFx workflow for a rapid and green chemistry based extraction, analysis, and isolation of polar compounds from styrax resin products. The protocol is easily adaptable to compounds of similar polarity and offers an environmental friendly alternative to conventional set-ups. Striking advantages of SFx technologies, which will have a lasting impact on future applications, include low solvent consumption, easy solvent removal, as well as high efficiency and selectivity.
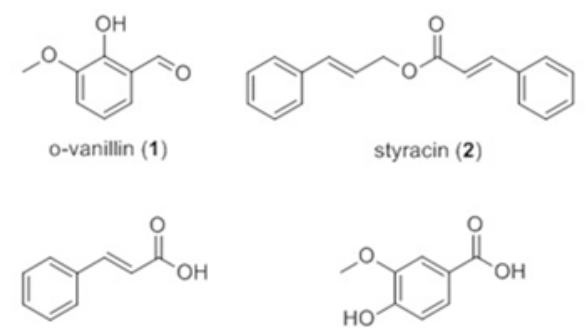

trans-cinnamic acid (4)

Fig. 1 styracin (2)
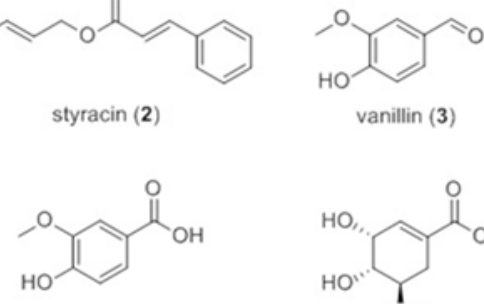

vanillic acid (5)

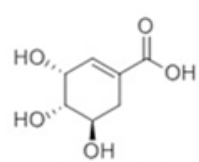

shikimic acid (6) 
This work was supported by the Austrian Science Fund (FWF: P24587).

[1] Hartmann A, Ganzera M. Planta Med 2015; 81: 1570 - 1581

[2] Scheuba J, Wronski V-K, Rollinger JM, Grienke U. Planta Med 2017; http:// dx.doi.org/10.1055/s-0043 - 105499

\section{Mo-Poster Session 1-PO-151 Comparison of the phytochemical composition and antioxidant activity of seeds from different Hypericum species}

Authors Heinrich $\mathrm{M}^{1}$, Lorenz $\mathrm{P}^{1}$, Stintzing $\mathrm{F}^{1}$, Kammerer $\mathrm{D}^{1}$ Institute 1 WALA Heilmittel GmbH, Department of Analytical Development \& Research, Section Phytochemical Research, Bad Boll/Eckwälden, Germany DOI 10.1055/s-0037-1608198

$H$. perforatum is widely used in phytomedicine, among others, for the treatment of depressive episodes [1]. The aerial parts of Hypericum species are known for their complex spectrum of secondary metabolites [2]. Interestingly, subterraneous parts received less attention. Especially data for seeds are scant in literature. The aim of the present study was to systematically compare the seeds of two species, i. e. $H$. perforatum and $H$. tetrapterum, with regard to their metabolite profile. For this purpose, seeds were extracted with dichloromethane and methanol and analyzed by HPLC-DAD-MS ${ }^{n}$ and GC-MS. Moreover, the free radical scavenging capacity of methanolic seed extracts was quantified using the DPPH antioxidant assay. $H$. perforatum and $H$. tetrapterum yielded a fatty oil fraction amounting to $30.5 \%$ and $18.0 \%$ of the seed weight, respectively. Xanthone derivatives, i.e. tetrahydroxyxanthones (THX), xanthone-glycosides and xanthone-sulfonates, were assigned in the methanolic extracts of both species. For structure elucidation, one representative xanthone, namely 1,3,6,7-THX, was synthesized. Total THX contents were quantitated, resulting in $1.25 \mathrm{~g} / \mathrm{kg}(H$. perforatum) and $0.27 \mathrm{~g} / \mathrm{kg}(H$. tetrapterum). $H$. perforatum extracts $\left(\mathrm{IC}_{50}=8.73 \mathrm{mg} / \mathrm{L}\right)$ and 1,3,6,7-THX $\left(\mathrm{IC}_{50}=3.02 \mathrm{mg} /\right.$ $\mathrm{L})$, exhibited good DPPH free radical scavenging activity compared to trolox $\left(I C_{50}=6.61 \mathrm{mg} / \mathrm{L}\right)$. The seeds of Hypericum were found to be a rich source of fatty oil and phenolic compounds exhibiting antioxidant activity, which was attributed to the remarkable xanthone contents. The results indicate, that Hypericum seeds might have promising potential in cosmetic and medicinal products.

[1] Rodríguez-Landa JF, Contreras CM. Phytomedicine 2003; 10:688 - 699.

[2] Brolis M, Gabetta B, Fuzzati N, Pace R, Panzeri F, Peterlongo F. J Chromatogr A 1998; 825: 9-16.

\section{Mo-Poster Session 1-PO-152 Essential oil composition and polar fraction analysis of Tanacetum macrophyllum (Waldst. et Kit.) Schultz Bip.}

Authors Frezza $C^{1}$, Venditti $A^{2}$, Sciubba $F^{2}$, Cianfaglione $K^{3,4}$, Maggi $F^{4}$, Bianco $A^{2}$, Serafini $M^{1}$

Institute 1 University of Rome "La Sapienza” -Department of Environmental Biology, Rome, Italy; 2 University of Rome "La Sapienza” -Department of Chemistry, Rome, Italy; 3 Université de Bretagne Occidentale - EA 2219 Géoarchitecture, UFR Sciences \& Techniques, Brest, France; 4 University of Camerino - School of Pharmacy, Camerino, Italy

DOI 10.1055/s-0037-1608199

Tanacetum macrophyllum (Waldst. et Kit.) Schultz Bip, also known as Tansy, is a perennial herbaceous plant belonging to the Asteraceae family. This species is typical of the Balcan area but is punctually spread in other European countries as a rare species [1]. In Italy, it is found mainly within forests [1, 2]. This species is often erroneously confused with Achillea grandifolia Friv. [1, 2]. In this work, a comprehensive phytochemical analysis on the volatile components and polar fraction of T. macrophyllum growing in central Italy was carried out. Flowers and leaves were separately analyzed for the essential oil composition and were characterized by oxygenated monoterpenes (39.4\%) and oxygenated sesquiterpenes (28.0\%) and sesquiterpene hydrocarbons (39.3\%) and oxygenated monoterpenes (25.4\%), respectively. The phyto- chemical analysis conducted on the ethanolic extract of the total aerial parts evidenced the presence of twelve compounds: apigenin, cirsimaritin, apigenin-7-O-glucoside, apigenin-7-O-glucuronide, kaempferol-7-O-glucoside, kaempferol-7-O-glucuronide, 3,5-dicaffeoylquinic acid, 4,5-dicaffeoylquinic acid, chlorogenic acid, shikimic acid, quinic acid and 4-O- $\beta$-D-glucopyranosyl-vanillic acid. Most of these compounds were reported for the first time in the species while three of them are new phytochemicals for the Tanacetum genus. The presence of all these compounds provides a phytochemical rationale for the botanical classification of this species and encourages further ethno-pharmacological studies just like for T. parthenium [3].

[1] Pignatti S. Flora d'Italia. Bologna, (Italy): Edagricole; 1982, 3, 99.

[2] Cerutti GV, Motta A. Inf Bot Ital 2011; 43: 148.

[3] Pareek A, Suthar M, Rathore GS, Bansal V. Pharmacog Rev 2011; 5: 103 110.

\section{Mo-Poster Session 1-PO-153 Simultaneous HPTLC identification of Fig, Senna and P. hybridus using Ion Exchange Chromatography}

Authors Nägele $B^{1}$, Wüthrich $\mathrm{J}^{1}$, Toff $S^{1}$, Schenk $A^{1}$

Institute 1 Department of Analytical Development, Max Zeller Söhne AG,

Romanshorn, Switzerland

DOI 10.1055/s-0037-1608200

The commercial product fig film coated tablets with Senna contains fig powder and dry extracts of Senna and P. hybridus as active pharmaceutical ingredients. Until now each active ingredient was identified in a separate analysis. Therefore, a new HPTLC technique for simultaneous identification for fig, Senna and P. hybridus was developed for fig film coated tablets with Senna. Fructose, sennosides A/B and cichoric acid were chosen as suitable markers for the corresponding active ingredients fig, Senna and $P$. hybridus. Due to the fact that these three markers have different chemical characteristics, a prepurification step must be performed to separate the active compounds. After extraction using a basic methanol solution, the resulting solution is applied to a cation exchange column. Using $\mathrm{pH}$ defined solutions, the three markers are eluted into two different fractions. After application of the fractions onto an HPTLC plate and development of the plate (mobile phase: formic acid:water:2butanone:ethyl acetate $(10: 10: 40: 40)(\mathrm{V}: \mathrm{V}: \mathrm{V}: \mathrm{V}))$, three different staining solutions are used for visualization. During the first staining, the plate is immersed in natural product reagent followed by PEG400. This step visualizes cichoric acid, which is the marker of P. hybridus, at $366 \mathrm{~nm}$. The second immersion step into thymol reagent visualizes the markers fructose and sennosides $A / B$ of fig and Senna, respectively, at Vis light and $366 \mathrm{~nm}$. With this method we are, therefore, able to analyze all relevant markers on one HPTLC-Plate. These markers only elute in the corresponding fractions and no co-elution is observed. The technique allows identification of all chosen markers in newly manufactured fig film coated tablets as well as stability samples of the product. Validation of this analytical procedure shows that it is selective, specific and robust.

\section{Mo-Poster Session 1-PO-154 Chemical Constituents from the Formosan Octocoral Nephthea columnaris}

Authors Whuang $\mathrm{TY}^{1,2}, \mathrm{Wu} Y \mathrm{~J}^{3}$, Sung PJ ${ }^{1,2}$

Institute 1 Graduate Institute of Marine Biology, National Dong Hwa University, Pingtung, Taiwan; 2 National Museum of Marine Biology and Aquarium, Pingtung, Taiwan; 3 Department of Biological Technology, Meiho Universtiy, Pingtung, Taiwan

DOI 10.1055/s-0037-1608201

In the studies on the chemical constituents of octocoral Nephthea columnaris, collected off the coast of Southern Taiwan, had led to the isolation of 11 metabolites, including ten sterols, columnaristerols A-C (1-3), litosterol (4),

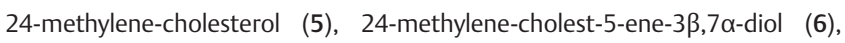

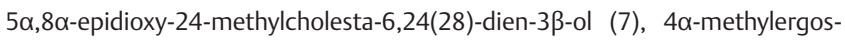


ta-22(E),24(28)-dien-3ß-ol (8), 24-methylcholesta-5,24(28)-diene-3ß,19-diol-

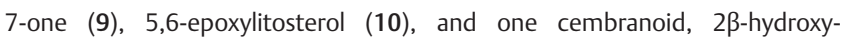
$11 \alpha, 12 \beta$-epoxynephthenol (11). Among these natural products, columnaris-

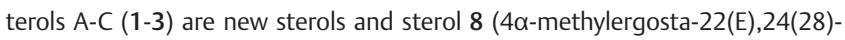
dien-3 $\beta$-ol) was first being reported from a natural source. The structures of above metabolites were determined by spectroscopic methods. Columnaristerol A (1) was proven to be a rare natural 19-norsterol possessing a $10 \beta$ hydroxy group and this compound was found to exhibit cytotoxic effects toward MOLT-4 and SUP-T1 cells.
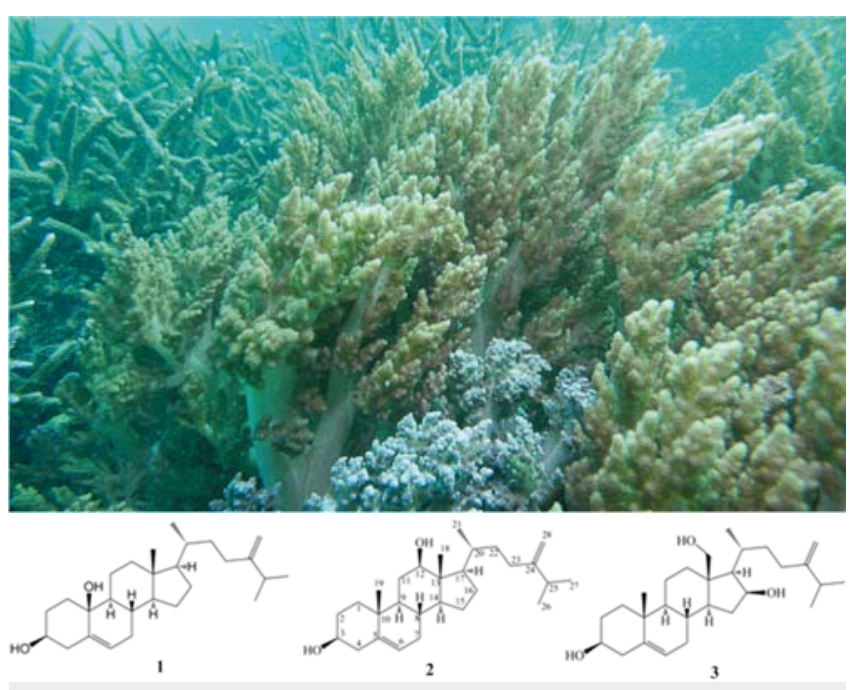

Fig. 1

\section{Mo-Poster Session 1-PO-155 VARIATION OF LIPIDS INDEXES IN POLLEN WITH ITS BOTANICAL ORIGIN}

Authors Anjos $\mathrm{O}^{1,2}$, Campos Maria $\mathrm{G}^{3,4}$, Dias $\mathrm{T}^{5}$, Estevinho $\mathrm{LM}^{5,6}$

Institute 1 Instituto Politécnico de Castelo Branco, Castelo Branco, Portugal; 2 Centro de Biotecnologia de Plantas da Beira Interior, Castelo Branco, Portugal; 3 Observatory of Herb-Drug Interactions/Faculty of Pharmacy, University of Coimbra, Heath Sciences Campus, Coimbra, Portugal; 4 Coimbra Chemistry Centre (CQC, FCT Unit 313) (FCTUC) University of Coimbra,

Coimbra, Portugal; 5 Agricultural College of Bragança Polytechnic Institut of Bragança, Bragança, Portugal; 6 Center of Molecular and Environmental Biology, University of Minho, Braga, Portugal

DOI 10.1055/s-0037-1608202

Bee pollen is one of nature's healthful food products with promising nutritional and therapeutic properties due to its chemical composition, particularly its protein content, which includes almost all the essential amino acids. Nevertheless the composition in lipids it is not completely known and supposedly will be highly variable depending on the floral origin. As such, this parameter must be ascertained in line with the research for bioactivity [1].

The aim of this work was to evaluate some health-related lipid indexes of bee pollen, namely omega-6/omega-3 fatty acid ratio (n-6/n-3); polyunsaturated fatty acid/saturated fatty acid ratio (PUFA/SAT), atherogenic index (AI) and thrombogenic index ( $\mathrm{TI})$ in samples harvested in Portugal. The selected parameters were calculated from the Fatty-Acid Profile, which was determined as previously reported by Bárbara et al. [3].

Bee pollen samples, after harvest, were cleaned and frozen at $-20{ }^{\circ} \mathrm{C}$ and were codified according the predominant pollen. Figure 1 associates the lipid indexes of the different samples with their botanical origins.

$\mathrm{n}-6 / \mathrm{n}-3$ and PUFA/SAT ratios were in within the limits recommended by World Health Organization (below 4.0 and above 0.45 , respectively), suggesting that bee pollen is a good product with the nutritional point of view, with potential beneficial effects for the consumer's health. Also, both $\mathrm{Al}$ and $\mathrm{TI}$ indexes of this natural product were low, even though this effect depended on bee pollen's botanical origin (Figure 1).

[1] Campos MG, Olena L. and Anjos O. 2016. Chapter 3, Chemical Composition of Bee Pollen. In Cardoso SM \& Silva AMS, Chemistry, biology and potential applications of honeybee plant-derived products, Bentham Science Publishers, United Arab. Pp. 67 - 88 (22)

[2] Bárbara, M.S, Machado, C.S, Sodré, G.D.S, Dias, L.G, Estevinho, L.M, \& de Carvalho, C.A.L. Molecules, 2015,20, 12525 - 12544

\section{Mo-Poster Session 1-PO-156 EXTRACTIVE METHOD OPTIMIZATION FOR Ligustrum lucidum THAT LEADS TO A BETTER FREE RADICAL SCAVENGING ACTIVITY}

Authors Delgado $T^{1}$, Campos Maria $\mathrm{G}^{2,3}$, Farinha $\mathrm{N}^{1}$, Estevinho $\mathrm{LM}^{4,5}$, Anjos $\mathrm{O}^{1,6}$

Institute 1 Centro de Biotecnologia de Plantas da Beira Interior, Castelo Branco, Portugal; 2 Observatory of Herb-Drug Interactions/Faculty of Pharmacy, University of Coimbra, Coimbra, Portugal; 3 Coimbra Chemistry Center (CQC, FCT Unit 313) (FCTUC) University of Coimbra, Coimbra, Portugal; 4 Portugal Center of Molecular and Environmental Biology, University of Minho, Braga, Portugal; 5 Agricultural College of Bragança Polytechnic Institut of Bragança, Bragança, Portugal; 6 Instituto Politécnico de Castelo Branco, Castelo Branco, Portugal DOI 10.1055/s-0037-1608203

Ligustrum lucidum Aiton is a plant with important biological properties and its berries are used in Chinese medicine with tonic effects, used as a protection against some diseases due to the presence of antioxidants [1]. No research has been done in Portuguese samples and the aim of this work was to optimize the extraction process that gives the best antioxidant activity for L. lucidum berries from two different regions in Portugal (Region1: Lisbon and Region 2: Castelo Branco).

Over the years several extraction methods have been developed in order to obtain the best antioxidant extract and their choice depends on the chemical structure of the active components. For this propose three different solvents were tested: boiling water ( $45 \mathrm{~min}$ ); $100 \%$ ethanol (shacked 6, 12 and 24 hours) and 50\% ethanol-water (v/v) (shacked 6, 12 and 24 hours). All experiments were performed in duplicate, at room temperature in the dark.

For berries growing in region 2 the higher extraction yields were obtained with $100 \%$ ethanol and $50 \%$ ethanol-water. No significant differences were observed between different extraction solvents or between extraction times for region 1.

In terms of antioxidant activity, the screening method chosen was 2,2-diphenyl-1-picrylhydrazyl UV quantification at $517 \mathrm{~nm}$. The extracts with higher antioxidant activity, measured as EC50 ( $\mathrm{mg} / \mathrm{ml})$, were performed with water and $50 \%$ ethanol-water. Nevertheless, with no significant differences on average, the antioxidant activity increased a little with the increase of the extraction time and the variability decrease. Given this results we propose 15 hours of extraction for all extracts performed with water and $50 \%$ ethanol-water. These results showed also that $L$. lucidum berries growing in Portugal have antioxidant potential. However, their activity varies among these two regions. [1] Li ML, Lui ML, Feng WH. Zhongguo Zhongyao Zhazhi 1994, 19: 504.

\section{Mo-Poster Session 1-PO-157 Marker Compounds of Microbial Infection in Allium cepa}

Authors Balash $\mathrm{A}^{1}$, Keusgen $\mathrm{M}^{1}$

Institute 1 Philipps-Universität Marburg, Marburg, Germany

DOI 10.1055/s-0037-1608204

Common onion (Allium cepa) is considered as one of the most important vegetables. ${ }^{1}$ Some bacteria and fungi can infect the onion and spoil the bulbs within a short period of time. ${ }^{2}$ The early stages of infection cannot be detected in onions by conventional analytical methods. It can be assumed that the pattern of chemical compounds is altered by the action of pathogenic 
bacteria and fungi. In this project, four types of microorganisms (Fusarium oxysporum, Fusarium proliferatum, Penicillium sp, Botrytis aclada) were tested in onion bulbs to elucidate and identify the volatile chemical markers after infection. Healthy and infected onion bulbs were extracted by ethyl acetate. The extracts have been analysed by HPLC to define the infection markers. Two different new peaks were identified in the chromatograms that can refer to marker compounds. Fractions were investigated to prove its chemical structure by HPLC/MS, NMR and HRMS. According to the obtained data, the two compounds are 2-hexyl-5-methyl-3(2 H)-furanone and 2-octyl-5-methyl-3 $(2 \mathrm{H})$-furanone (Fig.). Because these compounds only occur after infection, they can be considered as infection markers.

[1] Keusgen M. Volatile Compounds of the Genus Allium L. (Onions). Marburg: University of Marburg; 2010: 2 - 3.

[2] Shrestha H. A Plant Monograph on Onion (Allium cepa L.). Nepal: Pokhara University; 2007: 31.

\section{Mo-Poster Session 1-PO-158 Fingerprint profiling of traditional Uzbekistan medicine Eryxin by gas chromatography- mass spectrometry}

Authors Akbarov $\mathrm{U}^{1}$, Shikov $\mathrm{A}^{2}$, Laakso $\mathrm{I}^{3}$, Pozharitskaya $\mathrm{O}^{2}$, Makarov $\mathrm{V}^{2}$, Vuorela $\mathrm{H}^{3}$

Institute 1 Tashkent Medical Academy, Tashkent, Uzbekistan; 2 Saint Petersburg Institute of Pharmacy, St-Petersburg, Russia; 3 University of Helsinki, Helsinki, Finland

DOI 10.1055/s-0037-1608205

Eryxin represents a highly purified, deprotonated $1 \%$ aqueous hydrolysate prepared from the biomass of snakes of the Eryx genus. It is used in the traditional medicine of Uzbekistan as anti-inflammatory, hepatoprotective and immunomodulatory drugs which have been found effective in cases of rheumatological diseases, tuberculosis, brucellosis, acute obstructive or chronic bronchitis and dermatosclerosis [1].

However, the chemical composition of the Eryxin is not well investigated. The aim of the study was to analyze the composition of Eryxin by gas chromatography-mass spectrometry (CC-MS). The GC-MS analysis was carried out using a Hewlett-Packard Series 5890 gas chromatograph (Hewlett-Packard, PA, USA) coupled to an HP 5970 quadrupole mass selective detector operating in EI mode. An Rxi-5 ms column ( $15 \mathrm{~m} \times 0.25 \mathrm{~mm}$ ID, $0.25 \mu \mathrm{m}$ phase, Restek, PA, USA) was used with helium as the carrier gas. Dried extracts were silylated with $50 \mu \mathrm{L}$ of $\mathrm{N}$-methyl- $\mathrm{N}$-trimethylsilyl-trifluoroacetamide containing $1 \%$ trimethylchlorosilane.

Thirty seven compounds, constituting more than $95 \%$ of samples, were characterized. Typical major compounds found in Eryxin were 5-aminovaleric acid and its lactam, isovaleric, butanoic, succinic and hydrocinnamic acids. Alanine, valine, tryptamine and leucine were the most abundant amino acids. Other short-chain organic acids such as valeric, caproic, hydroxybutyric, aminobutyric, hydroxihexanoic, pipecolinic and pyrotartaric acids were detected in minor concentrations only. Phosphoric acid, together with palmitic and stearic acids suggested the occurrence of phospholipids.

To our knowledge, this is the first approach to the chemical profiling of Eryxin by using GC-MS. Future detailed studies are necessary for more clear explanation of pharmacological effects.

[1] Asatova S.S, Komarin A.S, Karimov M.S, Akbarov S.V. (2003). Pharm Chem J, $37,63-66$.

\section{Mo-Poster Session 1-PO-159 Only horses in danger?}

Authors Goerick $C^{1}$, Melzig $M^{1}$

Institute 1 Institute of Pharmacy, Freie Universität Berlin, Berlin, Germany DOI 10.1055/s-0037-1608206

Some Acer species like box elder (Acer negundo L.) and sycamore (Acer pseudoplatanus L.) are known as cause of atypical pasture myopathy in Europe [1]. Hypoglycin A is described as a constituent in the seeds of several members of
Aceraceae and Hippocastanoideae [2]. It is a toxic amino acid. The danger for intoxication is high by ingestion of the fruits and seeds especially in autumn and possible in blossoms or in the seedlings in the spring. Veterinarians recommend removing these plants near of pastures because of this illness of horses. One question is if other animals for example squirrels, wild boar, or insects also can be damaged after consumption. Simple extraction and high performance liquid chromatography method with UV detection at room temperature were developed to measure the presence of Hypoglycin A in plant extracts. Material of various parts of different Acer species was measured. A rapid detection of Hypoglycin A by HPTLC is not possible because of the presence of Leucin and Isoleucin in the samples with similar chromatrographic properties producing false positive results [3].

[1] Bochnia M. et al. PLOS ONE 2015, 10: e0136785;

[2] Frowden L, Pratt H Phytochemistry 1973, 12

[3] Baise E et al. Equine Veterinary Journal 2015, 1 - 4

\section{Mo-Poster Session 1-PO-160 Biopharmaceutical classification of Cimicifuga racemosa extract Ze 450}

Authors Disch $\mathrm{L}^{1}$, Siewert $\mathrm{B}^{1}$, Forsch $\mathrm{K}^{1}$, Drewe $\mathrm{J}^{1}$, Fricker $\mathrm{G}^{2}$

Institute $1 \mathrm{Max}$ Zeller Söhne AG, Romanshorn, Switzerland; 2 RuprechtKarls-University, Heidelberg, Germany

DOI 10.1055/s-0037-1608207

For classification of Cimicifuga racemosa extract Ze450 into the Biopharmaceutics classification system (BCS), up to four different substances of the main constituent class triterpene glycosides were selected to serve as analytical marker. The $\mathrm{pH}$-dependent thermodynamic solubility was determined via shake-flask method and apparent permeability $\mathrm{P}_{\text {app }}$ was determined using Caco-2 monolayer assay. The solubility profiles of tested triterpene glycosides actein, 23-epi-26-deoxyactein, cimiracemosid $\mathrm{C}$ and 4-O-acetyl-hydroshengmanol-4-O- $\beta$-D-xylopyranosid showed $\mathrm{pH}$ dependent solubility with a maximum at $\mathrm{pH} 7.5$ and a minimum at acidic $\mathrm{pH}$ values. In the scope of Caco-2 monolayer assay, 23-epi-26-deoxyactein was tested. It showed a high $\mathrm{Papp}_{\text {app }}$ (apparent permeability) from apical to basolateral which was found to be lower in the opposite direction. These findings imply a good uptake of 23epi-26-deoxyactein associated with a low efflux transport. Due to the high solubility in combination with high permeability, triterpene glycosides can be classified into BCS class I. Furthermore, dissolution performance of a herbal medicinal product containing Cimicifuga racemosa extract Ze450 was assessed, using actein and 23-epi-26-deoxyactein as analytical marker. Both showed rapid dissolution according BCS criteria. The results suggest that dissolution is no critical step in liberation process of triterpene glycosides. Based on this assumption, good bioavailability of triterpene glycosides can be postulated. However, results allow no prediction of the bioavailability of the whole Cimicifuga racemosa extract Ze450.

\section{Mo-Poster Session 1-PO-161 Phytochemical study of Juglans regia $\mathrm{L}$. leaves}

Authors Schwindl $S^{1}$, Kraus $B^{1}$, Heilmann $J^{1}$

Institute 1 University of Regensburg, Institute of Pharmaceutical Biology,

Regensburg, Germany

DOI $10.1055 / \mathrm{s}-0037-1608208$

Juglans regia L. is a deciduous tree which most likely is native to Central Asia, Anatolia, northern parts of Iran and Himalaya. Nowadays it is cultivated nearly all over the world for walnut production [1]. Its aromatic odd-pinnate leaves are used in folk medicine for treatment of i. e. venous insufficiency, diarrhea, hypoglycemia, hypotension, skin tuberculosis, inflammations of mouth and marginal blepharitis [2 -4]. The leaves contain phenolics like naphtoquinones, tannins, flavonoids, phenolcarboxylic acids and diarylheptanoids as well as monoterpenes, sesquiterpenes and megastigmanes [4-6]. In order to find potential new active secondary metabolites an EtOAc soluble fraction of a methanolic J. regia leaf extract was fractionated by various chromatographic 
techniques. Finally, ten hitherto unknown megastigmane glucoside derivatives (juglanionosides A-K, 1-10) and six tetralone glucoside derivatives (juglanosides J-O, 11-16) along with 24 already known compounds were isolated. 16 of them are reported to be isolated from the genus Juglans for the first time. Their structures were elucidated on basis of 1D and 2D NMR and mass spectrometric analysis.

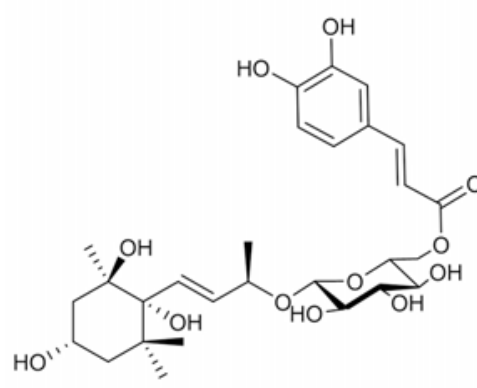

juglanionoside $A$

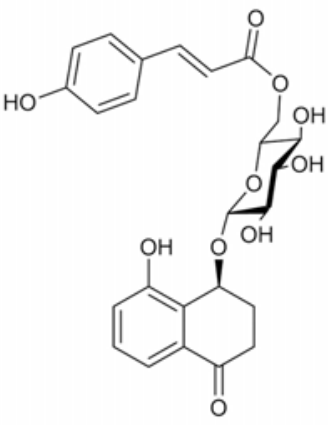

juglanoside $J$
Fig. 1

[1] Schaarschmidt, H. Die Walnussgewächse: Juglandaceae, 3 rd ed; Westarp Wissenschaften: Hohenwarsleben, 2006.

[2] Bruneton, J. Pharmacognosie, phytochimie, plantes médicinales, 2nd ed; Lavoisier: Paris, 1993.

[3] Wichtl, M.; Czygan, F.-C, Eds. Teedrogen und Phytopharmaka: Ein Handbuch für die Praxis auf wissenschaftlicher Grundlage, 4th ed; Wiss. Verl.-Ges: Stuttgart, 2002.

[4] Eisenman, S.; et al. Medicinal plants of Central Asia: Uzbekistan and Kyrgyzstan; Springer: New York, 2013.

[5] Bou Abdallah, l.; et al J Essent Oil Res 2016, 28, 545-550.

[6] Forino, M.; et al. J Funct Foods 2016, 26, $731-738$.

\section{Mo-Poster Session 1-PO-162 Chemical Constituents and Anti-inflammatory Principles from the Fruits of Forsythia suspense}

Authors Ping-Chung $\mathrm{K}^{1}$, Hsin-Yi $\mathrm{H}^{1}$, Tian-Shung $\mathrm{W}^{1}$

Institute 1 1. School of Pharmacy, National Cheng Kung University Hospital, College of Medicine, National Cheng Kung University, Tainan 701, Taiwan,

Tainan, Taiwan

DOI 10.1055/s-0037-1608209

Fifty compounds were isolated from the fruits of Forsythia suspensa, including 13 new compounds characterized as eight new diterpenoids (1-8), three new lignans (9-11), a new iridoid (12), and a new triterpenoid (13). Their structures were established on the basis of spectroscopic and spectrometric analysis. Most of the isolated compounds were examined for their anti-inflammatory activity in vitro. The results showed that several compounds displayed significant inhibition of $\mathrm{FMLP} / \mathrm{CB}$-induced superoxide anion generation and elastase release, with $\mathrm{IC}_{50}$ values ranging from $0.6 \pm 0.1$ to $8.6 \pm 0.8 \mu \mathrm{g} / \mathrm{mL}$ and from $0.8 \pm 0.3$ to $7.3 \pm 1.1 \mu \mathrm{g} / \mathrm{mL}$, respectively.

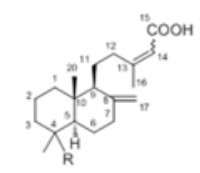

$113(\mathrm{Z}), \mathrm{R}=-\mathrm{CH}_{2} \mathrm{OH}$

2 13(E), $\mathrm{R}=-\mathrm{CH}_{2} \mathrm{OH}$ $413(Z), \mathrm{R}=-\mathrm{CH}_{2} \mathrm{OCHO}$ $5 \quad 13(Z), R=\cdots \mathrm{CO}_{2} \mathrm{H}$
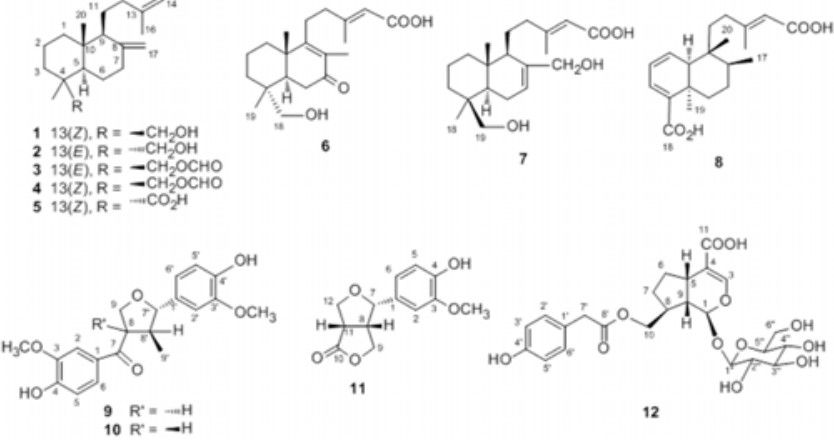

11

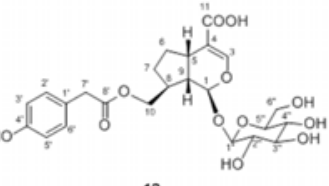

$\begin{array}{ll}9 & R^{\prime}=\ldots+H \\ 10 & R^{\prime}=-H\end{array}$

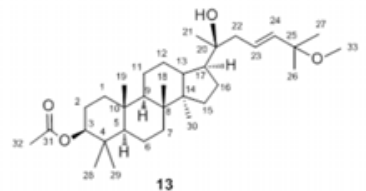

Fig. 1: Structures of compounds $1-13$.

\section{Mo-Poster Session 1-PO-163 Simple non-specific enzymatic oxidation method for studying the bioactivity of phenolic compounds in plants}

Authors Kim J ${ }^{1}$, Pälijärvi $\mathrm{M}^{1}$, Karonen $\mathrm{M}^{1}$, Salminen JP ${ }^{1}$

Institute 1 Natural Chemistry Research Group, Department of Chemistry,

University of Turku, Turku, Finland

DOI 10.1055/s-0037-1608210

A new oxidation method was developed to reveal species- and tissue-level bioactivity of plants. The method combines an existing alkaline oxidation method [1] with an in situ enzymatic oxidation step. Plant samples are incubated in an oven after freezing, enabling polyphenol oxidase (PPO) and other oxidizing enzymes getting into contact with phenolic compounds in the plant, resulting in oxidation of the compounds. As the results are analyzed using total phenolics assay on well plate reader and ultrahigh-performance liquid chromatography coupled to a diode array detector and mass spectrometer (UHPLC-DAD-MS), alterations in the levels of total phenolics and individual compounds can be observed. Depending on the available equipment in a laboratory, either of the analysis methods or both can be utilized.

Oxidation of individual compounds during in vitro alkaline oxidation tended to be consistent, whereas in situ enzymatic oxidation resulted in varying levels of oxidation of the same compound depending on the species and plant tissues. For example, a well-known PPO substrate 5-O-caffeoylquinic acid (5-CQA) all but disappeared from Menyanthes trifoliata L, but in Prunus padus it remained (Fig. 1), indicating that $P$. padus does not contain much PPO at least in active state.

The method provides a new way to inspect bioactivity of plants and the phenolic compounds they contain. The method is physiologically relevant, since the plant's own phenolics are oxidized by the plant's own enzymes, instead of phenolic extracts being oxidized by commercial enzymes, or extracted enzymes presented with substrates not necessarily present in the plant. 

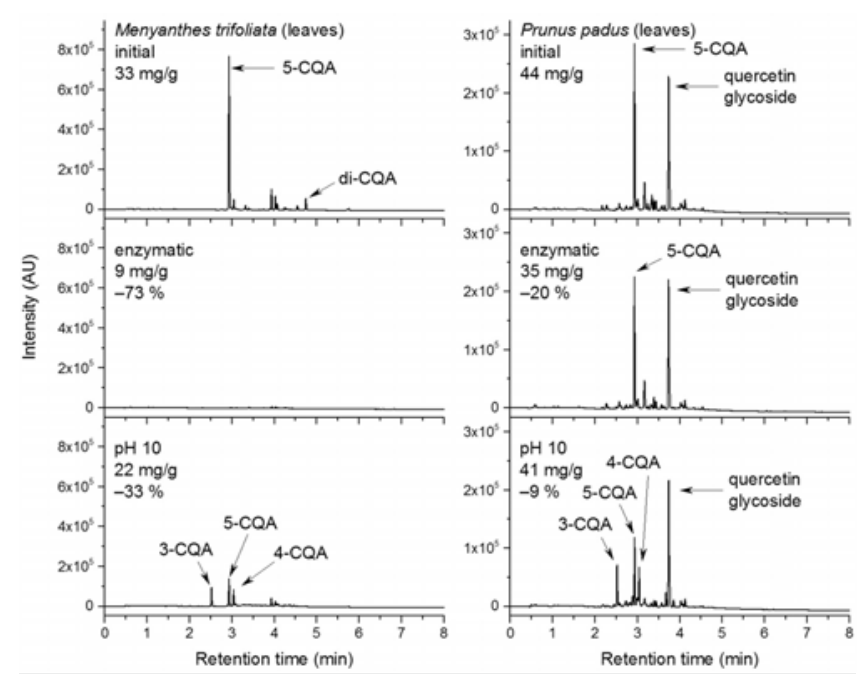

Dig. 1 UV chromatograms at $280 \mathrm{~nm}$ of Menyanthes trifoliata and Prunus padus. Top: Initial state. Middle: after enzymatic oxidation. Bottom: after alkaline oxidation. \% refer to total phenolics assay result.

[1] Vihakas M, Pälijärvi M, Karonen M, Roininen H, Salminen J-P. Phytochemistry 2014; $103: 76-84$.

\section{Mo-Poster Session 1-PO-164 Differences in the Alkaloid Composition of Glaucium leiocarpum Boiss. Growing in Northern and Southern Areas of Turkey}

Authors Demır $S^{1}$, Ulkar $D^{1}$, Kaya GI ${ }^{1}$, Onur MA ${ }^{1}$, Unver SN ${ }^{1}$, BOZKURT B ${ }^{1}$, Institute 1 Department of Pharmacognosy, Faculty of Pharmacy, Ege University, Bornova, Izmir 35100, Turkey, izmir, Turkey

DOI 10.1055/s-0037-1608211

The genus Glaucium (Papaveraceae) is represented by seven species in Turkey. One of these seven species Glaucium leiocarpum Boiss. grows in many places in Anatolia (1). These species are rich in alkaloid content (2). Glaucium species commonly contain glaucine alkaloid, which is used in some European countries for its antitussive effect (3). In this study, the specimens of Glaucium leiocarpum were collected from Bucak-Burdur (Southwest part of Turkey) and Torul-Gümüşhane (Northeast of Turkey). Aerial parts of these plants were analyzed by GC-MS (Gas chromatography-Mass spectrometry). GC-MS is a fast and reliable method for determination of the components in complex alkaloid mixtures. The alkaloids were detected by comparing their mass spectral fragmentation with standard reference spectra from the NIST MS search 2.0, or by GC/MS co-chromatography with previously isolated authentic standards. Moreover, data obtained from literature were used for the identification of the alkaloids. Totally seventeen alkaloids were determined in Glaucium leiocarpum alkaloidal extracts. In the specimen, collected from Torul-Gümüşhane, glaucine was the major component (\% 82.17) and other important alkaloids were identified as $\mathrm{N}$-Methyl laurotetanine and corydine. In the other specimen, collected from Bucak-Burdur region, isocorydine was the major component (\% 87.56) and glaucine was identified in quite low amount (\% 1.57).

This study was financially supported by TUBiTAK (Project No: 3155064 ) and EBILTEM (Project No: 2016/BiL/002). We thank Ege University, Faculty of Pharmacy, the Research Laboratory of Pharmaceutical Sciences (FABAL) for facilitating GC-MS analysis.

[1] Cullen J. "Glaucium A." in Davis P.H. (ed). Flora of Turkey and the East Aegean Islands 1, 214, Edinburgh: University Press; 1965.

[2] Gözler T. Planta Med 1982; 46: $179-180$.

[3] Cortijo J, Villagrasa V, Pons R, Berto L, Marti-Cabrera M, Martinez-Losa M, Domenech T, Beleta J, Morcillo EJ. Brit J Pharmacol 1999; 127: 1641 - 1651.

\section{Mo-Poster Session 1-PO-165 Phytochemical and biological research on Herniaria hirsuta}

Authors Peeters $L^{1}$, Bijttebier $S^{1}$, Foubert $K^{1}$, Van der Auwera $A^{1}$, Pieters $L^{1}$ Institute 1 Natural products \& Food Research and Analysis (NatuRA), Department of Pharmaceutical Sciences, University of Antwerp, Antwerp, Belgium

DOI 10.1055/s-0037-1608212

Urinary stone disease is considered as an important healthcare problem which affects $10-15 \%$ of the population. In the absence of prophylactic therapies, recurrence rates are as high as $50 \%$ within 10 years. An aqueous extract of the aerial parts of Herniaria hirsuta (Caryophyllaceae) is a widely used herbal medicine. Despite its proven activity against urolithiasis, little is known about the active compounds and the mechanism of action. $(1,2)$ Previous phytochemical research on Herniaria species revealed a limited amount of compounds including saponins, flavonoids and coumarins. (2)

As a first step in the quest for the active constituents, phytochemical research on the aerial parts of the dried herb was performed, using a comprehensive extraction. (3) An aliquot was analysed with ultra-high performance liquid chromatography-photodiode array-high resolution mass spectrometry (UHPLC-PDA-HRMS). Multiple flavonoids and saponins were (tentatively) identified with analytical standards or based on spectral and chromatographic data. Besides the known saponins, 7 new saponins were tentatively identified as glycosides of acetylated medicagenic acid, zanhic acid and medicagenic acid.

It is suggested that gastro-intestinal and/or hepatic metabolites of phytochemicals present in $\mathrm{H}$. hirsuta (most likely saponin metabolites) are responsible for the beneficial effects. (2) An in vitro gastro-intestinal model will be used to simulate the biotransformation of the extracts in the stomach, small intestine and colon, to avoid extensive in-vivo studies. The well-characterized metabolised extract will be evaluated in vitro for its activity against urinary stones.

[1] Atmani F, Slimani Y, et al. J Ethnopharmacol. 2004;95:87 - 93.

[2] van Dooren I, Foubert K, et al. Planta Med. 2016;82:1576 - 83.

[3] Bijttebier S, Van der Auwera A, et al. chimica acta. 2016;935:136 - 50.

\section{Mo-Poster Session 1-PO-166 Antioxidant and Acetylcholinesterase Inhibitory Activities of Prenylated Dihydrostilbenes from Macaranga heynei}

Authors Kamarozaman Aisyah $\mathrm{S}^{1,2,3}$, Ahmat $\mathrm{N}^{2,3}$, Nik Azmin Nik $\mathrm{F}^{2,3}$, Hafiz Zetty $Z^{2,3}$, Adenan Mohd $\mathrm{I}^{2,3}$

Institute 1 Centre of Foundation Studies, Universiti Teknologi MARA,

Selangor Branch, Dengkil Campus, 43800 Dengkil, Selangor, Malaysia;

2 Faculty of Applied Sciences, Universiti Teknologi MARA, 40450 Shah Alam, Selangor, Malaysia; 3 Atta-ur-Rahman Institute for Natural Product Discovery, Universiti Teknologi MARA, Selangor Branch, Puncak Alam Campus, 42300

Bandar Puncak Alam, Selangor, Malaysia

DOI 10.1055/s-0037-1608213

Macaranga which comprises of 300 species has abundant source of flavonoids, stilbenoids and terpenoids [1,2]. The chemical constituents isolated have demonstrated broad spectrum of pharmacological properties [2,3]. Hence, $M$. heynei was selected to examine the phytochemical study and bioactivities. The powdered leaves $(2 \mathrm{~kg})$ was macerated in methanol at room temperature for 24 hours to obtain methanolic crude extract $(300 \mathrm{~g})$ which was dissolved in $80 \%$ aqueous methanol followed by partition with hexane and ethyl acetate. The ethyl acetate crude extract $(150 \mathrm{~g})$ was fractionated by using vacuum liquid chromatography $(\mathrm{VLC})$ with a gradient mixture of hexane:ethyl acetate to give seven combined fractions ( $\mathrm{MH} 1-7)$. Fraction $\mathrm{MH} 2(1 \mathrm{~g})$ was subjected to radial chromatography (RC) twice with gradient eluent of hexane:ethyl acetate and chloroform:ethyl acetate subsequently to afford two pure compounds (1) $(10 \mathrm{mg})$ and $(2)(12 \mathrm{mg})$. By using VLC (hexane:ethyl acetate), column (chloroform:acetone) and preparative thin layer chromatography 
(hexane:ethyl acetate) on fraction $\mathrm{MH} 3(10.6 \mathrm{~g})$, a pure compound (3) $(14.7 \mathrm{mg})$ was successfully isolated. Based on spectroscopy analyses, the compounds were characterized as laevifolins A (1) and B (2) as well as a new compound $X(3)$. These compounds exhibited strong activity on DPPH radical scavenging $(<5 \mathrm{uM})$ as well as moderate activity for acetylcholinesterase inhibitory (<20 uM) (Figure 1).<smiles>[R]c1c(O)c(Br)cc(CCc2c(O)cc(O)c(CC=C(C)C)c2CC=C(C)C)c1Br</smiles>

(1) $R_{1}$ -

$$
\text { 年 }
$$

$\mathrm{R}_{2} \cdot \mathrm{OH}$

$\mathrm{R}_{3} \cdot \mathrm{H}$

$\mathrm{IC}_{50}$ (DPPH) $-4.80 \mu \mathrm{M}$

IC 50 (AChe) - $17.3 \mu \mathrm{MI}$

$$
\begin{aligned}
& \text { (2) } \mathrm{R}_{1} \cdot \mathrm{H} \\
& \mathrm{R}_{2} \cdot \mathrm{OH}
\end{aligned}
$$

(3) $\mathrm{R}_{1} \cdot \mathrm{H}$

$\mathrm{R}_{3} \cdot \mathrm{H}$

$\mathrm{IC}_{50}(\mathrm{DPPH})-4.98 \mu \mathrm{ul}$ $\mathrm{IC}_{50}$ (AChe) $->20 \mu \mathrm{M}$
Fig. 1

Greatest appreciation to Society for Medicinal Plant and Natural Product Research for the GA travel grant, MOHE (RAGS/1/2014/SG01/UITM/4) and Faculty of Applied Sciences for the financial support.

[1] Whitmore TC. Malayan Nature Journal 1967; 20: 89-99

[2] Lim TY, Lim YY, Yule CM. Food Chemistry 2009; 114: 594 - 599

[3] Magadula J]. Journal of Medicinal Plant Research 2014; 8:489 - 503

\section{Mo-Poster Session 1-PO-167 Polyalongirin A-B, two novel linked compounds from the twigs of Polyalthia longifolia}

Authors Yun-Sheng $L^{1}$, Chia-Yi $G^{2}$

Institute 1 Department of Biological Science and Technology, Meiho University, Pingtung, Taiwan; 2 Department of Biological Science and Technology, National Pingtung University of Science and Technology, Pingtung, Taiwan

DOI 10.1055/s-0037-1608214

Two novel linked compounds, Polyalongirin A (1) and Polyalongirin B (2), have been isolated from the twigs of Taiwanese Polyalthia longifolia. They were composed of a clerodane diterpene linked to an aprophine alkaloid, is an unprecedented natural N-1 and C-16 linked compound. The stuctures of 1 and 2 and their absolute configurations were elucidated by detail analysis of spectroscopic, including 1D and 2D NMR $(1 \mathrm{H}, 13 \mathrm{C}, 1 \mathrm{H}-1 \mathrm{H}$ COSY, HMQC, HMBC and NOESY) spectroscopic data and Kappa CCD Detector.
1

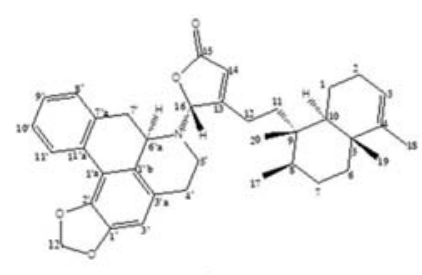

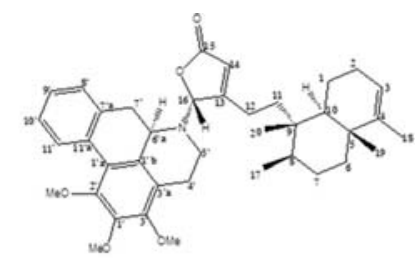

2

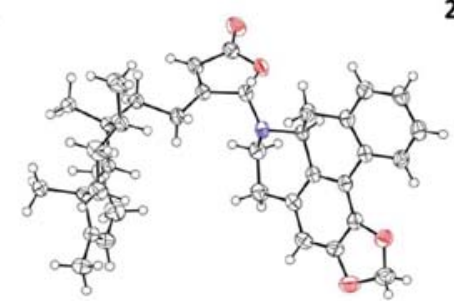

X-ray Structure of Polyalongirin A (1)
Fig. 1

\section{Mo-Poster Session 1-PO-168 Combined determination of sennosides, monomeric hydroxyanthacene glycosides and anthraquinone aglycones by UHPLC-DAD}

Authors Schenk $A^{1}$, Ziegler $L^{1}$, Meier $N^{2}$, Peter $S^{2}$, Wolfram $E^{2}$ Institute 1 Department of Analytical Development, Max Zeller Söhne AG, Romanshorn, Switzerland; 2 Department of Life Sciences of Zurich University of Applied Sciences, Wädenswil, Switzerland

DOI 10.1055/s-0037-1608215

Using a coreshell column, the new UHPLC-DAD method allows almost a baseline separation of seven sennosides and the monomeric hydroxyanthracene glycosides rhein 8-glycoside and aloe emodin 8-glycoside in senna pod dry extract and in a senna-fig syrup preparation. UV and mass spectrometry studies show that none of the peaks of interest is underlaid by flavonoids, a fact which, in the case of other LC methods for sennosides, requires a sample processing by means of SPE. Sennosides are determined by sennoside B, monomeric hydroyanthracene glycosides by rhein 8-glycoside as external standard. The combined determination of all hyrdoxyanthracene glycosides is important for pharmaceutical stability testing because sennosides are partly degraded to rhein- and aloe emodin 8-glycoside. In the posterior part of the chromatogram the aglycones rhein and aloe emodine can be determined. The complete assay runs $19 \mathrm{~min}$.

\section{Mo-Poster Session 1-PO-169 Synthesis and thymidine phosphorylase inhibition studies of 5-chlorobenzothiazole derivatives}

Authors Arbin $\mathrm{M}^{1,2}$, Ahmat $\mathrm{N}^{1,2}$, Taha $\mathrm{M}^{1,2}$

Institute 1 Universiti Teknologi MARA (Faculty of Applied Sciences), Shah Alam, Malaysia; 2 Universiti Teknologi MARA (Atta ur Rahman Institute for Natural Product Discovery), Puncak Alam, Malaysia DOI 10.1055/s-0037-1608216

Benzothiazole and their derivatives have been previously reported to exhibit antitumor, antimicrobial, antiviral and anticonvulsant activities [1]. Thymidine phosphorylase inhibitors have attracted great attention due to their ability to suppress the tumors formation. In our ongoing research, a series of 5-chlorobenzothiazole derivatives $(1-10)$ have been synthesized in good to excellent yields (80-90\%) and their thymidine phosphorylase inhibition potential has also been evaluated. The synthesized compounds showed moderate thymidine phosphorylase inhibitory activity with IC 50 values ranging from 19.60 \pm 0.45 to $93.50 \pm 2.88 \mu \mathrm{M}$, and 7- deazaxanthine (7DX) was used as a standard 
(IC50 38.68 \pm 4.42 ). Compound 1, 4-(5-chlorobenzo[d]thiazol-2-yl)benzene1,3-diol and compound 2, 5-chloro-2-(4-chlorophenyl)benzo[d]thiazole showed the lowest IC 50 values of $19.60 \pm 0.45 \mu \mathrm{M}$ and $23.40 \pm 0.68 \mu \mathrm{M}$, compared to the standard inhibitor. These compound showed better phosphorylase inhibition activity as compared to 7-deazaxanthine [2].

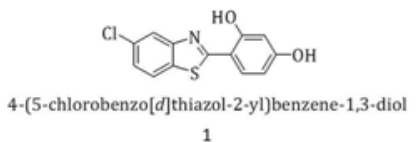

1

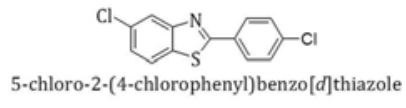

Fig. 1: Structure compound of Benzothiazole

Faculty of Applied Sciences, Universiti Teknologi MARA (UiTM) and Atta ur Rahman Institute for Natural Products Discovery, are acknowledged for providing laboratory facilities.

References:

[1] Srivastava SK, Yadav R, Srivastava SD. Indian J Chem 2004; 43: $399-405$.

[2] Sohail AS, Shahzad A, Yar M, Bajda M, Jadoon B, Khan ZA, Naqvi SAR, Shaikh AJ, Hayat K, Mahmmod A, Mahmood N, Filipek S. Bioorganic \& Medicinal Chemistry 2014; 22: $1008-1015$

\section{Mo-Poster Session 1-PO-170 Flavonoids from the twigs of Cynometra cauliflora Linn}

Authors Nik Azmin Nik F ${ }^{1}$, Ahmat $N^{1}$

Institute 1 Universiti Teknologi MARA (Faculty of Applied Sciences), Shah

Alam, Malaysia

DOI $10.1055 / \mathrm{s}-0037-1608217$

Cynometra cauliflora Linn, a member of the bean family Fabaceae, with vernacular name 'nam-nam', was believed to be native of Malaysia and cultivated in Indonesia and India[1]. C. cauliflora is a small, much-branched perennial tree growing to $5 \mathrm{~m}$ tall. It is a typical underutilized fruit tree that has the medicinal values in folk traditional medicine in treating several diseases and cultivated as an ornamental plant in the village. A decoction of the leaves is traditionally used for treating diabetes and hyperlipidemia, the fruits for curing the lost of appetite, and the seed oil is used to cure skin diseases. Previous works have been done on anti and pro-lipase activity which lead to the isolation of kaempferol-3-O-rhamnoside as an active constituents from the plant leaves [2]. Other study reported their chemical profile with antiacetylcholinesterase, antityrosinase, antioxidant and $\alpha$-glucosidase inhibitory activities of its leaves extract [3]. This study focused on investigating the constituents from the twigs part of this plant. The dried twigs of Cynometra cauliflora L. was macerated in acetone to obtain crude extract and fractionated using vacuum liquid chromatography (VLC). The isolation and purification of the chosen fractions by a combination of multiple radial and preparative thin layer (PTLC) chromatographic techniques afforded four pure compounds which consist of three flavones, luteolin (1), acatecin (2), 3',4',7-trihydroxyflavone (3) and one flavanone, eriodictyol (4). The structures of $1-4$ were elucidated on the basis of 1D NMR and 2D NMR correlations and comparison with previous literature.<smiles>[R]c1ccc(-c2cc(=O)c3c([R])cc(O)cc3o2)cc1[R2]</smiles>

$$
1 ; \mathrm{R}_{1}=\mathrm{R}_{2}=\mathrm{OH}, \mathrm{R}_{3}=\mathrm{H}
$$
$2 ; R_{1}=\mathrm{OH}_{2}, \mathrm{R}_{2}=\mathrm{H}_{2}, \mathrm{R}_{3}=\mathrm{CH}_{3}$ $3 ; R_{1}=R_{3}=H_{1}, R_{2}=O H$

Fig. 1

[1] Seidemann J. World spice plants. Springer: Berlin-Heidelberg, 2005, 131
[2] Ado MA, Abas F, Mohammed AS, Ghazali HM. Molecules2013, 18:14651 14669.

[3] Ado MA, Abas F, Ismail IS, Ghazali HM, Shaari K. J Sci Food Agric: Wiley2014, $1-8$

\section{Mo-Poster Session 1-PO-171 Oligomer resveratrols from Shorea bracteolate}

Authors Nor Izan $\mathrm{N}^{1}$, Ahmat $\mathrm{N}^{1,2}$, Syah Yana $\mathrm{M}^{3}$

Institute 1 Universiti Teknologi MARA (Faculty of Applied Sciences) 40450 UiTM, Shah Alam, Malaysia; 2 Universiti Teknologi MARA (Atta ur Rahman Institute for Natural Product Discovery), Puncak Alam, Malaysia; 3 Institut Teknologi Bandung, Bandung, Indonesia

DOI 10.1055/s-0037-1608218

Shorea bracteolate is a big forest tree and locally known as 'Meranti paang'. These Shorea species are known to contain different natural compounds such as terpenoids, flavonoids, coumarins, and resveratrol oligomers. The existing data showed that the most isolated compounds are resveratrol oligomers. The chemistry of known organic compounds such as terpenoids, coumarin and resveratrol oligomers is interrelated with biological activities. However, the knowledge of the other isolated compounds is very limited. Resveratrol oligomers isolated from the genus Shorea and its extracts show good biological activities including brine shrimps lethality bioassay, anti HIV-activity [1], antibacterial activity, $5 \alpha$-reductase inhibitor activity [2], antihepatotoxic activity, and cytotoxicity [3]. The air-dried stem bark of S. bracteolate was extracted with $6 \mathrm{~L}$ of acetone and concentrated at $60^{\circ} \mathrm{C}$ with rotary evaporator to afford $47.7 \mathrm{~g}$ of free tannin crude extract. The crude extract was fractionated using vacuum liquid chromatography (VLC). Isolation and purification of compounds used several chromatographic methods including radial, column and preparative thin layer chromatografies to obtaine three known resveratrol oligomers namely laevifonol (1), hemsleyanol D (2) and diptoindonesin F (3). The structure of the isolated compound was determined based on analysis of spectroscopic data, including NMR, UV, IR and comparison with previous reported data.

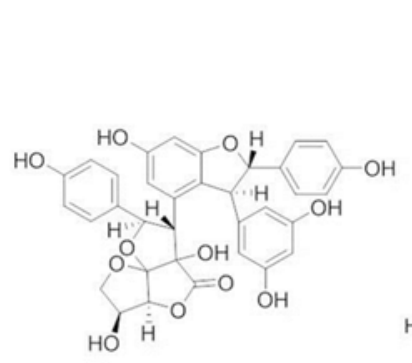

1
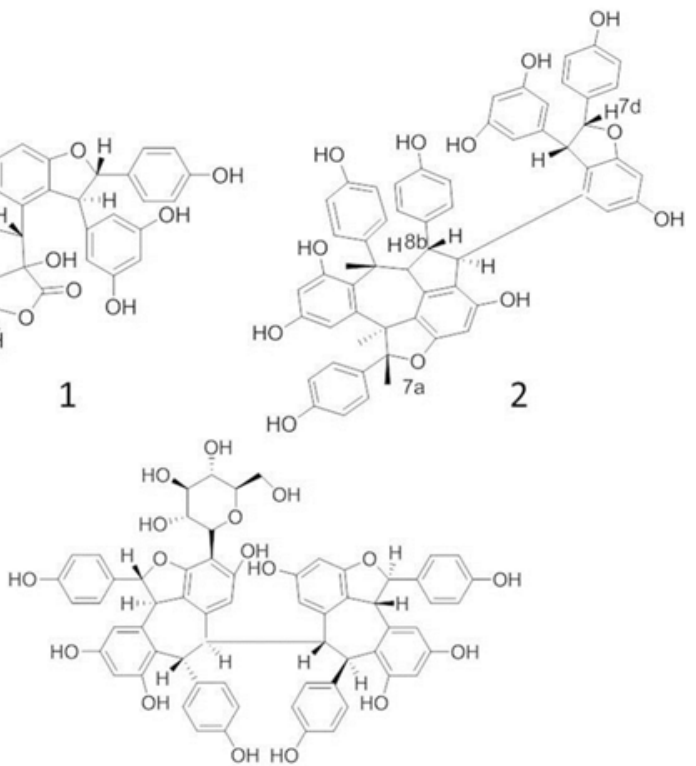

3

Fig. 1

Universiti Teknologi MARA is acknowledged for financial and technical supports.

[1] Dai, J.R, Hallock, Y.F, Cardellina II, J.H.; Boyd, M.R. J. Nat. Prod, 1998, 61, $351-353$ 
[2] Hirano, Y, Kondo, R, and Sakai, K. J. Wood Sci, 2001, 47, 308-312.

[3] Patcharamun, W, Sichaem, J, Siripong, P, Khumkratok, S, Jong-aramruang, J, Tip-pynag, S, 2011, 82, $489-492$

\section{Mo-Poster Session 1-PO-172 Chemical Constituents of Shorea macroptera Dyer.}

Authors Mohd Nazri Nur Ainaa $A^{1}$, Ahmat $N^{1,3}$, Kamarozaman Aisyah $\mathrm{S}^{1,2,3}$ Institute 1 Faculty of Applied Sciences, Universiti Teknologi MARA, 40450 Shah Alam, Selangor, Malaysia; 2 Centre of Foundation Studies, Universiti Teknologi MARA, Selangor Branch, Dengkil Campus, 43800 Dengkil, Selangor, Malaysia; 3 Atta-ur-Rahman Institute for Natural Product Discovery, Universiti Teknologi MARA, Selangor Branch, Puncak Alam Campus, 42300 Bandar Puncak Alam, Selangor, Malaysia

DOI 10.1055/s-0037-1608219

Shorea is the largest and economically most important genus in Dipterocarpaceae family which consists of 150 species [1]. This genus is known to contain abundant sources of oligostilbenoids which possesses broad spectrum of biological activities [2]. Shorea macroptera which is known by the locals as 'melantai' was selected to examine the phytochemical study. The dried powdered $(6 \mathrm{~kg})$ stem bark of was macerated in acetone at room temperature and repeated for 72 hours. The extract was filtered and dried by using rotary evaporator to yield acetone crude extract. The acetone crude extract (300 g) was subjected to vacuum liquid chromatography (VLC) with the eluent hexane:ethyl acetate:methanol to give six combined fractions. Fraction $1(6.2 \mathrm{~g})$ was fractionated by VLC with the same eluent to afford ten subfractions. Subfraction 1-3 was combined and washed with hexane to obtain a pure compound (1) (50 mg). Fraction 5 (33.9 g) was isolated by the same chromatographic technique with chloroform:methanol to give five subfractions. Subfraction $4(27.41 \mathrm{~g})$ was subjected to VLC again with the gradient eluent hexane:ethyl acetate:acetone to obtain six subfractions. Subfraction 5 (394.7 mg) was further purified by radial chromatography with the solvent system chloroform:acetone:methanol in increasing polarity to afford a pure compound (2) $(7 \mathrm{mg})$. Based on the spectroscopic analyses, the compounds were characterised as (E)-pentacosyl 3-(3-hydroxy-4-methoxyphenyl) acrylate (1) and gnetol (2).

Greatest appreciation to Society for Medicinal Plant and Natural Product Research for the GA travel grant, MOHE (RAGS/1/2014/SG01/UITM/4) and Faculty of Applied Sciences for the financial support.

[1] Cronquist A. An Integrated System of Classification of Flowering Plants. New York: Columbia University Press, 1981

[2] Seo EK, Douglas KA. Studies in Natural Products Chemistry 2000; 23: $531-561$

[3] Symington CF. Foresters' Manual of Dipterocarps. Kuala Lumpur: Universiti Malaya;1974

\section{Mo-Poster Session 1-PO-173 Isomer of bergenin and phytosterol from the stem bark of Mallotus leucodermis}

Authors Mohd Yusoff Aiza $\mathrm{S}^{1}$, Ahmat $\mathrm{N}^{1,2}$, Naz $\mathrm{H}^{2}$, Kamarozaman Aisyah $\mathrm{S}^{1,2,3}$

Institute 1 Faculty of Applied Sciences, Universiti Teknologi MARA, 40450 Shah Alam, Selangor, Malaysia; 2 Atta-ur-Rahman Institute for Natural Product Discovery, Universiti Teknologi MARA, Selangor Branch, Puncak Alam Campus, 42300 Bandar Puncak Alam, Selangor, Malaysia; 3 Centre of Foundation Studies, Universiti Teknologi MARA, Selangor Branch, Dengkil Campus, 43800 Dengkil, Selangor, Malaysia

DOI 10.1055/s-0037-1608220

Mallotus is one of the most diverse and richest genera of the Euphorbiaceae family, and consists of approximately 150 species distributed in tropical and sub-tropical regions of Asia [1]. Mallotus species are well known to possess medicinal properties and has been reported to be rich in flavonoids, terpenoids, phenolic compounds, and phloroglucinol derivatives [2]. Mallotus leu- codermis is commonly known as "balik angin bopeng" in Malaysia, and is used to treat skin complaints [3]. A phytochemical study was conducted on the stem bark of $M$. leucodermis collected from National Park Kuala Keniam, Pahang, Malaysia. The dried powder of the stem bark $(1.3 \mathrm{~kg})$ was macerated with acetone to yield $66.0 \mathrm{~g}$ of crude extract. The crude extract was fractionated using vacuum liquid chromatography to give six fractions. Fraction 5 was further purified using radial chromatography to afford an isomer of bergenin (1) $(23.0 \mathrm{mg})$. Purification of fraction 1 using radial chromatography gave a mixture of stigmasterol and $\beta$-sitosterol $(2.9 \mathrm{mg})$. The structure of compounds was elucidated by spectroscopic methods including $1 \mathrm{D}$ and 2D NMR, UV-Vis, FTIR, MS and by comparison with literature data.

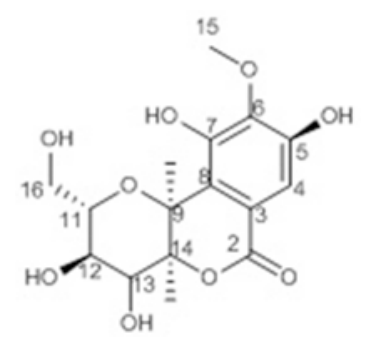

(1)

$$
\text { Fig. } 1
$$

Greatest appreciation to Society for Medicinal Plant and Natural Product Research for the GA travel grant, MOHE (RAGS/1/2014/SG01/UITM/4) and Faculty of Applied Sciences for the financial support.

[1] Thakur HA, Patil DA. Journal of Experimental Science 2011; 2:37 - 46

[2] Riviere C, Hong VNT, Hong QT, Chataigne G, Hoai NN, Dejaeger B, Tistaert C, Kim TNT, Heyden YV, Van MC and Quetin-leclercq J. Phytochem Rev 2010; 9:217- 253

[3] Faridah H, Nurulhuda H. J. Trop. Agric. Sci. 1999; 22: 85 - 94

\section{Mo-Poster Session 1-PO-174 Synthesis and docking studies of dihydrobenzofuran neolignan analogues as antileishmanial agents}

Authors Bernal $\mathrm{F}^{1}$, Kaiser $\mathrm{M}^{2,3}$, Schmidt $\mathrm{T}^{1}$

Institute 1 Institut für Pharmazeutische Biologie und Phytochemie (IPBP), University of Münster, Münster, Germany; 2 Swiss Tropical and Public Health Institute (Swiss TPH), Basel, Switzerland; 3 University of Basel, Basel,

Switzerland

DOI 10.1055/s-0037-1608221

Many thousands of new cases of Leishmaniasis, a worldwide distributed parasitic disease, are recorded every year. New effective and low-cost treatments are urgently needed. Natural products, as a well-known source of biologically active compounds, constitute a very important starting point. Specifically, dihydrobenzofuran neolignan analogues have demonstrated antileishmanial activity [1]. Therefore, and continuing our research on antileishmanial neolignans, a set of 15 dihydrobenzofurans were synthesized and tested in vitro against axenic amastigotes of Leishmania donovani. Cytotoxicity was also determined and selectivity indexes calculated.

The synthesized compounds included variations in the size of the lateral ester chains and the substitution on the aromatic moieties (Figure 1). In general, the former one affected stronger the antileishmanial activity than the latter. Even though no specific SAR's could be established so far, the experimentally measured activity was found to be linearly correlated with that previously predicted by 3D-QSAR modeling [2]. 


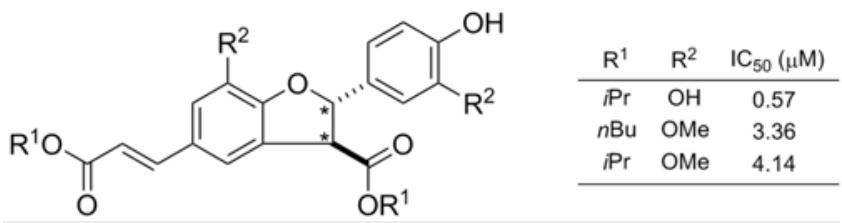

Fig. 1: Chemical structures and activity data of the three most active compounds. ${ }^{*}$ All compounds were synthesized as racemic mixtures.

In search for potential molecular targets, a docking study was carried out using a set of ten different protein structures from $L$. donovani and one from L. major. Several of the docked compounds $(n=75)$, showed relatively favorable scores, suggesting potentially strong interactions with some of the proteins and possible inhibition. Most interestingly, the experimental activity of the available compounds was linearly correlated with the docking scores for $\mathrm{N}$ myristoyl transferase, indicating a possible mode of action. Nevertheless, further SAR and mechanistic studies are still required.

[1] Van Miert S, Van Dyck S, Schmidt T], Brun R, Vlietinck A, Lemière G, Pieters L. Bioorg Med Chem 2005; 13: 661 - 669.

[2] Bernal FA, Schmidt T]. Planta Med 2016; 82(S 01): S1-S381.

\section{Mo-Poster Session 1-PO-175 Lupane Triterpenes and Sterol from the Stem Bark of Scaphiummacropodum (Miq.) Beumee}

Authors Al Muqarrabun Laode Muhammad $R^{1,2}$, Ahmat $N^{1,2}$, Syed Aris Sharipah $\mathrm{R}^{3}$, Kamarozaman Aisyah $\mathrm{S}^{1,2,4}$

Institute 1 Atta-ur-Rahman Institute for Natural Product Discovery, Universiti Teknologi MARA, Selangor Branch, Puncak Alam Campus, 42300 Bandar Puncak Alam, Selangor, Malaysia; 2 Faculty of Applied Sciences, Universiti Teknologi MARA, 40450 Shah Alam, Selangor, Malaysia; 3 Faculty of Education, Universiti Teknologi MARA, Selangor Branch, Puncak Alam Campus, 42300 Bandar Puncak Alam, Selangor, Malaysia; 4 Centre of Foundation

Studies, Universiti Teknologi MARA, Selangor Branch, Dengkil Campus, 43800 Dengkil, Selangor, Malaysia

DOI $10.1055 / \mathrm{s}-0037-1608222$

Scaphium macropodum, is a Malaysian medicinal plant known as malva nut (English) or Kembang Semangkuk Jantung (Malaysian). Species of S. Macropodum (Sterculiaceae) grows in tropical rain forests of Malaya, Cambodia, Thailand, Sumatera, and Borneo [1]. The seed forms a large quantity of gelatine which can be used to treat intestinal infections, diarrhoea, throat aches, asthma, dysentery, fever, coughs, inflammation, and urinary illness [2]. Several classes of compounds have been reported from the plants of family Sterculiaceae, including flavonoids, coumarins, alkaloids, terpenoids and cyanogenic glycosides [3]. Up until now, there have been no reports about the chemical constituents of S. macropodum. In this study, the isolation of the chemical constituents from S. macropodum was carried out using standard approaches commonly used in natural products chemistry. Ground, air-dried stem bark was macerated using methanol. The methanolic extract was then subjected to rotary evaporator under reduced pressure to give crude extract. Several chromatographic techniques, i.e. vacuum liquid chromatography (VLC) and radial chromatography (RC) were applied in the separation of the chemical constituents. Three compounds were isolated from the samples. The structures of the isolated compounds were determined using several spectroscopic methods, i.e. UV-Vis, FT-IR, 1D and 2D NMR, and MS. Two triterpenes, lupeol (1) and lupenone (2), and stigmasterol (3) were obtained from the stem bark of $S$. macropodum.

Greatest appreciation to Society for Medicinal Plant and Natural Product Research for the GA travel grant, MOHE (RAGS/1/2014/SG01/UITM/4) and Faculty of Applied Sciences for the financial support.

[1] Kochummen KM. Sterculiaceae. In T. C. Whitmore (Ed.), Tree Flora of Malaya. Kuala Lumpur: Longman; 2012: 2: $353-382$
[2] Lim, TK. Edible Medicinal and Non-Medicinal Plants. Dordrecht: Springer. pp; 2012: $189-191$

[3] Jalal MAF, Collin HA. Phytochem 1977; 16: 1377 - 1380

\section{Mo-Poster Session 1-PO-176 Oligostilbenoids from Dipterocarpus cornutus}

Authors Wan Mohd Zain Wan $Z^{1,2}$, Ahmat N ${ }^{1,3}$, Kamarozaman Aisyah $S^{1,3,4}$ Institute 1 Faculty of Applied Sciences, Universiti Teknologi MARA, 40450 Shah Alam, Selangor, Malaysia; 2 Faculty of Plantation and Agrotechnology, Universiti Teknologi MARA Jasin, 77300, Melaka, Malaysia; 3 Atta-ur-Rahman Institute for Natural Product Discovery, Universiti Teknologi MARA, Selangor Branch, Puncak Alam Campus, 42300 Bandar Puncak Alam, Selangor, Malaysia; 4 Centre of Foundation Studies, Universiti Teknologi MARA, Selangor Branch, Dengkil Campus, 43800 Dengkil, Selangor, Malaysia

DOI $10.1055 / \mathrm{s}-0037-1608223$

Dipterocarpus or commonly known as 'keruing' is an important source of dammarane, which contributes to a highly valued economic plant in Southeast Asia [1]. Preliminary study revealed that this plant is proven to produce a variety of chemical constituents such as volatile oil, triterpenoid, flavonoid, arylpropanoid, and oligomeric resveratrol [2]. Thus, in our continuing investigation on this family we wish to report the stem bark of $D$. cornutus which was subjected to explore more phytochemical constituents. The samples were collected from UiTM Jengka Forest Reserve, Pahang and extracted in methanol and acetone via cold maceration method. Isolation was done using multiple techniques of chromatography and the structural elucidation of the compounds were characterized using spectroscopic techniques as well as literary comparison. Phytochemical investigation led to the discovery of six oligostilbenoids namely davidiol A (1) and stenophyllol B (2), which were the first occurrence in Dipterocarpus genus along with hemsleyanol $D(3), \varepsilon$-viniferin (4), laevifonol (5) as well as ampelopsin F (6).

Greatest appreciation to Society for Medicinal Plant and Natural Product Research for the GA travel grant, MOHE for the SLAB-UITM scholarship and research grant (RAGS/1/2014/SG01/UITM/4) as well as Faculty of Applied Sciences for the financial support.

[1] Symington CF. Foresters' Manual of Dipterocarps. Kuala Lumpur: University Malaya Publication; 1974: 1-356.

[2] Wibowo A, Ahmat N. Biochemical Systematics and Ecology 2015; 59: 31 35

\section{Mo-Poster Session 1-PO-177 Pitfalls in the +ESI mass spectrometry of Solanum steroid glycosides}

Authors Sendker J ${ }^{1}$, Abdel-Hamid Alaa El-Din $E^{2}$, Jimenez-Negro $E^{1}$, DanneRasche $\mathrm{N}^{1}$, Salah El-Din $\mathrm{R}^{2}$

Institute 1 Institute of Pharmaceutical Biology and Phytochemistry, University of Münster, Hittorfstraße 48, 48149 Münster, Münster, Germany;

2 Department of Pharmacognosy, Faculty of Pharmacy, Cairo University, 11562 Cairo, Egypt, Cairo, Egypt

DOI 10.1055/s-0037-1608224

Plants of the Solanum genus contain steroid saponins and steroid alkaloid glycosides of which members with spirostan skeleton predominate i.a. in the species of $S$. dulcamara and S. villosum. Due to variations in their sugar chain and aglycon modifications, these compounds can occur in large numbers and cover a wide range of molecular weights, which impedes the planning of MS/MS scans in metabolic profiling of these species using HPLC-qTOFESIMS. In addition, protonated or ammoniated molecules of steroid saponins often show low relative abundancies and are therefore frequently ignored by data-dependent MS/MS procedures, whereas the very abundant protonated molecules of steroid alkaloid glycosides have proven themselves surprisingly stable and give only poor product ion yields. Using the steroid saponin degalactotigonin and the steroid alkaloid glycoside $\alpha$-tomatine, we studied high resolution mass and product ion spectra of different precursor ions which for 
their abundance are likely to be selected by data-dependent MS/MS procedures. The spectra were analyzed for their informative value towards characterizing sugar chains and aglycons. Problems and pitfalls in the interpretation of the spectra are highlighted and include biased isotope patterns, isobaric product ions, and internal residue loss in the dissociation of the sugar chain. The presented results are intended to aid the metabolic profiling of Solanum samples by HPLC-MS.

\section{Mo-Poster Session 1-PO-178 Metabolomic approach to predict new anti-inflammatory extracts with in vivo outstanding mechanism of action}

\author{
Authors Alcântara BGV ${ }^{1}$, Zanini $\mathrm{LBL}^{1}$, Fernandes $\mathrm{VHC}^{1}$, Casoti $\mathrm{R}^{2}$, \\ Soares $\mathrm{MG}^{1}$, Dias $\mathrm{DF}^{1}$, Oliveira $\mathrm{RB}^{3}$, Chagas-Paula $\mathrm{DA}^{1}$ \\ Institute 1 Federal University of Alfenas, UNIFAL-MG, Alfenas, Brazil; \\ 2 Universtiy of São Paulo, FCFRP-USP, Ribeirão Preto, Brazil; 3 Federal \\ Technological University of Paraná, Santa Helena, Brazil \\ DOI 10.1055/s-0037-1608225
}

Novel inhibitors able to inhibit both cyclooxygenase (COX) and lipoxygenase (LOX) pathways are currently desirable, since they could display high efficacy and low side effects. Some extracts from Asteraceae family showed in vitro dual inhibition of COX-1 and 5-LOX, and biomarkers correlated with this feature were determined [1]. Thus, some of the active extracts (EtOH-H2O 7:3, v/ v) were evaluated through in vivo anti-inflammatory assays (Ethical animal committee approved, \#16/2016), which can evaluate exactly both main inflammatory pathways: COX and LOX (inhibition of ear oedema and neutrophil recruitment, respectively; $\mathrm{n}=8,0.5 \mathrm{mg} / \mathrm{ear}$ ). Prestelia eriopus, Viguiera robusta, Viguiera trichophylla and Tithonia diversifolia proved to display in vivo outstanding mechanism of action by inhibition of both COX and LOX pathways, similarly positive controls ( $P>0.05$ compared to indomethacin and dexamethasone, respectively), and different of negative control (vehicle, $P<0.05$ ). These active extracts and other six inactive extracts were analysed by high performance liquid chromatography coupled to high resolution mass spectrometry (HPLC-HRMS) analysis, and subjected to in silico studies using O2PLS-DA. The biomarkers of in vivo dual activity were determined, and new model of prediction of active extracts were created and validated $\left(R^{2}\right.$ and $\left.Q^{2}>0.5\right)$. Twenty new extracts from a different family (Lauraceae) were also evaluated in the same run at HPLC-HRMS, together with extracts used to train the prediction model. The model was efficient to predict new biologically active extracts, which have determined biomarkers, even if they are from different families. One of predicted active extracts proved to be able to do in vivo inhibition of COX and LOX pathways. Thus, this new model can be useful to predict new anti-inflammatory extracts, which have innovative mechanism of action, using only HPLC-HRMS data.

CAPES, CNPq, FINEP, FAPEMIG and Asterbiochem.

[1] Chagas-Paula DA. et al. Planta Medica 2015; 81: 450 - 458.

\section{Mo-Poster Session 1-PO-179 Screening and identification of characteristic components in big traditional Chinese medicine prescriptions using HPLC-ESI-Q/TOF-MS}

\footnotetext{
Authors Xie $Y^{1}$, Wang $Y^{1}$, Luo $G^{1}$

Institute 1 Department of Chemistry, Beijing, China

DOI 10.1055/s-0037-1608226

In the present study, a powerful and rational strategy was proposed to offer higher quality structural information of components for the characterization of the complex big traditional Chinese medicine prescriptions (TCMPs). The self-built compounds database was established on the basis of the in-house screening and identification of each comprising herbal medicines using the same analytical method as the determined big TCMPs, including the same chromatographic and MS analysis, and also the same sample pretreatment methods. The herbs used for the self-built compounds database should be the
}

raw materials using in the manufacture of big TCMPs whenever possible to avoid other interference factors, including the botanical origin, producing area, collecting seasons, concocted processing of medicinal materials. The retention times, ultraviolet absorption spectrums obtained by diode-array detector, accurate mass within 5 ppm error and mainly characteristic MS fragmentation ions of components identified in each comprising herbal medicines were listed in the self-built compounds library. Then the extraction ion chromatography (EIC) of the corresponding components from each herbs and equivalent dose of formula were compared one by one on the condition of guaranteeing the stability of instruments based on HPLC-ESI-Q/TOF-MS. Then the components in big TCMPs would be identified by comparing the characteristic product ions, fragmentation patterns with corresponding components in the comprising single medicinal materials. A total of 152 components deriving from the all 16 comprising medicinal herbs of NDQG were identified with the proposed components identification strategy here. The complementary multi-level structural information provided by LC/ion trap MSn as well as reference standard comparison analyses were also accompanied in this analysis. Moreover, the analytical approach developed herein is expected to provide an effective and reliable pattern for comprehensive and systemic characterization of TCM systems and other complicated mixtures.

\section{Mo-Poster Session 1-PO-180 Efficient Dereplication of Fungal Antimicrobial Principles by Tandem MS and NMR Database}

Authors Lee $\mathrm{MS}^{1}$, Yang $\mathrm{YL}^{2}$, Chen $\mathrm{YL}^{3}$, Tzean $\mathrm{SS}^{3}$, Lee $\mathrm{TH}^{4}$

Institute 11 School of Pharmacy, Taipei Medical University, Taipei, Taiwan; 22 Agricultural Biotechnology Research Center, Academia Sinica, Taipei,

Taiwan; 33 Department of Plant Pathology and Microbiology, National Taiwan University, Taipei, Taiwan; 44 Institute of Fisheries Science, National Taiwan University, Taipei, Taiwan

DOI 10.1055/s-0037-1608227

The continuous re-isolation of the known and non-applicable compounds are critical problems in the discovery of biologically active compounds from natural resources that is wasting time and resources. To efficiently solve this problem, HPLC-DAD-microfractionation directed by disk agar diffusion assay was performed, and the active compounds were further dereplicated using the tandem mass spectrometry-based molecular networking [1]. Moreover, utilizing the ${ }^{13} \mathrm{C}$ NMR spectral database to deal with the problem of diastereoisomers and precisely determined the chemical structures from the results of dereplication among the numerous active samples. In this case, we were able to verify the presence of all the bioactive entities in the early research stage, no matter they are new or known compounds. The methanolic extracts of Phoma herbarum PPM7487, Cryptosporiopsis ericae PPM7504, and Albifimbria sp. PPM945 showed significant antimicrobial activity against the Candida albicans and Cryptococcus neoformans among 150 fungal strains in a preliminary agar diffusion assay. Off-line antimicrobial activity-based HPLC profiling of these extracts enabled a precise localization of the active compounds in the chromatogram. The purified active compounds were dereplicated based mainly on MS/MS database [2], and the stereoisomers were further identified by $13 \mathrm{C}$ NMR spectra in comparison with the literature values. In addition to seventeen known compounds 2-18, a new trichothecenoid analogue, namely trichoverrin C (1), was isolated and identified through this protocol. This report has demonstrated the combination of HPLC microfractination, antimicrobial assay, and dereplication with tandem MS assisted by ${ }^{13} \mathrm{C}$ NMR for speeding up the antimicrobial natural products discovery process. 


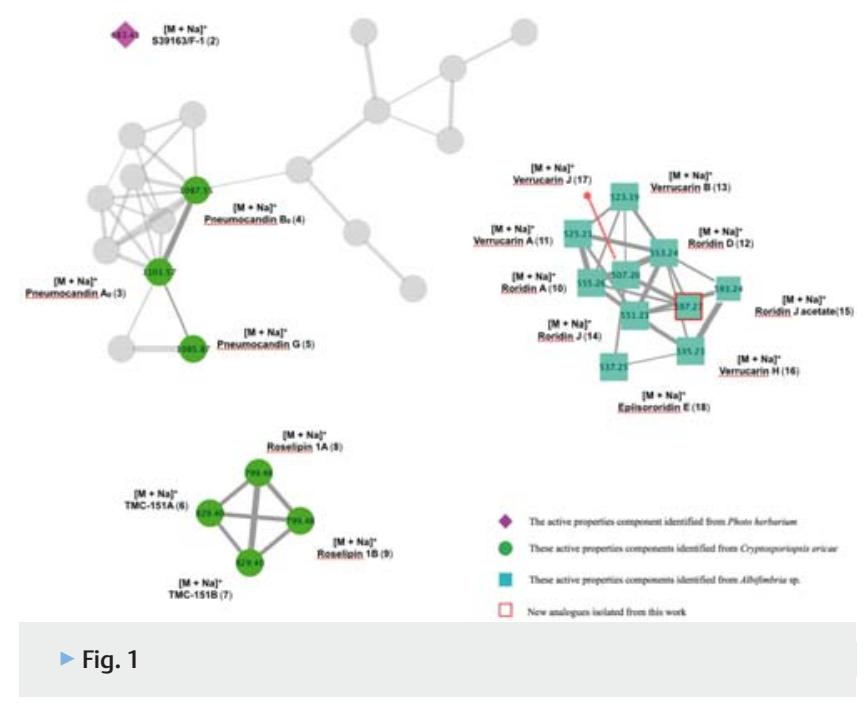

[1] Yang JY, Sanchez LM, Rath CM, Liu X et al. J. Nat. Prod. 2013; 76: 1686 1699.

[2] Wang M, Carver J], Phelan VV, Sanchez LM et al. Nat. Biotechnol. 2016; 34 : $828-837$.

\section{Mo-Poster Session 1-PO-181 Phenolic compounds from the aerial parts of Clematis viticella $\mathrm{L}$.}

Authors Kirmizibekmez $\mathrm{H}^{1}$, Inan Mumin $\mathrm{Y}^{1}$, Goren Ahmet $\mathrm{C}^{2}$, Yesilada $\mathrm{E}^{1}$ Institute 1 Department of Pharmacognosy, Faculty of Pharmacy, Yeditepe University, 34755, Kayisdagi, Istanbul, Turkey; 2 Chemistry Group Laboratories, TUBITAK UME, Gebze, Kocaeli, Turkey

DOI 10.1055/s-0037-1608228

The genus Clematis L. (Ranunculaceae) comprises around 300 species worldwide. Some of its species are used for the treatment of inflammation-associated diseases, chronic skin diseases as well as treating wind-cold rheumatism in various traditional medicines [1]. Saponins, flavonoids and lignans were reported as the major chemical classes of the genus Clematis. Some triterpenoid saponins and phenolic glycosides from Clematis species were reported to possess cytotoxic, anti-inflammatory and antioxidant activities $[1,2]$. In the flora of Turkey, the genus Clematis is represented by five species. In Turkish traditional medicine some of these species are used as a remedy to relieve joint pains [3]. As a part of our ongoing phytochemical studies on Turkish medicinal plants, we have investigated the chemical composition of the aerial parts of $C$. viticella which has never been studied in terms of its chemical constituents. From the $n$-buOH subextract of the crude EtOH extract, isoorientin, isoorientin 3'-O-methyl ether, quercetin 3,7-di-O- $\alpha$-L-rhamnopyranoside and manghaslin were isolated while, chrysoeriol 7-O- $\beta$-D-glucopyranoside, quercetin 7-O- $\alpha$-L-rhamnopyranoside, hydroxytyrosol, caffeic acid as well as a mixture of (E)-p-coumaric acid and $p$-hydroxybenzoic acid were obtained from the EtOAc subextract. The structures were elucidated on the basis of NMR experiments and HR-MS spectra. All compounds are being reported for the first time from $C$. viticella while, isoorientin 3'-O-methyl ether, quercetin 3,7-di-O- $\alpha$-L-rhamnopyranoside, manghaslin and chrysoeriol 7-O- $\beta$-D-glucopyranoside are new for the genus Clematis. The isolated flavonoids might have chemotaxonomic values for the genus Clematis within Ranunculaceae family and might be utilized for the chemotaxonomic evaluation of the genus.

[1] Chawla R, Kumar S, Sharma A. J Ethnopharm 2012; 143: 116 - 150.

[2] Zhao M, Ma N, Qiu F, Tia, X, Zhang Y, Tang H, Liu X. Fitoterapia 2014; 97 : $234-240$.

[3] Yesilada E, Kupeli E. J. Ethnopharm 2007; 110: 504 - 515.

\section{Mo-Poster Session 1-PO-182 Four New Iridoids isolated from the stem of Neonauclea reticulate}

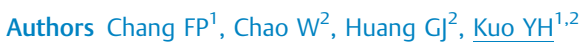

Institute 1 The Ph.D. Program for Cancer Biology and Drug Discovery, China Medical University, Academia Sinica, Taichung, Taiwan; 2 Department of Chinese Pharmaceutical Sciences and Chinese Medicine Resources, China Medical University, Taichung, Taiwan

DOI 10.1055/s-0037-1608229

Neonauclea reticulate (Rubiaceae) is a large evergreen tree and only one species found in Taiwan. It was an important Lanyu folk plant for built traditional canoe in Tao people culture. Previous investigations of some Neonauclea plants resulted in the isolation of alkaloids, anthraquinones, iridoids, triterpenes and saponins. Iridoid was a cyclic monoterpene type structure and exhibited a wide range of bioactivity in previous study, such as: antihepatotoxic, antiinflammatory, antispasmodic, antitumor, anti-diabetes and antiviral et al. In this study, stem of Neonauclea reticulate extracted with methanol. The methanol extracts suspended in water and partition with ethyl acetate. The water suspension partition with butanol. Each steps repeat three times. Four different layers assay for lung and liver tumor cells cytotoxicity ability. The result showed that the methanol extract owning a dose-dependent cytotoxicity against to the human hepatoma (Нер3B) cell line $\left(\mathrm{IC}_{50}=500 \mu \mathrm{g} / \mathrm{mL}\right)$. Investigation of the active layer of N.reticulate led to the isolation of three new dimeric iridoids:reticunin C (1) reticunin D (2) and reticunin E (3) and one new iridoid: neonanin $D(4)$, along with four known iridoids including siphonosetegiol (5), isoboonein (6), floribundano B (7) and alyxialactone (8). The structure of these compounds were determined through spectral analyses including extensive 2D-NMR data and the new compounds were identify by ESI-MS. In this symposium we described the four structural elucidations of 1 , 2, 3 and 4. Biological activities of pure compounds were all in progress.

\section{Mo-Poster Session 1-PO-183 HPTLC and TLC method development preliminary studies for damask rose}

Authors Agar Osman $\mathrm{T}^{1}$, Gokbulut $\mathrm{A}^{2}$, Demirezer Lutfiye $\mathrm{O}^{3}$ Institute 1 Department of Pharmaceutical Botany, Faculty of Pharmacy, Hacettepe University, Sihhiye, 06100, Ankara, Turkey; 2 Department of Pharmacognosy, Faculty of Pharmacy, Ankara University, Tandogan, 06100, Ankara, Turkey; 3 Department of Pharmacognosy, Faculty of Pharmacy, Hacettepe University, Sihhiye, 06100, Ankara, Turkey DOI 10.1055/s-0037-1608230

Rosa damascena Mill. (Rosaceae) has several pharmacological properties including anti-HIV, antibacterial, antioxidant, antitussive, hypnotic, antidiabetic, and relaxant effect on tracheal chains. Petals contain mainly essential oil and flavonoids [1].

The aim of this study is to develop TLC and HPTLC methods for quality control of marketed preparations. In this study, we identified the optimal solvent system and suitable reference molecules as biomarkers after several trials of extracts from Rosa damascena petals.

Extracts of $R$. damascena petals prepared by using the solvents in different polarity were tested with several mobile phases in thin layer chromatography. Subsequently, a simple, precise, accurate and rapid high performance thin layer chromatographic method was developed. Precoated silica gel 60 F254 was used as stationary phase. 9 mobile phase and 11 reference substance (kaempferol, kaempferol 3-O- $\beta$-D-glucopyranoside, kaempferol 3-O- $\beta$-D-galactopyranoside, kaempferol 3-O- $\beta$-D-gentiobioside, quercetin-3-O-rhamnopyranoside, quercetin-3-O-arabinopyranoside, quercetin 3-O- $\beta$-D-glucopyranoside, quercetin 3-O- $\beta$-D-galactopyranoside, rutin, RD 1 (structure elucidation has not been carried out yet), gallic acid) were tested to find optimal mobil phase and marker substance.

According to the results, ethyl acetate: formic acid: acetic acid: water $(100: 11: 11: 10, v / v / v / v)$ was determined as the optimal solvent system and kaempferol 3-O- $\beta$-D-glucopyranoside, quercetin 3-O- $\beta$-D-glucopyranoside, 
quercetin 3-O- $\beta$-D-galactopyranoside as biomarkers. The detection of spots was carried out at $254,366 \mathrm{~nm}$ and vanilin/ $\mathrm{H}_{2} \mathrm{SO}_{4}$ was used as revelator. The method validation in terms of linearity, accuracy, precision and specificity will be performed in further studies. The proposed method can be successfully used to determine the drug content of marketed preparations.

[1] Aslam M, Siddiqui A. Eur J Biomed Pharm Sci 2016; 3: 432- 435.

\section{Mo-Poster Session 1-PO-184 On-line hyphenation of counter-current chromatography and medium pressure liquid chromatography for preparative separation of euphorbia factors from Euphorbia lathyris L.}

Authors Song $\mathrm{K}^{1}$, Kim Yeong $\mathrm{S}^{1}$

Institute 1 Natural Products Research Institute, College of Pharmacy, Seoul National University, Seoul, Korea, Republic of (South)

DOI 10.1055/s-0037-1608231

Euphorbia lathyris L, one of the medicinal plants of the family Euphorbiaceae, contains a series of diterpenoids known as euphorbia factors which show pharmacological activities against HIV-1 replication, vasoconstriction, and cancer proliferation [1]. However, its chemical complexity and diversity make pure compounds isolation difficult. This study presents a developed method, two-dimentional hyphenation of counter-current chromatography (CCC) and medium pressure liquid chromatography (MPLC) for the preparative separation of euphorbia factors from Euphorbia lathyris. The extraction solution of Euphorbia lathyris seeds was directly and continuously injected into the CCC column (CCC-DCI, [2]) and concentrated target compounds were firstly separated by gradient elution of CCC. The CCC and MPLC were hyphenated via tenport injection valve and the overlapped mixture from CCC separation was secondly isolated by MPLC. Euphorbia factors L1 $(58 \mathrm{mg})$, L2 $(35 \mathrm{mg}), \mathrm{L} 3$ (62 mg), $\mathrm{L}_{7 \mathrm{a}}(4 \mathrm{mg}), \mathrm{L}_{7 \mathrm{~b}}(18 \mathrm{mg}), \mathrm{L} 8(11 \mathrm{mg})$, and L 9 (3 mg) were successfully obtained in a single CCC-MPLC separation. The purity determination and structural identification of the isolated compounds were performed by LCUV, LC-MS/MS, and 1D NMR.

[1] Jiao W, Fang DM, Wu Z], Chen JZ, Shao HW. Rapid Commun Mass Spectrom 2013; 27: $276-280$

[2] Song K, Lee KJ, Kim YS. J Chromatogr A 2017; 1489: 107 - 114

[3] Li Y, Wang N, Zhang M, Ito Y, Zhang H, Wang Y, Guo X, Hu P. Anal Chem 2014; 86: $3373-3379$

\section{Mo-Poster Session 1-PO-185 A strategy for identification of oplopane- and bisabolane-type sesquiterpenoids from Tussilago farfara L. by multi-scanning mode of triple quadrupole mass spectrometry}

Authors Song $\mathrm{K}^{1}$, Kim Yeong $\mathrm{S}^{1}$

Institute 1 Natural Products Research Institute, College of Pharmacy, Seoul National University, Seoul, Korea, Republic of (South)

DOI 10.1055/s-0037-1608232

A liquid chromatography-electrospray ionization tandem mass spectrometry (LC-ESI-MS/MS)-based dereplication method has been developed for identifying oplopane- and bisabolane-type sesquiterpenoids from Tussilago farfara L. $[1-3]$. This method used sequential MS scanning modes to identify fragmentation patterns of common skeletons and their derivatives. In the positive ion mode, product ion scanning for ions with $\mathrm{m} / \mathrm{z} 215,217,229$, or 231 yield same fragmentation pattern, respectively, indicating that ions with $m / z 215$ and 217 were diagnostic cation of oplopane sesquiterpnoids, and m/z 229 and 231 were those of bisabolane sesquiterpenoids. The difference of two cations ( $\mathrm{m} / \mathrm{z} 215$ versus 217 or $\mathrm{m} / \mathrm{z} 229$ versus 231 ) is the number of the esterlinkages. Since the cleavage of ester linkage dominantly undergoes a double bone reduction, the cations with $\mathrm{m} / \mathrm{z} 217$ (oplopane) and 231 (bisabolane) were caused from two cleavages of ester bond and the cations with $\mathrm{m} / \mathrm{z} 215$ (oplopane) and 229 (bisabolane) were derived from three cleavages. Secondly, precursor ion and neutral loss scanning for the cation with $\mathrm{m} / \mathrm{z} 215,217,229$, and 231 yield diagnostic fragmentation patterns and identified which moieties is linked to the common skeletons. The fragmentation patterns suggest parental and intermediate cations with specific $\mathrm{m} / \mathrm{z}$ values.

[1] Kikuchi M, Suzuki N. Chem Pharm Bull 1992; 40: 2753 - 2755

[2] Yaoita Y, Suzuki N, Kikuchi M. Chem Pharm Bull 2001; 49: 645 - 648

[3] Li W, Huang X, Yang XW. Fitoterapia 2012; 83: 318-322

\section{Mo-Poster Session 1-PO-186 Isolation and Structure Elucidation of four new polycyclic Acylphloroglucinols from Hypericum hirsutum L.}

\section{Authors Ziegler J', Heilmann J'}

Institute 1 University of Regensburg, Institute of Pharmaceutical Biology,

93053 Regensburg, Germany

DOI 10.1055/s-0037-1608233

Hypericum hirsutum L. (Hypericaceae) is a perennial herb with hirsute green parts that is native to Northern Africa, Europe and temperate Asia [1]. The chemical composition of the plant, especially the prenylated acylphloroglucinols which are very popular secondary metabolites in many species of the genus Hypericum (Hypericaceae), is so far mostly unknown. Chemical investigations of the petroleum ether raw extract of aerial parts of Hypericum hirsutum L. yielded four new polycyclic polyprenylated acylphloroglucinols with a rare tricyclo[4.3.1.1] undecane skeleton. Such complex caged benzophenone derivatives are hitherto unknown for the whole section 18 Taeniocarpium. Similar substances were already isolated from Hypericum sampsonii Hance (Hypericaceae, Sect. 9 Sampsonia) [2, 3] and Clusia obdeltifolia (Clusiaceae) [4]. The acylphloroglucinols were isolated by using Diaion ${ }^{\circledR}$ HP-20 column chromatography, silica gel flash chromatography, centrifugal partition chromatography, as well as semi-preparative RP-18 HPLC techniques. On basis of 1D and 2D NMR data the structures could be elucidated and with MS experiments they were confirmed.

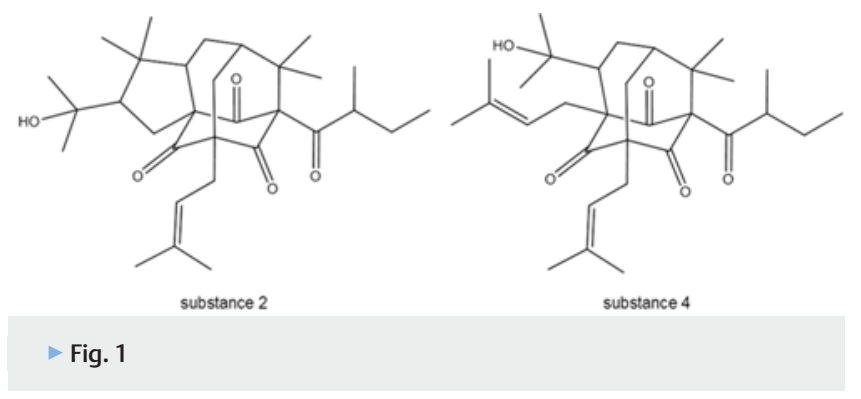

[1] Robsen N. K. B. Phytotaxa 2010, 4, $203-207$

[2] Hu, L.-H.; Sim, K.-Y. Tetrahedron Letters 1998, 39, 7999 - 8002

[3] Tian, W.--.; Qiu, Y.-Q.; jin, X.-J. Tetrahedron 2014, 70, 7912- 1916

[4] Cruz, F.; Teixeira, J. Journal of the Brazilian Chemical Society, 2004, 15, $504-508$ 


\section{Mo-Poster Session 1-PO-187 Isolation of atractyloside from Xanthium sibiricum PATR. and evaluation of its in vitro cytotoxicity together with carboxyatractyloside and decoctions}

\section{Authors Schiller $K^{1}$, Heilmann J $J^{1}$, Manns $D^{2}$, Franz $G^{1}$}

Institute 1 University of Regensburg, Institute of Pharmacy, Department of Pharmaceutical Biology, 93053 Regensburg, Germany; 2 Federal Institute for Drugs and Medicinal Devices (BfArM), 53174 Bonn, Germany DOI 10.1055/s-0037-1608234

Xanthium or cockleburr fruit (Xanthium sibiricum PATR, Asteraceae) is used in Traditional Chinese Medicine for nasal or sinus irritations with a viscous discharge and related headache. ${ }^{[1]}$ Fixed oil, volatile oil, phenolic acids (e.g. chlorogenic, dicaffeoylquinic acid) and sesquiterpenes ${ }^{[1-3]}$ are mentioned in literature as chemical constituents. Due to apparent hepatoxicity, also diterpene glycosides, like atractyloside (ATR) and carboxyatractyloside (CATR), are known to be present in the herbal drug material. ${ }^{[1,4,5]}$ ATR was isolated from Xanthii fructus using Sephade ${ }^{\circledR} \mathrm{LH} 20$, centrifugal partition chromatography (CPC) and preparative HPLC. The cell viability in vitro, using the human hepatoma cell line Huh-7 cells with the MTT assay, was examined with isolated ATR, commercial CATR (both 25 to $500 \mu \mathrm{M}$ ) and specially prepared decoctions of Xanthii fructus (100 to $750 \mu \mathrm{g} / \mathrm{mL}$ ). All three, ATR and CATR and decoctions inhibited, in this in vitro system slightly the cell viability in a dose dependent manner, indicating a low cytotoxicity. As literature data reported of an obviously higher toxic effect of CATR in comparison to ATR ${ }^{[6]}$ further comparative pharmacological analyses of both compounds are mandatory.

[1] Chinese Herbal Medicine: Materia Medica, Eastland Press Inc, Seattle, Washington 2004

[2] T. Han, H.-L. Li, Y. Hu, Q.-Y. Zhang, B.-K. Huang, H.-C. Zheng, K. Rahman, L.-P. Qin, Journal of Chinese Integrative Medicine 2006, 4, 194.

[3] T. Han, H.-L. Li, Q.-Y. Zhang, P. Han, H.-C. Zheng, K. Rahman, L.-P. Qin, Phytomedicine 2007, 14, 825.

[4] R. J. Cole, B. P. Stuart, J. A. Lansden, R. H. Cox, Journal of Agricultural and Food Chemistry 1980, 28, 1330.

[5] Y. Wang, T. Han, L.-M. Xue, P. Han, Q.-Y. Zhang, B.-K. Huang, H. Zhang, Q.L. Ming, W. Peng, L.-P. Qin, Die Pharmazie 2011, 66, 445.

[6] S. Luciani, N. Martini, R. Santi, Life Sciences 1971, 10, 961.

\section{Mo-Poster Session 1-PO-188 Development and validation of an HPLC-DAD method for iridoid glycoside screening in fresh plant parts from Plantago lanceolata $\mathrm{L}$.}

Authors Lotter $\mathrm{E}^{1}$, Heilmann $\mathrm{J}^{1}$, Kammerer $\mathrm{D}^{2}$, Stintzing $\mathrm{F}^{2}$

Institute 1 University of Regensburg, Institute of Pharmacy, Department of Pharmaceutical Biology, 93053 Regensburg, Germany; 2 WALA Heilmittel

GmbH, 73087 Bad Boll/Eckwälden, Germany

DOI 10.1055/s-0037-1608235

Plantago lanceolata L. is a long-known medicinical plant, exhibiting multiple favorable properties like anti-bacterial and abbiritative effects. ${ }^{[1]}$ Its drug has been thoroughly analyzed. However, scientific data of the fresh plant are still rare. Therefore, an HPLC-DAD fingerprint method for fresh plant parts from $P$. lanceolata L. was developed and validated. This method enables simultaneous screening of two substance classes characteristic of Plantago: the phenylethanoids and the iridoid glycosides. According to literature, P. lanceolata herb contains five phenylethanoids ${ }^{[2]}$ : the predominant phenylethanoid acteoside, its isomer isoacteoside, plantamajoside, lavandulifolioside and cistanoside $\mathrm{F} ;{ }^{[2]}$ as well as six iridoid glycosides: the main iridoid glycosides aucubin ${ }^{[3,4]}$ and catalpol $^{[3,4]}$, together with asperuloside ${ }^{[3,5]}$, globularin ${ }^{[3]}$, verbenalin ${ }^{[4]}$ and the methylester of deacetylasperuloside acid ${ }^{[3]}$. Among the predominant representatives the following constituents were identified in the fresh plant: the phenylethanoids isoacteoside and plantamajoside as well as the iridoids ver- benalin and asperuloside. During validation linearity, intra- and interday precision, robustness, recovery, LOD and LOQ were determined for aucubin at $195 \mathrm{~nm}$, previously being detected at $204 \mathrm{~nm}$ or higher wavelength $\mathrm{s}^{[6,7]}$. Due to the aromatic nature of the phenylethanoids, their detection can easily be performed at $330 \mathrm{~nm}$. Consequently their validation was abandoned. The obtained results confirm the validity of the novel HPLC-DAD method and underline its potential applicability in the routine quality control of iridoid containing plant raw materials and preparations derived therefrom.

[1] Ganz, Schweiz. Ztschr. Ganzheitsmed. 2014, 26, 17 - 18.

[2] Büechi and Wegener, Schweiz. Ztschr. Ganzheitsmed. 2005, 17, 167 - 170.

[3] Ronsted et al, Phytochemistry 2000, 55, $337-348$.

[4] Suomi et al, Electrophoresis 2001, 22, 2580 - 2587.

[5] Bianco et al, J. Nat. Prod. 1984, 47, $901-902$.

[6] Kartini and Azminah, Asian J. Pharm. Clin. Res. 2012, 5, 191 - 195.

[7] Kim et al, Chromatographia, 2009, 69, 11, $1397-1400$.

\section{Mo-Poster Session 1-PO-189 Rapid quantitative and qualitative analysis of anthocyanins using UHPLC-3Q-MS/MS}

Authors Suvanto $\mathrm{J}^{1}$, Salminen JP ${ }^{1}$

Institute 1 Natural Chemistry Research Group, University of Turku, Turku, Finland

DOI 10.1055/s-0037-1608236

A new method for quantifying anthocyanins using ultra-high performance liquid chromatography-triple quadrupole mass spectrometry (UHPLC-3Q-MS/ MS) was developed. The method utilizes controlled and optimized in-source fragmentation, which enables group-specific detection and subsequent quantification of all anthocyanins derived from a single anthocyanidin using common MRM transitions (Figure 1). The method works for a wide variety of different sample types, including crude plant extracts, simplifying and speeding up the analysis of anthocyanins.

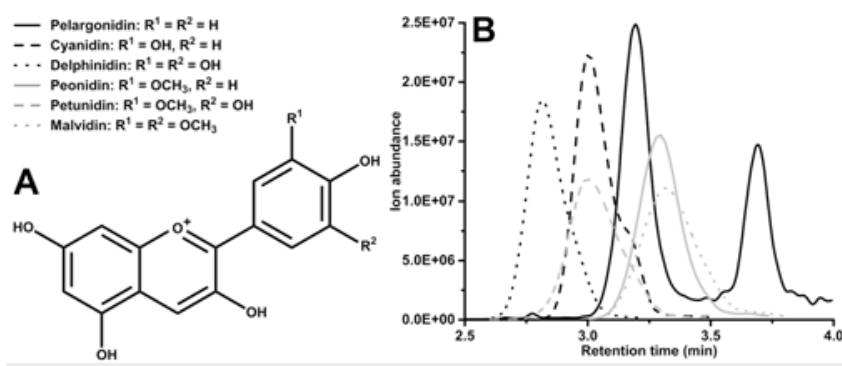

Fig. 1: Anthocyanidins included in the method (A) and example MRM chromatograms combined from two berry extracts (B).

Typical analysis methods for anthocyanins include methods based on (U)HPLCUV/Vis and conventional compound-specific tandem mass spectrometry. For the UV/Vis-based methods, separation of all compounds is naturally needed, and the compound-specific MS/MS methods require several SRM or MRM transitions to comprehensively quantify all anthocyanins derived from one anthocyanidin aglycone. As described, the developed method bypasses the limitations of the latter by using in-source fragmentation, and different cone voltages are used in the method to ensure the efficient cleavage of different types of glycones.

The method complements similar earlier analysis methods published for several other groups of polyphenolic compounds [1,2]. These previous methods can be combined with our new methods allowing the quantification of all major polyphenolic compound groups in one UHPLC-3Q-MS/MS injection.

[1] Engström MT, Pälijärvi M, Fryganas C, Grabber JH, Mueller-Harvey I, Salminen JP. J Agric Food Chem 2014; 62: 3390 - 3399

[2] Engström MT, Pälijärvi M, Salminen JP. J Agric Food Chem 2015; 63: $4068-4079$ 


\section{Mo-Poster Session 1-PO-190 Computer Assisted Structure Elucidation of Two Biflavonoids from the Leaves of Ochna Mauritiana}

Authors Dziwornu GA ${ }^{1}$, Toorabally NR², Bhowon $\mathrm{MG}^{2}$, Jhaumeer-Laulloo $\mathrm{S}^{2}$, Sunassee $\mathrm{SN}^{1,3}$, Moser $\mathrm{A}^{4}$, Salbert $\mathrm{T}^{5}$, Argyropoulos $\mathrm{D}^{6}$

Institute 1 Chemistry Department, University of Cape Town, Cape Town, South Africa; 2 Department of Chemistry, University of Mauritius, Reduit, Mauritius; 3 South African Medical Research Council, Drug Discovery and Development Research Unit, University of Cape Town, Cape Town, South Africa; 4 Advanced Chemistry Development (ACD/Labs), Inc, Toronto, Canada; 5 Advanced Chemistry Development (ACD/Labs) Germany GmbH, Frankfurt am Main, Germany; 6 Advanced Chemistry Development (ACD/Labs) UK Ltd, Bracknell, United Kingdom

DOI 10.1055/s-0037-1608237

As part of a phytochemical investigation of the leaves of $O$. mauritiana, we have isolated, using a combination of silica gel flash chromatography and preparative layer chromatography (PLC), two biflavonoid compounds ( 1 and 2) from the organic extract of this plant. Approximately $12.5 \mathrm{mg}$ of 1 and $2.5 \mathrm{mg}$ of 2 were placed in NMR tubes and dissolved with DMSO-d6 in order to elucidate their structures. High-Resolution Mass Spectra were also recorded in order to establish the molecular formulae.

Both compounds were relatively proton deficient resulting in few correlations in the long range 2D NMR experiments. The repeated ring structure made some of the $13 \mathrm{C}$ peaks appear very close together which also limited the usefulness of the conventional 2D experiments (Fig. 1). Together with the presence of many exchangeable protons the elucidation of the structures proved to be rather challenging. Here we describe the advanced computation method used for elucidating the structures using a series of $1 \mathrm{D}$ and $2 \mathrm{D} N M R$ experiments.
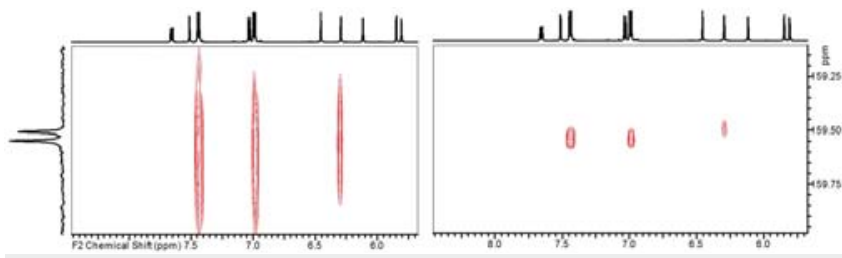

Fig. 1: Comparison between the conventional (left) and band-selective (right) $1 \mathrm{H}-\{13 \mathrm{C}\} \mathrm{HMBC}$ spectrum of compound 1 in the $\mathrm{F} 1$ region around $159.5 \mathrm{ppm}$.

Computer Assisted Structure Elucidation (CASE) together with band-selective versions of the $\mathrm{HMBC}$ experiment were employed to assign the NMR data and unequivocally determine the structures, confirming the new molecules. Several closely related structures were ruled out either by the poor agreement between the predicted and experimental $13 \mathrm{C}$ chemical shifts or by additional derivatisation experiments.

\section{Mo-Poster Session 1-PO-191 Identification of antioxidant polyphenolics from Jasione montana based on a preliminary LC-MS profiling}

Authors Jabłonowska M¹, Mutryn $\mathrm{K}^{2}$, Bazylko A ${ }^{3}$, Grochowski D ${ }^{4}$, Strawa J4, Tomczyk $\mathrm{M}^{4}$

Institute 1 Students' Scientific Association, Department of Pharmacognosy, Faculty of Pharmacy, Medical University of Bialystok, Bialystok, Poland; 2 Students' Scientific Association, Department of Pharmacognosy and Molecular Basis of Phytotherapy, Faculty of Pharmacy, Medical University of Warsaw, Warsaw, Poland; 3 Department of Pharmacognosy and Molecular Basis of Phytotherapy, Faculty of Pharmacy, Medical University of Warsaw,
Warsaw, Poland; 4 Department of Pharmacognosy, Faculty of Pharmacy, Medical University of Bialystok, Bialystok, Poland DOI 10.1055/s-0037-1608238

Jasione montana (Campanulaceae) known as sheep's bit scabious is biennial, rarely annual plant, occurring commonly throughout Europe [1]. Ethnopharmacological report emphasizes its role in sleep disorders in children, although its biological activity have not been yet assessed [2]. According to the literature, little is also known about the presence of undefinable phytochemicals in J. montana [3]. For the first time antioxidant activities of fractions obtained from sheep's bit scabious using different in vitro antioxidant models were also evaluated. The ethyl acetate fraction showed highest antioxidant activity, both for DPPH $\left(\mathrm{IC}_{50}=19.03 \mu \mathrm{g} / \mathrm{mL}\right)$ and for O2-. $\left(\mathrm{IC}_{50}=5.77 \mu \mathrm{g} / \mathrm{mL}\right)$ assays. An analytical approach based on LC-ESI-MS was applied to rapidly obtain a metabolite profile of all fractions obtained from the aerial parts of J. montana. Finally, in all investigated fractions ten polyphenolic compounds (1-10) were identified. Among them, eight rare derivatives of luteolin have been reported. Till now, two known flavonoids namely luteolin (1) and luteolin 7-glucoside (2) have been isolated and their structures have been unambiguously elucidated by extensive chromatographic and spectroscopic methods, including UV and NMR experiments. Isolation and identification of further flavonoid compounds from the investigated fractions is being carried out.

This study was financially supported by the Grant (No. N/ST/ZB/16/004/ 2212) from the Medical University of Bialystok (Poland). The study was conducted with the use of equipment by the Medical University of Bialystok as part of the OP DEP 2007-2013, Priority Axis 1.3, contract No. POPW.01.03.00 - $20-001 / 12$.

[1] Parnell JAN. J Ecol 1985; 73: 341 - 358;

[2] Trojanowska A. Analecta 2007; 1 - 2: 7 - 53;

[3] Zapesochnaya GG, Nikolaeva VG, Ban'kovskii Al. Chem Nat Comp 1972; 8: 112

\section{Mo-Poster Session 1-PO-192 Application of a novel UPLC-HRMS-based plasma metabolomics approach reveals differences between male and female mice following i.p. administration of trans-crocin-4.}

Authors Karkoula $\mathrm{E}^{1,4}$, Gikas $\mathrm{E}^{3}$, Baira $\mathrm{E}^{3}$, Kokras $\mathrm{N}^{1,2}$, Dalla $\mathrm{C}^{1}$, Skaltsounis $\mathrm{AL}^{3}$, Tsarbopoulos $\mathrm{A}^{1,4}$

Institute 1 Medical School, National and Kapodistrian University of Athens, Department of Pharmacology, Athens, Greece; 2 Medical School, National and Kapodistrian University of Athens, First Department of Psychiatry, Athens, Greece; 3 Department of Pharmacy, National and Kapodistrian University of Athens, Athens, Greece; 4 GAIA Research Center, The Goulandris Natural History Museum, Bioanalytical Department, Kifissia, Greece DOI 10.1055/s-0037-1608239

Trans-crocin 4 (TC4) is an important carotenoid constituent of saffron showing remarkable activity against Alzheimer's disease due to its antioxidant and antiamyloidogenic properties. Metabolomics is an emerging scientific field that enhances biomarker discovery and reveals underlying biochemical mechanisms aiming towards the early subclinical diagnosis of diseases. So far, there have been scarce data demonstrating changes induced to mice plasma metabolome after TC 4 administration. Thus, an untargeted UPLC-HRMS metabolomics approach has been employed to determine the alteration to the metabolic fingerprint after i.p administration of TC 4 in male and female mice. Therefore, blood samples from 58 mice administered with TC 4 including control animals were analyzed by UPLC-HRMS (Orbitrap Discovery XL). Statistical evaluation of the results was achieved by multivariate analysis (MVA) i.e. principal component analysis (PCA), projections to latent structures-discriminant analysis (PLS-DA) in order to discover the variables that contributed to the discrimination between treated and untreated groups whereas the variables contributing to the discrimination were identified using comparisons to online databases (e.g. Metlin) along with software manipulations e.g. adduct 
and fragment identification, covariance searching etc. It should be noted that due to the high variability imposed by various factors e. g. sex, dose and time points, the multilevel PLS-DA e.g. splitting variation to each individual component, has proven to be the only effective approach. By this methodology, the time sequence of metabolome changes due to the administration of TC4 has been made apparent. Furthermore, a sex-related effect on the metabolome has been proven to exist, denoting that the administration in both genders is indispensable in order to acquire safe conclusions as reliable metabolome pictures.

\section{Mo-Poster Session 1-PO-193 New terpenoid glycosides from Eriocoelum microspermum}

Authors Pertuit $D^{1}$, Mitaine-Offer $A C^{1}$, Miyamoto $T^{2}$, Tanaka $C^{2}$, Delaude $C^{3}$, Lacaille-Dubois MA $^{1}$

Institute 1 PEPITE EA 4267, Laboratoire de Pharmacognosie, UFR des

Sciences de Santé, Université de Bourgogne Franche-Comté, Dijon, France; 2 Graduate School of Pharmaceutical Sciences, Kyushu University, Fukuoka, Japan; 3 Centre de Recherche Phytochimique, Université de Liège, Institut de Chimie-B6, Sart Tilman, Liège, Belgium

DOI 10.1055/s-0037-1608240

The family of Sapindaceae includes more than 2000 species which are distributed into three sub-families and fourteen tribes. A chemical investigation of the hydro-ethanolic extract of the stems of Eriocoelum microspermum Radlk. ex Wild (Sapindoideae sub-family and Cupianeae tribe) from the Democratic Republic of Congo, led to the isolation of six new glycosidic compounds. Their structures were established mainly by extensive 1D and 2D NMR and mass spectroscopic analysis. Three new triterpene saponins and a known one were characterized as hederagenin derivatives, glycosylated by 3 to 5 sugars at the C-3 of the aglycone. The partial sequence 3-O- $\alpha$-L-rhamnopyranosyl-(1 - 2)- $\alpha$ $\mathrm{L}$-arabinopyranosylhederagenin was shown to possess additional glycosylations by $\alpha$-L-rhamnopyranosyl and/or $\beta$-D-xylopyranosyl units. This sequence already encountered in other genera than Eriocoelum, particularly Sapindus, Lecaniodiscus, Ellatostachys, Smelophyllum, may represent a chemotaxonomic marker for the Sapindoidae sub-family [1],[2]. Furthermore, two new glycosides of farnesol, an acyclic sesquiterpene alcohol, with 6 sugars (glc, rha, ara, $\mathrm{xyl}$ ), were isolated. The partial sequence $\mathrm{rha}^{1}-{ }^{6} \mathrm{glc}^{1}{ }^{1}{ }^{1}$ farnesol rarely described in the literature, was already found in three species of the genus Sapindus (Sapindoideae). This presentation shows that two classes of terpenoid glycosides characterized in Eriocoelum microspermum are relevant for the chemotaxonomy of the Sapindoideae.

[1] Delaude, C. Bull. Soc. Roy. Sci. Liège 1993, 62: 93-120.

[2] Voutquenne-Nazabadioko, L. Ethnopharmacologia 2010, 45: 49- 52.

\section{Mo-Poster Session 1-PO-194 Porcine small intestine, a good ex vivo model to investigate absorption and metabolism of natural products}

Authors Houriet J $]^{1}$, Arnold $\mathrm{YE}^{1}$, Petit $\mathrm{C}^{1}$, Kalia $\mathrm{YN}^{1}$, Wolfender $\mathrm{JL}^{1}$ Institute 1 School of Pharmaceutical Sciences, University of Geneva, University of Lausanne, CMU - Rue Michel Servet 1, Geneva, Switzerland DOI 10.1055/s-0037-1608241

Herbal extracts used in folk medicine are complex mixtures of natural products (NPs). Their efficacy in vivo after ingestion depends on their uptake (and sometimes their metabolites) in the gastrointestinal tract. The correlation of bioactivities measured in vitro with efficacy in vivo is a challenge since bioavailability and biotransformation can have an impact.[1] We have validated an ex vivo model using viable porcine small intestine to elucidate the effects of absorption and intestinal metabolism on drug uptake (manuscript in preparation). Porcine intestine closely resembles its human counterpart in terms of physiology and function; active and passive transport mechanisms are observed and similar metabolizing enzymes are present.[2] Representative NPs and their parent herbal extracts were selected and the permeation across porcine jejunum, harvested immediately after slaughter, was studied using an Ussing chamber system.[2] Aliquots from the donor and acceptor compartments were analyzed by non-targeted UHPLC-HRMS to enable metabolite profiling and the permeation of the NPs was quantified by targeted UHPLCMS-MRM. Results with Pueraria lobata indicated that, in contrast to observations with PAMPA [3] the C-glycoside puerarin and the aglycone daidzein were able to permeate; the different behavior was most likely due to differences in molecule partitioning and possibly to active transport. However, the corresponding O-glycoside, daidzin, showed no permeation but was extensively metabolized. This was observed for the pure constituent and the decoction - demonstrating the importance of pre-systemic metabolism. Tests with other NPs confirmed that this ex vivo model was well-suited to investigating the bioavailability of isolated NPs and components from complex extracts. Although not a high throughput system, it enables a much deeper understanding of the roles and impact of permeability and metabolism on bioavailability.

[1] Butterweck V, Nahrstedt A. Planta Medica: 2012; 78: 747 - 754.

[2] Sjögren et al. Eur ] Pharm Sci: 2014; 57: $99-151$.

[3] Petit C et al. Planta Medica:2016; 82: 424 - 431.

\section{Mo-Poster Session 1-PO-195 Separation of 1'S-1'-acetoxychavicol acetate from a Alpinia galanga rhizome extract by fast centrifugal partition chromatography}

Authors Sumthong Nakmee $\mathrm{P}^{1}$, Schwaiger $\mathrm{S}^{2}$, Stuppner $\mathrm{H}^{2}$

Institute 1 Faculty of Science at Sriracha, Kasetsart University, Sriracha Campus, 199 Moo 6, Sukhumvit Road, Sriracha, 20230, Chonburi, Thailand;

2 Institute of Pharmacy/Pharmacognosy, University of Innsbruck, CCB-Center for Chemistry and Biomedicine, Innrain 80-82/IV, 6020, Innsbruck, Austria DOI 10.1055/s-0037-1608242

Fast Centrifugal Partition Chromatography (FCPC) is one of the modern techniques for separation of medicinal plants with multiple benefits. Alpinia galanga rhizome was used for seasoning and traditional medicine with various biological activities. The separation of a of Alpinia galanga rhizome hexane extract by an optimized water-free FCPC method enabled the successful isolation of 1 'S-1,-acetoxychavicol acetate in a purity of $>93 \%$ and a yield of $76.47 \%$. The compound inhibited the growth of the bacterium Bacillus thuringiensis and the fungus Beauveria bassiana pathogens causing the silkworm disease with MIC values of 62.5 and $\leq 2 \mu \mathrm{g} / \mathrm{mL}$.

\section{Mo-Poster Session 1-PO-196 Isolation and identification of polyphenolic compounds from aerial parts of Corispermum marshallii Steven}

Authors Pawłowska $\mathrm{K}^{1}$, Wilczek $\mathrm{M}^{2}$, Granica $\mathrm{S}^{1}$

Institute 1 1Department of Pharmacognosy and Molecular Basis of Phytotherapy, Faculty of Pharmacy, Medical University of Warsaw, Warsaw, Poland; 2 Laboratory of NMR Spectroscopy, Faculty of Chemistry, University of Warsaw, Warsaw, Poland

DOI 10.1055/s-0037-1608243

Corispermum marshallii Steven is an herbaceous annual plant material belonging to the Amaranthaceae family. In Poland this plant is a domesticated species occurring in open, gravelly or sand habitats. In the literature there are no studies considering the polyphenolic composition of the aerial parts of any Corispermum species. Available research shows, that plant materials belonging to this genus contain ecdysteroids and some isoquinoline alkaloids including salsoline and salsolidine.

The aim was to isolate and characterise major flavonoids occurring in aerial parts of $C$. marshallii.

The plant material was collected on Vistula river banks, then dried aerial parts were extracted with acetone-MeOH-H2O. Solvents were evaporated under reduced pressure and water residue fractionated by liquid-liquid extraction 
with chloroform, diethyl ether, ethyl acetate and n-butanol. Ethyl acetate and n-butanol fractions were separated using column chromatography and preparative HPLC.

Thirteen major polyphenols were isolated. Their structures were established based on 1D and 2D NMR experiments and MS/MS analysis. All obtained compounds were classified as flavonoids - quercetin, patuletin or spinacetin derivatives. Hyperoside (1) and patuletin 3-O- $\beta$-D-galactopyranoside (2) were found as dominating constituents. Additionally, six other patuletin glycosides were isolated, including: 3-O- $\beta$-D-galactopyranosyl-7-O- $\beta$-D-glucopyranoside (3), 3-O-(6"'-O- $\beta$-D-glucopyranosyl)- $\beta$-D-galactopyranoside (4), 3-O-(6"-O- $\alpha$ L-arabinopyranosyl)- $\beta$-D-galactopyranoside (5), 3-O-(2"'-O-(5'”-O- $\alpha$-L-arabinopyranosyl)- $\beta$-D-apiofuranosyl)- $\beta$-D-galactopyranoside (6), 3-O-(2"'-O- $\beta-D-$ apiofuranosyl)- $\beta$-D-galactopyranoside (7) and 3-O- $\beta$-D-galactopyranosyl-7-O(6"'-O-feruloyl)- $\beta$-D-glucopyranoside (8). Moreover, three spinacetin derivatives -3-O- $\beta$-D-galactopyranoside (9), 3-O- $\beta$-D-galactopyranosyl-7-O- $\beta-D-$ glucopyranoside (10) and 3-O- $\beta$-D-galactopyranosyl-7-O-(6"'-O-feruloyl)- $\beta$ D-glucopyranoside (11) were identified. Other identified glycosides were 6methoxykaempferol 3-O- $\beta$-D-galactopyranoside (12) and naringenin 8-C- $\beta$-Dglucopyranoside (13).

All compounds were detected in the analysed plant material for the first time. Nine of the isolated flavonoid glycosides (compounds $3-11$ ) are new natural products.
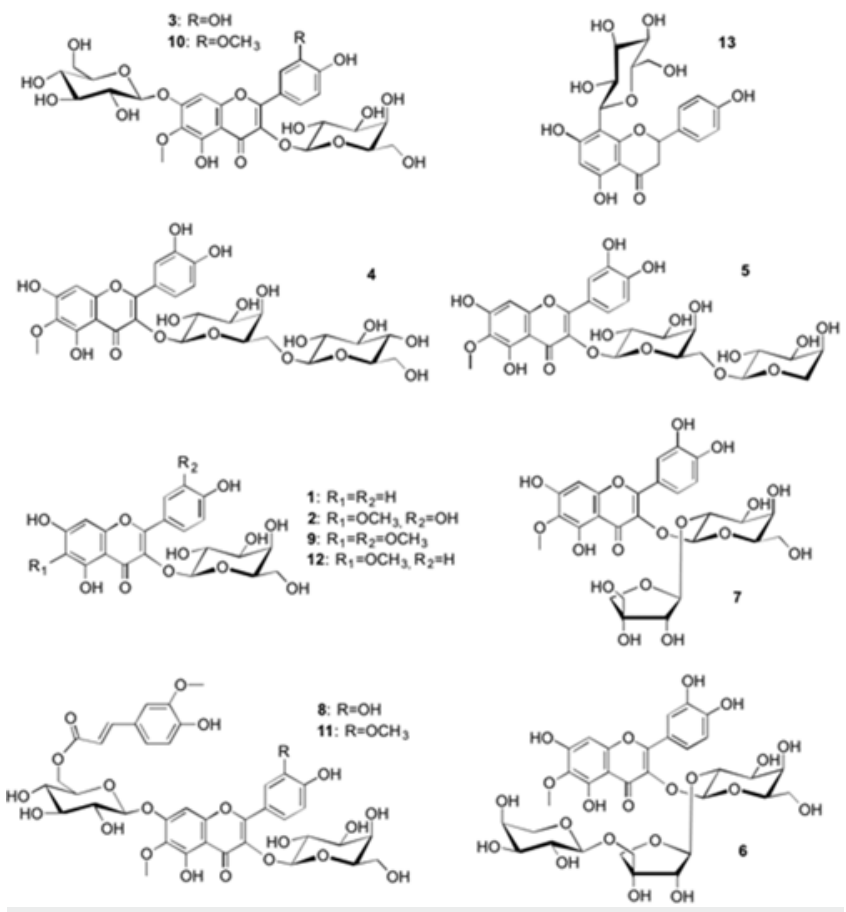

Fig. 1

\section{Mo-Poster Session 1-PO-197 Isolation of further alkaloids from Galanthus cilicicus}

Authors Kaya $\mathrm{Gi}^{1}$, Uzun $\mathrm{K}^{2}$, Demir $\mathrm{S}^{1}$, Bozkurt B ${ }^{1}$, Onur MA ${ }^{1}$, Unver Somer $\mathrm{N}^{1}$ Institute 1 Department of Pharmacognosy, Faculty of Pharmacy, Ege University, Bornova, Izmir, Turkey; 2 Department of Pharmacognosy, Faculty of Pharmacy, Erciyes University, Melikgazi, Kayseri, Turkey

DOI 10.1055/s-0037-1608244

Galanthus L. species are among the most important native flower bulbs exported from Turkey, therefore they have an economical importance. They are known to possess Amaryllidaceae alkaloids with interesting chemical structures and biological activities. Among these alkaloids, galanthamine is used for the treatment of mild to moderate Alzheimer's disease [1]. Galanthus cilicicus Baker, an endemic species among Turkish Galanthus taxa, is distributed in southern Turkey mainly in the province of Içel [2]. In the course of our ongoing phytochemical studies on Turkish Galanthus species, previously, we have reported the alkaloidal profile of G. cilicicus by GC-MS and the major alkaloids found in this species [3]. In the present study, two minor alkaloids were isolated from G. cilicicus. fractionation of the crude alkaloidal extract, using column chromatography and then further fractionation and purification procedures including preparative column and thin layer chromatography yielded two known phenanthridine-type Amaryllidaceae alkaloids, namely norismine and $\mathrm{N}$-methylcrinasiadine. The structures of the compounds were elucidated by means of spectroscopic methods (1D NMR, and MS). To the best of our knowledge, this is the first report of the occurrence of norismine and $\mathrm{N}$ methylcrinasiadine, in the genus Galanthus. The phytochemical studies carried on this endemic species revealed that it is a promising source for alkaloids with interesting structural diversities.

This study was financially supported by Ege University, Research Fund (16/ ECZ/032).

[1] Heinrich M, Teoh, HL. J Ethnopharm 2004; 92:147 - 162.

[2] Davis AP. The Genus Galanthus-Snowdrops in the Wild, In: Bishop M, Davis AP, Grimshaw J. (Eds.) Snowdrops, A Monograph of Cultivated Galanthus. Cheltenham: Griffin Press Publishing Ltd. 2006: 9- 63.

[3] Kaya GI, Uzun K, Bozkurt B, OnurMA, Somer NU, Glatzel DK, Fürst R. South African Journal of Botany 2017; 108: $256-260$.

\section{Mo-Poster Session 1-PO-198 In situ MALDI-HRMS-imaging of Maytenus senegalensis during the germination process reveals the ecological role of the important anticancer compound maytansine}

\author{
Authors Kusari $\mathrm{S}^{1}$, Eckelmann $\mathrm{D}^{1}$, Spiteller $\mathrm{M}^{1}$ \\ Institute 1 Institute of Environmental Research (INFU), TU Dortmund, \\ Dortmund, Germany \\ DOI 10.1055/s-0037-1608245
}

The ecological role of maytansine, an important antineoplastic and antimicrobial compound with high cytotoxicity $[1,2]$, particularly as a chemical defense compound has remained elusive since its discovery in the $1970 \mathrm{~s}$ in Celastraceae plants such as Maytenus and Putterlickia species [3]. After more than four decades of discovery of this important drug used against breast cancer, we provide a proof-of-concept of ecospecific and tissue-specific production and in situ spatial/temporal distribution of maytansine in Maytenus senegalensis plants. We have used MALDI-HRMS-imaging to visualize the occurrence and spatial/temporal distribution of maytansine in the leaves, stems, and roots of M. senegalensis plants, seeds obtained from the mother plants, through the germination process, and finally to the establishment of new seedlings or daughter plants. The mother plant was devoid of maytansine in all tissues. However, maytansine was produced and distributed in the cotyledons and the endosperm of the seeds with an augmented accretion towards the seed coat. Furthermore, maytansine was always detected in the emerging seedlings, particularly the cortex encompassing the radicle, hypocotyl, and epicotyl (Figure 1). 


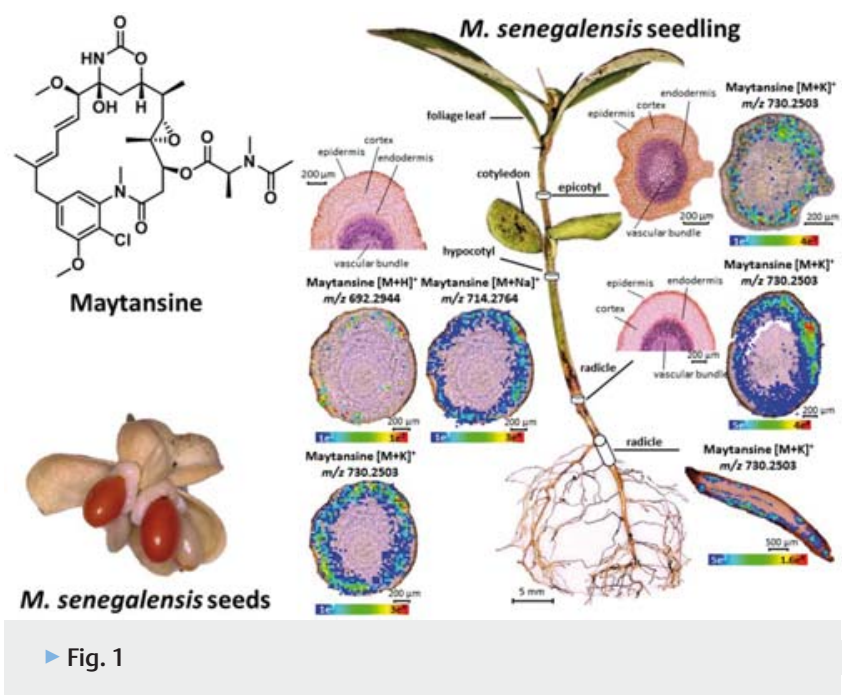

The typical pattern of production and accumulation of maytansine not only in the seeds but also during germination provides evidence that $M$. senegalensis is ecologically primed to trigger the production of maytansine in vulnerable tissues such as seeds during plant reproduction. By utilizing maytansine as chemical defense compound against predators and/or pathogens, the plant can ensure viability of the seeds and successful germination, thus leading to the next generation of daughter plants with an evolutionary advantage of survival [4].

[1] Kusari et al. J. Nat. Prod. 2014; 77: $2577-2584$

[2] Kusari et al. RSC Adv. 2016; 6: $10011-10016$

[3] Kupchan et al. J. Am. Chem. Soc. 1972; 94: $1354-1356$

[4] Eckelmann et al. Fitoterapia 2017; 119: 51 - 56

\section{Mo-Poster Session 1-PO-199 A New Sesquiterpene Isolated From The Aerial Parts of Ferula caspica Bieb.}

Authors Kahraman $\mathrm{C}^{1}$, Topcu $\mathrm{C}^{2}$, Bedir $\mathrm{E}^{3}$, Akdemir $\mathrm{ZS}^{1}$

Institute 1 Department of Pharmacognosy, Faculty of Pharmacy, Hacettepe University,TR-06100, Ankara, Turkey; 2 Department of Pharmacognosy,

Faculty of Pharmacy, Bezmialem Vakif University, TR- 34093, Istanbul, Turkey; 3 Department of Bioengineering, Faculty of Engineering, Izmir Institute of Technology, TR- 35430, Izmir, Turkey

DOI 10.1055/s-0037-1608246

Ferula (Apiaceae) is represented by 23 species, 13 of which are endemic to Turkey $[1,2]$. Ferula species used for diabetes, stomach ache, gynecological disorders and hypercholesterolemia, also known to be immunostimulant, demulcent, digestive, carminative, antihelmentic and aphrodisiac in Traditional medicine [3] are important biological sources to investigate. Aim of this study was elucidation of structures of secondary metabolites from F. caspica Bieb. Powdered aerial parts of the plant were extracted with petroleum ether, chloroform and methanol respectively. Chromatographic studies on chloroform extract resulted in the isolation of 2,3-dihydro-7-hydroxy-2,3-dimethyl-2[4', 8'-dimethyl-3',7'-nonadienyl]-furo[3,2,c]coumarin (1), 1-(2,4-dihydroxyphenyl)-3,7,11-trimethyl-3-vinyl-6(E),10-dodecadien-1-one (2), 3-(4',8'-dimethylnona-3',7'-dienyl)-2,3-dimethyl-2,3-dihydrobenzofuran-6-ol (=caspican) (3), laserine/2-epilaserine isomeric mixture (4A/4B) and stigmasterol/ $\beta$ sitosterol mixture (5A/5B) [2]. The structures of compounds were elucidated by spectroscopic methods ( $1 \mathrm{H}-\mathrm{NMR}, 13 \mathrm{C}-\mathrm{NMR}, \mathrm{HMQC}, \mathrm{HMBC}, \mathrm{COSY}$ and Mass Spektrometry). Compound 1 (=caspican) was structurally identified to be a new compound obtained from nature; the other compounds were isolated from this plant for the first time.

[1] Davis PH, Flora of Turkey and the East Aegean Islands, Vol 4. Edinburgh: Edinburgh University Press; 1972: 440 - 453

[2] Kahraman C. PhD thesis, 2016: 1 - 8
[3] Altundag E, Ozturk M. Procedia Soc Behav Sci 2011; 19:756 - 777

\section{Mo-Poster Session 1-PO-200 Multi informative molecular network to explore the chemodiversity and bioactivity of an atypical natural resource: entomopathogenic microrganisms}

Authors Pellissier $\mathrm{L}^{1}$, Toure $\mathrm{S}^{2}$, Allard PM $\mathrm{M}^{1}$, Eparvier $\mathrm{V}^{2}$, Wolfender $\mathrm{L}^{1}$ Institute 1 School of Pharmaceutical Sciences, EPGL, University of Geneva, University of Lausanne, Rue Michel Servet 1, CH-1211, Geneva, Switzerland; 2 CNRS, Institut de Chimie des Substances Naturelles UPR 2301, University Paris-Saclay, 1 Avenue de la Terrasse,91198, Gif-sur-Yvette, France DOI $10.1055 / \mathrm{s}-0037-1608247$

Entomopathogenic microorganisms (principally fungi and bacteria) are natural pathogens of insects involved in the regulation of host populations, representing an underexplored field of natural products (NPs) research. They have been shown to produce a wide range of bioactive secondary metabolites and thus appear as a promising source of new agents of biological, pharmaceutical and ecological interests (1). Our objective was to explore the chemodiversity and simultaneously investigate antimicrobial and insecticidal compounds within a unique collection of 57 enthomopathogenic strains collected on living infected insects. Recently, molecular networking (MN) has been established as a useful tool for the chemical exploration of complex extracts, since it enables the clustering of detected metabolites as structural families according to the similarity of their fragmentation patterns (tandem $\mathrm{MS}^{2}$ spectra) (2). Here, a multi-informative $\mathrm{MN}$ was built by overlaying different information layers (taxonomy, bioactivity) on top of the MN generated from the full collection of entomopathogenic fungi. This method allowed the organisation of 203'928 $M^{2}$ spectra into 821 clusters containing 5'784 nodes. By annotating the constructed MN against experimental and in silico spectral NPs databases (3), we could identify molecular families previously described in those organisms like beauveriolides or eniatins. Additionally, the exploration of the MN via the bioactivity mapping allowed to highlight clusters containing nodes from bioactive extracts. Compounds found in some of these clusters could be linked to previously isolated larvicidal and antibiotic secondary metabolites. The approach permitted to easily pinpoint unknown analogues of those compounds that are likely to be bioactive and the data generated will be used to target their isolation.

[1] Beemelmanns et al. Beilstein J Org Chem. 2016;12:314 - 27.

[2] Wang M et al. Nat Biotech. 2016;34:828 - 37.

[3] Allard PM et al. Anal Chem. 2016;88:3317-23.

Photo credits: ${ }^{\circ}$ Andreas Kay, 2016, https://www.flickr.com/photos/andreaskay/31115643631 (accessed March 2017)

\section{Mo-Poster Session 1-PO-201 Phytochemical investigation of the roots of Sesamum alatum Thonn.}

Authors Saraux $\mathrm{N}^{1}$, Christen $\mathrm{P}^{1}$, Cuendet $\mathrm{M}^{1}$

Institute 1 School of Pharmaceutical Sciences, University of Geneva, Geneva, Switzerland

DOI $10.1055 / \mathrm{s}-0037-1608248$

In the course of the investigation of plants used in traditional medicine in Niger, Sesamum alatum Thonn. (Pedaliaceae) was selected. This plant is traditionally used against women's sterility, diarrhea and fever [1]. A $20 \mu \mathrm{g} / \mathrm{mL}$ dichloromethane root extract showed a complete inhibition of multiple myeloma cancer stem cells (MM-CSCs) growth and a $90 \%$ inhibition of NF-kB. It also showed an inhibition $>80 \%$ at $10 \mu \mathrm{g} / \mathrm{mL}$ of the parasites Leishmania donovani and Trypanosoma brucei rhodesiense. Very few studies on Sesamum alatum have been reported so far [2]. A bioassay-guided fractionation of the extract was performed using a reverse phase semi-prep HPLC system and afforded 28 fractions. At $20 \mu \mathrm{g} / \mathrm{mL}$, sixteen fractions exhibited an inhibition of MM-CSCs growth $>90 \%$ and six showed an inhibition of NF-kB $>50 \%$. Three fractions were selected for further purification. The major compounds were 
isolated and identified as anthrasesamone $\mathrm{A}$ and hydroxysesamone. These compounds are reported for the first time in the roots of Sesamum alatum and their biological activity is under investigation.<smiles>CC(C)=CCCc1ccc2c(c1O)C(=O)c1ccccc1C2=O</smiles>

Anthrasesamone $\Lambda$

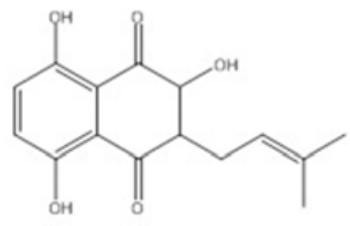

Hydroxysesamone
Fig. 1

[1] Eklu-Natey RD, Balet A. Pharmacopée africaine, Dictionnaire et monografie multilingues du potentiel médical des plantes africaines, Editions d'en bas: Genève; 2012: 2.

[2] Potterat O, Hostettmann K, Stoeckli-Evans H, Saadou M. Helv Chim Acta 1992; 75: $833-841$.

\section{Mo-Poster Session 1-PO-202 Dual high-resolution $\alpha$-glucosidase/PTP1B bioassays coupled with HPLC-HRMS-SPE-NMR for investigation of 'Insulin plants' (Myrcia sp.) as new medicines for type 2 diabetes}

Authors Lima Rita de Cassia $L^{1}$, Kato $L^{2}$, Kongstad $K T^{1}$, Jäger $A K^{1}$, Staerk $D^{1}$ Institute 1 Department of Drug Design and Pharmacology, University of Copenhagen, Copenhagen, Denmark; 2 Instituto de Química, Universidade Federal de Goiás, Goiânia, Brazil

DOI 10.1055/s-0037-1608249

Myrcia species or 'insulin plants' are used by indigenous communities in Brazil to treat type 2 diabetes [1]. Therapeutic targets for T2D include $\alpha$-glucosidase- and protein tyrosine phosphatase 1B (PTP1B) inhibitors [2]. Herein, we report the screening of six Myrcia species for $\alpha$-glucosidase and PTP1B inhibitory activity - M. guianensis ( $\left(\mathrm{C}_{50 \mathrm{AG}}=7.80 \mu \mathrm{g} / \mathrm{mL} ; \quad \mathrm{IC}_{50 \mathrm{PTP} 1 \mathrm{~B}}=3.26 \mu \mathrm{g} / \mathrm{mL}\right)$, M. rubella $\left(I C_{50 A G}=4.36 \mu \mathrm{g} / \mathrm{mL} ; \quad I C_{50 P T P 1 B}=9.84 \mu \mathrm{g} / \mathrm{mL}\right), M$. torta $\left(I C_{50 A G}=\right.$ $\left.5.80 \mu \mathrm{g} / \mathrm{mL} ; \quad \mathrm{IC}_{50 \text { PTP } 1 \mathrm{~B}}=27.00 \mu \mathrm{g} / \mathrm{mL}\right), \quad$ M. variabilis $\quad\left(\mathrm{IC}_{50 A G}=3.17 \mu \mathrm{g} / \mathrm{mL}\right.$; $\left.\mathrm{IC}_{50 \text { PTP } 1 \mathrm{~B}}=1.10 \mu \mathrm{g} / \mathrm{mL}\right)$, M. vestita $\left(\mathrm{IC}_{50 \mathrm{AG}}=13.60 \mu \mathrm{g} / \mathrm{mL} ; \mathrm{IC}_{50 \text { PTP } 1 \mathrm{~B}}=2.83 \mu \mathrm{g} /\right.$ $\mathrm{mL})$, and $M$. virgata $\left(\mathrm{IC}_{50 \mathrm{AG}}=6.30 \mu \mathrm{g} / \mathrm{mL} ; \mathrm{IC}_{50 \mathrm{PTP} 1 \mathrm{~B}}=3.00 \mu \mathrm{g} / \mathrm{mL}\right)$. M. rubella was chosen for further analyses, and the crude ethyl acetate extract was submitted to dual high-resolution $\alpha$-glucosidase and PTP1B profiling for identification of bioactive constituents. Extensive analyses using HPLC-HRMS-SPENMR [3] led to the identification of 36 compounds including the $\alpha$-glucosidase inhibitors $-4,5$-dicaffeoylquinic acid (IC50AG $=1.98 \mu \mathrm{M}$ ), isoquercitrin (IC 50$A G=22.4 \mu \mathrm{M})$, quercetin 3-O- $\beta$-D-glucuronide $\left(I C_{50} A G=21.30 \mu \mathrm{M}\right)$, and quercetin 3-O-(6"-malonylglucoside) $\left(\mathrm{IC}_{50} \mathrm{AG}=6.60 \mu \mathrm{M}\right)$. PTP1B inhibition was associated with triterpenes such as arjunolic acid and 3,4,3'-tri-O-methylellagic acid.

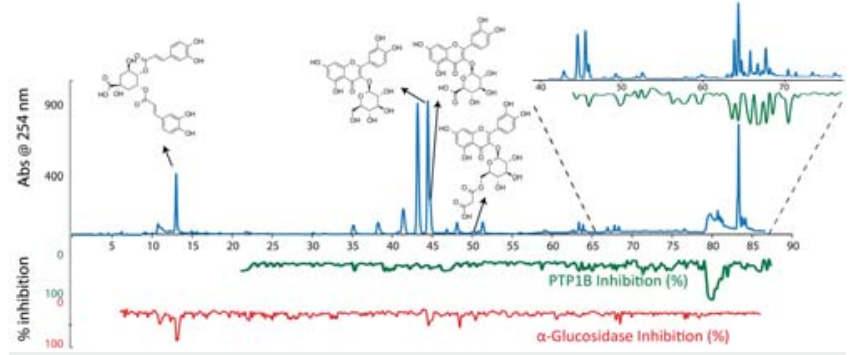

Fig. 1

[1] Cascaes MM, Guilhon GMSP, Andrade EHA, Zoghbi MGB, Santos LS. Int ] Mol Sci 2015; 16: $23881-23904$.
[2] Zhang S, Zhang Z-Y. Drug Discov Today 2007; 12: 373 - 381.

[3] Wubshet SG, Tahtah Y, Heskes AM, Kongstad KT, Pateraki I, Hamberger B, Møller BL, Staerk D. J Nat Prod 2016; 79: 1063 - 1072.

\section{Mo-Poster Session 1-PO-203 High-resolution $\alpha$-glucosidase inhibition profiling and HPLC-HRMS-SPE-NMR for phytochemical investigation and identification of antidiabetic constituents in Picramnia glazioviana}

Authors Gimenes $L^{1,2}$, da Silva Maria Fatima das Gracas $F^{1}$, Vieira Paulo $C^{1}$, Fernandes João $B^{1}$, Staerk $D^{2}$

Institute 1 Chemistry Department - Federal University of São Carlos, São Carlos, Brazil; 2 Department of Drug Design and Pharmacology, University of Copenhagen, Copenhagen, Denmark

DOI $10.1055 / \mathrm{s}-0037-1608250$

Type 2 diabetes (T2D) is a chronic metabolic disorder, and the incidence of T2D increases at an alarming rate and constitutes a global health problem. In this context, the use of plants as a source for inhibitors of $\alpha$-glucosidase, an enzyme involved in carbohydrate metabolism in the brush border of the small intestines, seems an attractive approach [1]. The genus Picramnia shows high diversity of secondary metabolites, such as anthrones, oxanthrones, anthraquinones, coumarins, and triterpenes [2]. In our on-going screening programme for antidiabetic compounds from plants, the ethanolic extract of $P$. glazioviana showed an inhibitory concentration $\left(\mathrm{IC}_{50}\right)$ of $27.31 \mu \mathrm{g} / \mathrm{mL}$ towards $\alpha$-glucosidase, and was therefore selected for further investigation. This extract was subjected to liquid-liquid partitioning, and the ethyl acetate and dichloromethane fractions showed $\mathrm{IC}_{50}$ values of $15.11 \mu \mathrm{g} / \mathrm{mL}$ and $38.44 \mu \mathrm{g} /$ $\mathrm{mL}$, respectively. The ethyl acetate fraction was investigated with high-resolution $\alpha$-glucosidase inhibition profiling combined with LC-HRMS. This disclosed two flavonoids, catechin and epicatechin, which have previously been reported to have $\alpha$-glucosidase inhibitory activity [1]. The dichloromethane fraction was investigated with a combination of HPLC-HRMS-SPE-NMR and preparative-scale HPLC. This disclosed six new nortriterpenes named picravianes A-F. The results show that plants of the genus Picramnia are a valuable source of new and bioactive compounds.

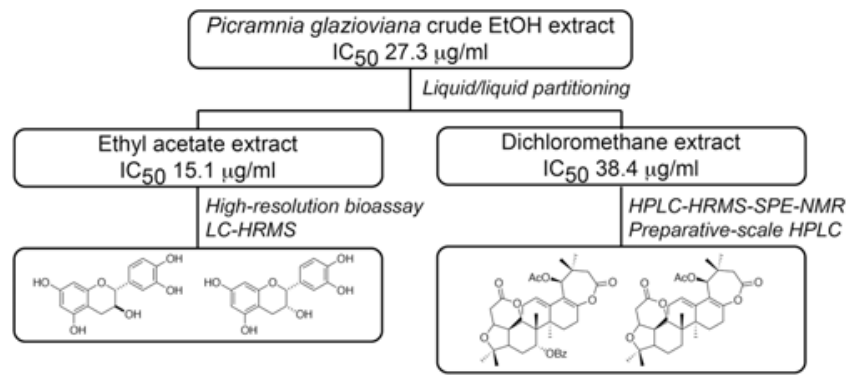

Fig. 1

FAPESP is acknowledged for a sandwich PhD scholarship to Leila Gimenes and Arife Önder is thanked for technical assistance.

[1] Kim TH. J Nat Med 2016; 70:811-815.

[2] Rodríguez-Gamboa T, Fernandes JB, Filho ER, Da Silva MFGF, Vieira PC, Barrios Ch M, Castilho OC, Victor SR, Pagnocca FC, Bueno OC, Hebling MJA. J Braz Chem Soc 2001; 12:386 - 390. 


\section{Mo-Poster Session 1-PO-204 High-resolution $\alpha$-glucosidase inhibition profiling combined with HPLC-HRMS-SPE-NMR for identification of $\alpha$-glucosidase inhibitors in Machilus litseifolia (Lauraceae)}

Authors $\mathrm{Li} \mathrm{T}^{1}$, Kongstad $\mathrm{KT}^{1}$, Staerk $\mathrm{D}^{1}$

Institute 1 Department of Drug Design and Pharmacology, Faculty of Health and Medical Sciences, University of Copenhagen, Copenhagen, Denmark DOI 10.1055/s-0037-1608251

Type 2 diabetes is a chronic multifactorial disease affecting millions of people worldwide, and new drug leads with selective $\alpha$-glucosidase inhibitory activity are urgently needed [1]. In this study, the crude ethyl acetate extract of branches of Machilus litseifolia S.K.Lee showed inhibitory activity of $\alpha$-glucosidase $\left(I_{50}=8.0 \pm 1.0 \mu \mathrm{g} / \mathrm{ml}\right)$, and was therefore investigated using high-resolution $\alpha$-glucosidase inhibition profiling combined with high-performance liquid chromatography-high-resolution mass spectrometry-solid-phase extractionnuclear magnetic resonance spectroscopy (HR-bioassay/HPLC-HRMS-SPENMR) [2]. Results from the high-resolution inhibition profile of the crude extract were used to guide preparative-scale HPLC towards five fractions correlated with bioactivity (Figure 1). Fraction 1, 4 and 5 were subjected to HRbioassay/HPLC-HRMS-SPE-NMR using complementary analytical-scale $C_{18}$ and pentafluorophenyl columns for separation as previously shown by Lima and coworkers [3]. With fraction 1, this led to trapping of 17 compounds correlated with bioactivity, of which the material eluted with peaks 7 and 8 were identified as the new compounds tamarixetin-3-O- $\alpha-\mathrm{L}-(2$ ", 4 "'-di-O-cis-coumaroyl)-rhamnopyranoside and tamarixetin-3-O- $\alpha$-L-(2"'-O-cis-coumaroyl-4"-Otrans-coumaroyl)-rhamnopyranoside, respectively. Structural identification of the remaining bioactive constituents from fraction 1, 4 and 5 using HPLCHRMS-SPE-NMR is in progress.

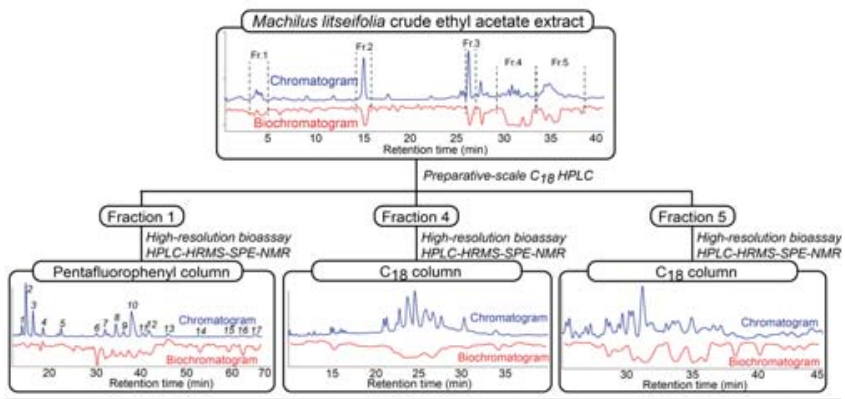

- Fig. 1

Tuo Li acknowledges the Chinese Scholarship Council for a scholarship. Arife Önder is thanked for technical assistance.

[1] International Diabetes Federation, IDF Diabetes Atlas, 7th ed, 2015

[2] Wubshet SG, Tahtah Y, Heskes AM, Kongstad KT, Pateraki I, Hamberger B, Møller BM, Staerk D. J Nat Prod 2016; 79: 1063 - 1072.

[3] Lima R, Gramsbergen S, Van Staden J, Jäger AK, Kongstad K, Staerk D. J Nat Prod 2017; 80: 1020 - 1027.

\section{Mo-Poster Session 1-PO-205 A new flavonoid isolated from Lotus aegaeus (Gris.) Boiss.}

Authors Yuzbasioglu $\mathrm{M}^{1}$, Simon $\mathrm{A}^{2}$, Toth $\mathrm{G}^{2}$, Kuruuzum-Uz $\mathrm{A}^{1}$

Institute 1 Department of Pharmacognosy, Faculty of Pharmacy, University of Hacettepe, 06100, Ankara, Turkey; 2 Department of Inorganic and Analytical Chemistry, Budapest University of Technology and Economics, Szt. Gellért tér 4, H-1111, Budapest, Hungary

DOI 10.1055/s-0037-1608252

The genus Lotus (Fabaceae) is represented in the Turkish Flora by 20 species [1]. These species can fix nitrogen through the root nodules so they can use as fodder, human food or green manure [2]. Lotus aegaeus (Gris.) Boiss. is known as "devre otu, nohudak, yonca " in Central Anatolia and used as animal forage and antimicrobial agent for treatment the animal injuries. Ethnobotanical studies indicate that it's poisonous and harmful to animals if it is consumed before the vernal equinox [2]. Proanthocyanidins, cyanogenic glycosides, flavonoids, sterols and triterpenes were isolated from these species so far [3]. In our previous studies, phytochemical content of L. aegaeus (Gris.) Boiss. was investigated for the first time and 7 compounds of the $n$-butanol extract of aerial parts were determined as quercetin-3-O- $\beta$-D-glucopyranoside-7-O- $\alpha-\mathrm{L}$ rhamnopyranoside, quercetin-3-O- $\beta$-D-galactopyranoside-7-O- $\alpha$-L-rhamnopyranoside, kaempferol-3-O- $\beta$-xylopyranosyl-( $1 \rightarrow 2)$ - $\alpha$-L-rhamnopyranosyl-7O- $\alpha$-L-rhamnopyranoside, $\quad$ kaempferol-3-O- $\beta$-D-galactopyranosyl-7-O- $\alpha$-Lrhamnopyranoside, saponins: wistariasaponin $C$ and $D$ and a cyanogenic glucoside: linamarin, based on literature data. Our following liquid chromatography studies resulted as yielded 2 more flavonoids and their structures identified as kaempferol 3-O-(5"-acetyl) apiofuranoside 7-O-rhamnopyranoside and a new compound for the nature, kaempferol 3-0-(3",5"-diacetyl) apiofuranoside 7-O-rhamnopyranoside by using spectroscopic (1D-2D NMR and MS) methods. Our biological activity studies are contiuoning on these compounds. [1] Heyn CC. Lotus L. in: Davis PH, editor. Flora of Turkey and the East Aegean Islands. Edinburgh,: University Press; 1970, 3: 518 - 531.

[2] Kargıŏlu M, Cenkci S, Serteser A, Konuk M, Vural G. Human Ecology 2010; 38: $429-450$.

[3] Gruber M, Skadhauge B, Yu M, Muir A, Richards K. Can J Plant Sci 2008; 88: $121-132$.

\section{Mo-Poster Session 1-PO-206 HPLC-DAD studies on different Turkish oaks as potential polydatin resource}

Authors Yuzbasioglu $\mathrm{M}^{1}$, Sohretoglu $\mathrm{D}^{1}$, Kuruuzum-Uz A

Institute 1 Department of Pharmacognosy, Faculty of Pharmacy, University of Hacettepe, 06100, Ankara, Turkey

DOI 10.1055/s-0037-1608253

Resveratrol is now gaining much attention because of its anti-aging, antioxidant, anticancer and antioxidant effects on human health. Polydatin is a natural precursor of resveratrol. When compared with resveratrol, Polydatin has a better antioxidant effect and higher bioavailability. Enzymatic, microbiologic or chemical methods are used for transforming polydatin to resveratrol. Polygonum cuspidatum, blueberry, peanut and wine have the highest level of resveratrol [1].

In our study potential Quercus spec. are investigated as a new resource for polydatin. The genus Quercus (Fagaceae) has 23 species in the Flora of Turkey, 4 of them are endemic. In traditional Turkish medicine Quercus species have been used as an antiseptic, antidiarrheal, hemostatic, wound healing, and stomachic agent as well as a remedy for poisoning from alkaloids, copper, lead and heavy metal salts. So far flavonoids, tannins, triterpenes ionon, phenols, lignans catechin derivatives have been isolated from this species [2 - 3]. In our study, methanol and water extract of 4 Quercus spec. (Quercus cerris L. var. cerris L, Quercus macranthera Fisch. and Mey. ex Hohen subsp. syspirensis (C. Koch.), Quercus aucheri Jaub. and Spac and Quercus coccifera L.) are investigated for their polydatin content for the first time and a new HPLCDAD method has been developed for these species. Our results showed that methanol extract of the Quercus coccifera L. have the most polydatin content $(16,95 \%)$ and it could be used for the polydatin resource and for yielding resveratrol.

[1] Su D, Cheng Y, Liu M, Liu D, Cui H, Zhang B, Zhou S, Yang T, Mei Q. PloS one 2013; 8: e54505

[2] Genc Y, Yuzbasioglu M, Harput Üş, Kuruuzum-Uz A. FABAD J Pharm Sci 2012; 37: $17-22$

[3] Söhretoglu D, Kuruüzüm-Uz A, Simon A, Patócs T, Dékány M. Records of Natural Products 2014; 8: 323 


\section{Mo-Poster Session 1-PO-207 Miscellaneous phenolic compounds from Peucedanum chryseum (Boiss. \& Heldr.) Chamberlain}

Authors Gürbüz $\mathrm{P}^{1}$, Yuzbasioglu $\mathrm{M}^{2}$, Demirezer $\mathrm{LO}^{2}$, Guvenalp $\mathrm{Z}^{3}$, Kuruuzum$\underline{U z} A^{2}$

Institute 1 Department of Pharmacognosy, Faculty of Pharmacy, University of Erciyes, 38039, Kayseri, Turkey; 2 Department of Pharmacognosy, Faculty of Pharmacy, University of Hacettepe, 06100, Ankara, Turkey; 3 Department of Pharmacognosy, Faculty of Pharmacy, University of Ataturk, 25240 -, Erzurum, Turkey

\section{DOI 10.1055/s-0037-1608254}

The genus Peucedanum (Apiaceae) comprising more than 120 species that are distributed in Europe, Asia, Africa and North America and 21 species with $42 \%$ endemism in the Flora of Turkey [1,2]. Peucedanum species have been used in traditional folk medicine for treatment of various ailments such as cough, cramps, pain, rheumatism, asthma and angina. There are several records on the chemistry of essential oil of the genus and different classes of coumarins, flavonoids and simple phenolics were reported from the genus. Previously only monoterpene hydrocarbons and fatty acids were isolated from Peucedanum chryseum (Boiss. \& Heldr.) Chamberlain [3]. In this study, Peucedanum chryseum, an endemic specie for Turkey was phytochemically investigated for its phenolic compounds. The methanolic extracts of $P$. chryseum was first suspended in water and partitioned with n-hexane, chloroform and $n-\mathrm{BuOH}$ respectively. Further chromatographic analyzes of $n-\mathrm{BuOH}$ sub-extract was led to the isolation of four phenolic compounds $(1-4)$ : a phloroacetophenone glycoside A (1), a linear dihydropyrano chromon; cimifugin (2), a 1,3,6,8 tetrahydroxy xhanthone (3) and a benzoyl quercetin glycoside (4). The structures of the compounds were elucidated by extensive 1D-and 2D-NMR spectroscopic analysis in combination with MS experiments and comparing with relevant literature[4-6]. To our knowledge all these phenolic compounds were reported from the genus Peucedanum for the first time.

[1] Sarkhail P. Journal of Ethnopharmacology 2014; 156: 235 - 270.

[2] Davis PH. Flora of Turkey and East Aegean Islands. Edinburgh: University Press; 4: 475 - 476.

[3] Ağalar HG, Kürkçüoğlu M, Duran A, Çetin Ö, Başer KHC. Natural Volatiles \& Essential Oils 2015; 2: 4 - 10.

[4] Chosson E, Chaboud A, Chulia AJ, Raynaud J. Phytochemistry 1998; 47: $87-88$.

[5] Sasaki H, Taguchi H, Endo T, Yosioka I. Chemical and Pharmaceutical Bulletin 1982; 30: 3555 - 3562.

[6] Elix JA, Gaul KL, Lumbsch HT. Australian Journal of Chemistry 1987; 40: $1031-1033$.

\section{Mo-Poster Session 1-PO-208 Fluorene Derivatives and Selaginellins Isolated from Roots of Selaginella tamariscina (Beauv.) Spring}

Authors Woo $\mathrm{S}^{1}$, Kim J $\mathrm{J}^{1}$, Sung Sang $\mathrm{H}^{1}$

Institute 1 College of Pharmacy and Research Institute of Pharmaceutical Sciences, Seoul National University, Seoul, Korea, Republic of (South) DOI 10.1055/s-0037-1608255

Plants within the genus Selaginella are widely distributed around the globe including more than 700 species [1]. Among the genus, aerial part of Selaginella tamariscina (Beauv.) Spring has been used as human cancer traditionally. Hundreds of compounds including biflavonoids, selaginellins and terpenoids have been reported from S.tamariscina. However, chemical constituents of the roots have been rarely studied owing to its difficulty for collection, compared to other parts of this plant. The recently reported rare naturally occurring fluorene derivatives exhibit extraordinary biological activities [2,3].

We report herein the phytochemical studies of roots of $90 \%$ ethanol extract of S.tamariscina. The extract was evaporated to give a residue. The residue was suspended in water, and partitioned by $n$-hexane, $\mathrm{CHCl}_{3}$ and EtOAc. The
EtOAc was fractionated by column chromatography using a silica gel, then further purified by RP-HPLC to yield eight compounds: selaginpulvilin A (1), selaginpulvilin $K(2)$, selaginpulvilin L (3), selaginellin (4), selaginellin $M(5)$, selaginellin S (6), breynioside A (7) and amentoflavone (8). Compounds 1-3 containing an 9,9-diphenyl-1-(phenylehynyl)-9H-fluorene moiety were isolated for the first time from S.tamariscina. The structure elucidation was carried out by ESI-Q-TOF-HRMS, UV and extensive NMR spectroscopic analysis ( ${ }^{1} \mathrm{H}$ NMR, ${ }^{13} \mathrm{C}$ NMR, ${ }^{1} \mathrm{H}-{ }^{1} \mathrm{H}$ COSY, HSQC, HMBC and ROESY). Compounds 4-7 were also isolated for the first time from this plant.

[1] Nguyen PH, Zhao BT, Ali MY, Choi JS, Rhyu DY, Min BS, Woo MH. J Nat Prod 2015; 78: $34-42$

[2] Huang YY, Liu X, Wu DY, Tang GH, Lai ZW, Zheng XH, Yin S, Luo HB. Biochem Pharmacol 2017; 130: 51 - 59

[3] Zhang JS, Liu X, Weng J, Guo YQ, Li Q], Ahmed A, Tang GH, Yin S. Org Chem Front 2017; 4: $170-177$

\section{Mo-Poster Session 1-PO-209 Bioactivity and Secondary Metabolome Profiling of Marine Fungi Associated with the Seagrass Zostera marina}

Authors Petersen $\mathrm{LE}^{1}$, Marner $\mathrm{M}^{1}$, Rouger $\mathrm{C}^{1}$, Labes $\mathrm{A}^{1}$, Tasdemir $\mathrm{D}^{1}$ Institute 1 GEOMAR Centre for Marine Biotechnology, Research Unit Marine Natural Product Chemistry, GEOMAR Helmholtz Centre for Ocean Research Kiel, Am Kiel-Kanal 44, Kiel, Germany

DOI 10.1055/s-0037-1608256

Fungi have played a historical role in discovery and development of life-saving drugs, such as antibiotics and anticancer drugs. Despite being an important portion of marine microbiome, chemical composition and the biotechnological potential of fungi from marine resources have so far remained underexplored. The eelgrass Zostera marina forms wide marine landscapes in the Northern Hemisphere and play a significant role in the welfare of marine ecosystems. In continuation of our work on seagrasses and marine fungi $[1,2]$, we have investigated fungi associated with $Z$. marina and its surrounding sediment. Fourteen fungal strains, with a high proportion of Penicillium sp. (10 sp.) were obtained. The organic extracts of liquid cultures in five different culture media (with/without shaking) were tested for bioactivity. All extracts showed strong (up to $100 \%$ at $100 \mu \mathrm{g} / \mathrm{mL}$ concentration) anti-quorum sensing activity against Allivibrio fischerii and antimicrobial activity against marine pathogens Pseudoalteromonas elyakovii and Vibrio anguillarum. HPLC-DADMS-based secondary metabolome (SM) analyses of the extracts indicated the high influence of culture conditions on the SM profiles. Chemical diversity was high and dereplication was successful. For example, among 66 compounds detected in all Penicillium sp. belonged to the classes of alkaloids, polyketides, and terpenoids and 45 were successfully assigned to known compounds. Analysis of SM relatedness in culture conditions by Hierarchical Cluster Analysis revealed generally low similarity. Specifically, one Penicillium strain (P-85) formed a very distinct group in terms of its SM. Further Principal Component Analysis biplots revealed the strong effect of medium selection on SM profiles of P-85. This strain is now being cultivated in large-scale for indepth metabolomics and bioactivity-guided isolation studies.

[1] Orhan I, Sener B, Brun R, Perozzo R, Tasdemir D. Phytomedicine, 2006, 13, $388-393$.

[2] Silber ], Kramer A, Labes A, Tasdemir D. Mar Drugs 2016; 14, doi: 10.3390/ md14070137.

\section{Mo-Poster Session 1-PO-210 Synthesis of new, potentially bioactive chalcones as protoflavone analogues}

Authors Gonda $T^{1}$, Ötvös Sándor $B^{2,3}$, Hunyadi $A^{1,4}$

Institute 1 Institute of Pharmacognosy, University of Szeged, Szeged,

Hungary; 2 Institute of Pharmaceutical Chemistry, University of Szeged,

Szeged, Hungary; 3 MTA-SZTE Stereochemistry Research Group, Hungarian 
Academy of Sciences, Szeged, Hungary; 4 Interdisciplinary Centre for Natural Products, University of Szeged, Szeged, Hungary

DOI 10.1055/s-0037-1608257

Protoflavones represent a rare, unique class of naturally occurring bioactive flavonoid derivatives. Recent publications revealed their antiviral, anti-angiogenic and cytotoxic activity, moreover they have also been found to interfere with crucial DNA damage response mechanisms ${ }^{[1-3]}$. The pharmacophore of these compounds is the non-aromatic B-ring - the unusual $p$-quinol moiety ${ }^{[3]}$. The formation of this moiety can be synthetically achieved from 4 ' -hydroxyflavones applying hypervalent iodine reagents.

Chalcones - also abundant in nature - exert anti-inflammatory, antioxidant, antibacterial and anticancer activity; anti-angiogenic effect of 4-hydroxychalcone 3 was reported lately ${ }^{[4]}$.

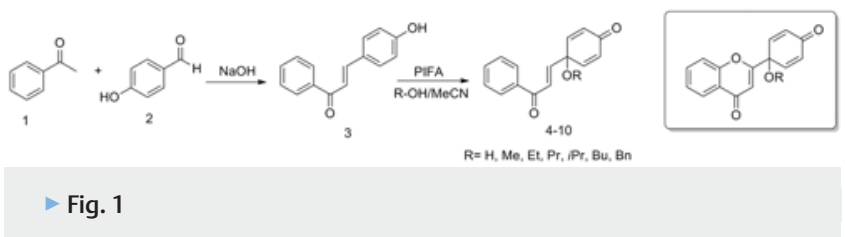

During the current project our aim is - in order to broaden our knowledge on structure-activity relationships concerning protoflavones - to build a compound library by synthesizing and characterizing variously substituted chalcone analogues bearing the $p$-quinol moiety.

The key intermedier 4-hydroxychalcone 3 was synthesysed via Claisen-Schmidt condensation. Seven new compounds (4-10) were subsequently obtained by oxidative dearomatization performed with PIFA in various solvent mixtures. These compounds are expected to exert potent antitumor activity; their bioactivity testing is currently in progress.

This work was supported by the NKFIH, Hungary (K119770), the EU-funded Hungarian grant EFOP-3.6.1-16-2016-00008, and GINOP-2.3.2-152016 - 00012. A.H. acknowledges the János Bolyai fellowship of the Hungarian Academy of Sciences and the Kálmán Szász Prize.

[1] Danko B et al. Anticancer Res 2012; 32: $2863-2870$.

[2] Wang HC et al. Mol. Cancer Ther 2012; 11:1443 - 1453.

[3] Hunyadi A et al. Phytochem Rev 2014; 13: 69- 77.

[4] Varinska L et al. Eur J Pharmacol 2012; 691: 125-133.

\section{Mo-Poster Session 1-PO-211 Sclerocarya birrea cortex ethanolic extract - Chemical characterisation and NOX4 inhibition (anti-ageing property)}

\footnotetext{
Authors Mncwangi $\mathrm{N}^{1}$, Waltenberger $\mathrm{B}^{2}$, Baraldo $\mathrm{C}^{3}$, Jansen-Dürr $\mathrm{P}^{3}$, Viljoen $\mathrm{A}^{1}$, Stuppner $\mathrm{H}^{2}$

Institute 1 1. Department of Pharmaceutical Sciences and SAMRC Herbal Drugs Research Unit, Tshwane University of Technology, Private Bag X680, Pretoria, South Africa; 2 2. Institute of Pharmacy/Pharmacognosy and Center for Molecular Biosciences Innsbruck (CMBI), University of Innsbruck, Innrain 80 - 20, 6020, Innsbruck, Austria; 3 3. Institute for Biomedical Aging Research and CMBI, University of Innsbruck, Rennweg 10, 6020, Innsbruck, Austria DOI 10.1055/s-0037-1608258

Sclerocarya birrea (A. Rich) Hochst (Marula), commonly known as the tree of life, belongs to the Anarcadiaceae family and is widely distributed in southern African regions including South Africa, Zimbabwe, Botswana, Mozambique, Zambia, and Namibia. Generally, stem bark and leaves are used for medicinal purposes and fruits in the food and beverages industries. Known constituents of $\mathrm{S}$. birrea include gallotannins, flavonoids, alkaloids, steroids ( $\beta$-sitosterol), coumarins, triterpenoids, sesquiterpenes, ascorbic acid, oleic, myristic and stearic acids, amino acids, gallic acid, myricetin, (-)-epigallacatechin-3-O-galloylester and (-)-epicatechin.1 Known biological activities of S. birrea include antidiabetic, anti-inflammatory, antiplasmodial, antimalarial, antiproliferative, antimicrobial, and apoptopic effects. 2 In the current study, analysis of an
}

ethanolic extract of S. birrea bark revealed a strong NOX4 inhibitory effect (66\%). TLC and HPLC-MS were used to chemically characterise the extract. Furthermore, the extract was subjected to liquid-liquid partitioning using solvents of different polarity. Biological testing on NOX4 showed that the butanol and the ethylacetat fraction had the most superior inhibitory activity, $84 \%$ and $79 \%$, respectively. Thus, all isolation work was performed on these two fractions. Major compounds were isolated and purified using various chromatographic techniques including column chromatography, flash chromatography, and semi-preparative HPLC. Isolated compounds were identified and characterised using MS and NMR. The following compounds were isolated from the ethylacetate fraction, (+)-catechin $(290.27 \mathrm{~m} / \mathrm{z}),(-)$-epicatechin ( $290.27 \mathrm{~m} / \mathrm{z})$, (-)-epigallocatechin-3-O-gallate (EGCG) $(458.37 \mathrm{~m} / \mathrm{z}),(-) 4^{\prime}-\mathrm{O}-$ methylepigallocatechin-3-O-gallate (4'-O-methyl-EGCG) $(472.40 \mathrm{~m} / \mathrm{z})$, and ()-epicatechin-3-O-gallate (ECG) $(442.37 \mathrm{~m} / \mathrm{z})$. To our knowledge, (-)4'-Omethylepigallocatechin-3-O-gallate (4'-O-methyl-EGCG) $(472.40 \mathrm{~m} / \mathrm{z})$ was identified and isolated for the first time in this study from $\mathrm{S}$. birrea bark. Supported by the EU-Marie-Curie Actions (H2020-MSCA-RISE project 691158 "MediHealth").

[1] Jiménez-Sánchez C, Lozano-Sánchez ], Gabaldón-Hernández J.A, SeguraCarretero A, Fernández-Gutiérrez A. Ind Crops Prod 2015; 7:214 - 234.

[2] Mariod A.A, Abdelwahab S.I. Food Rev Int 2012; 28:375 - 388.

\section{Mo-Poster Session 1-PO-212 Marker tannins of the hydrolysable tannin biosynthetic pathway can be detected rapidly and selectively by tandem mass spectrometry}

Authors Saha $\mathrm{S}^{1}$, Hauta $\mathrm{L}^{1}$, Karskela $\mathrm{T}^{1}$, Salminen JP

Institute 1 Laboratory of Organic Chemistry and Chemical Biology, Department of Chemistry, University of Turku, Turku, Finland

DOI 10.1055/s-0037-1608259

Hydrolysable tannins (HTs) are immensely distributed complex bioactive polyphenols with numerous defensive and medicinal activities. Plant produces HTs via a biosynthetic pathway, which can be segmented into 13 different major branches and each branch can produce several tannins with varying structures and bioactivities $[1,2]$.

It has always been very crucial and demanding for phytochemists to determine what types of HTs are produced by various plant species because of their diversified chemical structures, large range of molecular weights, complex biosynthetic pathways, and sometimes also the low amount in the plant sample [3]. Tandem mass spectrometry with multiple reaction monitoring (MRM) is the most selective quantitation tool so far utilized for modern tannin analysis. Unfortunately, compound-specific MRM methods have not been developed to be used widely in the tannin research. Newly developed extensive methods would enable all QqQ and Orbitrap users to detect the chosen tannins rapidly and selectively. Moreover, accurate quantification of HTs is very much possible with the presence of suitable standards. At present, the quantification of individual HTs is very limited and being carried out with trivial methodologies. Our ultimate target is to develop a modern UPLC-MS ${ }^{2}$ method that will selectively and precisely show in less than 10 mins per sample, which biosynthetic branches of the HT pathway are used by the tested plant species. In this poster, we will show how the created advanced tandem mass spectrometry method functions rapidly and selectively with tens of model species with known HT composition. In addition, we show its potency to be used even with randomly selected species.

[1] Moilanen J, Koskinen P, Salminen, J-P. 2015. Phytochemistry 2015; 116:188 - 197.

[2] Engström MT, Karonen M, Ahern JR, Baert N, Payré B, Hoste H, Salminen JP. J Agric Food Chem 2016; 64: 840 - 851.

[3] Moilanen J, Sinkkonen J, Salminen J-P. 2013. Chemoecology 2013; 23:165-179. 
Mo-Poster Session 1-PO-213 Comparative HPLC and CE studies on the formation of 20-hydroxyecdysone metabolites from base-catalyzed autoxidation and Fenton reaction

\author{
Authors Issaadi Halima $\mathrm{M}^{1}$, Csábi J $]^{1}$, Németh $\mathrm{K}^{2}$, Hunyadi $\mathrm{A}^{1,3}$ \\ Institute 1 Institute of Pharmacognosy, University of Szeged, Szeged, \\ Hungary; 2 Institute of Organic Chemistry, Research Centre for Natural \\ Sciences, Hungarian Academy of Sciences, Budapest, Hungary; \\ 3 Interdisciplinary Center for Natural Products, University of Szeged, Szeged, \\ Hungary \\ DOI 10.1055/s-0037-1608260
}

Phytoecdysteroids are known to exert a broad range of biological activities in mammals accounting for a general strengthening, adaptogenic, and anabolic activity. Our research group has recently reported the synthesis of various autoxidized bioactive metabolites of 20-hydroxyecdysone (20E) [1]. Time dependency of the oxidation was monitored by HPLC after neutralizing the $\mathrm{pH}$, and the above-mentioned metabolites were also detected when biomimetic oxidation of $20 \mathrm{E}$ using the Fenton reaction was performed and the product mixture was monitored by HPLC after sample pre-purification by solid phase extraction on silica [2]. In contrast with HPLC, capillary electrophoresis (CE) does not require sample pre-treatment, and it is also less time and solvent consuming.

The aim of the present study was to compare results obtained by HPLC and CE in order to gain insight on possible effects of the pre-purification concerning (i) the monitoring of base-catalysed autoxidation of $20 \mathrm{E}$ and (ii) the analysis of the crude Fenton reaction.

Oxidation of 20E through base-catalysis or by Fenton reagent was performed similarly as before, and direct analysis of the crude reaction mixtures was achieved by CE without sample preparation. Autoxidation showed a similar qualitative fingerprint and time dependency to that found before [1], with a significantly higher maximum possible yield of 14,15-dihydro- $14 \alpha$-hydroxycalonysterone and isocalonysterone than before, i. e. $70 \%$ and $50 \%$, respectively. Concerning the Fenton reaction, however, none of the autoxidized metabolites could be identified by CE, suggesting that the previous observation on the formation of these compounds [2] was an artefact arising from subsequent oxidation during the sample preparation.

This work was supported by the NKFIH, Hungary (K119770), the EU-funded Hungarian grant EFOP-3.6.1-16-2016-00008, and GINOP-2.3.2-152016 - 00012. A.H. acknowledges the János Bolyai fellowship of the Hungarian Academy of Sciences and the Kálmán Szász Prize.

[1] Csábi J et al. J Nat Prod 2015; 78: 2339 - 2345.

[2] Dankó B et al. Planta Med 2015; 81: SL2C_02.

\section{Mo-Poster Session 1-PO-214 Establishment the fingerprints of Phyla nodiflora by electronic nose (e-nose)}

Authors Tsu WC ${ }^{1}$, Ko $\mathrm{HH}^{1,2}$

Institute 1 Department of Fragrance and Cosmetic Science, Kaohsiung Medical University, Kaohsiung 80708, Taiwan, Kaohsiung, Taiwan; 2 Research Center for Natural Products and Drug Development, Kaohsiung Medical University, Kaohsiung 80708, Taiwan, Kaohsiung, Taiwan

DOI 10.1055/s-0037-1608261

The consistency of phytochemical profile might be affected by different geography conditions and post-growth factors. Thus, a systematic quality criterion for controlling the quality is imperative. Application of electronic nose (enose) based on fast GC system is one of the selected method and it allows identification of volatile organic compounds fingerprints. Phyla nodiflora is a perennial herbs containing various chemical constituents, such as flavonoids, monoterpenes, sesquiterpenes, triterpenoids and volatiles, and with herbal and minty odour. Althought, the HPLC-fingerprints of P. nodiflora has been figured out the odour-profile of this plant has not been investigated. In this present study, the preliminary analytic method will be discussed. To date, the e-nose chromatograms of the volatile complex of P. nodiflora indicated that 1 octen-3-one, limonene, $\delta$-nonalactone might contribute the odour of P. nodiflora. The appropriate analytic methods to indentify constitutions and establish the complete fingerprints of P. nodiflora by e-nose will be studied continually.

\section{Mo-Poster Session 1-PO-215 Chemical constituents from the groundnuts of Apios americana}

Authors Jeon $\mathrm{JH}^{1,2}$, Seo Young $\mathrm{H}^{1,2}$, Jeon Won $\mathrm{K}^{1,2}$, Kim $\mathrm{MS}^{1,2}$, Lee $\mathrm{D}^{3}$, Ryu Seung $\mathrm{M}^{3}$, Jang Dae $\mathrm{S}^{4}$, Lee $\mathrm{J}^{1,2}$

Institute 1 Herbal Medicine Research Division, Korea Institute of Oriental Medicine, Daejeon, Korea, Republic of (South); 2 2Convergence Research Center for Diagnosis, Treatment and Care System of Dementia, Korea Institute of Science and Technology, Seoul, Korea, Republic of (South); 3 Department of Biosystems and Biotechnology, Korea University, Seoul, Korea, Republic of (South); 4 Department of Pharmaceutical Science, Graduate School, Kyung Hee University, Seoul, Korea, Republic of (South)

DOI 10.1055/s-0037-1608262

Phytochemical investigation of the groundnuts of Apios americana has resulted in the isolation of three new isoflavonoids (1-3) along with seventeen known compounds $(4-20)$ by chromatographic separation methods [1-5]. The structures of all compounds were elucidated by spectroscopic methods including 1D-NMR $\left({ }^{1} \mathrm{H},{ }^{13} \mathrm{C}\right), 2 \mathrm{D}-\mathrm{NMR}$ (DEPT, HSQC, COSY, HMBC, and NOESY), and HRESIMS. The absolute configuration of chiral centers in compound 1 was determined by electronic circular dichroism (ECD) study. Three of these compounds $(4,5$, and 20$)$ were identified in and isolated from this species for the first time. Biological activities of the compounds (1-20) were also evaluated in this study.

[1] Ichige M, Fukuda E, Miida S, Hattan J, Misawa N, Saito S, Fujimaki T, Imoto M, Shindo K. J Agric Food Chem 2013; 61: $2183-2187$

[2] Haruna K, Koda M, Saito S, Imoto M, Kawada M, Yamazaki Y, Momose I, Shindo K. Biosci Biotech Biochem 2016; 80: $774-778$

[3] Zhao S, Zhang L, Gao P, Shao Z. Food Chem 2009; 114: 869 - 873

[4] Selepe MA, Drewes SE, van Heerden FR. Tetrahedron 2011; 67: 8654 8658

[5] Shibuya Y, Tahara S, Kimura Y, Mizutani J. J Biosci 1991; 46: 513- 518

\section{Mo-Poster Session 1-PO-216 Extraction Natural Dyes from Actinomycetes using Microwave Assisted Extraction}

Authors Wan Mohd Fathil Wan $Y^{1}$, Sharifah Aminah Syed $M^{1}$ Institute 1 Faculty of Applied Sciences, Universiti Teknologi MARA, 40450 Shah Alam, Selangor, Malaysia DOI 10.1055/s-0037-1608263

Microwave assisted extraction (MAE) is regarded as the simplest and cheapest techniques for the natural dyes extraction. The major advantages of this are considerable reduction in time and solvent consumption with better extraction yield (1). In MAE, the sample and solvent are contained in a sealed extraction container under controlled pressure and temperature conditions which allows the temperature of the solvent to rise above the boiling point which enhances extraction efficiency and shorten the extraction time $(2,3)$. This study focused on extraction of natural dyes from four selected actinomycetes using microwave assisted extraction. The extracted dyes of actinomycetes were treated with $\mathrm{FeSO} 4$ prior dyeing to increase dye-uptake. The dyeing of silk fabrics were done using exhaust dyeing techniques at $80^{\circ} \mathrm{C}$ for 1 hour. The evaluation of the dyed fabrics was carried out using colour spectrophotometer and colourfastness assessment. The dyes treated with mordant produces deeper shades on dyed silk fabrics and colours obtained are in the brown colour group. The results recorded for colourfastness showing good to 
excellent rating of all fastness properties. Thus, it was shown that MAE produces high quantity of dyes as well as eco-friendly and time saving.

\begin{tabular}{|c|c|c|c|}
\hline \multicolumn{2}{|c|}{ G1A39 } & \multicolumn{2}{|c|}{ G1A45 } \\
\hline Untreated & Treated & Untreated & Treated \\
\hline White smoke & Flaxen & White smoke & Buff \\
\hline \multicolumn{2}{|c|}{ L4E3 } & \multicolumn{2}{|c|}{ X1F1 } \\
\hline Untreated & Treated & Untreated & Treated \\
\hline Ghost white & Biscotti & White smoke & Clay \\
\hline
\end{tabular}

Faculty of Applied Sciences, Universiti Teknologi MARA, Shah Alam for providing the laboratory facilities to do the research.

[1] Wang, L, and Weller, C. L. Trends in Food Science and Technology 2006, $17,300-312$.

[2] Mason, T. J, Chemat, F. and Vinatoru, M. Current Organic Chemistry 2011, $15,237-247$.

[3] Chemat, F, Abert-Vian, M. and Zill-e-Huma, Y, J. Microwave assisted separations: green chemistry in action. In: Pearlman JT (ed) Green chemistry research trends. Nova Science Publishers, New York 2009, 33 - 62.

\section{Mo-Poster Session 1-PO-217 Profiling, anti-radical activity and bioactivity of Thymus mastichina, Thymus $x$ citriodorus and Thymus vulgaris aqueous extracts}

Authors Taghouti $\mathrm{M}^{1,3}$, Félix $\mathrm{L}^{1,2}$, Martins-Gomes $\mathrm{C}^{1,3}$, Santos $\mathrm{JA}^{1}$, Nunes $\mathrm{FM}^{3}$, Silva $\mathrm{AM}^{1}$

Institute 1 Centre for Research and Technology of Agro-Environmental and Biological Sciences (CITAB), University of Trás-os-Montes and Alto Douro (UTAD), Vila Real, Portugal; 2 Laboratory Animal Science (LAS), Institute for Research and Innovation in Health (i3S), University of Porto (UP), Porto,

Portugal; 3 Chemistry Center - Vila Real (CQ-VR), UTAD, Vila Real, Portugal DOI 10.1055/s-0037-1608264

Many plants belonging to the genus Thymus are endemic to the Mediterranean area. Cultivation of some Thymus species has increased in the past years due to their use in culinary and for essential oil extraction.

In this study, we aimed to characterize the chemical composition, the antiradical activity and bioactivity against human cell lines of aqueous extracts (decoctions) of lyophilized aerial parts of Thymus mastichina (T.m.), T. x citriodorus (T.xc.) and T. vulgaris (T.v.). Extracts were analyzed by HPLCDAD; three colorimetric methods were used to study extracts antiradical activity against ABTS., $\mathrm{OH}$. and NO. radicals; and cell viability was assessed in Caco-2 and $\mathrm{HepG} 2$ cell lines, using alamar blue assay (metabolic activity indicator).

Extraction yields ranged between 10\% (T.m. and T.xc.) and 25\% (T.v.). Extracts showed moderate hydroxyl radicals inhibition $(\mathrm{OH} \cdot)$ ranging between $28.23 \%$ (T.m.) and $42.58 \%$ (T.v.). Extract of T.v. showed the highest activity against NO. radicals, with $74 \%$ inhibition, while T.m. had the highest ABTS-inhibition (0.09 mmol Trolox equivalent/g dry plant). Caffeic acid and some of its derivatives (e.g, rosmarinic and salvianolic acids), luteolin and apigenin, and some of their derivatives, are amongst the phenolic acids and flavonoids identified by HPLC-DAD. Eriodictyol derivatives were also identified in all extracts.

Concerning to the bioactivity of these extracts, we observed a time- and concentration-dependent reduction of cell viability, indicating anti-proliferative effect.

Our results provide an overview of the phenolic compounds present in aqueous extracts of studied plants, of their potential in scavenging different radicals and anti-proliferative activity, suggesting a rich source of bioactive compounds and potential to use as food condiments or nutraceuticals.

UTAD Botanical Garden, FCT (grant to M.T.(PD/BD/52563/2014), INTERACTproject-NORTE-01 - 0145-FEDER-000017, ISAC, co-financed by ERDF through NORTE2020, (BI/UTAD/INTERACT/ISAC/203/2016, to L.F.).

\section{Mo-Poster Session 1-PO-218 Lupeol and resveratrol from the stembark of Shorea ovalis (Dipterocarpaceae)}

Authors Rosmawati Abdul $A^{1,2}$, Norizan $A^{1,2}$, Sharifah Aminah Syed $M^{1}$ Institute 1 Faculty of Applied Sciences, Universiti Teknologi MARA, 40450

Shah Alam, Selangor, Malaysia; 2 Atta-ur-Rahman Institute for Natural Product Discovery, Universiti Teknologi MARA, Puncak Alam Campus, 42300 Bandar Puncak Alam, Selangor, Malaysia

DOI 10.1055/s-0037-1608265

Dipterocarpaceae is a plant family of large three that is widely distributed in the tropical rain forest of Southeast Asia, such as Borneo and Peninsular Malaysia. Shorea is the largest genus of Dipterocarpaceae [1]. This genus is the most economically important in Malaysia. The timbers produced are very diverged, ranging from light to very heavy, and are characterized by the presence of intercellular resin canals with color ranging from white, yellow, pink, dark-red to brown.In Malaysia, Shoreais known as Balau, Meranti Pa'ang, Meranti Damar Hitam and Red Meranti. Shorea ovalis is the species included in the group of Red Meranti. Locally it is known as Meranti Kepong. So far, very limited chemical constituents have been reported from Shorea ovalis. In this study, a phytochemical study was conducted on the stem bark of Shorea ovalis. The stem bark was obtained from Hutan Simpan, Jengka, Pahang. The cleaned, chopped and dried stem-bark was grinded into powder. The powder was extracted using acetone for several times. The separation of the components was carried out using vacuum liquid chromatography (VLC) followed by radial chromatography and preparative thin layer chromatography method. The structure of the pure compounds was elucidated by spectroscopic methods including nuclear magnetic resonance (NMR), UV-Vis, and comparison with literature. Two compounds were isolated and identified as lupeol [2,3] and resveratrol. These compounds were reported for the first time from this plant.

We thank the Faculty of Applied Sciences, Universiti Teknologi MARA, Shah Alam for providing the laboratory facilities to do the research.

[1] Noviany. Modern Applied Science 2009; 3: 45 - 51

[2] Pattamadilok D, Suttisri R. J Nat Prod 2008; 71: $292-294$

[3] Jamal Ak, Yaacob WA, Din LB. Journal of Physical Sciences 2008; 19: 45 50

\section{Mo-Poster Session 1-PO-219 Phenolic compounds characterization and anti-oxidant activity of Thymus pulegioides L. extracts}

Authors Taghouti $\mathrm{M}^{1,2}$, Martins-Gomes $\mathrm{C}^{1,2}$, Schäfer $\mathrm{J}^{3}$, Santos $\mathrm{JA}^{1}$, Bunzel $\mathrm{M}^{3}$, Silva $\mathrm{AM}^{1}$, Nunes $\mathrm{FM}^{2}$

Institute 1 Centre for Research and Technology of Agro-Environmental and Biological Sciences (CITAB), University of Trás-os-Montes and Alto Douro (UTAD), Vila Real, Portugal; 2 Chemistry Center - Vila Real (CQ-VR), UTAD, Vila Real, Portugal; 3 Department of Food Chemistry and Phytochemistry, Institute of Applied Biosciences, Karlsruhe Institute of Technology (KIT), Karlsruhe, Germany

DOI 10.1055/s-0037-1608266

Thymus pulegioides L. is widespread in Europe and its aerial parts are used in culinary, as condiments, and in traditional medicine to treat gastro-intestinal and respiratory infections. Although it is used, data on the detailed composition of its extracts still needs further investigation as compared to that of essential oils.

This work aims to give a detailed composition of T. pulegioides phenolic compounds obtained by two extraction methods (decoction and repeated 
hydro-ethanolic extraction (HE)) and to assess their anti-oxidant activity. Total phenolic, total flavonoids and ortho-diphenols contents were evaluated using colorimetric methods. For phenolic compounds profile characterization and quantification, extracts were analyzed by HPLC-DAD-ESI-MSn negative mode). Three colorimetric in vitro methods were used to assess antiradical activity of extracts against: ABTS., OH. and NO-radicals.

Results indicate that, $\mathrm{HE}$ extract was richer than decoctions in total phenolics ( $56.11 \pm 5.6$ vs. $26.12 \pm 0.91 \mathrm{mg} \mathrm{CAE} / \mathrm{g}$ dry plant), total flavonoids (61.75 \pm 12.58 vs. $37.23 \pm 1.29 \mathrm{mg} \mathrm{CE} / \mathrm{g})$ and ortho-diphenols $(24.7 \pm 1.63$ vs. $14.95 \pm 0.57 \mathrm{CAE} / \mathrm{g})$. Phenolic profile of both extracts was characterized by the presence of rosmarinic acid as major compound $(8.53 \pm 1.56$ and 32.51 $\pm 3.58 \mathrm{mg} / \mathrm{g}$ dry plant, in decoction and $\mathrm{HE}$, respectively), eriodictyol derivatives, luteolin hexuronide, chrysoeriol hexoside and caffeic acid. HE extract was rich in phenolics, presenting compounds not detected in decoction (e.g. salvianolic acid I). HE extracts presented a 2-fold higher ABTS. radical scavenging activity than decoction. However, decoction had higher $\mathrm{OH}$. radical inhibition in site specific assay. Overall, studied extracts had moderate antiradical activity against $\mathrm{OH}$. and $\mathrm{NO}$. radicals comparing to other Thymus species. In conclusion, these data show that T. pulegioides could be considered as potential source for functional ingredients for food and pharmaceutical industries.

UTAD-Botanical-Garden, to FCT (grant to M.T.(PD/BD/52563/2014)), INTERACT-project-NORTE-01-0145-FEDER-000017 (ISAC), co-financed by ERDF through NORTE2020.

\section{Mo-Poster Session 1-PO-220 Secondary metabolites from Excoecaria formosana and their anti-hepatocellular carcinoma activity}

Authors $\mathrm{Wu} \mathrm{HC}^{1}$, Chen $\mathrm{JT}^{2}$, Fan $\mathrm{YH}^{2}$, Yen $\mathrm{CH}^{1,3}$, Chen $\mathrm{YM}^{3}$, Chang $\mathrm{HS}^{1,2,3}$ Institute 1 Graduate Institute of Natural Products, College of Pharmacy, Kaohsiung Medical University, Kaohsiung, Taiwan; 2 School of Pharmacy, College of Pharmacy, Kaohsiung Medical University, Kaohsiung, Taiwan; 3 Center for Infectious Disease and Cancer Research (CICAR), Kaohsiung Medical University, Kaohsiung, Taiwan

DOI 10.1055/s-0037-1608267

Glycine $\mathrm{N}$-methyltransferase (GNMT) is a tumor suppressor gene for hepatocellular carcinoma (HCC). The agents that enhance the expression of GNMT would have a beneficial effect on liver and utility in anticancer therapies. Previous studies also proved that GNMT might improve the antitumor activity of standard chemotherapeutic drugs, and expression of GNMT is highly downregulated in the livers of patients at risk of developing HCC such as those with hepatitis $C$ virus and alcohol-induced cirrhosis. So GNMT could be an excellent target in drug screening for anti-HCC medicine.

Approximately 1,400 species of Formosan indigenous plants have been screened for the GNMT promoter activity, and Excoecaria formosana (Hay.) Hay. (Euphorbiaceae) is the most potential species. E. formosana is a shrub and mainly distributed in Tonkin, Indo-China, the southern part of Taiwan in thickets and forests along the seashores. Till now, no investigation of chemical constituents and bioactivity from E. formosana have been reported, so it is potential for research.

Previously, we reported one new steroid: 6'-(stigmast-5-en-7-hydroperoxy-3O- $\beta$-glucopyransidyl)hexadecanoate (1) and thirteen known compounds including four steroids, three benzenoids, a mixture of cerebrosides, one coumarin, and one diterpene from the active ethyl acetate-soluble layer of this plant. Continuing investigation led to the isolation of one new coumarinolignan: excocoumarin A (2), one new diterpene: excoterpenol A (3) and twenty-one known compounds, including one apocarotenoid, three benzenoids, one coumarin, six coumarinolignans, two diterpenes, one flavonoid, two steroids and five tannins. The structures of these new compounds (1-3) were elucidated by 1D, 2D NMR, UV, IR, ESIMS, and HRESIMS analysis. The
GNMT promoter activity of these constituents have been evaluated, and the isolation of the active subfractions of this plant is still in progress.

\section{Mo-Poster Session 1-PO-221 Modern bioautography - a fast analytical tool to discover active compounds in plant extracts used for cosmetics}

Authors Oberle $\mathrm{M}^{1}$, Engemann $\mathrm{J}^{1}$, Klingelhöfer $\mathrm{I}^{2}$, Morlock $\mathrm{G}^{2}$

Institute 1 Merck KgaA, Life Science, Frankfurter Str. 250, 64293 Darmstadt, Germany; 2 Justus Liebig University Gießen, Institute of Nutritional Science, and Interdisciplinary Research Center, Heinrich-Buff-Ring 26-32, 35392 Gießen, Germany

DOI 10.1055/s-0037-1608268

Today's high-performance thin-layer chromatography (HPTLC) combines the advantage of a robust chromatographic separation with high sample throughput and the possibility of simple coupling to additional analytical techniques, like mass spectrometry (MS) or bioassays [1]. This increases the amount of information for a fast and efficient activity-based screening and the identification of raw materials for new cosmetic products.

Lengthy, time-consuming clean-up processes for first activity tests are avoided by adapting the activity test directly on the HPTLC plate. Assays for special target activities can be performed for many samples in parallel after chromatography. The transfer of discovered active substance zones to the mass spectrometer allows the direct characterization of the target substance without additional clean-up steps. Examples for such an "activity based" screening of plant extracts will be presented.

[1] Morlock, G. and Schwack, W. 2010. J. Chromatogr. A, 1217:6600-6609

\section{Mo-Poster Session 1-PO-222 New Approach to the Synthesis of 6-Geranylnaringenin via Ruthenium-Catalyzed Cross-Metathesis}

\author{
Authors Burger $\mathrm{T}^{1}$, Urmann $\mathrm{C}^{1}$, Riepl $\mathrm{H}^{1}$ \\ Institute 1 Hochschule Weihenstephan-Triesdorf, Straubing, Germany \\ DOI 10.1055/s-0037-1608269
}

Prenylated flavanones exhibit a broad spectrum of biological activities: neuroprotective, anti-cancerogenic, anti-fungal, anti-bacterial and estrogen-like activity as well as promotion of neuronal differentiation [1, 2]. Among C-6 and C-8 prenylated species, selective syntheses, especially for members bearing long terpene moieties, primarily exist for the C-8 prenylated flavanones. To examine the biological activities of C- 6 substituted species, a synthetic approach providing access to the various C- 6 substituted flavanones (e. g. prenyl, geranyl, farnesyl) was therefore designed.

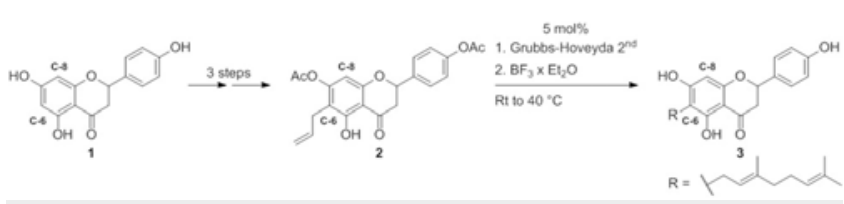

Scheme 1: General reaction sequence for C-6 prenylated flavanones

In this study, a method was designed, including cross-metathesis (CM) as key step and 6-allylnaringenin (6-AN, 2) [3] as general precursor (Scheme 1). This offers the benefit of a general synthesis instead of individual syntheses of desired C-6 prenylated flavonoids with different terpene moieties. Starting from naringenin (1), cross-metathesis between 6-AN (2) and 2,6-dimethyl-6hepten-2-ol and subsequent dehydration of the tertiary alcohol promoted by $\mathrm{BF}_{3} \cdot \mathrm{OEt}_{2}$ provided 6-geranylnaringenin (3) with a yield of $54 \%$. A major advantage now is that olefins used for cross-metathesis can be varied broadly, thus opening a library of different natural products for screening.

[1] E. Oberbauer, C. Urmann, C. Steffenhagen, L. Bieler, D. Brunner, T. Furtner, B. Bäumer, C. Bandtlow, S. Couillard-Despres, F. J. Rivera, H. Riepl, L. Aigner, J. Nutr. Biochem. 2013, 24, 1953 - 1962. 
[2] Y. Wang, W. Tan, W. Z. Li, Y. Li, J. Nat. Prod. 2001, 64, 196 - 199.

[3] S. Tischer, P. Metz, Adv. Synth. Catal. 2007, 349, 147 - 151.

[4] G. H. Posner, E. M. Shulman-Roskes, C. H. Oh, J.-C. Carry, J. V. Green, A. B. Clark, H. Dai, T. E. N. Anjeh, Tetrahedron Lett. 1991, 32, 6489-6492.

\section{Mo-Poster Session 1-PO-223 “Unnatural” natural products obtained by direct halogenation of crude plant extracts: the case study of Arrabidaea brachypoda}

Authors Pellisier $\mathrm{L}^{1}$, Quintino da Rocha $\mathrm{C}^{3}$, Marcourt $\mathrm{L}^{1}$, Randazzo $\mathrm{C}^{2}$, Rudaz $S^{2}$, Wolfender $\mathrm{LL}^{1}$, Ferreira Queiroz $\mathrm{E}^{1}$

Institute 1 Phytochimie et Produits Naturels Bioactifs, École de Pharmacie

Genève-Lausanne, Section des Sciences Pharmaceutiques, Université de Genève, rue Michel Servet 1, CH-1211 Genève 4, Switzerland, Geneva, Switzerland; 2 Sciences analytiques, École de Pharmacie Genève-Lausanne, Section des Sciences Pharmaceutiques, Université de Genève, rue Michel Servet 1, CH-1211 Genève 4, Switzerland, Geneva, Switzerland; 3 Centro de Ciência e Tecnologia-CCET, Departamento de Química. Universidade Federal do Maranhão. Av dos Portugueses, 1966. Bacanga, Cep: 65080 - 805-São LuísMA, Brasil, Bacanga, Brazil

DOI 10.1055/s-0037-1608270

While natural products (NPs) continue to be a source of inspiration for drug development in a majority of cases these compounds should be derivatised to be drugable. It is also estimated that around 20 percent of all therapeutic small molecule drugs are halogenated.1 Carbon-halogen bond lead to a wide range of effects, including an increase in thermal and oxidative stability and an increased biological membrane permeability. In this context we have recently developed a strategy for the generic halogenation of plant extracts to build libraries of original halogenated NPs for the search of new antimicrobial agents [1]. First the halogenation (bromination) reaction was performed and optimized with a series of NP standards from different chemical classes [2]. Once the reaction was successfully achieved, selected plant extracts were submitted to the same generic bromination procedure.

In the present work, the bromination reaction was applied to the dichloromethane extract of Arrabidaea brachypoda's roots $(250 \mathrm{mg})$. This plant extract contains unusual dimeric flavonoids possessing strong in vivo and in vitro activities against Trypanosoma cruzi. The reaction was monitored by UHPLCTOF-HRMS. After $24 \mathrm{hs}$ significant modification of the metabolites profile were observed suggesting the formation of new halogenated products. In order to target the isolation of the halogenated NPs, the analytical metabolite profiling conditions were geometrically transferred to semi-preparative HPLC. Using this approach a series of 11 halogenated NP analogues were isolated in one step and fully characterized by NMR and HRESIMS analyses. A chemical space analysis of these unnatural natural NPs demonstrated that the physico-chemical parameters were strongly modified and thus lead chemical novelty. Evaluations of the biological activity of the isolated compounds against Trypanosoma cruzi is under way.

[1] Mendez L. et al., ACS Comb Sci, 2011. 13:200 - 204.

[2]Bernini R. et al, New Journal of Chemistry, 2015. 39:2980 - 2987.

\section{Mo-Poster Session 1-PO-224 The correlation between BDNF in SH-SY5Y cell lines and GC-MS metabolomic profiling of Melissa officinalis, Hypericum perforatum and Passiflora incarnata}

\author{
Authors Gonulalan $\mathrm{E}^{1}$, Bayazeid $\mathrm{O}^{1}$, Nemutlu $\mathrm{E}^{2}$, Yalçın $\mathrm{F}^{1}$, Demirezer $\mathrm{Ö}^{1}$ \\ Institute 1 Hacettepe University, Faculty of Pharmacy, Dept. of \\ Pharmacognosy, ANKARA, Turkey; 2 Hacettepe University, Faculty of \\ Pharmacy, Dept. of Analytical Chemistry, ANKARA, Turkey \\ DOI 10.1055/s-0037-1608271
}

Pharmaceutical products prepared from Melissa officinalis, Hypericum perforatum and Passiflora incarnata are used in cases of mild depression [1]. Brainderived neurotrophic factor (BDNF) levels may play a key role in the patho- physiology of depression, and regulation of BDNF levels may contribute to antidepressant effect [2]. In this study, the metabolite profiling analysis and their correlation with BDNF expression in the SH-SY5Y cell line of the extracts were investigated. $P$. incarnata $\mathrm{MeOH}$ extract increased the BDNF level by $35 \%$, $74 \%(p<0.001)$ and $56 \%(p<0.05)$; H. perforatum $\mathrm{MeOH}$ extract by $40 \%, 102 \%$ $(p<0.0001)$, and $45 \%$; M. officinalis $\mathrm{MeOH}$ by $35 \%, 107 \%(p<0.001)$, and $140 \%$ $(p<0.0001)$ at 10,30 and $100 \mu \mathrm{g} / \mathrm{mL}$ concentrations respectively. The metabolomics analysis has been performed using gas chromatography-mass spectrometry (GC-MS) [3]. After deconvolution and aligned of the chromatograms, 721 mass spectral features have been detected and 181 of them were annotated using retention index libraries. The univariate, multivariate and correlations analysis were applied to evaluate correlation between metabolomic profiling and BDNF expression in order to investigate relations between activity and full of metabolomic profiling. The three plants have showed similar metabolomic profiling. 79 metabolites were found to have negative correlation between metabolites levels and BDNF activity, while 56 of highly positive correlation $(r>0.75)$. And also there was a concentration dependent activity between metabolites level, higher metabolite levels lowering effect on activity.

This study was supported by grant from Hacettepe University Scientific Research Projects (Project No: THD-2016 - 9171).

[1] Schwartz JP, Nishiyama N. Brain Res Bull 1994; 35: 403 - 407.

[2] Hashimoto K, Shimizu E, lyo M. Brain Res Rev 2004; 45:104 - 14.

[3] Issaq H], Abbott E, Veenstra TD. J Sep Sci 2008; 31:1936 - 1947.

\section{Mo-Poster Session 1-PO-225 The effect of the extraction method in Thymus carnosus Boiss. polyphenolic profile}

Authors Martins-Gomes $\mathrm{C}^{1,2}$, Taghouti $\mathrm{M}^{1,2}$, Schäfer $J^{3}$, Bunzel $\mathrm{M}^{3}$, Silva $\mathrm{AM}^{2}$, Nunes FM ${ }^{1}$

Institute 1 Chemistry Centre, Vila Real (CQ-VR), UTAD, Quinta de Prados, Vila Real, Portugal; 2 Centre for Research and Technology of Agro-Environmental and Biological Sciences (CITAB), UTAD, Vila Real, Portugal; 3 Department of Food Chemistry and Phytochemistry, Institute of Applied Biosciences, Karlsruhe Institute of Technology (KIT), Karlsruhe, Germany

DOI 10.1055/s-0037-1608272

Due to small occurrence of Thymus carnosus Boiss. in wild, it is considered a near threatened species. Apart from some individuals growing in botanical gardens, it can only be found in Portuguese south and southwest shore, and in Huelva, Spain. Only the essential oils of this species were analysed in previous works, with borneol being the main component, followed by camphene and terpinen-4-ol. The polyphenolic composition is still unknown, being the purpose of this work to identify and quantify the polyphenols in this thyme. Plants were harvested in UTAD botanical garden, in flowering stage, lyophilised and grounded. Then, two extraction methods were chosen: decoction, and repeated hydroethanolic extraction (HE), aiming to extract all extractable polyphenols. Extracts were frozen and lyophilised. Extract yield was higher in HE $(24.67 \pm 0.58 \%)$ than in decoction $(21.24 \pm 1.57 \%)$. Total phenols, flavonoids and ortho-diphenols were quantified. Although there were no significant differences in total phenols, the flavonoids and ortho-diphenols content was higher in HE than in decoctions.

The extracts were analysed by HPLC-DAD and HPLC-ESI-MS ${ }^{n}$ to assess their polyphenolic composition. Phenolic acids, as rosmarinic and caffeic acids, and derivatives of some flavonoids, as eriodyctiol, quercetin, luteolin and apigenin, were found in both extracts and are commonly found in Thymus genus. The three main components of the extracts were rosmarinic acid, a salvianolic acid $A$ isomer and salvianolic acid $K$, these two salvianolic acids are rarely described in thyme species. Salvianolic acid A, ursolic acid and salvianic acid A were also identified but are present in lower concentrations. Their quantification revealed significant differences between extraction methods, with $\mathrm{HE}$ as the best method, extracting the highest amount of polyphenols. 
UTAD Botanical Garden, FCT (grant to M.T.; PD/BD/52563/2014); INTERACTproject-NORTE-01 - 0145-FEDER-000017, in its research line entitled ISAC, cofinanced by ERDF through NORTE2020.

\section{Mo-Poster Session 1-PO-226 Photostability of sennosides in solution and their degradation products 1}

Authors Meier $\mathrm{N}^{1}$, Meier $\mathrm{B}^{1}$, Peter $\mathrm{S}^{1}$, Josic $\mathrm{G}^{1}$, Wolfram $\mathrm{E}^{1}$ Institute 1 Zürich University of Applied Sciences, Institute of Chemistry and Biotechnology, Research Group of Phytopharmacy and Natrual Products, Wädenswil, Switzerland

DOI $10.1055 / \mathrm{s}-0037-1608273$

The leaves and pods of Alexandrian and Tinnevelly senna were used for centuries as laxatives. Their main active constituents are the dianthron glucosides (sennoside A - D and A1) and anthraquinone glucosides [1]. The photochemical instability of sennoside A was published in 1980 [2]. The authors postulated a degradation to rhein. However, the degradation products were not well examined and only one solvent was used in the study. The aim was to test the photostability of sennosides A, B and C in different solvents, to detect the degradation products as well as to examine if and when the aglycones aloeemodin and rhein are formed.

Sennosides were dissolved in different solvents and exposed to light (Fluora ${ }^{\circledR}$ growth lamps), samples were taken over a period of 14 days to 1 month and analysed by UHPLC-PDA/QDa.

After $18 \mathrm{~h}$ of light exposure $50-60 \%$ of the sennosides degraded in a solution with methanol/0.1\% sodium bicarbonate $(70: 30 \mathrm{v} / \mathrm{v})$ into other sennosides and rhein-8-glucoside. The effect is less distinct in solution of $0.1 \%$ sodium bicarbonatewith a degradation of $\sim 10 \%$ and did not occur with storage at room temperature and $4{ }^{\circ} \mathrm{C}$ while protected from light. Also, further degradation was stopped without light exposure. A formation of the aglycones aloeemodin or rhein was not measured at any time, in contrary, in a senna extract exposed to light rhein degraded as well.

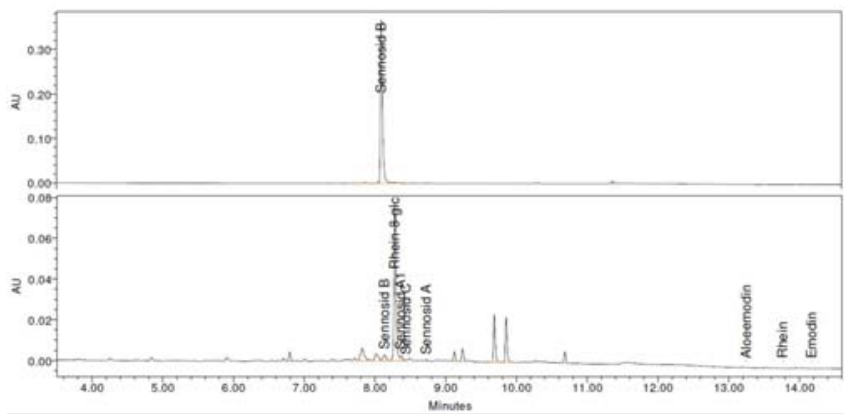

- Fig. 1: Chromatograms at $270 \mathrm{~nm}$ of sennoside B in methanol/ $0.1 \%$ $\mathrm{NaHCO}_{3}(70: 30 \mathrm{v} / \mathrm{v})$ before (upper) and after 14 days of light exposure (lower)

[1] Hänsel, R. \& Sticher, O. Pharmakognosie - Phytopharmazie 9th edition. Springer Medizin Verlag Heidelberg; 2010

[2] Nabil Aboul-Enein M, Wasser G.M, El-Difrawy S.M. Fitoterapia 1980; 51: $157-159$.

\section{Mo-Poster Session 1-PO-227 New isoprenoids with rare scaffolds from Salvia hydrangea}

Authors Tabefam $\mathrm{M}^{1,2}$, Moridi Farimani $\mathrm{M}^{1}$, Ramseyer $\mathrm{J}^{2}$, Potterat $\mathrm{O}^{2}$, Hamburger $\mathrm{M}^{2}$

Institute 1 Department of Phytochemistry, Medicinal Plants and Drugs Research Institute, Shahid Beheshti University, G. C, Evin, Tehran, Iran;
2 Division of Pharmaceutical Biology, University of Basel, Klingelbergstrasse

50, 4056 Basel, Switzerland

DOI 10.1055/s-0037-1608274

The genus Salvia (Lamiaceae) is a rich source of structurally diverse isoprenoids. As part of a project aimed at the discovery of structurally new bioactive metabolites from Iranian Lamiaceae, we studied Salvia hydrangea, a species commonly known in Persian as "Gol-e Arooneh". The flowers of S. hydrangea are used in popular Iranian medicine as an anthelmintic and antileishmanial drug. We previously identified several new antiplasmodial isoprenoids from this species [1,2]. A further phytochemical investigation of the aerial parts of S. hydrangea was conducted to identify minor isoprenoids. Fractionation of the $n$-hexane extract by a combination of open column chromatography on silica gel and preparative and semi-preparative RP-HPLC afforded five new isoprenoids. Their structures were established on the basis of extensive spectroscopic analysis, including MS, 1D and 2D NMR, and X-ray crystallographic analysis and ECD for determination of absolute configurations. Four compounds, including 1 , possess a six membered ring fused at C-8, C-9, and C24 with other carbocycles of the scaffold while compound 2 represents a new scaffold bearing a five membered ring instead. These isoprenoids are assumed to result from the cycloaddition of a diterpene with a monoterpene, followed by subsequent steps of oxidation and cyclization, and they have been found so far only in the genera Salvia and Perovskia.

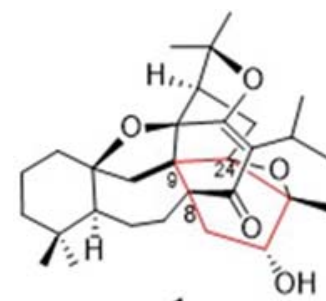

1

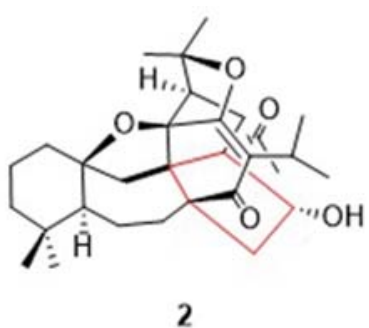

2
Fig. 1

[1] Farimani MM, Taheri S, Ebrahimi SN, Bahadori MB, Khavasi HR, Zimmermann S, Burn R, Hamburger M. Org Lett 2012; 14: 166 - 169

[2] Farimani MM, Bahadori MB, Taheri S, Ebrahimi SN, Zimmermann S, Burn R, Amin GR, Hamburger M. J Nat Prod 2011; 74: 2200 - 2205

\section{Mo-Poster Session 1-PO-228 CPC driven isolation and identification of Pistacia vera pericarp secondary metabolites: New source of rare and biologically active triterpenic acids}

Authors Popp $\mathrm{J}^{1,2}$, Petrakis $\mathrm{E}^{1}$, Angelis $\mathrm{A}^{1}$, Pisimisi $\mathrm{I}^{1}$, Halabalaki $\mathrm{M}^{1}$, Stuppner $\mathrm{H}^{2}$, Skaltsounis $\mathrm{AL}^{1}$

Institute 1 Division of Pharmacognosy and Natural Products Chemistry, Department of Pharmacy, National and Kapodistrian University of Athens, Panepistimiopolis Zografou, Athens, Greece; 2 Institute of Pharmacy/ Pharmacognosy, Center for Molecular Biosciences, University of Innsbruck, Innsbruck, Austria

DOI 10.1055/s-0037-1608275

Pistacia vera L. is considered the only species in the genus Pistacia (family Anacardiaceae) that produces edible tree nuts, which are widely known as pistachios [1]. The sensory, nutritional, and health attributes of this popular snack food make it increasingly valued and of significant economic importance for several countries including Greece, the sixth largest producer worldwide. Thus, valorization of the main side product derived from pistachio processing, the pericarp or outer hull, is of great interest and can be determined by the content in valuable phytochemicals that merit utilization [2]. In this study, the ethyl acetate extract of pericarp, obtained by means of ultrasound-assisted extraction (UAE), was submitted to centrifugal partition chromatography (CPC) for the fractionation and separation of secondary metabo- 
lites. A step-gradient method was employed, in reversed-phase mode, using a series of four biphasic solvent systems consisted of $n$-hexane/EtOAc/EtOH/ $\mathrm{H}_{2} \mathrm{O}$ in order of decreasing polarity of the aqueous mobile phase (i.e. from 8:2:5:5 to 8:2:9:1 $\mathrm{v} / \mathrm{v} / \mathrm{v} / \mathrm{v})$. The separation was performed in preparative (1000 $\mathrm{mL}$ ) CPC column, leading rapidly ( $<5$ hours) to the effective fractionation of $5 \mathrm{~g}$ of the initial extract. The comprehensive analysis of selected fractions by high resolution NMR and MS revealed that Greek pistachio pericarp comprises an excellent source of triterpenic acids, anacardic acids, and alkyl phenols. Interestingly, this process resulted in high amounts of $3 a$-(iso)masticadienolic and (iso)masticadienonic acids. The presence of 3a-(iso)masticadienolic acids in the Pistacia genus is reported herein for the first time, with the proposed CPC methodology enabling their efficient recovery.
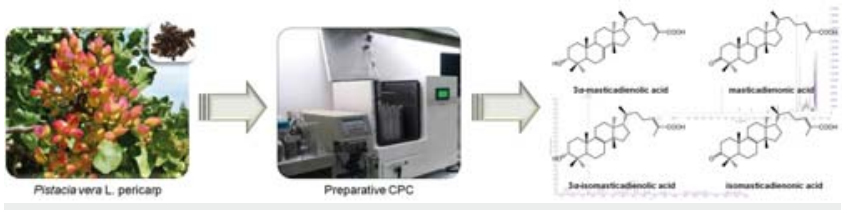

Fig. 1

[1] Alasalvar C, Shahidi F. Tree nuts: composition, phytochemicals, and health effects. Boca Raton: CRC Press; 2008: 295 - 304.

[2] Erşan S, Güçlü Üstündağ Ö, Carle R, Schweiggert RM. J Agric Food Chem 2016; 64: $5334-5344$.

\section{Mo-Poster Session 1-PO-229 Purification of alkaloids from Catharanthus roseus by $\mathrm{pH}-$ Zone Refining Centrifugal Partition Chromatography: process intensification and scale up}

Authors Kotland $\mathrm{A}^{1,2}$, Chollet $\mathrm{S}^{5}$, Autret $\mathrm{JM}^{4}$, Calmels $\mathrm{G}^{4}$, Diard $\mathrm{C}^{4}$, Hubert $\rfloor^{1}$, Marchal $\mathrm{L}^{3}$, Renault J $\mathrm{H}^{1}$

Institute 1 ICMR, UMR CNRS 7312, Reims, France; 2 Laboratoire de Génie Chimique UMR INPT, UPS, CNRS 5503, Toulouse, France; 3 GEPEA, UMR CNRS 6144, CRTT, Saint-Nazaire, France; 4 Institut de Recherche Pierre Fabre, Gaillac, France; 5 CPC Eng. - Capacités, Saint-Nazaire, France DOI 10.1055/s-0037-1608276

Centrifugal Partition Chromatography (CPC) is a versatile support free chromatographic technique involving the distribution and transfer of solutes between at least two immiscible liquid phases according to their partition coefficient, that avoids irreversible adsorption of the analytes due to the absence of solid support and allows a total recovery of various injected samples. The introduction of the so-called displacement mode dedicated to the purification of ionizable compounds, as well as the development of modeling approaches [1] taking into account both hydrodynamic and mass transfer aspects have attracted increasing interest to the area.

This communication describes the use of CPC in the $\mathrm{pH}$-zone refining mode for the purification of two indolomonoterpenic alkaloids, namely vindoline and catharanthine, from an industrial extract of Catharanthus roseus. The scale up of the separation on various pilot-scale columns [2] was achieved thanks to a numerical model allowing the prediction of chromatograms, highlighting the potential of CPC for the industrial purification of high added value compounds.

[1] Kotland A, Chollet S, Diard C, Autret JM, Marchal L, Renault JH. J. Chromatogr. A, 2015, 1391:80 - 87.

[2] Kotland A, Chollet S, Diard C, Autret J.-M, Meucci ], Renault J.-H, Marchal L, J. Chromatogr. A, 2016, 1474, $59-70$.

\section{Mo-Poster Session 1-PO-231 Monitoring of olive biophenols in 23 Olea europaea cultivars grown under the same agronomical conditions}

Authors Sarikaki $G^{1}$, Touvleliou $K^{1}$, Kourkouli $A^{1}$, Skaltsounis $L^{1}$,

Halabalaki $\mathrm{M}^{1}$, Mitakou $\mathrm{S}^{1}$

Institute 1 Division of Pharmacognosy and Natural Products Chemistry, Department of Pharmacy, University of Athens, PanepistimioupoliZografou,

15771 Athens, Greece, Athens, Greece

DOI 10.1055/s-0037-1608277

Olive oil based products are gaining increasing interest over the past few decades and the world becomes more diet-conscious. The investigations of olive biophenols are attracting increased scientific attention so as to shed light to the diverse range of bioactivities such as antioxidant, cardioprotective, antimicrobial and anticancer [1]. Olive fruits, olive leaves, olive processing residues constitute an enticing source of these compounds [2]. Additionally, there is an observed increased demand regarding their use in foodstuffs, food additives and functional foods. As the phenolic content of olive products is significantly influenced by the tree variety, the main objective of this study was the determination of composition of the phenolic compounds in leaves, fruits and olive oil as well as in olive oil production by-products. The phenolic composition was studied in twenty-three cultivars grown in the same grove under the same agronomical and environmental conditions in an effort to examine the effects of tree cultivar and determine possible varietal indicators. These indicators could be used as tools in order to confirm the provenance of unknown samples. Towards this direction UHPLC-HRMS \& HRMS/MS was employed for qualitative and quantitative purposes. The results indicated qualitative and quantitative differences in phenolic composition among cultivars. To our knowledge, this is the first study investigating the effect of olive oil variety alone to biophenols composition.

[1] Obied, H.K. et al, Analytica Chimica Acta, 2007, 603, 176-189.

[2] Taamalli, A. et al, Journal of Food Science, 2012, 77, R83- 92.

\section{Mo-Poster Session 1-PO-232 Phytochemical investigation of the Chilean berries Aristotelia chilensis and Ugni molinae. Evaluation of their anti-ageing effects employing a Drosophila model.}

Authors Sakavitsi $\mathrm{ME}^{1}$, Evangelakou $Z^{2}$, Faúndez Catherine $\mathrm{A}^{3}$, Lemus Belen Ringele $G^{3}$, Argyropoulou $A^{1}$, Kesternich $V^{3}$, Trougakos $I^{2}$, Halabalaki $M^{1}$, Skaltsounis Leandros $A^{1}$

Institute 1 Department of Pharmacognosy and Natural Products Chemistry, Faculty of Pharmacy, University of Athens, 15771, Athens, Greece;

2 Department of Cell Biology and Biophysics, School of Biology, University of Athens, 15784, Athens, Greece; 3 Laboratory of Organic Synthesis and Natural Products Chemistry, Department of Chemistry, University Católica de Norte, 0610, Antofagasta, Chile

DOI 10.1055/s-0037-1608278

Maqui (Aristotelia chilensis, Elaeocarpaceae [Molina] Stunz) and Murta (Ugni molinae, Myrtaceae Turcz), are small trees native to South Chile, deeply rooted in traditional culture of indigenous. Maqui and Murta berries have drawn research attention due to their potential health benefits, among them antioxidant, anti-inflammatory, anti-diabetic and anti-obesity activities, which are largely attributed to their rich polyphenolic content. The findings that diet interventions can increase lifespan, constitute dietary plants a promising source of chemical entities that promotes healthy span and/or delay ageing ${ }^{1}$. A high-throughput comparative phytochemical investigation of methanol and water extracts of both plants and specifically of their edible part, the fruits, was performed. Fingerprinting procedures using HPTLC showed that both plants had a rich chemical profile, while phenolic compounds prevailed. Profiling and dereplication procedures employing hyphenated techniques (HPLC) UHPLC-PDA, LC-(ESI)-HRMS/MS) were also applied and various putative bioactive compounds were identified. Hence, analytical and preparative techniques 
afforded compounds belonging to anthocyanins, flavonoids, flavonoid glucosides and phenolic acids, followed by structure elucidation (1D \& 2D NMR). The in vivo evaluation of anti-oxidant and anti-ageing properties of maqui and murta berries water extracts in Drosophila melanogaster flies revealed, that oral administration of both activate the Nrf2/Keap1 antioxidant pathway and proteasome peptidase activities. At the gene expression level, the administration of the above, altered the expression of well-known $\mathrm{CncC} / \mathrm{Nrf2}$ transcriptional targets, namely the antioxidant gstd 1 and $\operatorname{trxr} 1$ genes, the $\mathrm{CncC} / \mathrm{Nrf2}$ suppressor gene keap1 and the proteasomal genes $\alpha 7$ and $\beta 5$.

Conclusively, Maqui and Murta water extracts could be directly applicable for the development of anti-oxidant/anti-ageing nutraceuticals, while both extracts could be an important source of antioxidants.

[1] Argyropoulou, A, Aligiannis, N, Trougakos, I. P. \& Skaltsounis, A.-L. Natural Product Reports 2013,30, 1412.

\section{Mo-Poster Session 1-PO-233 Furochromones produced by Preussia sp.: an endophytic fungus from Casearya sylvestris var. lingua (Flacouteaceae)}

Authors Araujo Angela $\mathrm{R}^{1}$, Somensi $\mathrm{A}^{1}$, Biasetto Carolina $\mathrm{R}^{1}$, Ratsbone $\mathrm{Y}^{1}$, Cavalheiro Alberto $]^{1}$, Camargo Ilana $\mathrm{LBC}^{2}$, Abdalla Viviani $\mathrm{CP}^{3}$, Bolzani Vanderlan $S^{1}$

Institute 1 NuBBE - Nucleus of Bioassays, Biosynthesis and Ecophysiology of Natural Products, State University of São Paulo, Institute of Chemistry, Department of Organic Chemistry, Zip Code 14800 - 900, Araraquara, São Paulo, Brazil, Araraquara, Brazil; 2 São Carlos Institute of Physics, University of São Paulo, Zip Code 13566 - 590, São Carlos, São Paulo, Brazil, São Carlos, Brazil; 3 Federal University of São Carlos, Botany Department, Zip Code 13565 - 905, São Carlos, São Paulo, Brazil, São Carlos, Brazil

DOI 10.1055/s-0037-1608279

Endophytic fungi are widespread in nature and attract considerable attention as prolific sources of new natural products with diverse biological properties and structures. Fungi of the genus Preussia sp. belong to Sporormiaceae, family (Ascomicota), have shown to be potential sources of new secondary metabolites, mainly with biological activities for drug discovery. In our continual search for bioactive compounds from endophytic fungi from Brazilian plants we have investigated Preussia sp. isolated from Casearia sylvestris var. lingua (Flacourtiaceae). Preussia sp. was cultured in corn for 28 days at $25^{\circ} \mathrm{C}$ under static mode. The culture was extracted with $\mathrm{MeOH}$. After evaporation of the solvent, the crude $\mathrm{MeOH}$ extract was partioned using water and AcOEt by liquid partitioning. The EtOAc fraction was evaporated resulting in crude extract. This extract was dissolved in $\mathrm{CH}_{3} \mathrm{CN}$ and defatted with hexane by liquid partitioning. Following, the $\mathrm{CH}_{3} \mathrm{CN}$ fraction was evaporated to give $1,100 \mathrm{~g}$ of the crude extract. The $\mathrm{CH}_{3} \mathrm{CN}$ extract was subjected to chromatographic separation by CC on a silica gel and preparative HPLC (reversed-phase) yielding the isolation of three compounds, (Sporormiellin B, C e D) including new compound, Sporormiellin D. The structure elucidation of these compounds were achieved by spectroscopic data, including HREIMS, 1D and 2D NMR (HMQC, HMBC, COSY, NOESY). The Sporormiellin B showed phytotoxicity activity and Sporormiellin D eradication of biofilm formed by the bacterium S. epidermidis ATCC 34984 and presented bioactivity.

CNPq, FAPESP, and CAPES.

[1] XIONG, H. et. al. Sporormiellin A, the first tetrahydrofuran-fused furochromone with an unprecedented tetracyclic skeleton from Sporormiella minima. Royal Society of Chemistry Advances, 4, 24295 - 24299, 2014.

[2] ZHANG, F. et al. Journal of Natural Products, 75, 230-237, 2012.

\section{Mo-Poster Session 1-PO-234 SIMULTANEOUS DETECTION AND CHARACTERIZATION OF METABOLITES FROM MIKANIA LINDLEYANA DC (ASTERACEAE) LEAVES USING ESI-LC-MS}

\author{
Authors da Silva $A^{1}$, Piazzini $V^{2}$, Gomes Ana $C^{1}$, Bergonzi Maria $C^{2}$, Bilia \\ Anna $\mathrm{R}^{2}$, Barbosa Wagner $\mathrm{L}^{1}$
}

Institute 1 Faculty of Pharmaceutical Sciences, Institute of Health Sciences, Federal University of Para, Belém, Para, Brazil; 2 Department of Chemistry,

University of Florence, Sesto Fiorentino, Florence, Italy

DOI 10.1055/s-0037-1608280

The tea from leaves of Mikania lindleyana DC. (Asteraceae), popularly known as "sucuriju" or "sucurijuzinho", native in the Amazon region [1], is the main popular form of use of the plant to treat gastritis and inflammation [2]. $100 \mathrm{~g}$ of dried and minced herbal material were used to obtain the herbal preparation by decoction in $1 \mathrm{~L}$ of water, by boiling for 15 minutes. After cooling, the extract was filtered and frozen at $-45^{\circ} \mathrm{C}$ and lyophilized. Lyophilized aqueous extract (AEML) was fractionated aiming the isolation of chemical substances for characterizing this plant species. The fractionation was performed on Sephadex LH-20 $(25 \times 500 \mathrm{~mm})$ using EtOH/H2O $(1: 1 \mathrm{v} / \mathrm{v})$ as eluent and flow $2 \mathrm{~mL} / \mathrm{min}$ to give 33 fractions that were further analysed (TLC, LC-DAD-MS). For HPLC-MS analysis, MeOH/H2O (1:1 v/v) solutions of AEML and fractions were prepared at a final concentration of $3 \mathrm{mg} / \mathrm{mL}$ and $1 \mathrm{mg} / \mathrm{mL}$, respectively, and centrifuged before injection. Each reference standard was quantitatively weighed, dissolved in methanol, separately, at a concentration of $1.0 \mathrm{mg} / \mathrm{mL}$ and the stock solutions obtained stored under refrigeration $\left(-20^{\circ} \mathrm{C}\right)$. The working solutions prepared from the stock solutions are adjusted to a concentration of $500 \mu \mathrm{g} / \mathrm{mL}$ of each standard substance. Identification of constituents was performed by LC-DAD based on their UV-spectra and full scan MS-spectra, registered in the negative and positive modes. The signals [M-H]- and [2 M-H]as well as the masses of the fragments obtained permit propose 13 substances, namely phenolic acids, nucleotides, derivatives of catechin and one amino acid. Constituents were quantified using a calibration curve drawn with external standard.

[1] BERG, M. Plantas Medicinais na Amazônia-Contribuição ao seu conhecimento sistemático. CNPQ/PTO, Belém, Pará; 1982: 191.

[2] Barbosa, W. L. R. Etnofarmácia: fitoterapia popular e ciência farmacêutica; 2011

\section{Mo-Poster Session 1-PO-235 Absolute configuration of sesquiterpene lactones with potent immunosuppressive activity}

Authors Reinhardt $\mathrm{JK}^{1}$, Klemd $\mathrm{AM}^{2}$, De Mieri $\mathrm{M}^{1}$, Smiesko $\mathrm{M}^{1}$, Bürgi $\mathrm{T}^{3}$, Gründemann $C^{2}$, Hamburger $M^{1}$

Institute 1 Department of Pharmaceutical Sciences, University of Basel, Basel, Switzerland; 2 Institute of Environmental Health Sciences and Hospital Infection Control, University Hospital Freiburg, Freiburg (im Breisgau), Germany; 3 Department of Physical Chemistry, University of Geneva, Geneva, Switzerland

DOI 10.1055/s-0037-1608281

In a screening of Artemisia argyi (Asteraceae) and subsequent HPLC-based activity profiling canin (1) and the seco-tanapartholides 2 and 3 (stereoisomers) were identified as compounds with potent immunosuppressive activity in vitro. These isoprenoids were first discovered in 1969 and 1982, respectively, and have been extensively studied in the past for various biological activities[1]. Although many studies have been published examining their molecular structure and relative configuration by means of NMR and X-ray [2], the absolute configuration remained unresolved. We here established the absolute configuration of compounds 1-3 by a combination of electronic circular dichroism spectroscopy (ECD) and vibrational circular dichroism spectroscopy (VCD). 
ECD spectra of $1-3$ were measured and compared to spectra calculated $a b$ initio for different possible stereoisomers. Thereby, the commonly described relative stereoisomer of canin (1) was established as $(1 R, 2 S, 3 R, 4 S, 5 S, 6 S, 7 S, 10 R)$-canin. For compounds 2 and 3 the ECD data lowered the number of possible configurational isomers to four stereoisomers. The absolute configuration was finally established by VCD. Compound 2 was identified as (4R,5R,6S,7S)-seco-tanapartholide, and 3 as $(4 S, 5 S, 6 S, 7 S)$-secotanapartholide. The combination of ECD and VCD can thus be considered as a powerful approach in resolving the absolute configuration of conformationally flexible molecules.

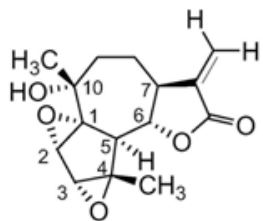

1

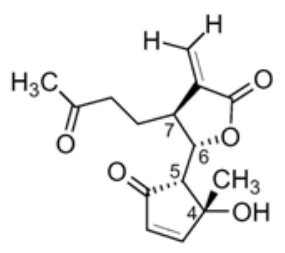

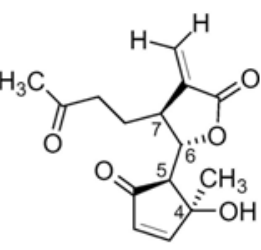

Fig. 1

[1] Knight DW. Nat Prod Rep 1995; 12: $271-276$

[2] Hewlett M], Begley tIM], Groenewegen WA, Heptinstall S, Knight DW, May J, Salan DWU, Toplis D. J Chem Soc, Perkin Trans 1 1996: 1979-1986

\section{Mo-Poster Session 1-PO-236 Beneficial effects of Lavender (Lavandula angustifolia Mill.) essential oil application prior a stressful situation in horses}

Authors Poutaraud $A^{1}$, Guilloteau $L^{2}$, Gros $C^{3}$, Lobstein $A^{4}$, Meziani $S^{5}$, Steyer $\mathrm{D}^{3}$, Moisan $M \mathrm{P}^{6}$, Foury $A^{6}$, Lansade $L^{7}$

Institute 1 Laboratoire Agronomie et Environnement, INRA, UMR 1121 Colmar, France; 2 URA, INRA, Nouzilly, France; 3 Twistaroma ${ }^{\circledR}$, Colmar,

France; 4 Laboratoire d'Innovation Thérapeutique, Faculté de Pharmacie, UMR 7200, Strasbourg, France; 5 Institut Européen des Antioxydants, Vandoeuvre les Nancy, France; 6 Nutrition and integrative neurobiology, INRA, UMR 1286, Bordeaux, France; 7 Unité Physiologie de la Reproduction et des Comportements, INRA, UMR85, Nouzilly, France

DOI 10.1055/s-0037-1608282

Horses are gregarious and fearful animals that undergo many stressful situations by humans. Simple and soft means are needed to manage their stress. This study was set up to investigate the relaxing effects of some EO on horses. The calming and soothing effects of Lavender (Lavandula angustifolia Mill.) essential oil (LaEO) have been widely demonstrated. The LaEO mode of action is essentially due to two major active molecules: linalool and linalyl acetate. The objective of this study was to develop an experimental protocol to highlight the effects of EO on equine stress response and to validate the effects of LaEO applied ante stress. Twenty-eight Welsh fillies were divided into two groups: one treated with $2 \mathrm{ml}$ of vegetable oil (control), the other treated with $10 \%$ LaEO applied with a roll-on around the nostrils. The fillies were subjected to a series of tests of 30 min during which the heart rate (HR) and different behavioral indicators of stress were noted. Salivary cortisol was also measured. Indicators of stress such as HR, alert postures and defecations measured during some tests are significantly lower in the LaEO group than in the control group. These results and salivary cortisol content show that the application of LaEO reduced the level of stress of animals placed in stressful situations. Furthermore, blood samples taken from 6 horses show that the pharmacokinetics of linalool in plasma presented a peak 20 min after LaEO application, agreeing with the effect of LaEO during the tests. In addition, a peak of antioxidant effectiveness appeared in the plasma 50 min after LaEO application. These data should encourage the use of this protocol with LaEO in further studies to optimize the effective dose, and possibly to assess other EO which could help horses to manage stressful situations.
Mo-Poster Session 1-PO-237 In vitro screening of the antispasmodic effect of Citrus extract based product and its main flavonoids on porcine intestine

Authors Mendel $\mathrm{M}^{1}$, Pettersen Hultén $\mathrm{E}^{1}$, Benarbia $\mathrm{A}^{2}$

Institute 1 Warsaw University of Life Sciences Faculty of Veterinary Medicine Division of Pharmacology and Toxicology, Warsaw, Poland; 2 Nor-Feed SAS 3 rue AMEDEO AVOGADRO, Beaucouzé, France

DOI 10.1055/s-0037-1608283

Since Citrus flavonoids have been proved to possess pronounced antispasmodic properties $[1,2]$, it seems reasonable to verify if they might be applicable as potential mitigation agents in swine diarrhoea by acting as spasmolytic agents on intestine smooth muscle. Nor-Spice $A B^{\circledR}$, a product containing Citrus extract, is currently used in pigs for its prebiotic effect but its impact on gut motility remains unrecognized. Therefore, the aim of this study was to evaluate the antispasmodic effect of Nor-Spice $A B^{\circledR}$ and main flavonoids of Citrus extract, hesperetin and naringenin, on segments of the jejunum collected from domestic pigs.

The experiments were performed on jejunum tissues collected from routinely slaughtered pigs. The experiments were conducted under isometric conditions.

The results showed that Nor-Spice $A B^{\circledR}$, hesperetin and naringenin cause a dose-dependent modification of carbamoylcholine (CCh)-induced contractility. If used at low concentrations, i. e. $0.01-1 \mu \mathrm{L} / \mathrm{mL}$ and $0.1-1 \mu \mathrm{M}$ for NorSpice $A B^{\circledR}$ and flavonoids, respectively, all agents generate significant increase in the contractile activity. In contrast, if applied at higher doses both Citrus extract product and individual phytoconstituents reduce CCh-evoked contraction of the smooth muscle.

Tab. 1

\begin{tabular}{|c|c|c|c|}
\hline Test substance & Concentration range & Induced effect & $\begin{array}{l}\% \text { of CCh-induced } \\
\text { contraction (control) } \\
\text { Mean values } \pm S D\end{array}$ \\
\hline \multirow{3}{*}{$\begin{array}{l}\text { Nor-Spice } \\
A B^{\circledR}\end{array}$} & $0.001 \mu \mathrm{L} / \mathrm{mL}$ & myorelaxant & $77.5 \pm 21.5$ \\
\hline & $0.01-1 \mu \mathrm{l} / \mathrm{mL}$ & contractile & $100.8 \pm 12.9-120.3 \pm 20.8$ \\
\hline & $10-100 \mu \mathrm{L} / \mathrm{mL}$ & myorelaxant & $58.9 \pm 15.4-82.7 \pm 10.1$ \\
\hline \multirow[t]{2}{*}{ Hesperetin } & $0.1-1 \mu \mathrm{M}$ & contractile & $106.6 \pm 15.8-160.3 \pm 14.9$ \\
\hline & $10-100 \mu \mathrm{M}$ & myorelaxant & $32.5 \pm 38.2-94.9 \pm 38.4$ \\
\hline \multirow[t]{2}{*}{ Naringenin } & $0.1-1 \mu \mathrm{M}$ & contractile & $97.5 \pm 25.6-149.2 \pm 16.8$ \\
\hline & $10-100 \mu \mathrm{M}$ & myorelaxant & $62.6 \pm 10.6-91.4 \pm 7.1$ \\
\hline
\end{tabular}

In conclusion, the obtained data suggest that hesperetin and naringenin might be responsible for the effect of Nor-Spice $A B^{\circledR}$ on jejunum contractility. The desired antispasmodic effect can be expected only if Citrus extract based product is used as feed additive in relatively high doses.

[1] Gharzouli K, Holzer P. Pharmacology 2004; 70: 5 - 14

[2] Yang ZH, Pan A, Zuo WL, Guo JH, Zhou WL. J Ethnopharmacol 2014; 155: $1177-1183$.

\section{Mo-Poster Session 1-PO-238 Verification of flavonoids effect on bovine cytochrome P450 activity in liver $\mathbf{S} 9$ fraction}

Authors Mendel $\mathrm{M}^{1}$, Karlik $\mathrm{W}^{1}$, Chłopecka $\mathrm{M}^{1}$, Dziekan $\mathrm{N}^{1}$ Institute 1 Warsaw University of Life Sciences Faculty of Veterinary Medicine Division of Pharmacology and Toxicology, Warsaw, Poland DOI 10.1055/s-0037-1608284

Flavonoids are very promising feed additives in dairy cows undergoing oxidative stress during the peripartum period and could potentially be used to prevent or minimalize the course of fatty liver syndrome. However, there is no data of their effect on hepatic metabolism in cows. Thus, the aim of the study was to verify the effect of some dietary flavonoids on the activity of cytochrome P450 (CYP450) in bovine liver S9 fractions.

The experiments were conducted on hepatic S9 fractions from routinely slaughtered adult cows. The effect of apigenin, quercetin and luteolin on 
7-ethoxycoumarin O-deethylase (ECOD) activity, measured as the amount of created 7-hydroxycoumarin (7-OH), was used to monitor CYP450 activity. The production of 7-OH in the absence of flavonoids amounted to 413.2 $\pm 83.8 \mathrm{pmol} / \mathrm{mg}$ of protein $/ \mathrm{min}$. All three flavonoids used in the concentration range of $1-100 \mu \mathrm{M}$ induced significant decrease of ECOD activity. The strongest inhibition of ECOD was observed when flavonoids were used in the highest dose, i.e. $100 \mu \mathrm{M}$. The amount of produced 7-OH amounted then to 34.1 $\pm 5.5,49.8 \pm 3.3$ and $51.7 \pm 3.9 \%$ of $7-\mathrm{OH}$ produced in the absence of flavonoids, for apigenin, quercetin and luteolin, respectively. If used in the lowest dose $(1 \mu \mathrm{M})$ apigenin inhibited ECOD activity significantly weaker $(87.5 \pm 2.7 \%$ of the control reaction) than quercetin and luteolin $(65.3 \pm 4.7$ and 60.9 $\pm 4.5 \%)$.

In conclusion, apigenin, quercetin and luteolin proved to be potent inhibitors of ECOD and thereby CYP450 activity. However, the observed effect was neither dose-dependent nor variable between flavonoids.

The study was supported by a research grant from the National Science Centre Poland No. UMO- 2012/05/D/NZ9/01610.

\section{Mo-Poster Session 1-PO-239 Analysis of anti-inflammatory effects of carvacrol and curcumin in vitro using the IPEC-J2 cell culture model}

\author{
Authors Kaschubek $\mathrm{T}^{1}$, Mayer $\mathrm{E}^{1}$, Schatzmayr $\mathrm{G}^{1}$, Teichmann $\mathrm{K}^{1}$ \\ Institute 1 BIOMIN Research Center, Technopark 1, 3430 Tulln, Austria \\ DOI 10.1055/s-0037-1608285
}

During weaning, piglets are exposed to a number of different stress factors accompanied by disproportionate inflammatory processes in the intestine, which can lead to an impairment of the intestinal barrier integrity and development of gastrointestinal disorders such as diarrhea. The pro-inflammatory transcription factor NFKB is considered as one of the key players in the development of inflammation and the control of NFKB is critical to overcome weaning-associated disturbance of animal health and reduced growth performance [1].

Formerly used antibiotic growth promoters have been banned in the EU since 2006 and there is a great demand for alternatives like plant-derived feed additives. This study evaluated the anti-inflammatory characteristics of the phytogenic feed additives carvacrol $[50 \mu \mathrm{g} / \mathrm{ml}]$ and curcumin $[1 \mu \mathrm{g} / \mathrm{ml}]$ in an intestinal porcine epithelial cell line (IPEC-J2) via the luminescence based NFKB reporter gene assay.

Cells were seeded in white 96 -well plates and incubated at $39^{\circ} \mathrm{C}$ and $5 \% \mathrm{CO}_{2}$. After 24 hours, cells reached approximately $70 \%$ confluence and were transfected with the NFKB reporter vector. Following 24 hours incubation time, cells were either pre-incubated with non-toxic test sample concentrations or just cultivation medium (DMEM/Ham's F12) in case of the cell control for another 24 hours. On the next day, the pro-inflammatory transcription factor NFKB was activated by stimulation with TNF- $\alpha$ for 5 hours and luminescence values were measured, which are directly proportional to the amount of activated NFKB.

After TNF- $\alpha$ stimulation, carvacrol and curcumin were both able to inhibit the activation of NFKB by $21 \%$ and $25 \%$ respectively, compared to TNF- $\alpha$ stimulated control cells. These results suggest promising inflammation restricting properties of carvacrol and curcumin in IPEC-J2. Further confirmation of the anti-inflammatory activities of both phytogenic compounds on mRNA and protein level is in progress.

[1] Gessner DK, et al. Acta Vet Scand 2013; 55: 18

\section{Mo-Poster Session 1-PO-240 Milk thistle extract showed antioxidant properties and protective effect on the gut barrier function in a porcine in vitro model}

\author{
Authors Novak B ${ }^{1}$, Kaschubek $\mathrm{T}^{1}$, Stelzer $\mathrm{J}^{1}$, Schatzmayr $\mathrm{G}^{1}$, Mayer $\mathrm{E}^{1}$ \\ Institute 1 BIOMIN Research Center, Department of Cell Biology, Tulln, \\ Austria \\ DOI 10.1055/s-0037-1608286
}

Milk thistle (Silybum marianum) is known for its broad range of positive properties in human and animal health and especially as hepatoprotective substance [1].

In our study, we investigated an ethanolic (70\%) milk thistle (MT) extract for its effect on gut barrier integrity and against the formation of reactive oxygen species (ROS) in a porcine intestinal epithelial cell line (IPEC-J2). Differentiated IPEC-J2 were grown in non-coated Transwell ${ }^{\circledR}$ membrane inserts $\left(1.12 \mathrm{~cm}^{2}\right.$; $0.4 \mu \mathrm{m}$ pore size) for 7 days, allowing the formation of tight junctions (TJs) and the build-up of a representative intestinal monolayer. On day 7, cells were pre-treated with an ethanolic MT extract, followed by addition of a stressor substance on day 8 . The barrier integrity was examined by measuring the transepithelial electrical resistance (TEER) by a Volt-Ohm-Meter after $24 \mathrm{~h}$, $48 \mathrm{~h}$, and $72 \mathrm{~h}$ of incubation. With the help of a fluorescence assay (DCFHDA assay), the cellular ROS amount, which is indicative for increased oxidative stress, was determined. Therefore, hydrogen peroxide $\left(\mathrm{H}_{2} \mathrm{O}_{2}\right)$ was used to increase the ROS amount. Then the MT extract was added to counteract the negative effect of $\mathrm{H}_{2} \mathrm{O}_{2}$.

The non-toxic concentration of $500 \mu \mathrm{g} / \mathrm{mL}$ MT extract increased the stressorimpaired TEER value by $11 \%$ ( $24 \mathrm{~h}$ incubation) and $7 \%$ ( $48 \mathrm{~h}$ incubation), respectively. Even compared to the cell control, MT could increase the TEER value by $3-10 \%$. In the DCFH-DA assay, $4 \mu \mathrm{g} / \mathrm{mL}$ MT extract reduced the $\mathrm{H}_{2} \mathrm{O}_{2}$-induced (set to $100 \%$ ) ROS amount by $12 \%$ after $1 \mathrm{~h}$ of incubation.

MT extract has been shown to act positively on the gut barrier integrity and the antioxidant status. The latter was the most effective parameter considering the applied low concentration in the DCFH-DA assay.

\section{Mo-Poster Session 1-PO-241 The effectiveness of natural claw spray in the treatment of Mortellaro disease}

Authors Kissels $\mathrm{W}^{1,2}$, Drint $\mathrm{M}^{3}$, Lievaart $\mathrm{J}^{3}$, van Hierden $\mathrm{Y}^{1}$

Institute 1 Ecostyle B.V, Oosterwolde, Netherlands; 2 Kissels Consulting B.V, Bilthoven, Netherlands; 3 Nederlands KlauwGezondheidscentrum, Bathmen, Netherlands

DOI 10.1055/s-0037-1608287

Digital dermatitis (DD), known as Mortellaro disease, is endemic in most dairy farms. The acute M2 stage is painful. In this study, an antibiotic free spray based on natural products was used instead of standard treatment with antibiotic spray.

Mortellaro disease causes many problems. Besides serious economic losses also animal welfare factors are at stake. The usually applied antibiotic-containing spray does not fit in the current policy of resistance prevention and reduction of antibiotic usage.

Objective was to test a treatment according a protocol of Mortellaro M2 lesions with Klausan $^{\odot}$-spray ${ }^{1}$ aimed at an outcome cure rate of $\geq 60 \%$.

The required number of cows with an $\mathrm{M} 2$ lesion was 97 divided over 5 average size farms. Farms were selected according to defined criteria. The empiric methodology was equal to a clinical trial by Utrecht University ${ }^{2}$. Assessment was executed in accordance with the international system of Döpfer et al. ${ }^{3}$. A total of $111 \mathrm{M} 2$ lesions have been assessed, treated and monitored. This involved 93 animals on 5 farms. The results reveal that treatment delivers up to the hypothesis, namely a minimum cure rate of $60 \%$ of treated Mortellaro M2 lesions. The average cure rate was $73 \%(52-82 \%)$. 


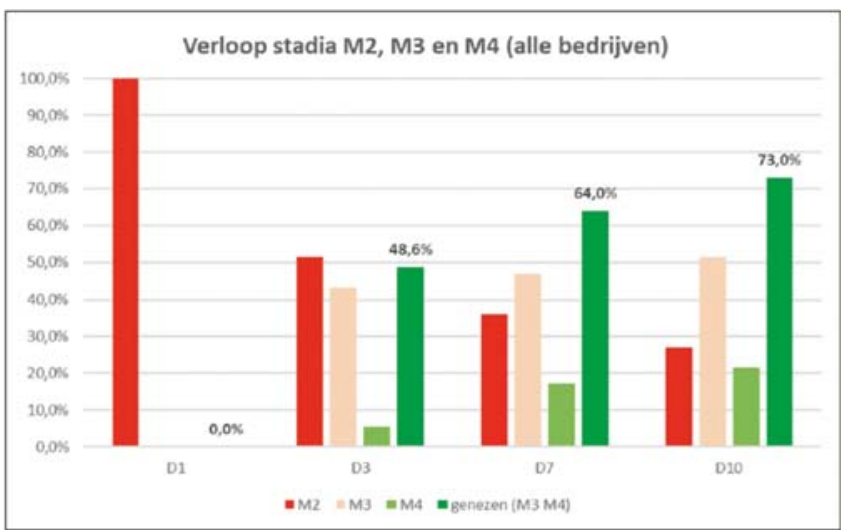

Fig. 1: Development stages M2, M3 and M4 (all farms)

The treatment protocol followed did meet expectations. Klausan-spray is an attractive antibiotic-free alternative for existing therapies. Treatment of Mortellaro remains farm tailored. Differences indicate that also other factors are of influence and can affect cure rate. Further studies should focus on exploring more in depth influencing factors.

[1] Klausan ${ }^{\odot}$ (Calendulae flos, Chamomillae flos, Terebinthina laricina, Cortex Quercus, Balsam Peruvianum), Ecostyle, Netherlands.

[2] Jorritsma R et al. University of Utrecht, Faculty of Veterinary Medicine.2013.

[3] Döpfer D et al. Veterinary Record. 1997; 140:620 - 623. Source: Kissels et al.Tijdschrift voor Diergeneeskunde.2017.

\section{Mo-Poster Session 1-PO-242 Effects of Wood Vinegar on the Protection of Diarrhea in Weaning Pigs}

Authors Kupittayanant $\mathrm{P}^{1}$, Kupittayanant $\mathrm{S}^{2}$

Institute 1 School of Animal Production Technology, Institute of Agricultural Technology, Suranaree University of Technology, Nakhon Ratchasima,

Thailand; 2 School of Preclinical Science, Institute of Science, Suranaree University of Technology, Nakhon Ratchasima, Thailand

DOI $10.1055 / \mathrm{s}-0037-1608288$

The aim of the study was to examine the effects of wood vinegar on the protection of diarrhea in weaning pigs. Weaning pigs were divided into 4 groups each group contained 24 animals. Group 1-4 was orally fed with distilled water, antibiotic (enrofloxacin), $5 \%$ wood vinegar, and $5 \%$ acetic acid $1 \mathrm{~mL} /$ daily for 3 weeks after weaning (started on day 28 after birth), respectively. Feces samples were collected and were subjected to bacterial culture (using Nutrient Agar, Lactobacillus MRS Agar and EMB Agar) and bacterial colonies counted. The results showed that $5 \%$ wood vinegar administration significantly increased the number of Lactobacillus spp. and significantly decreased Escherichia coli and total bacterial count when compared to the control group (group 1). Interestingly, the effects were the same as that of antibiotic administration and significantly better than those of administered by $5 \%$ acetic acid. Taken together, the data suggest that wood vinegar has protective effects against diarrhea by increasing Lactobacillus spp. and reducing Escherichia coli in weaning pigs and that the effects are as good as the use of antibiotics. Wood vinegar therefore may be used as an agent to promote a healthy gut in weaning pigs.
Mo-Poster Session 1-PO-243 Developing a single application formulation with a Solanum glaucophyllum extract for milk fever prevention. I: plasma kinetics in dry cows.

Authors Bachmann $\mathrm{H}^{1}$, Lanz $\mathrm{M}^{2}$, Rambeck $\mathrm{W}^{3}$

Institute 1 Herbonis AG, Augst, Switzerland; 2 Fachhochschule

Nordwestschweiz, Basel, Switzerland; 3 Lehrstuhl für Tierernährung Ludwig-

Maximilians-Universität, München, Germany

DOI 10.1055/s-0037-1608289

Milk fever is a metabolic disease in milk producing cows around calving. Clinical signs are muscular paralysis mainly of the legs with immobility of the affected animal. High milk producing breeds and older animals are most likely affected, and have a high chance of recurrence. An incidence of $5-10 \%$ has been reported. The disease is caused by an acute drop in blood calcium (Ca) when initiating milk production depletes the blood Ca too fast for repletion from bone mobilization and intestinal uptake. With the understanding of the underlying mechanism, Ca supply management and vitamin-D (VD) supplementation became prime candidates for MF prevention and therapy. Several strategies have been developed for MF prevention e.g. peri-parturient administration of large doses of calcium or an ante-parturient feeding regime known as DCAD. VD as the main calcium regulating agent has also been used. While the efficacy of 1,25D and of the plant Solanum glaucophyllum (SG) in Ca mobilization and MF prevention has been shown, hypocalcaemia has occasionally been observed after cessation of application. Here we present a system which may overcome this limitation by a single bolus containing an immediate and a slow release formulation of a SG extract (SCE).

Tab. 1

\begin{tabular}{|c|c|c|c|c|c|c|}
\hline & $\begin{array}{l}\text { Serum } \\
1,25 D^{1)}\end{array}$ & $\begin{array}{l}\text { Serum } \\
\mathrm{Ca}\end{array}$ & $\begin{array}{l}\text { Serum } \\
\text { P }\end{array}$ & $\begin{array}{l}\text { Serum } \\
\mathrm{Mg}\end{array}$ & $\begin{array}{l}\text { urine } \\
\mathrm{Ca} / \text { crea }\end{array}$ & $\begin{array}{l}\text { urine } \\
\mathrm{P} / \text { crea }\end{array}$ \\
\hline control & 1 & 1 & 1 & 1 & 1 & 1 \\
\hline I,25D & 2.89 & $1.07^{* *}$ & $1.20^{*}$ & $1.05^{*}$ & 6.98 & 1.62 \\
\hline irSGE & 3.13 & $1.08^{*}$ & $1.30^{* *}$ & $1.01^{\mathrm{ns}}$ & 7.21 & 1.53 \\
\hline srSGE & $\begin{array}{l}4.21 \\
\mathrm{~ns}\end{array}$ & $\begin{array}{l}1.12^{* *} \\
\text { 2) }\end{array}$ & $\begin{array}{l}1.41^{* * *} \\
\text { 2) }\end{array}$ & $\begin{array}{l}1.04^{\mathrm{ns}} \\
\text { 2) }\end{array}$ & 5.40 & 3.93 \\
\hline \multicolumn{7}{|c|}{$\begin{array}{l}{ }^{1)} \text { Standard deviation not calculated due to pooled serum } \\
\text { samples. } \\
\text { 2) } \text { p-values Mann-Whitney U Test ( } 2 \text {-sided) versus control: } \\
{ }^{* *} 0.01,{ }^{*} 0.05 \\
\text { Post hoc power calculation: } 100 \% \text { for Ca AUC; } 100 \% \text { for P AUC, } \\
\text { except for } 1.25 \mathrm{D} \text { vs. control ( } 93 \% \text { ); } 15 \% \text { (srSGE vs. control), } 6 \% \\
\text { (irSGE vs. control) and, } 68 \% 1,25 \mathrm{D} \text { vs. control for Mg AUC. }\end{array}$} \\
\hline
\end{tabular}

A single oral dose was given to 5 non-lactating cows either as synthetic 1,25D, as SGE or as a slow-release form of SGE (srSGE). Ca, P, Mg and 1,25D was measured in serum. Area under the curve (AUC) for 11 days over control for serum Ca, Phosphor and magnesium and for urine $\mathrm{Ca}$ and $\mathrm{P}$ is given below. AUCs were calculated and normalized to the value of the control group (set to 1).

In the present study shows the retard-release form of the tested srSGE the highest AUC within 11 days after a single application for serum $\mathrm{Ca}, \mathrm{P}$ and $1,25 \mathrm{D}$.

\section{Mo-Poster Session 1-PO-244 Prophylactic feeding of medicinal herbs for sustainable health improvement during the rearing of calves at a dairy farm}

Authors Spielberger $\mathrm{U}^{1}$, Brendieck-Worm $\mathrm{C}^{1}$

Institute 1 GGTM, Schallstadt, Germany

DOI 10.1055/s-0037-1608290

Respiratory diseases and diarrhoea are the main causes for rearing losses in dairy calves. However, the application of antibiotics, which is the most widespread method to combat these problems, is nowadays in the focus of public concern. To ensure animal health and reduce the use of antibiotics, alternative prophylactic treatments are needed. The present study evaluates the effect of a prophylactic application of medicinal herbs on the health status of rearing calves under field conditions. 
The study was conducted at a bio-dynamic dairy farm with 65 Brown Swiss dairy cows and calf rearing. During the 17 month period prior to our study, 33 calves (51\%) needed antibiotic (Cefquinom, Tildipirosin) and anti-inflammatory (NSAID) treatments due to severe respiratory diseases and diarrhoea. In order to decrease the rate of diseases and reduce antibiotics, prophylactic application of an herbal feed supplement (PlantaPulmin S Konzentrat, PPSK; SaluVet GmbH, Germany) was carried out between June 2014 and April 2016. The treatment consisted a $250-500 \mathrm{ml}$ infusion of $5 \mathrm{~g}$ of PPSK, which contains thyme, liquorice, gentian and wormwood. The infusion was administered orally to 54 calves for 40 days, starting at the third day of life. The health status of all calves was monitored until they were 4 months of age.

During the 23 month period of PPSK supplementation, only 12 calves suffered from diarrhoea. Eleven were cured by dietetic electrolytes, their body weight development was not impaired (ø $890 \mathrm{~g} / \mathrm{d}$ ). Antibiotic and NSAID treatments were unnecessary. Respiratory diseases occurred in only 3 of the 54 calves during the first 6 months of supplementation. This did not require the use of antibiotics.

These results clearly demonstrate that a prophylactic application of PPSK improved the health status of the calves and might have the potential to reduce the necessity of antibiotic treatments in these cases.

\section{Mo-Poster Session 1-PO-245 Replacing the coccidiostats: defining cost-effective surrogates}

Authors Roussel $\mathrm{P}^{1}$, Arnaiz $\mathrm{V}^{2}$, Rodríguez $\mathrm{V}^{3}$, García JM ${ }^{1}$, Honorio Javes $\mathrm{C}^{3}$, Chicoteau $\mathrm{P}^{1}$

Institute 1 Nor-Feed, Beaucouzé, France; 2 MONTANA, Lima, Peru; 3 Private University Antenor Orrego, Trujillo, Peru

DOI 10.1055/s-0037-1608291

The poultry industry will soon face new challenges, notably drastic restrictions on coccidiostats, which have been discredited due to their residues persisting in certain food animals and the emergence of antimicrobial-resistant pathogens [1]. The demand for meat continues to grow, encouraging agricultural intensification and thereby an increase in the incidence of production diseases. The annual cost of coccidiosis is estimated at over 3 billion dollars and, today, the disease represents the main threat for the poultry industry worldwide [2]. Thus the worst is yet to come if sustainable solutions are not found before the regulations change.

Saponins, naturally produced by some plants, are capable of lysing coccidia [3]. To determine whether coccidiostats can be replaced by our saponin-based product, an assay was performed on 75 broiler chickens. Two groups received the standard diet supplemented either with coccidiostats (starter, grower: Clopidol $500 \mathrm{~g} / \mathrm{T}$; finisher: Salinomycin $600 \mathrm{~g} / \mathrm{T}$ ) or with saponins (plants rich in sarsapogenin and diosgenin, $250 \mathrm{~g} / \mathrm{T}$ ); the control group did not receive any feed supplementation. On the $14^{\text {th }}$ day, the chickens were inoculated with coccidia (Eimeria acervulina, E. maxima, E. tenella, E. necatrix, E. brunetti, E. praecox).

The saponins significantly reduced the oocyst loads and the lesion scores, and augmented body weight compared to the control group. They were as effective as the coccidiostats and provide a better return on investment.

Cost-effective and effective in preventing coccidiosis, our saponin-based product proves to be a good surrogate. Contrary to the coccidiostats currently used, it is safe for all species (no toxicity, no microbial resistance, no residue in meat) and it is $100 \%$ natural.

[1] Center for Disease Dynamics, Economics \& Policy. State of the World's Antibiotics. CDDEP: Washington, D.C. 2015

[2] Williams RB. Avian Pathol 2002;31:317 - 353

[3] Pasaribu T, Astuti DA, Wina E, Setiyono A. Int J Poultry Sci 2014;13:347 352

\section{Mo-Poster Session 1-PO-246 In vitro antimicrobial activity of essential oils against Malassezia pachydermatis isolated from canine ear swabs}

Authors Bismarck D ${ }^{1}$, Dusold $\mathrm{A}^{2}$, Müller $\mathrm{E}^{1}$

Institute 1 Laboklin GmbH \& Co. KG, Bad Kissingen, Germany; 2 Hochschule für angewandte Wissenschaften Coburg, Coburg, Germany

DOI 10.1055/s-0037-1608292

The yeast Malassezia pachydermatis (MP) can cause canine otitis. The in vitro efficacy of 22 commercially available essential oils (EO) was tested against 15 isolates of MP rom canine ear swabs derived from routine diagnostic of Laboklin using the agar disk diffusion test (aromatogram).

Briefly, an agar plate is inoculated with MP and a paper filter disk with $10 \mu \mathrm{l}$ of the $\mathrm{EO}$ is placed in the middle of the plate. After incubation ( 4 days, $36^{\circ} \mathrm{C}$ ) the zone of inhibition of yeast growth is determined. The in vitro efficacy of EO was classified as follows: not sensitive $(<8 \mathrm{~mm})$, slightly sensitive $(8-13 \mathrm{~mm})$, moderately sensitive $(14-19 \mathrm{~mm})$, very sensitive $(\geq 20 \mathrm{~mm})$ and extremely sensitive, if total growth was inhibited. The EOs were also tested in a dilution of $1: 5$ in sesame oil and in case of a growth inhibiting effect in this dilution in a 1:10 dilution as well.

Lemon grass, winter savory, oregano and rose geranium oil showed mainly extremely inhibitory effects. Palamarosa, indian melissa, Thyme thymol 19\%, thyme, thyme linalool, cinnamon leaf, clove and coriander seed oil were effective against all tested isolates, but in different degrees. Manuka, fennel, tea tree, lavandin super, lavender fine, lemon, clary sage and angelica root oil showed inhibiting activity in some cases. Ravintsara and neroli oil were not effective. In a dilution of 1:5 only some EOs had inhibitory effects: Lemon grass, oregano, palmarosa, cinnamon leaf and winter savory oil were effective in all cases. Clove, indian melissa and rose geranium oil had an inhibitory effect in some cases. In a dilution of 1:10 only lemon grass oil was effective, thus being the most potent of the tested EO.

These in vitro results identify some EO as a promising candidate for therapy of MP associated canine otitis.

\section{Mo-Poster Session 1-PO-247 The aromatogram - the method matters}

Authors Bismarck D ${ }^{1}$, Kraus $\mathrm{L}^{2}$, Martinez $\mathrm{Y}^{1}$

Institute 1 Laboklin GmbH \& Co. KG, Bad Kissingen, Germany; 2 Hochschule für angewandte Wissenschaften Coburg, Coburg, Germany

DOI 10.1055/s-0037-1608293

For specific treatment with essential oils (EO) an aromatogram can be used as diagnostic tool. Hereby, agar disk diffusion is carried out to determine in vitro antibacterial activity of EO.

Briefly, a filter paper disk containing the $\mathrm{EO}$ in question is placed on an agar plate previously spread with the pathogenic bacteria. After incubation the diameter of the inhibition zone of bacterial growth is determined. Often pictures of the aromatogram depict testing of several oils on one agar plate, which reflects time and cost-effective routine diagnostic procedure. However, due to synergistic effects between EO, testing of several EO on one agar plate may lead to different results in comparison to single tests.

13 commercially available pure EOs (cinnamon leaf, clary sage, coriander seed, lavender fine, lemon grass, manuka, indian melissa, oregano, palmarosa, ravintsara, tea tree, thyme linalool, thyme thymol) were tested in different aromatogram layouts for their antibacterial activity against clinical isolates of Staphylococcus pseudintermedius, Escherichia coli and Pasteurella multcocida derived from canine or feline samples from routine diagnostic.

Different aromatogram layouts were tested. In the first three layouts several combinations of EO on one agar plate were analyzed, in the fourth layout only one EO per agar plate was tested.

Results show that testing of several EO on one agar plate can lead to larger inhibition zones and thus overestimation of their in vitro activity. This is especially true for gram-positive bacteria like Staphylococcus pseudintermedius and 
generally sensible bacteria like Pasteurella multocida. Gram- negative bacteria e.g. Escherichia coli seem to be less affected.

In conclusion, analyses of several EO on one agar plate can have an influence on the outcome of the aromatogram, especially testing gram-positive bacteria. A general procedure for laboratories carrying out aromatograms to obtain comparable and reliable results should be established.

\section{Mo-Poster Session 1-PO-248 Assessment of antimicrobial activity of ethnoveterinary anti-mastitis herbal formulation on field isolated pathogens using scanning electron microscopy (SEM)}

Authors Natesan $\mathrm{P}^{1}$

Institute 1 EVM Herbal Centre, VUTRC, Tamil Nadu Veterinary and Animal Sciences University, Thanjavur 613403 Tamil Nadu, India, Thanjavur, India, Trans-Disciplinary University, Bangalore, 560064 India

DOI 10.1055/s-0037-1608294

This is the study on the anti-bacterial activity from the extracts obtained from different solvents (Aqueous, ethanol and ethyl acetate) of an anti-mastitis ethno-veterinary herbal fresh formulation containing Aloe vera, Curcuma longa and calcium hydroxide, tested against field isolates of three types of microbes obtained from the clinical samples collected by the VUTRC, Thanjavur. Preliminary phytochemical screening revealed the presence of essential secondary metabolites such as flavanoids, tannins, saponins, terpenoids etc. The minimum inhibitory concentration (MIC) using agar well diffusion method was observed for the three extracts with the support of image representation (fig 1) by scanning electron microscopic technique (SEM). The scanning electron microscopy determined the morphology of the bacteria with and without using anti-mastitis herbal fresh formulation. After $24 \mathrm{~h}$ SEM images of Escherichia coli, Staphylococcus aureus and Pseudomonas aeruginosa not treated with extract, showed normal morphology with a multi-layered surface consisting of the outer membrane. But the organisms, after 24 hrs of the addition of the herbal preparation, showed various phases of the process in cell death. The above SEM images shows cell disruption, partial disappearance of the cytoplasmic membrane and plasmolysis [1,2]. This suggest that the fresh herbal preparation validated and used successfully in clinical situations across India, owes its action partially to its antimicrobial activity.

[1] Pandey A, Jagtap JV, Patil AA, Joshi RN, Kuchekar BS. Journal of Chemical and Pharmaceutical Research2010, 2: 182 - 186.

[2] Gupta A, Mahajan S, Sharma R. Biotechnology Report, 2015;6:51 - 55

\section{Mo-Poster Session 1-PO-249 Fresh herbal preparation in the treatment of a heifer with fractured and infected hind limb - A case report}

Authors Natesan $P^{1}$, Punniamurthy $N^{1}$, Vijay Anand $]^{1}$, Elamurugan $A^{1}$, Nair MNB ${ }^{2}$

Institute 1 EVM Training and Research Centre, Thanjavur, TANUVAS, 613 403, Thanjavur, Tamil Nadu, India, Thanjavur, India; 2 Trans-Disciplinary University, 74/2, Jarakabandekaval, Bangalore 560064, Bangalore, India

DOI 10.1055/s-0037-1608295

A successful case of treating a fractured and infected limb of a heifer, with natural herbal formulations in a stand-alone mode is presented. A cross bred Jersey heifer aged 2 years, ran over by a two wheeler at the distal end of the left hind limb, the animal was treated locally by a veterinarian with POP cast for 3 weeks. The bandage was removed and physical examination revealed a fracture in the first phalanx of left hind limb. The fractured area was swollen up to the hock region. The bone tissue was visible. The animal could not bear weight on the fractured limb. The purulent discharge and debris was removed by pressure and treated daily, externally with a herbal preparation containing Ocimum basilicum, Acalypha indica, Allium sativum, Curcuma longa and Centella asiatica in coconut oil [1,2]. Cissus quadrangularis was given orally for 14 days [3]. Animal was able to bear weight on the fractured limb after 30 days of herbal treatment and complete healing of wound surface was noticed after 45 days.

[1] Peng LH, et al. J Ethnopharmacol. 2010; 131:282 - 289.

[2] Ayyanar M, Int J Appl Res Nat Product 2009; 2: 29 - 42.

[3] Siu WS, et al. Evid Based Complement Alternat Med 2015; 905270.

\section{Mo-Poster Session 1-PO-250 Selective in vitro anthelmintic efficacy of Diospyros whyteana (Ebenaceae) active compounds derived using antifungal activity to guide fractionation}

Authors Sakong B ${ }^{1}$

Institute 1 University of Pretoria, Pretoria, South Africa

DOI 10.1055/s-0037-1608296

Nematodes are economically important parasites in livestock. Control of these infections relies mainly on synthetic anthelmintic drugs, against which development of resistance is a major problem. Many farmers, particularly in rural areas, use ethnoveterinary preparations comprising medicinal plants to combat endoparasitism. As anthelmintic assays are difficult and time-consuming, the aim of this study was to investigate if antifungal assays can be used as a model to isolate anthelmintic plant compounds, based on potential correlations in the two activities. In the anthelmintic egg hatch assay (EHA) and the larval development assay (LDA), the Diospyros whyteana acetone leaf extract had good activity with $\mathrm{EC}_{50}$ values of 4.43 and $5.58 \mu \mathrm{g} / \mathrm{ml}$ respectively. The extract was active against Candida albicans with MIC of $40 \mu \mathrm{g} / \mathrm{ml}$, and this organism was used as a model to isolate antifungal compounds from $D$. whyteana. Bioactivity of the fractions as well as cytotoxicity $\left(\mathrm{IC}_{50}\right.$, tested using Vero cells in the colorimetric MTT assay) was assessed during fractionation, which resulted in isolation of three active compounds. $\mathrm{EC}_{50}$ values of the compounds ranged from 0.11 to 3.30 in the EHA and 1.5 to 4.35 in the LDA, with promising SI values above 28 . The three compounds had the same MIC of $160 \mathrm{ug} / \mathrm{ml}$ against C. albicans. Results showed a good correlation between the EHA and LDA as well as good antifungal activities, indicating that the use of the antifungal assay assisted with the isolation of anthelmintic compounds. These compounds are being identified in current work.

\section{Mo-Poster Session 1-PO-251 Phytogenic Substances in a Model for Intestinal Barrier Function after Tight Junction Disruption}

Authors Bachinger $D^{1}$, Mayer $\mathrm{E}^{1}$, Teichmann $\mathrm{K}^{1}$

Institute 1 BIOMIN Research Center, Tulln, Austria

DOI 10.1055/s-0037-1608297

For humans and animals alike, gut integrity is crucial for gut health and performance. Amongst others, the intestinal barrier is composed of epithelial cells and intercellular tight junctions. Phytogenic substances are plant-derived feed additives commonly used in animal nutrition for health and productivity. The objective of this experiment was to explore the influence of selected phytogenic substances on the recovery of the barrier function after disturbance.

Intestinal porcine epithelial cells (IPEC-J2) were cultivated in 12-well Transwell ${ }^{\circledR}$ plates. After removal of the cell culture medium and a washing step, $2 \mathrm{mM}$ EGTA was added as a chelator. This caused a disruption of the tight junctions between the cells. After washing, the cells were further incubated for 24 hours with either cell culture medium alone (negative control), an additional MAPkinase inhibitor (positive control) or additional phytogenic extracts. The transepithelial electrical resistance (TEER) was measured after 0, 6, and 24 hours. After EGTA addition, the TEER of all cultures dropped below $0.2 \mathrm{k} \Omega / \mathrm{cm}^{2}$, indicating a complete loss of barrier function. After 6 hours, the cell control TEER increased to $3.29 \pm 0.83$, while it was significantly enhanced $(p<0.001)$ by a liquorice extract at $1000 \mu \mathrm{g} / \mathrm{ml}(5.35 \pm 0.96)$. After 24 hours, the cell control showed a TEER of $7.55 \pm 0.71$ and it was significantly enhanced $(p<0.05)$ by the liquorice extract at $1000 \mu \mathrm{g} / \mathrm{ml}(9.93 \pm 0.65)$, a herbal mix- 
ture at $80 \mu \mathrm{g} / \mathrm{ml}(8.66 \pm 0.27)$, and one of its constituents, angelica root at $80 \mu \mathrm{g} / \mathrm{ml}(8.74 \pm 0.31)$.

Glycyrrhizic acid, the sweet tasting principle of liquorice, and its metabolite glycyrrhetinic acid, did not influence the recovery of epithelial integrity when tested in corresponding concentrations as found in the liquorice extract.

Additional experiments on tight junction proteins are ongoing, in order to further elucidate the mode of action of phytogenic substances in this intestinal barrier model.

\section{Mo-Poster Session 1-PO-252 Allium sativum L. for prophylaxis of diarrhea in weaned piglets - how to find the right dosage?}

\author{
Authors Ayrle $\mathrm{H}^{1}$, Nathues $\mathrm{H}^{1}$, Mevissen $\mathrm{M}^{1}$, Walkenhorst $\mathrm{M}^{1}$ \\ Institute 1 Forschungsinstitut für biologischen Landbau (FiBL), Frick, \\ Switzerland
}

DOI 10.1055/s-0037-1608298

In piglets an infection with enterotoxigenic Escherichia coli strains leads to high economic losses as a result of a constant high morbidity and mortality. The incidence of neonatal diarrhea in piglets depends on concentration of antibodies in sow's colostrum. While piglets are protected by the antibodies in sow's milk, the predisposition for postweaning diarrhea increases with weaning. Additional factors to the immunological gap, including abrupt changes in diet, an increase in stomach $\mathrm{pH}$, and changes in the enzymatic and cellular configuration of the intestine lead to dysbiosis.

Several medicinal plant species are known for their antibacterial properties. Allium sativum L. (AS) exhibits antibacterial, antidiarrheal, anti-inflammatory and immunomodulatory effects, therefore it may be used for prophylaxis and treatment of diarrhea [1]. Based on a systematic review [1], 16 peer-reviewed references, 2 textbooks on veterinary phytotherapy, ESCOP and Commission E monographs were screened regarding scientific proven or recommended daily dosages of dry plant equivalent (DEV). Due to a lack of given data, for only 3 peer-reviewed references (pigs: 1; cattle: 1 ; poultry: 1 ) a dosage calculation was possible. To include data from studies with human and different animal species, the amount of used DEV was calculated in $\mathrm{g} / \mathrm{kg}$ metabolic body weight (MBW) per day (table 1). Daily dosages range from a minimum of $30 \mathrm{mg}$ to a maximum of $1052 \mathrm{mg}$ DEV AS/kg MBW (mean: $520.8 \mathrm{mg}$; median: $557 \mathrm{mg})$.

Tab. 1: Dosages of Allium sativum $\mathrm{L}$ in dry plant equivalent (DEV) in $\mathrm{mg} / \mathrm{kg}$ metabolic body weight (MBW; $\mathrm{kg}^{0,75}$ ) and re-converted for a $15 \mathrm{~kg}$ piglet

\begin{tabular}{|c|c|c|c|c|}
\hline \multirow[t]{2}{*}{ reference } & \multirow{2}{*}{$\begin{array}{l}\text { specification of the reference } \\
\text { (dr: dosage recommendation) }\end{array}$} & \multirow{2}{*}{$\begin{array}{l}\text { animal } \\
\text { species }\end{array}$} & \multicolumn{2}{|l|}{ daily dosage } \\
\hline & & & $\begin{array}{l}\text { per kg MBW } \\
\left(\mathrm{mgDEV} / \mathrm{kg}^{0,75}\right)\end{array}$ & $\begin{array}{l}\text { per } 15 \mathrm{~kg} \\
\text { piglet } \\
\text { (mg DEV) }\end{array}$ \\
\hline Kommission $\mathrm{E}$ & monograph (dr) & human & 30 & 239 \\
\hline Aichberger et al, 2012 & textbook (dr) & livestock & 120 & 900 \\
\hline ESCOP & monograph (lower $\mathrm{dr}$ ) & human & 247 & 1877 \\
\hline Grela et al, 2007 & clinical trial (lower dosage) & swine & 526 & 4000 \\
\hline Reichling et al, 2015 & textbook (lower $\mathrm{dr}$ ) & swine & 526 & 4000 \\
\hline ESCOP & monograph (upper dr) & human & 588 & 4470 \\
\hline Ghosh et al, 2010 & clinical trial & cattle & 664 & 5053 \\
\hline Reichling et al, 2015 & textbook (upper $\mathrm{dr}$ ) & swine & 789 & 6000 \\
\hline Hanieh et al, 2010 & clinical trial & poultry & 973 & 7400 \\
\hline Grela et al, 2007 & clinical trial (upper dosage) & swine & 1052 & 8000 \\
\hline
\end{tabular}

The authors gratefully thank the Swiss retailer Migros for funding this study. [1] Ayrle, H, Mevissen, M, Kaske, M, Nathues, H, Grützner, N, Melzig, M, Walkenhorst, M, BMC Veterinary Research 2016, 12:89; DOI 10.1186/ s12917-016-0714-8

\section{Mo-Poster Session 1-PO-253 Quantitative determination of Allicin in commercially available garlic-containing feedingstuff of dogs \& comparison with toxic doses}

Authors Hahn I , Fuhrmann I ${ }^{1}$, Zitterl-Eglseer $\mathrm{K}^{1}$

Institute 1 1 Institute for Animal Nutrition and Functional Plant Compounds, Department for Farm Animals and Veterinary Public Health, University of Veterinary Medicine Vienna, Veterinärplatz 1, Vienna, Austria

DOI 10.1055/s-0037-1608299

The use of six different, commercially available garlic containing feed materials and supplement feed of dogs were evaluated and discussed concerning possible benefits (against gastrointestinal parasites and ticks) and harmful effects. Quantitative analysis of Allicin content by means of HPLC (Tab. 1) showed quite good quality of three tested products ("Anibio" garlic powder, "PerNaturam" and "Lunderland" both garlic granules). Levels as high as in Ph. Eur. 8 required for garlic powder (Allii sativi bulbi pulvis: at least $0.45 \%$ Allicin in dried material for pharmaceutical use) were achieved - although these products are feedingstuff and not herbal remedies. Three other products contained a smaller amount of garlic and therefore also a lower level of Allicin.

Various data and recommendations from feed industry have been compared with recommended doses of veterinary practice (unfortunately scientific literature about dose rates is lacking). However, health hazards for dogs can be excluded in these dosages because toxic thresholds ( $5 \mathrm{~g}$ fresh garlic/kg body mass dog/day for seven days [1] - this is equal to $1.25 \mathrm{~g}$ dried garlic) are not reached in dogs.

Reported efficacy for prophylaxis and treatment of endoparasitosis and tick infestation with garlic powder in folk medicine is not scientifically proven invivo in dogs and could not be confirmed so far - further studies are required.

Tab. 1: Content of garlic (Allium sativum) and Allicin in different feedingstuff of dogs, including fresh garlic and garlic spice granulate (HPLC, triple determination).

\begin{tabular}{l|l|l}
\hline Product & $\begin{array}{l}\text { Content } \\
\text { Allium sativum }\end{array}$ & Mean value Allicin in \% \\
\hline Anibio Pulver & $100 \%$ & 0.55 \\
\hline PerNaturam Granulat & $100 \%$ & 0.52 \\
\hline Lunderland Granulat & $100 \%$ & 0.46 \\
\hline Grau Tabletten & $95 \%$ & 0.28 \\
\hline Canina Pulver & $13 \%$ & 0.06 \\
\hline Trixie Granulat & $5 \%$ & $\begin{array}{l}\text { determination influenced by adjuvants, } \\
\text { exact specification not possible }\end{array}$ \\
\hline Garlic fresh & $100 \%$ & 0.52 \\
\hline Garlic spice granulate & $100 \%$ & 0.66
\end{tabular}

\section{Mo-Poster Session 1-PO-254 A phytogenic uterus instillation shows a high potential to replace routine antibiotic uterus flush after artificial insemination in horses}

Authors Ryhner $\mathrm{T}^{1}$, Graf-Schiller $\mathrm{S}^{3}$, Mayer $\mathrm{P}^{3}$, Ammer $\mathrm{S}^{2}$, Walkenhorst $\mathrm{M}^{2}$ Institute 1 Nationales Pferdezentrum, Bern, Switzerland;

2 Forschungsinstitut für biologischen Landbau (FiBL), Frick, Switzerland;

3 SaluVet GmbH, Bad Waldsee, Germany

DOI 10.1055/s-0037-1608300

Artificial insemination (Al) is conducted in more than half of modern sport breeding mares in several European countries today. Al is still of rising importance. Several factors including bacterial infection can cause a persistent breeding-induced endometritis [1]. Antibiotic uterus flush (ABUF) is a common post-insemination routine to avoid this important disease complex [1]. In order to find a suitable alternative, one of the three largest Swiss Centers for Al in horses changed the routine 2012 from ABUF to an uterus instillation with a registered veterinary drug solely based on plant extracts from Eucalyptus globulus Labill, Melissa officinalis L, Calendula officinalis L. and Origanum majorana L. (PEUI; EucaComp PlantaVet). 
In total $1389 \mathrm{Al}$ and 624 early pregnancy diagnoses (ePD) were recorded from 2010 to 2014. Additionally the post-insemination treatment such as ABUF $(N=517)$, PEUI $(N=538)$ or no treatment $(N=334)$ were taken into account. In the data analysis with SAS 9.3 a chi-square test was used to compare the frequency distributions of the different treatments between the studied years. The level of significance was defined as $p<0.05$. The percentage of positive from all ePD remains stable around $50 \%$ without significant differences between the years. The post-insemination ABUF decreases significantly from more than $70 \%$ (2010 and 2011) to less than $10 \%$ (2012 - 2014) (figure 1), while the number of therapeutic antibiotic uterus flushes given independent from Al remain stable(2010: 50, 2011: 30, 2012: 9, 2013: 27, 2014: 10).

Well-known antimicrobial, anti-inflammatory and immunomodulatory effects of the medicinal plants used in PEUI show a potential to recover the uterus before the conceptus reaches it. Even if the meaningfulness of post Al treatment in horses is controversial discussed [1], PEUI might be an interesting alternative to ABUF.

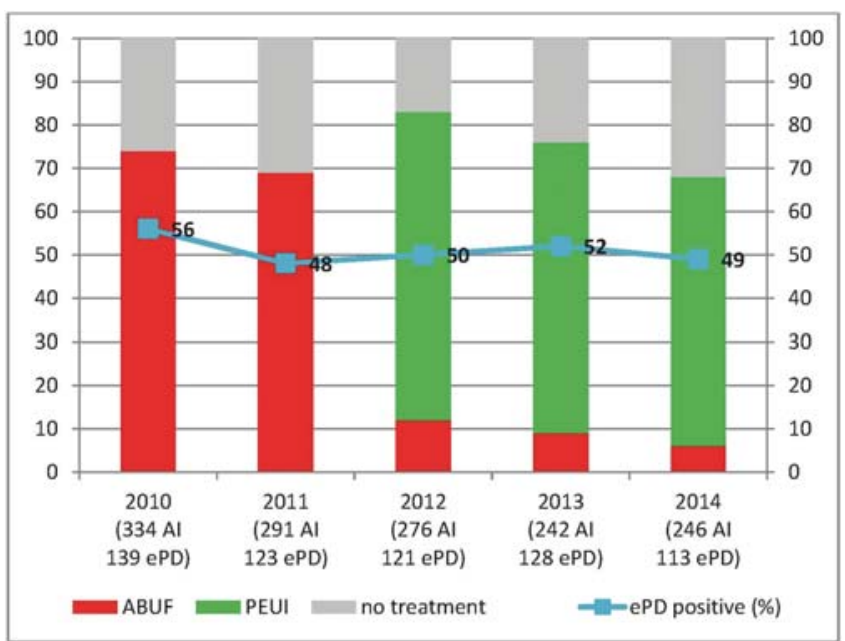

Fig. 1: Development of the outcome of early pregnancy diagnoses (ePD) after artificial insemination (Al) in mares in the largest Swiss centre for Al during a change in post-insemination treatment routine from antibiotic Uterus flush (ABUF) to an Uterus Instillation with a registered veterinary drug solely based on plant extracts (PEUI)

[1] Woodward, E.M. and Troedsson, M.H, Journal of Equine Veterinary Science 2013, 33; $673-682$.

\section{Lecture Session - ESA Silver Award Lecture} Tuesday, 5th September, 2017

Montreal - 09:00 - 09:45

\section{Tu-Egon Stahl Silver Award Lecture Ecdysteroids: from insect molting hormones to potent chemo-sensitizers in cancer}

Authors Hunyadi $A^{1}$

Institute 1 Institute of Pharmacognosy, University of Szeged, Szeged,

Hungary; Interdisciplinary Centre for Natural Products, University of Szeged,

Szeged, Hungary

DOI 10.1055/s-0037-1608301

Phytoecdysteroids are herbal analogs of the insect molting hormone and play a highly complex ecological role among different Kingdoms of Nature [1]. In mammals, beneficial bioactivities have been attributed to these compounds. Non-hormonal anabolic and adaptogenic effects are among such bioactivities, and these properties led to the production and worldwide marketing of ecdysteroid-containing plant extracts as food supplements [2].
In our recent studies on commercial extracts of Cyanotis arachnoidea, a particularly rich source of structurally diverse natural ecdysteroids, several compounds could be isolated in sufficient amounts to permit various semi-synthetic transformations.

We discovered that certain ecdysteroids can strongly sensitize multi-drug resistant (MDR) and susceptible cancer cell lines to chemotherapeutics [3]. Based on these results, a wide variety of chemical modifications of selected ecdysteroids have been performed in order to further extend and explore the available chemical space. Such modifications included base-catalyzed autoxidation, oxidative side-chain cleavage, oxime, oxime-ether and lactame formation, fluorination, and preparation of dioxolane rings. SAR for more than 120 compounds revealed that a C-20,22 side-chain cleavage together with introducing a 2,3-dioxolane moiety confers ecdysteroids a strong chemo-sensitizing activity against MDR cancer cell lines, without any detectable functional inhibition of drug efflux [4], which could lead to various side effects during possible in vivo applications. Based on our results, several promising lead compounds have been identified that can be further developed to be used in the treatment of MDR cancer.

Support from the NKFIH, Hungary (K119770), EFOP-3.6.1-16-201600008, GINOP-2.3.2-15-2016-00012, the János Bolyai fellowship of the Hungarian Academy of Sciences, and the Kálmán Szász Prize are acknowledged.

[1] Hornok et al. Sci. Rep. 2016, 6: 23390

[2] Hunyadi et al. Sci. Rep. 2016, 6: 37322

[3] Martins et al. J. Med. Chem. 2012, 55: $5034-43$

[4] Hunyadi et al. Molecules 2017, 22: 199

Lecture Session - Phytopharmacology/

Extract Pharmacology I

Tuesday, 5th September, 2017

Montreal - 11:00-12:301

\section{Tu-Phytopharmacology/Extract Pharmacology II-SL-01 Activities and Prevalence of Proteobacteria Members Colonizing Echinacea purpurea Fully Account for Macrophage Activation Exhibited by Extracts of This Botanical}

Authors Pasco $\mathrm{D}^{1,2}$, Pugh $\mathrm{ND}^{1}$, Haron $\mathrm{MH}^{1}$, Jackson $\mathrm{CR}^{3}$, Tyler $\mathrm{HL}^{4}$, Maddox $\mathrm{VR}^{5}$, Moraes $\mathrm{RM}^{1}$

Institute 1 National Center for Natural Products Research, University, United States; 2 Department of BioMolecular Sciences, Research Institute of Pharmaceutical Sciences, School of Pharmacy, University, United States; 3 Department of Biology, The University of Mississippi, University, United States; 4 Crop Production Systems Research Unit, USDA Agricultural Research Service, University, United States; 5 Department of Plant and Soil Sciences, Mississippi State University, Starkville, United States

DOI 10.1055/s-0037-1608302

Our previous work identified a fraction from Echinacea that potently activated macrophages in vitro through a Toll-like receptor 2-dependent mechanism. The active component was later identified as bacterial Braun-type lipoproteins. This agent together with bacterial LPS were responsible for over $97 \%$ of the macrophage activation potential exhibited by extracts of Echinacea and other immune enhancing botanicals. Subsequent studies indicated that $\sim 50 \%$ of the variation in in vitro macrophage stimulating activity exhibited by $E$. purpurea extracts could be accounted for by differences in total bacterial load within the plant material. We then tested the hypothesis that the type of bacteria, in addition to bacterial load, was necessary to fully account for extract activity. Bacterial community composition within commercial and freshly harvested (wild and cultivated) E. purpurea aerial samples was determined using high-throughput $16 \mathrm{~S}$ rRNA gene pyrosequencing. Bacterial isolates representing 38 different taxa identified to be present within E. purpurea 
were acquired, and the activity exhibited by the extracts of these isolates varied by over 8,000 -fold. Members of the Proteobacteria exhibited the highest potency for in vitro macrophage activation and were the most predominant taxa. Furthermore, the mean activity exhibited by the Echinacea extracts could be solely accounted for by the activities and prevalence of Proteobacteria members comprising the plant-associated bacterial community. The efficacy of $E$. purpurea material to treat respiratory infections may be partially determined by the Proteobacterial community composition of this plant, since ingestion of bacteria (probiotics) and other extracts containing bacterial components is reported to have a protective effect against this health condition. This research was partially funded by grants from the National Institutes of Health R21AT01207, R01AT002360 and R01AT007042 (NCAAM) to DSP and by the USDA, Agricultural Research Service Specific Cooperative Agreement No. 58 - 6408-7-012.

Winner of the Planta Medica Best Paper Award.

\section{Tu-Phytopharmacology/Extract Pharmacology II-SL-02 Organic food consumption during pregnancy is associated with different food intake and health-related characteristics: the KOALA Birth Cohort Study}

Authors Simões-Wüst APS $^{1,2}$, Moltó-Puigmartí $C^{3}$, Jansen $E^{4}$, van Dongen $\mathrm{M}^{3}$, Dagnelie $\mathrm{P}^{3,5}$, Thijs $\mathrm{C}^{3}$

Institute 1 Department of Obstetrics, University Hospital Zurich, Zurich,

Switzerland; 2 Research Department, Clinic Arlesheim, Arlesheim,

Switzerland; 3 Maastricht University, Department of Epidemiology, CAPHRI

School for Public Health and Primary Care, Maastricht, Netherlands; 4 Centre for Health Protection, National Institute for Public Health and the Environment, Bilthoven, Netherlands; 5 Maastricht University, CARIM School for

Cardiovascular Disease, Maastricht, Netherlands

DOI 10.1055/s-0037-1608303

To find out how the consumption of organic food during pregnancy is associated with macro- and micronutrient intakes and levels of several maternal blood biomarkers.

Pregnant women were recruited at midwives' practices and through channels related to consumption of food from organic origin. For this cross-sectional description, participants who filled in questionnaires on food frequency in gestational week $34(n=2786)$ were considered; thereof 1339 donated a blood sample and could be considered in the biomarkers analysis.

Compared with participants consuming no organic food (reference group), intake of retinol, carotene, tocopherol and folate was higher among participants consuming organic food (organic groups), whereas intake of vitamin D and $\mathrm{B}_{12}$ was lower. Furthermore, intake of trans fatty acids from natural origin (vaccenic acid and conjugated linoleic acids, CLA) was higher in the organic groups than in the reference group, whereas intake of elaidic acid, a marker of the intake of trans fatty acids found in industrially hydrogenated fats, was lower. Comparable differences were seen when the plasma biomarkers were investigated in the subgroup of participants who donated a blood sample. Here, a marker of dairy products intake (pentadecanoic acid) as well as vaccenic and rumenic acid (the major CLA) were higher in the organic groups, and elaidic acid was lower. Levels of homocysteine and 25-hydroxyvitamin D were lower in the organic groups than in the reference group. Most differences were seen even in groups with low consumption of organic food. Various types of food intake are associated with the consumption of organic food during pregnancy and are in line with different levels of plasma biomarkers. Food patterns accompanying the consumption of organic food are likely to play a major role in the observed associations.

\section{Tu-Phytopharmacology/Extract Pharmacology II-SL-03 Drug discovery approaches against Madurella mycetomatis - one of the most neglected tropical diseases (NTDs)}

Authors Khalid $S^{1}$ Institute 11 Faculty of Pharmacy, University of Science and Technology, P. O. Box 477 Omdurman, Sudan. 2 Faculty of Pharmacy, University of Khartoum, P. O. Box 1996, Khartoum, Sudan, Khartoum, Sudan

DOI 10.1055/s-0037-1608304

Madurella mycetomatis is a severe eumycotic infection caused by a stubborn fungus. It is characterized with uniques clinical manifestation involving the skin and subcutaneous tissue. The infection can keep spreading until it ereaches the bones and distant sites through the blood and lymph which eventually leads to destruction, deformity and loss of function [1]. Due to the increadsing burden of $M$. mycetomatis and the multiple gaps in our knowledge about this disease, the $69^{\text {th }}$ World Health Assembly (WHA) adopted an important resolution in May 2016 adding mycetoma to the list of NTDs and calling on al actors to join forces to control the public-health impact of this disfiguring and life threatening disease.

Prolonged treatment of $M$. mycetomatis with almost all currently available antifungal agents proved to be of disappointing clinical efficacy which usually associated with severe side effects and frequently results in amputation [2]. This warranted us to collect a dozen of clinical strains M. mycetomati from Sudanese patients followed by culturing and profiling of their susceptibility against eight known antifungal agents representing five azoles (ketoconazole, itraconazole, voriconazole, posaconazole, fluconazole) besides an allylamine (terbinafine), a polyene macrolide (amphotericin B), and a lipopeptide echinocandin (caspofungin).

The validity of our inhouse established in vitro screening method [3], was further consolidated by subjecting a series of plant secondary metabolites including, terpenoids, polyphenols and alkaloids. Among the later class, the protoberberine (e.g. berberine), benzophenanthridine (e.g sanguinarine), and $\beta$-carboline (e.g. harmine) alkaloids exhibited appreciable activities. Sanguinarine exhibited a remarkable in vitro activity with MIC 50 and MIC 90 of 1 and 4 , respectively.

[1] Fahal AH, 1992, British Journal Surgery, 79, 1138 - 1141.

[2] Abbott PH, Transactions of the Royal Society of Tropical Medicine and Hygiene. 1956; 50: $11-24$.

[3] Khalid SA, 2014, ResNet NPND workshop, Nov. 25-28th, 2014, Rio de Janeiro, Brazil.

\section{Tu-Phytopharmacology/Extract Pharmacology II-SL-04 Effects of Gypenosides on memory deficits in an MPTP-lesioned mouse model of Parkinson's disease treated with L-DOPA}

Authors Park Hyun $]^{1}$, Kim Kyung $S^{1}$, Zhao Ting $T^{1}$, Lee Kyung $E^{1}$, Lee Myung $\mathrm{K}^{1}$

Institute 1 College of Pharmacy, Chungbuk National University, Cheongju-si, Korea, Republic of (South)

DOI 10.1055/s-0037-1608305

Gynostemma pentaphyllum (GP) mainly contains approximately 90 dammarane-type triterperpene glycosides, which are named by gypenosides (GPS). This study investigated the effects of ethanol extract from GP (GP-EX) and GPS on memory deficits in 1-methyl-4-phenyl-1,2,3,6-tetrahydropyridine (MPTP)-lesioned mouse model of Parkinson's disease (MPTP-lesioned mice). MPTP (30 mg/kg/day, 5 days)-lesioned mice showed deficits of habit learning memory and spatial memory, which were further aggravated by treatment with L-3,4-dihydroxyphenylalanine (L-DOPA) $(25 \mathrm{mg} / \mathrm{kg}, 21$ days). However, treatment with GP-EX (50 mg/kg, 21 days) or gypenosides (GPS) $(50 \mathrm{mg} / \mathrm{kg}$, 21 days) ameliorated memory deficits in MPTP-lesioned mice treated with L- 
DOPA (25 mg/kg): GP-EX and GPS improved decreases in retention latency time of passive avoidance test and tyrosine hydroxylase-immunopositive cells and dopamine levels in the nigro striatum. GP-EX and GPS also reduced increases in retention transfer latency time of elevated plus-maze test and expression of $\mathrm{N}$-methyl-D-aspartate (NMDA) receptor, and improved decreases in phosphorylation of extracellular signal-regulated kinase (ERK1/2) and cyclic AMP-response element binding protein (CREB) in the hippocampus in the same models. These results suggest that GP-EX and GPS ameliorate habit learning memory deficits by activating dopaminergic neurons and spatial memory deficits by modulating NMDA receptor-ERK1/2-CREB system in MPTP-lesioned mice treated with L-DOPA. GP-EX and GPS may serve as an adjuvant phytonutrient for memory deficits in Parkinson's disease.

\section{Tu-Phytopharmacology/Extract Pharmacology II-SL-05 Baccharis dracunculifolia decreases nociception, depressive-like behaviour and supraspinal activated microglia in rats with experimental monoarthritis}

Authors Laranjeira ${ }^{1}$, Apolinário $E^{1}$, Amorim $D^{2,3}$, Silva-Filho $A^{4}$, PintoRibeiro $\mathrm{F}^{2,3}$, Dias $\mathrm{A}^{1}$

Institute 1 CITAB-Centre for the Research and Technology of Agro-

Environmental and Biological Sciences, Department of Biology, University of Minho, Campus de Gualtar, 4710 - 057 Braga, Portugal, Braga, Portugal; 2 Life and Health Sciences Research Institute (ICVS), School of Medicine, Campus de Gualtar, University of Minho, 4710 - 057 Braga, Portugal, Braga, Portugal;

3 ICVS/3B's - PT Government Associate Laboratory, Braga/Guimarães,

Portugal, Braga, Portugal; 4 Faculdade de Farmácia e Bioquímica,

Departamento Farmacêutico, Universidade Federal de Juiz de Fora, Juiz de

Fora, MG - Brasil, Braga, Portugal

DOI 10.1055/s-0037-1608306

In arthritic disorders both inflammation and the progressive degeneration of joints persistently activate nociceptors, in periarticular structures, leading to the development of persistent pain and comorbid emotional impairments. Arthritis-induced peripheral sensitization leads to increased release of nociceptive molecules by primary afferents that activate neurones e glial cells in the spinal cord and supraspinal pain modulatory areas such as the amygdala (AMY) and the periaqueductal grey matter (PAG).

Baccharis dracunculifolia DC (Asteraceae) (Bd) is a medicinal shrub from the brazilian flora, popularly known as "Alecrim do Campo", considered to be an important source of active anti-inflammatory and antinociceptive compounds. Adult 8 weeks old ovariectomized female rats (Rattus norvegicus, vr. Albinus, Wistar) weighting $210 \pm 17 \mathrm{~g}$ were divided in four groups ( $\mathrm{n}=6$ per group): (i) SHAM, (ii) ARTH, (iii) ARTH treated with B. dracunculifolia $(50 \mathrm{mg} / \mathrm{kg}$ ), and (iv) ARTH treated with $B$. dracunculifolia $(100 \mathrm{mg} / \mathrm{kg})$.

Mechanical hyperalgesia in ARTH animals was assessed using the pressure application measurement apparatus, anhedonia using the sucrose preference test and learned helplessness using the forced swimming test. Activated microglia was stained with IBA-I and quantified in a subset of brain slides containing the target areas, the amygdala and the periaqueductal gray matter. A three-week oral treatment with Bd extract reversed ARTH-induced mechanical hyperalgesia and partly reserved depressive-like behaviour. Concomitantly, Bd treatment decreased the number of activated microglia in the AMY and PAG of ARTH animals.

This study was supported by grants from INTERACT project - "Integrative Research in Environment, Agro-Chains and Technology", no. NORTE-010145-FEDER-000017 and the Portuguese North Regional Operational Program (ON.2 - O Novo Norte) under the National Strategic Reference Framework (QREN), through the European Regional Development Fund (FEDER), in its line of research entitled ISAC.

\section{Tu-Phytopharmacology/Extract Pharmacology II-SL-06 Beneficial effects of black bean (Rhynchosia volubilis) on ocular surface damage induced by benzalkonium chloride in mouse model of dry eye}

Authors Kim Kyung $A^{1,2}$, Kang Suk $W^{1,3}$, Lee Chung $\mathrm{H}^{4}$, Yang Sung $\rfloor^{4,5}$, Kang Tae $K^{1,7}$, Kim TJ ${ }^{6}$, Jung Sang $H^{1,2}$

Institute 1 Natural Products Research Center, Korea Institute of Science and Technology (KIST), Gangneung, Korea, Republic of (South); 2 Division of BioMedical Science \&Technology, KIST School, Korea University of Science and Technology, Seoul 02792, Republic of Korea, Seoul, Korea, Republic of (South); 3 Research School of Chemistry, Australian National University, Canberra,

Australia; 4 Department of Ophthalmology, University of Ulsan, Gangneung Asan Hospital, Gangneung, Korea, Republic of (South); 5 Department of Ophthalmology, Oregon Health \& Science University, Portland, United States; 6 Department of Biological Sciences, Pusan National University, Busan, Korea, Republic of (South); 7 Department of Marine bio-technology, GangnungWonju National University, Gangneung, Korea, Republic of (South) DOI 10.1055/s-0037-1608307

We investigated the beneficial effect of standardized ethanol extracts of Rhynchosia volubilis (EERV) on an animal model of dry eye (DE). Experimental DE was induced by treatment of $0.2 \%$ benzalkonium chlroride (BAC) on cornea in mice [1]. Various concentrations of EERV were daily administered, orally, for 14 after BAC treatment. Treatment of EERV significantly reduced fluorescein staining and increased tear break-up time (BUT). Moreover, treatment of EERV improved morphologically corneal surface regularity compared to DE group. Squamous metaplasia and apoptosis in the cornea were inhibited by treatment of EERV. In addition, treatment of EERV down-regulated the expression of Bax and cytochrome $\mathrm{c}$, while that of $\mathrm{BCl}-2$ was up-regulated against $\mathrm{DE}$. These results demonstrate that EERV has protective effects against BAC-induced corneal damage, and could be as a beneficial agent for treatment of dry eye.

\section{Experimental Dry eye model}

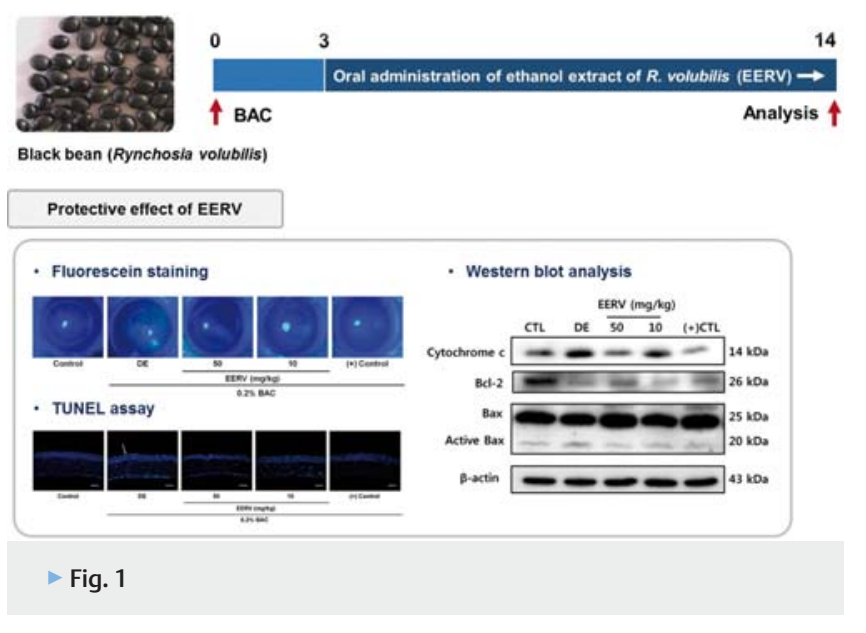

[1] Zhang, Z. et al. Invest. Ophthalmol. Vis. Sci. 2014; 5: $2963-2974$ 
Lecture Session - Biosynthesis \& Sustainable Use \& YRW Award Lectures Tuesday, 5th September, 2017

Singapore $-11: 00-12: 30$

\section{Tu-Biosynthesis \& Sustainable Use \& YRW Award Lectures-SL-01 Successive xanthone prenylation in Hypericum sampsonii}

Authors Nagia $\mathrm{M}^{1,2}$, Gaid $\mathrm{M}^{1,2}$, Beuerle $\mathrm{T}^{1}$, Beerhues $\mathrm{L}^{1,2}$ Institute 1 Institute of Pharmaceutical Biology, Technische Universität Braunschweig, Mendelssohnstraße 1, Braunschweig, Germany; 2 Center of Pharmaceutical Engineering (PVZ), Technische Universität Braunschweig, Franz-Liszt-Straße 35 A, Braunschweig, Germany DOI 10.1055/s-0037-1608308

Hypericum species exhibit affluence of bioactive polyprenylated metabolites derived from various chemical nuclei including acyl- and benzoylphloroglucinol, flavonoid and xanthone ${ }^{[1]}$. Prenylated xanthones receive special attention due to their biological impact on human health. Their biosynthesis is achieved through two stages, (i) formation of the xanthone nucleus, (ii) decoration by side chains. Aromatic prenyltransferases catalyse the introduction of prenyl groups to an electron-rich aromatic substrate. Metabolic profiling of Hypericum sampsonii, whose extracts are used in TCM to treat swellings, burns, backache and in Taiwan as antitumor drug, showed abundance of pharmacologically active prenylated xanthones. Patulone inhibits the exogenous platelet activating factor, induces hypotension in mice, and inhibits COX-1 enzyme ${ }^{[2]}$. Hypericumxanthones ${ }^{[3]}$ and Hyperixanthone $A^{[4]}$ are antibacterial metabolites from $H$. sampsonii.

This work presents the complete elucidation of the biosynthetic pathway of patulone. Two prenyltransferases from $\mathrm{H}$. sampsonii, which are responsible for the decoration of 1,3,6,7-tetrahydroxyxanthone to produce patulone, were studied at the gene level. Constructs of PECS-URA harbouring the individual genes were created for yeast expression. Activity screening of the recombinant proteins against potential substrates from different chemical classes revealed their narrow substrate specificity, the strict regiospecificity to C-8 of the 1,3,6,7-tetrahydroxyxanthone skeleton and absolute dependence on $\mathrm{Mg}^{2+}$ ions. The sequential prenylation of 1,3,6,7-tetrahydroxyxanthone was proved through determination of the kinetic parameters. The product of HsPT8PX is used as substrate for HsPTpat to form the gem-diprenylated patulone. When fused to YFP, both enzymes showed subcellular localization to chloroplasts. Our results inaugurate a novel chemo-enzymatic production approach for pharmacologically important trace natural products.
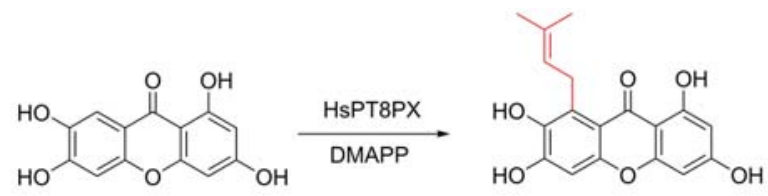

1,3,6,7-trihydroxyxanthone 8-prenyl-1,3,6,7-trihydroxyxanthone

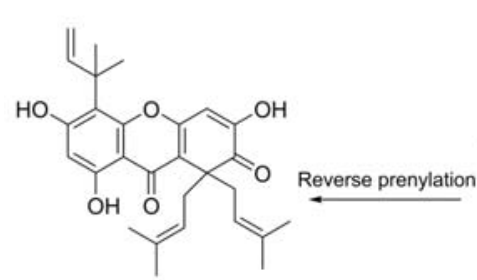
Hyperixanthone A

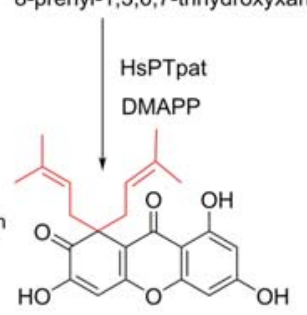

Patulone

Fig. 1: Successive xanthone prenylation in Hypericum sampsonii

[1] Avato, P. A Survey on the Hypericum Genus. Elsevier; 2005: 603 - 634.

[2] Yamakuni, T, et al, Neurosci. Lett, 2006; 394: $206-210$.
[3] Xin, W.-B, et al, Phytotherapy Res, 2011; 25: 536 - 539.

[4] Xiao, Z.Y, et al, Pharmaceut. Biol, 2008; 46: 250 - 253.

\section{Tu-Biosynthesis \& Sustainable Use \& YRW Award Lectures-SL-02 Generation of novel bioactive "unnatural" natural products through biotransformation by the enzymatic secretome of Botrytis cinerea}

Authors Gindro $\mathrm{K}^{1}$, Schnee $\mathrm{S}^{1}$, Righi Davide $\mathrm{R}^{2}$, Marcourt Laurence $\mathrm{M}^{2}$, Nejad Ebrahimi Samad Nejad $E^{3}$, Massana Codina Josep Massana $C^{1}$, Voinesco Francine $V^{1}$, Michellod Emilie $\mathrm{M}^{1}$, Wolfender $\mathrm{JL}^{2}$, Ferreira Queiroz $\mathrm{E}^{2}$ Institute 1 Agroscope, Institute for Plant Production Sciences IPS, Route de Duillier 50, P.O. Box 1012, 1260 Nyon, Switzerland, Nyon, Switzerland;

2 School of Pharmaceutical Sciences, EPGL, University of Geneva, University of Lausanne, CMU, 1, Rue Michel Servet, 1211 Geneva 4, Switzerland, Geneva, Switzerland; 3 Department of Phytochemistry, Medicinal Plants and Drugs Research Institute, Shahid Beheshti University, G. C, Evin, Tehran, Iran, Tehran, Iran

DOI 10.1055/s-0037-1608309

Biotransformation can be defined as the use of an intact whole organism or an isolated enzyme system to induce chemical modifications in organic compounds. Biotransformation has a number of advantages when compared to classical organic chemistry. The reactions can occur in mild conditions, near neutral $\mathrm{pH}$, ambient temperatures, and atmospheric pressure, and protection of certain functional groups is often not necessary. For performing biotransformation of NPs, or NP extracts, the protein secretome from fungi could be considered as a promising tool. Fungi are indeed of interest since they synthetized diverse and complementary extracellular proteins to permit host penetration and further successful colonization. This group of secreted proteins released in the extracellular space is defined as the secretome and constitutes the pathogenicity factors in the host-pathogen interaction mechanisms. ${ }^{1}$ In the present work, the protein secretome of Botrytis cinerea was used to perform the biotransformation of resveratrol, pterostilbene, and a mixture of both. The reactions were optimised with minimal amounts of NPs for monitoring at the analytical scale. Metabolite profiling by UHPLC-HRMS revealed the presence of compounds with unusual molecular formula, suggesting the existence of new products. The reactions were scaled-up and twenty-one analogues were isolated and fully characterized by NMR and HRESIMS analyses. The reaction with pterostilbene afforded five new compounds, while the reaction with a mixture of pterostilbene and resveratrol afforded seven unusual stilbene dimers. The antifungal properties of these compounds were evaluated using in vitro bioassays against Plasmopara viticola. Some of the isolated compounds present a strong antifungal activity showing that an inactive molecule, such as resveratrol, can be modified into more toxic compounds after enzymatic biotransformation. Our results suggest that biotransformation by usage of crude fungal secretomes represents an efficient way to create chemodiversity with enhanced biological activity.

[1] Gonzalez-Fernandez, R. et al V. Front Plant Sci 2015, 6, 839.

\section{Tu-Biosynthesis \& Sustainable Use \& YRW Award Lectures-SL-03 Biotransformation of diterpenes from Copaifera sp. oleoresins using filamentous fungi}

Authors Sousa IP ${ }^{1}$, Chellegatti MASC ${ }^{1}$, Veneziani RCS ${ }^{2}$, Ambrósio SR ${ }^{2}$, Furtado $\mathrm{NAJC}^{1}$

Institute 1 University of São Paulo, School of Pharmaceutical Sciences of Ribeirão Preto - Av. do Café, s/n, 14040 - 903, Ribeirão Preto, Brazil;

2 University of Franca, Nucleus of Research in Sciences and Technology - Rua Dr. Armando Salles de Oliveira, 201, 14404 - 600, Franca, Brazil DOI $10.1055 / \mathrm{s}-0037-1608310$ 
The labdane diterpenes ent-polyalthic (PA) and ent-dihydroagathic (DA) acids are secondary metabolites found in several plants including the trees of $\mathrm{CO}_{0}$ paifera sp. (Fabaceae-Caesalpinioideae), from which is extracted an active oleoresin rich in diterpenes [1]. Although these oleoresins are widely used in folk medicine [2], little is known about their metabolism. Biotransformation studies are an important approach to understand in vivo transformations of bioactive compounds [3]. The metabolism of two major constituents of Copaifera duckei oleoresin was investigated using filamentous fungi. An amount of $120 \mathrm{mg}$ of PA and $150 \mathrm{mg}$ of DA were incubated with an initial inoculum of $1 \times 10^{6}$ spores $/ \mathrm{mL}$ of Aspergillus brasiliensis and Cunninghamella echinulata, respectively. The biotransformation processes were carried out in flasks containing Koch's $\mathrm{K} 1$ medium, at $30^{\circ} \mathrm{C}$ and $120 \mathrm{rpm}$ for five days. After the incubation period, the culture broth was filtered and partitioned with ethyl acetate to obtain the crude extracts ( $161 \mathrm{mg}$ and $244 \mathrm{mg}$ of PA and DA biotransformation's extracts, respectively). The biotransformation metabolites were isolated by chromatographic procedures and identified using UHPLC-ESI-MS with Orbitrap mass analyzer and NMR. Both fungi promoted hydroxylation reactions in different positions of the labdane nucleus. These results can contribute to the understanding of the metabolism of Copaifera sp. medicinal oleoresins. Capes, FAPESP (grants 2016/25201 - 7 and 2011/13630 - 7) and CNPq.

[1] Borges CHG. et al. Chem Biodivers 2016; 13: $1348-1356$.

[2] Leandro LM. et al. Molecules 2012; 17: $3866-3889$.

[3] Paludo CR. et al. Eur J Drug Metab Pharmacokinet 2017;42: 213 - 220.

\section{Tu-Biosynthesis \& Sustainable Use \& YRW Award Lectures-SL-04 The controlled cultivation of Cannabis sativa at VitaPlant}

\section{Authors Chaanin $\mathrm{A}^{\top}$}

Institute 1 VitaPlant AG, Uttwil, Switzerland

DOI 10.1055/s-0037-1608311

Cannabis sativa belongs -behind alcohol- to the world's most commonly used drugs. On the other hand positive effects in the therapeutic use of cannabis preparations were recorded.

The law in Switzerland bans the cultivation and consumption as well as the trade of cannabis with a total content of THC (tetrahydrocannabinol) of over $1 \%$. Cannabis with THC levels above this level are considered as narcotics and the use is subject to a specific authorization requirement.

VitaPlant has an operating license from the canton of Thurgau and is required to apply for a special exemption from the Swiss Federal Office of Public Health (BAG) for every third party production. Research on cannabis has a long tradition at VitaPlant and several clones have been developed with specific active ingredients over the years. A main focus in the development of Cannabis at VitaPlant is the two cannabinoids: $\triangle 9$-THC ( $\Delta 9$-tetrahydrocannabinol) and CBD (cannabidiol).

Currently, VitaPlant is working on several projects for Swiss companies with respect to the production of cannabis in various grades. It is primarily concerned with the composition of the cannabinoids and the ratio of THC and $\mathrm{CBD}$, but also with various kinds of harvesting. Two different forms of harvesting are used in the preparation of the drug: flowering branches (i.e. herba) or as pure flower (flos) which has higher content of active compound (THC or (BD) in comparison to the harvest of flowering branches. In all cases the female flower is the main product. Depending on the harvested part the amount of THC and CBD differs considerably. However, the compounds of THC and THC-A or CBD and CBD-A are not stable. The time of harvesting and the maturity of flowers as well as the drying and processing of the raw material have an effect on the amount of both compounds.

\author{
Lecture Session - Veterinary \\ Phytomedicine \& Animal Health \\ Tuesday, 5th September, 2017 \\ Osaka/Samarkand - 11:00-12:30
}

\section{Tu-Veterinary Phytomedicine \& Animal Health-SL-01 Anthelmintic effect and intake dynamics of oak (quercus spp.) and walnut (juglans regia) foliage in goats}

Authors Heckendorn $\mathrm{F}^{1}$, Lèbre $\mathrm{A}^{1}$, Destailleur $\mathrm{V}^{2}$, Bouy $\mathrm{M}^{3}$, Spiegler $\mathrm{V}^{4}$, Lehmann $\mathrm{S}^{4}$, Hensel $\mathrm{A}^{4}$

Institute 1 Antenne FiBL France, Divajeu, France; 2 EPLEFPA Carmejane, Le Chaffaut St Jurson, France; 3 Cabinet Vétérinaire ANTIKOR, Barbières, France; 4 University of Münster, Münster, Germany

DOI 10.1055/s-0037-1608312

Oak and Walnut are both of ethnoveterinary importance, especially for anthelmintic treatment of ruminants [1,2]. $\mathrm{H} 2 \mathrm{O}$ and EtOH-water (1:1) extracts of oak and walnut foliage were used for phytochemical profiling and for in vitro assays with Caenorhabditis elegans and gastrointestinal nematodes (GIN). Oak extracts contained mainly tannins, walnut extracts were dominated by flavonoids. Oak EtOH extract caused significant lethal effects in adult $\mathrm{C}$. elegans (LC50 $1.5 \mathrm{mg} / \mathrm{mL}$ ); motility of GIN L3 larvae was reduced by the EtOH extract but at substantially higher concentrations. Egg hatching was inhibited by both, the oak $\mathrm{H} 2 \mathrm{O}$ and EtOH extract. In vivo: 42 lactating Alpine goats carrying natural GIN infections were allocated to 3 feeding groups: oak, walnut and control. Freshly harvested branches with foliage were offered to goats of the respective groups twice a day for a total of 17 days. Individual foliage intake and fecal egg counts (FEC) was performed every $2-3$ days. Oak and walnut foliage feeding did not lead to a significant reduction of FEC. Time sampling data revealed a substantial variability in leaf intake between goats for both, oak and walnut, which however, did not coincide with the hypothesis that goats with heavy GIN infections consume more leaf material than animals with light infections. Summarizing these results suggests an anthelmintic activity of oak due to the presence of tannins which points to a preventive effect by limiting development of pasture dwelling GIN parasitic stages. Adult GIN within the host do not seem to be affected with the quantities used in the study. Abu Taha M (2011). African Journal of Microbiology Research 5, 5796 - 5805 Paolini V (2004) Parasitology, 129, 69- 77

\section{Tu-Veterinary Phytomedicine \& Animal Health-SL-02 Spontaneous intake of essential oils during the early rearing phase of chicks and long-term effects on performance and transcriptome regulation}

Authors Guilloteau $L^{1}$, Anne $C^{1}$, Foury $A^{2}$, Helbling $J C^{2}$, Koch $A^{1}$, Crochet $S^{1}$, Cailleau-Audouin $\mathrm{E}^{1}$, Constantin $\mathrm{P}^{3}$, Lagarrigue $\mathrm{S}^{4}$, Désert $\mathrm{C}^{4}$, Mercerand $\mathrm{F}^{5}$, Rat $\mathrm{C}^{5}$, Delaveau $\mathrm{J}^{5}$, Grimaud $\mathrm{I}^{5}$, Ledru $\mathrm{E}^{5}$, Moisan $\mathrm{MP}^{2}$, Leterrier $\mathrm{C}^{3}$

Institute 1 URA INRA, 37380 NOUZILLY, France; 2 NutriNeuro INRA, 33076 BORDEAUX, France; 3 PRC CNRS IFCE INRA Université de Tours, 37380 NOUZILLY, France; 4 Pegase Agrocampus Ouest INRA, 35590 SAINT-GILLES, France; 5 PEAT INRA, 37380 NOUZILLY, France

DOI $10.1055 / \mathrm{s}-0037-1608313$

To mitigate the effects of stressful factors during the perinatal period in broiler chicks, we have tested their ability to drink spontaneously essential oils (EO) in addition to water. Chicks were either immediately placed in the rearing facility (C group), or submitted to a $24 \mathrm{~h}$-delayed period before their placement (S group) mimicking transport delay in commercial conditions. In experiment 1 , half of each group had only access to water and the other half to water and one EO (cardamom, marjoram or verbena) from D1 to D13. In experiment 2, half of each group had only access to water and the other half was offered water and the $3 \mathrm{EO}$ simultaneously. 
In experiment 1 , EO intake was higher in $S$ group than in $C$ group from $D 1$ to D 6 whatever the EO. In experiment 2, from D1 to D6, the marjoram intake was the highest compared to the other EO whatever the treatment. EO intake did not allow to overcome the reduced growth in $\mathrm{S}$ group but tended to overcome the reduction of the relative percentage of Pectoralis major muscles. Transcriptome analysis of blood cells showed long-term effects of the delay to placement on gene expression at slaughter age. Overexpression of transcription factors in $\mathrm{S}$ group was related to oxidative stress in males and to oncogenesis in females. EO intake reduced the overexpression of several genes involved in oxidative stress and inflammation in $S$ groups.

In conclusion, chicks are able to make spontaneous choices for EO according to their post-natal experience. These choices had a few beneficial effects on their performance, and long-term regulatory effects on blood transcriptome which could participate to maintain chicken welfare and health.

This study was supported by a grant from the Integrated Management of Animal Health metaprogram of INRA for the 'GISA - WHELP' project (www. gisa.inra.fr/en).

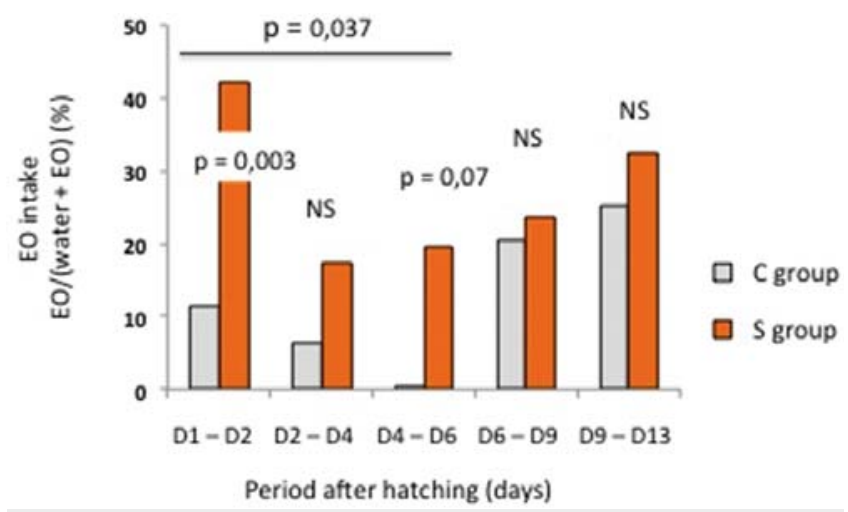

- Fig. 1: Spontaneous Intake of essential oils by chicks placed immediately after hatching in rearing facility ( $\mathrm{C}$ group) or submitted to a $24 \mathrm{~h}$-delayed period before thcir placement ( $\mathrm{S}$ group)

\section{Tu-Veterinary Phytomedicine \& Animal Health-SL-03 Developing a single application bolus with Solanum glaucophyllum extract (SGE) for milk fever prevention. II: Application at calving.}

\author{
Authors Bachmann $\mathrm{H}^{1}$, Lanz $\mathrm{M}^{2}$, Rambeck $\mathrm{W}^{3}$ \\ Institute 1 Herbonis AG, Augst, Switzerland; 2 Fachhochschule \\ Nordwestschweiz, Basel, Switzerland; 3 Ludwigs Maximilian Universität, \\ München, Germany \\ DOI 10.1055/s-0037-1608314
}

Milk fever (MF) is a metabolic disease in milk producing cows around calving. Clinical signs are muscular paralysis mainly of the legs with immobility of the affected animal. High milk producing breeds and older animals are most likely to be affected, and have a high chance of recurrence. An incidence of $5-10 \%$ has been reported. The disease is caused by an acute drop in blood calcium (Ca) when initiating milk production depletes the blood Ca too fast for repletion from bone mobilization and intestinal uptake. With the understanding of the underlying mechanism, Ca supply management and vitamin-D (VD) supplementation became prime candidates for MF prevention and therapy. In continuing the first study, tablets containing the Solanum glaucophyllum extract (SGE) in a instant-release (irSGE) and a slow-release form (srSGE) were combined into one bolus and applied to 31 cows $72-48$ hours before calving. Total dose for the SGE bolus was $10 \mathrm{~g}$ SGE containing $50 \mathrm{~m} \mathrm{~g} / \mathrm{g}$ 1,25D. as positive control acted 25 cows which received 4 calcium boli according to suppliers' instruction and a untreated control group of 12 cows was included in the study.
The course of serum 1,25D, Ca and $P$ is shown in figs. 1, 2 and 3 . The occurrence of milk fever too low for statistical evaluation. The control animals $(n=12)$ showed the expected reduction of serum Ca and $P$ around calving. Treatment with high doses of Ca $(n=25)$ was not able to prevent this reduction except at day 5 after calving for serum Ca. In contrast, treatment with one bolus SGE ( $n=23$ calving's within the application window of -72 to -24 hours a.p.) could increase 1,25D, Ca and P highly significant.

In conclusion, the present experiment showed that a single bolus of SGE was effective to prevent peri-parturient hypocalcaemia and -phosphataemia.

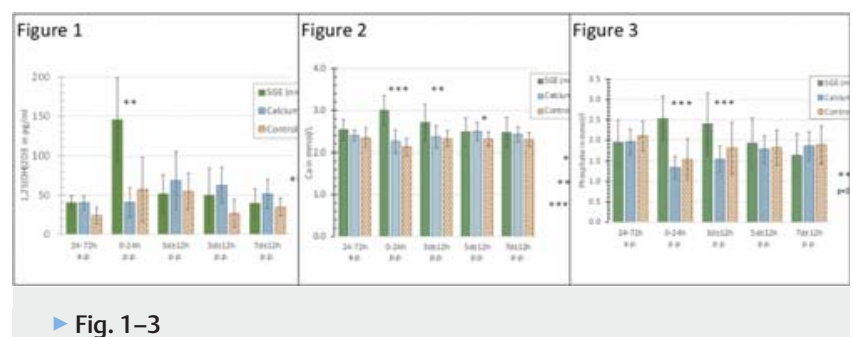

\section{Tu-Veterinary Phytomedicine \& Animal Health-SL-04 Ethno-veterinary medicine for prevention and treatment of bovine mastitis}

\section{Authors Nair $B^{1}$}

Institute 1 Trans-Disciplinary University, Bangalore, India; 1. Nair, Balakrishnan, Mannoor Narayanan, [Presenter], 2. Natesan, Natesan, Punniamurthy, 3. Kempanna, Kumar, Seethakempanahalli, DOI 10.1055/s-0037-1608315

Mastitis affects a large number of dairy cattle throughout the world. Mastitis in India increased 115 times in the last 50 years and the loss of revenue is about Rs. 71655 million annually [1]. The incidence of resistant mastitis in dairy cows in Tamil Nadu, India is 56.I \%- Escherichia coli (50.64\%), Staphylococcus aureus (44.25\%) and Methicillin resistant Staphylococcus aureus (5.11\%) [2]. Freshly prepared fine paste of Aloe vera leaves, Curcuma longa rhizome and calcium hydroxide applied 10 times per day for 5 days cured mastitis. If it is chronic mastitis add two pieces of Cissus quadrangularis with the above mixture and grind and apply for at least three weeks. A clinical trial using parameters like $\mathrm{pH}$, Somatic Cell Count and Electrical Conductivity recorded as pre and 6 days post treatment with EVP in 27 animals indicated that all selected parameters became normal within 6 days of treatment. This formulation can be used to prevent mastitis by applying this paste for three times a day during last trimester of pregnancy. Field study indicates herbal formulation used for all types of mastitis (sub-clinical, clinical and chronic) in dairy cattle have $91.7 \%$ efficacy. In Ayurveda mastitis is known as Sthanavidhradi, a disease of pitta origin, the drugs used in this formulation are potent pittashamaka. This formulation consists of Aloe vera, Curcuma longa and Calcium hydroxide which are having the properties of Krimighna (anti-microbial), Vranashodaka (Wound cleanser), Vranaropaka (Wound healing) and Shothahara (anti-inflammatory). Hence mastitis can be efficiently managed with this formulation.

[1] NDRI News April-June 2012 17, 1.

[2] D Chandrasekaran, et.al. 2015. India. J. Applied and Nat. Sce. 7: $304-308$ 


\section{Tu-Veterinary Phytomedicine \& Animal Health-SL-05 EVM as one stop solution for mastitis: validated through clinical and reverse pharmacology}

Authors Nair $\mathrm{B}^{1,2}$, Punniamurthy $\mathrm{N}^{1}$, Mekala $\mathrm{K}^{1}$, Nair MNB ${ }^{2}$, Ramakrishnan $\mathrm{N}^{1}$ Institute 1 EVM Herbal Centre, VUTRC, Tamil Nadu Veterinary and Animal Sciences University, Thanjavur 613403 Tamil Nadu, India, Thanjavur, India; 2 Trans-Disciplinary University, FRLHT, Bangalore, India DOI 10.1055/s-0037-1608316

Mastitis continues to be a major problem in dairy industry causing severe economic loss to the farmers. A wide variety of antimicrobials have been extensively used through parenteral as well as intramammary route to treat the infectious cause. Ethnoveterinary formulation for mastitis comprising Aloe vera, Curcuma longa and calcium hydroxide has been clinically evaluated and has been found to be successful as a stand-alone treatment mode against mastitis. The phytochemical screening of the formulation revealed the presence of alkaloids, saponins, steroids, carbohydrates, flavonoids, terpenoids, triterpenoids, sterols, emodin and diterpenes. TLC autobiography revealed the antioxidant activity of flavonoids, phenols and glycosides (ICAR Report 2016). Critical analysis of the published literatures provides ample evidence for the antimicrobial, anti-inflammatory and immunomodulating potential of the formulation. It can be concluded that after topical application of the formulation the pharmacologically active components penetrate into the skin and eliminate the infectious agents, effectively reduce the inflammation by suppressing the release of inflammatory mediators, provide analgesic effect, prevent fibrosis and further modulate the immune system of the host [1] resulting in recovery of udder swelling and infection as well as milk production.

[1] Kshirsagar, A. D, P. V. Panchal, U. N. Harle, R. K. Nanda and H.M. Shaikh, 2014. Int. J. Inflam, 12. http://dx.doi.org/10.1155/2014/690596

\section{Tu-Veterinary Phytomedicine \& Animal Health-SL-06 Effect of thyme oil dietary supplementation on thymol and thymol sulfate concentrations in duodenal wall, liver and plasma of chickens}

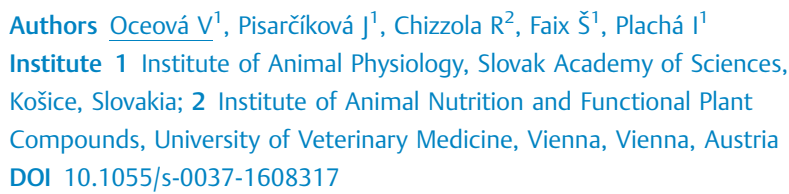

Phytogenic feed additives containing bioactive plant compounds have a beneficial impact on animal health and production. Plant compounds undergo a biotransformation in the animal organism and the resulting conjugates may bear different biological activities than the parental compounds. The study of intestinal absorption (IA) of individual compounds, their distribution within the body and biotransformation is fundamental for understanding their mode of action and the prediction of the optimal dosage. The goal of the current experiment was a quantification of thymol (the main compound of thyme oil) and thymol sulfate (product of thymol biotransformation) in duodenal wall, liver and blood plasma of broiler chickens fed a diet containing Thymus vulgaris L. essential oil (TEO) in different concentrations. Thirty-two 1-day old Ross-308 hybrid broiler chickens were divided into dietary treatments with TEO addition: 0\%; 0.01\%; 0.05\%; $0.1 \%(w / w)$. Samples of tissues and blood plasma (BP) were collected after 4 weeks of supplementation. Concentrations of thymol were analysed after the enzymatic cleavage of thymol metabolites by the method SPME-GC-MS. Thymol sulfate was quantified without enzymatic hydrolysis using UHPLC-ESI-QTOF-MS. Concentrations of thymol and thymol sulfate in duodenal wall, liver and BP significantly increased with the highest TEO supplementation. A significant correlation was observed between the thymol and thymol sulfate concentrations in duodenal wall. Results probably outline the relation between thymol IA and the subsequent biotransformation. The proportion of thymol and thymol sulfate in liver in comparison to the duodenal wall decreased with the increasing TEO supplementation. Ob- servations could support the assumption that the liver could adapt to a higher thymol supplementation with an intensive rate of the biotransformation and the subsequent excretion. Our results confirmed the thymol IA and the presence of its metabolite in BP and tissues on all dietary levels. The research was supported by the Slovak Scientific Agency VEGA, project 2/0078/16.

\section{Lecture Session - WHO Strategy Session \\ Tuesday, 5th September, 2017 \\ Osaka/Samarkand - 14:00 - 16:00}

\section{Tu-WHO Strategy Session-KNL-01 Herbal medicines - a global trend in patients and consumers}

Authors Stange $\mathrm{R}^{1}$

Institute 1 Immanuel-Krankenhaus Rheumaklinik Berlin-Wannsee und Zentrum f. Naturheilkunde Charité-Universitätsmedizin, Berlin, Germany DOI 10.1055/s-0037-1608318

Herbal products play a very significant role in medical care as a whole, and thus also in complementary and alternative medicine. The importance of these products can be captures by various measuring instruments. E.g. in Germany, several surveys have demonstrated sufficiently reliable results on the application of herbal medicinal by the population. They show e.g. that $63 \%$ of the population show a life time prevalence of the use of naturopathic therapies. A nationwide survey dealing with the practice of prescription shows the significantly higher popularity of phytotherapy in primary care $(88 \%)$ than in specialized medical practice (54\%).

Also marketing data can be used to describe trends reliably. Figures from China show that $70-80 \%$ of the population use TCM products, and that annual growth rates in this segment are $10-25 \%$. The underlying global trend supports the call for globally accepted standards for quality, safety and efficacy of herbal products.

\section{Tu-WHO Strategy Session-KNL-02 The WHO traditional medicine strategy - a way to better medical care}

Authors Kraft $\mathrm{K}^{1}$

Institute 1 Center for Internal Medicine, Universitätsmedizin Rostock,

Rostock, Germany

DOI 10.1055/s-0037-1608319

In continuation of earlier approaches, the WHO Traditional Medicine Strategy 2014 - 2023 aims to support Member States in developing proactive policies and implementing action plans that will strengthen the role of traditional medicine in their health care system. Herbal medicinal products (HMPs), which have an already large but still increasing relevance in medical care for a growing and ageing world population, are an integral part of it.

In many developing countries this strategy is already followed, and public funding is available for support of educational and research activities in products, practices and practitioners in traditional medicine. However, in a considerable number of industrialized countries, such as Germany, this strategy up to now has been of nearly no concern to national health policy.

For changing this and fostering integration of traditional medicine and especially herbal medicine into the national health care system e.g. in Germany, it is necessary to more vigorously popularize the WHO strategy. This will also result in an improved collaboration of all stakeholders involved in manufacturing, studying, prescribing, recommending and using these products. The reimbursement of HMPs by health insurances, e.g. in Switzerland and various East Asian countries, and the inclusion of a considerable number of HMPs into national medical guidelines e.g. in Germany [1] and, in exceptional cases, even in international guidelines [2], are recent success stories showing that there is more room for improvement. 
[1] Klose P, Kraft K, Cramer H, Luche H, Dobos G, Langhorst J. Forsch Komplementärmed 2014;21:388 - 400;

[2] Stanghellini V, Chan F K L, Hasler WL, Malagelada J R, Suzuki H, Tack ], Talley NJ Gastroenterology, 2016; 150: 1380 - 1392

\section{Tu-WHO Strategy Session-KNL-03 The WHO guidelines - a way to better quality of herbal products}

Authors Lorenz $\mathrm{M}^{1}$

Institute 1 PhytoConsult, Darmstadt, Germany

DOI $10.1055 / \mathrm{s}-0037-1608320$

The WHO is since many years issuing guidance documents on all aspects of the manufacturing and quality control as well as the therapeutic use of herbal products. One of the most influencial of these guidelines is on Good Agricultural and Collection Practices (GACP) for Medicinal Plants. This document is providing general technical guidance on obtaining medicinal plant materials of good quality for the sustainable production of herbal products classified as medicines. These guidelines are also related to WHO's work on the protection of medicinal plants, aiming promotion of sustainable use and cultivation of medicinal plants [1].

Accordingly, the main objectives of these guidelines are: to contribute to the quality assurance of medicinal plant materials used as the source for herbal medicines to improve the quality, safety and efficacy of finished herbal products; to guide the formulation of national and/or regional GACP guidelines and GACP monographs for medicinal plants and related standard operating procedures; and to encourage and support the sustainable cultivation and collection of medicinal plants of good quality in ways that respect and support the conservation of medicinal plants and the environment in general.

These guidelines concern the cultivation and collection of medicinal plants and include certain post-harvest operations. Good agricultural and collection practices for medicinal plants are the first step in quality assurance, on which the safety and efficacy of herbal medicinal products directly depend. These practices also play an important role in protection natural resources of medicinal plants for sustainable use.

Also the WHO monographs on selected medicinal plants, which were published in between 1999 and 2010, need to be mentioned in this context. The general limitation of all these documents is that they are not legally binding on a national level. This hampers the establishing of uniform standards for natural products globally.

WHO Guidelines on Good Agricultural and Collection Practices (GACP) for Medicinal Plants (2003).

\section{Tu-WHO Strategy Session-KNL-04 Herbal medicinal product regulation - how to find ways to better harmonization}

Authors Anquez-Traxler $C^{1}$

Institute 1 Regulatory and Scientific Affairs, AESGP - Association of the European Self-Medication Industry, Brussels, Belgium

DOI 10.1055/s-0037-1608321

The World Health Organization estimated that $80 \%$ of people worldwide rely on herbal medicines for some part of their primary health care. The ways herbal medicines are regulated are nonetheless still quite different. The different medical systems, cultures and traditions have imprinted their mark on the legislations of phytomedicines in the different regions.

In Europe, the different national systems around traditional herbal medicines and remedies have been harmonized by Directive 2004/24/EC which created an EU-wide definition of 'traditional herbal medicines', defined a simplified authorisation system and set-up a full-fledged committee at the EMA level which issues monographs and gives opinions. Although the increasing number of decentralised and mutual recognition procedures in the EU may also be a sign of this growing harmonization, differences subsist. Regulatory systems in some non-European countries like Canada and Australia to some extent follow similar approaches. In the US, herbal medicines are rare and dominated by food supplements containing botanicals, while East Asian countries rely on their own traditions like TCM and Kampo.

This situation complicates bringing products onto the market for companies and meeting rising patients' needs and customers' demand for high-quality herbal products with accepted safety and efficacy.

The talk will attempt to provide a tour d'horizon of the main regulatory systems related to herbal medicines around the globe, particularly reflecting on the situation in Europe, and will raise a number of questions. Is it feasible to further harmonization e.g. through regulatory bilateral or multilateral discussions, cooperation, training, etc.? Does the lack of harmonization bear an impact on innovation? Could there be downsides to harmonization?

\section{Tu-WHO Strategy Session-KNL-05 Global principles for better herbal products -a way to best serving patients and consumers}

Authors Canigueral $S^{1}$

Institute 1 University of Barcelona, Institute for Pharmacy, Unitat de Farmacologia i Far-macognòsia, Facultat de Farmàcia, Barcelona, Spain DOI 10.1055/s-0037-1608322

Despite growing demand, there are yet no globally accepted standards governing herbal products. On the one hand, this can led to questions in health professionals, patients and consumers. On the other hand, this has led to a great heterogeneity of regulatory approaches, which makes the development and manufacturing of herbal products difficult and complex. Before this background, it is important reconcile on key principles, which are the basis of successful serving the needs all stakeholders. These need to cover quality, safety, efficacy, sustainability, and access and benefit sharing. By focusing our efforts in these key areas, we are establishing a foundation for global standards supporting future success in the herbal products area:

Quality is inextricably linked to efficacy and safety. Good Agricultural Collection Practices are essentials for the success of herbal products.

Safety is based on multiple factors, which need to be regularly updated according to scientific progress. These include an appropriate quality control, toxicological proof, an effective pharmacovigilance system, as well as sound information to health care professionals, patients and consumers.

Efficacy of herbal products is relying on long-standing use and therapeutic experience.

Sustainability is the basis for long-term success of herbal products, and precondition is responsible sourcing respecting the natural habitat and sustainable cultivation methods, for being able to meet supply needs also in the future.

Fair access and benefit sharing helps to establish and maintain fair and sustainable collaboration with all stakeholders. Frame is the Nagoya Protocol, which needs now to find its way to daily practice in the development of herbal products.

The focus on these key areas will establish a foundation that can be the basis of the future global success in the field of herbal products, both from the manufacturers as from the patients and consumers point of view.

\section{Tu-WHO Strategy Session-KNL-06 Panel discussion}

Authors Canigueral $\mathrm{S}^{1}$

Institute 1 University of Barcelona, Insti-tute for Pharmacy, Unitat de

Farmacologia i Far-macognòsia, Facultat de Farmàcia, Barcelona, Spain

DOI 10.1055/s-0037-1608323

Panel Discussion with all Speakers and the audience. 
Lecture Session - Bioactive Natural

\section{Products II}

Tuesday, 5th September, 2017

Montreal - 14:00 - 16:00

Tu-Bioactive Natural Products II-SL-01 Influence of the structure of prenylated and non-prenylated stilbenoids on the NF-KB/AP-1 signalling pathway and cyclooxygenases/lipoxygenase inhibition

Authors Hošek J ${ }^{1}$, Leláková $\mathrm{V}^{1}$, Jabubczyk $\mathrm{K}^{2}$, Landa $\mathrm{P}^{2}$, Stammer $\mathrm{T}^{3}$, Temml V ${ }^{3}$, Schuster $D^{3}$, Šmejkal $K^{4}$

Institute 1 Department of Molecular Biology and Pharmaceutical Biotechnology, Faculty of Pharmacy, University of Veterinary and Pharmaceutical Sciences Brno, Brno, Czech Republic; 2 Laboratory of Plant Biotechnologies, Institute of Experimental Botany, Academy of Sciences of the Czech Republic, Prague, Czech Republic; 3 Institute of Pharmacy/

Pharmaceutical Chemistry and Center for Molecular Biosciences Innsbruck (CMBI), University of Innsbruck, Innsbruck, Austria; 4 Department of Natural Drugs, Faculty of Pharmacy,? University of Veterinary and Pharmaceutical

Sciences Brno, Brno, Czech Republic

DOI 10.1055/s-0037-1608324

Stilbenes are important components of food, such as peanuts, grapevine and different types of berries. [1] Anti-inflammatory activity have been determined for a number of stilbenes, including the widely known resveratrol, its analogues, e.g, piceatannol [2], and other non-prenylated and prenylated stilbenes. [3]

We investigated 32 different natural and synthetic stilbenes for their effect on the pro-inflammatory nuclear factor-KB (NF-KB)/activator protein 1 (AP-1) signalling pathway. As a model for determination of anti-inflammatory potential, the THP1-XBlue ${ }^{\mathrm{TM}}$-MD2-CD 14 cell line (Invivogen; USA) was used. Lipopolysaccharide (LPS) stimulation of Toll-like receptor 4 (TLR4) in this cell line induces a signalling cascade leading to the activation of NF-KB/AP-1. The ability of these compounds to attenuate the production of pro-inflammatory

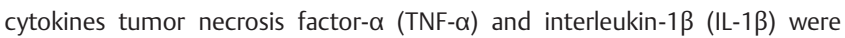
evaluated in LPS-stimulated THP-1 macrophages (European Collection of Cell Cultures; UK). The direct inhibiting effect on cyclooxygenase (COX)-1 and COX-2 and 5-lipoxygenase (5-LOX) were also determined. Molecular docking for the most active stilbenoids was performed to elucidate the interaction between compounds and enzymes.

Several compounds (e.g, piceatannol, TTH-16) affected the production of NFKB/AP-1 and TNF- $\alpha$. Some compounds isolated from Thailand's plant Macaranga siamensis S.J.Davies (Euphorbiaceae) affected the activation of NF-KB/AP1 and secretion of IL-1 $\beta$ more than prednisone, which was used as a positive control for the assay. We observed that prenylated stilbenes are in general more cytotoxic and more active inhibitors of NF-kB/AP-1 than their non-prenylated analogues. Significant anti-COX/LOX activity was also detected for several compounds.

Elucidation of the structure-activity relationship could contribute to develop potent, natural product-based anti-inflammatory drugs.

This project is supported by the Grant agency of the Czech Rublic, grant No $16-071935$.

[1] Chong J. et al. Plant Sciences 2008; 177: $143-155$.

[2] Piotrowska H. Mutation Research 2012; 750: 60 - 82.

[3] Pailee P. et al. Tetrahedron 2015; 77: 5562 - 5571.

\section{Tu-Bioactive Natural Products II-SL-02 Natural products upregulating Smac/DIABLO genes expression from Ficus deltoidea and their role in prostate cancer chemoprevention.}

\section{Authors Mohd Hanafi Mohd $\mathrm{M}^{1}$, Afzan $\mathrm{A}^{2}$, Yaakob $\mathrm{H}^{3}$, Aziz $\mathrm{R}^{3}$, Sarmidi} Mohamad $\mathrm{R}^{3}$, Wolfender $\mathrm{JL}^{2}$, Prieto Jose $\mathrm{M}^{1}$

Institute 1 Department of Pharmaceutical and Biological Chemistry, UCL School of Pharmacy, London, United Kingdom; 2 School of Pharmaceutical Sciences, EPGL, University of Geneva, University of Lausanne, Geneva, Switzerland; 3 Institute of Bioproduct Development (IBD), Universiti Teknologi Malaysia, 81310 UTM, Johor Bahru, Malaysia

DOI 10.1055/s-0037-1608325

This study aims to evaluate the in-vitro cytotoxic and anti-migratory effects of Ficus deltoidea L. plant on prostate cancer cells, identify the active compound/ $s$ and characterise their mechanism of actions. The crude methanolic extract was partitioned into n-hexane (FD1 h, FD 2 h) chloroform (FD 1c, FD 2c) and aqueous extracts (FD1a, FD2a). Active fractions were further fractionated. Active compound/s were dereplicated based on UHPLC-HRMS/MS. In-vitro mechanistic studies on PC 3 cells included: Annexin V-FITC staining, MMP depolarization measurements, activity of caspases 3/7, nuclear DNA fragmentation, cell cycle analysis, modulation of Bax, Bcl-2, Smac/Diablo and Alox-5 mRNA gene expression by RT-PCR. 2D migration and 3D invasion were tested by exclusion assays and modified Boyden chamber [1]. The expression of VEGF-A, CXCR4 and CXCL12 in PC3 cells were analysed by RT-PCR. FD 1c and $F D 2 c$ extracts induced cell death $(P<0.05)$ via apoptosis as evidenced by nuclear DNA fragmentation accompanied by an increase in MMP depolarization $(P<0.05)$, and activation of caspases $3 / 7(P<0.05)$. All active plant extracts up-regulated Bax and Smac/DIABLO, and down-regulated Bcl-2 $(P<0.05)$. Active plant extracts inhibited both migration and invasion of $P C 3$ cells $(P<0.05)$. These effects were accompanied by down-regulation of both VEGF-A and CXCL-12 gene expressions ( $P<0.001)$. UHPLC-HRMS/MS dereplication identified isovitexin in FD1c; and oleanolic acid, moretenol, betulin, lupenone and lupeol in FD2c. In conclusion, FD1c and FD2c were able to induce apoptosis by activating the intrinsic pathway, inhibit both migration and invasion by modulating the CXCL 12-CXCR4 axis, and inhibit angiogenesis by modulating VEGF-A expression in PC 3 cell line.

I would like to thank the Foundation Plants for Health for awarding me with a research subsidy and giving me the opportunity to collaborate with Prof JeanLuc Wolfender in completing this project.

[1] Ridley, Anne J, et al. Science 302.5651 (2003): 1704 - 1709.

\section{Tu-Bioactive Natural Products II-SL-03 Isolation of 2-arylbenzofurans and stilbenes with potential anti-inflammatory activity and their metabolism in model of colon microbiota}

Authors Šmejkal K${ }^{1}$, Maršík $\mathrm{P}^{2}$, Veselý $\mathrm{O}^{2}$, Hanáková Z ${ }^{1}$, Gazdová Mำ Institute 1 Department of Natural Drugs, Faculty of Pharmacy, UVPS Brno, Brno, Czech Republic; 2 Department of Quality of Agricultural Products, Faculty of Agrobiology, Food and Natural Resources, Czech University of Life Sciences Prague, Prague, Czech Republic

DOI 10.1055/s-0037-1608326

Stilbenes and benzofurans are common components of some food or content compounds of medicinal plants with antioxidant, chemoprotective and antiinflammatory effect. The occurrence of the most well-known stilbene resveratrol is described for example in wine, peanuts, cocoa powder and different berries, the rich source of benzofuran derivatives is for example mulberry root $[1,2]$.

Their activity is connected with interaction with multiple cellular targets, however, only limited information is known about their metabolism and interaction with gut microbiota. Therefore, our study is aimed to isolate natural 
stilbenes and benzofurans from plants, and to analyze their metabolism in a model of colon microbiota.

Several benzofurans and stilbenes were isolated from Morus alba root bark and Artocarpus heterophyllus using combination of different chromatographic techniques including preparative TLC and HPLC. These compounds were tested to analyze their anti-inflammatory activity, together with some commercially available stilbenoids.

Several stilbenoid compounds were subjected to metabolism in model of colon microbiota. The results obtained via LC-MS analysis of metabolic products showed the formation of total 19 metabolites. Surprisingly, no monocyclic stilbene metabolites were detected. The most intensive was metabolism of resveratrol and batatasin III, the most pronounced was formation of dihydroresveratrol from trans-resveratrol. The most common reaction observed was reduction of one meta localized hydroxy group, demethylation, double bond (bridge) oxidation or double bond reduction.

Authors thank to support of Grant Agency of Czech Republic (GAČR 16 07193S).

[1] Shen T, Wang XN, Lou HX. Nat Prod Rep 2009; 26: 916 - 935

[2] Khanam H, Uzzaman S. Eur J Med Chem 2015; 97: 483 - 504

\section{Tu-Bioactive Natural Products II-SL-04 Unusual pro-apoptotic benzo[j]fluoranthene metabolites from a mangrove-derived endophytic fungus Annulohypoxylon sp.}

Authors Daletos $\mathrm{G}^{1}$, Liu $\mathrm{Y}^{1,2}$, Kurtán $\mathrm{T}^{3}$, Wesselborg $\mathrm{S}^{4}$, Stork $\mathrm{B}^{4}$, Proksch $\mathrm{P}^{1}$ Institute 1 Institute of Pharmaceutical Biology and Biotechnology, Heinrich Heine University, Universitaetsstrasse 1, D-40225 Duesseldorf, Germany, Duesseldorf, Germany; 2 Key Laboratory of Marine Drugs, The Ministry of Education of China, School of Medicine and Pharmacy, Ocean University of China, Qingdao 266003, P. R. China, Qingdao, China; 3 Department of Organic Chemistry, University of Debrecen, P. O. B. 20, 400, 4002 Debrecen, Hungary, Debrecen, Hungary; 4 Institute of Molecular Medicine I, Medical Faculty, Heinrich Heine University, Universitaetsstrasse 1, D-40225

Duesseldorf, Germany, Duesseldorf, Germany

DOI 10.1055/s-0037-1608327

Four new compounds, including three new benzo[j]fluoranthene derivatives, namely, daldinones $\mathrm{H}-\mathrm{J}$ [1], and an unusual ten-membered macrocyclic lactone, together with six known metabolites were isolated from the endophytic fungus Annulohypoxylon sp. that was obtained from the Mangrove plant Rhizophora racemosa, collected in Cameroon. The structures and absolute configurations of the new compounds were elucidated on the basis of comprehensive NMR, MS, and ECD spectroscopic data analyses. Several attempts were undertaken to influence the pattern of fungal metabolites through cocultivation of the fungus with a panel of Gram-positive bacteria. Accordingly, co-cultivation of Annulohypoxylon sp. either with Streptomyces lividans or with Streptomyces coelicolor resulted in an up to 38 -fold increase in the accumulation of the constitutively present metabolite 1-hydroxy-8-methoxynaphthalene. On the other hand, no significant effect on metabolite accumulation was observed when the fungus was co-cultivated either with Bacillus subtilis or with Bacillus cereus. In a second set of experiments, feeding of the fungal culture with 1,8-dihydroxynaphthalene (DHN) resulted in a marked dose-dependent induction of the main metabolite daldinone $B$, which shows excellent agreement with its proposed biosynthesis involving oxidative coupling of two DHN units [1]. Among the isolated compounds, the new metabolite daldinone I exhibited the strongest activity against Ramos and Jurkat J16 cells with $\mathrm{IC}_{50}$ values of 6.6 and $14.1 \mu \mathrm{M}$, respectively. The distinct cytotoxicity of daldinone I against human lymphoma and leukemia cell lines was determined to be caused by induction of intrinsic apoptosis. Further mechanistic studies showed that daldinone I potently blocks autophagy, a potential pro-survival pathway for cancer cells, thus highlighting its potential as a promising lead for anticancer drug development.
[1] Liu Y, Stuhldreier F, Kurtán T, Mándi A, Arumugam S, Lin W, Stork B, Wesselborg S, Weber H, Henrich B, Daletos G, Proksch P. RSC Adv 2017; 7 : $5381-5393$

\section{Tu-Bioactive Natural Products II-SL-05 An alternative rapid screening technique to detect $\beta$-lactamase inhibitor from mangrove actinomycete extracts}

Authors Suhaidi $A^{1,2}$, Sharifah Aminah Syed $M^{1}$, Mohd Faiz Foong $A^{1}$, Norizan $A^{1}$

Institute 1 Faculty of Applied Sciences, Universiti Teknologi MARA, 40450

Shah Alam, Selangor, Malaysia; 2 Faculty of Applied Sciences, Universiti

Teknologi MARA, Perak Branch, Tapah Campus, 35400 Tapah Road, Perak, Malaysia

DOI 10.1055/s-0037-1608328

The study aims to develop a new screening technique for the detection of inhibitory metabolites from local mangrove actinomycetes toward $\beta$-lactamase (1). This new screening technique is believed to contribute a novel bioactivity. The oxacillin-resistance Staphylococcus aureus ATCC 43300 was used as test organism and the presence of $\beta$-lactamase enzyme from this pathogen was detected by using nitrocefin (2). Among 41 marine-derived actinomycetes, two isolates designated as KMS 1 and THH1 consistently exhibited the production of metabolites that can act as an inhibitor agents. For that reason, one of the active isolates, KMS 1 was grown in a large volume to extract and check the presence of metabolites as inhibitors of $\beta$-lactamase. This confirmation test was effectively done by using oxacillin-susceptible Staphylococcus aureus ATCC 25923 and penicillinase. Therefore, this study has successfully developed a new screening technique for a novel $\beta$-lactamase inhibitor bioactivity from local mangrove actinomycetes.

The authors thank the Institute of Graduate Studies and Faculty of Applied Sciences, Universiti Teknologi MARA (UiTM) Shah Alam, Selangor for providing the funds and facilities for this study. The credit also goes to Dr. Mohamad Ali Yusuf (UiTM Perak Branch, Tapah Campus) who helped in the editing work. [1] Ariffin, S, Abdullah, M. F. F, \& Sharifah, A. S. M. International journal on Advanced Science, Engineering and Information Technology, 2017, 7, 71 - 77. [2] Bredholt, H, Fjæruik, H, Johnsen, G, \& Zotchev, S. B. Marine Drugs, 2008, 6, $12-24$.

[3] Sarker, S. D, Nahar, L, \& Kumarasamy, Y. Methods, 2007, 42, 321 - 342.

\section{Tu-Bioactive Natural Products II-SL-06 Inhibition of Histone Deacetlases Using Flavonoids from Hops}

Authors Urmann $\mathrm{C}^{1}$, Chia-Leeson $\mathrm{O}^{1}$, Märkl $\mathrm{S}^{1}$, Stark $\mathrm{T}^{1}$, Kirchinger $\mathrm{M}^{1}$ Institute 1 Weihenstephan-Triesdorf University of Applied Sciences,

Straubing, Germany

DOI 10.1055/s-0037-1608329

Humulus lupulus L. is a traditional medicinal plant and was already mentioned by Mesue (died 1015). Beside bitter acids, prenylated flavonoids are partly responsible for manifold biological activities such as anti-cancer, neuroprotective activity as well as induction of differentiation. A possible link between these effects is the epigenetic and, in particular, the histone deacetylase (HDACs) [1]. The group of HDACs deacetylates the lysine on the terminal end of histones and therfore prepare the DNA for subscription.

In this study the inhibition of HDACS by prenylated flavonoids was determined. In the screening, the sum of HDACs were used, which were obtained by nuclear extraction of HeLa cells. 


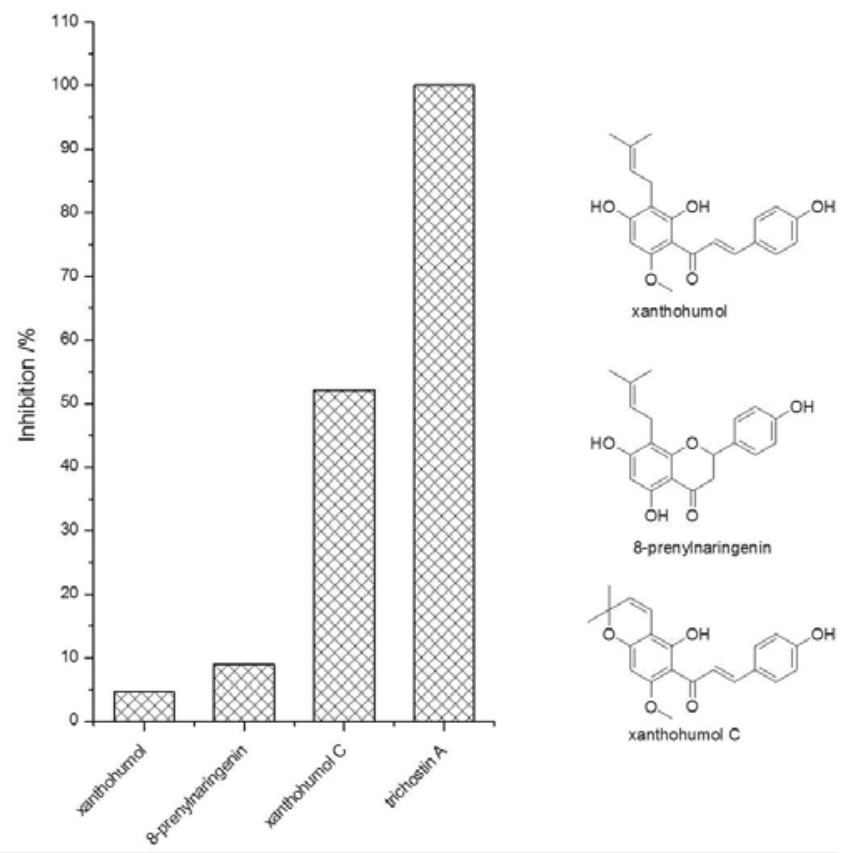

- Fig. 1: Inhibition of HDACs of HeLa cells nuclear extract by prenylflavonoides $(c=50 \mu \mathrm{M})$ compared to Trichostin $\mathrm{A}(\mathrm{c}=10 \mu \mathrm{M})$ and structures of compounds tested.

Xanthohumol, which is known as a potent influencer of cancerogensis shows the lowest inhibition rate. 8-prenylnaringenin [2], the most active phytoestrogen known thus far, shows an inhibition of approximatley $10 \%$. The most potent inhibitor of the HDACs is xanthohumol $C$ which was recently identified as a potent inducer of neurogenesis in neuronal stem cells [3]. Since it is known, that the induction of differentiation and neuroprotection is partly linked to the activity of different HDACs, the effect of xanthohumol $\mathrm{C}$ on several recombinant enzymes was further investigated.

[1] Marks P, Richon V, Rifkind, R. J Natl Cancer 2000; 92: 1210 - 1216.

[2] Oberbauer E, Urmann C, Steffenhagen C, Bieler L, Furtner T, Humpel C, Baumer B, Bandtlow C, Couillard-Despres S, Rivera FJ, Riepl H, Aigner L. J Nutr Biochem 2013; 24: 1953 - 1962.

[3] Urmann C, Oberbauer E, Couillard-Despres S, Aigner L, Riepl H. Planta Med 2015: 4: $305-311$.

\section{Tu-Bioactive Natural Products II-SL-07 Can obliquumol isolated from Ptaeroxylon obliquum be a new framework molecule for future antifungal antibiotics?}

Authors Eloff $]^{1}$, van Wyk $C^{1}$, Ramadwa $T^{1}$, Botha $\mathrm{F}^{1}$, Apostolides $Z^{2}$ Institute 1 Phytomedicine Programme Faculty of Veterinary Science University of Pretoria, Pretoria, South Africa; 2 Department of Biochemistry Faculty of Natural and Agricultural Sciences University of Pretoria, Pretoria, South Africa

DOI 10.1055/s-0037-1608330

Fungal infections are difficult to control in plants, animals and humans. Especially in immunocompromised patients fungal infections cause major problems. There is an increasing resistance by several fungi against some fungicides. It appears that the last new antifungal framework molecule was identified 60 years ago. Since then new antifungals were mainly derivative compounds. Searching for new antifungal compounds based on traditional use has been counterproductive probably because traditional healers generally have only water available as an extractant. The antimicrobial compounds we isolated from plants were all relatively non-polar. We consequently determined the activity acetone leaf extracts of 537 South African tree species against eight important bacterial and fungal pathogens including Candida albicans. In addition to finding extracts of species with high activity, we investigated whether predictions could be made which taxa had high activity. [1]. One of the species with high activity against Candida albicans, Ptaeroxylon obliquum (Ptaeroxylaceae) a tree occurring mainly in southern Africa was investigated in depth. A novel compound obliquumol closely related to eranthin was isolated. The compound had higher activity against Candida albicans than amphotericin B. Many natural products have high activity but may be too toxic for general use obliquumol had higher cellular safety against several cell lines than amphotericin B. It occurs in high concentrations in the tree leaves and we developed a procedure to isolate relatively large quantities. The compound also had good activity against other fungi and other promising biological activities. The low water solubility causes difficulties and derivatives have to be synthesized to overcome this problem. A preliminary docking study indicates that it targets different sites than amphotericin B. The information has been patented in Europe and the United States.

[1] Pauw and Eloff BMC Complementary and Alternative Medicine 2014, $14: 317$

\section{Tu-Bioactive Natural Products II-SL-08 BINDING MODE OF SOME NATURALLY-OCCURRING NEOLIGNANS INTO PAF-RECEPTOR: EXAMINATION AND VALIDATION}

Authors Coy-Barrera $\mathrm{E}^{1}$

Institute 1 Laboratorio de Química Bioorgánica, Facultad de Ciencias Básicas y Aplicadas, Universidad Militar Nueva Granada, Cajicá, Colombia

DOI 10.1055/s-0037-1608331

Platelet-activating factor (PAF) is a well-known regulator to be involved in the body's inflammatory response as well as vascular diseases such as thrombosis [1]. Currently, the use of computational techniques has been widely used particularly in the pharmacology field to establish the conceivable interaction mode of a molecule within the active site of a protein [2]. Such approach becomes as useful tool for establishing the interaction/activity of compounds within the active site of PAF-receptor [3]. Thus, as part of our research on PAFreceptor inhibitors, 85 naturally-occurring neolignans were in-vitro and in-silico evaluated (molecular docking, molecular dynamics and 3DQSAR) in order to establish their binding mode and determine its potentiality as PAF-antagonists. Stability of complexes between PAF-receptor and neolignans were investigated through Vina scores, selected residues interactions, and RMSD and RMSF profiles. Good Vina scores were obtained for receptor-ligand complexes interactions at different levels as well as good stabilization across $15 \mathrm{~ns}$ for several neolignans. Best poses were found to be different for each type of neolignan as well as the active site residues involved into the ligand-receptor binding. $I C_{50}$ values, docking scores and quantum properties were used as molecular descriptors along with the steric and electrostatic field values for PLS analysis. CoMFA model exhibited good statistical measure from PLS $\left(q^{2}=0.821\right)$ indicating as an useful tool for the prediction of test set as well as newly designed/homologous structures against PAF-receptor based on neolignans. This is the first study to provide an explanation at atomic level of binding of neolignan-related compounds within this receptor. Work derived from Project IMP-CIAS-2293 financed by Vicerrectoría de Investigaciones at UMNG - Validity 2017.

[1] Ishii S. et al. Prostag Other Lipid Mediat 2002; 68 - 69:599 - 609;

[2] Sliwoski G. et al. Pharmacol Rev 2014; 66:334-395;

[3] Gui C. et al. Proteins 2007; 67:41 - 52. 
Lecture Session - Analytical Studies \& Natural Products Chemistry II

Tuesday, 5th September, 2017

Singapore - 14:00 - 16:00

\section{Tu-Analytical Studies \& Natural Products Chemistry II- SL-01 Establishment of a quality control mixture for benchmarking LC-MS based dereplication protocols in natural product research}

Authors Dounoue-Kubo M ${ }^{1,2}$, Allard PMA ${ }^{1}$, Wolfender $\mathrm{JL}^{1}$

Institute 1 School of Pharmaceutical Sciences, EPGL, University of Geneva, University of Lausanne, Geneva, Switzerland; 2 2Faculty of Pharmaceutical Sciences, Tokushima Bunri University, Tokushima, Japan

DOI 10.1055/s-0037-1608332

In natural products (NPs) research, recent dereplication workflows are usually based on the treatment of data obtained from generic untargeted metabolite profiling by UHPLC-HRMS/MS of crude extracts [1]. When applied on large collection of complex NPs extracts, the amount of information generated by such approaches is extremely important and requires to finely tune the acquisition parameters. Additionally, various computational solutions, each presenting a great numbers of parametrical options, exists to mine the generated data. In order to assess the overall quality of the NPs profiling and dereplication workflows we propose to use a well-defined Quality Control (QC) sample mixture prepared from the combination of 5 extensively described plants of the European Pharmacopeia for which extensive phytochemical studies have been performed. Plants were selected by mapping the chemical space of their constituents against the space occupied by all NPs known to date for the best coverage and extended polarity range. This mixture was then analyzed in different LC gradient conditions and MS/MS acquisition modes on different MS platforms. By using MS-DIAL [3], for example, it could be observed that the number of identified features increased by a factor ranging from 2 to $15 \%$ when the measurement time increased by 2 min steps starting from a $5 \mathrm{~min}$ gradient. The quality of automated annotations was also assessed in various chromatographic regions and over a large dynamic range in data dependent or data independent modes.

The results suggested that this plant mixture easily generable in any laboratory could become a universal QC mix for the quality evaluation of NPs profiling and annotation workflows. Evaluation of various dereplication strategies in terms of number of annotations and their quality and acquisition modes will be discussed.

[1] Wolfender J-L, et al. J Chromatogr A 2015; 1382:136 - 164.

[2] Tsugawa H, et al. Nature Methods 2015; 12: $523-52$.

\section{Tu-Analytical Studies \& Natural Products Chemistry II- SL-02 The Comprehensive Screening of Traditional Chinese Medicine Using a Novel LC/MS Informatics Platform}

Authors Yuk J ${ }^{1}$, Isaac $G^{1}$, Nikles $S^{2}$, Wrona $M^{1}$, Bauer $R^{2}$

Institute 1 Waters Corporation, Milford, United States; 2 Institute of

Pharmaceutical Sciences, Department of Pharmacognosy, University of Graz, Graz, Austria

DOI 10.1055/s-0037-1608333

To fully understand the effectiveness of traditional medicines (TM), it is vital to investigate the chemical components of the raw herbal materials. This is a difficult task due to the complexity of the sample which can contain one or multiple herbs. LC/MS is a widely-used analytical technique as it is highly sensitive and is able to separate and identify the diverse chemical components in the TM. However, due to the large amounts of chemical information, the major challenge is actually screening the datasets and generating results rapidly and confidently. Here, we present a novel workflow that enables re- searchers to quickly identify chemical ingredients from a well-known traditional Chinese medicine, Yu Ping Feng San from a single LC/MS injection. Many active compounds such as furocoumarins, furochromones, isoflavonoids, triterpene saponins, and sesquiterpenes were detected in the different solvent extracts. These compounds were found to have many anti-proliferative, antioxidant and anti-inflammatory properties and through bioactivity tests, have shown to have significant inhibition for the expression of TNF- $\alpha$, IFN- $\gamma$ and IL$1 \beta$ in cells. This presentation will demonstrate a thorough investigation of the complex LC/MS data-set and deduction of the chemical components using an in-depth data analysis of identifying known knowns, unknown knowns, and unknown unknowns.

\section{Tu-Analytical Studies \& Natural Products Chemistry II- SL-03 Triple high-resolution $\alpha$-glucosidase/ $\alpha$-amylase/PTP1B inhibition profiling combined with HPLC-HRMS-SPE-NMR for identification of anti-diabetic constituents from Morus alba L.}

Authors Zhao $\mathrm{Y}^{1}$, Kongstad $\mathrm{K}^{1}$, Jäger $A K^{1}$, Staerk $\mathrm{D}^{1}$

Institute 1 Department of Drug Design and Pharmacology, Faculty of Health and Medical Sciences, University of Copenhagen, Copenhagen, Denmark DOI 10.1055/s-0037-1608334

Morus alba L. is one of the most well-known and widely distributed trees of the family Moraceae, and many parts of this plant are used for anti-diabetic purposes [1]. In the present study, the crude EtOAc extract of $M$. alba root bark was found to possess potent inhibition of $\alpha$-glucosidase, $\alpha$-amylase and protein-tyrosine phosphatase $1 \mathrm{~B}$ (PTP1B) with $\mathrm{IC}_{50}$ values of $1.70 \pm 0.72,5.16$ \pm 0.69 and $5.07 \pm 0.68 \mu \mathrm{g} / \mathrm{mL}$. The extract was subsequently investigated by triple high-resolution $\alpha$-glucosidase/ $\alpha$-amylase/PTP1B inhibition profiling combined with HPLC-HRMS-SPE-NMR [2]. This lead to the identification of six natural Diels-Alder adducts and seven isoprenylated flavonoids as potent $\alpha$ glucosidase and $\alpha$-amylase inhibitors with $I C_{50}$ values in the range of 0.60 \pm 0.09 to $27.15 \pm 7.10 \mu \mathrm{M}$ (for $\alpha$-glucosidase) and $1.22 \pm 0.34$ to 69.38 $\pm 8.58 \mu \mathrm{M}$ (for $\alpha$-amylase) respectively. These compounds were together with two 2-arylbenzofurans found to exhibit potent PTP1B inhibition with $\mathrm{IC}_{50}$ values ranging from $4.04 \pm 0.38$ to $21.67 \pm 3.38 \mu \mathrm{M}$ as well. This is the first report of all these compounds as $\alpha$-glucosidase, $\alpha$-amylase and/or PTP1B inhibitors. These findings provide the scientific rationale for developing M. alba into a polypharmacological herbal remedy.

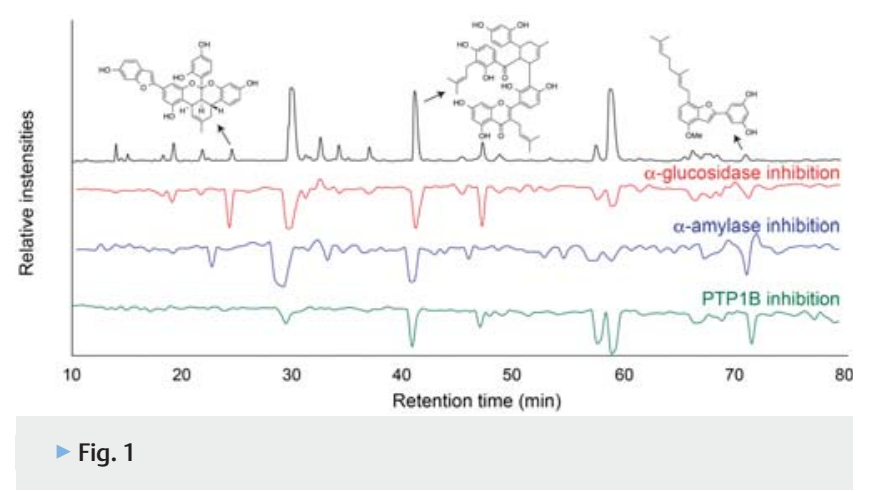

Yong Zhao acknowledges the Chinese Scholarship Council for a scholarship. Arife Önder is thanked for technical assistance.

[1] Grajek K, Wawro A, Kokocha D. Int J Pharm Sci Res 2015; 6: 3110 - 3122. [2]Wubshet SG, Tahtah Y, Heskes AM, Kongstad KT, Pateraki I, Hamberger B, Møller BM, Staerk D. J Nat Prod 2016; 79: 1063 - 1072. 


\section{Tu-Analytical Studies \& Natural Products Chemistry II- SL-04 Ultra-High Performance Supercritical Fluid Chromatography Applications for Natural Products Analysis}

\author{
Authors Isaac $\mathrm{G}^{1}$, Wang $\mathrm{M}^{2}$, Yuk $J^{1}$, Plumb $\mathrm{R}^{1}$, Wang $\mathrm{YH}^{2}$, Avula $\mathrm{B}^{2}$, \\ ElSohly $\mathrm{M}^{2}$, Khan $\mathrm{I}^{2}$ \\ Institute 1 Waters Corporation, Milford, United States; 2 University of \\ Mississippi, Oxford, United States \\ DOI 10.1055/s-0037-1608335
}

Ultra-high performance supercritical fluid chromatography (UHPSFC) is a separation technique that provides an increase in selectivity, efficiency and sensitivity. UHPSFC uses compressed $\mathrm{CO} 2$ as the primary mobile phase to leverage the chromatographic principles and selectivity of normal phase chromatography while providing the ease of use and method development simplicity of normal-phase LC. The use of CO2 as primary solvent results in significant savings in solvent costs and solvent waste disposal. The miscibility of $\mathrm{CO} 2$ with a wide range of polar and non-polar organic solvents has made the liquid $\mathrm{CO} 2$ based mobile phase versatile enough to separate a much wider variety of natural product compounds. UHPSFC in combination with sub- $2 \mu \mathrm{m}$ particles and either diode array ultraviolet, evaporative light scattering, or mass spectrometric detection has been shown to be a valuable technique for the analysis of natural products. In this study, sub- $2 \mu \mathrm{m}$ particle $\mathrm{CO} 2$ based UHPSFC was investigated for the separation and analysis of different natural products including terpene lactones and ginkgolic acids in Ginkgo biloba, cannabinoids in cannabis and cannabis products, volatiles in traditional medicine, beta-carotene, lipids and other compounds in different extracts. The method development and optimization involved the investigation of different columns, modifiers and instrument conditions. The benefit of UHPSFC will be highlighted and discussed compared to the traditional gas chromatography and normal phase chromatography for natural product analysis. For most of the applications better separation and selectivity was obtained with significant reduction in analysis time compared to alternative techniques such as GC which requires derivatization.

\section{Tu-Analytical Studies \& Natural Products Chemistry II- SL-05 Molecular Networking for Identification of Enhanced Chemical Inventories of Baltic Marine Fungi}

\section{Authors Parrot $\mathrm{D}^{1}$, Oppong-Danquah $\mathrm{E}^{1}$, Fan $\mathrm{B}^{1}$, Tasdemir $\mathrm{D}^{1}$}

Institute 1 GEOMAR Centre for Marine Biotechnology, Research Unit Marine Natural Product Chemistry, GEOMAR Helmholtz Centre for Ocean Research Kiel, Am Kiel-Kanal 44, Kiel, Germany

DOI 10.1055/s-0037-1608336

Molecular networking (MN) has revitalized natural product dereplication and metabolomics studies. By organizing the MS/MS spectra based on their fragment similarity, $\mathrm{MN}$ efficiently annotates the unknown derivatives of known compound clusters and reveal putative new molecular families [1]. In this study, over 220 fungi from Baltic Sea environment have been isolated and identified by $18 \mathrm{~S}$ and ITS sequencing. In order to activate biosynthetic gene clusters (BGCs) that often remain silent under artificial culture conditions, we used OSMAC (One-Strain-MAny-Compounds, changing culture conditions) and direct co-cultivation (against a competitive microorganism on the same Petri dish) techniques. The EtOAc extracts of the fungi (mono- and co-cultures) were tested against a large panel of phytopathogens and cancer cell lines. We comparatively profiled the secondary metabolome of the most active extracts by UPLC-QTOF-MS/MS-based MN, employing the open source GNPS database and XCMS statistical methods. Co-cultures of an Aspergillus species and the phytopathogen Botrytis cinerea showed increased cluster sizes of quinolones and echinulin-type indole alkaloids with a number of putative unknown analogues. Additionally, three putatively unknown clusters were identified. The seaweed-associated fungus Cadophora malorum was cultivated in four different media. Only the Potato Dextrose Broth (PDB) medium extract demonstrated activity against HepG2 liver cancer cell line $\left(\mathrm{IC}_{50}\right.$ value $11 \mu \mathrm{g} /$ $\mathrm{ml}$ ). The MN analysis showed this strain to be dominated by an enniatin-type cyclohexadepsipeptide cluster, plus two unknown molecular families. MN provided a visible and analyzable platform for quick identification and comparison of enhanced chemical inventories of large marine fungal extract libraries. In combination with bioactivity, it enabled us prioritizing the most promising extracts for subsequent bioassay-guided isolation studies. Hence, MN has great potential for accelerating the discovery of new, bioactive fungal metabolites.

[1] Quinn RA, Nothias LF, Vining O, Meehan M, Esquenazi E, Dorrestein PC. Trends Pharmacol Sci 2017; 38: $143-154$

\section{Tu-Analytical Studies \& Natural Products Chemistry II- SL-06 Comprehensive profiling of potential sources of cosmeceutical agents: Streptomyces griseus, Pseudonocardia alni and Flammulina velutipes}

Authors Tchoumtchoua $\mathrm{J}^{1}$, Hubert $\mathrm{J}^{1}$, Martinez $\mathrm{A}^{1}$, Borie $\mathrm{N}^{1}$, Bakiri $\mathrm{A}^{1}$, Nuzillard JM ${ }^{1}$, Ouazzani $\mathrm{J}^{2}$, Skaltsounis $\mathrm{AL}^{3}$, Fokialakis $\mathrm{N}^{3}$, Renault $\mathrm{JH}^{1}$ Institute 1 Institut de Chimie Moléculaire de Reims, UMR CNRS 7312, SFR CAP'SANTE, Université de Reims Champagne-Ardenne, 51097, Reims, Reims, France; 2 Centre de Recherche de Gif, Institut de Chimie des Substances Naturelles ICSN, Centre National de la Recherche Scientifique, CNRS, Avenue de la Terrasse, 91198, Gif-sur-Yvette, Cedex, Gif-sur-Yvette, France; 3 Division of Pharmacognosy and Natural Products Chemistry, School of Pharmacy, University of Athens, Panepistimioupoli Zografou, 15771, Athens, Athens, Greece

DOI 10.1055/s-0037-1608337

The FP-7 European project MICROSMETICS aims to discover and develop novel cosmeceutical agents from the microbial diversity. Within this context, more than 110 potential candidate strains including fungi and actinomycetes were selected from a Rational Drug Design Tool based on a functional prediction model, virtual screening and similarity search. Several extracts were generated and biologically evaluated for their skin-protecting, skin-lightening, and antioxidant properties.

The in-depth studies of three potential cosmeceutical strains Streptomyces griseus, Pseudonocardia alni and Flammulina velutipes are presented in this work. At first, the methanol and ethyl acetate extracts of each species were thoroughly investigated by applying a dereplication strategy combining ${ }^{13} \mathrm{C}$ NMR and LC-HRMS analysis without any isolation procedure. Specifically, a single ${ }^{13} \mathrm{C}$ NMR spectrum was recorded and a recently developed computeraided identification method was applied to identify the major compounds and the main classes of metabolites in the crude extracts [1]. The principle of this method is based on the calculation of a score resulting from a database searching method where predicted ${ }^{13} \mathrm{C}$ NMR chemical shift values of known natural metabolites are compared to experimental ${ }^{13} \mathrm{C}$ NMR signals of the crude mixture. In parallel, a fast LC-ESI-QTOF-MS methodology was applied in both positive and negative modes. Several minor components were identified on the basis of chromatographic and spectrometric features (Rt, UV, accurate $\mathrm{m} / \mathrm{z}$, proposed EC, DBE). In order to provide a detailed chemical profile of the active substances of the mixtures, all extracts were fractionated by Centrifugal Partition Chromatography. Overall, the global metabolite profiles of all six extracts revealed highly diverse molecular structures belonging to low molecular weight metabolites involved in primary metabolism (amino acids, nucleosides, fatty acids) as well as a diversity of secondary metabolites specific of each species and related to their biological activity.

[1] Bakiri et al., J Nat Prod. Apr 17 


\section{Tu-Analytical Studies \& Natural Products Chemistry II- SL-07 ${ }^{13} \mathrm{C}$ NMR-based Dereplication of Natural Products: A New Computer-Aided Method for Quick Metabolite Identification}

\author{
Authors Bakiri $\mathrm{A}^{1,2}$, Hubert $\mathrm{J}^{1}$, Reynaud $\mathrm{R}^{2}$, Lambert $\mathrm{C}^{2}$, Renault $\mathrm{JH}^{1}$, \\ Nuzillard $\mathrm{JM}^{1}$ \\ Institute 1 Institut de Chimie Moléculaire de Reims, Université de Reims \\ Champagne-Ardenne, Reims, France; 2 Givaudan, Active Beauty, Pomacle, \\ France \\ DOI 10.1055/s-0037-1608338
}

Recent advances in the development of dereplication strategies have brought new perspective to speed up natural product screening programs. Here is presented a ${ }^{13} \mathrm{C}$ NMR-based dereplication method dedicated to the chemical profiling of natural metabolite mixtures. Starting from a single ${ }^{13} \mathrm{C}$ NMR analysis and without need for fractionation, the method is based on an algorithm that compares the ${ }^{13} \mathrm{C}$ NMR chemical shifts obtained from the single spectrum of a crude natural extract with a set of predicted NMR data stored in a natural metabolite database. The matching between experimental chemical shifts and predicted data is evaluated by a score function reflecting the probability for a metabolite to be present within the extract. As an example, this method was applied on a crude alkaloid extract obtained from the leaves of Peumus boldus, resulting in the identification of eight major alkaloids including isocorydine, rogersine, boldine, reticuline, coclaurine, laurotetanine, N-methylcoclaurine, and norisocorydine, as well as three monoterpenes including $p$ cymene, eucalyptol, and $\alpha$-terpinene.

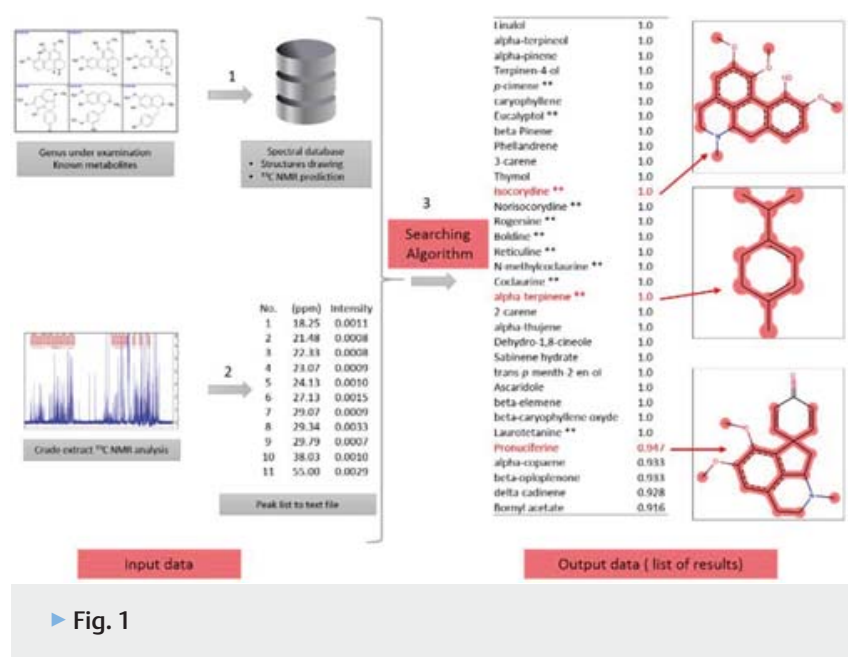

\section{Tu-Analytical Studies \& Natural Products Chemistry II- SL-08 Automated comparative metabolite profiling of large LC-ESIMS datasets in ACD/Labs, and data clustering on a new open-source web platform FreeClust}

Authors Bozicevic $\mathrm{A}^{1}$, Dobrzyński $\mathrm{M}^{2}$, De Bie $\mathrm{H}^{3}$, Gafner $\mathrm{F}^{4}$, Garo $\mathrm{E}^{1}$, Hamburger $\mathrm{M}^{1}$

Institute 1 Department of Pharmaceutical Sciences, University of Basel, Basel, Switzerland; 2 Institute of Cell Biology, University of Bern, Bern, Switzerland; 3 Advanced Chemistry Development, Inc, Toronto, Canada; 4 Mibelle Biochemistry, Mibelle AG, Buchs, Switzerland

DOI 10.1055/s-0037-1608339

The technological development of LC-MS instrumentation has led to significant improvements of performance and sensitivity. Complex samples, such as plant extracts can now be analyzed in high-throughput mode. Software tools allow efficient deconvolution of LC-MS chromatograms to obtain comprehen- sive information on single constituents. However, the systematic and unbiased comparative metabolite profiling of large numbers of complex LC-MS chromatograms remains a challenge. Existing software tools for comparative processing have certain limitations, such as black-box approach, lack of user friendliness, or limited options for data sharing.

We developed a two-step protocol comprising a comparative metabolite profiling tool integrated in ACD/Labs, and a web platform developed in R language designed for clustering and visualization of chromatographic data. Initially, all relevant chromatographic and spectroscopic data (retention time, molecular ions with the respective ion abundance, and sample names) are automatically extracted and assembled in an Excel spreadsheet. Afterwards, the file is loaded into an online web application equipped with various statistical algorithms where the user can compare and visualize the results in intuitive 2D heat maps.

Here we applied this processing workflow to LC-ESIMS profiles obtained with 69 honey samples. Within few hours of calculation with a standard PC, the LCESIMS chromatograms were deconvoluted. Honey samples were organized in clusters based on their metabolite profile similarities, thereby highlighting the common metabolite patterns and distributions among samples. Implementation in the ACD/Labs software package enables ulterior integration of other analytical data and in silico prediction tools for modern drug discovery.

\section{Poster Session}

Tuesday, 5th September, 2017

San Francisco - 16:00 - 18:00

\section{Tu-Poster Session 2-PO-01 Effect of rice bran extract on pancreas of rats fed a high-fat diet}

Authors Parklak $\mathrm{W}^{1}$, Munkong $\mathrm{N}^{2}$, Somnuk $\mathrm{S}^{1}$, Sompara $\mathrm{N}^{3}$, Naowaboot $\mathrm{J}^{3}$, Yoysungnoen $\mathrm{B}^{3}$, Lerdvuthisopon $\mathrm{N}^{3}$

Institute 1 Office of Graduate Studies, Faculty of Medicine, Thammasat University, Pathumthani 12120, Thailand; 2 Department of Pathology, School of Medicine, University of Phayao, Phayao 56000, Thailand; 3 Institute of Preclinical Science, Faculty of Medicine, Thammasat University, Pathumthani 12120, Thailand

DOI 10.1055/s-0037-1608340

As the water extract of rice bran $(R)$ was shown to ameliorate the pre-diabetes found in rats fed a high-fat diet [1], the effect of $R$ on the pancreas of the animal was investigated. Male Sprague Dawley rats were arranged into 5 groups, 8 rats each. Four groups of high-fat (65\% of total calories from fat) fed rats (HF) were studied against a control group (C) those were fed with standard chow. Leaving a group as the high-fat feeding alone, the other three were also co-fed with either 2,205 mg (HFR1) or 4,410 mg (HFR2) R/kg rat weight/day or $19.1 \mathrm{mg}$ metformin/kg rat weight/day (HFM). The HF showed a significant increase in mean \pm SEM of body weight gain, relative weight of visceral fat and liver but a decrease in that of pancreas when compared to $C$ $(0.49 \pm 0.01$ versus $0.58 \pm 0.01 \mathrm{~g}$ per $100 \mathrm{~g}$ rat $w \mathrm{t} ; \mathrm{p}<0.05)$. The values were improved in HFR1, HFR2 and HFM. The relative gene expression of insulin receptor substrate-2, glucose transporter-2 and glucokinase were significantly lower in $\mathrm{HF}$ than $\mathrm{C}$. Both $\mathrm{R}$ and $\mathrm{M}$ were able to increase the relative expression of the two former genes. Hematoxylin-eosin staining of the pancreas showed larger islets area in $\mathrm{HF}$ than $\mathrm{C}$. Senescence associated beta-galactosidase staining showed the denser stained materials in $\mathrm{HF}$ than $\mathrm{C}(0.488 \pm 0.012$ versus $0.407 \pm 0.007 ; p<0.001)$ indicating pancreatic senescence in HF. The staining in HFB1, HFB2 and HFM $(0.421 \pm 0.008,0.446 \pm 0.012$ and $0.430 \pm 0.009$, respectively) were significantly less than $\mathrm{HF}(p<0.05)$. In conclusion, there were substances in $R$ those can reduce pancreatic senescence found in highfat feeding rats. Therefore, further verification of those substances in $R$ is worth conducted.

[1] Kandee, et al, Thammasat Med J 2009;9:140 - 7. 


\section{Tu-Poster Session 2-PO-02 Kampferol-3 - 0 -glucopyranoside identified as the major Anti-oxidant glycoside from the Red kidney Bean (Phaseolus vulgaris) seed commonly consumed in Eastern Nigeria.}

Authors Umeokoli Blessing $\mathrm{O}^{1}$, Onyegbule $\mathrm{FA}^{1}$, Obi $\mathrm{C}^{1}$

Institute 1 Department of Pharmaceutical and Medicinal Chemistry, Nnamdi Azikiwe University, Awka, Nigeria, Awka, Nigeria

DOI 10.1055/s-0037-1608341

Plants and food consumed by man represents sources for human disease management. Aqueous and aqueous Methanolic extracts ( $80 \%$ Methanol) of the Nigeria grown red kidney beans, both showed the presence of one major compound. In this study, Kampferol-3 - 0 -glucopyranoside was isolated as the major and active compound. The structure was determined based on UV, MS, $1 \mathrm{H}-\mathrm{NMR}$ and 13C-NMR spectroscopic data. The HPLC chromatogram of both extracts and compounds reveals a single identical significantly major peak which is the major glycoside. In the bioassay with DPPH and superoxide scavenging activity, both water and aqueous methanolic extract with the purified compound showed significant activity; having IC-50 of 310, 187 and $10 \mu \mathrm{g} / \mathrm{ml}$ respectively; as against $25 \mu \mathrm{g} / \mathrm{ml}$ of standard antioxidant used. Oxygen free radicals, the reactive oxygen species (ROS) are also involved in cancer, cardiovascular diseases and various chronic liver diseases. Potent antioxidants quench these ROS aggravated degeneration. Our results revealed an insight for exploration of Kampferol-3-0 -glucopyranoside as an active compound for future anti-cancer therapeutics.

\section{Tu-Poster Session 2-PO-03 Inhibition of alpha-glucosidase and lipase activities of Phyllanthus emblica and Alpinia galanga}

Authors Chansriniyom $\mathrm{C}^{1}$, Likhitwitayawuid $\mathrm{K}^{1}$

Institute 1 Department of Pharmacognosy and Pharmaceutical Botany,

Faculty of Pharmaceutical Sciences, Chulalongkorn University, Phatumwan, Thailand

DOI 10.1055/s-0037-1608342

Two culinary anti-hyperlipidemic herbs, Phyllanthus emblica fruits and Alpinia galanga rhizomes, were evaluated for inhibition of alpha-glucosidase and lipase activities. The activities were determined using 4-nitrophenol- $\alpha$-D-glucopyranoside [1] and 4-methylumbelliferone oleate [2] as substrates for $\alpha$-glucosidase and lipase, respectively. The ethanolic extracts of $P$. emblica and $A$. galanga exhibited stronger anti- $\alpha$-glucosidase activities than acarbose $\left(\mathrm{IC}_{50}\right.$ of $168.380 \pm 1.227 \mu \mathrm{g} / \mathrm{mL}$ ), a positive control, with $\mathrm{IC}_{50}$ values of $0.333 \pm 0.001$ and $14.059 \pm 0.187 \mu \mathrm{g} / \mathrm{mL}$, respectively. Regarding anti-lipase activity, A. galanga ethanolic extract $\left(\mathrm{IC}_{50}\right.$ of $\left.35.654 \pm 0.656 \mu \mathrm{g} / \mathrm{mL}\right)$ showed stronger activity than $P$. emblica ethanolic extract $\left(\mathrm{IC}_{50}\right.$ of $\left.119.367 \pm 2.075 \mu \mathrm{g} / \mathrm{mL}\right)$; however, these extracts were much less active than the drug orlistat $\left(\mathrm{IC}_{50}\right.$ of 0.007 $\pm 0.000 \mu \mathrm{g} / \mathrm{mL}$ ). These results suggest that ingestion of $P$. emblica fruits and $A$. galanga rhizomes is beneficial for anti-diabetes and anti-hyperlipidemia.

[1] Limpanit R, Chuanasa T, Likhitwitayawuid K, Jongbunprasert, V, Sritularak, B. Rec Nat Prod 2016; 10: 609-616.

[2] Duangjai A, Ingkaninan K, Limpeanchob N. Nat Prod Res 2011; 25: 341 352.

\section{Tu-Poster Session 2-PO-04 Antimicrobial and Anti-Inflammatory Effects of Platycodon Tablets Added Platycodon grandiflorum root Fermented by Lactobacillus plantarum}

Authors Lee Ka $S^{1}$, Seong Bong $J^{1}$, Kim Sun $I^{1}$, Jee Moo $\mathrm{G}^{1}$, Kim Hyun $\mathrm{H}^{1}$, Song Young $\mathrm{E}^{2}$, Kang Jeong $\mathrm{H}^{3}$, Park Shin $\mathrm{Y}^{4}$

Institute 1 Ginseng \& Medicinal Plant Research Institute, CNARES, Geumsangun, Korea, Republic of (South); 2 Jeollabukdo Agricultural Research \& Extension Services, Iksan, Korea, Republic of (South); 3 Jellanamdo
Agricultural Research \& Extension Services, Naju, Korea, Republic of (South); 4 Fermented Food Science Division, National Institute of Agricultural Science, RDA, Wanju, Korea, Republic of (South)

DOI 10.1055/s-0037-1608343

Platycodon grandiflorum root (PGR) is one of the phlegm-relieving herb that helps reduce bronchial disease from the past. The saponin in PGR, platycoside compounds, has functions such as neuroprotective, antimicrobial, anti-inflammatory, anti-cancer, anti-allergy, improved insulin resistance and cholesterollowering properties.

In order to developing a tablet product that improved the functionality and preference of PGR, it was fermented using lactic acid bacteria (Lactobacillus plantarum N76-10 and 56-12), then freeze-dried and powdered. The tablets were created by PGR powder (PGRP) mixed with fermented PGR powder (FPGRP, 0, 1.0, 2.5, 5.0, 7.5, and 10\%). It was made of the $50 \%$ glucose, $35 \%$ PGRP in constant ratio of FPGRP, $2.0 \%$ hydroxypropyl methyl cellulose, and 3\% lemon flavor concentrate powder. The tablets were put into investigation for their preference of taste, antimicrobial and anti-inflammatory in accordance with amount of FPGRP. When it comes to preference of taste, the most favor is adding $5.0 \%$ of FPGRP. The antimicrobial activities were investigated for bacteria inducing bronchus diseases (Corynrbacterium diphtheriae, Klebsiella pnneumoniae, Staphylococcus aureus, and Streptococcus pyogenes) with tablets made in constant ratio of FPGRP. Tablet added 10\% FPGRP exhibited a strong microbial anti-proliferation in all four kinds of bacteria and was higher antimicrobial activity than tablet without FPGRP. Anti-inflammatory activity of tablets was investigated through inhibitory effect on the production of inflammatory meditator, nitric oxide (NO), tumor necrocis factor-alpha(TNF- $\alpha$ ), interleukin-6(IL-6) in lipopolysaccharide(LPS)-stimulated RAW264.7 macrophage. The Inhibition effect of LPS-induced NO production steadily increases up to adding $10 \%$ of FPGRP, and then the inhibition effect of pro-inflammatory cytokines such as TNF- $\alpha$ and IL- 6 slightly increases up to adding $5 \%$ of FPGRP, there was no remarkable rise in further samples.

Thus, A PGR tablet was made by adding FPGRP (5.0 10.0\%) and it was increased organoleptic quality, antimicrobial activity and anti-inflammatory. These studies may provide new product development for effective utilization on Platycodon grandiflorum root.

\section{Tu-Poster Session 2-PO-05 Anti-diabetic Effect of Fermented Loquat (Eriobotrya japonica Lindl.)}

Authors Kang Jeong $\mathrm{H}^{1}$, Lee You $\mathrm{S}^{1}$, Lee Sun $\mathrm{K}^{1}$, Kim Hee $\mathrm{K}^{1}$, Lee Ka $\mathrm{S}^{2}$, Song Young $\mathrm{E}^{3}$, Park Sin $\mathrm{Y}^{4}$

Institute 1 Jellanam-do Agricultural Research \& Extension Services, Naju, Korea, Republic of (South); 2 Ginseng \& Medicinal Plant Research Institute, CNARES, Geumsan, Korea, Republic of (South); 3 Jeollabuk-do Agricultural Research \& Extension Services, Iksan, Korea, Republic of (South); 4 National Institute of Agricultural Science, RDA, Wanju, Korea, Republic of (South) DOI 10.1055/s-0037-1608344

The loquats (Eriobotrya japonica Lindl.) have been well known for its medicinal effects on expectorants, diuretics and anti-edema. It was reported that loquats leaves contained large amounts of terpenoids and flavonoids with strong antioxidant, anti-inflammatory, and anticancer functions while loquats seed part exhibited hypoglycemic effect and anti-obesity (1). In this study, loquat fruits were fermented using Leuconostoc mesenteroides and/or Lactobacillus brevis $(2 \%, w / v)$ for 3 days at $37^{\circ} \mathrm{C}$ to reduce their strong sweetness and improve anti-diabetic effect. Fermented loquat fruits were evaluated their reducing sugar, corosolic acid contents by HPLC analysis, and anti-diabetic effect using a-glucosidase inhibition assay. As results of analysis, loquat fruits fermented by combined of Leuconostoc mesenteroides and Lactobacillus brevis showed the lowest content of reducing sugar $(4,733 \mathrm{mg} / 100 \mathrm{~g})$. The corosolic acid content, the representative anti-diabetic component, was increased by $47 \%(8.38 \mathrm{mg} / 100 \mathrm{~g})$, compared to the control (no fermentation). The a-glucosidase inhibition of fermented loquat fruits was $61.8 \% \sim 67.7 \%$. Especially, 
loquat fruits fermented by Leuconostoc mesenteroides and Lactobacillus brevis showed 2.3 times higher inhibition of a-glucosidase compared those of control loquat fruits.

[1] Eun Kim, Min-Sook Kim, Dong-Young Rhyu, Oh-jin Min, Hum-Young Baek, Yung-Jae Kim, and Hyeon-A Kim. Korean J. Food \& Nutr. 2009. 22: 159 165.1034.

\section{Tu-Poster Session 2-PO-06 Antioxidant and Antimicrobial Effects of Fermented Tumeric (Curcuma longa L.)}

Authors Kang Jeong $\mathrm{H}^{1}$, Ji Soo $\mathrm{H}^{1}$, Jo Gyeong $\mathrm{S}^{1}$, Lee Ka $\mathrm{S}^{2}$, Song Young $\mathrm{E}^{3}$, Park Sin $\mathrm{Y}^{4}$

Institute 1 Jellanam-do Agricultural Research \& Extension Services, Naju, Korea, Republic of (South); 2 Ginseng \& Medicinal Plant Research Institute, CNARES, Geumsan, Korea, Republic of (South); 3 Jeollabuk-do Agricultural Research \& Extension Services, Iksan, Korea, Republic of (South); 4 National Institute of Agricultural Science, RDA, Wanju, Korea, Republic of (South) DOI 10.1055/s-0037-1608345

Tumeric (Curcuma longa L.) belongs to the ginger (Zingiberaceae) and is yellowish and spicy ingredient for curry and has been treated as a medicinal herb for its strong anti-inflammatory function. The curcumin in turmeric is one of flavonoid with various beneficial functions such as anti-oxidant, antimicrobial activities and tonic effect (1). In this study, tumeric powder was fermented using Lactobacillus plantarum $(2 \%, w / v)$ for 3 days at $37^{\circ} \mathrm{C}$ to increase its curcumin content, antioxidant activity, and anti-microbial effect. Fermented turmeric was analyzed its curcumin content by HPLC analysis, antioxidant effect via DPPH radical scavenging activity, and the antimicrobial effect using agar diffusion method. The analysis results revealed that fermented tumeric with Lactobacillus plantarum contained a $11 \%$ higher curcumin content (22.8 mg/ $100 \mathrm{~g}$ dried weight) and a 3.1 times stronger DPPH radical scavenging activity compared to no fermented tumeric. Fermented tumeric also inhibited the growth of food poisoning bacteria such as Escherichia coli, Bacillus cereus, and Staphylococcus aureus. Especially Staphylococcus aureus and Bacillus cereus were strongly inhibited by treatment of fermented turmeric with the clear zone of $25.3 \sim 28.6 \mathrm{~mm}$.

[1] Hae-Yeon Choi. J. Korean Soc Food Sci Nutr. 2009. 38: 1202 - 1209

\section{Tu-Poster Session 2-PO-07 Method of separation of major off-odor component and change of components of Gastrodia elata Blume fermented by lactic acid bacteria}

Authors Young Eun $S^{1}$, In Sok $L^{1}$, Eun Ju $S^{1}$, Min Kyung $C^{1}$, Hyu $A$ h $H^{1}$, So Hee $S^{1}$, So Ra $C^{1}$, Sang Young $S^{1}$, Ki Kwon $L^{1}$, Ka Soon $L^{2}$, Jeong Hwa $K^{3}$, Shin Young $\mathrm{P}^{4}$

Institute 1 Jeollabuk do Agricultural Research \& Extension Services, Iksan, Korea, Republic of (South); 2 Ginseng \& Medicinal Plant Research Institute, CNARES, Geumsan, Korea, Republic of (South); 3 Jellanamdo Agricultural Research and Extension Services, Naju, Korea, Republic of (South);

4 Fermented Food Science Division, National Institute of Agricultural Science, RDA, Wanju, Korea, Republic of (South)

DOI 10.1055/s-0037-1608346

Gastrodia elata Blume often has been used for the treatment of headache, convulsions, hypertension and neurodegenerative diseases. The main active ingredients are gastrodin, 4-hydroxybenzyl alcohol, vanillyl alcohol, 4-hydroxybenzylaldehyde and parshin A, B, C, E. But Gastrodia elata has these unpleasant tastes and odors which are mainly caused by $\rho$-cresol (4-methylphenol) and butanal. For this reason, there is a need to reduce off-odor of Gastrodia elata as well as improve utilization as functional food materials using fermentation. This study found the method of separation of major off-odor component ( $\mathrm{p}$-cresol) of Gastrodia elata using various lactic acid bacteria. Methods to separate p-cresol known as the off-odor component of Gastrodia elata were as follows. First, the volatile component extracted by simultaneous steam distillation and extraction (SDE) followed by silica chromatography with n-pentane: diethyl ether as solvent fraction was used. For flavor test result on each fraction, strong off-odor, strong swine barn-like in n-pentane:diethyl ether (20:80) extraction fraction was attributed to p-cresol of Gastrodia elata through GC and GC-MS. In result of analysis of p-cresol of fermented Gastrodia elata, when fermented by Lactobacillus. sakei for 2 days reduced to up $54.7 \%$ compared with an unfermented sample. The parishin derivatives of Gastrodia elata were analyzed by HPLC to investigate change of them during fermentation of Gastrodia elata by lactic acid bacteria. Compared with the parishin derivatives of an unfermented Gastrodia elata, those of fermented Gastrodia elata by Lactobacillus. sakei were increased up to $18.3 \%$ for 2 days.

\section{Tu-Poster Session 2-PO-08 Genoprotective Effects of Crude Thai Bee Pollen and Its Extracts Against Doxorubicin, A Potent Genotoxic Compound in Human Lymphocytes}

Authors Ratanavalachai $T^{1}$, Thitiorul $S^{2}$, Jenkhetkan $W^{3}$, Jansom $C^{4}$, Itharat $\mathrm{A}^{5}$

Institute 1 Division of Biochemistry, Department of Preclinical Science, Faculty of Medicine, Thammasat University, 12120, Pathumthani, Thailand; 2 Division of Anatomy, Department of Preclinical Science, Faculty of Medicine, Thammasat University, 12120, Pathumthani, Thailand; 3 Ph.D. graduate program in Biochemistry and Molecular Biology, Faculty of Medicine, Thammasat University, 12120, Pathumthani, Thailand, Pathumthani, Thailand; 4 Research Center, Faculty of Medicine, Thammasat University, 12120 , Pathumthani, Thailand; 5 Department of Applied Thai Traditional Medicine, Faculty of Medicine, Thammasat University, 12120, Pathumthani, Thailand DOI 10.1055/s-0037-1608347

Bee pollen is traditionally used as a food supplement for strengthening the body and for medicinal uses to treat burn wounds and prostatitis (1). However, its genoprotective studies in human cells are still limited. This study evaluated the antigenotoxicity of Mimosa pudica L. Crude bee pollen (CBP) $(0.005-5 \mu \mathrm{g} / \mathrm{ml})$ from Chiangmai, Thailand, against doxorubicin; DXR $(0.1 \mu \mathrm{g} /$ $\mathrm{ml})$, a potent genotoxic chemotherapeutic agent in human lymphocytes by in vitro sister chromatid exchange (SCE) assay. The lipid fractions (LF) (11.3 g\% CBP) and defatted fractions (DF) (85 g\% CBP) obtained from CBP-Soxhlet extraction using diethyl ether were also analyzed. Results demonstrated that CBP pretreatment at $5 \mathrm{mg} / \mathrm{ml}$ induced cytotoxicity as few mitotic index was shown. Pretreatments of CBP at only $0.5 \mu \mathrm{g} / \mathrm{ml}$ and DF at only the highest dose of $5 \mu \mathrm{g} / \mathrm{ml}$ decreased DXR-induced SCE level by $26 \%$ and $28 \%$, respectively $(p<0.05)$. LF pretreatment at only the lowest dose of $0.00125 \mu \mathrm{g} / \mathrm{ml}$ slightly decreased the DXR-induced SCE level by $16 \%$, while LF pretreatments at higher doses $(0.0125-1.25 \mu \mathrm{g} / \mathrm{ml})$ did not. The data indicated that all CBP, LF, and DF at appropriate doses protected human lymphocytes from genotoxic damage induced by DXR. Its differential protective effects might result from different ratios of the active compositions. Phenolic content was 8-fold higher in DF than in LF. However, only LF contained $\alpha$-tocopherol, and it had 14-fold higher amount of ferulic acid than DF did. Further investigation of the effective dosage and its mechanism for genoprotective effects in humans is needed.

This work is supported by Research Fund, Thammasat University 2016.

[1]Komosinska-Vassev K, Olczyk P, Kazmierczak ], Mencner L, Olczyk K. Evid Based Complement Alternat Med 2015:297425.doi:10.1155/2015/297425 


\section{Tu-Poster Session 2-PO-09 Gelation Properties of Okra's Extract And Its Application In Strawberry Jam}

Authors Sompongse $\mathrm{W}^{1}$, Kamdee $\mathrm{S}^{1}$, Pornprasop $\mathrm{V}^{1}$

Institute 1 Department of Food Science and Technology, Faculty of Science and Technology, Thammasat University, Klong Luang, Pathumthani, 12120,

Pathumthani, Thailand

DOI 10.1055/s-0037-1608348

The mucilage of okra (Abelmoschus esculentus (L.) Moench), containing high amount of gum and pectin, possesses the structure of rhamnogalacturonan which has thickening properties [1,2]. Pectin in okra is a low-methoxy type, which forms gel with calcium ion at low sugar concentration. The aim of this research is to study the application of okra's extract as a thickening agent and to reduce sucrose content in strawberry jam. Firstly, the optimum temperature for the extraction of the okra was investigated. Physical analyses were determined by \% yield, flowing distance (viscosity) and color values (Hunter L* $\left.a^{*} b^{*}\right)$. It was found that at four different extracting temperatures, i.e, 50, 60, 70 and $80^{\circ} \mathrm{C}$, the optimum temperature for the extraction was $60^{\circ} \mathrm{C}$. Then, gelation properties of the okra's extract were investigated in 3 different concentrations of sucrose at 50, 60 and 70\% (by weight of okra's extract) and 3 different concentrations of calcium chloride at 2, 2.5 and 3\% (by total weight). Secondly, the physical analysis results showed that the sugar content at $50 \%$ and the calcium chloride concentration at $2 \%$ had the highest viscosity. Finally, an appropriate ratio of the okra's extract to reduce sucrose content in strawberry jam was further studied at either $60: 40$, or 40:60, or 80:20 (by sucrose weight) and the pectin content was varied at 0.5 and $1 \%$ (by total weight). The jam with the ratio of sucrose to okra's extract at 40:60, and of pectin at $0.5 \%$, possessed the highest spreadability and the lowest viscosity. Additionally, at this concentration the sensory scores in appearance and spreadability were higher than those of the control.

[1] Woolfe, M.L, Chaplin, M.F. and Otchere, G. J. Sci. Fd Agric. 1977; 28: 519 529.

[2] Bhat, U.S, Tharathan, R.N. Starch 1987; 39: 165 - 167.

\section{Tu-Poster Session 2-PO-10 Protective effect of Aronia melanocarpa (Chokeberry) extracts from benign prostatic hyperplasia in rats.}

Authors $\mathrm{Kim} \mathrm{NH}{ }^{1}$, Heo JD ${ }^{1}$, Kim JH${ }^{1}$, Hwang $\mathrm{KH}^{1}$, Jeong $\mathrm{E}^{2}$

Institute 1 Gyeongnam Department of Environment \& Toxicology, Korea

Institute of Toxicology, $660-844$, Jinju, Korea, Republic of (South);

2 Gyeongnam National University of Science and Technology, College of Life Science and Natural Resources, 660 - 758, Jinju, Korea, Republic of (South) DOI $10.1055 / \mathrm{s}-0037-1608349$

The target of the study is Aronia melanocarpa (Chokeberry), which is originate from the east province of North America and rich of phenolic compounds. In this study, Aronia extracts were treated orally in benign prostatic hyperplasia (BPH) rats for identifying its protective effects.

Accelerated solvent extraction is applied for the extraction of Aronia melanocarpa. Four extract samples (M2, M4, M9 and M10) at $30^{\circ} \mathrm{C}$ with $60 \%$ ethanol were selected, which showed the potent inhibition on 5 -alpha reductase activity in vitro. For in vivo experiments, 8-week old male SPF wistar rats were purchased $(n=30)$. All animals were castrated, then randomly divided into 6 groups (VC; vehicle control, PC; Saw palmetto extract, T1; M2, T2; M4, T3; M9, T4; M10). After a week of recovery phase, $100 \mathrm{mg} / \mathrm{kg}$ of the test materials were orally administered once-a-day for 6 weeks as well as subcutaneous injection of $3 \mathrm{mg} / \mathrm{kg}$ testosterone. Prostate glands were collected and measured at necropsy for calculating the Prostate Index. Blood serume chemistry, ELISA and qPCR were also perfomed.

As for the Prostate Index, those of M4 group showed the least value, although it was not statically significant compare to the VC. M2 group was revealed that tissue DHT levels were significantly decreased $(141.42 \pm 27.75 \mathrm{pg} / 50 \mathrm{mg}$ tissue) and prostatic 5-alpha reductase level showed the lowest in M2 group
(167.50 \pm 20.48 ), but not statistically significant. In the qPCR results, M2 and M4 group showed sharp decrease of AR, PCNA and PSA expression, but only PCNA levels were significantly different (VC: $55.08 \pm 43.87 \%$, M2: 12.05 $\pm 3.92 \%, M 4: 12.02 \pm 12.10 \%$ normalize with GAPDH). Additionally, administration of all four Aronia extracts could significantly lower the serum AST level. M9, M10 and Saw palmetto extracts treatment could not contribute to anymeaningful effects.

In this study, it was somewhat insufficient result since many parameters were statistically unclear. However, it was performed with only low concentration of extracts, so that it still have potentials of lowering the risk of BPH.

\section{Tu-Poster Session 2-PO-11 Radish germplasm with fusarium wilt-resistance as material for breeding disease-resistant varieties}

Authors Jeon YA ${ }^{1}$, Rhee $\mathrm{JH}^{1}$, Sung $\mathrm{JS}^{1}$, Baek $\mathrm{HJ}^{1}$, Hur OS ${ }^{1}$, Rho NY ${ }^{1}$

Institute 1 National Institute of Agricultural Sciences, Rural Development Administration, Jeonju, Korea, Republic of (South)

DOI 10.1055/s-0037-1608350

Radish, Raphanus sativus L, is used as food and also as medicine worldwide. The radish sprout exhibited the higher antioxidant activity than L-ascorbic acid1. Fusarium wilt of radish is a problem in continuous cropping in green houses2. Radish germplasm of 98 accessions collected from 15 countries was screened for resistance to fusarium wilt. Roots of 2-week-old seedlings of each accession were inoculated with Fusarium oxysporum f. sp. raphani isolate KACC 48265. The percentages of resistant seedlings which showed no disease symptoms or had discolored root and those of susceptible seedlings which severely wilted or died were investigated. Fusarium wilt-resistance for each accession ranged from $21 \%$ to $95 \%$ and 20 accessions showed the higher values than $80 \%$. Susceptibility for each accession ranged from 0 to $79 \%$ and 23 accessions showed the lower values than 10\%. The accession 'Zimska Crna' (IT32725) introduced from Macedonia showed 92\% resistance and zero susceptibility. Fusarium wilt-resistant radish accessions selected through mass screening of germplasm would provide new materials for breeding diseaseresistant varieties.

This study was carried out from the support of "Research Program for Agricultural Science \& Technology Development (Project No. PJ01192201)", National Institute of Agricultural Sciences, Rural Development Administration, Republic of Korea.

[1] Yoshiaki T, Yoshihito K, Tadashi F, Masatake N. J. Agricult Food Chem 2003; 51: $8061-8066$

[2] YS Lee, MW Lee. Biological control of plant diseases. Haworth Press Inc; 2007: 305

\section{Tu-Poster Session 2-PO-12 Comparison of Fatty Acid Compositions and Tocopherols in Perilla germplasm of South Korea}

Authors Sung JS $S^{1}$, Jeong $Y J^{1}$, Kim DJ', Lee $Y Y^{2}$, Jeon $Y A^{1}$, Ko $\mathrm{HC}^{1}$, Hur OS ${ }^{1}$, Ro $\mathrm{NY}^{1}$, Rhee $\mathrm{JH}^{1}$, Lee $\mathrm{MC}^{1}$, Baek $\mathrm{HJ}^{1}$

Institute 1 National Institute of Agricultural Sciences, Rural Development Administration, Jeonju, Korea, Republic of (South); 2 National Institute of Crop Science, Rural Development Administration, Jeonju, Korea, Republic of (South) DOI 10.1055/s-0037-160835

To select potential plant resources as natural antioxidants and functional materials, the evaluation of oil contents, fatty acids and tocopherols on 205 perilla accessions collected from South Korea was conducted. Total oil contents and fatty acid compositions were analyzed by Soxhlet extraction and gas chromatography [1]. The tocopherols were determined by high performance liquid chromatography [2]. The oil contents ranged from $33.9 \%$ to $48.6 \%$ with average content of $41.9 \%$. Linolenic acid had an average content of $72.4 \%$ and ranged from $52.8 \%$ to $76.7 \%$ content. IT178424 and IT185633 showed the highest linolenic acid content with $76.7 \%$ respectively. The tocopherol average 
concentrations of perilla were $6.4 \mu \mathrm{g} / \mathrm{g}$ of $\alpha$-tocopherol, $0.5 \mu \mathrm{g} / \mathrm{g}$ of $\beta$-tocopherol, $133.3 \mu \mathrm{g} / \mathrm{g}$ of $\gamma$-tocopherol, and $1.0 \mu \mathrm{g} / \mathrm{g}$ of $\delta$-tocopherol. IT105396 $(206.3 \mu \mathrm{g} / \mathrm{g})$ and IT103368 $(205.5 \mu \mathrm{g} / \mathrm{g})$ had high content of $\gamma$-tocopherol whereas IT191136 had the lowest $\gamma$-tocopherol $(60.5 \mu \mathrm{g} / \mathrm{g})$. Although there was a positive correlation $\left(r=0.739^{*}\right)$ between $\gamma$ - and $\delta$-tocopherol, there was no significant difference between oil compositions and tocopherols. IT178424, IT185633, IT105396 and IT103368 will be available as useful plant resources for natural antioxidants and functional foods.

This study was carried out from the support of "Research Program for Agricultural Science \& Technology Development (Project No. PJ012493)”, National Institute of Agricultural Sciences, Rural Development Administration, Republic of Korea.

[1] Ciftci, O. N, Przybylski, R, Rudzińska, M. Eur. J. Lipid Sci. Technol, 2012, 114: 794 - 800. doi:10.1002/ejlt.201100207

[2] Y.Y. Lee, H.M. Park, C.K. Lee, S.L. Kim, T.Y. Hwang, M.S. Choi, Y.U. Kwon, W.H. Kim, S.J. Kim, S.C. Lee, Y.H. Kim. Journal of Food Composition and Analysis. 2012, 27:70-80

\section{Tu-Poster Session 2-PO-13 The effect of Korean red ginseng on chronic non-bacterial prostatitis}

Authors Kim Su K${ }^{2}$, Ban Ju $Y^{1}$

Institute 1 Pharmacology of Dental, School of Medicine, Dankook University, Cheonan, Korea, Republic of (South); 2 Kohwang Medical Research Institute, School of Medicine, Kyung Hee University, Seoul, Korea, Republic of (South) DOI 10.1055/s-0037-1608352

Chronic prostatitis typically shows aging men. Urinary symptoms including frequency of passing urine and pain on passing urine are observed in patients with chronic prostatitis. In recent study, several studies showed that Korean red ginseng (KRG) has various effects of prevention and treatment to various diseases. We investigated whether KRG may repress the development of chronic non-bacterial prostatitis (CNP) in male Wistar rats.

7 weeks male Wistar rats were and divided into 5 groups (normal group, CNP group, positive group, KRG group $(0.25 \mathrm{~g} / \mathrm{kg})$, and KRG $(0.50 \mathrm{~g} / \mathrm{kg})$. In order to induce CNP in rats, rats were castrated and Beta-estradiol was injected subcutaneously everyday (s.c, $0.25 \mathrm{mg} / \mathrm{kg}$ ). After 4 weeks, all rats were sacrificed and their prostate and serum were analyzed.

Compared to the testosterone group as positive group, the KRG group showed similarly protective effect on CNP in histopathologic morphology of prostate and inflammation cytokines in serum. Results of immunohistochemistry showed decreased expressions of VEGFA, IL 6, IL-1ß, and TNF-alpha were also decreased in KRG group $(0.25 \mathrm{~g} / \mathrm{kg})$ and KRG $(0.50 \mathrm{~g} / \mathrm{kg})$.

These results suggested that KRG inhibited the development of CNP and might a useful herbal treatment or functional food for CNP.

\section{Tu-Poster Session 2-PO-14 Comparative study of antioxidant potential and cytotoxic effects of methanolic extracts from Lycium barbarum and Lycium ruthenicum berries}

Authors Castro $V^{1}$, Aierken $A^{2}$, Zhang $X^{2}$, Dias $A^{3}$

Institute 1 DYNAMIKLIZARD LDA, Avenida Alves Figueiredo, 1100. 4780-163 Santo Tirso, Santo Tirso, Portugal; 2 College of Veterinary Medicine, Northwest A\&F University, Yangling, Shaanxi, 712100 China, Yangling, Shaanxi, China; 3 Centre for the Research and Technology of Agro-Environment and Biological Sciences (CITAB-UM), AgroBioPlant Group, Department of Biology, University of Minho, Portugal, Braga, Portugal

DOI 10.1055/s-0037-1608592

Lycium barbarum (Lb) and Lycium ruthenicum $(\mathrm{Lr})$ are two species of goji and their fruits are commonly named as red goji and black gogi berries, respectively. These berries have been widely used as Chinese herbal medicines, foods, and teas. The aim of this study is to characterize methanolic fruit extracts of $L b$ and $L r$ and their in vitro antioxidant proprieties, using several in vitro tests (DPPH scavenging, iron chelation, inhibition of nitric oxide and superoxide production). Additionally, we further assessed their cytotoxic effects using HepG2 cells (hepatocytes). Results showed that Lb and Lr phytochemical HPLC profiles are very different. In terms of antioxidant activites, $L r$ revealed the best potential, compared with $L b$. Namely, in antiradicalar assays, DPPH and superoxide assay showed that extract from $L r$ have the highest radical scavenger capacity, with EC50 of 226.6 and $859.8 \mu \mathrm{g} / \mathrm{ml}$, respectively. Results for iron chelating activity revealed as well that $L r$ have the best capacity to chelate iron with an EC50 of $394.6 \mu \mathrm{g} / \mathrm{ml}$, almost two times more than $L b$. $L r$ also exhibited the greatest ability to inhibit nitric oxide production (EC50 $58.82 \mu \mathrm{g} / \mathrm{ml}$ ). We can conclude that extracts from these two species could act as a good antioxidant resources, namely $L r$, and might protect cells from damage caused by oxidative stress. So, first of all, we tested the cytotoxic effects of extracts on HepG2 cells. Results did not show any cytotoxic effects on HepG2 cells for the different concentrations tested (MTS test) up to $1 \mathrm{mg} /$ $\mathrm{ml}$. We can conclude that extracts from these two species are good antioxidants without significant cell toxicity.

This work was supported by Fundação para a Ciência e Tecnologia (FCT), project PEst- OE/AGR/UI4033/2014, INTERACT project -NORTE-01-0145-FEDER-000017, HEALTHKAKI, N

010672, Programa Operacional Regional do Norte, Portugal 2020. We acknowledge the Key Construction Program of International Cooperation Base in S\&T, Shaanxi Province (2015SD 0018), China.

\section{Tu-Poster Session 2-PO-15 Five Selected Underutilized Leafy Vegetables as Functional Foods in management of induced Myocardial Infarction in Male Wistar Albino Rats.}

Authors Okpuzor J $]^{1}$, Salisu $\mathrm{T}^{1}$

Institute 1 Department of Cell Biology \& Genetics, University of Lagos, Yaba, Lagos, Nigeria

DOI 10.1055/s-0037-1608353

These selected five leafy vegetables (Solanum nigrum L. var. virginicum (Black nightshade), Basella alba Linn. var. alba, Crassocephalum crepidioides (Benth.) S. Moore, Senecio biafrae Oliv. \& Hiern. and Launaea taraxacifolia Amin Ex. C. Jeffrey (Wild lettuce) are eaten in villages of South-Western Nigeria. This study evaluated their bio-protective potential in Isoproterenol-induced Myocardial Infarction in male Wistar albino rats.

Dried aerial parts of the selected vegetables were extracted with cold distill water using maceration techniques.

The isoproterenol-induced (ISO) myocardial infarction model was evaluated in 42 male Wistar albino rats (150 g to $200 \mathrm{~g}$ ), randomized into 7 groups of 6 rats each. Group 1(normal control) received saline, groups 2 to 6 were pretreated with $200 \mathrm{mg} \mathrm{kg}^{-1} \mathrm{bw}^{-1}$ of each plant extract as well as the mixture $(1: 1: 1: 1: 1)$ (group 7) while group 8 served as ISO control for 28 days. Then ISO drug dose of $20 \mathrm{mg} \mathrm{kg}^{-1}$ bw $^{-1}$ was subcutaneously administered on day 29 and 30 in groups $2-7$. The animals were sacrificed on day 31 and subsequently Lactate dehydrogenase (LDH) and $\mathrm{C}$ - reactive protein (CRP) were assayed using the supernatant from heart homogenates. Statistical package for social sciences (SPSS) software version 20 was used for the statistical analyses.

The highest concentrations of LDH and CRP were recorded in ISO control group not pre-treated with the vegetables extracts. A significant decline were apparent $(\mathrm{P}<0.05)$ in the parameters monitored in groups $2-7$ compared to controls. This result submits that the normal levels of LDH and CRP obtained from the vegetables pre-treated animals may be crucial in mitigating the impact of ISO and thus these leafy vegetables would be useful as functional foods and may be a good candidate for further studies. 


\section{Tu-Poster Session 2-PO-16 Establishment of Efficient Protocol for Rapid and Massive Micropropagation of Greek oregano (Origanum heracleoticum L.)}

Authors Hristozkova $\mathrm{M}^{1}$, Zayova $\mathrm{E}^{1}$, Stancheva $\mathrm{I}^{1}$, Geneva $\mathrm{M}^{1}$, Dimitrova $\mathrm{L}^{1}$, Petrova $\mathrm{M}^{1}$

Institute 1 Institute of Plant Physiology and Genetics, Bulgarian Academy of Sciences, Sofia, Bulgaria

DOI 10.1055/s-0037-1608354

Greek oregano (Origanum heracleoticum L, Lamiaceae) was used from ancient times in folk medicine. Presently is largely applied in pharmaceutical and food industry as a source of important biologically active substances with antioxidant and antimicrobial properties. The efficient and low-cost protocol for large-scale multiplication of this aromatic plant was developed using young stem tips explants. The explants were cultured on full and half strength Murashige and Skoog medium containing indole-3-butyric acid. Maximum shoot and root number were obtained on $1 / 2$ Murashige and Skoog medium supplemented with $0.1 \mathrm{mg} \mathrm{L}-1$ indole-3-butyric acid at the same time after four weeks of culture. The number of shoots per explant, shoot height, rooting percentage, the number of roots per plant and root length were collected and considered after the same cultural period. The highest percentage of survival $85 \%$ for this medicinal plant was recorded in a mixture of soil, sand and perlite $(2: 1: 1 \mathrm{v} / \mathrm{v} / \mathrm{v})$. This substrate mixture was most suitable for acclimatization of all propagated plants. After the adaptation period, the plants were transferred to the field. The plants bloomed within three months after transplantation without any phenotypic variations. An average of $90 \%$ of the acclimatized plants survived after transferring into the field. All the plants displayed normal development under the field conditions. Developed in vitro techniques could provide a promising alternative tool for large-scale field cultivation of in vitro propagated homologous Origanum heracleoticum plants.

This study was conducted with financial support from National Science Fund at the Bulgarian Ministry of Education and Science, Project DN06/7 17.12.16.

\section{Tu-Poster Session 2-PO-17 Elicitation of Bioactive Compounds by Yeast Extract in Shoot Culture of Dioscorea birmanica Prain \& Burkill}

\author{
Authors Jirakiattikul $Y^{1}$, Rithichai $P^{1}$, Jirapongpatta $R^{1}$, Itharat $A^{2}$ \\ Institute 1 Division of Agricultural Technology, Faculty of Science and \\ Technology, Thammasat University, Rangsit campus, Pathumthani, Thailand; \\ 2 Department of Applied Thai Traditional Medicine, Faculty of Medicine, \\ Thammasat University, Rangsit campus, Pathumthani, Thailand \\ DOI 10.1055/s-0037-1608355
}

Dioscorea birmanica Prain \& Burkill is a medicinal plant which its rhizome has been used with other medicinal plants for the treatment of cancers, septicemia diseases and AIDS [1]. It has been reported that the regenerated shoots of $D$. birmanica can synthesize and accumulate lower amount of bioactive compounds than those of mother rhizomes [2]. Therefore, the enhancement of bioactive compounds in the regenerated shoots by elicitation with yeast extract (YE) at different times of culture period was investigated. The 6-weeks old regenerated shoots were cultured on MS medium supplemented with or without $3 \mathrm{~g} / \mathrm{l}$ YE for 1, 2, 4 and 6 weeks. It was indicated that the shoots cultured on medium supplemented with $3 \mathrm{~g} / \mathrm{I}$ YE for 2 weeks contained the highest total saponin content of $1.23 \pm 0.05 \mathrm{mg}$ diosgenin $/ \mathrm{g}$ DW or 1.93 times higher than that of the non-YE shoots cultured for 2 weeks. However, YE had no effect on total phenolic content and DPPH radical scavenging activity of $D$. birmanica shoots cultured under aseptic conditions.

[1] Tewtrakul S, Itharat A. Bioorg Med Chem 2006; 14: 8707 - 8711

[2] Jirakiattikul Y, Rithichai P, Songsri O, Ruangnoo S, Itharat A. Acta Physiol Plant 2016; 38: 249.

\section{Tu-Poster Session 2-PO-18 Evaluation of Antiplasmodial and Immunomodulatory activities of Extracts of Endophytic fungi Isolated from four Nigerian Medicinal Plants}

Authors Ujam Nonye $\mathrm{T}^{1}$, Eze Peter $\mathrm{M}^{1}$, Umeokoli Blessing $\mathrm{O}^{2}$, Abba Chika $\mathrm{C}^{2}$, Okoye Festus $\mathrm{BC}^{2}$, Esimone Charles $\mathrm{C}^{1}$

Institute 1 Department of Pharmaceutical Microbiology and Biotechnology, Nnamdi Azikiwe University, Awka, Nigeria, Awka, Nigeria; 2 Department of Pharmaceutical and Medicinal Chemistry, Nnamdi Azikiwe University, Awka, Nigeria, Awka, Nigeria

DOI 10.1055/s-0037-1608356

Endophytic fungi are the most promising source for new drugs to combat the problem of antimicrobial resistance. Twelve fungal endophytes were isolated from leaves and stems of Azadirachta indica, Psiduim guajava, Newbouldia laevis and Agerantum conyzoides collected from southeastern Nigeria using standard methods. Solid state fermentation of the isolated fungi were carried out on rice media for 21 days at $22{ }^{\circ} \mathrm{C}$ and the fungal secondary metabolites were extracted using ethyl acetate. The extracts were tested for antiplasmodial activity in vivo against Plasmodium berghei (ANKA strain) using Peter and Reyley's curative test method while in vitro activity against Plasmodium falciparum was determined using WHO standardized microtest system. Immunomodulatory activity of the extracts was evaluated by cyclophosphamide induced myelosuppression. Active extracts were further subjected to DelayedType Hypersensitivity Response (DTHR) and Haemagglutination Inhibition Assay using SRBCs as antigens. The extracts were subjected to HPLC-DAD analysis for the detection of bioactive components. The extracts showed varying degrees of antiplasmodial activity. Growth of $\mathrm{P}$. berghei was significantly $(p<0.001)$ inhibited, curative effect ranges from 59.09-100\%. Schizont maturation of P. falciparum isolates was inhibited and the highest level of inhibition was observed at $1 \mathrm{mg} / \mathrm{ml}(\mathrm{p}<0.05)$. Seven $(58.33 \%)$ of the fungal extracts showed significant counteracting effect to cyclophosphamide-induced reduction in total WBC, \% neutrophil, lymphocyte and monocyte level $(p<0.05)$. There was significant inhibition of DTHR and heamagglutination induced by SRBC in rats up to 78.49 and $69.23 \%$ respectively. Correlation was positive and statistically significant between malaria and immunomodulatory activities observed $(P=0.036, r=0.786)$. HPLC-DAD analysis revealed the presence of ferulic acid, luteolin, peperine, phenylacetic acid, rocaglamid and epicatechin which have been reported to have antiplasmodial and immunomodulatory activities. Extracts of endophytic fungi isolated from these $\mathrm{Ni}$ geria medicinal plants possess antiplasmodial and immunomodulatory activities which portends a novel approach for malaria therapy.

\section{Tu-Poster Session 2-PO-19 Isolation and characterization of a novel $O$-methyltransferase from the tropical liana Triphyophyllum peltatum}

Authors Passolt $L^{1}$, jindaprasert $A^{2}$, Le Tran $T^{1}$, Seupel $R^{3}$, Bringmann $G^{3}$, Fester $\mathrm{K}^{1}$

Institute 1 Department of Pharmaceutical Biology, Institute of Pharmacy, Leipzig University, 04103, Leipzig, Germany; 2 Faculty of Agroindustry, King Mongkut's Institute of Technology Ladkrabang, Bangkok, Thailand; 3 Institute of Organic Chemistry I, Würzburg University, Am Hubland, 97074, Würzburg, Germany

DOI 10.1055/s-0037-1608357

The 'part-time' carnivorous plant [1] Triphyophyllum peltatum (Dioncophyllaceae) produces a large variety of naphthoquinones and naphthylisoquinoline alkaloids. These secondary metabolites show promising bioactivities [2] such as antimalarial and antitumoral effects. $O$-methylations are important steps in the biosynthetic pathway of these natural products. Our aim was to investigate involved $\mathrm{O}$-methyltransferases (OMTs).

In the course of an expressed sequence tag analysis of shoot cultures of Triphyophyllum peltatum, several partial sequences of putative OMTs were 
found. One of these sequences, TPOMT1, was completed by RACE-PCR and the full-length cDNA was expressed in E. coli. A cladistic analysis identified the isolated OMT as a member of group II S-adenosyl-I-methionine-dependent OMTs. It is currently unknown in which metabolic pathways the isolated OMT is involved since it does not methylate the examined precursor naphthoquinones and naphthylisoquinoline alkaloids. Instead, caffeic acid, which is a key component of plant metabolism, as well as orcinol and resorcinol are methylated by the newly discovered OMT.

[1] Bringmann G, Schlauer J, Wolf K, Rischer H, Buschbom U, Kreiner A, Thiele F, Duschek M, Aké Assi L. Carniv PI Newsl 1999; 28: 7 - 13

[2] Ibrahim SR, Mohamed GA. Fitoterapia 2015; 106: $194-225$

\section{Tu-Poster Session 2-PO-20 Malonyl-coenzyme A:21-Hydroxypregnane 21-O-Malonyltransferase Isoforms in Digitalis lanata}

Authors Meitinger $\mathrm{N}^{1}$, Fink $J^{1}$, Tropper $\mathrm{M}^{1}$, Kreis $\mathrm{W}^{1}$

Institute 1 FAU Erlangen-Nürnberg, Department Biology, Staudtr. 5, 91058 Erlangen, Germany, Erlangen, Germany

DOI 10.1055/s-0037-1608358

Recent research on elucidating cardenolide biosynthesis focused on the formation of the butenolide ring characteristic for cardenolides. It was suggested that the butenolide ring is formed from appropriate pregnane-21-O-malonyl hemiesters without participation of an enzyme [1].

Malonyl-coenzymeA:21-hydroxypregnane-21-O-malonyltransferase (21MaT) is an enzyme that catalyzes the formation of pregnane-21-O-malonyl hemiesters by transferring a malonyl moiety from malonyl-coenzymeA onto suitable 21hydroxypregnane derivatives. Even though $21 \mathrm{MaT}$ was characterized and purified from Digitalis purpurea [3], genes encoding 21MaTs have not yet been identified.

We here reinvestigated enzymatic pregnane 21-0-malonylation and found that more than one $21 \mathrm{MaT}$ isoform is present in $D$. lanata. $21 \mathrm{MaTs}$ convert a broader substrate range than previously assumed [2] and can be distinguished on the basis of their acyl-acceptor specificity. Fractionated ammonium sulfate precipitation separates two isoforms, one preferring 53-21-hydroxypregnane derivatives and one preferring the corresponding $5 \alpha$ - and $\Delta^{4}$-derivatives. Subsequent chromatofocusing of the fractions indicated the existence of a third isoform. The different isoforms prefer either $3 \beta$ - $O$-acetyl- $5 \beta$-pregane-14 $\beta, 21$ diol-20-one (21MaT1; IEP app. pH 5.5), desoxycorticosterone (21MaT2, IEP app. pH 5.3) or 5 3 -preganane-3,20-dion-21-ol (21MaT3, IEP app. pH 5.0) as substrate. $21 \mathrm{MaTs}$ may hence have a role not only in cardenolide metabolism, which is also supported by demonstrating $21 \mathrm{MaT}$ activity in the cardenolidefree species Arabidopsis thaliana [3]. Different 21MaT isoforms are now further characterized and purified focusing on the purification of $21 \mathrm{MaT2}$.

[1] Pádua, R. M, et al. Tetrahedron 2016, 72: 4556 - 4563.

[2] Kuate, S. P, et al. Phytochemistry 2008, 69: 619-626.

[3] Höhn, S. Dissertation, in preparation

\section{Tu-Poster Session 2-PO-21 Microbial Biotransformation of Ruscogenins}

Authors ÖZÇıNAR ÖZGE ${ }^{1}$, Özgür TA², Yusufoglu Hasan $S^{3}$, Kivçak B $B^{1}$, Bedır E ${ }^{4}$ Institute 1 Department of Pharmacognosy Ege University Faculty of Pharmacy, izmir, Turkey; 2 Bionorm Natural Products Production \& Marketing Corp, Izmir, Turkey; 3 Department of Pharmacognosy, Salman Bin Abdulaziz University, Al Kharj, Saudi Arabia; 4 Department of Bioengineering, Izmir Institute of Technology, Izmir, Turkey

DOI 10.1055/s-0037-1608359

Microbial transformation plays a vital role in the preparation of new oxygenated derivatives. Cunninghamella genus that has been widely used in transformation of steroids, also has frequently been used as microbial model of mammalian drug metabolism [1]. Endophytes that colonize living internal tissues of plants without causing any disease symptoms can produce novel bioactive compounds and interact with their hosts via special enzymes and chemicals. Therefore, in recent years, they have been considered for their use as biocatalysts [2]. Based on several clinical studies, oral supplements of Ruscus aculeatus is recommended to treat chronic venous lymphatic insufficiency. But there is a big gap in literature in regards to the metabolism studies of ruscogenins, the major active constituents of Ruscus aculeatus L. rhizomes [3]. Together with our previous study [4], microbial biotransformation of ruscogenins (Ruscogenin and Neoruscogenin) by C. blakesleeana, endophytic fungi Neosartorya hiratsukae and Alternaria sp. afforded twenty five new compounds. The structures were elucidated by HR-MS, 1D- and 2D NMR analyses. As far as can be ascertained from the literature, a few studies carried out with ruscogenin but these are the first microbial transformation studies performed on neoruscogenin.

This project is supported by Ege University Scientific Research Project $15 E C Z 011$ and we are very grateful to Bionorm Natural Products for providing Ruscogenins.

[1] Donova MV, Egorova OV. Applied Microbiology and Biotechnology 2012; 94: 1423 - 1447

[2] Borges WD, Borges KB, Bonato PS, Said S, Pupo MT. Current Organic Chemistry 2009; 13: 1137 - 1163

[3] Masullo M, Pizza C, Piacente S. Planta Med 2016; 82: 1513 - 1524

[4] Özçinar Ö, Tağ Ö, Kivçak B, Bedir E. Planta Med 2016; 82: 727

\section{Tu-Poster Session 2-PO-22 In vitro effects of Trichoderma secondary metabolites on Phaeoacremonium aleophilum}

Authors Carro-Huerga $G^{1}$, Mayo $S^{1}$, Rodríguez-González Á , Suárez Villanueva $\mathrm{V}^{1}$, González-López $\mathrm{O}^{1}$, Gutiérrez $\mathrm{S}^{2}$, Casquero $\mathrm{PA}^{1}$

Institute 1 Research Group of Engineering and Sustainable Agriculture, Research Institute of Environment, Natural Resources and Biodiversity, Universidad de León, Leon, Spain; 2 Research Group of Engineering and Sustainable Agriculture, Area of Microbiology, University School of Agricultural Engineers, Universidad de León, Ponferrada, Spain

DOI 10.1055/s-0037-1608360

Trichoderma species have an important relevance as biocontrol or biological agents inhibiting phytopathogenic fungi growth. Some of the secondary metabolites Trichoderma produces have shown antifungal activity. Phaeoacremonium aleophilum has been described as one of the first fungi colonizers which cause the Grapevine Trunk Disease.

The aim of this study is to evaluate the efficiency of an array of trichoderma species mainly isolated from old vineyards in controlling this important grapevine wood pathogen.

This in vitro antifungal experiment was carried out using a celophane membrane over PDA growth medium. The membrane allows secondary metabolites to pass through whereas fungi mycelium is retained ${ }^{1}$.

Results show (Figure 1) T100 to be the strain with the highest inhibitory capacity percentage, being well over $50 \%$. The following group of strains in the ranking, T077, T075, T084, T115, T127, T120, T080 and T074 exhibit a capacity between 30 and $40 \%$. Finally, the strains coming after this group continue in a decreasing succession reaching an inhibitory capacity less than $10 \%$. 


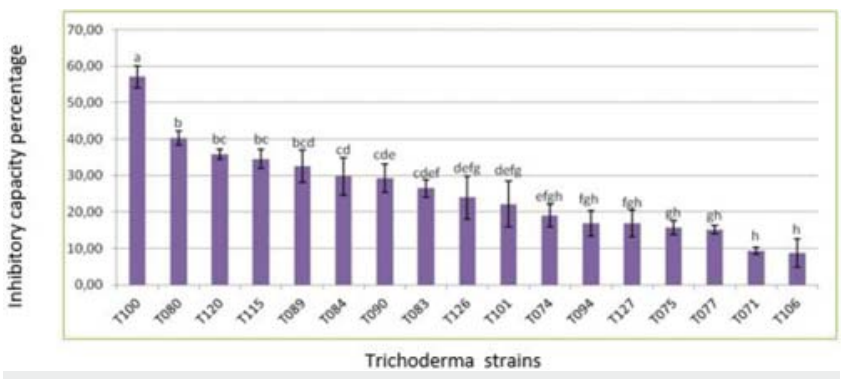

Fig. 1: Isolated Trichoderma strains with inhibitory capacity against $P$. aleophilum. Error bars are depicted. Different letters show significant differences. (Fisher's LSD. $\mathrm{p}<0.05$ ).

Pathogen growth is decreased by trichoderma secondary metabolites, stronly depending on trichoderma strain. Further trials are needed to identify the most efficient trichoderma strains at controlling Phaeoacremonium aleophilum. [1]. Mayo, S, S. Gutiérrez, M.G. Malmierca, A. Lorenzana, M.P. Campelo, R. Hermosa, and P.A. Casquero. 2015. Front. Plant Sci. 6: 1- 11.

\section{Tu-Poster Session 2-PO-23 Induced production of metabolites from the endophytic fungus Chaetomium sp. through co-culture with autoclaved Pseudomonas aeruginosa}

Authors Ancheeva $\mathrm{E}^{1}$, Akone Sergi $\mathrm{H}^{1,2}$, Mándi $\mathrm{A}^{3}$, Kurtán $\mathrm{T}^{3}$, Daletos $\mathrm{C}^{1}$, Proksch $P^{1}$

Institute 1 Institute of Pharmaceutical Biology and Biotechnology HeinrichHeine-University, Universitaetstrasse 1, Geb. 26.23, 40225 Duesseldorf, Germany, Duesseldorf, Germany; 2 Faculty of Science, Department of Chemistry University of Douala PO Box 24157, Douala, Cameroon, Douala, Cameroon; 3 Department of Organic Chemistry University of Debrecen PO Box 400, 4002 Debrecen, Hungary, Debrecen, Hungary

DOI 10.1055/s-0037-1608361

Co-cultivation of the endophyte Chaetomium sp. with an autoclaved Gramnegative bacterium Pseudomonas aeruginosa on rice medium afforded six new compounds, including cheatoisochorismin (1), shikimeran B (2) and four new butenolide derivatives (3-6) [1]. Among the isolated compounds, chaetoisochorismin (1) features a rare 3-carbomethoxy-phenylpyruvate core structure, which is proposed to be biosynthetically derived from isochorismic acid $[2,3]$. The structures of the new compounds were elucidated by HRESIMS, 1D and 2D NMR analysis. The absolute configurations of 1 and 2 were established by ECD calculations as well as by the modified Mosher's method. In addition, cocultivation of Chaetomium sp. with $P$. aeruginosa resulted in an up to 33-fold increase of the accumulation of constitutively present fungal secondary metabolites compared to the axenic fungal control. Notably, the employed experimental protocol included autoclavation of $P$. aeruginosa after four-day preincubation, which offers a safer experimental alternative in comparison with mixed fermentation employing living human pathogenic bacterial cultures. Thus, the results of this work support the potential of mixed co-cultivation experiments with $P$. aeruginosa and serve as a further example of the successful application of co-culture as an important tool to trigger biosynthesis of new compounds and accumulation of constitutively present metabolites.
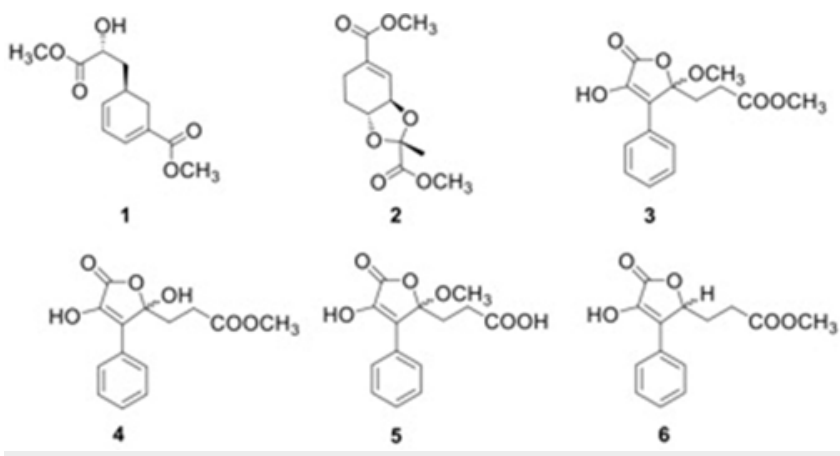

Fig. 1

[1] Ancheeva E, Küppers L, Akone S, Ebrahim W, Liu Z, Mandi A, Kurtan T, Lin W, Orfali R, Rehberg N, Kalscheuer R, Daletos G, Proksch P. Eur J Org Chem 2017; [Accepted manuscript]

[2] Zamir L O, Nikolakakis A, Bonner C A, Jensen R A. Bioorg Med Chem Lett 1993; 3: 1441 - 1446 .

[3] DeClue M S, Baldridge K K, Kast P, Hilvert D. J Am Chem Soc 2006; 128: $2043-2051$.

\section{Tu-Poster Session 2-PO-24 New approaches to handle old compounds - the generation of microcystin and nodularin derivatives with "clickable" features}

Authors Moschny $\mathrm{J}^{1}$, Schneider $\mathrm{P}^{2}$, Lorenzen $\mathrm{W}^{3}$, Jahns $\mathrm{S}^{3}$, Enke $\mathrm{H}^{3}$, Kramer $\mathrm{D}^{3}$, Niedermeyer Timo $\mathrm{HJ}^{1}$

Institute 1 Institute of Pharmacy, RG Biogenic Drugs, Martin-LutherUniversität Halle-Wittenberg, Hoher Weg 8, 06120 Halle (Saale), Germany; 2 Interfaculty Institute of Microbiology and Infection Medicine, EberhardKarls-Universität Tübingen, Auf der Morgenstelle 28, 72076 Tübingen, Germany; 3 Cyano Biotech GmbH, Magnusstr. 11, 12489 Berlin, Germany DOI 10.1055/s-0037-1608362

Lately, new crosslinking techniques have been developed to study the function of compounds in cellular processes. These techniques became known as bioorthogonal or so-called "click" reactions [1]. They are biorthogonal because they involve functional groups not found in nature. Thus, a semisynthetic approach is required to introduce the "clickable" groups into natural products - a sometimes difficult and time-consuming process.

The cyanobacterial cyclic peptides microcystins and nodularins are thoroughly studied natural products, but still their function within cyanobacteria remains unclear [2]. They have also been discussed as new, potent anti-cancer agents due to their efficient inhibition of eukaryotic protein phosphatases. However, their high toxicity potentially makes them unsuitable for clinical use [3]. Microcystin and nodularin derivatives with "clickable" features might help to gain new insights into the physiology of cyanobacteria and the biological function of these specialized molecules. Additionally, this might make them amenable for clinical use, e.g. by allowing to construct antibody-drug conjugates based on these toxins. Thus, we established rapid and feasible techniques to introduce azide- and alkyne groups into microcystins and nodularins, e. g. precursor directed biosynthesis: Supplementation of azide and alkyne functionalized tyrosine derivatives to a microcystin-YR producing Microcystis sp. strain resulted in their incorporation into this microcystin congener.

[1] Patterson DM, Nazarova LA, Prescher JA. ACS Chem. Biol. 2014; 9: 592 605

[2] Kaplan A, Harel M, Kaplan-Levy RN, Hadas O, Sukenik A, Dittmann E. Front. Microbiol. 2012; 3: 138

[3] McCluskey A, Sim ATR, Sakoff JA. J. Med. Chem. 2002; 45: 1151 - 1175 


\section{Tu-Poster Session 2-PO-25 The identification of tetrahydrocannabinolic acid synthase in the non-aqueous secretions of the storage cavities from Cannabis sativa glandular trichomes}

Authors Rodziewicz $\mathrm{P}^{1}$, Loroch $\mathrm{S}^{2}$, Feldmann $\mathrm{I}^{2}$, Schumbrutzki $\mathrm{C}^{2}$, Kayser $\mathrm{O}$ Institute 1 Technische Universität Dortmund, Dortmund, Germany;

2 Leibniz-Institut für Analytische Wissenschaften - ISAS - e.V, Dortmund, Germany

DOI 10.1055/s-0037-1608363

Cannabis sativa is an important herbaceous species cultivated since ancient times due to its unique medical and recreational properties, but also as a source of valuable seed oil and high quality fibre. Among numerous phytochemicals synthesized in this herb, cannabinoids represent the unique class of secondary metabolites, which largely contribute to the pharmacological properties of this species. More than 100 cannabinoids have been described in the literature, but $\Delta^{9}$-tetrahydrocannabinolic acid (THCA), cannabidiolic acid (CBDA) and cannabichromenic acid (CBCA) are present in the largest quantities in this plant. Cannabinoids are synthesized in glandular trichomes present mainly on female flowers and their main reservoirs are storage cavities of these hair-like structures [1].

Tetrahydrocannabinolic acid synthase catalyzes the unique oxidative cyclization of cannabigerolic acid (CBGA) into THCA, which is a direct precursor of the mind-affecting compound $-\Delta^{9}$-tetrahydrocannabinol (THC). Thus, it is considered to be a key enzyme controlling the psychoactive properties of $C$. sativa. In previous research it was suggested that THCA synthase is secreted into the storage cavities of glandular trichomes, where it might also synthesize the final product - THCA [2]. However, no direct evidence on protein level was presented.

To examine the putative extracellular location of THCA synthase, the storage cavity secretions from $C$. sativa trichomes were obtained and submitted for proteomic analysis. By using mass spectrometry we were able to identify the THCA synthase in the non-aqueous content of the storage cavities from $C$. sativa glandular trichomes.

[1] Andre CM, Hausman J-F, Guerriero G. Front. Plant Sci. 2016; 7:19

[2] Sirikantaramas S, Taura F, Tanaka Y, Ishikawa Y, Morimoto S, Shoyama Y. Plant Cell Physiol. 2005; 46, 1578 - 1582

\section{Tu-Poster Session 2-PO-26 Foliar Spraying of Salicylic Acid Alters Secondary Metabolites and Radical Scavenging Activity of Water Stressed Thymus vulgaris $\mathrm{L}$.}

Authors Salama $\mathrm{O}^{1}$, Khalil $\mathrm{N}^{1}$, Fekry $\mathrm{M}^{2}$, Bishr $\mathrm{M}^{3}$, El-Zalabani $\mathrm{S}^{2}$

Institute 1 Faculty of Pharmaceutical Sciences and Pharmaceutical Industries, Future University in Egypt, Cairo, Egypt; 2 Faculty of Pharmacy, Cairo, Egypt; 3 Mepaco Medifood, Al-Sharqeya, Egypt

DOI 10.1055/s-0037-1608364

Polyphenolic compounds are known to be one of the most valuable secondary metabolites produced in plants [1]. They have several medicinal and pharmacological importance [2]. They are known to play a physiological resistance mechanism in plants to help them face different types of stresses [3]. The present study aims to investigate the effect of different molar concentrations of the plant growth regulator; salicylic acid (SA), on some secondary metabolites of Thymus vulgaris $L$. exposed to decreasing amounts of irrigating water. The accumulation of flavonoids and polyphenols in the alcoholic extracts in different plant treatments was measured. The total flavonoid content in control plants was $6.135 \pm 0.327$ rutin equivalent/g dry weight and increased in drought plants (receiving $25 \%$ field capacity) sprayed with $3 \mathrm{mM}$ SA to reach $32.055 \pm 0.095 \mathrm{mg}$ rutin equivalent $/ \mathrm{g}$ dry weight. Also, total phenolic content increased from $8.476 \pm 0.344$ to $68.510 \pm 1.241 \mathrm{mg}$ gallic acid equivalent $/ \mathrm{g}$ dry weight in the same plant treatment. Radical scavenging activity on DPPH free radical was measured for the different plant treatments. IC 50 for control plants was $74.36 \pm 0.39 \mu \mathrm{g} / \mathrm{ml}$ and decreased to reach $36.55 \pm 0.86 \mu \mathrm{g} / \mathrm{ml}$ in drought plants sprayed with 3mM SA. Comparison between the polyphenolic content in control plants and plants with highest total polyphenol content was carried out using LC/MS. Several flavonoids and polyphenolic compounds were identified.

[1] Lin D, Xiao M, Zhao J, Li Z, Xing B, Li X, Kong M, Li L, Zhang Q, Liu Y, Chen H, Qin W, Wu H, Chen S. Molecules 2016; 21: 1374.

[2] Alu'datt M, Rababah T, Alhamad M, Al-Mahasneh M, Almajwal A, Gammoh S, Ereifej K, Johargy A, Alli I. Food Chem 2017; 218: $99-106$.

[3] Mazid M, Khan T, Mohammad F. Biology and Medicine 2011; 3: 232 - 249.

\section{Tu-Poster Session 2-PO-27 Regeneration of Plantago species plants from explants useable for Agrobacterium tumefaciens-mediated transformation}

Authors Klein J', Munkert J $]^{1}$, Stadler R²

Institute 1 Pharmaceutical Biology, Department of Biology, FAU, Erlangen,

Germany; 2 Molecular Plant Physiology, Department of Biology, FAU,

Erlangen, Germany

DOI 10.1055/s-0037-1608365

The genus Plantago is worldwide distributed and several of its members contain bioactive metabolites such as iridoids and polysaccharides, having anitoxidant and anti-inflammatory acitivity and the genus Plantago is therefore used as medicinal plants [1]. There are only few reports on regeneration of Plantago species as well as Agrobacterium tumefaciens-mediated transformation [2]. We here describe regeneration protocols for $P$. major, media and lanceolata as well as successful genetic transformation of $P$. lanceolata. Shoots were best regenerated from stem explants with around $54 \%$ and $98 \%$ efficiency for $P$. major and $P$. media, respectively. P. lanceolata was best regenerated from mature leaf explants ( $60 \%$ efficiency). T-DNA transfer was achieved by infiltration or incubation of $P$. lanceolata mature leaf explants with $p 9-355$ GUS in YNB Medium with acetosyringon $(40 \mathrm{mg} / \mathrm{L}$ ) and antibiotics (spectinomycin + rifampicin). Successful T-DNA-transfer was verified by GUS staining directly after co-cultivation. After $24 \mathrm{~h}$ pre-culture, 30 min incubation and $9 \mathrm{~d}$ co-cultivation all explants showed GUS-stained areas. The established regeneration and transformation protocol now offers a tool to study Plantago species in more detail with regard to natural product synthesis especially iridoids, which is the target pathway we are interested in.

[1] Zhou Q, Lu W, Niu Y, Liu J, Zhang X, Gao B, Akoh CC, Shi H, Yu LL. J Agric Food Chem 2013; 61: $6693-6702$

[2] Pommerrenig B, Barth I, Niedermeier M, Kopp S, Schmid J, Dwyer RA, McNair RJ, Klebl F, Sauer N. Plant Physiol 2006; 142: 1427 - 1441

\section{Tu-Poster Session 2-PO-28 Biotechnological development and biological activity of Balkan endemic Sideritis scardica Griesb.}

Authors Danova $\mathrm{K}^{1}$, Wolfram $\mathrm{E}^{2}$, Pedrussio $\mathrm{S}^{2}$, Koleva $\mathrm{P}^{1}$, Aneva $\mathrm{I}^{3}$, Evstatieva $\mathrm{L}^{3}$

Institute 1 Institute of Organic Chemistry with Centre of Phytochemistry, Bulgarian Academy of Sciences, Sofia, Bulgaria; 2 Zürcher Hochschule für Angewandte Wissenschaften, Department of Life Sciences and Facility Management - Institut für Chemie und Biotechnologie, Wädenswil, Switzerland; 3 Institute of Biodiversity and Ecosystem Research, Bulgarian Academy of Sciences, Sofia, Bulgaria

DOI 10.1055/s-0037-1608366

In vitro culture of Balkan endemic medicinal plant Sideritis scardica was previously initiated and different approaches for influencing the productivity of polyphenolics have been applied [1]. The present work discusses the spectrophotometric and HPTLC phenolics and flavonoids phytochemical profiling, DPPH radical scavenging activity and lipase and acetylcholinesterase inhibition assay of total methanol extract of plant material of the in vitro cultured plant. 
The following plant growth regulators were added to the control medium: $0.2 \mathrm{mg} / \mathrm{L}$ benzyl adenine (BA) $+0.02 \mathrm{mg} / \mathrm{L}$ 1-Naphthaleneacetic acid (NAA) (Sm); $0.2 \mathrm{mg} / \mathrm{L}$ BA $+0.5 \mathrm{mg} / \mathrm{L}$ NAA (Sr_1), $0.2 \mathrm{mg} / \mathrm{L}$ BA $+1.0 \mathrm{mg} / \mathrm{L}$ NAA (Sr_2), $0.5 \mathrm{mg} / \mathrm{L} \mathrm{BA}+0.5 \mathrm{mg} / \mathrm{L} \mathrm{NAA}\left(\mathrm{Sr}_{-} 3\right.$ ) and $0.5 \mathrm{mg} / \mathrm{L} \mathrm{BA}+1.0 \mathrm{mg} / \mathrm{L} \mathrm{NAA}$ (Sr_4). As a general observation all treatments increased significantly the survival rate of explants, as well as polyphenolics productions as compared with the non-treated control. Strong relation between polyphenolic content and radical scavenging capacity were recorded and one probable lipase and and 2 - 4 components with acetylcholinesterase inhibition activity were identified. As a result of the work in vitro culture systems for optimal biomass production and secondary metabolites yield were selected.

This work was financed by the PhytoBalk project (BSRP, grant No. IZEBZO_142989).

[1] Koleva P, Petrova N, Krumova S, Velikova V, Aneva I, Evstatieva L, Wolfram E, Danova K (2016) Planta Med 2016; 82(S 01): S1-S 381

\section{Tu-Poster Session 2-PO-29 Effect of growth regulators and photoperiod on endogenous phytohormonal levels and polyphenolic production in Artemisa alba cell aggregate cultures} Dobrev $\mathrm{P}^{3}$, Danova $\mathrm{K}^{2}$

Institute 1 Zürcher Hochschule für Angewandte Wissenschaften, Department of Life Sciences and Facility Management - Institut für Chemie und Biotechnologie, Wädenswil, Switzerland; 2 Institute of Organic Chemistry with Centre of Phytochemistry, Bulgarian Academy of Sciences, Sofia, Bulgaria; 3 Institute of Experimental Botany, Czech Academy of Sciences, Prague, Czech Republic

DOI 10.1055/s-0037-1608367

Cell aggregate lines of Artemisia alba were selected and developed in liquid cultures: ER_3 $\left[0.1 \mathrm{mg} / \mathrm{l} N^{6}\right.$-benzyladenine $(B A)+1.5 \mathrm{mg} / \mathrm{l}$ indole-3-butyric acid (IBA)] and ER_3_NAA $[0.1 \mathrm{mg} / \mathrm{l}$ BA $+1.5 \mathrm{mg} / \mathrm{l}$ naphtylacetic acid (NAA)], grown in the dark, gyratory shaker, 100 rpm; ER_3_hv and ER_3_NAA_hv were grown in the same conditions, but at $16 / 8 \mathrm{~h}$ photoperiod. Spectrophotometric assay of the total phenolic and flavonoid compounds was performed and the samples were also tested by HPTLC analysis with chlorogenic acid, as well as 1,3-, 1,5-, 3,5-, 3,4- and 4,5-dicaffeoylquinic acids, scopoletin and fraxidin-8-glucoside as reference compounds. The analysis showed general similarity in the tested samples. Photoperiod, however seemed to have an influence on glucosylation of the samples. While dark grown suspensions exhibited biosynthesis of both the aglycons (scopoletin and fraxidin) as well as their respective glucosides (scopolin and fraxidin glucoside), the $16 / 8$ photoperiod suspensions produced predominantly the glucosylated derivatives. These data were confirmed also by the UHPLC analysis. 3,5-dicaffeoylquinic acid was the predominant component in all samples. In terms of endogenous cytokinin production, a significant drop of their levels was observed in suspensions, where NAA represented the exogenously added auxin. The latter samples were also characterized by a significant stimulation of polyphenolics, comparable to those produced by the differentiated shoot cultures of the plant. The obtained results are of practical interest for the targeted biotechnological delivery of scopoletin and fraxidin, which represent a higher phytopharmacological potential as compared with their glucosylated derivatives.

Joint Scientific Research Project between the CAS and BAS (BAS-17 - 17).

\section{Tu-Poster Session 2-PO-31 Optimization and preparation of Methylcellulose edible film combined with of Ferulago angulata essential oil (FEO) nanocapsules for food packaging applications}

\section{Authors Esmaeili $\mathrm{A}^{1}$, Ebrahimzadeh $\mathrm{FM}^{1}$}

Institute 1 Department of Chemical Engineering, North Tehran Branch, Islamic Azad University, PO Box 19585/936, Tehran, Iran, Tehran, Iran DOI 10.1055/s-0037-1608368

The films based on methylcellulose with biodegradable and antioxidant activity incorporated with nano capsule suspension containing F. angulata essential oil were developed. Oil extraction and identification of $\mathrm{F}$. angulata essential oil compounds was done. Nano capsule suspension containing F. angulata essential oil was prepared by ultrasonic bath. The films were prepared by a casting method in three different ratios. The mechanical properties, colour, light transmission, antioxidant activity and release rate characteristics of the films were studied. The addition of nano capsule suspension to methylcellulose films decreased the thickness, tensile strength but increased the percentage elongation at break (\%) and lightness. High antioxidant activity and a prolonged release of $F$. angulata essential oil were also reported. Five factors design of Response Surface Methodology were used to optimize the thickness, holding time and anti-oxidant effect of edible film based on methylcellulose incorporated with nano capsule suspension containing F. angulata essential oil. Design of experiments was carried out by the software: Minitab 17 (Sigma package). Optimization of thickness, 2, 2-diphenyl-1-picrylhydrazyl radical scavenging and holding time would yield the best mixture proportions of methylcellulose and nano capsule suspension 30\%, 30\% and 70\%; oil.

\section{Tu-Poster Session 2-PO-33 In vitro release and biological activities of Carum copticum essential oil (CEO) loaded chitosan nanoparticles}

Authors Esmaeili $A^{1}$, Asgari $A^{1}$

Institute 1 Department of Chemical Engineering, North Tehran Branch, Islamic Azad University, PO Box 19585/936, Tehran, Iran, Tehran, Iran DOI 10.1055/s-0037-1608369

In recent years, the unparalleled and functional properties of essential oils have been extensively reported, but the sensitivity of essential oils to environmental factors and their poor aqueous solubility have limited their applications in industries. Hence, we encapsulated CEO in chitosan nanoparticles by an emulsion-ionic gelation with pantasodium tripolyphosphate (TPP) and sodium hexametaphosphte (HMP), separately, as crosslinkers. The nanoparticles were analyzed by Fourier transform infrared spectroscopy (FT-IR), Ultravioletvisible spectroscopy (UV-Vis), Differential scanning calorimetry (DSC), Scanning Electron Microscope (SEM) and Dynamic light scattering (DLS). The encapsulation efficiency (EE) and loading capacity (LC) of CEO in chitosan nanoparticles increased with the increase of initial CEO amount. The nanoparticles displayed an average size of $30-80 \mathrm{~nm}$ with a spherical shape and regular distribution. In vitro release profiles exhibited an initial burst release and followed by a sustained CEO release at different pH conditions. The amount of CEO release from chitosan nanoparticles was higher in acidic $\mathrm{pH}$ to basic or neutral $\mathrm{pH}$, respectively. The biological properties of $\mathrm{CEO}$, before and after the encapsulation process were evaluated by 2, 2-diphenyl-1-picrylhydrazyl radical (DPPH) and agar disc diffusion method, respectively. The results indicated the encapsulation of CEO in chitosan nanoparticles could be protected the quality. 
Tu-Poster Session 2-PO-34 Reducing cytotoxicity and fabricating mucoadhesivity of pluronic F127 in situ gels by xyloglucan (a natural polysaccharide)

Authors Tantishaiyakul $V^{1}$, Sai Laxmi Rangabhatlaa $\mathrm{A}^{2}$, Boonrat $\mathrm{O}^{2}$, Hirun $\mathrm{N}^{3}$, Ouiyangkul $P^{2}$

Institute 1 PSU Center of Excellence for Drug Delivery System, Faculty of Pharmaceutical Sciences, Prince of Songkla University, 90110, Songkhla,

Thailand; 2 Department of Pharmaceutical Chemistry, Faculty of

Pharmaceutical Sciences, Prince of Songkla University, 90110, Songkhla,

Thailand; 3 School of Pharmacy, Walailak University, 80161, Nakhon Si

Thammarat, Thailand

DOI 10.1055/s-0037-1608370

Xyloglucan from the seed of Tamarindus indica (TSX) is a natural polysaccharide widely used as a food additive and a cosmetic ingredient. It comprises (1 -

$4)$ - $\beta$-D-glucan backbone, with three of every four glucose residues substituted with $(1-6)$ - $\alpha$-D-xylose and some xylose residues are substituted with (1 - 2)$\beta$-D-galactose. TSX has various pharmacological activities including antitumor and immune stimulating activity, it can reduce UV-induced repression of the delayed type hypersensitivity response and promote skin regeneration by direct influence on cell proliferation. Unmodified TSX cannot form gel but it has suitable properties for use in pharmaceutical formulations such as biocompatibility, biodegradability and mucoadhesivity. The aim of this study was to prepare the blends of TSX and Pluronic F-127 (PF) to produce mucoadhesive in situ hydrogels with possibility to reduce cytotoxicity of PF. Aqueous solutions of PF (> $15 \mathrm{wt} \%$ ) are known to undergo thermogelling upon heating. However PF at high concentrations that forms gel is cytotoxic. The thermoresponsive in situ hydrogels were prepared from co-solutions of 18 and $20 \mathrm{wt}$ \% PF with 0.1 wt \% TSX (18PF/0.1TSX and 20PF/0.1TSX). Based on MTT assay on MC 3T3-E1 cells, extractables released from PF/0.1TSX are cytocompatible with similar cell viability to the control (culture medium) whereas those from PF are cytotoxic with cell viability of $54-60 \%$ compared with the control. Therefore the addition of 0.1 TSX results in a significant decrease cytotoxicity of PF. Furthermore PF/0.1TSX gels exhibit improved mucoadhesive strength against mucin compared with PF gels. According to micellization and gelation temperatures analyses using test tube inversion, DSC and rheological measurements, 18PF/0.1TSX and 20PF/0.1TSX are solution at room temperature and can turn to gel at body temperature. Therefore these PF/0.1TSX blends may be useful as injectable thermally responsive mucoadhesive gel for sustained drug delivery.

\section{Tu-Poster Session 2-PO-35 The raft forming chewable tablets of curcumin for stomach specific delivery}

Authors Kerdsakundee $\mathrm{N}^{1,2}$, Mahattanadul $\mathrm{S}^{2,3}$, Wiwattanapatapee $\mathrm{R}^{1,2}$ Institute 1 Department of Pharmaceutical Technology, Faculty of Pharmaceutical Sciences, Prince of Songkla University, Hat Yai, 90112, Songkhla, Thailand; 2 Phytomedicine and Pharmaceutical Biotechnology Excellence Research Center, Faculty of Pharmaceutical Sciences, Prince of Songkla University, Hat Yai, 90112, Songkhla, Thailand; 3 Department of Clinical Pharmacy, Faculty of Pharmaceutical Sciences, Prince of Songkla University, Hat Yai, 90112, Songkhla, Thailand

DOI 10.1055/s-0037-1608371

Curcumin has been widely used in healthcare for a long time because of the promising pharmacological activities and good safety profile. However, its poor aqueous solubility and rapid metabolism are major challenges in designing a suitable dosage form. Novel raft forming chewable tablets incorporating curcumin-Eudragit $^{\circledR}$ EPO solid dispersions are developed to deliver the curcumin in stomach for treatment of gastric ulcers. The solvent evaporation method was used for preparing the curcumin-Eudragit ${ }^{\circledR}$ EPO solid dispersions. Then, the curcumin-Eudragit ${ }^{\circledR}$ EPO solid dispersions were incorporated in raft forming chewable tablets compressed by direct compression method. The developed raft forming chewable tablets were orange in colour, with a convex surface. They floated within 5 to $14 \mathrm{~s}$ and maintain floating more than $24 \mathrm{~h}$ in acidic medium. The amount of curcumin released was between 40 to $70 \%$ in $8 \mathrm{~h}$ depending on the tablet composition. The selected formulation mainly comprises $100 \mathrm{mg}$ of sodium alginate, $50 \mathrm{mg}$ of calcium carbonate, $90 \mathrm{mg}$ of curcumin solid dispersion and other tablet excipients. This study demonstrates the successful development of the novel raft forming chewable tablets containing curcumin solid dispersions as a promising carrier for a stomachspecific delivery of lipophilic compounds.

[1] Prajapati VD, Jani GK, Khutliwala TA, Zala BS. J Control Release 2013; 168: $151-165$

[2] Kerdsakundee N, Mahattanadul S, Wiwattanapatapee R. Eur J Pharm Biopharm 2015; 94: $513-520$

[3] Patel M, Tolia P, Bhimani B, Patel U. Int J Pharm Res Bio Sci 2014; 3: 580 597

\section{Tu-Poster Session 2-PO-36 Preclinical Studies of Antileishmanial Activity of Caesalpinia spinose and Potential Utility in the treatment of patients with Cutaneous Leishmaniasis}

Authors Robledo $\mathrm{SM}^{1}$, Barrera $\mathrm{YA}^{2}$, Arboleda $\mathrm{M}^{3}$, Quintero $\mathrm{J}^{1}$, Restrepo $\mathrm{AM}^{1}$, Vélez ID ${ }^{1}$

Institute 1 Programa de Estudio y Control de Enfermedades TropicalesPECET, Facultad de Medicina, Universidad de Antioquia, Medellin, Colombia; 2 Bionest, Duitama, Colombia; 3 Instituto Colombiano de Medicina Tropical, Medellín, Colombia

DOI 10.1055/s-0037-1608372

The World Health Organization has prioritized the validation of traditional medicine products to strengthen the role this medicine plays in keeping populations healthy. Caesalpinia spinose is used in the management of ulcers for their astringent, antiseptic, anti-inflammatory and healing properties. A gum extract of $C$. spinose was formulated as Alyeyuba ${ }^{\circledR}[1]$. The antileishmanial activity of the gum extract was tested in Leishmania braziliensis amastigotes using flow cytometry and the cytotoxicity was analyzed in human U937 cells by MTT (3-(4,5-Dimethylthiazol-2-yl)-2,5-Diphenyltetrazolium Bromide) method [2]. The in vivo therapeutic response was determined in the experimental model for cutaneous leishmaniasis in hamster [2]. A preliminary evaluation of the therapeutic potential of these formulations as antileishmanial treatment in humans was done in seven patients with uncomplicated cutaneous leishmaniasis. Alyeyuba ${ }^{\circledR}$ showed moderate in vitro activity against amastigotes of Leishmania and no cytotoxicity in U-937 macrophages. Five of 6 hamsters (83.3\%) infected by L. braziliensis treated with Alyeyuba ${ }^{\circledR}$ cream and $6 / 6$ hamsters $(100 \%)$ treated with Alyeyuba ${ }^{\circledR}$ lotion, both administered twice per day for 28 days, cured at the end of the study. Indeed, seven patients treated with a combination of cream (twice a day) and lotion (three times a day) during 28 days showed complete cure 45 days after the end of treatment without adverse reactions. All patients remain cured. Alyeyuba ${ }^{\circledR}$ may represent an option for treating uncomplicated $\mathrm{CL}$ and therefore will be an opportunity to evaluate the efficacy and safety of this phytotherapeutic by randomized controlled trials to have an alternative for the treatment of $\mathrm{CL}$.

[1] https://es.unibrander.com/colombia/3665674CO/alyeyuba.html

[2] Mesa LE et alRev Soc Bras Med Trop. 2017; 50: 52 - 60.

\section{Tu-Poster Session 2-PO-37 Enhancement of antibacterial activity against microorganisms involved in diabetes foot ulcer by virgin coconut oil}

Authors Sungkharak $S^{1,2}$, Maneenuan $D^{1,2}$, Ditsopa $B^{1}$, Ungphaiboon $S^{1,2}$ Institute 1 Department of Pharmaceutical Technology, Faculty of Pharmaceutical Sciences, Prince of Songkla University, Hatyai 90112, Songkhla, Thailand; 2 Drug Delivery System Excellence Center, Faculty of 
Pharmaceutical Sciences, Prince of Songkla University, Hatyai, 90112, Songkhla, Thailand

DOI 10.1055/s-0037-1608373

The purpose of this study was to evaluate the enhancement of the antibacterial activity by virgin coconut oil (VCO) against microorganisms involved in diabetes foot ulcer including Methicillin resistant Staphylococcus aureus (MRSA) ATCC 43300, S. aureus ATCC 6538 and Escherichia coli ATCC 8739. Virgin coconut oil cream (VCOC) was developed and composed of all ingredients following non-ionic buffered cream base pH 5.5 (Thai National Hospital Formulary, 2016) (NBCB), except $10 \% \mathrm{w} / \mathrm{w}$ of liquid paraffin was replaced with VCO. Chitosan ( $10 \% \mathrm{w} / \mathrm{w})$ as microparticle form, prepared by the ionic gelation with the use of sodium tripolyphosphate, and gentamicin sulfate $(0.1 \% \mathrm{w} / \mathrm{w})$ were incorporated into both creams. The antibacterial activities of all creams were evaluated by agar well diffusion method.

It was found that VCOC as well as NBCB did not showed antibacterial activities against all bacteria tested as they produced no zone of inhibition. On the other hand, the antibacterial activities of chitosan and gentamicin incorporated in VCOC were significantly increased compared to the NBCB used. The inhibition zone diameter of $10 \% \mathrm{w} / \mathrm{w}$ chitosan in VCOC against MRSA, S. aureus and $E$. coli were $8.23 \pm 0.06 \mathrm{~mm}, 8.43 \pm 0.15 \mathrm{~mm}$ and $8.87 \pm 0.12 \mathrm{~mm}$, respectively, while there were no inhibition zone of the chitosan in NBCB. In addition, $0.1 \%$ $\mathrm{w} / \mathrm{w}$ gentamicin in VCOC had better antibacterial activities than the gentamicin in NBCB. The inhibition zone diameters of the gentamicin in VCOC against MRSA, S. aureus and E. coli $(25.73 \pm 0.42 \mathrm{~mm}, 26.17 \pm 0.92 \mathrm{~mm}, 24.80$ $\pm 0.80 \mathrm{~mm}$, respectively) were significantly higher than those of antibiotic in NBCB (and $21.57 \pm 1.72 \mathrm{~mm}, 20.67 \pm 0.76 \mathrm{~mm}, 21.60 \pm 0.35 \mathrm{~mm}$, respectively) ( $p$ value $<0.05$ ). The findings of this study indicated that VCO could enhance the antibacterial activity of chitosan and gentamicin.

\section{Tu-Poster Session 2-PO-38 Anti-hyperglycemic activity of anthocyanins loaded in non-ionic niosomes}

Authors Balcazar $N^{1}$, Acin $\mathrm{S}^{1}$, Ruiz $\mathrm{F}^{2}$, Lopera $\mathrm{Y}^{2}$, Colorado $\mathrm{D}^{1}$

Institute 1 Full Professor, School of Medicine, Universidad de Antioquia, Calle 62 \# 52 - 59, Medellin, Colombia; 2 AOXLAB SAS, Calle 32F \# 74B-122,

Medellin, Colombia

DOI 10.1055/s-0037-1608374

Anthocyanins (ACNs) are polyphenols that possess antioxidant and anti-inflammatory effects [1] In addition, it has been reported that the ACNs affect hyperglycemia and insulin secretion in diabetic animal models [2]. However, there is some controversy regarding their bioavailability. In the present study, the anti-hyperglycemic activity of oral delivery of ACN-loaded niosomes was investigated and evaluated.

Anthocyanins extracted from Vaccinium Meridionale Sw (Mortiño) by the Supercritical Fluid Extraction (SFE) method, and loaded into Niosomes, were prepared by agitation and sonication using non-ionic surfactant vesicles. The prepared niosomal formulation was characterized by its morphology using Transmission Electron Microscopy (TEM) and administrated in drinking water to obese, insulin resistant mice. We evaluated the effect of ACN-loaded niosomes on hyperglycemia, glucose and insulin intolerance and insulin blood levels in C57BL/6 J mice fed with a high-fat diet (HFD). After 2 weeks of treatment, the animals were sacrificed, and blood samples were taken to evaluate glucose and insulin plasma levels and lipid profile.

We demonstrate that $\mathrm{ACN}$-loaded niosomes were more effective in improving insulin resistance and glucose intolerance in mice fed an HFD, than ACN alone. Data showed no change in triglycerides, HDL and LDL cholesterol levels. However, administration of ACN-loaded niosomes reduced insulin, glucose and total cholesterol in mice fed with an HFD.

[1] Kim, H. J, Tsoy, I, Park, J. M, Chung, J. I. et al, FEBS Lett. 2006, 580, 1391 1397.

[2] Sasaki, R, Nishimura, N, Hoshino, H, Isa, Y. et al, Biochem. Pharmacol. 2007, 74, $1619-1627$.

\section{Tu-Poster Session 2-PO-39 Glucan particles as anti-inflammatory agent and drug delivery system for natural compounds in experimentally induced intestinal inflammation in vivo}

Authors Rotrekl D ${ }^{1}$, Vochyánová Z ${ }^{1}$, Paráková L L , Salo I ${ }^{3}$, Šalamúnová P³, Hanuš $]^{3}$, Štěpánek $F^{3}$, Hošek J ${ }^{1}$

Institute 1 Department of Molecular Biology and Pharmaceutical Biotechnology, Faculty of Pharmacy,? University of Veterinary and

Pharmaceutical Sciences Brno, Brno, Czech Republic; 2 Department of Human Pharmacology and Toxicology, Faculty of Pharmacy,? University of Veterinary and Pharmaceutical Sciences Brno, Brno, Czech Republic; 3 Department of Chemical Engineering, University of Chemistry and Technology Prague,

Prague, Czech Republic

DOI 10.1055/s-0037-1608375

$\beta$-Glucans, polysaccharides consisting of glucose polymers, have been described for their immunomodulatory and anti-inflammatory potential in many in vitro and in vivo studies [1]. Glucan particles (GPs) - hollow porous Saccharomyces cerevisiae cell walls consisting mainly of $\beta$-1,3-d-glucans, can moreover be used as drug delivery system [2]. The aim of our study was to confirm antiinflammatory effect of GPs on experimentally induced colitis and their role as drug delivery system for natural anti-inflammatory drugs.

In our experiment, we used glucan particles prepared by the partial extraction of yeast cell components as described in literature [3] and then loaded them with curcumin. Dextran sodium sulphate induced colitis was used as a model of intestinal inflammation. The activity of disease was monitored, the colonic tissue was evaluated macroscopically, histologically, and by performing immunodetection to determine levels of proteins associated with inflammation and antioxidant enzymes.

Encapsulated curcumin exerted better therapeutic effectivity than curcumin alone. It delayed onset of disease and reduced the disease activity index. Encapsulated curcumin affected the expression of interleukins, TNF- $\alpha$, CAT and SOD-2. Glucan particles alone were also able to significantly reduce inflammation.

Obtained results indicated the strong potential of GPs as carrier for antiinflammatory drugs and combined with curcumin they seem to be promising therapeutic strategy for treatment of inflammatory diseases.

This work was supported by Ministry of Health of the Czech Republic, grant no $16-27522 \mathrm{~A}$.

[1] Du, B, et al. Trends in Food Science \& Technology 2015, 41: 49 - 59

[2] Soto, E, et al. Polymers 2010, 2:681 - 689

[3] Salo, I, et al. Food and Bioproducts Processing 2016, 99: 128 - 135

\section{Tu-Poster Session 2-PO-40 Novel self-emulsifying controlled release tetrahydrocurcumin pellets coated with an aqueous-based system}

Authors Wiwattanapatapee $\mathrm{R}^{1,2}$, Setthacheewakul $\mathrm{S}^{1,2}$, Ketjinda $\mathrm{W}^{1}$, Mäder $\mathrm{K}^{3}$

Institute 1 Department of Pharmaceutical Technology, Faculty of Pharmaceutical Sciences, Prince of Songkla University, 90112, Hat Yai, Thailand; 2 Phytomedicine and Pharmaceutical Biotechnology Excellence Research Center, Faculty of Pharmaceutical Sciences, Prince of Songkla University, 90112, Hat Yai, Thailand; 3 Department of Pharmaceutics and Biopharmaceutics, Institute of Pharmacy, Martin-Luther- University, WolfgangLangenbeck-Str.4, D-06120, Halle, Germany

DOI 10.1055/s-0037-1608376

The objective of this study was to develop the controlled release self-emulsifying pellets of tetrahydrocurcumin (THC) with aqueous-based film coating. The use of self-emulsifying system provided better solubility and absorption of hydrophobic THC whereas the controlled release system could prolong the drug release. The composition comprising $1.18 \%$ of THC and $13.27 \%$ of the liquid self-emulsifying system (Cremophor EL, Labrasol, Labrafac PG, and Ca- 
pryol 90) mixed with $59.72 \%$ of solid pellet excipients (pregelatinized starch and microcrystalline cellulose), and the pellets were coated with $25.83 \%$ of polymers (hydroxypropyl methylcellulose and ethycellulose). The sub-coating layer I of a water-soluble hydroxypropyl methylcellulose polymer with the topcoating layer II of water insoluble ethycellulose polymer could refine the control of the in vitro release of THC in an oil-in-water microemulsion, with a particle size of less than $50 \mathrm{~nm}$. The optimum formulation provided a controlled release of $60 \%$ of THC over a $24 \mathrm{~h}$ period, which was significantly higher than that of unformulated THC (only $30 \%$ ). The formulation approach based on an aqueous solvent system offers the possibility of formulating and controlling the in vitro release of poorly water-soluble herbal drugs from solid-lipid dosage forms with economy and environmental safe.

[1] Serratoni M, Newton M, Booth S and Clarke A. Eur J Pharm Biopharm 2007; 65: $94-98$.

[2] Yang QW, Flament MP, Siepmann F, Busignies V, Leclerc B, Herry C, Tchoreloff P and Siepmann J. Eur. J. Pharm. Biopharm 2010; 74: 362 - 370.

\section{Tu-Poster Session 2-PO-41 ANTISEPTIC EFFECT OF AN ALCOHOL-FREE CHITOSAN-CURCUMIN MOUTHWASH: A PROPHYLACTIC ALTERNATIVE IN CANCER AND IMMUNOCOMPROMISED PATIENTS}

Authors Mahattanadul $\mathrm{S}^{1}$, Mustafa Mian $\mathrm{W}^{1}$, Ungphaiboon $\mathrm{S}^{2}$, Sungkharak $\mathrm{S}^{2}$ Institute 1 Department of Clinical Pharmacy, Faculty of Pharmaceutical Sciences, Prince of Songkla University, Songkhla, Thailand; 2 Department of Pharmaceutical Technology, Faculty of Pharmaceutical Sciences, Prince of Songkla University, Songkhla, Thailand

DOI 10.1055/s-0037-1608377

A non-staining, non-irritant (alcohol free) curcumin mouthwash was developed in order to be used for prevention the oral cavity infections in cancer and immunocompromised patients. Physiologically compatible formulation was formulated with acceptable solubility, stability as well as safety of curcu$\min (0.1 \% \mathrm{w} / \mathrm{v})$ and chitosan $(0.5 \% \mathrm{w} / \mathrm{v})$ using polyethylene glycol (PEG) 400 as a solvent [1]. Antimicrobial activity of the developed chitosan-curcumin mouthwash against planktonic organisms and candida was evaluated and compared with that of standard $2 \% \mathrm{w} / \mathrm{v}$ chlorhexidine mouthwash. Curcumin powder (with no volatile oil content) was active against all free floating test organisms with the highest antiseptic efficacy against $C$. albicans (MIC $=16 \mu \mathrm{g}$ / $\mathrm{mL}$ ) and the lowest antiseptic efficacy against $E$. coli and $K$. pneumoniae; $\mathrm{MIC}=250 \mu \mathrm{g} / \mathrm{mL}$ ). Chitosan (10 kDa) was mainly active against $E$. coli, $K$. pneumoniae, P. aeruginosa and S. salivarius ( $\mathrm{MIC}=500 \mu \mathrm{g} / \mathrm{mL}$ ) whereas chitosan (100 kDa) was active only against C. albicans (MIC $=250 \mu \mathrm{g} / \mathrm{mL}$ ). Chlorhexidine solution inhibited the growth of all test organisms with complete eradication. However, the anti-candida activity of curcumin $(\mathrm{MIC}=16 \mu \mathrm{g} / \mathrm{mL})$ is higher than that of chlorhexidine (MIC $=400 \mu \mathrm{g} / \mathrm{mL}$ ) [2]. It was found that curcumin at the concentration of $0.1 \% \mathrm{w} / \mathrm{v}$ with chitosan at the concentration of $0.5 \%$ $\mathrm{w} / \mathrm{v}$ (chitosan-curcumin mouthwash) had distinctive advantage over pure $0.1 \% \mathrm{w} / \mathrm{v}$ curcumin or $0.5 \% \mathrm{w} / \mathrm{v}$ chitosan mouthwash against C. albicans growth, both in free-floating form and in biofilms. The anti-candida activity of the developed mouthwash was comparable to that of a standard chlorhexidine mouthwash. This clinically effective anti-candida property of the nonirritant chitosan-curcumin mouthwash recommended its potential prophylactic alternative to prevent candidiasis in high risk patients such as cancer and immunocompromised patients.

[1] Anjana D, et al. Am J Infect Dis 2012; 8: 41 - 49.

[2] Lamfon, H. et al. J Antimicrob Chemother 2004; 53: 383 - 385.

\section{Tu-Poster Session 2-PO-42 Potential and bioaccessibility of Pueraria mirifica-loaded nanostructure lipid carriers for its impact on the biopharmaceutical application}

\author{
Authors Surassmo $\mathrm{S}^{1}$, Suktham $\mathrm{K}^{1}$, Yostawonkul J', Saengkrit $\mathrm{N}^{1}$,
} Ruktanonchai $U^{2}$

Institute 1 Nano-Delivery System Laboratory, National Nanotechnology Center, National Science and Technology Development Agency, Thailand Science Park, Pathumthani, Thailand; 2 National Nanotechnology Center, National Science and Technology Development Agency, Thailand Science Park, Pathumthani, Thailand

DOI 10.1055/s-0037-1608378

Pueraria mirifica (PM) is a natural herb which has anti-allergic properties and stimulates estrogenic activity. PM extracted-loading into nanostructure lipids carriers (NLCs) was exploited to enhance solubility and develop effective formulations for topical administration. The different lipids and concentration of surfactants were chosen to prepare NLCs using high pressure homogenization $(\mathrm{HPH})$ and evaluated to optimize NLCs formulation. The colloidal dispersions characterized for particle size, zeta-potential, in vitro release and entrapment efficiency due to explore for active ingredients permeation properties. PMloaded NLCs were arounds $150-200 \mathrm{~nm}$ in diameter, negatively charged and high entrapment efficiency more than $80 \%$. Furthermore, we investigated the effects of PM isoflavone (puerarin, daidzein, genestin) in crude extract and Pueraria mirifica extracted-loading into nanostructure lipids carriers (PMloaded NLCS) on the anti-proliferation potential of fibroblast (CRL-2522) and breast cancer cells (MCF-7 and MDA-MB-231). The results revealed that after treatment with PM and PM-loaded NLCs, a dose dependent inhibition of cell growth occurred in CRL-2522, MCF-7 and MDA-MB-231 cell lines. It was initiated that PM-loaded NLCs inhibited proliferation of the breast cancer cells at $50 \%$ cell growth concentration $\left(\mathrm{IC}_{50}\right)$ of $0.69 \mathrm{~g} / \mathrm{mL}$ with the low toxicity on CRL-2522 cell lines. Meanwhile, the results from cell cycle distribution and apoptosis assays revealed that PM-loaded NLCs induced cell apoptosis through caspase-3-depndent pathway. The results demonstrated that PM-loaded NLC showed an estrogenic effect on MCF-7 cell growth and a clear antagonistic effect with estradiol (E2) at high concentration. From these results, it was suggested that PM-loaded NLCs could enhance topical delivery property and it may act as a chemotherapeutic agent against cancer by reducing cell viability and inducing apoptosis. This is poised to significantly increase impact on the biopharmaceutical applications.

\section{Tu-Poster Session 2-PO-43 The Green Synthesis, Characterization of Silver Nanoparticles Synthesized from Anthemis tricolor Boiss. Methanol and Chloroform Extracts}

Authors Sümer Tüzün $B^{1}$, Özçınar Ö$^{1}$, Kıvçak $B^{1}$ Institute 1 EGE UNIVERSITY FACULTY OF PHARMACY DEPARTMENT OF PHARMACOGNOSY, izmir, Turkey

DOI 10.1055/s-0037-1608379

Anthemis L. (Asteraceae), one of the largest genus with 210 species, is represented in Cyprus by 10 species belonging to two subgenera, five sections and Anthemis tricolor Boiss. is one of the endemic species of Cyprus. [1]. Nanoparticle synthesis is usually applied some methods such as pyrolysis, laser ablation, vapor deposition, sol gel and lithography electro-deposition. The green nanoparticles synthesis from plant extracts is more easy, economic, efficient and fast than chemical and physical methods [2,3]. Here we present data on the green synthesis of silver nanoparticles of $A$. tricolor methanol and chloroform extracts, their physical characterization. (SEM-EDX) Scanning electron microscopy/Energy-dispersive X-Ray spectroscopy, particle size (PS) and XRay diffraction analysis (XRD) were used to characterize the formation of silver nanoparticles (AgNPs). SEM is used to see microstructure and detailed mor- 
phological studies, which showed spherical silver nanoparticles. XRD results represent cubic and monoclinic crystals. Particle size of silver nanoparticles from methanol extract $146 \mathrm{~nm}$ and from chloroform extract $300 \mathrm{~nm}$. Further studies are warranted to investigate their bioactivities.

\section{Tu-Poster Session 2-PO-44 Characterization of a Neurodifferentiation Inducing Flavonoid/ Cyclodextrin Inclusion Complex}

Authors Kirchinger $\mathrm{M}^{1}$, Bieler $\mathrm{L}^{2}$, Couillard-Despres $\mathrm{S}^{2}$, Riepl $\mathrm{H}^{1}$, Urmann $\mathrm{C}^{1}$ Institute 1 Organic and Analytical Chemistry, $S$ cience Center Straubing, Schulgasse 1694315 Straubing, Straubing, Germany; 2 Institute of

Experimental Neuroregeneration Paracelsus Medical University, Salzburg, Strubergasse 21, 5020 Salzburg, Salzburg, Austria

DOI 10.1055/s-0037-1608380

Humulus lupulus $L$. is a well known traditional medicinal plant. Recently, pyranoflavonoids of hops were identified as potent inducers of neuronal differentiation in adult neuronal stem cells.[1,2] Unfortunately, the lipophilic side chain results in low water solubility and this makes an application in vivo difficult. To overcome this hurdle, (2-hydroxypropyl)- $\beta$-cyclodextrin is used to enhance solubility.

This cyclodextrine is water-soluble, non-toxic and has already been applied in numerous pharmaceutical preparations.[3] The maximum solubility of xanthohumol $C$ in water is $2 \mu \mathrm{g} / \mathrm{mL}$. Using cyclodextrin the solubility can be increased to $1300 \mu \mathrm{g} / \mathrm{mL}$, confirmed by using HPLC analysis. In vitro, encapsulated xanthohumol C is as active as the pure flavonoid, making the cyclodextrin complex a suitable form of application for future in vivo studies.

[1] Oberbauer E, Urmann C, Steffenhagen C, Bieler L, Furtner T, Humpel C, Baumer B, Bandtlow C, Couillard-Despres S, Rivera FJ, Riepl H, Aigner L. Journal of Nutritional Biochemistry 2013, 24: $1953-1962$.

[2] Urmann C, Oberbauer E, Couillard-Despres S, Aigner L, Riepl H. Planta Medica 2015, 4: 305 - 311.

[3] T. Irie, K. Uekama, Journal of pharmaceutical Science, 86 (1996) 147 - 162.

\section{Tu-Poster Session 2-PO-45 LIPOSOME VEHICULATION OF THE ESSENTIAL OILS OF SALVIA TRILOBA AND ROSMARINUS OFFICINALIS FOR THE TOPICAL DELIVERY}

Authors Risaliti $L^{1}$, Kehagia $A^{2}$, Daoultzi $E^{2}$, Lazzari $D^{2}$, Bergonzi Maria $C^{1}$, Bilia Anna $\mathrm{R}^{1}$

Institute 1 Department of Chemistry, University of Florence, Sesto Fiorentino, Italy; 2 Aristotle University of Thessaloniki, Department of Pharmacy,

Thessaloniki, Greece

DOI 10.1055/s-0037-1608381

The vesicular systems represent useful strategies to improve the skin permeation of essential oils because of their instability to light and their volatile structure [1]. Essential oils have been widely used for their health and medicinal properties, principally as bactericidal, virucidal, fungicidal, insecticidal [2]. This study was focused on the development of versatile nanovesicles for the delivery of essential oils [3]. Liposomes were prepared and loaded with the essential oils of Salvia triloba and Rosmarinus officinalis. The essential oils were obtained by steam distillation and their main constituents were eucalyptol and camphor. The vesicles were prepared by Thin Layer Evaporation with a $1 \% \mathrm{v} / \mathrm{v}$ of oil. Nanocarrier batches were characterized for their size, polydispersity index, Zeta potential, recovery, encapsulation efficiency (EE\%), release and morphology. Results showed that all liposomes exhibit average sizes about $200 \mathrm{~nm}$, the Z-potential varied from $-35 \mathrm{mV}$ to $-20 \mathrm{mV}$, the PDI was under 0,25 , EE\% were $56,71 \pm 8,82 \%$ for $S$. triloba and $64,56 \pm 8,41 \%$ for R. officinalis. The stability test was carried out, after storing at $4{ }^{\circ} \mathrm{C}$ for 20 days for $\mathrm{S}$. triloba and R. officinalis, by monitoring changes in their mean size, PDI and EE\% values. In addition, the Skin PAMPA test (Parallel Artificial Membrane Permeability Assay) which is highly predictive for skin permeation was carried out for the developed formulations. The findings suggest that these formulations can decrease the volatility of the essential oil and optimise the delivery inside the skin.

[1] Bilia AR, Guccione C, Isacchi B, Righeschi C, Firenzuoli F, Bergonzi MC. Evid Based Complement Alternat Med 2014:651593. doi: 10.1155/2014/651593. [2] Bilia AR, Piazzini V, Guccione C, Risaliti L, Asprea M, Capecchi G, \& Bergonzi MC. Planta Medica; 2017;83:366 - 381. doi: 10.1055/s-0043 - 102949.

[3] Asprea M, Leto I, Bergonzi M.C, Bilia A.R. LWT - Food Science and Technology, 2017; 77: 497 - 502.

\section{Tu-Poster Session 2-PO-46 ESCIN-BASED NANOVESICLES TO IMPROVE BERBERIN TOPICAL DELIVERY}

Authors Vanti $\mathrm{C}^{1}$, Giovannetti $\mathrm{L}^{1}$, Bani $\mathrm{D}^{2}$, Bergonzi Maria $\mathrm{C}^{1}$, Bilia Anna $\mathrm{R}^{1}$ Institute 1 Department of Chemistry, University of Florence, Sesto Fiorentino (FI), Italy; 2 Department of Experimental and Clinical Medicine, University of Florence, Florence, Italy

DOI $10.1055 / \mathrm{s}-0037-1608382$

The study was focused on the development of nanovesicles based on phosphatidylcholine, cholesterol and Escin (ESN) and loaded with Berberine Chloride (BRB $\mathrm{HCl}$ ), to obtain a therapeutic synergism. Hence, ESN was selected for both structural and functional activity. ESN exhibits many pharmacological activities, such as anti-inflammatory, anti-edematous and veno-tonic properties [1]. BRB HCL, a natural isoquinoline alkaloid, is used in medicine because of numerous activities, principally cardiovascular, anti-inflammatory, antimicrobial, and anti-cancer effects [2]. Diverse nanovesicles were developed using different combinations of these compounds, in order to select the formulation with the best biopharmaceutical parameters. All the developed nanovesicles were fully characterized in terms of dimension and morphology, zeta potential, polydispersion and drug loading as previously reported [3,4]. The optimized nanovesicles enclose were prepared with phosphatidylcholine, ESN and $\mathrm{BRB} \mathrm{HCl}$ in the ratio $33: 5: 1.3 \mathrm{mg} / \mathrm{ml}$, respectively. The nanovesicles displayed an average diameter of $208 \mathrm{~nm}$, polydispersity index was 0.18 , \ potential was -24.8. Vesicles had spherical shape. In vitro release of $\mathrm{BRB} \mathrm{HCl}$ showed an initial burst effect, followed by a prolonged release for $24 \mathrm{~h}$.

Vesicles subjected to in vitro permeation studies, using a system of parallel artificial membranes (PAMPA) had a better permeation profile in comparison with free $\mathrm{BRB} \mathrm{HCl}$, also confirmed by an ex vivo permeation assay performed with rabbit ear skin.

[1] Sirtori CR. Pharmacol Res. 2001, 44:183 - 93.

[2] Imenshahidi M, Hosseinzadeh H. Phytother Res. 2016, 30:1745 - 1764.

[3] Isacchi B, Bergonzi MC, lacopi R, Ghelardini C, Galeotti N, Bilia AR. Planta Med. 2017, 83:412 - 419.

[4] Leto I, Coronnello M, Righeschi C, Bergonzi MC, Mini E, Bilia AR. ChemMedChem. 2016, 11:1745 - 51.

\section{Tu-Poster Session 2-PO-47 BRAIN-TARGETED DELIVERY OF ANDROGRAPHOLIDE USING SOLID LIPID NANOPARTICLES: IN VITRO AND IN VIVO EVALUATION}

Authors Graverini $\mathrm{G}^{1}$, Piazzini $\mathrm{V}^{1}$, Landucci $\mathrm{E}^{2}$, Pantano $\mathrm{D}^{3}$, Nardiello $\mathrm{P}^{3}$, Casamenti $F^{3}$, Pellegrini-Giampietro $D^{2}$, Bilia Anna $R^{1}$, Bergonzi Maria $C^{1}$ Institute 1 Department of Chemistry, University of Florence, Sesto Fiorentino, Florence, Italy; 2 Department of Health Sciences, Section of Clinical Pharmacology and Oncology, University of Florence, Florence, Italy; 3 Department of Neuroscience, Psychology, Drug Research and Child Health Division of Pharmacology and Toxicology, University of Florence, Florence, Italy DOI 10.1055/s-0037-1608383

Andrographolide (AG) is the major diterpenoid of Andrographis paniculata (Burm. f.) Wall. ex Nees (Acanthaceae) and have been reported to exhibit a wide spectrum of activities, including protection against oxidative stress, inflammation-mediated neurodegeneration and against cerebral ischemia [1]. However, AG shows low bioavailability and high chemical and metabolic in- 
stability. Moreover, up to now, there is no experimental evidence of AG crossing the blood brain barrier (BBB) in sufficient amount to achieve therapeutically relevant concentrations in the brain. To overcome these drawbacks the aim of this study was the preparation of solid lipid nanoparticles (SLNs) for brain delivery of AG. AG-SLNs have been developed using Compritol 888 ATO as solid lipid and Brij 78 as surfactant by emulsion/evaporation/solidifying method [2]. SLNs showed spherical shape and mean diameter below $300 \mathrm{~nm}$ with narrow size dispersion (Pdl lower than 0.2 ) and z-potential around -35 $\mathrm{mV}$; encapsulation percentage was $92 \%$ and exhibited in vitro AG controlled and sustained release profile for at least $72 \mathrm{~h}$. In vitro transport studies using artificial membranes (Parallel Artificial Membrane Permeability Assay) and hCMEC/D 3 cells revealed that SLNs were successful in enhancing the permeation of AG. Fluorescent-SLNs were also developed using fluorescein isothiocyanate for in vivo test in healthy rats. SLNs were administrated by intravenous route and were detected in the brain parenchyma twenty-four and seventytwo hours after administration, by confirming their ability to overcome the BBB.

Financial support was granted by Ente Cassa di Risparmio di Firenze.

[1] Serrano, F. G, Tapia-Rojas, C, Carvajal, F. J, Hancke, J, Cerpa, W, \& Inestrosa, N. C. Molecular Neurodegeneration 2014; 9: 61.

[2] De Vringer, T, \& De Ronde, H. A. G. Journal of pharmaceutical sciences 1995; 84: $466-472$.

\section{Tu-Poster Session 2-PO-48 LIPOSOMES LOADED WITH BERBERINE HYDROCHLORIDE: DEVELOPMENT, OPTIMISATION AND IN VITRO CITOTOXICITY}

\author{
Authors Giovannetti $\mathrm{L}^{1}$, Piazzini $\mathrm{V}^{1}$, Coronnello $\mathrm{M}^{2}$, Mini $\mathrm{E}^{2}$, Bergonzi \\ Maria $C^{1}$, Bilia Anna $\mathrm{R}^{1}$
}

Institute 1 University of Florence, Department of Chemistry, Sesto Fiorentino, Florence, Italy; 2 University of Florence, Department of Health Sciences, Clinical Pharmacology and Oncology Section, Florence, Italy DOI 10.1055/s-0037-1608384

In continuing our studies on the development of nanoliposomes [1], we now report three formulations containing different amount of cholesterol for the delivery of berberine hydrochloride (BRB). BRB, an isoquinoline alkaloid, is present in many plants and it is characterized by several pharmacological activities [2]. However, BRB shows low bioavailability, so we evaluated whether liposomes could be used for the delivery of the compound. In the present study we also evaluated cellular uptake of liposomes loaded with BRB using K562 and MCF-7 cells line by flow cytometry. Polydispersion of the vesicles showed values from 0.16 to 0.32 , indicating the homogeneity of the formulations. Regarding $\zeta$-potential, all vesicles displayed a negative surface charge ranging from -32.3 to $-40.3 \mathrm{mV}$. The $\mathrm{EE} \%$ values of these liposomes ranged from 21 to $41 \%$. TEM analyses revealed that all formulations had a spherical shape, with a good size distribution. Cellular uptake studies using K562 cells showed fluorescence ratio from 66 to 51; the assays conducted using MCF-7 cells showed fluorescence ratio from 96 to 39. Liposomes with low concentration of cholesterol showed the best fluorescence ratio, so this formulation was chosen for cytotoxicity studies. There was no significant difference of IC 50 between free BRB and BRB-loaded liposomes. Developed vesicles could provide for an improved biopharmaceutical profile through reduced toxicity, favourable pharmacokinetic behaviour and an enhanced therapeutic index in comparison to the free drug.

[1] Leto, I, Coronnello, M, Righeschi, C, Bergonzi, M. C, Mini, E, \& Bilia, A. R. ChemMedChem 2016; 11: 1745 - 1751.

[2] Ferraroni, M, Bazzicalupi, C, Bilia, A. R, \& Gratteri, P. Chemical Communications 2011; 47: 4917 - 4919.

\section{Tu-Poster Session 2-PO-49 Antiproliferative activities of Citrus aurantium against breast (MCF7), cervix (HeLa) and urinary bladder (EJ138) carcinoma cells}

Authors Segun Peter $\mathrm{A}^{1,2}$, Ismail Fyaz $\mathrm{MD}^{1}$, Ogbole $\mathrm{OO}^{2}$, Nahar $\mathrm{L}^{1}$, Evans $A R^{1}$, Sarker Satyajit $D^{1}$

Institute 1 Liverpool John Moores University, England and University of Ibadan, Nigeria, Liverpool, United Kingdom; 2 Department of Pharmacognosy, Faculty of Pharmacy, University of Ibadan, Ibadan, Nigeria

DOI 10.1055/s-0037-1608385

Citrus aurantium L. (Rutaceae), commonly known as bitter orange, is used in folklore medicine for the treatment of cancer, diabetes, malaria, painful swellings, typhoid and wounds [1]. In our search for cytotoxic agents from natural sources, we have recently carried out an ethnobotanical survey of plants used for cancer treatment amongst the ljebu ethnic group of South-Western Nigeria, and C. aurantium has been frequently mentioned as a cancer remedy by several traditional healers.

The ground bark of this plant was Soxhlet-extracted, successively, with nhexane, dichloromethane and methanol. As the dichloromethane extract showed significant cytotoxic activity $\left(\mathrm{IC}_{50}=11.5-14.3 \mu \mathrm{g} / \mathrm{mL}\right)$ against the breast (MCF7), the cervix (HeLa) and the urinary bladder (EJ138) carcinoma cells in the MTT assay, it was subjected to Vacuum Liquid Chromatography (VLC) to obtain six (F1-F6) different fractions. Since F2-F3 displayed the highest activities amongst the fractions $\left(\mathrm{IC}_{50}=8.55-10.38 \mu \mathrm{g} / \mathrm{mL}\right)$, the combined fractions were subjected to column chromatography and repeated preparative thin layer chromatography on silica gel leading to the isolation of an acridone alkaloid, citracidone-1. The structure of the alkaloid was elucidated by spectroscopic means. It could be assumed that citracidone-1, at least to a certain extent, accounts for the cytotoxic activity of $C$. aurantium, as its cytotoxic activity was documented in a previous study[2]. This study has demonstrated the cytotoxic property of $C$. aurantium and further investigations on citracidone- 1 in relation to its anticancer potential and possible mechanism of action are underway.

[1] Arias BA, Ramón-Laca L. J. Ethnopharmacol. 2005; 97: 89 - 95

[2] Teng WY, Huang YL, Shen CC, Huang RL, Chung RS, Chen CC. J. Chin. Chem. Soc. 2005; 52: $1253-1255$

\section{Tu-Poster Session 2-PO-50 Antiadhesive activity and phytochemical characterisation of the aerial parts of Tropaeolum majus L.}

Authors Vollmer $\mathrm{P}^{1}$, Sendker $]^{1}$, Scharf $\mathrm{B}^{1}$, Lipowicz $\mathrm{B}^{2}$, Hensel $\mathrm{A}^{1}$ Institute 1 University of Münster, Institute of Pharmaceutical Biology and Phytochemistry, Münster, Germany; 2 Repha GmbH, Langenhagen, Germany DOI 10.1055/s-0037-1608386

Tropaeolum majus L. is used within rational phytotherapy against urinary and respiratory tract infections due to its content of the antibacterial mustard oil benzylisothiocyanate, which is formed by enzymatic conversion from the glucosinolate glucotropaeolin. This study aims at elucidating other potentially antibacterial compounds in the herbal material.

Powdered plant material was fermented and its metabolic profile compared with that of an unfermented $70 \%$ methanol extract by using UHPLC-qTOFESIMS and multivariate data analysis. Besides glucotropaeolin, other compounds were found to be decomposed during fermentation. In total, 30 different compounds were identified or widely characterized on the basis of these data.

In vitro antibacterial activity of unfermented extract and an enzyme preparation of T. majus against uropathogenic Escherichia coli (UPEC) strain UTI89 was tested. Both samples showed no antibacterial activity (1 to $2000 \mu \mathrm{g} / \mathrm{mL}$ ). The unfermented extract did neither influence cell viability of T24 bladder cells. The adhesion of UPEC strain NU14 to T24 bladder cells was significantly inhibited by about $25 \%$ when the bladder cells were coincubated with bacteria and $1000 \mu \mathrm{g} / \mathrm{mL}$ of unfermented extract. 


\section{Tu-Poster Session 2-PO-51 Protective effect against oxidative neuron cell injury of Amaryllidaceae alkaloids}

Authors Cortes $\mathrm{N}^{1}$, Osorio Edison $\mathrm{H}^{2}$, Alzate $\mathrm{F}^{3}$, Cardona-Gomez Gloria $\mathrm{P}^{4}$ Osorio $\mathrm{E}^{1}$

Institute 1 Grupo de Investigación en Sustancias Bioactivas, Facultad de Química Farmacéutica, Universidad de Antioquia UdeA, Calle 70 No. 52 - 21, Medellin, Colombia; 2 Departamento de Ciencias Básicas. Universidad Católica Luis Amigó, SISCO. Transversal 51A No. 67B-90, Medellin, Colombia; 3 Grupo de Estudios Botánicos, Facultad de Ciencias Exactas y Naturales, Universidad de Antioquia UdeA, Calle 70 No. 52 - 21, Medellin, Colombia; 4 Cellular and Molecular Neurobiology Area, Group of Neuroscience of Antioquia, Faculty of Medicine, University of Antioquia, Calle 70 No. 52 - 21, Medellin, Colombia DOI 10.1055/s-0037-1608387

Oxidative stress is an excessive production of reactive species and insufficient activity of antioxidant defense mechanisms have been implicated as one of the important player in the pathogenesis of Alzheimer disease (AD), where the disturbance in systems employing the excitatory neurotransmitter L-glutamate may underlie part of its pathogenic mechanisms [1]. Amaryllidaceae alkaloids are capable of penetrating the blood-brain barrier, decrease oxidative damage and chronic neurodegeneration [2]. However, its antioxidant mode of action is not clear. Species of the family Amaryllidaceae (Clivia miniata, Eucharis bonplandii, Eucharis caucana) were collected in Colombia and their respective alkaloid fractions were obtained. The antioxidant activity was evaluated by Oxigen Radical Absorbance Capacity and Ferric Reducing Antioxidant Power assays, where the alkaloidal fraction of $E$. bonplandii presented the highest antioxidant capacity by both methods with values of 4538.96 \pm 140.00 and $574.66 \pm 7.77 \mu \mathrm{mol}$ Trolox/g alkaloidal fraction, respectively. The cytotoxicity and neuroprotective activity of the fractions was evaluated in a model of glutamate excitotoxicity in primary cultures of neurons at 0.75 , $1.5,3.0$ and $6.0 \mu \mathrm{g} / \mathrm{mL}$ concentrations. E. bonplandii protected the neurons after the toxic stimulus at $6.0 \mu \mathrm{g} / \mathrm{mL}$. This neuroprotection was related by the formation of Thiobarbituric Acid Reactive Substances, where E. bonplandii reduced significantly the oxidation of the cell membrane $45.45 \%$ with respect to the oxidation generated by L-glutamate. This evidence of antioxidant activity and lipid peroxidation may be involved in the neuroprotective activity, where the stabilization of free radicals should be associated with the ability of alkaloids to donating the hydrogen atom of the enol group.

[1] Dong X, Wang Y, Qin Z: Acta Pharmacol Sin 2009;30:379-387.

[2] Traykova M, Traykov T, Hadjimitova V, Krikorian K, Bojadgieva N: Z Naturforsch C 2003;58:361 - 5 .

\section{Tu-Poster Session 2-PO-52 Membrane stabilization and kinetics of carbohydrate metabolizing enzymes ( $\alpha$-amylase and $\alpha$-glucosidase) inhibitory potentials of Eucalyptus obliqua L. ethanolic leaf extract}

Authors Ashafa $\mathrm{O}^{1}$, Sabiu $\mathrm{S}^{1}$

Institute 1 University of the Free State, Qwaqwa Campus, Harrismith, South Africa

DOI 10.1055/s-0037-1608388

Eucalyptus spp. are important herbs finding therapeutic application in many parts of the world against a number of disorders including diabetes mellitus. Inhibitors of $\alpha$-amylase and $\alpha$-glucosidase offer an effective strategy to modulate levels of postprandial hyperglycemia via control of starch metabolism. The GC-MS analysis, membrane stabilization and mechanism(s) of inhibitory potential of Eucalyptus obliqua ethanolic extract on $\alpha$-amylase and $\alpha$-glucosidase were evaluated. The $\alpha$-amylase inhibitory potential of the extract was investigated by reacting different concentrations of the extract with $\alpha$-amylase and starch solution, while $\alpha$-glucosidase inhibition was determined by pre-incubating $\alpha$-glucosidase with different concentrations of the extracts followed by the addition of p-nitrophenylglucopyranoside. The mode(s) of inhibition of the enzymes were determined using the Lineweaver-Burke plot. The extract exhibited potent and moderate inhibitory potential against $\alpha$ amylase and $\alpha$-glucosidase, respectively. The inhibition was dose-dependent with respective half-maximal inhibitory concentration (IC50) values of 2.19 and $0.45 \mathrm{mg} \mathrm{mL}-1$. The extract also had respective membrane stabilization potentials of $86.58 \%$ and $76 \%$ on bovine erythrocytes against hypotonic solution and heat-induced hemolysis. The effect elicited by the extract at the investigated doses could be ascribed to the presence of active metabolites as revealed by the analytical chromatogram. Overall, the non-competitive mechanism of action of $E$. obliqua extract is due to its inhibitory effects on both $\alpha$-amylase and $\alpha$-glucosidase. This concomitantly extenuates the rate of starch hydrolysis leading to ameliorated glucose levels, thus lending support to hypoglycemic candidature of $E$. oblique.

\section{Tu-Poster Session 2-PO-53 Pterodon pubescens Benth: Isobolographic analysis of the Antinociceptive Interaction between enriched fractions of sesquiterpene, geranylgeraniol and voucapan compounds}

Authors Grando $\mathrm{R}^{1}$, Souza Vanessa $\mathrm{HS}^{1}$, Figueiredo Mariana $\mathrm{C}^{1}$, Zelioli Ícaro Augusto $\mathrm{M}^{1}$, Tarkany Basting $\mathrm{R}^{1}$, Oliveira Sousa Ilza $\mathrm{M}^{1}$, Queiroz Nubia de $\mathrm{A}^{1}$, Moreira Spindola $\mathrm{H}^{1}$, Foglio $\mathrm{M}^{1}$

Institute 1 Faculty of Pharmaceutical Science-UNICAMP, Faculty of Dentistry School, Medical Science, CPQBA, Campinas, Brazil

DOI 10.1055/s-0037-1608389

Pterodon pubescens Benth fruits are commercially available in the Brazilian medicinal plant street market. The crude alcoholic extracts of this plant are used in folk medicine as anti-inflammatory, analgesic, and anti-rheumatic preparations. Several studies have attributed antinociceptive activity of this species to the presence of enriched fractions containing geranylgeraniol and vouacapan compounds. The aim of this study was to evaluate synergistic effect of these fractions. Individual dose-response curves in acetic acid-induced abdominal writing test were measured for the separate fractions determening $\mathrm{ED}_{50}$. All treatments decreased significantly and in a dose dependent manner the writhing behavior with $\mathrm{ED}_{50}$ values of 102,34; 101,96; and $63,83 \mathrm{mg} \cdot \mathrm{kg}^{-1}$, for sesquiterpene, geranylgeraniol and voucapan fractions, respectively (ANOVA p < 0.05). An isobolographic analysis allowed the characterization of the pharmacological interaction produced by a fixed ratio combination of 3:1, 1:1 and 1:3 of equi-effective doses of these fractions. Theoretical antinociceptive ED 50 values of sesquiterpene/vouacapan fraction were 92,71; 83,09 and $73,46 \mathrm{mg} \cdot \mathrm{kg}^{-1}$, respectively. Experimentally antinociceptive $\mathrm{ED}_{50}$ values were 126,73; 69,96 and 57,91 mg. $\mathrm{kg}^{-1}$ (ANOVA $\mathrm{p}<0.05$ ), respectively. These results showed that: in the 3:1 proportion there is a antagonic interaction for these fractionswhereas with $1: 1$ and $1: 3$ proportion there was a synergic interaction. Theoretical antinociceptive $\mathrm{ED}_{50}$ values of geranylgeraniol/vouacapan fractions were $(92,43 ; 82,90$ and $73,36 \mathrm{mg} . \mathrm{kg}-1$, respectively) lager than those observed experimentally $\left(68,53 ; 41,51\right.$ and $37,21 \mathrm{mg} \cdot \mathrm{kg}^{-1}$ (ANOVA $p<0.05$ ), respectively) reporting synergic interaction in this association. Data suggest a synergic action between sesquiterpene and vouacapan fractions. Geranygeraniol and the vouacapan fractions also presented synergistic interactions in the acetic acid-induced abdominal writhing test.

\section{Tu-Poster Session 2-PO-54 Antiviral potential against influenza A virus of crude extracts from five South African medicinal plants}

Authors Mehrbod $\mathrm{P}^{1,2}$, Ali Abdalla $\mathrm{M}^{3}$, Fotouhi $\mathrm{F}^{2}$, Nicolaas Eloff $\mathrm{J}^{3}$, McGaw $\mathrm{LJ}^{3}$, Fasina Folorunso $\mathrm{O}^{1}$

Institute 1 Department of Veterinary Tropical Diseases, University of Pretoria, Pretoria, South Africa; 2 Influenza and Other Respiratory Viruses, Department, Pasteur Institute of IRAN, Tehran, Iran; 3 Phytomedicine Programme, 
Department of Paraclinical Sciences, University of Pretoria, Pretoria, South

Africa

DOI 10.1055/s-0037-1608390

Influenza infection remains a major health threat for animals and humans which crucially requires effective antiviral remedies. The usage of herbal medications as a readily available alternative and potentially fewer side effects compared to chemical treatments and their compatibility with body has become popular globally.

The aim of this study was to test in vitro anti-influenza activity of five South African medicinal plants, namely Tabernaemontana ventricosa, Cussonia spicata, Rapanea melanophloeos, Pittosporum viridiflorum and Clerodendrum glabrum.

The plant species were selected based on their use in traditional medicine and other biological activities. Methanol, ethanol, acetone, hot and cold water extracts of the dried, powdered plants were prepared and tested for cytotoxicity. The values below $50 \%$ cytotoxic concentration $\left(\mathrm{CC}_{50}\right)$ were tested for antiviral activity against influenza $A$ virus (IAV) in different types of simultaneous, pre-penetration and post-penetration combined treatments in the span of 1 hour incubation time. The effect of extracts on viral surface glycoproteins and viral titer were tested by $\mathrm{HI}$ and $\mathrm{HA}$ virological assays, respectively.

None of the extracts showed physical interaction with virus hemagglutinin glycoprotein. However, the most effective extracts against IAV were $R$. melanophloeos methanol extract $\left(\mathrm{EC}_{50}: 113.331 \mathrm{\mu g} / \mathrm{ml}\right)$ and $P$. viridiflorum methanol, $100 \%$ and $30 \%$ ethanol and acetone extracts ( $E_{50} \mathrm{~s}: 3.648,19.245,3.440$, $82.305 \mu \mathrm{g} / \mathrm{ml}$, respectively) in all types of combined treatments especially in pre- and post-penetration combined treatments with highly significant effect against viral titer.

The outcomes offer for the first time the scientific basis for the use of extracts of Rapanea melanophloeos and Pittosporum viridiflorum against IAV. They are worth to focus for finding effective active compounds in the next step and detecting the mechanism of action. The other three plant species were ineffective in vitro in this study.

\section{Tu-Poster Session 2-PO-55 Searching for extracts with a potential anti-inflammatory activity conducted using in vitro models}

Authors Michalak B ${ }^{1}$, Granica $S^{1}$, Piwowarski J , Waltenberger B ${ }^{2}$, Stuppner $\mathrm{H}^{2}$, Kiss $\mathrm{A}^{1}$

Institute 1 Department of Pharmacognosy and Molecular Basis of Phytotherapy, Medical University of Warsaw, Warsaw, Poland; 2 Department of Pharmacognosy, University of Innsbruck, Innsbruck, Austria

DOI 10.1055/s-0037-1608391

Based on ethnomedical and metabolomic knowledge of Asteraceae family species we screened Eupatorium cannabinum L, Inula helenium L. and Helianthus annuus $L$. on their biological activity in vitro. All plants selected for this screening were rich in sesquiterpene lactones showing antibacterial, immunological and anti-inflammatory properties [1].

The aim of this study was to examine the effect of the extracts on proinflammatory functions of stimulated neutrophils such as reactive oxygen species (ROS), interleukin 8 (IL-8), tumor necrosis factor alpha (TNF $\alpha$ ) production/release and expression of adhesion molecules CD 11b/CD 18.

The inhibition of ROS production by neutrophils was determined using luminol- dependent chemiluminescence. The effect on IL-8 and TNF $\alpha$ production by neutrophils was measured by an enzyme-linked immunosorbent assay. The expression of adhesion molecules CD11b on neutrophils was analyzed with flow cytometry.

The ethanolic extracts were shown to modulate lipopolysaccharide (LPS)-triggered production of IL-8 (at $25-100 \mu \mathrm{g} / \mathrm{mL}$ ) and TNF- $\alpha$ (at $25-100 \mu \mathrm{g} / \mathrm{mL}$ ). They were also active towards $\mathrm{f}-\mathrm{MLP}$ induced reactive oxygen species production (at $25-100 \mu \mathrm{g} / \mathrm{mL}$ ). Moreover, only Eupatorium cannabinum L. extract was also shown to decrease the expression of integrin CD11b. A major ses- quiterpene lactone isolated from Eupatorium cannabinum L. - eupatoriopicrin inhibited (IC $50<1 \mu \mathrm{M}$ ) IL-8 and TNF- $\alpha$ release by human neutrophils in response to lipopolysaccharides (LPS). Eupatoriopicrin displayed significant effects against $\mathrm{f}-\mathrm{MLP}$-induced reactive oxygen species production at $1 \mu \mathrm{M}$. The effects of eupatoriopicrin were compared with quercetin and clarithromycin used as a positive control.

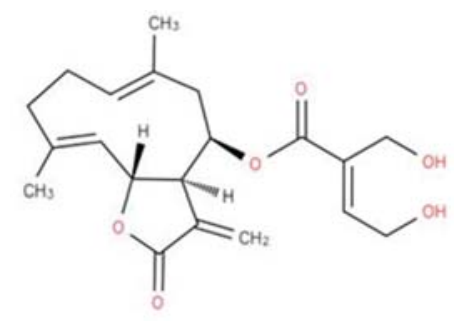

Eupatoriopicrin

Fig. 1

Our observations suggest that Eupatorium cannabinum L. can specifically modulate inflammatory functions of neutrophils. Moreover, eupatoriopicrin may contribute to beneficial health effects and can be the lead compound for further in vivo studies.

[1] Bessada SMF, Barreira JCM, Oliveira MPP. Ind Crops Prod 2015; 76 : 604 615

\section{Tu-Poster Session 2-PO-56 In vitro bioavailability of fatty acids and fatty acid esters of two saw palmetto preparations}

Authors Suter $A^{1}$, Engelhart-Jentzsch $K^{2}$

Institute 1 A. Vogel Bioforce AG, Roggwil, Switzerland; 2 BioTesys GmbH, Esslingen, Germany

DOI 10.1055/s-0037-1608392

The latest HMPC monograph on saw palmetto (Serenoa repens) differentiates by use of either ethanol or hexane as extractant between products for well established and traditional use. The hexane extract is further specified as it should contain $92 \%$ of fatty acids (free or esterified) and $2 \%$ of an unsaponifiable part. Ethanolic extracts contain more esterified fatty acids than hexane extracts, nevertheless in total is hardly ever more than $15 \%$ of all fatty acids [1].

If the differences in the chemical composition are relevant in the clinical situation is not clear so far. Therefore we carried out experiments with a hexane $\left(\right.$ Permixon $\left.^{\circledR}\right)$ and an ethanolic extract $(96 \% \mathrm{~V} / \mathrm{V})\left(\right.$ Prostasan $\left.^{\circledR}\right)$ in a validated in vitro model. It contains an artificial digestive process which simulates the enzymatic conditions as well as $\mathrm{pH}$ changes during gastrointestinal passage and then the transport of substances through a Caco-2 layer simulating the penetration from the intestinal lumen into the interior of the body.

The two extracts were similar in their content of fatty acids but the ethanolic extract contained $11.8 \%$ fatty acid esters compared to $2.7 \%$ of the hexane extract. After simulated gastric digestion, there were still fatty acid esters detectable in the serum in similar amounts for both extracts. After intestinal digestion and then also after passage through the Caco-2 layer there were for both extracts no fatty acid esters detectable but only free fatty acids in similar concentrations.

These in vitro experiments show that after digestion of lipophylic saw palmetto extracts only free fatty acids are available and absorbed into the human body.The amount of fatty acid esters in a saw palmetto extract should thus not serve as a marker to predict limited efficacy as it was warranted for example by the HMPC [2].

[1] Habib FK, Wyllie MG. ProstateCancer Prostatic Dis. 2004;7:195 - 200.

[2] EMA. Assessment report on Serenoa repens (W.Bartram) Small, Fructus. EMA/HMPC/137250/2013. Nov 2015. 


\section{Tu-Poster Session 2-PO-57 Herbal components myrrh, chamomile flower and coffee charcoal influence chemokine IL 8 and CXCL 10 secretion pattern from cytokine-challenged Caco-2 cells.}

\author{
Authors Vissiennon $\mathrm{C}^{1}$, Paulo $\mathrm{S}^{1}$, Hammoud $\mathrm{D}^{2}$, Goos $\mathrm{KH}^{3}$, Nieber $\mathrm{K}^{1}$, \\ Arnhold J ${ }^{1}$ \\ Institute 1 University of Leipzig, Leipzig, Germany; 2 Inter-Regional \\ University of Industrial Engineering, Biotechnologies and Applied Sciences \\ (IRGIB Africa University), Cotonou, Benin; 3 Repha GmbH Biologische \\ Arzneimittel, Langenhagen, Germany \\ DOI 10.1055/s-0037-1608393
}

A herbal combination consisting of myrrh, chamomile flower extract and coffee charcoal is used for the treatment of inflammatory bowel disease (IBD) - a chronic condition with an aberrant intestinal immune response driven by a dysregulated cytokine/chemokine homeostasis. Despite existing clinical data, pharmacological data supporting the observed efficacy remain insufficient.

The present study aims to investigate the influence of the single herbal components and a combination thereof on chemokine signalling of cytokine-challenged intestinal epithelial cells.

Thus, the influence of ethanolic myrrh (MY), chamomile flower (KA), coffee charcoal extract (CC) and budesonide (positive control) on chemokine (IL 8; CXCL 10) release from cytokine-(10 ng/mL TNF $\alpha ; 5 \mathrm{ng} / \mathrm{mL} \mathrm{IL} 1 \beta ; 10 \mathrm{ng} / \mathrm{mL}$ IFNY)-challenged Caco2 cells was investigated using an ELISA test system. To characterize the combined effect, concentration-response relations of single components and the combination were compared and IC 50 values derived. Interpretation of the data was based on a dose reduction index (DRI;= IC 50single/IC 50-comb.) which reported $x$-fold reduction of the plant extract concentration when applied in combination.

The single plant components inhibited cytokine induced $\mathrm{CXCL} 10$ release from Caco2 cells: MY (IC $50=41 \mu \mathrm{g} / \mathrm{ml} ; 93 \%$ max. inhib.), KA (364 $\mu \mathrm{g} / \mathrm{ml}$; $68 \%$ max. inhib.) and CC ( $447 \mu \mathrm{g} / \mathrm{ml} ; 56 \% \mathrm{max}$. inhib.), whereby relatively high concentrations are required. However, applied in combination, the IC 50 of the plant extracts were significantly reduced: MY $(I C 50=25 \mu \mathrm{g} / \mathrm{ml}), \mathrm{KA}(124 \mu \mathrm{g} / \mathrm{ml})$ and CC $(124 \mu \mathrm{g} / \mathrm{ml})(99 \%$ max. inhib.), resulting in DRI of $M Y=1.7, C C=3.6$ and $K A=2.9$. IL 8 release was inhibited after MY (IC $50=3 \mu \mathrm{g} / \mathrm{mL} ; 28 \%$ max inhib.) and CC (IC 50 $=218 \mu \mathrm{g} / \mathrm{mL}$; $75 \% \max$ inhib.) but increased after KA treatment (EC 50 $=39 \mu \mathrm{g} / \mathrm{mL} ; 29 \% \max$ stimul.). Treatment with all three plant extracts resulted in a moderate IL 8 inhibition with an inverted U-shape concentrationresponse curve (IC 50: MY $=56 \mu \mathrm{g} / \mathrm{ml} ; \mathrm{CC}=281 \mu \mathrm{g} / \mathrm{ml} ; 77 \%$ max. inhib.).

The herbal components myrrh, chamomile flower and coffee charcoal influence chemokine signalling of stimulated intestinal epithelial cells. Additive effects between the plant extracts justify the herbal combination and its usage in IBD.

\section{Tu-Poster Session 2-PO-58 In vitro effect on myometrial contractility by a combination of Bryophyllum pinnatum juice and atosiban}

Authors Santos $\mathrm{S}^{1,2}$, Haslinger $\mathrm{C}^{1}$, Hamburger $\mathrm{M}^{2}$, Mennet $\mathrm{M}^{3}$, Potterat $\mathrm{O}^{2}$, Schnelle $\mathrm{M}^{3}$, Mandach $U$ von ${ }^{1}$, Simões-Wüst $A P^{1}$

Institute 1 Department of Obstretics, University Hospital Zürich, Zürich, Switzerland; 2 Department of Pharmaceutical Sciences, University of Basel, Basel, Switzerland; 3 Clinical Research, Weleda AG, Arlesheim, Switzerland DOI 10.1055/s-0037-1608394

The herbal medicine Bryophyllum pinnatum has been used as a tocolytic agent in anthroposophic medicine and, recently, in conventional settings alone or as an add-on medication with tocolytic agents such as atosiban. We investigated the effects of $B$. pinnatum leaf press juice and atosiban, alone and in combination, on the contractility of myometrial tissue obtained from women undergoing Cesarean section.
Myometrial biopsies were collected during elective Caesarean section. Myometrial strips were placed under tension into a myograph chamber, and spontaneous contractions were recorded. After a $30 \mathrm{~min}$ period of regular contractions, B. pinnatum juice $(0.08 \%$ or $0.25 \%)$, atosiban $(0.53$ or $0.27 \mu \mathrm{g} /$ $\mathrm{mL}$ ), or the combination of both (Comb1 $=0.08 \%+0.53 \mu \mathrm{g} / \mathrm{mL}$; Comb2 $=0.25 \%+0.27 \mu \mathrm{g} / \mathrm{mL}$ ) were added to the chamber. Area under the curve (AUC) of contractions was determined for each contraction, and results were expressed as percentage of initial.

Results show that all test substances inhibited myometrium contractility, i.e. they led to significantly lower AUCs compared to control (in all cases $p<0.05$ ). When Comb1 was tested, contractions intensity lowered to $55.6 \pm 13.0 \%$ $(n=158)$. This was not significantly different from atosiban effect alone $(46.3 \pm 20.8 \% ; n=17)$. When Comb2 concentrations were tested, $B$. pinnatum juice decreased contractions down to $73.0 \pm 8.8 \%$, atosiban to $86.0 \pm 4.9 \%$, and the combination to $55.2 \pm 12.6 \%$. Thus, the inhibitory effect of Comb2 was significantly higher than atosiban alone at the correspondent concentration $(\mathrm{p}<0.05)$.

In conclusion, $B$. pinnatum and atosiban inhibit spontaneous myometrial contractions in vitro. The inhibitory effect of the combination is dependent on the concentrations used, and at a high B. pinnatum to atosiban ratio (Comb2), the effect is significantly higher than atosiban alone.

\section{Tu-Poster Session 2-PO-59 Recovery effects of winged bean (Psophocarpus tetragonolobus (L.) DC.) seed oil on menopausal symptoms in ovariectomized rats}

Authors Kupittayanant $S^{1}$, Kupittayanant $\mathrm{P}^{2}$, Thaeomor $\mathrm{A}^{1}$, Kusolrat $\mathrm{P}^{3}$ Institute 1 School of Preclinic, Institute of Science, Suranaree University of Technology, Nakhon Ratchasima, Thailand; 2 School of Animal Production Technology, Institute of Agricultural Technology, Nakhon Ratchasima, Thailand; 3 Department of Biology, Faculty of Science and Technology, Nakhonratchasima Rajabhat University, Nakhon Ratchasima, Thailand DOI 10.1055/s-0037-1608395

It has been reported that winged bean (Psophocarpus tetragonolobus (L.) DC.) seeds are the second largest phytosterols next to soybean, [1] which may be effective in recovery of menopausal symptoms. However, the effects have not yet been investigated. The aims of this research were therefore; 1 ) to study the estrogenic property of winged bean seed oil (WBSO), 2) to investigate the effects of WBSO on bone metabolism, and 3) to examine the effects of WBSO on lipid profile. Wistar female rats were divided into 4 groups of 5 animals each. Group 1 was sham-operated rats received $10 \%(\mathrm{v} / \mathrm{v})$ tween 80 whereas Group 2 to 4 were ovariectomized rats (OVX) received $10 \%(\mathrm{v} / \mathrm{v}), 17 \beta$-estradiol $(0.02 \mathrm{mg} / \mathrm{kg} \mathrm{BW})$, and WBSO $(1,000 \mathrm{mg} / \mathrm{kg} \mathrm{BW})$, respectively. Administration process started on day 60 after ovariectomy and lasted for 6 weeks. Data were analyzed by SPSS software using One-way analysis of variance. WBSO produced an increase in relative uterine weight and percentage of OVX rats having vaginal cornification. In addition, WBSO increased bone mineral density as well as decreased serum and urine Ca. Furthermore, WBSO decreased total cholesterol, LDL-cholesterol, but increased HDL-cholesterol. Our data suggest that WBSO could recover menopausal symptoms in ovariectomized rats and may be beneficial to help relief menopausal symptoms in women. [1] Yang, J, Tan, H. (May 2011). “Winged Bean Milk”. International Conference on New Technology of Agricultural Engineering, Zibo: 814-817. 


\section{Tu-Poster Session 2-PO-60 Cytotoxic Activity of Four Medicinal Plants and Four Food Plants Occurring in Thai Gynaecological Scriptures on Two Cancers Cell Lines}

\author{
Authors Jaiarree $\mathrm{N}^{1}$ \\ Institute 1 Department of applied Thai traditional medicine,Faculty of \\ medicine,Thammasat university, Pathumtani, Thailand \\ DOI 10.1055/s-0037-1608396
}

Medicinal plants found in Thai gynaecological scriptures are still used in the treatment of ovarian and breast cancer and some medicinal plants are used daily in Thai food. They are Boesenbergia rotunda (L.) Mansf, Piper nigrum Linn, Zingiber officinale Roscoe, Piper sarmentosum Roxb, Zingiber cassumunar Roxb, Zingiber zerumbet (L.) Smith, Oroxylum indicum(L.) Kurz. and Dioscorea birmanica Prain \& Burkill. The objective was to investigate cytotoxicity of these plants. Sample plants were extracted similarly to that practiced by Thai traditional practitioners (ethanol extraction). Bioassay-guided fractionation was used for isolating the pure compound from D.birmanica extract and elucidated by spectrophotometry. All extracts were tested for their cytotoxic activity against ovarian(SKOV-3) and breast(MCF-7) cancer cell lines and keratocyte cells (Hacat) by sulforhodamine B assay, involving eight herbal extracts plus the purified compound named Prosapogenin A of dioscin, which was purified from the $95 \%$ ethanolic extract of D.birmanica. Prosapogenin A of dioscin was cytotoxic against both ovarian and breast cancer cells with $\mathrm{IC}_{50}$ values of 7.30 \pm 0.05 and $5.36 \pm 0.26 \mu \mathrm{g} / \mathrm{ml}$. but not toxic to keratocyte cells with $\mathrm{IC}_{50}$ value of $94.76 \pm 1.25 \mu \mathrm{g} / \mathrm{ml}$. Z.zerumbet had the highest cytotoxicity against both ovarian and breast cancer cells with $\mathrm{IC}_{50}$ values of $5.57 \pm 0.47$ and 0.25 $\pm 0.32 \mu \mathrm{g} / \mathrm{ml}$. but toxic to keratocyte cells with $\mathrm{IC}_{50}$ value of $1.86 \pm 0.23 \mu \mathrm{g} /$ $\mathrm{ml}$. Z.cassumunar, P.nigrum, B.rotunda, O.indicum, Z.officinale and P.sarmentosum showed toxicity against only breast cancer cells with $I_{50}$ values of 11.14 $\pm 1.48, \quad 18.24 \pm 2.77, \quad 19.20 \pm 0.32,23.22 \pm 1.97,24.19 \pm 1.34$ and 24.84 $\pm 2.03 \mu \mathrm{g} / \mathrm{ml}$, respectively. They were not toxic to keratocyte cells with $\mathrm{IC}_{50}$ values of $48.53 \pm 4.35,36.13 \pm 1.41,34.25 \pm 0.62,>100,35.63 \pm 0.12$ and $40.39 \pm 0.98 \mu \mathrm{g} / \mathrm{ml}$, respectively.In conclusion, medicinal and food plants showed high toxicity against both ovarian and breast cancer cells, while not damaging normal cells. In particular, B.rotunda, Z.cassumunar and D.birmanica were highly toxic against breast cancer cells.

\section{Tu-Poster Session 2-PO-61 Investigation of potential anti-diabetic effect of Mucuna pruriens (L) DC (Fabaceae) aqueous leaf extract.}

\author{
Authors Akpoveso $\mathrm{OO}^{1}$, Olivier $\mathrm{G}^{1}$, Chatterjee $\mathrm{P}^{1}$, Olajide $\mathrm{O}^{3}$, Tumbas \\ Šaponjac $\mathrm{V}^{2}$ \\ Institute 1 University of Brighton, Brighton, United Kingdom; 2 Faculty of \\ Technology, University of Novi Sad, Novi Sad, Serbia; 3 University of \\ Huddersfield, Huddersfield, United Kingdom \\ DOI 10.1055/s-0037-1608397
}

Mucuna pruriens (L.) DC is an herbal plant popularly used in parts of Africa for the treatment of anaemia and in some parts of Asia for treatment of diabetes $[1,2]$. The antidiabetic effect of alcoholic leaf extract of Mucuna pruriens (MP) has been studied previously in diabetic rats [3]; however, the potential antidiabetic effect of an aqueous extract has not been evaluated.

MP leaves were identified and stored at the herbarium in the International Centre for Ethnomedicine and Drug development, Nigeria, West Africa. The specimen identification number is: InterCEDD-16018. We investigated the effect of an aqueous extract of Mucuna pruriens leaves (MPLE) on glucose uptake in rat NRK-52E renal cell line. Diethyl ether fractions from acid hydrolysis of MPLE (DE and NDE) and MPLE were also evaluated for insulin mimetic activity in differentiated 3T3-L 1 adipocytes. Phenolics in MPLE were detected using HPLC-UV/VIS. The peaks where identified by comparison with reference peaks obtained for known flavonoids and phenolic acids.
Identified phenolics included Epicatechin, Rutin, Gallic acid, Caffeic acid, Coumaric acid, and Ellagic acid. $1 \mathrm{mg} / \mathrm{ml}$ MPLE inhibited glucose uptake in NRK$52 \mathrm{E}$ cell lines by $35.5 \%$. This effect was comparable to $1 \mathrm{mM}$ Phloridzin (a standard sodium glucose transporter inhibitor). The extracts at 50 and $100 \mu \mathrm{g} / \mathrm{ml}$ stimulated glucose uptake by $65.97 \%$ and $78.25 \%$ of $15 \mathrm{ng} / \mathrm{ml}$ Insulin respectively.

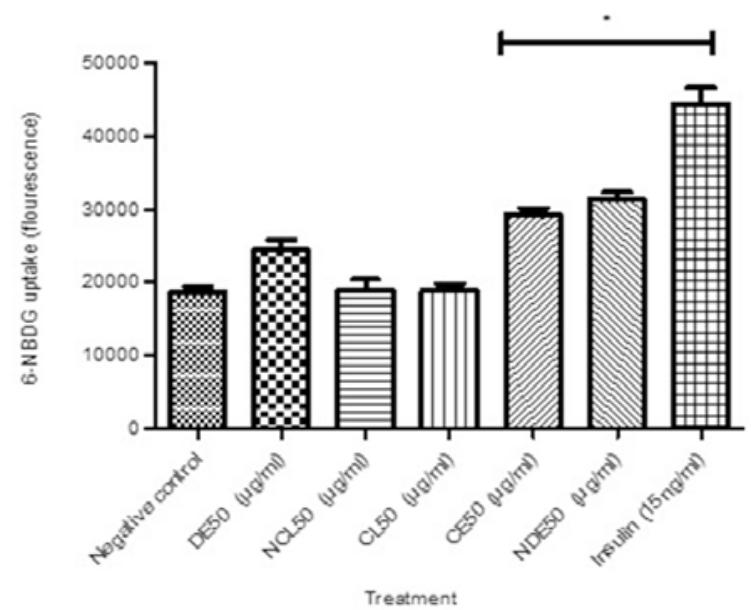

Fig. 1: Comparison of the effect of $50 \mu \mathrm{g} / \mathrm{ml} \mathrm{MPLE}$ and $50 \mu \mathrm{g} / \mathrm{ml}$ MPLE acid hydrolysed fractions on glucose uptake in 3T3-L1 adipocytes. ${ }^{*}$ Shows significant difference in glucose uptake stimulatory effect compared to the negative control at $p<0.05$ (one way ANOVA followed by a Bonferroni's post test). Data represents mean value \pm S.D 8 replicates $(n=8)$.

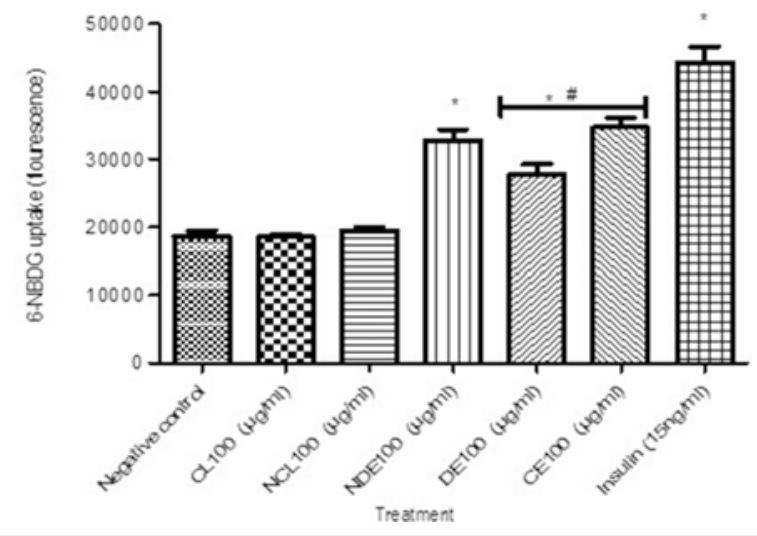

- Fig. 2: Comparison of the effect of $100 \mu \mathrm{g} / \mathrm{ml}$ MPLE and $100 \mu \mathrm{g} / \mathrm{ml}$ MPLE acid hydrolysed fractions on glucose uptake in 3T3-L 1 adipocytes. * Shows significant difference in glucose uptake stimulatory effect compared to the negative control at $\mathrm{p}<0.05$ \# shows significant difference in glucose uptake stimulatory effect between DE $100 \mu \mathrm{g} / \mathrm{ml}$ and $\mathrm{CE} 100 \mu \mathrm{g} / \mathrm{ml}$ at $\mathrm{p}<0.05$ (one way ANOVA followed by a Bonferroni's post test). Data represents mean value \pm S.D 8 replicates $(n=8)$. 


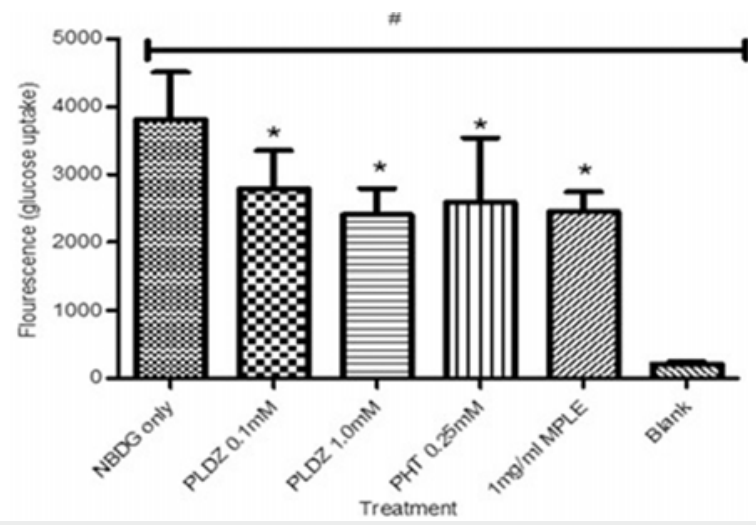

Fig. 3: Effect of MPLE on glucose uptake in NRK-52E cell lines using. *Shows significant difference compared to 2-NBDG only. \# shows significance compared to the blank measured at $\mathrm{p}<0.05$ (one way ANOVA followed by Dunnett's multiple comparison test). Data represents mean \pm SD of 9 replicates $(n=9)$.

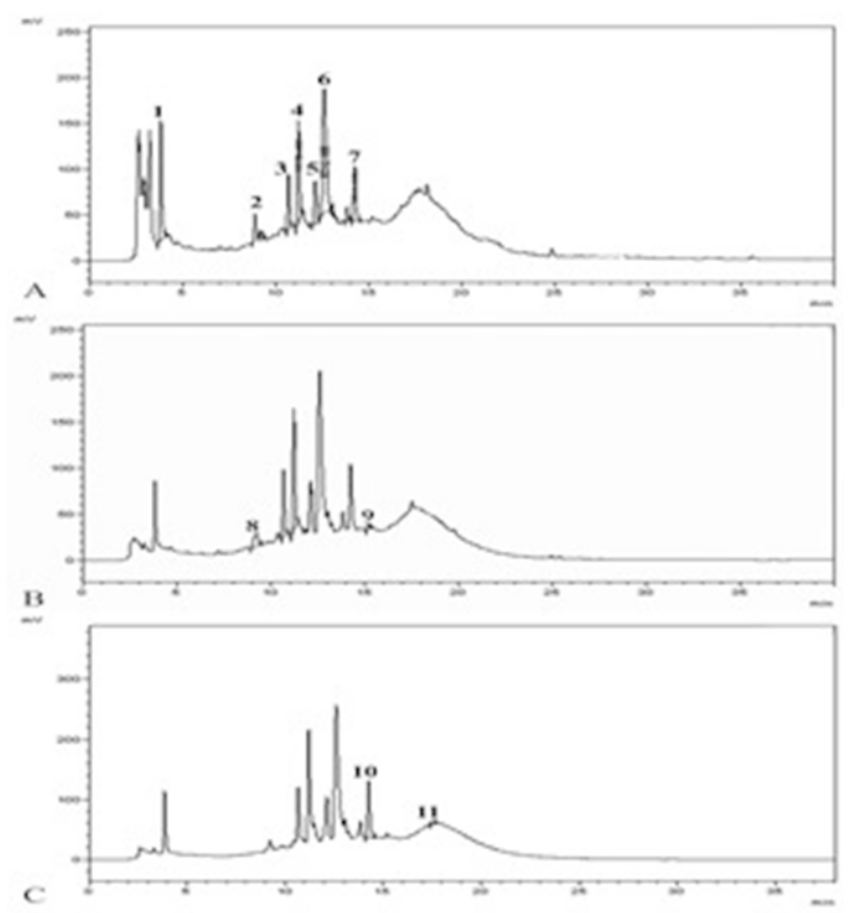

Fig. 4: Detection and quantification of known Phenolics in AMPLE using HPLC/UV-vis recorded at $280 \mathrm{~nm}$ (A), $320 \mathrm{~nm}$ (B), $360 \mathrm{~nm}$ (C). Peak Identification: I - Gallic acid, 2 - Vanillic acid, 3 - Catechin, 4 Protocatechuic acid, 5 - Caffeic acid, 6 - Epicatechin, 7 - Ellagic acid, 8 - Coumaric acid, 9 - Ferulic acid, 10 - Rutin, 11 - Myricetin

MPLE could exert anti-diabetic effects via multiple mechanisms of action. [1] Lampariello LR, Cortelazzo A, Guerranti R, Sticozzi C, Valachi G. J. Tradit Complement Med 2012; 2: 331 - 339

[2] Akindele AJ, Busayo FL. Nig Q Hosp Med 2011; 21: 93 - 98

[3] Murugan M, Reddy CUM. J Pharm Sci Technol 2009; 1: 69-73

\section{Tu-Poster Session 2-PO-62 Establishment of a simple method to search natural products that suppress $\alpha$-glucosidase amount in intestinal epithelial cell}

\author{
Authors Noda $\mathrm{K}^{1}$, Kato $\mathrm{E}^{1}$, Kawabata $]^{1}$ \\ Institute 1 Graduate School of Agriculture, Hokkaido University, Sapporo, \\ Japan \\ DOI 10.1055/s-0037-1608398
}

Diabetes is a chronic disease characterized by elevated blood glucose level. $\alpha$ Glucosidase is an enzyme involved in digestion of carbohydrates. Inhibition of $\alpha$-glucosidase suppresses rapid and large increase in post-prandial blood glucose level, which is effective for the treatment of diabetes patients. On the other hand, reduction of $\alpha$-glucosidase amount may work in the same way to treat diabetic patients. However, not much study is taking this approach to treat diabetes patients. Therefore, we established a simple method to examine the amount of $\alpha$-glucosidase expressed by the small intestinal epithelial cells, which is useful to search natural products that decrease $\alpha$-glucosidase amount.

Caco- 2 cell is frequently employed as a model of intestinal epithelial cell, and is known to express $\alpha$-glucosidase when differentiated. a-Glucosidase amount expressed by Caco-2 cell can be measured indirectly through the enzyme reaction rate. Addition of fluorescent substrate to Caco-2 cell induced a time dependent increase in fluorescence which reflects the enzyme reaction rate. With this simple method, several plant materials were screened for an activity to reduce $\alpha$-glucosidase amount. Among the screened plants, Perilla frutescens var. crispa, and Calluna vulgaris were found to show reduction of $\alpha$ glucosidase reaction rate in a dose dependent manner.

To confirm whether reduction of enzyme reaction rate reflect the reduction of $\alpha$-glucosidase amount, $\alpha$-glucosidase mRNA expression and protein amount were quantitated by real-time PCR and Western blotting. The extracts of P. frutescens and C.vulgaris suppressed sucrase-isomaltase, one of the intestinal $\alpha$-glucosidase complexes, at mRNA and protein level, demonstrating that this method is useful to search natural products that suppress $\alpha$-glucosidase amount.

\section{Tu-Poster Session 2-PO-63 Metabolization of the herbal combination STW-5 by human gut microbiota in vitro}

Authors Pferschy-Wenzig $\mathrm{EM}^{1,3}$, Roßmann $\mathrm{A}^{1,3}$, Koskinen $\mathrm{K}^{2,3}$, Abdel-Aziz $\mathrm{H}^{4}$, Moissl-Eichinger $\mathrm{C}^{2,3}$, Bauer $\mathrm{R}^{1,3}$

Institute 1 University of Graz, Institute of Pharmaceutical Sciences, Department of Pharmacognosy, Universitaetsplatz 4, 8010 Graz, Austria; 2 Medical University Graz, Department of Internal Medicine, Stiftingtalstraße 24/3/40, 8010 Graz, Austria; 3 BioTechMed, Krenngasse 37, 8010 Graz,

Austria; 4 Medical \& Clinical Affairs Phytomedicines, Steigerwald

Arzneimittelwerk GmbH, Bayer Consumer Health, Havelstr. 5, 64295

Darmstadt, Germany

DOI $10.1055 / \mathrm{s}-0037-1608399$

The herbal medicinal product STW- 5 is a fixed combination of 9 different plant extracts that was shown to be effective in functional gastrointestinal diseases like functional dyspepsia and irritable bowel syndrome. Many STW- 5 constituents are polar compounds. Due to the low oral bioavailability of such substances, they potentially reach the distal gut where they can interact with the local microbial community. Human gut microbiota are known to possess enormous metabolic capacity. This can lead to the production of new, potentially bioactive metabolites from plant constituents. The aim of this study was to investigate how gut bacteria metabolize STW- 5 constituents, and whether incubation with STW- 5 has an influence on gut microbial communities.

Lyophilized STW- 5 dissolved in buffer $(0.45$ and $2.25 \mathrm{mg} / \mathrm{ml})$ was incubated with human fecal suspension (HFS) under anoxic conditions. Samples were taken $0.5,4$ and $24 \mathrm{~h}$ after STW- 5 or vehicle addition, and subjected to LCDAD-HRMS analysis to detect changes in the extract's metabolic profile, and 
to next generation sequencing analysis (Illumina, MiSeq) to detect changes in the microbiome.

Results revealed dramatic modifications of the metabolic profile of STW- 5 incubated with HFS. Major phenolic constituents like rosmarinic acid, and liquiritin- and eriodictyol glycosides were metabolized so fast that they were not detectable anymore after $0.5 \mathrm{~h}$ of incubation. Instead, their putative metabolites dihydrocaffeic acid, liquiritin and eriodictyol were found to be enhanced after $0.5 \mathrm{~h}$, but further degraded at later time points. Triterpene glycosides, another major group of STW- 5 constituents, remained stable within the first $30 \mathrm{~min}$ but were found to be degraded subsequently.

These fast metabolic reactions were accompanied by rapid and dramatic changes in microbiome community composition. Notably, in particular Enterococcus species were found to be massively enhanced upon STW- 5 addition. We thank Steigerwald Arzneimittelwerk $\mathrm{GmbH}$, Bayer Consumer Health (Darmstadt, Germany) for financial support.

\section{Tu-Poster Session 2-PO-64 Interactions between hawthorn extract WS ${ }^{\circledR} 1442$ and human intestinal microbiota in vitro}

Authors Pferschy-Wenzig EM ${ }^{1,3}$, Roßmann $\mathrm{A}^{1}$, Koskinen $\mathrm{K}^{2,3}$, ArdjomandWoelkart $\mathrm{K}^{1}$, Meng $\mathrm{C}^{4}$, Koch $\mathrm{E}^{4}$, Moissl-Eichinger $\mathrm{C}^{2,3}$, Bauer $\mathrm{R}^{1,3}$

Institute 1 University of Graz, Institute of Pharmaceutical Sciences, Department of Pharmacognosy, Universitaetsplatz 4, 8010 Graz, Austria; 2 Medical University Graz, Department of Internal Medicine, Stiftingtalstraße 24/3/40, 8010 Graz, Austria; 3 BioTechMed Graz, Krenngasse 37, 8010 Graz, Austria; 4 Dr. Willmar Schwabe GmbH \& Co. KG, Willmar-Schwabe-Str. 4, 76227 Karlsruhe, Germany

DOI 10.1055/s-0037-1608400

Extracts from different parts of hawthorn species (Crataegus sp, Rosaceae) are therapeutically used mainly for the treatment of cardiovascular disorders. Phenolic constituents like procyanidins, flavanes and flavonoid glycosides are supposed to be the main bioactive constituents. Many of these compounds are not absorbed in the upper intestinal tract. Thus, their metabolization by gut microbiota may lead to formation of potentially bioactive metabolites. The aim of this study was to investigate, which compounds are metabolized by gut microbiota, and whether the extract has an influence on gut microbial community composition. WS ${ }^{\circledR} 1442$, a special hydroethanolic extract from leaves with flowers of officinal hawthorn species, was incubated with $10 \%$ human fecal suspension (HFS) under anoxic conditions at two different concentrations ( 0.45 and $2.25 \mathrm{mg} / \mathrm{ml}$ HFS). At $0.5,4$ and $24 \mathrm{~h}$ of incubation, samples were taken and analysed by LC-DAD-HRMS and by 16S RNA gene sequencing.

The degree of microbial degradation was found to be strongly dependent on compound class and extract concentration. Flavanes and procyanidins were readily metabolized, and at 4 and $24 \mathrm{~h}$, their putative metabolites dihydroxyphenyl-y-valerolactone, dihydroxyphenylvaleric acid and 3-(3-hydroxyphenyl)-propionic acid were detectable [1,2]. Flavonoid-O-glycosides were metabolized much faster than C-glycosyl-flavones. The degradation of C-glycosylflavones was strongly influenced by extract concentration. At $0.45 \mathrm{mg} / \mathrm{ml}$, the C-glycosidic bond was cleaved to a much higher degree than at $2.25 \mathrm{mg} / \mathrm{ml}$, indicating that too high extract concentrations might inhibit relevant microbial enzymatic activity.

The observed changes in the extract's metabolic profile were accompanied by shifts in microbial community composition. In particular, WS ${ }^{\circledR} 1442$ addition supported the increase of sugar-degrading beneficial microorganisms, like Faecalibacterium and Bacteroides.

We thank Dr. Willmar Schwabe GmbH \& Co. KG for financial support.

[1] Appledoorn, et al, J Agric Food Chem 2009, 57, 1084 - 92.

[2] Tagakaki, A, Nanjo, F, J Agric Food Chem, 2013, 61, 4927 - 35.

\section{Tu-Poster Session 2-PO-66 Sclerocaryabirrea fruit pulp and fruit peel protect against acute cadmium-induced testicular damage.}

Authors Sewani-Rusike $C^{1}$

Institute 1 Walter Sisulu University, Department of Human Biology, Faculty of Health Sciences, P. Bag X1, Mthatha, South Africa, Mthatha, South Africa DOI 10.1055/s-0037-1608401

Sclerocaryabirrea (marula) forms an important component of medicinal practices and diet in Southern Africa. The fermented fruit pulp beverage locally called "mukumbi" isused to treat male impotence and infertility. However, the peel tends to bediscarded in most traditional uses favouring the fruit pulp and tree bark. The aim of this study was to compare the effects of S. birrea fruit pulp and peel extracts on cadmium (Cd) induced testicular damage. Twenty four adult male Wistar rats were divided into four treatment groups ( $n=6$ ) group): Control, Cd only, Cd simultaneously treated with $\mathrm{S}$. birreafruit pulp or peel $(200 \mathrm{mg} / \mathrm{kg})$. Cd (1 mg/kg body weight as $\mathrm{CdCl} 2)$ was given intraperitoneally dissolved in normal saline for 3 consecutive days (day $1-3$ ) and treatment continued for a total of 10 days. Sperm count, testicular total antioxidant capacity, lipid peroxidation, superoxide dismutase activity (SOD), proinflammatory cytokine tumor necrosis factor-alpha (TNF- $\alpha$ ), serum reproductive hormones (T, FSH and $\mathrm{LH}$ ) and testis histology were assessed. Results showed significant adverse effects on male reproductive system after acute $\mathrm{Cd}$ exposure with reduced sperm count, deranged serum reproductive hormones and in testicular homogenates: increased malondialdehyde (MDA) content, decreased superoxide dismutase (SOD) activity, and upregulated levels of the proinflammatory cytokineTNF- $\alpha$. Histology showed an amorphous acellular material filling the seminiferous tubules in place of the regularly arranged cellular dynamics observed in control animals. Significant alleviation of these toxic effects was observed with both $\mathrm{S}$. birrea fruit pulp and fruit peel treatment. The fruit peel was more effective in alleviating testicular damage than fruit pulp especially for testicular structural protection as observed histologically. Mechanism of action of these extracts may involve inhibiting lipid peroxidation, restoration of SOD activity, reduction of inflammation by reducing TNF- $\alpha$ and protection of testis-blood barrier. Our data suggests that $\mathrm{S}$. birrea extracts exerted protective effects against $\mathrm{Cd}$-induced damage through antioxidant and anti-inflammatory effects.

\section{Tu-Poster Session 2-PO-67 Phytochemical and functional studies on the roots of Armoracia rusticana}

Authors jimenez Negro $\mathrm{E}^{1}$, Sendker J ${ }^{1}$, Scharf $\mathrm{B}^{1}$, Kleinwächter $\mathrm{M}^{2}$, Lipowicz $B^{2}$, Hensel $A^{1}$

Institute 1 University of Münster, Institute of Pharmaceutical Biology and Phytochemistry, Corrensstrasse 48, 48149 Münster, Germany; 2 Repha GmbH, Alt-Godshorn 87, 30855 Langenhagen, Germany DOI 10.1055/s-0037-1608402

Roots of horseradish (Armoracia rusticana G.Gaertn, B.Mey. \& Scherb.) are used within rational phytotherapy against infections of the upper respiratory tract and the urogenital system due to their content of glucosinolates, which by enzymatic catalysis decompose to the antibacterial isothiocyanates, also known as mustard oils. In order to investigate if other compounds than mustard oils or their progenitors contribute to the antibacterial activity, a $70 \%$ methanol extract was prepared of horseradish roots and fractionated on Sephadex LH20, silica, and RP18. Prior to functional testing within different in vitro assays, the extract and the respective fractions were fermented by using a protein extract from horseradish roots to release mustard oils and allow for the potential conversion of other natural compounds. As expected, strong antibacterial activity against uropathogenic Escherichia coli (UPEC, strains UTI89 and NU14) and Pseudomonas aeruginosa (strains ATCC 9027 and ATCC 27853) was found with fermented extract, whereas the unfermented extract was inactive. Unfermented extract however showed a significant antiadhesive effect by about $50 \%$ against UPEC NU14 and does not influence cell viability of 
T24 bladder cells. Phytochemical analysis of extracts and fractions by UHPLCqTOF-ESIMS indicated the presence of flavonoids, glucosinolates, and phospholipids hitherto not described for the genus Armoracia. In total, 60 different compounds were identified or widely characterized on the basis of these data. Differences in the flavonoid profiles of main and lateral roots and of fermented and non-fermented horse radish extract were identified by means of multivariate statistics.

\section{Tu-Poster Session 2-PO-68 Anti-elastase and anti-tyrosinase activities of selected traditional Chinese preparations}

Authors Brantner $\mathrm{A}^{1}$, Zhao $\mathrm{Y}^{2}$, Alajlani $\mathrm{M}^{1}$, Zhou $\mathrm{H}^{2}$, Baolin $\mathrm{B}^{2}$ Institute 1 Department of Pharmacognosy, Institute of Pharmaceutical Sciences, Karl-Franzens-University Graz, Universitaetsplatz 4, 8010, Graz, Austria; 2 Institute of Chinese Materia Medica, China Academy of Chinese Medical Sciences, 100700, Beijing, China

DOI 10.1055/s-0037-1608403

Skin aging can be related to the damage of the connective tissue and melanin over production. They may lead a loss of skin elasticity and pigmentation that are major signs of skin aging. Four traditional Chinese formulations were investigated for their anti-elastase and anti-tyrosinase properties. Methanolic extracts of the four traditional preparations Ermiao Wan (EW), Fang Feng Tong Sheng San (FFTSS), Si Miao San (SMS), and BuShenYiShou Capsules (BSYS) were evaluated along with standards by in-vitro enzymatic assays. The $\mathrm{IC}_{50}$ of the elastase inhibition was $0.004,0.330,0.028$ and $0.830 \mu \mathrm{g} / \mathrm{mL}$ for $\mathrm{EW}$, FFTSS, SMS and BSYS, respectively while the $\mathrm{IC}_{50}$ of the reference quercetin dihydrate was $0.480 \mu \mathrm{g} / \mathrm{mL}$. On the other hand the $I_{50}$ of the tyrosinase inhibitory activity was $0.77,0.27,0.98$ and $1.68 \mu \mathrm{g} / \mathrm{mL}$ for EW, FFTSS, SMS and BSYS, respectively compared to $\mathrm{L}(+)$-ascorbic acid $\left(\mathrm{IC}_{50} 38.89 \mu \mathrm{g} / \mathrm{mL}\right)$. The most potent formulation among the selected traditional preparations was Ermiao Wan with a 100 fold stronger inhibitory activity of elastase and over a 50 fold higher inhibitory potential of tyrosinase compared to references. This study confirms not only the mode of action for traditional Chinese herbal formulations as anti-aging medicines but also proves that the treatment with extracts can be more effective as a single compound treatment. It would potentially establish new alternative therapeutics for skin disorders based on traditional medicines.

Financial support from the Austrian Federal Ministry of Science, Research and Economy and the China Academy of Chinese Medical Sciences is gratefully acknowledged.

\section{Tu-Poster Session 2-PO-69 Lupinus mutabilis extract improves insulin secretion in Diabetic Goto-Kakizaki rats}

Authors Zambrana $\mathrm{S}^{1,2}$, Mamani $\mathrm{O}^{1}$, Catrina $\mathrm{SB}^{2}$, Gonzales $\mathrm{E}^{1}$, Ostenson $\mathrm{CG}^{2}$ Institute 1 Instituto de Investigaciones Farmaco Bioquimicas, Universidad Mayor de San Andres, La Paz, Bolivia; 2 Department of Molecular Medicine and Surgery, Karolinska Institutet, Stockholm, Sweden

DOI 10.1055/s-0037-1608404

The prevalence of type 2 diabetes is increasing worldwide and therefore investigation to develop novel therapeutic strategies is needed. Based on the use of Bolivian traditional foods, the aim of this study is to evaluate the antidiabetic nutraceutical property of Lupinus mutabilis (LM) [1]. The type 2 diabetic model, Goto-Kakizaki rats (GK), was used. In the Oral Glucose Tolerance test (OGTT) LM (2000 mg/kg b.w.), by single oral administration, improved glucose tolerance, expressed by the area under the glucose curve (AUC), $1005.0 \pm 57.59 \mathrm{mM} / 120 \mathrm{~min}$ vs. placebo $1379.7 \pm 132.5 \mathrm{mM} / 120 \mathrm{~min}$ $(p<0.05)$. The $L M$ long-term treatment $(1000 \mathrm{mg} / \mathrm{kg}$ b.w.), for 21 days, improved the AUC under the OGTT $(827.3 \pm 24.80 \mathrm{mM} / 120 \mathrm{~min})$ vs. value before treatment $(1050 \pm 26.96 \mathrm{mM} / 120 \mathrm{~min})(\mathrm{p}<0.05)$. Moreover, $L M$ long-term treatment increased serum insulin levels, after $30 \mathrm{~min}$ of glucose challenge, 2.4-fold at day $20(58.2 \pm 2.3 \mu \mathrm{U} / \mathrm{ml})$ vs. GK placebo $(24.6 \pm 0.8 \mu \mathrm{U} / \mathrm{ml})$ $(p<0.0001)$. Glycated hemoglobin, HbA1c, was reduced at day 20 by $30 \%$ vs. placebo group $(p<0.01)$. In vitro studies showed that $L M(10 \mathrm{mg} / \mathrm{ml})$ stimulates insulin secretion in GK batch incubated islets, at $16.7 \mathrm{mM}$ glucose, 3.3-fold increase $(118.9 \pm 4.8 \mu \mathrm{U} /$ islets/h vs. $35.1 \pm 3.7 \mu \mathrm{U} /$ islet/h, untreated islets) $(p<0.0001)$. The $L M$ mechanism of action was evaluated using inhibitory compounds of insulin secretion pathway. Insulin secretion of $L M(104.7$ \pm 6.9 ) was reduced $42 \%$ by Diazoxide $(60.4 \pm 3.2 \mu \mathrm{U} /$ islet/h) $(\mathrm{p}<0.001), 70 \%$ by calcium-blocker Nifedipine $(31.2 \pm 3.1 \mu \mathrm{U} /$ islet/h) $(\mathrm{p}<0.001), 86.7 \%$ by protein kinas A (PKA) blocker, $\mathrm{H} 89(13.8 \pm 1.0 \mu \mathrm{U} /$ islet/h) $(\mathrm{p}<0.0001), 70.8 \%$ by PKC-blocker Calphostine-C $(30.6 \pm 1.5 \mu \mathrm{U} /$ islet/h) $(\mathrm{p}<0.0001)$ and $93 \%$ by Pertussis toxin, inhibiting insulin exocytosis $(7.3 \pm 0.9 \mu \mathrm{U} /$ islet/h) $(p<0.0001)$. Our findings provide evidence of $L M$ anti-diabetic effect explained by stimulation of insulin release, dependent on L-type calcium channels, PKA and PKC system and insulin exocytosis.

[1] Fornasini, M, et al, Nutricion Hospitalaria, 2012. 27:425 - 433.

\section{Tu-Poster Session 2-PO-70 Systematic Review and Meta-analysis of Derris scandens (Roxb.) Benth. for pain treatment.}

Authors Puttarak $\mathrm{P}^{1,2}$, Sawangjit $\mathrm{R}^{3}$, Chaiyakunapruk $\mathrm{N}^{4}$ Institute 1 Faculty of Pharmaceutical Sciences, Prince of Songkla University, Hatyai, Songkhla, Thailand. 90112, Hatyai, Thailand; 2 Phytomedicine and Pharmaceutical Biotechnology Excellence Center, Faculty of Pharmaceutical Sciences, Prince of Songkla University, Hatyai, Thailand; 3 Clinical Pharmacy Research Unit, Department of Clinical Pharmacy, Faculty of Pharmacy, Mahasarakham University, Mahasarakham, Thailand; 4 Center of Pharmaceutical Outcomes Research, Department of Pharmacy Practice,

Faculty of Pharmaceutical Sciences, Naresuan University, Phitsanulok, Thailand DOI $10.1055 / \mathrm{s}-0037-1608405$

Derris scandens (Roxb.) Benth. was recommended in Thailand National List of Essential Medicines (NLEMs) for musculoskeletal pain treatment as herbal medicine [1] with no summarized of clinical effect and safety. Our study aimed to determine the clinical effects and safety of $D$. scandens for musculoskeletal pain treatment compared with nonsteroidal anti-inflammatory drugs (NSAIDs). International and Thai databases were searched. Comparative clinical studies investigating D.scandens for pain were included. Outcomes of interest were visual analog of pain scale and adverse event. Mean changes of the outcomes from baseline were compared between $D$. scandens and NSAIDs by calculating mean difference. From 42 articles identified, 4 studies involving a total of 414 patients were included for efficacy analysis. The effects of $D$. scandens on reducing pain score were not different from NSAIDs in any time points $(3,7,14,28$ days and overall) without evident of heterogeneity $\left(I^{2}=0.0 \%\right)$. When compared the adverse events (AEs), D.scandens showed not different relative risk with NSAIDs. The major adverse event was gastrointestinal symptoms. D. scandens may be considered as an alternative for musculoskeletal pain reduction.

[1] National drug committee. www.nlem.in.th,The national list of essential drug, A.D. 2015 [in Thai]. The royal gazette (132), 2015 Aug 10.

\section{Tu-Poster Session 2-PO-71 AMELIORATION EFFECT OF CLINACANTHUS NUTANS ON LPS-INDUCED NEUROINFLAMMATION IN RATS}

Authors Azam $A^{1}$, Ismail Intan $S^{1}$, Shaikh Mohd $F^{2}$, Abas $F^{1}$, Shaari $K^{1}$ Institute 1 Laboratory of Natural Products, Institute of Bioscience, Universiti Putra Malaysia, Serdang, Malaysia; 2 2Jeffrey Cheah School of Medicine and Health Sciences, Monash University Malaysia, Subang Jaya, Malaysia DOI 10.1055/s-0037-1608406

Neuroinflammation is a pathological hallmark of many neurological disorders associated with mental health conditions. In the stages of brain immune trauma or infections, an adaptive response in behavior which resulted from 
the conserving energy to fight acute inflammation was observed. We examined the chemical intervention effects on the rat serum metabolites, and associated with the physiological sickness behavior such as anxiety, exploration and reduction of locomotor. Metabolic dysregulation in serum of LPSinduced rats was observed at 0 and 14th day after treatment with Clinacanthus nutans (CN) aqueous leaf extracts. CN is a well-known herbal plant used for its inflammatory remedies. Yet there is no discovery on the brain inflammation. Hence, 25 rats were undergone unilateral stereotaxic injection of $10 \mu \mathrm{LPS}(1 \mathrm{mg} / \mathrm{ml})$, a toxin potent inducer of inflammation, into the substantia nigra pars compacta. Another ten rats were induced with phosphate buffer saline (PBS, $10 \mu \mathrm{l}$ ) as control. Observation on 29 parameters of the rat's behavior related to sickness was tracked with device software (SMART 3.0.1, Panlab Harvard Apparatus) for pre and post- treatment. The impact of pharmacological interventions of LPS, CN extracts $(250,500$ and $1000 \mathrm{mg} / \mathrm{kg}$ of rats BW) and control drug (dextromethorphan hydrobromide, $5 \mathrm{mg} / \mathrm{kg}$ of rats $\mathrm{BW}$ ) was observed in the $1 \mathrm{H}$ NMR serum metabolites variation by using metabolomic approach. All collected data were analyzed using Multivariate Data Analysis (SIMCA) from which pattern trend of related group was documented using orthogonal partial least squares (OPLS). The results revealed that both behavior and metabolite changes correlated with each other $(R 2=0.79)$. A similar ameliorated striking pattern of correlation between improvement in physiological sickness behavior and anti-inflammatory biomarker in $1 \mathrm{H} \mathrm{NMR}$ sera were detected.

\section{Tu-Poster Session 2-PO-72 Nephroprotective effect of Clinacanthus nutans against cisplatin-induced human kidney cell (PCS-400 - 010)}

Authors Ismail I', Mahmod Ilya I'

Institute 1 Laboratory of Natural Products, Institute of Bioscience, Universiti Putra Malaysia, Serdang, Malaysia

DOI 10.1055/s-0037-1608407

Clinacanthus nutans (C. nutans) Lind. locally known as Belalai Gajah or Sabah snake grass is a medicinal plant from family of Acantaceae. In Southeast Asia, this plant is traditionally used for treating skin rashes, insects and snake bites, diabetes mellitus, fever and has diuretic effect. C. nutans has been reported to possess anti-oxidant, anti-inflammation and anti-viral activity. Our previous studies have shown that, this plant could improve the cell viability due to cisplatin exposure on rat kidney cell line (NRK-52E). Subsequently, we have explored the protective effect of the $C$. nutans leaves on primary human kidney cell (PCS-400-010). Eight different solvent extracts of Clinacanthus nutans leaves were tested against cisplatin induced PCS-400 - 010. The aqueous extract showed a protective effect against the induced cell line based on the improvement of the percentage viability in mitochondrial dehydrogenase activity (MTT) and lactate dehydrogenese (LDH) assay. The effect was observed after $24 \mathrm{~h}$ pretreatment of the extract and $48 \mathrm{~h}$ cisplatin exposure afterwards. Among the cisplatin concentrations, 2.5, 5, 10, 20, and $30 \mathrm{uM}$, used for induction, 5 and 10 uM exhibited significant changes. LCMS based metabolomic approach coupled with multivariate data statistical analysis was used to assess the changes in the intracellular metabolic profile in PCS-400 010 , due to the pretreatment of the extract in the presence or absence of cisplatin.

\section{Tu-Poster Session 2-PO-73 Development of a Capsicum Extract for large scale production and its pharmacological characterisation}

\author{
Authors Erdelmeier $C^{2}$, Koch $E^{2}$, Palea $S^{1}$ \\ Institute 1 UROsphere, Faculté des Sciences Pharmaceutiques, Toulouse, \\ France; 2 Dr.Willmar Schwabe Arzneimittel, Preclinical Research, 76227 \\ Karlsruhe, Germany, Karlsruhe, Germany \\ DOI 10.1055/s-0037-1608408
}

Chili pepper fruits (Capsicum spp.) are not only consumed as a pungent spice but are also employed for medicinal purposes. Thus, the use of Capsicum extracts has a long tradition in folk medicine as rubefacient and topical analgesic for the treatment of various neuropathic and musculoskeletal pain conditions. In the European Pharmacopoeia (Capsici Fructus), the crude drug is defined as ripe fruits of $C$. annuum var. minimum Miller (Heiser) and small fruit varieties of $C$. frutescens $L$. Capsaicin, dihydrocapsaicin and further minor capsaicinoids are the main active ingredients of Capsicum fruits. It was the aim of our studies to develop a capsaicinoid-rich extract suitable for industrial scale production. Due to the rather lipophilic character of capsaicinoids, apart from ethyl acetate low-polar ethanol-water and acetone-water solvent mixtures were initially used for extraction. However, resulting extracts proved to be very inhomogeneous and required large quantities of excipients to yield dry and powdery materials. More polar extraction solvents were therefore tried and the best result was obtained with $70 \%(w / w)$ ethanol. The capsaicin content of such extracts amounts to about $2 \%$ of capsaicin and dihydrocapsaicin with an extract yield of ca. $20 \%$.

The analgesic effect of capsaicin is due to a blockade of TRPV1 receptors on nociceptive sensory nerves. As it is known that TRPV1 receptors are important in the pathogenesis of overactive bladder diseases and their modulation can reduce symptoms, the ethanolic extract was tested in a cyclophosphamideinduced rat model of interstitial cystitis/painful bladder syndrome (IC/PBS). Oral administration $(1,3,10$ and $75 \mathrm{mg} / \mathrm{kg})$ of the extract for 4 days reversed the induced visceral allodynia and hyperalgesia in a dose dependent-manner. The obtained data indicate that this Capsicum extract may be an interesting oral treatment option for complex pain conditions for which no standard therapy is yet available.

\section{Tu-Poster Session 2-PO-74 Ameliorative Effect of Laminarials Brown Algae Distributed in Korea on Diabetic Zebrafish Models for Type 1 and 2}

Authors Jeon $\mathrm{Y}^{1}$, Nam Youn $\mathrm{H}^{2}$, Kang Tong $\mathrm{H}^{2}$, Kwak Jong $\mathrm{H}^{1}$ Institute 1 School of Pharmacy, Sungkyunkwan University, Suwon, Korea, Republic of (South); 2 Department of Oriental Medicinal Biotechnology, College of Life Sciences, Kyung Hee University, Gyeonggi-do, Korea, Republic of (South)

DOI 10.1055/s-0037-1608409

Phlorotannins, belonging to a special class of natural polyphenols, are isolated from several Laminariales brown algae. Phlorotannins have been reported to show various biological activities, such as anti-inflammatory, hepatoprotective, anti-diabetic, antioxidative and anticancer activities [1]. Ten Laminariales brown algae were collected in Korea. We evaluated the anti-diabetic activity of the extracts from the algae in zebrafish models for type 1 and 2 diabetes. Type 1 diabetes zebrafish model was induced by alloxan, which cause pancreatic $\beta$ cell necrosis [2]. Type 2 diabetes zebrafish model was induced by insulin. Exposure to excess insulin can induce insulin resistance typical of type 2 diabetes [3]. Following alloxan or insulin treatment, pancreatic islet size and fluorescence intensity were measured. Most of the extracts showed potent anti-diabetic activities for type 1 and 2. Among ten Laminariales algae, Saccharina japonica and Agarum clathratum subsp. yakishiriense revealed the strongest ameliorative effect on pancreatic islet damage in zebrafish for type 1 and 2 diabetic models, respectively. The extracts were investigated inhibitory activities on $\alpha$-glucosidase, PTP1B and DPP-4. PTP1B and $\alpha$-glucosidase were 
significantly inhibited by Eisenia bicyclis, A. clathratum subsp. yakishiriense, Ecklonia kurome, E. stolonifera and E. cava.

This work was supported by a grant from Marine Biotechnology Program (PJT200620, Genome Analysis of Marine Organisms and Development of Functional Applications) funded by Ministry of Oceans and Fisheries.

[1] Kwak JH et al. Chem Commun 2014; 50: $13045-13048$

[2] Desgraz R et al. Trends Endocrin Met 2011; 22: 34 - 43

[3] Yang X et al. J Endocrinol 2014; 221: $469-480$

\section{Tu-Poster Session 2-PO-75 ARTEMISIA ANNUA ESSENTIAL OIL ACTIVITY AGAINST MALASSEZIA SPP}

Authors Pini $G^{1}$, Santomauro $F^{2}$, Sacco $C^{2}$, Donato $R^{2}$, Ascrizzi $R^{3}$, Bilia Anna $\mathrm{R}^{4}$

Institute 1 Department of Experimental and Clinical Medicine, University of Florence, Florence, Italy; 2 Department of Human Health Sciences, University of Florence, Florence, Italy; 3 Department of Pharmacy, University of Pisa, Pisa, Italy; 4 Department of Chemistry, University of Florence, Sesto Fiorentino (FI), Italy DOI 10.1055/s-0037-1608410

Artemisia annua L. (Asteraceae) is the source of the unique sesquiterpene endoperoxide lactone artemisinin used in the treatment of the chloroquineresistant and cerebral malaria. The essential oil, rich in mono- and sesquiterpenes, represents a by-product with medicinal properties. Besides significant variations in its percentage and composition have been reported (major constituents can be camphor (up to $48 \%$ ), germacrene D (up to $18.9 \%$ ), artemisia ketone (up to $68 \%$ ), and 1,8 cineole (up to 51.5\%)), numerous studies support exciting antibacterial and antifungal activities of the essential oil [1].

In continuing our studies on A. annua essential oil [2,3] we investigated the activity against some Malassezia species closely related to many skin disorders in man and animals. Tests were carried out either in liquid phase and in vapor phase. Essential oil of A. annua showed strong antimicrobial activity in both tests and towards every species of Malassezia analyzed.

The MFC results demonstrated a strong fungicidal activity of A.annua EO on most of Malassezia strains tested, with values from $0.78 \mu \mathrm{L} / \mathrm{mL}$ to $1.56 \mu \mathrm{L} / \mathrm{mL}$. Only three strains of M.sympodialis have required a higher concentration of $3.125 \mu \mathrm{L} / \mathrm{mL}$.

Overall the MICs obtained by the vapor diffusion assay were lower than those obtained by the liquid method. For example, by comparing for each species the average values of MIC obtained by the two methods at $72 \mathrm{~h}$, they are higher (1.3 to 8 times) in liquid method, respect to those in the vapor phase. [1] Bilia AR, Santomauro F, Sacco C, Bergonzi MC, Donato R. Evid Based Complement Alternat Med. 2014;2014:159819.

[2] Santomauro F, Donato R, Sacco C, Pini G, Flamini G, Bilia AR. Planta Med. 2016 Jul;82:1016 - 20.

[3] Tasdemir D, Tierney M, Sen R, Bergonzi MC, Demirci B, Bilia AR, Baser KH, Brun R, Chatterjee M. Planta Med. 2015 Aug;81:1029-37. doi: 10.1055/s0035 - 1546125.

\section{Tu-Poster Session 2-PO-76 Equisetum hyemale L. efficacy of organic extract in function of phenolic and flavanol content, antioxidant activity, reducing ability and allelopathy}

Authors Flores Xavier $V^{1}$, Gonçalves Rodrigues Das Dores $\mathrm{R}^{1}$, Silva e Souza $C^{2}$, Vieira Braga $\mathrm{T}^{3}$

Institute 1 Universidade Federal de Ouro Preto, OURO PRETO, Brazil;

2 Universidade Federal de Viçosa, Viçosa, Brazil; 3 Universidade Federal de Minas Gerais, Belo Horizonte, Brazil

DOI 10.1055/s-0037-1608411

This study aimed to evaluate organic extracts in function of biomass production, organic extracts yields, phenolic, flavonoids, flavanols and antioxidant capacity levels and the allelopathic potential of Equisetum hyemale L. The extracts were made in ethanol, methanol and ethyl acetate by maceration. The determination of phenolic by Folin Ciocalteu, flavanols by vanillin acid, flavonoids by aluminum chloride, antioxidant activity by DPPH and reducing power by ferric chloride, allelopathy test front lettuce seed and biotoxicity $\left(D_{50)}\right.$ by Artemia salina. The effectiveness of the vegetable extract under analysis is linked to the quality of the chemical properties of the extract. In all treatments color and odor of the extracts remained similar to that of the in natura species, after obtaining the dry residue. The highest yields of extracts, phenolic compounds and flavonoids were in methanolic extract (EH-MEOH), flavanols and reducing potential in the ethyl acetate extract (EH-ACOET), efficacy of obtaining the dry extract and antioxidant activity in ethanolic extract (EH-ETOH). In the bioassays, the lowest concentrations of all treatments did not lead to inhibition of growth and the lower toxicity can be attributed to the extract in ethyl acetate (EH-ACOET). Regarding the antioxidant properties can been directly related to bioflavonoids, and ethyl acetate and ethanol extracts were low toxicity.

\section{Tu-Poster Session 2-PO-77 Search for effective plant materials for Alzheimer's disease}

Authors Murata $\mathrm{K}^{1}$, Akiyama $\mathrm{R}^{1}$, Noro $\mathrm{I}^{1}$, Nishio $\mathrm{A}^{1}$, Nakagawa $\mathrm{S}^{1}$, Yoshioka $\mathrm{Y}^{2}$, Deguchi $\mathrm{T}^{1}$, Matsuda $\mathrm{H}^{1}$, Ishida $\mathrm{Y}^{1}$

Institute 1 Faculty of Pharmacy, Kindai University, Higashiosaka, Japan; 2 INABATA KORYO CO,LTD, Osaka, Japan

DOI 10.1055/s-0037-1608412

Alzheimer's disease became a threatening disease. We performed an extensive screening for effective plant materials for dementia, especially Alzheimer's disease utilizing $\beta$-secretase, acetylcholinesterase and butyrylcholinesterase. As results, we found that some extracts obtained from spices used in Asian countries would be effective to Alzheimer's disease in long-term consumption. In addition, the active principles were revealed as various compounds including terpenes, phenylpropanoids, alkaloids and lignans ${ }^{1)}$.

In this study, we have extended our screening source to ingredients of incence stick used in Japan. We have screened the ingredients consisting of the incence stick including star anise (fruit of Illicium verum), turmeric (rhizome of Curcuma longa), clove (bud of Syzygium aromaticum), patchouli (above ground part of Pogostemon cablin) and frankincense (rosin of Boswellia sp.) extracts showing $17.0,26.4,24.6,31.7$, and $24.4 \%$ of inhibitions, respectively, against acetylcholinesterase at $100 \mu \mathrm{g} / \mathrm{ml}$. On the other hand, basal material of incence stick: hexane extract from leaf of Cryptomeria japonica showed $31.4 \%$ of inhibition at $100 \mu \mathrm{g} / \mathrm{ml}$. Activity-guided purification of active principles led us to isolate kaur-16-ene, nezukol and ferruginol.

The heartwood of Chamaecyparis obtusa, a well-known conifer as C. japonica, was also tested for the inhibitory activity against acetylcholinesterase. Ethyl acetate extract of C. obtusa showed $37.7 \%$ on inhibition at $100 \mu \mathrm{g} / \mathrm{ml}$. One of the active principles was determined as hinokinin.

These results suggest that aromatic materials can be a promising screening source for effective agents for therapy of Alzheimer's diseases.

[1] Murata K, Matsumura S, Yoshioka Y, Ueno Y, Matsuda H.: J. Nat. Med,69, $123-129$ (2015).

\section{Tu-Poster Session 2-PO-78 Oral treatment with the Ginkgo biloba extract EGb $761^{\circledR}$ prevents sodium azide-induced memory deficits in rats}

Authors Nöldner $\mathrm{M}^{1}$

Institute 1 Preclinical Research Dr. Willmar Schwabe Pharmaceuticals, Karlsruhe, Germany DOI 10.1055/s-0037-1608413

EGb $761^{\circledR}$ is a special extract from leaves of Ginkgo biloba L. and is one of the most popular herbal medicines in the world. Its therapeutic use includes the treatment of cognitive impairment and neuropsychiatric symptoms in dementia. Alzheimer's disease (AD) is a neurodegenerative disorder that accounts for 
$50-70 \%$ of all cases of dementia. Although the etiology of the disease is still unknown considerable evidence suggests the involvement of mitochondrial damage. Reduction of mitochondrial complex IV activity mimics pathological processes involved in $A D$ and can be evoked in rats by chronic administration of sodium azide (NaN3). In fact, chronic infusion of NaN3 for one month via subcutaneously implanted osmotic minipumps induced deficits in learning performance of rats in the passive avoidance paradigm (PA). In comparison to control rats, animals receiving NaN3 showed a significantly shorter latency time indicating a diminished potential to remember previous aversive stimuli. Daily concomitant oral administration of EGb $761^{\circledR}(25,50$ or $100 \mathrm{mg} / \mathrm{kg})$ reversed the NaN3-induced learning deficit in the PA model which was statistically significant already for the lowest dose and was comparable to that of the acetylcholinesterase inhibitor donepezil $(0.3 \mathrm{mg} / \mathrm{kg} /$ day $)$.

Tab. 1: Effect of EGb $761^{\circledR}$ and donepezil on NaN3-induced cognitive impairment in rats as measured by the latency time (seconds) in the passive avoidance model

\begin{tabular}{|c|c|c|c|c|}
\hline $\begin{array}{l}\text { Dose } \\
\text { (mg/kg, p.o.) }\end{array}$ & $\begin{array}{l}\text { Vehicle + } \\
\mathrm{NaCl}\end{array}$ & $\begin{array}{l}\text { Vehicle + } \\
\text { NaN3 }\end{array}$ & $\begin{array}{l}\text { EGb } 761^{\circledR}+ \\
\text { NaN3 }\end{array}$ & $\begin{array}{l}\text { Donepezil + } \\
\text { NaN3 }\end{array}$ \\
\hline 0 & $180 \pm 0$ & $44 \pm 29 *$ & & \\
\hline 0.3 & & & & $145 \pm 61 \#$ \\
\hline 25 & & & $160 \pm 56 \#$ & \\
\hline 50 & & & $147 \pm 64 \#$ & \\
\hline 100 & & & $177 \pm 7$ \# & \\
\hline
\end{tabular}

These results substantiate earlier experimental findings demonstrating neuroprotective activity of EGb $761^{\circledR}$ and support its therapeutic use in the prevention and treatment of $\mathrm{AD}$.

\section{Tu-Poster Session 2-PO-80 Evaluation of Antiplasmodial and Immunomodulatory Activities of Extracts of Endophytic Fungi Isolated from four Nigerian Medicinal Plants}

Authors Ujam $\mathrm{N}^{1}$, Eze $\mathrm{P}^{1}$, Umeokoli $\mathrm{B}^{2}$, Abbah $\mathrm{C}^{2}$, Okoye $\mathrm{F}^{2}$, Esimone $\mathrm{C}^{1}$ Institute 1 Department of Pharmaceutical Microbiology and Biotechnology, Faculty of Pharmaceutical Sciences, Nnamdi Azikiwe University, Awka-Nigeria, Awka, Nigeria; 2 Department of Pharmaceutical and Medicinal Chemistry, Faculty of Pharmaceutical Sciences, Nnamdi Azikiwe University, Awka-Nigeria, Awka, Nigeria

DOI 10.1055/s-0037-1608414

Endophytic fungi are the most promising source for new drugs to combat the problem of antimicrobial resistance. Twelve fungal endophytes were isolated from the leaves and stems of Azadirachta indica, Newbouldia laevis, Psidium guajava and Agerantum conyzoides using standard methods. Solid state fermentation of the isolated fungi was carried out on rice media for 21 days at $22^{\circ} \mathrm{C}$ and the fungal secondary metabolites were extracted using ethyl acetate. The extracts were tested for antiplasmodial activity in vivo against Plasmodium berghei (Anka strain) using Peter and Reyley's curative test methods while in vitro activity against Plasmosium falciparum was determined using standardized WHO microtest system. Immunomodulatory activity of the extract was evaluated by cyclophosphamide unduce myelosuppresion. Active extracts were further subjected to Delayed Type Hypersensitivity Response (DTHR) and Heamaglutination Inhibition Assay using SRBCs and antigens. The extracts showed varying degree of antiplasmodial activities. Growth of P.berghei was significanlty $(P<0.001)$ inhibited, curative effect range from $59.09-100 \%$. Schizont maturation of P.falciparum isolates was inhibited and the highest level of inhibition was observed at $1 \mathrm{mg} / \mathrm{ml}(P<0.05)$. Seven (58.33\%) of the fungal extracts showed significant counteracting effect to the cyclophosphamide-induced reduction in total WBC, \% neutrophil, lymphocyte and monocyte $(p<0.05)$. There was a significant inhibition of the DTHR and heamagglutination induced by SRBC in rats up to 78.49 and $69.23 \%$ respectively. Correlation was positive and statistically significant between malaria and Immunomodulatory activities.observed $(P=0.036, r=0.786)$. HPLC$\mathrm{DAD}$ analysis revealed the presence of ferulic acid, luteolin, peperine, rocagla- mid, epicatechin and phenylacetic acid which have been reported to have antiplasmodial and immunomodulatory activities. Extracts of endophytic fungi isolated from these Nigerian Medicinal plants possess antiplasmodial and immunomodulatory activities which portends a novel approach for malaria therapy.

\section{Tu-Poster Session 2-PO-81 The efficacy of combined plant extracts of STW 5 on intestinal neurotransmission and motility in an experimental animal model}

Authors Sibaev $\mathrm{A}^{1,2}$, Abdel-Aziz $\mathrm{H}^{3}$, Kelber $\mathrm{O}^{3}$, Storr $\mathrm{M}^{4}$

Institute 1 Department of Internal Medicine II, Ludwig Maximilians University of Munich, München, Germany; 2 Walter-Brendel-Zentrum, Ludwig Maximilians University, Munich, Germany; 3 Innovation \& Development, Steigerwald Arzneimittelwerk GmbH, Bayer Consumer Health, Dar, Germany; 4 Zentrum für Endoskopie, Starnberg, Germany

DOI 10.1055/s-0037-1608415

The phytomedicine STW 5 (Iberogast ${ }^{\circledR}$ ) is a fixed combination of the fresh plant fluid extract of Iberis amara totalis and the fluid extracts of eight herbal drugs, Angelica root, Matricaria flower, Caraway fruit, St. Mary's thistle fruit, Balm leaves, Peppermint leaves, Greater Celandine herb and Liquorice root. Its clinical efficacy in functional gastrointestinal diseases was shown by several randomized controlled clinical studies and more than 50 years of clinical experience. The aim of this study was to clarify, whether combined plant extracts of STW 5 act on contractions elicited after electrical field stimulation (EFS) in the small intestine of mice in vitro.

In organ bath experiments contractions were elicited in the small intestine of mice using electrical field stimulation EFS. Single components of STW 5 were tested alone in dilutions of 1:1000, 1:500 and 1:100 and in presence of Iberis amara in a dilution of 1:500 (20 min. preincubation).

Iberis amara alone had not significant effect in 1:500 dilution on EFS-induced contractions in murine small intestine. The combinations of Iberis amara with Angelica roots, Caraway fruits or Greater Celandine herbs was more effective in reducing EFS contractions compared to the single extracts alone. No significant differences were seen in combination with Peppermint leaves, Matricaria flower, St. Mary's thistle fruits, or Lemon balm leaves. Liquorice roots increased EFS contractions alone (n.s.), the combination with Iberis amara significantly reduced this effect.

The combination of different extracts of STW 5 with Iberis amara shows potentiating effects on some compounds (Dual Principle of Action). Iberis amara presence seems to be crucial for the overall effect of STW 5.

\section{Tu-Poster Session 2-PO-82 Immunotoxicological safety and observational studies of homeopathic preparations from mycelium cultures of Laricifomes officinalis}

\section{Authors Bader $\mathrm{G}^{1}$, Wiethoff $\mathrm{K}^{1}$}

Institute 1 Sanum-Kehlbeck GmbH \& Co. KG, Hoya (Weser), Germany DOI 10.1055/s-0037-1608416

Fruiting bodies of Laricifomes officinalis (Vill.: Fr.) Kotl. \& Pouz. (Polyporaceae) are used in Traditional European Medicine (Fungus laricis, Agaricus albus, German Pharmacopoeia Supplement ErgB 6) as well as in Homeopathy (Laricifomes officinalis, Boletus laricis, German Homeopathic Pharmacopoeia HAB). Homeopathic preparations from the mycelium of the strain DSM 5211 of this fungus are evaluated in the view of safety.

The biotechnological produced biomass from the mycelium culture is purified from fermentation medium and then mechanically opened through a cell mill. After different purification steps, the water-soluble filtrate undergoes sterile filtration and is freeze-dried. The resulting lyophilisate is named "e volumine cellulae (lyophil, steril.)”, synonym: “e volumine mycelii (lyophil, steril.)”. 
Acute toxicity and possible immunotoxic effects after repeated oral or subcutaneous application were tested in various studies with GLP compliance in genetic defined mice and guinea pigs. These studies include general immunotoxicity, mitogenic effects of naive T-cells, proliferation of antigen-stimulated T-cells, delayed type hypersensitivity reactivity (DTH), antigen-specific antibody production, acute systemic anaphylaxis induction, and skin sensitisation studies. The tested preparations for oral use can be regarded as immunotoxicologically safe in potencies D 3, D 4 and D 5. For the subcutaneous administration, DTH reactions were detected in individual animals (potency D4); however, no systemic effects of immediate hypersensitivity were observed for the therapeutically used potency D 5. This data is valid only for the investigated fungus strain as well as for the specific, GMP controlled manufacturing process.

An observational study with oral and parenteral used homeopathic preparations (D4 capsules, D 5 drops and D 5 injection of the Larifikehl-line) was carried out. This study showed a very good safety profile with no side effects. The mentioned dosage forms of Larifikehl are authorised in Switzerland for the homeopathic treatment of fever, lung diseases and gastrointestinal inflammations.

\section{Tu-Poster Session 2-PO-83 Cytotoxic activity of medicinal plants selected from Euphorbiaceae family against Hepatocellular carcinoma cell}

Authors Ruangnoo $S^{1,2}$, Kuropakornpong $\mathrm{P}^{1,2}$, Itharat $A^{1,2}$

Institute 1 Faculty of Medicine, Thammasat University, Pathumthani,

Thailand; 2 Center of Excellence in Applied Thai Traditional Medicine Research, Faculty of Medicine, Thammasat University, Pathumthani, Thailand DOI 10.1055/s-0037-1608417

Euphorbiaceae is the large flowering plant families. A variety of Euphorbiaceae-based phytochemicals including alkaloids, flavonoids, saponins, tannins, phenols and essential oils, it would appear that their diverse medicinal properties. In this study we investigated the cytotoxic activity of the selected medicinal plants from Euphorbiaceae family against hepatocellular carcinoma cell line (HepG2) by using sulphorhodamine B (SRB) assay. The aqueous and ethanolic extract from Ricinus communis Linn, Jatropha multifida Linn, Euphorbia antiquorum Linn, Trigonostemon reidioides (Kurz) Craib and Bridelia ovata Decne. were tested for screen their cytotoxic activity against HepG2. The results found that only 3 ethanolic extracts with potent cytotoxic activity $\left(I C_{50}<50 \mu \mathrm{g} / \mathrm{ml}\right)$. The ethanolic extract of $T$. reidioides showed the highest cytotoxic activity against $\mathrm{HepG} 2$ which $\mathrm{IC}_{50}$ of $0.016 \pm 0.002 \mu \mathrm{g} / \mathrm{ml}$ following by $E$. antiquorum $0.43 \pm 0.02 \mu \mathrm{g} / \mathrm{ml}$. And the ethanolic extract of J. multifida showed less cytotoxic activity against HepG2 which $I C_{50}$ of $26.20 \pm 1.15 \mu \mathrm{g} /$ $\mathrm{ml}$. In conclusion, this study obtained the potent natural source for anti-liver cancer from $T$. reidioides and $E$. antiquorum. It should be continuously isolate cytotoxic compounds from this plant.

\section{Tu-Poster Session 2-PO-85 Puerariae radix extract suppresses $\mathrm{H}_{2} \mathrm{O}_{2}$-induced ARPE-19 cell death and permeability via phosphorylation of p38MAPK and JNK}

Authors Kang Nu $\mathrm{R}^{1,2}$, Kim $\mathrm{CS}^{1,2}$, Lee $\mathrm{Ik} \mathrm{S}^{1}$, Kim Young $\mathrm{S}^{1,2}$, Kim Jin $\mathrm{S}^{1,2}$ Institute 1 Korean Medicine Convergence Research Division, Korea Institute of Oriental Medicine (KIOM), Daejeon, Korea, Republic of (South); 2 Korean Medicine Life Science, University of Science Technology (UST), Daejeon, Korea, Republic of (South)

DOI 10.1055/s-0037-1608418

Puerariae radix (root of Pueraia lobata; Leguminosae) is one of the most important herbs in Korean traditional medicine and has been frequently used for the treatment of diarrhea, diabetes, cardiovascular disease and osteonecrosis. Retinopathy is a major cause of vision loss among middle aged and elderly people. The retinal pigment epithelium (RPE) plays an important role in the maintenance of photoreceptors in the retina, and RPE cells (ARPE-19) are used to investigate the pathology and physiology of retinopathy.

The roots of $P$. lobata were collected in Kyonggi-do, Korea, in April 2005 and were identified by Professor J.-H. Kim. The major compounds, puerarin, daidzin and daidzein, were isolated from the EtOH extract of Puerariae radix (PR). We evaluated whether the EtOH extract of PR and its major compounds could prevent cell death and decrease the membrane permeability in oxidative stress-induced ARPE-19. PR significantly protected against $\mathrm{H} 2 \mathrm{O} 2$-induced cell death, whereas its major compounds did not inhibit H2O2-induced intracellular ROS generation.

The effect of PR on the RPE barrier was conducted by measuring paracellular permeability of dextran in ARPE-19. PR showed significant decrease in the membrane permeability. Paracellular permeability is related with the alteration in tight junction protein expression. PR also attenuated the decrease in the expression of ZO-1 and occludin. ZO-1 expression was altered in response to oxidative damage. To identify the signaling pathway through which PR exhibits its effects in $\mathrm{H}_{2} \mathrm{O}_{2}$-induced cells, we examined the effects of PR on phosphorylation of ERK, P38MAPK, and JNK. PR significantly suppressed the phosphorylation of pERK, p38 MAPK, and JNK in ARPE-19 cells, indicating that PR significantly suppressed $\mathrm{H}_{2} \mathrm{O}_{2}$-induced ARPE- 19 cell death and permeability via phosphorylation of pERK, p38MAPK and JNK.

Taken together, these results suggest PR to be a potential alternative for preventing the progression of oxidative stress-related retinopathy.

\section{Tu-Poster Session 2-PO-86 Antiviral effect of Inonotus obliquus (Pers.:Fr.) Pilat extract against herpes simplex virus type 1 in vitro}

Authors Kapp $\mathrm{K}^{1}$, Püssa $\mathrm{T}^{2}$, Vuorela $\mathrm{H}^{1}$, Välimaa $\mathrm{H}^{3}$

Institute 1 Division of Pharmaceutical Biosciences, Faculty of Pharmacy, University of Helsinki, Helsinki, Finland; 2 Department of Food Hygiene, Institute of Veterinary Medicine and Animal Sciences, Estonian University of Life Sciences, Tartu, Estonia; 3 Department of Virology, Medical Faculty, University of Helsinki and Helsinki University Hospital, Helsinki, Finland DOI 10.1055/s-0037-1608419

The anti-herpes simplex virus type 1 (anti-HSV-1) activity of polypore fungus Inonotus obliquus (Pers.:Fr.) Pilat extract was studied in vitro.

The fungus material was obtained from Pakuso LLC, Finland and collected from birch trees grown in Finland above the Arctic Circle. The extract was obtained by treating the certified fungus material with an alcohol-water extractant that was at the end stripped to dryness. The dry extract was then dissolved into water.

For the experiment, wild type HSV-1 strain F was incubated with I. obliquus extract or mock control i.e. water undergone the same preparation process as the I. obliquus extract. Vero cell cultures in 24-well plates were infected with the virus and incubated. Infected cells were identified by immunoperoxidase staining. The effect of $I$. obliquus extract on the host cell viability was tested by a resazurin assay. Vero cells were cultured in a 96-well plate in the presence of I. obliquus extract or mock control in the concentrations of $5.6 \times 10^{-6}$ $7.5 \times 10^{0} \mathrm{mg} / \mathrm{ml}$. Wells containing no cells filled with cell culture media were used as a control. Triton X-100 was used as a positive control for cytotoxicity at two concentrations.

I. obliquus extract in the concentrations of $4.5 \times 10^{-5}-7.5 \times 10^{0} \mathrm{mg} / \mathrm{ml}$ had clear anti-HSV-1 effect. Extract concentration of $2.25 \times 10^{-5} \mathrm{mg} / \mathrm{ml}$ and lower did no longer have anti-HSV-1 effect. Mock control had no anti-HSV-1 effect. I. obliquus extract showed viability of Vero cells by $8.1 \%, 15.5 \%$ and $55.8 \%$ respectively at the concentrations of $7.5 \mathrm{mg} / \mathrm{ml}, 3.75 \mathrm{mg} / \mathrm{ml}$ and $1.88 \mathrm{mg} /$ $\mathrm{ml}$. Lower concentrations possessed no cytotoxic effect. Mock control showed no cytotoxicity to the Vero cells.

The results demonstrate the anti-HSV-1 effect of I. obliquus, indicating need for further experiments to determine the active compounds and the mechanism of action. 


\section{Tu-Poster Session 2-PO-87 Inhibitory effects of Ponciri Fructus on testosterone-induced benign prostatic hyperplasia in rats}

\author{
Authors Jeon $W Y^{1}$, Kim Ohn $S^{2}$, Seo $\mathrm{CS}^{1}$, Jin Seong $E^{1}$, Kim JA $A^{3}$, Shin $\mathrm{HK}^{1}$, \\ Kim $\mathrm{YU}^{4}$, Lee $M Y^{1}$ \\ Institute 1 K-herb Research Center, Korea Institute of Oriental Medicine, \\ Daejeon, Korea, Republic of (South); 2 KM Convergence Research Division, \\ Korea Institute of Oriental Medicine, Daejeon, Korea, Republic of (South); \\ 3 School of Pharmacy, College of Pharmacy, Yeungnam University, \\ Gyeongsan-si, Korea, Republic of (South); 4 Department of Pharmaceutical \\ Engineering, College of Biomedical Science, Daegu Haany University, \\ Gyeongsan-si, Korea, Republic of (South) \\ DOI 10.1055/s-0037-1608420
}

Benign prostatic hyperplasia (BPH) is non-cancerous condition of enlargement of the prostate, a common occurrence in older men. The immature fruits of Poncirus trifoliata (L.) Rafinesque (Rutaceae), Ponciri Fructus are widely used in traditional oriental medicine for the therapy of various diseases. However, little is known about the mechanism underlying the pathogenesis of BPH. In the present study, we investigated the protective effects of a Ponciri Fructus extract (PFE) on the development of $\mathrm{BPH}$ in a in a rat model of $\mathrm{BPH}$ induced by testosterone propionate (TP).

Male Sprague Dawley rats were used as a model of BPH after its induction by daily subcutaneous injections of TP/corn oil, for a period of four weeks. PFE was administrated daily $1 \mathrm{~h}$ before TP/corn oil injection by oral gavage at a dose level of $200 \mathrm{mg} / \mathrm{kg}$ during the 4 weeks of TP/corn oil injections. All rats were sacrificed at the end of the experiment, we measured the relative prostate weight, the levels of testosterone and dihydrotestosterone (DHT), histological changes, activities of antioxidant enzymes (catalase, glutathione peroxidase, glutathione reductase, and superoxide dismutase), and expression of proliferating cell nuclear antigen (PCNA). In addition, we also measured the inhibition (\%) of $5 \alpha$-reductase in the prostatic tissue.

Our findings indicate that PFE significantly inhibited the development of $\mathrm{BPH}$; decreased the relative prostate weight, the level of testosterone and DHT in serum and prostatic tissue, prostatic hyperplasia, expression of PCNA, and increased the antioxidant enzymes. Moreover, PFE showed a weak inhibitory activity on $5 \alpha$-reductase.

These results suggest that PFE may be used as a therapeutic agent for BPH via antiproliferative and antioxidant effects.

\section{Tu-Poster Session 2-PO-88 Quantitative analysis and biological efficacies regarding the neuroprotective and antineuroinflammatory actions of the herbal formula SCD-B-033}

Authors $\mathrm{Kim} \mathrm{Yu} \mathrm{J}^{1,3}$, Lim $\mathrm{HS}^{1}$, Kim BY${ }^{1}$, Seo $\mathrm{CS}^{2}$, Jeong SJ ${ }^{1,4}$

Institute 1 Herbal Medicine Research Division, Korea Institute of Oriental Medicine, Daejeon, Korea, Republic of (South); 2 K-herb Research Center, Korea Institute of Oriental Medicine, Daejeon, Korea, Republic of (South); 3 College of Pharmacy, Chungnam National University, Daejeon, Korea, Republic of (South); 4 Korean Medicine Life Science, University of Science \& Technology, Daejeon, Korea, Republic of (South)

DOI 10.1055/s-0037-1608421

SCD-B-033 (B33) is a traditional herbal formula that comprises seven medicinal herbs and is broadly utilized to treat hypertension[1], dementia[2], and headache[3] in Korea, Japan, and China. However, the effects of B33 and its herbal components on neurodegenerative diseases in general have not been reported. The common cellular events of neurodegenerative diseases, such as Alzheimer's, Parkinson's, and Huntington's diseases, include neuronal cell death and neuroinflammation. In our study, we examined the inhibitory effects of B33 and its seven components on neuronal cell death and inflammation using HT22 hippocampal cells and BV-2 microglia, respectively. In HT22 cells that were damaged by hydrogen peroxide $\left(\mathrm{H}_{2} \mathrm{O}_{2}\right)$ treatment, $\mathrm{B33}$ signifi- cantly reversed cell viability compared with cells treated with $\mathrm{H}_{2} \mathrm{O}_{2}$ alone. Among its seven herbal components, Uncaria sinensis, Chrysanthemum morifolium, Zingiber officinale (ZO), Pinellia ternate (PT), Citrus unshiu (CU), and Poria $\operatorname{cocos}(\mathrm{PC})$ exhibited significant neuroprotective effects. In lipopolysaccharidestimulated BV-2 cells, B33 significantly suppressed the production of the inflammatory cytokines tumor necrosis factor-alpha and interleukin-6, indicating the antineuroinflammatory activity of B33. In addition, the herbal extracts from ZO, Panax ginseng (PG), PT, CU, and PC, exhibited inhibitory effects on the inflammatory response in microglia. These data imply that the B33 effect on neurodegeneration occurs via coordination among its seven components. To establish a quality control for B33, a simultaneous analysis using five marker compounds identified hesperidin $(37.892 \pm 1.228 \mathrm{mg} / \mathrm{g})$, a marker compound of $\mathrm{CU}$, as a major compound of B33. Further investigation of the combinatorial activities of two or more marker compounds will be necessary to verify their antineurodegenerative regulatory mechanisms.

[1] Shimada Y, Yang Q, et al. Am J Chin Med 2003; 31: 79 - 85.

[2] Watanabe H, Zhao Q, et al. Pharmacol Biochem Behav 2003; 75: 635 643.

[3] Dohi K, Aruga T, et al. Headache 2004; 44: 375.

\section{Tu-Poster Session 2-PO-89 Protective effect of Saururus chinensis extract against an endocrine disruptor in mouse testicular germ cell line}

Authors Kim Hyung $D^{1,2}$, Kim $G S^{1}$, Lee $S E^{1}$, Lee Dae $Y^{1}$, Seo Kyung $\mathrm{H}^{1}$, Lee $\mathrm{YS}^{1}$, Lee Jae $\mathrm{W}^{1}$, Choi Doo $\mathrm{J}^{1}$

Institute 1 Department of Herbal Crop Research, NIHHS, RDA, Eumseong, Korea, Republic of (South); 2 Department of Biochemistry, School of Life Sciences, Chungbuk National University, Cheongju, Korea, Republic of (South) DOI 10.1055/s-0037-1608422

This study was performed to evaluate the protective effect of Saururus chinensis ethanol extract (SCE) against styrene toxicity in mouse testicular germ cells. Cytotoxicity in mouse testicular germ cells was measured using 3-(4,5dimethylthiazol-2-yl)-2,5-diphenyltetrazolium bromide (MTT) assay. Generation of reactive oxygen species (ROS) was determined using 2',7'-dichlorodihydrofluorescein diacetate (DCF-DA) assay. Semi-quantitative reverse transcription polymerase chain reaction (RT-PCR) and western blotting were performed to quantify the mRNA and protein expression levels, respectively, of stress or apoptosis-related genes. The results of the MTT assay showed that $50 \mu \mathrm{g} / \mathrm{ml} \mathrm{SCE}$ did not affect cell viability. ROS generation in mouse spermatocyte cells increased by treatment with $100 \mu \mathrm{M}$ styrene, and decreased by cotreatment with SCE. SCE repressed the mRNA expression of stress-related genes, which increased by styrene treatment. In addition, SCE inhibited the apoptosis of mouse testicular germ cells by ameliorating mRNA and protein levels of apoptotic genes that were altered by styrene treatment. These results suggest that SCE may alleviate styrene toxicity in mouse testicular germ cells by reducing ROS stress and regulating genes related to styrene toxicity.

\section{Tu-Poster Session 2-PO-90 Rhinacanthins-rich extract: A potent superoxide scavenger and advanced glycation end-product formation inhibitor}

\footnotetext{
Authors Shah Muhammad A ${ }^{1}$, Muhammad $\mathrm{H}^{2}$, Khalil $\mathrm{R}^{3}$, Ul-Haq $\mathrm{Z}^{3}$, Panichayupakaranant $\mathrm{P}^{1,4}$

Institute 1 Department of Pharmacognosy and Pharmaceutical Botany, Faculty of Pharmaceutical Sciences, Prince of Songkla University, Hat-Yai, Songkhla 90112, Thailand; 2 Department of Chemistry, Federal Urdu University of Arts, Science \& Technology, Gulshan-e- Iqbal Campus, Karachi75300, Pakistan; 3 Dr. Panjwani Center for Molecular Medicine and Drug Research, International Center for Chemical and Biological Sciences, University of Karachi, Karachi 75270, Pakistan; 4 Phytomedicine and Pharmaceutical
} 
Biotechnology Excellence Center, Faculty of Pharmaceutical Sciences, Prince of Songkla University, Hat-Yai, Songkhla 90112, Thailand

DOI 10.1055/s-0037-1608423

Rhinacanthins-rich extract (RRE), containing $60 \% \mathrm{w} / \mathrm{w}$ rhinacanthin- $\mathrm{C}$ (RC), is a semi purified extract from Rhinacanthus nasutus leaf, a medicinal plant in Thai traditional medicine and an herbal drink in Taiwan and China. RRE was prepared and standardized by the previously described method [1] with some modifications using a microwave assisted extraction and an alternative green solvent. RC was isolated from the RRE using a silica gel column chromatography. In the current study, RRE and RC were determined for their superoxide reduction activity using a cyclic voltammetric method [2]. Furthermore, the in vitro inhibitory assay of RRE and RC was performed on human albumin glycation [3]. RRE and RC exhibited a potent and equal superoxide scavenging activity (via $\mathrm{E}_{\mathrm{r}} \mathrm{C}_{\mathrm{i}}$ mechanism), with the $\mathrm{IC}_{50}$ values of 8.0 and $9.6 \mu \mathrm{g} / \mathrm{mL}$ as well as antiglycation activity, with the $\mathrm{IC}_{50}$ values of 39.7 and $37.3 \mu \mathrm{g} / \mathrm{mL}$, respectively. The structure activity relationship of RC was explained by molecular docking studies that identified the interaction of RC with albumin to mask it from non-enzymatic glycation (Fig. 1). The potent superoxide scavenging and significant antiglycation activities of RRE streamlined its potential role in diabetic complications.

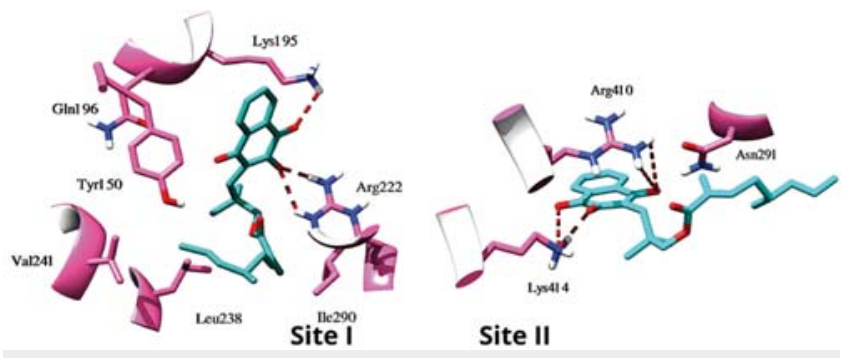

Fig. 1: The visual depicting the binding mode of rhinacanthin- $\mathrm{C}$ in the Sudlow's sites of human serum albumin.

TEH-AC PhD Award 2014 by Prince of Songkla University Hat Yai Thailand, and GA Travel Grant, 2017.

[1] Panichayupakaranant P, et al. J Chromatogr Sci 2009; 47:705 - 708.

[2] Muhammad H, et al. J Electroanal Chem 2016; 775:157 - 162.

[3] Khan MK, et al. Med Chem 2011; 7:572 - 580.

\section{Tu-Poster Session 2-PO-91 Antioxidant potential of fresh leaves of Motherwort herb (Leonurus sibiricus L.)}

Authors Gonçalves Rodrigues Das Dores $R^{1}$, Silva Souza $C^{2}$, Flores Xavier $V^{1}$, Ferreira Guimarães $S^{3}$, Santos Almeida Bastos Juliana $C^{1}$, Vieira Braga $T^{3}$ Institute 1 Universidade Federal de Ouro Preto, OURO PRETO, Brazil; 2 Universidade Federal de Viçosa, Viçosa, Brazil; 3 Universidade Federal de Minas Gerais, Belo Horizonte, Brazil

DOI 10.1055/s-0037-1608424

Motherwort herb or Macae or Rubim (Leonurus sibiricus L.) is used in folk medicine in the form of teas for postpartum bleeding, excessive menstruation as well as against edema, abscesses and renal, cardiovascular and liver problems. The infusion of leaves and flowers are used to combat vomiting and diarrhea. The objective of this study was to determine the concentration of total phenolic compounds, derivates flavonoids, reducing ability and antioxidant activity of fresh leaves of L. sibiricus. Fully expanded leaves picked in orchards, in a shading area and high irrigation, in October, in Mariana (Brazil). Voucher specimen of propagative material was prepared and is deposited in the UFOP Herbarium (OUPR). Eat the fresh leaves made up the ethanolic extract was made by remaceration, until exhaustion. The solvent $\left(40^{\circ} \mathrm{C}\right)$ was then evaporated to give the dried extract. The yield of the extract was calculated as a function of the initial mass of fresh leaves and solvent. Phenolic compounds were determined by Folin-Ciocalteu, flavonoid derivatives by hy- drochloric vanillin, antioxidant by DPPH and reducing power by ferric chloride and $\beta$-carotene by linoleic acid. The yield of the ethanolic extract of fresh leaves of L. sibiricus was $10.80 \%$. The contents of phenolic compounds, flavonoids, antioxidant activity and reducing power were $P C=160.3733$ $\pm 5.7607 \mathrm{mg} \cdot \mathrm{g}-1$ (R2 = 0.99); Flav $=27 \pm 5,5740 \mathrm{mg} \cdot \mathrm{g}-1 \quad(\mathrm{R} 2=0.998)$, AAT0 $=$ $61.87 \pm 0.69 \%$ and $\quad$ AAT3 $=81.90 \pm 0.59 \%$. $\quad \beta$-carotene $=66.45 \pm 0.84 \%$ $(\mathrm{BHA}=87.06 \pm 2.31 \%$ control). Low concentrations of fresh leaf extract of L. sibiricus had antioxidant activity equivalent to BHA control, thus being able to prevent the oxidation of free radicals, corroborating popular use with the potential of the species as cardioprotective and hepatoprotective, hypocholesterolemic and hypoglycemic.

\section{Tu-Poster Session 2-PO-92 Ananas comosus extract mediates uterotonic effect through serotonergic pathway}

Authors Monji $\mathrm{F}^{1}$, Adaikan $\mathrm{PG}^{1}$, Lau Lang $\mathrm{C}^{1}$, Siddiquee Abrar $\mathrm{AM}^{1}$, Said Baharudin $\mathrm{B}^{1}$, Choolani Mahesh $\mathrm{A}^{1}$

Institute 1 Department of Obstetrics and Gynaecology, Yong Loo Lin School of Medicine, National University of Singapore, Singapore, Singapore DOI 10.1055/s-0037-1608425

Ananas comosus is traditionally used to act as an abortifacient. We have previously shown that $A$. comosus extract can stimulate the uterine motility in vitro [1]. However, the in vitro technique may not reflect the complexity of cellular interactions occurring in vivo. This study is expected to provide information on uterotonic effect of $A$. comosus extracts in vivo as well as the underlying molecular mechanisms.

The ethanolic crude extract of $A$. comosus was prepared and fractionated through a series of liquid-liquid partitions. Total proteins were isolated from uterine strips following the functional studies for western blot analysis. The PhysioTel transmitter (PA-C40) was implanted under aseptic techniques in pregnant SD rats to measure intrauterine pressure (IUP).

Similar to serotonin, fraction 4 initiated the signal transduction through activation of ERK1/2, which resulted in the production of MMP-13. This data indicated the contribution of serotonergic pathway in the uterotonic activity of A. comosus extract. In pregnant animals (E18 and E19), F4 (1.5 g/100 g body weight), ergometrine $(1 \mathrm{mg})$ and vehicle control $(5 \mathrm{~mL})$ did not stimulate the uterine contraction following oral administration. The lack of efficacy of the F4 in rat model is possibly due to low level of estradiol and as a consequent low 5-HT receptors at the time of administration. In contrast, IUP increased 20 hour following injection of mifepristone $(15 \mathrm{mg})$ on E18 which caused premature labor in SD rats. In postpartum rats, oral administration of F4 and ergometrine showed significant increase in maximal IUP to 4.3 and 4.9 folds of basal IUP respectively ( $p<0.05$, student's $t$ test, $n=8$ ) suggesting that the active principle in aqueous fraction can serve as a drug lead in postpartum haemorrhage.

[1] Monji, F, Adaikan, P.G, Lau, L.C, Said, B.B, Gong, Y, Tan, H.M, Choolani, M, ] Ethnopharmacol 2016; 193: $21-29$.

\section{Tu-Poster Session 2-PO-93 Inhibition of advanced glycation end-products formation and antioxidant properties of Ficus deltoidea var. intermedia}

Authors Mohd Dom Nur $\mathrm{S}^{1}$, Adam $\mathrm{Z}^{2}$, Hamid $\mathrm{M}^{1}$

Institute 1 Department of Microbiology, Faculty of Biotechnology and Biomolecular Sciences, Universiti Putra Malaysia, 43400 Serdang, Selangor, Malaysia, Serdang, Selangor, Malaysia; 2 Malaysian Nuclear Agency, Bangi 43000, Kajang, Selangor, Malaysia, Kajang, Selangor, Malaysia DOI $10.1055 / \mathrm{s}-0037-1608426$

Increased protein glycation and build-up of tissue advanced glycation endproducts (AGEs) have been implicated in diabetic complications. By inhibiting the formation of AGEs would certainly prevent the occurrence of diabetic complications. Compounds with combined antiglycation and antioxidant 
properties may offer thereapeutic potential and possibly help on minimizing the complications of diabetes. The present study was performed to evaluate the in vitro antiglycation activity of Ficus deltoidea var. intermedia using fructose as a reducing sugar. The antiglycation activity was analyzed based on the inhibition of AGEs formation (24 hours, 14 and 28 days), the level of fructosamine, level of thiol group and inhibition of protein carbonyl. The antioxidant activity was measured using total phenolic content, DPPH radical scavenging activity and ferric reducing power assay. The plant extract was incubated with BSA and fructose for 24 hours at $60^{\circ} \mathrm{C}$ and the $\mathrm{IC}_{50}$ value of theinhibition of AGEs formation was found at $1.708 \pm 0.06 \mathrm{mg} / \mathrm{ml}$. During 4 weeks of study, Ficus deltoidea var. intermedia extract $(78.1-5000 \mu \mathrm{g} / \mathrm{ml})$ significantly inhibited the formation of fluorescence AGEs. The plant extract also decreased the level of fructosamine, the thiol group and inhibited the formation of protein carbonyl. This promising antiglycation activity may be attributed by the amount of phenolic compounds presence in the extract showed 188.58 $\pm 9.36 \mathrm{mg} \mathrm{GAE} / \mathrm{g}$ of extract. Meanwhile, for DPPH assay, the extract showed $\mathrm{IC}_{50}$ value at $78.96 \pm 6.25 \mathrm{mg} / \mathrm{ml}$ and ferric reducing power measured at $700 \mathrm{~nm}$ depicted $0.453 \pm 0.04$ ( $p<0.05$ ) compared to ascorbic acid. This study suggested that Ficus deltoidea var. intermedia have the ability to inhibit the formation of AGEs, decrease oxidative stress and may have the potential to be developed as new therapeutic agents to prevent the pathogenesis of diabetic complications.

\section{Tu-Poster Session 2-PO-94 A single oral dose of a Valeriana officinalis $L$ standardized extract reduced muscular strength without affecting neuromuscular tone in mice}

\author{
Authors Caudal $D^{1,2}$, Guinobert $\mathrm{P}^{3}$, Lafoux $\mathrm{A}^{1,2}$, Bardot $\mathrm{V}^{3}$, Cotte $\mathrm{C}^{3}$, \\ Ripoche $\mathrm{I}^{4}$, Chalard $\mathrm{P}^{4}$, Huchet $\mathrm{C}^{1}$
}

Institute 1 Laboratoire de Thérapie Génique, INSERM UMR U1089, Institut de Recherche Thérapeutique 1, Université de Nantes, Nantes, France;

2 Therassay, CAPACITES, 26 boulevard Vincent Gâche, 44200, Nantes, France;

3 Groupe PiLeje, 37 Quai de Grenelle, 75015, Paris, France; 4 Université

Clermont Auvergne, CNRS, SIGMA Clermont, ICCF, F-63000, Clermont-Ferrand, France

DOI 10.1055/s-0037-1608427

Valeriana officinalis L. is a medicinal plant traditionally used for its calming properties and also for muscle relaxation [1] but unlike data on smooth muscle, those on skeletal muscle are scarce and inconsistent [2]. We assessed whether a standardized extract of the fresh plant (EPS V. officinalis, PiLeje) had myorelaxant effects by decreasing skeletal muscle strength and/or neuromuscular tone.

Mice received a single dose of the $V$. officinalis extract at 2 (EPS 2) or $5 \mathrm{~g} / \mathrm{kg}$ (EPS 5) p.o.; tetrazepam (TTZ) at $10 \mathrm{mg} / \mathrm{kg}$ i. p.; or corresponding vehicles. Maximal muscle strength was measured using a grip test, while global skeletal muscle function (endurance and neuromuscular tone) was assessed in a wire hanging test.

In the grip test, both doses of the extract induced a significant decrease in maximal absolute and relative strength versus controls (relative strength: $18.2 \%$ at $2 \mathrm{~g} / \mathrm{kg}, \mathrm{p}<0.001$ and $-14.5 \%$ at $5 \mathrm{~g} / \mathrm{kg}, \mathrm{p}<0.01$; Figure $1 \mathrm{~A}$ ). Decrease was similar with TTZ (relative strength: $-23.3 \%, p<0.001$ ).

In the wire hanging test, both extract doses decreased the 100 seconds score ( $20 \%$; Figure $1 \mathrm{~B})$ and the impulse parameter $(\sim 14 \%)$ but these decreases were not significant. By contrast, both the 100 seconds score $(-83.3 \%$, $\mathrm{p}<0.001)$ and the impulse parameter $(-67.3 \%, \mathrm{p}<0.001)$ were strongly decreased with TTZ.

This study provides clear evidence that the standardized extract of $V$. officinalis tested decreases skeletal muscle strength without any significant effects on endurance and neuromuscular tone. With a selective milder effect than standard drugs, this extract could be an interesting alternative myorelaxant particularly in terms of side effects.
Figure $1 \mathrm{~A}$

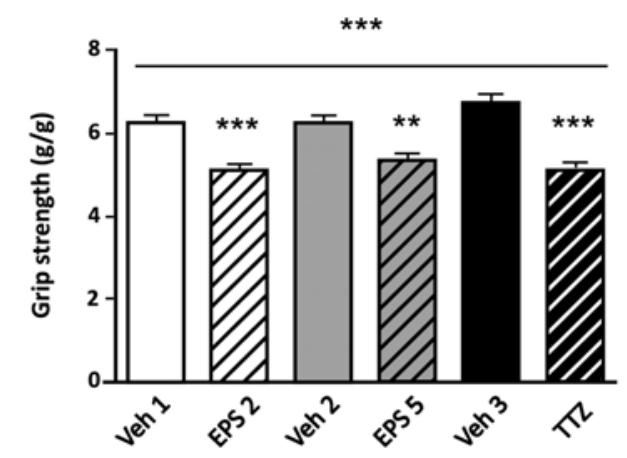

Figure $1 \mathrm{~B}$

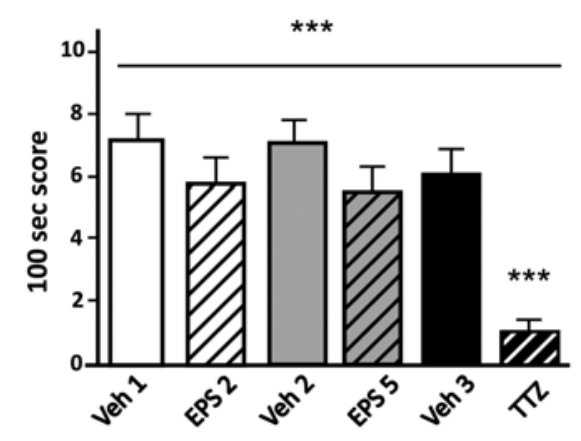

Fig. 1

[1] Occhiuto F et al. J Pharm Pharmacol 2009; 61: $251-256$

[2] Hattesohl M et al. Phytomedicine 2008; 15: 2 - 15

\section{Tu-Poster Session 2-PO-95 Astragalus mongholicus hydroethanolic root extract: HPTLC characterization and effects on telomere elongation}

Authors Guinobert $I^{1}$, Bardot $V^{1}$, Cotte $C^{1}$, Dubourdeaux $M^{1}$, M'Kacher $R^{2,3}$ Institute 1 Groupe Pileje, 37 Quai de Grenelle, 75015, Paris, France; 2 Cell Environment, Champigny sur Marne, France; 3 APHP-Hopital Paul Brousse Université Paris Sud/ESteam Paris Inserm UMR 935, Villejuif, France DOI 10.1055/s-0037-1608428

Telomeres, the protective ends of linear chromosomes, play a major role in the maintenance of genome integrity and stability. Telomere shortening is involved in age-related disorders such as cardiovascular diseases and cancer [1]. Astragalus mongholicus, depending on the type of extract, is known to stimulate telomerase activity that compensate telomere shortening $[1,2]$. We determined the phytochemical composition of a hydroethanolic extract of fresh A. mongholicus root (HRE; marketed under the brand name EPS Astragalus) and assessed its effects on telomere elongation.

High performance thin layer chromatography (HPTLC) analysis showed that amino acids, sugars and flavonoids were strongly present in the HRE unlike terpenoids and phenolic acids that generated a lower signal. Astragaloside specific analysis revealed the presence of astragalosides I to IV.

Cytotoxicity assay was performed after treatment of lymphoblastoide cell lines and circulating lymphocytes from 10 patients with $1 \mu \mathrm{l} / \mathrm{ml}$ of HRE for $72 \mathrm{~h}$. High cellular viability (> 80\%) and increased mitotic index compared to controls were observed after HRE treatment. A moderate toxicity was observed only for two patients. Quantitative fluorescence in situ hybridization (Q-FISH) technique was used to quantify telomere length of circulating lymphocytes of 10 patients with telomere dysfunction (high proportion of short telomeres) after HRE treatment. For 7 patients, we demonstrated that the HRE treatment 
significantly induced telomere elongation (from 0.05 to $2.5 \mathrm{~kb} ; \mathrm{p}<0.01$ ) and was associated with a decrease in the frequency of cells with very short telomeres.

The flavonoid- and astragaloside-rich A. mongholicus HRE tested could increase telomere length suggesting that the extract could be interesting in the management of age-associated diseases and telomeropathies. The advantages and risks associated with the modification of telomere length will be assessed.

[1] Molgora B et al. Cells 2013; 2: $57-66$.

[2] Jager K, Walter M. Genes (Basel) 2016; 7: 1 - 24

\section{Tu-Poster Session 2-PO-96 Safety issues concerning common ragweed}

Authors $\underline{\text { Csupor } \mathrm{D}^{1,2}}$, Kiss $\mathrm{T}^{1,2}$, Oszlánczi $\mathrm{G}^{3}$, Lukács $\mathrm{A}^{3}$, Tímár $\mathrm{Z}^{4}$,

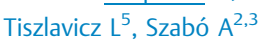

Institute 1 University of Szeged, Faculty of Pharmacy, Department of

Pharmacognosy, Szeged, Hungary; 2 University of Szeged, Interdisciplinary Centre for Natural Products, Szeged, Hungary; 3 University of Szeged, Faculty of Medicine, Department of Public Health, Szeged, Hungary; 4 SOLVO

Biotechnology, Szeged, Hungary; 5 University of Szeged, Faculty of Medicine, Department of Pathology, Szeged, Hungary

DOI 10.1055/s-0037-1608429

The spreading of common ragweed, accelerated by climate changes seems to be unstoppable. This plant is becoming part of our diet and medication accidently or intentionally. It appears in human food as contaminant, and as an easy accessible and cheap plant, A. artemisiifolia is already in use as basic ingredient of few food supplements. Although the high allergenic potential of this herb is well-known, the effect of long-term consumption has not been studied, yet.

The aim of our work was to study the subacute toxicity of $A$. artemisiifolia. Repeated-dose toxicity of a ragweed puree was tested on 24 male Wistar rats. The puree was administered in low dose $(500 \mathrm{mg} / \mathrm{kg}$ b.w.) and high dose $(1000 \mathrm{mg} / \mathrm{kg}$ b.w) ragweed in cookie balls. Animals in the control group received plain cookie dough without ragweed. Clinical symptoms, blood chemical parameters, body weight and organ weights of the rats were measured. After 28 days of treatment animals were over-anaesthetized and dissected.

In the high dose group the liver function enzymes (AST, ALT) and the carbamide level have been significantly reduced (Fig A, B). In the low dose group decreased triglyceride level was observed (Fig C). In both groups the weight of the liver relative to body weight was significantly reduced, while the brain weight relative to body weight was significantly elevated in both groups. Repeated use of ragweed resulted in toxic effects in rats and these results question the safety of long-term human consumption of A. artemisiifolia-containing products.

This research was supported by the European Union and the State of Hungary, co-financed by the European Social Fund in the framework of TÁMOP 4.2.4.A/ 2-11-1-2012-0001 'National Excellence Program'. This paper was supported by the János Bolyai Research Scholarship of the Hungarian Academy of Sciences, the National Research, Development and Innovation Office (115796) and the project GINOP-2.3.2-15-2016-00012.

\section{Tu-Poster Session 2-PO-97 Bioactivity in Australian native willow: comparative analysis of leaf extracts on cell viability}

Authors Patil $\mathrm{A}^{1}$, Fitzgerald $\mathrm{M}^{2}$, Shaw Paul $\mathrm{N}^{1}$, Parat $\mathrm{MO}^{1}$

Institute 1 School of Pharmacy, The University of Queensland, Brisbane,

Australia; 2 School of Agriculture and Food Sciences, The University of

Queensland, Australia, Brisbane, Australia

DOI 10.1055/s-0037-1608430

Australian native willow, (Pittosporum angustifolium Lodd.), known also as gumby gumby, has been traditionally used as a medicinal plant in Australian Aboriginal communities, anecdotally, to treat various skin conditions including cancer (1). A small number of in vitro studies have examined the cytotoxicity of $P$. angustifolium Lodd. leaf extracts on breast, lung, and glioblastoma cancer cells. The aim of the present study was to examine the cytotoxic effects of a series of extracts from $P$. angustifolium Lodd. leaves collected from three geographically different locations (GG1, GG2, and GG3). Extraction was accomplished either by decoction (GGD) or mechanical juicing (GGJ). The cell viability was assessed using the MTT (3-(4, 5-dimethylthiazol-2-yl)-2,5-diphenyltetrazolium bromide) assay on squamous cell carcinoma (SCC25) and noncancerous human keratinocyte (HaCaT) cell lines. SCC25 and HaCaT (6000 and 3000 cells/well, respectively) cells were treated with sterile filtered decoction (GGD 1, GGD2, and GGD 3) or juice (GGJ1, GGJ2, and GGJ3) extracts over a range of concentrations $(5-100 \mu \mathrm{g}$ extract $/ \mathrm{mL}$ ). The dose-response curve of all the extracts significantly showed decreases in cell viabilities for both SCC 25 and $\mathrm{HaCaT}$ cells $(p<0.0001)$. In conclusion, a comparative analysis of extracts is useful to examine the bioactivities, their cancer cell selectivity and the phytochemical variation(s) of plant extracts.

The contribution and assistance of Dale Chapman (First Foods) are gratefully acknowledged.

[1] Cayzer LW, Crisp MD, Telford IRH. Revision of Pittosporum (Pittosporaceae) in Australia. Australian Systematic Botany. 2000;13:845 - 902.

\section{Tu-Poster Session 2-PO-98 The furocoumarin content of Foeniculum vulgare The furocoumarin content of Foeniculum vulgare}

Authors Csupor $\mathrm{D}^{1,2}$, Kerekes $\mathrm{D}^{1,2}$, Gosztola B ${ }^{3}$, Zámbori-Németh É ${ }^{3}$ Institute 1 Department of Pharmacognosy, University of Szeged, Szeged, Hungary; 2 Interdisciplinary Centre for Natural Products, University of Szeged, Szeged, Hungary; 3 Department of Medicinal and Aromatic Plants, Szent

István University, Budapest, Hungary

DOI $10.1055 / \mathrm{s}-0037-1608431$

The harmful effects of linear furocoumarins have been demonstrated in several studies. The most typical toxic activity of these compounds is phototoxicity, however, hepatotoxic and carcinogenic effects have also been reported in animal experiments. Three furocoumarins - bergapten, imperatorin and psoralen - were previously described in fennel (Foeniculum vulgare Mill.), a widely used medicinal plant and spice, however no reliable quantitative data are available on the occurrence of these compounds in the fruits of the plant.

The aim of our work was to develop a highly selective and sensitive method for the determination of furocoumarins from fennel and to quantify these substances in several samples of different origin representing a wide range of genotypes. The bergapten, imperatorin and psoralen content of 33 fennel samples was analyzed using an LC-MS method. The measured quantities were compared to the safety recommendations of scientific guidelines in order to assess whether the furocoumarin content of fennel should be considered as a potential risk to the human organism in case of therapeutic application. The furocoumarin content of the fruits if applied at the highest therapeutic dose described in the monograph of the European Medicines Agency [1] ranged between $0.01-1.22 \mu \mathrm{g}$ which is below the most restrictive recommendations [2]. Based on this finding, the therapeutic use of fennel could be considered as safe at least based on its low furocoumarin content.

[1] EMEA/HMPC Foeniculum vulgare subsp. vulgare var. dulce (Miller) Thellung, fructus fennel fruit, sweet EMA/HMPC/411665/2008

[2] EMEA/HMPC Reflection paper on the risks associated with furocoumarins contained in preparations of Angelica archangelica L. EMEA/HMPC/317913/ 2006 


\section{Tu-Poster Session 2-PO-99 Bioactivities of Verbascum bugulifolium and isolation of secondary metabolites}

Authors Gokmen $\mathrm{A}^{1}$, Kúsz $\mathrm{N}^{2}$, Karaca $\mathrm{N}^{3}$, Demirci F $\mathrm{F}^{4}$, Hohmann J Kirmizibekmez $\mathrm{H}^{1}$

Institute 1 Department of Pharmacognosy, Faculty of Pharmacy, Yeditepe University, 34755, Kayisdagi, Istanbul, Turkey; 2 Institute of Pharmacognosy, Szeged University, Eötvös u 6, H-6720, Szeged, Hungary; 3 Department of Pharmacognosy, Graduate School of Health Sciences, Anadolu University, TR26470, Eskisehir, Turkey; 4 Faculty of Health Sciences, Anadolu University, TR26470, Eskisehir, Turkey

\section{DOI 10.1055/s-0037-1608432}

The genus Verbascum (mullein) comprises about 250 species in the flora of Turkey. Some species are used for the treatment of rheumatic disorders, eczema, spasmodic coughs, as an antiseptic and expectorant agent in different traditional systems [1]. In the continuation of our phytochemical and bioactivity studies on Turkish medicinal plants, we have investigated the antioxidant, anti-inflammatory and antimicrobial activities of the crude $\mathrm{MeOH}$ extract from the aerial parts of Verbascum bugulifolium as well as the subextracts obtained thereof, for the first time. Among the tested extracts, EtOAc and $n-\mathrm{BuOH}$ subextracts exerted the best antioxidant activity in DPPH, ABTS and CUPRAC assays $\left(\mathrm{IC}_{50}=0.02-0.09 \mathrm{mg} / \mathrm{mL}\right)$. The in vitro anti-inflammatory activities of the extracts were evaluated using a LOX inhibition assay where $n$ $\mathrm{BuOH}$ subextract caused a $35.7 \%$ inhibition at $50 \mu \mathrm{g} / \mathrm{mL}$. The antimicrobial activities of the extracts were also tested against three bacteria and three fungal strains. Only the $n$-hexane subextract showed antibacterial activity against Pseudomonas aeruginosa ATCC 10145 (MIC $=0.125 \mu \mathrm{g} / \mathrm{mL}$ ), while $\mathrm{CHCl}_{3}$ subextract exerted moderate activity against Candida krusei ATCC 6258 with a MIC value of $0.25 \mathrm{mg} / \mathrm{mL}$, which is followed by $n-B u O H$ and and $n$-hexane subextracts with a MIC of $0.5 \mathrm{mg} / \mathrm{mL}$. Due to activity of the $n-\mathrm{BuOH}$ and EtOAc subextracts, chromatographic purifications were conducted, where five iridoid glycosides catalpol, specioside, ajugol, ajugoside, 8-O-acetylharpagide, two phenylethanoid glycosides, verbascoside and glucopyranosyl-(1-Gi6)-martynoside, three flavonoids, apigenin 7-O-rutinoside, luteolin 7-O- $\beta$-Dglucopyranoside, luteolin 7-O-rutinoside were purified from the $n-\mathrm{BuOH}$ extract, while luteolin were obtained from the EtOAc subextract.

[1] Alipieva KI, Erdoğan Orhan I, Tatli Cankaya I, Emanuela P, Kostadinova, EP, Georgiev MI. Phytochem Rev 2014; 13: 417 - 444.

\section{Tu-Poster Session 2-PO-100 Anti-inflammatory activity of Hops extracts (Humulus lupulus L.) in human gastric epithelial cells: a bio-guided fractionation}

Authors Sangiovanni $\mathrm{E}^{1}$, Fumagalli $\mathrm{M}^{1}$, Taglialatela-Scafati $\mathrm{O}^{2}$ Santagostini $\mathrm{L}^{3}$, Forino $\mathrm{M}^{2}$, Piazza $\mathrm{S}^{1}$, Colombo $\mathrm{E}^{1}$, Fico $\mathrm{G}^{4}$, Dell'Agli $\mathrm{M}^{1}$ Institute 1 Dept. of Pharmacological and Biomolecular sciences, Università degli Studi di Milano, Milan, Italy; 2 Dept. of Pharmacy, University of Naples Federico II, Naples, Italy; 3 Dept. of Chemistry, Università degli Studi di Milano, Milan, Italy; 4 Dept. of Pharmaceutical Sciences, Università degli Studi di Milano, Milan, Italy DOI 10.1055/s-0037-1608433

Gastritis and ulcers are common inflammatory-based diseases mainly caused by Helicobacter pylori. During gastric inflammation, immune cells release different pro-inflammatory mediators including TNF $\alpha$, which in turn lead to IL-8 release by gastric epithelial cells through the NF-kB pathway. The side effects of the current anti-H. pylori therapy support the search for new therapeutic approaches. Besides the wide use in the food industry, female inflorescences of Humulus lupulus L, (hop strobiles) show anti-inflammatory and anti-H.pylori activities; however, no studies regarding the anti-inflammatory activity at gastric level have been reported so far.

The aim of this study was to evaluate the anti-inflammatory effect of the water (WE) and hydro-alcoholic (HE) extracts from hops in human gastric epithelial cells (AGS).
Extracts were prepared from $H$. lupulus cv. Cascade (1:10 ratio drug/solvent) and tested on AGS cells challenged with TNF $\alpha$. Reporter plasmids were used to assess the NF-KB driven transcription and the IL-8 promoter activity, while IL-8 secretion was evaluated by ELISA assay.

Both WE and HE inhibited IL-8 promoter activity $\left(\mathrm{IC}_{50}: 3.59\right.$ vs. $2.34 \mu \mathrm{g} / \mathrm{mL}$, respectively) and the effect was confirmed on NF-KB driven transcription (IC $\mathrm{C}_{50}$ : 2.25 vs. $2.44 \mu \mathrm{g} / \mathrm{mL}$, respectively), but only $\mathrm{HE}$ inhibited IL-8 release (IC 50 : $3.95 \mu \mathrm{g} / \mathrm{mL})$.

The in vitro gastric digestion of $\mathrm{HE}$ slightly reduced IL-8 secretion ( $\mathrm{IC}_{50}$ : $6.40 \mu \mathrm{g} / \mathrm{mL}$ ). HE was separated on a MPLC silica column eluted with a stepwise gradient of $n$-hexane to EtOAc, and then to $\mathrm{MeOH} ; 11$ fractions (A-M) were collected. The most active fraction (D) was further purified on a HPLC Gemini $10 \mu \mathrm{m}$ column eluted with $\mathrm{H}_{2} \mathrm{O}-\mathrm{MeOH}(2: 8)$ to obtain pure xanthohumol, which showed anti-inflammatory activity as well.

The present research firstly demonstrates the anti-inflammatory activity of hops at gastric level; if confirmed by future studies, hops extracts may be used to treat or prevent gastric inflammatory diseases.

\section{Tu-Poster Session 2-PO-101 Evaluation of Rumex crispus extracts as novel matrix metalloproteinase- 8 (MMP-8) inhibitors}

Authors Demirezer Lutfiye $\mathrm{O}^{1}$, Uzun $\mathrm{M}^{1}$

Institute 1 Department of Pharmacognosy, Faculty of Pharmacy, Hacettepe University, 06100, Ankara, Turkey

DOI 10.1055/s-0037-1608434

Matrix metalloproteinases are responsible for breaking down extracellular matrix and MMP- 8 is an interstitial collagenase, modulates TNF- $\alpha$, which may explain neuroinflammation [1]. It plays crucial roles on various cancer types and diseases such as obesity and rheumatoid arthritis [1 - 2]. In such diseases, inhibition of MMP-8 could be a part of treatment strategy, so we aim to inhibit MMP-8 by Rumex crispus extracts. Rumex species (Polygonaceae) contain anthranoids, tannins, flavonoids and naphthalenes as secondary metabolites and have various biological effects [3].

Standardized n-hexane, dichloromethane, ethyl acetate, ethanol and ethanol: water (70:30) extracts were prepared from roots, leaves and fruits and inhibitory effects on MMP-8 were investigated. NNGH was used as potent MMP-8 inhibitor. Inhibitor activities of all extracts were measured in $50 \mu \mathrm{g} / \mathrm{mL}$, $100 \mu \mathrm{g} / \mathrm{mL}, 200 \mu \mathrm{g} / \mathrm{mL}, 300 \mu \mathrm{g} / \mathrm{mL}, 400 \mu \mathrm{g} / \mathrm{mL}$ and $800 \mu \mathrm{g} / \mathrm{mL}$ concentrations at $412 \mathrm{~nm}$. The aqueous alcoholic extracts showed the highest inhibitory effect among other extracts at all concentrations. Inhibition values were $86.25 \%$ for root extracts, $84.48 \%$ for leaf extracts and $87.30 \%$ for fruit extracts, while NNGH was $93.88 \%$. This study shows that aqueous alcoholic extracts of Rumex crispus may be potential novel MMP-8 inhibitors.

This study was supported by The Scientific and Technological Research Council of Turkey (TUBITAK) (Project No:SBAG-214S 147).

[1] Lee E], Han JE, Woo MS, Shin JA, Park EM, Kang JL, Moon PG, Baek MC, Son WS, Ko YT, Choi JW, Kim HS. J. Immunol 2014; 193: 2384 - 2393

[2] Foda HD, Zucker S. Drug Discov Today 2001; 6: 478 - 482

[3] Vasas A, Orban-Gyapai O, Hohmann J. J Ethnopharmacol 2015; 175: 198 228 


\section{Tu-Poster Session 2-PO-102 Inhibitory activity of Cranberry extract on the bacterial adhesion in human urine: an ex vivo study}

Authors Scharf $B^{1}$, Dobrindt $U^{2}$, Sendker $]^{1}$, Hensel $A^{1}$

Institute 1 WWU Münster, Institute for Pharmaceutical Biology and

Phytochemistry, Corrensstraße 48, Münster, Germany; 2 WWU Münster,

Institute for Hygiene, Robert-Koch-Str. 41, Münster, Germany

DOI 10.1055/s-0037-1608435

The initial step of urinary tract infections (UTIs), frequently caused by uropathogenic Escherichia coli (UPEC), is the adhesion of bacteria to urothelial cells.

Extracts from Cranberry fruits (Vaccinium macrocarpon Ait.) have long been associated with the prevention of UTIs.

Within ex vivo experiments in a pilot study, a significant inhibition of bacterial adhesion of UPEC NU14 to human T24 bladder cells was achieved using urine from 4 volunteers after consumption of standardized Cranberry extract (CE). The antiadhesive effect of the extract was directed only against the bacterial adhesin FimH, not against P-fimbriae dominated UPEC [1].

To verify these data, a further in vivo study included 18 volunteers, $900 \mathrm{mg}$ CE daily over 8 days. The obtained urine exerted a time-dependent, significant inhibition of the adhesion of UPEC UTI89 to human T24 bladder cells ex vivo, but did not affect the adhesion of E. coli 2980.

To investigate the effect of the Cranberry metabolites on UPEC transcriptome analysis of UTI89 by Next Generation Sequencing was performed. The transcriptome of bacteria grown in urine collected after 7 days of cranberry intake indicated no relevant differences compared to that obtained from bacteria grown in control urine.

Therefore, the antiadhesive activity of urine after CE intake can be explained by an effect on bacterial adhesin assembly or a direct interaction of cranberry metabolites with bacterial adhesins, probably of the FimH class.

LC-MS/MS study of the urine was performed to correlate Cranberry-related metabolites with the antiadhesive effects.

[1] Rafsanjany N, Sendker J, Brandt S, Dobrindt U, Hensel A. J Agricult Food Chem 2015; 63: $8804-8818$

\section{Tu-Poster Session 2-PO-103 Synergistic in-vitro anthelmintic potentials of Vernonia amygdalina Delile stem and Carica papaya Lin. seeds}

Authors Quartey $\mathrm{A}^{1,3}$, Oppong $\mathrm{A}^{1}$, Ayensu $\mathrm{I}^{3}$, Apenteng $\mathrm{J}^{2}$, Mintah $\mathrm{D}^{1}$, Ikeani $\mathrm{C}^{1}$

Institute 1 Department of Medicinal Chemistry and Pharmacognosy, School of Pharmacy, Central University, Accra, Ghana; 2 Department of

Pharmaceutics, School of Pharmacy, Central University, Accra, Ghana;

3 Department of Pharmaceutical Chemistry, Faculty of Pharmacy and

Pharmaceutical Sciences, Kwame Nkrumah University of Science and

Technology, Kumasi, Ghana

DOI 10.1055/s-0037-1608436

Helminthiasis is among the most common infections and a major public health concern to humans and livestocks ${ }^{[1]}$. It affects $60 \%$ to $80 \%$ of the world's population of which a large percentage is in developing countries such as Ghana ${ }^{[2]}$. Vernonia amygdalina and Carica papaya are medicinal plants used ethnobotanically for treatment of various conditions including helminthes. Ethanol extracts of $V$. amygdalina stem (EVAS) and C. papaya seeds (ECPS) and chloroform extracts of $V$. amygdalina stem (CVAS) and C. papaya seeds (CCPS) were evaluated for synergistic in-vitro anthelmintic activity.

Preliminary phytochemical screening was carried out using methods described by Ayensu and Quartey [3]. The effects of concentrations (18.75, 37.5, 75, $150,300 \mathrm{mg} / \mathrm{ml}$ ) of each extract were evaluated for their In-vitro Anthelmintic activity on the paralytic and death times of Pheretima posthuma with piperazine citrate $(15 \mathrm{mg} / \mathrm{ml})$ and albendazole $(20 \mathrm{mg} / \mathrm{ml})$ as reference standards.
Synergistic effect was assessed by adding the ethanol extracts of both plants in the ratio of $1: 1$, and their chloroform extracts also in the same ratio. The presences of phytochemicals such as tannins, saponin glycosides, reducing sugars, alkaloids, steroids and flavonoids were observed in all the plant extracts (EVAS, ECPS, CVAS and CCPS), including the presences of terpenoids in EVAS and ECPS but its absence in CVAS and CCPS. There was the absence anthraquinones, cardiac glycosides and cyanogenetic glycosides in all the extracts (EVAS, ECPS, CVAS and CCPS). The extracts demonstrated a concentration dependent anthelmintic activity as (EVAS + ECPS) $>($ CVAS + CCPS) $>$ ECPS $>$ CCPS > EVASB > CVASB.

[1] Wasswa P, and Olila D. Afr. J. Traditional CAM. 2006; 3, 94-103.

[2] Cappello M. Pediatr Infect Dis ]. 2004; 23:663-4.

[3] Ayensu I, Quartey A.K. WJPPS 2015; 4, 76 - 90.5

\section{Tu-Poster Session 2-PO-104 Neutrophil gelatinase-associated lipocalin and kidney injury molecule-1, an acute kidney injury biomarkers were affected by herbal medicine}

Authors Seo $\mathrm{CS}^{1}$,

Institute 1 Korea Institute of Oriental Medicine, Daejeon, Korea, Republic of (South)

DOI 10.1055/s-0037-1608437

Nephrotoxicity is one of the initial kidney disorder cascade [1]. Acute kidney injury (AKI) biomarkers has recently been reported on normal kidney cell lines provoked by cisplatin. To resolve the renal toxicity, the aim of this study was to initially increase cell viability in normal kidney cell line without herbal medicine's toxic effect and to measure the activities of acute kidney injury biomarkers, neutrophil gelatinase-associated lipocalin (NGAL) and kidney injury molecule-1 (KIM-1). HK-2 human kidney cell lines were treated with cisplatin (10 $\mathrm{uM}$ ) and then modulated by herbal products at dose range of $0.1-0.5 \mathrm{mg} /$ $\mathrm{mL}$. The levels of nephrotoxicity biomarker were also measured by ELISA assay in those cell lines. Treatment of cisplatin at concentration (10 uM) decreased the cell viability. Induction of NGAL and KIM-1 as nephrotoxicity biomarker in HK-2 cells after treated by cisplatin for $24 \mathrm{~h}$. The results indicated that NGAL and $\mathrm{KIM}-1$ were decreased in dose dependent manner by Schisandra chinensis (SC) from $30 \%$ through $60 \%$ which showed the therapeutic potential on AKI biomarker in HK-2 cells (Figure). SC had a potential as an effector on acute kidney injury through inhibiting the activities of NGAL and KIM-1 below the toxic dosage range. Further in vivo proof-of-concept study is needed to elucidate their efficacies on both acute and chronic kidney disorders.

This study was supported by the Traditional Korean Medicine R\&D program funded by the Ministry of Health \& Welfare through the Korea Health Industry Development Institute (KHIDI, Grant \# HI16C 0948).

Yao X, Panichpisal K, Kurtzman N, Nugent K. Am J Med Sci 2007; 115 - 124.

\section{Tu-Poster Session 2-PO-105 Antimicrobial screening of two alien invasive plant species against opportunistic pathogens causing infections in the central nervous system}

Authors Omokhua $A^{1}$, Van Staden $]^{2}$, McGaw L ${ }^{1}$

Institute 1 Phytomedicine Programme, Department of Paraclinical Sciences, University of Pretoria, Private Bag X04, Onderstepoort 0110, South Africa,

Pretoria, South Africa; 2 Research Centre for Plant Growth and Development, School of Life Sciences, University of KwaZulu-Natal, Private Bag X01,

Scottsville 3201, South Africa, Pietermaritzburg, South Africa

DOI 10.1055/s-0037-1608438

Infections of the central nervous system (CNS) of animals and humans by opportunistic pathogens such as bacteria and fungi remain a global health issue, resulting in high morbidity and mortality rates. This challenge is further complicated by the rise in resistant microbial strains as well as emergence of 
new infectious pathogens, especially in developing countries. Plants (indigenous and alien) play a crucial role in the global health system for the treatment of opportunistic infections in animals and humans as they may possess natural products with medicinal properties. This study investigated the antimicrobial activity of leaf extracts of two alien invasive plants, namely Tithonia diversifolia (Hemsl) A.Gray (Asteraceae) and T. rotundifolia (Mill) F.S. Blake (Asteraceae) against some pathogens implicated in causing infections in the CNS. Phytochemicals that may be responsible for such activity and toxicity were also investigated. Antimicrobial activity was evaluated against six pathogenic bacterial and three fungal strains using the microdilution assay. Phytochemicals were detected through standard methods of analysis, while toxicity tests were carried out using the colorimetric MTT assay and the Ames test. Both species exhibited antimicrobial activity against the tested strains with minimum inhibitory concentration values ranging between 0.01 to $2.5 \mathrm{mg} / \mathrm{ml}$ and between 0.07 to $2.5 \mathrm{mg} / \mathrm{ml}$ for bacteria and fungi respectively. The leaf extract of both plants were rich in phenolics, flavonoids and tannins, although phenolics was significantly higher in $T$. diversifolia. Only $T$. diversifolia was cytotoxic while none of the extracts of both plants were mutagenic. The above results suggest that both species may be exploited as sources of antimicrobial treatment for opportunistic infections in animals and humans. However, T. diversifolia must be used with caution as it is a toxic plant and further toxicity screening is recommended.

\section{Tu-Poster Session 2-PO-106 Anthelmintic, antifungal, genotoxicity and cytotoxic activities of acetone leaf extracts, fractions and isolated compounds from Ptaeroxylon obliquum}

\section{Authors Ramadwa $\mathrm{T}^{1}$, McGaw $\mathrm{L}^{1}$, Adamu $\mathrm{M}^{1}$, Madikizela $\mathrm{B}^{1}$, Eloff $\rfloor^{1}$} Institute 1 University of Pretoria, Pretoria, South Africa

DOI 10.1055/s-0037-1608439

Ptaeroxylon obliquum (Rutaceae) is traditionally used in South Africa to treat many ailments in humans and animals, including parasitic infections. In this study the antiparasitic, antifungal, genotoxic and cytotoxic activities of acetone leaf extracts of P. obliquum, fractions and isolated compounds were determined. The in vitro anthelmintic activity was investigated against Haemonchus contortus ova and larvae using egg hatching and larval development assays. The antifungal activity was determined by serial microplate dilution method against Aspergillus fumigatus, Cryptococcus neoformans and Candida albicans. The mutagenic activity was determined using the Ames test with Salmonella typhimurium (TA98, TA100 and TA102) strains. Cellular toxicity was determined against Vero African green monkey kidney and human liver (C 3A) cell lines.

Three compounds were isolated from the chloroform fraction using silica gel open column chromatography and were characterized as obliquumol, eranthin and a mixture of lupeol and $\beta$-amyrin. The anthelmintic activity of the extract and obliquumol against $\mathrm{H}$. contortus ova and larvae indicated that the compound was more effective than the crude extract with an EC 50 as low as $95 \mu \mathrm{g} / \mathrm{ml}$ against the larvae. Obliquumol and eranthin had excellent antifungal activity against the fungi with minimal inhibitory concentrations (MIC) values ranging from $2-16 \mu \mathrm{g} / \mathrm{ml}$. All the tested samples were non-genotoxic against the tested Salmonella strains. The acetone crude extract was toxic with an IC 50 value as low as $14.2 \mu \mathrm{g} / \mathrm{ml}$ against the Vero cells. However, the isolated compounds were not toxic against either Vero or C 3 A cells and had good selectivity index values against the opportunistic fungal pathogens. The results of the anthelmintic activity indicated potential in the management of $\mathrm{H}$. contortus which may explain the ethnoveterinary use of this plant species. Further exploration of the activity of the purified compounds, particularly their promising antifungal activity, is under way.

\section{Tu-Poster Session 2-PO-107 ANTI-INFLAMMATORY AND ANTIOXIDANT ACTIVITIES OF TWO DIFFERENT GINKGO BILOBA L. EXTRACTS IN HUMAN ENDOTHELIAL CELLS: A COMPARATIVE STUDY}

Authors Piazza $S^{1}$, Pacchetti $B^{2}$, Fumagalli $M^{1}$, Sangiovanni $E^{1}$, Dell'Agli $M^{1}$ Institute 1 Department of Pharmacological and Biomolecular Sciences University of Milan, Milan, Italy; 2 LINNEA SA, Riazzino, Switzerland DOI 10.1055/s-0037-1608440

Ginkgo biloba L. leaves extracts are widely used for their beneficial effects in cardiovascular diseases prevention and therapy or to improve mild cognitive impairment and cerebrovascular insufficiency ${ }^{1}$. In some countries, the amount of acetone allowed in food supplements is stricter than ethanol, thus implying that the usage of standardized ethanol extracts is preferred.

The aim of the present study was to 1 ) to chemically profile acetone (G24) vs. ethanol (G4E) extracts from Ginkgo biloba leaves similarly standardized to identify differences among components; 2) to investigate if the extracts may be considered comparable as anti-inflammatory and antioxidant agents. Both the extracts were prepared and standardized by LINNEA SA (Riazzino, Switzerland). Biological activities were evaluated in human endothelial cells (HUVEC) challenged with TNF $\alpha(20 \mathrm{ng} / \mathrm{mL})$.

Both the extracts inhibited the soluble adhesion molecules ICAM-1, VCAM-1 and $\mathrm{E}$-selectin release by endothelial cells ( $\mathrm{IC}_{50}$ reported in Tab. 1); the effect was due to the impairment of NF-kB pathway since both G4E and G24 extracts inhibited the NF-kB nuclear translocation in a concentration dependent fashion (IC 5070.4 and $77.5 \mathrm{mg} / \mathrm{ml}$, respectively).

ROS release plays a crucial role in the endothelial inflammatory processes; G4E and G24 inhibited the intracellular ROS formation induced by oxidative stress $\left(\mathrm{H}_{2} \mathrm{O}_{2} 1 \mathrm{mM}\right)$ with $\mathrm{IC}_{50} 57.6 \mathrm{mg} / \mathrm{ml}$ and $38.8 \mathrm{mg} / \mathrm{ml}$, respectively.

Since the extracts have the same standardization in flavonoid glycosides and terpene lactones and comparable $\mathrm{IC}_{50} \mathrm{~s}$, these molecules seem to be responsible for the anti-inflammatory and anti-oxidant effects. Our results show that acetone and ethanol Ginkgo biloba extracts have comparable anti-inflammatory and antioxidant activities in human endothelial cells. This information will be useful for future commercialization of the extracts.

Tab. 1: $I C_{50}$ of the extracts on the adhesion molecules

\begin{tabular}{|l|l|l|l|}
\hline $\mathrm{IC}_{50}(\mu \mathrm{g} / \mathrm{ml})$ & ICAM-1 & VCAM-1 & E-SELECTIN \\
\hline G4E & 209 & 1.1 & 170 \\
G24 & 129 & 2.0 & 137 \\
\hline
\end{tabular}

[1] Tisato V, Mediators Inflamm 2013, 2013, 423-407.

\section{Tu-Poster Session 2-PO-108 GC-MS ANALYSIS OF VIBURNUM OPULUS (L) EXTRACT AND ITS TOXICITY STUDIES IN RATS}

Authors Adebayo $\mathrm{A}^{1}$, Alade $\mathrm{A}^{1}$, Yakubu $\mathrm{O}^{1}$

Institute 1 Biochemistry and Molecular Biology Unit, Department of

Biological Sciences, Covenant University, Ota, Ogun State, Nigeria

DOI 10.1055/s-0037-1608441

This study was aimed at establishing the antimicrobial and phytochemical profiles of Viburnum opulus $(\mathrm{L})$ as well as the safety potential of the extract in albino Wistar rats. Ethanol, n-hexane, ethyl acetate, butanol and water fractions were prepared for both phytochemical assessment using gas chromatography-mass spectrum analysis (GC-MS) ${ }^{1}$. Five groups of seven rats were used for the study. Group A received distilled water (control), while groups B to $E$ were treated respectively with $250,500,1000$ and $1500 \mathrm{mg} / \mathrm{kg}$ body weight of $V$. opulus extract by abdominal canulisation for 28 days $^{2}$. Blood samples were obtained for biochemical analyses and the liver tissues were further processed for histological studies. The GC-MS spectra revealed the existence of various phytoconstituents such as neophytadiene, germaciene, caryophyllene among others. High density lipoprotein and albumin were significantly $(p<0.05)$ elevated in animals administered with 500, 1000 and 
$1500 \mathrm{mg} / \mathrm{kg}$ bw of the leaf extract. Ethanol, butanol and water fractions of the leaf of $V$. opulus showed antimicrobial action against most of the organisms used in this study. The result indicates the $V$. opulus leaf extract contains a wild range of fatty acids and heterocyclic compounds with antimicrobial efficacy and no hepatic damage.

[1] Adebayo AH, Aliyu R, Gatsing D, Garba IH. Int. J. Pharmacol 2006; 2: 618 622.

[2] Adebayo AH, Abolaji AO, Opata TK and Adegbenro IK. Afr. J. Biotechnol 2010; 9: $2145-2150$.

\section{Tu-Poster Session 2-PO-109 INFLUENCE OF CO-ADMINISTRATION OF ARTEMISININ AND RICINODENDRON HEUDELOTII LEAF EXTRACT ON ANTIOXIDANTS AND LIVER FUNCTION IN MALE RATS}

\section{Authors Yakubu $\mathrm{O}^{1}$, Adebayo $\mathrm{A}^{1}$, Okechukwu $\mathrm{E}^{1}$, Adeyemi $\mathrm{O}^{1}$, Iweala $\mathrm{E}^{1}$,} Zhang $Y]^{2}$

Institute 1 1Biochemistry and Molecular Biology Unit, Department of Biological Sciences, College of Science and Technology, Covenant University, PMB 1023, Canaan land, Ota Ogun State, Nigeria; 2 State Key Laboratory of Phytochemistry and Plant Resources of West China, Kunming Institute of Botany, \#132, Lanhei Road, Heilongtan, Kunming China, Kunming, Nigeria DOI 10.1055/s-0037-1608442

Startling rate of malaria parasite resistance to Artemisinin and its derivatives has led to possible herb-drug combination therapy ${ }^{1}$. This study assessed the effect of co-administration of artemisinin and Ricinodendron heudelotii leaf extract on certain antioxidants and liver parameters in rats and also phytochemical analysis of different fractions of the extract. 40 male rats were randomly grouped into four and administered: distilled water (Group A), artemisinin only (Group B), artemisinin with $R$. heudelotii extract (Group C) and $R$. heudelotii extract only (Group D). Alanine amino transferase (ALT), Aspartate aminotransferase, Alkaline phosphatase (ALP). Total protein were determined using standard methods. Reduced glutathione (GSH), catalase and malondialdehyde (MDA) were determined spectrophotometrically while the qualitative and quantitative phytochemical analysis was also carried out. The results revealed that total protein increased significantly in group $C$. The activities of ALT and AST were significantly increased $(p \leq 0.05)$ in the group administered artemisinin only while their activities were regulated to control level both in group C and D. The liver GSH concentration decreased in the group administered artemisinin only compared to the other treatment groups. Similarly, group A exhibited significant increase $(p \leq 0.05)$ in MDA while group $C$ and $\mathrm{D}$ showed decrease in MDA and catalase concentration. Histological examination showed that few hepatocytes were necrotic in the group administered artemisinin only while group $\mathrm{C}$ only showed mild to moderate central venous congestion and periportal cellular infiltration. The study indicates that the bioactive constituents in the $R$. heudelotii extract might have a regulatory effect on artemisinin toxicity or collaboratively enhance its activity. Such bioconstituents can further be isolated and characterized for drug development to tackle Plasmodium falciparum resistance.

[1] Adedosu OT, Akinola NA, Jelili AB, Gbadebo EA, Adebola OA, Samuel AO. Asian J. of Sci \&Tech 2015; 6:1338-1343

\section{Tu-Poster Session 2-PO-110 TOXICOLOGICAL EVALUATION OF HEPACARE ${ }^{\circledR}$ - A NIGERIAN HERBAL FORMULATION}

Authors Adebayo $\mathrm{A}^{1}$, Ashano $\mathrm{E}^{2}$, Yakubu $\mathrm{O}^{1}$, Okubena $\mathrm{O}^{3}$

Institute 1 Medicinal Plant Research Group, Department of Biological

Sciences, College of Science and Technology, Covenant University, P.M.B.

1023. Canaanland, Ota, Ogun State, Nigeria; 2 National Biotechnology
Development Agency, PMB 5118, Abuja, Nigeria, Abuja, Nigeria; 3 Health

Forever International, Ikeja, Lagos, Nigeria

DOI 10.1055/s-0037-1608443

Hepacare ${ }^{\circledR}$ is a widely marketed herbal drug in Nigeria which is used for treating chronic liver ailments ${ }^{1}$ and in this study, it is evaluated for its safety as well as pro-inflammatory and genotoxicity effects in mice. The effect of the drug was estimated in a 28-day study in which twenty five mice were randomly divided into five groups and administered orally at 250, 500, 750 and $2500 \mathrm{mg} / \mathrm{kg}$ body weight. Biochemical and haematological parameters were determined. The relative organ weights were estimated and histopathology of the liver and kidney tissues were also conducted. Furthermore, mRNA expression of pro-inflammatory cytokines, TNF- $\alpha$ and IL- 6 were also estimated by RTPCR in acute toxicity experiment. ${ }^{2}$ There was a significant increase $(p<0.05)$ in the activity of ALP in the group treated with $750 \mathrm{mg} / \mathrm{kg}$ b.w.; while mice treated with $2500 \mathrm{mg} / \mathrm{kg}$ showed significant increase in the levels of AST, ALT, ALP, total bilirubin and total protein as compared to the control group. However, there was a significant dose related increase in monocytes counts in the groups treated with 750 and $2500 \mathrm{mg} / \mathrm{kg}$. There was no significant difference ( $p>0.05)$ in mRNA expression of TNF- $\alpha$, IL- 6 and genotoxicity studies in all the treatment groups as compared to the control. However, some hepatic and nephro-pathological derangements were observed in the groups treated with higher doses of the drugs. In conclusion, the study established that the herbal drug may not induce significant pro inflammatory toxic responses and genotoxic effects but prolonged intake may cause severe biochemical and clinical abnormalities especially at higher doses.

[1] Ishola IO, Akinyede AA, Robert AK, Omilabu SA. Drug Research. 2014; 65 : $30-39$.

[2] Schneider CA, Rasband WS, Eliceiri KW. Nature Methods. 2012; 9: 671 675

\section{Tu-Poster Session 2-PO-111 Echinacea purpurea extract can reverse respiratory virus induced secondary bacterial infection}

Authors Vimalanathan $S^{1}$, Schoop $R^{2}$, Suter $A^{2}$

Institute 1 University of British Columbia, Vancouver, Canada; 2 A. Vogel Bioforce AG, Roggwil TG, Switzerland

DOI 10.1055/s-0037-1608444

Respiratory virus infections, including influenza (IVA), respiratory syncytial virus (RSV), rhinovirus (RV) and parainfluenza virus 3 (PI3), predispose airways to secondary bacterial infections leading to bronchitis, pneumonia, otitis media and sepsis, which cause high morbidity and lethality. The medicinal herb Echinacea purpurea has been used to prevent viral respiratory infections and support immune functions.

In this in vitro study, we investigated the effect of standardized Echinacea extract (Echinaforce, EF) on respiratory virus-induced bacterial receptors (ICAM-1, CEACAM-1, PAFr), and adhesion of Haemophilus influenzae and Streptococcus pneumonia on human bronchial epithelial cells and primary bronchial epithelial cells from pediatric patient (6y). Our results revealed that IVA, RSV and RV infection augment adhesion of $H$. influenzae and S. pneumonia, and that $\mathrm{EF}$ reduced bacterial adhesion by down regulating bacterial receptors. Our results indicate that $E$. purpurea has the potential to reduce the risk of respiratory complications by preventing virus-induced bacterial adhesion providing a rational basis for benefits observed in clinical studies and meta-analysis [1].

[1] Schapowal A, Klein P, Johnston SL. Adv Ther. 2015;32:187 - 200 


\section{Tu-Poster Session 2-PO-112 Phenolic compounds and anti-HIV1 potential of Merostachys neesii Rupecht (Bambusoideae)}

\author{
Authors Anselmo Moreira $\mathrm{F}^{1}$, Grombone-Guaratini Maria $\mathrm{T}^{2}$, Furlan \\ Cláudia $\mathrm{M}^{1}$ \\ Institute 1 University of São Paulo, São Paulo, Brazil; 2 Botanic Institute, São \\ Paulo, Brazil
}

DOI 10.1055/s-0037-1608445

Bamboos have great economical and medicinal importance. Brazil presents the greatest diversity of bamboos in the New World, with Merostachys Spreng. being the genus most representative in number of species. However, phytochemical and pharmacological studies with native species are scarce. This study aimed to evaluate the contents of phenolic compounds of leaves and culms from Merostachys neesii Ruprecht, an endemic Brazilian bamboo species, and their anti-HIV1 potential. Plant material was collected, dried at $40{ }^{\circ} \mathrm{C}$, and powered. Vegetal material was extracted by maceration in $70 \%$ ethanol and by infusion. Leaf ethanol extract (LEE) and Culm ethanol extract (CEE) were partitioned resulting in four phases: hexane (P1), dichloromethane (P2), ethyl acetate (P3), and hydromethanolic (P4) phases. All extracts and phases were analyzed by high performance liquid chromatography (HPLC). Results were expressed as $\mathrm{mg} \mathrm{g}^{-1}$ equivalent to apigenin or chlorogenic acid. Extracts and phases were also tested against the HIV1 transcriptase reverse (RT) activity using a colorimetric kit (Roche Diagnostics) according to the manufacturer's instructions. Results were expressed in minimum concentration to inhibit $50 \%$ of RT activity $\left(\mathrm{MIC}_{50}\right)$. Culms showed higher flavonoids and phenolic acids contents than leaves. UV-visible spectra analyses revealed predominance of chlorogenic acid derivatives and flavones. For leaves and culms, P2 (3.98 and $17.88 \mathrm{mg} \mathrm{g}^{-1}$ ) and P3 (16.43 and $18.73 \mathrm{mg} \mathrm{g}^{-1}$ ) exhibited the highest contents of phenolic acids and flavonoids, respectively. Culm infusion extract showed the lower $\mathrm{MIC}_{50}\left(844.05 \mu \mathrm{g} \mathrm{mL} \mathrm{m}^{-1}\right)$, followed by leaf infusion extract $\left(1310.39 \mu \mathrm{g} \mathrm{mL}^{-1}\right)$. Although flavones and chlorogenic acids have been reported as substances with anti-HIV1 potential [1], M. neesii extracts and phases did not present anti-HIV activity. Isolation of the $M$. neesii phenolic constituents is in progress.

[1] Andrae-Marobela K, Ghislain FW, Okatch H, Majinda RRT. Curr Drug Metab 2013; 14: $392-413$

\section{Tu-Poster Session 2-PO-113 Anti-inflammatory and anti-oxidant effects of Korean ginseng berry extract in LPS-activated RAW 264.7 macrophages}

\author{
Authors Byun $J^{1}$, Jin Seul $A^{1}$, Ban Ju $Y^{1}$ \\ Institute 1 Department of Dental Pharmacology, College of Dentistry, \\ Dankook University, Cheonan, Korea, Republic of (South) \\ DOI 10.1055/s-0037-1608446
}

This study aims to investigate whether Korean ginseng berry extract (KCB) attenuate lipopolysaccharide (LPS)-induced inflammation in RAW264.7 macrophage cells. The nitric oxide (NO) production and inducible NO synthase (iNOS) expression induced by LPS was dose dependantly reduced by preatreatment with $K G B$, which was measured by Nitrite assay and RT-PCR, respectively. The levels of prostaglandin $E_{2}\left(\mathrm{PGE}_{2}\right)$ and cyclooxygenase-2 (COX-2) also were inhibited by treatment of KGB, as shown by RT-PCR. Furthermore, KGB significantly suppressed the LPS-induced gene expression and production of cytokines such as interleukin-1 $\beta$ (IL-1 $\beta$ ), IL-6, IL-10, and tumor necrosis factor- $\alpha($ TNF- $\alpha)$, as shown by ELISA, and western blot analysis and RT-PCR, respectively. Nuclear translocation of nuclear factor-KB (NF-KB) p65 subunit induced by LPS was also inhibited by treatment with KGB. KGB contributed to the downregulation of mitogen-activated protein kinases (MAPK) including extracellular signal-regulated protein kinase $1 / 2$ (ERK 1/2), c-Jun N-terminal protein kinase (JNK), and p38. Additionally, in the KGB-treated RAW264.7 cells, we observed the reduction of reactive oxygen species (ROS) generation, and antioxidant enzymes activities such as superoxide dismutase (SOD), glu- tathione peroxidase (GSH-Px), and catalase (CAT), as assessed by using a dichlorofluoroscein diacetate (DCF-DA) fluorescent probe, and ELISA, respectively. Taken together, these results indicate that KGB possesses anti-inflammatory and anti-oxidant effects via the inhibition of MAPK signaling pathway in LPS-activated RAW264.7 macrophages, suggesting that KGB may be a potential therapeutic agent for inflammatory diseases and oxidative stress related diseases.

\section{Tu-Poster Session 2-PO-114 Protective effect of Korean ginseng berry on hydrogen peroxide $\left(\mathrm{H}_{2} \mathrm{O}_{2}\right)$-induced oxidative damage in SH-SY5Y cells}

Authors jin Seul $A^{1}$, Byun J ${ }^{1}$, Ban Ju $Y^{1}$

Institute 1 Department of Dental Pharmacology, College of Dentistry, Dankook University, Cheonan, Korea, Republic of (South)

DOI 10.1055/s-0037-1608447

It has been known that the ginseng berry contains nutrients higher than roos of ginseng, and has higher ginsenoside content than of the root. However, until now, many studies have focused on the effect of the ginseng root. This study investigated the effect of Korean ginseng berry extract (KGB) on hydrogen peroxide $\left(\mathrm{H}_{2} \mathrm{O}_{2}\right)$-induced neuronal damage and potential mechanisms in human SH-SY5Y neuroblastoma cells. Our results revealed that pretreatment with $\mathrm{KGB}$ dose-dependantly inhibited $\mathrm{H}_{2} \mathrm{O}_{2}$-induced apoptotic cell death, which was measured by MTT assay and Hoechst 33342 staining. $\mathrm{H}_{2} \mathrm{O}_{2}$-induced increases of reactive oxygen species (ROS) generation and mitochondrial dysfunction were attenuated by KGB pretreatment. KGB effectively suppressed the up-regulation of Bax and down-regulation of $\mathrm{BCl}-2$, and caspase- 3 activation. KGB also blocked the $\mathrm{H}_{2} \mathrm{O}_{2}$-induced phosphorylation of mitogen-activated protein kinases (MAPK) including extracellular signal-regulated protein kinase $1 / 2$ (ERK 1/2), c-Jun N-terminal protein kinase (JNK), and p38. $\mathrm{H}_{2} \mathrm{O}_{2}$ induced induction of HSP70 was enhanced by KGB. Moreover, KGB enhanced the activities of antioxidant enzymes such as superoxide dismutase (SOD), glutathione peroxidase (GSH-Px), and catalase (CAT), and decreased the malondialdehyde (MDA) content. These results show that KGB has a protective effect against $\mathrm{H}_{2} \mathrm{O}_{2}$-induced oxidative stress in SH-SY5Y cells via the inhibiting of MAPK signalling pathway and enhancing HSP70 production. Therefore, KGB may be development potential as a preventive and therapeutic agent for neurodegenerative diseases and other disorders caused by oxidative stress.

\section{Tu-Poster Session 2-PO-115 Gynura Procumbens (Lour.) Merr Inhibits the Glutamate Induced Toxicity in Neuronal Cell Lines.}

Authors Lee Hyeon $\mathrm{W}^{1}$, Ryu Ga $\mathrm{H}^{1}$, Yang Woo $\mathrm{S}^{1}$, Ma Choong J $\mathrm{J}^{1,2}$

Institute 1 Biomaterials Engineering, College of Biomedical Science, Kangwon National University, Chuncheon, Korea, Republic of (South); 2 Institute of Bioscience \& Biotechnology, Kangwon National University, Chuncheon, Korea, Republic of (South)

DOI 10.1055/s-0037-1608448

Gynura procumbens is traditionally used in South East Asia to lowering the high blood pressure, and cholesterol level. It is also known to have antioxidant, antidiabetic and anti-inflammatory properties. The objective of the study was to confirm the neuroprotective effects of $80 \%$ methanol extract of Gynura procumbens. Glutamate is a well-known excitatory neurotransmitter but overexpression of glutamate causes brain cell damage due to oxidative stress. HT22 mouse hippocampal cells are cell lines that don't have ionotropic glutamate receptors so it can be used to screening the neuroprotective bioactive substance. In this study, Gynura procumbens significantly decreased the glutamate-induced cell death in HT22 cells by reducing the intracellular ROS and $\mathrm{Ca} 2+$ level and maintaining the mitochondrial membrane potential level. Meanwhile, total glutathione, glutathione reductase and glutathione peroxide activities are increased. Therefore, we can conclude $80 \%$ methanol extract of Gynura procumbens has significant neuroprotective effect in HT22 cell. 


\section{Tu-Poster Session 2-PO-116 Neuroprotective Effect of $80 \%$ Methanol Extract of Lysimachia Christinae Hance in HT22 Cells}

\begin{abstract}
Authors Lee Hyeon $W^{1}$, Ryu Ga $H^{1}$, Yang Woo $S^{1}$, Ma Choong J1,2 Institute 1 Department of Medical Biomaterials Engineering, College of Biomedical Science, Kangwon National University, Chuncheon, Korea, Republic of (South); 2 Republic of Korea, 2Institute of Bioscience \& Biotechnology, Kangwon National University, Chuncheon, Korea, Republic of (South) DOI 10.1055/s-0037-1608449

Lysimachia christinae Hance was traditionally used as Chinese traditional medicine. It is known to ease off the hyperuricemia and possess antipyretic and anti-inflammatory activity. Excessive glutamate contributes to excitotoxicity in brain and this can be etiological cause of the neurodegenerative diseases. The purpose of the study was to determine the neuroprotective effect of $80 \%$ methanol extract of Lysimachia christinae Hance against glutamate-induced oxidative stress in HT22 cells. In this experiment, intracelluar concentration of ROS and $\mathrm{Ca} 2+$ was decreased against glutamate treatment and this kept the normal mitochondrial membrane potential level. In addition, total glutathione amount and glutathione related antioxidant enzymes activities are increased. Through the whole mechanisms, Lysimachia christinae Hance significantly survived at the cell viability test. Consequently, Lysimachia christinae Hance is expected to be a good neuroprotective material.
\end{abstract}

\section{Tu-Poster Session 2-PO-118 Taraxacum officinale leaves aqueous extract-mediated glucose lowering effect in STZ-induced diabetic rats.}

\author{
Authors Sarkodie J ${ }^{1}$, Asare $C^{1}$, Debrah $\mathrm{P}^{1}$, Oppong Bekoe $\mathrm{E}^{1}$ \\ Institute 1 University of Ghana, Accra, Ghana \\ DOI 10.1055/s-0037-1608450
}

The use of traditional medicine in treating and managing chronic diseases is currently a common practice. Taraxacum officinale (dandelion), commonly used as food, has been reported to have several pharmacological properties including blood glucose lowering effects in diabetes mellitus. In the present study, the hypoglycemic property of the leaves of $T$. officinale was investigated.

The leaves of $T$. officinale were pulverized and extraction was done using distilled water. Phytochemical analysis was performed on the extract to find the probable active ingredient responsible for the pharmacological activity. The hypoglycemic effects of the extract were studied in streptozotocin (STZ)induced diabetic rats at three doses $(10 \mathrm{mg} / \mathrm{kg}, 30 \mathrm{mg} / \mathrm{kg}$ and $60 \mathrm{mg} / \mathrm{kg}$ ). Linear mixed model technique was used to analyze the multiple glucose measurements per rat. This model acknowledges the presence of dependency by introducing rat-specific random effect(s) which measures the individual rat variations either at baseline (random intercept) or at the different time point (random slope).

Saponins and polyphenols (may be responsible for the activity) were found to be present in the extract. The most appropriate statistical technique model estimated the overall trajectory of blood glucose levels. A random-intercept model was found to be sufficient to model the effect of treatment evolution. The results also showed that aqueous extract of $T$. officinale decreased the blood glucose levels of STZ induced diabetic rats compared to an untreated group after a 6 hour and a 28 day period. In the 28-day treatment, the reduction in blood glucose levels was dose dependent and comparable to the positive control (insulin treated group).

The study has provided evidence that aqueous extract of $T$. officinale has hypoglycaemic property comparable to insulin over a period of time.

\section{Tu-Poster Session 2-PO-119 In vitro screening of acute hepatic cytotoxicity of pyrrolizidine alkaloids in human and rodent hepatic cell lines}

\author{
Authors Forsch $\mathrm{K}^{1}$, Siewert $\mathrm{B}^{1}$, Disch $\mathrm{L}^{1}$, Unger $\mathrm{M}^{1}$, Drewe $\mathrm{J}^{1}$ \\ Institute 1 Max Zeller Söhne AG, Romanshorn, Switzerland \\ DOI 10.1055/s-0037-1608451
}

Pyrrolizidine alkaloids (PAs) are secondary plant ingredients formed in many plant species to protect against predators. PAs are generally considered acutely hepatotoxic, genotoxic and carcinogenic. Up to now, only few in vitro and in vivo investigations were performed to evaluate their relative toxic potential. The aim of this work was to develop a predictive screening tool of their relative hepatotoxicity. Methods: Different human and rodent hepatocyte cell lines (H-4-II-E, HepG2, HepaRG) were used to assess cytotoxicity of lasiocarpine, seneciphylline, and monocrotaline with WST-1 assay. Results: Incubation over $72 \mathrm{~h}$ at concentrations from $25 \mu \mathrm{M}$ up to even $2400 \mu \mathrm{M}$, resulted in no toxic effects in neither cell line. In a galactose-based culture medium $(11.1 \mathrm{mM})$ which increases cell susceptibility to mitochondrial toxicants, showed a significant toxicity at $900 \mu \mathrm{M}$ in $\mathrm{H} 4-\mathrm{II}-\mathrm{E}$ and HepG2 cells. Inhibition of carboxylesterase-mediated PA detoxification (specific carboxylesterase 2 inhibitor loperamide $(2.5 \mu \mathrm{M})$ and unspecific carboxylesterase inhibitor bis-pnitrophenyl-phosphate (BNPP, $100 \mu \mathrm{M})$ ) revealed that loperamide enhanced toxic effects of lasiocarpine in both cell lines, whereas BNPP had a weaker effect. Inhibition of glutathione-mediated detoxification by etacrynic acid $(100 \mu \mathrm{M})$ did not enhance toxicity. Comparison of lasiocarpine, seneciphylline and monocrotaline in galactose-based medium with loperamide showed the following rank order of toxicity in HepG2 cells: lasiocarpine > seneciphylline $\geq$ monocrotaline. Conclusion: If no toxicity is observed under standard conditions, sensitisation with galactose is useful to assess relative acute cytotoxicity of PAs in different cell lines. The results also suggest that carboxylesterases are involved in the detoxification of PAs.

\section{Tu-Poster Session 2-PO-120 Molluscicidal and Antimalarial Properties and Isolation of Alkaloids from Crinum jagus (Thomps) Dandy.}

Authors Elusiyan $\mathrm{C}^{1}$, Akinyele $\mathrm{S}^{1}$, Omisore $\mathrm{N}^{2}$, Adewunmi $\mathrm{C}^{1}$

Institute 1 Drug Research and Production Unit, Faculty of Pharmacy, Obafemi Awolowo University, Ile-Ife, Nigeria; 2 Department of Pharmacology, Faculty of Pharmacy, Obafemi Awolowo University, Ile-Ife, Nigeria

DOI 10.1055/s-0037-1608452

Crinum jagus fresh bulb and leaf methanol extracts were subjected to molluscicidal test using adult Biomphalaria glabrata snails and antimalarial prophylactic and suppressive tests in-vivo against chloroquine-sensitive (NK 65) Plasmodium berghei according to established experimental protocols $(1,2)$. The more active bulb extract was fractionated by vacuum liquid chromatography (VLC) into alkaloidal and non-alkaloidal fractions and also investigated for antimalarial property. The active alkaloidal fraction was further purified using various chromatographic techniques. The isolated alkaloids were identified using spectroscopic methods including NMR, FTIR and MS. The oral median lethal dose (LD50) of both leaf and bulb extracts were greater than $10000 \mathrm{mg} / \mathrm{kg}$ body weight (b.wt.) according to Lorke's 1983 (3) acute toxicity test. The leaf extract showed weak antimalarial and molluscicidal activities (40\% snail mortality) at $1000 \mathrm{mg} / \mathrm{L}$ while reference niclosamide $(0.5 \mathrm{mg} / \mathrm{L})$ gave $100 \%$ mortality. The bulb extract had a chemo-suppression of $76.55 \pm 2.76 \%$ and prophylactic effects of $90.49 \pm 2.70 \%(p<0.05)$ at the maximum concentration (1000 mg/kg b.wt). Similarly, the alkaloidal fraction had a chemo-suppression of $71.36 \pm 12.54 \%$ while the non-alkaloidal fraction was $51.49 \pm 10.46 \%$ $(p>0.05)$. Chloroquine and pyrimethamine standards had $80.26 \pm 3.09 \%$ chemosuppression and $50.39 \pm 6.80 \%$ prophylactic effects respectively. The two alkaloids isolated from the antimalarial active fraction were identified as lycorine [1] and crinamine [2]. 
[1] Duncan J. and Sturrock R. F. (1987). Laboratory Evaluation of Potential Plant Molluscicides. In: Mott K. E. (ed.). Plant Molluscicides. A Wiley medical publication. ISBN 047191228 X. Chapter 9, 251- 265.

[2] Peters W. (1980). Chemotherapy of Malaria. In: Malaria. Kreier J. P. (ed.), Vol. 1. Academic, New York, Pp 145-283.

[3] Lorke D. Archives Toxicology. 1983, 54:275 - 287

\section{Tu-Poster Session 2-PO-121 Lacistema pubescens Mart.: A species with antinociceptive activity}

Authors Conegundes J $]^{1}$, Fernandes Maria $\mathrm{F}^{1}$, Mendes $\mathrm{R}^{1}$, Pinto $\mathrm{N}^{1}$, Silva $\mathrm{J}^{1}$, Scio $E^{1}$

Institute 1 Laboratory of Bioactive Natural Products, Department of Biochemistry, Federal University of Juiz de Fora, Juiz de Fora, Brazil DOI 10.1055/s-0037-1608453

Lacistema pubescens MART. (Lacistemataceae) is a plant species originally from Brazil. The Lacistema genus is traditionally used to treat several diseases, including rheumatism, body aches and fever [1]. The objective of this study was to evaluate the antinociceptive activity of its dichloromethane extract (DE) in the writhing, formalin, and tail flick tests [2]. Those tests are behavioral, so to verify that DE did not have sedative action, the open-field test was performed [3]. Besides not affecting the animals locomotion, DE was effective in all doses, 100, 200 and $300 \mathrm{mg} / \mathrm{kg}$, inhibiting abdominal contortions in 67, 71 and $83 \%$, respectively, when compared to the negative control group, in a way statistically equal to indomethacin. This model is sensitive to centrally or peripherally acting analgesic compounds with several different mechanisms of action [3]. In the formalin test, DE (100, 200 and $300 \mathrm{mg} / \mathrm{kg})$ reduced the paw licking time in the initial phase $(0-5 \mathrm{~min})$ in 56,54 and $48 \%$, and in the late phase in 29,38 and $44 \%$, respectively, when compared to the negative control group. The nociceptive effect triggered in this assay is biphasic, with the first phase showing neurogenic nociception, while the second phase shows inflammatory nociception [2]. No effect was observed using the tail flick test, suggesting that DE presented no effect on the opioid system. Thus, these findings reinforce the popular use of the genus and strongly suggest that $L$. pubescens presented antinociceptive potential acting in neurogenic and inflammatory nociception.

This work was supported by the grants from FAPEMIG, CAPES and CNPq.

[1] Silva JM, Conegundes JL, Mendes RF et al. J Pharm Pharmacol 2015; 67:1613-1622

[2] Pinto A, Duque AP, Pacheco NR, Scio E. Pharm Biol 2015; 53:1 - 6

[3] Yin Z, Li L, Chu S, MA Z, Gu X. Sci Rep 2016; 6:27129

\section{Tu-Poster Session 2-PO-122 Sun Protection Factor (SPF) and antioxidant capacity of Lavandula angustifolia L.}

\section{Authors Gonulalan Ekrem $\mathrm{M}^{1}$, Ozenver $\mathrm{N}^{1}$, Demirezer $\mathrm{LO}^{1}$}

Institute 1 Department of Pharmacognosy, Faculty of Pharmacy, Hacettepe University, 06100, Sihhiye, Ankara, Turkey

DOI 10.1055/s-0037-1608454

The essential oil from lavender is mostly used for cosmetic purposes. However, it is known that in recent years, Lavandula angustifolia L. (Lamiaceae) has been researched on the skin rejuvenating properties (1).

In this study was to determine SPF values and antioxidant capacity of methanolic extract from Lavandula angustifolia, linalool and linalool free L. officinalis. SPF were determined according to Mansur et al (2). 100 and $200 \mu \mathrm{g} / \mathrm{mL}$ concentrations of samples were scanned at wavelength $290-320 \mathrm{~nm}$ and three replicates were made. SPF values $(0.62-1.37)$ of Lavandula officinalis, linalool and linalool free L. officinalis were found considerably low.

Antioxidant capacity tests were performed at 5 different $(25,50,100,250$ and $500 \mu \mathrm{g} / \mathrm{mL}$ ) concentrations. The best results were obtained with $500 \mu \mathrm{g} / \mathrm{mL}$. The results of $500 \mu \mathrm{g} / \mathrm{mL}$ were given in the table below.
Tab. 1

\begin{tabular}{l|l|l|l|}
$\begin{array}{l}\text { Radical scavenging and } \\
\text { antioxidant capacity tests }\end{array}$ & Lavandula officinalis & Linalool & Linalool free L. officinalis \\
\hline DPPH & $69.09 \%$ & $12.20 \%$ & $46.64 \%$ \\
ABTS & $85.54 \%$ & $5.44 \%$ & $79.25 \%$ \\
NO & $37.57 \%$ & $38.81 \%$ & $36.33 \%$ \\
\hline Phosphomolybdate & $92.01 \%$ & $37.25 \%$ & $34.81 \%$
\end{tabular}

This study was supported by The Scientific and Technological Research Council of Turkey (TUBITAK) (Project No: SBAG-214S147)

[1] Baumann LS, 2007. Dermatol. Therapy 2007; 20:330 - 342.

[2] Mansur JS, Breder MN, Mansur MC, Azulay RD. An Bras Dermatol 1986; 61: $121-124$.

\section{Tu-Poster Session 2-PO-123 The Antiinflammatory Activity of $\mathrm{N}$-Hexane Insoluble Fraction of Plantago Lanceolata Leaves and Its Major Compound on Mice}

Authors Fakhrudin $N^{1,2}$, Astuti Eny $D^{1}$, Sulistyawati $R^{1,5}$, Santosa $D^{1,2}$, Susandarini $R^{4}$, Nurrochmad $A^{1}$, Franyoto Yuvianti $D^{1,3}$, Wahyuono $S^{1,2}$ Institute 1 Faculty of Pharmacy, Universitas Gadjah Mada, Yogyakarta, Indonesia; 2 Center for Natural Antiinfective Research, Faculty of Pharmacy, Universitas Gadjah Mada, Yogyakarta, Indonesia; 3 Sekolah Tinggi IImu Farmasi “Yayasan Pharmasi” Semarang, Semarang, Indonesia; 4 Faculty of Biology, Universitas Gadjah Mada, Yogyakarta, Indonesia; 5 Akademi Analis Farmasi Al Islam Yogyakarta, Yogyakarta, Indonesia DOI 10.1055/s-0037-1608455

Instead of a normal physiological process as body's response to infection and injury, inflammation is also known to underlay a wide variety of diseases, such as tumors, asthma, arthritis, hepatitis, gastritis, and atherosclerosis. Although many antiinflammatory drugs have been discovered, the efforts to develop antiinflammatory agents are still challenging. Medicinal plants provide a huge compound diversity and have inspired drugs discovery for decades. Plantago lanceolata has been used traditionally to cure various inflammatory-related disorders. Previous study found that dichloromethane extract of $P$. lanceolata leaves demonstrates in vitro antiinflammatory activity. Here, we evaluated the in vivo antiinflammatory activities of $\mathrm{n}$-hexane-insoluble fraction of $P$. lanceolata leaves dichloromethane extract (HIFPL) and its isolated major compound. HIFPL was prepared by $n$-hexane partition of the dichloromethane extract of $P$. lancelata leaves to remove chlorophylls and other non-polar inert constituents. The first in vivo experimental model employed mice paw edema induced by carrageenan (s.c.). The reduction of the paw edema and the expression of COX-2 were measured. The effectively of HIFPL as COX-2 inhibitor was also assessed in enzymatic assay. The second in vivo experimental model used mice leukocytes migration induced by thioglycollate (i.p.). The reduction of the leukocytes migration and the level of adhesion molecules were measured in the peritoneum lavage.

We demonstrated that HIFPL (doses 40, 80, $160 \mathrm{mg} / \mathrm{kgBW}$; p.o.) exerts antiinflammatory activities in mice. It decreased the paw edema volume and the COX-2 expression level. Nevertheless, HIFPL showed unselective COX-2 inhibitor as it also inhibited COX-1 with a higher potency. Interestingly, HIFPL effectively inhibited leukocyte migration, at least partly, by inhibiting the expression of MCP-1 and IL-8. We also found that ursolic acid is the major compound in HIFPL. In line with HIFPL, ursolic acid exerted antiinflammatory activity in the mice leukocytes migration assay by lowering the level of MCP-1 and IL-8. 


\section{Tu-Poster Session 2-PO-124 Mass production method of Korea ginseng(Panax ginseng C.A. Meyer) leaf and inhibitory effect of extracts on fat accumulation}

Authors Jang Myeong $\mathrm{H}^{1}$, Kwon Tae $\mathrm{R}^{1}$, Nam Ju O², Lee Seul $\mathrm{C}^{2}$ Institute 1 Punggi Ginseng Institute, Yeongju, Korea, Republic of (South); 2 2Department of Food Science and Biotechnology, Kyungpook national University, Daegu, Korea, Republic of (South)

DOI 10.1055/s-0037-1608456

We have mostly used the root part of Korean ginseng as a fresh ginseng, red ginseng and white ginseng. Top part of ginseng is known that it has $3 \sim 5$ times more saponin than the ground part. Meanwhile, in terms of medicinal effect the value of the top part has been approved, but it hasn't been used due to the rearing relation of the ground part and the use of chemicals. In this study, we aimed to find out how to develope the mass production method of Korea ginseng's leaf that has a high practical value in facility and fat accumulation depressor effect to an animal. The mass production of the ginseng leaf was tried for the first time in Korea and we could mass-produce ginseng leaf in a vinyl greenhouse facility shaded $85 \%$ of the light with the density of seeding in 300 400plant/ $1.62 \mathrm{~m}^{2}$ by installing $100 \mathrm{~cm}$ width, $50 \mathrm{~cm}$ height, and $18 \mathrm{~m}$ length of a left and right direction movable cultivation bed. The yield of the top part of 3-year old ginseng was $3,471 \mathrm{~kg} / \mathrm{ha}$, which is the highest in 350 plant $/ 1.62 \mathrm{~m}^{2}$ process. It significantly reduced the amount of weight, epididymis and abdominal fat tissue in injection of the extract from Green leaf (GL) and Aging leaf (AL) to a high fat diet (HFD) inducement rat. AL extract restrained fat formation of 3T3-L 1 fat cell through the revelation control of the lipogenetic regulator like PPAR-y and CCAAT/enhancer-binding protein (C/EBP)- $\alpha$. In terms of the application of Korean ginseng leaf, the inhibition function of fat accumulation, which is not only fresh leaf but also aging leaf with chlorophyll-destroyed, will have considerable implications for ginseng cultivation and utilization.

\section{Tu-Poster Session 2-PO-125 Protective effect of Lagerstroemia speciosa against dextran sulfate sodium induced ulcerative colitis in C57BL/ 6 mice}

Authors subramanya $S^{3}$, Chaudhary $G^{1}$, Mahajan $U^{1}$, Goyal $S^{1}$, ojha $S^{2}$, Patil $C^{1}$

Institute 1 Department of Pharmacology, R. C. Patel Institute of Pharmaceutical Education and Research, Dhule, India; 2 Department of Pharmacology and Therapeutics, College of Medicine and Health Sciences, Al Ain, United Arab Emirates; 3 Department of Physiology, College of Medicine and Health Sciences, United Arab Emirates University, Al Ain, United Arab Emirates

DOI $10.1055 / \mathrm{s}-0037-1608457$

The protective effect of methanolic extract of Lagerstroemia speciosaleaves (LS) was evaluated against dextran sulfate sodium (DSS) induced ulcerative colitis in C57BL/ 6 mice. The administration of DSS (2.5\% in drinking water ad libitum) in C57BL/ 6 mice induced ulcerative colitis in 7 days. The LS was orally administered for 7 days at daily doses of 100 and $200 \mathrm{mg} / \mathrm{kg}$. At the end of 7 days of treatment the animals were sacrificed, colonic tissues were removed and processed for further analysis of oxidative stress, and histopathology. In DSS treated mice the oxidative stress markers were elevated compared to controls. There was also significant reduction in the anti-oxidant defense levels marked by reduced cellular glutathione, catalase, and superoxide dismutase. The DSS-induced damage to the colon epithelium was evident from a significant increase in the lipid peroxidation. The histology of colon sections revealed inflammatory changes and marked impairment in the integrity of the mucosal lining with inflammatory changes. Both the doses of LS significantly prevented DSS-induced inflammatory and ulcerative damages of the colon, reduced lipid peroxidation and also restored the levels of innate antioxidants in the colon tissue. These findings indicate the protective effects of LS against the DSS- induced inflammatory and oxidative damage in the mouse colon. Further investigation involving bioactivity guided fractionation of the LS can yield potent constituent which may have a significant role in the treatment of inflammatory bowel disease and ulcerative colitis.

\section{Tu-Poster Session 2-PO-126 Small concentrations of Lippia spp. can help in the control of the bean weevil, Acanthoscelides obtectus Say}

Authors Suárez-Villanueva $V^{1}$, Rodríguez-Conzález Á ${ }^{1}$, Da Silva $F^{2}$, Mayo $S^{1}$, Carro-Huerga $\mathrm{G}^{1}$, González-López Ó ${ }^{1}$, Álvarez-García S ${ }^{1}$, Casquero PA ${ }^{1}$ Institute 1 Research Group of Engineering and Sustainable Agriculture, Research Institute of Environment, Natural Resources and Biodiversity, Universidad de León, Leon, Spain; 2 Federal University of Reconcavo de Bahia, Rui Barbosa 710, CEP 44380 - 000, Cruz das Almas, Spain

DOI $10.1055 / \mathrm{s}-0037-1608458$

Acanthoscelides obtectus (Coleoptera: Chrisomelidae: Bruchidae) is an insect pest which attacks the common bean (Phaseolus vulgaris, L.). It has been suggested that plant products, e. g. essential oils, may be a suitable alternative for controlling insect pests ${ }^{1}$, including $A$. obtectus ${ }^{2}$. The objective of this study was to determine the capacity of essential oils of Lippia spp. to control $A$. obtectus adults under laboratory conditions. One millilitre of solution $(6 \mu \mathrm{L}$ of Lippia orinanoides and Lippia alba diluted in $994 \mu \mathrm{L}$ of ethanol) was sprayed (Potter tower) directly on Petri dishes ( $90 \mathrm{~mm}$ in diameter) covered with sterile filter paper. One hour after, twenty $A$. obtectus adults were placed in these Petri dishes. Four replicates were used for each Lippia spp. Daily monitoring of insect mortality was performed for 15 days.

Results (Figure 1) show L. orinagoides accumulated an insect mortality of $54 \%$, significantly different from L. alba, which achieved a mortality of $43 \%$. Both Lippia spp. treatments were significantly different from control treatment, with an accumulated mortality of $16 \%$.

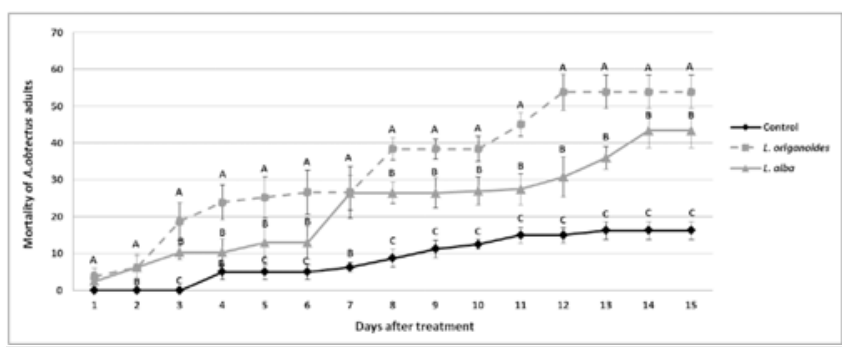

Fig. 1: Accumulated daily A. obtectus adults mortality by Lippia spp. essentials oils (day 1 represents 24 hours after treatment). Upper and lower error bars are represented. (Fisher's LSD. p<0.05).

The ability of both essential oils of Lippia spp. to paralyze the adults and kill them make these essential oils a new suitable tool for the control of adults of this insect pest in small storages.

[1] Pavela RInd. Crops Prod. 2011, 34: 888 - 892.

[2] Papachristos DP, Karamanoli KI, Stamopoulos DC, Menkissoglu-Spiroudi U. Pest Manag. Sci. 2004, 60: 514-520.

\section{Tu-Poster Session 2-PO-127 Control of Xylotrechus arvicola (Coleoptera: Cerambycidae) larvae population by inoculating Trichoderma spp. in vine wood}

Authors Rodríguez-González Á ${ }^{1}$, Suárez Villanueva $V^{1}$, Mayo $S^{1}$, CarroHuerga $\mathrm{G}^{1}$, González-López $\mathrm{O}^{1}$, Gutiérrez $\mathrm{S}^{2}$, Peláez $\mathrm{H}^{3}$, Casquero PA ${ }^{1}$ Institute 1 Research Group of Engineering and Sustainable Agriculture, Research Institute of Environment, Natural Resources and Biodiversity, Universidad de León, Leon, Spain; 2 Area of Microbiology, University School of Agricultural Engineers, Universidad de León, Ponferrada Campus, Av. Astorga 
s/n, 24401, Ponferrada, Spain; 3 Freelance, Vicente Aleixandre 24, 47008,

Valladolid, Spain

DOI 10.1055/s-0037-1608459

Xylotrechus arvicola (Coleoptera: Cerambycidae) is a grapevine (Vitis vinifera) pest in the Iberian Peninsula causing the spread of wood diseases. Trichoderma (Teleomorph: Hypocrea) is a fast growing opportunistic fungus, which produces antimicrobial compounds ${ }^{1}$. Moreover, it can be used as biological control of pests ${ }^{2}$. This study's aim is to evaluate Trichodema spp. efficacy on controlling $X$. arvicola larvae, simulating field conditions. One millilitre of spore suspension $\left(1 \times 10^{7} \mathrm{esp} / \mathrm{ml}\right)$ of Trichoderma spp. was applied directly to vine with a manual diffuser. One hour after, four $X$. arvicola larvae were placed on the vine. Vines were opened 135 days later to count larvae and extract wood samples. Four samples per treatment from rhytidome and galleries per vine in four replicates were incubated on Petri dishes with Rose Bengal-Chloramphenicol Agar medium at controlled conditions during 7 days.

Tab. 1: X. arvicola dead larvae with Trichoderma spp., number of isolations and percentage of isolates showing growth of Trichoderma spp. from vine wood (rhytidome and galleries)

\begin{tabular}{|l|l|l|l|l|l|}
\hline $\begin{array}{l}\text { Trichoderma } \\
\text { treatment } \\
\text { (Isolated } \\
\text { number) }\end{array}$ & $\begin{array}{l}\text { Mortality } \\
\text { (\%) of } \\
\text { X. arvicola } \\
\text { larvae }\end{array}$ & $\begin{array}{l}\text { Rhytidome } \\
\text { isolations }\end{array}$ & $\begin{array}{l}\text { Rhytidome } \\
\text { (\%) with } \\
\text { Trichoderma }\end{array}$ & $\begin{array}{l}\text { Galleries } \\
\text { isolations }\end{array}$ & $\begin{array}{l}\text { Galleries (\%) } \\
\text { with } \\
\text { Trichoderma }\end{array}$ \\
\hline $\begin{array}{l}\text { T. harzianum } \\
\text { (T-019) }\end{array}$ & $81.25 \pm 6.25 \mathrm{~A}$ & 21 & 76.19 & 16 & 75.00 \\
\hline $\begin{array}{l}\text { T. gamsii } \\
\text { (T-066) }\end{array}$ & $68.75 \pm 23.66 \mathrm{~A}$ & 17 & 29.41 & 8 & 100.00 \\
\hline $\begin{array}{l}\text { T. gamsii } \\
\text { (T-067) }\end{array}$ & $31.25 \pm 11.96 \mathrm{~B}$ & 16 & 37.50 & 12 & 33.33 \\
\hline $\begin{array}{l}\text { T. gamsii } \\
\text { (T-070) }\end{array}$ & $75.00 \pm 10.21 \mathrm{~A}$ & 11 & 54.54 & 4 & 100.00 \\
\hline $\begin{array}{l}\text { T. gamsii } \\
\text { (T-071) }\end{array}$ & $81.25 \pm 11.97 \mathrm{~A}$ & 12 & 33.33 & 11 & 45.45 \\
\hline Control & $6.25 \pm 6.25 \mathrm{~B}$ & 9 & 0.00 & 6 & 0.00
\end{tabular}

Different capital letters indicate significant differences among fungal isolates of dead larvae with Trichoderma spp. (Fisher's LSD. $\mathrm{p}<0.05$ ).

$100 \%$ of T. gamsii (T-066) and T. gamsii (T-070) galleries isolates showed Trichoderma growth, confirming the ability of this fungus to move through the vine wood by the galleries created by X. arvicola larvae. T. gamsii (T-071) and T. harzianum (T-019) treatments showed the highest control over insect larvae with a $81.25 \%$ mortality. These results indicate Trichoderma should be considered for further studies to evaluate its efficacy to control $X$. arvicola on field conditions.

[1] Mayo S, Gutiérrez S, Malmierca MG, Lorenzana A, Campelo MP, Hermosa R, Casquero PA. Front. Plant Sci 2015, 6, 685.

[2] Abdul-Wahid, OA, Elbanna, SM. Afr. J. Microbiol. Res. 2012, 6: 1024 - 1032.

\section{Tu-Poster Session 2-PO-128 Nurr1-Activating Daphnane Diterpenes from Daphne genkwa and its Neuroprotective Effect in an Animal Model of Parkinson's Disease}

Authors Han $\mathrm{BS}^{1}$, Kim $\mathrm{KS}^{2}$, Kim $\mathrm{YJ}^{3}$, Van Minh $\mathrm{N}^{3}$, Jung $\mathrm{HY}^{1}$, Sohn MJ ${ }^{3}$, Kim Won $\mathrm{G}^{3}$

Institute 1 Functional Genomics Research Center, Korea Research Institute of Bioscience and Biotechnology, Daejeon, Korea, Republic of (South);

2 Laboratory Animal Resource Center, Korea Research Institute of Bioscience and Biotechnology, Daejeon, Korea, Republic of (South); 3 Superbacteria Research Center, Korea Research Institute of Bioscience and Biotechnology, Daejeon, Korea, Republic of (South)

DOI 10.1055/s-0037-1608460

Nurr1, an orphan nuclear receptor involved in the differentiation of dopaminergic neurons in the brain, is a new attractive target for Parkinson's disease (PD).[1,2] During the screening for Nurr1 activators from natural sources using cell-based assay systems, an methanol extract of the combined stems and roots of Daphne genkwa was found to activate the transcriptional function of Nurr1. Eight daphnane-type diterpenes were isolated as active components from the hexane layer of the extract. The percentage of yuanhuacine (1) in the extract was highest among the diterpenes. Compound 1 and yuanhuadine most strongly enhanced the function of Nurr1. Nurr1-specific siRNA abolished the activity of 1 , strongly suggesting that transcriptional activation by 1 occurred through the modulation of Nurr1 function. Additionally, treatment with 1 inhibited 6-hydroxydopamine (6-OHDA)-induced neuronal cell death and lipopolysaccharide (LPS)-induced neuroinflammation. Importantly, the ethanol extract of D. genkwa improved behavioral deficits in a 6-OHDA-lesioned rat model of PD at oral administration $(100 \mathrm{mg} / \mathrm{kg} /$ day $)$ for 2 weeks. Morever, intraperitoneal administration of $1(0.5 \mathrm{mg} / \mathrm{kg} /$ day $)$ for 2 weeks significantly improved behavioral deficits and reduced tyrosine hydroxylase (TH)positive dopaminergic neuron death induced by 6-OHDA injection, and had a beneficial effect on the inflammatory response in the brain. Accordingly, Daphne genkwa extract and its active compounds are potential candidates for the treatment of PD.

[1] Kim CH, Han BS, Moon J, Kim DJ, Shin J, Rajan S, Nguyen QT, Sohn M, Kim WG, Han M, Jeong I, Kim KS, Lee EH, Tu Y, Naffin-Olivos JL, Park CH, Ringe D, Yoon HS, Petsko GA, Kim KS. Proc Natl Acad Sci USA 2015; 112: 8756 - 8761 [2] Kadkhodaei B, Ito T, Joodmardi E, Mattsson B, Rouillard C, Carta M, Muramatsu S, Sumi-Ichinose C, Nomura T, Metzger D, Chambon P, Lindqvist E, Larsson NG, Olson L, Bjorklund A, Ichinose H, Perlmann T. J Neurosci 2009; 29: $15923-15932$

\section{Tu-Poster Session 2-PO-129 Screening of Selected Sudanese Medicinal Plants for In vitro Activity Against Protozoal Neglected Tropical Diseases}

Authors Mahmoud $\mathrm{A}^{1,2}$, Mäser $\mathrm{P}^{1}$, Kaiser $\mathrm{M}^{1}$, Hamburger $\mathrm{M}^{3}$, Khalid $\mathrm{S}^{2,4}$ Institute 1 Department of Medical Parasitology and Infection Biology, Swiss Tropical and Public Health Institute, Basel, Switzerland; 2 Faculty of Pharmacy, University of Khartoum, Khartoum, Sudan; 3 Department of Pharmaceutical Biology, University of Basel, Basel, Switzerland; 4 Faculty of Pharmacy, University of Science and Technology, Omdurman, Sudan DOI 10.1055/s-0037-1608461

A number of 235 crude extracts originally obtained from plants reputed as antiparasitic in the traditional medicine in Sudan have been solicited from the depository of the Faculty of Pharmacy, University of Science \& Technology. The extracts were screened against Plasmodium falciparum, Trypanosoma brucei rhodesiense, T. cruzi trypomastigote forms, and Leishmania donovani. Assays were based on Alamar blue protocols for T. brucei and L. donovani, $\beta$ glactosidase assay for T.cruzi, and $[3 \mathrm{H}]$-hypoxanthine incorporation protocol for P. falciparum. Assays were performed in 96-well format, and standard reference drugs for each parasite were used as positive controls [1]. Of the 235 plant extracts tested, 161 samples showed inhibitory activity $>80 \%$ at $10 \mu \mathrm{g} / \mathrm{ml}$, and $>50 \%$ at $2 \mu \mathrm{g} / \mathrm{ml}$ against one or more of the selected parasites. Among them, 23\% fulfilled activity criteria against T. b. rhodesiense, L. donovani and P. falciparum; $18 \%$ were active against both L. donovani and P. falciparum, and $17 \%$ were active against both T. b. rhodesiense and P. falciparum with few extracts ( $<3 \%$ ) exhibited activity against T. cruzi.

Ethyl acetate fractions of the leaves of Acacia nilotica, Guiera senegalensis, Ziziphus spina-christi, Anogeissus leiocarpus and the bark of Terminalia laxiflora were among the most active samples. In order to associate bioactivity of the extracts with their HPLC chromatographic profiles coupled with their corresponding on-line spectroscopic data, the bioactive samples were submitted to HPLC-based activity profiling [2]. The isolation and structure elucidation of the antiparasitic compounds is well under progress.

[1] Adams, M, et al, Natural product communications, 2009. 4: 1377-1381. [2] Potterat, O. and M. Hamburger, Planta medica, 2014. 80: 1171 - 1181. 


\section{Tu-Poster Session 2-PO-130 In vitro Protective Effect of Chemically-Characterized Flower Extracts of Clitorea ternatea L. Against Hydrogen Peroxide Induced Cytotoxicity in Human Keratinocytes (HaCaT)}

Authors Zakaria Nik Nur $\mathrm{A}^{1,2}$, Okello $\mathrm{E}^{1}$, Howes $\mathrm{M}^{3}$

Institute 1 School of Agriculture, Food and Rural Development (AFRD), NE1

7RU Newcastle Upon Tyne, UK, Newcastle Upon Tyne, United Kingdom;

2 Faculty of Agro-based Industry (FIAT), Universiti Malaysia Kelantan (UMK),

17600 Jeli, Kelantan, Malaysia, Kelantan, Malaysia; 3 Jodrell Laboratory, Royal Botanic Gardens, Kew, Richmond TW9 3AB, UK, Richmond, United Kingdom DOI 10.1055/s-0037-1608462

Clitoria ternatea (Leguminosae), commonly known as the butterfly pea, is easily recognizable by its deep blue flowers. The leaves and roots of the plants are well research. However, very little is known about the flower parts of the plant. In Malaysia and Thailand, the flower part was used as a food colorant in the traditional dish, drink as herbal tea and consumed as vegetable salad for health benefits, particularly as defence against skin aging. This study was conducted to investigate the protective effect of $\mathrm{C}$. ternatea extracts against hydrogen-peroxide induced cytotoxicity in $\mathrm{HaCaT}$ cells (human keratinocytes) as skin cells model. Two extracts (aqueous and $70 \%$ ethanol) were prepared to mimic the traditional methods of preparation. The antioxidant potential of the extracts was determined using diphenyl-picryl hydrazine (DPPH) and 2, 2'azino-bis (3- ethylbenzothiazoline-6-sulphonic acid (ABTS) assays, expressed as Trolox equivalent (TE)/mg extract. The protective effect against hydrogenperoxide induced cytotoxicity was determined by 3-(4,5-dimethylthiazol-2-yl)5-(3-carboxymethoxyphenyl)-2-(4-sulfophenyl)-2 $\mathrm{H}$-tetrazolium (MTS) assay. The antioxidant assays showed the $\mathrm{TE} / \mathrm{mg}$ extract for $\mathrm{C}$. ternatea ethanol extract (CTE) were significantly higher than $\mathrm{C}$. ternatea water extract (CTW) $(\mathrm{p}<0.05)$. HaCaT treated with CTW extract showed to have higher percentage viability compared to untreated $\mathrm{HaCaT}$ after exposure to $200 \mu \mathrm{M}$ hydrogen peroxide $(p<0.05)$. No protective effect was observed from CTE extract. High resolution liquid chromatography-mass spectrometry (LC-MS) revealed the detection of compounds assigned as flavonol glycosides (derived from quercetin and myricetin), anthocyanins (derived from delphinidin) and coumaric acid derivatives (coumaroylsucrose and coumaroylglucose) in CTE and CTW. The higher antioxidant potential in CTE, observed by higher antioxidant potential to inhibit free radicals but antagonistically prevented the protective effect against hydrogen peroxide-induced cytotoxicity as opposed to $\mathrm{HaCaT}$ treated with CTW may be due to variation in the relative levels of phytochemicals in the CTE and CTW extracts.

\section{Tu-Poster Session 2-PO-131 Effect of herbal syrup "Limonidin" at the functional non ulcer dyspepsia}

Authors Zhussupova $\mathrm{Al}^{1}$, Izatullayev EA ${ }^{2}$, Kamytbekova $\mathrm{KZ}^{2}$, Maulenova $\mathrm{AV}^{2}$, Nikolaeva $\mathrm{OV}^{2}$, Zhussupova $\mathrm{GE}^{1}$

Institute 1 Al-Farabi Kazakh National University, Almaty, Kazakhstan;

2 Kazakh Scientific Research Institute of Cardiology and Internal Diseases,

Almaty, Kazakhstan

DOI 10.1055/s-0037-1608593

The syndrome of the functional not ulcer dyspepsia (FNUD) can be met independently as well as in association with a number of widespread diseases of the digestive system. Main reasons for its emergence are the violations of the motor function of the stomach and duodenum, and also a hypersensibility of stomach wall receptors to stretching. Abundance of complaints, linked to stomach pathology, is very high and occurs in industrialized countries at 30 $40 \%$ rate of the population, and a half of all cases are the share of non ulcer dyspepsia.

Clinical trials of domestic herbal syrup "Limonidin" on a syndrome of FNUD are conducted on the basis of scientific and clinical diagnostic center of SRICID on 30 patients ( $63.3 \%$ of which are women), middle age of $41.55 \pm 11.82$. Patients were included in a research only from their informed consent. Observa- tion was conducted for 21 days. At all patients included in a research the endoscopic study of proximal departments of digestive tract and ultrasound examination of abdominal organs was conducted. In the presence of organic pathology patients were excluded from a research. From the results of the experiment it might be concluded that the studied herbal medicine possesses along with antiinflammatory and antiseptic properties spasmolytic influence on a smooth emasculation of a stomach. Physiological activity of syrup might be defined by presence of epigallocatechin-3,3,5,7,3',4',6'-hexahydroxyflavan, (-)- epigallocatechin-( $4 \beta \rightarrow 8)-2 R, 3 R(-)$-epigallocatechin-3, (-)-epigallocatechin$(4 \beta \rightarrow 8)-3,5,7,3^{\prime}, 4^{\prime}, 6^{\prime}$-hexahydroxyflavan, myricetin and a number of its glycosides.

\section{Tu-Poster Session 2-PO-132 The antioxidative intervention in colon cancer initiation by Detarium microcarpum and its fractions}

Authors Akinsanmi $A^{1}$, Johnson $T^{2}$, Aguiyi j $]^{1,3}$

Institute 1 African Center of Excellence in Phytomedicine Research and Development, Faculty of Pharmaceutical Sciences, University of Jos, Nigeria, Jos, Nigeria; 2 University of Jos, Faculty of Medical Sciences, Department of Biochemistry, Jos, Nigeria; 3 University of Jos, Faculty of Pharmaceutical Sciences, Department of Pharmacology, Jos, Nigeria DOI 10.1055/s-0037-1608463

Pathophysiological situations have shown that oxidative stress and inflammation are closely related. Evidences to show the simultaneous existence of chronic inflammation and oxidative stress in cardiovascular, neurodegenerative, diabetic, and chronic kidney diseases, do exist [1]. Whenever chronic inflammation takes place, pro-inflammatory molecules, are all upregulated. Thus, providing an enabling enviroment, for the exponential growth of malignant cells [2]. The uses of anti-inflammatory agents have been found to reduce the incidence and management of cancer. Three folkloric plants used in the treatment of inflammation and cancer were studied; Boswellia dalzielii, Detarium microcarpum, and Daniellia oliveri. Identification, authentication and voucher numbers were carried out at the Federal College of Forestry, Jos, Nigeria. For the selection phase, after the preliminary screening and antioxidative assays, DML had the highest inhibitory ability to prevent the formation of malondialdehyde in the liver, brain and colon; going by its low $\mathrm{IC}_{50}$. The in vitro antioxidative capacity was carried out among the Methanolic extract DML, Butanol fraction of DML (BfDML) and Ethyl acetate fraction of DML (EfDML).The result showed that EfDML had the highest antioxidative capacity, followed by DML and the least was BFDML. The EfDML was chosen and the acute toxicity was carried out as to determine the LD 50. The test was carried out following the method described by the Organisation for Economic Cooperation and Development, OECD [3]. From the clinical observations, there was no sign of morbidity, but the results from the haematological, serum Biochemistry and histopathological studies, indicated the $\mathrm{LD}_{50}$ for EfDML extract to be $\leq 1000 \mathrm{mg} / \mathrm{kg}$.

[1] Gülçin I, Bursal E, Sehitoğlu MH, Bilsel M, Gören AC. Food Chem Toxicol 2010; 48: 2227 - 2238

[2] Hofseth LJ, Ying L. Biochim Biophys Acta. 2006; 1765:74 - 84.

[3] OECD (2001) OECD guideline for the testing of Chemicals, Acute Oral Toxicity- Acute Toxic Class Method [Guideline 423 adopted 17th December 2001]. 


\section{Tu-Poster Session 2-PO-133 Potential of acetone leaf extract and fractions of Psychotria capensis (Rubiaceae) to manage co-morbidities of tuberculosis and helminthiasis}

\begin{abstract}
Authors Famuyide $\mathrm{I}^{1}$, Aro $\mathrm{A}^{1}$, McGaw L ${ }^{1}$, Eloff $\mathrm{J}^{1}$
Institute 1 Phytomedicine Programme, Department of Paraclinical Sciences,

Faculty of Veterinary Science, University of Pretoria, Private Bag X04,

Onderstepoort 0110, Pretoria, South Africa, Pretoria, South Africa

DOI 10.1055/s-0037-1608464

Co-infection of tuberculosis (TB) and helminths (worms) is an emerging global public health concern with both affecting about one-third of the global population. Studies shows that worm infected individuals have a high risk of contracting tuberculosis and failure of therapy or vaccination against TB during co- or pre-infection with helminths has an immunological basis. Plants are potential sources of antimycobacterial and anthelminthic compounds. In this study the antimycobacterial and anthelminthic activity as well as cellular toxicity of a crude acetone extract and fractions of Psychotria capensis were determined. The antimycobacterial activity of the extract and fractions were determined against three non-tuberculous mycobacteria ( $M$. smegmatis, $M$. fortuitum, M. aurum) and $M$. bovis BCG using a two-fold serial microdilution assay. The Egg Hatch Assay (EHA) was used to determine anthelminthic activity on eggs of Haemonchus contortus. Cytotoxicity was determined using a tetrazolium-based colorimetric assay against Vero kidney cells.

The minimum inhibitory concentration (MIC) values of the crude extract and fractions ranged from $0.039 \mathrm{mg} / \mathrm{mL}$ to $1.25 \mathrm{mg} / \mathrm{mL}$ with the crude having the best $(0.039 \mathrm{mg} / \mathrm{mL})$ compared to Isoniazid acid $(>100 \mu \mathrm{g} / \mathrm{ml})$.

In the EHA, the hexane fraction had the best inhibitory concentration (IC 50) of $0.16 \mathrm{mg} / \mathrm{mL}$, while the crude extract, $\mathrm{n}$-butanol and chloroform fractions had IC 50 of $0.23,0.63$ and $0.63 \mathrm{mg} / \mathrm{mL}$ respectively compared to $0.02 \mu \mathrm{g} / \mathrm{ml}$ of albendazole.

The hexane and chloroform fractions had low toxicity with LC50 values of 0.290 and $0.248 \mathrm{mg} / \mathrm{mL}$ respectively. The crude extract $(0.064 \mathrm{mg} / \mathrm{mL})$ and $\mathrm{n}$ butanol fraction $(0.071 \mathrm{mg} / \mathrm{mL})$ were moderately toxic. The SI values (LC50/ MIC) ranged from 0.1 to 6.3. The hexane fraction had the highest SI.

The hexane fraction had the best dual activity and was the least cytotoxic. Compounds responsible for activity may be present in this fraction. Further work is in progress to isolate compound(s) and determine their anti-TB and anthelminthic activities.
\end{abstract}

\section{Tu-Poster Session 2-PO-134 In vitro inhibition of acetylcholinesterase by Brazilian plant extracts from Rubiaceae family}

Authors Vicentini $\mathrm{A}^{1}$, Alencar $\mathrm{T}^{1}$, Leite $\mathrm{F}^{1}$, Carrijo $\mathrm{T}^{1}$, Severi $]^{1}$

Institute 1 Federal University of Espírito Santo -CCENS, Alegre, Brazil DOI 10.1055/s-0037-1608465

The neurotransmitter acetylcholine (ACh) plays a central role in the regulation of physiological functions related to the Central and Peripheral Nervous System. The inhibition of acetylcholinesterase (AChE), the major enzyme involved in the breakdown of $\mathrm{ACh}$, is recognized as one of the most promising strategy for the treatment of human and veterinary cholinergic system-related disorders, such as Alzheimer, Myasthenia gravis, Botulism, Tick paralysis, and others. Despite the relevance, there is a lack of AChE inhibitors approved for clinical use, the current drugs have limitations of efficacy, short half-lives and/ or unfavorable side effects. Brazilian flora represents an outstanding source for the discovery of bioactive molecules, which prompted us to screen plants belonging to the Rubiaceae family for their AChE inhibitory activity (AChEI). Twenty-eight species collected in a Atlantic forest fragment in the southeastern Brazil were dehydrated $\left(45^{\circ} \mathrm{C}\right)$, milled $(0.05 \mathrm{~mm}$ particle size) and extracted by maceration (methanol, $72 \mathrm{~h}$ ). Extract solutions were concentrated to dryness and properly subjected to qualitative [1] and quantitative [2] AChEI assays. Based on this approach, 17 species showed some AChEl activity, while
2 samples were considered as potent AChE inhibitors [3]. Phytochemical characterization is still in progress and suggests the occurrence of alkaloids as the main bioactive compounds in such plant extracts from Rubiaceae family. These findings reinforce the high potential for the discovery of new bioactive compounds useful for some cholinesterase disorders.

[1] A Marston, J Kissling, K Hostettmann. Phytochem An 2002; 13: 51 - 54

[2] IK Rhee, MV Meent, K Ingkaninan, R Verpoorte, M Okada, M Marimo Heterocycles 1997; 45: $2253-2260$

[3] B Vinutha, D Prashanth, K Salma, SL Sreeja, D Pratiti, R Padmaja, S Radhika, A Amit, K Venkateshwarlu, M Deepak J Ethnopharmacol 2007; 109: 359- 363

Tu-Poster Session 2-PO-135 The safety and efficacy of Chinese formula Salviae Miltiorrhizae and Ligustrazine hydrochloride solution injection in the acute ischemic stroke patients

Authors Yan $\mathrm{C}^{1}$, Zhang $\mathrm{R}^{1}$, Zuo $\mathrm{W}^{1}$, Wang $\mathrm{R}^{1,2}$, Ling $\mathrm{Y}^{3}$, Kobayashi $\mathrm{Y}^{4}$ Institute 1 Guizhou Baite Pharmaceutical Corporation, Guizhou, China; 2 Zhejiang CONBA Pharmaceutical \& Drug Research Development

Corporation, Hangzhou, China; 3 Medical Corporation Soujikai, Osaka, Japan; 4 Shimane University, Izumo, Japan

DOI 10.1055/s-0037-1608466

Danshen ligustrazine formula (DL; salviae miltiorrhizae and ligustrazine hydrochloride solution) has been used in China for the treatment of acute ischemic stroke patients. In the present study, the safety and efficacy of DL injection for the treatment of patients with acute ischemic stroke were evaluated.

129 acute ischemic stroke patients and their National Institute of Health stroke scale (NIHSS) scores were less than 15 were randomly divided into trial group $(n=65)$ and the standard control group $(n=64)$. DL was applied by intravenous infusion, $10 \mathrm{ml} /$ time, 1 times/day, with $5 \%$ glucose saline. As the standard control, citicoline sodium injection was applied intravenously, 0 . $25 \mathrm{~g} /$ day, diluted with $5 \%$ glucose saline; Treatment was continued for 7 to 10 days and efficacy was evaluated at 7 days index score were scored. Symptomatic intracranial hemorrhage, severe systemic bleeding, or other serious adverse events associated with the drug application and death due to any cause of the first 24 hours within the symptoms of intracranial hemorrhage was recorded.

Final NIHSS score for DL treatment was from $4.61+2.00$ to $1.85+2.33$ and that for citicoline was from $4.71+2.32$ to $1.84+1.77$. There was no significant difference between the two groups in NIHSS score, Barthel index and Rankin score $(P>0.05)$. No obvious side effect was observed by DL treatment.

Effects on scores of acute ischemic stroke patients treated with DL was not significantly different from those treated. Efficacy indicators as the modified Rankin score (mRS), NIHSS, Barthel with citicoline. Safe, convenient and well tolerated in the treatment of patients with acute ischemic stroke by DL was evident.

\section{Tu-Poster Session 2-PO-136 Effects of Sappan wood and Ginger as antithrombotic and their interaction with aspirin}

Authors Saputri Fadlina $C^{1}$, Nabila $N^{1}$, Purna Sari $S^{2}$, Mun'im $A^{3}$ Institute 1 Laboratory of Pharmacology, Faculty of Pharmacy, Universitas Indonesia, Kampus UI Depok, 16424, Indonesia, Depok, Indonesia;

2 Laboratory of Clinical Pharmacy, Faculty of Pharmacy, Universitas Indonesia, Kampus UI Depok, 16424, Indonesia, Depok, Indonesia; 3 Laboratory of Phytochemistry, Faculty of Pharmacy, Universitas Indonesia, Kampus UI Depok, 16424, Indonesia, Depok, Indonesia

DOI 10.1055/s-0037-1608467

Thrombosis have an important role in cardiovascular [1]. Our recent study demonstrates the potential effect of sappan wood and ginger to inhibit platelet function for the prevention of thrombosis [2]. This study aims to investigate the efficacy of the mixture of sappan wood and ginger, and their inter- 
action with Aspirin on the thrombosis model mice. Mice were divided into two experimental models. The acute pulmonary thromboembolism model was obtained by an intravenous injection of collagen and epinephrine. The efficacy and interaction was analysed by measuring bleeding time and survival rate. The mixture of sappan wood and ginger significantly prolonged the mouse tail bleeding time of mice but did not raise bleeding risk when combined with aspirin. They prevented death caused by pulmonary thrombosis and showed potentiate to enhance the antithrombotic effect of Aspirin. This study suggest that the mixture of sappan wood and ginger may be one of the promising herb for preventing thrombosis and have potentiate the antithrombotic effect of aspirin without raising bleeding risk.

Ministry of Research, Technology and Higher Education of Indonesia for financial support (Research Grand 2017)

[1] Kang WS, Lim IH, Yuk DY, Chung KH, Park JB, Yoo HS, Yun YP. Thrombosis Res 1999; 96: $229-237$.

[2] Saputri FC, Nabila N, Mun'im A. J Young Pharm 2017; 9: 46-48.

\section{Tu-Poster Session 2-PO-137 Cytotoxic and anti-inflammatory effects of Eryngium creticum Lam. growing in Izmir, Turkey}

Authors Toktas $\mathrm{U}^{1}$, Nalbantsoy $\mathrm{A}^{2}$, Durmuskahya $\mathrm{C}^{3}$, Kayalar $\mathrm{H}^{1}$ Institute 1 Ege University, Faculty of Pharmacy, Dept. of Pharmacognosy, Bornova, Izmir, Turkey; 2 Ege University, Faculty of Engineering, Department of Bioengineering, Bornova, Izmir, Turkey; 3 Izmir Katip Celebi University, Fac. of Forestry, Dept. of Forest Engineering, Cigli, Izmir, Turkey

DOI 10.1055/s-0037-1608468

The genus Eryngium L. from the Apiaceae (Umbelliferae) family has a distribution with about 250 species all over the world. This genus is the richest genus of the Apiaceae family with 34 sections and numerous subsections $[1,2,3]$. $E$. creticum has a broad spectrum of biological and pharmacological effects attributed to different parts and extracts, including anti-snake and anti-scorpion venom, antibacterial and antifungal and antileishmanial effects. It also shows antihyperglycemic, hypoglycemic and antioxidant activities [1].

In this study, in vitro cytotoxic and inducible nitric oxide synthase (iNOS) inhibitory activity of the hexane, chloroform and methanol extracts obtained from aerial parts of $E$. creticum were investigated at 0,$5 ; 5$ and $50 \mu \mathrm{g} / \mathrm{ml}$ concentrations.

The cell lines A549, CaCo2, HEK293, HeLa and MCF7 were used for cytotoxic activity assays. Hexane extract against HeLa cell line with $\mathrm{IC}_{50} \quad 4,6185$ $\pm 0.12 \mu \mathrm{g} / \mathrm{ml}$ and chloroform extract with $\mathrm{IC}_{50} 15.95 \pm 4.36 \mu \mathrm{g} / \mathrm{ml}$ against HEK293 cell line had the highest cytotoxic activity.

The anti-inflammatory activity of the extracts was assessed by evaluating the ability of the extracts to inhibit RAW 264.7 macrophage cell line from generating harmful nitric oxide (iNOS activity). All three extracts showed antiinflammatory activity. Hexane and chloroform extracts inhibited NO production with $\mathrm{IC}_{50}$ values of $0.2247 \pm 0.14 \mu \mathrm{g} / \mathrm{ml}$ and $0.2915 \pm 0.16 \mu \mathrm{g} / \mathrm{ml}$ respectively.

[1] Kikowska M, Dworacka M, Kedziora I, Thiem B. Revista Brasileira de Farmacognosia. 2016; 26:3: 392 - 399

[2] Wörz A. Turk. J. Bot. 2004; 28: 85 - 92.

[3] Pimenov MG, Leonov MV. The genera of the Umbelliferae. Kew: Royal Botanic Gardens, 1993

\section{Tu-Poster Session 2-PO-138 Vernonia condensata: an active medicinal plant against Staphylococcus aureus strains}

Authors Silva J', Bessa $\mathrm{M}^{1}$, Tomasco $\mathrm{V}^{1}$, Scio $\mathrm{E}^{1}$, Alves $\mathrm{MS}^{2}$

Institute 1 Bioactive Natural Products Laboratory, Department of

Biochemistry, Federal University of Juiz deFora, Rua José Lourenço Kelmer s/n, Campus Universitário, Bairro São Pedro, CEP 36036 - 900, Juiz de Fora, Brazil; 2 Cellular and Molecular Bioactivity Laboratory, Department of
Pharmaceutical Sciences, Faculty of Pharmacy, Federal University of Juiz de Fora, Rua José Lourenço Kelmer s/n, Campus Universitário, Bairro São Pedro, CEP 36036 - 900, Juiz de Fora, Brazil

DOI 10.1055/s-0037-1608594

Vernonia condensata Baker (Asteraceae Bercht. \& Presl) is traditionally used to relief of several diseases, including those related to infectious disorders and caused by Staphylococcus aureus strains $1-4$. So, this study focused on the investigation of its antistaphylococcal activity. The leaves were extracted with ethanol and then submitted to a liquid-liquid partitioning process with solvents in increasing order of polarity. For this study, the ethyl acetate partition (PA) was used. The antibacterial activity was verified by the Minimal Inhibitory Concentration (MIC) and Minimal Bactericidal Concentration (MBC)5 against five S. aureus ATCC ${ }^{\circledR}$ strains $(6538,25923,29213,33591$, and 33592) and five methicillin-resistant S. aureus (MRSA) clinical strains isolated from blood infections (1485279, 1605677, 1664534, 1688441, and 1830466). PA revealed antibacterial activity against all $S$. aureus strains tested, being more active against the ATCC $^{\circledR}$ strains, with MIC value of $625 \mu \mathrm{g} / \mathrm{mL}$. Considering the MRSA clinical strains, the MIC values were $1250 \mu \mathrm{g} / \mathrm{mL}$ (MRSA 1485279), $2500 \mu \mathrm{g} / \mathrm{mL}$ (MRSA 1688441 and MRSA 1830466), and $>5000 \mu \mathrm{g} / \mathrm{mL}$ (MRSA 1605677 and 1664534). Ampicillin and chloramphenicol were used as standard antibiotics. MBC was not found within the gradient concentration tested $(5000 \mu \mathrm{g} / \mathrm{mL}$ to $7.8 \mu \mathrm{g} / \mathrm{mL})$. Additionally, PA was considered bacteriostatic for all strains. In conclusion, it was possible to corroborate the potential of $V$. condensata as an antistaphylococcal agent, with bacteriostatic effect.

This work was supported by the grants from FAPEMIG, CAPES and CNPq.

[1] Frutuoso, V.S. et al Planta Medica, 60, 21 - 25, 1994.

[2] Risso, W. E. et al. Indian Journal of Experimental Biology, 48, 811 -816, 2010.

[3] Silva, J. B. et al. Journal of Molecular Sciences, 12, 8993-9008, 2011.

[4] Valverde, A. L. Phytotherapy Research, 15, 263 - 264, 2001.

[5] Clinical and Laboratory Standards Institute. CLSI Document M100-S21, 2012.

\section{Tu-Poster Session 2-PO-139 Anti-inflammatory effects of Lagerstroemia indica and protection effects on on reflux-esophagitis in rats}

Authors Nam Hyeon $\mathrm{H}^{1}$, Choo Byung $\mathrm{K}^{1}$

Institute 1 Chonbuk National University, Jeonju-si, Jeollabuk-do, Korea, Republic of (South)

DOI 10.1055/s-0037-1608469

Lagerstroemia indica be called crape myrtle, is a species of the family Lythraceae from China, Korea and Japan. Currently, crape myrtle varieties can fill every landscape need, from tidy street trees to dense barrier hedges to fastgrowing dwarf types[1]. The esophageal mucosal injury is complex, it may involve the reflux of gastric acid and related to inflammation[2]. In the study, we performed to determine the anti-inflammatory effects in LPS-induced RAW 264.7 cells and protection effects on reflux-esophagitis in rats from Lagerstroemia indica fruits. The anti-inflammatory effect of Lagerstroemia indica extracts was measured by NO production inhibitory activity and the expression of pro-inflammatory protein such as iNOS, COX-2 and NF-kB on lipopolysaccharide (LPS)-induced Raw 264.7 cells. The NO production and iNOS, COX-2 and NF-kB expression were increased by LPS were inhibited by the Lagerstroemia indica extracts. In the result of experimental reflux-induced esophagitis, the mucosal damage on normal rats was not apparent. The oral administration of Lagerstroemia indica extracts decrease contradistinction to the reflux-esophagitis control. These results indicated that ethanol extracts of Lagerstroemia indica fruits expected development possibility as a useful nutraceuticals through high anti-inflammatory and protection effects on refluxesophagitis.

[1] From Lagerstroemia - Wikipedia

[2] Norimasa Yoshida. J. Clin. BiochemNutr 2007; 40:13-23 


\section{Tu-Poster Session 2-PO-140 Therapeutic application of wet-ground bee pollen in benign prostatic hyperplasia}

Authors Pyeon $\mathrm{HI}^{1}$, Bak J', Seok JI ${ }^{1}$, Choi YS ${ }^{1}$

Institute 1 Catholic University Of Daegu, Gyeongsangbukdo, Korea, Republic of (South)

DOI 10.1055/s-0037-1608470

Bee pollen is produced by honeybees and is considered one of the most balanced and nourishing nutritional supplements available. Historically, bee pollen has been prescribed for its healing properties and consumed for its high energy supply. Recently, accumulating evidence suggest that bee pollen has diverse biological activities, such as antioxidant, anti-inflammatory, antibacterial and even anticancer effects. However, the outer membrane of the pollen grain, exine, is highly resistant to most acidic solutions, high pressure, and even digestive enzymes, and the resulting low bioavailability limits its nutritional and clinical applications. To overcome this limitation, we employed a wet grinding method to effectively destroy the exine then, examined the pollen's biological activity in benign prostatic hyperplasia. First, microscopic observations provided strong evidence that wet grinding destroyed the exine time-dependently. In addition, the content of polyphenols, well-known ingredients of bee pollen and used as internal standards for the quality control of commercial pollen preparations, increased up to 11-fold with wet grinding. Further, the antioxidant activity demonstrated on the ABTS antioxidant assay as well as the DPPH radical scavenging assay was also dramatically increased. The potential biological effect of wet-ground bee pollen was examined in animal model of benign prostatic hyperplasia. For this purpose we compared the effect of wet-ground bee pollen with dutasteride, a $5 \alpha$ reductase inhibitor. In this study, we found that the reduced size of prostate in wet-ground bee pollen-treated animals was comparable to that in dutasteride-administered animals. Finally, we found that bee pollen has immune-modulating effect in animal model by influencing differentiation of $\mathrm{T}$ cells as well as B cells. Taken together, the results presented here support a new therapeutic potential of wet-ground bee pollen in benign prostatic hyperplasia.

\section{Tu-Poster Session 2-PO-141 Anti-inflammatory effects of Viola yedoensis and the application of cell extraction for investigating bioactive constituents in RAW 264.7 cells}

\author{
Authors Jeong Yun $\mathrm{H}^{1}$, Oh $\mathrm{YC}^{1}$, Cho WK $\mathrm{WK}^{1}$, Ma Jin $\mathrm{Y}^{1}$ \\ Institute 1 Korea Institute of Oriental Medicine, Daegu, Korea, Republic of \\ (South) \\ DOI 10.1055/s-0037-1608471
}

Viola yedoensis (VY, Violaceae) is a popular medicinal herb used in traditional East-Asian medicine for the treatment of inflammation-related diseases including swelling, sores, boils, furuncles, carbuncles, snakebites, and acute and chronic hepatitis [1]. However, the anti-inflammatory activities and its inhibitory mechanisms of VY have not been demonstrated. In this study, we investigated the anti-inflammatory effects of VY ethanol extract (VYE) on macrophages and attempted to identify the bioactive components of VYE. We assessed the effects of VYE on secretion of nitric oxide (NO) and inflammatory cytokines including tumor necrosis factor (TNF)- $\alpha$, interleukin (IL)- 6 , and IL-1 $\beta$. In addition, we explored the expression of inducible nitric oxide synthase (iNOS), cyclooxygenase (COX)-2, and changes in heme oxygenase (HO)-1, nuclear factor (NF)-KB, and mitogen-activated protein kinase (MAPK) signaling pathways in lipopolysaccharide (LPS)-stimulated murine macrophages. In addition, a rapid and useful approach to identify potential bioactive components in VYE with anti-inflammatory effects was developed using murine macrophage cell extraction coupled with high-performance liquid chromatography tandem mass spectrometry (LC-MS). We found that VYE exerted anti-inflammatory activity by inhibiting the production of key inflammation mediators and related products, as well as suppression of $\mathrm{HO}-1, \mathrm{NF}-\mathrm{kB}$, and
MAPK signaling pathway activation in RAW 264.7 cells. In addition, we identified two compounds in VYE via the cell extraction. Our results revealed that VYE exerts anti-inflammatory activities and its detailed inhibitory mechanism in LPS-stimulated macrophages. Furthermore, we identified bioactive components of VYE.

[1] Editorial Committee of Chinese Pharmacopoeia. Pharmacopoeia of the People's Republic of China, 2010 edition. Beijing: China Medical Science and Technology Press; 2010: 317

\section{Tu-Poster Session 2-PO-143 Gastroprotective effects of HT074, combined extracts of Inula britannica and Paeonia lactiflora on experimental gastric ulcer models in Sprague-Dawley rats}

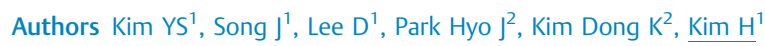
Institute 1 Department of Herbal Pharmacology, College of Korean Medicine, Kyung Hee University, Seoul, Korea, Republic of (South); 2 Korea Institute of Science and Technology for Eastern Medicine (KISTEM), NeuMed Inc, Seoul, Korea, Republic of (South)

DOI 10.1055/s-0037-1608472

The herbal formula HT074 is combined extracts of Inula britannica flower and Paeonia lactiflora root. In traditional Korean medicine, the flower of I. britannica have been prescribed for the treatment of digestive disorders and inflammation. The root of $P$. lactiflora has been used for enhancing blood circulation and inhibiting inflammation. This study aimed to investigate the gastroprotective effects of HT074 in three different acute gastric ulcer rat models and to elucidate the potential protective mechanism.

Gastric lesions were induced in seven-week-old Sprague-Dawley rats by acidified ethanol $(\mathrm{HCl} / \mathrm{EtOH})$, indomethacin, and water-immersion and restraintstress. In each experiment, rats were randomly divided into 5 groups: vehicle control, omeprazole $20 \mathrm{mg} / \mathrm{kg}$ (positive control), HT074 30, 100, and $300 \mathrm{mg} /$ $\mathrm{kg}$. The gastric lesion area was measured by imageJ (National Institutes of Health, Bethesda, MD) program. Adhered gastric mucus was estimated by alcian blue binding assay.

We found that HT074 conferred profound gastric protection in three different models. In HCl/EtOH-induced gastric ulcer model, HT074 300 mg/kg administered orally at 30 minutes prior to inducer significantly reduced the gastric lesion by $99.0 \%$ compared to control. In indomethacin-induced gastric ulcer model, HT074 $300 \mathrm{mg} / \mathrm{kg}$ administered orally at 30 minutes prior to inducer significantly reduced the gastric lesion by $78.5 \%$ compared to control. In the water-immersion and restraint-stress-induced gastric ulcer model, HT074 $300 \mathrm{mg} / \mathrm{kg}$ administered orally at 1 hour prior to water-immersion significantly reduced the gastric lesion by $81.5 \%$ compared to control. Furthermore, the treatment with $\mathrm{HTO} 074$ increased gastric mucus dose-dependently in $\mathrm{HCl}$ / $\mathrm{EtOH}$ induced gastric ulcer model.

In conclusion, these results suggest that HT074 inhibits the gastric lesion development by stimulating mucus secretion. HT074 could be helpful for preventing gastritis and gastric ulcer.

\section{Tu-Poster Session 2-PO-144 Phytochemical profile, antioxidant activity and cytotoxicity of different extracts of Thymus vulgaris ssp. fragantissimus}

Authors Félix $L^{1,2}$, Teixeira $I^{3}$, Taghouti $\mathrm{M}^{1,4}$, Nunes $\mathrm{FM}^{4}$, Silva $\mathrm{AM}^{1,3}$ Institute 1 1-Centre for the Research and Technology of Agro-Environmental and Biological Sciences (CITAB), University of Trás-os-Montes and Alto Douro (UTAD), Vila Real, Portugal; 2 2- Laboratory Animal Science (LAS), Institute for Research and Innovation in Health (i3S), University of Porto (UP), Porto, Portugal; 3 3- Department of Biology and Environment, UTAD, Vila Real, 
Portugal; 4 4- Chemistry Center (CQ-VR), Chemistry Department, UTAD, Vila Real, Portugal

DOI 10.1055/s-0037-1608473

In the past years, the interest in natural products has increased involving the investigation of medicinal plants for biological activities and therapeutic potentials. Plants from genus Thymus (Lamiaceae family) in which most species are native to western Mediterranean area, have been used from ancient times and continues to be used in food, pharmaceutical and cosmetic industries. Among Thymus species, Thymus vulgaris ssp. fragantissimus, a citrusy orange peel scent and flavor, remains one of the least studied, with very little information related to its antioxidant and cytotoxic activity. In this context, respective phytochemical profile, antioxidant and cytotoxic activities of hydroethanolic (HE) and aqueous extracts (decoction (D) and infusion (I)) from T. vulgaris ssp. fragantissimus were investigated. Total phenolics, flavonoids, orthodiphenols, tannins, hydroxycinnamic acids, flavones and flavonols were assessed using spectrophotometric methods. Antioxidant activities of both extracts were determined using various in vitro methods against DPPH, ABTS ${ }^{+}$, hydroxyl radicals, nitric oxide, superoxide and hydrogen peroxide. Finally, cell growth activity of extracts was evaluated in vitro using two cell lines (HepG2, human hepatocellular carcinoma, and RAW264.7 murine macrophage). A similar phytochemical profile was observed between $\mathrm{HE}$ and aqueous extracts with slight variations. Results also showed similar activity $\left(\mathrm{IC}_{50}\right)$ in DPPH and ABTS assay. A significant reduction of superoxide scavenging activity was observed for the aqueous extracts compared to HE extract. Tested extracts showed significant anti-proliferative activity against HepG2 and RAW264.7 cells after 24 and $48 \mathrm{~h}$ exposure. Overall, a ranking for anti-proliferative activity was established for the representative extracts tested ( $D>\mid>H E)$. These results validate this plant as a promising source of bioactive compounds with potential applications in several industrial sectors and warrant further investigation.

INTERACT-project-NORTE-01 - 0145-FEDER-000017 (ISAC), co-financed by ERDF through NORTE-2020. And grants (BI/UTAD/INTERACT/ISAC/203/ 2016, to L.F.), (PD/BD/52563/2014, to M.T.).

\section{Tu-Poster Session 2-PO-145 Chemical composition of Crotalaria spectabilis seeds extracts and their action on different soybean nematodes}

Authors Zeraik $\mathrm{ML}^{1}$, Camargo $\mathrm{GL}^{2}$, Miyasaki AKP² , Scarminio IS ${ }^{1}$, HoffmannCampo $\mathrm{CB}^{2}$, Nunes $\mathrm{EO}^{2}$

Institute 1 Departament of Chemistry, State University of Londrina (UEL), 86051 - 990, Londrina, Brazil; 2 Brazilian Agricultural Research Corporation Embrapa Soybean, Londrina, Brazil

DOI 10.1055/s-0037-1608474

The quality of soybean production can be affected by plant-parasitic nematodes, such as Heterodera glycines and Meloidogine javanica, the main nematodes that are harmful to soybean [1]. Crotalarias spectabilis is used in agriculture as crop rotation system due to its antagonist effect on entomopathogenic nematodes [2]. Thus, the purpose of this study was to investigate the chemical composition of $C$. spectabilis seed extracts and their action on two different soybean nematodes. After alkaline extraction of the seeds $\left(\mathrm{NaHCO}_{3}\right.$ $5 \% \mathrm{~m} / \mathrm{v}$ ), the $\mathrm{pH}$ of the extract was adjusted to $7.1 \pm 0.2$. The suppression nematode assay consisted in exposing $H$. glycines and M. Javanica (J2) to the crude extract of $C$. spectabilis seeds, at five different concentrations (5.0 $40.0 \mathrm{mg} \mathrm{mL}^{-1}$ ). The control group was treated with distilled water. The samples were evaluated at two moments: $24 \mathrm{~h}$-exposure and after $24 \mathrm{~h}$-recovery. C. spectabilis seeds extract showed higher immobility rates for $H$. glycines (96\%) after $24 \mathrm{~h}$-exposure when compared to $M$. javanica (38\% of inactive nematodes), at $5.0 \mathrm{mg} \mathrm{mL}^{-1}$. After $24 \mathrm{~h}$-recovery the $\mathrm{LC}_{90}$ (lethal concentration) were $20 \mathrm{mg} \mathrm{mL}^{-1}$ for $\mathrm{H}$. glycines and $30 \mathrm{mg} \mathrm{mL}^{-1}$ for $M$. javanica, however the $\mathrm{LC}_{100}$ was at $40.0 \mathrm{mg} \mathrm{mL}^{-1}$ for both nematode species. The chromatographic profile of the $C$. spectabilis extracts obtained by HPLC-UV/DAD showed the presence of saponins, such as, steroids glycosides and polycyclic terpenes, which may be responsible for the nematicide effect of $C$. spectabilis seeds on $H$. glycines and $M$. javanica nematodes. Such type of studies may lead to the development of a new nematicide for the control of soybean nematodes, necessary to obtain a competitive agricultural system.

CNPq and FA (Fundação Araucaria-PR, Brazil) and Embrapa soybean.

[1] Weaver, D. B. et al. J Nematol. 1995; 27: 585 - 591.

[2] Chitwood, D. J. Rev. Phytopathol. 2002; 40: 221 - 249.

\section{Tu-Poster Session 2-PO-146 The roles of Valerenic and Acetoxy valerenic acids on antidepressant effect of Valeriana officinalis: BDNF levels in SH-SY5 cell lines}

Authors Gonulalan $\mathrm{E}^{1}$, Bayazeid $\mathrm{O}^{1}$, Yalçın $\mathrm{F}^{1}$, Demirezer $\mathrm{Ö}^{1}$

Institute 1 Hacettepe University, Faculty of Pharmacy, Dept. of

Pharmacognosy, ANKARA, Turkey

DOI 10.1055/s-0037-1608475

The roots and rhizomes of Valeriana officinalis are used as mild sedatives [1]. To date, Valerian extract and valerenic acid associations with the GABA receptor have been investigated to explain the effects of valerian on anxiety and insomnia [2]. Brain-derived neurotrophic factor (BDNF) has important roles in the central nervous system and has been involved with some psychiatric conditions such as depression [3]. In this study, effects of Valerian extracts and its components (Valerenic acid-VA and Acetoxy valerenic acid-AVA) on BDNF expression in SH-SY5Y cells were investigated. For this purpose, MeOH extract, VA-free extract and AVA-free extracts were prepared and BDNF expression of the extracts were determined. The normal $\mathrm{MeOH}$ extract increased intracellular BDNF levels by $12 \%, 71 \%(p<0.05)$, and $93 \%(p<0.001)$, where the concentrations were 10,30 and $100 \mu \mathrm{g} / \mathrm{mL}$ respectively. The VA free extract at $25 \mu \mathrm{g} / \mathrm{mL}$ decreased the level of BDNF by $1.3 \%$, while the VA itself at $25 \mu \mathrm{g} /$ $\mathrm{mL}$ increase the level of BDNF by $20 \%(p<0.0001)$. On the other hand AVA at $25 \mu \mathrm{g} / \mathrm{mL}$ only increase BDNF level by $2 \%$ while AVA-free extract appears to increase BDNF level 18\% $(p<0.0001)$. These results clearly indicate the VA's role of Valeriana extracts effect on BDNF expression in SH-SY5Y cells.

This study was supported by grants from Hacettepe University Scientific Research Projects (Project No: THD-2016-91711001) and The Scientific and Technological Research Council of Turkey (TUBITAK) (Project No: KBAG116Z414)

[1] ESCOP Monographs, $2^{\text {nd }}$. ed,Thieme, New York, NY (2003)

[2] Felgentreff F, Becker A, Meier B, Brattström A. Phytomedicine 2012; 19:1216-1222.

[3] Björkholm C, Monteggia LM. Neuropharmacology 2016; 102: 72 - 79.

\section{Tu-Poster Session 2-PO-147 Biological activity and phytochemical analysis of extracts obtained from Santolina rosmarinifolia $\mathrm{L}$.}

Authors Félix $\mathrm{L}^{1,2}$, Coutinho $\mathrm{T}^{3}$, Barejão $\mathrm{A}^{3}$, Taghouti $\mathrm{M}^{1}$, Silva $\mathrm{AM}^{1,3}$

Institute 1 Centre for the Research and Technology of Agro-Environmental and Biological Sciences (CITAB), University of Trás-os-Montes and Alto Douro (UTAD), Vila Real, Portugal; 2 Laboratory Animal Science (LAS), Institute for Research and Innovation in Health (i3S), University of Porto (UP), Porto, Portugal; 3 Department of Biology and Environment, UTAD, Vila Real, Portugal

DOI 10.1055/s-0037-1608476

Species from Asteracea family have long been used in traditional medicine being described as important sources of bioactive compounds. Within this family, Santolina spp. have been used due to evidenced medicinal properties. This genus is widely distributed in the Mediterranean area and there has been a renewed interest in screening plants from this genus for novel biologically active compounds in order to unveil future industrial applications. Santolina rosmarinifolia L, a perennial shrub with lime-yellow aromatic foliage, presents multiple medicinal properties. To address the potential of S. rosmarinifolia as a 
source of bioactive products, the phytochemical profile and biological activity of hydroethanolic and of aqueous (decoction and infusion) extracts was made. Phytochemical evaluation was performed though spectrophotometric methods (total phenolics, flavonoids, ortho-diphenols, tannins, hydroxycinnamic acids, flavones and flavonols). Antioxidant capacity was evaluated by determining DPPH and ABTS radical-scavenging activities, hydroxyl and nitric oxide radicals, and superoxide and hydrogen peroxide scavenging activity. Cytotoxicity against HepG2 and RAW264.7 cells was assessed, using alamar-blue assay. Results showed that phytochemical composition varied in aqueous extracts with infusion showing higher percentage of flavonoids and ortho-diphenols while decoction showed a lower amount of hydroxycinnamic acids. Regarding radical scavenging activities, aqueous extracts exhibited lower DPPH and ABTS radical-scavenging activity compared to hydroethanolic extract. Both cell lines were susceptible to tested extracts at 24 and $48 \mathrm{~h}$. However, lower concentrations were required to induce higher cytotoxic effects in prolonged extract exposure (48 h). In general, hydroethanolic extracts showed lower IC 50 compared to aqueous extracts (at 24 and $48 \mathrm{~h}$ exposure). Results presented here contribute to value this plant species as a possible source of bioactive phytochemicals with prospective physiological benefits, concerning to antioxidant and anti-proliferative activities.

INTERACT-project-NORTE-01-0145-FEDER-000017 (ISAC), co-financed by ERDF through NORTE-2020. And grants (BI/UTAD/INTERACT/ISAC/203/ 2016, to L.F.), (PD/BD/52563/2014, to M.T.).

\section{Tu-Poster Session 2-PO-148 Biochemical Effects of Aqueous Stem Bark Extract of Dialium guineense on Oxidative Status of Normal Wistar Rats}

Authors Onoagbe $\mathrm{I}^{1}$, Abu $\mathrm{O}^{1}$

Institute 1 Biochemistry Department, University of Benin, Nigeria, Benin City, Nigeria

DOI 10.1055/s-0037-1608477

Dialium guineense (velvet tamarind) belongs to the Leguminosae family (1). Various parts of the plant have medicinal properties and are used to treat a variety of diseases. The pathogenesis of a number of diseases is linked to free radical-induced oxidative damage.

This study was undertaken to determine the effects of aqueous stem bark extract of Dialium guineense on antioxidant enzymes (superoxide dismutase, catalase, glutathione peroxidase and G6PDH), malondialdehyde and total protein concentrations in normal Wistar rats. Ten male rats were divided into two groups of five rats each. One group served as normal control while the other group received $1000 \mathrm{mg} / \mathrm{kg}$ body weight of aqueous extract. The experiment lasted twenty-eight days and assays were performed on weekly basis. Results of phytochemical analysis of the plant revealed the presence of tannins (3.76 $\pm 0.31 \%$ ), alkaloids ( $1.22 \pm 0.06 \%)$, phenols $(1.40 \pm 0.03 \%)$, flavonoids $(0.92$ $\pm 0.03 \%)$, saponins $(0.18 \pm 0.02 \%)$, glycosides $(0.04 \pm 0.01 \%)$ and steroids $(0.04 \pm 0.01 \%)$. There was no significant change $(p>0.05)$ in the concentrations of total proteins compared with control. The malondialdehyde concentrations were significantly reduced $(p<0.05)$ compared with control while $\mathrm{G} 6 \mathrm{PDH}$, superoxide dismutase, catalase and glutathione peroxidase activities were significantly increased $(p<0.05)$ compared with control. The extract seems to potentiate the activities of all the measured anti-oxidant enzymes. Measurement of G6PDH activity provides an indirect assessment of glutathione reductase activity as the reaction catalysed by it in the pentose phosphate pathway in the erythrocyte membrane and in cells of other sensitive tissues generates the coenzyme NADPH which furnishes the hydride ion (or hydrogen) needed to keep or maintain glutathione in the reduced state where it is active as a free radical scavenger.

Arogba, S.S, Ajiboro, A. and Odukwe, I. Journal of the Science of Food and Agriculture 2006; 66:533-534.

\section{Tu-Poster Session 2-PO-149 Antibacterial Activity of Anthemis tricolor Boiss. from Cyprus}

Authors Gulsoy Toplan $\mathrm{G}^{1}$, Tuysuz $\mathrm{M}^{2}$, Mat $\mathrm{A}^{1}$, Sariyar $\mathrm{G}^{3}$ Institute 1 Department of Pharmacognosy, Faculty of Pharmacy, Istanbul University, Istanbul, Turkey, ISTANBUL, Turkey; 2 Department of Microbiology, Faculty of Pharmacy, Istanbul University, Istanbul, Turkey, ISTANBUL, Turkey; 3 Faculty of Pharmacy, Cyprus International University, Lefkosa, Turkish Republic of Northern Cyprus, Lefkosa, Cyprus

DOI 10.1055/s-0037-1608478

The genus Anthemis (Asteraceae) is widely distributed in Europe, the Mediterranean region and Western Asia, with approximately 195 species [1]. Anthemis species contain essential oil, flavonoids, polyphenolic acids, terpene and sesquiterpene lactones [1,2]. Many of them have been used for centuries as a folk medicine for the treatment of various diseases [3]. There are many studies on the biological activities of some species of the genus especially antibacterial, antifungal, antispasmodic, anti-inflammatory and cytotoxic activities [1,2]. In present work, chloroform and methanol extracts obtained by Soxhlet apparatus the aerial parts of Anthemis tricolor Boiss, an endemic species collected from Turkish Republic of Northern Cyprus, were investigated for their antibacterial activity against Staphylococcus aureus, Staphylococcus epidermidis, Enterococcus faecalis, Escherichia coli, Klebsiella pneumoniae, Pseudomonas aeruginosa, Proteus mirabilis and Candida albicans using microbroth dilution method. According to the results, the extracts of Anthemis tricolor Boiss. showed moderate to weak inhibitory effects. The minimum inhibitory concentration (MIC) values were also ranged from $78 \mu \mathrm{g} / \mathrm{mL}$ to $1250 \mu \mathrm{g} / \mathrm{mL}$ for all the tested microorganisms. The strongest inhibitory effect was detected in chloroform extract against Enterococcus faecalis with a MIC value of $78 \mathrm{mg} / \mathrm{mL}$.

[1] Jassbi AZ, Firuzi O, Miri R, Salhei S, Zare S, Zare M, Masroorbabanari M, Chandran JN, Schneider B, Baldwin IT. Pharm Biol, 2016; 54: 2044 - 2049.

[2] Uzel A, Guvensen A, Çetin A. J Ethnopharmacol, 2004; 95: 151 - 154.

[3] Baytop T. Therapy with Medicinal Plants in Turkey, Past and Present, 2nd ed, Istanbul, Nobel Medical Publishing; 1999.

\section{Tu-Poster Session 2-PO-150 Geopropolis from Melipona quadrifasciata anthidioides Lepeletier: a source of antioxidant natural products of floral origins}

Authors Brandão Torres Luce $\mathrm{M}^{1}$, Uehara Daniella $\mathrm{E}^{1}$, Galdino $\mathrm{J}^{1}$, Oliveira Fidalgo $\mathrm{A}^{2}$, Fernandes Pinto da Luz $\mathrm{C}^{3}$

Institute 1 Núcleo de Pesquisa em Fisiologia e Bloquímica - Instituto de Botânica, SP, São Paulo, Brazil; 2 Núcleo de Pesquisa em Sementes - Instituto de Botânica, SP, São Paulo, Brazil; 3 Núcleo de Pesquisa em Palinologia Instituto de Botânica, SP, São Paulo, Brazil

DOI 10.1055/s-0037-1608479

Melipona quadrifasciata anthidioides Lepeletier is a stingless bee found in MogiGuaçu, São Paulo, Brazil that produces honey, geopropolis and accumulates pollen. The geopropolis is a mixture of floral origin pollens, waxes, plant resins, soil and salivary secretions ${ }^{1}$. The plant resins from geopropolis collected by Melipona quadrifasciata have several origins, and geopropolis of other regions have been reported to possess a great variety of biological activity ${ }^{2}$. The geopropolis samples (05) were collected for study in the year of 2015 at $13 / 01,09 / 03,15 / 04,10 / 05$ and $10 / 06$. The aim of this work was to study the chemical composition of hydroalcholic (70\%) and hydrocetonic extracts (70\%), to evaluate the antioxidant activity and quality of accumulated dominant pollens ( $>45 \%$ ) of geopropolis, a source of natural products. The results showed potential antioxidant activity when evaluated by DPPH (2, 2-diphenil1-picril hidrazil). The phytochemical screening showed that geopropolis is a mixture of phenolic compounds when derivated by $\mathrm{FeCl}_{3}$ and NP/PEG reagents, but the gallic acid is not the main natural product (HPLC-UV and gallic acid standard). This study showed that the dominant pollens of the geopropolis from Melipona quadrifasciata anthidioides were obtained from of genera 
Vernonia (Asteraceae collected at 10/06/2015), Pinus (Pinaceae, collected at $09 / 03 / 2015$ ) Eucaliptus (Myrtaceae at 13/01, 15/04 and 10/05/2015), and from Melastomataceae, Combretaceae and Malpiguiaceae families. These data suggest that the natural products present in the hydroalcoholic and hydrocetonic extracts are of floral origins of the geopropolis samples collected from the species belonging to the genera and families of plants that were visited during the collection periods.

[1] Dutra RP, Nogueira AMC, Marques RRO, Costa MCP, Ribeiro MNS. 2008 Rev. Bras. Farmacogn.18:557 - 562.

[2] Cunha, M.G; Rosalen, P. Luiz; Franchini, M.; Alencar, S. M.; Ikegaki, M.; Ransom, T.; Beutler, J.A. 2016. Planta Medica 82: 190.

\section{Tu-Poster Session 2-PO-151 Zanthoxylum zanthoxyoides root extract inhibits hemozoin-induced neuroinflammation in BV2 microglia}

Authors Ogunrinade Folashade $A^{1}$, Aderogba Mutalib $A^{2}$, Sarker Satyajit $D^{3}$, Olajide $\mathrm{O}^{1}$

Institute 1 Department of Pharmacy, University of Huddersfield, Queensgate, Huddersfield, HD 1 3DH, United Kingdom, HUDDERSFIELD, United Kingdom; 2 Department of Chemistry, Obafemi Awolowo University, Ile-Ife, Nigeria, IleIfe, Nigeria; 3 School of Pharmacy and Biomolecular Sciences, Liverpool John Moores University, Byrom Street, Liverpool, L3 3AF, United Kingdom,

Huddersfield, United Kingdom

DOI 10.1055/s-0037-1608480

Zanthoxylum zanthoxyloides (Z. zanthoxyloides) is a plant used in West Africa to treat diseases such as stomach ache, toothache and malaria. Extract and bioactives from the rootbark of $Z$. zanthoxyloides have been suggested to have anti-inflammatory activity (1 and 2 ). In cerebral malaria, the accumulation of malaria pigment hemozoin induces inflammation leading to excessive production of pro-inflammatory cytokines which contributes to its pathology (3). However, whether Z. zanthoxyloides could suppress hemozoin-induced neuroinflammation remains unclear. In this study, we investigated the effect of Z. zanthoxyloides in hemozoin-induced BV2 microglia. Cultured BV2 microglia cells were treated with 4,6 and $8 \mu \mathrm{g} / \mathrm{ml}$ of Z. zanthoxyloides 30 minutes before stimulation with hemozoin $(400 \mu \mathrm{g} / \mathrm{ml})$ for a further 24 hours. Levels of TNF $\alpha$, IL-1 $1 \beta, I L-6, I L-10$ released were measured using ELISA while PGE 2 production was measured by enzyme immunoassay. Nitric oxide (NO) production was determined using the Griess assay. Z. zanthoxyloides $(4,6$ and $8 \mu \mathrm{g} / \mathrm{ml}$ ) significantly $(p<0.05)$ reduced the production of TNF $\alpha(40 \pm 5 \%), I L-1 \beta$ (40 $\pm 2 \%)$, IL-6 (50 $\pm 2 \%)$, NO (40 $\pm 3 \%)$ and $\mathrm{PGE}_{2}(40 \pm 2 \%)$. Western blot experiments showed that $Z$. zanthoxyloides reduced levels of iNOS (45 $\pm 5 \%$ ), COX-2

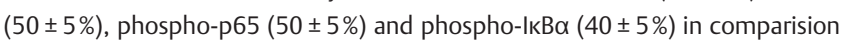
with hemozoin control. These results suggest that Zanthoxylum zanthoxyloides might be inhibiting neuroinflammation in hemozoin induced microglia cells by targeting NF-kB signalling. These results have significant implications in cerebral malaria.

[1] Prempeh, A.B.A. and Mensah-Attipoe, J, 2009. Ghana Medical Journal 43.

[2] Oriowo MA. (1982) Planta Medica 44: 54 - 56

[3] Sultan, S. and Irfan, S.M, 2015. Blood Research, 50, $72-72$.

\section{Tu-Poster Session 2-PO-152 The influence of the multicomponent herbal preparation STW5 on intestinal inflammation and motility}

Authors Marx $L^{1}$, Lehnerts $S^{1}$, Grundmann $D^{1}$, Schreiber $D^{1}$, Braun $A^{1}$, AbdelAziz $\mathrm{H}^{2}$, Kelber $\mathrm{O}^{2}$, Schäfer $\mathrm{KH}^{1}$

Institute 1 Department of Biotechnology, University of Applied Sciences Kaiserslautern, Zweibrücken, Germany; 2 Medical \& clinical affairs phytomedicine, Steigerwald Arzneimittelwerk GmbH, Bayer Consumer Health, Darmstadt, Germany

DOI 10.1055/s-0037-1608481

The multi-herbal preparation STW5 is a clinically proven drug to ameliorate symptoms of functional gastro-intestinal diseases[1]. Both, anti-inflammatory and motility-modulating effects are part of its mechanism of action.

In the present study, an in-depth investigation of the impact of STW5 on intestinal inflammation was performed in the small intestine of adult mice, based on full thickness gut wall samples and isolated myenteric plexus preparations.

For that purpose, a pro-inflammatory cytokine mixture (IL 1ß, TNFo, IFNY) was injected into the supporting blood vessel arcades of small intestinal segments with or without additional STW 5 luminal perfusion. Subsequently various cytokines were analyzed in the gut wall homogenates using multiplex-ELISA. Inflammation was induced in myenteric plexus cultures in vitro using the same cytokine cocktail. Cytokines in cell culture supernatants from cultures with and without treatment with STW5 were compared.

Multiplex analysis showed a higher amount of the pro-inflammatory cytokines G-CSF, IL-6 and LIF under inflammatory conditions in both, gut wall homogenates and myenteric plexus cultures. Treatment with STW5 leads to a reduction of the cytokine concentrations in the homogenates and myenteric plexus culture supernatants. The cytokines RANTES, GM-CSF and IL-22 could be reduced only in the gut wall homogenates, when treated with STW-5.

In conclusion, the multicomponent herbal drug STW5 leads to a reduced release of pro-inflammatory cytokines in the small intestinal gut wall and shows also a direct anti-inflammatory effect on the enteric nervous system under induced inflammation.

[1] Michael S, Abdel-Aziz H, Weiser D, Muller CE, Kelber O, Nieber K. NaunynSchmiedeberg's Archives of Pharmacology 2012; 385: 411-421

\section{Tu-Poster Session 2-PO-153 Cytotoxicity and Anti-trypanosomal Properties of Compounds isolated from Ethylacetate fraction of Zanthoxylum leprieurii}

Authors Eze $\mathrm{F}^{1}$, Krause $\mathrm{R}^{2}$, Noundou $\mathrm{X}^{2}$, Osadebe $\mathrm{P}^{1}$, Uzor $\mathrm{P}^{1}$ Institute 1 Department of Pharmaceutical and Medicinal Chemistry University of Nigeria, Nsukka, Nigeria; 2 Department of Chemistry, Rhodes University, Grahamstown, South Africa

DOI 10.1055/s-0037-1608482

Zanthoxylum leprieurii find wide application in African Traditional Medicinal Practice (ATMP) for treating various diseases. Solvent extraction was carried out on the stem back of $Z$. leprieurii using $95 \%$ aqueous methanol by cold maceration. The extract was concentrated in a rotary evaporator at $40 \mathrm{oC}$ to yield $22 \%$ dry crude extract. Toxicity studies by the Lorke's method revealed high safety margin with LD $50>5000 \mathrm{~g} / \mathrm{kg}$. The crude extract was subjected to solvent-guided fractionation by gradient elution on silica gel to obtain n-hexane (HF), ethylacetate (EAF), absolute methanol (MF) and water fractions (WF) respectively. Phytochemical analysis revealed the presence of only alkaloids and flavonoids in EAF, saponins, flavonoids and alkaloids in ME, alkaloid and tannins in WF and mainly fatty acids in HF. The EAF was subjected to TLC on silica gel plates eluted with 5: 1 mixture of diethylether and ethyl acetate to give six distinct bands B1-B6. The isolated compounds were assessed for overt cytotoxicity on Hela (Human cervix adenocarcinoma) cells. Compounds B2 and B3 showed significant activities with IC $50<50 \mu \mathrm{g} / \mathrm{mL}$. The trypanosoidal activity of EAF carried out on Trypanosoma brucei (T.b.) revealed IC 50 of $45 \mu \mathrm{g} / \mathrm{mL}$. Results showed that stem bark of $Z$. leprieurii can be used to manage trypanosomoiasis and certain forms of cancer. 


\section{Tu-Poster Session 2-PO-154 Cacao extract enriched in polyphenols prevents insulin-resistance and dyslipemia in a rat model}

Authors Rios $\mathrm{LL}^{1}$, Villagarcía $\mathrm{H}^{2}$, Castro María $\mathrm{C}^{2}$, González Arbeláez L ${ }^{3}$, Massa María $\mathrm{L}^{2}$, Schinella $\mathrm{G}^{4}$, Francini $\mathrm{F}^{2}$

Institute 1 Department Pharmacology, Faculty of Pharmacy, University of Valencia, Burjassot, Spain; 2 CENEXA (UNLP-CONICET), La Plata, Argentina; 3 CIC Centro de Investigaciones Cardiovasculares (UNLP-CONICET), La Plata, Argentina; 4 Cátedra de Farmacología Básica, Facultad de Ciencias Médicas (UNLP-CIC-PBA), La Plata, Argentina

DOI 10.1055/s-0037-1608483

Cocoa-derived foods are rich in polyphenols obtained from the fermented, roasted and industrially processed seeds of Theobroma cacao L. (Sterculiaceae). Its biological activities are due to procyanidins and flavanols [1]. Administration of a sucrose rich diet (SRD) to normal rats generates insulin resistance, oxidative stress, inflammation and liver dysfunction similar to those observed in human metabolic syndrome [2]. Our objective was to evaluate the effects of a cocoa extract enriched in polyphenols (CEP) in preventing the endocrinemetabolic alterations produced by SRD.

Male Wistar rats were fed with a standard commercial diet and drinking water (Blank); 10\% sucrose in water alone (SRD, negative control) or treated with CEP $(250 \mathrm{mg} / \mathrm{kg}$, all days) or $\mathrm{N}$-acetyl-cysteine (NAC, $50 \mathrm{mg} / \mathrm{kg}$, last 5 days) as positive control. At the time of sacrifice blood glucose, insulin, triglycerides and serum transaminases were determined. In liver, we measured: a) oxidative stress markers (GSH and protein carbonyls); b) glycogen content, glucokinase and glucose-6-phosphatase activities; and, d) protein levels of Akt/pAkt, eNOS/p-eNOS, iNOS and COX-2.

Insulin, triglicerides and glycogen were significantly reduced vs. control, whereas glucose-6-Pase activity was reduced. Glucose and glucokinase activity did not suffer significant changes. Serum transaminases showed no differences thus demonstrating no hepatotoxicity. SRD rats showed a decrease in GSH level, a value that increased significantly in CEP animals (36.0 vs. 12.9 $\mathrm{mmol} / \mathrm{g}$ ). No changes were observed in protein carbonyl levels. P-Akt and PeNOS levels were significantly reduced in SRD animals. iNOS and COX-2 were significantly increased in these animals. CEP administration prevented the mentioned changes.

In conclusion, CEP co-administration is effective in preventing the endocrinemetabolic changes induced by a SRD. This prevention is mediated through PAkt/P-eNOS-dependent signaling pathway.

[1] Andújar et al. Oxid Med Cell Longev. 2012, 2012, 906252.

[2] Francini et al. Life Sci. 2010; 86: 965 - 971.

\section{Tu-Poster Session 2-PO-156 Acute Toxicity, Phytochemical, and Antimicrobial Properties of Selected Nigeria Medicinal plants on Amoxicillin-resistant Microorganisms}

\author{
Authors Eze $\mathrm{F}^{1}$, Osadebe $\mathrm{P}^{1}$, Uzor $\mathrm{P}^{1}$ \\ Institute $\overline{1}$ Department of Pharmaceutical and Medicinal Chemistry \\ University of Nigeria Nsukka, Nsukka, Nigeria \\ DOI 10.1055/s-0037-1608484
}

Antimicrobial studies were carried out on the aqueous methanolic extracts of stem bark of Zanthoxylum leprieurii, Anthocleistha vogelli, Mesembryanthemum crystallinum and leaves of Milletia aboensis. These plants find wide applications in African Traditional Medicinal Practice (ATMP). The plant materials were subjected to cold maceration using $95 \%$ methanol and the extracts concentrated in a rotary evaporator at $40{ }^{\circ} \mathrm{C}$. Acute toxicity studies revealed that the plants have high safety margin with LD $50>5000 \mathrm{~g} / \mathrm{Kg}$ except M. Crystallinum which shows LD 50 of $2900 \mathrm{~g} / \mathrm{Kg}$. Qualitative phytochemical analysis confirms the presence of most of the secondary plant metabolites on all the plants tested. Antimicrobial screening was carried out using agar diffusion method. All the extracts, except that of $M$. aboensis showed fairly good inhibitory zone diameters on most of the tested organisms with the activities residing mainly on the n-hexane fractions.

\section{Tu-Poster Session 2-PO-157 Antibacterial activities of Teucrium kotschyanum from Turkey}

Authors Gulsoy Toplan $\mathrm{G}^{1}$, Tan $\mathrm{N}^{1}$, Bilgin $\mathrm{M}^{2}$, Gulec $\mathrm{M}^{1}$, Tan $\mathrm{E}^{2}$ Institute 1 Department of Pharmacognosy, Faculty of Pharmacy, Istanbul University, Istanbul, ISTANBUL, Turkey; 2 Department of Pharmaceutical Microbiology, Faculty of Pharmacy, Istanbul Yeni Yuzy? I University, ISTANBUL, Turkey DOI 10.1055/s-0037-1608485

Teucrium is the important genus of the Lamiaceae family with 260 species known worldwide [1]. There are 34 species of Teucrium in Turkey, 16 of them are endemic [1]. Members of this genus are commonly known as 'hemorrhoid plant' due to treatment of hemorrhoid disease. They have been used as a folk medicine for treatment of various diseases for more than 2000 years, mainly as diuretic, digestive, analgesic and antispasmodic agent and they also possess antibacterial, antifungal, antidiabetic, antihelmintic, antioxidative and anti-inflammatory activity [2]. Teucrium species are rich sources of diterpenoids and also contain monoterpenes, sesquiterpenes, triterpenes, iridoids, flavonoids and essential oil [3].

In this study, the aerial parts and also root of Teucrium kotschyanum collected from Izmir (Odemiş) were extracted with different solvents such as petroleum ether, ethyl acetate, methanol and infusion of plant was also prepared. All extracts were studied against standard Staphylococcus aureus, Staphylococcus epidermidis, Enterococcus faecalis, Escherichia coli, Klebsiella pneumoniae, Pseudomonas aeruginosa, Proteus mirabilis, Candida albicans and also Methicillinsusceptible S. aureus, Methicillin-resistant S. aureus, Methicillin-resistant coagulase negative Staphylococcus sp. Besides, the extracts were also tested against clinically isolated strain of Klebsiella pneumonia, Escherichia coli and Acinetobacter baumanii using microbroth dilution method. The extracts showed strong to moderate inhibitory effects. The minimum inhibitory concentration (MIC) values were also ranged from $9.7 \mu \mathrm{g} / \mathrm{mL}$ to $5000 \mu \mathrm{g} / \mathrm{mL}$ for all the tested microorganisms. Among tested extracts, petroleum ether extract from the root of $T$. kotschyanum showed strongest activity against Methicillinsusceptible S. aureus with a MIC value of $9.7 \mu \mathrm{g} / \mathrm{mL}$.

[1] Ozcan T, Dirmenci T, Coskun F, Akcicek E, Guner, O. Turk J Bot 2015;39:310 - 317.

[2] Baytop T. Therapy with Medicinal Plants in Turkey, Pastand Present, 2nd edition. Istanbul: Nobel Medical Publishing; 1999.

[3] Piozzi F, Bruno M, Rosselli S, Maggio A.Heterocycl 2005; 65: 1221 - 1234.

\section{Tu-Poster Session 2-PO-158 Evaluation of estrogenic activity of Trifolium pratense L. and Cimicifuga racemosa L. plant extracts and formulations using the planar-YES assay}

Authors Bräm $S^{1}$, Wolfram $E^{1}$

Institute 1 Zurich University of Applied Sciences, Institute of Chemistry and Biotechnology, Phytopharmacy and Natural Products Research Group,

Waedenswil, Switzerland

DOI 10.1055/s-0037-1608486

Supplementation with natural estrogens, known as phytoestrogens are desirable for the treatment of women suffering from climacteric complaints after menopause. However, in patients with hormone-dependent cancers, such as specific types of breast cancer, the intake of phytoestrogens may be contraindicated, since some compounds are claimed to trigger the formation of metastasis-initiating cancer cells [1].

HPTLC is an analytical tool in quality control of medicinal plants extracts. Separated compounds from multicompound extracts are fixed on the solid silica phase like a compound library. By direct coupling of the Yeast Estrogen 
Screen (YES) on the HPTLC plate, this compound library can also be used for screening of estrogenic activity.

The reporter gene YES assay encoding the human estrogen receptor alpha (ER $\alpha$ ) was adapted for the screening of Trifolium pratensis L. and Cimcifuga racemosa $\mathrm{L}$. extracts and formulations [2,3]. The method is rapid and simple with no need for special sample preparation. Excipients such as microcrystalline cellulose, carboxymethylcellulose or polyvinyl pyrrolidone did not interfere with the method.

In samples of $T$. pratensis, pronounced fluorescent zones of Genistein and Biochanin A could be identified, indicating estrogen receptor affinity. The half maximal effective concentration ( $\mathrm{EC}_{50}$ ) of Genistein and Biochanin A was 2.13 and $3.34 \mathrm{ng}$, respectively.

Cimicifugin showed weak estrogenic activity visible as fluorescent zones at applied masses of $4 \mu \mathrm{g}$. However, no ER $\alpha$ affinity was detected in $C$. racemosa samples. Formononetin interacted with ERa but was not detected in C. racemosa samples or commercial products.

[1] Virk-Baker MK, Nagy TR, Barnes S. Planta medica 2010; 76: 1132 - 1142.

[2] McDonnell DP, Nawaz Z, O'Malley BW. Mol Cell Biol 1991; 11: 4350 - 4355.

[3] McDonnell DP, Nawaz Z, Densmore C, Weigel NL, Pham TA, Clark JH, O’Malley BW. J Steroid Biochem Mol Biol 1991; 39: 291 - 297.

\section{Tu-Poster Session 2-PO-159 Bioavailability study and impact of Chios mastic supplementation on oxidative stress in healthy humans}

Authors Brieudes $\mathrm{V}^{1}$, Kallergis $\mathrm{E}^{1}$, Papada $\mathrm{E}^{2}$, Gioxari $\mathrm{A}^{2}$, Kaliora $\mathrm{A}^{2}$, Mitakou $\mathrm{S}^{1}$, Skaltsounis $\mathrm{L}^{1}$, Halabalaki $\mathrm{M}^{1}$

Institute 1 Divisionof Pharmacognosy and Natural Products Chemistry, Department of Pharmacy, National and Kapodistrian University of Athens, Athens, Greece; 2 Laboratory of Food Chemistry-Biochemistry, School of Health Science and Education, Department of Dietetics and Nutritional Science, Athens, Greece

DOI 10.1055/s-0037-1608487

Chios mastic, is the resinous secretion obtained from the wounds of the trunk and branches of Pistacia lentiscus L. var. Chia, which is endemic to the Greek island of Chios. Traditional usage of mastic dates back to more than 2500 years for gastrointestinal disorders [1]. More recently, mastic showed promising results in active Crohn's disease patients [2] as well as lipid- and glucoselowering properties [3], antioxidant and anti-inflammatory properties [4]. In this work, 17 healthy volunteers were participated receiving per os a single dose of Chios mastic. Blood samples were collected at specific time intervals (e.g. 0, 1, 2, 4, 6 and 24 hours). After pretreatment plasma samples were analyzed by UHPLC-tQ-MS and the main constituents namely isomasticadienonic acid (IMNA) and masticadienonic acid (MNA) were directly quantified. MNA and IMNA are the most characteristic triterpenic acids of Chios mastic resin. Both compounds are not commercially available and have been isolated by an SFC-MS based method newly developed in house. MNA and IMNA were detected and $C_{\max }$ plasma values were ranging from 29 to $768 \mathrm{ng} / \mathrm{mL}$ for IMNA and from 52 to $1390 \mathrm{ng} / \mathrm{mL}$ for MNA. Maximum concentration times were different depending on the subjects between 2 and 4 hours after intake. Moreover, oxidative stress markers in a postprandial phase were evaluated. Serum resistance time to oxidation increased after intake while the same trend was observed regarding the \% change in oxLDL. This study offers for the first time information on the availability of bioactive components following ingestion of Chios mastic in humans together with evidence of its potency for oxidative stress reduction.

[1] S. Paraschos et al., Current Medicinal Chemistry. 2012, 19, $2292-2302$.

[2] A.C. Kaliora et al., World J. Gastroenterol. 2007, 13, 748 - 753.

[3] A. Kartalis et al., Eur J Prev Cardiol. 2016, 23, $722-729$.

[4] A.N. Assimopoulou et al., Food Chemistry. 2005, 92, $721-727$.

\section{Tu-Poster Session 2-PO-160 3-DEOXYANTHOCIANIN AND FLAVONOIDS COMPOUND OF A. CHICA IN RATS ORAL BIOAVAILABILITY EVALUATION}

Authors Sousa Ilza $M^{1,2}$, Jorge $M^{2}$, Cabral Elaine $C^{2}$, Figueredo $M^{1,2}$, Queiroz Nubia de $C^{2}$, Tinti $S^{2}$, Basting $R^{1,2}$, Eberlin $M^{3}$, Carvalho João $E^{2}$, Foglio Mary $A^{1,2}$

Institute 1 Faculty of Medical Science. University of Campinas. São Paulo Brazil, Campinas, Brazil; 2 Faculty of Pharmaceuthical Science University of Campinas, Campinas, Brazil; 3 Chemistry Institute University of Campinas. São Paulo Brazil, Campinas, Brazil

DOI 10.1055/s-0037-1608488

Arrabidaea chica (Humb. \& Bonpl.) B. Verlot (Bignoniaceae), is employed in folk medicine for wound healing, inflammation, and gastrointestinal colic. Twenty four Wistar rats $(\sim 250 \mathrm{~g})$ were orally administrated with $100 \mathrm{mg} / \mathrm{kg}$ A.chica standardized extract. Plasma and urine were collected throughout $8 \mathrm{~h}$ after oral administration. Blood samples were taken from the vein femoral of rats into heparinized Eppendorf tubes every half hour after oral administration. and analyzed by HPLC-MS². Methanol was added to samples, mixed in vortexed and further centrifugation at 14,000 rpm for $5 \mathrm{~min}$. The supernatant was transferred to a clean tube, dried under nitrogen. An $10 \mu \mathrm{L}$ sample was injected into LC-MS/MS (TOF) mass analyzers system. The results, in positive mode, showed carajurin for urine. In plasma highlighted compounds with $\mathrm{m} / \mathrm{z}$ 711 and m/z 647 after 30 min but no carajurin was observed. Further analysis with HPLC-MS ${ }^{2}$ and selected ion monitoring are needed for better understanding.

[1] Shi,P.; Lin, X.;Yao, Hong.. Xenobiotica.2015; 45; 999 - 1008.

[2] Q. Li et al. J. Chromatogria. A 2015, 1395, 116 - 128.

\section{Tu-Poster Session 2-PO-161 Cytotoxic Activity, Seasonal Variation and GC/MS-based Metabolomics of Three Callistemon species Coupled to Chemometrics}

Authors Ayoub I ${ }^{1}$, Gad H ${ }^{1}$

Institute 1 Department of Pharmacognosy, Faculty of Pharmacy, Ain Shams University, 11566, Cairo, Egypt

DOI 10.1055/s-0037-1608489

Genus Callistemon, commonly known as bottlebrush, is recognized in folk medicine for its anti-bronchitis, anti-cough, and insecticidal effects. The essential oils have been used as antimicrobial and antifungal agents. In this study, interspecies and seasonal variations in the essential oil composition of three Callistemon species was assessed using GC-MS coupled to multivariate data analysis. The essential oils obtained by hydrodistillation of the fresh leaves of $C$. citrinus, C. rigidus and C. viminalis (Myrtaceae) were characterized by GC and GC/MS analysis. A total of 31 compounds were annotated accounting for $93.37 \%$ to $98.68 \%$ of the whole volatile constituents in four seasons. Eucalyptol represented the major component in the three Callistemon species $(51.62 \%-80.72 \%)$, followed by $\alpha$-pinene $(1.11 \%-20.75 \%)$, linalool $(0 \%$ $13.8 \%)$ and $\alpha$-terpineol $(2.13 \%-10.27 \%)$. Principal component analysis (PCA) and hierarchical cluster analysis (HCA) were applied as pattern recognition techniques in an attempt to discriminate the three species (three replicates for each species) based on their total ion content to investigate their metabolic variations for each season individually. The PCA plot was able to discriminate between the three Callistemon species in different seasons. They were clustered into three main groups, where each species was positioned in a separate quadrant. The loading plot showed that the main discriminating makers were eucalyptol, $\alpha$-pinene and linalool, showing the highest influence on the segregation between the samples. The dendogram obtained by applying HCA confirmed the results of PCA. Furthermore, PCA was able to partially segregate individual species in the different seasons. Moreover, cytotoxic activity of Callistemon essential oils was assessed using MTT assay on Caco-2 cell lines. The results revealed that $C$. citrinus essential oil showed very potent 
cytotoxicity with $\mathrm{IC}_{50}$ equals $0.80 \mu \mathrm{g} / \mathrm{mL}$ followed by $C$. rigidus and $C$. viminalis, exhibiting $\mathrm{IC}_{50}$ values of 2.0 , and $2.84 \mu \mathrm{g} / \mathrm{ml}$, respectively.
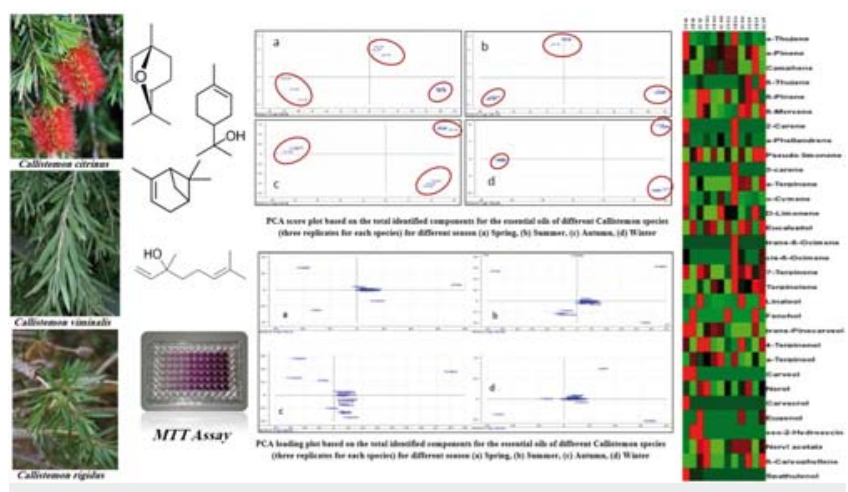

- Fig. 1

\section{Tu-Poster Session 2-PO-162 STW 5 prevents stress-induced changes in intestinal permeability in mice in-vivo}

Authors Aubert $\mathrm{P}^{2}$, Chevalier $\mathrm{J}^{2}$, Durand $\mathrm{T}^{2}$, Bessard $\mathrm{A}^{2}$, Kelber $\mathrm{O}^{1}$, AbdelAziz $\mathrm{H}^{1}$, Neunlist $\mathrm{M}^{2}$

Institute 1 Innovation \& Development, Steigerwald Arzneimittelwerk GmbH, Bayer Consumer Health, Darmstadt, Germany; 2 Inserm U1235-Institut des Maladies de l'Appareil Digestif du CHU de Nantes, Nantes, France DOI 10.1055/s-0037-1608490

The fixed-combination herbal preparation STW 5 (Iberogast) has been reported to increase intestinal chloride secretion in-vitro. However, the ability of STW 5 to modulate paracellular and transcellular permeability remains currently unknown. Therefore, we aimed to study the ability of STW 5 to modulate intestinal permeability under basal and repeated acute stress conditions.

C57-bl6 mice were gavaged for 14 days with STW 5 ( $3 \mathrm{~mL} / \mathrm{kg}$ ). After 10 days of treatment, mice were subjected to water avoidance stress (WAS) during 4 consecutive days. In-vivo permeability to FITC -Sulfonic Acid (FSA, $400 \mathrm{Da}$ ) and Horse Radish Peroxydase (HRP, 44KDa), total transit time and colonic transit (fecal pellet output - FPO) were assessed at Day 0 (D0), D 10 and D 14 of IB treatment. Ex vivo permeability to FSA and HRP was assessed on jejunum, ileum, proximal colon and distal colon at D14 using Ussing chambers. Corticosterone blood level was measured at D11 and D14.

In-ivo permeability to FSA and HRP as well as total transit time were not modified by STW 5 in basal and WAS conditions. However, STW5 prevented the increase in permeability to FSA induced by WAS in the distal colon ex vivo. Conversely, STW 5 prevented the increase in permeability to HRP induced by WAS in the jejunum and proximal colon. Furthermore, while STW 5 tended to increase colonic transit as compared to control in basal conditions, it prevented the increase in colonic transit induced by WAS. Finally, STW 5 did not modify the changes in corticosterone induced by WAS.

Our study suggests that STW 5 can prevent WAS induced changes in paracellular and transcellular permeability in specific regions of the gastrointestinal tract. Such effects could contribute to the therapeutic effects of STW 5 in irritable bowel syndrome and support novel therapeutic indications for pathologies in which barrier functions are altered.

\section{Tu-Poster Session 2-PO-163 The herbal medicine STW 5 is efficacious in functional dyspepsia also in the elderly - a meta-analysis of randomized controlled trials}

\author{
Authors Müller $\mathrm{J}^{1}$, Fink $\mathrm{C}^{1}$, Rabini $\mathrm{S}^{1}$, Kelber $\mathrm{O}^{2}$, Nieber $\mathrm{K}^{3}$, Kraft $\mathrm{K}^{4}$, \\ Storr $\mathrm{MA}^{5}$
}

Institute 1 Medical and Clinical Affairs Phytomedicines, Innovation and Development, Phytomedicines Supply and Development Center, Bayer

Consumer Health Division, Steigerwald Arzneimittelwerk GmbH, Darmstadt, Germany, Darmstadt, Germany; 2 Innovation and Development,

Phytomedicines Supply and Development Center, Bayer Consumer Health Division, Steigerwald Arzneimittelwerk GmbH, Darmstadt, Germany,

Darmstadt, Germany; 3 Pharmaceutical Institute, Leipzig University, Leipzig, Germany, Leipzig, Germany; 4 Chair for Naturopathy, Center for Internal Medicine, University Medicine Rostock, Rostock, Germany, Rostock, Germany; 5 Center for Endoscopy, Starnberg, Germany

DOI 10.1055/s-0037-1608491

With the increasing affinity of patients and consumers to natural treatment options, well proven herbal medicinal products gain increased attention also in functional gastrointestinal diseases. One of these is STW 5 (Iberogast), for which more than 55 years of therapeutic experience in more than 73 Mio patients are available. For answering the question, whether its efficacy is comparable in patients of different age groups, respective sub-group analyses were conducted.

After meta-analyses of randomized placebo-controlled double blind trials have shown compliance to modern standards for a proof of efficacy, in a second step, sub-group analyses were conducted. The analyses were based on the original patient data from the trials, including demografic data and primary endpoints (ANCOVA).

As the primary outcome variable, the validated gastrointestinal symptom score (GIS) [1] were identical in all trials. Also the therapeutic dose $(3 \times 20 \mathrm{drops} /$ day $)$ was the same, so that a uniform evaluation was possible. The full analysis set (FAS) included 557 patients (272 resp. 285 for placebo resp. verum). For the GIS the difference between placebo and verum after 28 days of treatment showed a highly significant $(p<0.0001)$ difference between placebo and verum (6.7 resp. 4.7 points). The analyses in different age groups (adults up to $30,30-40,40-50,50-60$, above 60 ) did show a comparable efficacy.

After meta-analyses have clearly shown the efficacy of STW 5 (Iberogast), now also its therapeutic usefulness in elderly patients was demonstrated. Given also the very good tolerability shown by the study and by pharmacovigilance data, STW 5 is very well suitable also in self-medication. Additional insights can be expected from additional sub group analyses, as e.g. the evaluation of subgroups with specific predominant symptoms.

[1] Adam et al. Aliment Pharmacol Ther 2005; 22: 357 - 363

\section{Tu-Poster Session 2-PO-164 Anti-inflammatory mechanisms in functional gastrontestinal diseases: STW 5 vs. NSAIDs}

\author{
Authors Kelber $\mathrm{O}^{1}$, Nieber $\mathrm{K}^{2}$ \\ Institute 1 Innovation and Development, Phytomedicines Supply and \\ Development Center, Bayer Consumer Health Division, Steigerwald \\ Arzneimittelwerk GmbH, Darmstadt, Germany, Darmstadt, Germany; \\ 2 Pharmaceutical Institute, Leipzig University, Leipzig, Germany, Leipzig, \\ Germany \\ DOI 10.1055/s-0037-1608492
}

Recent studies have shown that systemic responses of increased circulating lymphocytes and elevated proinflammatory cytokines and subtle inflammation may accompany the onset and persistence of symptoms of functional gastrointestinal diseases like functional dyspepsia (FD) and irritable bowel syndrome (IBS). The complexity of these diseases indicates that there are 
different pathophysiological mechanisms involved. We hypothesised that STW 5, a phytomedicinal product used in FD and IBS patients has anti-inflammatory effects like NSAIDs, but without GI side effects.

Data from in vitro studies were revealed and analysed for elucidating possible mechanisms of action underlying the anti-inflammatory effects of STW 5.

STW 5 activated COX-1, but had no effect on COX-2 mRNA expression in contrast to the control substances, like ASS and diclofenac, which inhibited COX-1 and COX-2 mRNA expression [1]. STW 5 inhibited the increased gene expression and reduced significantly the release of TNF-alpha by activation of adenosine A2A receptors in LPS $(100 \mathrm{ng} / \mathrm{ml})$-stimulated human monocytes, while having no effect in untreated cells [2]. Radioligand binding assays confirmed the affinity of STW 5 to adenosine A2A receptors.

The mechanism of action of STW 5 as an anti-inflammatory medication without involving COX-1 or COX-2 inhibitory properties may be an important reason for the very good tolerability of this medicinal product in patients with FD and IBS, as indicated by clinical trials and therapeutic use.

[1] Michael, S, et al. 2012. Inflammatory Bowel Disease 3: 41; 2. Bonaterra, G. A, et al. 2008. Z. Phytotherapie 29: S22

\section{Tu-Poster Session 2-PO-165 Intestinal permeability induced by psychological stress: Action of STW 5}

Authors Aubert $\mathrm{P}^{1}$, Chevalier $\mathrm{J}^{1}$, Durand $\mathrm{T}^{1}$, Bessard $\mathrm{A}^{1}$, Kelber $\mathrm{O}^{2}$, AbdelAziz $\mathrm{H}^{3}$, Neunlist $\mathrm{M}^{1}$

Institute 1 Inserm U1235-Institut des Maladies de l'Appareil Digestif du CHU de Nantes, Nantes, France; 2 Innovation and Development, Phytomedicines Supply and Development Center, Bayer Consumer Health Division, Steigerwald Arzneimittelwerk GmbH, Darmstadt, Germany, Darmstadt, Germany;

3 Medical and Clinical Affairs Phytomedicines, Innovation and Development, Phytomedicines Supply and Development Center, Bayer Consumer Health Division, Steigerwald Arzneimittelwerk GmbH, Darmstadt, Germany DOI 10.1055/s-0037-1608493

STW 5, a herbal medicinal product, has been shown to stimulate intestinal chloride secretion. Whether it can modulate paracellular and transcellular permeability remains currently unknown. Therefore, we did study the ability of STW 5 to modulate intestinal permeability under basal and repeated acute stress conditions.

C57 bl6 mice were gavaged for 14 days with STW 5 ( $3 \mathrm{~mL} / \mathrm{kg}$ ). After 10 days of treatment, mice were subjected to water avoidance stress (WAS) during 4 consecutive days. In vivo permeability to FITC -Sulfonic Acid (FSA, $400 \mathrm{Da}$ ) and Horse Radish Peroxydase (HRP, 44KDa), total transit time and colonic transit (fecal pellet output - FPO) were assessed at Day 0 (D0), D 10 and D14 of IB treatment. Ex vivo permeability to FSA and HRP was assessed on jejunum, ileum, proximal colon and distal colon at D 14 using Ussing chambers. Corticosterone blood level was measured at D11 and D14.

While in vivo permeability to FSA and HRP as well as total transit time were not modified by STW 5 in basal and WAS conditions, STW 5 prevented the increase in permeability to FSA induced by WAS in the distal colon ex vivo. STW 5 prevented the increase in permeability to HRP induced by WAS in the jejunum and proximal colon. While STW 5 tended to increase colonic transit as compared to control in basal conditions, it did not influence the increase in colonic transit induced by WAS. STW 5 did not modify the changes in corticosterone induced by WAS.

STW 5 (Iberogast) obviously can prevent WAS induced changes in paracellular and transcellular permeability in specific regions of the gastrointestinal tract. Such effects could contribute to the therapeutic effects of STW 5 in irritable bowel syndrome and support its use in indications in which barrier functions are altered.

\section{Tu-Poster Session 2-PO-166 The herbal preparation STW 5 positively influences changes in intestinal microbiota induced by functional dyspepsia or ulcerative colitis in rats.}

Authors Khayyal $\mathrm{MT}^{1}$, Wadie $\mathrm{W}^{1}$, El-Sayed $\mathrm{S}^{1}$, Abdel-Tawab $\mathrm{N}^{2}$, Kelber $\mathrm{O}^{3}$, Abdel-Aziz $\mathrm{H}^{3}$

Institute 1 Department of Pharmacology, Faculty of Pharmacy, Cairo

University, Cairo, Egypt; 2 Department of Microbiology, Faculty of Pharmacy, Cairo University, Cairo, Egypt; 3 Steigerwald Arzneimittelwerk GmbH, Bayer Consumer Health, Darmstadt, Germany

DOI 10.1055/s-0037-1608494

The herbal preparation STW5 (Iberogast ${ }^{\circledR}$ ) is a multi-component multi-target herbal preparation consisting of hydro-alcoholic extracts of bitter candytuft, lemon balm, chamomile, caraway fruit, peppermint leaf, Angelica root, milk thistle, celandine herb, and licorice root. It has been shown to be clinically effective in irritable bowel syndrome1 and functional dyspepsia2 as well as experimentally in inflammatory bowel disease3. Since many clinical gastrointestinal conditions have been linked with changes in intestinal microbiota, we have studied such changes in models of functional dyspepsia and ulcerative colitis. The effect of STW5 on gut microbiota in normal rats and in established FD and colitis rat models was studied by assessing changes in selected major bacterial phyla using quantitative Real Time-PCR (qPCR). Ulcerative colitis was induced in rats by feeding them $5 \%$ DSS while functional dyspepsia was induced by subjecting rats first to neonatal maternal separation followed by restrained stress at maturity. In normal animals STW5 dramatically increased the relative abundance of Bacteroidetes, Bacteroides and Prevotella phyla of rat gut microbiota but a decrease in Bifidobacteria, Actinobacteria and Clostridium. Treatment with STW5 induced a dramatic increase in Lacobacillus and Mathanobrevibacter populations. Changes in the microbiota Prevotella and Enterococcus induced by the stress models were prevented by STW5 administration. The findings lend support to the use of STW5 in the gastrointestinal conditions mentioned by influencing favourably the derangements in the intestinal microbiota caused by them.

[1] Schmulson M], Nature Clinical Practice Gastroenterology \& Hepatology, 2008, 5, 136 - 137,

[2] Madisch A et al, Aliment Pharmacol Ther, 2004, 19: 271 - 279,

[3] Wadie W et al, Int J Colorectal Dis, 2012, 27:1445- 53.

\section{Tu-Poster Session 2-PO-167 A new solubilized formulation of Curcumin, Boswellia and Xanthohumol extract markedly enhances anti-inflammatory activity}

Authors Khayyal MT ${ }^{1}$, Tawab $\mathrm{M}^{2}$, Behnam D ${ }^{3}$, El-Sabbagh WA ${ }^{4}$, El-Hazek RM ${ }^{4}$ Institute 1 Pharmacology Department, Faculty of Pharmacy, Cairo University, Cairo, Egypt; 2 Zentrallaborato-rium Deutscher Apotheker, Eschborn, Germany; 3 Aquanova AG, Darmstadt, Germany; 4 Department of Drug Radiation Research, National Centre for Radiation Research and Technology, Atomic Energy Authority, Cairo, Egypt

DOI 10.1055/s-0037-1608495

Native extracts of Curcumin and Boswellia are known to exert anti-inflammatory properties, but have poor bioavailability when given orally. Through advanced micellation technology, it has been possible to produce stable solubilisates of these extracts, thereby markedly enhancing their bioavailability and consequently reducing the orally administered dose and reducing their potential adverse effects. In the present study, we compared the chronic anti-inflammatory activities of native curcumin and boswellia extracts with the solubilized form of both in the adjuvant arthritis model upon daily administration for three weeks. Diclofenac was used as reference drug. The combination of solubilisates of curcumin and boswellia extracts showed a better anti-inflammatory effect than either one alone. The reduction in paw volume was reflected in corresponding changes in relevant parameters for mediators of inflammation: TNF- $\alpha$, IL-6. The findings confirm that the solubilsates of curcu- 
min and boswellia extracts allow the use of much lower doses than required in the native form to achieve a potent anti-inflammatory effect. Moreover the combination of curcumin and boswellia solubilisates show that they potentiate one another to produce a therapeutic effect equivalent to if not more potent than diclofenac. Much better results were obtained with the combination of solubilized xanthohumol and curcumin, which revealed an even more potent anti-inflammatory effect than diclofenac, which has been used as a reference drug, and the combination of solubilized curcumin and boswellia extracts.

\section{Tu-Poster Session 2-PO-168 Chemical composition and evaluation of the antimicrobial activity of Valeriana prionophylla Standl. from Guatemala}

Authors Perez Sabino Juan $\mathrm{F}^{1}$, Muñoz Wug Manuel $\mathrm{A}^{1}$, Mérida Reyes Max $\mathrm{S}^{1}$, Oliva Bessie $\mathrm{E}^{1}$, Taracena $\mathrm{E}^{1}$, Gaitán isabel $\mathrm{C}^{1}$, Martínez José $\mathrm{V}^{1}$, Ribeiro da Silva Antonio $]^{2}$

Institute 1 University of San Carlos of Guatemala, Guatemala, Guatemala; 2 Federal University of Rio de Janeiro, Guatemala, Guatemala

DOI 10.1055/s-0037-1608496

Valeriana prionophylla Standl. (Caprifoliaceae) is a perennial herb native of Guatemala and found usually over $2100 \mathrm{~m}$ at the western highlands of the country. The plant is traditionally used in Guatemala as antipyretic, anticonvulsive, spasmolytic, tranquilizer, sedative, among other uses. Root and rhizome samples of $V$. prionophylla were collected in July 2014 in populations at San José Ojetenam in the the western province of San Marcos at an altitude of $3350 \mathrm{~m}$. The essential oil was obtained by hydrodistillation for two hours (average yield: $1.55 \% \mathrm{w} / \mathrm{w}$ ). The oil components were identified by GC/MS through their mass spectra and retention indices and quantified based on their GC/FID peak areas, showing isovaleric acid, bornyl acetate and valerenic acid as main components (up to $60 \%$ ). The inhibitory activity of the essential oil against two Gram-positive bacteria (Staphylococcus aureus and Bacillus cereus), two Gram-negative bacteria (Pseudomonas aeruginosa and Escherichia coli) and one yeast strain (Candida albicans) was evaluated by the agar diffusion method (Kirby-Bauer method). The oil presented activity against all the tested organisms with inhibition halos of $25 \mathrm{~mm}$ (S. aureus) $29 \mathrm{~mm}$ (B. cereus), $21 \mathrm{~mm}$ (P. aeruginosa), $19 \mathrm{~mm}$ (E. coli) and $13 \mathrm{~mm}$ (C. albicans). The results suggest that the essential oil of $V$. prionophylla could be used as potential antimicrobial in the development of products in medicine and in the food industry.

[1] Nash DL, Dieterle JVA. Flora of Guatemala. Fieldiana Botany: 1976; 24: $302-303$.

\section{Tu-Poster Session 2-PO-169 Determination of 34 pyrrolizidine alkaloids (PA) as contaminants in various plant extracts using UHPLC-ToF-HRMS}

Authors Schenk $A^{1}$, Siewert $B^{1}$, Toff $S^{1}$, Drewe $]^{2}$

Institute 1 Max Zeller Söhne AG dep. of analytical development (1),

Romanshorn, Switzerland; 2 Max Zeller Söhne AG dep. of preclinical research

(2), Romanshorn, Switzerland

DOI 10.1055/s-0037-1608497

The very low PA regulatory threshold of a daily intake of $0.35 \mu \mathrm{g}$ proposed by the EMA/HMPC requires sensitive and reproducible methods for determination. After establishing an UHPLC-ToF-HRMS method to determine five natural occurring PAs in in a $\mathrm{CO}_{2}$ extract of $P$. hybridus leaves in 2014 (2), we now developed a contaminant assay for 34 PAs applicable for various extracts. To avoid matrix effects and to concentrate the analytes by the means of a second UHPLC pump and a trapping column, a 2D-chromatography system is integrated in the present UHPLC system. This replaces time-consuming sample preparation by means of SPE. Only the quantity of the sample to be injected is automatically purified and concentrated using autosampler and 2D-installation. The chromatographic separation avoids with two exceptions contem- poraneous elution of PA isomers. PAs are detected by ToF-HRMS (mass resolution $30000 \mathrm{FWHM}$ at $\mathrm{m} / \mathrm{z} 400$ ) in positive ionization mode. They are quantified using external standard series of all analyzed alkaloids. As a further important measure to avoid matrix effects, the standards are dissolved in a matrix solution of PA-free extracts. A detailed basic validation was executed with hops extract. For other matrices just linearity, precision, recovery and LoQ are validated. Due to fact that all external standards are dissolved in matrix solution the LoQs are slightly higher than with standards in pure solvents. Depending on the validated matrix they range from 10 to $20 \mathrm{ppb}$. Compared to methods without compensation of matrix effects, the measured values should be more close to the exact value.

[1] BfR (2013), Bestimmung von Pyrrolizidinalkaloiden (PA) in Pflanzenmaterial mittels SPE-LC-MS/MS. Methodenbeschreibung BfR-PA-Tee-1.0, http://www. bfr.bund.de/cm/343/bestimmung-von-pyrrolizidinalkaloiden.pdf, Last access: March 2015

[2] Schenk A, Siewert B, Toff S, Drewe, J (2015) UPLC TOF MS for sensitive quantification of naturally occurring pyrrolizidine alkaloids in Petasites hybridus extract (Ze 339)

\section{Tu-Poster Session 2-PO-170 Metabolic profiling of the Traditional Chinese formula Fang Feng Tong Sheng San by GC-MS}

Authors Brantner $A^{1}$, Zhao $Y^{2}$, Alajlani $\mathrm{M}^{1}$, Zhou $\mathrm{H}^{2}$, Baolin $\mathrm{B}^{2}$ Institute 1 Department of Pharmacognosy, Institute of Pharmaceutical Sciences, Karl-Franzens-University Graz, Universitaetsplatz 4, 8010, Graz, Austria; 2 Institute of Chinese Materia Medica, China Academy of Chinese Medical Sciences, 100700, Beijing, China

DOI 10.1055/s-0037-1608498

Traditional Chinese Medicines include a large number of component herbs. Each of the herbal constituents contains many compounds that may even with low concentrations be relevant to the medicinal activity. Some plants are rich in volatile ingredients. Fang Feng Tong Sheng San (FFTSS) consists of 17 components [1]. This reputed TCM formulation is recommended for all wind/heat that is showing signs of internal heat so as not to let pathogens penetrate deeper. This study is carried out to determine the major volatile compounds and their possible role in the effectiveness of FFTSS. The hexane extract of the grinded powder of FFTSS was analyzed by GC-MS with a capillary column $(30 \mathrm{~m} \times 0.25 \mathrm{~mm} \times 0.25 \mu \mathrm{m}$ film thickness). In this study an anaIytical procedure was developed for optimization and rapid analysis of the major volatile constituents in FFTSS. Optimal analytical conditions: initial temperature $60{ }^{\circ} \mathrm{C}$ (held for $1 \mathrm{~min}$ ), increased to $280^{\circ} \mathrm{C}$ at $4{ }^{\circ} \mathrm{C} \mathrm{min}-1$, final temperature was held for $15 \mathrm{~min}$.; carrier gas helium at a constant flow rate of 1.2 $\mathrm{mL} \mathrm{min}^{-1}$.The temperatures of the interface, ion source and quadrupole were 280,230 and $150^{\circ} \mathrm{C}$, respectively. Over 50 compounds have been identified ranging from alkanes, ketones to sterols. Seven compounds were considered as the most essential constituents in the FFTSS preparation (menthol, pulegone, butylidene phthalide, ligustilide, methyl linoleate, anthracenedione, isolongifolol). The proposed analytical method and the identified volatile compounds can contribute to the quality control of the FFTSS formula modernization and internationalization of this important preparation.

Financial support from the Austrian Federal Ministry of Science, Research and Economy, and the China Academy of Chinese Medical Sciences is gratefully acknowledged.

[1] Pharmacopoeia of the People's Republic of China, 10th edition, vol. 1, Chemical Industry Press, 2015 


\section{Tu-Poster Session 2-PO-171 DETERMINATION OF PHENOLIC COMPOUNDS IN Calendula officinalis $L$. FLOWER'S}

Authors Marques Fornseca Maira $C^{1}$, Gonçalves Rodrigues Das Dores $R^{2}$, Silva e Souza $C^{3}$, Nogueira Sedyama Maria $A^{1}$, Poltronieri $Y^{1}$

Institute 1 Empresa de Pesquisa Agropecuária de Minas Gerais, Viçosa, Brazil; 2 Universidade Federal de Ouro Preto, OURO PRETO, Brazil; 3 Universidade

Federal de Viçosa, Viçosa, Brazil

DOI 10.1055/s-0037-1608499

Calendula is cultivated in Brazil and widely used for medicinal purposes due to its healing, anti-inflammatory and antiseptic properties. Brazilian market does not provide vegetal drugs with biomarkers, within recommended range, thus, it is essential to study suitable technologies to produce high quantity and quality of vegetable raw. This work aimed to determinate the phenolic compound content in orange and yellow marigold flowers cultivated with increasing doses of cattle manure. The experiment was conducted at EPAMIG, Minas Gerais, Brazil. Marigold was cultivated in organic system using five doses of cattle manure $(0 ; 30 ; 60 ; 90 ; 120$ tha- 1$)$. Flowers were harvested, dried in air circulating oven $\left(40^{\circ} \mathrm{C}\right)$ and macerated in ethanol. The ethanol was evaporated $\left(40^{\circ} \mathrm{C}\right)$ to obtain the crude extracts. The phenolic compounds were determinate by spectrophotometry $(760 \mathrm{~nm})$. Crude extracts $(5 \mathrm{mg})$ were diluted in ethanol PA $(1000 \mu \mathrm{L})$, where, $200 \mu \mathrm{L}$ were added to Folin-Ciocalteu reagent $(1000 \mu \mathrm{L})$ and $7.5 \% \mathrm{Na} 2 \mathrm{CO} 3(1600 \mu \mathrm{L})$. The gallic acid was used in the standard curve and the results were expressed in $\mathrm{mg} / \mathrm{g}$. The mean contents of phenolic compounds were $679.20 \pm 173.65 \mathrm{mg} / \mathrm{g}(\mathrm{CV}=9.67 \%)$. Higher concentrations of phenolic compounds $(931.87 \mathrm{mg} / \mathrm{g})$ were found in orange flowers without fertilization (0tha- 1 ) and lower $(368.53 \mathrm{mg} / \mathrm{g}$ ) in yellow flowers fertilized with the highest dose of manure (120tha-1). In orange and yellow flowers extracts, treatments with fertilization of 30 t.ha- 1 and without fertilization had higher levels of phenolic compounds. Possibly, these compounds were produced in response to stress and adaptive need to the cultivation conditions. The dose-response curve was inversely proportional to manure doses in all treatments. Yellow flowers and high doses of manure (90 and 120tha-1) do not increase the production of phenolic compounds.

\section{Tu-Poster Session 2-PO-172 Cultivation of Black Cohosh: Non-targeted Chemical Profiling and Comparison to Wild}

Authors Bittner $\mathrm{M}^{1}$, Springer $\mathrm{A}^{2}$, Schenk $\mathrm{R}^{3}$, Melzig $\mathrm{MF}^{1}$ Institute 1 Freie Universität Berlin, Institute of Pharmacy, Pharmaceutical Biology, Berlin, Germany; 2 Freie Universität Berlin, Institute of Chemistry and Biochemistry, Core Facility BioSupraMol, Berlin, Germany; 3 HumboldtUniversität zu Berlin, Albrecht Daniel Thaer-Institute for Crop Science, Berlin, Germany

DOI 10.1055/s-0037-1608500

Black Cohosh (BC, Actaea racemosa (L.), Ranunculaceae) is a widely used herbal remedy. Wild-harvest in multi-tons leads to endangerment of the species. Efforts are made to establish cultivation. Nevertheless, different BC genotypes may exist [1], and physiography influences the rhizomes' chemical composition [2]. This can influence efficacy of herbal products.

In this study, we chemically profile BC rhizomes from cultivation and wildharvests. By non-targeted secondary metabolite profiling via LC/MS we explore differences between the two. We analysed methanol rhizome extracts of 42 specimen, from wild-harvests in the US $(n=8)$, and from worldwide sources $(n=26)$ together with clone-plants (vegetative propagation, $n=8$ ), both cultivated in Berlin. We generated metabolic data by high-resolution UPLC/ESI-QToF-MS analyses. For multivariate analysis, binning (bin-size $\mathrm{m} / \mathrm{z}$ 2) was performed [3].

Generally, composition of extracts from wild plants showed quantitative differences in comparison to cultivated. This is in line with previous findings [4]. In hierarchical cluster analysis (HCA), both entities grouped separately. Espe- cially, mass spectral bins representing triterpenes ( 37 bins with $\mathrm{FC} \geq 2$ /-LOG10 $(p) \geq 4$ (t-tests); i.e. bins $\mathrm{m} / \mathrm{z} 645 \_647(\mathrm{FC}=3.1 /$-LOG10 $(p)=10.3)$ or $661 \_663$ $(F C=2.3 /-\operatorname{LOG} 10(p)=6.2)$ were significantly more abundant in wild. Bins indicating fragments of polyphenols in (+)-ESI, like m/z 177_179 (FC=1.2/$\operatorname{LOG10}(p)=1.9)$ were not highly changed. In HCA of only cultivated plants samples clustered, too, supporting chemotype existence. Furthermore, clone-plants built a dense group, indicating homogeneity.

Growing conditions influence the compositions of extracts. Differences between i.e. wild/cultivated/chemotypes should be considered during clinical efficacy evaluation.

The authors thank Maged Sharaf, Ph.D. (AHPA) for provision of wild-harvested specimens.

[1] Motley T] et al. Proceedings of the Global Summit on Medicinal Plants 2004; 1: $112-118$

[2] Vickers A et al. Am J Plant Sci 2015;6:3272 - 3281

[3] Grace SC et al. BMC bioinformatics 2014;15Suppl11:S12

[4] Bittner M et al. Planta Med 2017;DOl:10.1055/s-0043- 108122

\section{Tu-Poster Session 2-PO-173 Profiling phenolic compounds in species of the genus Chuquiraga (Barnadesioideae, Asteraceae) by HPLC-DAD: species classification using chemometric models.}

Authors Ccana-Ccapatinta GV ${ }^{1}$, Da Costa FB ${ }^{1}$

Institute 1 AsterBioChem Research Team, Laboratory of Pharmacognosy, School of Pharmaceutical Sciences of Ribeira? o Preto, University of Sa? o Paulo, Ribeira? o Preto, Brazil DOI 10.1055/s-0037-1608501

Of the 22 species currently recognized for the genus Chuquiraga, at least 10 of them are used in the traditional medicine of several South American countries. Some species of this genus are widely commercialized, especially in markets of Ecuador and Peru. Event thought, little is known about the phytochemical composition of the genus Chuquiraga. This study aimed to profile phenolic compounds in commercial samples of three Chuquiraga species (C. jussieui, $C$. weberbaueri and C. spinosa) using HPLC-DAD and employ chemometric models (HCA and OPLS-DA) for species classification. A total of 127 samples were submitted to chromatographic analyses $(50 \% \mathrm{MeOH}$ extracts) on a Zorbax SBPhenyl column $(5 \mu \mathrm{m}, 4.6 \times 250 \mathrm{~mm})$ using a linear gradient of $\mathrm{H}_{2} \mathrm{O}(\mathrm{A})$ and $\mathrm{MeCN}(\mathrm{B})$, both with $1 \%$ acetic acid $(1.2 \mathrm{~mL} / \mathrm{min} ; 4 \% \mathrm{~B} \rightarrow 30 \% \mathrm{~B}$ in $50 \mathrm{~min}$, $100 \% \mathrm{~B}$, in $75 \mathrm{~min})$. The profiles acquired at $270 \mathrm{~nm}$ were selected and organized prior to baseline correction and alignment using the parametric time warping algorithm. HCA and OPLS-DA statistical analysis were then performed to evaluate similarities and classify the species. A set of 24 previously known pure phenolic compounds was used for peak identification (Fig. 1). The comparison of $R_{\mathrm{t}}$, UV spectra and co-injections enabled the identification of caffeic acid ester derivatives. On the HCA, three main clusters were observed: 1) samples of $C$. jussieui, from northern Peru and Ecuador, 2) samples of $C$. spinosa and $C$. weberbaueri, and 3 ) samples of $C$. jussieui, from southern Peru. Based on the OPLS-DA analysis, the three species were classified and two population of $C$. jussieui were discriminated. The occurrence of caffeic acid ester derivatives in the genus Chuquiraga is described for the first time. 


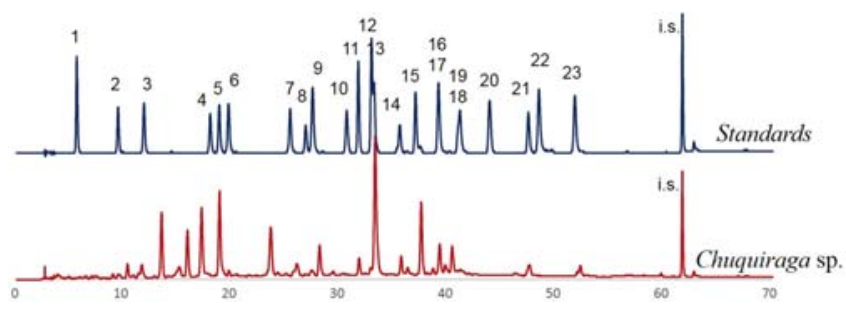

1 Galic acid

2 Protocatecuic acid

3 3-O-Caffeoylquinic acid

9 p-Coumaric acid

10 Ferulic acid

11 Rutin

12 Hyperoside

17 3,5-O-dicaffeoylquinic acid

18 Caffeic acid ester derivativ

19 4,5-O-dicaffeoylquinic acid

13 Quercetin-3-O-glucoronide 21 Methyl 3,4-O-dicaffeoylquinate

14 Caffeic acid ester derivative 22 Quercetin

5 5-O-Caffeoylquinic acid

6 Caffeic acid

15 3,4-O-dicaffeoylquinic acid 23 3-O-Methylquercetin

16 1,5-O-dicaffeoylquinic acid

i.s. Chrysin

Fig. 1 Set of phenolic compounds used for peak identification.

FAPESP (Grants\# 2014/ 16850 - 6 and 2014/26866 - 7) for funding.

\section{Tu-Poster Session 2-PO-174 Isolation of Antagonistic Microbes for Biological Control of Ginseng Root Rot}

Authors Lee KJ ${ }^{1}$, Jung $\mathrm{JS}^{1}$, Mo YM ${ }^{1}$, Yoon $\mathrm{YJ}^{1}, \mathrm{Kim} \mathrm{SI}^{1}$

Institute 1 Ginseng \& Medicinal Plants Research Institute, Gangwon

Agricultural Research \& Extension Services, Cheorwon, Korea, Republic of

(South)

DOI 10.1055/s-0037-1608502

Ginseng (Panax ginseng C.A. Meyer) is widely cultivated in Korea as a medicinal herb. Ginseng yield have recently been reduced by various factors, e.g. abnormal climate, disease and insect damage. Cylindrocarpon destructans, Fusarium solani and Sclerotinia sclerotiorum are major pathogens causing root rot disease in ginseng. Yield losses of ginseng reached up to $30-60 \%$ due to various diseases caused by $C$. destructans, F. solani and S. sclerotiorum during ginseng cultivation. It is well known that biological control using microorganism is eco-friendly method which can prevent disease in ginseng cultivation. Antagonistic microorganism can replace chemical agent, and reduce yield losses caused by several pathogens. In addition, antagonistic microorganism is also known to improve yield of ginseng by promoting the activity of plant root. For isolation of antagonistic microorganisms suppressing growth of pathogen, soil samples were collected from ginseng cultivation area and antimicrobial activities of isolates from soil were evaluated. Two strains that have antifungal activity against $C$. destructans, F. solani and S. sclerotiorum were isolated from soil. The optimum temperature ranges for growth of isolates were $25 \sim 30^{\circ} \mathrm{C}$ and both strains had protease activity. Analysis of the $16 \mathrm{~s}$ rDNA gene sequence revealed that these isolates belongs to genus Bacillus. 16 s rDNA gene sequence of each strain showed $99 \sim 100 \%$ of sequence similarity with B. subtillus and B. amyloliquefaciens, respectively. Ginseng root rot caused by pathogens was decreased $27.3 \%$ $56.7 \%$ by $B$. subtillus while decreased $18.9 \% \sim 67.5 \%$ by $B$. amyloliquefaciens (Table). These results suggested that these microbes could be used as biological control agent for prevention of major pathogens in ginseng cultivation.

Tab. 1: Rot symptom degree of ginseng root.

\begin{tabular}{|c|c|c|c|}
\hline Isolate & $C D^{a)}$ & FS & SS \\
\hline N.T. ${ }^{\text {b) }}$ & $3.0^{c)} \pm 00$ & $4.0 \pm 0.0$ & $3.7 \pm 0.5$ \\
\hline B. subtillus & $1.3 \pm 0.5$ & $1.7 \pm 0.5$ & $3.0 \pm 0.0$ \\
\hline B. amyloliquefaciens & $1.7 \pm 0.5$ & $1.3 \pm 0.5$ & $2.7 \pm 0.5$ \\
\hline
\end{tabular}

(deep brown color), 4 (severe rotted)

\section{Tu-Poster Session 2-PO-175 Growth characteristics and yield of ginseng (Panax ginseng C.A. Meyer) by irrigation set point in rain shelter house}

\author{
Authors Yoon $\mathrm{YJ}^{1}$, Jeong $\mathrm{HN}^{1}$, Jung $\mathrm{JS}^{1}$, Lee $\mathrm{KJ}^{1}$, Mo YM${ }^{1}$, Kim SI ${ }^{1}$ \\ Institute 1 Ginseng \& Medicinal Plants Research Institute, Gangwon \\ Agricultural Research \& Extension Services, Cheorwon, Korea, Republic of \\ (South) \\ DOI 10.1055/s-0037-1608503
}

Ginseng is used a worldwide well-known traditional herb. Recently, it has been reported that ginseng cultivation using rain shelter house can save workforce and reduce natural disaster damage compared with traditional shading structure. Especially, ginseng cultivation using rain shelter house is recognized more advantageous in northern region of Korea than southern region due to temperature. This study was conducted to investigate the growth characteristics and yield of ginseng by irrigation set point using rain shelter house in the northern region of Korea (Gangwon province). In order to compare the effect of irrigation set point on ginseng growth in rain shelter house, we measured growth characteristics and yield of 4 years old ginseng. Shading material of plastic film house was polyethylene film with two layered polyethylene net (PEF+PEN) $75 \%$ and the types of irrigation set point were 20,30 and $40 \mathrm{kPa}$. As a result, the average value of the raw weight of ginseng root was $66.8 \mathrm{~g}$ per root, and the yield of ginseng root was $3.61 \mathrm{~kg}$ per $1.67 \mathrm{~m}^{2}$ at $20 \mathrm{kPa}$ (Table). At the point of $20 \mathrm{kPa}$, the yield of ginseng root was the highest among the three set points and the miss-plated rate was the lowest. The order of tap root diameter and profit by irrigation set point was $20 \mathrm{kPa}>30 \mathrm{kPa}>40 \mathrm{kPa}$. We concluded that the $20 \mathrm{kPa}$ could be a good irrigation set point of rain shelter house for 4-year-old ginseng cultivation in northern region of Korea.

Tab. 1: Growth characteristics and yield of ginseng according to the irrigation set point

\begin{tabular}{|l|l|l|l|l|l|l|}
\hline $\begin{array}{l}\text { Irrigation } \\
\text { set point } \\
(\mathrm{kPa})\end{array}$ & $\begin{array}{l}\text { Miss-plated } \\
\text { rate } \\
(\%)\end{array}$ & $\begin{array}{l}\text { Root length } \\
(\mathrm{cm})\end{array}$ & $\begin{array}{l}\text { Tap root } \\
\text { diameter } \\
(\mathrm{mm})\end{array}$ & $\begin{array}{l}\text { Fresh weight } \\
(\mathrm{g} / \mathrm{root})\end{array}$ & $\begin{array}{l}\text { Root yield } \\
\left(\mathrm{kg} / 1.62 \mathrm{~m}^{2}\right)\end{array}$ & $\begin{array}{l}\text { profit } \\
(\text { Won/ } \\
\left.1.62 \mathrm{~m}^{2}\right)\end{array}$ \\
\hline 20 & $4.3 \pm 3.57$ & $33.6 \pm 4.29$ & $27.3 \pm 2.58$ & $66.8 \pm 16.4$ & $3.61 \pm 0.14$ & 104.724 \\
\hline 30 & $4.8 \pm 2.06$ & $31.2 \pm 3.97$ & $25.2 \pm 1.59$ & $61.5 \pm 20.1$ & $3.13 \pm 0.12$ & 90,569 \\
\hline 40 & $6.7 \pm 4.12$ & $33.3 \pm 5.65$ & $25.7 \pm 3.58$ & $60.9 \pm 19.7$ & $2.92 \pm 0.30$ & 84,544 \\
\hline
\end{tabular}

\section{Tu-Poster Session 2-PO-176 Emerging Application and Reflections of Cell Membrane Chromatography in the Quality Control of TCM: application study on three herbal medicines}

Authors Wang $Y^{1}$, Xie $Y^{1}$, Luo $G^{1}$

Institute 1 Tsinghua University, Beijing, China

DOI 10.1055/s-0037-1608504

Cell membrane chromatography (CMC) was first proposed by Professor He in 1996. As one of bioaffinity chromatography technique, CMC was a simple and convenient technology to study the interactions of active components in traditional Chinese medicines (TCMs) with membrane receptors in vitro, and screen active components from complicated TCMs. Recently, CMC technology was developed rapidly, which was widely applied in the discovery of lead compounds from nature product. Combining with our previous works in the analysis of the composition of complex substances, biochromatography and TLC bioautography for quality evaluation of TCM, a new holistic quality control strategy was proposed here, which could be summarized as the integration of screen (screening of quality control marker by CMC), macroscopic characterization (characterizing the chemical material basis by fingerprinting) and microscopic description (multi-component quantification). An antiplatelet aggregation activity related quality control method of salvianolic acids in Salviae Miltiorrhizae Radix Et Rhizoma (Danshen) were described here as an application example here. In this study, a platelet/CMC coupled with offline UPLCQTOF-MS/MS system was built to screen antiplatelet activity components from aqueous extract of Danshen, which serve as an agent of antiplatelet 
aggregation in Traditional Chinese Medicine. Rosmarinic acid, lithospermic acid salvianolic acid B were identified as the potential antiplatelet activity components. Moreover, rosmarinic acid, lithospermic acid, salvianolic acid B, salvianolic acid $C$ and danshensu were tested in platelet aggregation in vitro assay. The results suggested their retention time was closely related to the antiplatelet aggregation activities. Then rosmarinic acid, salvianolic acid $B$ and lithospermic acid were selected as "quality control markers" to evaluated the quality of Danshen and related extract. This study provides a rapid, effective and novel bioactivity related quality control strategy method of TCM, as well as a new method for screening the potential antiplatelet activity components from Chinese herb medicines.

\section{Tu-Poster Session 2-PO-177 Stability investigations on Rumex crispus ethanolic extracts}

Authors Demirezer Lutfiye $\mathrm{O}^{1}$, Uzun $\mathrm{M}^{1}$

Institute 1 1Department of Pharmacognosy, Faculty of Pharmacy, Hacettepe University, 06100, Ankara, Turkey

DOI 10.1055/s-0037-1608505

One of the most important quality control criteria is the stability of the botanical drugs [1]. According to FDA, it is necessary to protect $95 \%$ of the composition to say that it maintains the stability of a product or drug [2]. In this study, the quality control of Rumex crispus (Polygonaceae) was carried out by stability tests and HPLC, which were accepted by the authorities.

Ethanolic extracts of roots, leaves and fruits are subjected to accelerated stability tests at conditions of $40^{\circ} \mathrm{C} \pm 2{ }^{\circ} \mathrm{C}, 75 \pm 5 \% \mathrm{RH}$ and 6,000 lux $\pm 10 \%$. After 3 and 6 months the chemical profiles of the extracts were evaluated by HPLC. No microbiological contamination, weight loss and no change in physical properties were observed. All extracts protected physical stability and photostability. It has been found that all extracts maintain a chemical content of $95 \%$.

The fact that the extracts are cap-free has not been found to be detrimental to stability, but the change in the amount of the substance is much less when they are closed. All of the stability tests and HPLC analyzes showed that Rumex crispus was stable for 6 months.

This study was supported by The Scientific and Technological Research Council of Turkey (TUBITAK) (Project No:SBAG-214S 147.

[1] Connors KA, Amidon GL, Kennon L. Chemical stability of pharmaceuticals: a Handbook for Pharmacists: Wiley; 1979:367

[2] Fairweather WR, Lin TY, Kelly R. J Pharm Sci 1995; 84:1322 - 1326

\section{Tu-Poster Session 2-PO-178 Development of rapid method for detection of isomiroestrol in Pueraria candollei using immunochromatographic strip test}

Authors Chaingam $\mathrm{J}^{1,2}$, Kitisripanya $\mathrm{T}^{1,2}$, Tanaka $\mathrm{H}^{3}$, Putalun $\mathrm{W}^{1,2}$ Institute 1 Faculty of Pharmaceutical Sciences, Khon Kaen University, Khon Kaen, Thailand; 2 Research Group for Pharmaceutical Activities of Natural Products using Pharmaceutical Biotechnology (PANPB), National Research University-Khon Kaen University, Khon Kaen, Thailand; 3 Graduate School of Pharmaceutical Sciences, Kyushu University, Fukuoka, Japan

DOI 10.1055/s-0037-1608506

Pueraria candollei is a traditional herb widely used in Thailand as a source of phytoestrogen and was considered to be the standard herbal estrogen replacement therapy because of its highly estrogenic activity [1]. Isomiroestrol was a compound found in $P$. candollei. It has estrogenic activity the same as another miroestrol, deoxymiroestrol, and isoflavonoids thus isomiroestrol was used as an identical marker for P. candollei [2]. To date, LC-MS-MS, LCQ-Orbitrap/MS and enzyme-link immunoassay (ELISA) have been used to quantify isomiroestrol levels in plant samples which has several limitation such as long time-consuming and needed for expensive equipment [2-3]. Therefore, lateral-flow immunoassay using a colloidal gold-antibody probe was developed for the analysis of isomiroestrol in plant and product obtained from $P$. candollei. The competitive immunoassay was based on anti-isomiroestrol monoclonal antibody conjugated with colloidal gold-antibody (detection reagent). Secondary antibody was immobilized on one end of a nitrocellulose membrane (control line) and isomiroestrol-bovine serum albumin conjugate was immobilized on the other end (test line). Then eighteen different plant and products were tested using developed and validated method. The detection limit of isomiroestrol was $7 \mu \mathrm{g} / \mathrm{mL}$. Detection time was $15 \mathrm{~min}$. Our results demonstrated that the applied method was effective to detect isomiroestrol in $P$. candollei and its product.

[1] Chansakaow S, Ishikawa T, Seki H, Sekine K, Okada M, Chaichantipyuth C. J Nat Prod 2000; 63: $173-175$.

[2] Kitisripanya T, Jutathis K, Inyai C, Komaikul J, Udomsin O, Tanaka H, Putalun W. J Pharm Biomed Anal 2017; 137:229-234.

[3] Lee JH, Kim JY, Cho SH, Jeong JH, Cho S, Park HJ, Baek SY. J Chromatogr Sci 2017; 55: $214-221$.

\section{Tu-Poster Session 2-PO-179 Development of a colloidal gold nanoparticle based immunochromtographic strip for one step detection of puerarin and miroestrol}

Authors Krittanai $\mathrm{S}^{1,2}$, Kitisripanya $\mathrm{T}^{1,2}$, Udomsin $\mathrm{O}^{1,2}$, Putalun $\mathrm{W}^{1,2}$ Institute 1 Faculty of Pharmaceutical Science, Khon Kaen University, Khon Kaen, Thailand; 2 Research Group for Pharmaceutical Activities of Natural Products using Pharmaceutical Biotechnology (PANPB), National Research University-Khon Kaen University, Khon Kaen, Thailand

DOI 10.1055/s-0037-1608507

Pueraria candollei Wall. ex Benth (White Kwao Krua) is a Thai traditional plant that presents phytoestrogenic effect. Puerarin and miroestrol are bioactive compounds in this plant. Puerarin can also found in other plant in genus Pueraria including Pueraria lobata (Kudzu). However, miroestrol was found only in $P$. candollei. Therefore, this compound can be used as identical marker of $P$. candollei. Nowadays, various products derived from these plants were existed in the market especially in Asian country. Some counterfeit product can be problematic for the health of product consumer. The effective method for determination chemical marker of the plant should be applied to ensure the product quality. This study aims to investigate the amount of puerarin and miroestrol in plant samples and derived products of $P$. candollei and $P$. lobata using two immunoassay based methods. An immunochromatographic strip colloidal gold based detection system was developed in this study. On a strip membrane, anti-puerarin polyclonal antibody and anti-miroestrol monoclonal antibody [1] were equipped in the test spots. Therefore, both compounds can be detected in one step. In addition, indirect competitive enzyme-linked immunosorbent assay (icELISA) was performed to confirm reliability of the developed strip. The result showed high agreement between two methods. Among the plant samples, level of puerarin and miroestrol contents were fluctuated according to plant species, cultivated location, and the part of plant. In conclusion, the developed strip is an interesting alternative method for testing puerarin and miroestrol in samples. The strip is compact so it facilitate analysis in work field. Analysis procedure is straight forward. It is not required any complex sample preparation and result can be obtained within 10 minutes after dipped the strip into sample solution.

Kitisripanya, T, Krittanai, S, Udomsin, O, Jutathis, K, Komaikul, J, Juengwatanatrakul, T, Putalun, W. Planta Med 2017; http://doi.org/10.1055/s-0043102689. 


\section{Tu-Poster Session 2-PO-180 The behavior of some pesticide residues in stored medicinal plants samples}

Authors Drumea $V^{1}$, Olariu $L^{1}$, Nita $R A^{1}$

Institute 1 S.C. Biotehnos S.A, Otopeni, Romania

DOI 10.1055/s-0037-1608508

It is well known that there are many contaminants and residues that may cause harm to the consumers of herbal medicines. It is, therefore, essential to establish a convenient quality control method to assure the safety of herbal products (1). Knowing the behavior of residues in dried plants is a benefit for the direct consumer, for the processor, and for the analyst who must know the stability of samples during analytical methods validation and rutine sample testing. The purpose of testing was to evaluate the stability of 41 pesticides included in dried medicinal plants, at four time periods: one week, one month, 2 years and 4 years. The behaviour of residues was assessed by repeated analysis by comparing an initial fortified sample, properly stored, with a freshly prepared fortified sample. The quantitative analysis of samples was performed by QuEChERS methodology by a validated GC-MS method (2). Of each variant six repetitions were analyzed at each time. Statistical analysis was based on the comparison of the two averages obtained on each analysis time, by checking the significance of differences, calculating test statistic and comparing with the critical value (confidence level 95\%, N1+N2-2 degrees of freedom).The rate of pesticide movement has been evaluated for three calculated percent of remaining thresholds: below $20 \%$ : for some organophosphorous and organochlorine pesticides, between 20 - $80 \%$ interval: for some organophosphorous, organochlorine and acetamide pesticides and more than $80 \%$ : for some organochlorine and pyrethroid pesticides.

[1] Pérez-Parada A, Colazzo1 M, Besil N, Dellacassa E, Cesio V, Heinzen H. and Fernández-Alba R, Pesticides in the Modern World - Pesticide Residues in Natural Products with Pharmaceutical Use: Occurrence, Analytical Advances and Perspectives, Intech Open Sciences 2011: 357 - 390

[2] Łozowicka B, Jankowska M, Rutkowska E, Hrynko I, Kaczyński P, and Miciński J.: Journal of Natural Medicine 2014; $95-111$.

\section{Tu-Poster Session 2-PO-181 Effect of solar soil disinfection using Sudan grass blending in Ginseng Rain-sheltered Shade House}

Authors Won DY ${ }^{1}$, Choi Jin $\mathrm{K}^{1}$, Choi Yang $\mathrm{A}^{1}$, Jang Myeong $\mathrm{H}^{1}$, Jung Ji $\mathrm{Y}^{1}$, Kwon Tae $\mathrm{R}^{1}$

Institute 1 Punggi Ginseng Research Institute, Yeongju-si, Korea, Republic of (South)

DOI 10.1055/s-0037-1608509

The Ginseng Rain-sheltered Shade House is one of the efficient cultivation techniques as reducing the damages caused by natural disaster such as blight, high temperature \& humidity, dry, and heavy rain \& snow. However a soil description needs to be developed to reduce the period of re-cultivation because ginseng has to be cultivated at the only one ginseng rain-sheltered house without soil or cultivation change.

This study was carried out in the ginseng rain-sheltered house where ginseng had cultivated and harvested one time. Each section of cultivation of sudan grass+flooding, rice straw+flooding, and flooding was covered with vinyl and then, all areas were solarized through the summer season. The soil temperature was inspected at $5 \mathrm{~cm}, 10 \mathrm{~cm}, 15 \mathrm{~cm}$, and $20 \mathrm{~cm}$ below ground. As a result, the soil temperature of the place with vinyl covered and solarized was approached to $40^{\circ} \mathrm{C}$ repeatedly during the period of high temperature in July and August. In the sudan grass+flooding treatment, the number of days in which the soil temperature exceeded $40^{\circ} \mathrm{C}$ was 21 days at the depth of $20 \mathrm{~cm}$ underground. After the solar disinfection, the inspection result of Cylindrocarpon destructans spore density checked by Real time PCR was that $C$. destructans spores were not found at the place where sudan glass+flooding, and rice straw+flooding were used. However, the detected number of $C$. destructans spores in the inspection condition was roughly 9 at the soil samples from the fields which were flooded and solarized, and 33 at those from the repeatedly cultivated fields. The evaluations of aerial part growth by measurements were good in the following order: Sudan grass+flooding, rice straw+flooding and flooding. The evaluations of root weight by measurements were heavy in the following order: Rice straw+flooding, sudan grass+flooding and flooding. The incidence rate of the pathogens distantly decreased by the treatments of sudan glass+flooding and solar disinfection.

\section{Tu-Poster Session 2-PO-182 The Effect of Germanium Treatment on Rain Shelter House Cultivation of Korean Ginseng (Panax ginseng C. A. Meyer)}

Authors Kim DW ${ }^{1}$, Kim $\mathrm{CS}^{1}, \mathrm{Kim} \mathrm{HJ}^{1}$, Lee $\mathrm{YM}^{1}$, Kim HJ ${ }^{1}$, Kwon KB Institute 1 Jeollabukdo ARES Medicinal Resource Research Institute, Jinan, Korea, Republic of (South); 2 National Institute of Horticultural \& Herbal science, RDA, Eumseong, Korea, Republic of (South)

DOI 10.1055/s-0037-1608510

Ginseng (Panax Ginseng C. A. Meyer) is a perennial shade plant cultivated in Korea, China, U.S.A, Canada, and Germany. Ginseng is known to be effective in improving liver function, hypertension, diabetes, strengthening immunity, preventing aging, and improving memory. In general, Korean ginseng is cultivated in shading facilities covered with shade net. However, climate characteristics in Korea that include rainy season in the summer could lead to increase disease and pest in ginseng cultivation, and decrease production yield due to excess moisture injury. Therefore, cultivation technique using rain shelter house was developed as an alternative. Rain shelter house fundamentally can protect ginseng from rainwater which is the cause of disease and water injury resulting in increase of ginseng yield. We treated water-soluble germanium, which is known to enhance functionality of ginseng, to 3-yearsold ginseng grown in rain shelter house at a concentration of 500ppm and $1000 \mathrm{ppm}$. The weight of ginseng root treated with 500ppm and 1000ppm germanium solution were $26.7 \mathrm{~g}$ and $29.2 \mathrm{~g}$, respectively, while that of untreated group was $19.6 \mathrm{~g}$. The amounts of ginsenosides in Korean ginseng were higher in germanium treated group than untreated group. The contents of Rg1, Re, Rf, Rb1, Rc and Rb2 were $2.26 \mathrm{mg}, 4.10 \mathrm{mg}, 1.18 \mathrm{mg}, 3.83 \mathrm{mg} / \mathrm{Rc}$ $1.74 \mathrm{mg}$, and $1.53 \mathrm{mg}$, respectively(Figure). Total saponin contents were also higher in germanium treated groups, which show an increase of $44 \%$ and $40 \%$, respectively. These results represent that water soluble germanium might be a material to improve ginseng yield and saponin content in ginseng cultivation.

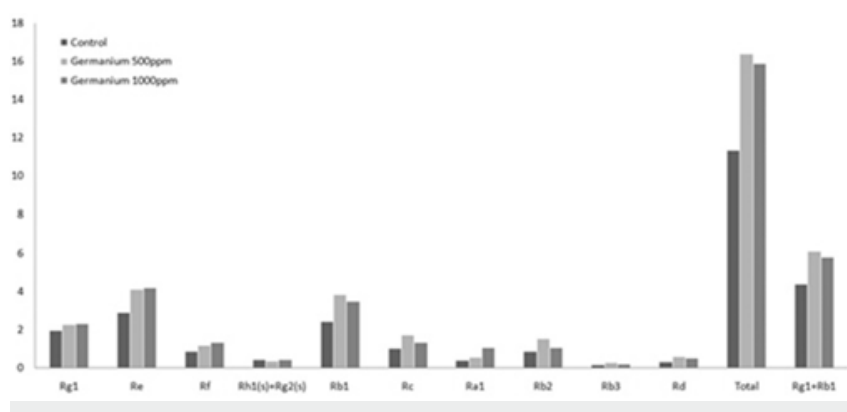

Fig. 1: Comparison of saponin| content in 3-year-old ginseng by treatment of germanium 


\section{Tu-Poster Session 2-PO-184 Effects of sowing time limitation and total rutin contents of buckwheat in Korea}

Authors Jung $\mathrm{CH}^{1}$, Kim SK

Institute 1 National Institute of Crop Science, RDA, suwon, Korea, Republic of (South)

DOI 10.1055/s-0037-1608511

Buckwheat is one of the traditional Korean crops which gains global attention as a health food due to its rich nutrition. Recent extreme weather events caused by climate change such as flooding and drought reduced crop production. Though, buckwheat grows during the fall season in Korea, flooding is one of severe environmental stress factors for buckwheat. The accumulated temperature of buckwheat was $1,093 \sim 1,515^{\circ} \mathrm{C}$ from sowing to harvest and $564 \sim 736^{\circ} \mathrm{Cfrom}$ flowering to harvest, respectively. Plant height, stem diameter, and dry matter weight were reduced as sowing date was delayed. The growing period of 'Yangjeol1' for autumn season was approximately 55 56 days. Total rutin content of the spring and the autumn buckwheat is 22.8 and $23.2 \mathrm{mg} / 100 \mathrm{~g}$, respectively, showing no difference in rutin content between both cultivation seasons. Quercetin content of the autumn ones was about $2.14 \mathrm{mg} / 100 \mathrm{~g}$ and was higher by about $30 \%$ than that of the spring ones. These results suggested that autumn buckwheat has more quercetin than spring one unlike rutin.

\section{Tu-Poster Session 2-PO-185 HPTLC general method for identification of polysaccharide containing herbal drugs and preparations thereof}

\section{Authors Oudkerk $S^{1}$, Vila $R^{1}$, Cañigueral $S^{1}$}

Institute 1 Unit of Pharmacology, Pharmacognosy and Therapeutics. Faculty of Pharmacy and Food Sciences. University of Barcelona, Barcelona, Spain DOI $10.1055 / \mathrm{s}-0037-1608512$

In the current edition of the European Pharmacopoeia (9 ${ }^{\text {th }}$ Ed.) (1), the chromatographic identification of polysaccharide rich herbal drugs (HD) and preparations thereof, such as mucilages and starches, is based on TLC analysis of monosaccharides released after hydrolysis. Nevertheless, this type of identification is lacking in a number of the monographs, HPTLC is not described in any of them, sample preparation is tedious and applies different conditions of hydrolysis, separation uses different mobile phases and three different detection reagents are applied. The aim of the present work was to establish an HPTLC harmonised and improved general method for identification of this type of HD and preparations, suitable for routine quality control, and adapted to the requirements of the new Ph.Eur. chapter 2.8.25 (1).

The following parameters were studied and harmonised: sample preparation (amount of sample, conditions of hydrolysis, post-hydrolysis sample treatment), HPTLC chromatographic separation (mobile phase, number of developments, and saturation of the chamber) and detection (four different reagents were tested). TFA $\left(100 \mathrm{~g} / \mathrm{L}, 120^{\circ} \mathrm{C}, 1 \mathrm{~h}\right)$ was selected for hydrolysis, and the posterior treatment was shortened thanks to the avoidance of the acid evaporation. An optimised separation was obtained using silicagel HPTLC plates, and double development with acetonitrile - water $(85: 15 \mathrm{v} / \mathrm{v})$ in unsaturated chamber. Diphenylamine - aniline - phosphoric acid reagent and observation under daylight was selected for detection. In addition, a system suitability test for plate qualification was established using the pair galactose and glucose.

The suitability of the method was positively assessed on more than 60 samples of $18 \mathrm{HD}$ and preparations, including acacia, spry- or roller-dried acacia, agar, carob gum, guar, guar galactomannan, Irish moss, ispaghula seed, ispaghula husk, kelp, psyllium seed and starches, as well as xanthan, of bacterial origin.

[1] EDQM. European Pharmacopoeia. $9^{\text {th }}$ Edition. Vol. 9.0. Strasbourg: Council of Europe (2016).

\section{Tu-Poster Session 2-PO-186 Development and validation of ultra-performance convergence chromatography method for quality control of Saposhnikoviae Radix}

Authors Kim Hyo $S^{1}$, Lee $A Y^{1}$, Choi $G^{1}$, Moon Byeong $C^{1}$

Institute 1 K-Herb Research Center, Korea Institute of Oriental Medicine,

Daejeon, Korea, Republic of (South)

DOI 10.1055/s-0037-1608513

Ultra-performance convergence chromatography $\left(\mathrm{UPC}^{2}\right)$ is an environmentally friendly analytical method that uses dramatically reduced amounts of organic solvents [1,2]. As the concept of "green chemistry" has grown more widely accepted, UPC ${ }^{2}$ has become an alternative and complementary method to the HPLC technique [1]. A rapid, sensitive, and environmentally friendly UPC method was developed for rapid quantification of four major chromones (cimifugin, 4'- $\beta$-D-glucosyl 5-O-methylvisamminol, s-O-glucosylhamaudol and prim-O-glucosylcimifugin) in Saposhnikoviae Radix using a Waters UPC ${ }^{2}$ Torus Diethylamine column $(2.1 \mathrm{~mm} \times 150 \mathrm{~mm}, 1.7 \mu \mathrm{m})$ with a runtime of $6.5 \mathrm{~min}$. This method validated according to the International Conference on Harmonization (ICH) guidelines for the validation of analytical procedures with respect to precision, accuracy, and linearity. The limits of detection of four chromones ranged from 0.01 to $0.03 \mu \mathrm{g} / \mathrm{mL}$, while the limits of quantitation ranged from 0.02 to $0.10 \mu \mathrm{g} / \mathrm{mL}$. The four chromones showed good regression $\left(r^{2}>0.999\right)$, excellent precision (RSD $<0.60 \%$ ), and acceptable recoveries (99.48-102.89\%). Comparison of system performance with conventional HPLC was made with respect to analysis time, efficiency and sensitivity. Quantification of four chromones in 13 commercial samples of Saposhnikoviae Radix was successfully performed using the developed UPC ${ }^{2}$ method. The identity and authenticity of Saposhnikoviae Radix were successfully monitored by the proposed UPC ${ }^{2}$ method. This study provided some references for quality control of Saposhnikoviae Radix.

[1] Geng Y, Zhao L, Zhao J, Guo B, Ma P, Li Y, Zhang T. J Chromatogr B 2014; 945: $121-126$

[2] Gong X, Qi N, Wang X, Lin L. Li ]. Food Chem 2014; 162: 172 - 175.

\section{Tu-Poster Session 2-PO-187 Distribution of secoiridoid glycosides in the root system of the medicinal plant Gentiana lutea L. subsp. aurantiaca}

Authors González-López Ó ${ }^{1}$, Mayo S ${ }^{1}$, Rodríguez-González Á1 , CarroHuerga $\mathrm{G}^{1}$, Suárez Villanueva $\mathrm{V}^{1}$, Berninger $\mathrm{T}^{2}$, Casquero $\mathrm{PA}^{1}$

Institute 1 Research Group of Engineering and Sustainable Agriculture, Research Institute of Environment, Natural Resources and Biodiversity, Universidad de León, Leon, Spain; 2 AIT Austrian Institute of Technology $\mathrm{GmbH}$, Center for Health \& Bioresources.Muthgasse 11, 1190, Vienna, Spain DOI 10.1055/s-0037-1608514

Gentiana lutea L. subsp. aurantiaca M. Lainz is an endemic medicinal plant from the Iberian Peninsula. Easily recognizable by the orange/reddish colour of its flowers, several other differences as regards habitat, genetics and concentration in bitter compounds have been reported when comparing subspecies aurantiaca with other $G$. lutea subspecies.

The present study evaluates the variability in the concentration of the main bitter compounds (secoiridoid glycosides): amarogentin, gentiopicroside, sweroside and swertiamarin, between the cortex and vascular tissues of gentian roots. Methanolic extracts from both tissues obtained from complete root systems of $G$. lutea subsp. aurantiaca wild collected in the Cantabrian Mountains (Spain) have been analysed by HPLC chromatography.

Obtained results show how the concentration of all analysed compounds is significantly higher in the cortex than in the vascular tissues, but as it can be observed in the Table 1 , the difference is much higher as regards the amarogentin. As known, with age the relative amounts of cortex and vascular tissues vary, and with it also the concentration of analysed compounds in the roots. These results underline the necessity of quality control measure- 
ments to ensure products with homogeneous characteristics and also suggest that the selection of optimal roots would increase the yield in secoiridoid glycosides.

Tab. 1: Secoiridoid glycosides (mg g of root dry weight) in the cortex/ vascular tissues in the roots of wild collected G. lutea L. subsp. aurantiaca. Different letters for every bitter compound are significantly different $(p \leq 0.05)$ according to Student's T test.

\begin{tabular}{|l|l|l|l|}
\hline & $\begin{array}{l}\text { Cortex } \\
\mathrm{mg} / \mathrm{g}\end{array}$ & $\begin{array}{l}\text { Vascular tissues } \\
\mathrm{mg} / \mathrm{g}\end{array}$ & $\begin{array}{l}\text { Variation } \\
\%\end{array}$ \\
\hline Gentiopicroside & $7.187 \mathrm{a}$ & $5.381 \mathrm{~b}$ & 33.6 \\
\hline Amarogentin & $0.088 \mathrm{a}$ & $0.024 \mathrm{~b}$ & 261.5 \\
\hline Sweroside & $0.174 \mathrm{a}$ & $0.108 \mathrm{~b}$ & 61.7 \\
\hline Swertiamarin & $0.370 \mathrm{a}$ & $0.309 \mathrm{~b}$ & 19.8
\end{tabular}

[1] Gonzalez-Lopez O. Polanco C. Gyorgy Z. Pedryc A. Casquero PA. Journal of Molecular Sciences 2014: 10052 - 10066

[2] Gonzalez-Lopez O. Carro G. Aiello N. Scartezzini F. Casquero PA. Planta Medica 2014; 80: 1489 - 1490

\section{Tu-Poster Session 2-PO-188 Phytochemical study of the Limonium leptophyllum plants and isolated substance}

Authors Zhussupova $\mathrm{A}^{1}$, Kalabaeva $\mathrm{A}^{1}$, Assemova $\mathrm{M}^{1}$, Shaheen $\mathrm{F}^{2}$, Zhussupova $\mathrm{G}^{1}$, Choudhary $\mathrm{I}^{2}$

Institute 1 Al-Farabi Kazakh National University, Almaty, Kazakhstan; 2 International Center for Chemical and Biological Sciences, Karachi, Pakistan DOI 10.1055/s-0037-1608595

From Limonium gmelinii a number of highly effecient medicinal preparations have been obtained possessing anti-inflammatory, antiviral and wound-healing activities with no allergic reactions and cumulative properties. Currently endemic plant Limonium leptophyllum was studied. Preparation of plant material collected in September, 2016 in Almaty region consisted in its drying and refinement to $3 \mathrm{~mm}$. In accordance with the requirements of the State Pharmacopoeia of the Republic of Kazakhstan, the authenticity for the studied type of medicinal plant material was established by the methods of macroscopy and microscopy. Quality indices were esteemed. Identification of various groups of biologically active compounds for substance standardization and assessment of active compounds for their comparative biological screening was carried out. The results obtained: humidity $-9.59 \%$; total ash $10.84 \%$; ash, insoluble in $10 \% \mathrm{HCl}-0.02 \%$; sulphated ash $-10.79 \%$. "Microbiological purity", "Heavy metals" and "Radionuclides" were established according to the norms for the medicinal plant materials. Pesticides and mycotoxins are not found. Varying the extraction conditions, a rational technological scheme was developed for obtaining a substance from the above-ground part of the plant species under study in the form of a dry extract. Extraction of the studied plant material was carried out twice with $50 \%$ ethanol at its ratio with the raw material of 1:6 for 6 hours at room temperature. The substance is a biologically active complex with a high hydrophilicity containing the main classes of biologically active natural compounds in the form of phenolic acids (gallic and ellagic), flavonols (quercetin and myricetin) both in free form and in the form of glycosides, hydrolyzed and condensed tannins, which are dimeric and oligomeric forms of flavan-3-ol. It also includes eight essential $\alpha$-amino acids, mono- and oligosaccharides, vitamins, microelements and other compounds. For the first time, aglikon apigenin is isolated from this genus.

\section{Tu-Poster Session 2-PO-190 Evaluation of transdermal drug-delivery system of capsaicin}

Authors Uzunovic $A^{1}$, Pilipovic $S^{1}$, Sapcanin $A^{2}$, Ademovic $Z^{3}$

Institute 1 Agency for medicinal products and medical devices of Bosnia and Herzegovina, Sarajevo, Bosnia and Herzegovina; 2 Faculty of Pharmacy,
Sarajevo, Bosnia and Herzegovina; 3 Faculty of Pharmacy, Tuzla, Bosnia and Herzegovina

DOI 10.1055/s-0037-1608515

Capsaicin has been employed topically to treat many diseases such as rheumatoid arthritis and nerve pain in diabetes. The short half-life of capsaicin by intravenous administration made topical application of capsaicin advantageous.

In this study, we have evaluated differences in the dissolution characteristics of capsaicin patch purchased from market, at different dissolution rotation speed.

The dissolution study was conducted using USP apparatus $5(n=6)$, ERWEKA DT800 dissolution tester and $500 \mathrm{~mL}$ of $\mathrm{pH} 7.4$ phosphate buffer as dissolution media. All dissolution studies were carried out at $32 \pm 0.5^{\circ} \mathrm{C}$ and different rotation speed $(50 \pm 5 ; 100 \pm 5$ and $150 \pm 5 \mathrm{rpm}) .5 \mathrm{ml}$ aliquots of samples were withdrawn at various time intervals (1, 4, 8 and 12 hours). Withdrawn were appropriate diluted and analyzed by HPLC.

The HPLC method proposed by Al Othman et al. (2011) has been optimized and validated for the separation and quantitation of capsaicin in a transdermal patch [1].

HPLC analysis was performed using ProntoSIL 120-3-C18AQ $125 \times 4,0 \mathrm{~mm}$ $(3 \mu \mathrm{m})$ column maintained at $60^{\circ} \mathrm{C}$. The mobile phase consisted of acetonitrile: water $(50: 50 \mathrm{v} / \mathrm{v})$, the flow rate of $0.9 \mathrm{~mL} / \mathrm{min}$, the injection volume $10 \mu \mathrm{L}$ and the detection wavelength $222 \mathrm{~nm}$.

The used HPLC method can be applied for fast (total run time was 4.0 minutes) and simultaneous analysis of capsaicin and dihydrocapsaicin in a transdermal patch.

We can conclude that the relative difference of dissolution rate of capsaicin after 12 hours was elevated by increase of dissolution rotation speed (100 rpm vs. 50 rpm: $87.1 \pm 12.1 \%$ and 150 rpm vs. 100 rpm: $39.6 \pm 8.7 \%$ ).

USP Apparatus 5 could be considered as a discriminatory test that would be able to point out the differences in the dissolution rate of capsaicin at different rotation speed.

[1] Al Othman ZA et al. Molecules 2011; 16:8919-8929.

\section{Tu-Poster Session 2-PO-191 Metabolite profiles of Labisia pumila and the discriminative analysis of the varieties using $1 \mathrm{H}$ NMR-based metabolomics approach.}

Authors Shaari $\mathrm{K}^{1}$

Institute 1 Laboratory of Natural Products, Institute of Bioscience Universiti Putra Malaysia, 43400 UPM Serdang, Selangor, Malaysia, Serdang, Malaysia DOI $10.1055 / \mathrm{s}-0037-1608516$

Medicinal plants and herbs have natural variation. The metabolite profile, and hence the biological properties of plant samples of different species, varieties, geographical origin localities or agronomic conditions, may vary quite significantly. Thus, the identification of crude herbs is crucial in order to ensure authenticity, quality, safety and efficacy of the raw material before it is converted to the final products. The chemical analysis techniques which should be unbiased, rapid, and reproducible, while requiring only simple sample preparation. Metabolomics is a comprehensive analysis of metabolites in biological samples using a combination of spectral platforms (e. g, NMR and mass spectroscopy) and multivariate statistical analysis. The approach has received a lot of attention due to its holistic nature and the information-rich results that can be obtained from it. In this paper, an NMR-based metabolomics approach was used to differentiate between three varieties of the medicinal plant Labisia pumila i.e L. pumila var alata, L. pumila var pumila and L. pumila var lanceolata. The medicinal herb is one of five herbs which have been prioritized as and Entry Point Project under the Agriculture NKEA (New Key Economic Area) program for Malaysia. The medicine plant is traditionally used to induce or facilitate labour and treat flatulence, dysentery, dysmenorrhoea, gonorrhoea, and bone sickness. L. pumila exerts an uterotrophic effect and regulates body 
weight gain by modulating secretion of leptin and resistin, and expression of the adipokines in adipose tissues. For mass cultivation of the plant, all three varieties were promoted. LCMS metabolite profiling of the varieties and NMRbased metabolomics discriminative analysis of the three varieties revealed that L. pumila var alata is differentiated from the other two varieties based on the presence of dexyloprimulanin. This paper will discuss the details of the analysis.

\section{Tu-Poster Session 2-PO-192 Case study of Allii Macrostemonis Bulbus: From chemical and metabolic profile to quality control by UPLC/Q-TOF-MS}

Authors Yao $Z^{1,2}$, Qin $Z^{1,2}$, Lin $P^{1,2}$, Hong $X^{1,2}$, Wang $L^{1,2}$, Dai $Y^{1,2}$, Yao $X^{1,2}$ Institute 1 Jinnan University, Guangzhou, China; 2 Guangdong Provincial Key Laboratory of Pharmacodynamic Constituents of TCM and New Drugs Research, College of Pharmacy, Jinan University, Guangzhou 510632, P.R. China; Guangzhou, China

DOI 10.1055/s-0037-1608517

Allii Macrostemonis Bulbus (AMB, named Xiebai in China) is a traditional Chinese medicine with medicinal values for the treatment of thoracic obstruction and cardialgia, and also a food additive. However, due to lack of available researches, the effective material of AMB still remains unknown and there is even no quantitative standard for $A M B$ recorded in the current edition of Chinese Pharmacopeia. In this study, we successfully investigate the chemical and metabolic profiles of AMB by ultra-performance liquid chromatography coupled with quadrupole time-of-flight tandem mass spectrometry (UPLC/QTOF-MS) using a "representative structure based homologous xenobiotics identification" strategy, which was proposed to address the challenges in complex chemical components and in vivo xenobiotics in our previous study. As a result, a total of 98 chemical components and 94 in vivo xenobiotics identified or tentatively characterized in AMB and its rat biosamples, respectively. To better understand metabolic characteristics, five representative single steroidal saponins were selected to investigate metabolite profiles. In addition, metabolic feature of saponins in AMB involved deglycosylation, glucuronidation, oxidation and dehydrogenation was proposed. Based on chemical and metabolic profile, fifty-four components were defined as quantitative markers for quality evaluation of AMB. The intra-day and inter-day precision test were less than $3.39 \%$ and $2.86 \%$, respectively. The repeatability, stability and recovery tests were all satisfactory. Furthermore, the validated method was successfully applied to evaluate the quality of multiple samples on the market. Among them, fifteen components were performed with exact quantification, while other thirty-nine components were conducted with semiquantification based on peak area. Taken together, our above study contributed to combining chemical and metabolic profile of AMB with multiple components quantitative analysis-based quality control method. Moreover, it would provide an evaluable example for the improvement of quality control of TCM on basis of the discovery of effective components.

\section{Tu-Poster Session 2-PO-193 Investigation of in vivo bioactive components and holistic quality control of Sarcandrae Herba}

Authors Yao $Z^{1,2}$, Lin $P^{1,2}$, Dai $Y^{1,2}$, Wei $Z^{1,2}$, Wang $Q^{1,2}$, Yao $X^{1,2}$ Institute 1 College of Pharmacy, Jinan University, Guangzhou 510632, P.R. China;, Guangzhou, China; 2 Guangdong Provincial Key Laboratory of Pharmacodynamic Constituents of TCM and New Drugs Research, College of Pharmacy, Jinan University, Guangzhou 510632, P.R. China;, Guangzhou, China DOI 10.1055/s-0037-1608518

Sarcandrae Herba (SH) is a common traditional Chinese medicine (TCM) for the treatment of inflammation and immunity related diseases. However, the current various quality control for SH do not really associate with bioactive makers due to its in vivo bioactive components have not yet been fully addressed, so that a universal international quality control cannot be developed.
In this research, an ultra-performance liquid chromatography coupled with quadrupole time-of-flight tandem mass spectrometry (UPLC/Q-TOF-MS) was adopted to describe the metabolic profiles after the oral administration of $\mathrm{SH}$ extract in rats and 24 prototype components and 26 metabolites were identified in biological samples of rats (plasma, urine, bile, feces). Then, peak areatime curves were adopted to preliminarily estimate the dynamic feature of 22 absorbed components in rat plasma and non-compartmental analysis (NCA) model were performed to calculate primary pharmacokinetic parameters by using WinNolin. Furthermore, a validated UPLC/Q-TOF-MS method was developed to study the plasma kinetics of the two bioactive and representative components (rosmarinic acid and isofraxidin) in rat plasma. Based on the in vivo bioactive components results, several components including coumarins, caffeoyl derivatives and flavonoids were selected as quality control components. Afterwards, a rapid identification method was established based on the Extractive Reference Substance (ERS), which covers the overall chemical profile of HPTLC for SH. A characteristic chromatogram containing principal components, anti-inflammatory active components and in vivo bioactive components as well as a Single Standard for Determination of Multiple components (SSDMC) method was developed and applied to the determination of six components of 38 batches of SH from 8 origins by UPLC. This study established a holistic quality control of $\mathrm{SH}$ on the basis of metabolic profiles and kinetics investigation, and it also proposed a strategy for the improvement of quality control for TCM based on the discovery of in vivo bioactive components.

\section{Tu-Poster Session 2-PO-194 Chromatographic Characterization of Hypophyllantin and Phyllantin in Methanolic Extracts from Three Common Phyllanthus Species in Malaysia.}

Authors Fazliyatun Shazlyana $\mathrm{M}^{1}$, Sharifah Aminah Syed $\mathrm{M}^{1}$, Emi Norzehan Mohamad $\mathrm{M}^{2}$

Institute 1 Faculty of Applied Sciences, Universiti Teknologi MARA, 40450

Shah Alam, Selangor, Malaysia; 2 Faculty of Applied Sciences, Universiti

Teknologi MARA, 35400 Tapah Road, Perak, Malaysia

DOI 10.1055/s-0037-1608519

Phyllanthus amarus, Phyllanthus niruri Linn and Phyllanthus urinaria are small medicinal herbs belonging to the Euphorbiaceae family which is widely distributed in tropical and subtropical countries [1]. The medicinal values possessed by this genus are due to the remarkable secondary metabolites present in order to treat various diseases such as liver cancer [2]. The objective of the study is to investigate the chromatographic profile of hypophyllanthin and phyllanthin from three commonly found Phyllanthus species in Malaysia by utilizing High Performance Liquid Chromatography (HPLC) technique to propose a reference standard. The chemical compounds were isolated from the whole plants of Phyllanthus samples by performing methanol extraction. The extracts then were subjected into reverse-phase chromatography by C-18 column to partitioning hydrophobic-lignan through gradient elution mode. The compounds in the extracts were identified by comparing their peaks with the same HPLC program run by the reference standard. The quantitative analysis for the quantification of both lignans contained in the Phyllanthus extracts showed that percentage of the both compounds ranged from $0.44 \%$ to $3.23 \%$ for hypophyllanthin and $2.98 \%$ to $18.51 \%$ for phyllanthin. Phyllanthus niruri Linn presented notable results in both recoveries of the markers. The reversed-phase HPLC method was validated by constructing graphs of linear calibration curve for both reference standards. The correlation coefficient (R2) values obtained were 0.9727 and 0.9257 for hypophyllanthin and phyllanthin, correspondingly. This investigation will aid the identification and authentication of species used in the commercialization of Phyllanthus-based products. [1] Webster, G. L. Ann Missouri Bot. Gard, 1994, 81:33 - 144.

[2] Sarin, B, Verma, N, Martin, J. P. and Mohanty, A. (2014). The Scientific World Journal, Volume 2014, Article ID 839172, 12 pages. 


\section{Tu-Poster Session 2-PO-195 Comparative quantitative and qualitative studies of extra virgin olive oil using HPTLC, HPLC-DAD, NMR, LC-HRMS \& MS/MS methods}

\begin{abstract}
Authors Beteinakis $S^{1}$, Stathopoulos $P^{1}$, Michailidis $D^{1}$, Angelis $A^{1}$, Argyropoulou $A^{1}$, Halabalaki $M^{1}$, Bonn $G^{2}$, Skaltsounis $\mathrm{AL}^{1}$

Institute 1 Department of Pharmacognosy and Natural Products Chemistry, Faculty of Pharmacy, University of Athens, Panepistimiopolis Zografou, Athens, Greece; 2 Institute of Analytical Chemistry and Radiochemistry, CCB-Center for Chemistry and Biomedicine, Innrain 80/82, 6020, Innsbruck, Austria DOI 10.1055/s-0037-1608520
\end{abstract}

Olive oil has a unique, mazy chemical composition that makes it a very complex and intriguing matrix for in-depth investigation [1]. Alterations in the concentration of certain constituents, dependent upon various factors, make olive oil a medley of compounds that corresponds differently in every assay even without changes in the experiment conditions. Biophenols, molecules in considerably low, yet with high impact, concentration in extra virgin olive oil, have gained increasing interest leading to a major health claim by the European Food Safety Authority (EFSA) in 2011 [2]. According to this claim, biophenols in olive oil are proven to have protective effects over LDL particles from oxidative damage. However, despite the numerous studies on its chemical composition, health benefits or proposed analytical techniques, there is still a large vacancy of major significance to be filled. The present study, aiming to be the key piece missing from the puzzle, will develop a series of comparative qualitative and quantitative novel methods of extra virgin olive oil's biophenols using state-of-the-art equipment comprised by Nuclear Magnetic Resonance (NMR) spectroscopy, High Performance Liquid Chromatography (HPLC) with Diode-Array Detection (DAD), Liquid Chromatography combined with High Resolution Mass Spectrometry and Mass Spectrometry/Mass Spectrometry (LC-HRMS and MS/MS) and High Performance Thin Layer Chromatography (HPTLC). Additionally, each technique displays both positive and negative elements, while preliminary data already available shows very promising results with high compliance between the different techniques.

[1] Angelis, A, Hamzaoui M, Aligiannis N, Nikou T, Michailidis M, Gerolimatos P, Termentzi A, Hubert J, Halabalaki M, Renault J-H, Skaltsounis A-L, 2017. Journal of Chromatography A 1491. 126-36. doi:10.1016/j.chroma.2017.02.046.

[2] EFSA. Scientific Opinion. EFSA Journal, 2011, 9, 2033. doi:10.2903/j. efsa.2011.2033

\section{Tu-Poster Session 2-PO-196 Chemical composition and antimicrobial activity relationship of essential oils from certain Myrtaceous plants using spectroscopic techniques coupled to chemometrics}

Authors El-Ahmady $\mathrm{S}^{2}$, Abdelrahman $\mathrm{E}^{1}$, Naumann $\mathrm{A}^{3}$, Schulz $\mathrm{H}^{3}$, Azzam $\mathrm{S}^{1}$, El-Kashoury ES ${ }^{1}$

Institute 1 Faculty of Pharmacy, Cairo University, Cairo, Egypt; 2 Faculty of Pharmacy, Ain Shams University, Cairo, Egypt; 3 Julius Kühn-Institut (JKI),

institute for Ecological Chemistry, Plant Analysis and Stored Product

Protection, Berlin, Germany

DOI 10.1055/s-0037-1608521

Antimicrobial resistance represents an increasingly serious global threat to public health as new resistance mechanisms emerge and spread all over the world. Special concerns are given to the emerging resistance rates to last-line antibiotics, especially in developing countries. In this context the evaluation of plant essential oils may serve as an alternative antimicrobial approach. Therefore, the essential oils from 15 species of three Myrtaceae genera (Syzygium, Eucalyptus, Melaleuca) harvested from botanical gardens (Egypt), were investigated regarding their individual chemical composition and antimicrobial activity. Their chemical profiles were analyzed using GC/FID, GC/MS, and ATR-IR and applying various chemometric algorithms (PCA and HCA). Eucalyptus camaldulensis and Corymbia citriodora showed the highest antimicrobial activity against Staphylococcus aureus ATCC 1578 (MIC 6 mg/ml), Staphylococcus aureus USA200 (12 \& $6 \mathrm{mg} / \mathrm{ml})$, MDR Enterobacter clocacae isolate $(12 \mathrm{mg} / \mathrm{ml})$, Klebsiella pneumoniae isolate (24 \& $12 \mathrm{mg} / \mathrm{ml})$, Bacillus subtilis ATCC CC33 (12, $1.5 \mathrm{mg} / \mathrm{ml})$ and E. coli ATCC $87(24 \mathrm{mg} / \mathrm{ml})$. Analysis by GC/FID and GC/MS allowed the identification of 46 compounds, representing 89 to $99.5 \%$ of the total oil composition. The main components were methyleugenol (0.24$95 \%)$, citronellal $(75-87.2 \%)$, 1,8-cineole $(0.19-77.7 \%)$, $\alpha$-pinene $(0.21-$ $64.8 \%)$, limonene $(0.18-44.1 \%)$, caryophyllene oxide $(0.23-42.8 \%)$, p-cymene $(0.19-34.9 \%)$ and crypton $(17.9-21.04 \%)$. E. camaldulensis was the richest species in $p$-cymene and crypton, while $C$. citriodora was the richest in citronellal. Therefore, citronellal, $p$-cymene and crypton could serve as potential candidates for developing novel antibiotics. Principal component analysis (PCA) and hierarchical cluster analysis (HCA) separated the 15 species essential oils into seven groups, each constituting a chemotype. ATR-IR analyses showed characteristic key bands that discriminated different chemotypes and correlated with those obtained by GC analysis. So, combination of vibrational spectroscopy and HCA could provide a fast and reliable method for chemotaxonomic characterization and control the purifying and redistilling processes of essential oils in the flavor and fragrance industry [1]

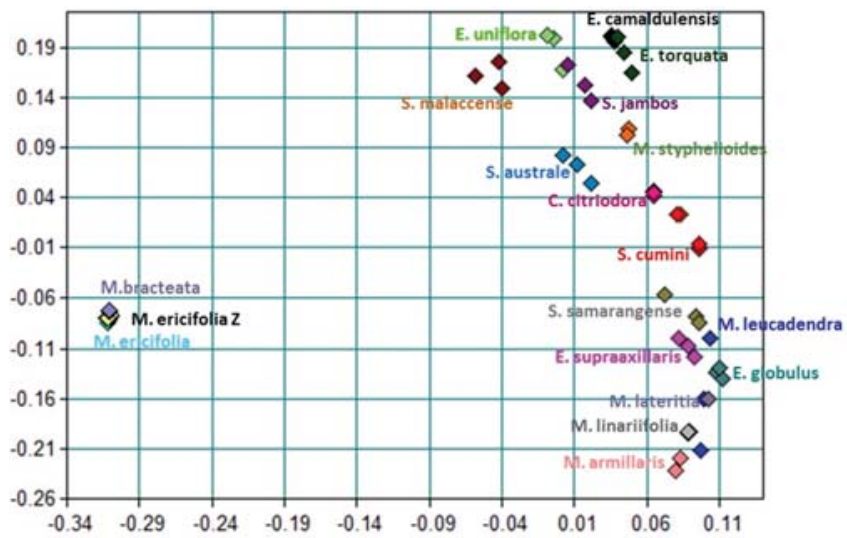

Fig. 1: Score 2 vs. Score 1

[1] Vibrational spectroscopy 2006; 42: 341 - 345

\section{Tu-Poster Session 2-PO-197 Microwave assisted extraction for determination of curcuminoid content in Curcuma longa rhizome}

Authors Sakunphueak $A^{1,2}$, Sribangrak $S^{1}$

Institute 1 Faculty of Pharmaceutical Sciences, Prince of Songkla University, Songkhla, Thailand; 2 Phytomedicine and Pharmaceutical Biotechnology Research Center, Faculty of Pharmaceutical Sciences, Prince of Songkla University, Songkhla, Thailand

DOI 10.1055/s-0037-1608522

Curcuma longa rhizome is a well-known herb that has been traditionally used in many countries. In Thailand C. longa have been added into the national drug list and widely used for treatment of dyspepsia instead of simethicone. Curcuminoid content in $C$. longa rhizome was used for control the quality of the raw material as described in Thai Herbal Pharmacopoeia (THP) [1]. The extraction method mentioned in THP was time consuming, it was performed by macerated C. longa powder in tetrahydrofuran for $24 \mathrm{hr}$ before analyzed with UV spectrophotometer. This study aimed to investigated the novel extraction method which are faster but still precise and accurate as well as the conventional method. Three different extraction methods including reflux extraction (RE), microwave assisted extraction (MAE) and ultrasonic assisted extraction (UAE), have been investigated and compared with the conventional method. It 
was found that MAE (900W, $30 \mathrm{~s}$ on/30 s off for 3 cycle) and UAE (90W, $37 \mathrm{kHz}$ for $30 \mathrm{~min}$ ) were able to extract curcuminoids as well as maceration for $24 \mathrm{hr}$, while RE for 30 min gave lower amount of curcuminoid content. Some parameters of MAE were optimized including power, cycle and extraction time. The optimized conditions were then evaluated on their intraday and interday precision in 40 samples both raw materials and commercial products. The result showed that the most appropriate conditions of MAE for extraction of curcumionoids were $900 \mathrm{~W}, 25 \mathrm{~s}$ on/ $30 \mathrm{~s}$ off for 4 cycle which showed \%RSD at $0.55 \%$ and $1.23 \%$ for intraday and interday precision, respectively. MAE was able to use as an alternative extraction method to the conventional one. The method is faster easier and more suitable for the routine analysis of curcuminoid content than the conventional method.

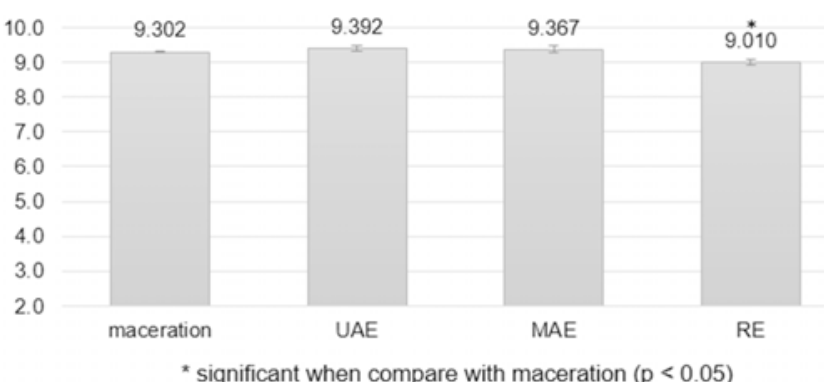

Fig. 1: curcuminoid content (\%)

[1] Department of Medical Sciences. Thai Herbal Pharmacopoeia, 1. Bangkok: Prachachon Co; 1995

\section{Tu-Poster Session 2-PO-198 Target- oriented Process design for the Identification and Isolation of Natural Products using Thin-Layer and Preparative Chromatography}

Authors Oberle $\mathrm{M}^{1}$, Sixt $\mathrm{M}^{2}$, Strube $\mathrm{J}^{2}$, Schulte $\mathrm{M}^{1}$, Uhlenbrock $\mathrm{L}^{2}$ Institute 1 Merck Life Science, Frankfurter Str. 250, 64293 Darmstadt, Germany; 2 Technical University Clausthal, Institute for Separation and Process Technology, Leibnizstrasse 15, 38678 Clausthal-Zellerfeld, Germany DOI 10.1055/s-0037-1608523

Thin-Layer Chromatography as well as Preparative Chromatography on silica sorbents are workhorses in natural product identification and isolation. Nevertheless, the process development using both techniques in combination is often based on trial and error and not examined in a systematical way. Only a few publications deal with the selection of chromatographic conditions and process development [1].

We will present general rules for the development of straightforward process design. Starting with the target-based screening, selection of appropriate solvents for extraction and analytical chromatography, the transfer of separations from the TLC-plate to process chromatography columns and the further optimisation of loading and throughput on the preparative HPLC-system. The importance of using the same silica selectivities will be shown as well as the use of TLC experiments to identify highly absorptive and labile compounds, which would be creating problems in the preparative separations.

[1] Weber,P, Hamburger,M, Schafroth,N, Pottereat, O, Fitoterapia 2011; 82: $155-161$

\section{Tu-Poster Session 2-PO-199 HPTLC fingerprint method for the detection of sennosides in Senna dry extracts}

Authors Meier $\mathrm{N}^{1}$, Meier $\mathrm{B}^{1}$, Peter $\mathrm{S}^{1}$, Wolfram $\mathrm{E}^{1}$

Institute 1 Zürich University of Applied Sciences, Institute of Chemistry and Biotechnology, Research Group of Phytopharmacy and Natrual Products, Wädenswil, Switzerland

DOI 10.1055/s-0037-1608524

In 2015 a new HPTLC method was proposed as part of the revision of the herbal drug monographs in the European Pharmacopeia Ph.Eur. of senna leafs and pods [1]. However, the proposed method was unspecific for the active constituents and showed difficulties with the repeatability in several laboratories. Therefore, alternative methods for fingerprinting were tested. The focus has been based on a fingerprint showing the active compounds which are mainly sennoside $A$ and $B$ and this in a fast and simple method.

The mobile phase used for senna folium in the pharmacopoeia of the people's republic of China in combination with potassium hydroxide reagent showed the best result in the separation and characteristic visualization of the sennosides. The sennosides show u-shaped zones which broaden with increasing application amount. The addition of acid to the mobile phase was tested but showed no improvement. The zones are under control by the applied amount. The sennosides $A$ and $D$ (zones [d]) are suitable as system suitability test for the method. However, sennosides D is not easily obtainable as reference substance; therefore, a senna dry extract CRS was used as cost-effective alternative. The chromatograms of senna leafs and fruits are comparable to the ones of the senna dry extracts shown.

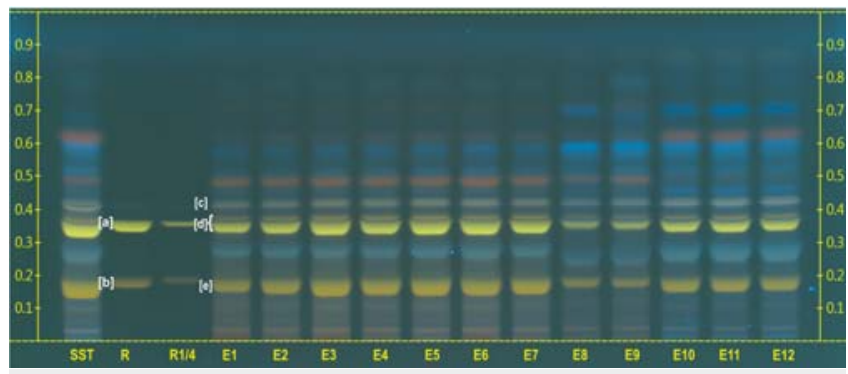

Fig. 1: Chromatograms of different senna dry extracts

SST: Senna extract CRS, R: sennoside A and B, R1 / 4: sennoside A and B diluted to 1/4, E1 - 7: different batches ethanolic fructus dry extract, E8/9: methanolic folia dry extracts, E10 - 12: aqueous fructus dry extracts

[1] EDQM. Draft for the revision of the monographs Senna leaf (0206), Senna pods, Alexandrian (0207) and Tinnevelly (0208) - PA/PH/Exp. 13A/T (15) 19 21 ANP. Pharmeuropa 27.3, $169-186$ (2015).

\section{Tu-Poster Session 2-PO-200 TLC as a screening and crosscheck method for natural products using the quantification of $\alpha$-and $\beta$-acids in hop as an example}

Authors Oberle $\mathrm{M}^{1}$, Engemann J', Pilakowski $\mathrm{V}^{1}$, Burholt $\mathrm{M}^{1}$

Institute 1 Merck KGaA, Life Science, Frankfurter Str. 250, 64293 Darmstadt, Germany

DOI 10.1055/s-0037-1608525

Hops are a very important base material for the production of beer. The substance Lupulin, isolated from the hops cones, is especially responsible for the bitterness and unique taste of a beer. That is why the amount of bitter acids in hops is very important for breweries. Lupulin contains various bitter acids [1], $\alpha$ - (humulone) and $\beta$ - (lupulone) acids, which vary in different types of hops. Caused by the content of bitter acids, hops are divided into aromatic 
hops ( $<10 \%$ of $\alpha$-acids) and bitter hops ( $>10 \% \alpha$-acids) [2]. In general, the $\alpha$ and $\beta$-acids are also divided into five homologues.

The investigation of different hop types has shown that the amount of $\alpha$-acid is both dependent on the hop category as well as on the region. It could also be determined that the $\beta$-acid is not related to these criteria and is similar in all hop samples regardless of whether the HPLC or TLC method was used. The amount of $\alpha$-acid, but not the $\beta$-acid, depends on the kind of hop (bitter or aromatic) and the region. Both methods, TLC and HPLC, were used in comparison to quantify $\alpha$ - and $\beta$-acids. With TLC a lot of samples can be analyzed in parallel in a short timeframe. Additional compounds can be visualized by using special derivatization reagents and all analytical information are available on the same TLC plate and is there for the quick screening and crosscheck method of choice. The HPLC had the better resolution and separated the hop acids in more homologues. Both methods have advantages for the analytical workflow and can be easily combined.

[1] Adrian Forster, Martin Biendl, Christiina Schönberger, Bernhard Engelhard, Andreas Gahr, Anton Lutz, Willi Mitter, Reinhard Schmidt (2012). Hopfen. Vom Anbau bis zum Bier, 1. Aufl. Carl, Nürnberg.

[2] B. Engelhard, a. Lutz, E. Seigner (2011). Hopfen für alle Biere der Welt.

\section{Tu-Poster Session 2-PO-201 Identification and quantification of herbal tea blend raw materials using hyperspectral imaging spectroscopy}

Authors Sandasi $\mathrm{M}^{1}$, Chen $\mathrm{W}^{1}$, Viljoen $\mathrm{A}^{1,2}$

Institute 1 Department of Pharmaceutical Sciences Tshwane University of Technology, Private Bag X680, Pretoria, South Africa; 2 SAMRC Herbal Drugs Research Unit, Tshwane University of Technology, Private Bag X680, Pretoria, South Africa

DOI 10.1055/s-0037-1608526

The consumption of herbal teas is increasing as consumers become more appreciative of the health benefits. Herbal tea blends, comprising of two or more plant species, are produced with the intention of improving taste and health effects through synergistic actions. As with foods, cosmetics and pharmaceutical products, quality control of herbal teas is important to ensure safety and efficacy. Chromatography-based techniques that are commonly used in quality control require sample preparation using solvents; thus, they are destructive. In this study, hyperspectral imaging spectroscopy is applied as a fast and non-destructive method for the quality control of herbal tea blends. The technique combines conventional spectroscopy and digital imaging to gather chemical information (spectral data) and visualise spatial distribution of chemical constituents within a matrix. Certified raw materials (Sceletium tortuosum and (yclopia genistoides) and herbal tea blends were sourced from a local supplier. Hyperspectral images of the raw materials and tea blends were captured separately on a sisuChema SWIR (short wave infrared) hyperspectral pushbroom imaging system. The images were analysed using Evince ${ }^{\circledR}$ multivariate analysis software 2.4.0. Principal component analysis (PCA) revealed $52.9 \%$ chemical variation between $S$. tortuosum and $C$. genistoides raw materials. Partial least squares-discriminant analysis (PLS-DA) models were developed to predict the plant species present in the blend and determine the relative proportions. Based on pixel classification it was possible to visualise the tea blend constituents as S. tortuosum and C. genistoides and quantitatively predict $C$. genistoides as the major constituent (>95\%) while S. tortuosum was present in relatively lower amounts $(<5 \%)$. The observed predictions are close to the company formulation and thus $\mathrm{HSI}$ in tandem with multivariate data analysis tools present a useful alternative in the quality control of herbal products.

\section{Tu-Poster Session 2-PO-202 Exploring the phytochemical variation of the "Pepper-bark" tree (Warburgia salutaris) using HPTLC and UPLC-MS}

\author{
Authors Leonard $C^{1}$, Chen $W^{1}$, van Vuuren $S^{3}$, Viljoen $A^{1,2}$
}

Institute 1 Department of Pharmaceutical Sciences, Faculty of Science, Tshwane University of Technology, Pretoria, South Africa; 2 SAMRC Herbal Drugs Research Unit, Faculty of Science, Tshwane University of Technology, Pretoria, South Africa; 3 Department of Pharmacy and Pharmacology, University of Witwatersrand, Johannesburg, South Africa DOI 10.1055/s-0037-1608527

Warburgia salutaris, commonly known as the "pepper-bark" tree, is an evergreen tree found in the southern parts of Africa and widely used for its antimicrobial properties. The bark is the most often used part and destructive practices such as "ring-barking" and excessive harvesting has resulted in Warburgia salutaris being classified as an endangered species. In this study, various methods were used to explore the phytochemical variation between wild-type and cultivated bark, root and leaf specimens from various geographical locations. Ultra performance liquid chromatography coupled to mass spectrometry (UPLC-MS) was used to generate data and subsequently analysed using multivariate data analysis methods to determine the variation, similarities and trends of the data. High performance thin layer chromatography (HPTLC) was used to develop chemical fingerprints to visually distinguish plant material (e.g. roots and leaves). Chemometric analysis of the chromatography data indicated that most of the variation could be attributed to seasonal variation, age of the plant the plant part and not whether the specimen originated from a wild or cultivated source. Bioactivity has mainly been attributed to the drimane sesquiterpenes, especially polygodial. In these specimens, the polygodial content ranged from 0.1 to $67.8 \mu \mathrm{g} / \mathrm{g}$ in wild bark and 0 to $0.6 \mu \mathrm{g} / \mathrm{g}$ in wild leaf. The cultivated bark specimens contained higher ranges of polygodial ranging from 11.0 to $174.0 \mu \mathrm{g} / \mathrm{g}$ while low values were documented for the cultivated leaf specimens ( 0 to $0.9 \mu \mathrm{g} / \mathrm{g}$ ). The HPTLC chemical fingerprint with distinguishing bands allowed for visual discrimination between plant parts. However, within geographical location groups, the phytochemical profiles for all specimens were conserved. The HPTLC method developed can easily be implemented as a screening tool to discriminate between large batches of bark and leaf material.

\section{Tu-Poster Session 2-PO-203 Development of an HPTLC identification method in new Ph Eur monograph for Rubi ideae folium (Raspberry leaves)}

Authors Wolfram $E^{1}$, Meier $N^{1}$, Peter $S^{1}$, Meier $B^{1}$

Institute 1 ZHAW, ICBT, Research Group of Phytopharmacy and Natrual

Products, Wädenswil, Switzerland

DOI 10.1055/s-0037-1608528

Rubi ideae folium, a traditional remedy for pain relief and smooth muscle relacation especially as support before and during child delivery has been recently assessed by the HMPC [1] and has not yet been monographed in the European Pharmacopeia. The development of the valid quality control methods for identification, contaminants and content assay is ongoing. This study developed an HPTLC method according to the novel.

Four different mobile phases have been tested until the ideal mix of anhydrous formic acid R, water R, ethylacetate $R(10: 10: 80, V / V / V)$ was chosen for good fingerprint separation. Hyperosid ( $2 \mathrm{mg} / 10 \mathrm{~mL}$ in methanol) und Chlorogenic acid (2 mg/ $10 \mathrm{~mL}$ in methanol) serve as System Suitability Solution for SST and Rutin (2 mg in $10 \mathrm{~mL}$ Methanol) applied as Rf reference value.

The results are a clear and well separated fingerprint - the prominent yellow band has not yet clearly identified. Results from TLC-ESI-MS and TLC-MALDI analysis give mass values which suggest the presence of quercitin glucoronide. However these results need to be confirmed by further in depth isolation and structure elucidation invetigations. 
[1] EMA (2012), Assessment report on Rubus idaeus L, folium. Commitee on Herbal medicinal products. EMA/HMPC/44209/2012

[2] EDQM (2017), 2.8.25. High performance thin-layer chromatography of herbal drugs and herbal drug preparations. Ph Eur Edition 9.0

\section{Tu-Poster Session 2-PO-204 Storage stability assessment of royal jelly skin care products by trans-10-hydroxy-2-decenoic acid (10-HDA) determination}

Authors Uzunovic $A^{1}$, Hatibovic $D^{2}$, Pilipovic $S^{1}$, Sapcanin $A^{3}$, Tahirovic ${ }^{4}$ Institute 1 The Control Laboratory of Agency for Medicinal Products and Medical Devices of Bosnia and Herzegovina, Sarajevo, Bosnia and Herzegovina; 2 Faculty of Pharmacy, Travnik, Bosnia and Herzegovina; 3 Faculty of Pharmacy, Sarajevo, Bosnia and Herzegovina; 4 Faculty of Science, Sarajevo, Bosnia and Herzegovina

DOI 10.1055/s-0037-1608529

Royal jelly is an important bioactive compound that possesses several health promoting properties. It has been widely used in commercial medical products, healthy foods and cosmetics in many countries. Trans-10-hydroxy-2decenoic acid (10-HDA) is the main fatty acid present in royal jelly and the 10HDA content has been proposed as a freshness parameter for royal jelly quality analysis [1]. This work has been aimed to assess the 10-HDA stability (storage temperature $2-8^{\circ} \mathrm{C}$ ) in 5 different home-made royal jelly skin care products, presented in Table 1.

Tab. 1: Composition of royal jelly skin care products

\begin{tabular}{|c|c|c|c|c|c|}
\hline Component & Sample 1 & Sample 2 & Sample 3 & Sample 4 & Sample 5 \\
\hline Frash royal jelly & $5 \%_{w / w}$ & & & $5 \%_{w / w}$ & $10 \%_{w / w}$ \\
\hline Lyophilized royal jelly & & $5 \% w / w$ & $5 \%$ w/w & & \\
\hline Cream base & & & $\mathrm{x}$ & & \\
\hline Sweet almond oil & $x$ & $\mathrm{x}$ & & $\mathrm{x}$ & $\mathrm{x}$ \\
\hline Cocoa butter & $\mathrm{x}$ & $\mathrm{x}$ & & $\mathrm{x}$ & $\mathrm{x}$ \\
\hline Beeswax & $\mathrm{x}$ & $\mathrm{x}$ & & $\mathrm{x}$ & $\mathrm{x}$ \\
\hline Distilled water & $\mathrm{x}$ & $\mathrm{x}$ & $\mathrm{x}$ & $\mathrm{x}$ & $\mathrm{x}$ \\
\hline Grape seed oil & $\mathrm{x}$ & $x$ & $x$ & $\mathrm{x}$ & $x$ \\
\hline Lavender oil & $\mathrm{x}$ & $\mathrm{x}$ & $\mathrm{x}$ & $\mathrm{x}$ & $x$ \\
\hline Bee pollen & & & & $2 \% w / w$ & \\
\hline Propolis & & & & $2 \%_{w / w}$ & \\
\hline Honey & & & & $2 \%$ w/w & \\
\hline
\end{tabular}

The HPLC method proposed by Kim et al. (2010) has been optimized and validated for the determination of 10-HDA in royal jelly skin care products [2]. All analyses were performed on a Waters Acquity UPLC system, equipped with a variable wavelength UV absorbance detector, operated with Waters EmpowerTM 2 software. UPLC analysis was performed using Agilent Poroshell $1202,7 \mathrm{um}(100 \mathrm{~mm} \times 4,6 \mathrm{~mm})$ column, adjusted to $25^{\circ} \mathrm{C}$ as a column temperature. The maximum absorbance of $10-\mathrm{HDA}$ was confirmed to be $215 \mathrm{~nm}$. The mobile phase was a mixture of methanol, water, and phosphoric acid $(55: 45: 2.7, \mathrm{v} / \mathrm{v} / \mathrm{v})$ and the flow rate was $1.0 \mathrm{~mL} / \mathrm{min}$. The total run time of each injected sample was $5 \mathrm{~min}$, as indicated in the figure below. 10-HDA content in home-made royal jelly skin care products after 80 days varies from $0.52 \pm 0.44$ to $91.61 \pm 1.73$ percent of the initial content of 10 -HDA $(2 \mathrm{mg} / \mathrm{g})$. According to the results obtained in this study, we confirm the impact of change in composition on stability of the finished products.

\section{Tu-Poster Session 2-PO-205 In vitro Cytotoxic Activity against Three Lung Cancer cells of Apaisalee Remedy Extracts and Its Plant Ingredients.}

Authors Itharat $\mathrm{A}^{1,2}$, Makaratat $\mathrm{N}^{3}$

Institute 1 Department of Applied Thai Traditional Medicine, Faculty of Medicine, Thammasat University, Pathumthani, Thailand; 2 Center of Excellence on Applied Thai Traditional Medicine Research, Thammasat University, Pathumthani, Thailand; 3 Student of Master of Science Program
(Applied Thai Traditional Medicine), Faculty of Medicine Thammasat University, Pathumthani, Thailand

DOI 10.1055/s-0037-1608530

Apaisalee [APSL] is a Thai traditional remedy in NLEM (National List of Essential Medicines) of Thailand. It used to treat COPD and reduce inflammatory in lung cancer. This remedy composed with 19 plant species[1]. However, there is no report on cytotoxic effect on lung cancer cell. Thus, the objective of this study is to investigate cytotoxic activity of the extracts of APSL and its plant ingredients against three types of human lung cancer cells such as A549, NCl-H226 and COR-L23. APSL and its plant ingredients were extracted by maceration in $95 \%$ ethanol and boiling in water. All extracts were tested for cytotoxic activity by sulforhodamine B (SRB) assay [2]. The ethanolic extract of APSL showed cytotoxic activity against A549, NCl-H226 and COR-L23 with $\mathrm{IC}_{50}$ values of $34.75 \pm 1.93,88.72 \pm 2.93$ and $10.16 \pm 1.67 \mu \mathrm{g} / \mathrm{ml}$, respectively, but the water extract of APSL had no cytotoxic activity $\left(\mathrm{IC}_{50}>100 \mu \mathrm{g} / \mathrm{ml}\right)$. The ethanolic extract of Clausena excavate showed the highest cytotoxic activity against A549, NCl-H226 and COR-L23 with IC 50 values of $0.01 \pm 0.00,0.014 \pm 0.003$ and, $1.18 \pm 0.06 \mu \mathrm{g} / \mathrm{ml}$, respectively. The water extract of Clausena excavate also showed the highest cytotoxic activity against A549 and COR-L23 with $\mathrm{IC}_{50}$ values of $28.12 \pm 2.25$ and $10.07 \pm 1.28 \mu \mathrm{g} / \mathrm{ml}$ respectively but it had no active on $\mathrm{NCl}-\mathrm{H} 226\left(\mathrm{IC}_{50}>100 \mu \mathrm{g} / \mathrm{ml}\right)$. The water extract of Syzygium aromaticum exhibited the highest activity against $\mathrm{NCl}-\mathrm{H} 266$ with $\mathrm{IC}_{50}$ as 28.39 $\pm 1.30 \mu \mathrm{g} / \mathrm{ml}$. These results support the use of APSL remedy in Thai traditional medicine for lung cancer treament and Clausena excavate is recommended as active plant ingredient of this remedy.

[1] Champasuri S and Itharat A Master thesis, Medical Sciences, Faculty of Medicine, Thammasat University, Thailand;2015.

[2] Skehan, P. et al J Natl Cancer Inst 1990; 82:1107 - 1112.

\section{Tu-Poster Session 2-PO-206 From ethnobotany to the laboratory: a computer aided ethnobotanic method for the identification of plants with interesting biological activities}

Authors Tittikpina $\mathrm{NK}^{1,2,4,5}$, Atakpama $\mathrm{W}^{2}$, Pereki $\mathrm{H}^{2}$, Fontanay $\mathrm{S}^{4,5,6}$, Nana $F^{4,5}$, Ejike Chukwunonso $\mathrm{ECC}^{7}$, Kirsch $\mathrm{G}^{4,5}$, Duval Raphael $\mathrm{E}^{4,5,6}$, Chaimbault $\mathrm{P}^{4,5}$, Karou Simplice $\mathrm{D}^{3}$, Batawila $\mathrm{K}^{2}$, Akpagana $\mathrm{K}^{2}$, Jacob $C^{1}$ Institute 1 Faculty of Pharmacy, Department of Bio-organic Chemistry, Building B 2.1, Room 1.13. Saarland State University, Campus D-66123, Saarbrücken, Germany; 2 Laboratoire de Botanique et Ecologie Végétale, Université de Lomé, BP 1515, Lomé, Togo; 3 Ecole Supérieure des Techniques Biologiques et Alimentaires (ESTBA-UL), Université de Lomé, Lomé, Togo; 4 Université de Lorraine, SRSMC, UMR 7565, 54001, Nancy, France; 5 CNRS, SRSMC, UMR 7565, ICPM, 1 boulevard Arago 57078, Metz, France; 6 ABC Platform $^{\circledR}$, Faculté de Pharmacie, F-54001, Nancy, France; 7 Department of Medical Biochemistry, Faculty of Basic Medical Sciences, Federal University Ndufu-Alike, Ikwo, PMB 1010, Abakaliki, Ebonyi State, Nigeria

DOI 10.1055/s-0037-1608531

The investigation of natural products used in Traditional Medicine in Africa is complicated as modern analytical and screening methods are often not available. Computer aided product identification from traditional usage records (CAPITURE) may provide an interesting alternative and has been evaluated in the context of an ethnobotanical survey on fungal diseases and their traditional treatment in Tchamba District (Togo). 53 traditional healers were interviewed and their knowledge, recorded [1]. Several indicators, the Use Value (UV), Plant Part Value (PPV), Specific Use (SU) value, Intraspecific Use Value (IUV) and Informant Consensus Factor (ICF), were applied to the data [2,3]. Those indices, in addition to a bibliographic review, were then fed into a computer-aided approach which predicted two interesting plants (out of the 43 species survey-recorded) and their specific activities: Pterocarpus erinaceus sap against ringworm, Daniellia oliveri sap against intertrigo and respectively their roots and barks against candidiasis. Subsequent laboratory studies have 
confirmed the predicted antimicrobial activities with MIC $(128 \mu \mathrm{g} / \mathrm{ml}$ to $30 \mathrm{mg} / \mathrm{ml}$ ) and without any notable toxicity on normal human cells (MRC-5 cells). Although such method may not be flawless, it provides first leads, and in the face of limited resources, is an attractive alternative worth considering.

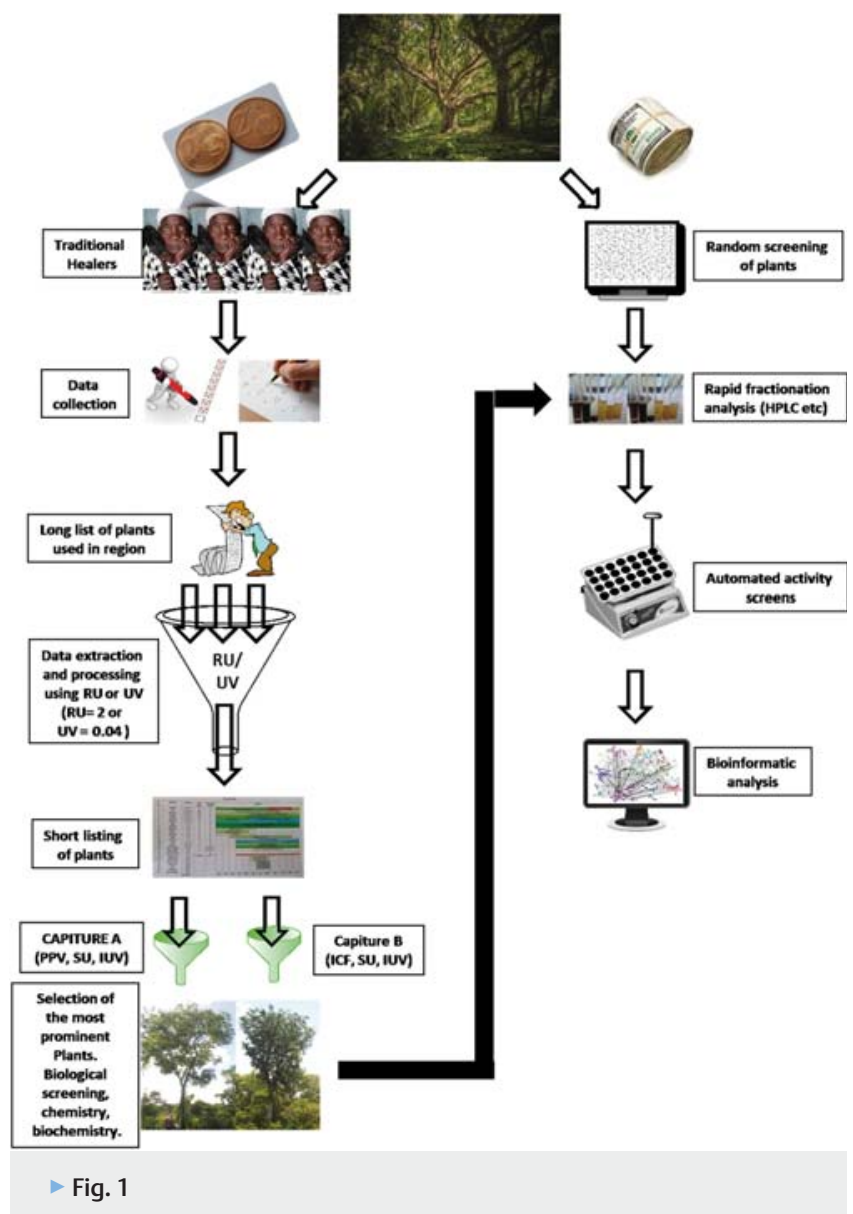

[1] Tittikpina, N. K. (2012). Contribution à l'évaluation des propriétés antimicrobiennes de: Pterocarpus erinaceus Poir (Fabaceae), Daniellia oliveri (Rolfe) Hutch. et Dalz (Fabaceae) et Anchomanes difformis (Blume) Engler (Araceae), utilisées en médecine traditionnelle dans la Préfecture de Tchamba (TOGO). [Th. D. Pharm]. [Univ. Lomé]: 89

[2] Gomez-Below, A. Economic Botany 2002, 56, 231 - 241.

[3] Gazzaneo, L. R. S, Paiva de Lucena, R. F, de Albuquerque, U. P. Journal of Ethnobiology and Ethnomedicine 2005, 1, DOI: 10.1186/1746 - 4269-1-9.

\section{Tu-Poster Session 2-PO-207 In vitro evaluation of the antimicrobial activity of fungus-growing termites Macrotermes bellicosus used in traditional medicine in Benin}

Authors Hammoud $D^{1}$, Chougourou $D^{2}$, Vissiennon $Z^{1}$, Ahyi $V^{1}$, Nieber $K^{3}$, Vissiennon $C^{4}$

Institute 1 IRGIB Africa University, Inter-Regional University of Industrial Engineering, Biotechnologies and Applied Sciences, Cotonou, Benin;

2 Department of Management of Environment, Polytechnic School of Abomey-Calavi (EPAC), University of Abomey-Calavi (UAC), Cotonou, Benin; 3 University of Leipzig, Institute of Pharmacy, Leipzig, Germany; 4 University of Leipzig, Medical Faculty, Institute of Medical Physics and Biophysics, Leipzig, Germany

DOI $10.1055 / \mathrm{s}-0037-1608532$
Background: Insects and insect-derived products have been widely used in folk healing in many parts of the world since ancient times. Among these insects, fungus-growing termites play an important role in nutrition and traditional medicine in various societies in tropical Africa.

Aim of the present study was to explore the traditional therapeutic uses of the fungus-growing termites Macrotermes bellicosus collected from different regions in Benin and subsequently investigate their antimicrobial potential.

An ethnomedicinal survey with active healers in Benin was conducted and the antimicrobial activity of ethanolic extracts of Macrotermes bellicosus against selected pathogenic microorganisms was investigated using the Kirby-Bauer disc diffusion method. Inhibition zone diameter (IZD) and minimum inhibitory concentrations (MIC) were derived to characterize the antimicrobial activity. Amoxicillin was applied as positive control.

The ethnomedicinal survey revealed that fungus-growing termites and its products (termites' mounds and fungus comb) are traditionally used in Benin to treat infectious and inflammatory diseases including digestive disorders, diarrhea and snake bites (Fig 1). Ethanolic extracts $(90 \% \mathrm{~V} / \mathrm{V})$ of species from Macrotermes bellicosus collected in Abomey-Calavi (T1S), Sekou (T2S) and Lokossa (T3S) showed antimicrobial activity comparable to amoxicillin $(25 \mu \mathrm{g} /$ disc) against Staphylococcus aureus (T1S:IZD $=15 \pm 0.1 \mathrm{~mm})$, Pseudomonas aeruginosa (T2S:IZD = $12 \pm 0.1 \mathrm{~mm}$ ) and Escherichia coli (T3S:IZD $=15 \pm 0.1 \mathrm{~mm}$ ), respectively. Additionally, the termite extract T3S have been showed to inhibite the activity of Klebsiella pneumonia (T3S:IZD = $14 \pm 0.1 \mathrm{~mm}$ ). The MIC values varied between extracts and test organisms used and ranged from 25 to $100 \mu \mathrm{g} /$ disc with T1S extract being the most effective on S.aureus (MIC $=25 \mu \mathrm{g} / \mathrm{disc}$ ). The chemical screening of Macrotermes bellicosus extract revealed the presence of terpenes, steroids, saponins, alkaloids, flavonoids and tannins.

Macrotermes bellicosus used as a traditional medicine is efficient against different bacterial strains and could be a therapeutic option for infectious diseases. The present finding confirmed the efficacy of traditional practices, which could bring new solutions to tackle increasing bacterial resistance to existing antibiotics.

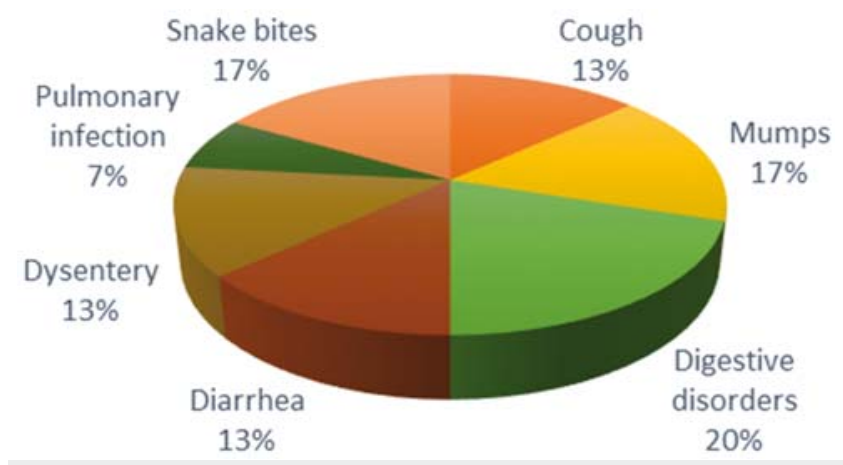

Fig. 1: Traditional therapeutic uses of fungus-growing termites Macrotermes bellicosus and their products in the traditional medicine of Benin. Results from an ethnomedicinal survey with active healers from Benin $(n=30)$.

\section{Tu-Poster Session 2-PO-208 Anti-inflammatory and analgesic activity of the water extract from Terminalia bellerica (Gaertn.) Roxb.}

Authors Sireeratawong $\mathrm{S}^{1}$, Jaijoy $\mathrm{K}^{2}$, Soonthornchareonnon $\mathrm{N}^{3}$ Institute 1 Department of Pharmacology, Faculty of Medicine, Chiang Mai University, Chiang Mai, Thailand; 2 McCormick Faculty of Nursing, Payap University, Chiang Mai, Thailand; 3 Department of Pharmacognosy, Faculty of Pharmacy, Mahidol University, Bangkok, Thailand

DOI 10.1055/s-0037-1608533 
Terminalia bellerica extract was investigated for its anti-inflammatory and analgesic activities in animal models. An anti-inflammatory activity test showed that $T$. bellerica extract inhibited ear edema of the rats induced by ethyl phenylpropiolate as well as inhibited carrageenan-induced paw edema. In contrast, the extract did not have an inhibitory effect on arachidonic acidinduced ear edema in rats. T. bellerica extract did not reduce granuloma weight, body weight gain and thymus dry weight in the cotton pellet-induced granuloma formation model. An analgesic activity study showed that $T$. bellerica extract decreased licking times in mice injected with $0.1 \%$ formalin in both the early and late phases, but exerted the most marked effect in the late phase. These results suggest that $T$. bellerica water extract may possess both analgesic and anti-inflammatory activities. The main mechanisms of action of $T$. bellerica extract may be due to the inhibitory effects on the synthesis and/or release of pain or inflammatory mediators.

\section{Tu-Poster Session 2-PO-209 A New Dosage Form of Licorice Based on Traditional Persian Medicine for Cough}

Authors Pasalar $\mathrm{M}^{1}$, Ghaemi $\mathrm{H}^{1}$, Mosaffa-Jahromi $\mathrm{M}^{1}$, Masoompour Seyed $\mathrm{M}^{2}$ Institute 1 Research Center for Traditional Medicine and History of Medicine, School of Medicine, Shiraz University of Medical Sciences, Shiraz, Iran, Shiraz, Iran; 2 Non-Communicable Diseases Research Center, Shiraz University of Medical Sciences, Shiraz, Iran, Shiraz, Iran

DOI 10.1055/s-0037-1608534

Cough is a nonspecific reaction to irritation anywhere from the pharynx to the lungs. This is the most prevalent complaint for which a medical consult is sought and the managing of cough demonstrates a physicians' challenge (1). There are some specific dosage forms for respiratory diseases available in traditional Persian medicine (TPM) pharmacopeia although they are not acceptable for precise clinical trials (2). Recent study carried out to evaluate the effect of a modified TPM formula for licorice in treatment of the cough. In a randomized double-blinded placebo controlled clinical trial, 70 patients with cough based on Leicester Cough Questionnaire total scores sorted in 2 equal groups and received licorice modified preparation (in the pastille form) and placebo for 2 weeks. Follow-up time was also arranged at the following 2 weeks. We introduced a new dosage form for licorice according to TPM instructions (3). The results at the follow-up time (week 4) showed a significant priority in cough severity in licorice pastille against placebo group $(p<0.05)$ (Table 1). The novel dosage form derived from TPM - licorice pastille could be a promising choice in treatment of cough severity.

Tab. 1: Data of cough severity score

\begin{tabular}{|l|l|l|l|}
\hline Time & Licorice pastille $(\mathbf{n}=\mathbf{2 9})$ & Placebo $(\mathbf{n}=\mathbf{3 1})$ & $\mathrm{p}$-value \\
\hline Beginning (Mean \pm SD) & $2.9 \pm 0.85$ & $2.7 \pm 0.85$ & 0.38 \\
\hline The end phase (week 2) (Mean \pm SD) & $1.4 \pm 0.93$ & $1.6 \pm 0.88$ & 0.44 \\
\hline Follow-up phase (week 4) (Mean \pm SD) & $1.2 \pm 0.93$ & $1.8 \pm 1.03$ & $<0.001$
\end{tabular}

[1] Ojoo JC, et al. Cough. 2013;9:9974- 9.

[2] Karegar-Borzi H, et al. Lauq: J Evid Based Complementary Altern Med. 2016;21:63- 70 .

[3] Sina HA. Al-Qanun fi al-Tibb [The Canon of Medicine]. Beirut, Lebonan: Alaalami Library; 2005.

\section{Tu-Poster Session 2-PO-210 Forty five years of Pharmacognostic Research on Panamanian Flora (1972 - 2017)}

Authors Gupta $\mathrm{M}^{1}$

Institute 1 Center for Pharmacognostic Research on Panamanian Flora, College of Pharmacy, Universidad de Panama, Panama, Panama DOI 10.1055/s-0037-1608535

Panamanian flora is one of the richest in the world. Its medicinal and economic potential has not been fully explored. The results of ethno-botanical surveys among the Kuna, Ngöbe-Buglé and Naso Amerindians of Panama will be presented. Results of bioassay-guided fractionation of plants within the framework of multinational collaborative projects executed during the last 45 years, supported by inter alia the Organization of American States, National Secretariat of Science, Technology and Innovation of Panama, European Union, CYTED Program and will be presented. Examples of successful collaborations with the University of Basel will also be highlighted.

SENACYT, EU and OAS.

\section{Tu-Poster Session 2-PO-211 Insights of traditional Persian medicine to polycystic ovary syndrome and its management}

Authors Hosseinkhani $\mathrm{A}^{1,2}$, Asadi $\mathrm{N}^{3}$, Pasalar $\mathrm{M}^{1}$, Zarshenas Mohammad $\mathrm{M}^{2}$ Institute 1 Research center for traditional medicine and history of medicine, Shiraz University of medical sciences, Shiraz, Iran; 2 Department of Phytopharmaceuticals (Traditional Pharmacy), School of Pharmacy, Shiraz University of Medical Sciences, Shiraz, Iran; 3 Maternal-fetal Medicine Research Center, Shiraz University of Medical Sciences, Shiraz, Iran; 4 Medicinal Plants Processing Research Center, Shiraz University of medical sciences, shiraz, Iran

DOI 10.1055/s-0037-1608536

Traditional Persian medicine (TPM) takes a holistic approach towards human health. Polycystic ovary syndrome (PCOS) is a common endocrine disorder in women of reproductive age. Common available treatments are suboptimal for this problem. It mainly represents itself by prolonged menstrual cycle intervals, hyperlipidemia and hyperglycemia [1]. The extant written documents of TPM contain a list of herbal medications used for centuries. Before further experimental research on any of these treatments, it is appropriate to study current related scientific evidence on their possible pharmacological actions [2]. This work aims to study PCOS and their herbal treatments in TPM. To collect data from medieval medicinal texts, six of the most famous TPM manuscripts were studied. Herbs which were involved in the treatment of prolonged intervals of menstrual bleeding were listed. Their authentications were confirmed. PubMed and ScienceDirect databases were searched for related pharmacological activities of the medicinal plants reported. Forty herbs in TPM books for the treatment of this problem were found. The majority of these herbs exhibited anti-hyperglycaemic (90\%) and anti-dyslipidaemic (77.5\%) effects. The effect of some of these were studied on ovulation induction and $27.5 \%$ had shown positive effects. It could be concluded that ancient physicians not only wanted to treat the irregular menstrual cycle but also their treatment options were aimed at ameliorating the related underlying metabolic dysfunctions. The recommended herbs, which have the most scientific proof for their related actions, can be studied further in experimental analyses.

[1] Celik, O. and O. Acbay, J Endocrinol Invest, 2012. 35: 905 - 10

[2] Hosseinkhani, A, et al, JEBCAM, 2017. 22: 334 - 343.

\section{Tu-Poster Session 2-PO-212 The Heat-clearing and Fire-purging Medicinal Composition for Combating Metastatic Cancer}

Authors Dai $\mathrm{YH}^{1,2}$, Yang $\mathrm{JC}^{1,2}$, Kuo $\mathrm{HH}^{2,3}$, Wu $\mathrm{YC}^{4,5,6}$

Institute 1 School of Pharmacy, China Medical University, Taichung, Taiwan; 2 Chinese Medicine Research and Development Center, China Medical University Hospital, Taichung, Taiwan; 3 Graduate Institute of Biomedical Science, China Medical University, Taichung, Taiwan; 4 Graduate Institute of Natural Products, Kaohsiung Medical University, Kaohsiung, Taiwan;

5 Research Center for Natural Products \& Drug Development, Kaohsiung Medical University, Kaohsiung, Taiwan; 6 Department of Medical Research, Kaohsiung Medical University Hospital, Kaohsiung, Taiwan DOI 10.1055/s-0037-1608537

Traditional Chinese Medicine (TCM) is widely used as alternative therapy for patients who suffer from cancer disease. Accumulating evidence support the 
notion that cancer may hijack systemic effectors to create microenvironment for cancer progression. This phenomenon is consisted with a theoretic hypothesis based on human systemic health/unhealthy homeostasis for altering disease environment. We learn from experience used of herbal medicine classified as heat-clearing and fire-purging medicine, which have the effect of clearing up internal heat due to yin deficiency. However, lack of scientific evidence to answer the question of how TCM affect cancer cells and alter systemic function is a challenge for translating TCM treatment into clinic. In this study, we look for folk medicine using TCM composition that can improve patients' outcome. A national health insurance (NHI) program was implemented in 1995. Over 22.6 million individuals from a total population of 23.0 million in Taiwan were enrolled in this insurance program. Observation cohort study was conducted to determine the association between used TCM containing Polygonaceae and Ranunculaceae materials and mortality among the bladder cancer patients. Patients treated with TCM over $135.65 \mathrm{~g}$ per year significantly reduce hazard ratio compared with Non-TCM user. Using scratch wound healing and time lapse imaging, we demonstrate that the combination of Polygonaceae and Ranunculaceae materials were successfully suppressed the migration of metastatic cancer cells. Furthermore, molecular mechanisms revealed that several molecules such as SOX8 and LHX3 may play an important role for treating metastasis by integrating mRNA and microRNA expression. Collectively, we found that heat-clearing and fire-purging medicinal composition containing two herbs may improve the outcome of metastatic cancer treatment.

\section{Tu-Poster Session 2-PO-213 Anti-hyaluronidase activity of traditional Uzbekistan medicine Eryxin}

Authors Akbarov $U^{1}$, Shikov $A^{2}$, Pozharitskaya $\mathrm{O}^{2}$, Demchenko $D^{2}$, Makarov $\mathrm{V}^{2}$

Institute 1 Tashkent Medical Academy, Tashkent, Uzbekistan; 2 SaintPetersburg Institute of Pharmacy, St-Petersburg, Russia DOI 10.1055/s-0037-1608596

Ibn Sina (Avicenna) argued that the use of snake meat prolongs life, strengthens forces, keep the sharp senses and youthfulness. A variety of remedies from snakes were used in Uzbekistan traditional medicine for centuries for the treatment of various diseases. And now there is an opinion that bouillon and dried meat of a snake, called kulvor-ilon, are a remedy for many ailments. A traditional medicine Eryxin represents an aqueous hydrolysate prepared from the biomass of sand boa snakes. It contains short-chain organic acids, aminoacids, phospholipids, inorganic salts. It was shown, that Eryxin among other activities has been effective for the treatment of rheumatoid arthritis [1].

We assessed the chondroprotective potential of Eryxin by examining its effects on the activities of hyaluronidase. Hyaluronidase activity was assayed by a modified version of Morgan-Elson method [2]. Percent of inhibition was calculated as follows:

Inhibition $(\%)=[(A-B)-(C-D)] /(A-B) \times 100$, where $A$ is the control absorbance; $B$ is the control blank absorbance; $C$ is the sample absorbance; and $D$ is the sample blank absorbance. IC 50 was calculated using the mean of three observations from each of the five concentrations for all the samples. Alflutop (BIOTEHNOS S.A, Romania) was used as positive control. The IC 50 values for Eryxin were $3.4-9.21 \mathrm{mg} / \mathrm{ml}$, while IC 50 values for Alflutop was $4.0 \mathrm{mg} / \mathrm{ml}$. In the present study, we evaluated the antihyaluronidase activity of Eryxin for the first time. The observed potent activity could be considered as one of mechanisms of anti-arthritis effects of Eryxin.

[1] Ubaidullaev A.M., Uzakova G.T., Akbarov S.V.. Med. Zh. Uzbek., 1999, 6, $88-90$.

[2] Samee H., Li Z.X., Lin H. et al.. J. Zhejiang University-SCIENCE B, 2009, 10, $147-153$

\section{Tu-Poster Session 2-PO-215 Alfa-glucosidase inhibiting activity of Four GUATEMALAN PLANTS used in the treatment of type 2 diabetes.}

Authors Andrade-Cetto $\mathrm{A}^{1}$, Cabello Hernández Christian A ${ }^{1}$, Cárdenas Vázquez René de $\mathrm{J}^{2}$

Institute 1 Universidad Nacional Autónoma de México Facultad de Ciencias. Laboratorio de Etnofarmacología, Ciudad de México, Mexico; 2 Universidad Nacional Autónoma de México Facultad de Ciencias. Laboratorio de Biología Animal Experimental, Ciudad de México, Mexico

DOI 10.1055/s-0037-1608538

Diabetes mellitus is defined as hyperglycemia associated with inadequate insulin secretion, either in the presence or absence of impaired insulin action. Type 2 diabetes is characterized by tissue insulin resistance combined with a relative deficiency in insulin secretion [1].

Among glucose-lowering medications, alfa glucosidase inhibitors (AGls) delay the absorption of ingested carbohydrates, reducing the postprandial glucose and insulin peaks [2]. In nature two main kind of glucosidases exist: Type 1 are present in yeast and insects, they show higher activity toward heterogeneous substrates as sucrose and none or low toward homogeneous substrates as malto oligosaccharides, Type II are present in molds, plants and mammals, they hydrolyse the homogeneous substrates more rapidly [3]. In our field studies among the mayan cakchiqueles we detect the use of Croton guatemalensis Lotsy. (CG), Hamelia patens Jacq. (HP), Solanum americanum Mill. $(\mathrm{SA})$, and Neurolaena lobata (L.) R. Br. (NL) to treat type 2 diabetes.

In previous studies, we demonstrated hypoglycemic activity in these plants. In the present work, we tested the alfa-glucosidase inhibitory activity of the plant extracts, in vivo by saccharose and maltose tolerance tests, and in vitro trough the inhibition of the enzymes isolated from Saccharomyces and from rat gut.

The tested plants did not reduce the hyperglycaemic peak under a maltose tolerance test, but AP, SA and NL did it under the saccharose test after 30 min. In vitro the extracts inhibit the glucosidase activity from Saccharomyces, but not that from rat gut (table 1). These results show that the plants can inhibit type 1 enzymes but not type 2 .

[1] ADA AD. Classification and Diagnosis of Diabetes. Diabetes Care 2015; 38: S8-S16 Available from: http://care.diabetesjournals.org/cgi/doi/10.2337/ dc15-5005

[2] Hanefeld M, Schaper F. In: Mogensen CE (ed.). Pharmacotherapy of Diabetes, New Developments. Springer, 2007: $143-152$

[3] Kimura A et al. Carbohydr Res 2004; 339: 1035

\section{Tu-Poster Session 2-PO-217 CHARACTERIZATION OF THE LIPID PEROXIDATION ACTIVITY OF METHANOLIC EXTRACTS OF Detarium microcarpum, Danielli oliveiri. and Boswellia dazielli LEAVES}

Authors Akinsanmi $\mathrm{A}^{1}$, Johnson $\mathrm{T}^{2}$, Longdet $\mathrm{I}^{2}$, Ojerinde $\mathrm{S}^{3}$, Oche $\mathrm{JR}^{2}$, Timothy $\mathrm{F}^{4}$, Ejembi $\mathrm{S}^{2}$, Aguiyi J $\mathrm{J}^{1,4}$

Institute 1 African Centre of Excellence in Phytomedicine Research and Development, University of Jos, Jos, Jos, Nigeria; 2 Department of Biochemistry, Faculty of Medical sciences, University of Jos, Jos, Jos, Nigeria; 3 Department of Pharmaceutical Chemistry, Faculty of Pharmaceutical sciences, University of Jos, Jos, Jos, Nigeria; 4 Department of Pharmacology, Faculty of Pharmaceutical Sciences, University of Jos, Jos, Jos, Nigeria DOI 10.1055/s-0037-1608539

It is a known scientific fact, that, oxidative stress is the leading cause of most degenerative diseases. A major source of reactive oxygen supply is the lipid peroxidation autocatalytic process [1]. Antioxidant defense system involves the antioxidant enzymes, both the essential trace elements and also amino acids constituents. These 3 leaves, Detarium microcarpum G. \& P. (DML), Danielli oliveiri $\mathrm{H}$. \& D. (DOL), and Boswellia dazielli $\mathrm{H},(\mathrm{BDL})$ have been used in Northern ethnomedicine for the treatment of inflammation and cancer [2]. 
The results revealed that BDL had the highest total phenol $(110 \pm 0.001 \mathrm{mg} /$ gGAE) and flavonoid content $(28.9 \pm 0.001 \mathrm{mg} / \mathrm{g}$ QUE) and DOL with the least values. The BDL had the highest reducing power, Nitric oxide, DPPH and $\mathrm{ABTS}^{+}$ free radical scavenging ability. Furthermore, the inhibition of lipid peroxidation by DML, in the brain, liver and colon using $\mathrm{FeSO}_{4} / \mathrm{SNP}$ as pro oxidants caused a significant increase in the Malondialdehyde (MDA) content, inhibitory effect at dose level $(15.33-77.83 \mathrm{mg} / \mathrm{mL})$ in the colon. This may be due to its having a high content of Selenium, Copper, Zinc, and Manganese; trace elements that boost antioxidant defense system. DML had a high content in amino acids that are components of antioxidant enzymes; glutamine, cysteine, glycine, and methionine [3]. Therefore, these findings indicate that the lipid peroxidation activity depends not only on the antioxidant capacity of the phytoconstituents, but most likely on the contents of essential elements and amino acids.

[1] Yin H, Xu L, Porter N. Chemical Reviews 2011; 10: 5944 - 5972

[2] Abubakar M, Musab A, Ahmeda A, Hussaini M. Journal of Ethnopharmacology 2007;11: $625-629$

[3] Liochev S, Fridovich I. J. Biol. Chem. 2000; 275: 38482 - 38485

\section{Tu-Poster Session 2-PO-218 Phenolic profile, antioxidant effects and phylogenetic characterization of Ficus natalensis subsp. natalensis from Benin - West Africa}

Authors Marquardt $\mathrm{P}^{1}$, Fester $\mathrm{K}^{1}$

Institute 1 Pharmazeutische Biologie Institut für Pharmazie Universität

Leipzig Johannisallee 21 - 23, Leipzig, Germany

DOI $10.1055 / \mathrm{s}-0037-1608540$

Medicinal plants used by traditional healers play an important role in the treatment of various diseases in Benin. Ficus natalensis subsp. natalensis, the coastal strangler fig, is used for the therapy of inflammatory conditions and wound healing(1). However, Ficus natalensis susbsp. natalensis is often confused with other Ficus species like Ficus thonningii and Ficus craterstoma due to similiar morphological features and phylogenetic studies propose an interbreeding process (2). A further analysis of the phylogenetic and phytochemical profile of Ficus natalensis subsp. natalenis from Benin is therefore fundamental. The presented research focuses on the analysis of the phenolic profile, phylogenetic characterization and the antioxidant effects of a methanolic leaf extract of Ficus natalensis subsp. natalensis from Benin. A combined approach of chromatographic profiling and antioxidant screening identified rutin, chlorogenic acid, neochlorogenic acid and kryptochlorogenic acid as the main constituents with antioxidant activities. The methanolic leaf extract showed a phenolic content of $1.43 \pm 0.07 \%$. A phylogenetic analysis based on internal transcribed spacer sequences of the collected plant material confirmed the collected Ficus species as Ficus natalensis subsp. natalensis. The compounds chlorogenic acid, neochlorogenic acid and kryptochlorogenic acid could be reported for Ficus natalensis subsp. natalensis for the first time. Our study suggests that presence of the antioxidant compounds rutin, chlorogenic acid and its isomers, supports the application of this plant for anti-inflammatory and wound healing purposes.

[1] Brink, M, and E. G. Achigan-Dako. "Fibres PROTA foundation." Wageningen, Netherlands 2012: $152-154$

[2] Burrows, J, \& Burrows, S, Figs of southern and south-central Africa. Hatfield, South Africa: Umdaus Press viii, 379 p.-illus, col. illus.. ISBN, 1919766243.

\section{Tu-Poster Session 2-PO-219 A systems biology approach for discovering the action mechanism of Niaoduqing particles in rats with chronic renal failure}

Authors Fan $\mathrm{X}^{1}$, Wang $\mathrm{Y}^{1}$, Luo $\mathrm{G}^{1}$

Institute 1 Tsinghua University, Beijing, China

DOI 10.1055/s-0037-1608541

Chronic renal failure (CRF) is a major public health problem worldwide, and Traditional Chinese medicine has a long history of dealing with diseases for several thousand years. Niaoduqing particles (NDQ) composed of 16 Chinese herbs, and has been proved to have remarkable effects on treating CRF in clinical. However, because of its complex prescriptions and unclear efficacy material basis, the mechanism of NDQ is still unclear.

In this study, combined with network pharmacology (target prediction), system biology research platform (lab study) was used to discover the mechanism of NDQ. In target prediction, 83 components identified by fingerprints technology (LC-MS) were subjected to target prediction and bioinformatics analysis, 39 signal pathways regulated by these components were obtained. In lab studies, the rat model with CRF was induced by adenine. The plasma protein and miRNAs expression levels were respectively detected by protein chip and miRNAs expression chip after NDQ intervention. 31 differentially expressed protein related pathways and 17 differentially expressed miRNAs related pathways were finally obtained.

Based on the above findings, a "component - miRNA - protein" topology network was built up and used to find the common pathways. Consequently, MAPK, Cytokine-cytokine receptor interaction, T cell receptor and Jak-Stat signaling pathways were screened. It is presumed that the four pathways are the main paths regulated by NDQ. Then, through reverse analysis, Radix Astragali, Rheum Officinale, Radix Salviae Miltiorrhizae, Radix Glycyrrhizae and Poria Cocos were found to play major regulatory roles in the four signal pathways, which indicated that the five herbs had an indispensable role in NDQ. In summary, based on the combination of laboratory research and target prediction, the action mechanism of NDQ was explored, and the herbal medicines played a major effect in the prescription were clear, which is worth further study.

\section{Tu-Poster Session 2-PO-220 Antimicrobial and antioxidant activities of Psydrax subcordata and Acioa dinklaglei}

Authors Agyare $C^{1}$, Boakye Yaw $D^{1}$, Obeng-Mensah $D^{1}$, Akplah Leslie $A^{1}$ Institute 1 Department of Pharmaceutics, Faculty of Pharmacy and Pharmaceutical Sciences, Kwame Nkrumah University of Science and Technology, Kumasi, Ghana

DOI 10.1055/s-0037-1608542

Psydrax subcordata (D. C. Bridson) (Family: Rubiaceae) and Acioa dinklagei (Engl.) (Family: Chrysobalanaceae) are plants widely distributed in the tropical region of West Africa. Leaves and stem bark of $P$. subcordata and the leaves of A. dinklagei are used to treat wound infections in West Africa. The aim of this study was to determine the antimicrobial and antioxidant activities of $P$. subcordata and A. dinklagei [1].

Preliminary phytochemical screening was carried out on the methanol leaf (PSLE) and stem bark (PSSE) extracts of $P$. subcordata and methanol leaf (ADLE) extract of $A$. dinklaglei to identify their phytochemical constituents [2]. The antimicrobial activity of PSLE, PSSE and ADLE were evaluated using the broth dilution method [3]. Antioxidant activities of the PSLE, PSSE and ADLE were determined using 1,1-diphenyl-2-picryl hyrdazyl [4].

MIC of PSLE was $12.5 \mathrm{mg} / \mathrm{mL}$ against test organisms. MIC of PSSE against test organisms was $25 \mathrm{mg} / \mathrm{mL}$ except $P$. aeruginosa $(12.5 \mathrm{mg} / \mathrm{mL})$. MICs of ADLE against $B$. subtilis, E. coli, S. pyogenes and P. aeruginosa were 2.5, 20, 10 and $5 \mathrm{mg} / \mathrm{mL}$, respectively (Table 1). PSLE, PSSE and ADLE exhibited antioxidant activity with $I C_{50}$ of $7.0 \pm 0.0016,99.0 \pm 0.0022$ and $96.0 \pm 0.017 \mu \mathrm{g} / \mathrm{mL}$. Tannins, saponins, flavonoids and glycosides were identified in PSLE, PSSE and ADLE.

PSLE, PSSE and ADLE possess antimicrobial and antioxidant activities.

Tab. 1: MICS of PSLE, PSSE and ADLE against test organisms

\begin{tabular}{|l|l|l|l|}
\hline Test organisms & MIC $(\mathbf{m g} / \mathbf{m L})$ & & \\
\hline & PSLE & PSSE & ADLE \\
\hline B. subtilis NTCC 10073 & 12.5 & 25.0 & 2.5 \\
\hline E. coli ATCC 25922 & 12.5 & 25.0 & 20.0 \\
\hline S. pyogenes (clinical strain) & 12.5 & 25.0 & 10.0 \\
\hline P. aeruginosa ATCC 4833 & 12.5 & 12.5 & 5.0 \\
\hline
\end{tabular}


[1] Agyare et al, J. Ethnopharmacol 2009; 125: $393-403$

[2] Evans, WC. Trease and Evans Pharmacognosy 16th ed. Williams \& Wilkins, Baltimore; 2009:1788

[3] Wiengand et al, Nat. Protoc 2008; 3:163-175

[4] Chizzola et al, J. Agric Food Chem 2008; 56:6897 - 6904

\section{Tu-Poster Session 2-PO-221 Antimicrobial and antioxidant activities of Duboscia viridiflora and Brachyachne obtusiflora}

\section{Authors Agyare $C^{1}$, Boakye Yaw D ${ }^{1}$, Obeng-Mensah $D^{1}$, Dodoo Deborah} Naa $A^{1}$, Akplah Leslie $A^{1}$

Institute 1 Department of Pharmaceutics, Faculty of Pharmacy and Pharmaceutical Sciences, Kwame Nkrumah University of Science and Technology, Kumasi, Ghana

DOI 10.1055/s-0037-1608543

Duboscia viridiflora (Family: Malvaceae) and Brachyachne obtusiflora (Family: Poaceae) are plants commonly found in west Africa. Leaves and stem bark of $D$. viridiflora as well as the leaves of $B$. obtusiflora are used to treat wounds and wound infections in Ghana [1]. Therefore the study sought to determine the antimicrobial and antioxidant activities of $D$. viridiflora and B. obtusiflora. Preliminary phytochemical screening was performed on the methanol leaf (DVLE) and stem bark (DVSE) extracts of D. viridiflora and methanol leaf extract of B. obtusiflora (BOLE) to determine the secondary metabolites [2]. Micro broth dilution method was used to determine antimicrobial activity of DVLE, DVSE and BOLE [3]. Antioxidant activities of the DVLE, DVSE and BOLE were determined using 1,1-diphenyl-2-picryl hyrdazyl [4].

MIC of DVSE was $3.13 \mathrm{mg} / \mathrm{mL}$ against test organisms. MIC of DVLE against $B$. subtilis, E. coli, and P. aeruginosa was $12.5,25.0$ and $25.0 \mathrm{mg} / \mathrm{mL}$, respectively. MIC of BOLEs against B. subtilis, E. coli, S. pyogenes and P. aeruginosa were 10.0, 20.0, 20.0 and $10.0 \mathrm{mg} / \mathrm{mL}$, respectively (Table 1). DVLE, DVSE and BOLE exhibited antioxidant activity with $I_{50}$ of $17.0 \pm 0.006,34.0 \pm 0.004$ and $127.0 \pm 0.059 \mu \mathrm{g} / \mathrm{mL}$, respectively. Tannins, flavonoids and glycosides were present in DVLE, DVSE and BOLE. Saponins were present in DVLE and DVSE but not BOLE.

DVLE, DVSE and BOLE possess antimicrobial and antioxidant activities.

Tab. 1: MICS of DVSE, DVLE and BOLE

\begin{tabular}{|l|l|l|l|}
\hline Test organisms & MIC(mg/mL) & & \\
\hline & DVSE & DVLE & BOLE \\
\hline B. subtilis NTCC 10073 & 3.13 & 12.50 & 10.00 \\
\hline E. coli ATCC 25922 & 3.13 & 25.00 & 20.00 \\
\hline S. pyogenes (clinical strain) & nd & nd & 20.00 \\
\hline P. aeruginosa ATCC 4833 & 3.13 & 25.00 & 10.00 \\
\hline nd: not determined & & & \\
\hline
\end{tabular}

[1] Agyare et al, J. Ethnopharmacol 2009; 125: $393-403$

[2] Evans, WC. Trease and Evans Pharmacognosy 16th edition. Williams \& Wilkins, Baltimore; 2009:1788

[3] Wiengand et al., Nat. Protoc 2008;3:163-175

[4] Chizzola et al., J. Agric Food Chem 2008;56:6897 - 6904

\section{Tu-Poster Session 2-PO-222 Preliminary chemical studies in Chrysobalanus icaco leaves and seeds from Panamá}

Authors Patiño $L^{1}$, Villagra $E^{1}$, Palermo $J^{2}$, de Guevara $V^{1}$

Institute 1 Centro de Investigación de Productos Naturales y Biotecnología, Facultad de Ciencias Naturales y Exactas, Universidad Autónoma de Chiriquí, Estafeta Universitaria 0427, Panamá, Chiriqui, Panama; 2 UMYMFOR -

Departamento de Química Orgánica - Facultad de Ciencias Exactas y Naturales - Universidad de Buenos Aires - Ciudad Universitaria, Pabellón 2 - (1428) Buenos Aires - Argentina, Buenos Aires, Panama

DOI 10.1055/s-0037-1608544

Panama has one of the highest biodiversity profiles in the world. In this context, studies in the chemistry of medicinal plants have been carried in the last fifty years. Attention has been focused on native and traditional knowledge, but still exists a lack of information about the occidental region of Panama. Chiriquí, is a province in Panama and has a wide range of climate and geographical features that make its flora and fauna unique ${ }^{1}$.

This study investigates some regional plants with medicinal uses that have no previous phytochemical and biological data in the country, starting with Chrysobalanus icaco L. (coco plum), a member of the Chrysobalanaceae family, widely distribute in America and Africa, which showed antimicrobial and hypoglycemic activity reports world wide ${ }^{2}$.

The chemical composition and biological activity of the polar and lipophilic extracts of the fresh leaves and seeds of $C$. icaco have been determined, through phytochemical screening, chromatographic techniques and antimicrobial test, respectively. The isolation of flavonoids, glycosides, triterpenes, diterpenes, tannins and saponins from leaves and fruits of this plant have been previously reported in the literature, with pomolic acid, glycoside myrcetin and quercetin derivates been the principal metabolites. In our study, flavonoids, cardiotonic glycosides and phenolic compounds have been identified from the methanolic extract, meanwhile; terpenes and flavonoids were detected in the hexanic one, as major secondary metabolites. From the treatment of the methanolic extract of the seed, a high content of sugar was identified.

[1] Robles V, G. R; Oliveira B, K; Villalobos S, R. (2000). Evaluación de los productos forestales no madereros en América Central. Departamento de Montes, Depósito de documentos de la FAO. Turrialba, Costa Rica.

[2] Ferreira-Machado, S; Gagliardi, R; Nunes, A; Rodrigues, M; Dantas, F; De Mattos, J; Peregrino, C; Moura, E; Caldeira-de-Araujo, A. J. Med. Plant. Res. 2014, 8, $52-57$.

\section{Tu-Poster Session 2-PO-223 Medicinal natural plant studies in Panama}

\section{Authors Patiño $L^{1}$}

Institute 1 Centro de Investigación de Productos Naturales y Biotecnología, Facultad de Ciencias Naturales y Exactas, Universidad Autónoma de Chiriquí, Estafeta Universitaria 0427, Panamá, Chiriqui, Panama

DOI 10.1055/s-0037-1608545

Panama has a huge floral diversity; around 10,400 vegetal species have been identified. Beyond the research efforts made in the last quarter century oriented to identifying, characterize and determining the medicinal plants chemical composition, it remains unclear the vast potential in the Panamanian flora. The region of Chiriqui is an example of this phenomenon, where the medicinal plant data is insufficient and disperse.

Actually, the medical field is going through the use of natural or ethnobotanical medicines, due to the active components studies in plants, bacteria, marine invertebrates and others, which already have been developed.

The problematic issue raised from these uses, is the poor information related to the effectivity, toxicity and side effects that could arise from them. Normally, it is just an inherited knowledge from generation to generation. This book, "Medicinal plants grown in Chiriqui" is a compilation of 90 medicinal plants already existing in the province, with international or national studies. This work contains geographic information, basic taxonomic description, chemical data and medicinal uses, that have been reported.

It began as a merely laboratory compilation, but thanks to the effort of botanic, chemical and many others individuals, was transformed into a book. It is the aim of this work to be used as a useful consulting manual, highlighting the importance of preserving all the floral biodiversity already existing. No one knows if here could be a miracle substance still waiting to be discovered.

[1] Ocampo, R. (1994). Domesticación de Plantas Medicinales en Centroamérica. Actas de la Reunión Técnica Centroamericana. CATIE, Turrialba, Costa Rica, [2] Martínez G, Libardo A. (2014). Plantas Medicinales nativas de Panamá y su potencial para el tratamiento de las patologías de mayor impacto en el país. Facultad de Ciencias, Pontificia Universidad Javeriana, 1-298. 


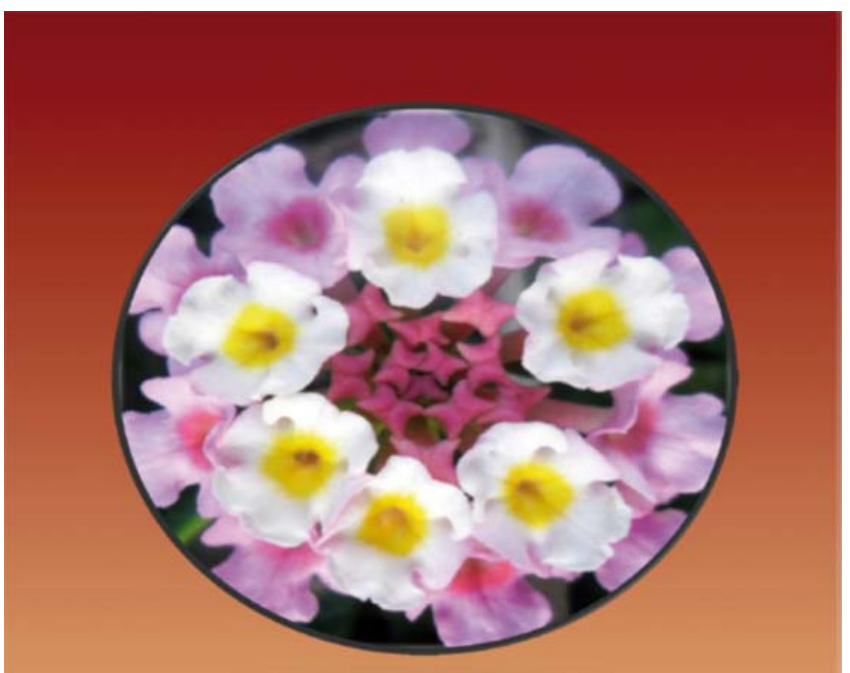

PLANTAS MEDICINALES CULTIVADAS EN CHIRIQUÍ:

COMPOSICIÓN QUÍMICA, USOS Y PREPARACIÓN.

\section{Tu-Poster Session 2-PO-224 Bio-guided isolation of new natural anti-venom agents from extracts reported on the work of the ancient Greek physician, Nicander}

\author{
Authors Michalea $\mathrm{R}^{1}$, Boznou $\mathrm{V}^{1}$, Dedousi $\mathrm{V}^{1}$, Kalpoutzakis $\mathrm{E}^{1}$, \\ Polychronopoulos $\mathrm{P}^{1,2}$, Aligiannis $N^{1,2}$ \\ Institute 1 Department of Pharmacognosy and Natural Products Chemistry, \\ Faculty of Pharmacy, University of Athens, Athens, Greece; 2 Greek Society of \\ Ethnopharmacology, Athens, Greece \\ DOI 10.1055/s-0037-1608546
}

Nicander was a famous poet-naturalist of the 2 nd century BC. Theriaca is his second surviving work, a Hellenistic poem, on the bites of venomous creatures, while a series of herbal remedies are also mentioned. The aim of this study was to evaluate the chemical content and biological properties of plants reported on Theriaca and in particular to investigate the possibility of their application as therapeutic agents for wound healing and neurodegenerative diseases. Plants were collected, dried and extracted by Accelerated Solvent Extraction (ASE) technique resulting in the creation of a 'theriaca extracts' library. Their chemical profile was analyzed by HPTLC and LC-MS. The antivenom potent of the most promising extracts was evaluated by enzymatic inhibitory assays. Hyaluronidase, phospholipase-A2 and acetylcholinesterase [1] are common molecular targets for the evaluation of anti-ophidian properties of natural products. Paeonia parnassica, a Greek endemic species, and three Aristolochia spp. were chosen for fractionation and separation of their chemical constituents by means of Centrifungal Partition Chromatography. The obtained fractions were evaluated for their ability to inhibit the enzyme targets where the initial extract was active. In parallel the chemical composition of each fraction was established by NMR-dereplication approach and analyzed by HPTLC. Finally, five anti-phospholipase agents corresponding to aristolochic acid derivatives from Aristolochia spp. and ten anti-acetylcholines- terase and anti-hyaluronidase components belonging to phenolics and flavonoids from Paeonia parnassica were identified.

[1] Molander M, Nielsen L, Søgaard S, Staerk D, Rønsted N, Diallo D, Chifundera KZ, Staden J, Jäger AK. J Ethnopharmacology 2014; 18:157:171 - 80

\section{Tu-Poster Session 2-PO-225 Studies on the antivenom activity of four plant species from Flora Ikaria: An ethnopharmacological approach}

Authors Polychronopoulos $\mathrm{P}^{1}$, Lyssaios $F A^{1}$, Michalea $\mathrm{R}^{1}$, Aligiannis $\mathrm{N}^{1}$ Institute 1 Department of Pharmacognosy and Natural Products Chemistry, Faculty of Pharmacy, University of Athens, Athens, Greece

DOI $10.1055 / \mathrm{s}-0037-1608547$

Theriaca is a poem of the ancient greek physician Nicander of Colophon ( $2^{\text {nd }}$ century $\mathrm{BC}$ ). This is his longest surviving work and is referred to antidotes of plant origin against bites from venomous creatures [1]. Flora Ikaria was chosen as the area where four species, mentioned in Theriaca, were collected [2]. These are Aristolochia hirta, Anchusa undulata, Echium plantagineum, Scorpiurus muricatus and are native to island Ikaria. Different parts of these species e.g. roots, stems, were extracted with different polarities. Twenty three extracts were obtained. The LC-MS and HPTLC profiling revealed the variety of metabolites. Also their anti-ophidian properties were evaluated using enzyme inhibition assays. Acetylcholinesterase, phospholipase A2 (sPLA2) and hyaluronidase are the examined biological targets since they are present in snake's venom. The hydroalcoholic extracts from the roots and aerial parts of Aristolochia hirta, an endemic species of East Aegean Sea, were selected for further phytochemical analysis. They are the most potent inhibitors of SPLA2 and AChe respectively during the preliminary screening and they are rich in different types of metabolites. The fractionation of the root extract was accomplished by Centrifugal Partition Chromatography. Several chromatographic techniques lead to the isolation of compounds from both extracts. NMR (1D,2D) spectroscopy permitted their identification. From the root extract nine compounds were obtained, belonging to categories of aristolochic acids (aristolochic acid I), hydroxycinnamic acids (p-coumaric acid) and their glucosylated derivatives (acretoside). From the aerial parts extract one cinnamoyl amide was isolated and was strong inhibitor of AChe. This work concluded to active agents against envenomations which may further be used as therapy against wounds or neurological diseases where the same biological targets are implicated.

[1] Gottlob J, Schneider G, Fleischer, Theriaca. Nicandri Colophonii, 1816 [2] Christodoulakis D, The flora of Ikaria (Greece, E. Aegean islands), Phyton (Horn, Austria) 1996; 36:63 - 91

\section{Tu-Poster Session 2-PO-226 Phytochemical profiles and antioxidant activity of Baccharis dracunculifolia and Baccharis trimera ethanolic extracts}

Authors Marques $B^{1}$, Silvana Alves $M^{2}$, Silva Filho $A^{2}$, Dias $A^{1}$ Institute $1 \mathrm{CITAB}$-Centre for the Research and Technology of AgroEnvironmental and Biological Sciences, Department of Biology, University of Minho, Campus de Gualtar, 4710 - 057 Braga, Portugal, Braga, Portugal; 2 Faculdade de Farmácia e Bioquímica, Departamento Farmacêutico, Universidade Federal de Juiz de Fora, Juiz de Fora, MG - Brasil, Juiz de Fora, Brazil

DOI 10.1055/s-0037-1608548

Baccharis sp. plants have been demonstrated antioxidant, neuroprotective and antiinflammatory properties. The goals of this work are to i- characterize the phytochemical profile of Baccharis dracunculifolia (BD) and Baccharis trimera (BT) extracts, and ii- evaluate their in vitro antioxidant activity.

Phytochemical profiles of BD and BT ethanolic extracts were obtained by HPLC-DAD. The phytochemical analysis showed that both plants are mainly composed by phenolic compounds (flavonoids and phenolic acids derivatives), which are known to have antioxidant properties. Antioxidant activity was 
evaluated using several in vitro models: free radical scavenging (DPPH method), iron quelation (ferrozine method), NO and SO scavenging, reduction power (FRAP method), and lipid peroxidation ( $\beta$-carotene bleaching assay); quercetin was used as a reference antioxidant standard. Both extracts showed antioxidant activity in all assays, except BT that did not display an efficient chelating iron activity. In general, BD exhibited better antioxidant activities (EC50 mg dw/ml; DPPH: $54.3 \pm 4.9$; SO: $11.1 \pm 2.2$; $\beta$-carotene: $60,0 \pm 5.3$ ) than BT. BD showed higher activities in NO scavenging (EC50, $217.1 \pm 19.5$ ) and FRAP assays (EC 50, $35.0 \pm 3.0$ ). The antioxidant activities of extracts were significantly different in all assays ( $p<0.05$ ), except for DPPH and FRAP assays. Remarkably, BD demonstrated significant higher activity in scavenging SO radical than quercetin, and both plants displayed better activity than quercetin in reducing ferric ions. Thus, our studies proved that $B D$ and $B T$ can be used as source of compounds with pharmacological properties, namely antioxidants, which could be helpful in neuroprotection and other oxidative stress-related diseases.

This work was supported by Fundação para a Ciência e Tecnologia (FCT), project PEst-OE/AGR/UI4033/2014, INTERACT project -NORTE-01-0145FEDER-000017, HEALTHKAKI, N 010672, Programa Operacional Regional do Norte, Portugal 2020

\section{Tu-Poster Session 2-PO-230 ANTI-ESTROGENIC PROPERTIES OF ETHANOLIC LEAF EXTRACT OF BOERHAVIA DIFFUSA ON FEMALE WISTAR RATS.}

Authors Olajide Omolola $C^{1}$, Okpuzor J ${ }^{1}$, Nnadozie Jane I ${ }^{1}$, Adewole Adeniyi $\mathrm{R}^{1}$

Institute 1 DEPARTMENT OF CELL BIOLOGY \& GENETICS, UNIVERSITY OF

LAGOS, NIGERIA, Yaba, Lagos, Nigeria

DOI 10.1055/s-0037-1608549

Boerhavia diffusa (BED) which belongs to the family Nyctaginaceae is found in India and Nigeria and is commonly known as Punarnava which means rejuvenates or renews the body. Boerhavia diffusa leaf has been used in folkloric medicine for the treatment of diverse ailments such as elephantiasis, night blindness and corneal ulcers, malaria, abdominal pain, jaundice, inflammation, as well as to treat infertility etc. This study assesses the estrogenic activity of ethanol extract of BED leaf in Cisplatin-induced infertility in female Wistar rats. Cisplatin is a chemotherapeutic drug used for the treatment of numerous human cancers, however with induction of infertility as one of its downsides. Twenty-four female albino rats weighing between $100-200 \mathrm{~g}$ were used for this study. They were distributed into five groups where the first group served as the control. The animals in group two received an intraperitoneal dose of Cisplatin ( $5 \mathrm{mg} \mathrm{kg}^{-1}$ body weight). Animals in groups three and four received a combination of BED ethanolic extract (100 mg kg $\mathrm{mg}^{-1}, 200 \mathrm{mg} \mathrm{kg}^{-1}$ body weight) and Cisplatin ( $5 \mathrm{mg} \mathrm{kg}^{-1}$ body weight) while animals in group five received an oral dose of BED ethanolic extract only (100 mg kg-1 body weight) for 14 days. At the end of the study period, two rats from groups 2, 3 and 4 were separated and mated with a male rat and blood samples were collected for hormonal analysis. After 8 weeks no pregnancy was achieved demonstrating that cisplatin can cause irreversible infertility.

A decrease in estradiol level $(p<0.05)$ in the treated groups was observed compared to control which may be an indication that Boerhavia diffusa possess anti-estrogenic properties.

\section{Tu-Poster Session 2-PO-231 Association of Pterodon pubescens and Cordia verbenacea in inflammation and thermal nociception models}

\author{
Authors Tarkany Basting $\mathrm{R}^{1,2}$, Maria de Oliveira Sousa $\mathrm{I}^{1,2}$, Moreira

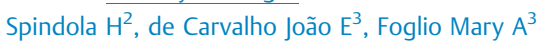 \\ Institute 1 Postgraduate Program in Medical Sciences - Faculty of Medical \\ Science - FCM/State University of Campinas - Unicamp, Campinas, Brazil; \\ 2 CPQBA, State University of Campinas - Unicamp, Campinas, Brazil;
}

3 Faculty of Pharmaceutical Sciences - FCF/State University of Campinas -

Unicamp, Campinas, Brazil

DOI 10.1055/s-0037-1608550

Pterodon pubescens (Pp) and Cordia verbenacea (Cv) species are traditionally used in Brazilian folk medicine for the known anti-inflammatory effects [1]. The purpose of this study was to assess the synergistic effect of the dichloromethane extract from the fruits of $\mathrm{Pp}$ with the essential oil from leaves of $\mathrm{CV}$ in paw edema induced by carrageenan, hot plate and tail flick models. All animals (male Swiss mice $n=6$ ) were orally treated with the association of the extracts based on their $E_{50}$, corresponding to: $A_{50}(32.5 \mathrm{mg} / \mathrm{kg}$ of $\mathrm{Pp}+$ $82.5 \mathrm{mg} / \mathrm{kg}$ of $\mathrm{Cv}) ; A_{100}(65 \mathrm{mg} / \mathrm{kg}$ of $\mathrm{Pp}+165 \mathrm{mg} / \mathrm{kg}$ of $\mathrm{Cv})$ and $A_{200}$ $(130 \mathrm{mg} / \mathrm{kg}$ of $\mathrm{Pp}+330 \mathrm{mg} / \mathrm{kg}$ of $\mathrm{Cv})$. All experimental animal procedures were approved by Ethics Committee $n^{\circ} 3181-1$. In hot plate and tail flick models, all associations tested did not show significant efficacy against nociception due to thermal stimulation. In paw edema induced by carrageenan, the 3 associations tested showed a significant reduction of edema from the fourth hour after carrageenan administration, the activity remaining after 48 hours. From the fourth hour of evaluation, $A_{200}$ showed $26 \%$ inhibition in the volume of edema when compared to the vehicle (negative control). After 6 hours of edema induction, $A_{50}$ and $A_{100}$ doses, respectively, showed inhibition of edema in $19 \%$ and $23 \%$, while $A_{200}$ showed reduction of approximately $39 \%$ when compared to vehicle. After 24 and 48 hours of edema induction, all associations showed reduction of approximately $32 \%$ when compared to vehicle. The results demonstrated that the associations showed a significant anti-edematogenic activity, but did not show significant results in thermal stimulus models. Therefore this extracts association may represent a potential therapeutic advantage for the clinical treatment of inflammation.

[1] Dutra, R.C. et al. Pharmacol Res 2016; 112, 4- 29.

\section{Tu-Poster Session 2-PO-232 On the in vitro Anti-Dengue Virus Activity of the Oleoresin Labdanum of Cistus creticus}

Authors Kuchta $\mathrm{K}^{1}$, Tung Nguyen $\mathrm{H}^{2}$, Ota $\mathrm{T}^{2}$, Rausch $\mathrm{H}^{3}$, Rauwald Hans $\mathrm{W}^{4}$, Morita $\mathrm{K}^{5}$, Shoyama $\mathrm{Y}^{2}$

Institute 1 Clinic for Gastroenterology and Gastrointestinal Oncology, Göttingen University, Göttingen, Germany; 2 Department of Pharmacognosy, Graduate School of Pharmaceutical Sciences, Nagasaki International University, Sasebo, Japan; 3 Phytochem Referenzsubstanzen, Neu-Ulm, Germany;

4 Department of Pharmaceutical Biology, Leipzig University, Leipzig,

Germany; 5 Department of Virology, Institute of Tropical Medicine, Nagasaki University, Nagasaki, Japan

DOI 10.1055/s-0037-1608551

During the epidemics of the mediaeval period, doctors in Byzantium and Italy developed the "Alipta muscata" as a preventive medicine against epidemics. When treating the "black death" doctors constantly used it for self-medication. The main ingredient of "Alipta muscata" was the oleoresin labdanum of Cistus creticus L. [1]. While the "black death" is mostly interpreted as Yersinia pestis, there is also an alternative interpretation as a viral haemorrhagic fever [2]. We tested several extracts and fractions of labdanum on their activity against the dengue virus (DENV-2 strain 00st-22A) in in vitro cultures on Vero cells (96-well-plates, 5 days). This haemorrhagic fever affects up to 500 million patients annually with no chemotherapeutic agent available and causes 20.000 deaths. Preliminary experiments with a labdanum full extract did not yield measureable results due to cytotoxic effects against Vero cells. In all following experiments, cell viability was constantly checked using the MTTtest. Fractionation of the dichloromethane raw-extract by liquid-liquid-extraction and column-chromatography on silica-gel (gradient elution with Hexane, EtOAc, $\mathrm{CHCl}_{3}, \mathrm{MeOH}$ ) succeeded in separating the anti-viral activity of labdanum from its cytotoxic effect. In the most active fraction GS5 at $30 \mu \mathrm{g} / \mathrm{ml}$, the dengue virus proliferation was $100 \%$ suppressed and cell viability over $90 \%$. Structural elucidation of major constituents of GS5 is currently ongoing. 
Claims concerning the antiviral activity of above ground parts of $C$. creticus have been made previously, but these generally ascribe this activity to hot water soluble polyphenols and propose an unspecific tanning effect of the viral surface proteins as the mechanism of action [3]. We describe an antiviral activity of a dichloromethane extract of labdanum against a virulent haemorrhagic fever like dengue for the first time.

[1] Husemann T. Archiv der Pharmacie. 1889;27:1075 - 1132

[2] Duncan C], Scott S. Postgrad Med J. 2005;81:315-20

[3] Ehrhardt C et al. Antiviral Res. 2007;76:38-47

\section{Tu-Poster Session 2-PO-233 The nematode strikes back - C. elegans' proline-rich response to treatment with oligomeric procyanidins}

\section{Authors Spiegler $\mathrm{V}^{1}$, Lubisch $\mathrm{M}^{2}$, Hensel $\mathrm{A}^{1}$, Liebau $\mathrm{E}^{2}$}

Institute 1 University of Münster, Institute for Pharmaceutical Biology \& Phytochemistry, Münster, Germany; 2 University of Münster, Institute for Animal Physiology - Molecular Physiology, Münster, Germany DOI 10.1055/s-0037-1608552

Human salivary proline-rich proteins are known to bind efficiently to tannins as a mechanism of detoxifications in mammals. However, no such response on protein level has been described for invertebrates [1]. Recent results from a microarray experiment indicated a remarkable concentration dependent increase in the expression of the hitherto uncharacterized gene T22D1.2 in Caenorhabditis elegans after treatment with oligomeric procyanidins (OPC) [2]. BLASTp analysis of the predicted protein sequence revealed a high similarity to the human Basic salivary proline-rich protein 2 (PRB2). For unambigious localization of the specific sites of gene expression and excretion of this protein, the promoter was fused to GFP either directly or with the sequence of the signal peptide inserted. In worms containing the GFP construct including the signal peptide following the promoter sequence, fluorescence immediately disappeared. However, a strong concentration dependent increase in fluorescence was observed in the worms' intestine after treatment for $6 \mathrm{~h}$ with an OPC enriched hydroethanolic (1:1) leaf extract $(0.2-2 \mathrm{mg} / \mathrm{mL})$ from Combretum mucronatum in $C$. elegans containing the promoter-GFP fusion. The negative control did not show any signs of fluorescence. These findings were additionally confirmed by qPCR experiments.

Due to its structural similarity to PRB2, its lack of constitutive expression and the strong up-regulation upon treatment with OPCs, this gene could likely be involved in the defense mechanism of $C$. elegans against tannins.

[1] Barbehenn RV, Constabel CP. Phytochemistry 2011; 72: 1551 - 1565.

[2] Spiegler V, Sendker ], Petereit F, Liebau E, Hensel A. Molecules 2015; 20 : $14810-14832$.

\section{Tu-Poster Session 2-PO-234 A COMPARATIVE STUDY OF THE PROXIMATE, ELEMENTAL NUTRIENT, FT-IR SPECTROSCOPIC OF Grewia lasiocarpa E.Mey. ex Harv.: INDIGENOUS SOUTHERN AFRICAN PLANT}

\footnotetext{
Authors Akwu N ${ }^{1}$, Naidoo $\mathrm{Y}^{2}$

Institute 1 University of Kwazulu Natal, Durban, South Africa; 2 University of Kwazulu-Natal, Durban, South Africa

DOI 10.1055/s-0037-1608553

The crude powder of the leaves, and stembark of Grewia lasiocarpa E.Mey. ex Harv, commonly known as forest raisins were analysed for proximate chemical composition (\% ash, \% carbohydrate, \% moisture, \% lipid, \% total protein) using the methods of Association of Official analytical chemist (AOAC), and the energy (Kcal) was derived. An analytical comparison of the Certified Reference Material (CRM) White Clover (BCR 402), gave a significant correlation, between the certified values of the analyte elements (uncertainty- $\mathrm{mg} / \mathrm{kg}$ ), and the data obtained using Energy dispersive X-ray fluorescence (EDXRF) microscopy. A Thermo Scientific Spectrophotometer system was employed to con-
}

duct the FT-IR; to detect the characteristic peak values, and their functional groups.

The proximate composition of the \% ash, \% carbohydrate, \% moisture, \% lipid, $\%$ total protein (Leaves/stembark) were $34.00 \% / 25.38 \%, 44.56 \% / 62.33 \%$, $6.84 \% / 6.04 \%, 2.67 \% / 2.00 \%, 11.93 \% / 4.25 \%$; while the derived energy values were $249.99 \mathrm{Kcal} / 284.32 \mathrm{Kcal}$ respectively. The EDXRF analysis revealed the presence of elements including the essential elements such as Calcium (Ca), Iron (Fe), Selenium (Se), Zinc ( $\mathrm{Zn})$, Copper (Cu), Chromium ( $\mathrm{Cr}$ ) in various proportions, dominant characteristics peak values in the FT-IR investigation, confirming the presence of aromatic compounds, carbonyl compounds, halogens, and other functional groups, in the crude powder of G. lasiocarpa E. leaves, and stembark.

The results obtained validate the potential that G. lasiocarpa is a plant with appreciable medicinal properties, although documentation on its ethnobotany is relatively limited.

\section{Tu-Poster Session 2-PO-235 Polyacrylamide and radiation-derived oligosaccharides induced regulation of physiological processes and essential oil production in Vetiveria zizanioides L. Nash}

Authors Khan $\mathrm{MMA}^{1}$, Uddin $\mathrm{M}^{2}$

Institute 1 Department of Botany, Faculty of Life Sciences, Aligarh Muslim University, Aligarh, India; 2 Botany Section, Women's College, Aligarh Muslim University, Aligarh, India

DOI 10.1055/s-0037-1608554

Vetiver (Vetiveria zizanioides L. Nash), a quintessential medicinal and aromatic plant (MAP), stockpiles its essential oil (EO) in the roots which serves as the well head of the key natural inputs to the proliferating cosmeceutical, pharmaceutical and perfumery industries. Radiation-processed marine polysaccharides (Figure) when exogenously applied, act as plant growth elicitors, regulating various biological activities. Polyacrylamide (PAM), on the other hand, is a remarkable soil conditioner known to accentuate root biomass. Therefore, a study was performed using pot experiment to find out the efficacy of the best screened combinations of PAM and irradiated sodium alginate (ISA) and irradiated chitosan (ICH) on various growth and physiological markers including EO production in vetiver. The structural characterization of the radiation-derived oligomers was done using complementary techniques like, GPC, SEM, FT-IR and UV-vis spectroscopy to develop an understanding of structure-property relationship. Of the various applied treatments, $120 \mathrm{mg}$ $\mathrm{kg}^{-1}$ PAM $+120 \mathrm{mg} \mathrm{L}^{-1} \mathrm{ICH}$ proved best for most of the parameters studied. It exceeded the net photosynthetic rate and chlorophyll fluorescence by $29.5 \%$ and $27.8 \%$, respectively over the control. Consequently, endogenous NO generation promoted the formation and elongation of roots in dose dependent manner. The linear increase in NO generation and nitrate reductase (NR) activity suggested that NR, rather than nitric oxide synthase (NOS), might be involved in oligomers induced root development and hence EO increase in the plant. The said treatment excelled the control in content and per plant yield of EO by $29.9 \%$ and $67.9 \%$ and that of khusimol by $60.9 \%$ and $170.4 \%$, respectively. In a nut-shell, combined application of these oligomers along with PAM was found more effective compared to their individual applications, thus, serving as an eco-friendly and economical way to improve and utilize the full potential of medicinal herbs like vetiver. 


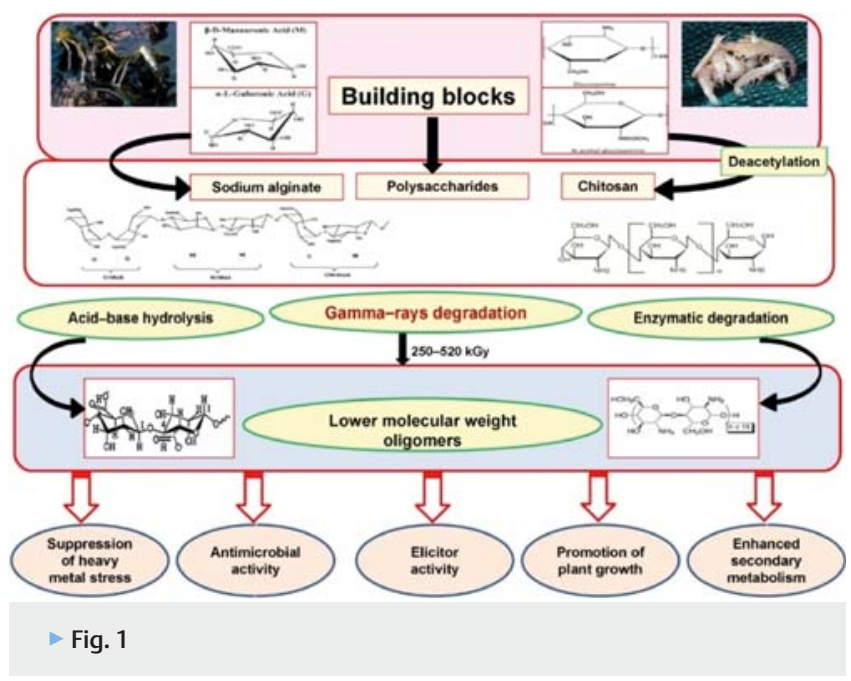

\section{Tu-Poster Session 2-PO-239 Determination of most prescribed herbal products in traditional medicine clinics affiliated to Tehran University of Medical \\ Sciences}

Authors Bozorgi $\mathrm{M}^{1}$, Rahimi $\mathrm{R}^{1}$

Institute 1 Department of Traditional Pharmacy, School of Traditional Medicine, Tehran University of Medical Sciences, Tehran, Iran, Tehran, Iran DOI 10.1055/s-0037-1608555

Traditional medicine (TM) has a long history in Iran and Iranian people have been use TM for varies reasons like efficacy and economical benefits [1]. In recent years, WHO strategies were focused on developing TM to ensure public health protection especially in developing countries. Based on these strategies school of Iranian TM and affiliated educational clinic and pharmacy were established by Tehran University of Medical Sciences (TUMS) in 2007 [2]. In this study herbal products prescribed by physicians during June to December 2016, were investigated. Data were collected from medical record information and analyzed using the SPSS version 23 software. As the result of this study, supplied herbal products are including capsules (58 types), tablets (24 types), syrups (36 syrups), majoons (semi solid product containing plants powder and honey) (22 types). All of the preparations are licensed by the food and drug administration, Ministry of Health and Medical Education. Five most widely prescribed samples are presented in table 1 . The most useful product is "kabed" capsule for improvement of liver function. Kabed capsule in addition to some of other medications showed beneficial effect for treatment of a patient with cryptogenic cirrhosis [3]. According to Iranian TM, all of these products have varies applications and some of them have not been studied yet. Investigation of the key usages can be subjected for future studies.

[1] Adhami HR, Mesgarpour B, Farsam H, Herbal Gram 2007; 74: 35 - 43.

[2] Rezaeizadeh H, Alizadeh M, Naseri M, Shams Ardakani MR. Iranian J Publ Health 2009; 38 (Suppl. 1):169 - 172.

[3] Shamsi Baghbanan H, Nazem E, Yarjoo S, Minaei B. Iran Red Crescent Med ] 2014; 16: e16548.

\section{Tu-Poster Session 2-PO-240 Antioxidant and anti-inflammatory Studies of mushrooms}

Authors Seo Kyung $H^{1}$, Park JY ${ }^{1}$, Kim HD ${ }^{1}$, Lee Dea $Y^{1}$, Choi Doo J', Lee Jea $W^{1}$, Lee $Y S^{1}$, Lee $S E^{1}$, Kim Geum $S^{1}$

Institute 1 Rual Development Adiministration, Eumseing, Korea, Republic of (South)

DOI 10.1055/s-0037-1608556

Oxidative stress and inflammation are closely related to chronic inflammatory diseases including arthritis, atherosclerosis, diabetes, and hypertension. We studied the effects of the antioxidants and anti-inflammatory activities of various mushroom. We extracted the mushroom with ethanol and water and investigated the antioxidant properties of mushroom extracts with the DPPH and ABTS radical scavenging activity. The extracts showed potent antioxidant activity compared with trolox and tocopherol. In macrophages, nitric oxide (NO) works as a key inflammatory mediator and releases an array of cytokines in inflammation. We also investigated that the inhibition effects of mushroom extracts on $\mathrm{NO}$ in anti-inflammatory activities in which the generation of NO was turned down than LPS-induced inflammatory group in Raw264.7 cells when treating with $100 \mu \mathrm{g} / \mathrm{ml}$ and $200 \mu \mathrm{g} / \mathrm{ml}$ concentration of ethanol extracts, such as Isaria japonica Yasuda, Phellinus linteus (Berk. EtCut.) Teng, Inontus obliquus and Umbilicaria esculenta. In addition, mushroom extracts downregulated the production and mRNA expression of proinflammatory cytokines including tumor necrosis factor (TNF)- $\alpha$, interleukin (IL)-6 and IL-1 $\beta$. These results all suggest that mushroom extracts have antioxidants and anti-inflammatory activity.

\section{Tu-Poster Session 2-PO-241 Neutralizing activity of Echinacea purpurea on Coronaviruses including highly pathogenic Middle-East-Respiratory Syndrome Virus (MERS-CoV)}

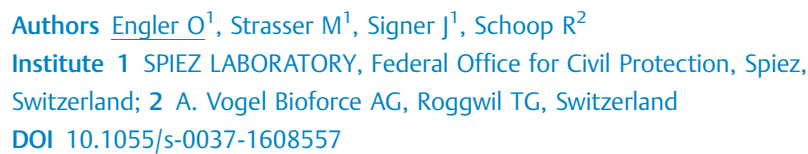

Coronaviruses ( $\mathrm{CoV}$ ) are able to infect the human respiratory tract and subtypes 229E, NL63, HKU-1 or OC 43 are frequently detected during common colds. Other CoV strains infect animals but recent history has seen two cases of animal-to-human transitions, leading to the epidemic of Severe Acute Respiratory Syndrom (SARS) in 2003 and in recent years to outbreaks of MiddleEast-Respiratory-Syndrome (MERS).

We tested the antiviral hydroalcoholic extract $(65 \% \mathrm{~V} / \mathrm{V})$ of freshly harvested Echinacea purpurea herb and roots (Echinaforce ${ }^{\circledR}$, EF) on Coronavirus $229 \mathrm{E}$ and also the highly pathogenic MERS-type. CoV $229 \mathrm{E}\left(10^{5}\right.$ tissue culture infectious dose, $\left.\mathrm{TCID}_{50}\right)$ and MERS-CoV $\left(1.6 \times 10^{4} \mathrm{TCID}_{50}\right)$ were incubated with $0,1,10$ and $50 \mu \mathrm{g} / \mathrm{ml} \mathrm{EF}$ for $1 \mathrm{~h}$ and remaining infectivity assessed by determining $\mathrm{TCID}_{50}$ on HuH-7 and Vero cells. EF showed a dose dependent reduction of CoV 229E infectivity with an IC $50=9+/-3 \mu \mathrm{g} / \mathrm{ml}$. Complete neutralization was achieved with $50 \mu \mathrm{g} / \mathrm{ml}$. Similar inhibition was observed for MERS-CoV, where $10 \mu \mathrm{g} / \mathrm{ml} \mathrm{EF}$ reduced $>99.9 \%$ and $50 \mu \mathrm{g} / \mathrm{ml}$ fully blocked infectivity. Interestingly, virus inhibition was only seen upon direct exposition of virus to the extract. Corresponding tests with other viruses showed that membranous RNA-viruses such as Influenza, Yellow fever virus were also neutralized by EF, while infectivity of DNA-viruses such as Parvo- and Vaccinia virus were not altered by EF.

In summary, pre- and post-incubation experiments show that EF has the potential of inhibiting infection rather than the dissemination of Coronaviruses. Our data are in agreement with the results of a placebo controlled study, where 4-months Echinaforce ${ }^{\circledR}$ prevention significantly reduced membranous virus infections overall $(p=0.0114)$, and Coronavirus infections in particular: 33 infections in $\mathrm{N}=362$ with placebo i.c. to 21 infections in $\mathrm{N}=355$ with $\mathrm{EF}$ [1].

[1] Jawad M, et al. ECAM. 2012: 841315. Epub 2012 Sep 16. 
Lecture Session - Bionorica Phytoneering Award Lecture

Wednesday, 6th September, 2017

Montreal - 09:00 - 09:45

\section{We-Bionorica Phytoneering Award Lecture Re-emergence of medicinal plant materials rich in ellagitannins?}

Authors Kiss AK ${ }^{1}$, Granica $S^{1}$, Piwowarski JP $P^{1}$

Institute 1 Department of Pharmacognosy and Molecular Basis of

Phytotherapy, Medical University of Warsaw, Warsaw, Poland

DOI 10.1055/s-0037-1608558

Ellagitannin-rich plant materials were for the long time beyond the mainstream of the natural product research. Despite many of them were used in Traditional European Medicine (TEM) and some have nowadays a monograph in European Pharmacopoeia, their phytochemical composition and biological activities were not an attractive scientific target. This was because ellagitannins (ETs) as high molecular weight polyphenols, were commonly considered to be difficult for isolation and structure elucidation, while their biological effects were attributed to unspecific protein binding properties.

Our studies were thus focused on so far underestimated ET-rich plant materials used in TEM for treatment of inflammation-associated diseases of i.a. skin, mucosa and gastrointestinal tract or as chemopreventive agents. During our investigations many ellagitannins were isolated. Complex comparative studies on human neutrophil ex vivo model have shown interesting differences in modulation of inflammatory response between extracts and isolated ellagitannins indicating also structure-dependent activities for single compounds. These results support the traditional use of chosen ET-rich plant materials, however due to doubtful bioavailability of ETs, they can only be referred to the topical application. In order to find out what could be the fate of ETs after oral administration, studies of extracts' and ETs' metabolism using human gut microbiota ex vivo cultures were conducted showing that they are transformed to the series of small-molecule, bioavailable compounds called urolithins. The urolithins were tested on various cellular models including neutrophils and macrophages. Obtained results have shown strong and specific inhibitory activities of urolithins towards various pro-inflammatory processes, which were observed at concentrations within their bioavailability.

The conducted investigations have proven, that the ET-rich plant materials used in TEM are attractive candidates for further research of novel anti-inflammatory and chemopreventive agents as well as indicated gut microbiota as a crucial factor determining their metabolism after oral administration.

\section{Lecture Session - Plenary Lecture 4}

Wednesday, 6th September, 2017

Montreal - 09:45 - 10:30

\section{We-Plenary Lecture Session 4-PL-01 Translational research - an integral part of rational herbal drug development}

\section{Authors Drewe J ${ }^{1}$}

Institute 1 Max Zeller Soehne AG, Romanshorn, Switzerland

DOI 10.1055/s-0037-1608559

In the past, herbal drug development was often based on ethnopharmacological experiences and traditional use. Due to over the years increasing regulatory demands, innovative new herbal drug development becomes more and more cost intensive and therefore less affordable. Therefore, there are only very few new herbal medicinal drugs that have been developed and registered during the last years.

To overcome these problems, new approaches need to adopted. Herbal extracts with recognized traditional or well-established use, however, possess often a well-defined and beneficial safety profile and mostly an established galenics. That minimizes the required preclinical effort, when these extracts are developed in new indications (use extension).

Systematic evaluation of pharmacological targets is a new promising way of rational herbal drug development in order to exploit the full therapeutic potential of medicinal plants. Examples of such translational research are discussed for Hypericum perforatum (HP) and Cimicifuga racemosa (CR) including treatment of neuropathic pain (HP) and antidiabetic and antiproliferative effects (CR), respectively.

\section{Lecture Session - Ethnopharmacology Wednesday, 6th September, 2017 Montreal - 11:00 - 12:30}

\section{We-Ethnopharmacology-SL-01 Anthelmintic activity of procyanidins from West African medicinal plants - Insights into phytochemistry and molecular targets}

Authors Spiegler $\mathrm{V}^{1}$, Liebau $\mathrm{E}^{2}$, Hensel $\mathrm{A}^{1}$

Institute 1 University of Münster, Institute for Pharmaceutical Biology \& Phytochemistry, Münster, Germany; 2 University of Münster, Institute for Animal Physiology - Molecular Physiology, Münster, Germany DOI 10.1055/s-0037-1608560

Oligomeric procyanidins (OPC) have been identified as the major anthelmintic components in hydroethanolic extracts from Combretum mucronatum leaves [1] and Paullinia pinnata roots [2] that are used as traditional remedies against soil-transmitted helminthiasis (STH) in West Africa [3]. The traditional use was rationalized by in vitro mortality assays against Caenorhabditis elegans and selected parasitic helminths (Toxocara cati, Trichuris vulpis).

To investigate the anthelmintic activity of OPCs on a molecular level, a transcriptome analysis was performed in $C$. elegans after treatment with purified OPCs from C. mucronatum (0.02 to $2 \mathrm{mg} / \mathrm{mL}$ ).

Highly significant changes in differential gene expression were observed mainly for proteins related to the intestine, many of which were located extracellularly or within cellular membranes. Among the up-regulated genes, several hitherto undescribed orthologues of structural proteins in humans were identified, but also genes that are potentially involved in the worms' mechanism of tannin detoxification. For example, T22D 1.2, an orthologue of human Basic salivary proline-rich protein (PRB) 2 or a nuclear localized metal responsive (numr-1) were found to be strongly up-regulated. Downregulated genes, were mainly associated with lysosomal activity, glycoside hydrolysis or the worms' innate immune response.

The current findings support previous hypotheses of OPCs interacting with intestinal surface proteins and provide the first insights into the nematode's response to OPCs on a molecular level as a base for the identification of future drug targets.

[1] Spiegler V, Sendker J, Petereit F, Liebau E, Hensel A. Molecules 2015, 20 : $14810-14832$.

[2] Spiegler V, Liebau E, Peppler C, Raue K, Werne S, Strube C, Heckendorn F, Agyare C, Stark T, Hofmann T, Hensel A. Planta Medica 2016, 82: 1173 - 1179. [3] Agyare C, Spiegler V, Sarkodie H, Asase A, Liebau E, Hensel A. J Ethnopharmacol 2014, 158PA: $255-263$.

\section{We-Ethnopharmacology-SL-02 History of medicinal plant use in Europe: A phylogenetic and organoleptic approach}

Authors Leonti $\mathrm{M}^{1}$, Casu $\mathrm{L}^{2}$, Staub $\mathrm{P}^{1}$

Institute 1 Department of Biomedical Sciences, University of Cagliari, Via Ospedale 72, 09124 Cagliari, Italy, Cagliari, Italy; 2 Department of Life and 
Environmental Sciences, University of Cagliari, Via Ospedale 72, 09124

Cagliari, Italy, Cagliari, Italy

DOI 10.1055/s-0037-1608561

Historical texts have emerged as a valuable tool for studying the therapeutic knowledge of past cultures. Dioscorides' De Materia Medica (DMM) written during the $1^{\text {st }}$ century $A D$ is one of the most influential historical texts dealing with the therapeutic use of herbal drugs in Europe and the Mediterranean. DMM summarizes medicinal knowledge for over 1000 plant, mineral and animal derived drugs. Despite the historical relevance of DMM for modern herbal medicine and phytotherapy the content of this work has hitherto not been assessed systematically [1]. Therefore, the herbal drugs described in DMM (ex Matthioli, 1568 [2]) were analysed using a multidisciplinary and quantitative approach. We analysed the correlation of smell and taste properties of herbal drugs assessed through an organoleptic tasting panel with their therapeutic uses described in DMM. A prevalence of uses for infectious diseases, a high frequency of root drugs, a marked use of Fabaceae seeds for dermatology, Apiaceae exudates for neurological problems and Apiaceae seeds as antidotes were noticed. Herbal drugs with higher flavour complexity also tend to have more therapeutic uses indicating that chemosensory complexity is linked with perceived therapeutic efficacy.

This project has received funding from the European Union's Seventh Framework Programme for research, technological development and demonstration under grant agreement no. 606895.

[1] Staub P, Casu L, Leonti M. Phytomedicine 2016; 23:1043 - 1052.

[2] Matthioli PA. I Discorsi di M. Pietro Andrea Matthioli. Sanese, Medico Cesareo, et del Serenissimo Principe Ferdinando Archiduca d'Austria \& c. Nelli Sei Libri Di Pedacio Dioscoride Anazarbeo della Materia Medicale. Vincenzo Valgrisi, Venezia, 1568. Reprinted in Facsimile. Rome: Stabilimento Tipografico Julia; $1967-70$.

\section{We-Ethnopharmacology-SL-03 Hepatic gluconeogenesis inhibition by four traditional used, hypoglycemic plants}

Authors Andrade-Cetto A ${ }^{1}$, Mata-Torres Valle $\mathrm{G}^{1}$, Espinoza-Hernández $\mathrm{A}^{1}$, Cárdenas-Vázquez René de $J^{2}$

Institute 1 Universidad Nacional Autónoma de México Facultad de Ciencias. Laborartorio de Etnofarmacología, Ciudad de México, Mexico; 2 Universidad nacional Autónoma de México Facultad de Ciencias. Laborartorio de Biología Animal Experimental, Ciudad de México, Mexico

DOI 10.1055/s-0037-1608562

Type 2 diabetes (T2D) is characterized by insulin resistance combined with a relative deficiency in insulin secretion [1]. In T2D the liver plays a key role in maintaining blood glucose levels during fasting by synthesizing glucose, mainly from pyruvate and amino acids (gluconeogenesis) [2]. In T2D with overt fasting hyperglycemia (>140 mg/dl,), an excessive rate of hepatic glucose output is the major abnormality responsible for the elevated fasting plasma glucose. In Mexico, besides the prescribed drugs for the treatment of Type 2 diabetes, people use medicinal plants attempting to control their hyperglycaemic state. Generally, they prepare infusions that are drunk during the day, between meals. We documented in our field work at different communities of Mexico, four medicinal plants used by the people to treat T2D; Ageratina petiolaris (AP), Bromelia karatas (BK), Rhizophora mangle (RM) and Smilax moranensis (SM). We previously proved that they exert a chronic and acute hypoglycemic effect in diabetic rats. We hypothesize that one possible mechanism of action for these plants is through inhibition of hepatic gluconeogenesis. We tested the effect of extracts from these plants on gluconeogenesis by performing pyruvate tolerance tests in fasted Wistar rats and analyzing their effect on the in vitro activity of glucose -6-phosphatase from hepatic rat microsomes.

The in vivo results showed that AP, RM and SM reduce the hyperglycemic peak at 30 min after the administration of pyruvate, with only AP and RM sustaining the effect up to 120 min, in vitro AP, RM and SM inhibited the activity of the enzyme (table 1). We conclude that the extracts of AP, RM and SM can inhibit gluconeogenesis both in vivo and in vitro.

[1] ADA AD. Classification and Diagnosis of Diabetes. Diabetes Care 2015; 38: S8-S 16.

[2] Guyton AC, Hall JE. Textbook of Medical Physiology. 11th ed. Philadelphia, Pennsylvania: Elsevier, 2006

\section{We-Ethnopharmacology-SL-04 Anti-mycobacterial, cytotoxicity and genotoxicity effects of five traditionally used anti-tuberculosis plants in South Africa}

Authors Madikizela $\mathrm{B}^{1}$, McGaw $\mathrm{L}^{1}$

Institute 1 Phytomedicine Programme, Department of Paraclinical Sciences, University of Pretoria, P/Bag X04, Onderstepoort 0110, Pretoria, South Africa DOI 10.1055/s-0037-1608563

Tuberculosis (TB) is a re-emerging bacterial infectious disease that infects both humans and animals (cattle). This disease has caused major health concerns and is responsible for about 1.4 million deaths per annum. TB can be cured, however, the emergence of drug-resistant strains has made the disease difficult to treat, thus establishing a need to search for new effective agents. Worldwide, plants are believed to serve as anti-TB agents. In South Africa, there is potential to find plants with anti-TB effects due to the great floral diversity found in this region. Based on a local ethnobotanical survey identifying 24 traditionally used anti-TB plants, and after scrutiny of available literature, Hypoxis colchicifolia, Heteromorpha trifoliata, Phymaspermum acerosum, Pterocelastrus echinatus and Pittosporum viridiflorum were selected for antimycobacterial activity. Acetone, $70 \%$ ethanol, cold and hot water extracts were tested for antimycobacterial activity against non-pathogenic Mycobacterium aurum, M. bovis BCG, M. fortuitum, M. gordonae and M. smegmatis. Extracts that demonstrated MIC values less than $1 \mathrm{mg} / \mathrm{ml}$ against at least three $\mathrm{Myco}$ bacterium strains were evaluated for cytotoxicity (againts Vero monkey kidney cells) and genotoxicity (against Salmonella typhimurium TA98 and TA100). The MIC values ranged from 0.020 to $2.500 \mathrm{mg} / \mathrm{ml}$, and $\mathrm{LC}_{50}$ values ranged from 0.001 to $17.739 \mathrm{mg} / \mathrm{ml}$. P. acerosum ethanol extract demonstrated the best MIC value of $0.020 \mathrm{mg} / \mathrm{ml}$ against $M$. smegmatis and $M$. bovis, whereas $P$. echinatus acetone extract was the most cytotoxic $\left(\mathrm{LC}_{50}=0.001 \mathrm{mg} / \mathrm{ml}\right)$. Almost all tested extracts were non-genotoxic with the exception of of $H$. colchicifolia and $P$. viridiflorum that were slightly genotoxic, as the number of revertant colonies increased with increasing concentrations. The results indicate that these plants could be investigated further against pathogenic and drug-resistant TB strains, which is underway.

\section{We-Ethnopharmacology-SL-05 Bioactive Compounds from Sumatran Medicinal Plants}

Authors Arbain $\mathrm{D}^{1}$

Institute 1 Faculty of Pharmacy/Sumatran Biota Laboratory, Andalas

University, Kampus Limau Manis, Padang 25163, West Sumatra Indonesia, Andalas University, Padang, Indonesia

DOI 10.1055/s-0037-1608564

Sumatra is the fourth largest island in the world and known to be very rich with varieties of tropical rainforest plants. Many of these plants have been used traditionally for centuries for many purposes such as medicines, coloring matters, food, spices, insecticides, and aromatics. Until the 1950 s the Sumatran flora was relatively untouched from a scientific point of view. Realizing the danger of losing these Sumatran plants and their related traditional knowledge and culture, our first inventory of Sumatran plants was carried out in my village near Bukittinggi and in Padang. Later inventories followed in more than 50 different ecotypes of Sumatran forests. Based on these inventories more than 130 flowering plants having traditional medicinal values have been in- 
vestigated for their chemical constituents, and a number of new and known compounds were isolated [1].

In recent years we have continued with collecting trips in Sumatra, with a focus on traditional uses and antimicrobial activities. Extracts were screened against human pathogenic bacteria, eg. Bacillus subtilis ATCC 6633, Enterococcus faecalis ATCC 29212, Staphylococcus epidermidis ATCC 12228 and NTCC 1224, Salmonella typhosa NCTC 786, Streptococcus mutans ATCC 25175, Micrococcus luteus ATCC 10240, Staphylococcus aureus ATCC 25923, Escherichia coli ATCC 25922 and NTCC 1224, Salmonella thypimurium ATCC 14028 and NTCC 12023, and methicilline resistant Staphylococcus aureus (MRSA). Phytochemical investigation of selected plants, such as Piper crocatum Ruiz \& Pav afforded new natural products, while the parasitic plant Balanophora elongata Blume, and ferns such as Sphaerostephanos polycarpus (BI.) Copel, Oleandra pistillaris (SW.) C. Chr, and Trichomanes javanicum L. yielded compounds with strong antibacterial activity. Attempts to conserve these useful Sumatran plants will be mentioned.

[1] Arbain D. Nat Prod Comm 2012; 7: 685-814

\section{We-Ethnopharmacology-SL-06 Use of herbal medicine in the management of trypanosomiasis in Angola}

Authors Vahekeni $\mathrm{N}^{1,2}$, Neto Pedro $\mathrm{M}^{3}$, Kayimbo Malilo $\mathrm{K}^{2}$, van Eeuwijk $\mathrm{P}^{1,7}$, Mäser $P^{1}$, João Pedro $G^{3,4}$, Théophile $J^{5}$, Wolfram $E^{6}$, da Costa $E^{3}$, Falquet $]^{8}$ Institute 1 Swiss Tropical and Public Health Institute (Swiss TPH), University of Basel, Basel, Switzerland; 2 Nacional Center of Scientific Investigation (CNIC), Luanda, Angola; 3 3Centro de Estudos e Investigação Científica de Botânica, University of Agostinho Neto, Luanda, Angola; 4 Engineering Faculty of Agostinho Neto University, Luanda, Angola; 5 Instituto de Combate e Controlo das Tripanossomíasses (ICCT), Luanda, Angola; 6 Zürich University of Applied Sciences (ZAHW), Phytopharmacy \& Natural Products, Institute of Chemistry and Biotechnology, Wädenswil, Switzerland; 7 Institute of Social

Anthropology, University of Basel, Basel, Switzerland; 8 Community Health

Association, Geneva, Switzerland

DOI 10.1055/s-0037-1608565

More than 70 per cent of the Angolan population relies on herbal remedies to treat various ailments. Angola is endemic with Human African Trypanosomiasis, a parasitic disease [1]. Though current chemotherapy is available to treat trypanosomiasis, the treatment is long and adherent, which complicates its implementation in remote rural communities. It is supposed that rural population who lacks access to biomedical medication, turns to folk medicine to cure sleeping sickness and its symptoms. There is extensive literature on antitrypanosomal activity of traditionally-used African medicinal plants [2], but to our current knowledge, there has not yet been a study reporting the use of local herbal remedies to heal trypanosomiasis and its symptoms in Angola.

We explored the use of herbal remedies by conducting structured and semistructured interviews within two distinct study populations. The first group comprises 30 patients who were biomedically diagnosed for trypanosomiasis and treated by reference treatment. The second group included 9 traditional practitioners who have already healed sleeping sickness.

Out of the patient group, first qualitative results show that almost half of the inquired lay people turns to folk medicine in the management of trypanosomiasis symptoms. From 30 patients, 12 use herbal remedies before visiting a biomedical doctor. Out of these 12,3 reported improvement of health condition.

Out of 9 traditional practitioners interviewed, 31 medicinal plants were cited to heal sleeping sickness. Among them, Vitex madiensis was the most cited medicinal plant. Roots and leaves are the most used plant parts and decoction is the mostly cited mode of preparation. Though species identification is being finalized, the most represented family in species used to manage sleeping sickness is Fabaceae.
[1] Simarro, P.P, et al. Negl Trop Dis 2012, 6, e1859

[2] Ibrahim, M.A, et al. J Ethnopharmacology 2014, 154, 26 - 54

\section{Lecture Session - Dermatology \& \\ Dermatocosmetics \\ Wednesday, 6th September, 2017 \\ Singapore - 11:00-12:30}

\section{We-Dermatology \& Dermatocosmetics-SL-01 A therapeutic strategy for psoriasis: STAT3 and NF-KB inhibition by tussilagonone as an Nrf2 activator in keratinocytes}

Authors Lee $J^{1}$, Lee $S^{2}$, Suh $Y_{G^{2,3}}$, Kim Yeong $S^{1}$

Institute 1 Natural Products Research Institute, College of Pharmacy, Seoul National University, Seoul, Korea, Republic of (South); 2 College of Pharmacy, Seoul National University, Seoul, Korea, Republic of (South); 3 College of Pharmacy, CHA University, Gyeongghi-do, Korea, Republic of (South) DOI 10.1055/s-0037-1608566

Psoriasis is a common inflammatory skin disorder with a prevalence of $2-3 \%$ in the world's population. It is characterized by abnormal keratinocyte hyperproliferation, resulting in thickening of the epidermis and stratum corneum [1]. In the present study, we investigated pharmacological effects of tussilagonone (TGN), a sesquiterpenoid isolated from Tussilago farfara, on the transcription factors related to pathogenesis of psoriasis. TGN upregulated the ARE-luciferase activity time- and dose-dependently (up to 19.1-fold and 17.7-fold, respectively). Also, it induced the protein expression of Nrf2 and its downstream target, heme oxygenase-1 (HO-1) in $\mathrm{HaCaT}$ cells. In addition, TGN treatment increased the nuclear translocation of Nrf2, suggesting that TGN activates Nrf2 pathway. To elucidate whether TGN could reduce the activation of STAT3 and NF-kB, crucial transcription factors involved in the pathogenesis of psoriasis, we conducted western blot analysis and luciferase assay. TGN inhibited IL-6-induced STAT3 phosphorylation and STAT3-dependent transcriptional luciferase activity, and also suppressed TNF- $\alpha$-induced NFKB activation. Knockdown of Nrf2 or HO-1 by small interfering RNA (siRNA) abrogated inhibitory effects of TGN on STAT3 and NF-KB activation, indicating that Nrf2 upregulation by TGN can regulate STAT3 and NF-KB pathway in keratinocytes. Moreover, proliferation assay revealed that $10 \mu \mathrm{M}$ TGN treatment inhibited IL-6-induced HaCaT cell proliferation and the protein expression of keratin 16, one of the markers for hyperproliferation. Taken together, these results suggest that TGN, as an effective Nrf2 activator which plays an important role in regulating STAT3 and NF-KB activation, can be a promising therapeutic candidate for treatment psoriasis.

[1] Alice B. Gottlieb. Nat Rev Drug Discov 2005; 4: 19-34

\section{We-Dermatology \& Dermatocosmetics-SL-02 Anti-inflammatory activity of Cannabis sativa L. extracts in an in vitro model of skin inflammation}

Authors Fumagalli $M^{1}$, Sangiovanni $E^{1}$, Pacchetti $B^{2}$, Piazza $S^{1}$, Dell'Agli $M^{1}$ Institute 1 Dept. of Pharmacological and Biomolecular Sciences, University of Milan, Milan, Italy; 2 Linnea SA, Riazzino, Switzerland

DOI 10.1055/s-0037-1608567

Skin is an important organ of human body with several functions, including the protection against different stressors, such as microbial pathogens, mechanical damage and UV irradiations. During cutaneous inflammatory diseases (such as psoriasis), keratinocytes, the most abundant cells in the epidermis, release a variety of pro-inflammatory mediators (for example IL-8 and MMP$9)^{1,2}$ to amplify and prolong the inflammatory process. IL-8 and MMP-9 expression in human keratinocytes is regulated through the activation of different transcription factors, including NF-kB. 
Cannabidiol (CBD) is one of the lipophilic cannabinoids occurring in Cannabis sativa L.; although previous study has demonstrated that CBD exerts antiinflammatory effects in an in vivo model of skin inflammation ${ }^{3}$, no studies on the anti-inflammatory and antioxidant effects in human keratinocytes have been reported so far.

The aim of this work was to evaluate the anti-inflammatory activity of the Cannabis sativa L. extracts, standardized in CBD and cannabidiolic acid (CBDA), in human keratinocytes.

Two Cannabis sativa L. extracts, each containing $1 \%$ or $50.8 \%$ CBD+CBDA, were prepared and standardized by LINNEA SA (Riazzino, Switzerland). IL-8 and MMP-9 release were analysed by ELISA assays, whereas NF-KB driven transcription was tested using a reporter plasmid.

Both extracts inhibited TNF $\alpha$-induced MMP-9 secretion in a concentration dependent manner with an $\mathrm{IC}_{50}$ of 5.54 and $1.78 \mu \mathrm{g} / \mathrm{mL}$ respectively; considering IL-8 secretion, only $1 \%$ extract reduced the chemokine release $\left(\mathrm{IC}_{50}\right.$ : $34 \mu \mathrm{g} / \mathrm{mL}$ ). The effect was ascribed to the impairment of the NF-KB pathway since both the extracts inhibited TNF $\alpha$-induced NF-KB driven transcription ( $\mathrm{IC}_{50}: 37.8 \mu \mathrm{g} / \mathrm{mL}$ for $1 \%$ extract, and $2.7 \mu \mathrm{g} / \mathrm{mL}$ for $50.8 \%$ extract).

These results suggest that Cannabis sativa L. containing CBD and CBDA may possess beneficial effects against inflammatory skin diseases.

[1] Barker J.N.W.N. et al. J Clin Invest 1990. 85:605-08

[2] Majtan J. et al. Arch Dermatol Res 2013. 305:619- 27

[3] Lodzki et al. J Control Release 2003. 93:377 - 387

We-Dermatology \& Dermatocosmetics-SL-03 Screening the dermatological potential of Plectranthus species components - antioxidant and inhibitory capacities over elastase, collagenase and tyrosinase

Authors Marçalo J ${ }^{1}$, Garcia $C^{1}$, Nicolai $\mathrm{M}^{1}$, Reis $\mathrm{C}^{2}$, Faustino $\mathrm{C}^{3}$, Rodrigues Luís $\mathrm{M}^{1}$, Rijo $\mathrm{P}^{1}$

Institute 1 Universidade Lusófona's Research Center for Biosciences and Health Technologies (CBIOS), Lisbon, Portugal; 2 Biophysics and Biomedical Engineering Institute (IBEB), Faculty of Sciences, University of Lisbon (ULisboa), Lisbon, Portugal; 3 Research Institute for Medicines (iMed.ULisboa), Faculty of Pharmacy, University of Lisbon, Lisbon, Portugal

DOI 10.1055/s-0037-1608568

Medicinal plants from Plectranthus genus are employed for skin ailments in traditional medicine [1], and have been validated for their antioxidant activity [2]. As far as we know, ROS can initiate complex molecular pathways for melanogenesis by enhancing tyrosinase activity and activating ECM enzymes, such as collagenase and elastase. Therefore, this study aims to perform a primary evaluation of Plectranthus spp. extracts and isolated compounds, for the synergistic treatment of skin disorders.

The compounds tested isolated from Plectranthus spp. using bioassay-guided fractionation of extracts (aqueous, acetone, methanol and ethyl acetate), were: $7 \alpha$-acetoxy-6 $\beta$-hydroxyroyleanone from $P$. grandidentatus; $6 \beta, 7 \alpha$-dihydroxyroyleanone and 6,7-dehydroroyleanone from $P$. madagascariensis; Parvifloron $\mathrm{D}, \beta$-sitosterol, stigmasterol, oleanolic and ursolic acids from $P$. ecklonii; (13S,15S)-6 $\beta, 7 \alpha, 12 \alpha, 19$-tetrahydroxy-13 $\beta, 16$-cyclo-8-abietene-11,14-dione from $P$. porcatus; $\left(11 R^{*}, 13 E\right)$-11-acetoxyhalima-5,13-dien-15-oic acid, Plectrornatin $\mathrm{C}, 1,6$-di-O-acetylforskolin and 1,6-di-O-acetyl-9-deoxyforskolin from $P$. ornatus; $\alpha$-amyrin and $\beta$-amyrin from $P$. neochilus; chlorogenic acid from $P$. saccatus and rosmarinic acid from the aqueous extracts of Plectranthus spp.

The ethyl acetate extracts of $P$. grandidentatus and $P$. ecklonii registered the highest antioxidant activity, most likely due to the presence of abietane diterpenes [3]. Concerning the skin-related enzymatic assays, all organic extracts of $P$. ecklonii, $P$. grandidentatus, $P$. madagascariensis and $P$. saccatus revealed highest tyrosinase and collagenase inhibition. Whereas abietane diterpenes and triterpenes highly inhibited tyrosinase and elastase.

Overall, the experimental results showed the powerful antioxidant and inhibitory action over tyrosinase, collagenase and elastase of Plectranthus spp. ex- tracts and isolated compounds, supporting their further research as bioactives for cosmetic formulations.

[1] Lukhoba CW, Simmonds MS, Paton AJ. J Ethnopharmacol 2006; 103: 1 24;

[2] Rijo P, Batista M, Matos M, Rocha H, Jesus S, Simões MF. Biomed Biopharm Res 2012;9: 225 - 235;

[3] Rosa S, Correia V, Ribeiro I, Rijo P, Simões MF, Saraiva N, Fernandes A. Biomed Biopharm Res 2015; 12: 59 - 67.

\section{We-Dermatology \& Dermatocosmetics-SL-04 Cosmetic application of Marula seed oil (Sclerocarya birrea): Clinical outcomes}

Authors Komane $B^{1}$, Viljoen $A^{1}$, Vermaak $I^{1}$, Kamatou $G^{1}$, Summers $B^{1}$ Institute 1 Department of Pharmaceutical Sciences, Tshwane University of Technology, Private Bag X680, Pretoria, 0001, South Africa, Pretoria, South Africa

DOI 10.1055/s-0037-1608569

Marula oil, extracted from the kernels of Sclerocarya birrea (Anacardiaceae), is traditionally used by southern African women as a moisturising agent on their faces, feet and hands. The seed oil is rich in mono-unsaturated fatty acids that have hydrating and moisturising properties. The oil is a popular ingredient in cosmetics such as skin lotions, lipsticks and foundations. The demand for marula oil produced by rural communities in Namibia has increased tremendously such that in 2008 almost US\$ 20 million was spent on the oil for cosmetic products by The BodyShop ${ }^{\circledR}$ and Aldivia ${ }^{\circledR}$. Despite its popularity, scientific evidence for efficacy and safety to support the traditional as well as cosmetic use is still lacking. This study evaluated the irritancy potential (safety), moisturising and hydrating effects as well as occlusivity properties (efficacy) of marula oil upon topical application. In addition, the oil composition was determined using two-dimensional gas chromatography. Healthy Caucasian adult female volunteers $(n=20)$ who complied with the inclusion and exclusion criteria for the irritancy patch and moisture efficacy tests participated in the study. A 2x magnifying lamp (Visual observation), Chromameter ${ }^{\circledR}$, Aquaflux ${ }^{\circledR}$ and Corneometer $^{\circledR}$ were used to evaluate and monitor the irritancy level, skin barrier function, trans-epidermal water loss, hydrating and occlusive effects, respectively. The results revealed that marula oil is nonirritating $(p<0.001)$, with hydrating properties $(p<0.001)$ when applied to a lipid-dry (xerosis) skin and moisturising $(p<0.001)$ when applied to normal skin. These findings may be linked to the easy absorption of the oil into the skin due to the high percentage of oleic acid (69\%) followed by palmitic acid (15.3\%), linoleic acid (9.2\%), palmitoleic acid (4.1\%) and stearic acid (1.5\%). The study provides scientific basis for inclusion of marula oil in cosmetic formulations as it is non-irritating and provides moisturising effects with moderate prevention of trans-epidermal water loss which may alleviate prematureaging.

\section{We-Dermatology \& Dermatocosmetics-SL-05 Sustainable cosmetics - berry-based ingredients for bioactive skin care products}

Authors Oksman-Caldentey $\mathrm{KM}^{1}$, Nohynek $\mathrm{L}^{1}$, Smolander $\mathrm{M}^{1}$, Alakomi $\mathrm{HL}^{1}$, Rischer $\mathrm{H}^{1}$, Puupponen-Pimiä $\mathrm{R}^{1}$

Institute 1 VTT Technical Research Centre of Finland Ltd, Espoo, Finland DOI 10.1055/s-0037-1608570

Cosmetics are moving towards pharma and cosmeceuticals are entering the market. Modern biotechnology offers many advantages to develop new type of ingredients for industrial applications in a sustainable way. Due to the arctic growth conditions Nordic wild berries are rich sources of natural biomolecules with multifunctional effects to the skin. We have shown that phenolic compounds in berries very efficiently inhibit the growth of many human pathogens including skin pathogens, such as Staphylococcus aureus, S. epidermis and Pseudomonas aeruginosa without effecting the growth of beneficial bacteria. 
Several mechanisms of action, including the weakening of the outer membrane of Gram- negative bacteria, are involved in the growth inhibition. More interestingly, various berry phenolics are shown to specifically block cell-to-cell signalling (quorum sensing) in a bacterial community. Thus these phytochemicals can act in cosmetic preparations in dual way, as natural preservatives to prevent spoilage of the product, and as stabilizers of healthy skin microbiota by inhibiting the growth of harmful skin microbes and by supporting the growth of beneficial microbes.

Bioprocessing including enzyme treatment and fermentation combined with special dry fractionation technologies of the berry material has resulted in increased bioactivities. Using plant cell and tissue culture technology whole biosynthetic capacity of the plant can be advantaged and even totally new beneficial compounds for the skin can be produced. The unusual phenolic profile of the cultured berry cells, as well as their fatty acid composition with a high proportion of $\alpha$-linolenic acid and high protein content makes them a unique and interesting alternative for cosmetic industry. Efficient utilization of the biomolecules requires controlled delivery systems into the skin, such as microcurrent patches developed at VTT, which are based on renewable enzyme catalysts and renewable materials. The possibilities using natural plant cell-derived ingredients combined with new delivery system will also be discussed.

\section{We-Dermatology \& Dermatocosmetics-SL-06 Actinobacteria of global biodiversity as a source of bioactive metabolites for the discovery and development of novel cosmeuceutical agents}

Authors Georgousaki K $\mathrm{K}^{1}$, Katsinas $\mathrm{N}^{1}$, Tsafantakis $\mathrm{N}^{1}$, Gumeni $\mathrm{S}^{2}$, OvesCostales $\mathrm{D}^{3}$, González $\mathrm{I}^{3}$, Almeida $\mathrm{C}^{3}$, Genilloud $\mathrm{O}^{3}$, Trougakos $\mathrm{IP}^{2}$, Fokialakis $\mathrm{N}^{1}$

Institute 1 Department of Pharmacognosy and Natural Products Chemistry, Faculty of Pharmacy, National and Kapodistrian University of Athens, Athens, Greece; 2 Department of Cell Biology and Biophysics, Faculty of Biology, National and Kapodistrian University of Athens, Athens, Greece; 3 Fundacion Medina, Granada, Spain

DOI 10.1055/s-0037-1608571

In the frame of MICROSMETICS EU project 57 potential candidate actinobacteria strains of global biodiversity were selected to be studied. In total 614 extracts were produced and a broad spectrum of cell-free bioassays have being incorporated for the evaluation of their anti-ageing activity, including analyses for anti-oxidant, skin-protecting and skin-whitening bioactivity [1].

Among the initial 614 extracts, 12 actinobacteria strains were selected as promising bioactive products and have been further evaluated in cell-based assays for their bleaching activity (i.e. tyrosinase inhibition) in mouse melanocytes (B16F10 cell line), as well as in normal human skin fibroblasts for their capacity to activate proteostasis ensuring anti-ageing mechanisms, namely the ubiquitin-proteasome and autophagy-lysosomal systems. The most bioactive strains CA-129531, CA-126581 and CA-218259 have been selected for dereplication, cultivation in larger scale and bioguided fractionation and identification of bioactive compounds.

The dereplication was performed using UPLC-HRMS while the isolation of the compounds contained in those extracts was performed using chromatographic methods like HPLC and FCPC. The full set of spectroscopic data (HRMS and NMR) was recorded for all isolated compounds in order to unambiguously elucidate their structure.

The evaluation of the isolated compounds in cell-free and mammalian cell based assays emphasized the anti-ageing effect of several metabolites produced by the selected strains. Special attention was given to isolated hydroxamic acid and histidine derivatives due to their remarkable skin-whitening activity and capacity to activate proteostasis as well as to deferoxamine and conjugates for their strong capacity to chelate iron, a free radical catalyst, known to be accumulated with age. Therefore, these extracts can be consid- ered as potential candidates for industrial development and can serve as a proof of concept that microbial ingredients can have successful applications in cosmeceutical industry.

[1] K. Georgousaki et al. Planta Medica 2015; 81 - PM_234.

\section{Lecture Session - Miscellaneous}

Wednesday, 6th September, 2017

Osaka/Samarkand - 11:00 - 12:30

\section{We-Miscellaneous-SL-01 Phytoequivalence of Therapeutic Cinnamon Barks and Extracts with Low Coumarin Levels}

Authors Leach $\mathrm{D}^{1}$, Fryganas $\mathrm{C}^{2}$, Mueller-Harvey $\mathrm{I}^{2}$, Wohlmuth $\mathrm{H}^{1,3,4}$

Institute 1 Integria Healthcare, Brisbane, Australia; 2 University of Reading, Reading, United Kingdom; 3 National Institute of Complementary Medicine, Sydney, Australia; 4 University of Queensland, Brisbane, Australia DOI 10.1055/s-0037-1608572

Past research has demonstrated that extracts of cinnamon increase insulin activity, enhance glucose breakdown and improve blood sugar concentrations $^{1}$; benefit women suffering from polycystic ovary syndrome ${ }^{2}$; are traditionally used for indigestion, nausea and diarrhoea ${ }^{3}$. Of the four main cinnamon sources (Cinnamomum cassia, C. verum, C. burmani and C. loureiroi) only the first two are approve for use in listed (low-risk) medicines in Australia. More recently the Therapeutic Goods Administration (TGA) has limited coumarin to a maximum of $0.001 \%$ in any finished products containing cinnamon. Barks can contain up to10,000 ppm coumarin hence additional steps in manufacture are now required to minimise this component. Production steps employed by some cinnamon manufacturers to produce low coumarin extracts meeting TGA requirements have severely impacted on the procyanidin profile and levels. Sourcing cinnamon bark extracts that now meet both the current coumarin restrictions with phytoequivalence of original cinnamon bark extracts used in existing commercial products has become problematic.

We have explored the use of DNA barcoding, volatiles (including coumarin) composition and HPTLC as a means of authenticating cinnamon raw materials. The limitations of these approaches will be highlighted and contrasted to a simplified LC-MS approach, using a suite of procyanidin dimers, trimers and tetramers that appears to provide a useful analytical approach for identity and quality control. A more advanced LC-MS approach following thiolytic degradation of procyanidins provides additional detailed compositional information to ensure phytoequivalence from source to finished products.

[1] Anderson, RA, Broadhurst, CL, Polansky, MM, Schmidt, WF, Khan, A, Flanagan, VP, Schoene, NW and Graves, DJ. J Agric Food Chem 2004, 52: 65 - 70. [2] Kort, DH and Lobo RA. Am J Obstet Gynecol 2014:487.e1 - 6.

[3] Cortex Cinnamomi. In WHO Monographs on Selected Medicinal Plants. Vol. 1. World Health Organization, Geneva, 1999.

\section{We-Miscellaneous-SL-02 STABILITY AND INTESTINAL ABSORPTION OF VINPOCETINE IN HUMAN EPITHELIAL CACO-2 CELLS}

Authors Piazza $S^{1}$, Sangiovanni $E^{1}$, Maldini $M^{1}$, Fumagalli $M^{1}$, Spezzano $R^{1}$, Giavarini $\mathrm{F}^{1}$, Pacchetti $B^{2}$, Caruso $D^{1}$, Dell'Agli $\mathrm{M}^{1}$

Institute 1 Department of Pharmacological and Biomolecular Sciences University of Milan, Milan, Italy; 2 Linnea SA, Riazzino, Switzerland DOI 10.1055/s-0037-1608573

The semi-synthetic alkaloid vinpocetine is widely used in Europe and Japan for the improvement of neurodegenerative diseases ${ }^{1}$. The compound is insoluble in water and slightly absorbed mainly by small intestine. Vinpocetine undergoes extensive metabolism into its hydrolysis product apovincaminic acid ${ }^{2}$. The poor bioavailability is only partially explained, since the effect of the 
intestinal digestive process on its stability has never been previously investigated.

The aim of the present study was to evaluate the stability and absorption of vinpocetine in human Caco-2 cells, before and after an in vitro simulated intestinal digestion.

The intestinal absorption of $50 \mathrm{mM}(17.52 \mathrm{ng} / \mathrm{mL})$ vinpocetine was evaluated after 1 hour treatment in differentiated Caco-2 cells (enterocytes) grown on Transwell ${ }^{\circledR}$ supports, before and after in vitro simulated intestinal digestion. Vinpocetine was applied at the apical side and its diffusion through the enterocytes barrier was detected by measuring the compound in the apical and basolateral compartments by LC-MS/MS analysis. Quantitative method was set up using tabersonine as internal standard. The separation was achieved using a reverse phase $\mathrm{C}-18$ column, while detection by triple quadrupole (TQ) after $\mathrm{ESI}^{+}$ionization.

The percentage of vinpocetine diffused through the enterocyte barrier was $8.1 \%$. After digestion, only $3.8 \%$ of vinpocetine was recovered indicating that the molecule is unstable at intestinal conditions. Interestingly, only $0.003 \mathrm{ng} /$ $\mathrm{mL}(0.017 \%)$ of the total amount of vinpocetine $(17.52 \mathrm{ng} / \mathrm{mL})$ recovered following intestinal digestion was absorbed.

The present study reports a suitable analytical method to quantify the intestinal absorption of vinpocetine; moreover, our results underline the importance of intestinal digestion in determining the poor bioavailability of vinpocetine.

[1] Patyar, S. Pharmacological Reports 2011, 63, 618-628.

[2] Szakacs, T. Pol. J. Pharmacol. 2001, 53, 623.

\section{We-Miscellaneous-SL-03 Combining LC-MS metabolomics and next generation sequencing to study the interactions between herbal medicines and human gut bacteria in-vitro}

Authors Pferschy-Wenzig $\mathrm{EM}^{1,3}$, Koskinen $\mathrm{K}^{2,3}$, Roßmann $\mathrm{A}^{1}$, ArdjomandWoelkart $\mathrm{K}^{1}$, Moissl-Eichinger $\mathrm{C}^{2,3}$, Bauer $\mathrm{R}^{1,3}$

Institute 1 University of Graz, Institute of Pharmaceutical Sciences, Department of Pharmacognosy, Universitaetsplatz 4, 8010 Graz, Austria;

2 Medical University Graz, Department of Internal Medicine, Stiftingtalstraße 24/3/40, 8010 Graz, Austria; 3 BioTechMed, Krenngasse 37, 8010 Graz, Austria

\section{DOI 10.1055/s-0037-1608574}

The microbial community that populates the human distal gut has a strong impact on human health and enormous metabolic capacity. Therefore, many constituents of herbal medicines that remain unabsorbed in the upper intestinal tract are available for metabolization by human gut microbiota. This might lead to the formation of potentially bioavailable and bioactive metabolites, an aspect that was often not considered in the past when searching for active principles of herbal medicines.

We have established an interdisciplinary platform that allows investigating the metabolization of herbal medicines by human gut bacteria, as well as their influence on gut microbial community composition. Herbal preparations were incubated with human fecal suspension under anoxic conditions. After 0.5, 4 and $24 \mathrm{~h}$, samples were taken and subjected to LC-HRMS and $16 \mathrm{~s}$ RNA sequencing analysis.

This approach has been applied to three approved herbal medicinal products: standardized willow bark extract, hawthorn leaves and flowers extract, and a fixed combination-preparation consisting of 9 herbal extracts.

In all cases, microbial degradation was found to be strongly dependent on compound class and extract concentration. Constituents like caffeoyl quinic acids, catechins, procyanidins and flavonoid-O-glycosides were usually metabolized very fast. Metabolization could also be shown for C-glycosylflavones and triterpene glycosides. Assignment of newly formed metabolites allowed the construction of putative degradation patwhays for many degraded compounds. Interestingly, the use of higher extract concentrations usually re- tarded the observed metabolic reactions, indicating that too high concentrations of certain compounds might inhibit microbial metabolic enzymes.

165 rRNA gene sequencing revealed significant changes in microbial community composition upon extract addition, and allowed the assignment of certain microbial taxa that are potentially involved in some of the observed metabolic reactions.

We thank Steigerwald Arzneimittelwerk $\mathrm{GmbH}$, Bayer Consumer Health and Dr. Willmar Schwabe GmbH \& Co. KG for financial support.

\section{We-Miscellaneous-SL-04 Quantification of antigenotoxic flavonoids in the stem bark of Erythrina latissima by an HPLC-UV method}

Authors Zarev $\mathrm{Y}^{1,3}$, Foubert $\mathrm{K}^{1}$, Naessens $\mathrm{T}^{1}$, Ionkova $\mathrm{I}^{3}$, Elgorashi $\mathrm{E}^{4}$, Verschaeve $L^{2}$, Pieters $L^{1}$, Apers $S^{1}$

Institute 1 Natural Products \& Food Research and Analysis (NatuRA), Department of Pharmaceutical Sciences, University of Antwerp, Antwerp, Belgium; 2 Toxicology, Scientific Institute of Public Health, Brussels, Belgium; 3 Department of Pharmacognosy, Faculty of Pharmacy, Medical University-

Sofia, Sofia, Bulgaria; 4 Toxicology and Ethnoveterinary Medicine; Food, Feed and Veterinary Public Health, ARC-Ondertsepoort Veterinary Institute,

Onderstepoort, South Africa

DOI 10.1055/s-0037-1608575

Erythrina latissima E. Mey (Leguminosae) is a tree up to $24 \mathrm{~m}$ tall and generally found in Southern Africa. The stem bark shows high potential for livestock industry since dietary supplementation might induce antimutagenic activity and subsequently decrease animal diseases and increase production of eggs, milk and meat [1 -3$]$. Five new and 18 known flavonoids were identified in the plant material and evaluation of their antigenotoxic properties against aflatoxin B1 induced genotoxicity revealed most flavonoids were active. Sigmoidin $A$ and $B$ showed an activity $\left(I_{50}=18.7 \mu \mathrm{g} / \mathrm{mL}\right)$ equivalent to that of curcumin $\left(\mathrm{IC}_{50}=18.4 \mu \mathrm{g} / \mathrm{mL}\right)$ [1]. Industrial use of E. latissima includes the necessity of the development and validation of a method for quantification of total flavonoids in the stem bark of E. latissima. The sample extraction method and the HPLC-UV conditions were optimized. The analytical validation was performed according to ICH guidelines. The calibration model was linear for both rutin and naringenin, used as a secondary standard for flavonoid glycosides and aglycons, respectively. Statistical analysis showed that for both glycosides and aglycons the method seems precise on different days and concentration levels. By mean of a recovery experiment, the method showed to be accurate concerning aglycons. This method is fully applicable for the quantification of non-glycosylated flavonoids of the stem bark of $E$. latissima (average amount $1.44 \%$ ) and indicative for the amount the glycosylated flavonoids (average amount 1.97\%). Ongoing In vivo experiments with the quantified extract of E. latissima will provide additional information on its use as a chemopreventive agent.

[1] Zarev Y; Foubert K, de Almeida VL, Anthonissen R, Elgorashi E, Apers S, lonkova I, Verschaeve L, Pieters L, Pytochemistry, submitted.

[2] Binder EM, Tan LM, Chin L], Handl J, Richard J, Anim Feed Sci Technol 2007; 137: $265-282$

[3] Pier AC, J Anim Sci 1992; 70: 3964 - 3967.

\section{We-Miscellaneous-SL-05 Bioactivity screening for xylopic acid, geraniin and its gut metabolites, ellagic acid and urolithin $A$, in cell based assays}

Authors Boakye $\mathrm{YD}^{1}$, Heiss $\mathrm{E}^{2}$, Agyare $\mathrm{C}^{3}$

Institute 1 Department of Pharmaceutics and, Kwame Nkrumah University of Science and Technology, KNUST, Kumasi, Ghana; 2 2Department of Pharmacognosy, University of Vienna, Vienna, Austria; 3 Department of 
Pharmacology, Kwame Nkrumah University of Science and Technology, KNUST, Kumasi, Ghana

DOI 10.1055/s-0037-1608576

Xylopia aethiopica and Phyllanthus muellerianus are medicinal plants which are widely used in folkloric medicine for the management of various conditions including inflammation, pain, wounds and infections in West Africa [1, 2]. The aim of the project was to identify potential molecular targets of xylopic acid (isolated from Xylopia aethiopica), the ellagitannin geraniin (isolated from Phyllanthus muellerianus) and its gut metabolites, ellagic acid and urolithin A in in vitro and cell-based assays. Reporter gene assays in CHO and HEK 293 cell lines were employed to determine the influence of xylopic acid, geraniin and its gut metabolites on the activation of Nrf2, FXR, RXR, LXR, PPARY, TGR5 and on the inhibition of NF-kB signaling. Test compounds were subjected to proliferation and migration assays in vascular smooth muscle cells (VSMC) and tested for their influence on vascular adhesion molecule (VCAM) and heme oxygenase (HO)- 1 expression.

At $30 \mu \mathrm{M}$ xylopic acid moderately reduced VCAM expression and showed inhibition of NFkB- and activation of Nrf2 signaling. Geraniin $(30 \mu \mathrm{M})$ and urolithin $\mathrm{A}(10 \mu \mathrm{M})$ increased the expression of HO1 protein levels in VSMC. Geraniin and ellagic acid at $30 \mu \mathrm{M}$ exhibited anti-migratory activity in VSMC. Additionally, $30 \mu \mathrm{M}$ urolithin A inhibited VSMC proliferation. Xylopic acid, geraniin and ellagic acid did not positively influence LXR, RXR, PPAR, FXR and TGR5 in reporter gene assays. Urolithin A was able to very weakly induce a RXR-dependent luciferase signal. Xylopic acid could be shown to possess a moderate anti-inflammatory activity as evident in reduced VCAM expression which is possibly due to activation of Nrf2 and inhibition of NFkB. Geraniin and/or its gut metabolites could be shown to significantly increase the expression of HO-1 protein levels in vascular smooth muscle cells and to interfere with VSMC migration and proliferation.

[1]Woode et al, J Pharm BioAll Sci 2012, 4: 291 - 301.

[2]Agyare et al, ]. Ethnopharmacol, 2009, 125: $393-403$.

Lecture Session - Plenary Lecture 5

Wednesday, 6th September, 2017

Montreal - 14:00 - 14:45

\section{We-Plenary Lecture Session 5-PL-01 Natural products based molecules for target and drug discovery in pharmaceutical research}

\section{Authors Petersen $\mathrm{F}^{1}$}

Institute 1 Novartis Institutes for Biomedical Resaerch, Basel, Switzerland DOI 10.1055/s-0037-1608577

Natural products with their specific structural features deliver chemical starting points in drug discovery to develop innovative therapies for diseases for which no or only unsatisfactory treatments exist. The evolved function of natural products in regulating a plethora of diverse biological pathways in nature makes them to a biologically biased and complementary source of chemical probes to identify novel mechanisms of molecular interactions. As there is a high need for specific modulators of new targets, the identification of new natural products and the elucidation of their mechanism of action are gaining an increasing attraction in today's drug research. The technological driving forces of synthetic biology, genome sequencing, and DNA-synthesis are currently changing the face of natural products research. Whole genome data and the identification of silent natural products pathways in-silico open the door to a so far inaccessible chemodiversity from natural sources. Selected examples will illustrate how the investigation of new chemotypes from natural products, natural products-inspired synthetics in phenotypic assays can be integrated to orthologous target identification technologies. The fragmentation of natural products is a further scientifically exciting example how the broad diversity of natural products can be leveraged in the science of drug discovery.

\author{
Lecture Session - Natural Products \\ Formulation \\ Wednesday, 6th September, 2017 \\ Osaka/Samarkand - 14:45-16:15
}

\section{We-Natural Products Formulation-SL-01 \\ Sapofectosid - an isolated triterpenoid saponin from Saponaria officinalis $L$. ensures non-toxic and universal gene delivery}

Authors Sama $\mathrm{S}^{1}$, Jerz $\mathrm{C}^{2}$, Melzig $\mathrm{M}^{1}$, Weng $\mathrm{A}^{1}$

Institute 1 Department of Pharmaceutical Biology, Institute of Pharmacy, Freie Universität Berlin, Königin-Luise-Straße 2-4, 14195 Berlin, Germany, Berlin, Germany; 22 Institute of Food Chemistry. Technische Universität Braunschweig, Schleinitz-Straße 20, 38106 Braunschweig, Germany, Braunschweig, Germany

DOI $10.1055 / \mathrm{s}-0037-1608578$

To this day, a variety of transfection methods are available on the market, promising a highly effective gene delivery into eukaryotes. As well as the enhancement of transfection efficiency, the toxicity of the reagents play a crucial role in the biochemical research. Particularly with regard to conducting in-vivo studies or even gene therapies, the transfection agents must preferably cause no self-toxicity. In the recent past, a number of studies were conducted, implying the great potential of triterpenoid bisdesmosidic saponins of Caryophyllaceae species for being a valuable non-toxic transfection enhancer. By disturbing the integrity of late endosomes and lysosomes during the endocytosis, these saponins are able to release the applied gene carriers into the cytosol before being degraded by lysosomal enzymes. In this study, we try to illustrate potential of the isolated triterpenoid saponin (molecular weight: $1862 \mathrm{Da}$ ) from Saponaria officinalis $L$. "sapofectosid" as the first agent of a new transfection method - sapofection. For this purpose, the herbal extract of $S$. officinalis $L$. underwent several purification steps as well as the chemical clarification in order to isolate the active compound. The isolated sapofectosid was tested on a number of transfection methods to underline the universal applicability as a non-toxic transfection enhancer for future gene therapies.

\section{We-Natural Products Formulation-SL-02 NeoSol ${ }^{\mathrm{TM}} \mathrm{RCL} 40$ is a novel Red Clover isoflavone aglycones extract preparation with improved solubility and increased availability of active compounds}

\author{
Authors Cosentino $\mathrm{M}^{1}$, Marino $\mathrm{F}^{1}$, Rasini $\mathrm{E}^{1}$, Bombelli $\mathrm{R}^{1}$, Luini $\mathrm{A}^{1}$, \\ Pacchetti $\mathrm{B}^{2}$ \\ Institute 1 Center for Research in Medical Pharmacology, University of \\ Insubria, Varese, Italy; 2 Linnea SA, Riazzino, TI, Switzerland \\ DOI 10.1055/s-0037-1608579
}

Red clover (Trifolium pratense L, Fabaceae; RCL), a perennial plant rich in isoflavones, is a natural alternative for menopausal symptoms, as well as antiaging and antioxidant. Isoflavone preparations usually contain aglycones and $\beta$ glycosides. Aglycones, the active moieties, are absorbed slowly and unevenly due to reduced water solubility and biotransformation from $\beta$-glycosides. NeoSol ${ }^{\mathrm{TM}} \mathrm{RCL} 40$ is a novel RCL isoflavone aglycones preparation based on active solubilization technologies [1].

Using in vitro models, we evaluated $\mathrm{NeoSol}^{\mathrm{TM}} \mathrm{RCL} 40$-induced isoflavones solubilization, estrogenic and antioxidative effects in comparison to a standard RCL extract (RCLE).

Isoflavones aglycones, either as RCLE or as NeoSol ${ }^{\mathrm{TM}} \mathrm{RCL} 40$, were dissolved in water $(100 \mathrm{mg} / 50 \mathrm{~mL})$. Total aglycones in solution, assayed by HPLC [2], were (mean \pm SD) $\quad 0.009476 \pm 0.001202 \mathrm{mg} / \mathrm{ml}$ with RCLE and 0.047401 $\pm 0.005760 \mathrm{mg} / \mathrm{ml}$ with $\mathrm{NeoSol}^{\mathrm{TM}} \mathrm{RCL} 40(\mathrm{n}=11-13, \mathrm{P}<0.0001)$. Estrogenicity, assessed in MCF-7 cells [2], was: potency $\left(\mathrm{EC}_{50}\right.$, mean dilution $\times 1000$ $[95 \% \mathrm{Cl}]) 1.237(0.981-1.558)$ with RCLE and $0.361(0.248-0.527)$ with 
NeoSol ${ }^{\mathrm{TM}} \mathrm{RCL} 40$, and efficacy ( $\mathrm{E}_{\mathrm{MAX}}$, \% of response to estradiol $0.1 \mathrm{nM}$ ) $116.0 \%$ (104.6-127.3\%) with RCLE and $110.6 \%$ (98.2-123.0\%) with NeoSol ${ }^{\mathrm{TM}} \mathrm{RCL} 40$. NeoSol ${ }^{\mathrm{TM}} \mathrm{RCL} 40$ was more antioxidant than RCLE (FRAP assay: $660.4 \pm 51.3$ vs. $328.3 \pm 46.3 \mu \mathrm{M} \mathrm{FeSO}_{4}$ equivalents, $\mathrm{n}=6, \mathrm{P}<0.0001$; DPPH assay: $69.6 \pm 5.3$ vs. $84.2 \pm 3.3$ arbitrary units, $n=5, P=0.0001$ ) [3].

Results support the ability of NeoSol ${ }^{\mathrm{TM}} \mathrm{RCL} 40$ to promote isoflavones solubilization and availability. The ratio NeoSol ${ }^{\mathrm{TM}} \mathrm{RCL} 40 / \mathrm{RCLE}$ for isoflavones solubilization was 5.0 and $\mathrm{NeoSol}^{\mathrm{TM}} \mathrm{RCL} 40$ had 3.4-fold higher estrogenic potency and was more antioxidant than RCLE. NeoSol ${ }^{\mathrm{TM}} \mathrm{RCL} 40$ is therefore an interesting novel preparation providing improved availability of active isoflavones aglycones.

[1] Cram DJ. Science 1988; 240: 760 - 767

[2] Spagnuolo P, et al. Fitoterapia 2014; 94: 62 - 69

[3] Cosentino M, et al. Pharmacol Res 2007; 56: 140 - 147

\section{We-Natural Products Formulation-SL-03 Preparation of Glucan Microparticles with Curcumin for Treatment of Idiopatic Inflammation Diseases}

Authors Salamunova $\mathrm{P}^{1}$, Salon $\mathrm{I}^{1}$, Hanus $\mathrm{J}^{1}$, Hosek J ${ }^{2}$, Stepanek $\mathrm{F}^{1}$, Rotrekl D², Plavcova $\mathrm{Z}^{2}$

Institute 1 UCHT Prague, Technická 3, 166 28, Prague 6, Czech Rebublic, Prague, Czech Republic; 2 University of Veterinary and Pharmaceutical Sciences Brno, Palackého 1946/1, CZ-612 42 Brno, Czech Republic, Brno, Czech Republic

DOI 10.1055/s-0037-1608580

Glucan microparticles are hollow porous shells, which are obtained from cell walls of Saccharomyces cerevisiae (baker's yeast). Organels are removed from baker's yeast by alkaline and acidic extraction steps. Cell walls of glucan particles are mainly composed of $\beta-1,3$-glucans. Lymphatic cells are mainly presented in inflammation area in organism where are responsible for progress of inflammation [1]. Glucan particles (GP) interact with lympatic cell's receptors on their surface and after that $\beta$-1,3-glucans are phagocytosed by the lymphatic cells. Therefore, GP represent an effective oral delivery system for transporting of biological active subtances. Curcumin is an anti-inflammatory natural product which occurs in plants of family Zingiberaceae. In our project, curcumin was delivered to an inflammation in organism using GP. Loading of curcumin into GP was enabled via precipitation of curcumin from organic solvents. Curcumin dissolved in organic solvents was added to the GP and through pores penetrated into cavity of glucan shell. Next, the solvent was removed and the curcumin was precipitated in glucan microparticles. GP with the flavonoid inside were observed by confocal microscopy and SEM (scanning electron microscopy). The achieved content of curcumin was successfully analyzed by other methods, which can not be mentioned directly due to article in preparation. These composites are promising materials for biomedical applications such as an efficient treatment of inflammatory diseases (e.g. idiopatic inflammatory bowel disease, rheumatoic arthritis).

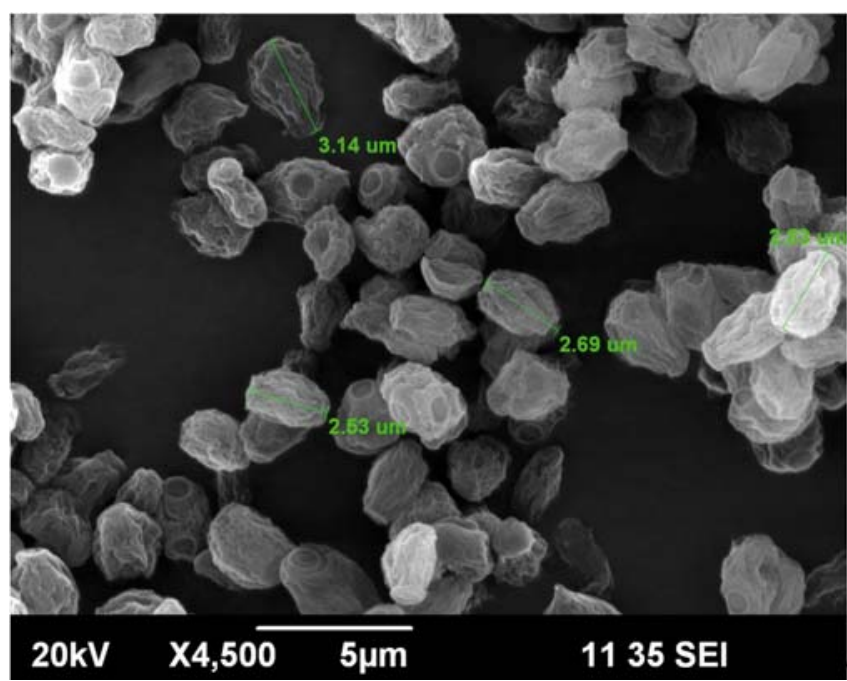

- Fig. 1: Scanning electron microscopy- Pure glucan particles

[1] Salo, I, Hanuš, ], et al. Food and Bioproducts Processing. Elsevier, 2016, 7, $128-135$

\section{We-Natural Products Formulation-SL-04 Encapsulation of Resveratrol into silk protein nanocarriers: Fabrication, Characterization and In vitro Toxicity Against Colon Cancer Cells}

Authors Suktham $\mathrm{K}^{1}$, Koobkobkruad $\mathrm{T}^{2}$, Wutikhun $\mathrm{T}^{3}$, Surassmo $\mathrm{S}^{1}$ Institute 1 Nano-Delivery System Laboratory, National Nanotechnology Center, National Science and Technology Development Agency, Thailand Science Park, Thailand, Pathumthani, Thailand; 2 Nano-Cosmeceutical Laboratory, National Nanotechnology Center, National Science and Technology Development Agency, Thailand Science Park, Thailand, Pathumthani, Thailand; 3 Nano-Characterization Laboratory, National Nanotechnology Center, National Science and Technology Development Agency, Thailand Science Park, Thailand, Pathumthani, Thailand

DOI 10.1055/s-0037-1608581

Resveratrol (RES)-loaded silk protein (SP) nanoparticles is a promising strategy to fabricate biocompatibility and bioviability nanocarriers. Resveratrol, a multitargeted anti-oxidative and anti-inflammatory natural polyphenol, was used as model drug in this study. Meanwhile, SP base nanoparticles are currently available for biodegradable drug carriers. Consequently, this research investigated the capability of RES-loaded SP nanoparticles which were prepared by non-solvent precipitation technique due to enhance functionality and properties both of resveratrol and silk protein nanoparticles. The nanoparticle size was approximately $200-400 \mathrm{~nm}$ and negative charges which was dependent on the silk protein concentrations $(0.1 \%, 0.6 \%$ and $1.0 \%(w / v))$. At $0.5 \%(w / v)$ of poloxamer surfactant could be an optimum condition to prepare SP nanoparticles. TEM and AFM topography of the particles exhibited the monodisperse, spherical shape and smooth surface morphology. The SP nanoparticles were non-toxic to normal skin fibroblast (CRL-2522) and colorectal adenocarcinoma (Caco-2) cells after performed cytotoxicity assay. Cell internalization of the SP nanoparticles showed the ability to transfect into the cell, and internalization level was response to the incubation time. Moreover, RES was loaded up to $75 \%$ into $0.6 \%(\mathrm{w} / \mathrm{v})$ silk protein nanoparticles which could be sufficient to assure required activity outcome. In vitro drug release study demonstrated the ability of silk protein nanoparticle to provide sustained release of RES over $72 \mathrm{~h}$. Cytotoxicity and cell viability assays of RES-loaded SP nanoparticles proved the toxicity against Caco-2 but non-toxic to CRL-2522 cells. In addition, anti-oxidative activity confirmed the activity of loaded RES. The study demonstrated the fabrication of silk protein nanoparticles to im- 
prove and sustain the bioviability of nanoparticles. This RES-loaded silk protein nanoparticles enhance the solubility and prolong the significantly sustained release of RES which can be applicable for further therapeutic and cosmeceutical applications.

\section{We-Natural Products Formulation-SL-05 SOLID LIPID NANOPARTICLES FOR ORAL DELIVERY OF SILIBININ: FORMULATION, CHARACTERIZATION AND EVALUATION USING PAMPA AND CACO-2 CELL MODELS}

Authors Piazzini $V^{1}$, Graverini $G^{1}$, Vanti $G^{1}$, Bigagli $E^{2}$, Cinci $L^{2}$, Luceri $C^{2}$, Bilia Anna $\mathrm{R}^{1}$, Bergonzi Maria $\mathrm{C}^{1}$

Institute 1 Department of Chemistry, University of Florence, Seso Fiorentino, Florence, Italy; 2 University of Florence, Department of Neuroscience, Psychology, Drug Research and Child Health, Section of Pharmacology and Toxicology, Florence, Italy DOI 10.1055/s-0037-1608582

The study is focused on the preparation and evaluation of naked and chitosancoated solid lipid nanoparticles (SLNs) containing silibinin (Sb) to increase its intestinal absorption. Sb is the key biologically active flavonolignan present in silymarin isolated from the fruits and seed of Silybum marianum L. Gaertn. (Asteracee). Sb is used for treatment of liver toxicities, inflammation, fibrosis, oxidative stress and different carcinomas. Recently Sb has been proposed to be beneficial in type 2 diabetes patients. However, its hydrophobicity nature limits bioavailability compromising in vivo biological effects [1]. In this work SLNs were prepared by emulsion/evaporation/solidifying method, using stearic acid and brij 78. In order to improve the delivery efficacy of SLNs we applied a chitosan coating. Based on cationic property chitosan-coated nanoparticles exhibit mucoadhesive features and prolonged residence time in small intestine, thus increasing the drug concentration at the site of absorption [2]. Developed SLNs exhibited particle size and z potential in the range of 150 $200 \mathrm{~nm}$ and $-48-+40 \mathrm{mV}$ respectively. Encapsulation efficiency and recovery were greater than $90 \%$. Moreover SLNs showed excellent stability in both simulated gastric fluid and simulated intestinal fluid with a small release of Sb during at least 24 hours. Further we demonstrated that SLNs were successful in increasing the permeation of Sb by Parallel Artificial Membrane Permeability Assay (PAMPA). Fluorescein isothiocyanate was selected as a model dye to analyse in vitro uptake and permeation of drug using Caco-2 cells. The cell line studies revealed higher uptake and apparent permeability of fluorescentSLNs than free probe. The obtained results suggest that the developed SLNs could improve the bioavailability of Sb.

[1] Pepping, J. American journal of health-system pharmacy 1999; 56: 1195 1197.

[2] Fonte, P, et al. Methods Enzymol 2012; 508: 295 - 314.

\section{Lecture Session - Quality Control \\ Wednesday, 6th September, 2017}

Singapore $-14: 45-16: 15$

\section{We-Quality Control-SL-01 Botanical Ingredient Adulteration - How Commonly Used Analytical Techniques Can Be Fooled}

Authors Gafner $S^{1}$, Upton $R^{2}$, Khan $I^{3}$, Cardellina $J^{4}$, Foster $S^{5}$, Blumenthal $\mathrm{M}^{1}$ Institute 1 American Botanical Council, Austin, TX, United States;

2 American Herbal Pharmacopoeia, Scotts Valley, CA, United States;

3 NCNPR, University of Mississippi, University, MS, United States;

4 ReevesGroup, Virginia Beach, VA, United States; 5 Steven Foster Group,

Eureka Springs, AR, United States

DOI 10.1055/s-0037-1608583

The chemically complex nature of botanically-derived ingredients calls for unique quality control processes by suppliers, manufacturers, and producers of herbal products. One of the universal regulatory requirements in industrialized nations is the appropriate testing for identity and authenticity of botanical materials that are to be used in consumer products. Nevertheless, there have been numerous recent cases of accidental or intentional adulteration. Intentional adulteration is also referred to as economically motivated adulteration (EMA), where raw materials are intentionally substituted or diluted with undisclosed lower-quality ingredients for financial gain of the seller. Another concern is the occurrence of extracts "spiked" with various exogenous compounds, including prescription pharmaceutical drugs, to create a false sense of efficacy. This reflects a significant challenge to the global botanical medicine marketplace and, in some cases, affects consumer safety.

Suppliers of intentionally adulterated botanical ingredients try to take advantage of the lack of specificity of certain analytical methods used for identification. Examples are currently being compiled by an independent consortium of nonprofit organizations consisting of the American Botanical Council (ABC), the American Herbal Pharmacopoeia (AHP), and the National Center for Natural Product Research (NCNPR) at the University of Mississippi as part of the ABC-AHP-NCNPR Botanical Adulterants Program. Such examples include, e.g, the addition of food dyes to St. John's wort extracts to enhance the UV/Vis absorption at the wavelength used to determine hypericin contents, or the addition of rutin- or quercetin-rich materials from extraneous sources to comply with the required contents for total flavonoids in ginkgo extracts.

The limitations of various analytical techniques, such as macroscopic, microscopic, chemical, and genetic assays will be discussed using actual adulteration cases occurring in the dietary supplement and herbal medicine industry. Since most of the available methods focus on the determination of marker/ active compounds, the emphasis will be on the chemical analysis.

\section{We-Quality Control-SL-02 Quality assessment of Ginkgo biloba supplements based on a single HPLTC Method}

Authors Frommenwiler $\mathrm{D}^{1,2}$, Booker $\mathrm{A}^{3,4}$, Heinrich $\mathrm{M}^{3}$, Reich $\mathrm{E}^{1}$, Cañigueral $S^{2}$

Institute 1 CAMAG, Muttenz, Switzerland; 2 Department of Pharmacology and Pharmacognosy, University of Barcelona, Barcelona, Switzerland; 3 UCL School of Pharmacy, University of London, London, United Kingdom;

4 Division of Herbal and East Asian Medicine, Department of Life Sciences, University of Westminster, London, United Kingdom

DOI 10.1055/s-0037-1608584

Ginkgo biloba L. (GB) is one of the top-selling herbal products in the world. The extract of GB used in commercial preparation is often standardized to $24 \%$ of flavonol glycosides and $6 \%$ terpene lactones. Being a high-value herbal (medicinal) product, GB extracts may be a subject for economically driven adulteration.

In [1] we reported that of 35 GB products (containing extracts and plant material) from the UK market only two show the HPTLC fingerprint that described in the Ph. Eur. and USP monographs for Ginkgo extract. All others contain high levels of quercetin, rutin, or additional zones not characteristic of GB.

Aiming at a better characterization of the adulterants, additional detection modes were evaluated and another derivatization step was added to the original HPTLC method for identification. Beside added rutin and/or quercetin, the modified method detects added Buckwheat and Styphnolobium japonicum (L.) Schott (SJ - syn.: Sophora japonica L.) fruit prior to derivatization as well as S) flower after derivatization. Additional HPTLC methods were established to verify the levels of quercetin (NMT $0.5 \%$ ) and the presence of genistein as marker for SJ fruit.

Samples were also assayed for total flavonoid glycosides by HPLC according to the USP and Ph. Eur monographs on Ginkgo leaves and extract. Twelve samples were in compliance with the total amount of flavonol glycoside specified in the monographs. However, ten of those had elevated levels of quercetin in 
HPTLC fingerprint. This was confirmed with an additional HPLC limit test for rutin and quercetin described in the ginkgo extract monograph from USP. With the proposed modified HPTLC method for identification of GB several types of adulteration can be detected without additional chromatography. That significantly saves cost in a routine setting.

[1] Booker A, Frommenwiler D, Reich E, Horsfield S, Heinrich M. J Herb Med 2016; 6: $79-87$

\section{We-Quality Control-SL-03 Hyperspectral imaging in combination with chemometric data analysis - a novel approach in the quality control of herbal material}

Authors Vermaak $\mathrm{I}^{1,2}$, Tankeu $\mathrm{S}^{1}$, Djokam $\mathrm{M}^{1}$, Sandasi $\mathrm{M}^{1}$, Chen $\mathrm{W}^{1}$, Viljoen $\mathrm{A}^{1,2}$

Institute 1 Department of Pharmaceutical Sciences, Tshwane University of Technology, Pretoria, South Africa; 2 SAMRC Herbal Drugs Research Unit,

Tshwane University of Technology, Pretoria, South Africa

DOI 10.1055/s-0037-1608585

The quality control of herbal material is notoriously challenging due to the complex mixture of compounds present in plants. Hyperspectral imaging (HSI) integrates conventional spectroscopy and imaging to obtain spectral and spatial information from a sample. Once the method has been developed, the visual results are rapidly obtained and easy to interpret. In this study, the use of HSI in combination with chemometric data analysis in quality control was illustrated using three examples: 1) distinguishing between the whole dried fruit of Illicium verum (Chinese star anise) and Illicium anisatum (Japanese star anise); 2) S. tetrandra ('hang fang ji') and its substitution or adulteration with Aristolochia fangchi ('guang fang ji'); 3) determining the proportion of each constituent in a tea blend consisting of Aspalathus linearis (rooibos) and Agathoshma betulina ('buchu'). Hyperspectral images were captured using a shortwave infrared pushbroom imaging system in the wavelength range $920-2514 \mathrm{~nm}$. Evince ${ }^{\circledR}$ and/or Matlab ${ }^{\circledR}$ software were used to analyse the data. For the star anise example, a classification model was developed and used to accurately predict the identity of whole dried fruit of I. anisatum and I. verum. In the 'fang ji' example, the replicates for each plant species were predicted at a value $>99 \%$ for all the samples. Artificially adulterated samples were accurately predicted from as low as $10 \%$. In the herbal tea blend example, the classification model was applied to determine the relative proportions of each blend constituent in intact tea bags. With the increasing need to regulate herbal products and ingredients, emerging technologies are providing alternative methods that allow the holistic analysis of the samples. Hyperspectral imaging is ideally suited as a qualitative tool for the quality control of herbal raw material as it is a visual, rapid, accurate and non-destructive method with high prediction ability.

\section{We-Quality Control-SL-04 Hypericum perforatum - a comparison of commercial samples using DNA-barcoding and chemical approaches}

Authors Scotti $F^{1}$, Mali $P^{2}$, Masiero $E^{2}$, Booker $A^{1,3}$, Sgamma $T^{2}$, Howard $C^{4}$, Agapouda $A^{1}$, Frommenwiler $D^{5}$, Reich $E^{5}$, Slater $A^{2}$, Heinrich $M^{1}$ Institute 1 Research Cluster Biodiversity and Medicines/Centre for Pharmacognosy and Phytotherapy, UCL School of Pharmacy, WC 1N 1AX, London, United Kingdom; 2 School of Allied Health Sciences, De Montfort University, The Gateway, LE1 9BH, Leicester, United Kingdom; 3 Division of Herbal and East Asian Medicine, Department of Life Sciences, University of Westminster 3Division of Herbal and East Asian Medicine, Department of Life Sciences, University of Westminster, W1W 6UW, London, United Kingdom; 4 4BP-NIBSC Herbal Laboratory, National Institute for Biological Standards and
Controls, Blanche LAne, South Mimms, EN6 3QG, Potters Bar, United Kingdom; 5 CAMAG, Sonnenmattstrasse 11, 4132, Muttenz, Switzerland DOI 10.1055/s-0037-1608586

Adulterated herbal products are a serious concern and recently DNA-based techniques have come to a wider attention as a novel tool. The aim of the project is to establish a distinguishable method in the identification of plant material and in quality control. In this study we analysed 20 commercial Hypericum perforatum L. (HP; [1]) products, combining chemical and genetic techniques: NMR-based metabolomics combined with principal component analysis (PCA) and HPTLC analysis [2] and DNA-barcoding.

HPTLC analysis confirmed $50 \%$ of the samples to be HP, with the remainder differing from the HP chromatographic description and identified as "Chinese SJW". NMR-PCA analysis mostly agrees with the HPTLC findings, grouping the allegedly "Chinese SJW" together. DNA-barcoding confirmed the identity of three HP samples. Specific PCR tests gave a positive signal with a further three HP samples but also with three "Chinese SJW" samples.

While the chemical methods, as expected, agree with each other, DNA-barcoding identification was limited by the ability to extract viable DNA from all samples. The design of more specific primers to distinguish HP from "Chinese SJW" is now required. A combination of DNA and chemical methods will confer a higher degree of certainty and can be extended to other herbal medicines.

A charitable donation by Fa. Schwabe (Germany) is gratefully acknowledged. [1] McCutcheon, M. 2017. Hypericum perforatum Botanical Adulterants Bulletin January 2017. www.botanicaladulterants.org

[2] Booker, A, Jalil B, Frommenwiler D, Reich E, Zhai L, Kulic Z, Heinrich M. 2016. Phytomedicine 23: $754-762$

\section{We-Quality Control-SL-05 Efficient Workflow for NMR Metabolomics Screening of Natural Products}

Authors Fischer $\mathrm{C}^{1}$, Colson $\mathrm{K}^{2}$

Institute 1 Bruker BioSpin GmbH, Rheinstetten, Germany; 2 Bruker BioSpin, Billerica, MA, United States

DOI 10.1055/s-0037-1608587

NMR spectroscopy has significant qualities that make it an attractive tool for metabolomics and especially for natural products. These qualities include high reproducibility, simple sample preparation, compound specificity and quantitation. Traditionally, NMR has required sophisticated operators to operate and harvest valuable information from resulting NMR spectra. Also traditionally, studies of natural products rely heavily of purification strategies to simplify NMR spectra.

Our work focuses on the development of integrated NMR software solutions for evaluation of natural products metabolomics spectra, on crude extracts, with an aim to automate the spectral evaluation process. Dereplication and identification of key metabolites in crude extracts are accomplished in automation using various automatically defined line fitting algorithms. Limit of detection calculation are utilized to establish minimal reportable quantity of key components. Taxonomic classification is achieved using scaling function routines allowing for consistent or highly variable spectra. This allows customization for the species or material studied.

This poster will show the software development through a series of natural product examples. The aim is to develop an automated work flow for analysis of these spectra for operators of various skill levels including (1) metabolomics researchers and (2) quality control technicians. NMR assists the researcher with accurate component identification. NMR analysis of natural health products may lead to improved quality, labelling and product consistency. 
Lecture Session - Phytopharmacology/

Extract Pharmacology III

Wednesday, 6th September, 2017

Montreal - 14:45-16:15

\section{We-Phytopharmacology/Extract Pharmacology III-SL- 01 Influence of the St. John's Wort extract Ze117 on membrane fluidity, signal transduction, and mobility of the $\beta_{1}$-adrenergic receptor}

\author{
Authors Keksel $\mathrm{N}^{1}$, Bußmann $\mathrm{H}^{1}$, Unger $\mathrm{M}^{2}$, Drewe $\mathrm{J}^{2}$, Franken $\mathrm{S}^{1}$, \\ Häberlein $\mathrm{H}^{1}$ \\ Institute 1 Institute of Biochemistry and Molecular Biology, Rheinische \\ Friedrich-Wilhelms-Universität, Bonn, Germany; 2 Max Zeller Söhne AG, \\ Romanshorn, Switzerland \\ DOI 10.1055/s-0037-1608588
}

Besides specific ligand and receptor interactions plasma membrane properties also influence cellular signal transduction pathways [1]. Membrane fluidity which is one of these properties can be measured by fluorescence anisotropy. Here we used this technique to determine the influence of the St. John's Wort extract Ze117 on the plasma membrane fluidity of C 6 glioblastoma cells. For this purpose DPH (1,6-diphenyl-1,3,5-hexatriene) and TMA-DPH (1-(4-(trimethylamino)phenyl)-6-phenylhexa-1,3,5-triene) were applied probing the rotational microenvironment within the hydrophobic membrane core and close to the membrane/water interface, respectively.

Chronic exposure (6-8 days) of C 6 cells to Ze117 dose-dependently increased DPH and TMA-DPH fluorescence anisotropy, reflecting a membrane rigidification effect. The in parallel tested antidepressants desipramine and citalopram showed no effect on the membrane fluidity after chronic incubation.

Since changes in membrane fluidity are likely to affect membrane-associated GPCR signal transduction we examined the $\beta_{1}$-adrenergic receptor ( $\beta_{1}$-AR) stimulation-mediated CAMP formation after chronic exposure to Ze117. A concentration-dependent reduction in $\beta_{1}$-AR-mediated cAMP formation after stimulation with dobutamine was observed for the Ze117 extract [2].

This observation of a dose-dependent membrane rigidification suggests a reduced lateral receptor mobility, explaining the reduced $\beta_{1}$-AR signaling after chronic exposure of C 6 cells to Ze117. Thus, Ze117 might be suitable to reduce stress-mediated $\beta_{1}$-AR signaling by reducing the fluidity of plasma membranes.

[1] Escribá PV, Wedegaertner PB, Goñi FM, Vögler O. Biochim Biophys Acta 2007; 1768: $836-852$

[2] Data were generated by Anne Hage-Hülsmann at the Institute of Biochemistry and Molecular Biology, Bonn

\section{We-Phytopharmacology/Extract Pharmacology III-SL- 02 Dry cough associated with pharyngeal irritation:} Survey on Althea officinalis L.

\footnotetext{
Authors Fink $\mathrm{C}^{2}$, Müller $\mathrm{J}^{2}$, Kelber $\mathrm{O}^{1}$, Nieber $\mathrm{K}^{3}$, Kraft $\mathrm{K}^{4}$ Institute 1 Innovation and Development, Phytomedicines Supply and Development Center, Bayer Consumer Health Division, Steigerwald Arzneimittelwerk GmbH, Darmstadt, Germany; 2 Medical and Clinical Affairs Phytomedicines, Innovation and Development, Phytomedicines Supply and Development Center, Bayer Consumer Health Division, Steigerwald Arzneimittelwerk GmbH, Darmstadt, Germany; 3 Pharmaceutical Institute, Leipzig University, Leipzig, Germany; 4 Chair for Naturopathy, Center for Internal Medicine, University Medicine Rostock, Rostock, Germany DOI 10.1055/s-0037-1608589

According to a monograph of the European regulatory authority EMA, herbal preparations from Althaea officinalis L, radix (marshmallow root) are used as a demulcent preparation for the symptomatic treatment of oral or pharyngeal
}

irritation and associated dry cough (1). The two prospective, non-interventional studies reported here aimed on creating a better documentation of the users' impression of the effectiveness and tolerability and their satisfaction. In two independently performed surveys with altogether 822 consumers buying either lozenges or syrup of the aqueous Marshmallow root extract STW42 for treatment of their dry cough were recruited in pharmacies. They were asked to fill in a questionnaire covering a treatment duration of seven days for the documentation of the course of symptoms, and the global assessments of effectiveness, tolerability and satisfaction.

The users stated that both preparations showed a good effect with respect to the symptomatic treatment of oral or pharyngeal irritation and associated dry cough with a very rapid onset of effects, in the majority of cases within 10 minutes. The tolerability was very good ( 3 minor adverse events for the syrup).

The results of the surveys support the long-standing use of Marshmallow root syrop and lozenges for symptomatic treatment of dry cough.

[1] European Union herbal monograph on Althaea officinalis L, radix, EMA/ HMPC/436679/2015

\section{We-Phytopharmacology/Extract Pharmacology III-SL- 03 Activation of pregnane $X$ receptor (PXR) by herbal supplements and risk of Herb-Drug Interactions}

Authors Khan $\mathrm{S}^{1}$

Institute 1 National Center for Natural Products Research, Research Institute of Pharmaceutical Sciences, School of Pharmacy, University of Mississippi, Oxford, United States

DOI 10.1055/s-0037-1608590

Herbal supplements are used worldwide for treating many ailments as well as for general health benefits. In recent years the use of herbal supplements in combination with the prescription and over-the-counter drugs is on the rise to treat multiple disease conditions which is posing an increased risk of herb drug interactions ( $\mathrm{HDI})$. $\mathrm{HDI}$ is becoming an important safety concern for many herbal supplements. Since most of the clinically relevant pharmacokinetic drug interactions are known to occur due to the modulation of the activity of drug metabolizing enzymes (CYPs) and drug transporter (P-gP), PXRmediated induction of CYPs and P-gp has drawn considerable attention as one of the major mechanisms involved in drug interactions. St John's Wort (Hypericum perforatum) is a well known example of the herb causing clinically relevant herb drug interactions that is mediated through PXR activation and hypericin is known to be responsible for such effect.

We have screened some commonly used herbs and their constituents for activation of PXR to predict their potential for herb drug interaction using a reporter gene assay. Their effects on the expression of major drug metabolizing enzymes (3A4, 1A2, 2C9, 2B6) and efflux transporter (P-gp) were also determined by $q-P C R$ techniques and the enzymatic activities were measured using specific substrates for each enzyme isoform. Potent activation of PXR was observed with several herbs such as M. speciosa, E. californica, B. natalensis, and $G$. glabra which led to an increased expression of its target genes. Results will be discussed.

This study is partially supported by The United States Department of Agriculture, Agricultural Research Service, Specific Cooperative Agreement No. 58 6060 - 6-015 and the Food and Drug Administration "Science Based Authentication of Dietary Supplements” award number 2U01FD 004246 - 06. 


\section{We-Phytopharmacology/Extract Pharmacology III-SL- 04 Effects of Inonotus hispidus extracts and compounds on human immunocompetent cells}

Authors Gründemann $C^{1}$, Arnhold $M^{1}$, Meier $S^{3}$, Bäcker $C^{3}$, Garcia-Käufer $M^{1}$, Grunewald $F^{1}$, Steinborn $C^{1}$, Klemd Amy $M^{1}$, Wille $R^{2}$, Huber $R^{1}$,

Lindequist $\mathrm{U}^{3}$

Institute 1 Center for Complementary Medicine, Institute for Environmental Health Sciences and Hospital Infection Control, Faculty of Medicine, University of Freiburg, Freiburg, Germany, Freiburg, Germany; 2 ABNOBA GmbH,

Pforzheim, Germany, Pforzheim, Germany; 3 Institute of Pharmacy, Ernst-

Moritz-Arndt-University Greifswald, Greifswald, Germany, Greifswald, Germany DOI 10.1055/s-0037-1608591

Inonotus hispidus is used as a traditional medicine in China. Previous investigations revealed promising immunomodulatory activity of fruit body extracts of I. hispidus. Bioactivity-guided fractionation showed that hispolon and hispidin were active substances.

In this study, we analysed effects of $I$. hispidus extract (IHE) and selected constituents on different types of human immune cells and investigated the potential of IHE as a medicinal mushroom. The influence of IHE on activity and maturation of human T cells, purified NK cells and dendritic cells (DCs) was analysed using flow cytometric-based surface marker expression. The celldivision characteristics of the activated T cells were assessed by membranepermeable dye, and the function of NK cells was investigated by degranulation-CD 107a assay. Apoptosis induction was assessed by surface staining of phosphatidylserine, and camptothecin (CPT) and cyclosporine A (CsA) were used individually as controls. Phytochemical analysis, using TLC chromatograms and HPLC analysis, was conducted to characterize the IHE.

IHE increased the activation and diminished the proliferation of activated human T cells in the presence of apoptosis. NK cell activity and function were dose-dependently increased. Surface marker expression of DCs demonstrated that IHE has the potential to induce maturation. TLC and HPLC analyses showed that the extract contained hispidin and hispolon. Investigations using hispidin and hispolon demonstrated similar, albeit non-congruent results, with extracts on measured parameters.

The results indicate that extracts from 1 . hispidus and their constituents, hispidin and hispolon, interfere with function of multiple immune cells, thus providing a rationale for their potential as a medicinal mushroom. 


\section{Authors' Index}

A

Aati $\mathrm{H} \quad \mathrm{S} 60$

Abas F $\mathbf{S 1 3 2}$

Abba Chika C S116

Abbah C $\mathbf{S 1 3 5}$

Abdalla Viviani CP $\quad$ S91

Abdalla M S46

Abdel-Aziz H S15, S130, S135, S156, S159, S160

Abdelgadir HA $\mathrm{S} 53$

Abdel-Hamid Alaa El-Din E S72

Abdelrahman E S169

Abdel-Tawab N S160

Aboobaker Z S9

Abu O $\mathbf{S 1 5 5}$

Acin S $\quad S 122$

Acin-Martinez S S30

Acuram Lovely K S15

Adaikan PG $\mathrm{S} 138$

Adam Z $\mathrm{S} 138$

Adamu M S143

Adebayo A S143, S144

Ademovic Z $\mathrm{S167}$

Adenan Mohd I S68

Adenubi $\mathrm{O}$ S2

Aderogba Mutalib A $\mathbf{S 1 5 6}$

Adewole Adeniyi R S179

Adewunmi C S146

Adeyemi O $\quad \mathrm{S} 144$

Afzan A S106

Agapouda A 5191

Agar Osman T S74

Aguiyi J S150, S175

Agyare C S176, S177, S187

Ahmad Fasihuddin B S20

Ahmad Md I S41

Ahmat N S11, S68, S69, S70, S71, S72

Ahyi V S173

Aierken A S115

Akaberi M 549

Akbarov U S66, S175

Akdemir ZS S80

Akinsanmi A S150, S175

Akinyele S S146

Akiyama R $\quad \mathrm{S} 134$

Akone Sergi H S118

Akpagana K S172

Akplah Leslie A S176, S177

Akpoveso OO S129

Akwu N S180

Al Muqarrabun Laode Muhammad R S72

Al Salmani K S47

Al Taee H S42

Alade A S143

Alajlani M S132, S161

Alakomi HL S185

Alcântara BGV S73

Alencar T S151

Alfattani A 549

Alhabib I 547

Al-Harrasi A $\$ 47$

Ali Abdalla M S126

Aligiannis N S178

Alilou M 551

Allard PM S49, S80

Allard PMA S109

Almeida C S57, S186

Alonso Isabella G S32

Álvarez-García S S148

Alves Maria S 557

Alves MS S152

Alzate F S126

Ambrósio SR S101

Ammer S S97

Amorim D $\mathbf{S 1 0 0}$

Ancheeva E S118
Andersen Jeanette $\mathrm{H} \quad \mathrm{S} 11$

Andrade C S50

Andrade-Cetto A S175, S183

Aneva I $\$ 119$

Angelis A S89, S169

Anjos O $\mathbf{5 6 5}$

Anne C S102

Anquez-Traxler C S105

Anselmo Moreira F S145

Aparecida Chagas-Paula D S36

Apenteng \ $\mathrm{S} 142$

Apers S S61, S187

Apolinário E $\mathbf{S 1 0 0}$

Apostolides Z S108

Araujo Angela R S91

Arbain D $\mathrm{S} 183$

Arbin M S69

Arboleda M S121

Ardjomand-Woelkart K S131, S187

Aree K S42

Argyropoulos D $\quad \mathrm{S77}$

Argyropoulou A S90, S169

Arnaiz V $\mathbf{5 9 5}$

Arnhold J 5128

Arnhold M S193

Arnold YE S78

Aro A S46, S15

Asadi H S12

Asadi N S174

Asare C S146

Ascrizzi R $\quad$ S134

Asgari A S120

Ashafa O S126

Ashano E S144

Assemova M S167

Astuti Eny D S147

Atakpama W S172

Atanasov A S9

Aubert P S159, S160

Autret JM S90

Avula B $\quad$ S110

Ayensu I S142

Ayoub I $\mathbf{S 1 5 8}$

Ayrle $\mathrm{H} \quad \mathrm{S} 1, \mathrm{~S} 97$

Azam A $\mathrm{S} 132$

Azimullah S S42

Aziz R $\quad \mathrm{S} 106$

Azzam S S169

B

Bacher S S62

Bachinger D $\mathrm{S96}$

Bachmann H S94, S103

Bäcker C S193

Bader G S135

Bae JS S39, S40

Baek HJ $\mathrm{S} 114$

Baira E S77

Bak」 S153

Bakiri A S110, S111

Balash A 565

Balcazar N S122

Balcazar-Morales N S30

Ban Ju Y S115, S145

Bani D S124

Baolin B S132, S161

Baraldo G S48, S84

Barbosa Wagner L $\mathrm{S} 91$

Barboza Rodolfo S 519

Bardot V $\mathrm{S} 139$

Barejão A S154

Barina Z S46

Barrera YA S121

Basting R S158

Batawila K S172
Bauer R S21, S28, S109, S130, S131, S187

Bayazeid O S49, S88, S154

Bazylko A 577

Bedir E S49, S80

Bedır E S117

Beerhues L S101

Behnam D $\$ 160$

Benarbia A $\$ 92$

Béni Z S34

Bergonzi Maria C S91, S124, S125, S190

Bernal F S71

Berninger T $\mathrm{S} 166$

Bessa M S152

Bessard A S159, S160

Beteinakis S S169

Beuerle T S101

Bhambra A 547

Bhowon MG S77

Bianco A S64

Biasetto Carolina R $\mathrm{S91}$

Bieler L S124

Bigagli E S190

Bijttebier S \$59, S60, S61, S68

Bilgin M S157

Bilia Anna R S91, S124, S125, S134, S190

Bischoff I S29, S34

Bischoff T S3

Bishr M S119

Bisio A S25

Bismarck D $\mathrm{S95}$

Bittner M S162

Blanchet E S49

Blumenthal M S190

Boakye Yaw D S176, S177

Boakye YD S187

Bolzani Vanderlan S S91

Bombelli R S188

Bonn GK S169

Bonté $\mathrm{F} \quad \mathrm{S} 17$

Booker A S190, S191

Boonrat O S121

Borie N 5110

Botelho C S58

Botha F S108

Boueroy P S45

Bouy M S102

Boyle G S26

Bozicevic A $\$ 111$

BOZKURT B S68, S79

Boznou V S178

Bozorgi M S181

Bózsity N S38

Braca A S24

Bräm S S157

Brandão Torres Luce M S155

Brandt S S13, S16

Brantner A S132, S161

Braun A S156

Bräutigam 」 S34

Breinlinger S S50

Brendieck-Worm C S94

Breynaert A S61

Brieudes V S158

Brillatz T S37, S38

Bringmann G $\mathbf{S 1 1 6}$

Broeckx G S60

Broit N S26

Brun R S8, S25, S36, S61

Buchholz T S51

Bulmer A s9

Bunzel M S86, S88

Burger T S87

Bürgi T S91

Burholt M S170

Bußmann H S192 
Butsri S S44

Butterweck V S58

Byun J S145

$$
\text { C }
$$

Caballero-George C S59, S60

Cabello Hernández Christian A S175

Cabral Elaine C $\mathbf{S 1 5 8}$

Cailleau-Audouin E $\mathrm{S} 102$

Cakova V S17

Calmels G $\mathrm{S90}$

Camargo llana LBC S91

Camargo GL S154

Campos Maria G S65

Canigueral S S105

Cañigueral S S166, S190

Cardellina J $\$ 190$

Cárdenas Vázquez René de J $\$ 175$

Cárdenas-Vázquez René de J S183

Cardona-Gomez Gloria P S126

Carrijo T S151

Carro-Huerga G S117, S148, S166

Caruso D S186

Carvalho Joao E S32

Carvalho João E S158

Casamenti F S124

Casoti R S73

Casquero PA S117, S148, S166

Castagnola P S25

Castañon Maria C S55

Castro María C S157

Castro V S58, S115

Casu L S182

Catrina SB S132

Caudal D $\$ 139$

Cavalheiro Alberto J S91

Ccana-Ccapatinta GV S12, S162

Cerón-Carrasco José P S27

Četojević-Simin D S7

Chaanin A S12, S102

Chagas-Paula DA S73

Chaimbault P $\mathrm{S} 172$

Chaingam 」 S164

Chaiyakunapruk N S132

Chalard P $\$ 139$

Challal S S37

Chanchao C S36

Chang CC S46

Chang FP S74

Chang FR S28, S30, S31

Chang HS S46, S47, S48, S87

Chang HT S21

Chang TH S41

Chansriniyom C S112

Chao W S74

Chao YH S17

Charoenwiwattanakij P S56

Chatterjee P $\quad$ \$129

Chaudhary G $\$ 148$

Chave \ 449

Chedier L S55

Cheilari A S43

Chellegatti MASC S101

Chen $\mathrm{BH}$ S31

Chen $\mathrm{CH}$ S41

Chen CL S33

Chen CY S28

Chen IS S46, S47, S48

Chen IJ S41

Chen JT S87

Chen SR S28

Chen W S171, S191

Chen YC S18, S44

Chen YF S48

Chen YL S73

Chen YM S87

Cheng JC S40

Cheng MJ S47
Cheng TL S48

Cheng YB S28, S30

Chervin J S11

Chevalier 」 S159, S160

Chia-Leeson O $\$ 107$

Chiang HM S35

Chia-Yi G S69

Chicoteau P $\mathrm{S95}$

Chiranthanut N S52

Chiu CW S18

Chiyeon L S35

Chizzola R S104

Chłopecka M S5, S92

Chmara S S27

Cho WK $\$ 153$

Choi Doo J S32, S137, S181

Choi Jin K S165

Choi Yang A S165

Choi G S166

Choi YS S153

Chollet S S90

Choo Byung K S152

Choolani Mahesh A S138

Choudhary I S167

Chougourou D S173

Christen P $\mathrm{S} 80$

Cianfaglione K S64

Cimpan G S11

Cinci L S190

Ciubotaru M S37

Colombo E S141

Colorado D $\mathrm{S} 122$

Colson K S191

Conegundes J S147

Constantin P S102

Coronnello M S125

Corradi E S58

Cortes N S126

Cosentino M S188

Costa-Silva T S50

Cotte C $\$ 139$

Couillard-Despres S S124

Coutinho T S154

Coy-Barrera E S108

Crawford AD S37, S38

Crochet S S102

Crous P 59

Csábi] 585

Csizmadia V S38

Csorba A S45

Csupor D $\mathrm{S} 140$

Cuendet M S80

Culmsee C $\mathrm{S14}$

Custódio L S43

Czerwińska M S33, S41

$$
\text { D }
$$

da Costa E S184

da Costa F S13

Da Costa FB S12, S162

da Silva Maria Fatima das Gracas F S81

da Silva A $S 91$

Da Silva F S148

Dagnelie P $\mathrm{S99}$

Dai Y $\mathbf{S 1 6 8}$

Dai YH $\mathbf{S 1 7 4}$

Daletos G S107, S118

Dalla C 577

Danne-Rasche N S72

Danova K S119, S120

Danton O S49, S52

Daoultzi E S124

Dapra L S39

Datkhayev U S35

De Bie H S111

de Carvalho João E $\quad$ S179

de Guevara V S177

De Leo M S24
De Mieri M S58, S59, S91

De Tommasi N S24, S25

Debrah P $\mathbf{S 1 4 6}$

Dedousi V $\mathrm{S} 178$

Deguchi T S134

Dékány M S34

Delaude C $\mathbf{S 7 8}$

Delaveau J $\mathrm{S} 102$

Delecolle 」 $\mathrm{S} 17$

Delgado T S65

Dell'Agli M S141, S143, S184, S186

Demchenko D S175

Demir S S79

Demır S S68

Demirci F $\mathrm{S} 141$

Demirezer Lutfiye O S25, S26, S54, S74, S141, S164

Demirezer Ö S88, S154

Demirezer LO S55, S56, S83, S147

den Haan H S27

Deng $\mathrm{H} \quad \mathrm{S} 11$

Désert C S102

Destailleur V $\mathrm{S} 102$

Deutsch A S21, S28

Diallo D S32

Diard C 590

Dias A S58, S100, S115, S178

Dias DF $\mathrm{S73}$

Dias T S65

Diazgranados M S13

Dimitrova L S116

Dirsch V 59

Disch L S66, S146

Disler M S3

Ditsopa B S121

Djokam M S191

do Amaral Corrêa José O S57

Dobrev P S120

Dobrindt U S13, S142

Dobrzyński M S52, S111

Dodoo Deborah Naa A S177

Donato R S134

Dounoue-Kubo M S109

Drewe J S14, S66, S146, S161, S182, S192

Drint M S93

Drumea V S165

Duangprompo W S42

Dubourdeaux M S139

Dudek M S41

Duque Ana P S55

Durand T S159, S160

Durchschein C $\mathbf{S 2 8}$

Durmuskahya C $\mathbf{S 1 5 2}$

Dusold A $\mathrm{S95}$

Duval Raphael E $\mathbf{S 1 7 2}$

Dziekan N S92

Dziwornu GA S77

E

Ebel R S11

Eberlin M S158

Ebrahimzadeh FM S120

Echeverri F S31

Eckelmann D 579

Edrada-Ebel R $\mathrm{S} 63$

Edrada-Ebel RA S10

Efferth T S25, S26

Ejembi S $\mathbf{S 1 7 5}$

Ejike Chukwunonso ECC S172

El-Ahmady S $\$ 169$

Elamurugan A S96

ElGamal A S60

Elgorashi E S187

El-Hazek RM S160

El-Kashoury ES $\$ 169$

Eloff J S2, S108, S143, S151

El-Sabbagh WA S160

El-Sayed S S160 
El-Shazly M S31

ElSohly M S110

Elusiyan C S146

El-Zalabani S S119

Emi Norzehan Mohamad M S168

Engelhart-Jentzsch K S127

Engemann J S87, S170

Engler O S181

Engström M S12

Enke H S50, S118

Eparvier V S80

Erdelmeier C $\mathbf{S 1 3 3}$

Erdogan Orhan I S27

Esch S S16

Esimone Charles C S116

Esimone C $\$ 135$

Esmaeili A $\quad$ S120

Esmaeili $\mathrm{H} \quad \mathrm{S} 12$

Espinoza-Hernández A $\quad$ S183

Esposito F S38

Estevinho LM S65

Eun JuS S113

Evangelakou Z $\mathbf{S 9 0}$

Evans AR S125

Evans M S47

Evstatieva L S119

Eze Peter M S116

Eze F S156, S157

Eze P $\$ 135$

Faix Š $\quad 5104$

Fakhrudin N S147

Falquet J S184

Famuyide I S46, S151

Fan B S110

Fan X $\quad$ S176

Fan YH S87

Farinha N S65

Fasina Folorunso O S126

Faúndez Catherine A S90

Faustino C S43, S185

Fazliyatun Shazlyana M S168

Fekry M S119

Feldmann I S119

Félix L S86, S153, S154

Feller $\mathrm{R} \quad \mathrm{S} 2$

Fernandes João B S81

Fernandes Maria F S147

Fernandes Pinto da Luz C S155

Fernandes VHC 573

Fernandez M $\quad 554$

Ferreira Guimarães S S138

Ferreira Queiroz E S88, S101

Ferreira MJP S37

Ferreira PL S12

Fertig O $\quad \mathbf{S 2}$

Fester K S116, S176

Fico G S141

Figueiredo Mariana C S126

Figueiredo C S41

Figueredo M S158

Fimia Gian M S43

Fink C S159, S192

Fink J $\mathbf{S 1 1 7}$

Fink-Gremmels J S2

Finnie JF S53

Fischer C S191

Fitzgerald M S140

Florencio 」 557

Flores Xavier V S134, S138

Foglio Mary A S158, S179

Foglio M S126

Fokialakis N S43, S57, S110, S186

Fontanay S S172

Forgó P S38

Forino M S141

Forsch K S66, S146
Foster S $\mathbf{S 1 9 0}$

Foteinos S S43

Fotouhi F S126

Foubert K S59, S61, S68, S187

Fougere B S2

Fourbert K $\mathrm{S} 60$

Foury A S92, S102

Francini $\mathrm{F} \quad \mathrm{S} 157$

Franken S S192

Franyoto Yuvianti D $\quad$ S147

Franz G S76

Freitas Blanco Veronica S S32

Frezza C S64

Fricker G S66

Frish R S38

Frommenwiler D S190, S191

Fryganas C S186

Fuhrmann I S97

Fumagalli M S141, S143, S184, S186

Furlan Cláudia M S145

Fürst R S29, S34

Furtado NAJC S101

Gad H S158

Gafner F S63, S111

Gafner S S190

Gaid M S101

Gaitán isabel C S161

Galdino J 5155

Galuppo MK S37

Gan KH $\mathbf{S 4 8}$

Garcia C S43, S185

García JM S95

Garcia-Käufer M S193

Garo E S52, S111

Gazdová M S106

Geneva M S116

Genilloud O S43, S57, S186

Georgousaki K S43, S57, S186

Ghaemi H S174

Ghotge N S3

Giavarini F $\mathbf{S 1 8 6}$

Gikas E $\quad$ S77

Gille E S58

Gimenes L S81

Gindro K S101

Giovannetti L S124, S125

Gioxari A S158

Girón Rodríguezd F S27

Goerick C S66

Goffin V S14

Gokbulut A S74

Gokmen A S141

Goletti D S43

Gomes Ana C 591

Gomes Soares M S36

Gomes A S58

Gomes M S19

Gonçalves Rodriques Das Dores R S134, S138, S162

Gonda T S83

Gonulalan Ekrem M S55, S56, S147

Gonulalan E S88, S154

Gonzales E S132

González Arbeláez L S157

González I S43, S57, S186

González V S43

González-López Ó S117, S148, S166

Goodman Christopher D S32

Goos KH S128

Goren Ahmet C S74

Görick C S51

Gosztola B $\mathbf{S 1 4 0}$

Goyal S S148

Graf-Schiller S 597

Granchi C S24

Grando R S126
Granica S S8, S20, S33, S41, S78, S127, S182

Graverini G S124, S190

Grecco S 550

Greve H S8, S25

Grienke U S8, S63

Griesbeck C S39

Grimaud I S102

Grochowski D S77

Groh C S17

Grombone-Guaratini Maria T S145

Groot M S2

Groppo M S12

Gros C S92

Gründemann C S31, S91, S193

Grundmann D S156

Grunewald F 5193

Guh JH S47

Guillen A S30

Guilloteau L S92, S102

Guinobert I S139

Gulec M S157

Gulsoy Toplan G S155, S157

Gumeni S S43, S57, S186

Günther F S18

Gupta M S174

Gupta MP S59

Gürbüz P S83

Gutiérrez S S117, S148

Guvenalp Z S26, S54, S83

$\mathrm{H}$

Häberlein H S192

Hadian I S12

Hafiz Zetty Z S68

Hahn H S10

Hahn I 597

Hahnvajanawong C 545

Halabalaki M S89, S90, S158, S169

Halbsguth C S6

Hamburger M S3, S12, S24, S31, S49, S52, S58

S59, S89, S91, S111, S128, S149

Hamid M S138

Hammoud D S128, S173

Han Byung W S10

Han Chang K S42

Han BS $\$ 149$

Hanáková Z S106

Hansakul P S42

Hanus 」 $\mathbf{S 1 8 9}$

Hanuš ] 5122

Haris P 547

Haron MH $\mathrm{S98}$

Hashempour H S24

Haslinger C S128

Hatibovic D $\quad \mathrm{S} 172$

Hauta L S84

Heckendorn F S102

Heilmann J S7, S66, S75, S76

Heinrich M S64, S190, S191

Heintz D $\mathrm{S} 17$

Heiß E S9

Heiss E S34, S187

Helbling JC S102

Hensel A S13, S16, S102, S125, S131, S142, S180, S182

Heo JD S114

Herfati F S24

Hermans N S61

Hernandez C $\mathbf{S 1 5}$

Herrmann Fabian C S10

Hirosawa R S14

Hirun N S121

Hoang An T S32

Hoffmann-Campo CB $\mathrm{S154}$

Hohmann J S34, S37, S38, S46, S141

Hong X S168

Honorio Javes C S95

Hosek J S189 
Hošek J S106, S122

Hosseinkhani A S174

Houriet J S78

Howard C S191

Howes M S150

Hristozkova M S116

Hsin-Yi H S33, S67

Hsu LC S47

Huang CY S27

Huang GJ 574

Huang $\mathrm{HC} \quad \mathrm{S40}$

Huang HK S48

Huang YH S47

Huber R S193

Hubert J S90, S110, S111

Huchet C S139

Huefner A S28

Huffman M S4

Hunyadi A S37, S45, S46, S83, S85, S98

Hur OS S114

Hwang $\mathrm{KH} \quad \mathrm{S} 114$

Hwang SY S40

Hwang TL S41

Hyu Ah H S113

I

lanora A S38

Iguchi T S21, S22

Ikeani C S142

In Sok L S113

Inan Mumin Y S74

Indy Tamayose C S37

lonkova I S187

Isaac G S109, S110

Ishida Y S134

Ismail Fyaz MD S125

Ismail Intan S S132

Ismail Nor $\mathrm{H}$ S20

Ismail I S133

Issaadi Halima M S85

Itharat A S17, S42, S113, S116, S136, S172

Ittiudomrak T S36

Iwasaki S S57

Iweala E S144

Izatullayev EA S150

」

Jabłonowska M S77

Jabubczyk K S106

Jackson CR $\mathrm{S98}$

Jacmin M S37, S38

Jacob C $\mathrm{S} 172$

Jäger AK S81, S109

Jahns S S118

Jaiarree N S129

Jaijoy K S173

Jang Dae S S85

Jang Myeong $\mathrm{H}$ S148, S165

Jansen E S99

Jansen-Dürr P S48, S84

Jansom C S113

Jaspars M S11

Jas|'pińska 」 S58

Jee Moo G S112

Jee MG S19

Jenkhetkan W S113

Jeon Won K S85

Jeon JH S85

Jeon WY S137

Jeon $Y$ S51, S133

Jeon YA S114

Jeong Hwa K S113

Jeong Yun H S153

Jeong EJ S114

Jeong HN S163

Jeong S] S137

Jeong $\mathrm{YJ} \quad \mathrm{S} 114$

Jerz G S188
Jhaumeer-Laulloo S S77

ji Soo H S113

jiang $Y \quad$ S34

Jimenez Negro E S131

Jimenez-Negro E S72

Jin Seong E S137

jin Seul A S145

Jindaprasert A S116

jirakiattikul Y S17, S116

jirapongpatta R S116

Jo Gyeong S S113

João Pedro G S184

Johnson T S150, S175

Jorge M S158

Josic G 589

Joubert E S7

Jung Ji $Y$ S165

Jung Sang $H \quad S 100$

Jung GH S166

Jung HY S149

Jung JS S163

Junka A S27

Jürgenliemk G S7

K

Ka Soon L S113

Kaewtunjai N S19

Kahraman C S80

Kaiser M S8, S24, S25, S36, S61, S71, S149

Kalabaeva A $\mathbf{S 1 6 7}$

Kalia YN S78

Kaliora A S158

Kallergis E S158

Kalpoutzakis E $\mathbf{S 1 7 8}$

Kamarozaman Aisyah S S68, S71, S72

Kamatou G S185

Kamdee S S114

Kammerer D S64, S76

Kamytbekova KZ S150

Kang Jeong H S112, S113

Kang Kyo B S10

Kang Nu R S136

Kang Suk W S100

Kang Tae K S100

Kang Tong H S51, S133

Kao YC S33

Kapp K S136

Karaca N S141

Karami A S12

Karkoula E S77

Karlik W S92

Karonen M S12, S67

Karou Simplice D $\mathbf{S 1 7 2}$

Karskela T S84

Kaschubek T S93

Kato E S14, S130

Kato L S81

Katsinas N S186

Kauhl U S26

Kawabata 」 S14, S130

Kaya Gi $\quad$ S79

Kaya Gl $\mathbf{S 6 8}$

Kayalar H S152

Kayimbo Malilo K S184

Kayser O S119

Keach JE S41

Kehagia A S124

Keksel N S192

Kelber O S15, S135, S156, S159, S160, S192

Kerdsakundee N S121

Kerekes D S140

Keresztes F S18

Kesternich V S90

Ketjinda W S122

Keusgen M S65

Khalid S S99, S149

Khalil N S119

Khalil R S137
Khamma S S38

Khan I S110, S190

Khan MMA S180

Khan S S192

Khayyal MT S160

Khoddami M S51

Khong Q S39

Khonsung P S52

Ki Kwon L S113

Kim Dong K S153

Kim Geum S S181

Kim Hee K $\quad$ S112

Kim Hyo S S166

Kim Hyun H S112

Kim Hyung D S32, S137

Kim Jin S S27, S136

Kim Kyung A $\mathbf{S 1 0 0}$

Kim Kyung $S$ S99

Kim Ohn S S137

Kim Su K S115

Kim Sun I S112

Kim Won G $\$ 149$

Kim Yeong S S75, S184

Kim Young $S$ S136

Kim Yu J S137

Kim AR S52

Kim BY $\quad$ S137

Kim CS S136, S165

Kim DJ $\mathrm{S} 114$

Kim DW S165

Kim DY S39

Kim GS S32, S137

Kim H $\mathrm{S} 153$

Kim HD S181

Kim HG $\quad$ S19

$\mathrm{Kim} \mathrm{HH} \quad \mathrm{S} 19$

Kim HJ S165

Kim 」 S67, S83

Kim JA S137

Kim JH S114

Kim KS S149

Kim MS S85

Kim NH $\quad \mathrm{S} 114$

Kim SI S19, S163

Kim SK S166

Kim TJ $\quad$ S100

Kim YJ S149

Kim YS S153

Kim YU $\quad$ S137

Kimani N S61

Kincses A S46

Kirchinger M S107, S124

Kirmizibekmez H S74, S141

Kirsch G S172

Kiss A S20, S127

Kiss AK S8, S182

Kiss T $\quad \mathrm{S} 140$

Kissels W S2, S22, S93

Kitisripanya T S164

Kivçak B S117

Kıvçak B S123

Kleijer G S4

Klein J S119

Kleinwächter M S131

Klemd Amy M S193

Klemd AM S31, S91

Klingelhöfer I S87

Klungsaeng S S44

Ko HC S114

Ko HH S46, S85

Kobayashi Y S57, S151

Kočevar Glavač N S54

Koch A S102

Koch E S14, S62, S131, S133

Kohl R S48

Kokorin A $\quad$ S18

Kokras N S77

Koleva P S119 
Komane B S185

Kongpetch S S44

Kongstad K S109

Kongstad KT S81, S82

König S S16

Koobkobkruad T S189

Korinek M S31

Koskinen K S130, S131, S187

Kossack R S50

Kotland A $\$ 90$

Kourkouli A $\mathbf{S 9 0}$

Kovács B S34

Kowalczyk M S27

Kraft K S104, S159, S192

Kramer D S118

Krámos B S34

Krastel P S37

Kratz JM S8

Kraus B S66

Kraus L S95

Krause R S156

Kreft S S54

Kreis W S117

Kretschmer N S21, S28

Krittanai S S164

Krupitza G S38

Kuchta K S179

Kukongviriyapan V S44

Kulic Z S62

Kulmány Á S46

Kuo HH S174

Kuo YH S40, S46, S74

Kupittayanant P S94, S128

Kupittayanant S S94, S128

Kupper 」 S1

Kuroda M S22

Kuropakornpong P S136

Kurtán T S107, S118

Kuruuzum-Uz A S82, S83

Kusari S $\$ 79$

Kusolrat P S128

Kúsz N S38, S45, S46, S141

Kwak Jong Hwan K S52

Kwak Jong H S51, S133

Kweon KB S19

Kwon Tae R S148, S165

Kwon DY S32

Kwon IS S52

Kwon KB S165

Kyeremeh K S11$$
\text { L }
$$

Laakso I S66

Labes A S83

Lacaille-Dubois MA S78

Ladurner A S9

Lafoux A $\$ 139$

Lagarrigue S S102

Lago J S41, S50

Lahrita L S14

Lai HC S47

Lai KY S35

Lajter I S38

Lambert C S111

Lami R S49

Landa P S106

Landucci E S124

Lans C S4

Lansade L S92

Lanz M S94, S103

Laphookhieo S S7

Laranjeira I S100

Lau Lang C S138

Lauritano C S38

Lautenschläger T S23

Lazzari D $\mathrm{S124}$

Le Tran T $\$ 116$

Leach D S186
Lèbre A S102

Ledru E S102

Lee Chung $\mathrm{H} \quad \mathrm{S} 100$

Lee Dae Y S137

Lee Dea Y S181

Lee Hyeon W S145, S146

Lee Ik S S27, S136

Lee Jae W S32, S137

Lee Jea W S181

Lee Ka S S112, S113

Lee Kyung E S99

Lee Myung K S99

Lee Seul G S148

Lee Seung E S32

Lee Sun K S112

Lee You S S112

Lee AY S166

Lee CL S20, S35

Lee D S85, S153

Lee DY S32

Lee HS $\quad 540$

Lee $\mathrm{HZ} \quad \mathrm{S} 44$

Lee J S85, S184

Lee JI \$40

Lee $\mathrm{KJ} \mathrm{S} 163$

Lee KS S19

Lee MC S114

Lee MS S73

Lee MY S137

Lee $S$ S184

Lee SE S137, S181

Lee TH $\mathrm{S73}$

Lee $W \quad$ S10

Lee YM S165

Lee YS S32, S137, S181

Lee YY S114

Lehmann S S102

Lehnerts S S156

Leite F S151

Leláková V S106

Lemus Belen Ringele G S90

Leonard C S171

Leoni S S49

Leonti M S182

Leppä M S12

Lerdvuthisopon N S111

Leterrier C S102

Li S S57

Li T S82

Liebau E S180, S182

Lievaart J $\mathrm{S93}$

Likhitwitayawuid K S112

Liktor-Busa E S34

Lim HS S137

Lima Rita de Cassia L S81

Lima ML S37

Lin $\mathrm{CH} \quad \mathrm{S} 46, \mathrm{~S} 48$

Lin JW S24

Lin $\mathrm{P} \quad \mathrm{S} 168$

Lin $\mathrm{Y} \quad \mathrm{S} 57$

Lindequist $U$ S193

Ling $Y \quad S 151$

Lipowicz B S125, S131

Liu B S34

Liu Cl S17, S21

Liu $\mathrm{HH} \quad \mathrm{S} 44$

Liu Y S107

Liu Z S19

Lobstein A S17, S92

Longdet I S175

Lopera Y S122

Lorenz M S105

Lorenz P S64

Lorenzen W S118

Lorkowski G S15

Loroch S S119

Lotter E S76

Lu MC S18
Lu YY S28

Lubisch M S180

Luceri C S190

Luini A S188

Luiz Baldim J S36

Lukács A S140

Lunder M S54

Luo G S73, S163, S176

Lyssaios FA S178

M

Ma Choong J S145, S146

Ma Jin Y S153

Maddox VR S98

Mäder K S122

Madikizela B S143, S183

Maggi F S64

Mahajan U S148

Mahattanadul S S121, S123

Mahmod Ilya I S133

Mahmoud A $\$ 149$

Mahmoudi-Kordi F S24

Mai CW S20

Mair CE S8

Makaratat N S172

Makarov V S66, S175

Makhatov B S35

Maldini M S186

Mali P S191

Malterud Karl E S32

Mamani O S132

Mandach U von S128

Mándi A S118

Mandombe José L S23

Maneenuan D S121

Manns D 576

Marçalo J S43, S185

Marchal L S90

Marcourt Laurence M S101

Marcourt L S37, S38, S88

Maria de Oliveira Sousa I S179

Marino F $\mathrm{S} 188$

Märkl S S107

Marner M S83

Marquardt P S176

Marques Fornseca Maira C S162

Marques B S178

Maršík P S106

Martínez José V S161

Martinez A S110

Martinez Y S95

Martins A S46

Martins-Gomes C S86, S88

Marx L S156

Mäser P S149, S184

Masiero E S191

Masoompour Seyed M S174

Massa María L S157

Massana Codina Josep Massana C S101

Mat A S155

Matasyoh J S61

Mata-Torres Valle G S183

Matei A S37

Matkowski A S27, S58

Matsuda H S134

Maulenova AV S150

Mayer E S93, S96

Mayer M S3

Mayer P $\mathrm{S97}$

Mayo S S117, S148, S166

Mayorga O $\mathrm{S} 57$

McGaw L S2, S46, S142, S143, S151, S183

McGaw LJ S126

Md Yusoff Y 563

Meeran MFN S42

Meesakul P S7

Mehrbod P 5126

Meier B S3, S89, S170, S171 
Meier N S69, S89, S170, S171

Meier S S193

Meitinger N S117

Mekala K S104

Melzig M S3, S29, S66, S188

Melzig MF S18, S51, S61, S162

Melzig MM S18

Mendel M S5, S92

Mendes R S55, S147

Meng G S131

Mennet M S128

Mercerand F $\mathrm{S} 102$

Mérida Reyes Max S S161

Merle T S37

Mesquita JT S37

Mevissen M S1, S97

Meziani S $\$ 92$

Michailidis D $\mathbf{S 1 6 9}$

Michalak B S127

Michalea R S178

Michellod Emilie M S101

Mimaki Y S21, S22

Mimica-Dukić N S7

Min Kyung C S113

Min BS S40

Mini E S125

Mintah D S142

Minutolo F S24

Miranda Iranaia A S19

Mitaine-Offer AC S78

Mitakou S S90, S158

Miyamoto T $\mathrm{S} 78$

Miyasaki AKP $\mathbf{S 1 5 4}$

M'Kacher R S139

Mncwangi N S84

Mo YM S163

Moeslinger T S8

Moesslacher 」 $\mathbf{S 4 8}$

Mohd Dom Nur S S138

Mohd Fadhil Mohd F S58

Mohd Faiz Foong A S107

Mohd Hanafi Mohd M S106

Mohd Nazri Nur Ainaa A S71

Mohd Yusoff Aiza S S71

Moisan MP S92, S102

Moissl-Eichinger C S130, S131, S187

Moltó-Puigmartí C S99

Mölzer C S9

Mombekov S S35

Monge M S12

Monji F $\mathrm{S} 138$

Monteiro Rodrigues L S43

Montoya-Perez Guillermo L S30

Moon Byeong C S166

Moradi-Afrapoli F S49, S52

Moraes RM S98

Moreira Spindola H S126, S179

Moridi Farimani M S89

Morita K S179

Morlock G S87

Mosaffa-Jahromi M S174

Moschny \ 5118

Moser A S77

Motyka V S120

Mueller-Harvey I S186

Muhammad H S137

Müller E S95

Müller J S159, S192

Mun'im A S151

Munkert 」 S119

Munkong N S111

Munoz Diana L S30

Muñoz Wug Manuel A S161

Murata Ramiro M S32

Murata K S134

Musat MG S37

Mustafa Mian W 5123

Mutryn K S77
Mvumi B S2

Myung CS S39

$$
\text { N }
$$

Na Takuathung M S52

Na MK S39, S40

Nabila N S151

Naegeli H S1

Naessens T S187

Nägele B S64

Nagia M S101

Nagy N S38

Nahar L S125

Naidoo V S2

Naidoo $Y$ S180

Nair B S103, S104

Nair MNB S96, S104

Nakagawa S S134

Nalbantsoy A $\mathbf{S 1 5 2}$

Nam Hyeon H S152

Nam Ju O S148

Nam Youn H S51, S133

Nana F $\mathrm{S} 172$

Naowaboot J S111

Nardiello P S124

Natesan P S96

Nathues H S97

Naumann A $\$ 169$

$\mathrm{Naz} \mathrm{H} \quad \mathrm{S} 71$

Ndhlala Ashwell R S53

Nejad Ebrahimi Samad Nejad E S101

Nejad Ebrahimi S S12, S24

Németh K S85

Nemutlu E S88

Neoh CA S21

Neris Rômulo SL S19

Neto Pedro M S184

Neunlist M S159, S160

Ngo Bum E S37

Nguyen Dieu L S34

Nguyen T S50

Nicolaas Eloff J S126

Nicolai M S43, S185

Nieber K S128, S159, S173, S192

Niedermeyer Timo HJ S118

Niedermeyer T S50

Nik Azmin Nik F S11, S68, S70

Nikles S $\$ 109$

Nikolaeva OV S150

Nishio A S134

Nita RA S165

Nnadi CO S23, S36

Nnadozie Jane I S179

Noda K S130

Nogueira Sedyama Maria A S162

Nohynek L S185

Nöldner M S134

Nor Izan N S70

Norizan A S86, S107

Noro I $\$ 134$

Noundou X S156

Novak B S93

Nunes EO S154

Nunes FM S86, S88, S153

Nurficahyanti R S56

Nurrochmad A S147

Nuzillard JM S110, S111

Nwodo NJ S36

Nyahangare E S2

O

Obeng-Mensah D S176, S177

Oberhänsli T S59

Oberle M S87, S170

Obi C $\mathrm{S} 112$

Oceová V S104

Oche JR S175

Ogbole OO S125
Ogunrinade Folashade A S156

Oh YC S153

Ojerinde S S175

Ojha S S42, S148

Okechukwu E S144

Okello E S150

Okoye Festus BC S116

Okoye F $\mathrm{S} 135$

Okpuzor 」 S115, S179

Oksman-Caldentey KM S185

Okubena O S144

Olajide Omolola C S179

Olajide O S129, S156

Olariu L S165

Oliva Bessie E S161

Oliveira Da Silva J $\$ 49$

Oliveira Fidalgo A S155

Oliveira Sousa Ilza M S126

Oliveira RB S73

Oliver K S60

Olivier G S129

Omisore N S146

Omokhua A S142

Onoagbe I S155

Onur MA S68, S79

Onyegbule Felix A S19

Onyegbule FA $\quad \mathrm{S} 112$

Opatz T S26

Oppong Bekoe E S146

Oppong A S142

Oppong-Danquah E $\mathbf{S 1 1 0}$

Orazbekov Y S35

Orčić D 57

Osadebe P S156, S157

Osorio Edison H $\quad \mathrm{S} 126$

Osorio E S126

Ossman T $\mathrm{S} 17$

Ostenson CG $\mathrm{S} 132$

Østnes Hanssen K S11

Oszlánczi G S140

Ota T $\$ 179$

Ötvös Sándor B S83

Ouazzani 」 S110

Oudkerk S S166

Ouiyangkul P S121

Oves-Costales D S57, S186

Özçınar Ö S123

ÖZCINAR ÖZGE S117

Ozenver N S25, S26, S56, S147

Özgür TA $\$ 117$

Özsoy N S30

P

Pacchetti B S143, S184, S186, S188

Padilla-Gonzalez G S13

Padilla-Gonzalez GF S12

Paget V S11

Pagitz K S48

Palea S S133

Palermo J 5177

Pälijärvi M S67

Panfoli I S25

Panichayupakaranant P S41, S137

Pantano D S124

Panthong A S52

Papada E S158

Paráková L S122

Parat MO S140

Park Hyo J S153

Park Hyun J S99

Park Shin Y S112

Park Sin Y S112, S113

Park HS S39

Park IW S39

Park J S10

Park JY S181

Parklak W S111

Parrot D $\quad \mathrm{S} 110$ 
Parsons P S26

Pasalar M S174

Pasco D $\mathrm{S} 98$

Passolt L S116

Pastre Julio C S32

Patel R S47

Patil A S140

Patil C S148

Patiño L S177

Paulo S S128

Pawłowska K S41, S78

Peace D S11

Pecio $\longleftarrow$ S58

Pedrelli F S25

Pedro Costa Elias J S36

Pedrussio S S119

Peeters L S68

Peláez H S148

Pellegrini-Giampietro D $\quad \mathrm{S} 124$

Pellisier L S88

Pellissier L $\quad \mathbf{S} 80$

Peńa García J S27

Peng CY S20

Pereki H $\mathrm{S} 172$

Perez Garrido A S27

Perez Sabino Juan F S161

Pérez Sánchez H S27

Perron K S49

Pertuit D $\mathrm{S78}$

Pertz O S52

Peruzzi L S24

Peter S S69, S89, S120, S170, S171

Petersen F S37, S188

Petersen LE S83

Petit C S37, S78

Petrakis E S89

Petrova M S116

Petruccioli E S43

Pettersen Hultén E $\$ 92$

Peyrat LA S57

Pferschy-Wenzig EM S130, S131, S187

Phatchana R S45

Piazza S S141, S143, S184, S186

Piazzini V S91, S124, S125, S190

Pieters L S59, S60, S61, S68, S187

Pigat N S14

Pilakowski V S170

Pilipovic S S167, S172

Ping-Chung $K$ S33, S67

Pini G $\mathrm{S} 134$

Pinke G S38

Pinto N S55, S147

Pinto-Ribeiro F $\mathbf{S 1 0 0}$

Pisarčíková J S104

Pisimisi I S89

Piwowarski J S20, S127

Piwowarski JP S8, S182

Plachá I S104

Plavcova Z S189

Plumb R S110

Poltronieri Y S162

Polychronopoulos P $\mathbf{S 1 7 8}$

Pompermaier L S23

Pompimon W S19

Popp J S89

Pornprasop V S114

Pothitirat W S56

Potterat O S58, S59, S89, S128

Poutaraud A $\mathrm{S} 92$

Pozharitskaya O S66, S175

Prawan A S44

Prieto lose M S106

Proksch P S19, S107, S118

Prus|'A S41

Pudhom K S7

Pugh ND $\mathrm{S98}$

Punniamurthy N S96, S104

Purna Sari S S151
Püssa T S136

Putalun W S164

Puthong S S36

Puttarak P S132

Puupponen-Pimiä R S185

Pyeon $\mathrm{HI} \quad \mathrm{S} 153$

Pyne S S7

Pyo S S52

$$
\text { Q }
$$

Qin Z S168

Quartey A S142

Queiroz Nubia de A S126

Queiroz Nubia de C S158

Queiroz E S49

Queiroz EF S37, S38

Quintero J S121

Quintino da Rocha C S88$$
\mathrm{R}
$$

Rabenau M S14

Räber N S58

Rabini S S159

Rahimi R $\quad 5181$

Rainer B S39

Rajnochova Svobodova A S17

Ramadwa T S108, S143

Ramakrishnan N $\mathrm{S} 104$

Rambeck W S94, S103

Ramirez-Pineda Jose R S30

Ramseyer J S59, S89

Randazzo G S88

Rasini E S188

Rasol Nurulfazlina E S20

Rat C S102

Ratanavalachai T $\mathbf{S 1 1 3}$

Ratsbone Y S91

Rausch H S179

Rauwald Hans W S179

Rédei D S46

Reich E S190, S191

Reichel C S29

Reinhardt JK S31, S91

Reis C S43, S185

Remias D S39

Renault JH S90, S110, S111

Restrepo A S54

Restrepo AM S121

Revoltella S S48

Reynaud R S111

Rhee JH S114

Rho NY S114

Ribeiro da Silva Antonio 」 S161

Riepl H S87, S124

Righi Davide R $\quad$ S101

Righi D S38

Rijo P S43, S185

Rinner B S21, S28

Rios JL S157

Ripoche I S139

Risaliti L S124

Rischer H S185

Rithichai P S17, S116

Rivera Mondragón A S59

Rivera-Mondragón A S60

Ro NY S114

Robledo SM S31, S54, S121

Rodrigues Luís M S185

Rodrigues Maria 」 S43

Rodrigues Rodney Alexandre F S32

Rodríguez $\mathrm{V}$ S95

Rodríguez-González Á S117, S148, S166

Rodziewicz P S119

Rollinger JM S8, S63

Romagnoli A $\mathrm{S43}$

Romano G S38

Roque N S37

Rosa W S36
Roßmann A S130, S131, S187

Rosmawati Abdul A S86

Ross S S35

Rossberg F S52

Rotrekl D S122, S189

Rouger C S83

Roussel P S95

Roy M S49

Ruangnoo S $\$ 136$

Rudaz S S88

Ruffoni B S25

Ruiz F $\quad$ S122

Ruktanonchai U S123

Ryhner T S97

Ryu Ga H S145, S146

Ryu Seung M S85

Sabiu S S126

Sacco C $\mathbf{S 1 3 4}$

Saeed M S25, S26

Saengkrit N S123

Saha S S84

Şahin H S30

Sai Laxmi Rangabhatlaa A S121

Said Baharudin B 5138

Sakavitsi ME $\mathrm{S90}$

Sakong B 596

Sakunphueak A S169

Salah El-Din R S72

Salama O S119

Salamunova P S189

Šalamúnová P $\quad$ S122

Salbert T S11, S77

Salisu T S115

Salminen JP S12, S67, S76, S84

Salon I S189

Salo I $\$ 122$

Saltan Fatma Z S53

Sama S $\mathbf{S 1 8 8}$

Sandasi M S171, S191

Sang Young S S113

Sangiovanni E S141, S143, S184, S186

Santagostini L S141

Santana Caldas I S36

Santomauro F $\mathbf{S 1 3 4}$

Santos Almeida Bastos Juliana C S138

Santos A S41

Santos B S57

Santos JA S86

Santos R S22

Santos S S128

Santosa D $\mathrm{S} 147$

Sapcanin A S167, S172

Saputri Fadlina C S151

Saraux N S80

Sarı A $\$ 30$

Sarikaki G $\mathbf{S 9 0}$

Sariyar G S155

Sarker Satyajit D S125, S156

Sarkodie J S146

Sarmidi Mohamad R S106

Sartorelli P S41, S50

Sátori G $\$ 46$

Savchenko A S26

Sawangjit R S132

Scarminio IS $\$ 154$

Schäfer 」 S86, S88

Schäfer KH S156

Schärer H] S59

Scharf B S125, S131, S142

Schatzmayr G $\$ 93$

Scheideler M S21

Schenk A S64, S69, S161

Schenk R S162

Scheuba J $\mathrm{S63}$

Schiller K S76

Schinella G S157 
Schirmeister T S50

Schito Anna M S25

Schmid K S3

Schmidt Thomas 」 S10, S36

Schmidt N S58

Schmidt T S61, S71

Schmidt T] S8, S25

Schmitt E S37

Schmitz FJ S51, S52

Schnee S S101

Schneider H S62

Schneider P S118

Schnelle M S128

Schoop R S15, S144, S181

Schötz K S62

Schreiber D S156

Schulte M S170

Schulz H S169

Schumbrutzki C S119

Schuster D S106

Schwaiger S S23, S39, S51, S63, S78

Schwind S S66

Scio E S55, S147, S152

Sciubba F S64

Scotti F S191

Segun Peter A S125

Sehyun L S35

Semir J $\mathrm{S} 12$

Sendker J S72, S125, S131, S142

Senggunprai L S44

Seo Kyung H S32, S137, S181

Seo Young H S85

Seo CS S137, S142

Seok JI S153

Seong Bong J $\$ 112$

Seong BJ S19

Serafini M S64

Setthacheewakul S S122

Seupel R S116

Severi J S151

Sewani-Rusike C S131

Sezer Senol F S27

Sgamma T S191

Shaari K S132, S167

Shah Muhammad A $\$ 137$

Shaheen F S167

Shaikh Mohd F S132

Sharifah Aminah Syed M S58, S85, S86, S107, S168

Shaw Paul N S140

Sheu JH S27

Shih SP S18

Shikov A S66, S175

Shin Han J S42

Shin Young $P \quad S 113$

Shin HK S137

Shiow-Chyn H S33

Shoyama Y $\mathrm{S} 179$

Sibaev A S135

Šibul F S7

Siddiquee Abrar AM S138

Siewert B S66, S146, S161

Signer J S181

Silva e Souza C S134, S162

Silva Filho A $\mathrm{S178}$

Silva Souza C S138

Silva AM S86, S88, S153, S154

Silva J S55, S147, S152

Silva-Filho A $\mathbf{S 1 0 0}$

Silvana Alves M S178

Simões-Wüst AP $\quad$ S128

Simões-Wüst APS $\$ 99$

Simon A S82

Sireeratawong S S52, S173

Siti Norazura J S58

Sivakumar N S10

Sixt M S170

Skaltsounis Leandros A S90
Skaltsounis AL S77, S89, S110, S169

Skaltsounis L S90, S158

Slacanin I S37

Slater A S191

Ślusarczyk S S27, S58

Šmejkal K S106

Smiesko M S91

Smolander M S185

So Hee S S113

So Ra C S113

Soares M S41

Soares MG $\mathrm{S73}$

Sohn MJ S149

Sohretoglu D S82

Somensi A S91

Somnuk S S111

Sompara N S111

Sompongse W S114

Song Young E S112, S113

Song J 5153

Song K S75

Soonthornchareonnon N S52, S173

Sousa Ilza M S158

Sousa F $\quad$ S50

Sousa IP $\quad$ 101

Sousa O 557

Souza Vanessa HS S126

Sowa I S27

Spengler G S46

Spezzano R S186

Spiegler V S102, S180, S182

Spielberger U S94

Spiteller M S79

Springer A S162

Srashar S S13

Sribangrak S S169

Sriboonthai C S17

Sribuhom T S45

Srivedavyasasri R S35

Stadler R S119

Staerk D S81, S82, S109

Stallinger A S28

Stammer T S106

Stancheva I S116

Stange R S104

Stanisławska I S8, S20

Stark A S29

Stark T S107

Stathopoulos P S169

Staub P S182

Steinborn C $\mathbf{S 1 9 3}$

Stelzer J $\mathrm{S} 93$

Stepanek F S189

Štěpánek F $S 122$

Steyer D $\mathrm{S92}$

Stien D S49

Stierhof M S11

Stintzing F S61, S64, S76

Stochmal A S27, S58

Stork B S107

Stork C S13

Storr M S135

Storr MA S159

Strasser M S18

Strawa 」 $\mathrm{S} 77$

Strube J S170

Stucki K S3

Stuppner H S23, S39, S48, S51, S63, S78, S84 S89, S127

Su C] $\mathrm{S} 28$

Su JH S17, S18, S21

Suárez Villanueva V S117, S148, S166

Suárez-Villanueva V S148

subramanya S S148

Suh YG S184

Suhaidi A $\$ 107$

Suin C S35

Suktham K S123, S189
Sulistyawati R S147

Sümer Tüzün B S123

Summers B $\mathbf{S 1 8 5}$

Sumthong Nakmee P S78

Sunassee SN S77

Sung Sang H S10, S83

Sung IS S114

Sung PJ S18, S33, S64

Sungkharak S S121, S123

Surassmo S S123, S189

Susandarini R S147

Suter A S127, S144

Suthiwong J S44

Suvanto 」 S76

Świerczewska A S33

Syafni N S52

Syah Yana M S70

Syed Aris Sharipah R S72

Szabó A S140

T

Tabaza Y S10

Tabefam M 589

Tabudravu JN S11

Taghouti M S86, S88, S153, S154

Taglialatela-Scafati O S141

Taha M S69

Tahirovic I S172

Tähtinen P S12

Tai-Long P S33

Takeo E S36

Tamaru N S57

Tamm L S59

Tan Yuen $\mathrm{P}$ S26

Tan E $\mathrm{S} 157$

Tan N S157

Tanaka C S78

Tanaka H S164

Tankeu S S191

Tantishaiyakul V S121

Taracena E S161

Tarkany Basting R S126, S179

Tasdemir D S83, S110

Tawab M S160

Tcacenco C $\mathbf{S 5 0}$

Tchoumtchoua J S110

Teichmann K S93, S96

Teixeira I $\mathbf{S 1 5 3}$

Temml V $\$ 106$

Tempone A $\mathbf{S 5 0}$

Tempone AG S37

Teng WT S27

Thaeomor A S128

Théophile J S184

Thibane Vuyisile S S53

Thijs C S99

Thitiorul S S113

Thongsri $Y \quad S 44$

Thuerig B $\$ 59$

Tian-Shung W S33, S67

Tímár Z S140

Timothy F $\mathrm{S} 175$

Tinti S S158

Tipgomut C S36

Tiszlavicz L S140

Tittikpina NK S172

Tober C S15

Todorova M S120

Toff S S64, S161

Toktas U S152

Tomasco V S152

Tomczyk M S77

Tong Ming H S11

Toorabally NR S77

Topcu G S80

Tormo José R S43

Tosevska A S9

Tóth B S38 
Toth G S82

Tóth G \$46

Toure S S80

Touvleliou K S90

Trendafilova A S120

Trockenbacher A S39

Tropper M S117

Troppmair 」 S51

Trougakos I S43, S90

Trougakos IP S57, S186

Trouillas P S17

Tsafantakis N S43, S57, S186

Tsai JY S22

Tsai SH S22

Tsai YC S31

Tsarbopoulos A $\mathrm{S77}$

Tsu WC S85

Tu P S34

Tuccinardi T S24

Tuenter E S61

Tumbas Šaponjac V S129

Tung Nguyen H S179

Tuntiwechapikul W S19

Tuysuz M S155

Tyler HL S98

Tzean SS S73

U

Uddin M S180

Udomsin O $\mathrm{S} 164$

Uehara Daniella E S155

Uhlenbrock L S170

Ujam Nonye T S116

Ujam N S135

Ul-Haq Z S137

Ulkar D S68

Ulrichova J S17

Umeokoli Blessing O S19, S112, S116

Umeokoli B S135

Unger M S14, S146, S192

Ungphaiboon S S121, S123

Unver Somer N S79

Unver SN $\quad$ S68

Upegui YA S31

Upton R S190

Urgast Dagmar S S11

Urmann C S87, S107, S124

Uzor P S156, S157

Uzor PF S23

Uzun K $\mathrm{S79}$

Uzun M S54, S55, S56, S141, S164

Uzunovic A S167, S172

$$
\text { V }
$$

Vágvölgyi M S46

Vahekeni N S184

Valente Ligia MM S19

Välimaa H S136

Valizadeh H S24

van Asseldonk T S4

Van der Auwera A S68

van Dongen M S99

van Eeuwijk P S184

van Hierden Y S93

Van Minh N S149

Van Staden J S53, S142

van Vuuren S S9, S171

van Wyk C S108

Vander Heyden Y S60

Vanti G S124, S190

Ványolós A S34

Vasas A $\$ 38$

Vélez ID S31, S54, S121

Venditti A S64

Veneziani RCS S101

Vermaak I S185, S191

Verpoorte R S6

Verschaeve L S187
Veselý O S106

Vicentini A S151

Vieira Braga T S134, S138

Vieira Paulo C S81

Vijay Anand J S96

Vila R S166

Viljoen A S9, S84, S171, S185, S191

Villagarcía H $\quad$ S157

Villagra E S177

Vimalanathan S S144

Vissiennon C S128, S173

Vissiennon Z S173

Vochyánová Z S122

Vogel S S62

Vogl CR S3

Voinesco Francine $\mathrm{V} \quad \mathrm{S} 101$

Volkov I S7

Vollmer P S125

Vollstedt S S5

Vostalova J S17

Vuorela H S66, S136

W

Wadie W S160

Wagner $\mathrm{KH}$ S9

Wahyuono S S147

Walkenhorst M S1, S3, S97

Wallner M S9

Waltenberger B S23, S48, S84, S127

Wan Mohd Fathil Wan Y S85

Wan Mohd Zain Wan Z S72

Wang CS S48

Wang D $S 9$

Wang $\mathrm{H} \quad \mathrm{S} 19$

Wang $\mathrm{HC} \quad \mathrm{S} 24$

Wang L S168

Wang LC S31

Wang M S110

Wang Q S168

Wang R S57, S151

Wang SW S28

Wang SY S24

Wang Y S73, S163, S176

Wang YH S110

Wangensteen $\mathrm{H} \quad \mathrm{S} 32$

Warzecha $\mathrm{H} \quad \mathrm{S} 7$

Wattanalai R S56

Wei Z S168

Weise C S18

Wen ZH S33

Weng A S18, S188

Weng IT S57

Weng JR S39

Wesselborg S S107

Whuang TY S64

Wiethoff K S135

Wilczek M S78

Wille R $\mathrm{S} 193$

Williams C $\mathbf{S 2 6}$

Wiwattanapatapee R S121, S122

Wlodarczyk M S27

Wohlmuth H S186

Woith E S61

Wojciak-Kosior M S27

Wolber G S51

Wolfender JL S37, S38, S49, S78, S80, S88, S101,

S106, S109

Wolff T S19

Wolfram E S6, S69, S89, S119, S120, S157, S170,

S171, S184

Won DY S165

Wongnoppavich A S52

Wongprommoon A S36

Woo S 583

Wrona M S109

Wronski VK S63

Wu CC S22

Wu HC S48, S87
Wu X S22

Wu YC S18, \$20, \$28, S30, S31, S174

Wu YJ S21, S64

Wüthrich 」 S64

Wutikhun T S189

Xie W S29

Xie Y S73, S163

Y

Yaakob H $\mathrm{S106}$

Yakubu O S143, S144

Yalçın F S49, S88, S154

Yan G S151

Yang Sung 」 S100

Yang Woo S S145, S146

Yang DY S30

Yang JC S20, S174

Yang SS S47

Yang YL S73

Yao X $\mathrm{S} 168$

Yao Z S168

Yen $\mathrm{CH} \quad \mathrm{S} 87$

Yenjai C S44, S45

Yepes LM S54

Yesilada E S74

Yi 553

Yokosuka A S21, S22

Yoon YJ S163

Yoshioka Y $\$ 134$

Yostawonkul J S123

You WJ S17

Young Eun S S113

Young L S10

Yoysungnoen B S111

Yu ML S31

Yu Y S11

Yuk J S109, S110

Yun-Sheng L S69

Yusufoglu Hasan S S117

Yuzbasioglu M S82, S83

Z

Zahner C S6

Zakaria Nik Nur A S150

Zámbori-Németh É S140

Zambrana S S132

Zanini LBL S73

Zarev Y S187

Zarshenas Mohammad M S174

Zayova E S116

Zelioli Ícaro Augusto M S126

Zeng K S34

Zeraik ML S154

Zhang C S34

Zhang R S151

Zhang $X \quad S 115$

Zhang YJ S144

Zhao Ting T S99

Zhao Y S109, S132, S161

Zhou H S132, S161

Zhussupova A $\$ 167$

Zhussupova Al S150

Zhussupova G S167

Zhussupova GE S150

Ziak M S5

Ziegler J S75

Ziegler LN S69

Zielinska S S27

Zitterl-Eglseer K S97

Zomborszki Zoltán P S34, S45

Zoofishan Z S45

Zuchtriegel G S29

Zuo W S151

Zupkó I S34, S38, S46 